(n) (1)

(1)

(1)

(1)

- 


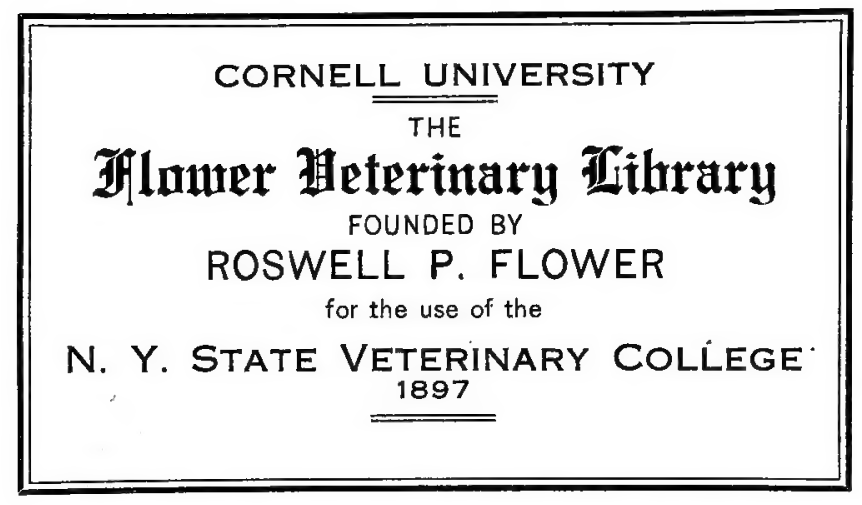




\section{RC 251 Cornell University Library}

\section{9}

Diseases of metabolism and of the blood,

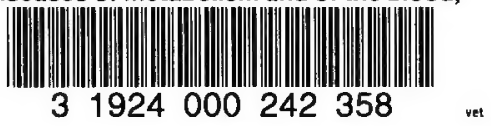




\section{Cornell University Library}

The original of this book is in the Cornell University Library.

There are no known copyright restrictions in the United States on the use of the text. 


\title{
DISEASES OF METABOLISM AND OF THE BLOOD
} ANIMAL PARASITES TOXICOLOGY

EDITED BY

\author{
RICHARD C. CABO'T, M.D.
}

INGTRUCTOR IN CLINICAL MEDICINE IN THE MEDICAL SCHOOL OF GARVARD UNIVERSITY

AN AUTHORIZED TRANSI ATION FROM "DIE DEUTSCHE KLINIK" UNDER THE GENERAL EDITORIAL SUPERVISION OF JUIIUS L. SALINGER, M.D.

WITH ONE COLORED PLATE AND FIFTY-EIGHT ILLUSTRATIONS IN THE TEXT

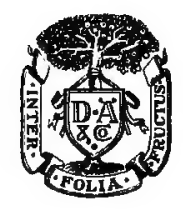

NEW YORK AND LONDON

D. A P P L T T N A N C CM PAN Y 


$$
\text { No.17459 }
$$

COPYRIGHT, 1906, 1909, BY

D. APPLETON AND COMPANY

$$
\begin{aligned}
& R C \\
& 251 \\
& C 11 \\
& 1909
\end{aligned}
$$

PRINTFD AT THE APPLETON PREgS

NEW YORK, U. S. A. 


\section{INTRODUCTION}

Altrodgr the statement of Prof. Hoppe-Seyler that "The process of life of the organism is, in the main, a complete mystery" is still true, much has been accomplished by the researches of modern physiologists and clinicians who have endeavored to find the key to this enigma. Perhaps in no realm of medicine has labor fructified so richly as in the recent study of metabolism. This has been of value not only in diagnosis but, even more so, from the standpoint of treatment. It may be confidently stated that the treatment of some diseases of metabolism, such as diabetes mellitus, gout, and obesity, now rests on a scientific basis. This advance is almost exclusively due to the labors of the modern German school of physiologists and internal clinicians.

What is true of the pathology of metabolism may also be asserted of diseases of the blood. The study of maladies whose etiology and pathology were formerly obscure has now been simplified, their relations classified, and the whole arranged in exact scientific order. The processes of blood formation and blood degeneration, while not yet forming an open book, have in part been clearly portrayed. That therapy has not kept pace with this advance affords an opportunity for the physician of the twentieth century, but much has already been accomplished, and many problems have been solved. For this progress also the medical world is indebted to modern research.

The object of this volume is to present a picture of diseases which were formerly designated as of "obscure causation," and to outline their treatment. Each article is the work of a master in his special field of labor.

In the translation an endeavor has been made to adhere as closely as possible to the individual style of each contributor without the subserviency of clearness in the text. 
I am indebted for much valuable service in the preparation of this volume to my secretary, Miss M. A. Clarke.

In anticipation of the fact that many points in regard to these affections will be searched for by the reader, and to facilitate this, an endeavor has been made to add a comprehensive and full general index.

Julius L. Salinger.

1729 North 42nd Street, Philadelphia. 


\section{EDITOR'S PREFACE}

WE have in English no discussion of the Constitutional Diseases which treats with any fulness the problems arising when we consider the reasons for what occurs in these diseases.

The rationale of the symptomatology, the rationale of treatment (i.e., in diabetes) is here admirably set forth by clinicians each of whom speaks with authority.

The writers have wisely confined themselves for the most part to thorough discussions of certain points in each of the diseases considered-making no fatuous attempt to compress a huge subject into narrow limits. They have brought their subjects up to date and given very frankly their personal views on the points at issue.

For the manner and for the matter of their work, therefore, we are indebted to our Teutonic brethren, and we are glad that the book will now find a large circle of readers.

Richard C. Сabot.

190 Marlborough Street, Boston, Mass. 



\section{LIST OF CONTRIBUTIONS}

The Quantitative Analysis of Disturbances of Metabolism in the Clinic. By W. WeIntraud, Wiesbaden.

Over-nutrition and Under-nutrition. By C. v. Noorden, Frankfort-on-Main.

Diabetes Mellitus. By B. NaUnyn, Strassburg.

Diabetes Insipidus. By D. GERHardT, Strassburg.

Gout. By W. Ebstein, Göttingen.

Obesity. By W. Ebstein, Göttingen.

Myxedema with special Reference to Organotherapy. By C. A. EwaLd, Berlin.

Addison's Disease. By L. Reiss, Berlin.

Acromegalia. By C. Benda, Berlin.

Chronic Articular Rheumatism. By W. His, Basel.

Pentosuria. By F. Blumenteral, Berlin.

Blood and Blood Examination. By A. Lazarus, Charlottenburg (Berlin).

The Anemias. By P. EhrLich, Frankfort-on-Main, and A. Lazarus, Charlottenburg (Berlin).

Chlorosis. By E. Grawitz, Charlottenburg (Berlin).

Leukemia. By W. von Leube, Würzburg.

Pseudo-leukemia (Hodgkin's Disease and Banti's Disease). By H. Senator, Berlin.

The Hemorrhagic Diatheses. By M. LiTten, Berlin.

The Animal Parasites of Man. By E. PeIPER, Greifswald.

Important Poisons and Their Treatment. By R. v. JAKSCH, Prague. 



\section{CONTENTS}

The Quantitative Analysis of Disturbances of Metabolism in the Clinic page

Introduction . . . . . 1

Consumption of Food in the Healthy . . 4 . 4

A. The Laws of Nutrition $\quad$. 4

Energy Metabolism per Square Meter of Body Surface 5

B. The Methods for Estimating the Calory Requirement of the Body 6

C. The Energy Requirement of the Healthy . . $\quad 9$

D. The Albumin Minimum . . . . . 13

E. The Requirements for Albumin Deposition . . . . $\quad 15$

The Food Requirement of the Sick . . . . . . . 21

The Degree of Oxidation . . . . . . 22

The Maintenance Diet and the Proteid Requirement of the Sick . . . 37

Disturbances in the Absorption of Food . . . . . 43

Concluding Remarks . . . . . . . . . . . $\quad$. 49

Over-Nutrition and Under-Nutrition . . . . . . . . 54

1. Conception of the "Condition of Nutrition" . . . . . 54

2. Increase of Flesh and Increase of Fat . . . . 55

3. Occurrence and Consequences of Under-Nutrition . . . . . . $\quad 59$

4. Occurrence and Consequences of Over-Nutrition . . . . . 61

5. Indications for Hypernutrition and Hyponutrition . . . . 62

A. Over-Nutrition . . . . . . . 63

B. Under-Nutrition . . . . . 67

6. Remarks Regarding the Technic of Over-Nutrition and Under-Nutrition 69

A. The Technic of Forced Feeding . 70

B. The Technic of Antifat Cures . . . . 72

Diabetes Mellitus

I. Glycosuria and Diabetes . . $\quad$. 77

II. The Various Forms of Diabetes; Diabetic Predispósition . . . 80

III. Mild and Severe Forms of Diabetes; Course, Symptomatology, and Complications . . . . . . . 83

IV. The Theory of the Treatment of Diabetes . . . . . . 87

V. Practical Therapy . $\quad$. . . . . . . . . . . 92

VI. Tables . . . . . . . . . . . . . 103

Short Popular Dietetic Rules for Diabetics . . . $\quad$. $\cdot$ ix 108 
DiABeTES INSIPIDUS

Symptoms and Clinical Course . . . . . . . . . . . 112

Diagnosis . . . . . . . . . . . . . . . . 121

Treatment . . . . . . . . . . . . 122 GovT

The Pathogenesis of Gout . . . . . . . . . . . . . . 126

Symptomatology . . . . . . . . . . . . . 132

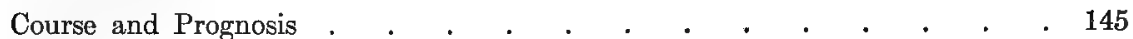

Diagnosis . . . . . . . . . . . . . . . . 146

Treatment . . . . . . . . . . . . . 146

OBEŚITY . . . . . . . . . . . . . . . . . . . 151

Etiology . . . . . . . . . . . . . . . 133

Symptomatology . . . . . . . . . . . . . 155

Diagnosis . $\quad . \quad$. $\quad . \quad . \quad . \quad . \quad . \quad . \quad 159$

Treatment . . . . . . . . . . 160

MyXedema with Spectal Reference to Organotherapy . . . . . . 179

History $\quad . \quad$. . . . . . . . . . . . 179

Etiology . . . . . . . . . . . . . . . . 180

Symptoms . . . . . . . . . . . . . . . . 180

Pathology . . . . . . . . . . . . . . . . . . . 186

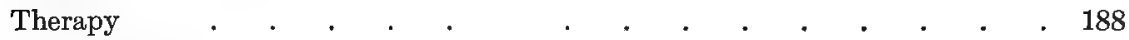

Addison's Disease $\quad . \quad$. . . . . . . . . . 199

Clinical Picture of Addison's Disease . . . . . . . . . 200

Grosser Pathologico-Anatomical Lesions in Addison's Disease . . . . 202

Anatomy and Physiology of the Adrenals . . . . . . 204

Pathologico-Anatomical Changes of the Nervous System in Addison's Disease 215

Diagnosis . . . . . . . . . . . . 222

Therapy . . . . . . . . . . . . . 223

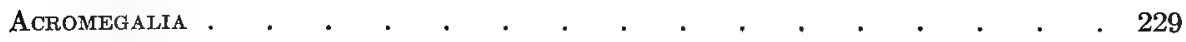

Etiology . . . . . . . . . . . . . 230

Symptoms . . . . . . . . . . . . . . . 231

Pathology . . . . . . . . . . . . . . . 234

Pathological Anatomy . . . . . . . . . . . . 237

Treatment . . . . . . . . . . . 242

Chronic Articular Rheumatism . . . . . . . . . . . . 243

Pathology . . . . . . . . . . . . 248

Clinical Symptoms . . . . . . . . . . . . . . 250

Treatment . . . . . . . . . . . . . 257

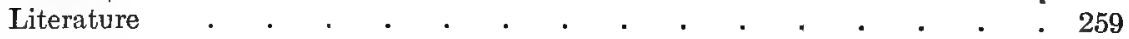

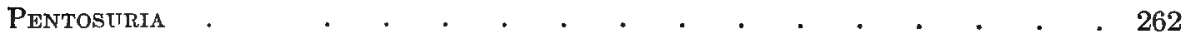

Blood and Blond Fxamination . . . . . . . . . . . . . 275

I. Hemoglobin . . . . . . . . . . . . . . 277

II. Counting the Blood-Corpuscles . . . . . . . . . 281 
III. Counting the Leukocytes

IV. Specific Gravity .

V. Alkalinity

VI. Volume of Red Cells

VII. Spectroscopic Examination

VIII. Agglutinative Reactions

IX. Bacteriological Examination

The Anemias .

Simple Anemia

Progressive Pernicious Anemia

The Course of the Disease

Therapy

\section{Chlorosis}

Symptoms .

The Blood

Pathological Anatomy

Frequency of Chlorosis with Regard to Sex, Age and Locality

Predisposing Influences

The Genesis of Chlorosis

Diagnosis .

Course of the Disease

Prognosis

Treatment

Prophylaxis

Leukemia

Composition of the Blood

Symptoms and Blood-Changes . . . . . . . . . . 348

Diagnosis .

Leukocytosis

Prognosis .

Treatment

Pseudo-Leukemia (Hodgkin's Disease and Banti's Disease) . . . . 370

Hodgkin's Disease . . . . . . . . . . . . . 371

Diagnosis . . . . . . . . . . . . . . . 375

Etiology . . . . . . . . . • . . . . . . 377

Course, Duration and Result . . . . . . . . . . . 378

Therapy . . . . . . . . . . . . . . . 378

Banti's Disease . . . . . . . . . . . . . 381

The Hemorrhagic Dratheses . . . . . . . . . . . 388

Scurvy . . . . . . . . • . . . . . . . 388

History

Etiology

Pathological Anatomy . . . . . . . . . . . . 399 


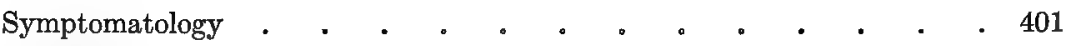

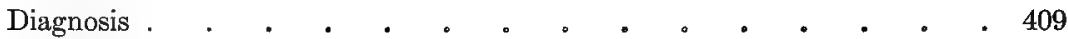

Prognosis . . . . . . . . . . . . . . 410

Prophylaxis and Treatment. . . . . . . . . . . 410

Hemophilia (Bleeder's Disease) . . . . . . . . . . 413

Pathological Anatomy . . . . . . . . . . . 430

Symptomatology and Course . . . . . . . . . . 434

Local Hemorrhages upon a Hemophilic Basis . . . . . . 442

Prognosis . . . . . . . . . . . . 451

Treatment . . . . . . . . . . 452

Morbus Maculosus Werlhofii (Purpura)

(Purpura Simplex, Hemorrhagica, Rheumatica seu Peliosis, Rheumatica Schoenleinii) . . . . . . . 457

Definition . . . . . . . . . 457

Etiology . . . . . . . . . . . . . 458

General Clinical Picture . . . . . . . . . . . . 469

Pathological Anatomy $\quad . \quad$. . . . . . . . . 487

Special Symptomatology . . . . . . . . . . 490

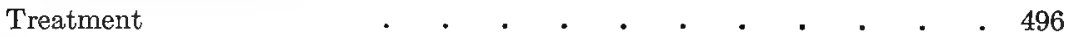

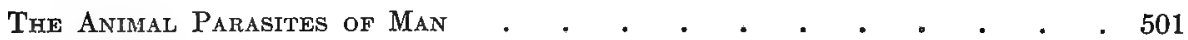

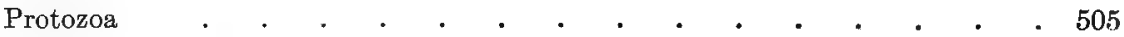

Trematodes $. \quad . \quad . \quad . \quad . \quad . \quad . \quad . \quad . \quad . \quad . \quad . \quad . \quad .508$

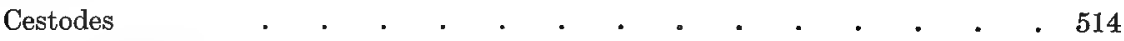

Prognosis . . . . . . . . . . . . . . . . . 524

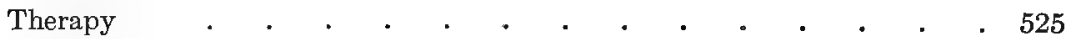

Echinococcus Disease . . . . . . . . . . . . . . 526

Symptoms . . . . . . . . . . . . . . . . 532

Treatment . . . . . . . . . . . . . . . . 533

Echinococci of Various Organs . . . . . . . . . . 534

Nematoda, Thread-Worms . . . . . . . . . . 544

Ankylostomiasis-Uncinariasis . . . . . . . . . . . 560

Arthropoda . . . . . . . . . . . . . . . 565

Fly Larvæ (Maggots) . . . . . . . . . . . 573

Important Poisons and Their Treatment . . . . . . . . . . 579 


\section{LIST OF ILLUSTRATIONS}

FIG.

1. Incipient Myxedema

2. Advanced Myxedema

3. Myxedema in the stage of recovery

4. Röntgen picture showing Heberden's nodes

5. Metatarso-phalangeal articulation with beginning chronic arthritis

6. Subcutaneous nodules upon dorsal tendon sheaths in chronic exudative polyarthritis .

7. Patellar cartilage after an attack of gout . . . . 253

8. Radiograph of hand of a gouty patient . . . . . . . . . . 254

9. Hemometer. (After v. Fleischl-Miescher) . . . . . 278

10. Hemometer. (After Sahli) . . . . . . . 279

11. Hemometer. (After Sahli) . 279

12-13. Hemophotograph. (After Gaertner) . $\quad 280$

14. Hemocytometer. (After Thoma) . . 282

15. Microscopic picture with blood-corpuscles. (After Landois) . . . 282

16. Absorption spectra of hemoglobin. (After luandois) . . . . . . 288

17. Relapsing Fever spirilli. (After v. Jaksch) . . . . . . 291

18. Use of forceps in blood examination . . . . . . . 294

19. Small copper kettle for staining solutions $\quad$. . . . . 294

20. Ehrlich's eye-piece with iris diaphragm $\quad . \quad$. . . . . . . . . 301

21. Case of Hodgkin's disease . . $\quad 373$

22. Genealogical tree of the bleeder family Mampel. (After Lossen) 416

23. Genealogical tree of a bleeder family. (After H. Gocht) . . . 417

24. Genealogical tree of a family showing Daltonism. (After Horner) . 419

25. Genealogical tree of a hemeralopic family. (After E. Ammann) . 420

26-28. Leydenia Gemmipara. (After von Leyden-Schaudinn) . . 505

29. Trichomonas Vaginalis. (After Künstler) . . . . 507

30. Balantidium Minutum. (After Schaudinn) . . . . . . . 508

31. Nyctotherus Faba. (After Schaudinn) . . . . . . 508

32. Distomum Hepaticum, Linné . . . . . . . $\quad$. 509

33. Distomum Westermanni and ovum. (After Katsurada) . . . 510

34. Distomum Spathulatum and ovum. (After Katsurada) . . 510

35. Ova of Distomum Hæmatobium . . . . . . . . 512

36. Ova of Tenia Solium . . . . . . . . . . . . . . 515 
FIG.

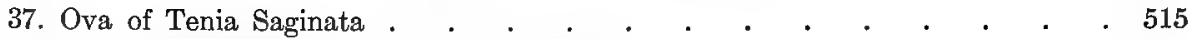

38-42. Tenia Confusa, Ward. (After Guyer) . • . . . . . . 517

43. Tenia Africana. (After v. Linstow) . . . . . . . . . . . 518

44. Tenia Asiatica. (After v. Linstow) . . . . . . . . . . 519

45. Cercocyst from Cyprinotus Incongruens, $1900 \quad$. . . . . . $\quad$. 519

46. Hymenolepis Lanceolata, Bloch . $\quad$. . . . . $\quad$. 520

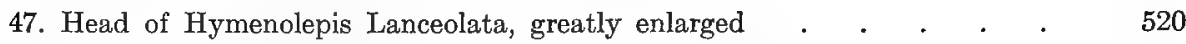

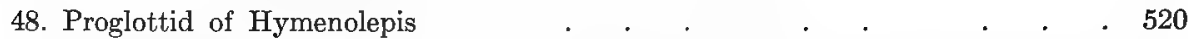

49. Bothriocephalus Grandis. Proglottid and ovum. (After Ijima and Kurimoto) 521

50. Tenia Echinococcus . . . . . . . . . . . . $\quad .527$

51. Head of Tenia Echinococcus . . . . . . . . . . . . 527

52. Expectorated Echinococcus membrane . . . . . . . . . . . 538

53. Filaria Embryos . . . . . . . . . . . . . 547

54. Ankylostoma Duodenale . . . . . . . . . . . . . . 561

55. Ova of Ankylostoma Duodenale . . . . . . . . . . . . . . 561

56. Larva of Ankylostoma Duodenale . . . $\quad$. $\quad . \quad$. $\quad$. 561

57-58. Sarcopsylla Penetrans, Sand-flea . . . . . . . . . . . $\quad$. 573

COLORED PLATE

Blood Cells . . . . . . . . . . . . . . . Facing 302 
DISEASES OF METABOLISM 



\title{
THE QUANTITATIVE ANALYSIS OF DISTURBANCES OF METABOLISM IN THE CLINIC
}

\author{
By W. WEINTRAUD, WIEsBaden
}

CoNTENTS: Introduction.-Normal metabolism: A. The laws of nutrition; $B$. The methods for estimating the calory requirement of man; $C$. The normal energy requirement; $D$. The albumin minimum; $E$. The requirements for storing up albumin.-The food requirement of the sick: The degree of oxidation; maintenance diet and albumin requirement; disturbances of absorption of food.-Concluding remarks.

\section{INTRODUCTION}

DURING the past decade the scientific researches of internal medicine have been made chiefly in the clinico-chemical laboratory.

Prepared and developed by physiology, the pathology of metabolism offered a fruitful field for study, hence it was not surprising that numerous young investigators soon found here their sphere of activity. A flood of researches in metabolism was the result. Clinical literature of the latest epoch bears the lasting impress of these workers. Many interesting questions concerning the laws of metabolism can be regarded as decided, chiefly by the theoretic conclusions based on these clinical studies, but an eminently practical issue has also been attained: Based upon these fundamental laws, a rational therapy. of nutrition has been evolved which affords an extraordinarily fruitful field for professional usefulness.

It is interesting to recount, in extenso, the valuable discoveries which have. come from the clinico-chemical laboratories, and have brilliantly justified. their raison d'être.

The problems which arose for solution, as soon as investigation passed beyond the mere examination of urine for albumin and sugar, werc manifold. Often they led into the realm of physiology, and thus it happened that many obscure points in the physiology of digestion and metabolism were brilliantly illuminated by the labors of clinicians, and experimental investigation in the chinical laboratory has especially enriched our knowledge of the physiology of metabolism.

With energy and thoroughness, therefore, researches into the pathology of metabolism were pursued clinically. The wards of the hospitals offered rich material and abundant opportunity for observing the course of metabolic processes under abnormal conditions, and the desire to study physiological questions at the bedside was as much promoted by stimulus from the physiologic laboratories of Hoppe-Seyler, Ludwig, Voit, Pflüger, Salkowski, and 
Baumann as by the desire to find a key to the comprehension of pathologic processes.

The practical result of these labors may be noted in the extraordinary interest manifested everywhere to-day in modern dietotherapy. For this reason it is worth while to cast a retrospective glance over the long road which has led to this satisfactory result.

The scientific study of metabolism began when Lavoisier, in 1780, designated chemical processes in the animal economy as the source of heat accompanying all processes of life. He taught us that the food products in the body utilized the oxygen breathed in with the inspired air and decomposed, producing carbonic acid; that this process is therefore analogous to the combustion of organic substances outside of the body, and is an oxidation process which is the most important source of animal heat.

A long time elapsed before the discovery of the law of conservation of energy also permitted new conceptions in the realm of biology ( $R$. Meyer). In opposition to the view that, besides combustion, still other independent sources of heat were present in the circulation of the blood (friction, etc.), the opinion became general that all expressions of force which are recognized in the living organism (i.e., production of heat and capacity for work) were susceptible of a uniform explanation. They originate from the energy which is furnished the body by the food. By tireless and long-continued original researches, Rubner proved conclusively that the law of the conservation of energy, the correctness of which R. Meyer and Helmholtz proved in the realm of physics, deserves proper recognition also in biology. The supply of energy contained in the food passes through the animal body without diminution.

Thus the law of the permutation of forces, which had its origin in Lavoisier's discovery of combustion as the source of energy for the organism, was conclusively demonstrated by Rubner.

It was an important epoch in the development of the laws of metabolism when Justus von Liebig demonstrated the identity of the proteids of the animal and vegetable organisms in their chemical properties, and explained the relations of the albumin products of the food to the nitrogenous products of decomposition in the urine. We owe to him the lnowledge that it is albumin, fat, and carbohydrates which are the basis of metabolic processes. He was the first to emphasize the fact of the unequal importance of the products necessary for structural formation in the animal organism. He assumed that the albumin introduced with the food alone reconstructs that which has been destroyed by the activity of the body, and called albumin the plastic (tissueforming) food product, contrasting with it the $\mathrm{N}$-free products (fat and carbohydrates) as respiratory products. These alone, he supposed, were attacked directly by the oxygen in the air, and by their combustion protected the plastic products, the albumin, from oxidation.

But this theory of a division of all food products into two opposite groups could not be maintained, and the theory of Liebig that the oxygen taken up in respiration caused the disintegration of the food products, and that its amount decided the degree of metabolism was also later proven to be unten- 
able. We know to-day that the causal relation between oxygen intake and food consumption as accepted by Liebig does not exist, but that in the living protoplasm of the cell exists the cause of the decomposition going on in the organism.

Food decomposition does not take place in the blood and fluids, as was assumed at that time, but in the tissues, where the decomposition is produced by the chemical activity of the cells. ${ }^{1}$

The type and rate of metabolism are determined neither by the presence nor by the absence of oxygen, but only the energies dormant in the protoplasm of the cells - energies whose multiplicity the investigations of our own times are constantly impressing upon us.

The amount of decomposition which takes place in the cell is decided alone by the energy requirement of the entire organism, so that the total consumption of the body is made up of the metabolism of all the cells. These cells, however, show a wonderful adjustment to the requirements of the organism, not only quantitatively but, on account of their specific properties, qualitatively as well.

It is not the oxygen that by its affinity to the individual constituents of the body dominates the chemical processes that occur there. If oxygen ruled, the readily oxidizable substances, such as uric acid, could not be so uniformly present in unaltered form in the excretions, while substances which are only oxidized with difficulty, such as the fats, are completely burned. It is the specific force contained in the protoplasm of the cells which causes the metabolic processes in the animal economy.

Among these processes the splitting up of complex chemical bodies into simpler bodies, while oxygen is at the same time taken up, plays an important rôle. But besides mere splitting up, and splitting up with absorption of water (hydrolytic dissociation), other processes of reduction and synthesis are largely active, and occasionally all are combined in a single cell.

Hence, as Rubner maintained, life cannot be described under the general idea of a process of combustion; we must rather say that while life exists there is constantly an employment of energy and a transference of the same into other forms (activity and heat).

Physiologic and pathologic chemistry will aid us still further in analyzing the chemical changes which occur during the processes of metabolism. The evolution of the individual protein substances contained in the cell, and the disclosure of their composition by the production of split-products, constitute the one method which has been followed with success in solving the problems here involved. But the protoplasm of the living cell and the proteid which we have produced from it by the aid of our chemical methods are quite different things. So also the products of decomposition which arise in the destruction of the cell protoplasm in the body are quite different from those which we make by splitting up albuminous substance in a test tube. In the one case dead albumin, in the other living albumin is decomposed, and as we appreciate this great difference we seem to drift yet further from the solution of the

1 Hofmeister, "Chemie der lebenden Zelle," Vortrag, 1901. 
problem of life. Yet even here the ingenuity of our scientists has found a way to probe more deeply into the cause of things, and, though still in the first stages, the most recent investigations upon organs kept alive outside the body (saturated with, blood, autolysis) have been followed by important results which permit us to expect more surprising conclusions in the future.

On the other hand, it must be emphasized that although our knowledge of the chemical processes of metabolism is still comparatively primitive, the laws of metabolism have been decidedly advanced by a more physical consideration of the processes. The dynamic conception of the transition of food products, thought out by Rubner, furnished a basis for labors in the clinical pathology of metabolism upon which a superstructure might be raised with ease and certainty, and upon this the imposing structure of modern laws of metabolism in the diseased body was soon erected.

If we take this as our actual theme, we note that in the clinical study of metabolism in the last few decades quantitative investigations take precedence.

The history of this begins with the publication of $\mathrm{E}$. Bischoff's book, "Urea as the Measure of Metabolism" (1853). The method of estimating the amount of urea by titration, first employed by J. v. Liebig, enabled Bischoff and Voit to undertake numerous investigations by which they determined the laws of nutrition in the carnivora. ${ }^{1}$

Chossat had previously, by careful experiments with starving pigeons, demonstrated the daily loss of weight and the amount of the excretions; and Bidder and Schmidt in their book, "The Digestive Fluids and Metabolism" (1852), described minutely the valuable results of their investigations regarding the metabolism of carnivora (cat) under the most varied conditions of nutrition. They were the first to relate in detail, after an investigation in which all requirements were fulfilled, how to find in the urine and the feces the nitrogen ingested in food in the form of meat, and upon this they founded the law of nitrogen equilibrium.

With the aid of the elementary analysis of urine and feces, and the careful estimation of the factors of respiratory metabolism which were made possible by the help of $v$. Pettenkofer, the study of the quantitative analysis of metabolism in Voit's physiologic laboratory in Munich soon attained great technical exactness. With this came the facilities for studying the alterations of decomposition under most varying circumstances, and particularly with food of different nature and quantity, and thus it became possible to determine the laws of the total animal economy.

\section{CONSUMPTION OF FOOD IN THE HEALTHY}

\section{A. THE LAWS OF NUTRITION}

The estimation of the amount of food required by the healthy human organism to maintain its equilibrium has been the subject of many experiments by Voit and his pupils.

1 Bischoff u. Voit, "Die Gesetze der Ernährung des Fleischfressers," 1880. 
The more complete the technic, and the more ingenious the investigation, the more certainly the results show that metabolism is determined by the activities of the body (heat production and force). Then by excluding all external factors which could increase metabolism, it is shown that the "resting-energy" 1 of metabolism is equivalent in its potential value to the amount of heat produced. This has unquestionably been proven by Rubner's calorimetric investigations.

In rest, the dissipation of heat is the only avenue for loss of force. Dissipation of heat, however, is a function of the surface of the body. If it determines the ratio of metabolism in rest, this metabolism must in turn be dependent upon the surface of the body (law of the development of the surface, Rubner).

There can be no doubt that this biologic law also exists in pathology. Where conspicuous differences in the amount of the resting-energy per kilogram of body-weight exists, an attempt should be made to explain it by the variation in the amount of 'surface before we accept the view that a special protoplasm, characterized by a more active or sluggish metabolism, plays a rôle (in nurslings, in obesity, etc.).

\section{TABLE I}

\section{ENERGY METABOLISM PER SQUARE METER OF BODY SURFACE?}

\begin{tabular}{|c|c|c|}
\hline & $\begin{array}{l}\text { Weight in } \\
\text { kilograms. }\end{array}$ & $\begin{array}{l}\text { Calories per } \\
\text { twenty-four } \\
\text { hours in one } \\
\text { square meter }\end{array}$ \\
\hline 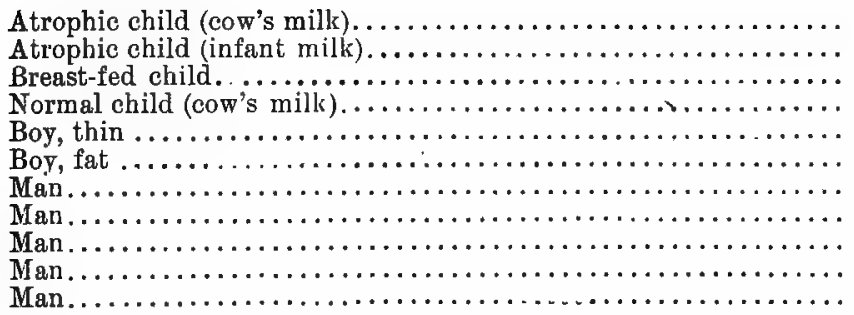 & $\begin{array}{l}3 \\
3 \\
5 \\
8 \\
26 \\
41 \\
49^{3} \\
58^{3} \\
6 r^{3} \\
71^{3} \\
99^{3}\end{array}$ & $\begin{array}{l}1090 \\
1036 \\
1006 \\
1143 \\
1290 \\
1279 \\
1180^{4} \\
1030^{4} \\
1066^{4} \\
1116^{4} \\
973^{4}\end{array}$ \\
\hline
\end{tabular}

The metabolism which takes place normally in the body of a mammal when in absolute rest, for the purpose of maintaining life, is always the same, without regard to the source whence it obtains the organic material to fulfil its needs-whether from albumin, fat, or carbohydrates.

For the maintenance of life the organic food products compensate according to their specific energy of tension (law of isodynamics). The amount of energy which the individual food products contribute to the processes of

1 German: Ruheverth.

2 Rubner, Beiträge zur "Ernährung im Knabenalter," Berlin, 1902, p. 62.

3 Weight with clothing.

4 Calculated from the carbonic acid values. 
metabolism that arise from them, and by their combustion in metabolism becomes living force, Rubner has calculated at 4.1 calories for one gram of albumin, 9.3 calories for one gram of fat, 4.1 calories for one gram of carbohydrate.

These are the figures which are utilized as standards in all clinical investigations in metabolism (the correspondence of the value for albumin and carbohydrate is quite accidental).

In these values force is expressed in units of the heat which is generated in the organism when by combustion of the individual food products their end products in metabolism are produced. The loss which occurs in the intestines by insufficient absorption of the food is not considered here. They are, therefore, gross values (large calories). To convert them into pure calories, Rubner has calculated a subtraction of 8 per cent. from the average heat value of the feces on a mixed diet. Hiis latest investigations, by means of the direct calorimetry in man, have shown that the calculation obtained from chemical analysis of the standard figures in derived heat corresponds almost perfectly with the amount of heat in calories taken directly from the calorimeter. The method commonly used in the clinic to calculate the energy transference from the calory value of the food introduced is therefore accurate, if no severe disturbances in absorption are present.

\section{B. THE METHODS FOR ESTIMATING THE CALORY REQUIREMENT OF THE BODY}

The method in use in Voit's laboratory for determining the degree of metabolism in a healthy individual under various conditions of nutrition is by the comparison of intake and output in a trial period of twenty-four hours. This presupposes that the body within this time rids itself of the total end products of metabolism that have arisen from the decomposition of organic substances. This supposition is quite correct for excretion in the respired air and in the urine. To estimate the contents of the feces (the discharges usually occurring later here), we need to mark in some way the beginning of the experimental period. This is usually accomplished without difficulty and with sufficient exactness by the administration of from 10 to 15 grams of powdered charcoal or 0.3 gram of carmin at the beginning and at the end of the metabolism investigation. This method in its high technical completion was the sine quâ non for the quantitative investigation of metabolism at the bedside.

An accurate estimate of the food ingested during the period of trial and of the carriers of energy contained in it (albumin, fat and carbohydrates) is necessary. The excretions, i. e., the urine, the feces and the expired air, are measured with exactness, and analyzed as to the amount of nitrogen and carbonic acid which they contain. From a comparison of ingesta and excreta the balance is obtained.

I shall quote an example of one of Voit's experiments: A workman weighing 69.5 kilograms took in the form of meat, egg, albumin, milk, bread, lard, wutter, flour and sugar a total of 137 grams of albumin, 117 grams of fat, and 
352 grams of carbohydrate, containing 19.5 grams of $\mathrm{N}$ and 315.5 grams of $\mathrm{C}$, besides 2,016 grams of water. He excreted in complete rest:

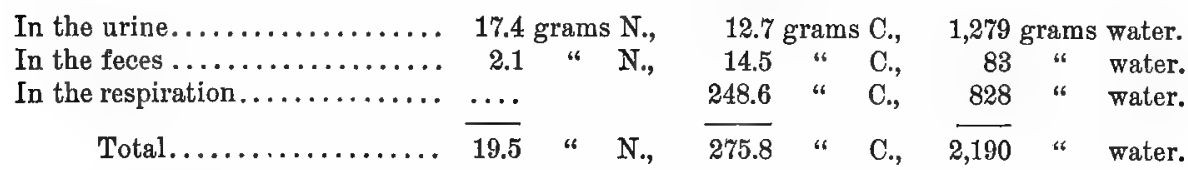

The body was, therefore, in N-equilibrium; excreted 174 grams of water and retained 39.8 grams of carbon, which corresponds to a deposition of 52 grams of fat.

This method gives us knowledge of the body-albumin during the investigation. This is estimated by means of the nitrogen balance, a method which, since that time, has come into general use.

One hundred grams of albumin contain 16 grams of $\mathrm{N}$. If-the entire amount of $\mathrm{N}$ due to the decomposition of albumin during the trial period were excreted in the urine, the $\mathrm{N}$ figure of the urine need only be multiplied by 6.25 to determine the amount of albumin metabolism. The other $\mathrm{N}$ losses of the body are slight. A small portion appears in the feces as a residue of the intestinal secretions and this is sufficiently large to be calculated in each investigation. The other $\mathrm{N}$ losses of the body (loss of hair, scales of the epidermis and sweat) are so insignificant that they may be ignored in a trial period lasting twenty-four hours.

Nitrogen equilibrium is then shown when the food- $\mathrm{N}=$ urinary- $\mathrm{N}+$ fecal-N.

Voit's quantitative method of estimating metabolism also permits us to recognize from the carbon balance whether the body's need of $\mathrm{N}$-free substance has been fulfilled by the food given during the trial period or not. The organism has its very definite calory requirement. With an insufficient administration of nourishment, the body takes a supply of energy from its own material component parts. When, in a body previously in equilibrium, carbon is retained or more excreted than is ingested, a corresponding accumulation or loss of fat is indicated. In the form of carbohydrates (glycogen) the body does not accumulate any great supply of energy.

In the quantitative estimation of metabolism in the clinic it has been necessary as a rule to proceed without determining the carbon balance. This require the estimation of the total $\mathrm{CO}_{2}$ excretion in the respired air, which can only be accomplished by means of a large respiratory apparatus such as that of Pettenkofer. Such an apparatus is expensive and very difficult to adjust. As many hours are required to make the experiment, it is inconvenient to employ this apparatus at the bedside.

To compensate for this, the condition of the body-weight has been employed as a control to determine whether, besides the proteid requirement, a sufficient energy supply for the needs of the organism is present in the food. If there is little variation in the material constituents of the body (albumin, fat and water), this is permissible, especially if the experimental period is not too brief. Instead of trials of twenty-four hours, therefore, trial periods of three to four days are usual in metabolism analyses of this type. 
If the test is made for still longer periods, the weight of the body alone, with minute estimation of the energy value of the food, may enable us to recognize the calory requirement.

Forster demonstrated in persons living under ordinary conditions the food values just sufficient to maintain weight and to enable them to follow their usual occupations. In numerous investigations with larger groups of men (in barracks, in prisons and in almshouses) by an analysis of the food ingested, valuable standards have been established for the amount of food required by healthy persons. If the individual continues as vigorous as usual and his body-weight remains unchanged while on a prescribed diet, the conclusion is justifiable that the amount of energy contained in the allotment of food is sufficient for the requirements of the body.

As the metabolism of the body depends upon the needs of all the cells in the body, and represents essentially a process of combustion, it is obvious that the intake of oxygen should also be considered in estimating metabolism in the body. But the estimation of oxygen consumption is not an accurate measure of the total metabolism, because the same amount of oxygen, according to whether it is utilized for the oxidation of albumin, fat or carbohydrates, corresponds to different quantities of heat; also because one gram of expired carbonic acid, according as it has been formed by combustion of one or another food stuff, corresponds to very different degrees of heat.

Therefore, only when the nature of the food-materials decomposed in the body remains the same are consumption of oxygen and excretion of carbonic acid a measure for changes in the entire consumption of products.

By means of the large respiratory apparatus of Hoppe-Seyler-Tigerstedt, the direct estimation of all the factors of the respiratory metabolism of gases is possible. The apparatus is difficult to manage, however, and the necessarily long stay in the chamber of the person experimented upon makes its use inconvenient; therefore, in the sick but few observations have been made on this plan.

It is much easier to estimate the absorption of oxygen and the production of carbonic acid with the apparatus of Zuntz and Geppert. In brief experiments, trials are usually made by means of a mouth-piece for only five to ten minutes, at most for one hour; the $\mathrm{O}_{2}$ and $\mathrm{CO}_{2}$ in the expired and inspired air and the amount of $\mathrm{O}_{2}$ intake and $\mathrm{CO}_{2}$ excretion per minute are calculated from this.

To calculate the total $\mathrm{O}_{2}$ consumption for an entire day from these figures would lead to error, on account of the great variation in the respiratory interchange of gases from hour to hour.

Their reciprocal relation, however, furnishes the respiratory quotient, which gives valuable information regarding the nature of the material decomposed at the time. It approaches the value of 1.0 in carbohydrate combustion, while during the combustion of albumin alone it amounts to 0.73 , and in combustion of fat to $0 . \%$.

For the quantitative estimation of the interchanges, the enormous amount of material gathered by Zuntz and his pupils in numerous healthy and sick persons contributes valuable points. In absolute rest of the body, and in the 
intervals of intestinal inactivity, which occur from six to eight hours after meals, the values for the intake of $\mathrm{O}_{2}$ and the excretion of $\mathrm{CO}_{2}$ per minute are quite constant for any healthy individual, and in different healthy individuals they vary only within certain limits. In these "fasting values" we have standards, variation from which in one or the other direction denotes pathologic change.

In conclusion, direct calorimetry must be considered as the exact method by which to determine the entire transference of energy in the resting body. This coincides, as already mentioned, with the total loss of heat, the coincidence of which with the heat-equivalent of the food stuffs consumed have been determined by Rubner in animals by masterly experiments. In man, and especially in the sick, direct calorimetric estimations to determine the quantity of the total metabolism have been carried out only in isolated cases (see below).

\section{THE ENERGY REQUIREMENT OF THE HEALTHY}

The quantity of food required by a workman while performing "moderate" labor Voit stated to be 118 grams of albumin, 50 grams of fat, and 500 grams of carbohydrates. This was based upon many experiences taken from daily life, and corresponds with the results of numerous exact analyses of metabolism carricd out, according to the method described above, in Voit's laboratory (comparison of the intake and output). For a long time Voit's principles existed almost as a dogma. Later the total calory value to which this diet corresponds ( 3,055 calories), as wcll as the large amount of albumin contained in it, became the subject of lively discussion, in which the clinical investigators took a prominent part.

If the performance of muscular work increases the energy requirement of the body above that of rest (and, in fact, it multiplies several times the heat equivalent to the work done), the calory requirement of the "workman" cannot be estimated otherwise than by considering the amount of work to be performed. The rich statistical material which is now at hand confirms the requirement of Voit for about 43 calories (which is the amount contained in the above quantity of food per kilogram of body-weight) for persons doing active muscular work.

From many observations Rubner calculates as the medium amount for a twenty-four-hour metabolism in the healthy adult, of average body size, and weighing 70 kilograms:

At rest Pure
calories. Calory per kilogram.

With slight muscular work.................... 2,445 $=34.9$

With moderate muscular work.................... 2,868 $=41.0$

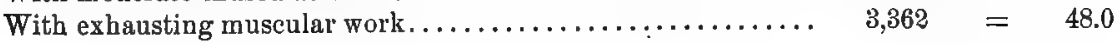

With a deduction of 8 per cent. from the 3,055 large calories which are contained in Voit's amount of food, this corresponds exactly with Rubner's calculations. 
With more justice, Voit's requirement of 118 grams of albumin has been the subject of dispute. In the discussion regarding this amount there has been much difference of opinion. In their conception of the main point in dispute, the views of different authors were diametrically opposed; and the conflicting opinions which arose in consequence of this have kept the discussion alive to the present time.

In the food of man, which consists of albumin, fat and carbohydrates, the albumin bodies grouped in contrast to $\mathrm{N}$-free substances are especially important. While the latter may be compensated for to a great extent by each other and also by albumin, according to the measure of their energy value, a certain quantity of albumin-in the daily food is irreplaceable and indispensable if the proteids of the body are to be maintained intact.

To determine this indispensable amount of albumin, the minimum which the body requires to maintain the living substance in a functioning condition has been the subject of much discussion.

For a time it was believed (Bidder and Schmidt) that this albumin minimum corresponded with the albumin-metabolism of persons kept in a state of starvation, i. e., that the nitrogen excretion in starvation furnishes a standard for the nitrogenous metabolism necessary to maintain life, that is, the actual albumin requirement of the body. All the albumin absorbed from the intestine in excess of this was to be considered "luxury," and was supposed, like the $\mathrm{N}$-free substances, to undergo prompt combustion in the blood without becoming organized at all.

Among clinicians, no less a one than Frerichs upheld this theory of "luxury combustion," propounded by C. G. Lehmann.

Voit opposed the "luxury" theory. In his "Handbook of the Physiology of Total Metabolism" (page 269) he devotes a special chapter to contradicting this, and furnishes convincing proofs.

According to experiments made in the dog, which of course are to be interpreted somewhat differently from human experiments and which in their general application to man have been attacked by some investigators, the smallest quantity of albumin which will maintain the nitrogenous equilibrium of the body (on a mixed diet with $\mathrm{N}$-free food substances) is usually $2 \frac{1}{2}$ to 3 times larger than the nitrogenous metabolism in starvation. In their comprehensive experiments E. Voit and Korkunoff ${ }^{1}$ still found the minimum albumin-requirement to be 111 per cent. greater than the nitrogenous metabolism in starvation (and even with very large amounts of starch in the food).

It may therefore be considered as proved that if we give a dog only the amount of proteid which is decomposed in a state of starvation, this amount is not sufficient to maintain the nitrogenous equilibrium of the body.

In man the same appears to be true. With sufficient food, the excretion of urea is increased decidedly above the starvation figures. From this experience, and upon the basis of investigations on the nutrition of a large number of workmen, Voit computed as a standard for any sufficient diet the above-

$1 E$. Voit and Korkunoff, "Die geringste zur Erhaltung des Stickstoffgleichgewichtes nöthige Menge von Eiweiss." Zeitschr. f. Biol., xxxii, p. 58. 
mentioned high requirement (hygienic albumin minimum in contrast to the physiologic albumin minimum).

It appears, however, that proteid metabolism during starvation, even in the same individual, has no constant height, at least not during the first days of fasting. Here it apparently depends upon the composition of the food which has preceded the experiment and on the food products retained in the body. It is high if, in the days preceding the fast, a large amount of albumin has been ingested, and decidedly smaller if for a long time prior to the starvation period the ingestion of albumin has been slight. Only after from one to three days will the nitrogen excretion in the urine, varying in the same individual, show constant figures. This constant figure apparently depends on the composition of the body, i. e., its albumin and fat condition. From this, during starvation, it must make up its losses.

The source of the albumin which is so rapidly decomposed during the first days of starvation is not, according to Voit, the albumin of the organs, but the much more easily decomposed and soluble albumin of the fluids, the "circulating albumin." This quantity, which is increased by a diet rich in albumin, also determines the proteid metabolism in the first days of starvation.

A deficiency of albumin in the food diminishes the amount of "circulating albumin," and as this is dependent upon the fixed or organic albumin it indirectly works mischief upon the organs.

A superfluous intake of albumin, on the other hand, increases the amount of the readily destroyed circulating albumin in the fluids, and hence proportional with the introduction of albumin there is an increased albumin decomposition which is dependent upon the amount of circulating albumin and the $\mathrm{N}$-excretion in the urine.

The amount of albumin consumed in a unit of time in the animal body is dependent to an astonishing degree upon the amount of the albumin ingested. The capacity of the normal adult organism to maintain its equilibrium with any amount of albumin-exceeding the minimum-is hardly limited (in so far as the body's capacity to take up albumin permits investigation). If the energy-requirement of the organism is met by the food, the proteid of food decomposes completely and with extraordinary rapidity. Scarcely absorbed in the fluids, it is soon destroyed, and in the briefest time its nitrogenous cleavage products appear in the urine for excretion in quantities corresponding exactly with the increased albumin intake. This fact forms one of the most important points in Voit's whole theory of nutrition; important both for pathology and for physiology. Hence it became at once the spur for many clinical investigations in metabolism.

Many endeavors have been made to explain these facts. They contradict completely the otherwise applicable law that the amount of decomposition in the animal body is equivalent to its requirement of energy. For Voit has shown that with a sufficient amount of food any additional amount of albumin, no matter how large, is at once decomposed, so that metabolism (transference of energy) is greatly increased.

The changes in the total metabolism by variation of the intake other than those referred to above are slight. The introduction of amounts of 
food whose heat-equivalent is not decidedly above the interchange of forces in the starving organism does not increase the total metabolism, and with a diet beyond the amount required by the needs of the body the introduction of extra $\mathrm{N}$-free material (fat and carbohydrates), no matter how great, increases the interchange of energy only to a certain percentage of the excess of food.

If the surplus is introduced in the form of fat, only 10.7 per cent. of it undergoes combustion; therefore almost 90 per cent. is deposited as fat in the body. If superfluous carbohydrates are introduced, only 15.9 per cent. goes to increase the total metabolism; 84.1 per cent. is accumulated in the body and is utilized for fat formation, since the body's capacity to increase its carbohydrate material (glycogen deposit) is a limited one. ${ }^{1}$

Since the amount of metabolism is relatively independent of variations in the ingestion of nourishment, we have the conditions which make possible a deposition of fat.

With an abundant introduction of albumin, however, proteid decomposition increases in exact ratio to the superfluous amount of albumin ingested. The entire superfluous supply is taken up in metabolism, and the $\mathrm{N}$-excretion in the urine shows a height corresponding to the increased administration.

In reality it is, of course, only the $\mathrm{N}$-contaiming complex in albumin which is excreted, and these products of excretion are at once thrown off. Of the non-nitrogenous components which remain after excretion, as well as also the superfluous fat and carbohydrates introduced, only a portion is subjected to decomposition, if the organism does not require thern to meet its energy requirements, while a considerable remainder (69.1 per cent.) is stored up in the body (fat formation from albumin). Only the elimination of nitrogen is complete.

For this reason albumin occupies an exceptional position among food substances; the organism requires it for its simple material maintenance. It is, therefore, difficult to understand why the organism should waste so much of, and so soon eliminate, this most valuable food product.

A number of explanations for this apparent waste have been offered. It has been assumed that owing to the circulation of albumin products in the fluids after albumin nutrition, the individual food stuffs take part in the total metabolism. On the other hand, a special chemical affinity of the cell substance for the individual food products has been assumed owing to the fact that the albumin is most readily absorbed, less speedily the carbohydrates, and last of all the fats.

Rubner ${ }^{z}$ has lately assumed that the divisibility of the individual food stuffs explains the different share taken by each in the processes of metabolism. These differences in divisibility have not been sufficiently considered until now: "A measure of these differences is the size of the molecules. In the case of fat this amounts to $8 \%$, in grape sugar, to only 180 . In what

1 This increase in metabolism by introduction of food was formerly explained by an increase of intestinal activity. Lately the correctness of this view has been doubted.

${ }^{2}$ Rubner, "Handbuch der Ernährungstherapie," Bd. i, p. 78. 
form the albumin products circulate is not known. Nevertheless, the view that albumin and allied substances are always bodies of high atomic weight, has suffered a setback. The albumoses have a molecular weight of from 1,200 to 2,100 ; the peptones, however, of only about 200 , similar to that of sugar. In a molecule of fat there are 8,1\%0 calories, in a molecule of grape sugar only 674 . The peptones vary very slightly from the latter value. The varieties of sugar and albumin, therefore, viewed from this standpoint, might predominate in decomposition without special forces coming into play."

Gruber ${ }^{1}$ looks upon the prompt splitting up of the albumin of food as a very necessary process, in fact as an arrangement which is quite indispensable to rid the organism as soon as possible of the great bulk of soluble albumin bodies which are unnecessary for its normal condition and which cannot be utilized. A further increase in the size of the organs in the adult animal is possible only to a slight degree after the body has reached the limit of growth fixed by its hereditary germinal predisposition (increase of the size of muscles as the result of body exercise). To replace the amount of organic albumin daily destroyed a small portion of the food proteid is sufficient. As the accumulation of the balance in the body would alter the function of the organs, its immediate decomposition is absolutely necessary in order to keep the composition and concentration of the body fluids unchanged in spite of any variation in the composition of the food.

The prompt splitting up of the food albumin is believed by Gruber to be the effect of the action of enzymes with which the cells of the adult healthy organism are richly supplied. This view has much to commend it. We can thus readily explain the enormous albumin accumulation which occurs in convalescence from severe diseases, after prolonged starvation, etc., and is almost independent of the administration of albumin. The absence of the albumin-splitting enzyme in body cells that have been damaged in their proteid constituents by disease explains the absence of that rapid splitting and excretion of proteids which occurs in health.

\section{THE ALBUMIN MINIMUM}

We have seen that Voit's requirement of 118 grams of albumin in the daily food of an adult performing moderate labor is a practical proposition for rational nutrition, and does not represent the physiologic albumin minimum which the mature body absolutely requires to supply its functioning organs. Incorrectly viewed, this stimulated a great number of researches in which the starting point was the question (although it can have only theoretic interest), How far may the albumin metabolism be decreased without the body suffering in any of its albumin constituents? And yet every new investigator in the solution of this question attempts to establish a standard and to proclaim a still lower value as the "albumin minimum." That man may exist with smaller quantities of albumin, and be capable of work, Voit himself readily determined in the analysis of metabolism in a vegetarian who

\footnotetext{
1 Gruber, "Einige Bemerkungen zum Eiweissstoffwechsel." Zeitschr. f. Biol., xlii, p. 407.
} 
weighed 57 kilograms. Choosing his diet freely this person consumed, year in, year out, only 54.2 grams of albumin (with 32.4 grams of pure albumin), 22 grams of fat and $55 \mathrm{r}$ grams of carbohydrates, and maintained the same body-weight.

The investigations in metabolism of Hirschfeld, Klemperer, Peschel, Kumagawa, Breisacher, and lately also of Siven and Albu, give even smaller values as the albumin minimum. The following table is a comparative compilation of the food values with which individual persons experimented upon were able to maintain their $\mathrm{N}$-equilibrium.

TABLE II

\begin{tabular}{|c|c|c|c|c|c|}
\hline AOTHOR. & $\begin{array}{l}\text { Body } \\
\text { weight. }\end{array}$ & Albumin. & $\begin{array}{l}\text { Albumin } \\
\text { per kgm. }\end{array}$ & $\begin{array}{c}\text { Large calories } \\
\text { per } \mathrm{kgm} .\end{array}$ & $\begin{array}{l}\text { Duration } \\
\text { of trial. }\end{array}$ \\
\hline 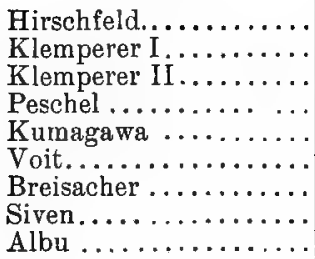 & $\begin{array}{l}73.0 \\
63.0 \\
65.0 \\
79.5 \\
48.0 \\
57.0 \\
57.0 \\
58.9 \\
37.5\end{array}$ & $\begin{array}{l}35.4 \\
25.1 \\
26.6 \\
34.2 \\
37.8 \\
32.4 \\
51.4 \\
28.3 \\
43.13\end{array}$ & $\begin{array}{l}0.485 \\
0.400 \\
0.41 \\
0.43 \\
0.788 \\
0.570 \\
0.900 \\
0.48 \\
0.90\end{array}$ & $\begin{array}{l}47.4 \\
80.0 \\
77.2 \\
47.1 \\
51.6 \\
47.5 \\
50.3 \\
41.4 \\
37.32\end{array}$ & $\begin{array}{l}8 \text { days } \\
8 \text { " } \\
8 \text { "6 } \\
8 \text { " } \\
9 \\
\text { Usual diet } \\
30 \text { days } \\
1 \text { day } \\
5 \text { days }\end{array}$ \\
\hline
\end{tabular}

The albumin interchange in the professional fasters, Cetti and Breithaupt (calculated from the $\mathrm{N}$-figures of the urine by multiplying by 6.25 ), gave on the other hand: ${ }^{1}$

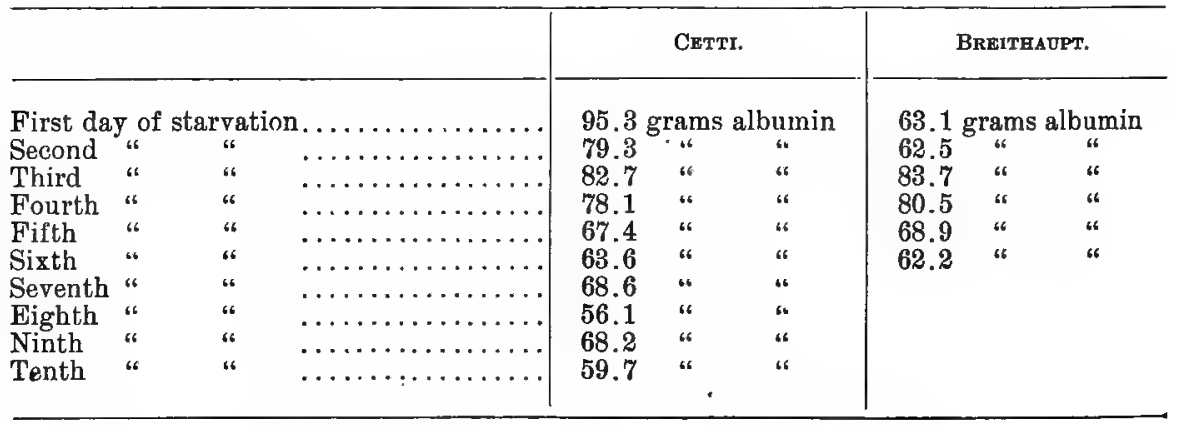

Therefore, it is evident from the table that with a sufficient calory administration in food products free of nitrogen the albumin requirement may fall far below 118 grams, and even below the metabolism under complete withdrawal of food.

Theoretically this fact, determined by such numerous, painstaking investigations, is of the greatest scientific interest. But caution has been quite properly enjoined upon us not to draw far-reaching conclusions from such

1 "Untersuchungen an zwei hungernden Menschen." Virchow's Arch., vol. cxxxi, Suppl.-Heft, pp. 21 u. 68. 
ief investigations of the practical laws of nutrition. We need not, with lüger, assign to albumin a pre-eminent importance " as the only and immeate source of muscular power," but, for the permanent nutrition of the althy and the sick, we must still insist upon greater amounts of albumin an proved sufficient to maintain the albumin condition of the body in the :periments above cited.

In fact it is hardly possible to transfer the conditions of an experiment - practice, and to find a diet that will give a sufficient degree of energy to ie body, that will agree, that will continuously be palatable and can be gested, yet will contain such slight amounts of albumin as were, for exame, present in the experiments of Siven.

This, however, may be seen from all of the previously mentioned experients-that the $\mathrm{N}$-equilibrium can only be maintained on such a low proteid iet when we have a sufficient calory supply. In most experiments the calory lue of the food reaches the amount which Voit requires for the "medium orker" (with nine to ten hours' daily labor), although the persons experitented upon did not perform very laborious work; in a few experiments decidedly exceeds this amount.

Therefore, only with a superfluous calory administration can the albumin tetabolism be diminished to such slight values.

The calory carriers, carbohydrates and fat, are therefore albumin savers, ad for practical nutrition this is perhaps the most important conclusion of sese investigations regarding the albumin minimum.

\section{E. THE REQUIREMENTS FOR ALBUMIN DEPOSITION}

According to Voit's law, the addition of albumin to a diet already suffiient increases albumin metabolism to such an extent that after a few days T-equilibrium is again reached, yet no decided accumulation of albumin ccurs. But we should note here in the increase of the energy value of the zod by the addition of fats and carbohydrates a process to diminish albumin retabolism, so that when albumin administration remains the same, albumin eposition must be the consequence. This gives us reason to hope to increase re body albumin by forced feeding.

Unfortunately the energy supply contained in the superfluous food which ; utilized in albumin deposition is not very great, as is shown by Voit's zvestigations in the dog. With the addition of carbohydrates upon an averge 91.5 per cent., and with the addition of fats upon an average 95 per znt. of the superfluous potential energy accumulates in the form of fat, and nly 8.5 (or in fact only 5 per cent.) in the form of albumin. In man ccording to the investigations of $\mathrm{v}$. Noorden, ${ }^{1}$ at most 10 per cent. of the rperfluous energy supply is utilized to store up albumin in the body.

The experiments of Krug ${ }^{2}$ upon himself, under v. Noorden's direction,

1 v. Noorden, "Verhandlung. der physiol. Geselschaft," im Arch. f. Anat. u. Physilogie, 1893.

2 Krug, "Ueber Fleischmast beim Menschen." v. Noorden's Beiträge z. "Lehre vom toff wechsel," Heft 2. 
have shown, however, that a slight accumulation of albumin day by day can be attained by giving a food of high calory value for a long time. With food which differed from that of the prior period (when he was in $\mathrm{N}$-equilibrium) by an increase of 1,710 calories, albumin remaining the same, Krug retained decided $\mathrm{N}$-amounts (a total of about 50 grams in fifteen days) and, what is remarkable, the gain during the last of the fifteen days of forced feeding equalled that of the first days.

Liüthje ${ }^{1}$ has lately obtained still larger $\mathrm{N}$-retention in his forced feeding experiments in which he increased the total energy administration of the food, like Krug, and also raised the quantity of albumin decidedly above the requirement (albumin administration up to 380 grams with 6,035 calories). The total, retention in twenty-six days amounted to 149.61 grams of $\mathrm{N}$.

This decided N-retention within a short time, even more than the small values formerly obtained, raised doubts as to whether the $\mathrm{N}$ retained in the body actually indicated a gain in "flesh."

Voit has never regarded the N-retention obtained by forced feeding as an increase of "organic albumin"; he held the view that the albumin saved under the influence of nutrition remained in the circulation until it was changed into organic albumin by the slow production of new tissue. As, however, under the influence of hypernutrition, the plasma of blood and lymph does not, so far as we know, become richer in albumin, v. Noorden proposed the hypothesis that the albumin which is saved, and not yet utilized for the structure of new tissue, is retained as a dead mass in the living protoplasm of the cells, analogous to the superfluous glycogen and fat, remaining there as " reserve albumin," though with decidedly different conditions of decomposition than are present in organic albumin. Correspondingly, he draws a sharp distinction between increase of "reserve albumin" and increase of muscle (by which he understands the increase of living cell albumin). $\mathrm{He}$ believes the latter to be a result of the specific growth energy of the cells, a function of cellular labor, and not a result of extra nutrition since, as we have seen, hypernutrition increases only the reserve albumin.

This hypothesis of $\nabla$. Noorden's is not in agreement with Pflüger's ${ }^{2}$ view regarding the meaning of the reserved and deposited albumin. In the latter's numerous publications on the subject, he maintained that the accumulated albumin is soon deposited as cell substance in the body of the animal, and therefore at once takes a prominent part in the total metabolism. Pflüger does not regard it as a dead cell inclusion, but as a "working mass," and he also thus explains the increase of albumin metabolism which occurs after proteid administration as an increase of the "working cell substance," upon the amount of which, in his opinion, the total metabolism is directly dependent.

1 Lüthje, Beiträge zur "Kenntniss des Eiweissstoffwechsels." Zeitschr. f. klin. Med., xliv, p. 21.

${ }^{2}$ Pflüger. Arch. f.. d. ges. Phys., Bd. lii, p. 1. "Ueber einige Gesetze des Eiweissstoffwechsels," Bd. liv, p. 333. "Ueber den Einfluss, welchen Menge und Art der Nahrung auf die Grösse des Stoffwechsels und der Leistungsfähigkeit ausüben." Pflüger's Arch., Bd. lxxvii, p. 425. 
But the enormous amount of $\mathrm{N}$-retention in the forced feeding investiations of Lüthje makes us hesitate to believe that the total amount of itrogen retained is really deposited as "albumin," especially as we should be rced to assume at the same time that the reserved albumin is deposited 'gether with a quantity of water, such as corresponds to the usual relation atween albumin and water in the cellular elements of the body (one gram $\mathrm{N}=6.25$ grams, albumin $=29.4$ grams of flesh).

According to this we should have to suppose that the $\mathrm{N}$-retention of 49.61 grams $\mathrm{N}$, which was attained by Lüthje within twenty-six days, corresonded to an albumin deposition of 935.06 grams of albumin, or a flesh eposition of 4,398.53 grams. The increase in the body-weight of the person xperimented on amounted to 6,070 grams during this time. If we estimate ue flesh accumulation from the $\mathrm{N}$-retention, more than two-thirds of the crease in body-weight (4,398 grams) must have been due to flesh deposion. The considerable calory excess contained in the administration of food 1 the days in question certainly must have resulted in a deposition of fat. 'he amount of this Liuthje calculates in the usual manner; from the total alory excess during the entire period (29.656 calories) he deducts the calory alue of the albumin which has accumulated $(935.06 \times 4.1)$, i. e., in round umber 3,838 calories, and the remainder, amounting to 25.818 caloies, is ascribed to fat accumulation. Twenty-seven hundred and seventy-six rams of fat correspond to this energy value. 'The flesh accumulation of ,398 grams, and a simultaneous fat deposition of 2,776 grams coincide ery imperfectly with an increase in weight of only 6,070 grams. This may, owever, be readily explained by the assumption that the body during the rasting period lost a weight of water, corresponding to the difference $(1,104$ rams).

In the feeding experiments of Krug, the same difference was noted. Vith an increase in weight of only 3,100 grams, the albumin accumulation of , 455 grams calculated from the retained $\mathrm{N}$, and the fat accumulation from xe calory excess after deducting the albumin calory, amounted to 2,254 rams. Krug assumed without more ado that the body lost 609 grams of ater.

This assumption is certainly justifiable, and a decrease in water during rrced feeding appears quite plausible if we remember that each increase of 1e albumin metabolism (for example, by a large albumin meal) is soon folwed by a decided excretion of water.

In my opinion the assumption of so large a loss of water is, however, unnecssary. In the previously mentioned calculations, we have always proceeded a the assumption that the total calory excess of the food not used to store $p$ albumin must be utilized in fat formation. It has, however, not been condered that an excess of food also invariably increases the total metabolism, ad, it may now be supposed, increases this out of proportion to the intestinal bor. A true luxury consumption of non-nitrogenous food substances takes lace. Only a portion of the superfluous food energy is utilized in accumution; in man another portion, probably showing individual variation, underoes combustion, and is utilized in heat production, the organism replying 
to this increase by an increased excretion of vapor. In the investigations in forced feeding, the fat accumulation, which can only be controlled by an accurate estimation of the carbon balance, may also be less than authors have assumed, judging by the caloric excess in the food.

In such a profuse $N$-retention, the supposition that the accumulation of nitrogen-containing end products of albumin metabolism may play a rôle appears to be quite unwarranted for the reason that there are no sufficient proofs that $\mathrm{N}$-containing products of intermediary albumin metabolism remain as such in large quantities in the body of the healthy. The view is much more likely that the $\mathrm{N}$ is retained as albumin, and that this albumin (as a native molecule, however, and without a corresponding participation of water) enters the cell. As evidence of this Lüthje points to the observation that in certain snails albumin is found included in the cells in a crystalline form.

It seems to me, however, that there is one phenomenon-which appears, so far as I am aware, in all investigations regarding albumin accumulation if the analyses in question are carried out-and which goes to show that the albumin accumulated in the protoplasm of the cells increases and rejuvenates it while assimilating itself with the general cell albumin. I refer to the simultaneous retention of phosphorus when $\mathrm{N}$ is retained, and its increased excretion (as $\mathrm{P}_{2} \mathrm{O}_{5}$ ) during albumin decrease. This is shown in the investigations of Kayser, and of Jacob and Bergell. It may be recognized also in Lüthje's metabolism figures, and only lately Kaufmann has called attention to the decided phosphorus retention which he observed during albumin accumulation in feeding with "egg albumin. The fact that any phosphorus containing albumin body especially favors albumin accumulation (as was shown by Röhmann and his pupils, and also by Zuntz and Caspari) favors this view.

Quite an analogous N-retention (which probably no one will doubt means an albumin accumulation) Rost ${ }^{1}$ has observed in his metabolism control experiments in growing animals.

The amounts of nitrogen of the food which were not excreted in the urine and feces of three young dogs were decidedly greater than could be accounted for by the increase of body-weight, if the $\mathrm{N}$-accumulation were reckoned in the formation of muscle albumin. Therefore, as in the investigations of Lüthje, either a loss of water occurred or the retained nitrogen was utilized to build up a cell protoplasm with higher nitrogen contents than are found in muscular substance.

When it became necessary, in forced feeding experiments in animals with meat free of fat, to promulgate a formula for the $\mathrm{N}$-accumulation (Pflüger, Cremer) certain difficulties naturally arose. The amount of carbon retained in the organs was greater in comparison with the nitrogen than would correspond to the composition of albumin.

If, with Pflüger, we reject the possibility of a fat formation from albumin, although accepted by Voit's adherents, we must be content with assum-

1 Rost, "Zur Kenntniss des Stoffwechsels wachsender Hunde." Veröffentlichungen des kaiserl. Gesundheitsamtes, Bd. xviii, p. 206. 
an "unknown tissue-building substance," and refer the N-retention to its umulation.

No matter in what form the nitrogen accumulates in the body in forced ling, all authors seem to be unanimous in the opinion that the organism eïves a valuable addition.

For this reason, we shall enumerate again the methods which lead to an rease of the body albumin.

We see N-retention without any increase in the administration of rogenous foods when we give a calory excess of $\mathrm{N}$-free substances (Krug), $\mathrm{l}$ a still more decided $\mathrm{N}$-accumulation when the food contains both an essive calory value and an excess of albumin (Lïthje).

That an excess of albumin alone in an adult healthy person without deed increase of the albumin-saving fats and carbohydrates could bring about albumin accumulation (apart from the slight $\mathrm{N}$-retention in the first is, until N-equilibrium is attained) has been looked upon as impossible, :ording to Voit's principles. Only special circumstances (growth, conescence, diminution in body albumin from preceding starvation) would ke it possible permanently to accumulate albumin in this way.

Bornstein, ${ }^{1}$ however, lately showed in an experiment on himself that it sossible in man by an increased administration of albumin alone to produce a lited increase of the albumin contents of the body. By adding to his diet grams of nutrose daily he accumulated in fourteen days 16 grams of $=100$ grams of albumin, just as, in Pflüger's experiments in feeding adult imals (dog and cat) with fat-free meat, a decided increase in weight was duced by flesh deposition. And as Pflitger looks upon the increase of the rumin mass of the body as an increase of power, so Bornstein looks upon : $\mathrm{N}$-accumulation as a sign of a previously sub-standard value of his musar and albumin apparatus, which was then improved by a deposition of rumin.

It is an interesting fact that stock raisers ${ }^{2}$ to-day also believe it possible bring about a production of flesh, in a restricted sense of the word and der certain circumstances, in adult animals to a more decided extent than s formerly thought possible. They regard an increased accumulation of sumin in the body of the adult animal as decidedly improving the quality the meat-an improvement which does not depend upon an increase in iscle bulk or new formation of muscle fibers, but is due to an increase the juices in which the muscular fibers are bathed.

For the physician these views regarding muscle food are very interesting, ssmuch as it is often necessary for him to treat persons below par in their iscular activity. In cases in which a further accumulation of fat is never sirable (anemia, neurasthenia, etc.), Bornstein's advice to adopt forced sding with a decided addition of albumin is certainly worthy of considera-

1 Bornstein, "Ueber die Möglichkeit der Eiweissmast." Berliner llin. Wochenschr., 18, vol. ix, p. 791.

2 Henneberg und Pfeiffer, Journal f. Landwirthschaft, Bd. xxxviii. 
tion. It increases the albumin contents of the body, and also the albumin decomposition. We know that a man called on to perform work beyond the usual amount always prefers a diet rich in albumin (the average albumin metabolism in athletes is about 200 grams of albumin) ${ }^{\mathbf{1}}$ and this coincides perfectly with the views of Pflüger that, " increased albumin decomposition is combined with that increased vitality which in the struggle for existence gains the victory." 2

More exact and recent investigations in metabolism show that the general tendency of working organs to attract albumin and to retain it for their growth is seen in the highest degree in the muscles. Caspari ${ }^{3}$ studied a dog that, with a certain diet, was in $\mathrm{N}$-equilibrium, yet, with precisely the same food, continuous $\mathrm{N}$-accumulation occurred when the dog was daily subjected to considerable muscular exertion. Bornstein ${ }^{4}$ was able to show that the accumulation of albumin attained by him with the addition of a larger amount of albumin to an already sufficient diet could even be increased by daily muscular exercise in moderation (without work about 16 per cent. of what is ingested is accumulated; with moderate labor about 22 per cent.). He therefore speaks of a true labor hypertrophy of the muscles, and the investigations in metabolism carried out upon a large scale by Atwater and Benedict ${ }^{5}$ confirm this view. Without increasing the nitrogenous food of their experimental persons during the period of labor, they produced an accumulation in albumin (in contrast to an N-loss in the period of rest), although in the period of labor the additional calories were given wholly in the form of carbohydrates without any additional albumin.

It is of course obvious that this labor hypertrophy has its limits. Even with continuous muscular work, an increase of albumin in the food, which is also limited by the person's digestive capacity, will not force a continuous accumulation of muscle. A condition of equilibrium is attained in which, however, the body now controls more organic working albumin than formerly, and possesses increased vitality.

For the treatment of the cases (constantly multiplying in our time) in which diminished capacity for work is one of the most prominent symptoms, these new studies in metabolism contain the most valuable hints. For who will maintain that every patient who comes to us with a body-weight corresponding to his age and his size controls an amount of the organic substances used in metabolism which corresponds to his maximum or even to his optimum?

With the recognition that we can build flesh by the simple addition of albumin to the food (a point on which Pflüger's views and Voit's teachings

1 Lichtenfeldt, "Ueber d. Nährstoffbedarf b. Training." Pfüger's Arch., Bd. lxxxvi, p. 177.

2 Pfïger's Arch. f. d. ges. Physiol., Bd. li, p. 319.

3 Caspari, "Ueber Eiweissumsatz und Ansatz bei Muskelarbeit." Pfüger's Arch., Bd. Ixxxiii, p. 6509 .

4 Bornstein, "Eiweissmast und Muskelarbeit." PAïger's Arch., Bd. Ixxxv, iii, p. 540.

5 Atwater and Benedict, "Experiments on the metabolism of matter and energy in the human body." Washington, 1899. Quoted by Caspari, loc. cit., p. 539. 
are sharply contrasted), the saving of albumin by a superfluous calory addition is not divested of its therapeutic importance. Where a simultaneous accumulation of fat is not undesirable, this high calory diet will, as a rule, be the easiest way of accumulating albumin. But here we should always remember that muscular work is an important auxiliary means of fixing the retained albumin in the organs of the body, and that a simultaneous highproteid diet is not superfluous or even unnecessary. (See the considerable albumin accumulation in the investigations of Lüthje.)

That it is not immaterial in saving albumin whether the calory surplus is brought about by fat or carbohydrates in the food was shown long ago by the investigations of Voit and Rubner. Clinical researches in metabolism have confirmed this. In Kayser's ${ }^{1}$ researches albumin was lost when he replaced the carbohydrates by an isodynamic amount of fat, and Tallquist. also demonstrated that the carbohydrates were capable in a higher degree than fat of protecting the albumin of the body from loss.

To understand these differences, which apparently cannot be reconciled with the laws of isodynamics, it is well to remember the experiments of Rubner, according to which the different food stuffs (in equal excess) increase the interchange of energy very differently because they are utilized in quite varying amounts, the fats undergoing combustion in metabolism, less so than the carbohydrates.

In the practical therapy of nutrition this preponderance of the carbohydrates in comparison with the fats as albumin savers must not permit us to abstain from the plentiful use of fat for the purpose of saving albumin, especially in those cases in which carbohydrates are not allowed, i. e., in diabetes. And since we see here that with an exclusive albumin fat diet (without immoderate excess of albumin) diabetics are able to preserve their albumin condition with a relatively slight administration of fat calories, we might almost believe that the fats under certain circumstances may almost equal the carbohydrates in their albumin-saving effect. In the very plentiful use made of fat in modern dietetics we see further evidence of this.

\section{THE FOOD REQUIREMENT OF THE SICK}

For all physicians a fair comprehension of the total food requirement of the diseased organism is the best foundation in prescribing a suitable diet. Modern nutritive therapy no longer limits itself to the prohibition of this or that food. It expresses positive opinions in ordering the diet, both as regards the nature and the quantity of the food.

Only in diseases which run a rapid course may the amount of food consumed be for the time disregarded. Here real starvation may be necessary on

1 Kayser, "Ueber die Beziehungen von Fett und Kohlehydraten zum Eiweissumsatz des Menschen." Diss., Berlin, 1893.

2 Tallquist, "Zur Frage des Einflusses von Fett und Kohlehydraten auf den Eiweissumsatz des Menschen." Arch. f. Hygiene, Bd. xli, p. 177. 
account of the altered functions of the stomach and intestines, and we may make up our minds regarding the quantity and choice of the food, quite independently of the actual food requirements of the body.

In convalescence from such maladies and in all protracted diseases, however, the quantity of the food is important. A proper appreciation of this in the dietetic treatment of chronic diseases will give the physician his best results.

The appetite which normally regulates the quantity of nourishment ingested, so that metabolism is always exactly covered in spite of all variations in regard to amount, is no measure for the amount of food required by the sick. Appetite is no guide when wasting diseases increase the food requirement of the body; appetite is equally deceptive when excessive corpulence makes it imperative to bring about a reduction of the body-weight.

Only an exact knowledge of the amount of total energy metabolism, the sum of the energy which the body requires and utilizes in the special case, is a guide to the physician for his dietetic orders in respect to quantity.

\section{THE DEGREE OF OXIDATION}

Regarding the degree of metabotism in disease, clinical researches in the last few decades have given us definite figures.

Without a knowledge of the amount of nourishment consumed, and without a proper consideration of the energy values contained in it, mere observation at the bedside, and the analysis of the excretions in the urine exclusively, have frequently led to entirely erroneous conclusions. French literature regarding azoturia, and the writings of Bouchard regarding the slowing of metabolism, contain numerous examples of this fallacy. Increase or slowing of metabolism had been frequently assumed in cases in which later control experiments in metabolism proved the contrary. When the exact methods of the German physiological schools (Voit, Pflüger, Zuntz) were employed in the analysis of the processes of metabolism in the diseased organism the former conclusions were reversed. In spite of this, we cannot yet claim clear insight into many pathologic conditions, but we may nevertheless say that many deeply rooted errors have been corrected by the methodic quantitative labors of our clinics.

Metabolism in the normal human adult, in complete rest, amounts to about one calory per kilogram of body-weight and per hour; therefore with a weight of 70 kilograms, in twenty-four hours it is 1,680 calories $(=24$ calories per kilogram). Even with the ordinary exertion of daily life a decided increase occurs (from about 2,450 to 3,000 calories, i. e., 35 to 42 calories per kilogram). Increased ingestion of food, bodily exercise (muscular labor) and the unavoidable giving off of heat while exercising are the factors to which this increase of transference may be attributed. The amount, the time limits and duration of the increase of metabolism resulting from exercise in healthy persons have been for this reason closely studied.

The hope that differences in the rate of interchange of force in the well and the sick while at rest could be measured by the estimated intensity of 
oxidation $\left(\mathrm{O}_{2}\right.$ decomposition and $\mathrm{CO}_{2}$ excretion) which characterizes individual pathologic conditions has only been fulfilled to a slight degree. On the other hand, later researches have furnished proofs that the healthy and the sick differ in the reaction of their organism to influences that increase metabolism (administration of food, work performed, and the amount of heat given off).

To appreciate these differences, the physiology of metabolism must be briefly considered.

The increase of the processes of oxidation and of heat production, under the influence of the intake of nourishment, which may be subjectively noted in a certain feeling of warmth, has been directly measured by calorimetry. The factors of the respiratory metabolism have also been indirectly controlled.

If a dog's food is increased upon three different days to 55 per cent. above its previously determined calory requirement by administering isodynamic amounts of albumin, fat and carbohydrates, its elimination of heat upon the albumin day rises 19.7 per cent., upon the fat day 6.8 per cent., and upon the carbohydrate day 10.2 per cent. (Rubner).

This coincides exactly with the figures which Magnus-Levy ${ }^{2}$ obtained in his numerous analyses of the interchange of gases after the administration of particular foods; he has referred to these in his valuable dissertation on the influence of nourishment upon respiratory metabolism. He also determined that the three principal food products increase $\mathrm{CO}_{2}$ excretion and $\mathrm{O}_{2}$ consumption in very different degrees. The proteids again take the first place; they increase the intensity of oxidation from 50 to 60 per cent.; then follow the carbohydrates, after the administration of which the interchange of gases is only increased 35 per cent. above the point reached when the stomach is empty; finally the fats, which, if administered in amounts that do not exceed the normal requirement, scarcely increase the consumption of $\mathrm{O}_{2}$ in man at all, and in the dog increase it only about 10 per cent.

Investigations in man with a freely chosen diet make the increase of metabolism after a meal very apparent. In the first four hours after breakfast the $\mathrm{O}_{2}$ intake, in comparison to that in a condition of rest, is increased $21,37,22$ and 10 per cent.; in the first five hours after the midday meal $36 \frac{1}{2}$, $30,20,18$ and 18 per cent., and in the first three hours after the evening meal 33, 31 and 18 per cent. According to this, the increase of the interchange of gases for a whole day, in consequence of the administration of food, amounts to 13 per cent. of the $\mathrm{O}_{2}$ intake, and $19 \frac{3}{4}$ per cent. of the $\mathrm{CO}_{2}$ excretion.

Hösslin found similar figures which are the basis of Voit's investigations in metabolism, and if the body's requirement does not decidedly exceed the intake of nourishment, we are generally right in counting about 10 per cent. as the increase of the oxidation processes due to this cause (Zuntz).

That this increase of oxidation is due principally to the increased labor

1 Magnus-Levy, "Ueber die Grösse des respiratorischen Stoffwechsels unter dem Einflusse der Nahrungsaufnahme." Pfüger's Arch., Bd. lv, p. 1. 
of digestion, and that with a superabundant nourishment these increases in metabolism can be pushed still further, was for a long time the predominant view (Zuntz and v. Mering). Yet lately authors have come more and more to the conclusion that the increase in the labor of the digestive organs is not sufficient to explain the heat production after intake of nourishment. Investigations on this point have been carried on in patients, and have shown that the increase in metabolism produced by the administration of food is very much greater in some individuals than in others. These results have increased our doubts regarding the correctness of this prevalent opinion (see below).

Physiologists have determined the increase of metabolism after physical exertion even more minutely than that following the introduction of food.

The promptness and delicacy of the changes in the amount of oxidation after muscular labor are almost astonishing.

Increase in the peristalsis of the smooth musculature of the intestine after the introduction of mineral salts and after the administration of food, changes of the mechanism of respiration, unconscious muscular effort in strained positions of the body or when the temperature of the skin is reduced, all of these factors influence very notably the interchange of gases. Of course a much more striking variation becomes apparent in tests with muscular activity of a type which can be mechanically measured.

Regarding the degree to which the process of oxidation may be increased, when the amount of work is increased, Zuntz and Katzenstein have given us exact figures. It is an absolute law that $\mathrm{O}_{2}$ consumption and $\mathrm{CO}_{2}$ formation increase with the amount of muscular labor. Accordingly, the interchange of gases is greater when the individual is standing than when he is in the recumbent posture. The increase is slight if the person is standing in a comfortable position; it amounts to about 20 per cent. of the "rest value" if the person is standing erect as in military drill.

In walking the consumption of products becomes from 2 to 4 times as great, according to the rapidity of the pace; in rapid hill climbing it is 5 times as great as the normal, and even more.

Proportionally to the interchange of gases the heat production is increased by work (in comparison to rest values), as Atwater and Benedict have determined by direct calorimetry. About two-thirds of the energy set free by increased decomposition is utilized in heat production, and under ordinary circumstances only one-third in mechanical work. (Under the influence of practice the utilization of energy for the performance of labor may increase just as it diminishes under the influence of exhaustion.)

These are the values which have been estimated in the healthy. As to the way in which labor increases the total metabolism in disease, and how much useful energy in the shape of chemical activity the diseased body gets from the increased decomposition and from the utilization of its own muscles, only very isolated reports are at hand.

From the reply to these questions, conclusions in regard to pathologic metabolism will inevitably result. 
The question most discussed has been whether the effects of cold and heat decidedly increase the processes of combustion in the body. That remaining in a cold atmosphere produces a great loss of heat will scarcely be doubted, and in the endeavor of the body to maintain its own temperature a decided loss in heat in consequence of cooling cannot be prevented, at any rate not without an increased consumption of its combustible material. The question to be chiefly considered is, whether the irritation of cold upon the surface of the body produces chemical changes in the interior, and thus directly increases the intensity of combustion. Speck disputes this, and considers that some physical regulation is responsible for the preservation of body temperature by limiting the loss of heat to the slightest amount. In his opinion, the involuntary muscular contractions which result as the effect of cold are the cause of the increase in the interchange of gases which has been observed.

As a matter of fact, Loewy, ${ }^{1}$ in his investigations of the influence of cold upon the respiratory metabolism, was unable to determine an increase of $\mathrm{O}_{2}$ consumption in all cases of intelligent persons who understood their bodily functions and who maintained completely flaccid muscles during the investigations. This corresponds with the experience of Wolpert, which is of practical importance, that "the temperature of the air of the work-room, during work within the limits of $5^{\circ} \mathrm{C}$. and $25^{\circ} \mathrm{C}$., exerts no especial influence upon the degree of $\mathrm{CO}_{2}$ excretion, either during rest or during strenuous corporeal labor." (In contrast to this are the views of Liebermeister, according to whom the heat loss from the effect of cold causes directly an increase of heat production.)

Regarding the increase of oxidation processes which are generated by the action of heat upon the body, when the conduction of heat by evaporation is prevented, we have some interesting figures obtained in the experiments on the respiratory metabolism which v. Mering had performed in his clinic by Winternitz, ${ }^{2}$ Lohse, ${ }^{3}$ and others. In contrast to the decided increase of oxidation produced by the hot-water bath, as shown by these investigations, the effect of a hot-air bath upon metabolism is quite insignificant in consequence of a prompt physical regulation (sweating) (Salomon). ${ }^{4}$

According to the few researches at hand (Schattenfroh, ${ }^{5}$ Wolpert ${ }^{6}$ ) we may assume that the influence of heat and cold in the metabolism of the sick is quite different from that in the healthy. In the obese, for example, at least

\footnotetext{
1 A. Loewy, "Ueber den Einfluss der Ablühlung auf den Gaswechsel des Menschen." Pflüger's Arch., Bd. xlvi, p. 189.

${ }^{2} \mathrm{H}$. Winternitz, "Ueber den Einfluss heisser Bäder auf den respiratorischen Stoffwechsel." Klin. Jahrbuch, 1899, Bd. vii.—“Ueber die Wirkung verschiedener Bäder auf den Gaswechsel." Hab.-Schrift, 1902, Nauenburg.

${ }^{3}$ Lohse, "Ein Beitrag zur Lehre von der Einwirkung des heissen Bades auf den menschlichen Stoff wechsel." Diss., Halle, 1900.

4 Salomon, "Ueber die Wirkung der Heissluftbäder und elektrischen Lichtbäder." Zeitschr. f. diät. u. physik. Therap., Bd. v, Heft 3.

${ }^{5}$,Schattenfroh, "Respirationsversuche an einer fetten Versuchsperson." Arch. $f$. Hyg., Bd. xxxviii, p. 93.

"Wolpert und Broden, "Respiratorische Arbeitsversuche bei wechselnder Luftfeuchtigkeit an einer fetten Versuchsperson." Arch. f. Hyg., Bd. xxxix, p. 298.
} 
under certain circumstances, the regulation is quite different from that in the lean person. In the latter, the greatly increased heat produced by hard physical exercise may be completely removed by radiation and conduction; in other words, by increasing the temperature of the skin, and this is attained by increased activity of the circulation of the blood. In the obese this method is unavailing, and the secretion of sweat must vicariously take the place of the insufficiently active blood-current. Even in rest and in sleep, the obese person, particularly in a very warm atmosphere, throws off more moisture in a humid atmosphere than in a dry one. As regards the endurance of high temperatures the person with great deposits of adipose tissue is easily overcome, even in rest, and especially so on exertion. Besides the insensible perspiration which must be counted in heat regulation, the obese loses greatly by the sensible perspiration which exudes from the skin, and there is no doubt that as a result of this his metabolism (through changes in the blood composition) is very different from that of the lean individual when exposed to changes of temperature.

The numerous and practical methods given us by physiology for estimating the degree of oxidation in health make it obvious that pathologic conditions should be studied and classified from the same point of view: Is the intensity of the processes of combustion increased or diminished, or unaltered?

It is well known that Bouchard ${ }^{1}$ has described an important group of diseases (diabetes, obesity, gout) as the consequence of a "slowing of metabolism," and lately has extended the scope of this disturbance of metabolism still further so as to include in this group other pathological conditions.

Formerly such facts as the increased excretion in the urine of incomplete products of combustion (uric acid, oxalic acid), maintenance of life on a lessened amount of food, the deposition of fat with a normal intake of food, decrease of body temperature at rest, etc., led him to ascribe the condition to a slowing of metabolism. To-day he attributes to a "slowing of metabolism" any disease which is shown by statistics to occur frequently in association with diabetes, obesity, or gout, or which he supposes to have a genetic relationship with these diseases.

Such a division, justified perhaps from the standpoint of clinical investigation, has absolutely no foundation in exact quantitative analyses of metabolism. Where exact analyses have been carried out we find diseases grouped in an entirely different manner. In quite a number of pathological conditions which are to be considered here, we have exact analytic data concerning the intensity of the processes of oxidation and it will be interesting to consider the results from this standpoint.

1 Bouchard, "Troubles préalables de la nutrition in Traité de Pathologie génêrale," Tome iii, p. 179. 
In the following table, the "rest values" for $\mathrm{O}_{2}$ consumption and $\mathrm{CO}_{2}$ excretion, in various diseases, have been compiled.

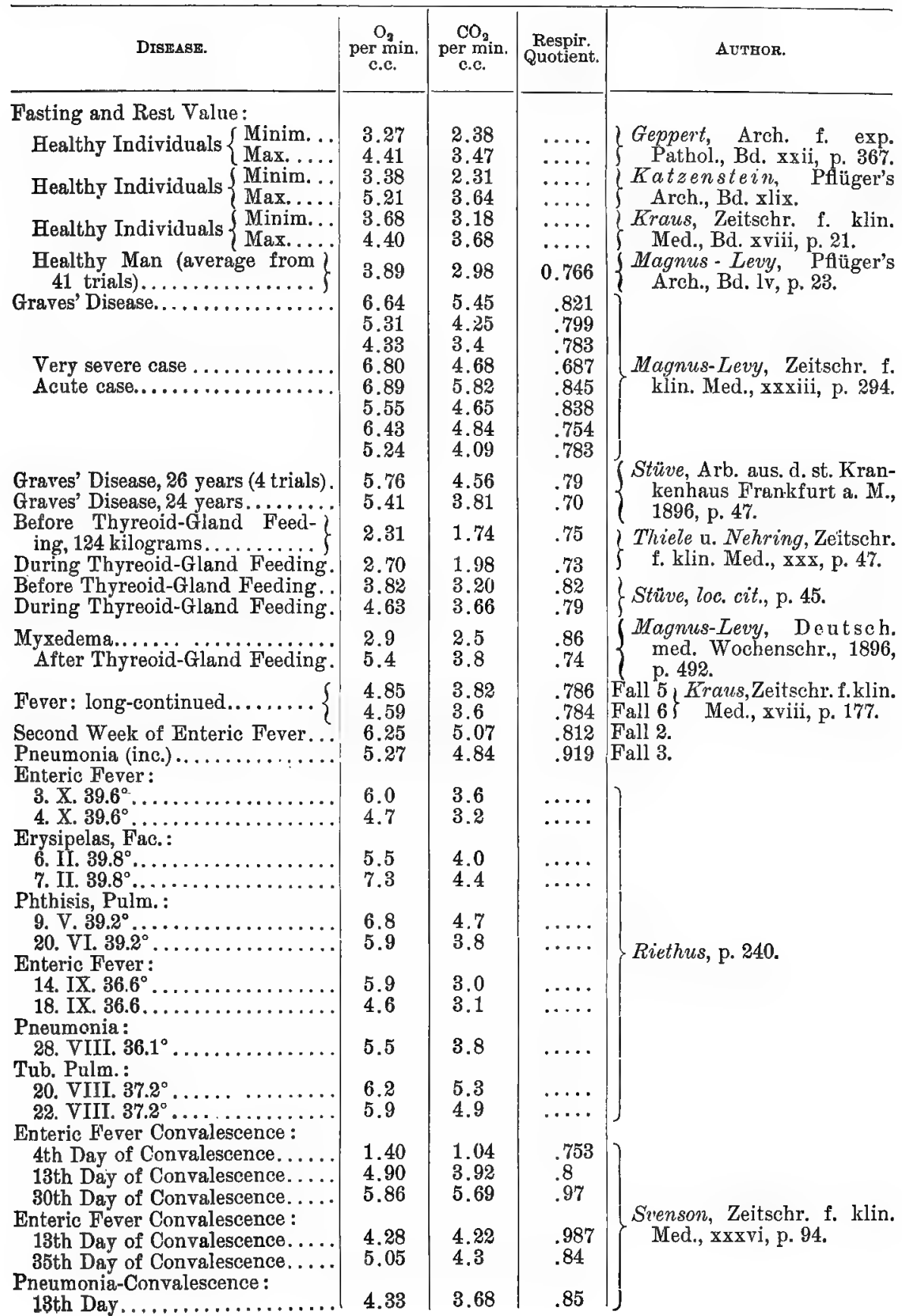




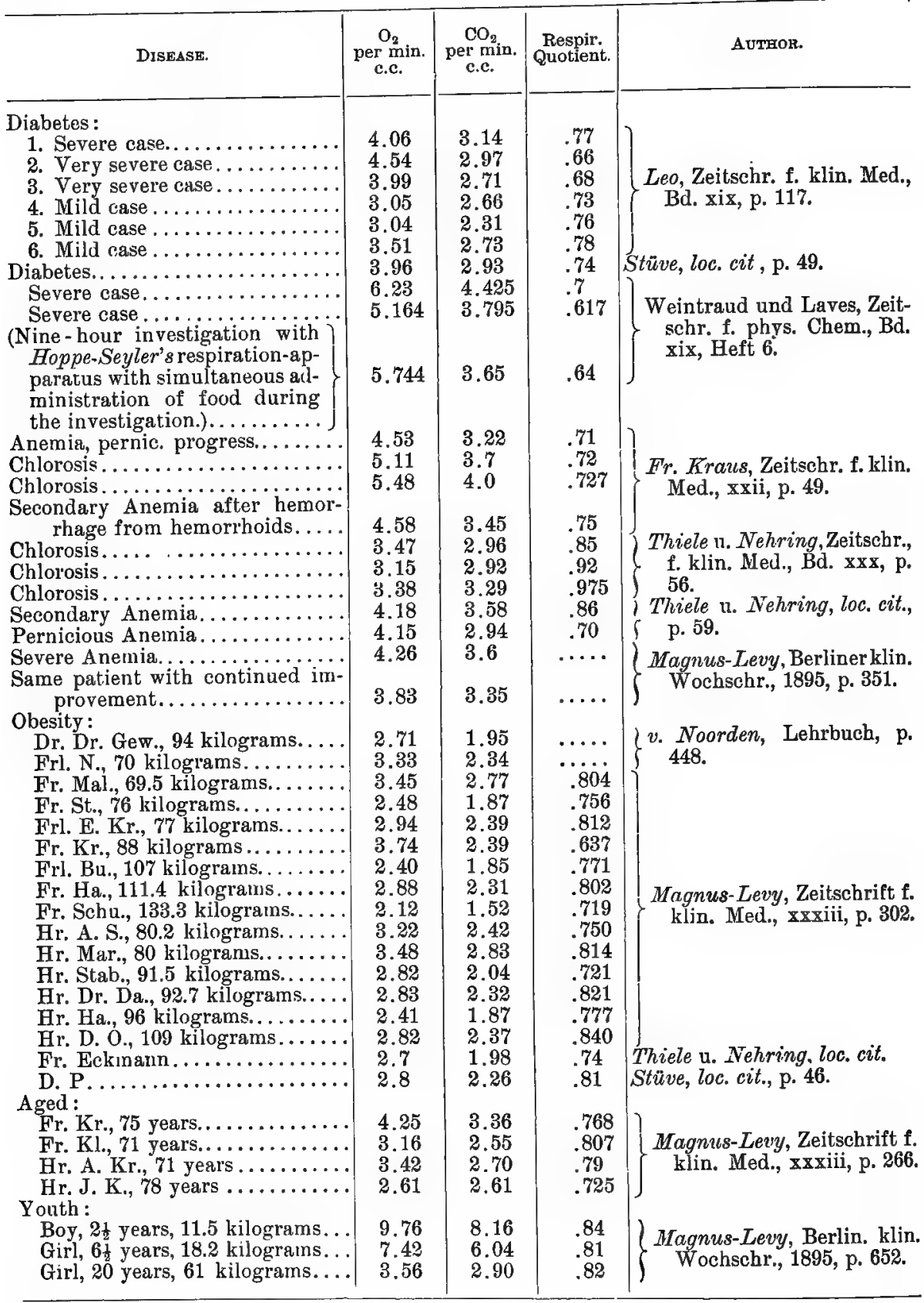

Among pathological conditions in which investigation of the respiratory metabolism has invariably shown an increase, GRAVES' DISEASE occupies the first place. 
Magnus-Lery, ${ }^{1}$ by his many valuable physiological researches in this realm, was the first to attempt to estimate the factors of respiratory metabolism in Graves' disease (Basedow's disease). He found the $\mathrm{O}_{2}$ intake and the $\mathrm{CO}_{2}$ excretion decidedly greater, even 50 per cent. greater, than the rest values of healthy individuals. At the same time his results showed that the administration of thyreoid extract in normal persons resulted in the same increase of the interchange of gases.

These investigations also furnished the first experimental proof that there are toxins in metabolism which cause increased activity in the cells of the body during rest, and this discovery furnished a clue for the understanding of the disturbance in metabolism observed in Graves' disease (emaciation, sweating, sensations of heat) as well as the clinical symptoms after thyreoid gland feeding, which are in many points similar.

A year before this practical use had been made of the effects of metabolism thus elucidated, when Leichtenstern ${ }^{2}$ determined the actual loss in weight of obese persons after thyreoid gland administration, and with this inaugurated a medical treatment for obesity.

The quantitative investigations in metabolism which followed the clinical advice of Leichtenstern, the loss of weight after thyreoid administration, and the attempts to analyze the constituents of this loss first demonstrated that there is a decided loss in proteids and water. But some of the investigators s soon observed that, even when $N$-equilibrium was completely maintained, there was decided loss in weight which could only be explained by loss of water and fat. Analysis of the respiratory metabolism showed an enormous increase in the intensity of the processes of oxidation, and thus confirmed the clinical observation that in the losses of weight which are observed during thyreoid feeding it is particularly the body fat which is subject to increased oxidation.

But it is certainly not on the body fat alone that the overflooding of metabolism with thyreoid secretions exerts its deleterious action in severe cases of Graves' disease.

Even if we take the largest increase over "rest metabolism" which has ever been observed in Graves' disease (about 50 per cent. or 1,800 calories), an additional consumption of 900 calories or about 100 grams of fat in the daily food would cover the loss. Yet as a matter of fact exact quantitative investigations in metabolism have shown that we are not always successful in maintaining the equilibrium of body-weight (Fr. Müller), ${ }^{4}$ even by a very profuse administration of nourishment, and only in mild cases, when conditions are favorable for a profuse intake of nourishment (good appetite), can the proteid constituents of the body be preserved (Scholz). ${ }^{\overline{5}}$

The etiologic relations and the clinical contrasts between Graves' disease

1 Magnus-Levy. Berl. klin. Wochenschr., 1895, Nr. 30.

2 Leichtenstern, "Ueber Myxödem und über Entfettungscuren mit Schilddrüsenfüitterung." Deutsche med. Wochenschr., 1894, Nr. 50.

${ }^{3}$ Grawitz, Münchener med. Wochenschr., 1896, Nr. 14.

4 Fr. Müller, Arch. f. klin. Med., vol. li, p. 36.

5 Soholz, Centralbl. $f$. inn. Med., 1895, Nr. 43. 
and MXXEDEMA (or sporadic cretinism) make it advisable to include these diseases here.

They are the only ones in which a diminution of the normal oxidation has been proven with certainty to be intimately connected with disease. The values which Magnus-Levy ${ }^{1}$ obtained in a case of sporadic cretinism (for $\mathrm{O}_{2}$ intake 2.8 to 3.0 c.c., for $\mathrm{CO}_{2}$ excretion 2.4 to 2.5 c.c., per kilogram and per minute) are near the lowest normal limits, and when taken in connection with the clinical symptoms (low temperature, diminished formation of sweat, inactivity of the muscles) the few analyses of metabolism which have been made justify the conclusion that in this instance a diminution of metabolism was actually present, and was due to the nature of the disease.

In no other diseases in which up to the present time the interchange of gases has been determined is the explanation of the results obtained so simple and clear as it is in Graves' disease and in myxedema. Nevertheless, the figures obtained have considerably modified the prevailing views regarding the intensity of the processes of combustion in special diseases, and by this means have broadened our insight into their pathogenesis, and have furnished us much help regarding their treatment.

For a long time it was supposed that in FEVER the processes of oxidation were increased. Nothing was more obvious than to connect the higher temperature of the body with an increased use of carbon-containing material in the body.

Regarding the degree of the increase of oxidation in the fever of human beings experimental investigations in animals could only give us imperfect standards $\left(\mathrm{May}^{2}\right)$. Researches which Kraus ${ }^{3}$ carried out in febrile human beings have, however, shown that fever is possible without decided increase of the oxidation processes (measured according to Zuntz's method which shows the relation of the factors of metabolism). This is particularly true of such individuals as have had fever for a long time-those suffering from inanition (Robin et Binet ${ }^{4}$ ).

In acute infectious fevers, the $\mathrm{O}_{2}$ consumption amounts to scarcely more than 20 per cent. above the normal. Previous to this, higher values have frequently been obtained for the increase in oxidation, but this has been due to accidental factors such as greater muscular activity owing to dyspnea, increased muscular tonus in the chill of fever, etc.

The-amount of increase in the interchange of gases, which Kraus and Riethus ${ }^{5}$ have shown to be attributable to some febrile infections, is suffi-

1 Magnus-Levy, Deutsche med. Wochenschr., 1896, p. 491.

2 May, "Der Stoffwechsel im Fieber," "Experimentalle Untersuchungen." Zeitschrift $f$. Biol., vol. xxx, p. 1.

3 Kraus, "Ueber den respiratorischen Gasaustausch im Fieber." Zeitschr. f. klin. Med., vol. xviii, p. 160 .

4 Robin et Binet, "Études cliniques sur le chêmisme respiratoire." Arch. génér. de méd., 1896, Juin et Octobre.

"Riethus, "Beobachtungen über den Gaswechsel kranker Menschen und den Einfluss antipyretischer Medicamente auf denselben." Arch.f. exp. Path. u. Pharm., vol. xliv, p. 239. 
ciently explained by the increase in proteid decomposition which forms part of every febrile process and, as it appears, bears a much more intimate relation to febrile processes than the increase in oxidation (Traube, Naunyn ${ }^{1}$ ).

The investigations which are constantly multiplying make it obvious that there is no constant and direct relation between the height of the temperature and the degree of the increase of oxidation. Febrile conditions occasionally show a conspicuously low consumption of oxygen, and afebrile cases conspicuously high.

The indications to be derived from this, in regard to the regulation of nutrition in fever patients, are obvious. It is not the absolute height of the fever which endangers the metabolism of the body, and for this reason it should not be our only care to study minutely the diet of our patients. On the contrary, it is the toxins produced by infection which prove injurious to the nutritive condition of the body, and in all cases in which the physician recognizes these deleterious consequences, he must arrange the dietary accordingly. Remembering this he will not disregard the dietetic indications of many afebrile infectious diseases (some forms of pulmonary tuberculosis).

In contrast with fever, in which the increased combustion causes much less difficulty than the toxogenous proteid decomposition (see below) is the nutrition of CONVALESCENTS. Here these difficulties are usually slight. Analysis of the diet, under which debilitated patients after long-continued under-nutrition are able to increase in weight, has frequently shown astonishingly low energy values. Fr. Müller ${ }^{2}$ in a case of stenosis of the esophagus after intoxication from caustic potash studied a fearfully emaciated patient, who gained 3.5 kilograms in three weeks, under a diet of only 24.7 to 30 calories per kilogram. Among G. Klemperer's ${ }^{3}$ patients there were some who throve very well on a diet containing only 13.5 to 18 calories per kilogram. A patient of Nebelthau's ${ }^{4}$ who was greatly emaciated in consequence of stubborn vomiting, accumulated very considerable amounts of albumin despite a quite insufficient proteid and calory administration after she had remained for four days without any food.

Without doubt the processes of oxidation are here decreased to a certain degree. The metabolism is diminished to the minimum which is absolutely necessary to maintain life. Correspondingly, the formation of heat is slight (tendency to chilliness), and the power of bodily activity is limited.

There is no objection to our assuming that this diminution of metabolism in chronic under-nutrition is a true slowing of metabolism. This makes it possible to maintain life even under very unfavorable external conditions, and is apparently produced gradually by adaptation and habit.

Sudden withdrawal of nourishment does not diminish the total metabolism

I Naunyn, Berliner klin. Wochenschr., 1869, Nr. 4.

2 Fried. Müller, "Stoffwechseluntersuchungen von Krebskranken." Zeitschr. f. klin. Med., vol. xvi, p. 496.

3 G. Klemperer, "Ueber Stoff wechsel und Ernährung in Krankheiten." Zeitschr. $f$. klin. Med., vol. xvi, p. 550.

${ }^{4}$ Nebelthau, "Ein Beitrag zur Kenntniss der Acetonurie." Centralbl. $f$. inn. Med., 1897, p. 977. 
to anything like the same degree. In the estimations which Zuntz and Lehmann ${ }^{2}$ carried out in the professional faster Cetti, the "rest-values" for $\mathrm{O}_{2}$ consumption and $\mathrm{CO}_{2}$ excretion during the days of starvation (on the average 4.78 and 3.34 c.c.) were but little lower than during the days preceding the fast $\left(5.35\right.$ c.c. $\mathrm{O}_{2}$, and 3.90 c.c. $\mathrm{CO}_{2}$ ). In investigations in the case of the faster Breithaupt, the average values prior to the fast and after it had begun were almost exactly the same.

Whether the ease with which patients convalescent from acute febrile disease compensate for their loss in weight depends upon the same capacity to economize in metabolism which enables patients in a state of chronic undernutrition to gain weight even on a diet of low calory value is a mooted question. Svenson ${ }^{2}$ has recently thrown light upon this question by investigations of metabolism in convalescence from pneumonia and from enteric fever.

His figures (see Table on page 27) do not show a tendency to economy in the processes of combustion. On the contrary, in the first afebrile days after a severe enteric fever, he found a slight diminution of the respiratory values; true convalescence with increase in weight was being characterized by high values, and metabolism was therefore increased. Hence the increase in weight cannot be explained by assuming a diminution of consumption. On the contrary, the balance is positive because the intake of nourishment with the usual increased appetite of convalescence is decidedly greater than the energy value of the average food of maintenance (60 to 90 calories per kilogram).

The "rest-value" for metabolism in convalescence then is in any case increased and this is particularly true of the metabolism after work, as was shown by Svenson's further researches. The convalescent is able to utilize in muscular work only a small portion of the energy set free by the processes of combustion, and therefore has a higher $\mathrm{CO}_{2}$ consumption per kilogram meter than the healthy man. There is no economy of metabolism here.

Diabetes MELlitus was for a long time looked upon as a disease in which metabolism was increased. The enormous ingestion of food which, because unsuitable, is often insufficient (in spite of its great energy value) to satisfy the appetite which accompanies diabetes mellitus, and to maintain nutrition, naturally led to this view, at a period when a deeper insight into the economy of metabolism of the diabetic was not yet possible.

The quantitative analyses of metabolism which, because of therapeutic experiments in diet, were carried out especially often in diabetics, soon showed, however, that with suitable food, the usual calory intake was sufficient to maintain the equilibrium of metabolism even in severe diabetes.

The diabetic perversion of metabolism cannot be due to an increased interchange of products since it is possible with a diet which contains only 25

1 Zuntz und Lehmann, Virchow's Arch., vol. exxxi; Suppl.-Heft, pp. 50 und 91.

2 Svenson, "Stoffwechselversuche an Reconvalescenten." Zeitschr. $f$. klin. Med., vol, xliii, p. 86 . 
calories per kilogram to maintain the patient for weeks in an active condition. ${ }^{1}$

On the contrary, observation proves that metabolism in the diabetic may occasionally be abnormally slow, and the experience accumulated by many careful investigations of the nutrition of diabetics demonstrates that, in many cases, it is in fact slower than the metabolism of a healthy person. I say in many cases, for this is by no means true of all. A pathologic diminution of the nutritive requirement is by no means the rule in diabetes. If, however, we give for a long time a diet which is apparently superfluous but, in reality, quite insufficient-because improper-under the influence of this chronic under-nutrition the same diminution in metabolism may occur that also occurs in other persons who are under-nourished (sce above). Hence it becomes possible to support life on less food than is necessary for healthy people and to increase the diabetic's weight with a diet which is only just sufficient for the needs of a normal person.

Correspondingly, the rest values for $\mathrm{O}_{2}$ consumption and $\mathrm{CO}_{2}$ excretion that have been determined in diabetes neither exceed nor fall below the limits which we have come to recognize in the normal individual (Voit and Pettenkofer, Leo, ${ }^{2}$ Weintraud and Laves, ${ }^{3}$ Stüve ${ }^{4}$ ).

The same is proven by the investigations of Magnus-Levy in Gour, which Bouchard, as is well known, includes with diabetes among diseases characterized by decreased metabolism.

In the various forms of ANEMIA (chlorosis, secondary and pernicious anemia) we might expect diminution of $\mathrm{O}_{2}$ intake, in view of the more or less marked diminution of the oxygen carriers in the blood, especially if we remember the fatty degeneration of organs, which is frequently observed in anemia.

Experimental investigation, however, has decided against this view. Indeed the values determined for $\mathrm{O}_{2}$ intake and $\mathrm{CO}_{2}$ output have been shown to be near the upper physiological limits. In those cases of pernicious anemia which are characterized by increased proteid decomposition, this limit has even been several times exceeded (Meyer, ${ }^{5}$ Magnus-Levy). ${ }^{6}$ A diminution of metabolism due to impoverished blood cannot therefore be assumed (Kraus, Thiele and Nehring). ${ }^{8}$

1 Weintraud, "Untersuchungen über den Stoffwechsel im Diabetes melitus." Bibl. med., Cassel, 1893.

2 Leo, "Ueber den respiratorischen Stoffwechsel und Diabetes." Zeitschr. f. klin. Med., vol. xix.

3 Teintraud und Laves, "Ueber den respiratorischen Stoff wechsel im Diabetes." Zeitschr. f. phys. Chemie, vol. xix.

4 Stüve, Arbeiten aus dem städt. Krankenhaus Frankfurt a. M., 1896, p. 49.

5 R. Heyer, "Ueber $\mathrm{O}_{2}$ Verbrauch und $\mathrm{CO}_{2}$ Ausscheidung bei Anämien." Dissert., Bonn, 1892.

6 Magnus-Levy, Berliner klin. Wochenschr., 1895, p. 351.

7 Kraus, "Ueber den Einfluss von Krankheiten, besonders von anämischen Zuständen, auf den respiratorischen Gaswechsel." Zeitschr. f. klin. Med., vol. xxii, p. 449.

${ }^{8}$ Thiele und Nehring, Zeitschr. f. Klin. Med., vol. xxx. 
It is remarkable that in certain forms of oBESITY no diminution of the physiologic processes of combustion can be determined. Clinicians have noted two classes of cases, $(a)$ those in which overeating and deficiency of muscular labor were the obvious causes of the accumulation of fat, and $(b)$ those in which even a normal amount of food resulted in accumulation of fat, and even with a decided diminution of nourishment it was impossible to bring about a reduction in weight.

v. Noorden ${ }^{1}$ reports the case of a man weighing 102 kilograms who-in spite of the fact that his food during three months did not exceed 1,720 calories per day, and that as an inspector of a country district he had every day plenty of exercise-lost only a kilogram of weight in three months. In a lady weighing 86 kilograms he failed to produce the slightest diminution in weight in the course of six weeks, though he reduced the food to 900 to 1,000 calories. In a woman weighing 145 kilograms, Stadelmann ${ }^{2}$ reports that with a diet containing but 1,500 calories per day she not only throve but even gained one kilogram per week in weight. Only when nutrition was reduced to 1,000 calories did she begin to lose weight, while on 1,200 calories she gained in weight for a time. (Compare the criticisms of these reports in Rubner.) ${ }^{3}$

In the light of these observations the question constantly arises, whether the tissue elements of obese persons do not require a slighter amount of material to perform their normal functions, and whether the very ready accumulation of fat is not due to the fact that the organs function more economically than under normal conditions. $\nabla$. Noorden was the first to study this question by exact experimental investigation, and in his excellent text-book, The Pathology of Metabolism, which stimulated so many later researches in metabolism, he worked out in two cases of obesity the first figures representing the consumption of oxygen and the excretion of carbonic acid. (See Table.)

Researches by Magnus-Levy followed these (Table). All the "minutekilo-values" taken with an empty stomach are low, in fact near the lowest limit of the normal standard figures. "But they are not so low that a diminished oxidation energy of the cells must be assumed, especially if we consider that the values per kilogram as calculated in the obese become lower the more fat the body accumulates. But in metabolism during quiet respiration this factor is not operative. In studies regarding the intensity of the process of combustion fat cannot be looked upon as of the same value as flesh and gland substance" (Magnus-Lery). Thus when it appeared that analysis of the respiratory metabolism (according to the Zuntz-Geppert method) would yield no support for the belief that there is a diminution of the processes of combustion in the obese, new researches by Jaquet ${ }^{4}$ suggested the method by which in the corpulent an economy of the food material introduced produces the gradual deposition of fat.

¿ $v$. Noorden, "Die Fettsucht," in Nothnagel's Handbuch, p. 31.

2 Stadelmann, Berliner klin. Wochenschr., 1901, Nr. 25.

3 Rubner, Beiträge zur "Ernährung im Kindesalter," Berlin, 1902, p. 31.

4 Jaquet und Svenson, "Zur Kenntniss des Stoffwechsels fettsüchtiger Personen." Zeitschr. f. klin. Med., Bd. xli, p. 375. 
According to a diagram proposed by Magnus-Levy ' the total metabolism of an individual may be considered to be composed of three factors:

1. The entire metabolism in rest and with an empty stomach.

2. The metabolism which is necessary to sustain the work (glandular and intestinal) that results in the assimilation of food introduced.

3. The metabolism which is necessary for useful or useless movements of the body.

Metabolism thus estimated amounts :

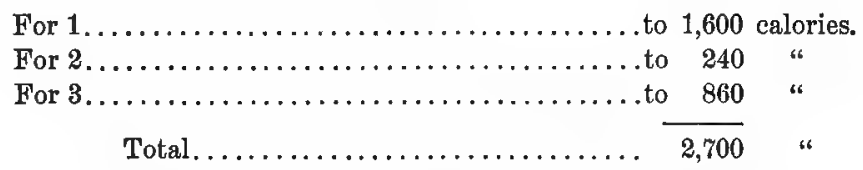

In fact it is unlikely that an obese person requires less energy than a healthy person for the maintenance of his vital functions, for normal activity of the heart and respiration, for the rest metabolism of the glandular activity and for the maintenance of body heat. v. Noorden mentioned the possibility that for 3 he saves something, because the development of heat which accompanies physical exercise per kilogram of working protoplasm and per kilogram-meter of physical exercise is less in him than in the average person, but Magnus-Levy expressed his doubts of this. It was therefore particularly interesting that Jaquet found the value for 2 diminished in his corpulent patient. The increase of the products of combustion due to ingestion of nourishment was decidedly less and of shorter duration in his three obese patients than in normal persons. It was shown that during a period of digestion reckoned as fourteen hours, the obese require 21.84 liters $\mathrm{O}_{2}$ less than a normal person under similar circumstances. With this amount of $\mathrm{O}_{2}, 11$ grams of fat can be burned, and a daily saving of 11 grams of fat corresponds to an accumulation of 4 kilograms of fat a year. Thus the analysis of respiratory metabolism opens a path for the understanding of the pathogenesis of constitutional obesity.

Practical rules for the nutrition of the sick cannot be immediately deduced from these clinical studies regarding the amount of respiratory metabolism in the diseases which have been mentioned, valuable as they are for our knowledge of disturbances of metabolism.

The knowledge of $\mathrm{O}_{2}$ consumption and $\mathrm{CO}_{2}$ excretion, conveyed to us in the clinical investigations which are almost exclusively carried out by Zuntz's method, with simultaneous estimation of albumin metabolism (by N-estimation in the urine) can give at most but approximate conclusions regarding the part played by individual food substances in combustion. The respiratory quotient gives us some information but the absolute value of the transference of energy in a unit of time cannot be calculated from the "minute-values" for $\mathrm{O}_{2}$ and $\mathrm{CO}_{2}$, because, as was mentioned above (page 8 ), the same amounts of carbonic acid and oxygen correspond to entirely different degrees of heat,

1 Magnus-Levy, Zeitschr. f. klin. Med., Bd. xxxiii, p. 299. 
according as we are dealing with combustion of albumin, of fat, or of carbohydrates.

A complete, correct calculation of force-transference and heat-transference is only possible if the excretion of carbon and of nitrogen is minutely investigated for a considerable period of time, and then from both of these the implication of proteids and of fat is estimated in metabolism. From the hour or minute values, fixed conclusions cannot be drawn regarding the total calory requirement; they are therefore not final. We may hope, however, that prolonged investigations of the interchange of gases (with Pettenkofer's or, even better, with Hoppe-Seyler's apparatus) will give us more valuable conclusions for pathology, and decidedly broaden our knowledge regarding total metabolism.

The cases in which the entire interchange of forces in pathologic conditions has been determined by sufficiently exact technical investigations are up to the present time very few. We owe them mostly to the labors of Rubner.

In his researches in artificial nutrition carried on with Heubner ${ }^{1}$ in a normal and in an atrophic nursling, intake and output were directly estimated in every way, and by calorimetric analysis it was possible to determine the actual figures for the interchange of forces. It was shown that there is no abnormal form of decomposition (force-transference) in the atrophic child. The diet necessary for maintenance in the atrophic child and in the healthy nursling correspond very well if we make allowance for small differences in digestion and in the temperament of the children (influence of body rest, motion and sleep). If they are given an excess of food, both utilize about the same percentage for heat production, and about the same percentage is deposited in the tissues. The only peculiarity of the atrophic child is a lessened power of intestinal absorption, and hence a more profuse production of feces.

In a technically complete investigation of metabolism in an obese child, Rubner ${ }^{2}$ was unable to determine ariy diminution of interchange of forces. Despite his pathologic predisposition, the boy required no less food than a healthy boy of the same weight tested by Camerer.

On the other hand characteristic differences in the processes of decomposition were found by Sonden and Tigerstedt ${ }^{3}$ in their investigations of metabolism in men of various ages. They conclude from these that there is a greater life energy during youth, in contrast to the metabolism of the aged, in which a lessened consumption may be recognized. The heat production amounted:

In boys of 35.2 kilos weight................. to 1,322 calories

In men of 67.8 kilos weight..................... to 1,016 "

In an aged man of 62.3 kilos weight............. to 924 "

1 Rubner und Heubner, "Die natürliche Ernährung eines Säuglings." Zeitschr.f. Biol., Bd. xxxvi, p. 1. "Die kïnstl. Ernährung eines normalen und eines atrophischen Säuglings." Zeitschr. f. Biol., Bd. xxxviii, p. 315 .

2 Rubner, Beiträge zur " Ernährung im Knabenalter mit besonderer Berücksichtigung der Fettsucht," Berlin, 1902.

3 Sonden and Tigerstedt, "Untersuchungen iber die Respiration und den Gesammtstoffwechsel des Menschen." Slkand. Arch. f. Physiol., 1895, Bd. vi. 
In view of the criticisms which Camerer and Rubner have devoted to these investigations we shall, however, withhold acceptance from the conclusions which Sonden and Tigerstedt have drawn from their researches in regard to the varying intensity of the processes of metabolism in the young and in the aged. (See Table.)

\section{THE MAINTENANCE DIET AND THE PROTEID REQUIREMENT OF THE SICK}

Besides the complete technic and investigations which can be carried on by means of complicated apparatus, we can use at the bedside a simpler method to determine the total metabolism, viz.: the calculation of the tension power, based upon the food which has been consumed. The demonstration in Rubner's studies that to supply suitable energy the food of man must be adapted to his condition of life permits the conclusion that if the individual, although ill, maintains his body-weight, the supply of force present in his food corresponds with his metabolism (interchange of force).

The calculation of the energy value of food from the standard figures given by Rubner is simple, and is in general use to-day. The only question that arises is whether the control of the body-weight and the frequent computation of the $\mathrm{N}$-balance is sufficient to indicate all important changes in the material condition of the body.

But for the clinical pathology of metabolism this is certainly sufficient, and with the proviso that this assumption is justifiable, the quantitative analyses of metabolism in patients, now coming into common use, give us valuable points for the administration of food suitable for the maintenance of the organism in special diseases, either hypernutrition or hyponutrition.

A few of these investigations have been mentioned. It would extend this article too much if I were to enumerate them all; and some of them will be referred to later. (See absorption investigations.)

v. Noorden's systematic tests of the energy value of food and his methods of producing a rapid increase in weight, will be described explicitly.

In eight women who were confined to their beds weight began to increase when the food reached the following values: $26,26.5,19,32,33,36,36,38.5$, 39 calories per kilogram and per day. Upon the average 32 calories per kilogram.

In five women who were out of bed for two to three hours during the day, increase in weight began when the value of the food reached $28,31.5,34,36.5$, 36.5, 40 calories per kilogram and per day. Upon the average 34 calories.

In four women who spent most of the day out of bed, and occasionally walked about in the garden, increase in weight began when the value of food reached $32,37,3 \%, 41$ calories. Upon the average 37 calories per kilogram.

Although these figures demonstrate that increase of weight occasionally occurs even when the calory value of the food is very slight ( $2 \%$ calories), nevertheless v. Noorden cautions us that food in which the calory value is less than 30 calories per kilogram should not be used. The increase in weight with small calory values is usually limited to patients in whom prolonged undernutrition has led to a decided diminution of the amount of water in the body, 
so that the increase in weight observed may be referred more to a satiation of the demand of the tissues for water than to an actual gain in substance. ${ }^{1}$

Yet it cannot be doubted, in view of these experiments, that, in emaciated individuals, administration of food of very slight calory value may compensate completely for the food requirement and the interchange of force, and may even permit an increase in weight. For if, in spite of a slight calory administration, $\mathrm{N}$-equilibrium has been maintained for a long time and albumin actually accumulated, there cannot be a calory deficit for any length of time. Later investigations in metabolism show that the $\mathrm{N}$-balance is to a great extent independent of the calory supply. The stimulation of albumin accumulation which results from muscular exertion as well as from growth makes possible an $\mathrm{N}$-retention even with an insufficient calory supply and an actual loss in weight (Caspari ${ }^{2}$ ).

But there are many observations showing that after prolonged undernutrition, such as occurs in disease, the body may, up to a certain point, adapt itself to a smaller amount of food. It limits itself to the functions absolutely necessary to maintain life, and under these conditions the slightest increase in food soon brings about an increase in weight as well as a retardation of the pathological processes, under the action of which the body has become so reduced. The lessening of body-weight, upon whose maintenance, according to Pflüger, the energy of metabolism is dependent, becomes here the direct cause of a diminished rate of metabolism.

The emaciation which is the invariable consequence of many diseases finds its chief expression in the decrease of body-weight, so that frequent weighings of the body in chronic diseases are, as Gerhardt says, almost as valuable as the temperature record in acute diseases. The variations may, however, be due to various causes.

When patients suffering from gastric disease are emaciated this is often due wholly to the poverty of the calory value of their food, as was first emphatically stated and clearly proven by $\nabla$. Noorden. The fear of pain, or a false estimation of the nutritive value of individual foods, may have aided in bringing this about. If food of normal calory value be given, the loss in weight ceases.

Other patients (Graves' disease), as we have noted, lose weight, in spite of a calcry intake sufficient for the healthy, because the intensity of the process of combustion is decidedly increased. If the capacity for taking food is great, as in many afebrile tubercular patients and in many cases of Basedow's disease, the calory contents of their food need only be increased beyond the usual needs of the healthy, and they will not only attain their N-equilibrium, but will very soon begin to accumulate fat.

This, however, does not succeed in all cases. Fr. Müller ${ }^{3}$ was the first

1 v. Noorden, "Stoffverbrauch und Nahrungsbedarf in Krankheiten." Arbeiten aus dem städt. Krankenhaus zu Frankfurt a. M., 1896, p. 3.

2 Caspari, "Leber Eiweissumsatz und Ansatz bei der Muskelarbeit." Pflüger's Arch., Bd. lxxxiii, p. 509.

з Fr. Müller, "Stoffwechsel bei Krebskranken." Zeitschr. f. klin, Med., Bd. xvi, p. 496 . 
to note in his cancer patients that in malignant cachexia, even with a plentiful calory supply (with sufficient albumin elements in the food), it was often impossible to attain $\mathrm{N}$-equilibrium. Gärtig ${ }^{1}$ confirmed these reports.

Here a toxin damages the protoplasm of the cells, and the albuminous débris of cells, decidedly beyond the requirements of the pathologic organism for albumin, enters the circulation, there to suffer complete destruction.

In fever patients, in whom the extraordinarily high urea figures obtained by former investigators (Traube and Naunyn) were for a long time emphasized, we now have numerous analyses of metabolism some of which show that the destruction of tissue in acute cases of fever cannot be entirely prevented by any mode of nutrition (v. Leyden and Klemperer ${ }^{2}$ ).

The same is also true of certain severe forms of anemia. Yet the increased decomposition of albumin does not always occur in impoverished conditions of the blood. We know this from the researches of $v$. Noorden, who conducted exact analyses in metabolism in severe chlorosis, which prove beyond doubt that such patients, with the ordinary calory supply and without particularly large amounts of albumin, are able to maintain their N-equilibrium (Lipmann-Wulff ${ }^{3}$ ). On the other hand, there are certain clinical cases characterized by a severe course, the so-called pernicious anemias, some of which are to-day explained by the presence of intestinal parasites; in these the proteid metabolism is pathologically increased. Even the first clinical observers of the disease (Strümpell, Eichhorst) found in these patients high urea values, though of course these figures, without simultaneous observation and analysis of the food, did not prove anything. Exact analyses of metabolism which Rosenquist lately carried out in patients with bothriocephalus-anemia have determined with certainty that, at any rate in this form of pernicious anemia, there is an increased decomposition of albumin. These researches constitute conclusive proof of the law of so-called toxogenous decomposition of albumin, for they illustrate how, shortly after the expulsion of the parasites (five to six days later), an accumulation of albumin, formerly impossible, was attained. As the blood changes here depend upon toxic action, after the removal of which normal regenerative changes take place, so also the pathologic decomposition of albumin may be readily stopped by expelling the parasites.

The indications for preventing the threatening tissue destruction in febrile and cachectic patients are much more obscure. Yet this is often of paramount importance in dietotherapeutic endeavors.

Practical experience shows that the two methods which improve the albumin balance in the healthy are also applicable here. By the administration of a liberal albumin diet, the N-loss may be diminished during fever (Bauer and Künstle); the same result can be obtained by giving a profuse calory supply of $\mathrm{N}$-free substances. For both processes excellent methods are found

1 H. Gärtig, "Untersuchungen über den Stoffwechsel in einem Fall von Carc. Esophagi." Diss., Berlin, 1890.

2 v. Leyden und G. Klemperer, "Ernährungstherapie in acuten Fieberkrankheiten." "Handbuch d. Ernährungsther.," Bd. ii, p. 408.

3 Lipmann-Wulff, "Ueber Eiweisszersetzung bei Chlorose." Diss., Berlin, 1891.

4 Rosenquist, Berl. klin. Wochenschr., 1901, p. 666. 
in the tables of v. Leyden and Klemperer (loc. cit.). That the carbohydrates in themselves are superior to the fats as albumin savers (see page.21) and that they are especially valuable in the diet of fever and cancer patients as compared with albumin and fat (the administration of which is frequently limited by an unconquerable repugnance and by difficulty in digestion), is clearly shown by experience at the bedside as well as by experimental study. In fever artificially produced May ${ }^{1}$ found a limitation of the combustion of albumin as a result of the profuse intake of carbohydrates.

In spite of the pathologically increased toxogenous decomposition of albumin the laws which normally control the metabolism of albumin are still active in wasting diseases. This fact should always be borne in mind, and measures based upon it should be employed in the dietetic treatment of such cases. Hirschfeld ${ }^{2}$ succeeded by forced feeding in attaining an $\mathrm{N}$-accumulation in tuberculous febrile cases. $\nabla$. Noorden, in his text-book, mentions quite a number of patients with temperatures of over $101.3^{\circ} \mathrm{F}$. in the late weeks of typhoid cases running a slow course, also patients with sepsis and pulmonary tuberculosis, who on a diet rich in albumin but not of particularly high calory value were able, at least for two weeks, to maintain their bodyweight.

Whether nitrogen-equilibrium actually existed in these cases cannot be determined from the figures given. There is a great difference as regards the $\mathrm{N}$-balances in febrile and those in non-febrile convalescents for, with the same calory supply, we can produce in afebrile cases a decided gain in albumin while in febrile cases we can scarcely maintain the nitrogen-equilibrium.

The "intoxication necrosis" of the tissues which occurs in febrile diseases as well as from under-nutrition, and the deleterious effects of which cannot be prevented by any known means, is followed in convalescence by a remarkable endeavor of the enfeebled cells to recuperate. They attempt to gorge themselves with albumin, and the balance of metabolism which terminated in a nitrogen deficit suddenly begins to show an N-retention (Dünschmann, v. Leyden and Klemperer, loc. cit.).

The great rapidity with which this $\mathrm{N}$-retention may occur despite a normal calory supply and normal proteid supply has been noted in numerous studies of metabolism. ${ }^{3}$ In this connection Lüthje's ${ }^{4}$ latest experiments in forced feeding have shown what can be done by means of abundant food during convalescence. In his studies of convalescents from enteric fever, $\mathrm{N}$-retention of from 10,12 to even 14 grams of $\mathrm{N}$ per day, corresponding almost to 100 grams of albumin within twenty-four hours, was repeatedly observed.

This $\mathrm{N}$-addition is all the more remarkable because, in my opinion, we must assume that the retained nitrogen is utilized for the production of living protoplasm, and therefore is utilized in the total metabolism.

1 May, "Der Stoffwechsel im Fieber." Zeitsohr. f. Biologie, Bd. xxx, p. 41.

2 Hirsohfeld, Deutsch. Arch. f. klin. Med., 1882, Bd. xxx, p. 28.

$3 A l b u$, "Ueber den Eiweissstoffwechsel bei chronischer Unterernährung." Zeitschr. f. klin. Med., Bd. xxxviii, p. 250 ; here also the other literature.

4 Lïthje, Beiträge zur "Kenntniss des Eiweissstoffwechsel." Zeitschr. f. klin. Med., Bd. xliv, p. 22. 
With such an amount of $\mathrm{N}$-retention there is no possibility that we are producing only a temporary retention of $\mathrm{N}$-containing products of metabolism (proteid destruction). Further such an enormous increase cannot take wholly the form of circulating albumin (in Voit's sense). Neither can we call it "reserve albumin" (v. Noorden), if this term is meant to imply that the albumin accumulated during convalescence is a different kind of albumin from, and subject to other laws of destruction than, the ordinary albumin of the organs.

In the person experimented upon by Lüthje, whose diet for twenty days averaged 62.5 grams of $\mathrm{N}, 1$ after a reduction to 16.7 grams $\mathrm{N}, \mathrm{N}$-equilibrium was attained, not at once indeed, but within three or four days; and even after eight days, when the research was stopped, but small losses of nitrogen were shown. ${ }^{2}$ In view of the obvious tendency toward $\mathrm{N}$-equilibrium in this case there is no reason to assume that, in the subsequent period, all the nitrogen (166.78 grams) which had accumulated during twenty days of the albumin period was again lost. At any rate, there was no loss in weight, although the patient after leaving the clinic and while living on a freely chosen diet probably did not consume a very large quantity of albumin.

Apparently then the amount of his living cell substance probably remained large. Consequently, in later investigations in the same person larger quantities of albumin were required to bring about a large N-retention, a phenomenon quite in accord with the views of Pflüger, according to which the degree of the albumin metabolism is dependent upon the amount of living cell substance of the body.

We are, therefore, justified in holding the view that the $\mathrm{N}$-retention in convalescents is not due to the retention of $\mathrm{N}$-containing urinary products of metabolism, but to an actual accumulation of albumin which is equivalent to an increase of living protoplasm and, in a certain sense, to an increase in the activity of the body. Moreover, we must not always consider the muscles of the body alone, and for this reason the designation, "muscle-food," had better be avoided. Richness in albumin (increase of the juices of the flesh) is only the necessary pre-requisite for great power in the musculature, and it is exercise and only exercise which increases power, on which capacity for work is directly dependent.

On the other hand a decided retention of nitrogen, which -does not mean an accumulation of albumin (or an increase of living protoplasm), is frequently met with in renal diseases. Disturbance of the nitrogen-equilibrium is usual in these affections.

It is evident without further elucidation that in nephritis which is a consequence of disease of the excretory organs urinary substances are prone to remain in the body, and we are therefore not far wrong, when we find relatively small amounts of nitrogen in the urine (in comparison with the

1 Corresponding to the enormous administration of 390 grams of albumin per day.

2 For which the further decrease of $\mathrm{N}$-administration to 15.6 grams $\mathrm{N}$ is to be made partly responsible. 
amount of nitrogen administered in the food), in concluding that the cause is deficient excretion of nitrogenous products of albumin metabolism (Fleischer, ${ }^{1}$ v. Noorden and Ritter ${ }^{2}$ ).

Because of this fact, as well as because days with increased $\mathrm{N}$-excretion often follow these periods of decided N-retention, the N-elimination in nephritis is in a very " unaccountable and bizarre state which, according to v. Noorden, characterizes the metabolism of renal patients."

But we are here dealing wholly with disturbances of excretion; there are no changes in the amount of albumin metabolism (no toxogenous albumin decomposition).

In investigations of the metabolism of gouty patients we are often confronted with irregularities in the excretion of urea, apparently in consequence of functional insufficiency of the organs of excretion, which is natural if we remember that renal disease often complicates gout (Vogel, ${ }^{3}$ Schmoll ${ }^{4}$ ).

It is interesting to note that Rosemann ${ }^{5}$ lately found in a healthy, or apparently healthy, young man during a long investigation in metabolism carried out for a different purpose, a decided $\mathrm{N}$-retention with subsequent increased excretion of urea. In this case there were no sufficient grounds for the assumption of any renal disease. On the other hand, the person experimented upon had suffered for years from a cutaneous affection (occasional attacks of urticaria), and it is conceivable that, in consequence of this, the excretion of water through the kidneys had in the course of years become diminished in favor of that through the skin; and that, therefore, the $\mathrm{N}$-elimination by means of the urine had suffered. However, the excretion of water in the urine during the period of $\mathrm{N}$-retention was never so slight in this case that the urine could not have excreted more nitrogen. Rosemann, therefore, expressed the opinion that a prolonged ingestion of iodin was responsible for the disturbance of $\mathrm{N}$-excretion (the person experimented upon had suffered from a cutaneous affection for years, and up to within fourteen days of the test had taken potassium iodid), but at the present time proofs are wanting that the secretory activity of the kidneys can be thus influenced by the use of iodin, even for a long time.

I believe that every one who has made many quantitative researches in metabolism has occasionally met with healthy persons in whom, in spite of the most careful regulation of the amount of the food, it was impossible to attain $\mathrm{N}$-equilibrium. In such persons the daily $\mathrm{N}$-excretion constantly varies up and down when we are attempting to get at the average figures. Occasionally there are periods of relatively low excretion lasting several days

1 Fleischer, Klinische und pathologisch-chemische Beiträge zur "Lehre von den Nierenkrankheiten." Deutsches Archiv f. klin. Med., vol. xxix, p. 129.

$2 v$. Noorden und Ritter, "Untersuchungen über den Stoff wechsel Nierenkranker." Zeitschr. f. klin. Med., vol. xix, p. 197.

3 Vogel, v. Noorden's Beiträge zur "Lehre vom Stoffwechsel," 1894, Heft 2, p. 113.

4 Schmoll, "Stoff wechselversuche an einem Gichtkranken." Zeitschr. f. klin. Med., vol. xxix, p. 510 .

5 Rosemann, "Ueber die Retention von Harnbestandtheilen im Körper." Pflüger's Archiv, vol. lxxii, p. 467 . 
followed by similar periods of increased $\mathrm{N}$-excretion. This phenomenon is particularly frequent and familiar when the subject is on a régime rich in albumin (more than 20 grams of $\mathrm{N}$ in the daily food).

In contrast with these irregularities of the excretion of nitrogen in the urine which occasionally occur in the healthy, and more frequently in renal disease and in gout, we have the N-retention which is observed in cachectic patients during the formation of transudates and edemas.

Concerning this quite a number of investigations have been made, particularly in patients with hepatic cirrhosis. Several times during the reaccumulation of an ascites (after paracentesis) the nitrogen balance has been estimated (Schubert, ${ }^{1}$ Marischler and Ozarkiewicz ${ }^{2}$ ). That the $\mathrm{N}$-retention which is noted in these cases does not indicate an actual albumin accumulation is clear. The retained nitrogen is not utilized as albumin to build up tissue but goes to form edema or ascites. Authors have therefore often spoken of a "pathological" N-retention, and explained it by the hypothesis that while the organism normally possesses the faculty of destroying albumin and digesting it, this function is now lost. Most probably, however, purely mechanical disturbances of absorption here play a rôle in causing the retention of $\mathrm{N}$. These disturbances depend upon changes in the amount of mineral salts in the fluids of the body, the osmotic changes being dependent upon variations in the concentration of these salts which cooperate with the active properties of the endothelia to govern absorption.

There is no considerable interest in the often-discussed question whether the albumin in these re-accumulating transudates (ascites) comes from the albumin of the food, or whether the body albumin is utilized. The question becomes meaningless if we discard Voit's differentiation of two varieties of albumin in the body (organic albumin and circulating albumin). This decision cannot be arrived at by analysis of the mineral metabolism in such cases, for the albumin of the food has been absorbed, taken up into the fluids of the body, and thence also into the cell protoplasm; thence together with the organic albumin it issues in the fluids of pathological transudates.

\section{DISTURBANCES IN THE ABSORPTION OF FOOD}

In determining the food requirement of a patient, we must consider ( $a$ ) changes in the amount of oxidation due to the disease, $(b)$ consumption of albumin due to toxic influences, and (c) other factors, important among which are the losses of energy which the body suffers by giving off food substances which it should retain.

These losses are so manifold and so various that it is impossible to place them side by side as equivalents. Some of these losses are not susceptible to quantitative estimation; on the other hand, in so far as they are due to

1 Schubert, "Ueber den N- und Cl-Umsatz während der Bildung und nach der Punktion des Ascites bei Lebercirrhose." Dissert., Breslau, 1895.

2 Marischler und Ozarkiewicz, "Stoffwechsel bei abnehmendem und zunehmendem Ascites," Arch. f. Verdauungskrankh., vol. v, p. 222. 
faulty absorption of food-and this is to be especially discussed here-they can be well controlled and have in fact been thoroughly investigated.

The loss in material which the body suffers from profuse sweating is relatively slight. But the heat equivalent that corresponds to the evaporation of one liter of sweat amounts to no less than 580 calories. We are justified, therefore, in speaking of " exhausting sweats."

The food requirement of the patient is decidedly increased by muscular work. We know that even simple muscular tension, without any accompanying effort, is expressed in an increase of the respiratory metabolism. It is not essential, therefore, that there should be a pathologic tendency to movement which constantly keeps the entire musculature in action, as in many insane patients, or that severe convulsions should produce such shock. On the contrary, even the restlessness of a patient in bed with increased respiration and cardiac action and mild tremor, as frequently occurs in the susceptible patient, will bring about an increase of oxidation. The degree of such increase in metabolism cannot, however, be determined quantitatively.

The conditions are still more difficult if we attempt to estimate the loss of substance which the diseased body suffers in the discharge of transudates, in suppurating wounds, in profuse expectoration, in severe albuminuria, in long-continued hemorrhage, and the like. There can be no doubt that, in all cases in which there is loss of the body albumin, the diet must be arranged to compensate for it if loss of weight is to be prevented.

The conditions are much plainer when, in consequence of qualitative disturbances of metabolism, the affected organism suffers loss because the food products are not oxidized to their normal end products, and, in consequence, material which is still capable of oxidation is present in the blood, and is excreted unoxidized or imperfectly oxidized in the urine.

The sugar which the diabetic excretes in the urine is lost to the body as a source of power. If we fail to take account of this we overestimate the actual food-requirement and energy-interchange of the diabetic. With a suitable diet, in fact with a diet of the same calory values, we can produce equilibrium of metabolism in the diabetic as in the healthy. Only the food must be given to him in such a form that he can utilize it.

By other products also, which, in disturbances of metabolism, are found in the urine (acetone, aceto-acetic acid, $\beta$-oxybutyric acid, cystin, homogentisic acid, etc.) energy is occasionally lost to the body.

The organism always loses energy when there is insufficient assimilation of food in the intestines. This occurs in the healthy, but is frequently and decidedly increased in the sick. Its amount may be accurately determined.

In the healthy estimation of the calory value of feces such as are discharged by those on a mixed diet has led Rubner to assume a reduction of 8 per cent. from the raw calory value of the ingested food as the average amount of energy thus lost. This calculation, however, is valid only under conditions in which the feces are made up chiefly of the unabsorbed residue 
of intestinal secretions, and do not contain remnants of food. These conditions are realized only when absorption is normal and the food is suitable.

This assumption, however, is not true in health if the diet contains substances in a form difficult of absorption. In the sick, in whom the mechanism of digestion as well as the mechanism of absorption is disturbed, the estimation of the food lost in the feces is absolutely necessary, for we cannot estimate it by simple subtraction.

The analysis of feces is therefore very important in all quantitative clinical investigations of metabolism. The results, compared with an exact estimation of the food ingested, give a clear idea of the amount of food assimilated in the intestinal canal.

Voit demonstrated in his laboratory the energy-value of the food of healthy persons by thus estimating the part which remained undigested in the intestine and in the feces when the subject was given various diets, simple-or mixed. This forced us to investigate the manifold pathologic conditions which complicate the mechanism of digestion and the processes of absorption and to study the absorption of and utilization of food in these conditions.

The researches undertaken in this direction are extremely numerous, and only their most important results can be summarized here.'

In by far the majority of these studies we have limited ourselves to estimating accurately the nitrogen and the fat during the period of investigation. In view of the fact that the disappearance of carbohydrates during their passage through the intestinal canal is accomplished not only by absorption through the intestinal mucous membrane, but also by decomposition (fermentation), and hence that the residue of carbohydrates found in the feces does not give a reliable measure of the actual amount used, carbohydrate estimation in feces has been quite commonly neglected in these investigations. Only lately A. Schmidt ${ }^{1}$ has minutely investigated this question, and in his "fermentation test" has given a method for determining accurately those portions of the undissolved carbohydrates which are susceptible to the fluids of digestion, yet have escaped absorption. His proposition to utilize always the same trial meal (qualitative and quantitative) for the quantitative estimation of the absorption of albumin and fat in disease deserves the fullest consideration.

The investigation of the absorption of food in pathologic conditions, in so far as technic and quantitative estimation are concerned, has now reached a high degree of perfection.

Comparatively few of the numerous researches in metabolism have given noteworthy and positive results concerning the assimilation of food.

The researches of Fr. Müller ${ }^{2}$ in the metabolism in jaundice, which are the earlier quantitative investigations at the bedside, should be mentioned first, since they determine a very important decrease in fat absorption in cases of occlusion of bile from the intestine.

1 A. Schmidt, Deutsches Arch. f. klin. Iled., Bd. lxi, pp. 280 und 545; Verh. gr. f. innere Med., 1898 und 1899.

2 Fr. Müller, "Untersuchungen über Ikterus." Zeitschr. f. klin. Med., Bd. xii, p. 45. 
But also the negative results of many investigations which showed normal or almost normal absorption in such pathologic states-conditions in which with a certain degree of justice it might have been supposed that more serious disturbances were present-have given a basis of support for the dietetic treatment of such cases, and have shown the fallacy of many diet lists based upon erroneous ideas.

I refer to the excellent monograph of Ad. Schmidt and J. Strassburger, ${ }^{1}$ in which the results of their researches in this subject are compiled, and $\mathrm{I}$ shall limit myself to mentioning a few researches.

The mal-assimilation of food which Fr. Müller found in patients with stasis of bile is limited almost exclusively to fat. Müller found in the feces of his patients fat amounting to $8 \% .4$ per cent. (against 22 per cent. in the healthy). The loss in fat often amounted to 50 to 70 per cent. of the fat administered as food.

The same decided decrease of fat absorption was afterward observed in occlusion of the pancreatic juice from the intestine without complications (Deucher).$^{2}$ In a case of carcinoma of the head of the pancreas, in which no stasis of bile was present, only 17 per cent. of the fat introduced was absorbed; therefore, the simultaneous action of pancreatic juice and bile is necessary for a sufficient absorption of fat in man.

On the other hand, it appears from the reports of investigations that neither bile nor pancreatic juice is necessary for the complete splitting up of fats in the intestinal canal. Not only the fatty stools of the jaundiced patient (Fr. Müller), but also (according to Deucher) the feces in occlusion of the pancreatic duct contain fat which is chiefly in a split-up form (up to 80 per cent.) ; therefore, Deucher teaches that we cannot count upon the absence of free fatty acids in the feces as evidence of disturbances in the pancreatic function.

Predominance of free fatty acids, naturally, not at the cost of the neutral fats, but, on the contrary, at the expense of the soaps (Deucher), means presumably a decrease or absence of the pancreatic juice secretion.

By others ${ }^{3}$ in individual cases, a lessened fat splitting has also been observed in connection with occlusion of the pancreatic juice from the intestine. Volhard ${ }^{4}$ has shown that, although the decomposition of neutral fats is primarily the function of the pancreas, in cases of occlusion of the pancreatic juice, fat splitting occurs not only through the action of bacteria, but also through the action of the gastric juice which also contains a fat splitting ferment. There is need of further investigations with due consideration of this factor.

1 Ad. Schmidt und J. Strassburger, "Die Fäces des Menschen," Bonn, 1901.

2 Deucher, "Stoffwechseluntersuchungen bei Verschluss des Ductus pancreaticus." Correspondenzbl. f. Schweizer Aerzte, 1898, Nr. 11.

3 Fr. Müller, loc. cit., Weintraud, "Die Bedeutung des quantitativen Stoffwechselversuches für die Diagnostik innerer Krankheiten, insbesondere von Pankreaserkrankungen." Die Heilkunde, 1898, Heft 2.

\& Volhard, "Ueber das fettspaltende Ferment des Magens." Zeitschr. f. klin. Med., 1901, Bd. xliii, p. 397. 
Except in the cases just mentioned a decided diminution in the absorption of fat, e. g., as a result of disturbance of the gastric chemism, has not yet been observed. With insufficient or increased HCl in the gastric juice, and also in complete achylia (apepsia), the fat appearing in the feces does not amount to more, or to but very little more, than the usual percentage.

On the other hand, unusually large amounts of fat are found in the discharges in disease of the intestine (amyloid degeneration, ${ }^{1}$ tabes mesenterica, ${ }^{2}$ chronic intestinal tuberculosis with chronic tubercular peritonitis, ${ }^{3}$ fatty diarrhea [Biedert $\left.\left.{ }^{4}\right]\right)$.

Regarding the laws of albumin absorption in the sick, $\nabla$. Noorden ${ }^{5}$ has demonstrated the surprising fact that the absence of $\mathrm{HCl}$ in the gastric juice, in spite of its great importance for the peptonizing of the albumin bodies which is necessary for their absorption, nowise influences the assimilation of the latter. Patients with disease of the stomach, with anacidity and with hyperacidity, showwed a quite normal power of albumin absorption in the intestine.

But later, in cases of apepsia gastrica ${ }^{6}$ and of pernicious anemia ${ }^{7}$ in which not only $\mathrm{HCl}$ but also the digestive ferments of the gastric juice were absent, slight diminution in the absorption of albumin was observed. It must, however, be remembered that in these conditions there is often not only independent disease of the mucous membrane of the stomach, but (as the anatomical findings in individual cases have also shown) actual atrophy of the glands in the intestinal mucous membrane. Hence it is easily understood how, in the absence of any peptic effect upon the albumin in the stomach, the vicarious intestinal digestion which ordinarily occurs does not produce complete absorption.

The slight albumin losses in the feces, which amount to from 11 per cent. to 15 per cent., instead of 7 per cent. as in health, in these cases and also in complete occlusion of bile from the intestinal canal, are in sharp contrast with the great losses which occur in occlusion of the pancreatic juice (Deucher, Weintraud, loc. cit.).

In extensive disease of the intestinal mucous membrane (as in an atrophic nursling), ${ }^{8}$ in intestinal amyloid disease, ${ }^{9}$ in extensive intestinal tuberculosis ${ }^{10}$ great losses of nitrogen have been observed in the feces.

1 Fr. Müller, loc. cit.

2 Ad. Schmidt, loc. cit.

8 Weintraud, loc. cit.

* Biedert, Jahrbuch der Kinderheilkunde, Bd. xxviii, p. 21.

5 v. Noorden, "Die Ausnützung der Nahrung bei Magenkranken." Zeitschr. f. llitin. Med., Nr. 17, p. 137.

6 Strauss, "Untersuchungen über die Resorption und den Stoffwechsel bei Apepsia gastrica." Zeitschr.f. klin. Med., Bd. xli, p. 280.

7 Erben und Steyskal, "Klinisch-chemische Studien." Zeitschr. f. klin. Med., Bd. xl, p. 165.-Morazewski, "Stoffwechselversuche bei schweren Anämien." Virchow's Archiv, Bd. clix, Heft 2.

${ }^{8}$ Rubner und Heubner, "Die künstliche Ernährung eines normalen und eines atrophischen Säuglings.” Zeitschr. f. Biologie, Bd. xxxviii, p. 315 .

${ }^{9}$ Müller, loc. cit.

10 Weintraud, loc. cit. 
Conditions of stasis, as in disease of the heart, do not cause decided loss in albumin absorption (Grassmann ${ }^{1}$ ) neither are diarrheas of mild grade combined with a decrease in the assimilation of albumin in the intestine ( $v$. Hösslin).$^{2}$

We know from the researches of $\nabla$. Mering that the absorption of carbohydrates in the intestinal canal (in the form of sugar) does not take place so much by the chyle tracts as by means of the circulation, and that they are introduced through the roots of the portal vein into the general circulation. We might expect that circulatory disturbances in consequence of stasis of the portal vein and in uncompensated valvular disease would seriously interfere with the absorption of albumin.

The investigations of Grassmann in persons with valvular disease did not show any such influence from disturbance upon the circulation, and many later studies of assimilation have not demonstrated a decided decrease in carbohydrate absorption. Even in Deucher's investigations in patients with occlusion of the pancreatic juice from the intestines, the absorption of carbohydrates is complete. Nevertheless, the pancreatic juice contains the most active saccharifying ferment known.

The negative results obtained probably depend in part upon the unreliability of the method previously in use. It was therefore a matter of great importance when A. Schmidt with a uniform diet (test diet), and by means of a new method (fermentation test), taught us to discern the finer disturbances in the absorption of carbohydrates.

In numerous investigations undertaken in association with Strassburger ${ }^{3}$ he succeeded in recognizing a pathological condition (in which the clinical symptoms were not very pronounced, but, nevertheless, were sufficiently characterized by an incomplete digestion of starch) as a not uncommon disturbance of function of the small intestine (in a broader sense, including the pancreas and the upper large intestine). Only by a quantitative study of metabolism can a positive diagnosis be made (intestinal fermentative dyspepsia).

In conclusion we must refer to the great importance of quantitative clinical researches in absorption, in the healthy and in the sick, as helping us to decide upon the merits of the nutritive preparations with which chemical industry has flooded the market during the last ten years. The new albumin preparations, in particular, have thus instigated researches in the clinic, and the studies in metabolism upon which the employment of neutrose, eucasin, tropon, plasmon, and roborat are based are certainly not few. The degree of absorption as shown by quantitative investigations in metabolism, together with practical experience at the bedside, will always be the best measures of the actual value of such food preparations.

1 Grassmann, "Die Resorption der Nahrung bei Herzkranken." Zeitschr. f. klin. Med., Bd. xv, p. 183.

2 v. Hösslin, Experimentelle Beiträge zur " Frage der Ernährung fiebernder Kranker." Virchow's Archiv, Bd. 1xxxix, p. 95.

s Schmidt und Strassburger, Deutsches Arch. f. klin. Hted., Bd. lxix, p. 570. 


\section{CONCLUDING REMARKS}

In the pursuit of knowledge regarding metabolism in pathological conditions, also in the nutrition of the sick, in the last twenty years, it would be unjust to ignore the importance of the dynamic point of view in the investigation of the processes of metabolism, which to-day dominates pathology as well as physiology.

On the other hand, however, we cannot be too careful-when relying upon a law based upon the combustion value of individual food products-in considering the quantitative exclusively, or even to such an extent as has been the custom of the average student of metabolism in the last few years.

Primarily it is practical experience at the bedside which should dictate the diet, and not theoretical knowledge of food requirement, however well founded. The patient cannot be nourished with calories alone, and it would certainly restrict the further progress of our knowledge of the laws of nutrition if we should consider the caloric value of the individual foods more, and the individual digestibility and tolerance, the manner of preparation, etc., of foods less.

All honor to the calory reckoning of the food-it is of inestimable value in the treatment of chronic diseases-but we must beware of carrying it too far. In practice we have a sufficiently well-founded dietetic treatment, especially in acute disease, without calory reckonings.

But apart from this limitation of its value in practical dietetics, which is not to be misunderstood, the purely dynamic conception of processes of metabolism has not always influenced our scientific understanding and research in these problems in a fortunate way.

Upon one of the first pages of Hoppe-Seyler's Physiologic Chemistry, these words are italicized: "The process of life of the organism is, in the main, a complete mystery."

When we read, however, in modern clinical researches in metabolism, that in the form of albumin, fat or carbohydrates only such and such calories are to be allowed in the diet, or are to be eliminated from the diet, in order to increase or to diminish the proteids or fat of the body, or definitely to influence the activity of the organism in this or that direction, we might almost believe that the veil had long been lifted from the mystery, while in reality we are as far from a solution as we were in the period in which Hoppe-Seyler wrote the foregoing words.

In the modern pathology of metabolism the view is constantly becoming more prominent that the calory carriers of the introduced food are simply decomposed in the daily metabolism of the body without having become an integral constituent of the organism. This prevents us from studying the great problem of life, and the investigator gets his inspiration not from the hope of a speedy solution of the problem but purely in the exhilaration of steady work and steady progress upon the path already trodden; still the goal itself must never be lost sight of.

This, however, is the case if the view is accepted as final that the mystery 
of life was long ago solved by the hypothesis that the organism is a machine in which the food products undergo combustion somewhat as in an oven, thereby becoming a source for the production of heat and force.

This is what Liebig assumed for the N-free foods (carbohydrates and fats), which he therefore designated as respiratory materials; because they undergo combustion without taking part in the structure of the body he did not consider them true foods. In true metabolism, in the decomposition of products, in labor and in the regeneration of tissue destroyed thereby, they have no part according to his conception. And when Voit explained the decided increase of albumin decomposition upon adding albumin to an already sufficient diet by an increase of "circulating albumin," and (in place of the "luxury consumption" of albumin, which was the current theory) showed the special conditions of decomposition of circulating albumin, making it responsible for the increase in albumin decomposition, then it was proclaimed that albumin also may be decomposed without being taken up into the organic parts of the body. Thus the opinion became more deeply rooted, favored by the purely dynamic conception of the food products as carriers of energy, that without any entrance of the food into the tissue of the living cells, the condition of the body could be influenced by the food in the circulation, where the nutritive products were supposed to be built up by transference into higher stages of oxidation. In this manner the organism was supposed to be able to utilize its food and the energy resulting to cover its losses in heat and mechanical labor.

This conception found decided support in the demonstration by Voit that muscular labor does not go hand in hand with an increased albumin decomposition.

Many objections may be raised, however, to such a conception of the processes of nutrition. Since this conception occurs particularly in the articles on metabolism written by clinical pathologists it is probably to be explained by the historical development of the pathology of metabolism on the basis of Voit's law of metabolism and nutrition.

Certainly much would have been otherwise if the principles which Pflüger adrocated in his numerous publications had been accepted.

In many clinical researches of metabolism the subject of investigation can only be comprehended after considering its historical development.

I shall only refer to the question, still mooted, ${ }^{1}$ whether alcohol is a nutrient and an albumin saver. As a carrier of energy alcohol is of even greater importance than the carbohydrates, the recognized albumin savers (one gram of alcohol $=7$ calories, one gram of carbohydrates $=4.1$ calories).

How could this question have been so long disputed if alcohol, following the dynamic laws, simply undergoes combustion in the body, and in this manner offers its energy value to the organism? And why, in the numerous clinical investigations of metabolism in regard to the albumin-saving action

$1 R$. Rosemann, "Ueber die angebliche eiweisssparende Wirkung des Alkohols." Pftüger's Arch., Bd. lxxvii, p. 405; here also the other literature. 
of alcohol, does it so constantly strike us that the results of research are conflicting and permit different explanations?

Because the whole process is without doubt quite different from our ordinary dynamic conception of it. Alcohol, after absorption, is not only decomposed into carbonic acid and water, and thus converted into a definite amount of heat, but, moreover, it enters the protoplasm of the cells where all oxidation takes place, and there modifies the destruction of the protoplasm.

For the same reason the carbohydrates and fats do not act isodynamically in regard to albumin saving, as would be the case if the supply of heat represented by them became free in the circulation. In fact the carbohydrates are more active than the fats in the physiologic experiments.

By the appearance of acetone bodies after the complete withdrawal of carbohydrates, pathology indicates that not only quantitatively, but also qualitatively, the destruction of the cell protoplasm occurs in different ways, according to whether the carbohydrate molecule or the fat molecule is found in the cell which dominates the processes of decomposition.

It has already been mentioned that the newer facts regarding the conditions and the occurrence of the accumulation of albumin are very difficult to reconcile with Voit's laws of albumin metabolism.

The fact that gelatin, and as Mann ${ }^{1}$ has lately shown, also elastin, even although they are inferior to fat as calory carriers, are far superior as albumin savers, certifies beyond doubt that the nitrogen-containing food molecule of the body-cell in which decomposition takes place brings with it something else than the calories-something which influences metabolism, independently of the calories which it brings.

Analogous with this is the remarkable observation of Loewi, ${ }^{2}$ who fed a dog with $\mathrm{N}$-free starch and cane sugar as the sole carriers of nitrogen and with soluble products of continuous pancreas digestion until the complete disappearance of the biuret reaction. Thus he managed to produce nitrogen equilibrium. Therefore, the dog must have formed albumin synthetically from the nitrogen carriers in solution (from amido acids, ammonia, purin bases and hexon bases).

This proof was necessary to throw light upon many physiologic and pathologic processes of metabolism. I shall mention only one more:

According to the dominant teaching, it was formerly very difficult to answers questions which arose as to the origin of muscular power. Voit was the first to show that increased muscular labor is not expressed by a decided increase of albumin decomposition (in the balance of metabolism), and, in opposition to the views of Liebig who believed albumin decomposition to be inseparably combined with the activity of the organs, regarded the nitrogenfree food products as the source of energy from which the organs, without suffering in their substance, derived energy for their functions.

Now the newer investigations of Caspari actually show an accumulation

1 Mann, "Ueber das Verhalten des Elastins im Stoffwechsel des Menschen." Arch. f. Bygiene, Bd. xxxvi, p. 166.

2 Loewi, "Ueber Eiweisssynthese im Thierkörper." Centralbl. f. Physiol., Bd. xv, p. 590. 
of albumin (nitrogen retention) during forced muscular labor. Is it possible that the muscular machine acts so wonderfully that it is not subject to wear and tear, but actually develops during this condition, while it transfers the energy supplied by the carbohydrates into living force? Or are the conditions in reality. quite different, and are nitrogen-free substances really burned in the muscle during its work, or, on the contrary, does it decompose the specific albuminous protoplasm of the muscle cell so that the nitrogen also at once decomposes like the albumin of the muscle, and is utilized for the reconstruction of muscular albumin? Verworn assumes that in muscular activity the biogen molecule which represents living albumin decomposes into atom groups containing nitrogen and non-nitrogenous atoms, of which only the latter are excreted in metabolism, whereas the former regenerate to complete biogens. That we must recognize synthetic processes if we are to make such an assumption, cannot be thought remarkable after what has been said. For does not the animal organism evolve from inorganic iron such a highly complicated molecule as is represented by hemoglobin? It produces synthetically nuclear albumin from its components, etc.

If we dismiss the view that each molecule of food which is absorbed, and is required by the organism to maintain its vital functions, is at once in all its constituents decomposed into end-products of metabolism and quantitatively excreted, then many other points in the pathology of metabolism formerly difficult to understand will be made clear.

When nuclein decomposition occurs in the body, the phosphoric acid excretion is frequently not uniform with the excretion of alloxur bodies; apparently because phosphorus is retained for the purpose of nuclein synthesis, for the regeneration of the nuclein which has been lost.

After complete withdrawal of carbohydrates, the amount of sugar excreted in severe cases of diabetes (as has lately been frequently observed) may exceed the quantity which may be assumed to be formed from albumin. Before we speak of a sugar formation from fat, we should think of a renegeration of the $\mathrm{N}$-containing part of the albumin molecule after splitting off the carbohydrate group, perhaps with the aid of the atom groups taken from the fat molecule. This is, however, quite different from a direct sugar formation from fat expressed in chemical equation. It is simultaneous splitting and synthesis, a work of the living cell, whose effort toward regeneration becomes greater the more its protoplasm is involved in decomposition.

In the study of the pathology of metabolism, we must escape from the narrow confines of views which, in the discipline of student days, were of certain value to us, but which must no longer be a barrier to development.

The opinion must probably be discarded that the amount of decomposition of animal protoplasm (living substance), that is, the amount of metabolism necessary to maintain life, is equal in all individuals and at all times.

Of course the common estimation of the amount of total energy consumption in clinical metabolism investigations is not decisive in judging this question. Slight, and even scarcely noticeable differences in the activity of different individuals (greater muscular exertion, etc.) may produce varying degrees. Hence the difference of two values obtained under similar con- 
ditions of research should not have too great importance attached to it, as the identity of the conditions is only apparent.

Nevertheless the low nutritive values which are sufficient to maintain life after prolonged under-nutrition. and in some cases of diabetes are of no importance. That in some obese persons an extrcme diminution of the calory supply is actually necessary before they begin to lose weight also makes it likely that here a fundamental property of the living cells is changed, so that the degree of their energy of decomposition has been diminished. And the contrary experience that, in some perfectly healthy persons, even with a food very rich in calories, it is impossible to produce an increase in weight, makes us doubt whether in this case the amount of food is really the decisive factor in the interchange of energy. On the contrary, cases of this kind make it more likely that there is an increased energy of the cells and so an actual "luxury consumption."

In practice these individuals do not always present such conspicuous external signs of a lively temperament that the increase in metabolism can be explained by an increased activity of the muscles; and we are the more inclined to search for an individual anomaly of protoplasmatic activity, if such an assumption can be in consonance with the dominant teaching.

The dynamic theory, which has been of inestimable value in making us recognize in biology the force of the law of conservation of energy, naturally lays particular stress only upon the intake and output of the organism. But this theory does not exclude intermediary metaloolism from consideration in the future. The importance of inorganic salts will then appear in its proper light; they are indispensable in the food; nevertheless, because they do not bring tension power (calories) into the organism they have no part assigned to them in the dynamic conception of the processes of metabolism and nutrition.

Again we must remember that in the animal organism we have not only tension energy consumed, but sources of energy newly formed (ferments).

Although researches in the realm of the pathology of metabolism will become much more complicated, when all these facts are borne in mind the desirable result will be attained that clinical pathology of metabolism will become deeper but less expansive. 


\title{
OVER-NUTRITION AND UNDER-NUTRITION
}

\author{
By C. v. NOORDEN, Frankfort-on-MaIn
}

IN modern therapy, dietetic cures are becoming more important from year to year, particularly those which are intended to influence the entire nutritive condition. Originally confined to the domain of internal diseases, they have become familiar to the surgeon and gynecologist, and no less valuable to them than to the specialist in clinical medicine. It is well worth while to consider the salient features of these cures, without expecting that much that is new will be promulgated. For the fundamental laws are well known, though their application to practice is eternally new. When the individuality of each patient has to be considered, the rules lose their supposed uniformity and their diagrammatic aspect. Uniform and self-evident as the method is when formulated in theory, in practice each particular dietetic treatment brings with it new difficulties, new demands, and when the art of the physician has succeeded, he invariably experiences the gratification of an artistic triumph.

\section{CONCEPTION OF THE "CONDITION OF NUTRITION"}

When we speak of a person's "condition of nutrition" we mean, in the first place, his development of adipose tissue; but not only this is to be considered, for the state of the muscles is of no less importance. Their development is not always proportionate to the amount of fat. There are many persons who are deficient in fat and weak in muscle, and many who are rich in fat and strong in muscle. But there is a third class who are of strong muscular power and deficient in fat, and a fourth who are lacking in muscle and rich in fat.

Of most importance, of course, is the condition of the muscles, all the more so since with powerful muscles we expect a normal composition of the blood and of the most important glandular organs. In every treatment by which we hope to influence the "condition of nutrition" the protection of the muscles must be kept in mind, or, stated more generally-the living and functioning protoplasm of the body must be protected, and if possible improved, so that the maximum of its development may be attained.

How great is the amount of fat on the body-leaving out of consideration pathologic leanness, pathologic obesity and special instances (see below) -is of less significance. There is no generally accepted standard for a normal amount of fat. Between emaciation on the one hand and obesity on the 
other hand, there is a wide space for the conception of a "medium state of nutrition." Although this conception permits no positive statement as to the amount of superfluous fat, no definite body-weight, no distinct relation between weight and size, age and sex, that can be called normal, it must, nevertheless, be maintained that, for each individual, there is a definite standard for the condition of nutrition, particularly with regard to the amount of fat. To recognize this optimum is the duty of the physician, and presupposes much thought and experience. Good judgment will take into consideration the state of health, the competency of all the organs, and the entire mode of life. A few examples will illustrate this. Experience teaches us that persons who are predisposed to tuberculosis are endangered by leanness, and that a certain degree of corpulence protects them. Neurasthenics, as a rule, feel better if they have a large amount of adipose tissue. For patients suffering from disease of the heart, Bright's disease, and emphysema the slightest amount of superfluous fat is an evil, and in diseases that affect the organs of motion, obesity is, at least, the cause of great annoyance. Persons who perform hard labor continue in better health and are more capable of working if their adipose tissue is not too greatly developed. Those who have passed the prime of life, and are no longer compelled to work hard, bear a certain development of corpulency better than those just beginning the struggle for existence. These points will be amplified in the description of the indications for over-nutrition and under-nutrition.

It follows from what has been said that in all treatment by which we attempt to influence nutrition, we must first of all try to maintain and improve the muscles; in certain cases, however, we attempt nutritive cures in which increase or diminution of adipose tissue represents the sole aim of the treatment. Even in these, however, the state of the muscles must not be lost sight of. The importance of this must be emphasized, for very frequently it is forgotten. We sometimes see patients with weak muscles who in the process of "strengthening" are fattened by various means, and when the cure is done we find obese subjects with feeble muscles. Again we see corpulent persons subjected to such irrational antifat cures that not only the fat but also the muscles and blood are depleted, and the activity of their functions permanently injured.

Our theme will be the question: How far can and should the general condition of nutrilion, i. e., of muscle and fat, be influenced by dietetic treatment?

\section{INCREASE OF FLESH AND INCREASE OF FAT}

To accomplish the most important point, the accumulation of muscle, dietetic treatment has, unfortunately, but limited powers. Theoretically it appears to be most difficult to maintain the musculature of the body in antifat cures. The animal experiments of physiologists have invariably shown that with under-nourishment fat disappears from the body, but that albumin also decreases. To this loss of body albumin, that is to say, waste of muscle, we owe the serious consequences which frequently surprise those who carry out a too rapid antifat cure. Discussions regarding the admixture and 
amount of nourishment which will most surely produce a loss of fat without impairing the muscular condition have been carried on for years, and reached their acme in the debates at the Congress of Internal Medicine in the year 1885, and in the literary war waged between Ebstein and Oertel. The views were based rather upon theory than upon practical experience. Investigations regarding metabolism during antifat cures had not yet been attempted. A few years later when, independently of one another, F. Hirschfeld and v. Noorden-Dapper worked out this problem experimentally, contradictions at first resulted. The former found it almost impossible to produce a loss of fatty tissue without a simultaneous decrease of the body albumin. The other two authors proved by numerous investigations that this goal may be reached, and even without special difficulty, provided the leap from a plentiful diet to one of abstinence is not too sudden. All the authors who later devoted themselves to similar researches have confirmed this. The satisfactory result of these studies of metabolism has had, it appears to me, great influence upon the practical work of physicians, and has encouraged them-certainly not to the detriment of the patient-to attempt careful antifat cures, where previously they refrained from them because in every antifat cure-even if only transiently - the supply of body albumin was jeopardized. According to other investigations in metabolism as well as in my own, and also according to later researches of Dapper and myself, careful planning of the ingestion of nourishment so that the albumin supply of the body will not be decreased may obviate this danger, but by no means removes all other difficulties. For, in numerous cases, it is evident that the muscular power of the patient must not only be maintained but increased. This end also may be practically achieved, and will crown the success of a thoughtfully carried out antifat cure. It presupposes that the patient must become accustomed, from the beginning of the treatment, to an increasing amount of bodily exercise. We must utilize the well-known physiological fact that, if we exercise a muscle, it gains not only in strength but also in bulk. It is true that investigations of metabolism in which the combustion of albumin has been tested during dietetic antifat cures with and without systematic, muscular exercise have not yet been carried out; but we hardly require them, for clinical experience demonstrates how readily strength and size of muscles may be increased during dietetic antifat cures.

Formerly (in opposition to the facts) we regarded the preservation of the stability of the organic albumin in antifat cures as difficult, even impossible. Yet, at the same time, it was not doubted that by forced feeding it was very easy to accelerate not only the formation of adipose tissue but also of the animate protoplasm of the body, and particularly to strengthen the muscles. The proposition, however, is by no means so simple as it appears. That albumin metabolism is diminished and N-containing material accumulates in the body on over-feeding with albuminates, particularly with fat, and to a still greater degree with carbohydrates, had been long known from animal experiments by Bischoff and C. v. Voit and their pupils. Investigations in man-especially the researches of Bleibtreu and Krug-have confirmed this, and Bornstein and Lïthje in their latest studies in metabolism have demon- 
strated the surprising fact that the amounts of nitrogen which remain in the body in over-nutrition may be raised in an astonishing degree. There can be no doubt; therefore, that an increase in the body nitrogen may be secured by the quality and quantity of food. In all probability we may go further and say that this increase of nitrogen means increase of albumin, i. e., the $\mathrm{N}$ which remains accumulates in the body in the form of albumin. Here our knowledge for the present ceases. C. v. Voit has called attention to the fact that, in an adult animal, the albumin accumulated by excessive nutrition is stored up in a form readily decomposed and must therefore be differentiated from true organic albumin. Hence he proposed the name "circulating albumin." Since it is, however, extremely unlikely that this albumin actually "circulates," i. e., that it is present in the circulating fluids, and as it is very much more probable that it is deposited in the cells, I have suggested the term "reserve albumin." This term has now been generally accepted. It is meant to imply that the albumin, like the fat globules and glycogen granules, is absorbed into the body of the cell, and is deposited there for a time (as reserve material) without becoming an integral constituent of the cell protoplasm. According to this, in the investigations conducted with me by $\mathrm{Krug}$, and in my Text-Book of the Pathology of Metabolism, I have regarded it as unproven and improbable that the increase of body-albumin which may readily be attained is synonymous with increase of muscle. Increase of muscle should mean the increase of living cell-albumin. But the mass of blood-cells and gland-cells unquestionably is but little influenced, and this is especially true of muscle. With few exceptions (prominent among them E. Pflüger and Bornstein) later authors have accepted this view, and in a recent excellent and instructive, as well as critical, dissertation by Lüthje, who has produced by far the greatest increase of body-albumin, we find the same opinions expressed. If increase of albumin (so readily attained) were synonymous with increase of true muscle tissue weaklings could without great difficulty be transformed into robust, muscular people. But this is of course impossible. Every one knows that over-nutrition will produce corpulent persons, but not athletes.

Increase of muscle is a function of the specific growth energy of the cells, i. e., of cellular activity rather than of excessive nourishment. Hence we see plentiful and permanent increase of muscle:

1. In every growing body.

2. In the fully grown body when it is gradually accustoming itself to increased labor (work hypertrophy of the muscles).

3. When, from previously insufficient nourishment or from disease, the muscular tissue of the body has been diminished, and subsequently profuse nourishment makes up this loss. It is a fundamental error to look for the primary cause of this variety of muscle accumulation in food; it is an expression of the regenerative energy of the cells. This is a mighty power. It shows itself, as investigations in the metabolism of convalescence have demonstrated, even when there is no question of forced feeding, and indeed when the calory supply is so slight that fat must certainly be lost in the body, and even a healthy person would lose body albumin. 
Of course, under circumstances otherwise favorable for the accumulation of albumin, muscular development takes place more certainly and more rapidly with very profuse nourishment than with scant food. Usually, however, in adults otherwise healthy-provided we consider long periods of time and not brief intervals-muscle-increase is independent of an excess of food. Muscle growth is dependent on food supply only because the body is better supplied by over-nutrition with reserve products (glyeogen, fat, reserve albumin), and because better food and the somatic and psychical stimulation resulting from it produce greater capacity for work. To this greater capacity of the muscles and secreting glands for work and the stimulus for blood formation which arises thereby, the body owes its power of accumulating protoplasm as well as fat, and-what is more important-of permanently retaining it.

From this point of view, it appears that under special conditions (after wasting disease or after prolonged periods of hunger and under-nutrition) we may hope by over-alimentation to promote the formation of new protoplasm, and particularly of muscular substance. The natural regenerative endeavor of the organism will aid us. If, however, we are treating persons who, although weak, are sufficiently well nourished, the chances for increased muscular development are much more uncertain.

In such debilitated individuals, especially in convalescents, the accumulation of flesh will be greatly favored, if from the onset, or as soon as the strength at all permits it, forced feeding is combined with muscular exercise. This, however, contradicts a widely prevalent custom; for, in the original methods of Playfair-Mitchell, most patients who were to undergo forced feeding were advised either to go to bed or to keep as quiet as possible. In certain cases, for example, in very irritable nervous persons, this may be fully justified. Generally, however, it appears to me after years of experience that early and sufficient activity of the muscles is much more beneficial when we desire to nourish our patient. The increase in weight is not less than in rest cures, and it is gratifying to note that the patients gain in muscular strength and activity at the same time that they increase in weight and size. There is very little danger that, in consequence of muscular exercise, much of the fat which has been acquired with difficulty may be again lost, since the increase of appetite induced by muscular exercise easily makes good the material used up.

In view of what has been said it is obvious that in forced feeding, as well as in carefully and wisely conducted antifat cures (for obesity), the increase and strengthening of the muscles is almost independent of the loss or accumulation of fat. Insufficient nourishment in obesity favors the loss of muscle; superfluous nourishment both in normal nutrition and when it is below par favors the accumulation of muscle. But muscle-loss and muscle-increase are not in immediate dependence upon, or in exact proportion to, the quantity of food. An individual factor always intervenes; an elective property of the organism rather than the bulk of food produces muscle-accumulation.

The conditions are quite different in losses and accumulations of fat. Here the law may be definitely stated. When the supply of nourishment is 
less than the requirement for maintenance, fat is always lost; when it is greater than the requirement for maintenance fat is always accumulated. In the former case we speak of under-nutrition, in the latter case of overnutrition.

Although I shall be repeating what is known to most readers, it is necessary to devote a few lines to the definitions of food necessary for maintenance, of over-nutrition, and of under-nutrition.

In proportion to the requirement of the cells, according to the amount of work required of them externally and internally in heat production, etc., the body arranges the use of material for combustion. When the combustion value of the food is equal to the demand, the equilibrium of metabolism in the body is preserved. The amount of food which is necessary for this we call "food necessary for maintenance." Most normal persons, if left to themselves and following their own inclinations, usually take neither more nor less food than is necessary for maintenance; variations in this balance of nutrition may occur, but the deficiency of one day is made up the next. Consequently it is the rule that normal adults remain for years and decades at about the same weight. The proportion of food which is necessary for the equilibrium of metabolism, calculated per day and for each kilo of the bodyweight, must have a combustion value of from thirty to thirty-five calories in complete rest (in bed), thirty-five to forty calories with light exercise, forty to forty-five calories with moderate exercise, and forty-five to sixty calories in exhausting muscular labor.

For children these figures are to be raised about one-third, for the aged they are to be lowered about one-fourth. There are no decided differences between males and females. These figures relate to a "moderate condition of nutrition"; in the obese they are from twenty to twenty-five per cent. lower, in very thin persons they are just as much higher, for while fat increases the body-weight it takes no part in metabolism. This ratio will enable us to calculate the amount of food necessary in the individual case with sufficient exactness for practical purposes.

\section{OCCURRENCE AND CONSEQUENCES OF UNDER-NUTRITION}

As soon as the supply of food (i. e., its combustion value) falls below that required for maintenance we have a state of hyponutrition. The under-nourished body docs not accommodate its combustion processes to a lower scaleexcept perhaps in the most extreme marasmus and in the death agony. When the supply is less than the requirement, it lives upon its own body substance. In the obese, as we have scen, this process may be limited to the adipose tissue while the albumin, that is, the muscle, is preserved. In normal and mal-nutrition, however, apart from exceptional cases (in convalescence after acute diseases, or after periods of hunger), the supply of albumin in the body may also be slowly consumed, and the person not only loses fat but becomes weaker in muscle. We rarely resort to systematic under-nutrition except in the treatment of obesity. Whether or not treatment for obesity is indicated does not depend wholly upon the degree of corpulence, but also upon many 
other conditions, particularly upon the healthy state of certain organs, for example the heart and the kidneys. To this we shall refer later. But very often, without our interference and against our will, our food produces the characteristics of hyponutrition. This occurs in many diseases. We are unable to maintain the balance of nutrition either because patients refuse to take a sufficient supply, or because diseases of certain organs (for example, the stomach) necessitate a limitation. In acute diseases which run a rapid course the danger is not great, for what has been lost is rapidly regained in convalescence. But in chronic diseases the body is often more weakened and damaged by continued under-nutrition than by the disease itself. It then becomes one of the most important duties of the physician to increase the amount of food at least to the point of maintenance, and, if possible, to make up what has been lost by forced feeding. He will then often have the pleasure of noting that not only does the state of nutrition improve, but that this improvement acts favorably in the cure of the disease. In some maladies, for instance, in not too far advanced tuberculosis of the lungs, this is the rule.

At least as frequently as in actual disease we meet persons who are really not ill but under-nourished. This results from caprices of appetite, from unfounded fear of injury from this or that food (for example, fat substances), from disturbances of appetite which are slight or which are taken too seriously, from poverty, etc. Thus they have become accustomed to a too slight ingestion of nourishment. Sometimes this is habitual from youth, and such persons if left to themselves never attain the acme of nutrition (muscular and fatty tissue) of which they seem capable, judging from their constitution and build. Adipose tissue is seant, and the muscles, no matter how susceptible of development, and no matter what efforts are made to strengthen them by exercise, continue weak, for everything that is attempted is frustrated by the oxidation-processes of the body.

Others first manifest the symptoms of chronic under-nutrition after they have reached adult life. These are mostly persons in whom there is a neuropathic taint, and in whom the various forms of nervous dyspepsia develop. Still others have become neurasthenic and hysterical only in the struggle for existence against àdverse circumstances. Organic causes which would prevent a sufficient ingestion of food are not present, but manifold nervous disturbances interrupt the distinctive connection between actual food-requirements (tissue hunger) and appetite (gastric hunger). Gradual loss of weight, disappearance of adipose and muscular tissues, lessening. of the bodily and mental powers, are the inevitable consequences. These patients, to the detriment of their health, often seek a cure by unsuitable means, by gymnastic exercises and sports, by mountain climbing, by exhausting cold-water cures (frequently in so-called nature-cure institutions), where they hope to build up their nervous system. This acts at first like a whip, apparently increasing and stimulating their activities But the oats are lacking, and in a short time there is a relapse to the former condition. The treatment is all the more harmful because the food, during the time spent in these exhausting cures, is weakening rather than strengthening. The patients and their "NaturalHealer" proceed from the view that a too piofuse animal diet has disordered 
the nervous system. Meat and eggs are withdrawn and the patients are put upon a vegetable diet. To this no special objection can be raised, provided it is carefully chosen; but too frequently it is lacking in nourishment. This has been so abundantly proven as to need no further consideration at this point.

\section{OCCURRENCE AND CONSEQUENCES OF OVER-NUTRITION}

If the food (i.e., its combustion value) exceeds what is necessary for maintenance we have the condition of hypernutrition. In over-nutrition the organism does not increase its processes of combustion, or, at least, does so to a very slight extent. Perhaps the increase of oxidation which arises from over-nutrition has been for a time underestimated, as the latest investigations of Fr. Müller appear to prove. Theoretically this increase is interesting, but it is too slight to be of practical importance.

The increase of oxidation is not due to a stimulation of the cells to a greater katabolic activity, in other words, to a greater rapidity of metabolism, but only to the greater labor which is put forth by the mechanism of mastication, the stomach and intestines, the digestive glands, the organs of circulation and respiration, etc., in order to work up and utilize the greater mass of food. After deducting the slight amounts spent upon the increased labor of digestion, etc. (about 7 per cent. to 20 per cent. of the energy supplied by the food), a large residue from the superfluous food remains, which accumulates as reserve material, and serves to increase the body mass. We call this "food surplus" (= the difference between food ingested and food used up in metabolism).

Aside from slight differences it is of no importance to the processes of metabolism whether the surplus of food occurs from excess of albuminates. or of $\mathrm{N}$-free food, or whether the increased supply comes from one source only (the albumin or the fat or the carbohydrates). A surplus of carbohydrate nourishment favors almost exclusively the production of adipose tissue, provided special circumstances do not promote the increase of protoplasm (see above).

In an especial case (B. Krug's experiment upon himself carried out under my direction) the following calculations were made:

Dr. Krug (perfectly well and in a moderately good state of nutrition), after a period in which he had been abundantly nourished, took for fifteen days in addition to his ordinary food a daily total of 1,710 calories, consisting of fat and carbohydrates. This sum represented "surplus nourishment"; for the fifteen days it amounted to 25,650 calories. Of these 23,051 calories were utilized by the body.

1,720 calories $=7.46$ per cent. albumin accumulation, and 21,331 calories $=92.54$ per cent. accumulation of fat.

Whether the production of albumin was equivalent to accumulation of flesh could not be decided.

It follows from the preceding statements that after the ingestion of a surplus amount of food only an accumulation of fat can certainly be counted 
upon. It is quite uncertain whether an accumulation of muscle will take place; with an equal amount of surplus nourishment the gain in muscle varies from case to case. It is sometimes lacking, in other cases it occurs to a greater or less extent. But it may be prophesied with mathematical certainty that in over-nutrition fat will accumulate. How much is dependent upon the amount of food, and how much upon the amount of work performed by the body? A disproportion between the supply of food and the assimilation of food, to which the accumulation of fat (and ultimately obesity) owes its origin, can be brought about:

1. By an increase of the food above an arerage normal consumption;

2. By diminution of exertion (muscle laziness) with an average normal amount of food;

3. By a combination of superfluous food and diminished exertion.

We frequently meet with cases of over-nutrition, but we are not so often called upon professionally to combat this condition as we are to treat undernutrition. Occasionally, however, we are called to deal either with obesity or with persons who, by continuing their present mode of life, are in danger of becoming too fat. Every corpulent person has behind him a period of over-nutrition; not, perhaps, because he has eaten more than others who have not become fat, but he has eaten more than his individual constitution and bodily functions enabled him to utilize. It is immaterial whether this superfluous ingestion of food has arisen in consequence of a preference for albumin, for fat, for carbohydrates, or for alcohol ; we meet with obesity among decided meatand-fat eaters as well as among those who prefer starchy foods, sweets, and beer. We observe it among those who perform hard manual labor, but who more than compensate for their great metabolism and output of energy by a profuse intake of food; we observe it also in those who, although they eat little, consume an amount of food out of proportion to their slight physical activity, slight metabolism and slight output of strength. To elaborate this in individual instances would be to consider the etiology and pathogenesis of obesity, which is not the purpose of this article (the reader is referred to my monograph on obesity in Nothnagel's Handbuch der speciellen Pathologie und Therapie).

Whether in the development of corpulence there is an increase of the muscles depends, as we observe daily, upon external conditions. The rule holds good that only those obese persons who utilize and, in spite of their corpulence, exercise their muscles have strong and firm muscles. In such people we see the picture of the so-called "plethoric obesity." Those who live much indoors have flabby and weak muscles, often so weak that serious consequences may be the result. Distressing yet convincing proofs are thus furnished to show how loose is the connection between over-nutrition and increase of muscle.

\section{INDICATIONS FOR HYPERNUTRITION AND HYPONUTRITION}

Having explained the changes of metabolism which occur in the organism in under-nutrition and over-nutrition, we must briefly discuss the indications for forced feeding and for antifat cures. 


\section{A. OVER-NUTRITION}

Group 1. In chronic wasting diseases-Considered historically, overnutrition in pulmonary tuberculosis, or rather in tuberculosis of any type, is to be placed in the front rank. Even the old custom of administering cod liver oil in "scrofula" and tuberculosis belongs to this category. We have gradually been forced to acknowledge that its action is not specific but due to the fat which it contains, that is, to its high nutritive value. Brehmer was the first, and Dettweiler the next, systematically to employ over-nutrition in tuberculosis. The success of this method is surprising, as any one can see in his daily experience. By over-nutrition, as now carried out in all institutions for the cure of pulmonary tuberculosis, in hospitals, in the homes of the patients, or wherever it may be attempted, we do not attain a cure for tuberculosis, but the favorable results which have been attained up to now in treating tuberculosis are inseparably associated with over-nutrition. Here, better than anywhere else, it may be noted how beneficially the improvement in the general nutrition acts upon the resistance of the tissues. I have already alluded to this elsewhere, and, after further experience, I wish to reiterate that the modern treatment of pulmonary tuberculosis by over-nutrition is in danger of accomplishing too much. Pulmonary patients who had formerly been emaciated sometimes after treatment become corpulent; this is not to be regarded as desirable, but as a disadvantage. For obesity always throws increased work upon the respiratory and circulatory organs, and in this way makes demands upon the diseased organ which it should be spared. But apart from these extreme cases, over-nutrition in pulmonary tuberculosis may be designated as one of the most valuable agents in the realm of dietetic therapy.

Over-nutrition as a prophylactic measure produces even more favorable results than in tuberculosis that has already developed. It is especially valuable in children and adolescents who have suffered from a previous tuberculous disease of the glands, joints and bones-and hence are ljable to a later "pulmonary tuberculosis"-as well as in those who without such preceding affections are likely to become tuberculous on account of general weakness and hereditary predisposition. The majority of consumptives attacked in the second or third decades of life have been, as Brehmer once correctly remarked, small eaters from youth. Under circumstances such as these the early resort to forced feeding, combined with exercises to harden the muscles and properly develop the body, is one of the most beneficent and grateful tasks of the family physician, all the more grateful if this method be instituted early in life when the body is still in the stage of growth and the accumulation of muscular tissue takes place more readily than in later years.

Cures based on forced fecding practically play a less important rôle in all other chronic wasting diseases than in tuberculosis. Most important, perhaps, is over-nutrition in syphitis, a disease which frequently, and especially in its early stages, markedly influences nutrition, and in its subsequent course (in the so-called tertiary forms) almost invariably produces extensive tissuedisintegration. The old custom, originating centuries ago, and not yet rooted out, was to place these patients upon a scant, meager diet, and to weaken them 
by laxative drinks. In contrast to these old usages, an excessive or forced administration of nourishment has shown itself to be much more efficacious. Many protracted cases of tertiary syphilis can be cured only when the body has been thus strengthened by over-nutrition.

Diabetes mellitus may also be counted among the chronic wasting diseases, naturally within certain limitations, as there are numerous cases of diabetes mellitus in which, vice versa, definite relations to obesity exist. Where this is not the case (as in the severe forms of diabetes, particularly that occurring in youth), emaciation is combined with the disease, partly as the result of losses in sugar, and partly as the result of peculiarities of the diet. To interfere, to replace by over-nutrition not only what has been lost and if possible to raise the patient's state of nutrition above the average, is important, for experience teaches that a good state of nutrition will often avert many of the dangers of the disease.

Diabetes insipidus must also be mentioned. Unfortunately, we know little regarding the pathogenesis and the minute disturbances of metabolism which take place in this disease. The most conspicuous symptom in all severe cases is the decided emaciation and loss of strength, which cannot be reasonably explained from the loss of water by the body. If we leave out of consideration the gradual restriction of the intake of water, and a few purely symptomatic drug indications, the especial and systematic improvement of the nutrition by a long-continued, forced diet is the only measure which promises success. In view of the rarity of these cases, I shall briefly report a case under treatment by me three years ago for diabetes insipidus. The disease occurred in a man aged fifty, and was credibly reported to have followed a severe shock sustained in a railway accident; it had existed for about fifteen months. The patient's previously normal state of nutrition had suffered acutely during this time, the loss in weight amounting to more than 25 kilograms. The daily amount of urine varied between 9 and 13 liters. In this case, by a plentiful addition of cream and butter to his diet (between 250 and 300 grams of milk fat daily), weight was increased 20 kilograms within four months. After the first six weeks, the urine had already diminished to between 5 and 6 liters, and, after two months more, to 3 to 4 liters. The once markedly debilitated patient had, in the meantime, become strong again and fully capable of working. He continued strong until attacked the following winter by pneumonia to which he succumbed. According to the statements of his relatives there had been no increase in the amount of urine.

Finally, among the chronic wasting diseases, we must mention exophthalmic goiter (Basedow's disease). Probably from a thyreogenous autointoxication (Möbius, Fr. Müller, A. Magnus-Levy) an increase of the process of metabolism occurs, i. e., an increase both in the assimilation of albumin and in the combustion of fat. In consequence of this, if the patients take only the amount of food which is the average for normal persons, this proves insufficient, and they therefore consume their own body substance (protoplasm and fat). Only by over-nutrition can we safeguard them from this. By overnutrition it is possible at least to conserve the fat, or we may even hope to increase it. According to the researches of Fr. Müller, we cannot count on 
maintaining and developing the muscular tissue even by forced feeding so long as the disease progresses. Nevertheless, forced feeding carried on persistently is remarkably successful; the patients accumulate valuable reserve material, and experience shows that they are much better than when exposed to progressive emaciation by dietetic negligence. But though it is necessary to promote the nutrition of the patient, it is not to be supposed that a very energetic and rapid forced feeding is here indicated. Great care is necessary; for patients who present severe forms of Graves' disease, and whose nutritive condition has been correspondingly damaged, suffer also from cardiac weakness. To maintain their balance of nutrition, they require upon the average from 15 to 20 per cent. more food than a person in normal health of the same weight and build, and of course if we are to attain an increase in weight, still more food is necessary. But according to my experience, the cardiac difficulties frequently increase if we proceed too rapidly with forced feeding. Indeed the cardiac weakness which results may be dangerous. Last year an able author reported a case in which, in a short time, an increase of weight of 22 pounds was brought about by over-nutrition. In spite of this desirable result, collapse occurred in which the patient succumbed. I believe it cannot be doubted that this too rapid over-nutrition was responsible for the unfavorable outcome. I have, myself, not seen such serious results from over-nutrition in Basedow's disease, but I have observed exacerbations which made me fear collapse. Hence, there is need of proceeding slowly in all severe cases; we must be satisfied if at first only the rapid loss of weight is stopped. When this has been accomplished, and the weight maintained for several weeks, forced feeding should be again resorted to, but an increase of about a pound a week should not be exceeded.

Group 2. In functional nervous diseases.-We are indebted to S. Weir Mitchell for having pointed out the deleterious effects in neurasthenic and hysterical persons of a poor state of nutrition-whether this dated from the onset or developed in the course of the disease. He still further demonstrated, by innumerable and convincing examples, that with systematic over-nutrition (forced feeding) not only the state of the nutrition but also the activity of the nervous system could be restored.

Nearly twenty years have passed since the publication of these views by $\mathrm{S}$. Weir Mitchell and since that time forced feeding in the treatment of neurasthenia and hysteria has become the common method of nearly all physicians. The results thus attained are the foundation and the corner stone of the reputation which numerous clinics and sanatoria have acquired in the treatment of these cases. Retrospectively speaking, this therapeutic accomplishment which we owe to $\mathrm{S}$. Weir Mitchell is of the highest significance. The treatment of Weir Mitchell and Playfair, the latter of whom did a great deal to popularize the method, consists of several factors:

1. Isolation of the patient.

2. Rest in bed, lasting according to the circumstances of the case from one to several weeks.

3. Over-nutrition; beginning with small amounts of food, and gradually increasing to high values. 
4. Massage and faradization of the muscles during the rest in bed; careful hydrotherapy to stimulate the circulation.

For numerous cases, especially those in which excessive irritability of the nervous system is prominent among the clinical symptoms, no more valuable treatment can be outlined to-day than the Weir Mitchell-Playfair plan. But it has been demonstrated that the diversity of the cases necessitates numerous modifications. The other factors associated with hypernutrition- (especially isolation, rest in bed, massage, etc.) are frequently not only unnecessary, but actually a hindrance to success. The diet which Weir Mitchell proposed may be modified as occasion requires. Any one conversant with the laws of nutrition and with general therapy may with ease select what is suitable for each individual case.

My experience leads me to state that I frequently employ only limited isolation, i. e., the removal of the patient from his usual surroundings and activities, but rarely insist on prolonged rest in bed. In my opinion, the latter should be avoided whenever possible, for reasons previously mentioned.

The indications for forced feeding, according to the experiences of the last twenty years, are present in functional neuroses combined with malnutrition, whether the poor state of nutrition is to be looked upon as the cause of the neurosis, or whether the neurosis by its influence on the intake of nourishment and digestive processes produces secondarily a damage to the nutrition of the body. In the former case, we can well hope to remove all of the pathologic phenomena together with their cause. In the latter case we are at least combating a symptom from which the disease constantly receives new stimulus.

Here also, as in the case of exophthalmic goiter-although not for the same reasons-I must caution against immoderation. Many neurasthenics are easily fattened, and, after six to seven weeks, may show a gain of 30 or 40 pounds in weight. But during such rapid augmentation of body-weight new disturbances not infrequently appear which, in themselves unimportant, are over-estimated and exaggerated by the suspicious neurasthenic and hysterical patient. For this reason, I limit the acquisition of weight desired to about 20 pounds, which, according to the individual case, may take from four to five weeks. Then a halt must be called, and after an interval, forced feeding, if necessary, may be begun again.

Group 3. In all individuals with a poor condition of nutrition it is important to determine whether this faulty nutrition has been the hindrance to the full development of their power, to the natural building up of their bodies (in children and young persons), to recuperation after excessive bodily and mental labor, or to convalescence after diseases. The guide for the physician must be not only the present, but the thought of what the future may bring. The various special indications cannot be individually described without entering into the wide realm of general pathology. I should like to repeat (see above) that for every person there is a different optimum of nutrition. Only experience will enable the physician to estimate and recognize this optimum in individual cases. He who trains himself from this point 
of view possesses weapons against disease (existing and threatening) than which there are none more effective.

Of course in all of these cases, even more than in Groups 1 and 2, the development of the muscles must be considered, for by the accumulation of fat alone nothing is gained. Therefore, systematic muscular exercise must be added to forced feeding. The form which this exercise takes is of minor importance. I prefer exercise in the open air (walking, mountain climbing, gymnastic exercises, games, bicycling, etc.), provided we are certain of not overdoing, to massage or indoor gymnastics. Gymnastics with apparatus deserve consideration only when exercise in the open air is contra-indicated for special reasons, or when development of special muscle groups is desirable.

As a rule, it is wise to regulate forced feeding and muscular exercise in this third group of cases, so that only a gradual increase of weight results. We are then much more certain of a permanent and substantial gain. In cases belonging to the first and second group, increases in weight of about 12 to 15 pounds within four weeks-with certain exceptions (see above)-are worth striving for, and sometimes, as in neurasthenics, particularly rapid results are important as suggestive curative agents. But in cases of the third group it is better to have this increase in weight distributed over months instead of weeks. From this it may be further concluded that these persons, who need not so much an instant recovery from a morbid condition as a general strengthening of the body, should not be treated in hospitals or sanatoriums, or only temporarily. Few can bear removal from the ordinary routine of daily life for so long a time, for the air of the sanatorium is rich in psychical contagion. The treatment, and especially the regulations for forced feeding and slow increase of the body-weight, must therefore be planned according to the patient's circumstances, occupation, habits, and position in life. With strict regulations we are less likely to reach our goal than if the constraint of an institution is abolished. Of course a complete change from the previous mode of life which led to the unsatisfactory condition is the first step. Then we can easily learn whether a simple increase of food without any special regulation of its quality, quantity or distribution is sufficient, or whether any particular food must be added to the diet to make the success certain, and, lastly, whether this addition should come from the albumin group, from fats or from carbohydrates.

\section{B. UNDER-NUTRITION}

Antifat cures are undertaken almost exclusively in obese persons; rarely, if ever, in persons normally or insufficiently nourished. What rule is to guide the physician in the important problem: Is an antifat cure to be begun or not? I desire to refute the opinion so frequently expressed that antifat cures always belong to the so-called "weakening cures." The popular views in regard to this are very exaggerated. I maintain positively that an antifat cure begun for proper reasons-and provided that the choice of the method, the rapidity with which it is carried out, and the loss of weight are adjusted to the individual condition-never deserves the name of a "weakening cure," but may always be conducted without injury to the patient, and 
will always be beneficial to the whole body. It will be noted that this favorable opinion is based upon a number of provisos which, although apparently simple, require careful coinsideration and good judgment.

I shall mention only the most important of the indications as these have been elsewhere extensively discussed. ${ }^{1}$

1. Simple obesity in otherwise healthy persons.-(a) Extreme obesity is almost always an indication for an antifat cure. The dangers which may result from a further gain of fat are great, and we must endeavor to prevent them. Certain modifications are required by the age of the patient. In children and in adolescents up to about the twentieth year, we should be content with preventing an extreme development of obesity, and only from time to time, during short periods of from four to five weeks, should an effort be made to reduce the superfluous fat (intermittent antifat cures). In the aged antifat cures are prohibited, as almost invariably there is a rapid loss of strength.

(b) Moderate obesity (body-weight about 15 to 25 kilograms above the average for the age, sex and height) is most frequent and at the same time best suited for treatment. In most cases it can be let alone without any great danger to health, but it cannot be denied that corpulence brings many evils in its train; for if any acute disease occurs (infections, diseases of the heart, of the lungs, of the kidneys, the joints, etc.) or after unusual exertion (such as over-strain of the heart) the obesity may become dangerous. Here, also, the rule holds that in the aged this treatment must not be attempted, and in childhood and in youth only in slow tempo and with the greatest caution.

(c) Slight obesity (body-weight upon the average 5 to 15 kilograms above the normal) in healthy persons never necessitates an antifat cure, but, if the corpulence steadily increases, measures may be taken to check its further development. Nevertheless, very often, particularly in women, the aid of a physician is sought because of a desire to reduce weight and to attain slenderness. In many cases the physician will all the more willingly comply with the wishes of his patients as he may thus gain control of their entire mode of life, in which usually much should be amended. Although in the effort to reduce early obesity, and in the timely regulation of detrimental habits, vanity rather than bodily ill is sometimes the motive power, we as physicians should not criticise this. It may amuse us, but it must be welcomed as a therapeutic opportunity.

Care is always necessary. Many a woman, as the result of a too rapid and too far reaching antifat cure, acquires in exchange for her moderate corpulence various derangements of the abdominal organs, such as constipation, ruptures, gastrectasis, movable kidney, sometimes also of the uterus; or, after the disappearance of fat, the liver and gall-bladder are more exposed to the pressure of the corset, the flow of bile is impeded, and gall-stones result. All these evils are much more frequent in slight corpulence than when it is marked. In the latter the antifat treatment is rarely carried to such an extent that decided pressure changes occur in the abdominal cavity; enough fat always remains to protect the viscera and preserve them from injury.

1 v. Noorden, "Sammlung klinischer Abhandlungen," Heft 1, Berlin, 1900. 
It may be said in general that slight obesity does not especially demand slow and careful reduction of weight, although, to prevent excessive corpulence and for many other reasons, this is often very desirable.

2. Indications for antifat cures when obesity is complicated by other diseases.-Diseases of various kinds, from slight functional disturbances of vital organs to severe anatomical changes, may influence the physician in his decision of the antifat question. More frequently than otherwise, it is the complicating conditions that we meet which lead us to combat obesity.

Such complications may lead us to undertake an antifat cure when if we found a normal condition of all the organs there would be no indication for treatment.

Among the disturbances whose course and prognosis are influenced by obesity, and which for this reason may necessitate the reduction of moderate and even slight corpulence are the following:

Disturbances of the organs of circulation, such as valvular diseases, myocarditis, myocardiac degeneration of the heart, fatty heart, or better, cardiac weakness in obesity, arteriosclerosis, aortic aneurism, etc.

Renal diseases, particularly contracted kidney, on account of the implication of the heart and the arteries which is always associated with it.

Certain chronic diseases of the respiratory organs, such as chronic bronchitis, pulmonary emphysema, extensive bronchiectases, bronchial asthma, adhesive pleurisy; kyphoscoliosis also may be here included.

Chronic articular rheumatism.

Gout in all severe forms.

Other diseases characterized by loss or difficulty of motion or locomotion, for example, hemiplegias, chronic diseases of the spinal cord, some cases of peripheral paralysis, many surgical affections of the bones and joints, large varices, chronic ulcer formation upon the leg, deformities of the lower extremities, etc.

Diseases of the nervous system. That neurasthenics and hysterical persons in general are in better condition when their fat is somewhat profusely developed has already been mentioned; therefore these are the patients who frequently require forced feeding (see above). But as these neuroses do not always disappear under forced feeding, neither does corpulence always prevent their appearance. Where the neurosis is combined with corpulence, careful antifat treatment and the re-establishment of the normal nutritive condition may be indicated, and may be as potent in relieving the neurosis as forced feeding is in thin, poorly nourished persons.

\section{REMARKS REGARDING THE TECHNIC OF OVER-NUTRITION AND UNDER-NUTRITION}

It is an old axiom which constantly demonstrates itself anew that dietetic cures originating with this or that author, and advised for this or that disease, degenerate in practice into mere formulæ. The "cure," the method, is forced upon the patient, instead of the method being adjusted to the patient and its main principles applied to the unending variety of cases. Routine precludes 
success in this field, and frequently does more harm than good. There is no single "best method" of over-nutrition, and no single best method for the removal of fat, but there is for each individual case one and only one best method. It must be constructed by the physician upon the scientific laws of nutrition, whether a celebrated name is attached to the method or not. Hard and fast dietetic rules are most widely developed in the realm of antifat cures. There are obesity cures according to Harvey-Banting, Oertel, Ebstein, Hirschfeld, Schweninger, Kisch, Pastor Kneipp, etc.

I do not intend either in over-nutrition or in under-nutrition to dwell upon the individualities of the methods, but I shall emphasize the main points.

\section{A. THE TECHNIC OF FORCED FEEDING}

Over-nutrition presupposes that the ingestion of food is to be greater than the requirement. How great the surplus of food is to be depends upon the rapidity with which the process is to be carried out. Apart from particular indications (for example, in Graves' disease, see above) the capacity of the digestive organs should decide this question.

How the requirements of the patient can be approximately determined from the body-weight has been related.

According to the calculations which I made in a large number of cases treated by forced feeding I obtained the following results (I must admit in this connection that the figures had not been theoretically determined prior to beginning forced feeding, but were gained empirically during the treatment): With a daily excess of food of about 500 to 800 calories, we get an increase of weight of from 600 to 1,000 grams in a week; with a daily increase in food of 800 to 1,200 calories, weight increases from 800 to 1,200 grams per week; with a daily excess of food of from 1,200 to 1,800 calories, weight increases from 1,200 to 2,000 grams per week.

Quite properly the question arises, not only how many calories-should the food contain, but also how much albumin and $\mathrm{N}$-free substances are included in it.

The albumin bodies theoretically considered have no great value in increasing weight. The average albumin content of ordinary diet (100 to 110 grams) may be somewhat increased in forced feeding, but hardly more than 50 grams. Still larger quantities of albumin can be utilized only in exceptional cases as the bulk of the food becomes too great, and also because all albuminous foods produce satiety to a marked extent. Even the preparation of purified albumin products (casein, nutrose, tropon, plasmon, roborat, aleuronat, ergon, etc.) has not changed this. From 20 to 30 grams during the day are useful; larger quantities, if their use be prolonged, as a rule spoil the appetite, whether we administer them in milk, soups, sauces, pap, in the form of pastry or otherwise. No matter to what form we add the 50 grams of albumin, which I have designated as the most that can be used, it is certain that for increase of weight little is gained by this; it only furnishes about 205 calories.

I hold that in general, allowing for exceptions, the daily administration of from 120 to 130 grams of albumin is quite sufficient. My best results were arrived at by keeping within these limits. Nevertheless, it must be 
emphasized that occasionally the mere increase of the amount of albumin is beneficial; for example, in preparation for true forced feeding, or, if there is repugnance to great quantities of $\mathrm{N}$-free food, during a "vacation period" of from eight to fourteen days which the patient may have to take in his forced feeding treatment. Under these conditions temporary forcing of the albuminintake at the expense of the $\mathrm{N}$-free substances renders the body more receptive to the later action of the true forced feeding. For these purposes the purified albumin preparations, such as plasmon, etc., are more suitable than meat. From 50 to 60 grams of plasmon may be given with advantage during the day. There is no difficulty in administering this amount, as, in this period, we no longer increase the $\mathrm{N}$-free substance. During longer periods, however, little can be expected from such an exclusive increase of the albuminates.

Among the substances free from nitrogen we may choose between the administration of fats or of carbohydrates, or may administer both in almost equal proportions. In principle it is immaterial, as fat and carbohydrates compensate for one another according to the law of isodynamics. One hundred grams of fat are equal to 224 grams of carbohydrates, and vice versa. As a rule the marked increase of carbohydrates is preferable, provided always that they are better tolerated than fats. In some patients this is unquestionably true, but in the majority certainly not. If we desire to add about 1,200 calories of $\mathrm{N}$-free substances, we require either 129 grams of fat or 300 grams of carbohydrates; many other mixtures may be used; for example, 200 grams of carbohydrates and 40 grams of fat, or 100 grams of carbohydrates and 85 grams of fat.

For more than ten years I have preferred to increase the administration of fat, for I have found that a larger amount of carbohydrates-300 to 400 grams a day-is not well borne for any length of time. When, under the constraint of treatment in an institution, the patient is forced to take the large amounts of food which are necessary if we use the carbohydrates alone, we see only too frequently, after the restraint is removed, a period of anorexia during which much of the body-weight gained with so much difficulty is lost.

Accordingly, the composition of the nourishment during forced feeding should be about as follows:

120 to 130 grams of albumin (490 to 530 calories).

300 to 350 grams of carbohydrate (1,230 to 1,435 calories).

This nourishment (720 to 1,965 calories) forms the basis to which as much fat may be added as is necessary to make the proposed calory total: i. e., from 200 to 300 grams of fat (1,860 to 2,730 calories) or even more. Frequently a portion of the required calory excess may be given in the form of alcohol, whereby certain amounts of fat are protected from oxidation and preserved for the organism (9.3 grams of alcohol protect about 7 grams of fat from oxidation).

The amount of water to be added, provided special indications do not necessitate exact regulation, may be according to the desire of the patient. As a rule, if much carbohydrate is given the amount is greater than if much fat is administered. 


\section{B. THE TECHNIC OF ANTIFAT CURES}

In antifat cures the intake of nourishment must be smaller than the demand, and in the obese this cannot be estimated very readily. The practical need will be satisfied if for an obese person the average normal requirement, amounting to about 2,500 calories, is given and is combined with slight or moderate bodily exertion. On this basis I have proposed the following scale in antifat cures: ${ }^{1}$

First grade of antifat diet.-To this belong antifat cures in which the diet may be reduced to about four-fifths of the normal requirement, i. e., to about 2,000 calories. Success follows this only when we are treating robust persons who are able to take sufficient bodily exercise. The action is usually slow; we must not count in the beginning upon losing more than from three to four pounds, and later two to three pounds, of weight per month.

Second grade of antifat diet.-In this category the antifat cures belong in which the diet is reduced to three-fifths of the usual requirement, therefore, to about 1,400 to 1,500 calories. This diet may very readily be adapted to the mode of life of most patients, so that they can follow their occupations without hindrance. The rapidity of cure depends upon the amount of physical exercise they can take. If exercise is difficult or impossible (as in many women, in very lazy persons, in certain diseases, particularly in paralysis and with threatening signs of cardiac weakness), the action of diet alone is quite slow (about two to three pounds a month). In vigorous persons who, besides pursuing their daily occupations, can devote an hour to an hour and a half daily to mountain climbing, bicycling, rowing, exercise in the gymnasium, etc., at first losses in weight of four to six pounds, later of two to four pounds, a month are the result. For a time, as for instance during a vacation in the mountains, these losses may without excessive exertion be increased to 11 pounds a month or more.

Third grade of antifat diet.-To this belong antifat cures in which the diet is less than three-fifths, or even only two-fifths of the usual requirement, i. e., about 1,000 to 1,400 calories. In this class belong the well-known dietaries calculated to produce decided and rapid action, those of Banting-Harvey, Oertel, Ebstein, and others, as is shown by the following tables:

\begin{tabular}{|c|c|c|c|c|}
\hline DIET SCHEME ACCORDING TO & Albumin. & $\begin{array}{c}\text { Carbo- } \\
\text { hydrates. }\end{array}$ & Fat. & Calories. \\
\hline 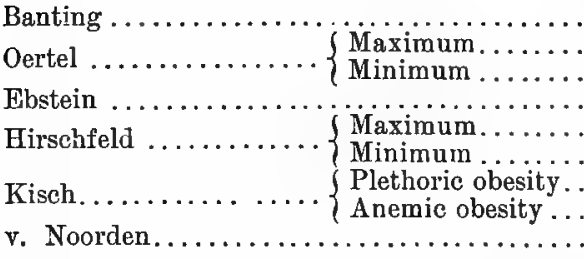 & $\begin{array}{l}172 \\
170 \\
156 \\
102 \\
139 \\
100 \\
160 \\
200 \\
155\end{array}$ & $\begin{array}{r}81 \\
120 \\
75 \\
47 \\
67 \\
50 \\
80 \\
100 \\
112\end{array}$ & $\begin{array}{r}8 \\
45 \\
25 \\
85 \\
65 \\
41 \\
11 \\
12 \\
28\end{array}$ & $\begin{array}{l}1,100 \\
1,600 \\
1,180 \\
1,300 \\
1,400 \\
1,000 \\
1,086 \\
1,116 \\
1,366\end{array}$ \\
\hline
\end{tabular}

1 v. Noorden, "Die Fettsucht" in Nothnagel's " Handbuch der speciellen Pathologie und Therapie," Wien, 1900, p. 110. 
A diminution of food to about two-fifths of the usual requirement is looked upon as the utmost that may be wisely attempted in the obese patient. The amount and rapidity of the loss of weight depend in the main on the accompanying conditions. If the patient is inactive, the loss in weight amounts to from $6 \frac{1}{2}$ to 13 pounds in a month. The greater reductions are noted in the medium, the slighter decreases in the most marked grades of obesity. If auxiliary measures, such as systematic muscular exercise, hydrotherapeutic agents, mineral spring cures, etc., are utilized, the loss in weight may readily be increased to 22 to 33 pounds in a month.

The following table showing the success which Dapper attained in his sanatorium at Kissingen, in which the diet scheme was based upon that proposed by me, is instructive:

\begin{tabular}{|c|c|c|c|c|}
\hline NUMBER. & $\begin{array}{c}\text { Men. } \\
\text { Women. }\end{array}$ & $\begin{array}{c}\text { Weight at } \\
\text { the onset } \\
\text { in kilograms. }\end{array}$ & $\begin{array}{l}\text { Duration of } \\
\text { the treatment } \\
\text { in weeks. }\end{array}$ & $\begin{array}{l}\text { Loss of weight } \\
\text { during the } \\
\text { time in } \\
\text { kilograms. }\end{array}$ \\
\hline 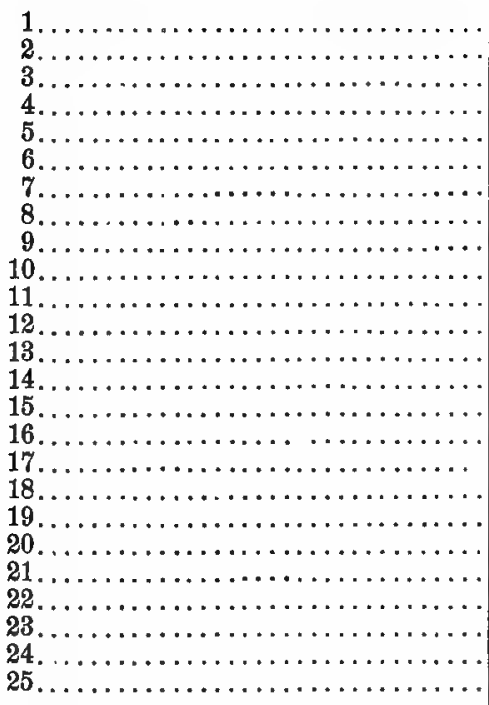 & $\begin{array}{l}\text { M. } \\
\text { W. } \\
\text { M. } \\
\text { M. } \\
\text { W. } \\
\text { M. } \\
\text { M. } \\
\text { M. } \\
\text { W. } \\
\text { M. } \\
\text { W. } \\
\text { M. } \\
\text { W. } \\
\text { M. } \\
\text { W. } \\
\text { M. } \\
\text { M. } \\
\text { W. } \\
\text { W. } \\
\text { M. } \\
\text { M. }\end{array}$ & $\begin{array}{r}129.5 \\
104.5 \\
99.5 \\
114.5 \\
104.5 \\
112.0 \\
104.5 \\
111.0 \\
74.0 \\
101.0 \\
97.0 \\
97.0 \\
112.0 \\
107.0 \\
116.0 \\
74.7 \\
96.0 \\
110.0 \\
128.0 \\
107.0 \\
106.7 \\
113.5 \\
133.7 \\
114.0 \\
105.8\end{array}$ & $\begin{array}{l}5 \\
4 \frac{1}{3} \\
2 \\
4 \\
5 \\
5 \\
3 \\
4 \frac{1}{2} \\
5 \\
5 \\
4 \\
2 \\
4 \frac{1}{2} \\
4 \\
4 \\
4 \\
3 \frac{1}{2} \\
4 \\
4 \frac{1}{2} \\
4 \\
4 \\
5 \\
5 \\
5 \\
4 \frac{1}{2}\end{array}$ & $\begin{array}{c}17.75 \\
9.5 \\
4.5 \\
15.0 \\
11.5 \\
10.75 \\
5.5 \\
11.5 \\
7.5 \\
10.0 \\
9.5 \\
4.5 \\
10.0 \\
9.0 \\
10.75 \\
5.0 \\
8.0 \\
9.5 \\
10.5 \\
11.5 \\
9.5 \\
10.5 \\
13.0 \\
13.5 \\
10.0\end{array}$ \\
\hline
\end{tabular}

In what proportions the main constituents of the food, albumin, fat, and carbohydrates, are to be arranged in the antifat diet of milder and medium grades is of minor importance. The restriction of food is not so complete that danger from uniformity of the diet is to be feared. On the other hand, the question as to how we are to act in antifat cures of the third degree has been much discussed. It cannot be considered of vital importance, for practical experience has sufficiently demonstrated that with a high intake of albumin (Banting, Oertel, Kisch, v. Noorden) as well as with a low intake of albumin (Ebstein, Hirschfeld) satisfactory results have been attained. The investigations in metabolism of the last few years are in accord with this. I regard the question as one of technic rather than of theory or principle. 
Experience appears to prove that in numerous patients the administration of large amounts of meat-equivalent to a high intake of albumin-is much better than small ones. Meat is not only the most important food for most persons, but also the one most desired. Nevertheless, there are many corpulent persons, especially women, who dislike large amounts of meat, and in whom any antifat dict that consists largely of meat will certainly suffer shipwreck. Nothing can be more unwise than, for the sake of theory, to force the administration of albumin up to a certain height; the requirements of the individual case and nothing else should be considered. But we must insist on not less than the minimum of about 100 grams of albumin. Above this, theoretically, the limit cannot be definite. In practice, about 180 grams of albumin per day should rarely be exceeded.

No less warm than the question of the administration of albumin is the discussion, whether, among the substances free from nitrogen, more fat or more carbohydrate should be given. The important point is, that the total of these, according to their calory values, must be diminished so that the total amount of food reaches the degree aimed at in the antifat diet. But both the fat and the carbohydrates must be reduced below the average amount if the administration of nourishment is to be limited to two-fifths or threefifths of the average requirement (see above). If only the one or the other is forbidden or limited-whether it be the fats or the carbohydrates-sufficient of the other remains in the diet to render the result dubious. Whether in the simultaneous limitation a little more fat (Ebstein, Hirschfeld) or a little more carbohydrate (Banting, Oertel, Kisch, v. Noorden) remains is of subordinate importance. Only the calory total of both, not the chemical constitution-whether fat or carbohydrates-is of significance. I regard it as only a question of technic how far the limitation of one or of the other is to be carried. The wishes, the manner of life, and the peculiarities of the patients, not the principles of treatment, are the determining factors. But from my own experience I should like to emphasize the fact that, as a rule, we attain better results if the fats are limited as much as possible, and somewhat more of carbohydrates are permitted in the food; for the carbohydrates furnish a larger volume, satisfy more readily, and permit a much greater variation in the diet.

Alcohol, of course, must not be given with a free hand in antifat cures. As already remarked, it saves fat and in this manner favors its accumulation. Many persons owe their excessive adipose tissue, primarily, to the immoderate use of alcohol. Nevertheless, almost all authors have ascribed to alcohol a subordinate place in antifat cures-and quite properly so, since in practice its stimulating effect cannot always be dispensed with. But where it is possible-particularly in young, robust people - it is wise to exclude alcohol on account of its high calory value.

In antifat cures the question of the allowance of water is usually discussed. Among laymen, and also among physicians, the opinion prevails that the intake of fluids favors the accumulation of fat. This is unquestionably incorrect. How easy and cheap would it be for the farmer if the administration of water favored fattening! On the other hand, the statement that antifat 
treatment is favored by limiting the amount of water cannot be pronounced unqualifiedly incorrect. We must be quite clear that this fact has no bearing on the relation between the administration of water and decomposition of fat-as taught by Oertel and Schweninger-but that water has its effect by influencing the intake of food. Many persons eat decidedly less when forbidden to take as much fluid as they have been accustomed to doing. They certainly eat less if the simultaneous ingestion of fluid and solid food is forbidden. The lessened ingestion of food will then promote the disappearance of body fat. But this result cannot always be counted upon, for among the obese we meet many whose appetite is not influenced in the least by limiting the intake of fluid. They naturally remain fat, or even deposit more fat, no matter how much they are tormented by the thirst cure.

I have formulated the present status of the question in the following statements: ${ }^{2}$

1. A limitation of the intake of fluid, according to Oertel and Schweninger, has not the slightest influence upon the decrease of body fat or upon the increase of fat metabolism.

2. The primary effect of limitation of fluid upon body-weight depends upon the losses of water from the blood and the tissues. This may be utilized therapeutically:

(a) To improve the circulatory conditions in endangered failure of the heart (in cases of valvular disease, discase of the heart muscle, arteriosclerosis, contracted kidney);

(b) In patients with a tendency to immoderate production of sweat, and for the purpose of combating hydrorrhea;

(c) In some patients it is a valuable auxiliary remedy through suggestion.

3. The influence of the limitation of water upon fat metabolism, particularly in decreasing the amount of fat, is only indirect, and comes into question only under special conditions, namely:

(a) If, by decreasing the fluid intake, the circulatory disturbances present are to be compensated; there may be then a possibility of favoring the combustion of fat by muscular exercise.

(b) When, by decreasing the fluid intake, the appetite of the person for fat-producing foods is diminished. This result may appear in a marked degree, more frequently in slighter degree, or not at all.

4. When no special indications are present the intake of water is not to be limited in the obese, as, without this, we attain the same end and the limitation of water in such cases is only an unnecessary hardship for the patient.

That, in antifat cures, under-nutrition is not the only agent to be employed, but that this is to be assisted by systematic muscular exercise, has been reiterated. I shall not enter into individual points, but will only refer to other chapters of this book in which the treatment of obesity has been explicitly described.

Here it is only necessary to indicate the important points that should 
be remembered in all curative methods in which over-nutrition and undernutrition come into play. The better the physician understands the general laws of these cures, the more readily will he be able to meet the indications of the individual case. The successes of dietetic therapy may only too readily become matters of routine. Many practitioners adhere too closely to definite diet schemes which have been proposed by this or that celebrated author, or they even hand to the patients a so-called diet list which refers only to the disease, and not to the patient, and which means the annihilation of individual dietetic therapy. Only the intimate union of practical experience with a comprehensive understanding of fundamental theoretic laws will enable the physician to choose rightly in every case. He must always bear in mind that, although there are many methods of securing over-nutrition and undernutrition, for the special case there is but one best method. 


\title{
DIABETES MELLITUS
}

\author{
By B. NAUNYN, Strassburg
}

Contents: I. Glycosuria and Diabetes. II. 'The Various Forms of Diabetes; Predisposition. III. Mild and Severe Forms of Diabetes; Course, Symptoms and Complications. IV. Theory and Treatment. V. Practical Management. VI. Tables; Diet Lists; Scheme of Food Values; Dietetic Rules for Diabetics.

\section{GLYCOSURIA AND DIABETES}

Glycosuria, Levulosuria, Lactosuria and Pentosuria. Physiologic and Alimentary Glycosuria. Alimentary Glycosuria e saccharo; Non-Diabetic and Diabetic Forms. Spontaneous Non-Diabetic Glycosurias.

By the term "glycosuria" we mean the appearance in the urine of true grape-sugar (dextrose, glucose), which is dextrorotary. We also speak of "levulosuria," when we mean the excretion of levulose, a fruit sugar which is levorotary. "Lactosuria" means the excretion of milk-sugar (lactose). A sugar containing five atoms of carbon, in its molecular composition known as pentose, may also appear, hence the term "pentosuria."

Lactosuria may occur during pregnancy and in nurslings, and has nothing in common with diabetes. Pentose may be found in the urine after the ingestion of cherries, plums and beer, and occasionally also in diabetic urine, but its rôle in diabetes is quite obscure.

On the other hand, there are cases of diabetes mellitus in which levulosuria takes the place of dextrosuria and levulose plays the part in the disease which ordinarily is assumed by dextrose. Such cases are, however, very rare, not more than half a dozen being known.

Glycosuria (dextrosuria) is the cardinal symptom of diabetes mellitus, but not every case of glycosuria is of a diabetic nature. There is a so-called physiologic glycosuria; in other words, a normal individual may excrete dextrose in his urine, but the quantity is always extremely small. Disregarding for the present so-called alimentary glycosuria, the percentage of sugar in the urine of healthy individuals is seldom more than 0.05 per cent., although it may reach 0.1 per cent. or even 0.2 per cent.

It is fortunate that the amount of sugar in normal urine does not react to the ordinary tests. Trommer's (or Fehling's) and also Fischer's test only give a positive reaction in urine when the amount of sugar present is abnor- 
mally high; i. e., over 0.1 per cent., and only when this quantity of sugar is present can it be quantitatively estimated. If on boiling urine in an alkaline solution of copper sulphate (Trommer's test) we immediately, not subsequently, obtain a red or reddish-yellow precipitate, or if on warming the urine with phenylhydrazin and acetic acid on a water-bath for half an hour, a distinct crystalline sediment is deposited, we may be sure that the glycosuria is not physiologic. As possible sources of error there may be mentioned the presence in the urine of lactose, pentose, and combinations of glycuronic acid (after the administration of chloral, chloralamid, etc.). Lactose and pentose as well as combinations of glycuronic acid have a considerable power of reduction, but we may readily distinguish them from dextrose by the fermentation test and by polarization.

In diabetes, then, we are concerned only with hyperglycosuria, or, in other words, with cases in which the sugar exists in such quantities as to react readily to the above mentioned tests. But hyperglycosuria may exist independently of diabetes. The so-called alimentary glycosuria is a case in point: a person whose urine does not contain sugar partakes of sugar or sugar forming substances (starchy material, dextrin) in sweetened foods and liquids, beer, bread or potatoes, etc.; if sugar then appears in the urine so that it may be detected by means of any of the ordinary quantitative tests, this condition is designated alimentary glycosuria.

In diabetics whose urine is temporarily free from sugar, this is of quite regular occurrence. It also takes place in non-diabetics, but with this difference, that in the diabetic the sugar producers in the food-the flour in bread, etc.-if consumed in large amounts give rise to glycosuria almost as surely as the sugar itself, while this condition only occurs in the non-diabetic from sugar and not from starchy substances.

Therefore, glycosuria after the ingestion of sugar need not be diabetic, but may be produced in normal persons even up to several per cent., provided the sugar (grape-sugar, milk-sugar, or cane-sugar) is given upon an empty stomach in amounts of 100 grams and over. If such a glycosuria occur after the consumption of 100 grams of sugar (usually grape-sugar is used) when the stomach is no longer empty, we are dealing with abnormal glycosuria, i. e., alimentary glycosuria e saccharo. This may indicate diabetes or be the first sign of the development of the disease; in other words, the person in question may develop diabetes sooner or later, but not necessarily; the individual need not be a diabetic, nor even become one.

Taking as a criterion the nature of the processes concerned, let us now attempt to differentiate between diabetic and non-diabetic glycosuria.

In the diabetic, the organs which are concerned in the consumption of sugar have suffered damage and are incapacitated for work; they fail to extract the sugar from the blood which is brought to them, or they return it to the circulation, as they are incapable of utilizing it. Thus a hyperglycemia and consequent glycosuria arises, provided the amount of sugar in the blood is more than 0.2 per cent.- 0.3 per cent., and this is a diabetic hyperglycemia and glycosuria. The condition depends upon obstruction to the normal drainage of sugar from the blood by the organs of sugar metabolism, and it is for 
this reason that sugar is excreted by the kidneys. But the organs whose function it is to burn sugar cannot take up from the blood and consume an unlimited amount of sugar, even if they and all the other organs are perfectly sound and function normally. It is true that a flooding of the blood with sugar can hardly occur in health through the ingestion of starches, even in the largest amounts, as these are absorbed too slowly, but it may readily occur after ingestion of large quantities of readily absorbable sugar solutions. That alimentary glycosuria (e saccharo) may be explained in this manner is evident from the fact that in most of these cases the sugar (for example, milk-sugar or levulose) is excreted in the same form in which it is introduced. This explanation is also favored by the circumstance that alimentary glycosuria occurs more readily when the stomach is empty, for, as is well known, absorption is then more rapid. This pathologic but nondiabetic alimentary glycosuria (e saccharo) is probably due, therefore, to an abnormally hastened absorption of the sugar solution.

Finally, non-diabetic glycosuria, i. e., glycosuria despite a normal condition of the sugar-consuming organs, may occur when the renal secretion has become so increased that sugar and other solids are drawn out of the blood in abnormally large quantities and excreted with the urine. Perhaps it may be possible to explain in this way the fact that, after the ingestion of much beer or champagne, sugar (even several per cent.) is occasionally found in the urine.

It is possible, however, that in these "beer glycosurias" the damaging influence of the alcoholic beverage upon the liver is also a factor; the possibility is obvious enough, since, in cirrhosis of the liver, alimentary glycosuria is frequent. In these cases we are then concerned with diabetic glycosuria, i. e., with the complication of cirrhosis of the liver and diabetes mellitus.

This example shows how difficult it is to separate alimentary glycosuria from the diabetic form, and there are many other illustrations of this diffculty. Thus, in traumatic neuroses true diabetes may occur. On the other hand, alimentary glycosuria (e saccharo) is a particularly frequent symptom in traumatic neuroses. And one cannot look upon this sign, in all cases, as indicating diabetes-at any rate in the overwhelming majority of cases such a condition does not arise. In exophthalmic goiter the same state of affairs exists. Taken all in all, in every alimentary glycosuria, including the "e saccharo" variety, it is necessary to observe great caution in deciding whether this is a sign of diabetes or not. Alimentary glycosuria following the ingestion of starch must always be looked upon as a sign of true diabetes.

"Experimental" glycosuria in the human being does not play a great rôle, although it occasionally occurs-for example, glycosuria after poisoning with coal gas, or with phloridzin for purposes of malingering. These conditions must be understood in order to recognize that they are not cases of diabetes.

In all cases of spontaneous glycosuria (non-alimentary) the greatest care is necessary before deciding whether they are to be looked upon as signs of diabetes or not. We must be sure that pentose or glycuronic acid in the urine is not mistaken for glycosuria; when lactosuria occurs in pregnancy or in 
the puerperal state, we must know that this has nothing to do with diabetes. As an example of true glycosuria appearing spontaneously in man, yet not the expression of diabetes, I can only mention the form occurring in cholera asiatica and cholera nostras.

All other forms (unless proofs are present to the contrary) should be looked upon as diabetic; but we need to know what is meant by diabetes, i. e., the disease of this name as it occurs in the human subject.

\section{THE VARIOUS FORMS OF DIABETES; DIABETIC PREDISPOSITION}

Acute Diabetes, the Acute Form Terminating Fatally and the Acute Form Resulting in Recovery-Transitory, Nervous (Cerebral Trauma), and Pancreatic Glycosuria Representing the Shortest Course of Acute Diabetes Mellitus Terminating in Recovery. Chronic Diabetes Mellitus as an Expression of an Hereditary Predisposition. Its Three Varieties: (a) The Pure Form, (b) Diabetes of the Aged, and (c) Organic Diabetes Mellitus. Etiology of Diabetes Mellitus and the Possibility of its Cure in the Light of Hereditary Predisposition-the Heredity of Diabetes Mellitus. One Form or Several Forms of Diabetes?

Diabetes is generally considered a chronic affection, but it may take an acute course and be either rapidly fatal or readily curable.

Well studied cases with an acutely fatal course have been reported; for instance, Wallach reports the case of a chemist who was accustomed to make weekly examinations of his own urine. The urine was always free from sugar until suddenly a severe glycosuria appeared, and within five weeks the man died in diabetic coma.

The question of acute cases that terminate in recovery is less simple.

Schmitz reports the case of a four-year-old child of a diabetic mother. The anxious mother had the urine of the child examined frequently, and it was always free from sugar up to November 22, 1871. On November 26th, the child was attacked by a gastric fever, and on the 2\%th the sugar contained in the urine amounted to 5.8 per cent. The child was put upon strict diet, and on the 13th of December the urine was again free from sugar. Although the patient gradually returned to the usual saccharine and amylaceous diet the urine remained normal for twenty years. Whether it is correct to say that the diabetes had already been cured upon the 13th of December is at least very questionable, for at that time, and for a long period afterward, the child lived upon a very strict diet, which later was even more restricted, so that very little was expected from its carbohydrate metabolism. On December 13 th, the recovery was probably only relative.

Zinn observed a child attacked by diabetes mellitus after scarlatina; the patient, after ten weeks, even upon "mixed diet" showed no sugar in the urine; unfortunately the observations in regard to the recovery were only continued for a few months.

Similar conditions may be observed in all cases of true diabetes in which recovery takes place.

Diabetes after cerebral injuries also occurs as an acute disease which may 
terminate in recovery. As a result of trauma of the brain we note cases of diabetes that are severe, even very severe; then there are cases which run a milder course similar to those of Schmitz and Zinn mentioned above; finally, there are cases after cerebral injuries, in which transitory glycosuria, without any further consequences, occurs and lasts but a few days. What right have we to deny that these transitory glycosurias represent the mildest forms of diabetes mellitus? The same lessons which are taught us by the study of diabetes after cerebral injury in the human subject are paralleled as regards pancreatic diabetes by experiments upon animals; extirpation of the pancreas produces the most severe forms of chronic diabetes; transitory lesions of the organ produce mild ephemeral glycosuria.

In my opinion we must admit that diabetes may occur as an acute affection, and this conclusion has some practical importance; spontaneous, true, acute diabetes is certainly very rare, but transitory, spontaneous (?) glycosuria may be explained in this manner:

Although the course of the disease is for the most part chronic, the conditions are not accurately designated if we call diabetes "a chronic disease"; in the majority of cases there is an innate hereditary predisposition, a weakness of sugar metabolism, which, sooner or later, in combination with other causes, or even without, such, may lead to insufficiency of sugar metabolism and thus give rise to the disease.

According to this, three forms of the disease may be differentiated:

1. Diabetes mellitus of young individuals (those between thirty and forty years of age) ("pure diabetes").

In this form the hereditary weakness of sugar metabolism, without the association of any special disease of an organ, progresses to insufficiency. This occurs at an early period of life, owing to a particular severity of the pathologic predisposition, and, in keeping with this, the cases are for the most part serious.

2. Diabetes of the aged, the usual mild diabetes of old persons; the saying that "the age of an individual depends upon the condition of his arteries" is also true in these cases; usually, arteriosclerosis is the underlying condition and with this all factors are operative which favor its development; luxurious living and, above all, the use of alcohol.

It is readily seen that the least severely predisposed cases are the ones in which this form of diabetes is most likely to appear; perhaps this accounts for its relatively mild course.

3. Organic diabetes; this refers to cases in which disease of a particular organ is the immediate cause of the affection. As examples, there may be mentioned: $(a)$ among the diseases of the liver, cirrhosis and, rarely, chronic cholelithiasis; (b) among diseases of the nervous system, apoplexy, chronic encephalomalacia, cerebral syphilis, dementia paralytica, tabes dorsalis, cerebral trauma of all kinds, the various functional neuroses, particularly the traumatic ones, and paralysis agitans; $(c)$ diseases of the thyreoid gland (Graves' disease), and $(d)$ diseases of the pancreas.

Experimental evidence would seem to show that the pancreas takes the first position among the organs which cause diabetes, since by extirpation or 
by a more or less extensive resection, true diabetes may be produced. Our investigations in man, however, only correspond with this in so far as extirpation of the pancreas and disease of the pancreas also cause diabetes in the human subject. On the other hand, diseases of the pancreas have only in rare instances been determined with certainty as the cause of diabetes; these affections are pancreatic calculi, pancreatic cirrhosis, neoplasms and cysts.

If we have disease in an organ which is very influential in sugar metabolism, as, for instance, the nervous system or pancreas, this suffices to produce diabetes, even without a previous predisposition; if, however, a predisposition exists, disease of one of the vital organs is not absolutely necessary. Slight general disturbances are sufficient to bring about an insufficiency in sugar metabolism. Thus the varying etiology of diabetes mellitus becomes comprehensible, and we learn to recognize as causes not only the above mentioned organic diseases, but also many severe or mild general affections: influenza, enteric fever, scarlatina, erysipelas, phlegmons, syphilis, and, finally, the most manifold forms of trauma, surgical operations, psychical disturbances, bodily or mental over-exertion, indigestion and excesses of all kinds; all of these are occasionally of etiological significance in diabetes mellitus.

This conception of diabetes mellitus as an expression of an hereditary weakness of metabolism makes clear at once what we can expect as regards the cure of diabetes.

One can speak of actual cure only in those cases in which the disease appears as the result of an affection of one of the principal organs; if an affection of the organs in question improves, then diabetes may actually be "cured." That this occurs is certain (in man) in the case of diabetes as the result of brain injuries.

If, however, as in most cases, the cause of diabetes must be referred to hereditary predisposition, we must at once assume a skeptical attitude in regard to the cure, all the more so if the predisposition is the only causal factor. Where other causes are associated with this, e. g., cirrhosis of the liver, infectious diseases, especially syphilis (we may remark in passing that syphilis plays but a very slight rôle in the etiology of diabetes), arterio-sclerotic circulatory disturbances, we have more reason to hope that, by treatment or by cure of the causes simultaneously present, sugar metabolism may again become sufficient, but the predisposition remains, and with it the danger that any fresh damage may bring about a relapse.

Many cases may be explained in this way; for example, an elderly person who may have suffered many years ago with diabetes, is apparently cured; i. e., he returns to his usual mode of living without paying much attention to his "cured" diabetes. The urine is free from sugar; he is then attacked with influenza, or undergoes some marked psychical disturbance, and the glycosuria at once returns quite severely; or he has to submit to an operation, and after the operation a coma ensues, coma diabeticum, the urine containing many per cent. of sugar.

We are justified in assuming (as we have) an hereditary predisposition for many cases of diabetes, apart from what has already been said, by the fact of the heredity of the disease. In most cases of diabetes, heredity is a factor; 
it has been proven in more than 30 per cent. of my cases, and becomes more frequent the more closely I investigate. This hereditary predisposition is true of all three varieties, and can be demonstrated in a like number of cases of the organic form, in diabetes of the aged, and in "pure diabetes." The hereditary factor in diabetes is related to the neuropathic taint and to the predisposition to gout and obesity. In many families gout or some variety of neurosis or psychosis occurs alternately with diabetes, and if, in such diabetic families, one of the members is conspicuously fat, this is very suspicious of diabetes. These are, incidentally, the cases for which the improperly used term "diabetes of the obese" should be reserved, the cases, namely, in which obesity points to diabetes.

It is often said that as the cases of diabetes are quite different, and as these etiologic differences correspond with differences in the course and the symptomatology, diabetes should no longer be looked upon as a single disease. We should no longer speak of "diabetes mellitus," but of "various forms" of diabetes mellitus. But in answer to this we must again emphasize in all forms of diabetes the hereditary predisposition. This is the common bond which holds these various forms together; it is operative in each of the varieties which are to be differentiated etiologically. In every type of the disease, in nervous diabetes, as well as in liver diabetes, in diabetes after infections, in diabetes of the aged, and in "pure" diabetes, 30 per cent. (or more) are herëditary cases.

Further, we do not know at present whether the pathogenesis of glycosuria, i. e., the disturbance in metabolism which causes it in the different cases, is as multiform as the etiology; on the contrary, the glycosuria which occurs in all the various types of diabetes mellitus (so far as this point has been investigated) is hyperglycemic in origin, i. e., the condition is due to an increase in the amount of sugar in the blood. This is only true, however, of diabetes mellitus as it occurs in man; we can produce in animals and in man an experimental glycosuria which is not hyperglycemic, i. e., the form due to phloridzin. In animals even the most marked phloridzin glycosuria produces no noteworthy increase of sugar in the blood.

We cannot, therefore, see any sufficient reason for speaking of "various forms" of diabetes, but we do distinguish a mild and a severe form of the disease. This distinction will be observed throughout the following description of the symptomatology and it will also be seen that we have taken full account of the differences in etiology between different groups of diabetic cases.

\section{MILD AND SEVERE FORMS OF DIABETES; COURSE, SYMPTOMATOLOGY AND COMPLICATIONS}

Mild and severe forms of diabetes mellitus. The importance of complicating organic disease in the symptomatology of the mild cases. Differentiation of the various symptoms according to whether they are due to the organic disease or to diabetes mellitus. Hyperglycemia as a cause of the latter. The albuminuria of the diabetic.

In practice we cannot avoid differentiating between mild and severe cases, or the mild and severe forms of diabetes mellitus. It is true that this is only 
a difference of degree; the nature of the affection depends upon the same process in the mild and in the severe cases, and transitional forms are by no means rare.

Well developed cases of the mild and of the severe variety resemble one another as little as an epileptic migraine resembles typical severe epilepsy, or even less. When, in a severe case of diabetes, 4 to 15 or more liters of urine are passed with a specific gravity of 1.025 to 1.040 , or even 1.060 , and a percentage of sugar from 5 per cent. to 12 per cent. daily (200 to 1,500 grams of sugar daily), it is obvious that there must be an enormously increased intake of nourishment; yet it is easy to understand that in spite of this the patient emaciates, becomes debilitated, and soon perishes. The course of the disease in such cases is necessarily brief. Often within a few weeks the bodyweight falls to 60 kilograms in a man or 50 kilograms in a woman, and in spite of all endeavors to prevent this, the patient's condition becomes progressively worse; in from one and a half to three years increasing cachexia, diabetic coma, or pulmonary tuberculosis, terminates life.

Mild cases of diabetes may exist undiscovered for years, even decades, until a complication or an accident leads to the discovery of the glycosuria; so vague may this condition be. In such cases only 10, 30 or 50 grams of sugar are excreted daily, with a normal or slightly increased amount of urine in twenty-four hours, and this state of things may go on indefinitely, although the patient before, as well as after, the discovery of the sugar pays but slight attention to his condition. If he limits the amount of beer, bread and starchy food and gives up sugar and potatoes entirely, this will suffice to keep his glycosuria within the limits above mentioned, whereas, in a severe case, even the strictest diet only brings about an amelioration, and the patient's whole life becomes a combat with glycosuria, a battle for existence. If glycosuria is a symptom in a mild case of diabetes, in a severe case it is the disease, the fatal disease itself. The loss of sugar in the urine, the impossibility of compensating for it by an increased nourishment, and the consequences, among which coma is common, are the conditions from which the patient suffers and to which he finally succumbs.

In the severe cases glycosuria itself is, in a certain sense, the main disease, since usually in cases of "pure" diabetes no disease of any special organ can be found. It is not necessary to discuss the question whether in these cases of "pure" diabetes we are justified in looking upon a disease of some special organ-generally the pancreas-as the cause of the disturbances of metabolism. On account of my own position in regard to pancreatic diabetes $I$ have particularly interested myself in this question; but it is impossible to prove in these severe cases, either from the symptoms and signs present during the life of the patient, or at the autopsy, anything which points to disease of the pancreas or of any other organ. There are, as has already been mentioned, cases of diabetes due to disease of the pancreas, pancreatic calculi, cirrhosis of the pancreas, etc., but they are rare.

The mild cases rarely belong to the type classified as "pure" diabetes; more frequently the symptoms point to hepatic diabetes, to nervous diabetes, or to diabetes of the aged (arterio-sclerotic diabetes). Nevertheless, "pure" 
diabetes may occasionally run a mild course, and cases of the other form may sometimes be severe; this latter course is most frequently seen in the diabetes which occurs after trauma of the brain.

Not infrequently the symptoms of the accompanying disease (hepatic, nervous, or arterio-sclerotic) are much more prominent than the slight glycosuria, and so it happens that the cirrhosis, or the tabes, in spite of the complicating diabetes, runs the same course which it would have assumed without the associated condition. In arterio-sclerotic diabetes, as the disease develops, the arterio-sclerosis may become more and more prominent, presenting circulatory disturbances, cardiac asthma, angina pectoris, dropsy, congested liver and albuminuria; in all of these cases we may note that, as they progress, the glycosuria decreases and finally disappears.

Although in many of these cases the glycosuria does not become prominent, nevertheless, the diabetes and the diabetic disturbances of metabolism usually do not fail to produce evil consequences; on the contrary, there is quite an array of complications and symptoms which appear frequently in mild cases ; pruritus pudendorum, vaginitis, vulvitis, balanitis, phimosis, urethritis, impotence, fermentation of the urine in the bladder (with pneumaturia), and as a consequence of this, cystitis and pyelonephritis; itching of the skin, urticaria, eczema, the most manifold ulcerating dermatoses, furunculosis, carbuncle, lymphangitis, boils, intermittent claudication, gangrene of the toes, necrosis of internal organs, pulmonary gangrene, pulmonary tuberculosis; also a host of nervous diseases; encephalomalacia, column degeneration of the spinal cord, neuritis, polyneuritis, neuralgia, peripheral paralyses (particularly paralysis of the facial nerve), malperforant, very frequently loss of the patella reflex which occasionally ushers in the remarkable picture of pseudo-tabes diabetica.

It is impossible to bring this confused array into any systematic arrangement; any one of these symptoms, or complications, may appear alone as the first sign of a diabetes, latent up to them; each of these conditions may remain the only one, or be succeeded in any order by any of the others.

For some of these conditions diabetes alone cannot be held responsible; thus arterio-sclerosis is the true cause of gangrene of the extremities with its occasional prodrome, claudicatio intermittens, perhaps also of malperforant. This is true, even though these lesions occur during the course of a case of diabetes. In the peripheral paralyses and neuralgias, the neuropathic predisposition doubtless often plays a part, and the diabetics that suffer from polyneuritis are, as far as I know, all alcoholics.

Naturally the question next arises whether diabetes has any influence at all in the production of these lesions; for example, in cardiac asthma or in angina pectoris of the diabetic; take the case of an old diabetic, with a glycosuria of from 3 per cent. to 4 per cent., from 50 to 60 grams of sugar per day, suffering from cardiac asthma or angina pectoris; there is slight venous stasis of the liver, occasionally slight albuminuria; nothing abnormal can be detected in the heart. Nevertheless, there is a well-grounded suspicion that a beginning cardiac insufficiency, perhaps due to myocardiac degeneration and perhaps to arterio-sclerosis, is present. Removal of the glycosuria 
helps but little; digitalis, however, soon brings relief. We must not be too certain that, in these complications of diabetes, glycosuria does not play a part, and we should never fail to attempt to remove the glycosuria, for there are cases, like the one just described, in which no result can be obtained without this method of treatment. It should remain a rule that wherever there is even a possibility that the symptom present is of a diabetic nature, the attempt must be made to reduce the glycosuria.

We are compelled, then, seriously to consider glycosuria as the cause of these symptoms. Glycosuria may produce symptoms in two ways: First, by the loss of sugar; of this we have already spoken and shall have to refer to it again frequently; in mild diabetes the loss of sugar is not important, and glycosuria as such is only to be considered as the cause of urinary fermentation and its consequences, such as pneumaturia, cystitis, pyelonephritis, pruritus pudendorum, balanitis, vaginitis, etc. In mild diabetes it is the hyperglycemia that we hold responsible for symptoms.

As long as the diabetic excretes sugar, the sugar contents of his blood is increased above the normal and there is hyperglycemia. In marked glycosuria (above 1 per cent.) the hyperglycemia amounts to over 0.2 per cent. and in the severe grades of glycosuria it may amount to 0.7 per cent.; if the glycosuria is slight ( $\frac{1}{2}$ per cent. to 1 per cent.), or if the urine of the patient is free from sugar, the hyperglycemia is very slight, scarcely amounting to more than 0.1 per cent. (which is almost normal), and then the condition is not serious.

Diabetic hyperglycemia may certainly be held responsible for the loss of resistance of the diabetic - even of the mild cases-toward infections; at least it has been experimentally proven that many of the pathogenic microbes flourish better in the tissues which contain sugar. I shall include, besides this, the cutaneous affections and the neuralgias as among the complications dependent upon the hyperglycemia, basing my opinion upon the results of the therapeutic removal of glycosuria. Removal, perhaps, implies too much; it would be more correct to say reduction, for to render the hyperglycemia innocuous, it is almost always sufficient to reduce the glycosuria to the limit mentioned above.

Regarding the symptomatology and complications of diabetes, I must limit myself to what has been previously mentioned. The other important symptoms not yet enumerated, for instance, coma, will be spoken of elsewhere; only in regard to albuminuria I should like to add a few words.

Albuminuria has one significance in mild and another in severe cases; in mild cases, the albuminuria is the expression of a renal affeetion independent of the diabetes, although in some cases arteriosclerosis or hepatic cirrhosis may represent a connection between these diseases and the diabetes. In severe diabetes, the albuminuria may be referred directly to the diabetes; it is the consequence of the excessive functional irritation from which the kidney suffers owing to the continued polyuria.

Perhaps the abnormal constitutents of diabetic urine, possibly the sugar itself, may irritate the renal elements. But this diabetic albuminuria is not nephritic in origin, i.e., it does not denote nephritis; and we should err 
greatly if we were to diagnosticate nephritis on this account. What is found at the autopsy is the large, slightly hyperemic " diabetic kidney" which shows none of the changes that we expect to find in nephritis.

The transition of diabetes into nephritis, which is frequently mentioned, is, therefore, very questionable; the true diabetic albuminuria of severe cases is not nephritic, and the albuminuria of the mild cases is not diabetic, but frequently due to an independent nephritis. It is true that a genuine nephritic albuminuria not infrequently takes the place of glycosuria; for when the arterio-sclerotic or other form of chronic nephritis which may appear becomes really severe, the albuminuria becomes more pronounced, whereas the sugar disappears from the urine.

\section{THE THEORY OF THE TREATMENT OF DIABETES}

Diabetic glycosuria has a tendency to increase during a decrease in the patient's tolerance, whereas during sugar-free periods the tolerance usually increases. Hypercompensatory hyperglycemia. Dietetic aglycosuria as a theoretic postulate. The relative importance of carbohydrates, albumin, fat, and alcohol for the diabetic. Difficulties in supplying sufficient nutrition; temporary under-nutrition not always avoidable. Acidosis diabetica with acetonuria and diaceturia. The secondary increase of albumin waste and the secondary decrease of the powers of oxidation in diabetics. Acidosis an expression of the disproportion between over-abundant tissue-destruction and the lessened powers of oxidation. Acidosis as a cause of the diabetic (dyspneic) coma.

The only method of treatment of real value in diabetes is the dietetic. (We shall refer later to the medical treatment.)

The foundation for the dietetic treatment in diabetes was laid one hundred years ago by an English physician, Rollo, but it is only of late years that this method of treatment has become general. Rollo discovered the correct method empirically, but, as is frequently the case, the theoretic foundation had to be worked out before his treatment obtained general recognition, and it required great labor to establish this theoretic foundation. But it is not for this reason that I intend to enter somewhat more minutely into these theoretic considerations of the treatment of diabetes, but because we cannot otherwise obtain such clear ideas of diabetes as are required for the practice of medicine.

THE FIRST FUNDAMENTAL PRINCIPLE in the treatment of diabetes to which I refer is this:

Diabetic glycosuria usually increases with time, while the tolerance of the patient decreases.

The tolerance of the diabetic depends upon the relation between the amount of sugar excreted and the quantity of sugar and sugar producers (particularly the carbohydrates) ingested.

In the following example, for the sake of simplicity, I shall estimate the entire amount of the carbohydrates in the food as bread.

A patient receives 500 grams of meat, 3 eggs, 400 grams of vegetables deficient in carbohydrates (salad, spinach, etc.), 100 grams of fat (cheese, sausage), in which there is some albumin which need not be taken into account, butter and fat in the food, 100 grams of wheat bread, 100 grams of cream and the necessary wine, coffee and water. He excretes two and a half liters of urine, with 3.5 per cent. sugar, that is, on the average, 
88 grams of sugar per day. If he lives for months, with this glycosuria, it is to be expected that with the same nourishment he will excrete more sugar; for example, 4 per cent., that is, upon an average, 100 grams per day; in other words, his tolerance (for carbohydrates) has decreased.

This lessening of tolerance, due to the glycosuria, may not take place if the glycosuria is very slight and does not amount to more than from ten to twenty grams in a day; but in cases in which the glycosuria shows such an increase as in the example just quoted, the patient's tolerance is bound to diminish within a short time.

THE SECOND FUNDAMENTAL PRINCIPLE which is a necessary complement to that mentioned above is as follows:

When the diabetic is free from sugar, his tolerance usually increases.

For example, a diabetic on the diet previously mentioned excretes 88 grams of sugar. To diminish the glycosuria his allowance of bread is reduced; this decrease in food, however, is not sufficient; bread must be withdrawn entirely to render the patient free from sugar; as soon as even ten grams of bread are allowed he begins to excrete sugar again, although only from six to eight grams per day. Next the patient remains for four weeks without bread and his urine becomes free from sugar. After this, if the physician is careful, from twenty to thirty or forty grams of bread may gradually be given without being followed by an excretion of sugar; the patient's tolerance, during the sugar-free period of four weeks, has been materially improved.

How may both these conditions be explained? The improvement of tolerance during the sugar-free "period exemplifies Hoffmann's law that "by lessening the work of any diseased function the latter may be improved," which law, however, if we are honest, is, in the case of diabetics, little more than a lengthy statement of the facts which are to be explained.

There is more hope of success in the attempt to explain the aggravation of the patient's intolerance of carbohydrates during every decided glycosuria. The amount of sugar in the blood, as is well known, is quite constant in man, at least it never falls below a certain point (about 0.8 per cent.); if, for example, after severe muscular exertion, sugar almost disappears from the blood, a fresh supply is sent from the carbohydrate storehouse (the liver); this may be called a process of compensation. If now, after severe sugar losses, it is desirable that a great amount of sugar be quickly brought to the blood, it appears (according to some facts the explanation of which I shall not give at this point) that a hypercompensation occurs. Hence arises the paradoxical conception that loss of sugar produces hyperglycemia! If this seeming paradox is really true, and if it holds good even for the diabetic with an already existing hyperglycemia, then a spontaneous increase of diabetic hyperglycemia by hypercompensation becomes comprehensible. Therefore, as long as sugar is lost by the urine, a steady increase of hyperglycemia leading to a still greater glycosuria is to be expected.

Whether these attempts at explanation are correct or not, the two laws themselves to which they refer are as near the truth, I think, as can be determined to-day. And as the aim of the therapy of diabetes they show us this: to render the patient free from sugar and to keep him aglycosuric.

This conclusion is identical with that arrived at in the end of the last chap- 
ter regarding the meaning of diabetic hyperglycemia. We there said that hyperglycemia is the cause of most of the complications and many of the dangers of diabetes mellitus, and the patient is safe only when he is excreting little or no sugar.

Therefore, there can be no question that in every case of diabetes the therapeutic indication is to render the patient aglycosuric, or sugar-free. It is obvious that the treatment is to be begun as soon as possible, so that little time may remain in which the disease may unfold its tendency to develop ad pejus. If there is any theoretical law as regards treatment which is found to succeed in practice it is this. The chances for successful treatment are, ceteris paribus, very much better in recent cases. It is just as important to insist that the treatment is an obvious necessity in the later course of the disease, and should also be attempted from the beginning of symptoms by means of dietetic treatment. For it has been determined only as regards the dietetic treatment, and not as regards the aglycosuria brought about by drug treatment, that it increases the tolerance of the diabetic.

There are no theoretic contra-indications (i. e., such as might be derived from our knowledge of the diabetic disturbances of metabolism) against carrying out this requirement, although the practical difficulties are frequently great.

The dietetic treatment of the diabetic gains its end by forbidding the use of sugar and sugar-producing foods as far as possible. The sugar-producing foods are the carbohydrates and albumin. Even from albumin sugar is produced in animal metabolism, and in no small quantity, as from 100 grams of albumin (which amounts to about 400 grams of raw meat) about 50 grams of sugar, or even more, may be formed. It is important to note that the organism also produces sugar from those varieties of albumin which contain no preformed sugar, i. e., those from which it is impossible to extract sugar by chemical means. An example of such an albumin containing no preformed sugar is casein, and from this substance the diabetic produces sugar in largé amounts.

Sugar production from fat does not play such an important rôle as to influence diabetic glycosuria to any notable extent. For this reason, and on account of its high calory value, fat is the most valuable food substance for the diabetic.

According to the very latest investigations (Bjierre) it may be looked upon as settled that alcohol has nutritive value (indeed, 1 gram of alcohol upon oxidation furnishes as much as 7 calories) and the diabetic does not form sugar from this. But its utilization as food is limited, for if it is administered in large amounts it acts as a poison to protoplasm, increasing albumin decomposition. It is believed that this toxic action of alcohol is to be feared when more than 50 grams of alcohol are taken per day.

That the patient must be sufficiently nourished, no matter how strict the diabetic treatment, is a well-known rule, but by this we do not mean to say that, temporarily, the patient may not be subjected to hyponutrition; I have already spoken of this. Under some circumstances, as we shall see, it is even then permissible for the patient to abstain completely from food for twenty- 
four hours in order that his urine may become free from sugar. Just so, it is occasionally necessary to give for a few days so little food that some of the patient's body substance is utilized. At the beginning of strict dietetic cures, it is often very difficult to overcome this consumption of tissue in diabetics who have previously lived upon a mixed diet and have excreted much sugar.

Let me again illustrate. Imagine, for example, a man weighing 65 kilograms, who, on a mixed diet, has been excreting 600 grams of sugar daily. He is put upon a diet not absolutely free from carbohydrates. According to Rubner, a man requires for his maintenance about 35 calories per kilogram, so that this man ought to have sufficient food to produce $35 \times 65$, or, in round numbers, 2,300 calories daily.

$\begin{array}{rlrl}80 \text { grams } & \text { wheat bread contain } 50 \text { grams starch } & =200 \text { calories. } \\ 500 " & \text { boiled or fried meat, medium fat (5 per cent. })=750 \\ 200 " & =600 \\ 200 " & \text { cream ( } 30 \text { per cent. fat) } \\ 100 " & \text { milk } & =120 & \\ & & \end{array}$

2,570 calories.

Besides this we will add about 300 to 400 grams of green vegetables, whose calory value, like that of the albumin partaken of in fat foods (bacon, cheese, fat sausage, etc.), may be disregarded.

According to this our patient would be plentifully fed, if he were free from sugar, for a diabetic who excretes no sugar does not emaciate, and consequently does not need any more food than a healthy person. Unfortunately, our patient continues on this diet to excrete his full 100 grams of sugar per day. The calory value of these 100 grams ( 400 calories) must be subtracted from the total calory value of the food he is ingesting; 2,570 calories -400 calories $=2,170$ calories, which is 130 calories less than the patient requires, according to Rubner.

In some cases, under favorable circumstances, it is possible to supply these missing 130 calories by the further addition of fat, but by no means always; at least, under ordinary circumstances, it is often quite difficult to make the patient take the 500 grams of meat, 200 grams of cream and 100 grams of fat mentioned in the dietary above; and we must always avoid throwing too great a burden upon the metabolism of the diabetic. In short, as a choice of evils, we must often allow our patient to remain underfed and lose weight, until his excretion of sugar decreases appreciably. Only when the glycosuria becomes so slight that the net calory value of the ingested food approaches the gross calory value, can we expect to avoid the evil of under-nutrition. We must risk under-nutrition quite often, in severe cases, e. g., when, in order to abolish the glycosuria, we forbid all bread, and reduce the albuminous (meat) food considerably.

I know of no successful treatment of severe cases without temporary undernutrition, but the physician must be very careful during these periods. We should be especially cautious in regard to long-continued hyponutrition, such as entails a loss of weight of more than two kilograms in subjects who have already reached the minimum weight of 130 pounds in men and 110 pounds in women, for this loss is difficult to regain.

In the consideration of the theory of treatment in diabetes, some mention of drabetic acidosis will not be out of place. By this term I mean the forma- 
tion of $\beta$-oxybutyric acid in the processes of metabolism. It never fails to occur in serious cases, and even in mild cases it appears quite often.

Acetonuria and diaceturia are part of acidosis, for both substances, acetone as well as acetoacetic acid (diacetic acid), originate from oxybutyric acid. I must insist upon this view which has many evidences in its favor, among them the fact that acetonuria and diaceturia only occur when oxybutyric acid is also found in the urine. I know very well that some prominent chemists have maintained the direct opposite, but I also know that very prominent chemists may be mistaken in their reports; for in the very cases in which they found no oxybutyric acid in spite of the presence of acetone and acetoacetic acid, and in the specimens which they kindly sent to me for examination in my laboratory, I succeeded in demonstrating oxybutyric acid in the urine.

Therefore, acidosis, including acetonuria and diaceturia, plays an important rôle in diabetes. In twenty-four hours oxybutyric acid may be excreted in amounts above 100 grams, and acetone + diacetic acid up to 15 grams. As these substances are easily oxidized, their excretion in such large amounts shows a deficiency in the power of oxidation possessed by the tissues in these cases of diabetes mellitus. As regards this decrease in the power of oxidation in the diabetic, the conditions are very similar to those involved in the increased decomposition of albumin. Neither is primarily due to the diabetic disturbances of metabolism, but since the albuminoid decomposition is only increased when sugar is wasted and passes out unutilized in the urine, this weakness of oxidation may be referred to the lack of oxidation of sugar.

The oxidation of sugar, however, does not fail because the general power of oxidation is diminished, but vice versa. Since for other reasons the sugar is not oxidized, the general power of oxidation becomes lessened; of all the products which are consumed in the organism, sugar is the most readily combustible, and in the fire which thus arises in normal metabolism, other less readily oxidizable substances are consumed, a process designated by physiologists as secondary oxidation. Owing to the facts just mentioned, the same substances which produce acidosis may occur in other conditions-in nondiabetic persons whenever carbohydrates and sugars are withheld entirely. In the diabetic, too, they usually appear when the carbohydrates are excluded from the food. They may be excreted, however, in severe diabetes, with marked glycosuria, even when carbohydrates are eaten in large amounts. Here acidosis indicates that the sugar metabolism of the body has fallen so low that in spite of the plentiful ingestion of carbohydrate food, very little of it is consumed. The fire which lights the secondary oxidation processes is almost extinguished, and thus the total power of oxidation of the organism is damaged. It must be remembered that this condition is due to the disproportion between the products ready for oxidation in metabolism and the power of oxidation. In diabetic patients tissue decomposition goes on too rapidly for their power of oxidation; their nourishment is both improper and too profuse, yet by limitation of their metabolism and careful diet it is often possible to restore to par the power of oxidation, and thus cause the acidosis to disappear.

It has been necessary to discuss acidosis quite in detail because of its rôle in diabetic coma. Acidosis is the only certainly known cause of this coma 
(that is, of many cases of it). We now recognize that it is oxybutyric acid which produces true dyspneic coma; that is, an acid coma, i. e., a coma which is an expression of an over-acid condition of the blood, and this hyperacidity is due to the presence of a large amount of oxybutyric acid (100 grams or more), which is formed and which enters the blood within twenty-four hours. This fact, and also the danger that by a too rapid withdrawal of carbohydrates one may cause acidosis, as well as coma, renders it necessary for the physician to be familiar with this process.

\section{PRACTICAL THERAPY}

Every therapeutic measure is to be used, but after the dietetic treatment mineral waters alone are of much service. Prophylaxis; obesity and diabetes mellitus. From the point of view of dietetic treatment, three forms of diabetes are to be distinguished: the medium severe, the mild, and the severe. The medium severe cases, their diagnosis and their importance in practice. The aim of treatment in cases of each form. For the proper dietetic treatment of every case a quantitative and qualitative estimate of the entire dietary is necessary. Preliminaries of treatment: Quantitative regulation without limitation; its result. Further steps toward the abolition of glycosuria and improvement of tolerance, maximal reduction of albuminous food and the twenty-four-hour fast. Diet lists for the diabetic; there are no foods absolutely permissible. Carbohydrate nutrition: Bread, cereals, vegetables, fruits; calculation of their relative advantages and dangers. Meat, fish, and eggs. Fatty foods. Sausage, cheese, bacon, butter, oil, cream. Drinks: Milk, wine, beer, whiskey, tea, coffee. Artificial foods. Hospital treatment. Dispensary treatment and "bath-cures." Disturbances and dangers arising during the treatment of diabetes.

I wish to speak first and chiefly of the dietetic treatment of diabetes, but of course every other therapeutic measure is to be welcomed and utilized. It should not be forgotten that since almost any disturbance of a diabetic's general health tends to aggravate the underlying disease, common sense teaches us to treat as carefully as we can any minor ailment from which the patient may suffer from time to time. If he is syphilitic, carefully planned specific treatment should be used, as also quinin in malaria, digitalis in circulatory disturbances, and appropriate treatment for nervous affections. By such treatment we may succeed in improving but, unfortunately, very rarely in curing the diabetes - that is to say, the glycosuria and the other symptoms which may be dependent on it.

It is just as evident that the treatment of diabetes must always be a treatment of the entire organism, i.e., that the mode of life of the patient must be properly arranged in all respects. From this standpoint, there are two factors to be considered-mental and cmotional rest, and sufficient muscular exercise; I say sufficient muscular exercise, but no more, for too much readily increases the glycosuria.

The main point, however, the alpha and omega in the care of the diabetic, is the dietetic treatment; besides this, mineral water cures (Carlsbad, Neuenahr, Vichy) also play a rôle, but not drugs. There is scarcely a physician familiar with diabetes who will resort to drugs to diminish the glycosuria. It is true there are many remedies which bring this about, and among them some which produce this effect without diminishing the demand for food or 
the actual intake of nourishment. But-they act only in those cases in which the dietetic treatment alone would suffice, and in which they are, therefore, superfluous. During the administration of drugs (if we do use them) the dietetic treatment should never be forgotten, else the case will fail to do well in the long run. For a few weeks, perhaps, here and there, by the administration of opium, occasionally also by the use of antipyrin and other nervines, the glycosuria may be limited or even removed. Then the action of these drugs ceases and glycosuria returns to its former height, sometimes even exceeding it. It appears, as I have already indicated, that the tolerance of the diabetic is not improved by a drug diminution of glycosuria; and even in an aglycosuric condition, in case this is brought about by drugs, the patient's tolerance does not improve as it does under diet.

That we shall ever find a specific remedy for diabetes, must be regarded as quite unlikely, especially by those who, like myself, look upon the disease as an expression of hereditary weakness of metabolism; at present we certainly do not possess such a remedy.

We shall now proceed to the discussion of the dietetic treatment in detail.

This method of treatment has its place not only in the care of confirmed, true diabetes mellitus, but also in prophylaxis.

In families in whom this disease is hereditary there should be great moderation in the use of carbohydrates in any form, i. e., limitation of the sugar metabolism should be made an unalterable law. But there should also be moderation in eating and drinking in general. There can be no doubt that habitual hypernutrition favors the outbreak of diabetes if the predisposition to it exists. The cases of diabetes mellitus complicating obesity (even in full-blooded individuals with arteriosclerosis) are almost all to be included in this category. Alcoholism, at least that form that goes hand in hand with over-nutrition, also plays a part in the etiology of diabetes.

In individuals with a hereditary predisposition and marked obesity due to over-nutrition, diabetes should always be watched for, and an occasional examination be made of the urine voided four hours after a breakfast in which at least 100 grams of bread and about 30 grams of sugar have been consumed; or, for greater certainty, a test for glycosuria alimentaria e saccharo should be made, and the case should then be judged with the necessary reserve.

In confirmed diabetes, dietetic therapy has for its object the elimination of glycosuria if possible. This occurs very readily in some cases, in others it is difficult, and in some it may be impossible; even when it may be accomplished, although with difficulty, there are, unfortunately, many cases in which the results scarcely compensate the physician and patient for the required trouble.

The amount of energy that should be put into the treatment varies in different cases, and much labor will be saved the physician and the patient if this fact is recognized from the onset; on this account it is convenient to divide diabetics into three groups, a method which I proposed ten years ago.

1. The moderately severe cases.

2. The mild cases.

3 . The severe or very severe cases. 
We have already learned to differentiate between mild and severe cases, but in gauging the case from a therapeutic standpoint we need to recognize also a group of moderately severe cases. The mild cases are so benign that they offer a splendid prospect for therapy and usnally require but little treatment. The severe and very severe cases, on the other hand, are so malignant that even after the most energetic therapy the results obtained are slight and unsatisfactory because incomplete or transitory. In the group of moderately severe cases are included a great many which occupy a middle position, being by no means so benign that they do not require continued observation and proper treatment, but by no means so malignant that they should be left to their fate; by a more or less strict plan of treatment, they may be preserved in fair health and moderate activity for many years. Among the cases which at the beginning of treatment must be included in this intermediate group, there are not a few which prove to belong to the mild form, but which, on account of unfavorable influences, have passed temporarily into the moderately severe form. In this group we also find some which belong to the severe or very severe forms, but have not yet reached the full development of the disease.

\section{Let us illustrate:}

A man aged fifty, with an inherited predisposition to diabetes. Ten days previously, he undertook a lengthy and fatiguing excursion in the Black Forest, ate and drank more than usual, and was thoroughly drenched by a heavy rain. Since that time, he has had a voracious appetite, extreme thirst, marked diuresis, and has lost much weight. His urine contained 8 per cent. of sugar(!). His daily dietary was restricted quantitatively to about one pound of meat, several eggs, coffee with rich cream but no sugar, green vegetables (no potatoes or beets), no cereals, 80 grams only of wheat bread, his meals to be prepared without flour; one bottle of wine was allowed. Within a few days his glycosuria had disappeared. The case has continued to run a mild course up to the present time (twelve years subsequently). Since the acute attack, the patient's diet, for the most part, has been restricted as above, although at times it has been relaxed to the extent of allowing sweet champagne; the sugar in the urine has never risen above 0.5 per cent.

At the beginning of treatment this case appeared very desperate, but it soon became a clear example of the mild form of diabetes.

To illustrate again:

A peasant, thirty-three years of age and of a diabetic family. For five weeks following an accident he complained of hunger, thirst, loss of flesh, lassitude, and cramps in the calves of his legs. Patellar reflexes present; no complications; weight 48 kilograms; internal organs sound. After eight days on regulated diet including fat meat, with 150 grams of milk, and 20 grams of wheat bread, the sugar in his urine was still 4 per cent. -5 per cent. ( 75 to 100 grams of sugar a day). Only after the complete withdrawal of bread and inerease of milk to 200 grams did the urine gradually become free from sugar. After this on a diet with 200 grams of milk and 50 grams of bread he remained aglycosuric for several months, but even then he had to rigidly conform to rules in order to prevent the reappearance of glycosuria. In this fashion he has now lived for years. His weight is 56 kilograms.

This is a typical case of the medium severe form; severe at its onset but under continuous, moderately strict dietetic treatment taking a rather favorable course. 
The following is another typical example of this group:

A bookbinder, seventeen years old. No family history of diabetes. For five weeks, without apparent cause, he was continually hungry, he lost weight, was extremely weak, and had a non-gonorrheal urethritis. Organs sound; knee-jerk normal; weight 45 kilograms. On a diet of 220 to 300 grams of meat, green vegetables, 250 grams of milk and 300 grams of bread, there was a glycosuria of 6 per cent. $=150$ to 200 grams of sugar a day. Only upon complete withdrawal of bread while still taking 25 grams of milk, did he become free from sugar. After a sugar-free period of one month, he could take more milk and a small quantity of bread without excreting sugar. He gradually became accustomed to a larger allowance of bread and milk, being careful never to take enough to produce glycosuria. This tolerance gradually increased until he could ingest 170 grams of bread and 100 grams of milk without sugar appearing in the urine.

The patient remained in this condition, outside the hospital, for two years. After this (probably because he did not adhere to the same diet) sugar was again excreted, and did not disappear on very strict diet-so that the case became severe.

The object in quoting these examples is to show clearly the existence of a moderately severe form of the disease. At the outset, cases like those just quoted may readily be looked upon as extreme, and thus great harm may result. Among the points on which the recognition of medium severe cases rests the most important is this, that after partial withdrawal of carbohydrates (for example, 200 grams of milk and 50 grams of bread), no matter how marked the glycosuria has previously been, it immediately and decidedly decreases, usually falling below 100 grams. Besides, in these cases of medium severity, the amount of acidosis as determined by the ferric-chlorid and acetone reaction is slight, and the amount of urine is not great ( 4 and, at most, 5 liters per day). All these conditions may also exist in severe cases.

After determining to which group a given case belongs, the physician must outline the method of treatment as follows:

1. Mild cases are to be made aglycosuric, and maintained in this condition until continuous observation for at least six months has demonstrated that the disease shows no tendency to progress. Later, a permanent increase of glycosuría above 0.5 per cent. should not be permitted. Such a transitory increase need cause no anxiety, but as soon as it becomes greater the case is to be treated as at first by strict limitation of the diet.

2. Cases of the medium severe form are to be made aglycosuric by strict treatment if this is necessary. Then, in case they do not prove to be mild cases, they are to be permanently maintained in this condition. Even a mild glycosuria is not to be permitted, and anything causing a transitory increase of glycosuria is to be absolutely avoided, as it threatens the already greatly endangered tolerance. It is possible that there are individual cases which at the onset belong to the medium severe group, but which may be rendered mild, and then the indulgences possible for cases of the mild forms may be permitted; but such cases are certainly not frequent.

3 . The nature of the severe and very serere cases becomes manifest when on the first attempt to produce aglycosuria the feebleness of their tolerance appears. In these cases, our primary object is to bring the patient to the point at which life can be maintained without a deficit; but this can rarely be brought about, unless we can limit, and that decidedly, the loss of sugar in 
the urine. We must make an attempt to diminish the glycosuria, and see how far we are successful. Now and then, we can accomplish more than we had hoped, and a better tolerance may follow a regulation of the diet and a reduction of the glycosuria, so that we may succeed in maintaining the patient's equilibrium of metabolism with a very slight glycosuria and with a fair condition of health for years, even after all hope had seemed to be gone.

This is the chief task of the physician, easy in some cases, more difficult in others, and in many almost impossible. Although the treatment of the different groups of cases varies, one general rule applies to all. We should determine the exact quantitative and qualitative diet for every diabetic who comes under treatment. Prout exaggerated when he stated (1820) that the quantity of the food, that is, the quantitative restriction, is more important for the diabetic than its quality; but his maxim that all of the diabetic's food (including meat) is to be determined quantitatively, holds good, because nothing more unfortunate can befall a diabetic than the overtaxing of his general powers of metabolism. I consider the restriction of labor of the whole metabolism to be an important advance in the therapy of diabetes. There are no foods which the diabetic can be allowed to eat in unrestricted amount; the quantity of each must be measured. We must see that he does not get too much carbohydrate food, or too much animal food, and that he has just enough fat, neither too much nor too little.

I am proceeding on the supposition that the patient is to be treated not in a hospital but in his own home. First of all, we must determine quantitatively for several days the amount of each food ingested. For this purpose it is only necessary to know the amount of each carbohydrate (flour, bread, sugar and milk) used in the preparation of his meals. If this plan is carried out, there is no difficulty later. Each variety of food should be served on a separate plate, and the amount weighed. Sauces are not considered, and bread is weighed separately. If the patient cannot afford this, or if he is unwilling to do it, he should either go to a hospital where others will look after his food, or we must relinquish the attempt to regulate the whole diet quantitatively. We may be able to get along without weighing the food in very mild cases in which a moderate reduction of the carbohydrates is sufficient to control the disease.

Still, most patients, even those in limited circumstances, can arrange to weigh their food. Usually a simple quantitative regulation of the diet, with liberal allowance for the wishes and inclination of the patient, and without too strict limitation, may be followed by good, even remarkably good results; at any rate, we may thus ascertain the maximum of the glycosuria on a given diet and thus establish the necessary basis for further dietary regulations.

An example will best serve to make this intelligible.

A woman, forty years of age, no hereditary history obtainable, has suffered for four months from extreme hunger and thirst; there is copious diuresis; loss of weight 20 kilograms. Present weight 58 kilograms; no organic disturbances or complications; no acidosis; reflexes normal. The patient asserts that she has been on a diet, that is, that she has eaten but little bread, etc. During the first three days of treatment, she ate as before and was found to be excreting $2 \frac{1}{2}$ to 3 liters of urine with 6 per cent. to 7 per cent. (= 150 to 200 grams) of sugar per day. Then the diet was regulated quantitatively, 
taking the wishes of the patient into consideration. She received daily 500 grams of milk, 100 grams of bread, 200 grams of meat, $4 \mathrm{eggs}, 200$ grams of vegetables with ten per cent. of fat, 50 grams of butter, and 75 grams of fatty cheese or sausage, etc., with tea, coffee, and water ad libitum, and a half liter of wine. This diet was abundant, and gave the patient 35 calories per kilo of body-weight. She remarked that she had not eaten much more before treatment, not evẹn of bread. During the following days she excreted $I_{\frac{1}{2}}$ to 2 liters of urine, and 3.5 per cent. ( $=50-70$ grams) of sugar daily. At the same time she felt stronger and improved each day. No acidosis.

This is, in itself, a truly remarkable result of quantitative regulation of the diet. Of course, there was some restriction, but the patient hardly noticed it.

In this case, the milk and the bread must be still further reduced until the sugar has disappeared entirely.

Our patient preferred not to have the milk in her diet reduced. The bread was therefore reduced to 40 grams, but after four days upon a diet containing 60 grams of bread she still excreted sugar, but when the milk was reduced to 300 grams and the bread to 40 grams there was no glycosuria. On this diet she remained aglycosuric, so that after fourteen days it was deemed possible to increase her food. For the sake of precaution, 100 grams of milk were withdrawn, but 10 grams of bread were added. She remained free from sugar. After three days 100 grams of milk were added, and the patient continued free from sugar; again after three days 10 grams of bread were added, and 100 grams of milk withdrawn. Thus she continued upon a diet of 500 grams of milk and 100 grams of bread for six weeks, when she was aglycosuric and in apparent full health, having attained a weight of 62 kilograms.

She remained under observation. Occasionally, as the result of an indiscretion in diet, a slight, transitory glycosuria occurred, and then the patient would live on 50 grams of bread for a few days, or would desist altogether from eating bread for a whole day, then return to 50 grams of bread for a few days, until finally she became perfectly aglycosuric on a diet of 100 grams of bread.

The removal of glycosuria is by no means always so easy and simple. Very often more stringent mothods must be employed; carbohydrates, flour foods, and even milk, must be wholly excluded before the patient becomes entirely free from sugar, and frequently even this is insufficient and nothing remains but the reduction of albuminous food. The albumin nutrition may then be restricted to 200 grams, even to 150 grams of cooked meat (reckoning albumin as meat), so that the patient does not receive more than 40 to 50 grams of albumin. In the severe cases this limitation of the albumin nourishment is one of the most important points. Its effect is often much greater than can be explained by the withdrawal of the sugar formed from the albumin. For example: With 100 grams of albumin (besides the necessary fat) 40 grams of sugar are excreted; after a reduction to 60 grams of albumin, sugar disappears in a few days. That 40 grams of sugar are formed from 40 grams of albumin is hardly possible; therefore, the aglycosuria cannot be due to the limitation of sugar intake (in albumin). I have long been convinced that it is the limitation of food and the resulting disencumbrance of the entire metabolism which brings about this favorable result. The metabolism of the diabetic is not deficient in only one respect, namely, that with which we are concerned, the working up of the sugar molecule and its preparation for oxidation. His metabolism is deficient as a whole-witness the weakness of the powers of oxidation as expressed unmistakably in the acidosis. 
In the severe cases which we are now considering it is almost always necessary to limit the entire amount of food (albumin and fat included) so that its total calory value will be lowered, even below the minimum required by Rubner ( 35 calories per kilo of body-weight). Under-nutrition need not be feared; my diabetics, at least those who are treated in the hospital, get along with 30 calories, and even less, per kilo, without losing body-weight.

When the excretion of sugar has fallen to the minimum, about 0.5 per cent., and not more than 10 grams of sugar per day are excreted, aglycosuria may finally be brought about by giving the patient no food for twenty-four hours. He may be allowed water ad libitum, for it is unnecessary in this condition that the patient should suffer from thirst. This procedure-a twentyfour-hour fast-was introduced by Cantani.

Both the reduction of the albuminous foods and the twenty-four-hour fast seem cruel, or at least drastic, measures, but they really are not so; at least the author has never had the least difficulty in enforcing them. More difficulty in the strict dietetic treatment of diabetes is encountered in other directions. We shall speak of this later on.

After glycosuria has been abolished, if we expect to increase the tolerance, we must maintain aglycosuria for at least two weeks before we can appreciably add to the food. After the patient has been finally brought to the daily allowance of food which is sufficient for him he must remain upon this diet and be under observation. It is also advisable at times to institute once a week a twenty-four-hour fast for carbohydrates, during which period the patient receives no bread.

Keeping the patient for a month or longer without bread, starchy foods or milk rarely succeeds, but the author knows of several diabetics who have lived for years on a strict albumin-fat diet with the addition daily of 200 to 400 grams of green vegetables (deficient in carbohydrates), and they have been able to attend to their business without taking unusual care of themselves. One, a man about forty years of age, held the responsible position of professor at a university.

We must, then, exert all our efforts and all our art properly to regulate the diet!

In what follows, I shall only mention briefly some of the main rules, the observance of which will enable the physician judiciously to handle his diabetics. We shall see, however, that in order to prescribe a diet, he must be familiar with the composition of the most common foods as regards fat and carbohydrates. All recent books on diabetes contain detailed data on this subject, and the most essential figures in this respect will be given at the conclusion of this article.

I shall attempt no distinction between what is absolutely (in any quantity) allowable for a diabetic, and what is absolutely forbidden. I consider such a distinction wrong and dangerous. No diabetic should be permitted the unrestricted use of any food; everything must be ordered him quantititively.

The amount of carbohydrates permitted is best considered in terms of wheat bread; and most patients prefer to take the amount of carbohydrates 
permitted them in the form of good wheat bread. Some, however, would rather have it as flour-cakes, and there is no objection to their having it in the form of any simple carbohydrate that they prefer. If the patients take their carbohydrates partly as wheat bread and partly in some other way, then we must subtract the latter amount from the total that is permissible. Suppose, for instance, that the patient is allowed 100 grams of wheat bread = about 60 grams of starch. He prefers to take part of his starch in another form, for instance, as oatmeal or rice, in soup, 10 grams being sufficient to make a large dish of $\frac{1}{4}$ of a liter; or he takes balls of oatmeal, or 25 grams of oatmeal alone. Oatmeal and rice contain about 75 per cent. of starch. We can consider the 10 grams of oatmeal as equivalent to wheat bread, and therefore we must subtract 10 grams (or to be quite accurate 12 grams) from the daily allowance (100 grams) of wheat bread. Twenty-five grams of grits or rice are equal to about 30 grams of wheat bread. Of course, the patient may have other forms of bread instead of wheat bread. Rolls are useful for this purpose, the amount of starch being almost the same as in wheat bread. The rolls may be cut into small slices and spread with butter. Rye bread is also serviceable; it contains 15 per cent. less of starch, but is heavier and more compact. Aleuronat bread contains only about half as much starch as wheat bread, but there are few diabetics who like this bread, although among all the varieties of so-called "diabetic bread," this has the most agreeable taste.

Among vegetables the diabetic should eat only those in which the amount of carbohydrates does not exceed 5 per cent. (compare the tables), and even this slight amount of carbohydrates must not be ignored in those cases in which the removal of glycosuria is difficult. With 300 grams of vegetables per day, it may amount to as much as 15 grams.

Fruits should be permitted the diabetic if possible. There are plenty of fruits whose carbohydrates do not exceed 6 per cent. (sugar + pectin). They must be well cooked and sweetened with saccharin. Almost any kind of fruit may be given, even such as contain sugar in large amounts, but the amount of carbohydrates they contain must be deducted from the amount of bread which is permitted. Only grapes and dried fruits, on account of their high sugar-contents, are to be excluded. The fact that the sugar contained in fruit is levulose modifies the case but little, for after prolonged use levulose becomes almost as injurious as dextrose. Many so-called "fruits for diabetics" are to be found on the market; they contain about 2 per cent. to 4 per cent. of sugar, therefore less than the fresh fruits, even those poor in sugar, but, unfortunately, many diabetics grow tired of them after a time.

In meat, besides the albumin, we must take into account the fat it contains. Considering only the percentage of albumin (25 per cent.), the calory value of stewed or broiled meat is only 1 . When the ordinary amount of fat is present (10 per cent.) the calory value becomes 2 , but we must remember that in the cooking of meat (broiling, stewing) part of the fat is lost; in fact, most of the fat is lost in cooking. Excluding the fat, all kinds of meat are of the same calory value. Liver is strictly forbidden; of other "glands" sweetbreads and brain, though not strictly meat, are of equal value on account of their high percentage of fat. Naturally, fat meat is always preferable for 
a diabetic; some fat meats and fat fish contain 20 per cent. of fat or more (see diet lists).

As a working principle, let us say that a diabetic should not eat more than 500 grams of meat (weighed after cooking), for most patients lose their appetite if they eat more; diabetics who are under close medical supervision may be permitted more, but it is usually of no benefit.

Eggs are very useful. On account of the large amount of fat in the yolk, each egg has a value of 75 calories. If we allow more than two eggs, we should subtract 50 grams for each egg from the amount of meat allowed.

Fat foods are of the greatest value, for fat, as a rule, does not increase glycosuria and has the enormous nutritive value of 9 calories, while starch and albumin have only 4. In good sausage (poor sausage often contains flour and but little fat!) there is 30 per cent. to 40 per cent. of fat; in good cheese from 20 per cent. to 30 per cent.; poor cheese contains but little fat. Butter contains 85 per cent. of fat; lard (butter or fat melted), bone marrow, and vegetable oils almost 100 per cent. Bacon varies greatly in regard to the amount of fat it contains; it averages about 92 per cent. An enormous calory value may be obtained in the following foods: 100 grams of Holland cheese (30 per cent. fat) gives 270 calories, without including the albumin; 100 grams of good butter, 720 calories. Fat and butter may be mixed with vegetables so as to make them contain 10 per cent., even 15 per cent. of fat; bacon and oil in salad, up to 20 per cent. to 40 per cent.

Cheese may be pulverized and added to soups and sauces, and also may be made into cheese cakes, cheese puddings, etc.

Cream contains 25 per cent. of fat, and is, therefore, very valuable; the slight amount of sugar it contains ( 3 per cent. milk-sugar) need scarcely be regarded. Some cream contains even more fat than this (up to 40 per cent.). If it is dairied in a very cleanly manner, it is at first very grateful to the taste, but after prolonged administration patients often do not like it.

Milk is in general use, and, even in the most severe cases, of great value. But it must not be supposed that the milk-sugar is "harmless" to the diabetic, for it is not much less so than dextrose and cane sugar; but milk contains very little sugar, only about 3 per cent. Its carbohydrate contents must be reckoned, but it also contains 4 per cent. to 5 per cent. of fat. There are several manufactured preparations of milk which contain a larger amount of fat, and in which the sugar is decreased; there are even artificial milks free from sugar-but my patients soon become tired of these preparations.

Other fluids are to be estimated according to their carbohydrate value; among wines only the southern wines and champagne contain more than 3 per cent.; these contain 12 per cent. In beer the carbohydrate value is rarely under 6 per cent. Beer is more harmful than this percentage would lead us to suppose, as it contains the greater part of its carbohydrates as maltose; this at once decomposes in the intestines into dextrose, and, for this reason, the diabetic ingests in beer the most dangerous, most readily absorbable form of carbohydrate food.

Among alcoholic liquors only rum, cognac, whiskey, etc., are to be con- 
sidered! No liqueurs! These, and often the so-called "bitters," contain enormous quantities of sugar.

Coffee and tea may be looked upon as harmless for the diabetic. Cocoa contains (without admixture) 15 per cent. of starch, and must be estimated accordingly.

If it is desirable to increase the menu still further, manufacturing chemists have placed many preparations on the market for the diabetic. The physician should assist the diabetic to make his life as comfortable as possible, yet the medical adviser must never allow the reins to be taken out of his hands; unfortunately, this often occurs.

Which diabetics are to be treated at a hospital (of course, one well appointed with reference to diabetic treatment), which may be treated at home, and which should be sent to Carlsbad, Vichy, Neuenahr, etc.?

The very mild cases, in which only sugar, potatoes and beer must be restricted in order to inake the urine free from sugar, do not need hospital treatment, though it is advisable for thern to undergo each year, or at least from time to time, a course of treatment at any one of the previously mentioned bath cures. Frequently these cases occur in elderly persons, in whom a cure of this sort is indicated on account of complicating arteriosclerosis, disease of the liver, or gout.

It is often wise in such cases, after sugar has been discovered, to send them at once to Carlsbad; they return free from sugar, and with a greater docility and willingness to carry out-further dietetic treatment.

If the case is severe, so that it is necessary to reduce to a minimum the allowance of bread in order to free the urine from sugar, the question whether the patient should be treated at home or in a hospital must be settled by the individual circumstances. If the patient is sensible, reliable, and in easy circumstances, treatment at home should be tried, but we must not be too optimistic as to results; as soon as signs of unreliability on the patient's part are detected, hospital treatment should be urged. If, under treatment at home, the sugar has been considerably reduced (to about 20-30 grams a day), we may send the patient to Carlsbad, whence he often returns sugar-free, and he may then more easily than before be kept aglycosuric; but this applies only to the mild cases.

Every diabetic whose case is not very mild, or who is not reliable or wellto-do, as well as every moderately severe and every severe case (that is, every patient from whose diet carbohydrates must be largely or wholly eliminated, if only for a time), must, to begin with, have hospital treatment. In a hospital we find out how much can be attained, and we determine the diet on which the patient will become sugar-free, or will have, at least, only a glycosuria of definite and known intensity. After this he must remain under supervision, and if the glycosuria again shows a progressive tendency he should return to the hospital. Poor patients with severe diabetes need hospital treatment at least every year, of six or eight weeks' duration the first year, and reduced, if necessary, to four weeks the next year.

In the author's experience, "bath cures" rarely benefit these patients, and often do harm. 


\section{DISTURBANCES AND DANGERS OCCURRING IN THE DIETETIC TREATMENT OF DIABETES}

That disturbances of digestion often occur during dietetic treatment has been known ever since the disease has been treated by a restriction of food. The more violent disturbances, such as used to occur when our knowledge was less, for instance, diarrhea, sometimes combined with vomiting, should now be prevented entirely. They may be avoided in trustworthy patients if the whole amount of food ingested is quantitatively determined, if too large a portion of meat is not allowed, and if we bear in mind the sensitiveness of the patient as regards fat and fatty foods. Some patients lose their appetite from the beginning, or as soon as carbohydrate food, especially bread, is restricted. This anorexia may be avoided by giving them fruits. If the patient likes the fruits prepared for diabetics he is very fortunate; otherwise cooked or uncooked fruits that contain but little sugar may be used. I am loath to forbid fruits altogether, and even in the severe cases I usually allow as much as 100 to 200 grams. Milk, also, is very valuable; $\frac{1}{2}$ to 1 liter of milk a day with a restricted fat-albumin diet often helps the patient over the period of anorexia without increasing the glycosuria to any extent, and without making the patient lose weight. With these precautions, we are not compelled to break off the dietetic treatment as soon as it is begun, but may go on with it cautiously as soon as the appetite has improved.

Every disturbance of appetite, however, must be seriously considered, for, on the whole, cases with anorexia are the most difficult to deal with. In some of these cases, psychic depression plays a certain part, and if their disease is not too pronounced, they often get along much better at Carlsbad or Neuenahr than if they stay at home, or go to a hospital.

In acidosis we have an especially formidable hindrance to strict dietetic treatment. In many of the moderately severe cases it appears as soon as the diet is restricted, or, if already present, it becomes more marked when the carbohydrates are limited. Then the patient is at once in danger, because acidosis may lead to coma. Formerly, this often happened when the diet was restricted too suddenly, and even to-day cases with acidosis are always in danger if severe gastric disturbances occur. In the absence of gastric irritability this danger may be averted by the administration of sodium bicarbonate. Enough should be given to make the urine feebly alkaline, and 50 or more grams in twenty-four hours may be necessary. With this precaution we may endeavor to reduce glycosuria by strict diet even where decided acidosis already exists. Of course all food and its calory value must be determined with quantitative exactness, for, to repeat, it is the disproportion between the quantity of the products prepared in metabolism for oxidation and the power of oxidation itself which finds its expression in acidosis. In cases with acidosis we must not think of withdrawing carbohydrates entirely, but should allow 60 to 80 grams of bread, $\frac{1}{2}$ a liter of milk, and as much as 200 grams of fruit. The latter is here particularly important in order to prevent indigestion.

We may count with certainty on the fact that if the acetone reaction becomes marked at the onset, the cause is simply the urinary sodium, that is, the 
alkalinity of the urine. Sometimes the diacetic acid (ferric chlorid) reaction also becomes marked; but this should not be allowed to occur after the first few days. In case the reaction becomes still more intense, so that the urine blackens on the addition of ferric chlorid, milk should be cautiously added to the diet, and its calory value subtracted from that of the meat, which should be correspondingly reduced. Otherwise, if the general condition of the patient remains good, we may maintain an expectant attitude until the ferric chlorid reaction becomes less marked, which usually occurs in eight to fourteen days, sometimes earlier or later. When this diminution of acidosis begins the danger from this source is over; acidosis gradually disappears and will not cause trouble, even should a further restriction of the carbohydrates be necessary.

Of course, if symptoms of coma appear we must increase the dose of sodium bicarbonate until the urine is alkaline. Sometimes 100 grams or more of sodium bicarbonate must be given in twenty-four hours. In almost every case of this kind, it is milk which answers our purpose best.

\section{TABLES}

Tables of foods: different kinds of meat, fish, eggs, sausages, meat-preserves, and various kinds of cheese, arranged according to the amount of fat they contain.-Fat, butter and varieties of milk, with percentages of fat and carbohydrates.-Bread, flour and vegetables, with percentages of carbohydrates.-Fruits and their carbohydrate (sugar + pectin) contents.-Beer, wine and spirits, with their amounts of alcohol and carbohydrates.-Plan for calculating the food-value of the diet in calories.-Short popular dietary instructions for diabeties.

MEAT WITH PERCENTAGE OF FAT

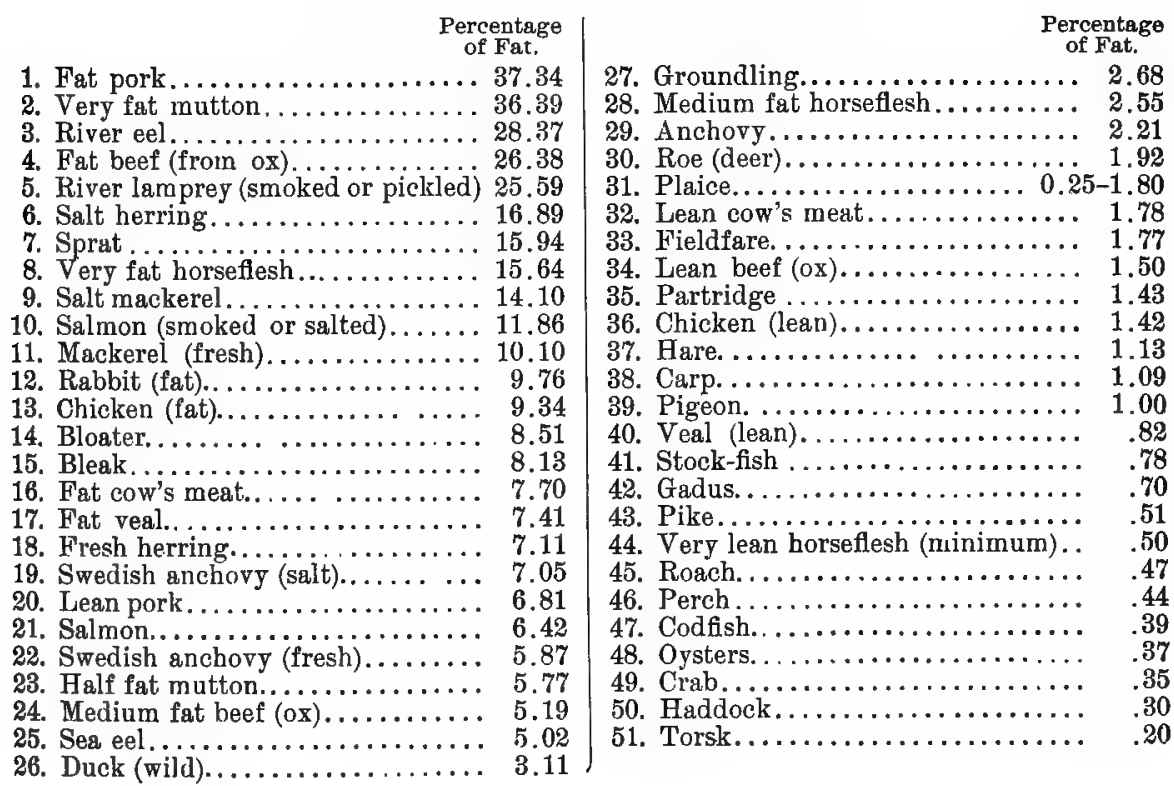



EGGS

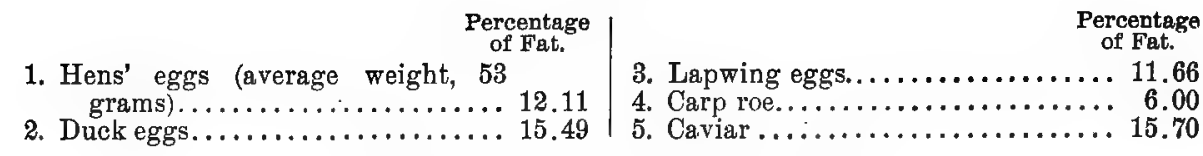

\section{SAUSAGES AND PRESERVED MEATS}

Percentage of Fat.

1. Clean, well-smoked, meat-free bacon 92.20

2. Westphalia sausage............ 39.88

3. Cervelat sausage............. 39.76

4. Frankfurters ............... 39.61

5. Westphalia ham.............. 36.48

6. Smoked beef-tongue.......... 31.61

\section{Percentage}

7. Pomerania goose-breast of Fat.

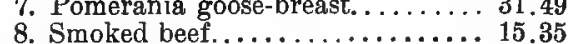

9. Tinned meat................ 12.63

10. Blood-sausage............. 11.48

11. Hard smoked sausage......... 11.40

12. Dried beef. . .............. 5.24

\section{CHEESE}

\begin{tabular}{|c|c|}
\hline $\begin{array}{l}\text { Percentage } \\
\text { of Fat. }\end{array}$ & $\begin{array}{c}\text { Percentage } \\
\text { of Fat. }\end{array}$ \\
\hline Neufchâtel .. & 12. Cheshire cheese. \\
\hline 34.55 & 13. Holl \\
\hline cchino cheese. & 14. Artificial oleomargarin \\
\hline rt cheese. & leese (ordinary). \\
\hline Brick cheese... & 16. Artificial fat cheese.... \\
\hline eddar cheese. & 17. Romadour \\
\hline lam cheese.... & 18. Camembert. \\
\hline sheese.... & 19. Brie \\
\hline mmenthal cheese. & 20. Parmesan cheese. \\
\hline & 21. Swiss caraway cheese.. \\
\hline ye & 22. Meager cheese.. \\
\hline
\end{tabular}

\section{DIFFERENT FATTY FOODS AND VARIETIES OF MILK \\ (Arranged according to their fat and carbohydrate-sugar-contents.)}

\begin{tabular}{|c|c|c|c|c|c|}
\hline P]a & Fat. & & 85 & Fat. & Carbohy. \\
\hline & 100.00 & & $\begin{array}{l}0.1 \\
9 .\end{array}$ & & \\
\hline $\bar{A}$ & 87. & & 10. Cor & & \\
\hline $\mathrm{B}$ & 83.2 & $0.8-0.58$ & 11. As & 1.64 & \\
\hline $\mathrm{L}$ & 100.00 & & rmilk......... & .9 & 3 \\
\hline $\begin{array}{l}\text { Cream .......... } \\
\text { Condensed cow's }\end{array}$ & $26 . ?$ & .52 & 13. Kumiss from cow's & & \\
\hline $\begin{array}{l}\text { without addition of } \\
\text { cane-sugar......... }\end{array}$ & 13.19 & 15.38 & 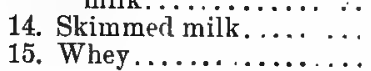 & 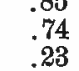 & 4.70 \\
\hline
\end{tabular}

The liver of fattened geese (Strassburg liver) should by no means be excluded from the diet on account of its supposedly high percentage of glycogen, for, in fact, this is slight, and does not amount to more than from 0.96 per cent. to 2.8 per cent. (of the fresh liver): I am unable to find any quantitative reports of the percentage of fat in these livers, but it may safely be assiumed to be about 30 per cent.

\section{BREAD, FLOUR AND VEGETABLES \\ (According to their percentage of carbohydrates.)}

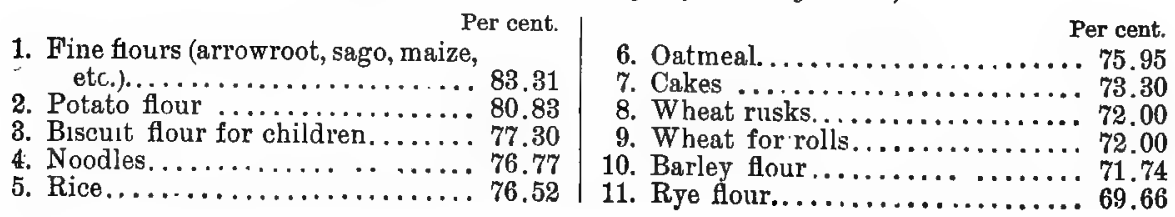


BREAD, FLOUR AND VEGETABLES (continued)

\begin{tabular}{|c|c|c|}
\hline $\mathrm{Pe}$ & & F cent. \\
\hline Barley bread................. 69.06 & 46. Other-wort. & \\
\hline $\begin{array}{l}\text { eat flour, including bran } \\
\text { aam bread...........66.00 }\end{array}$ & $\begin{array}{l}\text { 47. Carrots (large) } \ldots \ldots \ldots \ldots \ldots \ldots \ldots \\
\text { 48. Beet-root. }\end{array}$ & \\
\hline & 49. Scallion. & $\begin{array}{l}9.26 \\
9.08\end{array}$ \\
\hline 64.21 & 50. Radish. & $\begin{array}{l}9.08 \\
8.43\end{array}$ \\
\hline 64.05 & 51. Kohlrabi, underground (tuber)... & 8.18 \\
\hline 63.00 & 52. Carrots (small)..................... & 8.18 \\
\hline 53.00 & 53. Parsley. & 7.44 \\
\hline 49.25 & 54. Dill. & 7.43 \\
\hline 49.00 & 55. Green horse beans............... & \\
\hline 47.00 & 56. Pumpkin.................. & \\
\hline am bread.. & p leaves................... & 7.28 \\
\hline ake) 34 & $\ldots \ldots \ldots \ldots \ldots$ & 7.14 \\
\hline ead (after Ebslein) : : 27.50 & 59. French beans ${ }^{1} \ldots \ldots \ldots \ldots \ldots \ldots$ & \\
\hline$\ldots \ldots \ldots \ldots \ldots \ldots 52$ & bulb and root)........ & 6.53 \\
\hline $\begin{array}{lll}\ldots \ldots & \ldots \ldots & 48 \\
48\end{array}$ & 61. $\mathrm{R}$ & 6.25 \\
\hline 58 & 62. $\mathrm{B}$ & 6 . \\
\hline 43 & 63. $\mathrm{Cg}$ & 6.02 \\
\hline dry) & 64. K & 4.00 \\
\hline 29 & 65. W & 4.87 \\
\hline oms (air dry).. & 66. $\mathrm{C}$ & \\
\hline 列 & leaves) & 4.55 \\
\hline white). & 68. $\mathrm{Sp}$ & \\
\hline 20 & 69 & 4.29 \\
\hline 15 & 70. T & 4.07 \\
\hline & & \\
\hline (topinambur).. & salad (lettuce).. & 3.55 \\
\hline bas & $\ldots .$. & \\
\hline & 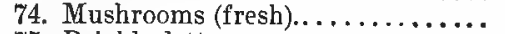 & \\
\hline & 75. Prickly & \\
\hline & 7b. A & \\
\hline & 77. E & \\
\hline & 78. Cucumbers.. & 2.28 \\
\hline Truffles (fresh) $\ldots \ldots \ldots \ldots \ldots \ldots \ldots$. 10.73 & 79. Headed lettuce................. & 2.19 \\
\hline
\end{tabular}

PERCENTAGE OF CARBOHYDRATES IN DIFFERENT FRUITS, ETC.

(Sum of sugar and non-nitrogenous [sugar-producing] extracts [pectin].)

1. Dates. Sugar. N.-free extracts.

2. Locust-bean. $\{$ Dextrin.... 3.4$\}$ $\{$ Sugar......

3. Dried prunes ¡ Cane-sugar

4. Raisins. Grape-sugar

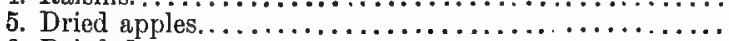
58.00 $\because 0.22$

$44.19\}$

54.56

42.83

49.79

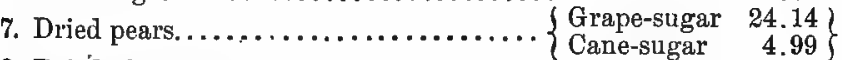

. Dried pears.

9. Bananas.

4.99

31.22

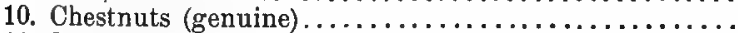

11. Sunflower seed................................

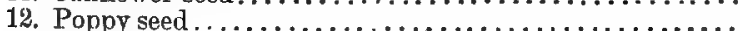

13. Wine grapes.

.....

$\ldots \ldots$

$\ldots \ldots$

14.36

13.00
67.67
17.69
7.48
11.40
$\ldots \ldots$
19.34
14.19
23.05
38.34
20.03
18.74
1.96

Total.

74.40

67.67

62.10

62.04

54.23

49.79

48.47

45.51

23.05

38.34

20.03

18.74

16.32

1 Ripe black salsify and sweet potato (topinambur) contain levulose almost exclusively. The unripe roots contain much dextrose, the proportion between levulose and dextrose in both is inconstant and variable, as the roots are not always quite ripe. In French beans (59), the substance given as being a carbohydrate is inosite, which is not a genuine carbohydrate, and is entirely harmless. 
PERCENTAGE OF OARBOHYDRATES IN DIFFERENT FRUITS (continued)

\begin{tabular}{|c|c|c|}
\hline & $\begin{array}{r}\text { Sugar. } \\
3.16\end{array}$ & $\begin{array}{c}\text { N.-free extracts. } \\
11.46\end{array}$ \\
\hline Mirabelle plums. $. \ldots \ldots \ldots \ldots \ldots, \ldots, \ldots, \ldots, \ldots, \ldots$ & 3.97 & 10.07 \\
\hline$\ldots \ldots \ldots \ldots \ldots \ldots \ldots, \ldots$ & 7.22 & 5.81 \\
\hline$\ldots \ldots \ldots \ldots \ldots \ldots \ldots$ & 10.24 & 1.76 \\
\hline$\ldots \ldots \ldots \ldots \ldots \ldots \ldots \ldots$ & 9.19 & 2.03 \\
\hline$\ldots \ldots \ldots \ldots . . . . \ldots$. & 8.26 & 3.54 \\
\hline ................ & 4.48 & 7.17 \\
\hline$\ldots \ldots \ldots \ldots \ldots$ & 6.15 & 4.02 \\
\hline 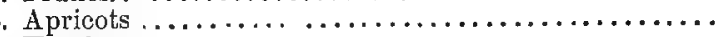 & & 6.35 \\
\hline $\ln u t s \ldots \ldots \ldots \ldots \ldots \ldots \ldots \ldots \ldots \ldots$ & & 3 \\
\hline ............ & 7.03 & 1.40 \\
\hline 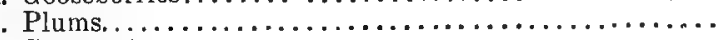 & 3.56 & 4.68 \\
\hline ut............................... & $\cdots$ & 8.06 \\
\hline 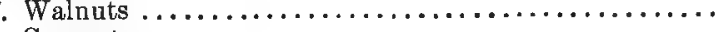 & $\therefore>0$ & 7.89 \\
\hline 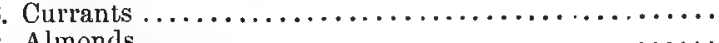 & 6.38 & $\begin{array}{l}0.80 \\
7.23\end{array}$ \\
\hline & 6.28 & 0.48 \\
\hline$\ldots \ldots \ldots \ldots \ldots \ldots \ldots \ldots$ & 5.02 & 0.87 \\
\hline$\ldots .$. & & 5.88 \\
\hline ...... & 4.1. & 1.44 \\
\hline & 4.59 & 0.95 \\
\hline . & 3.8 & 1.44 \\
\hline $\begin{array}{l}\text { eanuts..... } \\
\text { ranberries. }\end{array}$ & 1.53 & \\
\hline
\end{tabular}

\section{DIFFERENT KINDS OF BEER \\ (According to their alcohol and carbohydrate contents.)}

\begin{tabular}{|c|c|c|}
\hline & & \\
\hline & $\begin{array}{c}\text { hydrates. } \\
52.29\end{array}$ & $\begin{array}{c}\text { vol. per cent. } \\
2.96\end{array}$ \\
\hline eat beer & 10.45 & 0.70 \\
\hline ........ & 7.55 & 5.35 \\
\hline ek beer (Märzen, Saloon, double beer, etc.). & 7.20 & 4.74 \\
\hline e beer.$\ldots \ldots \ldots \ldots \ldots \ldots \ldots \ldots \ldots \ldots \ldots$ & 6.83 & 4.02 \\
\hline port beer. & 6.48 & 4.31 \\
\hline$\ldots \ldots$ & 6.03 & 4.89 \\
\hline er or summer beer. & 5.78 & 3.95 \\
\hline dish beer............... & 5.68 & 3.89 \\
\hline . $\ldots \ldots \ldots \ldots \ldots$ & 5.65 & 5.55 \\
\hline hter beer varieties (usual yeast and winter beers).......... & 5.49 & 3.46 \\
\hline eiss beer $(1878) \ldots \ldots \ldots \ldots \ldots \ldots \ldots \ldots \ldots \ldots$ & 4.28 & 3.33 \\
\hline elgian $k$ & 3.84 & 6.08 \\
\hline
\end{tabular}

PERCENTAGE OF CARBOHYDRATES (SUGAR + "EXTRACT") IN WINES AND SPIRITS

1. Russian Dorvy-vodka.

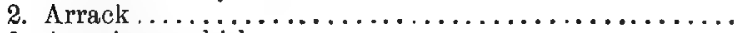

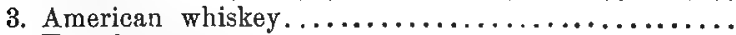

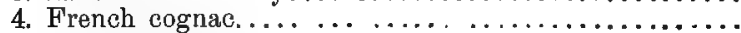

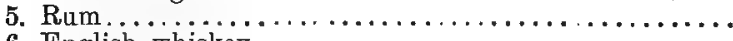

6. English whiskey......................

7. Ordinary brandy (s. schnaps)..................

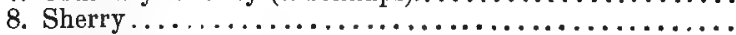

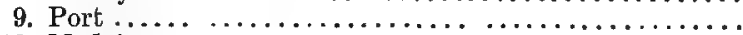

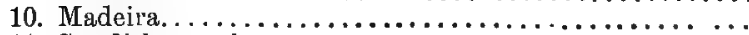

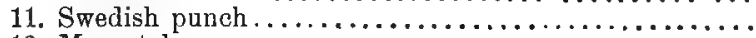

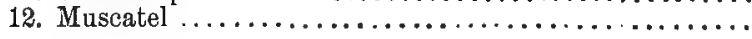

13. Greek wine.

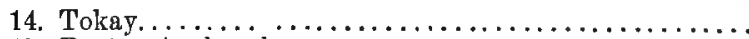

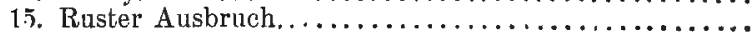

Alcohol, vol. per cent. 62.00

60.05

60.00

55.00

51.40

49.40

45.00

20.89

20.00

19.20

18.90

16.05

15.40\{

14.89

14.72
Carbohydrates (sugar + "extract").

$\ddot{0} \ddot{8}$

...

...

$\ldots$

$\ldots$

$\dot{3} . \dot{3}$

6.99

5.28

33.20

18.60

41.00

2.65

72.4

5.88

5.34
5.30

1.85
1.53

7.28

.23
6.76

5.89

8


PERCENTAGE OF CARBOHYDRATES IN WINES AND SPIRITS (continued)

$\begin{array}{cc}\text { AND SPIRITS } & \begin{array}{c}\text { (continued) } \\ \text { Alcohol, } \\ \text { Carbohydrates } \\ \text { (sugar + "extract "). }\end{array} \\ \text { vol. per cent. } & \begin{array}{c}17.27 \\ 14.22\end{array} \\ 13.86 & 3.63 \\ 12.57 & 3.67 \\ 12.09 & 1.99 \\ 12.06 & 1.804 \\ 11.55 & 2.4 \\ 11.45 & 2.3 \\ 11.16 & 2.2 \\ 11.07 & 2.0 \\ 11.07 & 1.7 \\ 10.35 & 16.7 \\ 10.34 & 2.0 \\ 10.30 & 1.9 \\ 10.14 & 1.7 \\ 11.08 & 3.0 \\ 9.938 & 1.8 \\ 9.90 & 2.5 \\ 9.78 & 2.265 \\ 9.67 & 1.2 \\ 9.66 & 1.4 \\ 9.56 & 1.972 \\ 9.55 & 3.06 \\ 9.49 & 2.7 \\ 9.44 & 10.70 \\ 9.40 & 2.3 \\ 7.85 & 2.2 \\ 4.28-5.65 & 2.75-4.75 \\ & \end{array}$

16. Malaga.

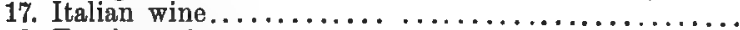

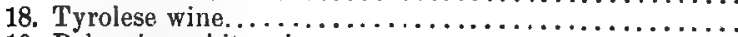

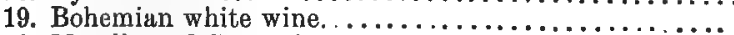

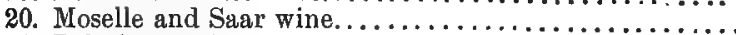

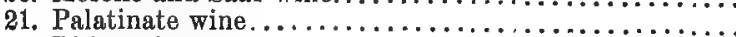

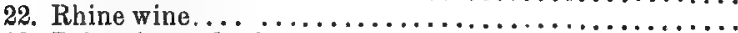

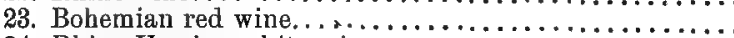

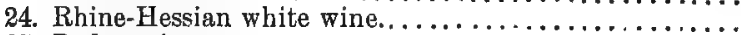

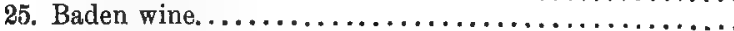

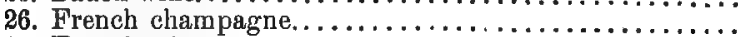

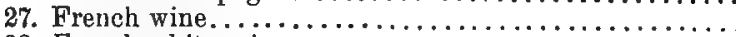

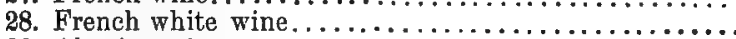

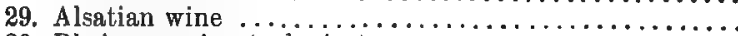

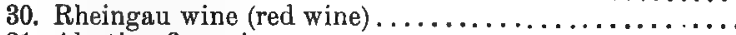

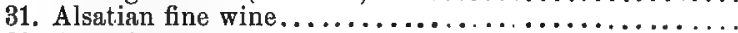

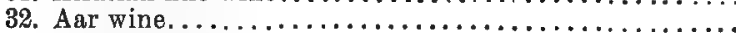

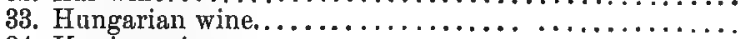

34. Hessian wine.................................

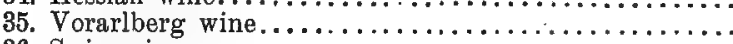

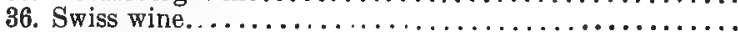

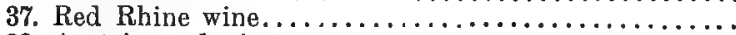

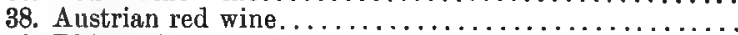

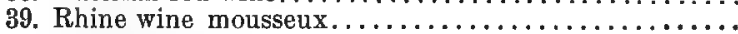

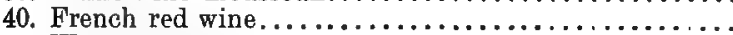

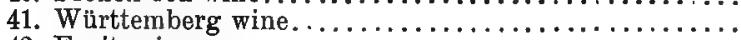

42. Fruit wine

FOR CALCULATING THE NUTRITION AND CALORY VALUE OF THE DIET

\begin{tabular}{|c|c|c|c|c|c|c|c|c|c|}
\hline \multicolumn{2}{|l|}{ FooD. } & \multicolumn{2}{|c|}{ AlbuMin. } & \multicolumn{2}{|c|}{ Fat. } & \multicolumn{2}{|c|}{ Санвон. } & \multicolumn{2}{|c|}{ Calorits. } \\
\hline & Amount. & Per cent. & Amount. & Per cent. & Amount. & Per cent. & Amount. & Per cent. & Amount. \\
\hline 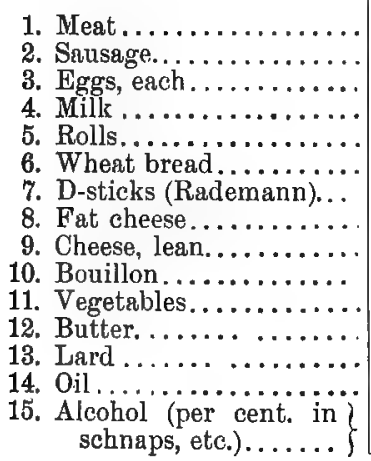 & & $\begin{array}{c}22.0 \\
15.5 \\
6.0 \\
3.1 \\
7.5 \\
6.25 \\
22.0 \\
25.0 \\
28.1 \\
0.63 \\
0.63 \\
\ldots \ldots . \\
\ldots \ldots . . \\
\ldots \ldots .\end{array}$ & & $\begin{array}{c}4.0 \\
48.0 \\
5.5 \\
3.0 \\
1.0 \\
3 . .0 \\
48.0 \\
30.0 \\
8.0 \\
0.10 \\
3-20 \\
87.00 \\
100.00 \\
100.00\end{array}$ & & 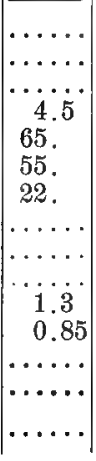 & & $\begin{array}{c}125 . \\
500 . \\
75 . \\
59 . \\
295 . \\
250 . \\
627 . \\
375 . \\
150 . \\
30-200 \\
800 . \\
900 . \\
900 . \\
700\end{array}$ & \\
\hline 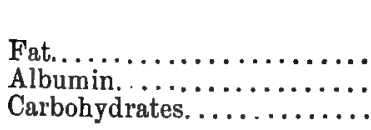 & 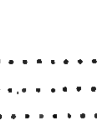 & $\begin{array}{ll} \\
\cdots \cdots \\
\cdots \cdots \cdots\end{array}$ & $\begin{array}{ll}\cdots \cdots \\
\cdots \cdots\end{array}$ & $\begin{array}{l}\cdots \\
\cdots \\
\cdots\end{array}$ & $\begin{array}{l}\text { Gram } \\
\ldots \ldots \\
\ldots \ldots \\
\ldots .\end{array}$ & & & $\begin{array}{c}\text { Calories. } \\
\ldots \ldots \ldots \\
\ldots \ldots \ldots \\
\ldots \ldots\end{array}$ & \\
\hline \multicolumn{5}{|c|}{ 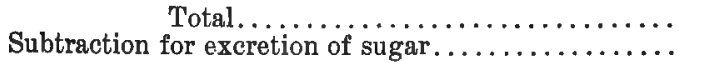 } & \multicolumn{3}{|c|}{$\ldots \ldots$} & \multicolumn{2}{|l|}{$\begin{array}{l}\bar{\ldots} \\
\cdots \cdots\end{array}$} \\
\hline \multicolumn{8}{|c|}{ 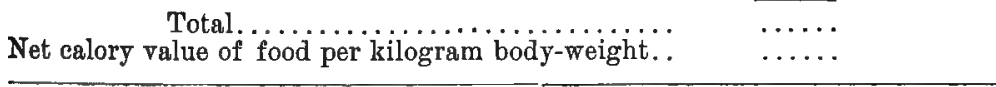 } & …… & \\
\hline
\end{tabular}




\section{SHORT POPULAR DIETETIC RULES FOR DIABETICS}

No food is to be given to a diabetic in unrestricted amount! The following rules are not, therefore, to be understood as permitting any food in indiscriminate quantity. The physician must decide in the beginning how much of each food may be eaten, and the patient must comply with his orders. These tables are, therefore, subject to variation.

1. Meats: bouillon or meat broth; meat of any kind, stewed, grilled, smoked or preserved. The addition of flour or bread in the preparation of meat is to be avoided; hence, Wiener schnitzel, stuffing of meat, stuffing of chicken, etc., are forbidden.

The kind of meat is of little importance except for the amount of fat it contains; the patient may take beef, pork, mutton, veal, goat, game, venison, birds, etc. The part of the body used for food is also of little account; muscle, kidney, pancreas, brain, giblets, are all allowed. On account of the glycogen it contains, liver forms an exception and is forbidden.

Flour and bread should not be used in the preparation of sauces.

2. Fish of any kind are allowed. Still, as they do not contain the same percentage of fat, they are of different values. The fattest are the eel, river lamprey and herring; most others are lean. Lobster and crab have the same value as lean fish. The same rules apply to their preparation as to that of meat. Shell-fish, oysters and snails are not advisable on account of their high percentage of glycogen.

3. Eggs and fat-foods. Eggs of any kind and preparation (without flour) are allowed; also good sausage (except liver sausage), ham, bacon, caviar, Strassburg goose-liver, sardines, fat, cheese, butter, oil, etc.

4. Milk preparations. Small quantities of not too sweet cream are allowed, also sour milk from which the milk-sugar has at least partially disappeared through fermentation. Regarding milk, special orders from the physician must be obtained.

5. Vegetables and starchy foods. Of vegetables we allow spinach, cauliflower, French beans, asparagus, lettuce, cucumbers, small quantities of celery and of crisp cabbage, mushrooms and other edible fungi (except truffles) and sauerkraut, as much fermented and as old as possible.

Prohibited are flour, rice, cereals, sago, tapioca, potatoes, peas, lentils, dried beans, green peas, yellow, white and red beets, horseradish, arrow-root, and corn-starch.

The orders of the physician must be strictly followed as to the amount of bread to be taken in twenty-four hours.

Gluten bread, Graham bread, aleuronat bread, which are recommended as substitutes for ordinary wheat and rye bread, are, on account of the larger or smaller amount of flour they contain, to be used only after consulting the physician.

6. Fruits, etc. Advisable are mandarins, oranges, currants, raspberries, gooseberries and strawberries, but especially cranberries; also nuts and almonds.

7. Beverages: Allowed are water, mineral water, coffee, tea, red wine 
which is not too sweet, or white wine, cognac, cherry brandy, and pure rye whiskey.

Prohibited are cocoa, chocolate, sweet wine, champagne, and also beer and liqueurs.

8. Spices. Pepper, also red pepper, and small quantities of English mustard are harmless.

Saccharin or crystallose is allowed in small quantities (0.1 gram per diem) to sweeten the food.

Sugar, also levulose, is forbidden in any form, as well as honey.

Every diabetic should be advised to eat as much fat and butter as he can without disturbing the appetite; hence, fat gravies, fat meat, vegetables, and salads prepared with considerable oil are recommended. 


\title{
DIABETES INSIPIDUS
}

\author{
By D. GERHardT, Strassburg
}

Drabetes insipidus belongs to those diseases which are of great interest to physicians, less because of the frequency of their occurrence than on account of the peculiarities of the pathological disturbances, and their definite analogy to physiological experiment.

Strictly speaking the disease has but one symptom: decided increase in the excretion of urine. Everything else that is mentioned among the symptoms is either a consequence of this disturbance or is only an inconstant accompanying symptom.

Increase of the excretion of urine occurs in quite a number of pathological conditions as a more or less regular symptom; thus, particularly in contracted kidney, in the convalescence of febrile diseases, and during the absorption of fluids from the cellular tissues or serous cavities. But we speak of diabetes insipidus only when none of these conditions is present.

This rare disease occurs at all ages, most frequently between the tenth and forticth years of life. The male sex is about twice as frequently affected as the female.

There are great variations in the severity of the clinical picture in different cases. In many persons the disease lasts for decades, and even for a lifetime; and beyond the inconvenience of frequent urination and the corresponding thirst, there are no disturbances of-the general health. In others nutrition suffers greatly, and there is a lessening in the capacity for work yet without any influence on the duration of life. In still other cases it is a severe disease which soon terminates in death.

This great difference in the severity of the symptoms makes it likely that there is no single disease corresponding to the name "diabetes insipidus," but that a number of different diseases in which the most conspicuous symptom is the profuse production of urine, are grouped under this name.

We know that an increase of urinary excretion may be artificially produced by increasing the intake of fluid, by raising blood-pressure, by irritation of certain areas of the central nervous system, by direct irritation of the kidneys with certain pharmacological remedies, and finally, by the introduction of so-called diuretics, which in their excretion through the kidneys carry with them profuse quantities of water.

It is probable that in the genesis of the clinical pictures which have been included under the name of diabetes insipidus, several of these factors and 
perhaps all of them have played a part, and it is possible that upon the basis of such difference in the manner of production a separation of the varying clinical pictures into individual groups may be made. In such an attempt, however, we can hope for nothing more than partial success.

One group of cases in which clinical observation or an anatomical finding shows organic disease of the brain can be sharply defined. Since Claude Bernard's celebrated experiments have shown that by injuring an area in the floor of the fourth ventricle (close to the glycogenic center) simple polyuria may be produced, many cases of this type of diabetes insipidus have been collected, and it has been determined that in the majority of these cases there has been a definite change in the posterior cerebral areas, particularly in the region of the sinus rhomboidalis.

These.cases are in harmony with the teachings of physiology; they form a well-defined, genetically connected group, and though we may be in doubt whether certain cases belong in this category or not, this doubt is due to the fact that in these instances the alterations attributed to the brain are indefinite.

Only a small proportion of all cases belongs to this group. Among the remainder, a group may be separated in which the increased extretion of urine is simply the result of an increased ingestion of fluid. In a number of cases it has been found that by limiting the ingestion of water, gradually or suddenly, we produce a short period of discomfort followed by cure of the disease. Here the obvious explanation is that the cause of the trouble is simply an increased ingestion of fluid whether due to an unexplained thirst or to habit; in short that polydipsia is the trouble.

Regarding many other cases it must remain questionable whether they should be included in this group, partly because some symptoms are opposed to this idea, partly because the decisive test of limiting the ingestion of fluid cannot be carried out.

Still other cases are proved by special characteristics in the clinical picture to depend upon an increased excretion of water by the kidneys, independent of the ingestion of fluid. The facts which justify this explanation are: First, a diminished excretion of water by the skin and lungs in spite of the great natural afflux of water to these parts and, as a more easily recognized expression of this condition, an excessive amount of urine as compared with the amount of fluid ingested; naturally the implied diminution of the amount of water normally ingested is at the expense of the water consumed with solid food. Secondly, we note the independence of the excretion of urine upon the ingestion of water, so that, for example, after a sudden stoppage of the intake of fluid, the production of urine continues unchanged, at any rate during the next few hours.

These conditions, polydipsia and true polyuria, are in practice very difficult to separate, and, in spite of much labor which some authors have bestowed upon this point during the last decades of the preceding century, the separation of these forms has not gained general acceptance. So it is with the attempt made by Schapiro and others to distinguish a form of diabetes insipidus due to disease of the abdominal sympathetic and to characterize it clinically; it is not convincing. 
To gain a comprehensive grasp of the manifold clinical phenomena of the disease, it is still most convenient to group the cases according to their apparent etiology. Thus the following forms may be described:

1. Diabetes insipidus in cerebral diseases,

2. Diabetes insipidus in functional neuroses,

3. Diabetes insipidus, idiopathic form.

Further description will show that under each of these divisions several very different clinical pictures are grouped together.

\section{SYMPTOMS AND CLINICAL COURSE}

The essential symptom is the large quantity of urine. The daily excretion is increased to 5, 10, even 20 liters, and in the severest cases it amounts to even more than this; cases have been reported in which the weight of the daily quantity of urine was as great as the body-weight. These cases occurred in children weighing $20 \frac{1}{2}$ and $27 \frac{1}{2}$ pounds; a patient of Trousseau voided 43 liters daily.

Of course the urine is greatly diluted, almost colorless, and feebly acid; its specific gravity is low, usually under 1.010, and it may even fall nearly to that of water.

As a rule the normal solids of the urine show no deviation from their ordinary amounts. It is true that the daily amounts of urea and sodium chlorid have been found abnormally large or abnormally small in a number of cases, but it is extremely probable that this was in consequence of a profuse or scanty ingestion of albumin and sodium chlorid in the food; diabetes insipidus in itself does not influence these values. Uric acid and kreatinin are found in the usual amounts; special importance has been attached to the occurrence of inosite in urine free from sugar; but according to the investigations of Strauss and Kiilz it is only the result of profuse excretion of urine such as may occur in the healthy after abundant ingestion of water.

The manner in which the urine is discharged varies in individual patients; in most, the number, in others, only the quantity, of the individual evacuations is increased. Many patients excrete more urine during the night than during the day, as in cases of contracted kidney; others do not show this peculiarity. The "morning flood of urine" (Quincke), that is, the production of a large amount of urine as soon as the patient wakens in the morning even when he stays in bed and takes no fluid, is quite similar to what occurs in the case of healthy persons. Further, as regards the influence of increased body warmth, the excretion of urine in diabetes insipidus appears to conform to the condition of health, i. e., in fever whether produced naturally or artificially (by injections of albumoses) it is decreased.

In the main the kidney functions more independently of the ingestion of water than in the healthy individual; after the ingestion of a large amount of fluid the amount of urine does not increase so rapidly as in health (though even to this peculiarity there are many exceptions). After a decreased ingestion of water it is true the urine decreases, but not so rapidly nor so markedly as in health. 
The kidneys apparently endeavor to excrete more water; they withdraw fluid from the body approximately at a uniform rate; if water is not supplied in plentiful amounts, inspissation of the blood occurs more readily than in healthy individuals. Thus, in a case studied by Strubell, in a patient whose thirst was unrelieved by water for several hours, the specific gravity of the blood rose from 1.055 to 1.071 .

Similarly to the condition in contracted kidney (though here the secretion of urine is increased from other causes) we find in cases of diabetes insipidus a diminution of the excretion of water through the skin and lungs. We are not prone to attribute this condition to disturbance of the functions of the skin and lungs but are rather disposed to assume that most of the water has been discharged through the kidneys, and hence there is not enough left for the skin and the lungs. Only after the disease has lasted a long time does the diminution in the activity of the skin attain a certain independence in the clinical picture, as it does in nephritis.

Insensible perspiration, as well as sweat formation, is diminished, and the fact that no sweating occurs in great summer heat has been noted in a number of clinical histories. Stoermer states that one of his paticnts showed a rise in temperature to $102.9^{\circ} \mathrm{F}$. on very hot days; on account of his inability to sweat, the patient apparently was unable to cool his body in opposition to the high external heat.

Naturally the increased excretion of water also requires an increased intake of water, and accordingly the normal desire for water is decidedly increased in all cases of diabetes insipidus. Decided thirst may be the first and most troublesome symptom of the disease, but the physician (in typical cases) sees in it only a result of the diabetes. How far a primary and pathological thirst with secondary increase in the amount of urine must be assumed in certain cases will be considered further on.

That the condition of nutrition in many cases is not influenced, and in other suffers more or less severely, has been already mentioned. This variation in the condition of the general health bears no direct relation to the amount of the urinary secretion.

As thirst is uniformly present so also the appetite is often enormous. Ehrhardt relates the case of a patient who consumed 12 beefsteaks for breakfast and 14 portions of meat at his midday meal. It is self-evident that in these individuals the composition of the urine will deviate from the ordinary in that the amount of the dissolved constituents will be decidedly greater than the normal. French authors describe diabète azoturique as an independent affection differing from the ordinary diabetes by an increase of $\mathrm{N}$-excretion. It is very probable that a large number of cases included in this group represent really such cases of diabetes insipidus as were blessed with an appetite of the variety just mentioned. At any rate, in recent times, when pathology has not only considered excretion but also metabolism as a whole, no cases of diabetes insipidus have been reported in which albumin decomposition was decidedly greater than the albumin intake.

Frequently with an improvement in the general condition, the abnormally great appetite also disappears. 
In contrast to these cases, there are others in which the amount of nourishment consumed is very slight, and in which nevertheless the body-weight is maintained. Quite a number of observations have been recorded which show that adults can maintain their N-equilibrium upon a diet containing remarkably small quantities of albumin ( 35 grams in a person weighing 55 kilograms).

In most cases, however, the quantity of nutriment is about the normal amount.

Quite frequently in patients suffering from diabetes insipidus, the temperature is found to be conspicuously low. Whether this is simply to be regarded as the result of a profuse ingestion of cool drink cannot be decided. In view of the diminished metabolism which may be observed in some of the patients, we must consider the possibility that the diminution in the body warmth is to be referred to a lessened oxidation, such as occurs in old people.

In contrast with this, an enormously increased body-weight has been reported in isolated cases.

Disturbances on the part of the organs of circulation, such as cardiac hypertrophy, apparently do not occur; on the other hand, the digestive apparatus is sometimes damaged by the profuse ingestion of fluids. Gastric catarrh, especially, develops not infrequently; true gastrectasis is only noted exceptionally.

The psychic functions do not appear to suffer serious disturbance, although hypochondriacal and melancholic attacks are frequently met with. In the cases which run their course with decided implication of the somatic condition, these psychic disturbances are the rule. Occasionally, in diabetes insipidus occurring in youth, a general retardation of mental development is observed.

Of other nervous disturbances, only sciatica is to be mentioned as a comparatively frequent complication of diabetes insipidus.

In the main, the diabetic does not appear more predisposed to general or organic disease than are healthy individuals. The reports that he is particularly susceptible to all sorts of infectious diseases, and that he is very liable to be attacked by tuberculosis, have not been confirmed by the study of more complete statistics.

Only with one disease is there a close connection which must be somewhat more minutely considered; I mean diabetes mellitus. This may be in various ways.

The connection is clearest in the cases of diabetes due to disease of the brain. A number of cases are on record in which, after trauma to the head, true diabetes mellitus with an increased urinary flow first appeared, the sugar, however, disappearing after a few weeks, and then for a long period, sometimes for years, a simple polyuria persisted. We know from Claude Bernard's experiments that the region, injury to which gives rise to polyuria, is situated only a few millimeters above the glycogenic area; later investigations by Eckhard have shown that in rabbits by lesions in the region of the sinus rhomboidalis, sometimes glycosuria, sometimes polyuria, may be produced. It is 
therefore quite plain that this cerebral form of diabetes insipidus is intimately related to diabetes mellitus.

But even in the non-cerebral cases of diabetes insipidus we can often recognize a relationship to saccharin diabetes, especially when we note the transition from the one disease to the other, and the occurrence of both diseases in members of the same family. Typical examples of such occurrence have been collected, particularly by Senator, and to these he has added cases observed by himself. It is quite probable that in these instances the neuropathic constitution represents the connecting link.

The results of animal experiments also suggest the possibility of a relation between diabetes insipidus and diabetes mellitus. During increased (experiment) diuresis, no matter whether it is due to drugs or to a simple injection of water, sugar is often found in the urine. Probably the sugar is simply swept along with the current. In man no well-attested examples of this purely secondary appearance of sugar in permanent polyuria have been observed, and the attempts to produce alimentary glycosuria in diabetes insipidus by an increased ingestion of sugar do not favor the view that such a washing out of sugar occurs readily in this disease.

As we go on from this sketch of the disease to the special varieties of the malady and their course, simple polyuria in cerebral diseases must be first considered.

This combination is not frequent; about 80 cases may be collected from literature. They are connected most often with injuries to the head; secondly with brain tumors, then with softening, while the rest are divided among various other cerebral affections, hemorrhage, encephalitis, meningitis, etc.

In the overwhelming majority of cases, the lesion is found to be in the posterior parts of the brain, mostly in the region of the pons or the fourth ventricle, i. e., especially in, or at least in the vicinity of, the area in which Claude Bernard and Eckhard were able, by experimental lesions, to produce in animals transitory, and Kahler permanent, polyuria. Kahler collected from literature 25 cases after trauma to the head, and 21 following other cerebral diseases, and added one of each variety from his own observations; among the 22 cases of the second group, 4 occurred in disease of the pons, 2 from compression of the pons, 2 from compression of the sinus rhomboidalis by tumor, 3 in diffuse disease of the medulla oblongata; 1 was probably due to a lesion of the medulla oblongata, 1 to a cerebellar tumor, 2 to syphilis of the brain, and $\%$ to disease of the corpora quadrigemina.

Less frequently, in cases of trauma to the head, the region of the sinus rhomboidalis is found to be the seat of the lesion; the description in the few necropsies is not clear. On the other hand, in all of these eight cases there occurred other cerebral symptoms (particularly paralysis of the abducens) which point with great likelihood to a lesion in the pons.

Hence, although diabetes insipidus is found especially in those cerebral diseases which affect the medulla oblongata, the pons, or the middle brain, nevertheless, upon accurate investigation of the individual observations, we find no single area in the brain which has been uniformly affected, and, on 
the other hand, a great number of observations show that every one of the previously mentioned areas may be affected without polyuria appearing.

We must, therefore, admit that although we can find cases which are in accord with Claude Bernard's celebrated experiments, and although the cases of diabetes mellitus occurring in connection with cerebral disease have turned out to be associated with a lesion of the very area in the floor of the fourth ventricle which was discovered in Bernard's experiments, nevertheless we cannot speak of diabetes insipidus in the same sense that we do of aphasia as a focal symptom, meaning that it occurs invariably in lesions of this area.

In the majority of cases, diabetes insipidus first appears at a relatively advanced stage of the cerebral disease, i. e., after other cerebral symptoms have developed. In some cases, on the other liand, polyuria has been the only symptom for months. This has been observed several times in tubercular meningitis, in tumors of the brain, and in cerebral softening.

A similar polyuria has been noted after trauma to the head, usually associated with other cerebral symptoms. Almost always the injuries are severe and are followed by complete loss of consciousness for several days. The increase of urine usually appeared for the first time after consciousness had returned; comparatively often the signs that the seat of the cranial lesion was in the posterior brain were paralyses of the muscles of the eye. Such a polyuria arising after injuries to the head sometimes disappears with the other cerebral symptoms, while in other cases it persists as the only morbid phenomenon for a varying period of time, even for many years.

The form of diabetes insipidus which occurs in connection with cerebral affections usually leads only to a moderate increase in the amount of the urine. In the traumatic cases the daily excretion of urine amounts to from 5 to 20 liters; in the form occurring in non-traumatic cranial disease, from 5 to 10 liters are excreted. There are no peculiarities in the behavior of the polyuria which distinguish these cerebral cases from other types of polyuria. Variations in the intensity of the urinary disturbances occur, but are for the most part slight; it is but little influenced by drugs or other therapeutic measures. The general health appears but slightly affected by the polyuria itself, apart from the influence of the cerebral disease.

There is only one peculiarity of the cerebral form, and this is its relation to diabetes mellitus, which, as we have already mentioned, is apparently more distinctive than in other forms of the affection.

Among the functional neuroses in which diabetes insipidus is frequently observed, two are of importance: epilepsy and hysteria.

In epilepsy, polyuria usually develops gradually, after the underlying disease has existed for years; more rarely it appears suddenly in connection with the attack. Usually it reaches but a moderate grade, and as a rule it is more susceptible to treatment than the cerebral form.

The manner in which epilepsy develops and its different stages are without any discernible influence on the polyuria. That diabetes insipidus occurs in connection with a particular form of epilepsy (e.g., one suspected to depend 
on an organic cerebral affection) has not been determined by any reliable evidence up to date.

The cases associated with epilepsy are not very frequent. In contrast to this, those combined with hysteria form a very large group. It is true that there are great differences of opinion in regard to our right to associate many of these cases with hysteria. The earlier writings on this subject were based on a careful collection of cases from literature, and a few cases were described in detail as a proof of the possibility of a combination of hysteria and diabetes insipidus; lately, with a number of authors, there is rather a tendency to class the bulk of such cases under hysteria, and we even read that practically all cases not due to organic cerebral lesions are of a hysterical nature. The latter view seems too extreme; but it is really difficult to draw the line correctly. We know that "normal" diabetes insipidus leads invariably to various psychical anomalies, slight in themselves (nervous irritation, hypochondriacal conditions and melancholia). Hence it may be difficult subsequently to determine how far symptoms of this kind should be referred to diabetes insipidus, and how far they should be ascribed to an hysteria which has existed for some time.

Diabetes insipidus develops in the course of hysteria either gradually or quite suddenly after nervous irritation, fright, or worry, or sometimes directly after a severe hysterical attack. In the latter cases it must be remembered that the hysterical attack is always followed by the profuse discharge of clear urine, typical urina spastica, that, therefore, a certain tendency to polyuria accompanies any such attack and is included in the conception of hysteria.

Some cases of diabetes insipidus occur in profoundly hysterical individuals whose disease has existed for years, and who exhibit severe spasms, paralyses, and similar symptoms. Examples of this form have been particularly described by French authors; curiously it is more often seen in men than in women.

More frequently, at least in Germany, polyuria appears in persons in whose previous history slight signs of hysteria are found, or in those in whom examination reveals hysterical stigmata, such as anesthesia, hyperalgesia of the skin, mucous membranes, or ovaries, limitation of the fields of vision, and increased reflexes.

That we are not dealing in such cases with a mere coincidence of two diseases entirely independent of one another, and that the diabetes insipidus may be a real symptom of the hysteria, has been demonstrated several times very clearly by the influence of hypnosis. By this means polyuria has been made to disappear suddenly, and later to reappear as before. Matthieu mentions a very significant case which was cured by the administration of powders containing, nothing but sodium chlorid, but which later relapsed when the patient discovered the deception.

In most of the hysterical cases, we are dealing apparently with pure polydipsia, as has already been mentioned, and in fact the majority of cases of polydipsia appear to belong to this group, viz. : to hysterical diabetes. But the reverse of this statement is not correct; cases of pure primary polyuria may also be caused by hysteria. 
Hysterical polyuria occurs most frequently in adults between the third and fourth decades of life. It is rare in adolescents and children, but, nevertheless, Terrier reports two undoubted instances in children aged one and a half and two and a half years respectively.

In this hysterical polyuria, as in the other forms, the patient is much more inconvenienced by constant thirst and the frequent desire to urinate than by actual pathologic symptoms; but there are exceptions to this rule. Some persons in whom the further course of the disease justifies the diagnosis " hysterical polyuria" emaciate decidedly as long as the diabetes exists. This is, however, unusual. As a rule, nutrition and the general well-being are not disturbed. Variations in the degree of polyuria are frequent; often the course of the polyuria is parallel with the severity of the other hysterical phenomena.

Hysterical polyuria may be cured, and sometimes with surprising rapidity. Occasionally this occurs spontaneously, more frequently after the employment of various drugs (valerian, antipyrin, etc.), by suggestion, under true hypnosis, or by means of powders of sodium chlorid and similar placebos. Indeed, it is often this rapid cessation of the malady after such slight external causes that stamps the condition with certainty as hysteria.

But by no means all cases of hysterical diabetes terminate so promptly. Many cases are protracted over long periods of time. In quite a number the history states only that at the time of the patient's discharge the diabetes continued unimproved. In such cases the disease appears to last for decades. In another series it disappears gradually in the course of a few weeks under appropriate antihysteric treatment, or more rarely it ceases spontaneously.

Diabetes insipidus in hysterical subjects shows very varying behavior. In some, the symptoms point decidedly to a primary increase of renal activity. This is especially shown by the dryness of the skin and the slight tendency to sweating. In other patients, on the contrary, perspiration is profuse, occasionally so free that they complain of it. If we are still in doubt, in such instances, whether the decided excretion of water through the skin can be explained by a primary renal insufficiency or whether the increased excretion of water is a phenomenon coordinate with the increased activity of the kidneys, the study of a further group of cases distinctly proves that this hysterical polyuria is really the consequence of an increased ingestion of water, i. e., a condition of primary polydipsia. A number of such cases are susceptible of cure by a more or less compulsory limitation of the intake of water, without the patients undergoing any great hardship. Sometimes we have only to quiet the thirst with simple remedies, such as stewed prunes or small quantities of lemonade, in order to control the polyuria. Such cases appear to represent scarcely more than a bad habit, and the number of these cases may in reality be much greater than can be determined from the clinical histories in literature because proof (i. e., the therapeutic test) cannot be obtained.

As the third main group the idiopathic form of diabetes insipidus will next be considered, i. e., that form in which no other organic or functional disturbance can be found as a cause for the polyuria. According to its mode of origin we may subdivide this group into: 
(a) A hereditary group,

(b) A group occurring in connection with acute diseases,

(c) The group of cases which appear after psychical irritation, known.

(d) A group of cases in which the causes are various or quite un-

The hereditary form has been observed only in isolated instances, but a few times in an extraordinarily striking way. The most remarkable series of observations we owe to Weil. He found 23 diabetics among 91 members of a family which embraced 4 generations, namely, the ancestor, 3 children, 7 grandchildren, and 12 great-grandchildren; the heredity was always a direct one, never skipping a generation; men and women were affected to a similar extent. Undue thirst was noted in some of these individuals even as nurslings; besides the breast-milk, it was necessary to administer water; in others the condition first arose between the second and fourth years of life. Almost all members of the family possessed a good constitution and enjoyed good health; many reached extreme old age.

There are also other records of the occurrence of diabetes insipidus in three or four successive generations, the individuals remaining mentally and physically healthy, apart from the anomaly in question.

With comparative frequency, i. e., in about 10 per cent. of all cases, diabetes insipidus appears after acute infectious diseases (scarlatina, measles, diphtheria, malaria, rheumatic fever, etc.); most of these cases (like those just discussed) appeared to cause but slight disturbance of health. They are of theoretic interest from the fact that convalescence in these same infectious diseases is quite commonly associated with an increase in the excretion of urine. It appears therefore that permanent polyuria may arise from a normal transitory polyuria, just as it does in hysterical individuals who have paroxysms with urina spastica. Similarly, permanent polyuria may appear after the temporary employment of diuretic remedies which cáuse a powerful diuresis.

In a number of cases diabetes insipidus has occurred as a sequel of various deleterious factors; extreme cold or heat and sunstroke are mentioned as causes. More frequently alcoholic excesses, bodily injuries (excluding head injuries), and psychical trauma are mentioned.

We include this entire group with the idiopathic form of diabetes. But in many cases doubt may arise whether they might not be grouped as well, or better, with hysteria. Even if none of the definite stigmata of hysteria is found, and if the course of the affection does not decidedly favor this diagnosis, we often meet with striking proofs that the patients belong to the category of neuropathic individuals. They react in this peculiar way to relatively feeble irritations which in the normal individual would be without effect, and, when once produced, the abnormal activity of the kidneys continues for a long time. We often succeed in discovering from the history that certain abnormalities have preceded these pathological reactions. Griffouillères points to the fact that in youth enuresis nocturna has often preceded the condition. Numerous therapeutic experiments lead us to suspect that the neuropathic element plays a prominent rôle in the etiology of these forms of diabetes. 
Hence, even if we do not consider this type of diabetes as belonging to hysteria, we must look upon it as closely related.

In this category we also include the cases in which diabetes insipidus occurs in pregnancy. A typical example of this kind is reported by Janzen from v. Mering's clinic, in which a woman in three successive pregnancies was attacked by a severe form of diabetes insipidus (passing 15 liters of urine, whereas she ordinarily voided but 2 to 3 liters).

The belief that such cases are closely related to the hysterical group is not simply a matter of theoretic classification but has an important influence on the choice of treatment to be employed and the energy with which it is to be carried out.

A number of cases remain in which none of the previously mentioned factors comes into question, and in which the malady progresses slowly, frequently becomes very severe, and comparatively often leads to decided loss of strength and to emaciation. These forms have mostly been looked upon as typical cases of diabetes insipidus. In the majority of these, observations regarding metabolism and the influence of varying quantities of fluid ingested have been made. For the most part such cases are serious; as a rule, attempts at cure are unavailing or have only a transitory effect. Nevertheless, life is preserved and the nutrition, which at first suffers severely, improves again without, however, reaching the normal.

Occasionally, decided permanent impairment of health has been observed, but marasmus increasing in severity until the disease terminates in death is rare. Earlier literature contains more frequent reports of an unfavorable outcome. Indeed, Trousseau believed diabetes insipidus to be almost invariably fatal. During the last decades, however, it seems that no instances of the kind have been published; perhaps a case reported by Strubell belongs to this group.

As a rule, in these severe cases of diabetes insipidus the constitutional condition appears to go hand in hand with the psychical. Permanent psychical depression, hypochondriacal and melancholic conditions, are frequent and almost constantly accompanying phenomena. We may even be impelled to ask whether a psychical anomaly is not the fundamental condition, and the diabetes insipidus only a symptom.

Nevertheless, such severe cases are rare; the majority of idiopathic cases lead a fairly comfortable life for years.

The cases of diabetes insipidus developing from syphilis appear to occupy a position quite apart. A number 'of instances of this kind are known in which, besides the diabetes, there were various symptoms pointing to organic disease of the brain, and in some of these the necropsy showed the presence of gummata or of meningitis. In addition to these, there are cases in which diabetes insipidus develops during the course of syphilis, sometimes many years after infection has taken place and without any other cerebral symptoms. By antisyphilitic treatment this form of the affection has several times been made to disappear promptly. It is therefore scarcely questionable that it was 
due to syphilis. Whether we are to assume in these cases a gumma of the brain (the simultaneous existence of destructive gummata of the skin in one of these patients appears to favor this view), or whether we are to assume a disturbance in the function of the kidney due to syphilis, is very difficult to determine at present. The practically important point is this, that in the course of syphilis, with or without cerebral symptoms, simple polyuria may occur, and that antiluetic treatment in both instances may bring about a cure of the diabetes insipidus.

\section{DIAGNOSIS}

The diagnosis of diabetes insipidus is usually easy; the important symptom, polyuria, can be readily determined, care being taken not to confound the disease with other conditions in which polyuria also occurs. Diabetes mellitus may easily be excluded by an examination for sugar. The question whether or not contracted kidney is present is not always so promptly solved. Cases of chronic nephritis in which the urine is at times free from albumin are not very rare; but the examination of the heart, and particularly the hardness of the pulse, will generally lead to a correct diagnosis. It is noteworthy that in diabetes insipidus, in spite of the fact that plethora serosa occurs frequently, hypertrophy of the heart and changes in the tension of the pulse never result.

Difficulties in the exclusion of nephritis may, however, arise in an opposite direction, namely, from the presence of slight quantities of albumin in the urine in diabetes insipidus. A number of clinical histories which describe otherwise typical cases of diabetes insipidus, some of the hysterical type, contain the statement that periodially small quantities of albumin could be detected in the urine. It is difficult to exclude nephritis in these cases as they have not come to autopsy; nevertheless the fact remains, that cases which in their course and especially in their uniform benignity do not differ from the ordinary picture of simple polyuria may from time to time show traces of albumin in the urine.

Amyloid kidney, less often than contracted kidney, may lead to confusion; although occasionally albuminuria is absent here, the general condition, the enlargement of the liver and spleen, make the diagnosis clear.

Arteriosclerosis more often leads to diagnostic difficulties, as it tends decidedly to increase the amount of the urine; but here also the action of the heart and pulse is conclusive.

Chronic pyetitis must also be considered in the differential diaghosis; in some few cases of diabetes insipidus described in literature, one cannot help suspecting that the writers were really portraying cases of pyelitis. The reports that the urine always contained slight traces of albumin and a purulent sediment are significant.

Finally, the possibility that diabetes insipidus may be mistaken for the results of a simple increase of the intake of fluid must be considered.

As a matter of fact this differentiation, at least for a time, may be simply impossible; the limits vary and are partially arbitrary. If after simply dimin- 
ishing the amount of fluid ingested, the polyuria ceases, and never recurs, as is sometimes the case in the hysterical form of diabetes insipidus, it depends ultimately upon the option of the observer whether the case is classed as one of bad habit, or as diabetes insipidus; for diabetes is so comprehensive an entity that such cases as the above can be included under it. In fact, literature contains quite a number of such examples.

\section{TREATMENT}

Treatment may be carried out in various ways. Where possible, we should search for the underlying condition which results in polyuria, and an attempt should be made to remove this.

In diabetes due to cerebral causes, such treatment is practicable only in isolated instances. Nevertheless, a case is on record in which, during the course of an apparently mild pulmonary tuberculosis, acute polyuria appeared, later followed by pain and stiffness in the cervical vertebræ, then paralysis of both arms and legs; here the diabetes was cured, simultaneously with the paralysis, by treatment with Glisson's suspension.

Otherwise, among the cerebral cases of diabetes insipidus, apart from the traumatic which not infrequently get well without treatment, only those depending upon cerebral syphilis are to be considered; in these cases and all others complicated by syphilis (whether a definite cerebral lesion exists or not), a cure may be effected by the use of iodin and mercury, as has been previously mentioned.

Somewhat more uncertain are the cases occurring in the course of hysteria. The fact that hypnotism sometimes brings about a prompt cure is a proof of the purely functional nature of these cases, and of the possibility of an "etiologic therapy" by purely psychical treatment. Whether or not in every hysterical case, or in every case that is suspected of being hysterical, hypnotism is to be employed, is a question that must be answered according to the confidence of the physician in the harmlessness of this remedial agent. In point of fact, in Germany no reports of cures of this kind have been made.

On the other hand a number of measures of a more innocent nature directed against the general nervous condition have often been quite successful. Among these are, first, general dietetic measures, sufficient exercise in the open air, and proper employment; occasionally, change of climate has been successful; often diabetes has been seen to disappear or become less marked in the course of hydrotherapeutic treatment. Regulated cold ablutions, douches, baths, in the milder cases can be carried out at the patient's own home, in the severe cases in hydropathic institutes; lukewarm baths appear to have a similar effect.

Among drugs which seem to be of use in a similar way, antipyrin (3 grams daily) and valerian are the favorites; the latter, given up to 20 grams of the powdered root, is sometimes decidedly beneficial; also potassium bromid, assafetida, and camphor, employed from the same point of view, have occasionally been very useful.

The remedies just mentioned are, a priori, most useful in the cases in 
which diabetes insipidus occurs in hysterical individuals. Regarding the prompt action of valerian, as well as of quite a number of remedies to be mentioned later on, the success of which is reported in the journals every year, it is unquestioned that diabetes insipidus is very often only a symptom of hysteria which, like other hysterical symptoms, may disappear after the use of the most varied remedies; as, for instance, after the employment of powders of simple sodium chlorid, provided only that simultaneously the necessary psychic influence is exerted. Many cases, perhaps most of those that come under treatment at all, occur in individuals whose malady cannot positively be included in the category of hysteria, yet is closely related to this group of affections, and these people are known as "nervous persons" in the ordinary sense of the term. In them, as well as in frankly hysterical cases, success comparatively often follows the use of the previously mentioned remedies.

Other drugs have been employed with the hope of influencing the circulation in the kidneys; the amount of urine in the main depends upon the height of the blood-pressure in the glomeruli, and the more contracted the small renal arteries are the less is the amount of blood which reaches the glomeruli. As the principal representative of such artery-constricting remedies, ergotin has frequently been administered in diabetes insipidus, particularly in the last few years, as its action has been much praised. Lead acetate and tannin are less useful.

Atropin and other belladonna preparations, which are supposed to act more directly upon the secretion of the kidneys, have also an inhibiting secretory influence upon most of the other glands. This influence, unfortunately, is more obvious in the salivary glands than in the kidneys; at least, increased dryness of the mouth and correspondingly great thirst have been observed more often than decrease in the amount of the urine.

Electricity has been employed for the sake of its (supposedly) direct influence upon the renal function. As irritation of the cervical cord diminishes renal secretion in animal experiments (on account of its powerful contraction of the vasa afferentia) the attempt has been made to diminish the amount of urine in man by galvanization of the cervical cord. More recently we have attempted to make the current act directly upon the kidneys (both electrodes in the renal region). Whether the electrical current thus applied has any real action is very difficult to decide; it is, however, a fact that this treatment is often quite successful.

Another method by which we have tried to diminish renal secretion is by stimulating the excretion of water by the skin. It has been frequently noted that the steam bath is conspicuously grateful to the diabetic, although it diminishes the secretion of urine; other patients report that they feel much better during the hot season than during the cold. Among the remedies which stimulate secretion by the skin, sweat baths and pilocarpin are to be considered ; the former certainly have transitorily an ameliorating effect. Permanent and complete cure has not been observed; but decrease in subjective discomfort and diminution in the daily amount of urine have been noted. The same is true of pilocarpin, polyuria decidedly decreasing with its employment (injections of from 0.01 to 0.015 on two successive days). It is true that in other 
cases the remedy has been ineffectual, and of course it will always be futile in persons who readily perspire.

Finally, that method of treatment must be considered which perhaps appears to be the most obvious, the limitation of the intake of fuid. This has often been tried by the patients themselves, but, as regards the propriety and success of such attempts at cure, the views of different authors are diametrically opposed.

In some cases withdrawal of water, strictly adhered to, acts with great rapidity; the patients are better almost from the first day, and after a few days may be looked upon as permanently cured. These are the cases which we have designated, upon the basis of this sort of improvement, as primary polydipsia. Very characteristic is a case described by Westphal: primarily for the purpose of studying her condition, the intake of fluid was limited, and at the. conclusion of the experiment the disease was cured. In other cases the same result has been attained by a gradual diminution of drink.

However, it is only with a small number of diabeties that we so readily reach the goal. Most patients cannot endure the tormenting thirst, and with or without the knowledge of the physician they will drink water. In such patients, this method of treatment can be carried out, and with it sometimes an actual cure attained, by isolation and observation. Geigel reports such a case of diabetes insipidus in which during the first few days of treatment severe disturbances appeared, but the withdrawal of water was persisted in, and after a short time the case could be looked upon as almost cured.

For the cases of diabetes insipidus, sensu strictiori, in which the polyuria actually depends upon increased renal secretion, such attempts at cure by the withdrawal of water are rarely successful. Indeed there is reason to fear that we do harm by a dangerous inspissation of the blood. Unfortunately it is often difficult in practice to decide between the two forms, and this is true of the majority of cases, i. e., of those that are symptomatic of hysteria or at least have some connection with it. Despite this difficulty, and in order to achieve success wherever possible, it is advisable to attempt with all patients of this type a methodical and cautious withdrawal of water. Of course this can hardly be carried out except in thoroughly organized hospitals. 


\section{GOUT}

\section{By W. EBSTEIN, Göttingen}

THE words "Gicht" and "Zipperlein" are genuine German words. The former is the equivalent of the old English masculine "ghida," which means "bodily pain in general." Zipperlein is the diminutive of "Zipper," formed from the verb "zippern," which in German means "jerking, restless, tripping." The term "Zipperlein" has been used in German for "Gicht" since the fifteenth century. It was invented by sufferers from this disease in a sort of grim humor. It is quite certain that the word "Gicht" is not, as was supposed, in etymological relation with the English word "gout," or with words of the same derivation in the Romanic languages (the French "goutte," the Italian "gotta," the Spanish "gôta"). These designations cannot be rejected, as they have become established by usage, but that they are not to be looked upon as appropriate every one must admit. They are based upon the old pathological conception that gout was caused by a peculiar humor distilled from the blood, which, drop by drop, was exuded into the joints, a view for the support of which no proof of any kind can be adduced. The designation "Zipperlein" for gout has quite properly been excluded from scientific nomenclature, as, in a symptomatologic connection, a uniform meaning is not attached to it. Among the ancients, the pathologic term "gout" was embraced in the collective term "arthritis," under which every form of arthritic inflammation was included. The term "arthritis" for "gout" embraces so much more than the purely symptomatologic conception that it includes a very important and active constituent of gout, namely, the "inflammation" of a joint. The words podagra, chiragra, gonagra, omagra, ischiagra and rachisagra, were applied by the ancients to joint pains in different regions of the body, and pains chiefly due to gouty and rheumatic affections were thus designated.

Certainly to-day, when we speak of podagra, we mean only a gouty affection of the foot. The word "arthritis" of the ancients alone discloses nothing as to the etiology of the joint inflammation. Later an attempt was made to separate the various arthritic inflammations according to their cause, and after we had demonstrated the causal relation of uric acid and its combination with alkalies to gout, the term arthritis urica, or uratica was given to gouty joint inflammations. As, however, there are still differences of opinion on this point, it would perhaps be wiser to speak only of gouty joint inflammations, or, for one who is fond of foreign designations, of gouty arthritis. 
The name gout has the advantage that it indicates nothing more than the pain which always plays a prominent rôle in gouty affections.

It must be remembered, however, that in gout we are not dealing with pain that is restricted to the joints, but with a general pain which is well described by Trousseau, "totum corpus est podagra." We shall refer to this more minutely when we consider the clinical aspect of gout.

Gout is certainly a disease of antiquity. It is not my intention here to discuss in detail the historical aspect of the disease. A. Delpech has recently written quite a voluminous work (678 pages) on the history of gout and of rheumatism, in which he dwells upon the antiquity of the disease, and brings it down to the time of Thomas Sydenham (1624-1689). In the study of the clinical history of gout, we, as a rule, only go back to the time of Sydenham, and, it appears to me, quite properly so. Sydenham's description is an account of his own illness, for, when he wrote his dissertation, he had already been suffering from gout for thirty-four years. All that had been written prior to Sydenham's time regarding the symptomatology of gout was of little value compared with what Sydenham communicated to us. The elucidation of the cause and the cure of gout Sydenham bequeathed to posterity.

\section{THE PATHOGENESIS OF GOUT}

The pathogenesis of gout has been studied from many points, and is now by preference our subject of investigation. It was the theme which occupied the attention of the Section on Internal Medicine of the XIIIth Medical Congress in Paris. I was unable to perform the task allotted to me, and give a synopsis of this subject at the Congress; but, at Easter-tide, 1900, I sent to the Secretary of the Section of Internal Medicine a compilation setting forth my views, and it may not now be out of place briefly to discuss the present standing of the question as set forth in this thesis. The views expressed by me were as follows:

1. Gout is a more or less chronic disease which develops upon the basis of a pathologic predisposition which is hereditary and generally congenital, and is usually designated the uric acid diathesis.

2. Regarding the ultimate cause of the uric acid diathesis, merely hypotheses can be at this time proposed. The intimate relations existing between the nucleins and uric acid make it appear likely that in the uric acid diathesis there is an abnormal composition of the cell nucleus; that is, of the protoplasm in the person in question. It is quite evident that an individual predisposition which may assert itself through entire families and races is a prominent factor in the uric acid diathesis.

3. Various circumstances appear to show that true gout develops from the uric acid diathesis. Regarding these circumstances the following may be mentioned:

(a) The more pronounced the uric acid diathesis in a person, the earlier, that is, the more decidedly, ceteris paribus, will gout develop in him.

(b) There are habits which favor the appearance of gout; among these are indolence and high living, especially the combination of these, and, of 
prime importance, the use of alcohol. In the main, the periodic variations which have been reported as to the frequency of gout may be referred to this.

(c) There are acute and chronic intoxications as well as infections, which, in combination with the uric acid diathesis, favor the development of gout, and in which bacterial toxins also may play a part. I shall first mention the relations of gout to rheumatism, to syphilis, and to lead intoxication. Influenza, also, appears to predispose to gout.

(d) Contagion, as was first asserted by no less an authority than Boerhaave, does not seem to play any part in gout.

(e) Climatic conditions apparently have no influence upon the development of gout.

4. The poison which causes gout, the actual materia peccans, appears to be uric acid. Whether, besides this, other products of metabolism of the group of alloxur and nuclein bases are operative, or, as some authors assume, a poison the nature of which is unknown, is still an open question. It is likely that only the endogenous nuclein substances of the human body, and not the uric acid originating from the nucleins of the food, have a direct influence upon the pathogenesis of gout. Whether an increased formation of uric acid occurs in gout has not been proven with certainty, nor do I believe this proof to be absolutely necessary. Nevertheless, I consider such increase to be probable. That an increased production of uric acid (which we assume from an increased excretion) does not alone give rise to gout is taught by the study of leukemia. In some cases of this disease, as is well known, more uric acid is excreted than in any cases of gout.

5. Uric acid is a chemical, but not a septic, poison. Its action is not uniform in all species of animals, at different ages in the same animal, nor in their different organs. Uric acid produces inflammation and necrotic changes in the affected tissues, and finally causes complete death of the tissue (necrosis). Only after the tissue is wholly necrotic does a deposit of crystalline uric acid take place in the form of acid sodium urate (monosodium urate, Tollens, sodium biurate, Roberts). According to Tollens, uric acid also circulates in the blood and in the alkaline juices of the tissue in the same alkaline combination, and not, as Roberts has assumed, as sodium-quadriurate, a combination which Tollens more technically designates as hemisodium-urate. It is admitted at once that monosodium-urate, Tollens (sodium-biurate, Roberts), under otherwise similar conditions may also be deposited as crystals in tissues, where not uric acid itself but another poison has caused necrosis.

6. To understand the pathogenesis of the different symptoms of gout, we must distinguish between $(a)$ primary arthritic gout and $(b)$ primary renal gout. The first is by far the more frequent, and the sufferers from this often reach extreme old age. Primary arthritic gout first develops under the influence of a localized uric acid stasis, i. e., it is limited to one or more parts of the human body. In primary renal gout we have from the onset a generalized uric acid stasis, which, therefore, affects all parts of the body, and is invariably due to a primary and severe disease of the kidney.

It is evident from the Transactions of the Section of Internal Medicine of the XIIIth International Medical Congress, that the opinions of the two 
reviews differed decidedly from my own, just mentioned, as I think I suffciently emphasized in my theses; yet, as the subject is a very difficult one, this diversity of views is not to be wondered at. Nevertheless, our views have so much in common that we may hope, sooner or later, to come to some agreement regarding the most important points in the pathogenesis of gout. I base my remarks upon the review in the "Semaine médicale," No. 30, 1900, pp. 202, 203, which reports the address of the reviewer, Dr. Le Gendre, and of the co-reviewer, Sir Dyce Duckworth.

Of course, if Sir Dyce Duckworth believes that the kidney is the only organ in which the synthesis of uric acid is carried out, i. e., in which uric acid is formed-in which opinion he and his countryman Luff occupy quite an isolated position not only among pathologists but also among physiologists - unanimity in regard to the most important question in the pathogenesis of gout is not to be arrived at. Sir Dyce Duckworth maintains, with Garrod, that uric acid is the materia peccans in gout and that the latter results from a hindrance to the excretion of uric acid through a faulty action of the kidney which can by no means be demonstrated from anatomically determinable changes. Sir Dyce Duckworth, therefore, in all cases of gout, assumes a uricemia from a faulty excretion of uric acid. Le Gendre laid great stress in his paper upon the influence of functional disturbances of the kidney in the production of gout and also referred to the functional disturbances of the nervous system; these result in nutritive changes in the tissue and cells, and thereby cause irregularity in the formation, that is, in the excretion, of toxic products of metabolism from the influence of which gout develops. In regard to these doubtful points in the pathogenesis, it appears to me advisable to wait for the publication of Le Gendre's complete paper. On the other hand, there are a number of factors which may be designated as occasional causes of gout, particularly family predisposition and the influence of the mode of life, etc., concerning which there is complete unanimity.

Instead of voluminous descriptions, it will best serve our purpose to cite a few cases from practice.

Orservation I.-Dr. X., from H., a physician about thirty-nine years of age, consulted me June 22, 1900, on account of gout. About fifteen years before, while he was a medical student, I had repeatedly treated him for this. As a jolly fraternity student, he probably drank more beer than was good for him, and had acquired a decided embonpoint at too early an age. In addition to a plentiful use of alcohol, he ate a great deal, and took very little exercise. In spite of these facts he was by no means dissipated, but passed a very good examination, and is now an extremely busy practitioner. The patient's family has a predisposition to gout; although his parents did not suffer from it, his maternal grandfather late in life had a severe form of gout, and two of his maternal uncles also suffered from it.

As early as between his sixteenth and eighteenth vears, the patient had repeated attacks of cramps in the calves, and he is inclined to regard as "gouty" an attack of "stiff knee" which appeared in his boyhood after a salt-water bath, and was accompanied by very severe pain. The first typical attack occurred in his twenty-first year, in the metatarsal phalangeal joint of the right great toe. The attacks recurred twice in the next year; the fourth attack, in June, 1885, which was accompanied with fever, was more severe than the preceding ones. The fifth attack, in September, 1885, which appeared three days after "turning" the left foot, was localized in the first metatarsal 
phalangeal joint. This ran its course within three days, was mild, and accompanied with only slight redness and swelling of the affected joint and no disturbance of his general health. After the patient began the practice of medicine he had no attack of gout for years. He lost his excessive weight, and when I met him in consultation a number of years ago I was astonished to find him rather thin. He followed minutely my dietetic regulations, and under this treatment only "premonitions" of his old trouble occurred. His constantly increasing, very extensive and exhausting practice now made it impossible for him to live in accordance with the rules previously followed. The attacks of gout recurred, and in the winter of 1899-1900 he suffered almost every two months from severe typical attacks, with violent onset, and constantly accompanied with fever. But while these formerly lasted for a few weeks, they now disappeared in a few days. These paroxysms were always associated with large effusions into the joints, and the seat of the attacks was in the toe and the talocrural joint upon both sides. The attacks were cured by the use of wine of colchicum seed, potassium iodid and sodium bicarbonate. Pain in the right shoulder joint, however, which had troubled him off and on for some time, now became chronic. The influence of trauma in the oecurrence of typical gout was shown when, upon April 30, 1900, the patient fell from his bicycle, causing a contusion of the right talocrural and of the left knee joint. As early as the next day, in both of the previously mentioned joints, a typical attack of gout developed which affected the patient greatly. He never had venereal disease; he smokes muchaccording to reports 8 mild cigars daily-and consumes a bottle of light wine daily, but no beer. The patient considers the wine necessary, as, without it, he has intermittent heart action. Appetite and digestion are good. His maximum weight was in 1883, when he weighed 209 pounds; now he weighs 176 pounds. During the attacks the urine is said to be scant and dark in color, but returns to the normal after the paroxysm has passed off. Albumin and sugar have never been present. The patient, when I examined him, had a pulse of 64 , cardiac dulness was not increased, the second aortic sound was loud but not quite clear. Organic changes were not evident. The patient was very much enfeebled, and as he desired to undergo a bath and mineral spring treatment, I advised him to go first to the Allgan Alps at Obersdorf. On the 2d of August, 1900, the patient wrote me from the "Nebelhornhaus" (2,251 meters high), stating that he felt very well there and that without much trouble he could climb quite high mountains. Besides the observance of a strict dietetic rêgime, abstinence from alcohol and a decided limitation of smoking were required.

The case just cited is in many respects interesting, and I shall take opportunity to revert to it later. At this time, only the factors which bear upon the pathogenesis of gout are to be considered. We note that the family predisposition of the patient was only in the ancestral line of the mother, the latter herself remaining free from the affection. The maternal grandfather and two of the maternal uncles, on the other hand, had gout. As the clinical history shows, the patient was inclined to date the beginning of his disease from his youth. It is evident that gout may come on early in life. A very skilful physician who had suffered from gout, but who died of a severe diabetes mellitus complicated by nephritis, told me that even in his ninth year he had seizures of so-called rheumatism in the ball of the great toe of his left foot. In children, attacks of gout frequently follow trauma, and it has been maintained that gout is the consequence of trauma. As a matter of fact, I have seen gout in adolescents, but always in boys, an observation which is confirmed by medical literature. The cramps in the calves with which my patient was affected between his sixteenth and eighteenth years may, in consideration of the course which the disease took, be regarded as premonitory gouty symptoms. From this, however, and also from numerous other experiences at hand, it 
cannot be doubted that the abuse of alcohol during his student days, as well as the previously mentioned obesity, conduced to the development of the gouty paroxysms. But it must be emphasized that, in my patient, the later disappearance of his corpulence did not lead to the cessation of the gout. Obesity is, therefore, not a prerequisite for the production of gout, although they may occasionally occur in combination, sometimes with diabetes mellitus as well. Of further interest in my patient's history is the fact, that in proportion as he deviated from the mode of life advised by me, the latent gout, i. e., the gouty diathesis, asserted itself by the appearance of repeated paroxysms, and that the last attack of this kind occurred after a fall from a bicycle whereby he sustained a contusion of the left knee-joint and of the right talocrural. I do not consider this occurrence of a gouty paroxysm after trauma as by any means accidental. Opposed to its being a mere coincidence is the frequency with which this combination appears. My patient is a physician in easy circumstances. We know that the wealthy minority, the so-called "upper ten thousand," are most frequently the subjects of gout. Among our hospital patients who, for the most part, belong to the poorer classes of the population, gout has heretofore been looked upon as a rare affection. I saw a poor tailor in the Hospital of the Home for Incurables in Breslau, who showed extensive, partly ruptured gouty tophi. Yet when I review the cases of gout accumulated during my long professional service in the Hospital, the number of the poor is quite small when compared with those in better circumstances whom I have seen in consultation and in my private hospital. In this connection, there has lately been a remarkable change in Germany, and one which is food for thought. Albert Fraenkel, in the Report of the Medical Division of the Hospital Urban in Berlin, from the year 1896 to 1897, and among 1,706 male patients, has observed 24 cases of arthritis uratica. In the hospitals of England, previous to this, the number of gouty patients was a striking one. In the years $18 \% 4$ to $18 \% 6$, in the Medical Division of St. George's Hospital in London, among 4,695 patients, not less than $9^{7 /}$ cases of gout were present, while, in the same period of time, in the Munich Medical Clinic, among 4,670 patients, there were only 11 cases of gout. We might say, it is true, that in this coincidence plays a leading rôle. But it must be observed that in the same period of time, and in nearly the same number of patients, in a London Hospital, there were almost nine times as many cases of gout as in the Munich Hospital. If it is true that in Munich, upon the average, more than 200 liters of beer per capita of the population are yearly consumed, the conclusion must be drawn that the influence attributed to Munich beer in the development of uric acid gout must be regarded as erroneous during the period just mentioned. The reports of His, Jr., and Magnus-Levy favor the increase of gout among the poorer population of Germany. Among the poor people of Leipsic the former not infrequently saw gout. The latter, in the Out-Patient Medical Clinic in Strassburg, made the same observation. Nevertheless, the social position, that is, the wealth or poverty of the individual-hence his mode of lifehas no fundamental influence upon the development of gout. The special disposition of the person which is noted in other members of the same family, even in entire races, and which may be hereditary, is the important point. It 
is evident that the presence of so-called predisposing, favoring factors is not alone sufficient for the development of gout.

In this connection, Thomas Oliver alludes in his valuable book, "Lead Poisoning," to an investigation regarding the workmen in the large lead works at Newcastle-on-Tyne, and this is very characteristic. As is well known, lead is a predisposing factor in the development of gout. Yet such of the workers in lead at Newcastle-on-Tyne as come from the north of England are only rarely attacked by gout, even when their kidneys are diseased, for in that portion of the population the predisposition to gout is absent; while those who come from the south of England, where the gouty predisposition is widely distributed, are frequently attacked with gout when employed in the previously mentioned lead works in the north of England. One problem referred to by me in the above named theses on the pathogenesis of gout requires further discussion: in what does the predisposition to gout consist, and how is the so-called gouty predisposition, the uric acid diathesis, to be conceived? The principal rôle is played by a faulty composition, not of the "humors," the juices of the human body, but of the protoplasm including the nuclei of the cells, in consequence of which uric acid, and perhaps still other bodies of the group of the alloxur and nuclein bases, are either formed in increased amounts or in an unusual region. I believe it to be very likely that the simultaneous occurrence of both these anomalies is the rule. The organs richest in nuclein play the most important part in this process, and I believe we may assume from the clinical history of gout that bone marrow and the great muscular masses of the human body, especially those of the extremities, are involved in this anomaly of uric acid formation. It is quite possible that in these tissues, already rich in nuclein substance, an increased formation of alloxur or nuclein bases, that is, of uric acid, takes place. My investigations have shown that uric acid is a chemical poison, and hence, until the same has been proven of the other previously named substances, and until the question has been answered whether there is a specific "gout poison," as is believed by some authors, uric acid may be looked upon as the materia peccans in gout. The uric acid, loosely combined with an alkali formed in the muscles and bone marrow, is dissolved, flows through the lymph and blood channels, and is for the most part excreted by the kidneys, unless other processes of decomposition take place during its course. If this process of excretion occurs smoothly and without hindrance, and if the gouty predisposition is not too decided, the consequences of the existing anomaly of metabolism will show themselves only after a certain time by a number of pathological phenomena, mild or severe, subjective and objective. But as soon as any intercurrent cause produces stasis of the juices rich in uric acid in the affected area-naturally at first in the peripheral parts, particularly in the lower extremities, in which stasis is especially prone to happen-engorgement will occur which, in consequence of this local stasis, also primo loco, in the affected parts of the body, will become more or less noticeable as a typical attack of gout. I call these cases primary arthritic gout. In Observation I, we have a case of primary arthritic gout which should more correctly be designated primary extremity gout, since not only the joints were implicated, but also the muscles, and especially the skin. 
Besides the extremities, in this form of gout the most distant parts of the body, i. e., those situated at the actual periphery, are implicated, as, for instance, the concha auris. For the explanation of these phenomena in primary extremity (arthritic) gout, an insufficiency of the kidneys which causes general uric acid stasis is by no means necessary. Following Garrod's reasoning, this is still frequently assumed, although the reasons mentioned by him have been shown to be incorrect. We shall revert to this later. Besides this primary extremity (arthritic) gout there is also a primary renal gout. Here, in consequence of a primary severe renal affection, most likely based upon a gouty predisposition, there is an incapacity on the part of the kidneys to excrete the waste of metabolism, i. e., the uric acid. Consequently a generalized uric acid stasis develops which affects all the parts of the body. The patients frequently die before a typical attack of gout occurs. They succumb, therefore, to the renal affection, usually while still young, whereas in primary extremity (arthritic) gout they will live to old age. In primary arthritic gout the kidneys suffer as well as all the other organs; but there are numerous cases of primary arthritic gout in which the patients die from intercurrent diseases, and in which the kidneys show absolutely nothing abnormal. Primary arthritic gout is fortunately by far the most frequent form of the disease, and the one we shall first consider. Formerly it was thought absolutely necessary to differentiate various forms of gout. I do not think it expedient to dilate upon this point, and will only mention that to-day designations are not infrequently used for some of these varieties, between which no important differences exist: thus we hear of latent gout, larval gout, pseudo-localized gout, retrocedent gout, etc. Yet I have not been satisfied with Garrod's differentiation of a regular and an irregular form; even in professional practice it does not simplify the condition.

\section{SYMPTOMATOLOGY}

We will now discuss the symptomatology of primary arthritic gout, and in order to make the condition clear I shall include the necessary pathologicoanatomical data. I shall retain the designation primary arthritic gout, as in the great majority of cases the joint symptoms are still the most prominent, and as I do not desire, by another designation, further to complicate a subject which is already rich in superfluous names. To avoid prolixity, in enumerating the possible limitations in the pathogenesis of gout, among the noxa which produce it I shall refer exclusively to uric acid as the materia peccans. This materia peccans which produces the disturbances, the totality of which, therefore, forms the gouty symptom-complex, is in part a purely functional disturbance, for which up to the present no constant pathologico-anatomical material lesion is known; in part, however, a disturbance for which the material substratum has been discovered. The latter depends in some degree upon inflammatory, also upon degenerative, changes in the organs and tissues. Suppurative processes are not produced by uric acid; it is not a septic, but chiefly a chemical, poison. The degenerative changes in gout from the influence of uric acid show nothing characteristic of gout until necrosis of the 
affected tissue occurs. If tissue necrosis has taken place, then acid sodium urate is deposited in the form of crystalline needles. That necrosis of the tissue is the primary condition is shown from the fact that the necrosed area is larger than the urate deposits. Around the necrotic areas, more or less round cell infiltration is often found. I have proven that such gout foci, i. e., necroses with crystalline urates (completely analogous processes), may be experimentally produced in birds. In these experiments urate deposits also occur to a greater or less extent in normal tissue, i. e., in tissue which has not become necrotic. Of course, this has absolutely no connection with gout, but merely explains the post mortem finding that, with cessation of life, the tissues are saturated with urates, and these are excreted in a crystalline form.

The symptoms of primary gout are as follows: In primary arthritic gout the first phenomena are the manifestations of the gouty predisposition. They may be designated as the premonitory or initial symptoms and they precede the first attack. It is possible that where the predisposition is not very great, and especially where an exciting cause is lacking, these so-called premonitory symptoms of gout may remain the only ones, so that a true gouty paroxysm does not develop at all. Generally, however, this is not the case. Under any circumstances, however, these premonitory symptoms may make it difficult properly to interpret the underlying condition. Not infrequently these prodromal symptoms, previously quite unintelligible to the physician, are at once explained by the sudden appearance of an attack of gout. Similar to these premonitory symptoms are the pathologic phenomena which have been designated by me as intermediate symptoms. They occur in the intervals between the attacks of gout, show remarkable similarity to the premonitory symptoms, but are much more difficult to explain, as the preceding gouty paroxysms have closed the path to their proper recognition. We shall later consider more minutely these premonitory and intermediate symptoms. It appears expedient first to direct attention to the typical condition in primary arthritic gout, namely, to the acute paroxysm of gout, the typical attack.

The clinical history of the physician suffering from primary arthritic gout (OBSERVATION I) shows that the first attack of gout may occur in very early life. I shall later demonstrate that this is also true of the appearance of extensive gouty tophi. As a rule, however, this is not the case. The first typical attack of gout usually occurs shortly before or after the fortieth year of life. The gouty attacks frequently recur at certain seasons of the year, especially at the beginning of spring and at the beginning of winter. Besides certain premonitory symptoms, the true attack may be preceded by prodromes in the part of the body that is to be the seat of the paroxysm, such as drawing pains, or slightly disagreeable sensations to which the patient pays no attention, and of which we only learn by careful questioning. Much more frequently the attacks begin suddenly, often coming like a thief in the night, and surprising the calm sleeper. As a rule, the first attacks are prone to be localized in the most peripherally situated parts of the body, and especially in the joints of the lower extremities. The joints of the feet, chiefly the first metatarsophalangeal joints, are most frequently attacked. The typical attack of gout is a more or less severe process characterized by an inflamma- 
tory but aseptic course, which is strikingly similar to an erysipelatous inflammation. Corresponding to the intensity of the inflammatory process, there is as a rule extraordinarily severe pain. Most patients declare it to be as though the affected parts of the body were tightly screwed in a vise. The skin is intensely red (purplish red or bluish red), sometimes even permeated by small hemorrhages; it appears as though suppuration were developing, and is tender on pressure, glistening, and swollen. The swelling is the consequence of edema; upon pressure there is pitting of the skin which only very slowly disappears when the pressure is relaxed. In the most severe cases active motion is impossible, and upon attempting passive movement of the painful joints, the patients resist. Under these circumstances, the patient is early confined to his bed.

Apart from these local pathologic phenomena, we also observe in acute paroxysms of gout disturbances of the general constitution. Especially noteworthy are the fever and the symptoms dependent upon this. In the milder attacks, fever may be absent. We note from Observation $I$ that, in the same individual, it was present in some attacks and absent in others. Occasionally there is a slight rise of temperature only in the first days, so that if the patient, for instance, comes under observation only upon the third day of the attack there may be no fever. Usually, the fever is moderate, a rise above $102.2^{\circ} \mathrm{F}$. being rarely reached at the height of the paroxysm. During the exacerbations so frequently observed in attacks of gout, the temperature rarely exceeds $101.3^{\circ} \mathrm{F}$., and when remission occurs, as a rule it at once falls to normal. The age of the patient seems to have some influence upon the temperature curve during the paroxysm, as, under otherwise similar conditions, the highest temperatures occur in young robust patients. Nevertheless, in the aged, during acute attacks, rises in temperature are by no means absent.

The further course of the gouty inflammation in the affected part of the body is generally typical. After the parra-articular phlegmons have existed for a few days, or several weeks and longer with remissions and exacerbations, the latter being sometimes so severe that an extensive joint suppuration seems imminent, the inflammatory symptoms ameliorate, the pain usually subsides, and the affected joint in many cases returns apparently to its normal condition. As in crysipelas, the skin becomes pale and desquamates. Nevertheless, decided sensitiveness to pressure may be noted in the affected joints, and this increases upon active and passive movement.

As a rule, the first attacks of gout are limited to one or several small joints of an extremity; rarely, for example, is the knee-joint implicated in the first attack of gout. Effusions, which are not at all rare in the gouty paroxysm, localize usually in the joints, and often they last for a long time. This causes the condition so frequently noted in the later course of the gouty process, in which two or more joints are attacked, one after the other, and thus the paroxysm is decidedly prolonged. An illustration of this and of the fact that in the course of gout numerous joints are always implicated may here be given.

OBSERVATION II.-X., a lawyer from B., forty-six years of age, consulted me on the 6th of July, 1896, on account of gout. His father-now over eighty years of age-and his younger brother are both said to have had an attack of gout. His mother died at 
the age of fifty-six of diabetes mellitus. The patient previously had two attacks of pyorrhea. He admits prior and oceasional marked abuse of alcohol; since practising his profession he drinks moderately. He has always been a great eater, and takes but little exercise. At the age of twenty-one or twenty-two he had a slight attack of gout in a joint. The attack eame on suddenly at five o'clock in the afternoon while in his office. In the following June the first severe attack oceurred, and confined him for almost three months to his room. Remedies-external and internal-were ineffectual until finally the American remedy (?) - potassium iodid, colchicum and colocynthitinbrought relief. The attack was in the left foot, and localized especially to the great toe. The paroxysms now recurred yearly, and the right foot also became implicated. In the year 1888, after a prolonged, exhausting ride upon the tricycle, an attack of gout occurred which was localized in the left lnnee-joint. In November, 1895, a severe attack of gout came on after drinking a somewhat larger quantity than usual of cognac with which the patient hoped to abort an attack of influenza. At first both feet and the knee-joints were implicated; the patient was compelled for almost five months to remain uninterruptedly in the recumbent posture. Relapses constantly occurred in which also the right elbow-joint was involved. He reports that, when free from attacks, the urine was dark and frequently contained sediment. The patient was under observation in my private hospital from the 6th to the 13th of July. He had tophi in the concha auris. The daily excretion of urine upon a mixed diet amounted to $0.6-0.8$ gram in twentyfour hours with an excretion of urea of about 40 grams and a phosphate excretion of from 3.1-2.3 grams. I regulated the diet and advised him to spend his summer in the Tyrol or in Switzerland. He did the latter. In the main, the patient apparently followed directions. That he rigidly adhered to the diet, and absolutely avoided alcohol, cannot, however, be asserted. The best proof of this is furnished by the reappearance of gouty paroxysms. In the first year during which he adhered to the dietetic rêgime, he had no attaeks.

I cannot enter into details here; the following fact will suffice. When the patient celebrated his fiftieth birthday, he thought he could not do without champagne. I know not how much he drank, but this I do know, that the patient had a very severe paroxysm of gout upon the following day.

The following case also is instructive. It proves that persons with a gouty predisposition can, by a proper mode of life, keep the disease in check. If they fail to do it, not only may severe attacks of gout appear in rapid sequence, but it is occasionally observed that the paroxysms soon attack a greater number of joints.

ObSenvation III.-The Commercial Councilor and City Councilman, X, aged fortynine, a very busy man, whose grandfather suffered greatly from gout, states that he comes from a family in the main very healthy. Toward the end of the sixth decade of the last century, he had Asiatic cholera in Vienna; as a young man he had a severe attack of gonorrhea. The patient was under observation in my private hospital from the 11th to the 14th of August, 1899. Regarding his gout the patient wrote for me the following history: "About six years ago I was attacked with gouty pains in the great toe, which appeared every six months and very rapidly passed away. I went to Kissingen every year, which benefited me greatly. Last year (1898) I had a very painful attack of gout in the foot, particularly in the small joints, and in September, 1898, I went to Wiesbaden. At the beginning of February of the following year (1899) another and also very severe attack of gout occurred, mainly in the right knee, but also in the small joints of the right foot, which kept me confined to bed for about three weeks. In May, 1899, I went to Aix-la-Chapelle, where I took the bath cure for four weeks, and used douches. Shortly after my return, gouty pains recurred, but disappeared in a few days. At the present time, upon prolonged sitting, slight pains occur in the joints of the right foot but soon pass away." This closes the report. The patie.2t had gouty tophi in the right ear. The urine showed a trace of albumin. The intestines appeared to be filled with feces. The gouty joints were sensitive to pressure. The patient relates that the 
attacks constantly appear, and so suddenly and so decidedly that even ten minutes after the onset of an attack he is unable to walk. Lately the patient has suffered from cramps in the calves. His nutrition is good. He is extremely excitable, and lives in constant dread of the appearance of a sudden attack. The patient tells me that the liqueur Laville helps him, and purges him decidedly. I ordered the observance of purely dietetic rules, and upon the 7 th of $M a r c h, 1900$, he wrote to me that he was adhering to my regulations, and since that time he has had no attack of gout. Nevertheless, I have reason to believe that the patient will not long continue this mode of life, although it is beneficial to him.

In regard to the gouty paroxysms and the relative frequency with which the individual joints are attacked by the gouty process, what has already been stated may be amplified by the following account. The joints of the knee, elbow, and vertebra are far less frequently attacked than the joints of the toes and the tarsal joint, but among these rarer localizations that of the kneejoint is perhaps the most frequent. I once saw in a patient aged fifty-four, after all the peripheral joints had been attacked by gouty paroxysms, an acute gouty inflammation of the left sternoclavicular joint. Any joint may be attacked by gout; even the small joints of the larynx have been found involved. This much is evidently certain, that gouty changes in the joints of the legs are not only earlier but also far more common than in the joints of the upper extremities. The joints of the hand and of the elbow are as a rule simultaneously involved. The premonitory as well as intermediate symptoms, to be discussed later, may become aggravated with the appearance of an acute paroxysm of gout.

In connection with this description of an acute attack of gout it appears necessary to say a few words in regard to the composition of the urine, especially as to the excretion of uric acid. Garrod assumed that during the acute attack the amount of uric acid excreted was diminished. He considered this to be due to a functional disturbance of renal activity, apparently because he was unable to find material changes in the kidneys. This diminished excretion of uric acid led Garrod, as already stated, to believe the gouty attack due to a retention of uric acid in the body, i. e., a generalized uric acid stasis. Garrod's view is vulnerable from more than one point. It must be first stated that a product of metabolism which may be still further decomposed in the body may, during this further decomposition, appear in diminished amounts in the urine; hence it cannot be concluded from its lessened excretion in the urine that it is also produced in diminished amount in the organism. Furthermore, if the view of Garrod were actually correct, it is incomprehensible why-as in the majority of cases-the attack of gout should be localized in the metatarsophalangeal joint of the great toe. Now, however, since the old, quite untrustworthy methods of estimating uric acid in the urine have been replaced by reliable ones, it has been demonstrated not only that the amount of uric acid excreted during an attack of gout is not decreased but that it is either normal or decidedly increased. It is by no means necessary that uric acid sediment or urate sediments should be found in the urine. An increased excretion of uric acid during an attack of gout is in the main due only to the attack itself. It has been shown to be independent of the nature of the food and of the total albumin metabolism. Thus the view expressed 
above gains support from the fact that, in the gouty process, an increased production of uric acid occurs, and plays an important rôle. In the cases of gout complicated with severe renal inflammation the complete retention of the excretory function of the kidney for uric acid is proven beyond doubt by the fact that, on the administration of food rich in nuclein, the amount of uric acid in the urine of such persons may be increased.

Associated with typical attacks of gout and occasionally without such attacks having preceded, in a certain proportion of gouty patients deposits of crystals which consist of acid sodium urate take place in the tissues. These prove that we are dealing with gout. Most frequently they are found in the joints in which one or more attacks of gout have occurred, and most commonly in the first metatarsophalangeal joint, in which the first and the most frequent paroxysms of gout are localized. However, it sometimes happens that these deposits are absent even when many typical attacks of gout have preceded. A prerequisite for the production of such urate deposits is tissue necrosis in the affected parts of the body. If the deposit of urate in the tissue occurs in a nodular form, gout NODULES (TOPHI) are spoken of. The number of these in the same individual may be quite large. The size of the tophi varies decidedly; small at the beginning, they may attain the size of a chestnut or even become larger. The analysis of two gouty nodules in the laboratory of my clinic shows that the first consisted mainly of uric acid (59.7 per cent.) and alkalies which formed urates with it (nearly 70 per cent.). These urates are to be looked upon as monosodium urates (Tollens), though Roberts designates them as biurates. There is also about 28 per cent. of animal matter present. Phosphoric acid, calcium and magnesium-all three in combination -were present only in traces. The analysis of the second gouty tophus showed in the main analogous conditions. Here also uric acid made up the highest percentage (61.27 per cent.) ; sodium oxid gave 12.28 per cent., and animal matter 26.45 per cent. For the practitioner, of course, these gouty tophi which are easily found and unquestionable are of chief interest from their diagnostic value. No error in diagnosis can be made in the case of a gouty patient with tophi in the ear, where they not infrequently develop in great numbers. Gouty tophi may, however, develop at very different parts of the body. The bursæ mucosa which are usually implicated in primary arthritic gout, and which are very frequently filled with urate containing material, become the seat of such tophi. Every fresh gouty inflammation which is localized there, as a rule, produces a paroxysmal increase of the tophus. The bursa over the olecranon process appears to be the preferred seat of such gouty concretions. Here in some patients the first gouty tophus develops. These uratic deposits sometimes form without pain. There is at first swelling from which, upon puncture, a white cream-like fluid consisting of acid sodium urate exudes. Later these tophi become hard and firm. They may disintegrate, and form gouty ulcers, the base of which is the previously mentioned urates. The ulcers are slow in healing and may, according to their localization, etc., lead to serious consequences when suppuration develops. Even when the urate masses separate and the ulcers finally heal, they show a constant tendency to again form. To illustrate the clinical history of gouty tophi some cases may be quoted: 
OBservation IV.-This record shows what I have already emphatically stated that, in comparatively young persons who are attacked by primary arthritic gout, an extensive tophus formation may occur. This patient was a merchant, aged twenty-five, from Cleveland (Ohio), who was evidently always a great eater, whereas he took scarcely any alcohol, nor was there any family predisposition. Even in his tenth year the patient suffered from decided cramps in the calves, and in his sixteenth year he had an attack of gout in the first left metatarsophalangeal joint. These attacks recurred frequently, and as time-passed the intervals became shorter. In these paroxysms not only the various small but also the large joints, occasionally several simultaneously, were involved. The severest attacks always occurred in spring and late autumn. About a year and a half after the first attack-in the patient's eighteenth year-the first gouty tophi appeared in the left ear. The paroxysms almost always came on suddenly; only rarely were drawing pains in the joints present as prodromes, and in these joints the attack was usually localized. Psychical disturbance was said to favor the appearance of the attacks. I saw the patient for the first time upon September 29, 1885. He weighed 124 pounds. His weight is said to have never been greater than this. He was a pale, mediumsized man and had a large number of gouty tophi in both ears. The third phalanx of the little finger of the left hand was thickened to more than double its size in consequence of gouty deposits. Gouty swellings were also found in several of the other joints of the finger. Between the first and second phalanges of the middle finger there was an extensor tendon with a movable, indolent gouty nodule. A somewhat smaller one was situated upon the radial side of the left index finger. Tophi were absent upon the right hand. Upon the third toe of the right foot a tophus about the size of a hazelnut was present. Upon the left foot there were two nodules which had ruptured. One of these gouty ulcers was situated upon the nail phalanx of the great toe, the other at the heel. The skin surrounding these ulcers was inflamed. Where these gout tophi had ruptured, white, chalky, urate masses exuded. About fourtcen days after the rupture of the tophus at the heel, almost all of the urate masses had been discharged. In the large joints no tophi were to be seen, nor were any deformities noted. The appetite of the patient continued good; the bowels were regular. About September 20, 1885, nine days before the patient came under my observation, he had an acute attack, running an afebrile course, not accompanied by disturbance of the appetite nor of the general system and localized in the left heel, the fingers of the right hand, and the right elbow-joint; amelioration began upon the 6 th of October. The internal, organs of the patient were normal and he was by no means cachectic. The urine did not contain albumin nor sugar. The daily excretion of urea at the height of the last mentioned attack varied between 23.3 and 27.1 grams in twenty-four hours. The uric acid estimations, at that time made by Heintz's method, can no longer be utilized. The examination of the sweat of the patient by means of the murexid test for uric acid gave a brown color with nitric

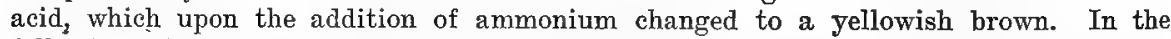
following winter, in Abbazia, the patient had an attack of gout which lasted for two weeks. In connection with this a gouty tophus developed upon the flexor side of the second phalanx of the ring finger of the right hand which the patient showed to me when he consulted me in the following May. After a stay in Abbazia, he also went to other places in Italy, and although he had no further attacks of gout he felt by no means well.

We see in this observation a case of primary arthritic gout which began early in life; paroxysms rapidly succeeded each other and very soon led to quite extensive tophus formation.

Gout shows a varying course. In regard to the paroxysm, it must be emphasized here that with a slight gouty predisposition combined with a suitable manner of life, the typical attacks occur only rarely and pursue a short and mild course. Attacks of gout which are of rare occurrence may assume a definite periodic type. I knew an army officer of high rank who lived to be over eighty years of age, and who, up to the time of his death, had each 
year an attack of gout. People with slight gouty predisposition, in whom gout develops under the influence of one or more favoring factors, may keep the disease in check provided these predisposing factors are removed. Thus, for example, if the patient modifies his mode of life he may conquer the existing pathological predisposition which would otherwise enslave him. If such persons have been exempt from gout for a longer period than usual, they flatter themselves that they have been permanently cured of gout, although the physician may have frequently cautioned them to the contrary; but they learn only too soon, if they return to their former manner of life, that this is by no means the case. A single excess is sufficient to reawaken the slumbering predisposition to the disease.

We now turn to the description of the PREMONITORY and INTERMEDIATE symptoms by which, as already remarked, I desire to have understood those symptoms which precede the individual paroxysms or are present between the attacks. When the individual attacks of gout have passed off, and during the period free from attacks, gouty patients are often troubled with a number of ailments. I shall here exclude entirely those symptoms which are due to the implication of the internal organs, and also others which not rarely complicate the pathological process; of these I shall speak later. In the period free from attacks, there is often more or less decided discomfort, referred particularly to the joints which have been affected, but also to those not attacked in the paroxysms; these disturbances often affect the muscles and bones of the surrounding areas. We must especially mention here that there is sometimes marked sensitiveness to pressure in the periosteum; this is elicited by pressure of the skin covering the bones, particularly the superficial bones such as the shin, but also the sternum, and more often the ribs. More troublesome, because often acute and ushered in with great severity, are the muscular symptoms, especially the painful cramps in the legs which recur frequently; no less unpleasant are the so-called rheumatic pains in the lumbar region called by the Germans "Hexenschuss," popularly known in English as "crick in the back." To this may be added vague, wandering, muscular pains and a sensation of extreme fatigue which have a particularly depressing effect upon the patient. Although these muscular symptoms are by no means pathognomonic of gout-for we observe analogous symptoms occasionally also in diabetes--they have, notwithstanding, a certain diagnostic value. Between gout, diabetes mellitus and obesity, there are intimate relationships. I have included these three diseases in a pathologic group as "general diseases of the protoplasm with a hereditary predisposition." 1

Similar to the intermediate symptoms are those which precede the attacks. These may exist for years before the outbreak of a paroxysm. According to my experience, such symptoms may be present in persons with slight gouty predisposition who are of active habits and live temperately; they remain the only indication of the affection and a gouty paroxysm never appears.

It is obvious that premonitory and intermediate symptoms may also occur,

1 Deutsche med. Wochenschr., 1898, Nr. 44, und Verhandlungen der deutschen Naturforscher und Aerzte, 70; Versammlung, 2. Hälfte, Leipzig, 1899, p. 73. 
soon or late, in consequence of implication of the internal organs in the gouty process, for, in primary arthritic gout, as already explained, the entire organism is more or less damaged in the course of time. These deleterious effects will show themselves earliest in persons whose gouty predisposition is severest and in those who are most exposed to the exciting causes of the disease. The acutest cases, however, are those in which several auxiliary causes become active in combination with a decidedly gouty predisposition. Under such circumstances it may rapidly become apparent that, as is true also in primary arthritic gout, "Totum corpus est podagra."

This leads us to the discussion of so-called visceral gout. This indicates nothing else than the implication of various other tissues and organs in the gouty process. In gout there is a decided tendency to catarrhal inflammation of the mucous membrane, and perhaps of the digestive canal, while even without this, many gouty patients throw too much labor upon their digestive organs, by immoderate eating and drinking, thus exposing them to inflammatory affections of this nature. We may mention, in passing, the frequent catarrhal affections of the mouth and pharynx in gout. The tongue, too, often takes part in this. Among the etiological factors in so-called psoriasis linguce, gout must be included. Worthy of note in the gouty is the loosening of the teeth which is often observed, and terminates in their premature falling out; an alveolar periostitis is looked upon as the essential cause of this very disagreeable symptom. Naturally the stomach is early implicated in gout, and it has been quite properly said that the stomach is in gout what the heart is in rheumatism. The gastric dyspepsias of the gouty play a great rôle in the symptomatology, even in those cases in which special dietetic errors cannot be proven. The causes of these dyspepsias are manifold as has been demonstrated by recent investigations. In the paroxysms of gout a decreased motility of the stomach and a decided diminution of the degree of acidity of the gastric juice has been observed; a deficiency in $\mathrm{HCl}$ is said to be especially common. It is certainly true that in gouty patients dyspeptic symptoms of all kinds, among which anorexia is perhaps in the front rank, are wont to appear. Just as frequent in gout are intestinal dyspepsias. Often in this condition we observe very troublesome and obstinate constipation, by the removal of which, after treatment of the stomach has been quite ineffectual, in many cases the gastric dyspeptic symptoms entirely disappear and even the symptoms of gout in the extremities are decidedly improved. To the symptoms in primary arthritic gout referred to the digestive canal, we may add that in this condition also functional disturbances of the activity of the liver are noted. That intimate co-relation exists between gout and the liver can hardly be doubted. Charcot and others have even looked upon gout as the result of a functional disturbance of the liver. However, it is not the purpose of this article to enter upon the discussion of these complicated and theoretic questions. On the other hand, the practical and important point in treatment is to be emphasized, that primary arthritic gout and cholelithiasis occur simultaneously with comparative frequency. Among other factors, the predisposition in gout of the mucous membrane of the biliary passages to inflammatory processes analogous to that of other mucous membranes may represent an important link in 
the chain connecting these pathologic processes. It may be here mentioned that in the etiology of the so-called hypertrophic hepatic cirrhosis gout apparently plays a part; indeed the liver appears to be implicated in primary arthritic gout, and even more frequently than is usually assumed.

However, the heart and the vascular system play a far more important rôle in gout than the liver, at least from the standpoint of the practitioner.

In regard to the heart, it may be remarked that in primary arthritic gout functional disturbances occur frequently; such are palpitation, intermittent beats, arrhythmia, and other irregularities of the heart's action, decided pain in the precordia, etc. These occur mostly as prodromal or intermediate symptoms, and as a rule diminish with the development of the gouty paroxysm. In general, they are not of serious import, and are often mild and transitory; nevertheless, they are frequently distressing to the patient. I have often observed such prodromal symptoms in gouty patients combined with stubborn intestinal disturbances, principally obstinate constipation, and have seen the cardiac symptoms disappear with a proper evacuation of the bowels after large encmata of oil. These functional cardiac disturbances which develop in primary arthritic gout are usually observed in persons under fifty years of age. In older persons, especially in such as have had a number of severe attacks of gout, we should bear in mind that these symptoms are caused by organic changes in the circulatory apparatus, even though by physical examination no gross disturbance can be determined. I am very careful not to say in such cases "The heart is normal," but content myself with the expression: "I can find nothing abnormal in the heart." Particularly serious in such cases are attacks of angina pectoris, although I know quite a number of gouty patients who, in spite of this, have lived for a long time. In the case of older gouty patients, especially those who suffer from attacks of angina pectoris, there is often disease of the coronary arteries. In fact we see, as the result of gout, all the different parts of the heart (myocardium, endocardium, valves, pericardium?) attacked, sometimes singly, sometimes in combination. Occasionally, deposits of urates have been found in the excrescences of the cardiac valves and in so-called ossification of the aorta. Apart from these cardiac disturbances, there is also gouty renal inflammation which occurs in primary arthritic gout after a longer or shorter time, and I shall revert to this later. There are no specific cardiac symptoms of gout; nevertheless, when investigating the etiology of attacks of angina pectoris, we should in the first place think of gout. How a cardiac affection can arise upon a gouty basis may perhaps be understood from the etiology. We can readily understand that, in the course of primary arthritic gout, the heart as well as the vascular system must become damaged because the organs of circulation are overwhelmed with blood laden with urates which do them injury. In the arteries, under these circumstances, there is developed a chronic inflammatory process, atheroma or an endarteritis; and phlebectases must also be explained in the same manner, at least in part.

Frequently disease of the urinary organs develops in the course of primary arthritic gout. I refer in the first place to the complication of gout with nephrolithiasis. This latter association has been denied by some, but incorrectly; by others it is declared to be a mere coincidence. But here, in $\mathrm{my}$ 
opinion, there may be a causal relation as well as in the not infrequent occurrence of gall-stones in gouty patients (see above) with or without simultaneous urolithiasis. (See my article, "Einige Bemerkungen über die Beziehungen zwischen der Gicht und den Steinkrankheiten," Aerztliche Praxis, 1901, Nr. 4, where I have discussed these questions at length.)

I shall now consider the condition of the kidneys in primary arthritic gout.

At the autopsy of those who have suffered from this disease, the kidneys may be found quite sound and normal, even in the cases in which decided gouty changes in the joints had taken place. In other cases, the kidneys are variously altered by the morbid process. We find chronic interstitial changes with especial frequency, usually the picture of genuine contracted kidney. With this, amyloid degeneration of the kidney is often found. Deposits of urates may be entirely absent in the kidney and in such cases we are unable to recognize the etiology from the anatomical changes. In other cases besides a more or less advanced nephritis, urate crystals are found in the uriniferous tubules. Even this condition I do not believe to be characteristic of gout. Finally, we may note in the degenerated kidneys typical foci of necrosis with urate deposits which closely resemble gouty tophi, both consisting of monosodium urate (Tollens). Nephritis due to gout, in the main, shows the same symptom-complex as when due to other etiologic factors. So-called gouty gonorrhea has been much discussed; it is certainly a rare affection, in which, in so far as I have been able to form an opinion, we are principally concerned with a catarrh of the excretory ducts of the prostate.

Inflammation of the mucous membranes of the respiratory organs is by no means rare in gout, and this is not remarkable in view of what has been stated regarding gouty disease of the mucous membranes. No further explanation is required concerning the way in which gout produces this complication, nor why in this disease there is a predisposition to nutritive disturbance of the lungs themselves, which favors the development of pulmonary emphysema. It is also evident that gout by no means excludes the development of pulmonary tuberculosis and other affections of the lungs. Pulmonary tuberculosis and gout not infrequently occur in combination. In the larynx specific gouty processes also occasionally take place.

The symptoms on the part of the nervous system are of special interest. As is well known, many celebrated clinicians have maintained the opinion, to which some still adhere, that gout is essentially a trophoneurosis, or a disturbance of the nutritive function. This need not be here discussed. Certainly disturbances in all parts of the nervous system often accompany gout, but many of the usual cerebral affections are due to vascular changes produced in the course of the malady. Diseases of the cerebral vessels have frequently been proven to be the cause of severe diseases of the brain, for example, of cerebral hemorrhage.

Often, however, in primary arthritic gout, so-called general neuroses are observed, i. e., those affections of the central nervous system for which no constant material substratum can be found. Among these I must first mention the severe neurasthenic conditions which sometimes reach the highest degree of hypochondriac depression, and which in some cases may even 
occur in paroxysms. In this group also belongs vertigo, which, however, as experience has taught me, may also be due to disease of the anditory apparatus in connection with gout. Here we must also mention migraine so commonly associated with gout, but which apparently develops under varying conditions. Gouty paralyses and neuralgias, among which we find the visceral form, are in many cases due to neuritis. The gouty affections of the organs of special sense, in which, perhaps, those of the eye fill a prominent place, require description by a specialist. It need only here be remarked that g7aucoma, so far as I am able to understand the condition, is usually due to gout.

That gout plays a prominent rôle in the etiology of cutaneous affections cannot be doubted. We have already described the gouty inflammation of the skin in the paroxysms; to these also belong the so-called copper nose which is not always due to gout, but arises so frequently in gouty patients that a causal relation between them may be looked upon as very likely. This appearance of "rhinagra," which usually runs a chronic course in this prominent portion of the human countenance, must be considered analogous to the gouty processes in the ear. However, within the limits of my experience, necrotic processes of the skin rarely occur in the nose, and, therefore, gouty tophi are absent there. In regard to gouty concretions in the skin and in the subcutancous connective tissue of the various regions of the body, it is unnecessary to speak further. Occasionally lymphangitis is seen as a consequence of gout. This condition does not give rise to difficulties according to the views which I have formed of the pathogenesis of primary arthritic gout. Gonty eczema and gouty psoriasis are frequently mentioned. It is at once evident that in the development of the former the irritation of the skin in gout, like the gouty inflammation of the mucous membranes, forms a predisposing factor. In psoriasis, of the cause of which we know so little, I have not succeeded in deducing any reliable connection between it and gout; I do not, however, by any means desire to deny the existence of such a connection, particularly as psoriasis and gout so often occur side by side. The same is also true of certain cutaneous affections which may be referred to vasomotor disturbance, among which I desire primarily to call attention to urticaria, which often appears as a chronic affection in gouty women. That these cutancous affections are invariably due to gout I naturally do not believe. Finally, it may here be mentioned that alopecia is sometimes found associated with gout, although in these cases quite a number of other etiologic factors may bring about the condition.

At this place only a review of the more frequent symptoms in so-called visceral gout can be given. In the consideration of the diseases which occasionally accompany gout, the question will and must occur whether there is actually a causal connection between them. If we reflect that in gout all the organs are subject to a certain tissue irritation, we shall be inclined to regard many diseases as due to gout. The fact is of interest that celebrated French investigators, such as Bazin and Ch. Bouchard, are of the opinion that cancer is the result of gout. The former has even said that gouty patients are particularly apt to succumb to cancer, especially to cancer of the rectum or of the bladder. I shall not enter into this in detail. On the other hand, the 
fact must be expressly pointed out that gout, obesity and diabetes mellitus are intimately related, which is evident from the circumstance that two of these diseases, or not infrequently all three, simultaneously occur in the same person. There is an extensive literature regarding the connection between obesity and gout. Some look upon obesity and gout as inseparable accompaniments of one another. Still I must emphatically state here that gouty patients are by no means all fat. Many are so, but even these may emaciate after they have become cachectic as the result of gout. Certainly there are, as has been stated, many fat gouty patients, and I may here refer to my article upon obesity, in which I mentioned such a case occurring in my practice, and took occasion to discuss the relation between gout and obesity somewhat more in detail. This observation is specially interesting because it concerns a lady who had well developed typical attacks of gout, which are by no means so rare as is usually supposed, but, on the contrary, in my experience, are frequent enough in women suffering from obesity. In discussing obesity, I referred to the development of flat-foot which we occasionally observe with increasing corpulence, particularly in persons with a hereditary predisposition to obesity and gout; these are mostly young individuals of either sex. The relations between gout and diabetes mellitus need not be here considered. But it may be noted that gouty and diabetic symptoms not infrequently alternate with one another, a fact which causes the diabetes to assume a somewhat intermittent character. The excretion of sugar is then slight, as a rule, and the so-called diabetes arthriticus frequently shows a course similar to that described by Johann Peter Frank as diabetes decipiens. A. Gilbert and Emil Weil attribute this form of diabetes mellitus to an insufficient or entirely absent function of the liver. Alimentary and rapidly disappearing, so-called simple, glycosurias, which I always look upon with suspicion, are not so rare in gout.

We must now consider the relation between gout and rheumatism. What is to be understood by this? First, there are a number of so-called cases of chronic arthritic rheumatism which should be considered as gout. When persons about fifty years of age, who have previously never suffered from any disease resembling acute articular rhcumatism, are attacked by an afebrile disease which particularly implicates or almost exclusively affects the small joints, which is accompanied by inflammatory symptoms, and which runs its course with acute exacerbations, gout should first be thought of. This is also true of the cases in which the joints show a certain deformity, and in which we usually recognize the presence of so-called deforming articular rheumatism, a disease which probably has no uniform etiology. An investigation of the family history certainly plays an important rôle in the diagnosis of such cases. Further, in considering the relation of rheumatism to gout it must be remembered that rheumatism, like all morbid processes that damage the joints, when combined with the gouty predisposition, furthers the development of gout, for anything that limits the free circulation of the fluids in the extremities favors the development of gout in persons who are predisposed.

Rheumatism not only attacks the joints but all their component parts, i. e., the muscles as well, and must be looked upon as a more or less active occasional cause of gout. In a similar manner connecting links of relationship may 
be found between gout and a number of other pathologic processes. From such points of view we may explain the well-known relations of syphitis to gout, which diseases frequently occur simultaneously. I believe that the explanation of this association may be that syphilis in a person predisposed to gout frequently precipitates an outbreak. It is a fact that in the beginning of the secondary stage in syphilitics the limbs, and particularly the joints, small and large, are especially implicated. Any one who fails sufficiently to consider this in treatment misses a very important curative guide. It is certainly difficult, from the nature of the changes in the joints, and when gouty tophi are not present, to say that this is a case of true gout and that is one of rheumatism. These difficulties at once disappear if we consider the history of the so-called Heberden's nodes. By this term we understand the thickening of the joints of the middle and end phalanges of the threc-jointed fingers. Some authorities consider these joint deformities as gouty; others look upon them as of rheumatic origin. In my experience such processes certainly may arise from a gouty basis; upon the other hand, we can by no means always prove that gout is the cause of these joint thickenings. We are not justified in concluding from the same external manifestation of a pathologic change a similarity in the cause of the disease. A case in point is the contracture of the aponeurosis palmaris which has been named, after the celebrated surgeon Dupuytren, Dupuytren's contracture. It was incorrectly maintained that this by no means rare affection was always due to gout. This may be the case: it is not, however, necessarily so. At all events such obscure combinations in practice are not to be underestimated, since diversity of symptoms always awakens interest, and for the affected patient, that is, for his etiologic treatment, the elucidation of such questions is of the greatest importance.

\section{COURSE AND PROGNOSIS}

The course of gout, as shown by the previous description, is subject to many variations, the majority of which may be understood by supposing that in individual cases there is a varying intensity in the predisposition to the disease, as well as in the influence exerted by manifold occasional causes in producing the condition. Gout is a disease of extremely chronic course. The earlier the vital organs, such as the heart, the vascular system, and the kidneys, are implicated the sooner does a fatal result ensue. So long as gout remains limited to the extremities, it is a comparatively harmless although very irksome disease. As it is often caused by the fault of the patient, it frequently makes him the sport of his associates and so irritates him. For this reason, the gouty patient with his "Weh und Ach so tausendfach" and his "Zippern" is rarely the recipient of sympathy. People forget entirely that even an apparently mild attack of gout may be very serious, either because it may throw itself upon the "internal parts," as the laity say, or, to use a more scientific expression, when it becomes retrocedent, because complications ensue or because the patients become cachectic. Nevertheless a gouty patient, in spite of periodically recurring attacks of moderate intensity, may. reach very old age provided no intercurrent affections arise. 


\section{DIAGNOSIS}

The diagnosis of primary gout depends upon the presence of two symptoms, namely, the typical attack and the gouty tophi. Either of these, provided that it has been determined with absolute certainty, may in itself be looked upon as proof positive. It is not necessary to repeat here by what means we may determine beyond doubt the presence of these two symptoms. I have endeavored in the description of the symptomatology to mention these factors briefly, but to characterize them definitely. Neither the joint phenomena, nor skiagraphy of the affected joints, are by any means such positive proof as to deserve the confidence of the physician in the diagnosis of primary arthritic gout. The same is true of the uric acid contents of the blood, of the serum from blisters, which is used in Garrod's so-called thread test, as .well as of the uric acid contents of the urine. Such findings are by no means to be undervalued; but the data gained in this way cannot as yet well be utilized in practice. They do not increase our diagnostic certainty. On the other hand the recognition of a family predisposition, as well as the history of the patient in other respects, is of inestimable value.

There are certain symptoms the consideration of which is important, particularly those which we have learned to recognize as premonitory or intermediate. These, too, are by no means positive, yet they often as pathfinders lead us to success. This is true, for example, of lumbago, especially if it occurs frequently without other determinable etiologic factor, and is quite persistent. It is likewise true of the frequently recurring severe cramps in the calves, and of neurasthenia. I have always laid stress upon the fact that a great number of neurasthenics owe their neurasthenia to a gouty predisposition which acts by limiting their energy. Special difficulty in diagnosis is often met with in women, when no typical well-developed attacks of gout occur. Here the characteristic gouty tophi are sometimes absent, but, on the other hand, we may find the Heberden nodes. I have previously mentioned that in women true typical gouty paroxysms occasionally occur even although they do not have the same acuteness and intensity as the gouty attacks of men. In such cases the investigation of the etiology is important. It must also be maintained that not infrequently gouty paroxysms can be recognized as consequences of trauma. To the category of these belong a number of joint affections which are called "sprain of the ankle," "stretching of the tendons," etc. Such errors are especially prone to happen in dealing with children as gout is usually not looked for in them. These mistakes may be explained by the fact that, as a rule, a slight trauma precedes the gouty attacks, and this is just as predisposing for the onset of the gouty attacks as the other etiologic factors that have been enumerated.

\section{TREATMENT}

For all practical purposes the treatment of primary arthritic gout may be divided into two parts. First, we will consider the treatment of gout as a whole, including the treatment of the gouty predisposition, and secondly, the 
treatment of the so-called acute gout, the typical gouty attack or the gouty paroxysm. In the treatment of the gouty process as a whole, prophylaxis plays a most important part. The latter appears simple enough, provided we are correct in assuming that gout depends for its development and existence upon a manner of life which deviates from the normal. Seneca, the celebrated Stoic philosopher, who lived in the first century, A.D., lamented that, in his age, the women who were living in the same opulent manner as the men showed the same tendency'to gout as the men, while in the age of Hippocrates women were almost exempt from gout. In general this is true, for Hippocrates says expressly that women do not suffer from gout except when their menses cease. There is, therefore, some truth in the words of Seneca, and since that time moderation has been continuously taught. I will quote the advice of Thomas Sydenham in regard to the treatment of gout. He says (Medical Works, Translated by J. J. Marstaler, Vienna, 1787, ii, p. 312) : "In the first place, then, moderation must be observed in meat and drink, so that the stomach will receive no more food than it can digest, and no fresh fuel be added to the disease. The other extreme, however, as I have found in my own person, is equally injurious. Abstinence weakens the parts by withholding their due proportion of that aliment which is necessary for supporting their strength and vigor."

In regard to the diet of gouty patients and of those persons who have a family predisposition to gout, the same rules are to be observed as in the nutrition of the obese. The view formerly maintained that the use of fat increases the formation of uric acid has been shown to be entirely erroneous by investigations carried out in regard to the ingestion of fat. The amount of albumin permitted to gouty patients must not be deficient. Everything that weakens the body and diminishes the activity of the gouty patient influences the gouty process unfavorably. On the other hand, the administration of large quantities of meat, as experience has shown, is decidedly deleterious to the gouty. I believe it, therefore, in the nutrition of gouty patients, to be not only advisable but even necessary to employ vegetable albumin in the manner mentioned by me in the treatment of obesity. I am even willing to maintain that for gouty patients a vegetarian diet with the necessary amount of vegetable albumin forms the most suitable plan of nutrition. Milk and milk products may furnish the variety which is grateful to the patient. Eggs also, if taken in moderation, may be permitted. White meat is looked upon as better than dark. Alcohol, no matter what its form, is poison for the gouty patient and for all those who are predisposed to the affection. Only when the physician for definite reasons declares it to be necessary, is the amount prescribed by him to be employed. The most suitable drink for gouty patients is ordinary good drinking-water or mild alkaline waters. I frequently order that from the Offenbach Kaiser Friedrich Spring, which in my opinion is preferable to all other alkaline mineral waters on account of its slight amount of alkali. For this reason, drugs containing vegetable acids that are excreted in the urine in the form of carbonates should be used in the treatment of gout. The cherry cure and the strawberry cure are not improperly named. These fruits contain alkalies rich in vegetable acid. On the other hand, much harm is 
done by the so-called lemon cure. Patients eat so much of this fruit that frequently troublesome dyspeptic symptoms arise. Neither do the grape cures bring about what was formerly expected of them. Sometimes they are followed by annoying gastric symptoms and even serious digestive disturbances. Moderation in eating and drinking is the first duty of the patient, but, notwithstanding this, the avoidance of all antifat cures, i. e., all measures liable to produce inanition, should be a guiding principle. In this connection the immoderate use of alkalies which are so frequently advised, chiefly on account of their uric acid solvent properties, is injudicious.

Mineral spring cures and bath cures are frequently resorted to in gout, and the thermal baths enjoy a great reputation. I usually advise them for patients who cannot take necessary muscular exercise such as gymnastics, mountain tours, etc. The same is true of baths as of the employment of mineral water and bath cures in obesity, which, as we have seen, often accompanies gout. The thermal baths are, in general, to be reserved for $(a)$ such cases of gout as occur in decrepit persons, (b) for old persons for whom energetic muscular activity is no longer possible, $(c)$ for cases of gout in which exudates have formed in the joints and in which resorption is to be brought about, and (d) for cases in which complications make such a treatment necessary. From this point of view peat baths and mud baths are in many cases very serviceable. The sulphur mud baths in Pistyan in Upper Hungary as well as the mud baths in Germany are highly recommended. In many such cases of gout it is noted that, when all other remedies fail, sweat baths in the Grotto of Monsummano or in the Grotto of Bormio give very excellent results. Carlsbad, Wiesbaden, Aix-la-Chapelle, Vichy, Wildbad and many other resorts are visited by gouty patients, but often without success unless they are assisted by suitable dietetic measures. It is of greater importance successfully to combat the morbid processes which in persons predisposed to gout form active auxiliary causes in its development, and decidedly favor its advance. Among these may be mentioned rheumatic affections and especially syphitis. In beginning a treatment of gout, it should always be determined whether syphilis has preceded it or not.

There are quite a number of so-called specific remedies for gout. Those which diminish uric acid formation, that is, which render the urates more soluble, play an important rôle. Of these remedies lithium has been for a long time in the van. It can only be administered as lithium carbonate, not as lithium chlorid. I have been unable to convince myself of the value of this remedy. Urotropin, introduced into practice by A. Nicolaier (twice daily a dose of 0.5 gram, $7 \frac{1}{2}$ grains, each tablet dissolved in a quarter of a liter of a slightly alkaline water), is better. But further investigations concerning its use are necessary. I have heard it praised by a number of old gouty patients who had tried almost everything. Piperazin (about 1 to 2 grams daily in an alkaline water) has proven useful, particularly for the "rheumatic" pains in chronic arthritic gout. The importance of treating the very defective intestinal function in the course of gout (constipation) is not to be underestimated. This disturbance of intestinal digestion must be relieved, and, as a rule, I employ large enemata of oil. That plentiful exercise is beneficial and neces- 
sary to the gouty patient, requires no further explanation. As long as his strength permits this must be kept up. I have already stated that in these cases I prefer muscular exercise to all other methods. If the gouty paroxysms do not occur so suddenly and are not so severe that the patient must go to bed, he should remain as long as possible upon his feet. When, however, the patient cannot walk, and the affected member cannot be moved, " patience and cotton," as many old gouty persons say, are the best remedies; others praise the Laville liqueur, which is probably beneficial on account of the colchicum which it contains. Colchicum is also administered as a tincture, or in combination with opium, purgatives, and potassium iodid. Pure crystalline colchicin has lately been recommended in doses of one milligram. Such powerful remedies should naturally only be taken under the advice and supervision of the physician. But only too frequently persons subject to gouty attacks refuse professional aid and attempt to cure themselves. The so-called antirheumatics, e. g., the salicylates, are also valuable occasionally for the relief of pain, although in the present status of our knowledge, they cannot be advised as a rational remedy for gout.

The various symptoms and complications of gout should be treated according to special indications. Here we will only consider the treatment of the numerous nervous symptoms, especially the therapy of gouty neuralgia with its related conditions, and the functional cardiac disturbances which so frequently occur in the gouty. Where there is chronic constipation, the treatment previously mentioned for the evacuation of the intestine filled with feces is of paramount importance. Even if chronic constipation is not present a useful auxiliary remedy in intestinal treatment is the employment of arsenic - and iron, preferably in the form of iron waters containing arsenic. In general it is advisable to begin with the mildest water of the Levico-Vetriolo in the Valsugana (South Tyrol). A residence in this region, which is easily accessible, is to be preferred to the use of the arsenicontaining mineral water at home, for the place is luxuriously furnished with all curative agents that are effective in gout, and is also advisable on account of its climate. There are many cases of so-called "larval" gout, which cannot readily be diagnosticated, yet are particularly benefited by such cures. Cures of this kind are also very suitable, mutatis mutandis, for such gouty patients as are already decrepit and have reached the so-called cachectic stage of gout. The many curative agents which nature and science there furnish, may be adapted in various ways by expert physicians to the individual needs of the patients. In these decrepit gouty patients no kind of so-called specific treatment is to be used, and colchicum especially is badly borne. Only tonic treatment-adjusted to the condition-is advisable. In the surgical treatment of gouty tophi, all the precautions of antiseptic surgery are of course to be observed.

A few words must be devoted to the consideration of primary renal gout, by which we mean the most serious form of gout, in which a generalized uric acid engorgement arises as the result of a severe renal affection and usually consists in a chronic and, particularly, in an interstitial inflammation of the kidneys. In this renal inflammation, the gout itself is an important pathogenetic factor. At all events, in primary renal gout the renal symptoms are 
the earliest ones, and the joint symptoms are secondary. When the latter develop, the nephritic process is usually so far advanced that the gouty nature of the affection can only be determined at autopsy, partly from the gouty changes in the kidney, partly from the gouty changes in the individual joints, particularly the first metatarsophalangeal joint. Fortunately this form of gout is not very frequent. The diagnosis during life is only possible when the conditions just described are recognized in the affected person. If the diagnosis of primary renal gout has been made, the prognosis is grave, as in all severe interstitial renal inflammations, since the renal parenchyma is destroyed. The therapy should be directed to the renal affection, for which diaphoresis and the dietetic treatment mentioned in primary arthritic gout are first to be tried. 


\title{
OBESITY
}

\author{
By W. EBSTEIN, Göttingen
}

WE understand by obesity an excessive infiltration of fat into the connective tissue in areas of the body where, also in healthy individuals, fat is found in varying quantities. The chief points of deposit of fat are the subcutaneous connective tissue and the mesentery. Special parts of the subcutaneous connective tissue are particularly involved, chiefly the abdomen. In the region of the breasts the deposit of fat may also reach enormous grades. The female breast has been known to attain a weight of 33 pounds. The highest grades of corpulence we designate obesity. In this condition fat also collects in regions where under normal conditions fat can hardly be perceived. Larrey, who accompanied Napoleon I as chief surgeon in all of his campaigns, saw in the Arabs in Syria scrota which attained, owing to fat formation, the size of a bulging cow's udder though under the skin of the normal scrotum only a very scanty loose connective tissue is found. Fat may, therefore, appear even in this region and in great quantity. In regard to the words in common use to designate obesity something may be added in the way of definition. Adipositas is a term often used and this designation is quite clear. A more general term is obesity; ${ }^{1}$ the French speak of obésité. The word is derived from "ob" and "edere," and means literally "to eat up quickly." In English the term corpulence is generally employed-a term which is also used by the Germans, but not to indicate the most marked grades of obesity. In the excellent book of William Osler: The Principles and Practice of Medicine, third edition (New York and London), 1898, page 439, the disease is described under the name of obesity, and it is mentioned that Lord Byron who was himself quite fat, as is well known, defined the condition-" oily dropsy." The designations "pimelosis" and "physconia" are but little employed. Like the other terms just discussed, pimelosis refers directly to the main factor of the disease, being derived from the Greek word " $\eta^{\prime} \pi t \mu \epsilon \lambda \eta^{\prime \prime}$

1 According to the Latin dictionary of Forcellinus, the word "obesitas" occurs in the writings of Columella (a contemporary of Celsus and Seneca), about the middle of the first century A.D., and in Suetonius (according to T'euffel's History of Roman Literature, about 65 A.D. to 160 A.D.). The former, in his "de re rustica" 6,24 , writes : "Ne steriles eas reddat, nimia corporis obesitas." The latter mentions in his biography of Domitian (18), that he was disfigured by a bald head as well as by "obesitate ventris." Further, Suetonius in his history of the life of Claudius (41) tells us that laughter was provoked "defractis compluribus subselliis obesitate cujusdam." In the Historia Naturalis, Pliny the Flder (born 23 A.D.) mentions $(17,27)$ that certain trees also "laborant obesitate." 
(= fat). Hyrtl (Text-Book of Human Anatomy, 20th edition, Vienna, 1889, p. 114) holds that the word constructed by pathologists, " pimelosis," is quite superfluous, since the Greek physicians already had a word for this disease,

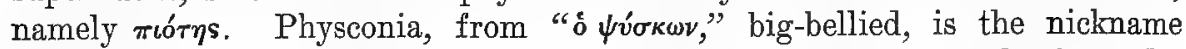
of Ptolemy the Eighth, Energetes the Second (170 в. c.), the classic predecessor of Banting. Occasionally polysarcia adiposa ( $\eta^{\prime} \sigma a ́ \rho \xi$, flesh) is used as a synonym for obesity. Strictly speaking, polysarcia should only be used for that stage of obesity in which the fat patient still retains his muscular power, as is occasionally observed at the beginning of the disease. But frequently such persons suffer from muscular weakness at the very onset of obesity. Among many other designations for obesity, lipomatosis universalis may be mentioned-a term which is used as a synonym for the highest grades of obesity. The fat which is normally present about the epicardium attains decided development, and, for example, in lipomatosis cordis, or true fatty heart, we note that the myocardium almost completely disappears before the proliferating fat which permeates it.

That fat people have existed since the coming of man is in itself probable, but this is also proven by the oldest records. In the Book of Judges, 3, 21, we read that Ehud (a left-handed man whom Jahve had raised up to deliver the Israelites from the bondage of Eglon king of Moab), in obedience to God's command put forth his left hand, and took the dagger from his right thigh, and thrust it into Eglon's belly, and the haft also went in after the blade, and the fat closed upon the blade, so that he could not draw the dagger out of his belly; for Eglon was a very fat man. In Psalms 73, 7, there is written, "Their eyes stand out with fatness: they have more than heart could wish," and, finally, in the Book of Job, 15, 2\%, we read: "Because he covereth his face with his fatness, and maketh collops of fat on his flanks." 1 Whether obesity was a matter for professional treatment among the Israelites cannot be determined from Biblical records; we shall revert to this later. In the Hippocratic writings we find the earliest rules regarding the treatment of obesity-rules which hold good even to-day. In the art of the Greek mythology, Silenus is usually represented as a bald-headed old man with a pug nose, small pig ears, and pot-bellied, a typical representation of obesity in the stage which I have designated as the "ludicrous." Rubens, in one of his pictures has graphically portrayed the plump, drunken Silenus, supported by a negro who walks behind him. That obesity during the time of the Roman Emperors excited ridicule is proven by the previously mentioned quotation from Suetonius, and Columella alludes delicately to the prohibitive influence of obesity upon feminine fertility. Pliny the Elder, who was almost contemporaneous with Columella, formulated rules for the treatment of obesity which later were generally accepted. These ancient views have lately been brought forth by some authors as the result of their individual investigations. I shall subsequently revert to this again.

Obesity is a very common disease. Indeed $\mathrm{L}$. Traube considers that a certain amount of stoutness (which the French and sometimes the Germans

1 W. Ebstein, "Die Medicin im Alten Testament," Stuttgart, 1900, pp. 10 and 150. 
refer to as "embonpoint") is normal. Healthy, strong children are usually plump when they are born. This fat which the child brings into the world normally increases during its early life, but later it decreases. We see from this that a certain amount of adipose tissue is to be looked upon as normal in the child at birth and for the first few years after birth; indeed not only is it normal but its absence indicates something abnormal. An excessive amount of body fat, however, is morbid and an evil. What produces such an excessive formation of body fat?

\section{ETIOLOGY}

The CAUSES OF OBESITY can be best understood if we consider for a moment the process of fattening animals, which forms an important chapter in the science of farming. The stock raiser, whenever he is raising animals for slaughter, endeavors to increase fat as well as to produce-meat. Many experiences with animals have shown that fodder deficient in fat as well as that rich in fat will, under some circumstances, produce a profuse accumulation of fat. Naturally, this presupposes that the food introduced exceeds the amount required to maintain the animal, and that albumin products are also present in the food in plentiful amounts. It is true that animals can be fattened with fat alone without the administration of any albumin, but in this case the animal is fed to death. Such experiments are made under conditions which do not come into question in the ordinary fattening of animals, or in the nutrition of the human subject. Apart from their fodder, the manner of life of the animals has a great influence upon the accumulation of fat, and especially the prevention of too great activity. It has been determined by numerous and careful experiments that sheep and oxen which have been fattened may be kept very fat for several months on a comparatively small amount of fodder if they are prevented from active body movements and protected from cold. But we should by no means infer from this evidence that in the development of human obesity too little muscular activity is the only important factor, even if we suppose that the fattening of animals may be regarded as parallel with the devclopment of obesity in man, of which however there can be no doubt. Of course we know that active muscular labor counteracts a too profuse accumulation of fat. Bunge believes that it is quite healthful and normal for man to eat everything that he likes and in any quantity that he pleases, and that in an otherwise normal mode of life this never leads to obesity. Bunge even declares it to be a portentous error to look for the cause of obesity in an over-profuse intake of nourishment or even in unsuitable food, i. e., in the too profuse intake of carbohydrates and fat. But his ideas by no means correspond with the facts. Bunge, it is true, does not deny that different persons are predisposed to obesity from different causes. This well-known fact need not even here be discussed in detail. But the familiar family predisposition to obesity cannot be adduced as a proof of Bunge's assertions. The objection is too obvious that laziness and an indisposition to take sufficient muscular exercise are also family traits, and also hereditary, and these may be more important than any predisposition to a too profuse fat deposit. An 
incontestable proof that a predisposition to obesity exists may be seen in the fact that there are many persons who eat whatever and as much as they wish, and nevertheless do not become obese. In what this predisposition to obesity consists is very difficult to say. I look upon obesity, gout, and diabetes mellitus as well, as forming a single group of interrelated diseases whose basis I designate as " a general disease of protoplasm resting on a hereditary predisposition." The epithet "hereditary" is not to be strictly construed. The predisposition may, but need not, be hereditary. By protoplasm I understand not only the cell body but also the cell nucleus, therefore the cell in toto. One may readily conceive that in the production of each of the three diseases just mentioned cell body and cell nucleus are implicated in different ways, but I shall not follow these hypotheses any further at present. The investigations so far published regarding the respiratory interchange of gases in the obese do not justify any definite and certain opinions, but we may assume that the consumption of fat in a person predisposed to obesity is slighter than in one not so predisposed provided both live under the same conditions. Further investigations into the respiratory interchange of gases in the obese made with the large Pettenkofer respiratory apparatus, and lasting longer than twenty-four hours, are much needed. Experiments of shorter duration do not justify us in forming any opinion.

The predisposition to obesity may become apparent at any time in the patient's life, and the manifestations vary much in intensity. Bunge believes that there is no predisposition to obesity which cannot be overcome by muscular activity. Whether this assumption be correct can, of course, never be proven with certainty. In my opinion there are cases which cannot be cured by exercise unless there is also a limitation of the ingestion of nourishment. The predisposition to obesity rarely shows itself in childhood to any unbecoming degree. I have already mentioned that even in utero and in the first years of life there is normally a certain plumpness which rarely increases so as to exceed physiologic limits. I have, however, seen several exceptions to this rule. Occasionally children with a monstrous development of fat are exhibited in shows. What I have seen of these makes me believe that in cases of this kind there is an excessive development of the whole body, a sort of gigantism, rather than a pure lipomatosis universalis, although the latter is favored by their very unnatural mode of life, for they pass their time almost exclusively in a sitting posture, and eat especially such food as decidedly promotes the accumulation of fat. Evidently this so-called polypionia infantum (which, in many cases at least, is associated with the condition described by Schönlein, called chlorosis gigantea) is quite rare. More frequent is an abnormal accumulation of fat in adolescents, especially in women at the period of puberty and in combination with various disturbances of menstruation. In boys also, when the normal development of the sexual organs at the time of puberty is retarded, an increased development of the body fat is not infrequently noted. That eunuchs are fatter than normal beings, although often maintained, appears to me to be insufficiently proven. In animals, however, castration favors fattening. On the other hand, there can be no doubt that women who were previously thin, after several labors, very frequently 
even after their first child, acquire a decided increase of their panniculus adiposis. The same also occurs at the menopause, and during the period of involution in woman. In some men we note that the body fat may increase enormously even during vigorous adult life. There can be no doubt that at this period an improper mode of life decidedly favors the accumulation of fat. Any one who goes to Munich, and watches the inhabitants, will be able to confirm this.

Of special interest is the previously mentioned obesity of women in the involution period, which, however, is by no means an invariable occurrence, but is observed in only about one woman in four during the menopause. Nevertheless, the obesity of women at this age has almost become proverbial. One speaks of "matronly proportions," and the Germans have a yet more significant phrase, "fat as an old woman." It is often asserted that this accumulation of fat is due to the cessation of menstruation, to the absence of function of the sexual glands which causes a decidedly diminished consumption of oxygen, and also that the decreased interchange of gases can be restored to normal and more than normal in a comparatively lirief space of time by the use of oöphorin, which compensates for the absence of ovarian activity. Further investigations will show whether, and how far, this tearhing is justified. At any rate this much is certain, that a decided increase of body fat above the normal is often observed without lesions of any special organ showing a decidedly determinable influence. Thus, for example, obesity may occur after recovery from severe illness, and is then usually explained by the long rest in bed during convalescence, and by the increase of nourishment above the normal after a long period of semi-starvation. We know that not infrequently, after recovery from severe enteric fever, the patient becomes quite stout, and that the corpulence acquired in this way is often permanent.

A factor of importance in the pathogenesis of obesity is the use of alcohol. I have already referred to the immoderate use of beer in Munich, and it is not necessary to quote further examples. Bunge also declares alcohol to be conducive to the accumulation of fat, and in the main explains this by supposing that alcohol, by its paralyzing action upon the brain, makes people lazy and unwilling to exert themselves.

\section{SYMPTOMATOLOGY}

In discussing the symptomatology of obesity I shall follow the division of obesity into three stages as proposed by me; if not pushed to a pedantic extreme, this plan simplifies matters very much. For practical purposes this division into three stages has shown itself to be very serviceable. In the first stage the obese man is an enviable person, in the second a ludicrous one, and in the third a pitiable one.

The FIRST STAGE may be identified with polysarcia. The flesh, the muscles, increase in due proportion to the fatty tissue. This is the type of obesity which conveys a majestic impression. The body becomes fuller, the figure fills out, the person reaches a proper embonpoint. This type is usually found in young persons whose youth is full of activity. In this stage obesity does 
not give rise to any inconvenience, and so long as the affected individuals maintain good muscular power no attempt should be made to remove or reduce the accumulation of fat. A certain amount of fat is a good reserve fund in time of need, for, not infrequently, even in this stage of corpulence, such unwelcome complications as gout and diabetes mellitus appear. Therefore, in this stage of obesity the condition is not to be combated, but any increase of it must be prevented.

The conditions are naturally different when the obese individual becomes a ludicrous figure. Such persons suffer from the ridicule of their companions, and form an excellent subject for the gibes of artists and poets. The portly Silenus has already been mentioned. The fat Falstaff, the popular representative of low comedy, has been glorified and immortalized by no less a one than Shakespeare. Not equal to bodily exercise on account of their great weight, obese persons in this SECOND STAGE have difficulty in dragging themselves along even upon a level, and upon the slightest bodily exertion they not only sweat profusely, but, as a rule, they must soon moderate or altogether give up their exercise on account of dyspnea. So far, however, the dyspnea, though it occurs upon slight bodily movement, is usually still of the "functional" type, i. e., it is not due to irreparable changes of the thoracic organs or to other gross organic lesions. The difficulty in breathing is due rather to disproportionate development of the abdominal cavity in which the enormous distention of the intestines by feces and gases due to atony of the bowel plays no insignificant rôle. Owing to this abdominal distention the thoracic space is decidedly narrowed. The diaphragm is forced up and its free excursion hindered, while the thoracic organs suffer a more or less decided compression limiting their function. Such obese persons, despite their difficulty in moving, show considerable fondness for all kinds of sport (hunting, horseback riding, etc.), particularly as they hope thereby to regain their health. But their motions and their whole appearance, which has so frequently been described and put into song, are so ludicrous that they provoke laughter. Yet we must not forget that even at this stage the complications and results of the immoderate accumulation of body fat often become alarming.

In the THIRD STAGE of obesity all the severe sequelæ develop which make the patient pitiable, and, in fact, he is commiserated. At this stage we meet especially the symptoms of severe disease of the heart, which I have just referred to, as a frequent accompaniment of obesity. This gives rise to dyspnea due to severe anatomical changes in the heart, and thus differing from the functional form above described. Besides this, a constant accompaniment of every case of marked obesity is a steadily increasing anemia. Prominent observers have contrasted the anemic form of obesity with a plethoric variety, but appearances are deceptive. I need only mention the obese chlorotics from the country with their very red cheeks. Even if we admit the possibility of plethora vera seu sanguinea (which in fact is a greatly disputed condition), we must specifically deny that any plethora occurs in well-developed obesity. Plethora could only happen in those forms of corpulence which belong to the first stage of the disease, i. e., that in which the muscular system is still in a normal condition. In general, anemia with all of its symptoms 
and consequences is typical of advanced obesity, especially of lipomatosis universalis.

We have already said that the other general "diseases of protoplasm based on hereditary predisposition" (gout and diabetes mellitus), frequently complicate the earlier stages of obesity, the enviable and the ludicrous stages. These unfortunate complications should not be regarded as direct sequelæ of obesity, but as diseases developed as the result of a pathologic condition of the body cells. The three diseases need not accompany or follow one another, but may arise independently. Hence we must infer that in each of them a separate abnormality of protoplasm exists. That uratic calculi often occur in the form of obesity which is commonly seen in combination with gout, and that the stones are caused by the gout, has been taught me often enough by my own experience. These stones rarely reach such a size that they cannot be spontaneously voided with the urine.

Atheromatous degeneration of the arteries, which is not infrequently noted in combination with obesity, I should not so much refer to the obesity as to the often coexisting gout, that is, to the uric acid diathesis. The obese, as a rule, enjoy an excellent appetite. Almost invariably they eat more than normal persons under otherwise similar conditions. Special stress is to be laid upon this in estimating the quantity of food which a corpulent individual consumes. Naturally we must not depend upon his own reports. In my experience obese individuals who are recognized as notorious gourmands, will, if questioned, deny that they consume an immoderate amount of food. They usually only say that their food agrees with them very well. Fat people often have, as I have already pointed out, a tendency to obstinate constipation. Hemorrhoids exist, or at least develop, very often. Upon the basis of the composition of the urine, A. Robin has differentiated two forms of obesity. In the one form there is profuse, in the other very slight, excretion of urea and phosphates. Robin believes that in the former group obesity is the result of increased, in the latter case of decreased, assimilation. He holds that this division is of great importance, especially in measuring the amount of fluid allowed in the dietetic treatment. That with the previously mentioned complications the urine shows corresponding changes, need only be indicated here. In advanced obesity there is usually a tendency to catarrhal conditions, especially of the pharynx and of the bronchial tree.

Obesity, if left to itself (or improperly treated), and when no conditions arise which produce emaciation-as, for example, severe acute or chronic affections-becomes a protracted disease which, as a rule, shows a tendency to progress. Yet I have not infrequently known persons who have become fat in middle age to lose their fat as they grew older without an apparent reason. Usually this is not the case. As regards longevity, obesity is a factor generally considered to shorten life. On this point we are indebted to the life insurance companies for valuable conclusions. Especially worthy of note, it appears to me, is what A. Hägler says in his book "On the Factors of Resistance and the Prognosis of the Duration of Life in Normal Individuals" (Basel, 1896, page 47 et seq.) regarding the PRognosis of OBEsity. All cases are by no means to be judged by the same scale. Age, heredity, manner 
of life and occupation must be carefully considered. Moreover, we should ascertain the patient's power of reaction against external influences, especially mountain climbing and other bodily exercise. Hägler requires that obese persons with an apoplectic or a diabetic family predisposition, with irregular pulse, with decided bradycardia, those who live in an improper manner, and those who are addicted to the use of alcohol, should be excluded from life insurance. No absolute norm for a maximal or minimal body-weight exists, but the rules laid down by Hägler are nevertheless noteworthy: "The insurance company at Basel is very cautious in its dealing with individuals of less than 340 grams, or more than 530 grams of weight per centimeter of bodyheight, and such are usually rejected. We have, however, reached the conclusion that in certain regions, especially among the very large, often giantsized, land owners and farmers of East Friesland, a higher body-weight is compatible with a normal duration of life." In my professional activity I have come across such persons, and in general may confirm this statement. But however true this may be, the risk of the obese person is to be estimated with caution. Even the enviable stage of obesity I look upon with suspicion, for it happens now and then that, without the ludicrous intervening, the pitiable stage arises, and. this is especially frequent, in my experience, in the cases in which obesity is associated with diabetes mellitus. Primary arthritic gout, even when it runs a severe course, produces, as I have seen, far less deleterious effects. Frequently the obese are threatened by lesions of the heart and the vascular system. In the heart itself in consequence of fatty deposits dilatation at first develops; sooner or later, when compensation for the damage to the cardiac muscle is no longer possible, the symptoms of muscular insufficiency supervene. The vascular disturbances are scarcely dependent upon obesity, but are to be referred to the uric acid diathesis, usually a factor in such cases. The arterial diseases of the obese usually cause effusions of blood into the brain, to which many fat persons succumb. It is of great practical importance in prognosis that persons who suffer from obesity, as is proved by experience, readily succumb to any infectious disease that attacks them. Among other factors which render the prognosis more serious in obesity, I must particularly emphasize the fact that as it progresses it makes the affected individual more and more sedentary, a habit to which the foot affections arising as the result of obesity also furnish their share. Flat-foot very often develops in the young, particularly in individuals hereditarily predisposed to obesity, when the body-weight increases. This usually affects those belonging to the higher classes of society, men as well as women. I should like to add here that I have observed such cases of flat-foot, mostly in those with a gouty predisposition. This is particularly worthy of note because such a deformity of the foot cannot be cured without considering gout as an etiologic factor. A sufficient amount of active muscular exercise is quite impossible on account of the affection of the joints of the foot and the resulting pain. But if the superfluous fat is removed by suitable dietetic regimen, with the slighter weight the diseased feet will also show a corresponding improvement.

At this point some remarks on the influence of increasing obesity upon 
the psychical life of the patient may be in order. It is commonly believed that fat persons have a more phlegmatic, and, associated with this, an especially good-natured disposition. That there is a necessary connection between obesity and a special temperament characterized by indifference and apathy is certainly not true. I wish to issue a warning against the adoption of this very common assumption as correct. [That this is the gencral view, not only among the laity but even among philosophers and poets, is well illustrated by the familiar quotation from Julius Cæsar, Act 1, Scene II, lines 192, et seq.:

Let me have men about me that are fat,

Sleek-headed men, and such as sleep o' nights:

Yon Cassius has a lean and hungry look;

He thinks too much: such men are dangerous. (J. L. S.)]

It is true, however, that the obese individual is usually distinguished by a less irritable nervous system. It is often declared that obesity hinders the development of great mental power. With necessary limitations, we must admit the truth of this assumption as regards the extreme types of obesity. One who has a great burden of body fat to carry about with him can hardly be expected to develop mental powers equal to those of persons not limited in this way. So long as obesity is not excessive, strong, energetic, and talented natures will be able to adjust themselves to the increase of their body fat and the resulting restriction of their bodily movements without suffering any considerable limitation of their mental functions.

\section{DIAGNOSIS}

The diagnosis of obesity is, as a rule, made correctly by the laity, for the condition is obvious, and may be determined whenever one finds an immoderate development of fat in the subcutaneous connective tissue. More perplexing is the decision whether the superfluous fat development has proven detrimental to other structures, for example, to the muscular tissue, as we should assume in all of the more marked grades of obesity. But for the physician this offers no great difficulty. Whether too much fat is formed and deposited in the areas of the subcutaneous connective tissue which are most accessible to examination is readily learned in most cases by observation of those parts of the body not covered by clothing, particularly the face. The cheeks, and especially the region of the chin, appear more massive than normal. The "double chin" is a familiar feature. Yet there are fat individuals in whose faces the fat does not reach decided proportions. The nude body, as a rule, shows accurately to what extent fat is accumulated in the subcutaneous connective tissue. All the landmarks of the body are displaced in obesity; they appear as if they had been forced downward or pushed laterally. There is great accumulation of fat in the subcutaneous tissue of the mammary region, not only in fat women but also in fat men; in the latter we often see the subcutaneous fat heap up until it produces masses that in point of size are not much less than the well-developed female mamma. Upon the abdominal walls thick transverse rolls of fat may often be seen. Upon the lower portions of the thorax, 
and particularly over the crests of the ilium, great collops of fat stand out. An enormous size is often attained by the buttocks which are thickly cushioned. with fat. These, however, do not appear rounded but assume a somewhat triangular form since the masses of fat hang downward and deviate laterally. Over the fold of the buttocks there is, therefore, a thick, shapeless mass formed by the superficial subcutaneous fatty tissue, which projects considerably beyond the lateral line of the thighs. In other cases the fat accumulates particularly in the sacral and lumbar regions. The remarkably great development at the fold of the buttock continues downward into the upper portion of the thigh, where the subcutaneous fatty tissue shows an increase that is scarcely less conspicuous. In very corpulent persons the subcutaneous fatty tissue upon the legs also forms prominent tumors. Of course, it is only at the autopsy of the obese that we can recognize the full extent of the enormous masses of fat which pack the internal cavities of the body, the mediastinal fat, the fat upon the pleura, the great accumulations of epicardial fat, the often excessive accumulation of fat in the omentum and mesentery, as well in the folds of the synovial membranes, etc.

J. P. Frank mentions a man observed by Boerhaave who, as the result of over-indulgence in food and drink, became so fat that the abdomen had to be carried in a sling which reached down from the shoulders, and the table at which he was accustomed to sit had to be cut away in a semicircle. His mesentery alone weighed 33 pounds.

Such fat people are generally looked upon as unsightly. Among the Moors, however, obesity in women is regarded as a great mark of beauty, and among the Kelowi in Central Africa a faultless odalisk must have the weight and circumference of a young camel, a circumference which she attempts to secure by a fattening process carried out with great perseverance. De gustibus non est disputandum!

I have already mentioned the conclusions of the Basel Life Insurance Company in regard to the proportion between size and body-weight as a factor in prognosis. In the diagnosis, however, we must determine not only that the individual in question is obese but also the degree of his obesity; we must recognize probable complications and the patient's capacity for work; we must decide to what extent he is anemic, etc. All these questions must be accurately determined before treatment is begun, and careful examination of the urine must be made in every case if gross errors are to be avoided.

\section{TREATMENT}

The treatment by which a patient is freed from his fat is often designated antifat treatment. Of course, the individual is not to be rid of all his fat and reduced to absolute leanness, but he is to have the surplus fat removed. In such an antifat treatment, it must never be forgotten that adipose tissue is a normal constituent of the body, and that its complete absence is not only unbecoming but a decided menace to the health of the person in question. There are people whose occupation necessitates that they be thin and remain so, guarding themselves against the over-accumulation of fat from any cause. 
This is attained by a process of training, so called, which consists in systematic and gradually increased exercise combined with a suitable diet. In this manner such persons fit themselves for various feats of strength. There is special training for riding, for marching, for swimming, for rowing, etc. We are all familiar with the training of race horses; by a special kind of treatment, by feeding and systematic muscular exercise, they are prepared for their task. By the same means men may prevent an increase of obesity, and eventually even become rid of it. In the well known processes of training, therefore, the first principles of the treatment of obesity are embodied. Nevertheless the detailed application of the latter is decidedly different, in that such tests of strength as are used in training are not suitable in the treatment of obesity, and, as a rule, would be dangerous in a corpulent person.

Nothing is easier than to make a person lean. Debove at a meeting of the Académie de Médecine at Paris on the 6th of March, 1900, showed a patient who had just passed through an emaciation cure (cure d'amaigrissement). To explain this method I will quote briefly a review of Debove's treatment published in the Semaine Médicale, No. 10, 1900, showing what this physician advises in the treatment of obesity.

I must remark at the outset that obesity cures and emaciation cures are by no means to be looked upon as synonymous. In the former only the superfluous fat is to be removed, while in the latter both fat and muscle are to be diminished. Leanness is attained by hunger; a treatment to produce leanness and a hunger cure are identical. Debove's obese patient, who suffered from urinary gravel, weighed before treatment (which lasted less than a year), 147 kilograms; after treatment he weighed only 94 kilograms. The patient, therefore, in less than a year lost 53 kilograms of body-weight. How was this attained? The patient was put upon a pure milk diet. He received daily for one month 21 liters of milk, during the second month only 2 liters, and in the third month only one liter per day. The patient, who was unable to leave his bed, lost in the first two months of this treatment 15, and in the third month 5, kilograms of body-weight. After the first three months he weighed only 127 lkilograms. During the next four months the patient received daily only one liter of milk. At the end of seven months from the beginning of the treatment he weighed only 105 kilograms. He then ceased to emaciate. But after a change of diet which permitted the patient to take cooked vegetables, salad and fruit in any quantity that he desired, he lost 12 kilograms more, so that he then weighed only 94 kilograms. As the result of this treatment Debove reports that the man not only was reduced in flesh but that he regained his health and his physical and moral capacity. Debove hopes that the good effects of this treatment will be maintained.

This case demonstrates that occasionally, by means of a starvation cure, we may obtain good results. In this case there was certainly a considerable degree of malnutrition, for 2.5 liters of milk per day, the amount which the patient received for the first month, is decidedly insufficient for nutrition, to say nothing of the fact that he had to content himself for months with one liter a day. Now it is certainly strange that, in this patient, loss of weight ceased 
after seven months in spite of continued insufficient nourishment. On the other hand, upon administration of a purely vegetable diet, especially after partaking of fruit in unlimited quantity, loss of weight again took place. I can only explain these facts by supposing that the patient, after the first seven months, became so hydremic that a further loss of weight was no longer possible. Subsequently, a decrease in weight could only be brought about when, by the diuretic influence of the vegetable acid salts resulting from the ingestion of large quantities of fruit, depletion of the fluids of the body occurred.

In obesity cures nowadays we naturally do not wish to use any method of treatment which brings about inanition. It is true that in every obesity cure, however rationally it is carried out, there must always be depletion of some kind. This depletion, however, must not go so far that muscle as well as fat is lost. A withdrawal of fat without loss of muscle can be brought about by a suitable change in the diet or by increased muscular activity, or-best of all-by a combination of both methods. By a diminution of the nourishment which has previously been taken in excess, and by an increase of muscular exercise which has previously been insufficient, the superfluous body fat will gradually be consumed. I do not doubt for a moment, and I have often emphatically said, that this goal may be reached by very simple means. Typical in this respect is the quaint tale of Johann Peter Hebel, which many an indolent, fat carouser may take as an example. This story is told of a rich, fat Amsterdamer, whose physician, living a hundred hours' journey from himand this was no less a personage than the celebrated physician Boerhaave, of Leyden-insisted that the patient visit him, and that he come on foot. He impressed upon him the necessity of a regular mode of life in order to crush the dragon which he carried around in his abdomen. The patient became an expert pedestrian, and then learned to saw wood; he restricted himself to the food that hunger required, became as healthy as a fish in water, and reached the age of eighty-seven years, four months and eighteen days. These effective means of preventing an excessive increase of body fat, or of causing superfluous fat to disappear, have met with little favor, though obviously they are so easy to carry out. We see that celebrated physicians like Johann Peter Frank almost maintain an attitude of hopelessness as regards measures for the removal of obesity. Frank joins in the old complaint of a Leipsic physician of his time that " a few succeed, by the aid of very strict diet, in wholly ridding themselves of their superfluous fat; as soon, however, as they have accomplished this (not without great loss of power), and attempt to recuperate by a less severe nutrition and mode of life, they regain their previous amount of fat, or, in its place are attacked by a pitiable condition of dropsical accumulation." It is obvious from this that J. P. Frank had starvation cures in mind, and that he was so daunted by these unfortunate experiences that he did not further pursue his endeavors. He mentions no special dietetic rules for the relief of obesity.

It seems to me best to present in chronological order a comprehensive review of the methods of treatment which have been employed in our time, and I desire expressly to state that much that has been claimed by individual 
authors, with great self-laudation, as the product of their own ingenuity, may be referred to very ancient sources.

First, then, as the earliest of the modern methods of treatment of obesity, the "no-fat cure" must be mentioned. This treatment prohibits the eating of fat, on the principle that under all circumstances fat will produce fat. The type of this is the so-called "Banting cure." Banting, a very corpulent Englishman, who lost his fat by this method under the treatment of his physician, Harvey, has made himself and his cure widely celebrated. Certainly it is to his credit that he described his disease and his treatment in a very charming manner. This made obesity cures popular for the first time, but they were in practice long before Banting's time. A Parisian physician, Dr. Léon, in the year 1839, was the first to translate and annotate the work of the English physician, Wadd. This book treated of corpulence and its cure, and at the same time emphasized the fact that fat people must learn to endure thirst as much as possible. Léon appropriated the ancient teaching of Pliny, to which I shall revert later. His views, however, did not appear to meet with general approval. Much greater publicity was attained by the methods of the English physician, Thomas $K$. Chambers. He prohibited most strictly the use of fats and sugar as well as starch in the form of potatoes; he limited decidedly the eating of bread, and permitted only very small quantities of fluids.

I shall here quote two of Chambers's diet-tables; it is obvious that the first is much more strict than the second. The conclusion may be drawn from these tables that the enforcement of such strict rules met with great opposition on the part of the patient. Perhaps, too, Chambers himself found that too great a limitation of $\mathrm{N}$-free foods is not well borne by patients.

1. Thos. K. Chambers, "Corpulence or Excess of Fat in the Human Body," London, 1850 , p. 126.

Brealcfast: Dry, toasted bread, or, better, snip biscuit; if severe muscular exercise is contemplated, a small piece of lean meat is also permitted.

Midday meal (one o'clock) : Some meat (without fat) ; with this stale bread or crackers, or a small quantity of maccaroni (cooked soft with some French mustard or stewed fruit), or some cracker pudding. Fluids may only be taken one-half hour after a meal.

Later in the day if a sensation of weakness appear, a piece of biscuit and a glass of water, otherwise no solid food is permitted; before going to bed a cup of gruel or a baked apple is allowed. Chambers believes ten ounces (= $300 \mathrm{grams})$ of solid food to be sufficient, but prescribes a small quantity of malt-extract at meal-time more effectually to quiet hunger.

2. Chambers, "Lectures on Corpulence," London, 1864, p. 542.

Breakfast (to be taken early): Two lamb chops carefully freed from fat, broiled or stewed, and ship biscuit. By way of variation a pigeon, game, or fish in corresponding amount. As fluid: Soda water, or, even better, ordinary water, perhaps a cup of tea without milk made in the Russian style with a thick slice of lemon.

Lunch (second breakfast): The same solid foods. Fluid: a glass of claret and Burgundy, half and half, with water.

Dinner (best about six o'clock): Soup and fish are to be avoided. Boiled lamb and beef are to form the principal constituents of diet. With this some biscuit with vegetables rich in chlorophyl and starch, such as cabbage, lettuce, spinach, beans, and celery in small amounts; no potatoes. Sweets, eggs, and beer are to be avoided like poison. Next to water, claret is the best drink. Champagne is the worst.

Evening: A cup of tea in the Russian style, or a glass of ice-water, or, better, a glass of soda water or ordinary water. 


\section{Banting's diet list is as follows:}

Brealffast: 120 to 150 grams of beef or lamb, kidneys, fried fish, ham, or any cold meat (only pork being absolutely prohibited), a large cup of tea without milk or sugar, some rusks, or 30 grams of toast without butter (total of 150 to 180 grams of solids and 240 grams of fiuid).

Besides, Banting first took a swallow of "Balsam of Life"-probably a kind of bitter-tonic.

Midday meal: 150 to 180 grams of fish (except salmon), or meat (no pork), or any kind of poultry or game, all sorts of vegetables (except potatoes), 30 grams of toasted bread or stewed fruit. Two to three glasses of red wine, sherry or Medoc (champagne, Port wine and beer are prohibited); total of 240 grams each of fluids and solids.

Afternoon: A cup of tea (without milk or sugar), 60 to 90 grams of fruit, one or two large biscuits; total of 60 grams of solid and 240 grams of fluid.

Supper: 90 to 220 grams of meat or fish (same varieties as at the midday meal), and one or two glasses of red wine; total 90 to 120 grams of solids and 180 grams of fluid. As a drink just before going to bed some grog (consisting of red wine or rum without sugar) or one or two glasses of red wine.

In this compilation it appears that Banting (who in respect to quantities did not exactly conform to this scheme) was allowed by his physician about 600 to 650 grams of solids and 500 to 1,000 grams of fluid per day. In Banting's letter describing his cure I cannot find the statement that he allowed an unlimited supply of water to persons predisposed to obesity-as Immermann and Cantani report.

The following rules of Robin may also be arranged among the antifat cures. Albert Robin (Revue de thérap. méd.-chir., 189\%, No. 24, quoted from the Correspondenzblatt für Schweizer Aerzte, 1898, page 96, No. 3) permits five meals daily.

1. Eight o'clock in the morning: A soft boiled egg, 20 grams of fish or lean meat taken cold without any addition, 10 grams of bread, a cup of weak tea as hot as possible and without sugar.

2. T'en o'clock: Two soft boiled eggs, 5 grams of bread, 160 c.c. of wine with water, or tea without sugar.

3. Twelve o'clock (midday meal) : Cold meat according to choice but without bread; as a substitute for bread, itttuce or water-cress with a little salt; if absolutely necessary at most 30 grams of bread. Exclusively green vegetables, particularly boiled lettuce ( 100 to 150 grams). The same quantity of raw fruit for dessert; as fluid one or two glasses 'of red wine with water.

A quarter of an hour after the meal a cup of weak tea without sugar.

4. Four o'clock P.M.: A cup of weak tea without sugar.

5. Evening, seven o'clock (supper): Same as in the morning at eight o'clock with the addition of hot meat with or without fish, at most 100 grams.

After every meal a walk lasting for one-half to three-quarters of an hour; besides, general hygienic measures, hydrotherapy with friction, steam baths and general massage are advised. Seven hours of sleep for adults, eight hours for children. No sleep during the day. Regarding Robin's advice as to the amount of fluid, mention will be made later.

Robin's diet regulations require much greater self-denial on the part of the obese than those of the English authors previously mentioned. I think it quite likely that Robin reckoned first of all with the manner of life and the dietetic peculiarities of his French compatriots. Cantani is more strict with 
his obese patients. He absolutely prohibits not only all fat, but also all foods made of flour, and also all sugar-containing materials.

Cantani probably realized that the dietary formulas which he published could scarcely be followed by the obese, or only endured for a brief while. In some of his patients, partly on account of the unconquerable aversion consequent upon consuming such great amounts of meat, partly on account of the incapacity of the stomach to digest so much animal food, and partly because of the great muscular debility which appeared after following his dietary rules, Cantani himself combined his rules with Harvey-Banting formulas in which a certain quantity of carbohydrates and fat were permitted. However, even the so-called Banting cure is by no means harmless. The large quantity of albumin required by it on the one hand, and the too great limitation of $\mathrm{N}$-free foods (fat and carbohydrates) on the other hand, are very badly tolerated by the patient, and, as professional experience has shown, not infrequently are productive of serious harm. We know, for example, to mention but one point, that no-fat cures of this kind may produce severe organic disease of the kidneys. The danger of such cures would be yet greater if the "lean meat" did not contain a certain proportion of fat; even lean beef contains about 2 per cent. of fat. Nevertheless, the amount of carbohydrates allowed by these tables is still insufficient. Cheese, of course, cannot be taken in a no-fat cure-it is expressly forbidden by Cantani-as there is no kind of cheese that is free from fat.

By this no-fat method of treatment, quite insupportable conditions are produced which even a very strong-willed person can at most endure for only a brief while. Yet these methods were those most commonly employed up to the beginning of the eighth decade of the nineteenth century. Since then, however, the "no-fat" method has more and more fallen into disuse. Of course, it must be admitted that the treatment by the withdrawal of fat is effectual and even to a certain degree rational, i. e., by such a system of nutrition a loss of fat may be brought about without damage to the muscle; but great care must be exercised. In the most favorable cases the method can be pursued only for a period of a few weeks, or at most a few months, provided all goes well. Further, this method can only be risked in obese patients who are still in good muscular condition; for only very strong constitutions can endure such a diet for any length of time. On account of this limited application, the "no-fat" cures do not fulfil the requirements which a truly rational cure of obesity calls for. In such a method, for instance, it is desirable that the patient should, without danger to his health and without too great privation, be able to continue the diet permanently after the result has been obtained, though perhaps with slight modifications. Such modifications have, however, not been proposed by any of the exponents of these methods if we except the advice of Cantani that, in case his dietary regulations prove impracticable, the Banting cure, i. e., the régime of Thomas K. Chambers, is to be substituted. I do not believe that such modifications of antifat cures are possible without violating and completely overthrowing their principle. In such a modification the amount of albumin which is to be consumed must be lessened, and either fat or carbohydrates substituted in a corresponding 
amount. Of course, under such circumstances, the diet loses its significance, which, after all, consists in the large amount of albumin which the patient is supposed to consume.

The shortcomings of the "no-fat" cures, especially the fact that at best they admit of but temporary employment, caused me to propose in the year 1882 a method for the treatment of obesity which is free from the objectionable features of those previously mentioned, and which, without losing its distinguishing characteristics, may be variously modified from time to time according to whatever conditions may arise. My method, which is by no means a modification of the Banting cure, permits a manner of life which differs but little from that of other plain and sensible persons. My plan can be continued indefinitely by the person in question without the exercise of too great self-denial. By this means alone is it possible to retain permanently what has once been achieved. The treatment of obesity is a rational one only when we endeavor to bring about a lasting cure, and not only a rapid transitory result. We can never succeed permanently with any method of treatment if the patient follows it only for a certain length of time and then returns to his former mode of life; that which has shown itself as curative must form a permanent and integral constituent of his future manner of life.

The conceptions which led me to propose for obese persons a diet which should be curative, and, in its main principles, could be maintained during life were chiefly the following: It is sufficiently proven by experience that even in fat persons the ingestion of a measured quantity of fat under certain circumstances fails to produce any accumulation of fat, and that the person in question may even rid himself of his superfluous fat provided that the carbohydrates are properly limited and that his manner of living is otherwise normal and in accordance with the fundamental laws of the modern physiology of nutrition. I have, therefore, abjured the fat-depletion cures and assigned to fat the place which it should occupy in the diet of the obese. The prohibition of fat is entirely opposed to the physiological laws of normal nutrition. Fat is a necessary food. No less prominent a physiologist than Donders refers to this as follows: "Too little fat undermines the organism, and lays a foundation for faulty nutrition, a poor admixture of the nutritive juices and of the tissues." That this law is not operative for the obese can neither be proven by scientific reasoning nor inferred from professional experience. Even Hippocrates advised for the obese the ingestion of foods prepared with fat, as, in this manner, the appetite was most rapidly satisfied. This observation of Hippocrates is perfectly correct.

It is, therefore, an ancient law of experience that by the addition of a certain amount of fat to the food the sensation of hunger is more lastingly removed than by food very deficient in fat, or by an equivalent amount of carbohydrates or of albumin. Fatty foods act in this way not because they spoil the appetite nor (as has been maintained by some) becausc. they produce dyspeptic symptoms. On the contrary we see that dyspeptic symptoms appear frequently after the ingestion of too large quantities of meat. That one may eat enormously of meat without a sensation of satiety is well known. Quite 
similar are the experiences in regard to carbohydrates. The question why fat satiates us most rapidly may be very easily explained by the observations of M. Matthes and E. Marquardsen (Verhandl. d. Congr. für innere ICed., 1898, XVI, p. 358 u. flg.). These observers have shown that fats remain in the stomach for a long time, and that large amounts of fat necessitate a very large expenditure of the regulatory powers of the stomach, and produce a decided encumbrance of the same. As in my diet regulations too great amounts of fat are not permitted the obese, the deleterious effects arising therefrom are not to be feared. Further, the inclusion of fat is by no means a cure by means of nausea, as has been stated by one author. It is in fact not a cure but a mode of living whereby fat simply assumes its proper function as a food.

To attain this purpose, no larger amount of fat is necessary than is permitted to non-obese persons, even to those who are subjected to the hardest labor. In the nutrition of the obese the limitation in the amount of carbohydrates is the chief point. Of course, this does not mean that an intolerable limitation of vegetable food is necessary. On the contrary, in the nutrition of fat persons a plentiful use is to be made of green vegetables rich in water and poor in carbohydrates, since their pre-eminent qualifications as satiating foods and fat carriers make them especially suitable. Only vegetables rich in starch, such as turnips, potatocs, ete., are to be avoided. By this method fat is administered to the patient in such wise as to cause no repugnance, and the carbohydrates may be limited by giving a bread richer in albumin than is usually taken-a point to which I called attention a number of years ago. Our ordinary bread contains only about 6 to 7 per cent. of albumin, and its nutritive product is almost exclusively starch. The starch may be decidedly reduced if the albumin contents of the bread are increased by the addition of vegetable albumin. In this manner it is easy to prepare, even at home, a very palatable bread which will contain from 20 to 30 per cent. of albumin. I have repeatedly published the formulas necessary for this purpose, last in the article by Schwalbe and myself on Diabetes Mellitus in the "Handbuch der praktischen Medicin," Stuttgart, 1901. For the preparation of such a bread we require pure vegetable albumin, which may be obtained from a number of plants. Vegetable albumin is easily digested, readily utilized by the human organism, and is not only very much cheaper than meat but, for many other reasons, is even preferable. Up to very recent times but two such pure vegetable albumins were at our disposal, one of which was discovered by Dr. Johannes Hundhausen of Hamm i. W., and manufactured from gluten, a patent albumin which the inventor calls "aleuronat." In addition to small quantities of salts ( 0.78 per cent.) and cellulose ( 0.45 per cent.) aleuronat contains at least 80 per cent. of albumin, about 7 per cent. of carbohydrates, and about 9 per cent. of water. The second vegetable albumin, placed at our disposal later than aleuronat, is the rice albumin "ergon" which is manufactured by Dr. Hensel \& Co., in the chemical laboratory at Lesum near Bremen.

This is not the place at which to enumerate the varied uses we may make of vegetable albumin in the nutrition of patients with diabetes mellitus. In 
obesity as well as in gout we utilize vegetable albumin almost exclusively. for the preparation of a bread rich in albumin, by which we may satisfy a greater albumin requirement in the individual than is possible by means of our ordinary bread. In this manner larger amounts of bread may be permitted than would otherwise be allowable, which is very acceptable to the patient. By the aid of these vegetable albumins we may produce a bread which is entirely unobjectionable; frequently, however, such a bread is distasteful. This is due to the fact that the necessary care has not been observed in its preparation. It therefore appears to me worth while to give some directions and a few recipes for the preparation of these breads rich in albumin, such breads as are useful in the dietetic treatment of obesity and gout. To ensure a faultless bread, absolutely necessary prerequisites are: 1 . Scrupulous cleanliness of all utensils and purity of all ingredients; 2. A pure, starch-free, compressed yeast (common, so-called baker's yeast may be adulterated with starch flour up to 50 per cent.) with good fermenting power-for the preparation of good aleuronat or ergon bread requires a larger quantity of yeast than other breads; 3. An exact following of the recipes in regard to the amount of fluid.

\section{RECIPE FOR THE PREPARATION OF WHEAT BREAD WHICH CONTAINS ABOUT 27.5 PER CENT. OF ALBUMIN}

\section{(Before the addition of any liquids.)}

600 grams of wheat flour,

150 grams of aleuronat or ergon,

10 grams of yeast,

$\frac{1}{2}$ liter of milk,

$5 \frac{1}{2}$ grams of common salt,

Proportion of aleuronat or ergon to wheat

About one gram of sugar (i. e., as much as

the yeast requires for fermentation).

The flour and aleuronat are to be well mixed in a pan previously warmed to about $30^{\circ} \mathrm{C}$. A small portion of the milk is warmed to about the same temperature, and after one gram of sugar has been dissolved in it the mixture is poured upon the crumbled yeast, and the whole set in a warm place at not over $30^{\circ} \mathrm{C}$., and allowed to stand until it ferments. This mixture is then poured into the center of the flour and aleuronat (or ergon) mixture in such a way that the outer borders of the flour are not moistened. Next the remainder of the warm $\left(30^{\circ} \mathrm{C}\right.$. $)$ milk and the salt are stirred in with a spoon, still keeping the outer portions of the dough dry. The vessel must then stand in a warm place $\left(30^{\circ} \mathrm{C}\right.$.) covered with it cloth until the dough rises; then it is to be mixed, first with a spoon and then lightly with the hands (firm kneading is to be avoided), with the dry flour still remaining at the edges until a soft dough is formed from the entire mass. It is then made into small loaves which are allowed to rise in a baking pan slightly warmed $\left(30^{\circ} \mathrm{C}\right.$.), greased with butter. After they have risen, the loaves are glossed over with melted butter and baked for one-half or three-quarters of an hour. Brushing the surface of the loaves with cold water before they are taken from the oven gives them greater luster. These loaves may be eaten on the day of baking, or even a day or two later, but are better if placed in the oven and re-baked. Bread containing fat, and very palatable, may be made by adding butter to the mixture of warm milk and table salt in a proportion of about 50 grams of butter to about one pound of dough. This bread also is to be smeared with butter, and baked in a pan. 


\section{RECIPE FOR TEE PREPARATION OF RYE BREAD WHICH CONTAINS ABOUT 27.5 PER CENT. OF ALBUMIN}

(Before the addition of liquids.)

1,200 grams of rye flour, 300 grams of aleuronat, 30 grams of sour dough, 1 About 12 grams of table salt, Proportion of aleuronat to rye flour as $1: 4$.

About 1.5 liters of lukewarm water, and lastly, some caraway seed (if desired).

On the evening before baking, flour and aleuronat (or ergon) having been previnusly warmed are thoroughly mixed in a vessel warmed to $30^{\circ} \mathrm{C}$. To this mixed flour add the sour dough which has been previously mixed with some of the lukewarm water, and is now poured into a hollow in the center of the mass of flour, the outer borders of the flour remaining dry and untouched. Now the dry aleuronat (or ergon) mixture at the edges is mixed with the watery mixture of sour dough in the center while we gradually add the rest of the water, stirring from the center to the periphery. After this is done, the entire mass in the vessel is sprinkled with the aleuronat (ergon) flour mixture, and the dough prepared in this manner is allowed to stand over night (about twelve hours), well covered and kept warm at about $30^{\circ} \mathrm{C}$. In the morning salt and caraway seed are added, and the mass is kneaded at once. If the dough is too stiff it may be made more spongy by the addition of lukewarm water, and if it is too sticky flour and aleuronat (or ergon), 1 to 1, may be kneaded in. Then the dough is placed in a shallow iron pan, covered with a linen cloth, and set in a warm place at about $30^{\circ} \mathrm{C}$. for 2 to $2 \frac{1}{2}$ hours, to rise. When light the loaves are brushed with melted butter, and baked for about 2 to $2 \frac{1}{2}$ hours. To test whether the bread is done, a splinter of wood may be introduced into a loaf, and if, on withdrawing it, no moist dough adheres to it the bread is thoroughly baked. It is advisable during the last part of the baking to turn the loaves in the pan. This bread should not be cut until the next day. It should be kept in a cool place, and in the summer in the refrigerator.

of course, bread containing less aleuronat or ergon may be made either with wheat flour, or with rye flour. Thus, for example, we can make bread which contains one and a half times as much or double the amount of albumin (in dry substance) contained in ordinary bread, which, as is well known, contains about ten per cent. of albumin (dry). To make bread containing about fifteen per cent. of albumin in the dried condition, add one part of aleuronat or ergon to about twenty parts of flour; to make bread containing about twenty per cent. of albumin (dry), add about one part of aleuronat or ergon to eight parts of flour. The smaller the amount of aleuronat or ergon the easier it is to make aleuronat or ergon bread. It is therefore advisable for an amateur in bread baking to begin with one of the weaker aleuronat or ergon breads according to the recipes just given. The breads containing larger quantities of albumin can then be made with greater certainty and ease.

Recently the number of vegetable-albumin preparations has greatly increased. Among these may be mentioned the product of Nicmöller in Gütersloh i. W. from grain, and called "roborat." For baking purposes this is exceedingly useful. Further experiences will have to be gathered in regard to these preparations, whose number will probably increase still further.

A formidable rival to these vegetable-albumin preparations is fresh casein. The investigations undertaken in my clinic by E. Schreiber (Centralbl. $f$. Stoffwechsel- und Verdaungskrankheiten, 1901, Nr. 5) have shown that there

( 1 Rye flour dough from a previous baking, which has been set aside for several days, and has turned sour. [J. L. S.]) 
is no cheaper, and at the same time more palatable, albumin for these purposes. Bread produced from fresh casein (casein bread) may of course be also utilized in the nutrition of gouty patients, for casein does not produce an increase in uric acid excretion. The bread made with this casein can be readily baked by any baker. I have often used it with advantage in the nutrition of the obese, of gouty patients, and particularly also in diabetes mellitus.

It is obvious that within certain limits leguminous vegetables may also be utilized in the nutrition of the obese. Besides a plentiful amount of albumin these vegetables contain much carbohydrate, but with a corresponding limitation of the other carbohydrates of the diet, they may be used with advantage. The discreet use of fruit not too sweet, and of stewed fruit without the addition of sugar, is permitted. Sugar, puddings, and starchy foods of other kinds, as well as potatoes and all dishes made from them, are absolutely prohibited for the obese. A small quantity of wine deficient in sugar and containing small amounts of alcohol may be given, provided the patient cannot get along without it. Beer I prohibit. This is generally one of the most severe trials which the obese patient is called upon to undergo. Brillat-Savarin is, however, quite correct when he says to the obese: "Avoid beer as you would the pest." But with regard to tobacco, provided there is no contraindication on account of the heart, I permit a moderate indulgence-two or three mild cigars a day.

Finally, regarding the proper quantity of albumin for corpulent individuals, of course all superfluous proteid must be reduced according to the individual requirements, as in the antifat methods of treatment. Certainly custom permits entirely too much latitude in this direction, and often the patients take far too much albumin. We have seen that by including vegetable albumin in the diet, a considerable amount of the albumin needed by the body is provided. On the other hand, I must emphasize that, in such a diet, the patients have all the variety necessary for those who live under normal conditions, and who do not perform excessive bodily labor. With this diet, wherein the fatty foods fill their proper place, we find that hunger is more easily satisfied than with an antifat diet, and that the thirst which occurs in all antifat cures is also avoided. If thirst were not a constant feature in "antifat cures" it would be unnecessary to make such statements as that the patients "must learn to endure thirst." The effect of fat in diminishing thirst has been observed and dwelt upon by so many competent authorities that it is unnecessary for me to enter more minutely into this subject. If the method of treatment proposed by me, or, let us say, the "proper manner of life for the obese," is maintained (controlled, of course, by an expert), eventually the superfluous fat, and only this, will gradually disappear. A too rapid loss of weight is injurious. Loss of fat should always occur slowly, and must be brought about with great care. The method must never be allowed to fall into a rigid routine, but must be arranged according to the individual conditions of each case; and it must also be so arranged that the patient may pursue his ordinary occupation without detriment.

A few examples will serve to illustrate briefly the manner of life advised 
by me. No hard and fast rules can be given because, as I have several times stated, the method should never become a routine affair. For example, in the first case (see below) if instead of the ordinary rye and wheat bread, a bread richer in albumin is chosen, the ration of bread must naturally be increased and the daily quantity of meat correspondingly diminished, etc. Three meals a day I believe to be the best rule. Yet even here some modification might become necessary. In spite of many slight, but important, variations the general principles remain the same.

OBservation I.-A man, forty-four years of age, moderately muscular, suffered from. increasing corpulence though he was otherwise healthy and had formerly been thin. He led an active life, but limited his exercise to that in the house. He was of very temperate habits, especially in the use of alcohol. The obesity had evidently developed as the result of a very profuse ingestion of albuminous food, with the careful avoidance of fat, but with a fondness for carbohydrates, particularly for sweets. The diet which I had the patient follow was about as follows:

1. Breakfast (in summer at six or half-past six o'clock) : A large cup-about 250 c.c. - of strong tea without milk or sugar; 50 grams of bread (wheat or rye bread), toasted, with 20 or 30 grams of butter.

2. Midday meal (between two and half-past two o'clock): Meat broth, frequently with bone mairrow in a solid form (the bone should be cooked for an hour to an hour and a half so that the marrow does not melt) or with an egg, or other suitable addition; 120 to 180 grams of boiled or broiled meat, preferably fat meat if it agrees with him; vegetables as mentioned above, preferably peas, lentils or beans. For dessert, some fresh fruit if obtainable, particularly strawberries, cherries, and, best of all, apples. A salad, according to the season; also apple-sauce freshly cooked or, if unobtainable, stewed dried or preserved fruit, always, however, without sugar.

As fluid :- Two to three glasses of a light Rhine wine.

Soon after this meal a large cup (about 250 c.c.) of strong tea without sugar.

3. Supper (between seven and a half and eight o'clock): In winter almost invariably, in summer occasionally, a cup of tea as in the morning and after the midday meal; an egg or roast meat, preferably fat, or some ham with fat, or Cervelat sausage, or fish, smoked or fresh; a total of 75 to 80 grams of meat, about 30 grams of wheat bread, and 15 to 20 grams of fat, this depending on the amount of fat in the meat, with more or less butter. Occasionally a small quantity of cheese and some fresh fruit or stewed dried fruit.

This diet was combined with moderate exercise in the open air; on Sundays he took walks usually lasting several hours, and these were continued during the summer holidays when a residence of several weeks at the seashore or in the Alps interrupted his ordinary work. The effect on the patient's bodily health and mental activity was very good. The diet agreed excellently. A second breakfast was never taken, but he was always hungry for the midday meal. There was a remarkable decrease in the previously extreme thirst. In the evening the craving for food was not very great, and was easily satisfied. In the course of six months he lost 22 pounds, gradually but steadily, as is desirable in all such cases, and in about nine months his waist measurement decreased $16 \mathrm{~cm}$. This diet, in the main, has been continued. As time passed the ingestion of fluid was still further decreased. Instead of a large cup of tea three times daily, he now took a small cup only in the morning, and in the evening coffee, which formerly had not been well borne; the daily use of wine had been stopped for years. Only at the beginning of the regulation of the diet was it necessary to weigh the allowance of the individual foods. He soon learned to cstimate them very readily. After the use of aleuronat became known, it was employed by this patient very largely.

OBSERVATION II.-While in the first observation we were dealing with a case of uncomplicated obesity, this one concerns a lady who suffered from obesity complicated by gout. The patient is thirty-seven years of age, the wife of an officer from $K$. The patient had no special family predisposition to obesity or gout. In her youth she was 
painfully thin, and began to menstruate at eleven years of age. She was apparently anemic, but otherwise strong and without any symptoms of importance. She married at nineteen, aborted after eight weeks, and within a few months became stout and remained so for almost nine months. During this time her husband died. As a widow she again became as thin as when she was a girl and remained so during her widowhood (seven years), during which time she was occasionally very anemic. After her second marriage, the patient soon became pregnant. After the birth of a girl (autumn, 1890) the patient became very stout, again lost some flesh in the next few years, but in 1893 , after a serious miscarriage, she again became very stout and under the influence of an injudicious mode of life she remained so up to March, 1898, when she consulted me for the first time. After this miscarriage an exudate formed in the pelvis, and she has since then suffered from obstinate constipation; it was on this account that she desired treatment. Besides this, the patient suffered from gout which had appeared for the first time in 1886, and was localized in the fingers, being diagnosticated by Professor Lücke in Strassburg. On this account Professor Liticke ordered a course of Vichy water at the Springs. At this time a dificulty in hearing appeared for the first time and gradually increased. In January, 1898, an acute attack of gout occurred in the great toe of the right foot, and lasted eight days. For some years cramps had occurred in the calves and feet, mostly at night when the patient went to bed, but also during the night, and they recurred almost every night, especially in the right leg. Early in Mareh, 1898, severe pains were suddenly felt in the great toe of the right foot. The toe swelled somewhat and became red. In about ten days, during which period the patient was unable to stand upon the foot for several days, the attack subsided. From the 14th to the 25th of April, 1898, the patient suffered from an attack of influenza, during the course of which the temperature rose to $102.2^{\circ} \mathrm{F}$., and she was much debilitated. About the 9 th of May of the same year, suddenly and during her menstrual period, an attack of gout supervened. This was localized in the little finger of the left hand, and ran a typical course in a very short time. Upon the 12th of the same month, the redness of the affected area had disappeared. At the beginning of June the patient complained of decided gouty pains in the feet. The first metatarsophalangeal joints of both feet were very sensitive to pressure. No typical attack developed, however. When the patient stopped treatment upon the $23 \mathrm{~d}$ of June, she was able to walk quite a distance without difficulty.

In this case the treatment was first directed against the obesity, gout, and also particularly the stubborn constipation. For evacuation of the bowels, large enemata of oil were used with good effect. A diet was instituted according to the principles laid down by me, and, in so far as gout permitted, walking was ordered for active bodily exercise, which was supplemented by massage of the body. As a drug, the prolonged use of 0.5 ( $7 \frac{1}{2}$ grains) urotropin in a quarter of a liter of alkaline water to be taken twice daily was prescribed. Her weight fell during this time from 199 pounds to 187 pounds. This success was evidently not due to the diet alone, which under the circumstances could not be strictly carried out. During this time is severe influenza occurred from which the patient slowly recovered, also quite severe gout which of course also decreased the body-weight. When I saw the patient again in October of the same year, tho constipation had been relieved by the continuance of the enemata, the gouty diffi. culties had not recurred, but there was a decided psychical depression which was increased by various external conditions. The weight had again risen to 194.5 pounds, and the patient had resumed her former manner of living, which was conducive to the accumulation of fat. The weight, when she decided to renew her treatment, had risen to 203 pounds. Treatment began at my private hospital upon the 28th of August, 1899, and ended upon the 5 th of December, 1899. This time the treatment was not hindered by disturbing intermediate maladies such as the attack of influenza and the two attacks of gout during the first period of treatment. The function of the intestine had been greatly improved. Of course, it was decidedly retarded by a very pendulous abdomen. The gouty pains had by no means completely disappeared. The use of urotropin was continued, besides the diet which was regulated according to my principles. The weight of the patient was as follows: Weight upon the 28th of August, 203.86 
pounds; upon the $2 d$ of September as well as upon the 9th of September, 203 pounds; upon the 15th of September as well as upon the 23d of September, 200 pounds; upon the 30th of September, 198 pounds; upon the 7 th of October, 196 pounds (health decidedly improved, can walk well); upon the 14th of October, 193 pounds; upon the 21 st of October, 193 pounds; upon the 28th of October, $19 \mathrm{I}$ pounds; November 3d, 190 pounds; upon November 11 th, 189 pounds; upon November 18 th, 187 pounds; upon November 25 th, 186 pounds; upon the 4 th of December, 185.51 pounds. The total decrease in weight amounted to 18.35 pounds. The patient lost weight slowly and steadily, about one pound a week. At the same time the general condition and bodily activity had greatly improved. The psychical depression had disappeared except for a few slight relapses. The gouty difficulties were present to only a slight extent, if at all. Later reports regarding the health of this patient have been very favorable.

A more or less slow result which in the end may perhaps prove fairly satisfactory occurs in those cases in which the patients do not exactly follow the regulations, but make changes of their own accord. I shall mention only one case of this kind. A high government official (lawyer), forty-five years old, weighing 221 pounds; circumference of the abdomen at the height of the navel $123 \mathrm{~cm}$. He believed that he could not get along without beer and a larger amount of bread-and so increased this to 160 grams of rye breadand took one liter of beer daily. In the main he followed directions, and to compensate for his deviation he walked every afternoon for an hour and a half. In the first six months he lost 31 pounds, in the second six months $6 \frac{1}{2}$ pounds, in the third six months 2.2 pounds, a total of $39 \frac{1}{2}$ pounds. The circumference of the abdomen diminished $34 \mathrm{~cm}$. Entirely satisfied with these results, the patient continued to live according to the principles under which he had gotten rid of his numerous difficulties.

Any one desirous of success with this method must have a certain knowledge of the modern physiology of nutrition, and must recognize the necessity of changes to meet the pathologic conditions peculiar to each individual and his general circumstances.

These are essentially the rules according to which an obese person must arrange his diet if he desires to-rid himself of superfluous fat and prevent its reaccumulation. To carry out this régime does not give rise to difficulties; the privations which are caused by it are as nothing in comparison with those which are caused by obesity and with the dangers which may in this way be aroided. It is presupposed that while carrying out these dietetic rules a suitable amount of exercise must be taken. One of the advantages of my method of treatment is that the patients may follow their usual occupations, and that thus the ordinary movements of the body may be made serviceable in the treatment. Especially exhausting muscular movements are inadvisable for persons who are both fat and anemic, since they give rise to certain difficulties, are almost always impossible to carry out, and are by no means so successful in reducing fat as they are often claimed to be. Banting in his letters upon corpulence relates very entertainingly how an excellent physician well known to him had advised, to prevent the increase of his corpulence, that before beginning his usual daily work he should take extra exercise. As the physician considered rowing very good for this purpose, Banting rowed a few hours early in the morning. He relates that by this means he gained muscular 
power-but simultaneously a voracious appetite, and adds ingenuously "As I yielded to this, I constantly increased in weight until my dear old friend (the previously mentioned physician) advised me to give up this kind of exercise." What I have previously advised in regard to exercise for the obese patient (Observation I) may in general be looked upon as sufficient.

Quite a number of other diet rules have been proposed by various authors according to the methods advised by me in the nutrition of the obese. Of course, I cannot and will not enumerate all of these, for in the treatment of obesity the most varied diet schemes may be based upon the foundation given. But I shall mention the régime proposed by Hirschfeld, which is elucidated by two illustrations. As is evident from these, Hirschfeld requires a "uniform" limitation of all kinds of food, and maintains that the appetite can be satisfied without any excess of food.

\section{Example I.}

Breakfast: Coffee, without cream or sugar; 1 roll (50 grams).

2d Breakfast : 2 eggs.

Dinner: Bouillon with about 30 grams of rice weighed raw; 250 grams of lean meat, weighed raw, either boiled or broiled with a little fat.

Afternoon: Coffee, without cream or sugar. Supper : 50 grams of cream cheese ; 100 grams of bread; 10 grams of melted fat (goose fat).

\section{Example II.}

Breakfast: As in Example I.

2d Breakfast: Bouillon and 2 eggs.

Dinner: Potato soup, 300 grams of meat, weighed raw.

Supper: 200 grams of lean ham, 100 grams of bread.

There is contained:

In diet No. $1 \ldots \ldots \ldots \ldots \ldots \ldots, \quad \begin{aligned} & \text { Albumin. } \\ & 95 \text { grams }\end{aligned}$

In $\operatorname{diet}$ No. $2 \ldots \ldots$.......... 134
Fat. 43 grams
Carbohydrates. 106 grams $=1,224$ calories

In women it will be possible to get along with even less food.

Incidentally, in speaking of "no-fat" cures, I mentioned that they (in contrast to my method of treatment) necessitate that the patient learn to endure his thirst. The withdrawal of water played a rôle in antifat cures even in antiquity. Pliny the Younger advised those who desired to become thin to refrain from fluids while eating, and even afterward to drink but little. This is not the place in which to follow in detail the history of these thirst cures. It may be only mentioned here that the father of the latest movement in this direction, which originated in Munich and for a time gave rise to much discussion, was the founder of the Naturheilansstalt Brunnthal, near Munich, Dr. J. Steinbacher (died 1868). In his booklet, "Asthma, Fatty Heart, Corpulence, etc.," he has laid special stress on ridding the body of a part of its fluid in the cure of obesity. Oertel, following him, has developed this method in great detail, and it has been named after him the "Oertel Cure." The withdrawal of fluid, upon which the greatest stress is laid, in this method may be attained in various ways. First, by the limitation of the fluid intake. This is hard. Thirst is much more difficult to endure than hunger. Most persons very soon become nervous under it, and will not listen to reason. Sec- 
ondly, much fluid may be withdrawn from the body by inducing sweating. In regard to this I need only mention the sun baths advised by Celsus for the obese. These depletion cures cause a loss of body albumin. Some of the reports of the so-called Oertel cure may be given here. Oertel permits the obese 156 to 170 grams of albumin, 75 to 120 grams of carbohydrates, 25 to 45 grams of fat. The upper limit Oertel allows only for those persons in whom the craving for food is great owing to hard muscular work, for example, mountain-climbing. On account of the importance which Oertel attaches to the ingestion of fluid, he has fixed the amount at 973 to 1,414 grams.

A sample diet list given by Oertel is as follows: Brealfast: fine wheat bread 35 grams, coffee 120 grams, milk 30 grams, 2 soft-boiled eggs 90 grams (100 grams of broiled meat), sugar 5 grams (butter 12 grams). Second brealffast: mild Rhine wine, or bouillon, or water 100 grams, or Port wine 50 grams, solid food 50 grams of cold meat and 20 grams of rye bread. Midday: light Rhine wine 250 grams, broiled beef 150 to 200 grams, salad or vegetables (cabbage) 50 grams, cereal 100 grams (bread 25 grams), fruit 100 grams. Afternoon: coffee, with milk and sugar as in the morning. Evening: mild Rhine wine or water 250 grams, caviar 12 grams (Kiel sprats 12 grams, smoked salmon 18 grams, 2 soft-boiled eggs $=90$ grams), game 150 grams, cheese 15 grams, rye bread 20 grams (fruit 100 grams).

The preceding diet list of Oertel's contains :

$\begin{array}{cccc}\text { Albumin. } & \text { Fats. } & \text { Carbohydrates. } & \text { Water. } \\ 160 \text { grams } & 42.5 \text { grams } & 117.5 \text { grams } & 1,440 \text { grams }\end{array}$

The withdrawal of fluid in the treatment of obesity appears to have found no favor in this latest plan. It is of especial interest to note that this method, originating in Munich (where it was ostentatiously advertised) is now vigorously condemned, as is evident in the criticism of R. v. Hoesslin based upon a very rich experience.

As the result of his observations $\nabla$. Hoesslin arrives at the conclusion that in the so-called Oertel cure we are using in fact a true hunger cure. v. Hoess.lin even believes it questionable whether in antifat treatment the reduction in the amount of fluid plays any part. He does not regard Oertel's method as a commendable one, and holds the same view in regard to the Banting cure; for though he looks upon its effect in depleting fat as good, its action upon the general condition he designates as bad. His method in the treatment of obesity consists of four parts, and depends upon nutrition by a pure fat-albumin diet, modified according to the principles proposed by me. Without here going into detail in regard to the modifications in v. Hoesslin's diet lists, I will simply emphasize the fact that he does full justice to fat in the treatment of obesity, and that he pronounces the results obtained by him through diet alone to be very satisfactory. It is true he lays stress upon the fact that these results do not equal those attained by diet combined with the other three components of his obesity treatment. These consist in the simultaneous stimulation of metabolism by hydrotherapy, the augmentation of oxidation processes by increased muscular exercise, and finally, the administration of thyreoidin tablets.

What of the treatment of obesity with thyreoidin tablets as mentioned by v. Hoesslin? I have subjected this method to an accurate test, and have pub- 
lished the results in the Deutsche medicinische Wochenschrift, 1899, p. 1. When this treatment was introduced it became highly popular, but it proved by no means so harmless as many at first maintained. I shall content myself with the following statement in regard to it. I do not advise thyreoid treatment in obesity because, in the first place, the loss of weight produced by it is quite inconstant, and always ceases at once with the discontinuance of the thyreoid preparation. Further, this method of treatment is not rational because the danger in the loss of body albumin is a considerable one. Finally, it is quite unnecessary because we are in possession of dietetic rules for the treatment of obesity which are as successful as they are devoid of danger. This view in regard to thyreoid gland treatment in obesity secms now likely to be generally accepted.

In regard to the two other curative factors made use of by $\nabla$. Hoesslin, namely, hydrotherapy and augmentation of the processes of oxidation by increased muscular exercise, there can be no objection to their use in conjunction with an appropriate diet. It must be admitted that when these curative agents are wisely regulated by an able and experienced hand, and are individualized, the result is not only hastened, but in many respects heightened. R. v. Hoesslin, as physician-in-chief of the institution in New Wittelsbach near Munich, certainly has the advantage of splendid facilities and has acquired great experience. But with the remedial agents just discussed it is as true as it is of the dietetic measures that a permanent result may only be expected when the persons in question continue their muscular exercise.

What is the character of the muscular movements which are of especial importance in the treatment of obesity? Those especially should be practised which will influence most favorably, and to the greatest extent, the total metabolism, i. e., muscular movements in which most or all of the muscles are brought into action, as in the so-called free exercises of German turners and in exercises with gymnasium apparatus. The former, because they call for only a comparatively slight use of power, are particularly suitable for persons with feeble muscles.

Muscular exercises of this kind practised in the open air, passing gradually and regularly from the light to the severe, are preferable as a rule to the exercise involved in games or sport. The latter may only be prescribed for the obese when they can be practised in moderation, and when they call for the most varied action of all the muscles. In permitting the practice of any sport we must make sure of course that there is a sound, competent heart and normal kidneys. This is especially true of bicycling, which is now in such vogue. But for obese persons of active habits, the best form of exercise is manual work performed in the field or the garden. This is, after all, the most natural. Besides this, walking, especially in a region of diversified scenery, mountain-climbing, and all sorts of mountain sports, provided always the heart is normal, are factors not to be underrated in the treatment of obesity. They are much preferable to spas and water cures, but need, nevertheless, to be carefully controlled. A weak heart which, even when the patient is at rest, does not function properly, cannot be expected to endure the strain of muscular exercise and exertions of this kind. When the heart is weak, passive 
muscular exercise, and, best of all massage, are especially valuable. By light clothing, the consumption of heat, and with it the metabolism of the obese, may be decidedly increased. By clothing of this kind muscular exercise is facilitated. In winter it is advisable to wear woolen underclothing. Unfortunately when it is washed it generally loses its porosity, and with this its greatest advantage, and thus becomes merely expensive. In summer, on account of their great tendency to excessive sweat formation, the obese mostly prefer porous cotton underclothing.

A sensible mode of life in which moderation in eating and drinking is maintained, and in which sufficient muscular exercise is secured, permits us to predict with some degree of certainty that no superfluous body fat will accumulate, and that, where already present, it will disappear. If an individual predisposed to obesity will, after ridding himself of his superfluous fat in this manner, adhere to the prescribed mode of life, he may expect that no renewal of the accumulation of fat will take place in later life.

I have endeavored in this article to suggest the methods known to us to-day of which we may avail ourselves in assisting the obese to regain their health without discomfort. Nevertheless, in the prognosis the physician is not to give way to illusions. In the course of years I have collected a considerable number of experiences which show that there are many obese persons who prove to be very satisfactory patients; who, regulating their mode of life in accordance with the condition of their bodies, improve, and may be entirely cured within a short space of time. It is not well, however, to be too sanguine. Often all hope is shattered by the epicurism and foolishness of these patients. Many of them, it is true, will submit for a certain time to the deprivations and obligations to which their condition subjects them, but will then return to the habits which have produced the obesity. If they belong to the minority who are in good circumstances, they go in the summer time to a "cure" which they expect in a few weeks to make them perfectly well. Instead of pursuing the course of life which experience has shown to be sensible, and by which they may avoid the downward path to which their condition inevitably leads, they quietly pursue their sluggish way. For eleven months of the year they commit all sorts of dietetic sins, and then in the twelfth month submit to a mineral spring treatment and bath cure which is to cleanse the body and wash away all its burdens. I adhere to-day to the opinion which I have always expressed that every cure of this kind, as well as every drug treatment, particularly by means of purgatives of any kind, is to be rejected in obesity. This is particularly true of the treatment which Kisch has designated as his method for the cure of the plethoric form of obesity (Kisch, "The Cure of Obesity," Berlin, 1891, p. 113, et seq.), and I am strengthened in my adverse opinion by the example which Kisch quotes to show the efficacy of his method, namely, a loss of 11 kilograms and 15 grams of weight within twentyeight days in an uncomplicated case of obesity in a man aged forty-two years and weighing 146 kilograms and 75 grams. This procedure is by no means to be recommended. The cure, as practised by Kisch, consisted (apart from the dietetic treatment which in the main corresponded to the no-fat treatment) in the use of Marienbad mineral waters internally, for bathing, in 
steam baths, exercise amounting to 25,000 steps daily, and hydrotherapeutic measures. It is true that Kisch has proven it to be possible by this method to become lean. But, a priori, can one doubt that in such a treatment (the patient lost on the first day one kilogram and 25 grams) muscle as well as fat is lost? The loss in weight by this treatment is not always so great as that which Kisch attained at Marienbad in four weeks. It varies between 3 and 13.2 per cent. of the patient's weight; on the average it amounts to 6.7 per cent., which, in my opinion, is more than is desirable in the rational treatment of obesity. It should therefore be avoided.

In conclusion, I must mention the dangers which confront all who use acids, particularly vinegar, a practice which, unfortunately, we find very common in young girls who, from vanity and a desire to remain slender, risk their health and even their lives. 
The patient, a woman aged fifty-five, has been known to me for six years; the symptoms of the disease developed slowly and insidiously, and for a long time they were so little characteristic that it was hardly possible to differentiate the condition from the ordinary cachexia of old age. But the behavior and appearance of the patient were, even at that time, so peculiar that I had a photograph of her taken which is here reproduced (see Fig. 1). Later, however, the typical symptoms appeared (see Fig. 2). They improved and nearly disappeared under specific treatment (thyreoid extract), but returned after some time, the patient having discontinued her treatment. Fig. 3 shows the patient in this stage of her disease. ${ }^{1}$

\section{ETIOLOGY}

Regarding etiology, as little could be determined in my case as in other cases of myxedema. A tendency to nervous diseases and depressing influences of a psychical nature are said to be conducive to the development of the disease. Whether alcohol, syphilis, and tuberculosis, those exterminators of the human race, play their part in this disease is very questionable, although several authorities (Pel, Greenfeld, and Byrom Bramwell) have called attention to the occurrence of tuberculosis in the families of myxedematous patients, or in the patient himself. Several cases in the same family have also been observed.

The sex of my patient confirms the general experience that women are attacked in the majority of instances, and Sir William Gull entitled his first communication, "On a Cretinoid State Supervening in Adult Life in Women." Full seven years after the first publication regarding myxedema, a male suffering from this affection was observed by Savage (1880). In 1894, in a compilation of $12 \%$ cases of myxedema, Heinsheimer found only 10 men, i.e., \%.8 per cent.; and this proportion would about correspond to the ratio if we tabulated all the cases that have been published up to the present time.

Typical myxedema is a disease of adults, occurring most frequently between the thirty-fifth and fiftieth years of life. It is, however, by no means limited to these ages, but may occur earlier, as the so-called infantile myxedema, or even later (which is quite rare).

\section{SYMPTOMS}

The symptoms of myxedema consist of:

1. Changes in the external integuments (skin, hair, nails), and the absence, or, more strictly speaking, the degencration of the thyreoid gland.

2. Disturbances of the cerebral and nervous functions.

3. Disturbances of nutrition (of metabolism) and of circulation.

1 The literature up to 1896 is almost complete in O.A. Ewald's "The Diseases of the Thyreoid Gland, Myxedema and Cretinism," in Nothnagel's "Special Pathology and Therapy," xxii, Part I; for later articles see Ewald's article "Organotherapy," in Mendel's Jahresbericht für Neurologie und Psychiatrie. 


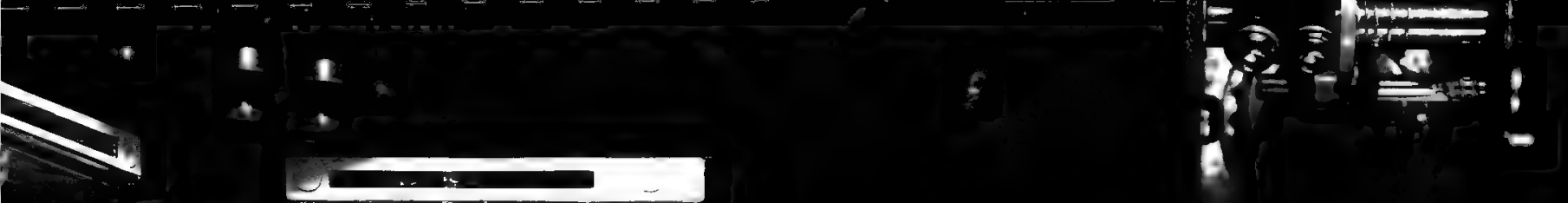

10ron to me for sis pow

nisidionsisy, and foon?

aradly possithe to idte

old age. But the blest

ame, in peculiar thall:

lluced (see Figg. 1). Is:

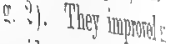

Teoid estract), but netre

ber treathent. Fig 3 :

mined in my mese a : I."

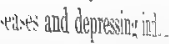

to the derelogpenat oitt.

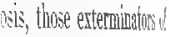

reary questionalle, athone?

Breantelli) have allit:

willes of nysgelenatous set.

the same farmily lare evi:

exal esperience that ron:

Prillian Gidle entith li:

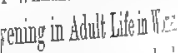

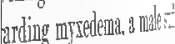

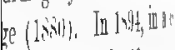

imper found only 10 㽞.

it correspond to the ridito

I up to the prceient tire

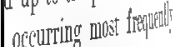

It is, borterer, by at?

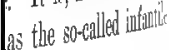

h, hair, nails. and the dis

.

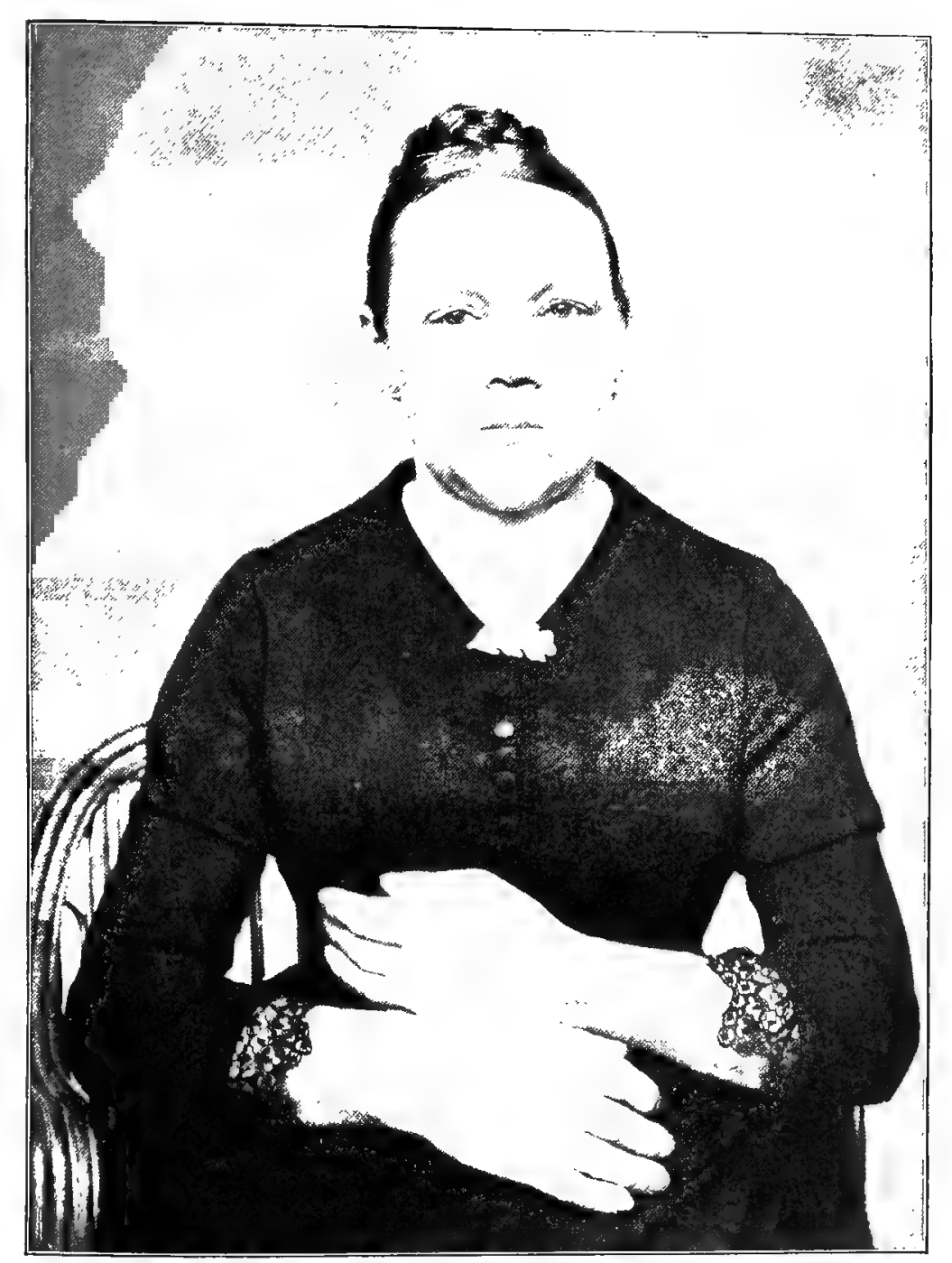

Fig. 1.-W. M., Aged Forty-eight; Ixcipient Mrxedema.

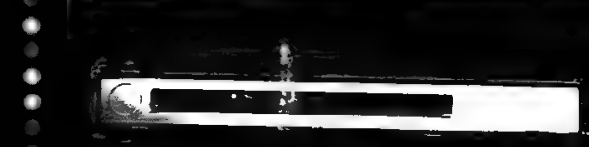

$+8$

1. 1 


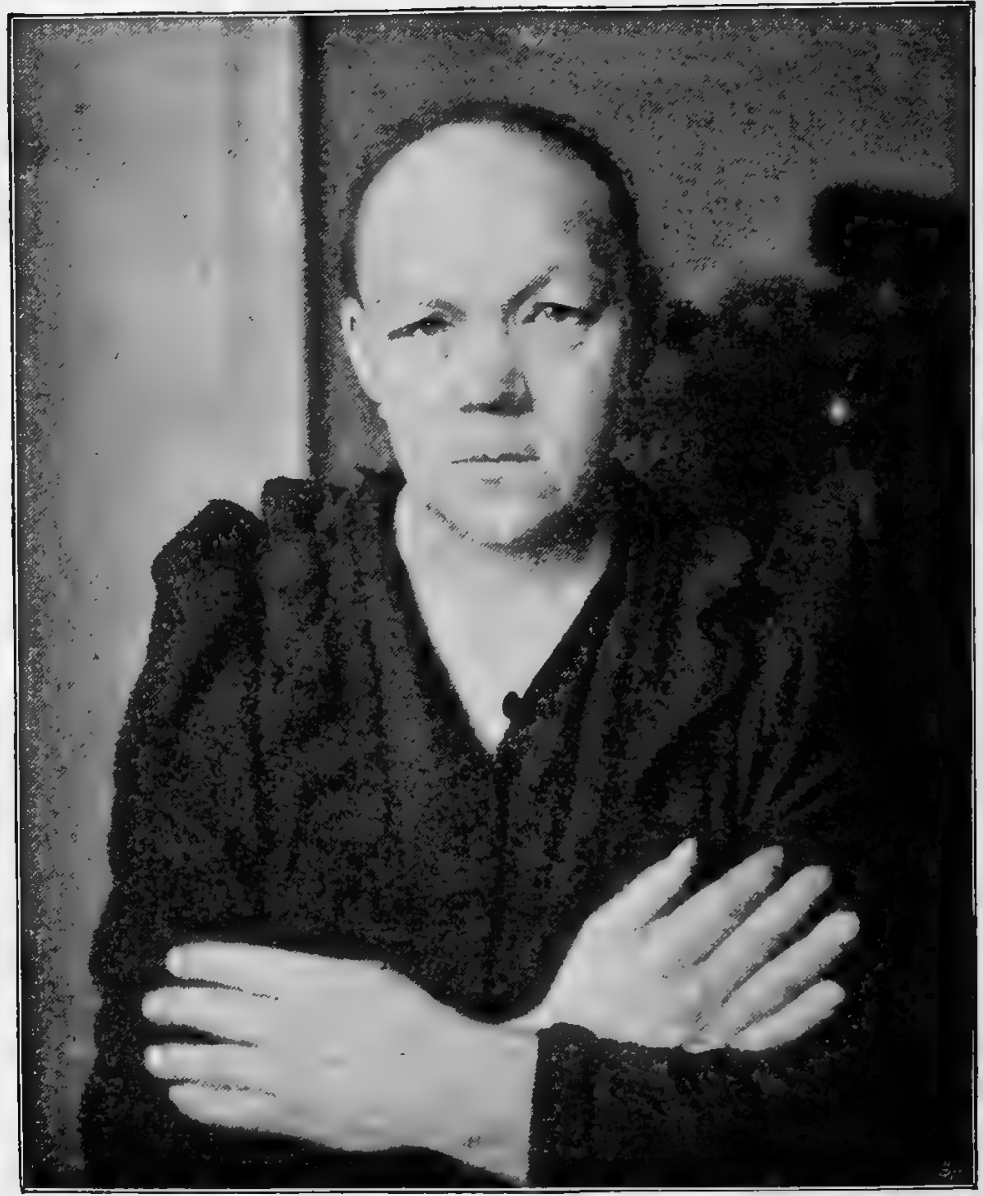

Fig. 2.-W. M., Aged Fifty-two; Advanced Mrxedema. 


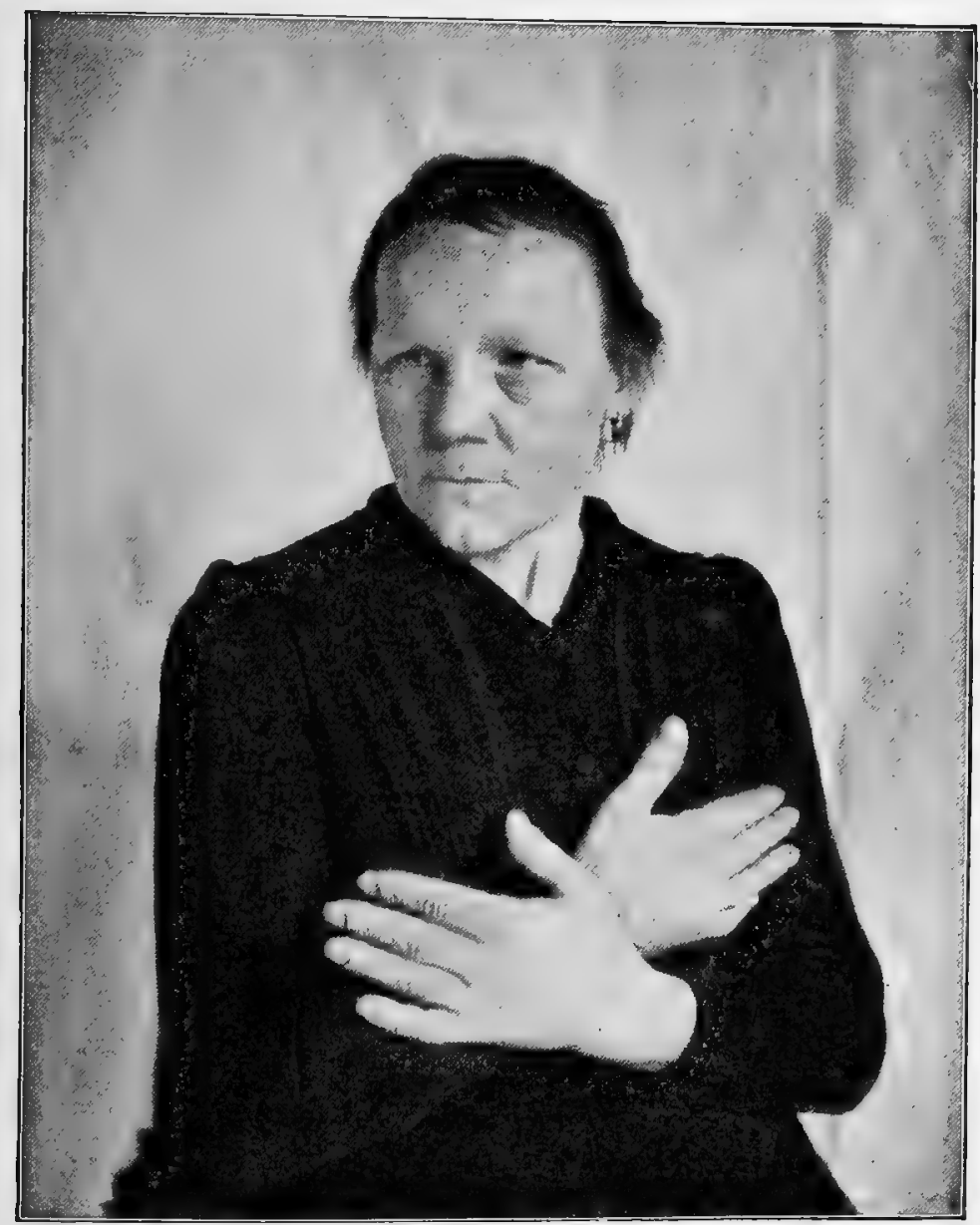

Fig. 3.-W. M., Aged Fifty-three; Mrxedema in the Stage of Recovery after the Employment of 39.2 Grams of Thyreoidin. 
These features are not equally developed nor even all present in every case, and my case was no exception to this rule.

Of greatest importance in the diagnosis of the disease are the cutaneous symptoms. They make it possible for us to recognize the malady, prima vista, so to speak, and after a comparatively brief examination of the patient. They give the patient the characteristic stamp which I attempted briefly to portray at the beginning of this article.

1. Most frequently the disease begins unnoticed and insidiously, with a gradual swelling of the skin, at first in the face, then in the hands, arms, feet and legs; finally and least noticeably upon the trunk. In the cheeks, around the eyelids, and upon the chin, puffiness is noticed; the lips and nose become swollen and thickened, the palpebral fissure is diminished by the swelling of the eyelids, and a hard, elastic edema of the whole face produces a stupid, dull expression. The tongue is thick, clumsy and too large for the mouth; the soft palate, the urula, and the postpharyngeal wall, as well as the larynz, are swollen. Hence the voice becomes rough and hoarse with a peculiar deep sound. The gums also swell, bleed readily, and retract from the teeth, which have a tendency to caries and frequently drop out without any change of structure. The lobes of the ear are also coarse and misshapen. Upon both sides of the neck above the clavicle, swellings appear, which are soft to the touch, and from about the size of a plum to that of a hen's egg. These pads are not due to swollen glands, but consist of fat, connective tissue, and conrolution of vessels (veins). The extremities appear swollen and shapeless, the hands like paws; wider shoes and larger gloves become necessary.

The skin is pale and looks anemic; occasionally it has a marbled appearance; to the touch it is cold, unelastic, hard and coarse. If pressure is made with the finger a slight impression is made, but the pitting does not remain. As a rule, this swelling is by no means uniformly distributed over the entire body. It attacks preferably either the face and the supraclavicular region or the extremities. Occasionally it disappears temporarily at the beginning of the disease (Ord), and then recurs, wandering here and there. The skin is dry, rough, desquamates decidedly, and not even by means of diaphoretics can sensible perspiration be induced, while insensible perspiration is lessened from 40 to 60 per cent. in comparison to the normal. The nails show longitudinal fissures and become brittle. The hair of the head and eyebrows falls out, and soon large areas are formed, which are perfectly bald.

Besides these striking changes there is a further defect which indeed cannot always be determined with certainty during life, but which in my patient, for example, was readily demonstrated; I mean the absence of the thyreoid. In place of the lobes of the gland normally situated upon both sides of the wind-pipe, the smooth wall of the trachea may be palpated from the cricoid cartilage to the jugulum. I shall revert later to this remarkable condition, and to its importance in pathogenesis.

2. The disturbances of the cerebral functions mostly set in with headache, sometimes with a feeling of anxiety, and a heaviness in the limbs. The patients move about slowly and with uncertainty. The power of co-ordination is diminished, as is frequently shown in the attempts at walking. The patient 
then conveys the impression of being under the influence of a high grade of nervous irritability with severe muscular tremor, so that it is impossible to maintain an erect attitude.

The patient's appearance is peculiarly quiet and suggests mental limitation, an impression which is increased by the fact that the head is mostly bent forward so that the chin is held, or, more correctly, falls upon the chest, for the patient cannot keep the head erect. The power of thought lessens, and its exercise is apparently difficult. There is loss of memory, particularly in regard to the true duration of the illness; now and then actual hallucinations appear. Speech itself is peculiar, being monotonous with a nasal or rough quality, and this, combined with a certain tendency of the patient after having once begun to speak to continue uninterruptedly like a wound-up clockwork, is looked upon by Ord as especially characteristic. I must admit that this latter condition has not yet been noticed by me in my very much more limited experience. Finally, actual convulsions and coma have been observed. The reports in regard to reflexes, cutaneous sensation, and electric irritability vary. In my case no disturbance of these functions was present. The tendon reflexes were prompt, and electric irritability both for the faradic and constant current was normal. The sensation of cold, and the lowered body temperature which is the cause of this, may be considered as due in part to the nervous disturbances. The temperature in the axilla varies between $96.8^{\circ} \mathrm{F}$. and $98.6^{\circ} \mathrm{F}$. The patients feel "as if they were living in an eternal winter." Unquestionably, this sensation of cold depends upon the inability of the patients to regulate the body temperature so as to correspond with variations in the external temperature; they suffer particularly in cold and frosty weather. On the other hand, there is deficient energy of oxidation, a diminished heat production, in the organism.

This leads us to the discussion of the last point:

3. Disturbances of metabolism and of the circulation. The former is decidedly decreased. The urea or $\mathrm{N}$-excretion as well as the respiratory metabolism (Magnus-Levy) is decreased. Albumin is occasionally found in the urine, sometimes only in traces, sometimes in larger amounts. According to the observations of Byrom Bramwell, as well as of Hun and Prudden, albumin is found in about 18 per cent. of the cases. In rare instances at the acme of the myxedematous stage, mucus is also found in the urine. In the blood there is a slight oligocythemia or a polycythemia. In women there is amenorrhea. The pulse is small, low and weak. Hemorrhages from the mucous membranes, particularly from the nose and mouth, are not rare.

I have now sketched the most important symptoms. I omit the rarer ones as, for example, synovitis of the knee-joint, premature climacteric, tremor of the eyelids, contractures of the hands and feet, salivation. The characteristic symptoms of the affection under discission are limited to tissue changes, particularly of two organs, the skin and the thyreoid gland. The connective tissue of the corium is loosened, its individual fibers thickened and hyperplastic. The cell nuclei and the fibrillary elements of the gelatinous substances between the individual fat lobes are increased. It seems as if the skin were saturated with a fluid or semi-fluid substance. Whether this actually con- 
sists of mucus, i. e., of an edematous fluid containing considerable amounts of mucin, is not yet certain. This much, however, is assured, that the degree of this infiltration may vary decidedly in individual cases. In my patient there is now only slight swelling of the eyelids and of the cheeks, but at the height of the disease the entire face, shoulder-girdle, hands, forearms, and feet showed swelling which was everywhere unmistakable, and here and there extreme. In a case observed by Kast (Ponfick) the thickening of the skin was so extreme that numerous fissures formed, with intertrigo-like excoriations which were followed first by a phlegmon of the arm and later by a general fatal sepsis.

In the internal organs usually no changes are found apart from the previously mentioned atrophy of the thyreoid gland, but occasionally cirrhotic processes in the liver and kidneys, endarteritis obliterans, and enlargement of the pineal gland have been noted.

The changes in the thyreoid gland are undoubtedly the most remarkable feature of this disease. In most cases, even during life, we observe that it is impossible to palpate the lobes of the thyreoid gland in the neck. It may be said that this condition is found in about 80 per cent. of the cases. In some instances, however, the atrophy of the gland cannot be determined with certainty, and in a few an enlargement has been noted. Yet in these cases, in so far as accurate observations have been obtained, there has always been found a strumous degeneration of the organ, which may also have resulted in a loss of function. I have been able to collect 36 reliable autopsy reports, in 33 of which, or 94.4 per cent. of the cases, there was atrophy of the thyreoid gland. The necropsy reports show that the gland substance had lost its characteristic structure, and had been changed into a more or less shrunken remnant, of hard, fibrous consistence and yellowish white color. Connective tissue proliferation leads to destruction of the parenchyma, so that only isolated remains of degenerated alveoli can be detected. Very recently Ponfick has reported an extremely careful histological investigation of the remains of the gland in a well-developed case of myxedema. He found " in wide areas almost total destruction of the follicles; here and there were the remains of rudimentary alveolar structures, all filled with colloid. Besides this atrophy there was an enormous increase and thickening of the connective tissue structure; in a word, a picture which markedly resembled the terminal stage of degenerative inflammatory processes, such as are met with so frequently in the kidneys, liver, etc."

\section{PATHOLOGY}

I need hardly say that this degeneration of the thyreoid gland is the key to the entire pathological condition. Atrophy of the thyreoid gland is not only one of the symptoms of the disease; it is the causative factor. This fact is absolutely certain in spite of occasional objections. Its recognition forms a glorious page in the history of pathology. We owe this to the united labors of physiologists and clinicians, the fruit of which is a therapy which, as regards the certainty of success, may be placed side by side with the most reliable remedies of our therapeutic armamentarium. 
I shall attempt to prove this in a few words, thereby taking an opportunity of referring to related conditions, namely, to sporadic and endemic cretinism, to infantile myxedema, and to the so-called cachexia strumipriva.

Two observations stand out prominently like landmarks in the great number of physiologic and clinical experiments and communications which resulted in the establishment of the importance of the thyreoid gland in the causation of myxedema. First, the experiment of M. Schiff, the results of which were for a long time discredited and even ridiculed, but at last completely proven. In this experiment an animal was subjected to thyreoidectomy, which under ordinary circumstances would have proved fatal, as Schiff had previously shown in 1859. But Schiff found that the animal did not succumb if the thyreoid gland were grafted into the abdominal cavity or under the skin, the specific constituents of the gland being thus preserved for the organism. Next came the important communications of Kocher and Reverdin who observed after the extirpation of the thyreoid gland in man a condition of general cachexia with physical and mental phenomena which show a marked similarity to the symptoms of myxedema just described, and also correspond accurately to the phenomena developing in an animal after extirpation of the thyreoid. In other words cachexia strumipriva, produced experimentally or (in man) as the result of an operation, is identical in nature with genuine myxedema. Now since in both cases the same defect is present, namely, absence of the thyreoid, it follows that the resulting insufficiency, that is, the complete loss of function of the thyreoid gland, is to be looked upon as the cause of these pathological phenomena.

I cannot here discuss the different phases which the evolution of this view has undergone. But one stage at least I should like to bring into prominence, for it shows how difficult the proper interpretation of an experiment may sometimes be, and how obscured by complicating conditions.

Some investigators did not succeed in producing the symptoms of cachexia strumipriva (or, more correctly, cachexia thyreopriva, as we are not speaking of the removal of a goiter) by thyreoidectomy, and consequently were justified in doubting the causal relation of the thyreoid gland. Then Gley showed that besides the great mass of the thyreoid there are in animals small, supernumerary glands, which had already been anatomically described by Sandstrom. These extra thyreoids, if not removed together with the gland, perform vicariously the function of the latter. Only after this circumstance had been taken into consideration could constant results be obtained. In the same manner we may explain those observations (which, however, are in the minority) which show that in some cases cachexia strumipriva did not follow thyreoidectomy in man. This is because parts of the gland were not removed at the operation or because a rapid reproduction of the gland took place. But even a few isolated and trustworthy cases of this kind would not overbalance the enormous number of positive observations to the contrary.

But let us proceed a step further. All over the world, as is well known, there are unfortunate individuals whose mental and bodily development is retarded, so that they present a stupid and misshapen appearance. They are called cretins, and their disease cretinism. From the fact that such ill-fated 
persons are met with in isolated regions, and are particularly numerous in certain localities, and inasmuch as the development and distribution of the disease are apparently dependent upon the condition of the soil and the watersupply of the district in which the cretins accumulate, we call these cases "endemic cretinism." In other parts of the world they are only occasionally met with, and we then speak of them as cases of sporadic cretinism. In these cases as in myxedema there is an insufficient development, or an atrophy and disturbance of function of the thyreoid gland. Hence we speak of them as an athyreosis chronica, in contrast to the results produced experimentally in animals, or after loss of the thyreoid by operation in man in whom this state may be called cachexia thyreopriva.

Accurate observation and study of the cases belonging to this group show that endemic cretinism is a condition of physical and intellectual degeneration, which occurs only where there are local predisposing causes, and a degeneration of the thyreoid gland produced by these or going hand in hand with them. The disease begins its development even in fetal life. Sporadic cretinism, on account of its resemblance to myxedema of adults, is in early life also designated as infantile myxedema. In these cases we find mental weakness even to idiocy. Like actual typical myxedema, it is an occasional affection of the thyreoid gland with consequent loss of function, i. e., a disease not limited to a definite region. The course of genuine cretinism, as well as myxedema, is a very chronic one, lasting for decades, so that a cretin may attain relatively old age provided he does not die of some intercurrent disease. Cases of sporadic cretinism (infantile myxedema) have, as a rule, a briefer duration of life, and if not ameliorated by treatment scarcely survive the third, at most the fourth, decade. Cachexia thyreopriva runs a subacute course, and unless relieved by medical aid terminates in death after a comparatively short time, at the longest four or five years!

\section{THERAPY}

This is not the place in which to discuss the clinical symptoms of these diseases; that degencration of the thyreoid gland is unquestionably the causative factor in all of them is the only circumstance important for us. This is clear not only from the general correspondence of the external pathologic phenomena, but, in particular,. from the results of therapy founded especially upon the knowledge of the causal rôle of the thyreoid gland in these cases. The results of thyreoid therapy have proven, a fortiori, that the common cause "of these diseases is an absence of function of the thyreoid, by which, it may be remarked in passing, they differ from goiter or struma.

Properly speaking, in these cases we are dealing with a substitutiontherapy, replacing the lost or deficient glandular secretion by the administration of the glandular substance or its extracts.

The surprising experiments of Schiff, previously mentioned, actually compelled the application of these principles in the human subject, and Bircher in 1890 by implantation of a human thyreoid in a woman with cachexia strumipriva first succeeded in obtaining a curative result. Shortly after 
Horsley proposed, in place of the human gland, to utilize the thyreoid of the sheep in myxedema and cretinism, and Bettencourt and Serrano noted a rapid improvement in a woman with myxedema, in whose abdomen they engrafted one-half of a sheep's thyreoid. When Murray, instead of employing the entire gland, subcutaneously injected its glycerin extract, and Mackenzie in 1892 administered the fresh gland or its extract internally with the same effect, and other observers showed that the dried glandular substance in tablet form acted in the same manner, the therapy of myxedema and of the related pathologic conditions previously mentioned became as simple as it was reliable. In fact, reports came from all quarters regarding the almost remarkable action of this "organotherapy," which was particularly effective in myxedema, in infantile myxedema, and in cachexia strumipriva, while endemic cretinism, owing to its nature, could only be benefited to a slighter extent. The tablets which were administered were so prepared as to dosage that each tablet corresponded to 0.25 gram of the glandular substance; these were given in increasing doses of from 3 to $\%$ or 10 tablets per day, until (early or later) symptoms of intoxication, extreme or mild (the symptoms of so-called thyreoidism) appear.

It cannot be denied that the use of the entire gland has certain disadvantages, on account of the varying amount of active substance in the individual gland or tablet, the admixture of products of decomposition, etc. Obviously it would be desirable to isolate and to utilize the specific active substance. There have been numerous trials in this direction. At one time it was hoped that the desired body could be obtained in the form of a ferment, at another time it was supposed that it could be secured as a fixed chemical combination from the group of proteid substances or even as an alkaloid. I must mention that S. Fraenkel, in 1895, isolated a substance belonging to the guanidin group with the empiric formula, $\mathrm{C}_{6} \mathrm{H}_{11} \mathrm{~N}_{3} \mathrm{O}_{5}$, which he called " thyreoantitoxin," and Drechsel and Kocher, Jr., isolated similar combinations from the gland. Then Baumann, in 1896, made the surprising discovery that the gland contained considerable amounts of iodin, and that this iodin was found in combination with organic substances, chiefly in his opinion with albumin, and to a smaller extent with globulin. This body Baumann called iodothyrin, later thyreoiodin, and showed that from the purest thyreoiodin 9.3 per cent. of iodin could be obtained in a crystalline form. Investigators are not yet quite unanimous as to the true nature of this thyreoiodin. Tambach assumes that the iodin is combined with various albumin bodies, and that iodothyrin forms only a part of the iodin-containing substances of the thyreoid gland. According to Oswald, the iodin combined with albumin has the character of globulin, for which reason he called it thyreoglobulin. From this, by long-continued pepsin digestion, iodothyrin and iodin-containing albumoses and peptones can be split off: Besides these, a nucleoproteid is said to occur which, however, does not possess the specific action of the gland. Blum denies the presence of iodothyrin as a primary constituent; he thinks it is not preformed but is an artificial cleavage-product. However this may be, Baumann in association with Roos seemed to prove that thyreoiodin possesses the specific properties of the natural gland, and acts as a substitute for its curative effects; it 
also possesses the same toxic action, but has the advantages of a more exact dosage and absolute purity. These opinions were soon confirmed by others (Hofmeister, Ewald, Honnig, Goldmann, and others), so that there can be no doubt that thyreoiodin may be employed in place of the natural gland. Whether it is actually an equivalent for the complete gland appears doubtful from the investigations of Gottlieb and Jaquet, who observed in a number of dogs after thyreoidectomy that thyreoiodin alone did not keep the animals alive, while Gottlieb by administering an extract from the complete gland (thyraden), and Jaquet by a substance called by him aiodin (a tannin precipitate of the extract of the gland with physiological salt solution) succeeded in sustaining them. I might also mention that Cunningham in England isolated a so-called colloid substance from the gland which is analogous to Baumann's thyreoiodin, i.e., the contents of the acini after previous peptic digestion of the gland tissue. $\mathrm{He}$ is consequently of the opinion that this colloid is the true active extractive product of the gland cell, while Oswald believes it to be a mixture of thyreoglobulin and nucleoproteid. In a similar manner, McLennan produced a preparation from iodoglobulin and thyreoiodin which has received the name thyreoglandin. In view of all this it is obvious that the nature of the specifically active substance of the gland has by no means been definitely decided; on the contrary, each day brings new reports and new views.

This is true also of another highly interesting discovery which science owes to Baumann, who was unfortunately too early removed from his sphere of activity. In a comparative estimation of the iodin contents of glands, some of which were procured from Freiburg and some from Hamburg and Berlin, it was shown that the iodin contents of the former were considerably less than in the glands brought from the two last mentioned cities. The glands obtained from Freiburg, in the same parts by weight, averaged 2.5 milligrams of iodin; those from Hamburg 3.83 milligrams, and those from Berlin 6.6 milligrams; in the investigations in North America made by Gideon Wells, the iodin contents of 20 glands, some from Chicago, some from Baltimore, Boston, New York, etc., averaged 10.79 milligrams. Now, in Freiburg goiter is endemic and frequent, in Berlin and Hamburg comparatively rare, in America almost unknown. What can be more reasonable than to connect the slight amount of iodin in the glands from Freiburg (where, as may be remarked in passing, goiter is found conspicuously often even in the new-born and in young children) with the prevalence there of goiter? For many years strumous degeneration has been referred to a lack of iodin in the water, in the air, in the food, though no convincing proof of this has been established. Here, for the first time, we seem to have analytic evidence of the influence of iodin upon the development of goiter, or, more correctly, the relations of the iodin contents of the thyreoid gland to goiter. I expressly say "seem to have evidence," for to make this assumption a certainty more comprehensive investigations are necessary, and I am forced to admit that Oswald made numerous analyses of glands from Switzerland and from the regions where goiter occurs endemically and has demonstrated the exact opposite; namely, that the amount of iodin in glands from this district was greater than 
in those obtained elsewhere, while, on the other hand, Rositzki's rescarches in Styria confirmed Baumann's reports.

No matter how diverse the manufactured preparations may be, their use evidently checks the previously described symptoms of myxedema, which give place to complete or almost complete health; therefore all must contain the active substance, though perhaps some more than others. The skin becomes soft, smooth and elastic; the edematous infiltration-the myxedema-disappcars; the hair which has fallen out is renewed. The general health returns to the norm, vigor and activity take the place of the preceding debility and indolence-briefly, so complete a transformation in the condition of the patient takes place that anything more extraordinary can hardly be imagined. The same is also true, mutatis mutandis, in the conditions previously described, viz.: infantile myxedema, cachexia thyreopriva, an abortive form of myxedema which has been designated "myxoedème fruste", and to some extent also in cretinism. To enter minutely into this subject would carry us too far, hence we will only remark that the influence of thyreoid preparations upon the length and caliber of the bones in infantile myxedema has been observed, and to a certain extent demonstrated, ad oculis, by means of radioscopy.

We noted the curative effect of this remedy upon myxedema in my patient. As time has gone on the preparations of various English and German firms have been administered, as well as thyreoiodin pastilles, all with good results which, however, lasted only as long as the drug was given.

After discontinuing the administration of the remedy for some time-in my case for a few months-we must always return to it, as signs of myxedematous cachexia always recur. These usually consist of chilliness, malaise, and slight swellings in the face; occasionally, however, severe symptoms of depression appear, particularly of a psychic nature. This is not to be wondered at, as our substitutiontherapy does not root up the evil, but only re-supplies some necessary products for metabolism, and for this reason the medication must be persistently carried on. At times the remedy becomes unneccesary, and it may be discontinued for a considerable interval, because a certain reserve accumulates, which, after the cessation of the specific therapy, is only gradually utilized and consumed by the organism. The same is also true, according to our present experiences, in cachexia strumipriva, while in infantile myxedema and cretinism this effect does not occur, and from the beginning we must carry on the thyreoid therapy almost continuously.

One of my patients, a highly cultured woman, who has been taking thyreoid extract for five years, wrote me as follows: "I may say that (inconceivable as it may appear) under organotherapy the system becomes decidedly accustomed to the taking of thyreoid gland, so that its therapeutic effect is weakened, and the doses must be constantly increased if improvement is to be expected. On the other hand, when we consider the toxic action, the debility and tiring of the heart, the damage to the stomach, etc., it appears that one does not become accustomed to it; the longer the drug is administered the greater the susceptibility." The latter remark points to the secondary effects of thyreoidtherapy, effects which, after prolonged use of the drug, appear to be more or less severe. 
This secondary condition has been designated thyreoidism; the disturbances may be separated into the nutritive and the nervous. The urine increases in amount, and its $\mathrm{N}$-containing constituents, as well as the chlorids and phosphates, are increased. The body-weight decreases and, as was first shown by Wendelstadt, not over $\frac{1}{6}$ of the loss in weight is of the body albumin; the remainder is a decrease of fat and water, so that the increased combustion of fat may be referred to increased oxidation (Magnus-Levy). In this process oxidation, during and for some time after the use of thyreoid, may rise 10 to 20 per cent. above the former values; there is therefore an increased metabolism. As a nervous symptom we note primarily a decided increase in pulse frequency and cardiac palpitation, which may be looked upon as a definite indication that the dose is too large. After this headache, nausea, vomiting, and general debility may occur. Sometimes these symptoms increase to an alarming extent, and formerly, when this condition was not sufficiently understood, even fatal cases were observed. Therefore, when these symptoms appear (which may be soon or late, according to individual susceptibility) thyreoid preparations must be immediately stopped, and the treatment be interrupted. These toxic symptoms will then shortly disappear without causing permanent injury. This teaches the lesson that these preparations should not be used except on the advice and under the supervision of a physician, and their sale by an apothecary except upon prescription should be prohibited. How far thyreoidism is a phenomenon of specific intoxication, and how far toxic products of decomposition generated during the manufacture of the preparations are concerned in it, is doubtful. Fortunately, we have lately become able to combat these symptoms of thyreoidism, or better, to prevent their appearance; as Mabille has proposed, we administer simultaneously with the thyreoid preparations small doses of arsenic, either as Fowler's solution or as arsenious acid. As a matter of fact, in my case I administered no less than 962 tablets without any deleterious effects, and during three months I gave 3 drops of Fowler's solution, i. e., upon the whole a minimal amount of 0.16 gram of arsenious acid.

We may consider it as definitely settled that we are able by means of substitutiontherapy and only in this way to influence the entire constitution to such a surprising extent, in the pathologic conditions which depend upon atrophic or degenerative changes in the thyreoid gland. We should mention here also the value of thyreoid extract in the abortive forms of myxedema which Hertoghe has described under the designation " myxoedème fruste."

The use of thyreoid preparations should also be considered in local disease of the thyreoid gland, namely, in the treatment of goiter. Although success in treatment is here limited to the so-called parenchymatous goiters of youth, i. e., to that form of goiter which is not so much the result of degenerative change as of a hypertrophic condition of the glandular tissue, their use is here of great value. v. Bruns was the first to employ thyreoid gland preparations in simple goiter with the idea of diminishing the labor of the hyperactive gland. For it is self-evident that in such cases there is an increased demand in the organism for the product of the secretion of the gland, either because, from some unknown disturbance of metabolism, more thyreoiodin was required 
and so consumed by the organism, or because the supply of iodin was deficient while the activity of the gland was especially active, thus leading finally to hypertrophy of the organ. v. Bruns, in fact, observed a decided diminution in the goiters after the administration of comparatively small doses, and Ewald, Stabel, and others in Germany obtained similar results. Wells in 1897 compiled 584 cases of simple goiter which were treated with different thyreoid extracts, of which cases 475 , i. e., about 82 per cent., were improved. Nevertheless, in my experience, the cure is never complete; that is, the goiter does not completely disappear, although $\mathrm{v}$. Bruns reports this result in about 8 per cent. of his cases. As has been already stated, the best results are seen in youthful individuals in whom the goiter has not existed for too long a time. The older the patient and his goiter the slighter the changes which may be observed in the gland in favorable cases even after four to six days of treatment with thyreoid preparations. Usually, however, this becomes noticeable only during the second and third weeks of treatment. A diminution in the circumference of the neck of from 4 to 6 centimeters is frequent; greater reduction than this is exceptional. Even in these cases, after discontinuance of the remedy relapses occur, so that small doses must be repeated from time to time.

I have the history of two girls, aged nineteen and twenty-one years respectively, the circumference of whose necks after the use of thyreoid preparations was reduced in one from $3 \% .6$ to 35.1 centimeters, and in the other from 33.5 to 32.1 centimeters. Symptoms of thyreoidism did not appear. I must not fail to mention that equally favorable results have been obtained with thymus preparations by Mikulicz and Reinbach.

The conditions are very different in another disease which also implicates the thyreoid gland: Graves' disease. In this affection enlargement of the thyreoid, as is well known, is one of the three cardinal symptoms of the disease-goiter, exophthalmos, tachycardia. But here we are no longer dealing with a vicarious or compensatory labor hypertrophy of the gland, but with an active increase of its secretion, which leads to the phenomena of thyreoidism occurring in the course of the disease. The use of thyreoid preparations in this malady is contraindicated, even if we omit entirely from consideration the fact that the cause of Graves' disease is not to be found in an affection of the thyreoid gland alone. We know that its cause is multiform and that other organs-as is shown by the cases without goiter-especially the sympathetic nervous system, are implicated. As a matter of fact all cautious and unbiased observers have been convinced of the complete failure of thyreoid therapy in Basedow's disease (exophthalmic goiter). Indeed it has been noticed that its use is not only useless, but often produces a decided aggravation of the symptoms.

Although we have up to this point discussed the administration of thyreoid preparations only as true substitutiontherapy in diseases in which a disturbance of function of the thyreoid can be demonstrated, yet the list of the uses of the remedy has by no means been exhausted. We know of other diseases, or disease groups, in which, based upon the pharmacodynamic action of thyreoiodin, this remedy has been employed with more or less success. I 
have stated before that the employment of thyreoid preparations increases metabolism, and causes a decrease in the fat of the organism; this has been experimentally proven. What was more natural than to attempt a cure of obesity by this means? Y. Davies in America, Leichtenstern and Ewald in Germany, were the first to publish favorable results of this kind. They obtained in obese persons reductions in weight of 5 kilograms and more in a few weeks without any special restriction of the food, and without producing marked debility, restlessness, fatigue, etc. This condition is due, as already remarked, to an increased combustion of carbohydrates and fat, while albumin decomposition is but little affected. Schrödt has lately published a case in which a loss of 16 kilograms of weight occurred in an obese person, and, as was shown by carefully conducted investigations of metabolism, this was almost wholly due to combustion of fat. The results are even better if the diet is regulated simultaneously, i. e., the fats and carbohydrates limited to a certain extent without, however, insisting on a dietetic cure for the reduction of fat in the strict sense of the word. Cabot has collected 145 cases of this kind, and reports favorable results in all but 6 cases. $\nabla$. Noorden, who is not well disposed to thyreoid treatment in obesity, nevertheless observed among 14 patients, who, in the course of four or five weeks, had taken upon the average 3 to 4 tablets per day, a reduction in weight of 5.4, 6.0, and 7.8 kilograms, and had only one complete failure. In thyreoiodin we showld, therefore, possess an almost ideal remedy for obesity if only it were always effective. Reports of failure are, however, not wanting, and in several cases, instead of the desired loss of weight, symptoms of thyreoidism have appeared. This latter condition, with or without a simultaneous reduction of body fat, has been apt to supervene when the laity, without professional advice, have used the thyreoid preparations either too long or in too large doses. In another article, in referring to a case of psoriasis treated by the thyreoid preparations, I have discussed the question asked by Hertoghe, whether success or failure in these cases does not depend upon the condition of the thyreoid gland of the affected individual; i. e., the treatment is only successful in cases in which the obesity (or the skin disease) is one of the manifestations of an affection produced by disturbance of the function of this organ, as the gland certainly has a decided influence upon metabolism.

There can be no doubt, as has been determined from observations in myxedema, of the effect of thyreoid in trophic processes of the skin. Hence thyreoid preparations are administered in various skin diseases, lichen planus, prurigo, adenoma sebaceum, ichthyosis, xeroderma, lupus, and, above all, in psoriasis; of course here also the success is varying and uncertain. In some quite chronic cases I have seen conspicuous improvement, and it is not too much to say a cure; in others there was no result in spite of treatment for several months. In the last case successfully treated, a spare man, aged fifty-three, whom I showed at the meeting of the Berlin Medical Society on July 18, 1900, the thyreoid gland could not be palpated. This man had a persistent, freely desquamating eruption, appearing in large blotches upon the trunk and extremities, for the cure of which various remedies had been unsuccessfully employed for years. After the administration for three months of thyreoiodin 
tablets with arsenic - he received during this time 450 tablets which contained 0.25 gram of iodothyrin and 0.16 gram of arsenious acid--the eruption almost disappeared, being replaced by small, isolated areas of about the size of a silver three-cent picee, and by natural, smooth, pink skin. Of 154 cases collected by Cabot, 63 were improved, 53 unimproved, and 22 aggravated. This is greatly in favor of my view previously expressed regarding the connection between these diseases of the skin and disturbances in function of the thyreoid gland; for Wells, in a case of scleroderma in a woman aged fifty-one who had suffered for about one year from the affection, found the thyreoid gland, post mortem, to be greatly atrophied, so that in the fresh condition it weighed only 14 grams and when dried only 3.23 grams. The total amount of iodin was only 2.94 milligrams, i. e., only about one-fourth of the normal. Microscopically the connective tissue was greatly increased, the intima of the glandular vessels was proliferated, the lumen of the acini, for the most part without colloid, changed into small cysts which were filled with colloid material. The hypophysis was hypertrophied, it weighed 0.7 gram, its acini were distended by colloid masses with uncommonly numerous eosinophile cells in the interacinous tissue. Wells remarks quite properly that if these changes could be determined constantly in scleroderma the fact would greatly favor a thyreogenous origin of this disease.

Actuated by the same reasons as in the therapy of obesity and psoriasis, etc., physicians have used thyreoid preparations in mental disease, in tetanus, chorea and progressive myopathy. Favorable results in isolated cases have even been reported, particularly by Bruce from the Royal Edinburgh Asylum, by Mabon and Babcock from the St. Lawrence Hospital, and by Reinhold, Levy-Dorn, Gottstein, and Bramwell in tetanus, etc.; but invariable and convincing results have been obtained more rarely in these affections than in the diseases previously mentioned. Gauthier, Quénu, Reclus, Ferria and others have employed thyreoid treatment also in fractures with delayed union of bones, on account of the fact that the thyreoid gland has a predominant influence upon the growth of bone; the results were apparently most gratifying, and the same reason led to the employment of this remedial agent in rachitis. But here the watchword for the present must be: temporize! Confirmations are wanting; and from this it may be concluded that the treatment has been tried by others, but without success.

We cannot close this discussion without referring briefly to an organ apparently in close connection with the functions of the thyreoid gland, and by embryologic and anatomic analogies also histogenetically parallel with it. This is the hypophysis, the pituitary body, the anterior glandular part of which is developed, like the thyreoid, from the ectoderm, a diverticulum of the posterior pharyngeal wall, the structure of which also consists of acini which unite and form follicles containing more or less of a colloid substance.

Unquestionably this gland has some relation to osseous growth, for it is almost invariably enlarged in acromegalia and giant growth. In isolated cases it has been known to attain the size of a hen's egg, and the sella turcica is abnormally developed. Nevertheless, this does not yet prove, as most authors too hastily assume, that the hypertrophy of the gland is the cause of these 
anomalies of growth. It may be.a mere coincidence, or vice versa, a resulting condition of disease of the bone, to a certain extent a reflex from the bone to the gland. After thyreoidectomy in animals the hypophysis is said to hypertrophy vicariously (Hoffmeister), and in a few cases of myxedema hypertrophy of the pituitary gland has been found. But Ponfick, to whom we owe two very accurately investigated cases of myxedema recently reported, found upon transverse section in one case the glandular portion of the hypophysis enlarged, in the other case a decided destruction of the organ so that the tubules of the gland were replaced to a great extent by a "worthless fibrous mass." Here also there are evidently contradictions and enigmas which still await solution, especially as we can state nothing definite in regard to possible therapeutic results.

Although we are completely in the dark in regard to these last-named affections, and for the present are compelled to await further investigations, this much is certain, that in thyreoid therapy we possess a remedy previously unknown with which to combat a number of chronic diseases, which even a short time ago were looked upon as incurable, and which it was thought impossible to influence therapeutically. This type of therapy has been designated organotherapy or opotherapy, ỏmós, juice), and, by a hasty generalization, the principles which are the foundation of thyreoid gland therapy have been misapplied to preparations from other organs. It has been forgotten that, in the case of the thyreoid gland, we are dealing with successes gained in conformity with well-founded physiologic and pathologic experiments and observations. If now we attempt to extend this substitutiontherapy to all the other organs which are looked upon (more or less correctly) as the cause and seat of certain diseases, corresponding extracts and preparations from these organs are thrown upon the market. But it must be remembered that, barring isolated exceptions, there is no reasonable foundation for any hope of success. Here the wish has been father to the thought, and views have sometimes become so extravagant that a certain resemblance to the notorious "similia similibus" of homeopathy resulted. As a matter of fact, treatment by organotherapy is by no means of recent origin, as is commonly supposed.

Park, in a very interesting article in the Buffalo Medical Journal, 1899, has shown that it may be traced to a very early epoch in the history of medicine.

In China, since remote antiquity, the juice from macerated pigs' lungs is said to have been an efficient remedy for pulmonary diseases; for headache, pigs' brains are taken; for dysentery and chronic diarrhea, pigs' intestines are administered; semen is reputed a potent tonic in chlorosis, anemia, impotence, etc. Tout comme chez nous!

Strictly speaking, the administration of ovarian extract is the only one of these attempts that is founded upon experimental proof as well as upon analogy. The administration of pancreatic substance in diabetes (on account of the experimentally proven relation of the pancreas to glycosuria and diabetes, is a therapeutic experiment which, critically examined, is based upon nothing more than analogy, and lacks direct experimental proof. Regarding ovarian therapy, i. e., the use of so-called oöphorin tablets, Loewy and Richter have demonstrated experimentally the interesting fact that in 
castrated bitches the consumption of oxygen falls about 20 per cent., the total metabolism of gases about 9 per cent. below normal, and that this alteration of respiratory metabolism continues for a long time after ovariotomy, even from six to twelve months. If such animals are fed with dried ovaries, the interchange of gases returns to its former height and even beyond it.

On the other hand, under the influence of oöphorin, no increased destruction of $\mathrm{N}$-containing substances takes place-no interchange more rapid than in normal animals-nor could similar results be obtained after ovariotomy in animals by the use of didymin or spermin. This explains Landau's successes with the administration of ovarian tablets in menstrual anomalies, during the climacterium, in chlorosis and hysterical conditions; these good results I can partially confirm from my own experience.

Other organic extracts and preparations are also known to produce certain effects upon the organism. Thus adrenal extract increases blood pressure, preparations made from the spleen and liver have an influence upon leukocytosis, etc. However, these are, as far as can at present be ascertained, only local effects limited to definite circumscribed areas, and may be compared to the action of digitalis upon the heart, or of atropin upon the pupil. We cannot claim for them any specific curative effect.

The successes reported with cerebrin in cerebral affections, with medullin in diseases of the bone, with didymin, with the extract or with the substance of the adrenals in Addison's disease, with pancreatic tablets in diabetes, etc., must be regarded as very exceptional, if indeed we credit them at all. These preparations have not as yet found general acceptance. Consequently, I do not intend to relate in detail what has been accomplished, or, better, what has been attempted by their use.

In this field of therapy the same phenomenon is met with which we find at present in other realms of therapy: professional activity and choice are influenced more than is desirable from a source outside of the profession. I mean by the manufacturing chemist. The chemical laboratories, especially of the anilin works, have lately thrown themselves zealously into the endeavor, laudable in itself, to produce or to combine chemical bodies whenever a theoretic consideration or, more rarely, an animal experiment appeared to show the existence of a pharmacodynamic property. These endeavors have flooded the medical market with an endless number of new preparations that are distributed for trial, and with the hope of a successful trial, to competent and incompetent judges. Now there is absolutely no curative agent, no matter how slight the foundation for its use, that does not have its firm believer, and serve for purposes of investigation. Frequently it is tested in only a few cases from private practice, and then its epithon ornans "clinical observation" is attached. The results, in the form of reprints, are then sent by the laboratory into the world by thousands. Unfortunately the opinions expressed in these reprints often depend upon autosuggestion or an uncritical spirit in the author: in other cases the effects produced by the new preparations are nowise better than the action of old and reliable remedies.

Who knows the preparations? Their name is Legion; they are sent out with "first-class recommendations"-this cornmercial expression is excusable 
for, in fact, it is in place here - are put upon the market, and, after a brief and ephemeral existence, are relegated to the orcus of the medicine closet, never to see again the light of day!

The natural reaction is a constantly increasing doubt in regard to new remedies. The fascinating reprints, of which every physician has a plentiful supply, owing to the activity of the manufacturer, are read or not read, but are followed very cautiously, for, otherwise, time and money would often be uselessly spent, even if other and greater injury to the patient does not result from their use.

Why do I mention all this?

Certainly not to check a spirit of rivalry in the production, nor in the testing of new remedies by competent physicians, and this $I$ desire to repeat with emphasis and to bring out prominently. The advantage which has already resulted from organotherapy is so obvious that many a failure would be amply compensated for-but I must advise those who busy themselves with this line of investigation to be more cautious in arriving at conclusions, and to exercise more patience in collecting and more care in utilizing their material.

It is not wise to cut the corn while it is yet green; harvesting also is an art. Whoever has not learned this, whoever works in a field which is too narrow, i.e., in other words, whoever lacks the necessary experience and the endowment of a faculty for scientific criticism, should refrain from giving opinions on such a subject.

To this group of over-hasty, uneritical, and, therefore, untrustworthy publications, belong those châteaux en Espagne which form the greater part of that "so-called" organotherapeutics with which we have been favored in the last few years. With alarming haste the manufacturing chemists have made preparations from all of the organs, and offered them to the medical profession for its kind consideration. 


\title{
ADDISON'S DISEASE
}

\author{
By L. REISS, BerLIN
}

IN recent pathological discussions attempts have been made to refer the clinical symptoms in a number of general affections to a so-called "autointoxication " produced by the decrease or absence of the "internal secretion" of an important organ of the body. Of course this view refers chiefly to those forms of disease in which clinical and pathologico-anatomical experience have taught us to recognize the invariable predominance of changes in an organ having a secretory character. An example which best illustrates this is the wonderful symptom-picture of myxedema, the direct dependence of which upon a cessation or absence of function of the thyreoid gland has been lately proven with all the certainty that can be desired.

To the general diseases, in which, at the first glance, it must be obvious that similar functional disturbances are causative, belongs also the remarkable symptom-group which, about the middle of the last century, was first accurately described by the English physician, Addison, and to which, therefore, his name has been attached. The relation of this clinical picture to a welldefined disease of the adrenal bodies which is present in the majority of cases has governed the views of observers from remote times regarding the nature of the disease, and in spite of the many differences of opinion which have become prominent in the last decades regarding the pathogenesis of Addison's disease, the great majority of physicians maintain that a cessation of the function of the adrenals is the essential basis of the affection. It is also in harmony with this view that lately, by a transference of the practical question of the treatment of this affection by organotherapy, favorable expectations have been aroused so that many trials have already been made of this method.

But nevertheless, upon closer observation, the conditions do not appear so simple. Many of the clinical symptoms cannot be referred without further consideration to a disturbed function of the adrenals; a constant group of clinical and anatomical findings cannot be said to have been found, and thus an accurate weighing of the experiences gathered up to this time leads to the conclusion that the exclusive dependence of Addison's disease upon disease of the adrenals, although in many respects quite likely, has not been absolutely proven, and does not suffice fully to explain the pathogenesis of the disease.

Under these circumstances, it is instructive to investigate the most important points and ascertain whether or not the weight of evidence points to a direct dependence upon disease of the suprarenal bodies. In the following 
survey of the present status of our knowledge of the pathology of the affection, this problem is our chief interest. We hope clearly to demonstrate what the investigations of the last half century have contributed in explanation of the disease, and what is left for future studies to elucidate.

A brief description of the clinical picture must precede the rest of my discussion.

\section{CLINICAL PICTURE OF ADDISON'S DISEASE}

The description need be but very brief for, in the portrayal of the clinical symptoms, the first description given by Addison in 1855 is practically exhaustive, and many of the later observations have but supplemented this in certain respects.

All typical cases of the disease show (simultaneously or serially) the following four groups of symptoms:

1. General adynamia; 2. gastric and intestinal disturbances; 3. general nervous symptoms; 4. abnormal pigmentation of the surface of the body.

I here emphasize the conception of "typical cases," and do not believe it advisable in describing the nature of the disease to deviate too far from this conception. In particular, I do not believe that we ought to include as cases of Addison's disease (as has often been done of late) those in which the most conspicuous symptom, the abnormal pigmentation of the skin, is absent, even though examples of this kind (of which more will be mentioned later on) may be important in the explanation of the nature and the pathogenesis of the disease. The differentiation of characteristic symptom-pictures can advance our clinical knowledge only when it is carried out with the necessary consistency.

The signs of adynamia as a rule develop very insidiously, showing themselves in general malaise with exhaustion upon slight exertion, gradually increasing to extreme muscular weakness, particularly of the lower extremities, so that walking may become very difficult. These symptoms cannot well be referred to a profound disturbance of nutrition of the muscles, for, as a rule, neither very marked emaciation nor muscular atrophy is present, at least in the early stages of the disease. A weakened condition of the heart muscle is also apparent, particularly in advanced stages of the disease. The pulse is often very small, and, at least in the earlier stages, more often slowed than accelerated. Subnormal temperature $\left(95^{\circ} \mathrm{F}\right.$. or a little above this) has been repeatedly observed, particularly in the terminal stages of the disease.

The muscular weakness cannot be attributed to a marked anemia, for the microscopic examination of the blood (which will be referred to more in detail later) has generally indicated even in a late stage of the disease only a moderate diminution of the red blood-corpuscles and leukocytosis while some recent observations have shown a conspicuously large number of erythrocytes.

The disturbances referred to the digestive canal show themselves in the earlier stages of the affection by general dyspeptic complaints: Loss of appetite (often extreme), occasionally bulimia; tendency to nausea and vomiting, sense of pressure in the epigastrium; also disturbance of intestinal activity 
which more frequently leads to diarrhea than to constipation. With this very often, even early, there are marked pains in the abdomen especially in the epigastrium, in both lumbar regions, and in the right and left hypochondrium, intermittent at first, subsequently often becoming continuous. All of these symptoms increase late in the course of the disease, and often become so severe that after a time the gastrointestinal disturbances, with- stubborn vomiting and often steady diarrhea, rapidly decrease the strength.

The symptoms which may be referred to the nervous system are slight at the onset of the disease, of a general character, and limited for the most part to psychical apathy, tendency to headache and the like. Later, attacks of vertigo and syncope are usually observed, not infrequently also epileptiform attacks and somnolence. Death occurs most often after coma and convulsive phenomena.

The most conspicuous symptom of the disease in all well marked cases is the discoloration of the skin which has given the affection the designation of " bronze disease." Like the other symptoms, this usually develops gradually from a light yellowish brown up to a dark brownish black (mulatto color, negro color). It often begins simultaneously with the general asthenia, but may precede this, in some cases even by a long time (in one recorded case twelve years). The bronzing appears most often on the places which are exposed to the air (face, neck, hands), and on those which normally show most pigmentation (the axillæ, the areola of the nipple, the linea alba, the genital region), and thence distributes itself over the rest of the skin. Upon the uniformly pigmented surface there often develop later circumscribed, still darker areas; in some cases, on the other hand, we find zones which are without pigment (leukoderma).

The simultaneous involvement of certain mucous membranes in this pigmentation has very properly been emphasized throughout the literature of Addison's disease as especially important in the differential diagnosis from other discolorations of the skin. Almost invariably the mucous membrane of the mouth is affected, usually in the form of irregular bluish black spots, their seat being chiefly upon the mucous membrane of the lips and palate. According to my experience, the tongue deserves more attention than is usually given to it. In the cases seen by me it often shows in different areas a very conspicuous inky stain. Sometimes pigment areas are found upon the vaginal mucous membrane; exceptionally also upon the conjunctiva palpebrarum and scleræ and upon the vocal cords.

The pigmentation, as a rule, gradually increases in intensity throughout the course of the disease. Only exceptionally has a diminution been noted prior to death. Somewhat more often transitory remissions of the bronzing have been reported. I myself have seen in two cases a bleaching of the skin lasting several months (with a simultaneous general improvement) and later increased discoloration.

The course of the symptoms just mentioned is decidedly chronic; only exceptionally is the onset acute and progress rapid. The severer symptoms follow the milder; not infrequently they come on some months or weeks prior to death, with a certain suddenness: Two years may be mentioned as the 
average duration of the disease, as determined from a large collection of cases. The termination is always fatal; in the few cases which have been described as cured the diagnosis is doubtful.

To complete the description it may be stated that Addison's disease (particularly in North Germany) is quite rare, that with the exception of earliest childhood and extreme old age it attacks all periods of life quite uniformly, and is more frequent in men (60 to 70 per cent.) than in women. Important etiologic factors cannot be determined; in a number of cases prolonged psychical depression (care and sorrow) appear to play a rôle.

Besides those diseases with which the malady is often associated (tuberculosis, and, to a lesser extent, carcinosis) I must mention certain cutaneous complications which have become known by experience, among which are especially scleroderma, and in rarer cases prurigo, mycosis fungoides, etc.

\section{GROSSER PATHOLOGICO-ANATOMICAL LESIONS IN ADDISON'S DISEASE}

As regards the pathological anatomy of the disease numerous investigations in the last few decades have confirmed the findings emphasized by Addison, i. e., the coincidence of the symptom-complex just described, with disease of the suprarenal bodies. In the overwhelming majority of the recorded cases, a profound change in these organs with destruction of the greater part of the normal parenchyma has been observed. Furthermore, experience has shown that Addison's disease is not produced by any and all the pathological changes of the adrenals but that a definite form of disease of the organ is usually present, particularly a form of chronic tuberculosis, in which the parenchyma is rapidly destroyed, partly by caseous degeneration, partly by fibrinous contraction. The macroscopic changes according to age and distribution: In the majority of cases the adrenals are enlarged (occasionally to several times the normal); upon section the tissue in part shows inflammatory swelling with foci of caseous softening; in other areas, contraction and induration with hard, cheesy, or calcified deposits. In a small number of cases processes of contraction are predominant, so that the organ is smaller, occasionally so small that it can only be found with difficulty. The disease of the suprarenal bodies in the majority of cases is bilateral but, as a rule, not equally developed on the two sides. In the interior of the individual organ the cortical and the medullary substance are as a rule uniformly implicated, so far as can be noted at the autopsy. Tubercle bacilli have lately been found by many observers in the foci of disease.

In a small number of cases the adrenals show other forms of disease. Among these, according to the latest investigations and reports, comparatively the most frequent seems to be simple atrophy of the organ, a change which in some instances is so extreme that the adrenal appears to be absent. But it is very likely that some of these atrophic cases represent only the remains of inflammatory processes after the absorption of softened foci. In a still smaller number of cases malignant neoplasms (carcinoma and sarcoma) have been found in the parenchyma of the organ. Still more rarely other diseases, such 
as amyloid degeneration, gummatous changes, echinococcus disease, etc., occur. Finally, destruction of the organ by hemorrhage has been occasionally mentioned (in cases running an acute course).

An important point is that in a large number of the accurately investigated cases the neighborhood of the adrenals has also been found changed, so that as a result of adhesions and abnormal connective tissue changes (apparently the remains of circumscribed peritonitis) the adrenals have coalesced and become embedded in the neighboring parts. Among the areas thereby affected the filaments and plexuses of the abdominal sympathetic (above all, of the solar plexus and of the celiac ganglia which are situated in the neighborhood of the suprarenals) have quite often been found embedded in thick masses of connective tissue.

In the purest cases the changes to be found in the adrenals and in their immediate surroundings are the only abnormal autopsy findings. Frequently, however, other changes take place, among them (naturally and primarily) tuberculous lesions which attack the lungs in about one-third of the cases. But Addison's disease does not often complicate cases of advanced phthisis. Similarly malignant neoplasms of the adrenals may exist alone or be combined with tumors of other abdominal organs.

So far I have purposely considered only the simple typical cases of the disease. This method I should like to continue for the present, excluding the cases above referred to, which present the clinical picture of Addison's disease but show no changes of the adrenals. The question must now be asked: May we, and must we, in such pure cases with a typical symptompicture and well-developed disease or destruction of the adrenals, refer the former directly to the latter?

Before deciding this question, two other questions, it appears to me, must first be solved: (1) May the main symptoms of Addison's disease be produced by certain general conditions which are present in the affection? and (2) Can we believe with our present knowledge of the nature and function of the adrenals that disease of these may produce the symptoms of Addison's disease?

In regard to the first question a number of maladies must be considered in which, as a result of a profound anemia and cachexia, with the general symptoms of asthenia (and eventually also gastric and nervous disturbances), an abnormal pigmentation of the skin may develop. To this group belong certain cases of malarial cachexia, pseudo-leukemia, pellagra (Neusser), the so-called vagrant's disease which is produced by deprivation of all kinds, advanced stages of tuberculosis and carcinosis, and others. But it is not likely that any disease of the adrenals exists in these disorders. Moreover, the discoloration of the skin differs in these cases most distinctly from that seen in Addison's disease. In the diseases just mentioned it is usually of slighter degree than in Addison's disease, and is far less generally distributed; it rarely shows, as in the latter malady, the formation of dark areas on a background of diffusely discolored skin, and above all the accompanying pigmentation of the mucous membranes is absent. On the other hand, in the general diseases enumerated above the principal pathogenic factor is a 
disturbance in nutrition and a change in the composition of the blood, which is generally absent in Addison's disease: The anemia, as a rule, is only developed to a moderate degree in Addison's disease; the red blood count, even in advanced stages, is rarely below 3,000,000; the hemoglobin also has been often found comparatively high. The increase of the erythrocytes, which has been lately determined, is not constant and is still unexplained; in one case from $6,800,000$ to $7,200,000$ were found, and, shortly before death, 6,500,000. The frequently accompanying leukocytosis, with the relative increase of lymphocytes which has been recently reported, was not marked enough to constitute a characteristic change in the blood composition. The pigmentation seen in tuberculosis is not of importance, inasmuch as changes resembling Addison's disease occur only in its late stages; typical cases of Addison's disease, however, are rarely the accompaniments of advanced stages of tuberculosis.

In answer to the other question, what consequences are, a priori, to be expected upon destruction of the adrenals, it is necessary to briefly review the reports concerning the anatomy and physiology of these organs.

\section{ANATOMY AND PHYSIOLOGY OF THE ADRENALS}

Formerly the adrenal was assumed to be essentially a part of the nerrous system, and its medullary substance was described as consisting almost exclusively of ganglion cells. But the newer histologic investigations are unanimous in this, that the cortical substance as well as the medullary substance contains chiefly parenchyma cells which show a varying size and form in different areas, are arranged in groups inside of a connective tissue structure, and contain very abundant pigment, chiefly in the intermediary zone (Virchow). The rich blood supply of the organs has long been emphasized; this is shown partly by the comparatively great number of afferent and efferent large vessel trunks, and partly by the very dense capillary network (particularly in the medullary substance). The parenchyma cells are everywhere in intimate relation with the walls of the capillaries, according to some reports in part directly within them. Between the cells, excreted "colloid" masses have of late been frequently observed (Auld). All authors, even the most recent, agree that the organs (especially their medullary substance) are particularly rich in nerve elements, i. e., in non-medullated (sympathetic) and medullated nerve fibers as well as also in ganglion cells deposited in the parenchyma. Numerous nerves unite the organs with the neighboring parts of the nervous system, particularly with the semilunar ganglion and the other parts of the solar plexus; they have even been followed further, and traced into the splanchnic nerve, the vagus and the phrenic nerve. Some authors have described small ganglion cell groups situated upon the external part of the capsule of the adrenal.

The conception of the embryologic development of the adrenals differs with various investigators; the majority of observers believe that there is an intimate relation between the medullary substance (which develops separately from the cortex) and the (sympathetic) nervous system, either assum- 
ing a direct development of the adrenal medullary substance from the ganglion of the sympathetic, or a later growth from the sympathetic ganglion cells and nerve fibers.

In spite of many points which are still obscure the complicated anatomical structure of the adrenals gives us the impression that they are (ductless) secretory organs, constructed with a comprehensive innervation apparatus.

Still more complicated, and in many respects uncertain, is our knowledge of the physiologic function of the adrenals.

For a long time they have been classed with the well known glandular ductless organs, being designated as blood-vessel glands serving an unknown purpose. Particular attention was drawn to the adrenals by the first descriptions of the Addison symptom-complex, and this caused a deeper scientific interest regarding their importance, and the investigation of their function. And thus, soon after the publication of Addison's views, a series of experimental researches were begun which had for their purpose the extirpation of the adrenals in animals, and the observation of symptoms thus produced, in order to determine the functional importance of these organs.

But the difficulty of such investigations was soon shown in the uncertainty of the results. This gave rise to the important question, whether the adrenals were to be looked upon as organs necessary to life. This question was answered definitely in the affirmative by the first investigator, Brown-Séquard; in a publication in 1856 he stated that after removal of both adrenals all the animals died in a short time (one to two days) with prostration and severe nervous symptoms. But his reports were at first not confirmed by other observers; on the contrary, there soon appeared a number of communications (among which I shall mention only those of Philippeaux, Gratiolet, Harley and Schiff), according to which certain animals, in spite of the loss of both adrenals, continued to live for a long time (several months), and from which it appeared likely that the death of the experimental animals was produced by lesions of neighboring parts (peritoneum, abdominal nerve plexus, etc.). At this point I must refer to a series of experiments by Nothnagel, who produced bilateral contusion of the adrenals in a great number of rabbits, a few of which were still living eighteen months later and showed no gross lesions.

Thus the vital importance of the adrenals was for a long time a subject of doubt. But a series of new investigations substantiated the original opinion of Brown-Séquard. These (conducted by Tizzoni, Abelous and Langlois, de Domenicis, Cybulski, Szymonowicz and others) showed that in a number of different animal species (dogs, guinea-pigs, frogs, etc.) death invariably resulted after a complete removal of both adrenals.

The period before death varied (according to the animal and according to certain auxiliary conditions) from a few hours up to several months. By careful control experiments it was shown that only the loss of the adrenals could be the cause of death. An opposite result has been obtained in some investigations, very few of them recent. This contradiction may be explained in various ways: Either the observation of the animals may have been too brief, or the removal of the adrenals was incomplete. 
Finally, as an explanation of this problem (as of many others to be mentioned later) we must remember the presence of the so-called accessory or supplementary suprarenal bodies to which Marchand was the first to call attention. These accessory adrenals are found particularly in the neighborhood of the internal genitalia, which have been experimentally proven to be capable of decided hypertrophy after removal of the adrenals. In conclusion it has lately been regarded as settled that after complete removal of the adrenals a continued healthy existence is impossible.

More difficult is the decision of the question, what are the disturbances of function in experimental animals after removal of the adrenals?

In the first place a number of symptoms in these animals may be referred to general nutritive disturbance (mostly diarrhea); further some observers have noted lowering of temperature and lowered blood-pressure. Such symptoms are not consequences of loss of the function of the adrenals, as has lately been often affirmed. On the contrary, they may find sufficient explanation in the severity of the operation and in various auxiliary circumstances. It must be emphasized, too, that up to the present time, no animal experiment has been successful in producing unmistakably the principal clinical symptom of Addison's disease, viz., the pigmentation of the skin.

In some more extensive series of experiments a few of the animals lived for a longer time and some developed areas of pigment upon the mucous membrane of the mouth or nasal cavity; but it is doubtful whether in these exceptional cases the operation produced the pigmentation.

On the other hand more conspicuous symptoms relating to the nervous system were observed in the experimental animals. In those that soon succumbed there were convulsions, symptoms of vertigo, rotary movements, and the like; among the animals which lived longer, paralytic conditions, contractures, etc., developed. More profound pathologico-anatomical changes of the nervous system have been described only by Tizzoni. In his cases there were lesions in the central nervous system, particularly the medulla oblongata, the cervical cord and cerebellum, as well as in the abdominal plexus of the sympathetic, and certain peripheral nerve trunks. In animals that died quickly the central nervous system showed hyperemia with hemorrhages and cell infiltration surrounding the vessels; in the cases running a chronic course the changes were more in the form of column degeneration and atrophy of the gray substance. The starting point of the process was apparently the abdominal sympathetic. But nothing analogous to this has been reported by other experimenters.

Brown-Séquard believed that he could determine important changes in the blood of these experimental animals, particularly a decided increase of pigment with pigment emboli in the organs. From this observation he inferred an intimate connection between the adrenals and pigment formation in the body. But confirmation of these reports by other observers is lacking. Reliable results were furnished by prolonged investigations with the injection of the blood of epinephrectomized animals into healthy animals by Brown-Séquard and by later observers (particularly Abelous, Langlois, etc.). These experiments showed, after removal of the adrenals, a conspicuous toxicity of the 
blood serum so that when it was injected into animals one of whose adrenals had been excised death was produced or hastened. From the sum of these investigations the observers conclude that, after the operation, substances circulate in the blood which under normal circumstances are rendered harmless by the adrenals; that the latter are therefore protective organs in relation to certain toxic products of metabolism. Further consideration caused a number of these observers to emphasize the similarity of the symptoms to those of curare poisoning. This was true of the animals operated upon as well as those animals into whom the blood was injected (particularly in regard to the action upon the nerves). It was also determined that after inoculation of the blood and serum of dogs into other animals whose muscles were exhausted by tetanizing action was produced similar to that after the removal of the adrenals. Regarding the substances which appear to be neutralized by the normal function of the adrenals, these experiments justify us in believing that they are the toxic products of the metabolism of muscular activity.

Finally I must mention the researches of Jacobi, which appear to prove an intimate relation between the adrenals and intestinal peristalsis. And this influence must be looked upon as an inhibitive action exerted on the gut by the adrenals. Extirpation of the adrenals acted like division of the splanchnic and produced active intestinal peristalsis while irritation of the adrenals caused the intestine to remain quiescent. The adrenals accordingly are looked upon as branches of the splanchnic, and the inhibitive tracts controlling the intestine considered to be fibers which lead from the adrenals to the semilunar ganglions. It is true these investigations have not as yet been thoroughly confirmed.

In the reports of experiments up to the present time, therefore, certain points of support are found for ascribing to the adrenals an important function in the organism.

This conviction is strengthened and confirmed by other recent investigations, in which the attempt was made to introduce the active constituents of the adrenals in the form of an organic extract (watery, glycerin-containing, etc.) into animals by injection, and to study the action thus produced. I shall only mention a few of the most important, and the conclusions which have been drawn from them regarding the function of the adrenals:

After a few earlier observers had determined the toxicity of adrenal extracts, noting the effect of injections in different animals who showed symptoms of general body and nervous weakness, Oliver and Schäfer (1894 and 1895) were the first to make minute studies regarding this condition. They found as the most conspicuous action of intravenous injections of the extract (particularly that obtained from the medullary substance) a transitory but very decided rise of blood-pressure. Further investigations (cutting the vagus, injection of atropin, destruction of the medulla oblongata and of the spinal cord, etc.) proved that this rise in blood-pressure is due to a direct increase of vascular tonus and general muscle-tone (particularly in the heart and in the vessels). The extract, besides strengthening the cardiac action, also slows it. Adrenal extracts which were taken from cases of Addison's disease were inactive. The observers conclude: That the adrenals secrete a 
substance which reaches the blood, and gives to the muscles the degree of tension necessary for their functions.

In a similar manner Cybulski and Szymonowicz proved the rise in bloodpressure (by contraction of the vessels) and the strengthening of the cardiac activity resulting from injections of suprarenal extract. But upon further investigation (after severing the vagus and spinal cord, injecting atropin, etc.) they obtained results which appeared to favor, not a peripheral action of the extract, but an influence upon the central nervous system, particularly the vasomotor centers of the cord. They also found marked irritation of the respiratory center. They therefore assume that the secretion furnished by the adrenals to the blood is intended to maintain the tonus of the cardiac, vasomotor and respiratory centers. But the majority of the later investigations have favored the assumption of a peripheral action of the adrenal extract. Only v. Cyon believes from his investigations that the rise in bloodpressure depends for the most part upon an irritation of the vasomotor centers. On the other hand, the researches of Velich favor the view that the vascular contractions which he observed after injection of adrenal extract into the tongue, conjunctiva, ear, intestine and kidneys depend upon an influence of the extract upon the peripheral vascular apparatus as well as upon the co-operation of spinal vaso-constrictor centers. Biedl obtained, after complete. destruction of the spinal cord, marked rise in blood-pressure by intravenous injections of suprarenal extract, so that an irritation of the peripheral apparatus may be assumed. Gottlieb, according to whom the increase in bloodpressure depends principally upon a strengthening of the cardiac activity, after investigations in chloralized animals, refers this to an energetic irritation of the motor ganglion groups which dominate cardiac movement. Lewandowski has further demonstrated in a convincing manner a peripheral action of the injected suprarenal extract upon the smooth muscles of the eye and of the orbit (dilatation of the pupil, prominence of the bulbus oculi, etc.), which is parallel to the vascular action. Finally I must mention the local vaso-constricting action of the suprarenal extract which has been lately recognized, and which has been used therapeutically upon the conjunctiva (by advice of Darier), upon the larynx and nasal mucous membrane, and in intestinal hemorrhages.

In several of the investigations it has been proven that the adrenal venous blood acts similarly to the extract (Cybulski, Dryer). The rise in bloodpressure which is thus attained is usually very decided. In some cases it has been raised above $300 \mathrm{~mm}$. of mercury. After destruction of the spinal cord, which reduces blood-pressure almost to zero, it can be raised to $160 \mathrm{~mm}$. by injection, and by repeated injection the pressure can be maintained for a long time at from 90 to $140 \mathrm{~mm}$. (Biedl). By using a very pure extract recently produced by Moore and Purinton $\frac{1}{4000000}$ of a gram is sufficient to maintain in a dog a persistent rise in blood-pressure of from 20 to $40 \mathrm{~mm}$. of mercury.

Interesting observations have recently been made which show that injection of adrenal extract into the abdominal cavity of guinea-pigs causes a destruction of red blood-corpuscles and produces in the spleen and lymph-glands 
hemosiderin deposits similar to those which occur in the so-called hemochromatosis (a pigmentation of the skin resembling Addison's disease) (Foà). A recent experiment is also to be mentioned according to which the subcutaneous (or intravenous) injection of adrenal extract in animals produces glycosuria in the majority of cases (Blum).

From these experiments it appears that we must admit an "internal secretion" of the suprarenals, and must recognize that these organs furnish to the blood a substance which acts primarily to raise blood-pressure.

Endeavors to isolate and more minutely to characterize the substance in the adrenals which raises blood-pressure have not been lacking. These have lately led to definite and fixed, even though not always uniform, results. But these endeavors, like the earlier and frequently repeated chemical investigations of the adrenal parenchyma, are not calculated to advance our knowledge regarding the function of these organs. The most important experiments are the following:

The earlier investigations (conducted by Vulpian and Virchow, and dating from the time when the clinical picture of Addison's disease was first made known) were made with a substance which is found in the medulla of the adrenals and which, with iron chlorid, gives a blackish green color. These observers first called attention to the similarity of the pigment seen in the parenchyma of the adrenals (particularly in the intermediary zone) to that of the rete Malpighii in pathologic discoloration of the skin. Furthermore, observers have made frequent attempts to isolate a peculiar coloring matter from the parenchyma of the adrenals but without reaching unanimous results. Some writers emphasize the brown coloration of the tissue (particularly in the intermediary zone) which occurs with salts of chromic acid. Several others have noted small globular structures in the blood of the adrenal veins. These granules can be colored with chromic acid, and have been looked upon as products of secretion of the organs. A chromogen with different derivatives has been found by several observers in the parenchyma; by some this has been believed to be identical with hemochromogen (McMunn).

Among the substances which have been demonstrated in the parenchyma of the adrenals are leucin and myelin which were referred to in earlier reports, lecithin which has been found in large amounts (Alexander), a related body resembling jecorin which contains sulphur and phosphorus (Manasse), and neurin. All these are substances from the presence of which certain relations between the adrenals and nervous system may be inferred. The substances which give a green color with iron have been found to be chemically closely related to pyrocatechin (Krukenberg), and from the extracts of the medullary substances it is possible to isolate a compound from which, by boiling with hydrochloric acid, pyrocatechin can be produced (Mühlmann). These findings also indicate a relation with pigmentation of the skin without furnishing definite proof of it.

The "substance forming a green color with iron" and the "substances resembling pyrocatechin" are identical with the products which cause a rise. in blood-pressure, and for use as substitutes for the adrenal extract in animal injections have been produced from the organs by complicated chemical proc- 
esses. Of the numerous investigations of this character which have recently been conducted three must be particularly mentioned: One of these bodies has become known as "sphygmogenin" (Fränkel) and is a syrupy substance which shows marked reducing property, is easily oxidized, and with a watery iron chlorid solution gives a dark green reaction. Another substance named "epinephrin" has been obtained by Abel from a watery extract of the adrenals as a benzoyl combination. As a result of elementary analysis Abel looks upon it as an alkaloid belonging to the pyrrhol or skatol group, and obtains from it by treatment with dilute alkali a dark pigment. Finally, v. Fürth, after prolonged study, believes he has found a still purer body which he calls "suprarenin," and designates as a dioxypyridin having a composition of $\mathrm{C}_{5} \mathrm{H}_{7} \mathrm{NO}_{2}$ or $\mathrm{C}_{5} \mathrm{H}_{9} \mathrm{NO}_{2}$. This substance also raises blood-pressure upon subcutaneous injection. How far the previously mentioned bodies actually represent the important secretions furnished by the adrenals to the blood must be decided by further physiologico-chemical investigations.

After this brief description of the results which have been obtained from experiments and chemical investigation in regard to the physiologic action of the adrenals, we return to the question: Can the clinical picture of typical Addison's disease be explained from the lesion, that is, from the destruction of these organs?

Up to a certain point an affirmative answer may at once be given. We can reconcile many important symptoms of the disease with a gradual decrease and final cessation of function which, from the experience we have gathered, may be ascribed to the adrenals, and we can find abundant analogies between the condition of the person attacked by Addison's disease and the experimental animal that has been deprived of its adrenals.

First of all we recognize in the steadily progressive constitutional disease which leads to death the same consequences of the loss of vital organs which we found in the experiments. That the duration and course here correspond but little with those in the animal experiments is not surprising. Rarely does experimental disease in the animal accurately simulate the human disease, and still less so when, as in this case, the sudden removal of the organs from the animal (even should the extirpation in part be undertaken at two different times) is contrasted with a chronic disease in the human being with very gradual loss of function. The duration of the disease in the human subject is often many years-indeed disturbances of the adrenals are sometimes of such long standing that in the last periods of life a complete loss of function of the organ must be assumed. Nevertheless we can sometimes find analogy for this in the circumstance that for many months, even for years, it is possible to keep the experimental animal alive. The nature of the general disease in both cases is similar: In both, emaciation is present, but is not the predominant symptom; in both the signs of true anemia are usually of moderate degree. Against this the general asthenia, the permanent and increasing weakness of most muscle groups, becomes particularly prominent in the clinical picture, and reminds us strongly of the prostration which appears so constantly in the experimental animal. 
This asthenia in Addison's disease may also be alleged as valuable proof of the theory of many observers that the function of the adrenals mainly consists in the maintenance of the muscular tonus.

The cardiac weakness so frequently noted in Addison's disease, which numerous authors have also observed in the animals operated upon, may in some respects be regarded as a part of the general muscular weakness. Obviously this must be looked upon as an expression of the fact that a disturbance of the adrenals has decreased or abolished the formation of a substance which increases blood-pressure and strengthens the heart, and which according to the investigations we have mentioned must be ascribed to the parenchyma of the organ.

In regard to the manifold disturbances originating in the digestive canal and its surroundings, practical experience at the bedside coincides with experimental results and theoretic deductions: The majority of the gastric symptoms, such as general dyspepsia, particularly loss of appetite, gastric oppression, eructations, nausea, vomiting, etc., may be referred to the general affection with its adynamia, anemia, etc. The epigastric, hypochondriac, and lumbar pains which are never absent, and occasionally form the most distressing of the subjective symptoms, may be directly due to pathologic processes in the adrenals themselves. Experimental investigators have repeatedly demonstrated that the adrenal, particularly its medullary substance, is very sensitive to pain. The frequency, too, with which the adrenal affection attacks the surrounding areas, in which are found signs of old local peritonitis in the form of adhesions and connective tissue masses, certainly points to a similar explanation of these pains. But though we cannot be sure of the connection between these pains and the chronic and progressive inflammation, a terminal stage of the Addison symptom complex resembling peritonitis has of late been frequently described (Ebstein). To explain the intestinal symptoms, the same factors may suffice, but we may also call to mind the above-mentioned experiments which show a nervous connection between the adrenals and the intestinal ganglia and suggest that inhibitive nerve impulses are normally transmitted from the adrenals to the intestine, so as to control intestinal movements. The diarrhea which appears in the terminal stage of the disease is a particularly strong argument for this view.

It is less easy to ascribe to a simple adrenal defect some of the many nervous phenomena which accompany the disease. The general nervous symptoms and the milder forms of cerebral implication such as apathy, headache, insomnia, vertigo, attacks of syncope and the like may be related to the adynamic and anemic general condition. Nevertheless, these symptoms are often much greater than can be accounted for by the general asthenic conditions; for example, the apathy often changes into increasing psychical depression with disturbance of the mental functions, showing itself partly by somnolence, partly by irritability. To this may be added the neuralgic conditions often observed in the earliest stages, especially affecting the extremities and the joints, and finally, the usually spasmodic, often epileptiform attacks, accompanied by extreme irritability and delirium which lead up to the final coma of the later course. If this picture is considered, it corresponds neither to a 
general nutritive disturbance nor to a simple chronic intoxication; on the contrary, in many particulars it points to a direct implication of various areas of the nervous system.

Most difficult of all to understand is the principal clinical symptom, the bronzing of the skin. It must first be pointed out that in many investigations of extirpation of the adrenals in animals, even in those animals which were kept alive a long time, this symptom could never be produced. But too much stress is not to be laid upon this point, for there is justification for the view, shared by many observers, that the comparatively early death of the animals experimented upon may have checked the deposition of pigment. Further, we do not know whether abnormal pigmentation of the skin occurs in animals as readily as in man.

But neither do our investigations furnish anything which can be looked upon as proof that by destruction of the adrenals pigmentation of the skin is directly caused or even favored. We may concede most readily the possible truth of the theory (which is frequently expressed) that under normal conditions there is a substance circulating in the body which furnishes material favoring pigmentation, but which is rendered inoperative by the internal secretion of the adrenals. That the chemical investigation of the parenchyma of the adrenals has furnished no positive points of support for this has already been mentioned. Such a connection, too, is untenable without a demonstrable pathologic change in certain fluids of the tissues and in the blood; for such demonstration, however, there is no further experimental evidence, for example, in the blood and in the urine.

Of the blood it has already been stated that an estimation of the bloodcorpuscles and the amount of hemoglobin shows for the most part but slight change, and upon exact counting of the leukocyte varieties no important deviation from the normal has been found. In these investigations signs of abnormal amount of pigment of the white blood-corpuscles or of the serum, analogous to melanemia, have never been observed, nor does the finding of cells containing blood-corpuscles and pigment granules in the marrow of the tubular bones, mentioned in one case, permit positive conclusions. Nor is much added to our knowledge by certain reports, according to which there is a qualitative change in the blood with increase of reduced hemoglobin and appearance of methemoglobin (Tschirkoff).

Even slighter deviations from the normal were shown by the urine in most cases. Apart from polyuria, which is not infrequently noted, in some patients an increase in indican, and in one a decided increase of urobilin, has been found. In one case an abnormal coloring material (urohematoporphyrin) was determined spectroseopically (McMunn). Furthermore, there are reports regarding decrease of urea and creatinin. Opposed to this, however, are a large number of reports of cases in which a normal condition of the principal urinary constituents, particularly of indican, of urea or nitrogen, has been determined. A new amin base (Ewald) obtained from the urine in a single instance has not been satisfactorily explained.

Nevertheless while there is no evidence which points with certainty to a direct importation of the pigment from the adrenals to the skin, the possibility 
of a process of this kind cannot as yet be excluded. For the formation of pigment in the organism is a complicated process, and no less so its deposition in the tissues, particularly in the skin. Regarding the latter process we are quite well informed by recent investigations, which shall briefly be referred to here.

According to these, the pathologic deposition of pigment in Addison's disease occurs in the same manner as physiologic discoloration of the skin (in dark races, in pregnancy, etc.) which is apparently distributed in an analogous manner also in all species of animals. The main seat of the pigment particles, all observers who have undertaken microscopic investigations of the bronzed skin find to be the cells of the lowest layers of the rete Malpighii. The previous view that the pigment development here is autochthonous (by metabolic activity of the cells) has been almost wholly abandoned. On the contrary, quite a number of recent investigators (Renaut, Nothnagel, Riehl, v. Kahlden and others) have proved conclusively that the pigment from the deeper layers of the corium is brought to the rete cells by motile cells, and that the pigment most probably originates in the blood. The findings resemble one another very much in individual cases; almost always, beneath the pigment layer of the rete, wandering cells are to be seen carrying pigment through the corium; in the deeper cutis layers these cells often accumulate in large groups around the vessels, partly also in their adventitia. A disease of the walls of the vessels has been described in several cases, in which swelling, cellular infiltration and small hemorrhages were conspicuous (Riehl), but this has not been discerned by other observers, and does not appear to play a rôle in the process. The mucous membrane of the mouth shows conditions analogous to those of the cutis.

It is obvious that this process of pigment-deposit in the skin favors the assumption of an abnormal coloring material admixed with the blood as the result of adrenal disease, a chromogen, for the existence of which, however (as I have previously said with emphasis), our knowledge up to this time gives no points of support. It must also be remembered that if there were such an over-flooding of the vascular system with pigment it would be very difficult to explain why no deposits of pigment are found in any areas of the internal organs. At any rate, it is obvious that besides the hypothesis of the transportation of pigment from the blood to the superficial layers of the skin we should also consider the possibility that the disease may be due to an implication of the nervous system, upon whose vasomotor and trophic tracts the walls of the vessels and the cells of the cutis are dependent to a high degree. Evidently the unusual formation of pigment substance from the hemoglobin (normal or modified), and the increased activity of the cells transporting the pigment, can scarcely occur without the nerve elements being subjected to an abnormal irritation. Also many irregularities of the pigmentation of the skin occurring in Addison's disease, particularly the not infrequent occurrence of leukoderma plaques, as well as the combination with other trophic cutaneous disturbances (sclerodema), point to varying local processes of innervation. Finally, it is not without significance to remember that in many species of animals characterized by conspicuous staining of the shin (chame- 
leons, frogs, etc.) the dependence of "chromoblasts" upon the nervous system (Raymond) has been proven.

Thus the most conspicuous symptom of Addison's disease points with more emphasis than any of the others to an implication of the nervous system. Therefore we are forced to the conclusion that even in the most typical cases a conception of the clinical picture as due to a cessation of suprarenal function is beset with difficulties.

The perplexities of explanation increase materially when we leave the realm of purely typical cases of Addison's disease, and consider the deviations which the clinical symptoms and pathologico-anatomical findings present under some circumstanices. Unfortunately, these variations from the normal type of the disease are not very rare. I mean by this, not the many quantitative differences in individual symptoms whereby it often occurs that one symptom, for example, gastro-intestinal phenomena, becomes more marked than all others, or that the bronzing of the skin is very intense in the early stages of the disease, while at other times, toward the termination of the affection, it is only very moderate, that at the autopsy the adrenals, contrary to rule, are found with lesions of fresh degeneration, and in part present well retained parenchyma and the like. All these variations depend upon the duration, course and complications of the disease, and partly upon auxiliary conditions which cannot be controlled. Much more important are the instances of a lack of coincidence between the main clinical symptom, the pigmentation of the skin, and the important factor of the pathologico-anatomical picturethe degeneration of the adrenals. Such exceptions were noted soon after the publication of Addison's fundamental description, and have multiplied in the course of years, so that many modern authors deny wholly the parallelism between the pigmentation of the skin and adrenal disease.

Two groups of cases are here to be distinguished, and by most observers have been noted separately: The cases in which in well developed Addison's disease with bronzing no affection of the adrenals has been shown, and upon the other hand, advanced changes in the adrenals without discoloration of the skin and even in some cases without any severe symptoms. The frequency of such exceptions naturally cannot be seen in a small group of cases, and full compilations have up to the present time been very rarely made (Averbeck, Greenhow, G. Lewin). Among the earlier reports of this kind Greenhow's statistics are to be referred to, in which among 172 cases of undoubted Addison's disease, disease of the adrenals was absent in 10 . The most complete compilation at present is that of Lewin, which includes 561 autopsies; according to this, typical Addison's disease with changes in the adrenals is noted in 88 per cent., without lesion in 12 per cent.; on the other hand there was adrenal disease with bronzing of the skin in $7_{2}$ per cent., without such in 28 per cent. of the cases. Now it must be stated, once for all, that most statistics of this kind which refer to carly cases are unreliable on account of the lack of definiteness in the descriptions of the pathologico-anatomical conditions of the adrenals. In some cases disease of the adrenals has doubtless been overlooked, in others erroncously assumed. Nevertheless the number 
of these deviations from the supposed pathology of Addison's disease is too great for us to ignore.

For us the exceptional cases in which the clinical picture of Addison's disease does not correspond with the adrenal affection are of the greatest importance. They are the ones which even in early years caused doubts to arise regarding the direct dependence of the clinical picture upon disease of the adrenals, and soon caused investigators to search for another explanation of many of the pathologico-anatomical findings. That the first point of attack was usually an investigation into the nervous system is not surprising when we consider the location and intimate connection of certain parts of the nervous system, particularly the abdominal sympathetic plexus, with the adrenals. At autopsies, special attention has apparently been directed to these parts by the frequent extension of the disease of the adrenals to the surrounding areas, often in the form of circumscribed inflammation and connective tissue development, more rarely in the form of tumor formation, particularly in important nerve areas. Thus in the last decades a great number of anatomical investigations, partly superficial, partly very thorough, have been made of the nervous apparatus surrounding the adrenals, and a great number of results have been obtained, the most important of which will now be briefly sketched.

\section{PATHOLOGICO-ANATOMICAL CHANGES OF THE NERVOUS SYSTEM IN ADDISON'S DISEASE}

The parts of the nervous system most frequently investigated, and most frequently found diseased, are the semilunar ganglia of the solar plexus. These are naturally implicated by a cireumscribed peritonitis or insidious connective tissue inflammation developing from the adrenals; and thus we find a decided percentage of cases in which these ganglia are reported as adherent to their surroundings, enclosed by connective tissue proliferation, or embedded in cicatricial tissue. It may also be assumed that these conditions are even more frequent than the records show since in the protocols of the earlier observers, adhesions and peritoneal remains have often been reported as present in the surroundings of the adrenals without the sympathetic plexus being mentioned.

In other cases it is stated that in spite of the connective tissue embedding, normal nerve elements have been demonstrated in the plexus; yet it remains questionable whether these findings included all areas of the nervous organs, and also whether nerve elements pressed upon by tissue of this kind may be looked upon as functionally normal. Such connective tissue proliferation is closely analogous to a compression of the sympathetic plexus by tumors (tuberculous, carcinomatous, or leukemic) or by aneurism of the aorta.

With or without such disease of the surrounding tissue, the semilunar ganglia have shown many macroscopic and microscopic changes. The macroscopic alterations have been noted either as. enlargement, thickening and swelling (and at this not always of the ganglia themselves but frequently also of the nerve bundles extending from them to the adrenals) with hyper- 
emia and abnormal succulence; also decrease in size, atrophy and induration. In some cases the ganglia have been implicated in a process of caseation (tumor formation is also mentioned). Histologic investigations have shown the signs of fresh or older inflammation and degeneration with their consequences, particularly interstitial connective tissue proliferation, cellular infiltration and thickening of the capsules of the ganglion cells and nerve sheaths; moreover, abnormal pigmentation of many tissue elements, fatty degeneration and pigment atrophy of the ganglion cells (occasionally reaching a very high grade). Finally there are observations of various forms of neuritis, atrophy and degeneration of the nerve fibers traversing the ganglia and proceeding from them. Conspicuous vascular changes (thickening and hyaline degeneration of the walls of the vessels, accumulation of cells and hemorrhages in the adventitia) have also been noted, particularly in the areas near the adrenals ( $\nabla$. Kahlden).

The same changes (thickening, contraction, degeneration of ganglion cells and nerve fibers) have also been frequently found in other parts of the solar plexus.

Various observers note far-reaching degeneration of the splanchnic nerve, partly in the form of fresh or older neuritis, partly as gray degeneration of the medullary fibers, the latter sometimes as consequences of compression; for example, by an aneurism of the aorta (Jürgens).

Similar neuritic disease has been found in the sympathetic system, somewhat removed from the adrenals, particularly in the boundary column of the sympathetic and in its cervical ganglia. The former has often been found conspicuously thickened and indurated, the latter decidedly swollen, and showing inflammatory changes. Fleiner was able to follow similar processes of the disease through a large part of the sympathetic nervous system, and also in two cases in the central nervous system. These findings are the more convincing as in both cases they were quite similar, while the nature of the disease differed decidedly (one was dependent upon tuberculosis of both adrenals, and the other upon sarcomatosis of an adrenal and its surroundings). In both instances a chronic process of inflammation could be demonstrated which extended from the diseased adrenals through the semilunar ganglion to the boundary column of the sympathetic with its thoracic and cervical ganglia into the pneumogastric, and was particularly well developed in the intervertebral ganglia of the posterior thoracic and upper lumbar portions of the vertebral column. In all of these areas there was found diffuse connective tissue proliferation, or focal accumulation of round cells in the neighborhood of vessels; besides this markedly intense pigment atrophy of the ganglion cells, and extensive degeneration of medullary fibers in the sympathetic, the splanchnics and also in the vagus. In the spinal ganglia the nerve fibers showed advanced degeneration, most markedly in the nerve branches leading to the sympathetic, less so in those leading to the posterior roots of the spinal cord. Finally, the same degenerative processes were found in a number of peripheral nerves, chiefly in the sensory cutaneous branches.

In the spinal cord the same observer found no important changes, but such have repeatedly been described by others (Burresi, Babes and Kalindero, etc.), 
and especially in the dorsal cord. The changes consist partly of hyperemia and round cell accumulation, partly of sclerosis with thickening of the neuroglia and of the walls of the vessels, also atrophy of the ganglion cells and neuritic processes in the spinal roots (particularly the posterior ones).

It must also be mentioned that by some authors special stress is laid upon disease of the small pericapsular adrenal ganglia which are frequently implicated by the local process.

Many of these reports have been met by the criticism that the importance of the pathologico-anatomical changes has been exaggerated, and that many of them do not go beyond what we have learned to recognize by more recent investigations as the consequence of general nutritive disturbances produced by many forms of anemia, cachexia and the like in the elements of the nervous system. This criticism can only refer to the mild degrees of such changes, as the atrophy of the ganglion cells and nerve fibers, the pigmentation of tissue elements, etc.; and I do not intimate that the importance of changes such as these is to be denied. It is, however, different with the severer findings reviewed above, in which, as a rule, advanced degenerative conditions of the cellular elements are combined with conspicuous infiammatory processes of the interstitial tissue. Such changes must be counted among the characteristic findings of the disease in question, and must be considered in judging of its pathogenesis.

It is true that our limited knowledge makes it difficult to estimate the relative frequency of such pathologic nerve findings. Judging from the material at hand, we cannot look upon them as constant. For quite a number of reports are diametrically opposed to those just mentioned, and show that, in the investigation of certain areas of the nervous system, no abnormality had been found. It is true a large number of these communications are far removed from being susceptible of general proof. First, there are some of them, particularly dating from earlier periods, in which the anatomical investigation regarding the more minute relation of the nerve elements was insufficient. It need only be mentioned here that in many of the earlier cases it was decided macroscopically whether, for example, the semilunar ganglia were of normal size, color, consistence and the like. Then the majority of these investigations refer only to small areas of the nervous system; few among them follow completely the important central parts of the sympathetic system, and investigate whether the neighboring areas of the central nervous system are also implicated. In the overwhelming majority of cases only the semilunar ganglion and solar plexus have been examined; much more rarely the splanchnies and the boundary column between the dorsal ganglion and the cervical ganglion of the sympathetic have been examined. The intervertebral ganglia, upon which lately special stress has been laid, as well as upon the spinal roots and the spinal cord, have been but very rarely investigated except in the few cases mentioned above. It may be added that these negative reports (as well as the positive nerve findings) occurred usually in cases in which the adrenals showed no conspicuous change.

Here, therefore, are flaws and doubts which can be removed only by continued and thorough histologic investigations of a large number of additional 
cases. Nevertheless, in judging these cases impartially, the impression is received from the reports which have been published regarding the condition of the nervous system, especially of the sympathetic, in Addison's disease, that there is decidedly more in favor than against the presence of important disease of these tissues. This opinion coincides with the results of a later English compilation (Thompson); here among $7 \%$ records which included minute histologic investigations of sympathetic nerve areas, important changes were noted in 60 .

The question must now be propounded whether, by the aid of these anomalies of the nervous system, determined in a large series of cases, we can better explain the atypical cases, in which, with true Addison's disease (clinically), no demonstrable adrenal affection is found to correspond. This question must without doubt be answered in the affirmative. As regards the bronzing of the skin it has already been indicated that for its development we must assume an implication of the nervous system, especially vasomotor and trophic functions, even in the cases with typical pathologico-anatomical findings. A disease of the essential parts of the abdominal sympathetic and neighboring nerve areas, and particularly changes in these processes with increased formation and transportation of pigment into the region of the cutis layers, may be very readily conceived. In this connection we may point especially to the spinal ganglion, whose influence in producing trophic changes of the skin, according to clinical and experimental investigations, has at various times been especially noted.

If then no additional knowledge concerning the connection of the adrenals with pigment formation in the body is obtained by further investigations, the pigmentation of the skin in typical Addison's disease (as well as in cases without demonstrable adrenal affection) will therefore probably be found related to changes in the nerve areas in question. What then is the relation of the other symptoms of the disease to the lesions of the nervous system? May they also under these circumstances be referred to nervous disturbance? Up to a certain point such an explanation does not appear unlikely: the characteristic lumbar and epigastric pains of Addison's disease are almost the same as those which have long been referred to irritation of the abdominal sympathetic ganglion. Furthermore the intestinal symptoms may be explained by irritation or paralyses of the abdominal nervous system, particularly of the splanchnic nerve, and the cachexia and other general symptoms may be in part referred to nutritive disturbance due to changed gastric and intestinal functions. Naturally the picture of this general disturbance (even without considering the pigmentation) remains a peculiar one, such as we are not accustomed to see in most cachexias. And if we look upon the symptoms of the disease as a result of disturbed suprarenal function (particularly the characteristic muscular weakness), there would be no barrier for the assumption in these cases of a functional disturbance of the adrenals as a consequence of disease of the nervous areas which influence glandular activity.

That such a disease of the nervous system, without (or only with a secondary) implication of the adrenals, is capable under certain circumstances 
of producing the picture of the disease, is evident from the rare cases which have been cited in which compression of the solar plexus, of the splanchnic nerves, etc., by tumors (aneurisms and the like) has been the basis of Addison's disease. But how often a similar affection of the nervous system is also present (perhaps late, perhaps from the outset) in the cases running their course with adrenal affection, cannot be decided at this time on account of our insufficient knowledge regarding the nervous findings. The usual course of the common affection will probably be this, that the affection, mostly inflammatory, starting from the adrenal attacks the surroundings; that is, first, the semilunar ganglion, later, the other parts of the sympathetic, eventually, the cerebrospinal nervous system. The frequency of this course of the discase is suggested also by the fact that those adrenal lesions in which there is an insidious inflammation or some similar process which attacks the adjacent areas are especially apt to cause Addison's disease. To this category belong especially the tuberculous affections of the adrenals, most of which probably depend upon atrophies due to older inflammatory processes; also certain tumors which cause metastases. With the majority of single tumors of the adrenal glands, therefore, there is no Addison's disease, and in the rare cases of adrenal tuberculosis without Addison's symptoms, disease in the surroundings of the organs has, as a rule, been absent.

Whether, however, the form of disease with the characteristics of Addison's disease, which, starting from the adrenal attacks the surrounding areas, does not also perhaps cause certain changes within the affected nerve areas so that they may develop independently, we cannot say on account of our lack of pathologico-anatomical knowledge. It must be assumed that under some circumstances an analogous but independent disease of the nervous system may take the place of adrenal disease and produce the same clinical picture entirely without implication of the adrenals or with only a late involvement of the glands.

Physiologic experiments have failed to determine the matter in question. It is true that repeated experiments by extirpation (also cauterization) of the semilunar ganglia have been attempted without, however, furnishing definite conclusions: Previous experiments of this kind (Foà) have been entirely without result; and in a more recent series of investigations (Lewin and Boer) in animals which lived thirty days or longer after the operation, only paresis of the intestines, usually with diarrhea, but with no pigmentation or other characteristic changes, was noted.

The researches next to be mentioned relate chiefly to cases of Addison's disease (with autopsy) in which the adrenals were not implicated, but, on the other hand, certain nerve areas were attacked. A few other cases remain in which the autopsy shows no important changes in the adrenals and none in the nervous system. The number of such cases is small, and from this group many must be excluded in which the diagnosis is questionable. of those remaining it is not quite certain from the reports that all of the nerve areas were considered in the investigation and that it was sufficient to exclude any disease. Whether, and how often, cases of this kind exist, minute histologic examination of similar doubtful cases must in the future decide. If we 
admit their occurrence, they cause great difficulty in explanation; for to assume a functional disturbance of the affected nerve areas, and perhaps also of the adrenals, without any anatomical substratum, would be very unsatisfactory. It may be assumed, however, that cases of this kind will always remain rare exceptions.

The group of cases in which adrenal disease is present without Addison's symptoms, particularly without bronzing of the skin, does not belong, as has already been stated, to the disease we are considering. Nor does it offer important reasons to vitiate the views which have been expressed above. In the first place, we usually find in such cases affections of the adrenals which are in marked contrast to typical cases of Addison's disease: The majority of them are tumors of the adrenal glands and the absence of Addison's symptoms is here the rule; thus a recent compilation of 26 cases of primary malignant tumors of the adrenals showed that the clinical picture of Addison's disease was invariably absent. In most of the other cases of this group it remains uncertain whether by tumor formation or other disease of the parenchyma of the adrenals the destruction is as marked as is the rule in most cases of tuberculosis; the areas surrounding the glands also appear to remain comparatively free. Finally, in explanation of these cases, an abnormally great development of the accessory adrenals may be thought of.

I believe the considerations above adduced will suffice to make it obvious that, according to the pathological and clinical material at our disposal, the clinical picture of Addison's disease does not depend exclusively upon a gradual destruction of the adrenals, nor exclusively upon a disease of the nervous system (i. e., the abdominal sympathetic and other nerve areas intimately associated with it), but that both these factors are simultaneously active in the development of the symptom-complex - a view in which I coincide with a number of earlier as well as more recent investigators (Fleiner, Neusser and others). How these forms of the disease merge into each other is very difficult to determine, and varies evidently to an extreme degree. A particularly frequent connection, according to the views mentioned above, appears to be a kind of ascending disease, which spreads from the adrenals to the neighboring, and, eventually also, to the more distant nerve tracts; that, however, opposite (descending) processes are also at work is at least likely, and has been mentioned previously. Moreover, there need be no parallelism between these pathologic processes; on the contrary, in well advanced changes of the adrenals very slight implication of the nerves may be found, and vice versa; in quite a number of cases according to present experience, the disease of one or both parts may be entirely absent. By this variation in the relations of the diseased areas, we may very readily explain the great variation in the intensity of individual symptoms and in the time of their appearance, so that, for example, the bronzing of the skin may occur years before the general symptoms, or, inversely, the general disturbance may exist for a long time without pigmentation.

If a definition of the pathologico-anatomical basis of Addison's disease is desired it may be given in the following form: The disease depends upon an insidious, partly inflammatory, partly degenerative affection (most frequently 
dependent upon tuberculosis), which impticates first the parenchyma of the adrenals and, secondarily, certain nerve areas which are in intimate relation to the adrenals (particularly the abdominal sympathetic and some nerve areas connected with it, perhaps also certain portions of the spinal cord). Since disease of both of these regions may be combined in various ways, and may also compensate for one another, the peculiar general affection develops which represents what is characteristic of Addison's disease.

I believe it is impossible to explain in a more definite manner the nature of this general disturbance on account of the missing links in our present knowledge. From experimental investigations, and also from the clinical observations which have been outlined, it is evident that from disease of the adrenals a deleterious element appears in the organism, and many theories have been offered to explain the nature of Addison's disease. Almost every one of these is plausible up to a certain degree, but not proven; and almost all explain only a few of the important phases of the clinical picture. Which of these is to be accepted as the most reasonable is merely a matter of opinion in the present status of our knowledge. I need indicate only a few of these: They may be divided in general into. the "toxic" and "nervous" theories, according to whether the changes in the adrenals or those in the nervous system have been assumed to be the more important. Of the first group, recent experiments have made it seem probable that after extirpation of the adrenals the development of substances resembling muscle poisons takes place in the organism. They assume accordingly that the normal "antitoxic action," which is said to be a function of the adrenals, declines or is entirely absent; hence the poisons circulating in the muscles and tissues are able to exert their harmful influence. According to the views of others, the diseased adrenal itself is supposed to furnish the toxins which, by their circulation in the fluids of the body, produce the general affection. Other theories which consider a regulation of cell nutrition to be the normal function of the adrenals ascribe the disease to the development of toxic products by an abnormal activity of the tissue cells which thus leads to the picture of a general intoxication. The theory is plausible that, in consequence of the absence of the normal function of the adrenals, a substance is missing in the fluids which should give to the muscular fibers and other tissue elements the tonus necessary for their normal functions. Opposed to this are the theories that the disease is one of the nervous system, especially of the sympathetic, and that Addison's disease arises as part of a general trophoneurosis. Such views emphasize vasomotor disturbances and an unequal distribution of blood in the body, whereby there occurs a hyperemia in the area of the splanchnic nerve with anemia of the peripheral parts; but these views are opposed by the absence in many cases of well developed anemia. Between these groups of opinions are earlier views according to which nothing but a general cachexia (which by some is designated as a tubercular cachexia) is said to be the basis of the clinical picture. The disease is sometimes designated as an "anematosis" (Pepper) depending upon a profound disturbance of blood production, but this view cannot be accepted because of the small number of cases which show blood changes. Many other theories might be mentioned. 
But I must repeat that the endeavor to propound such theories is, for the present, not worth the labor. On account of the uncertainty which still exists regarding the function, not only of the adrenals but also of individual areas of the sympathetic nervous system, there is but little hope of following the complicated pathologico-anatomical changes which produce the symptoms of the disease. I believe it, therefore, to be correct to adhere to this view in the explanation of the pathologic process: That the disease depends upon a pathologic modification or cessation of the normal relation to the organism of the necessary functions of the adrenals and of the nerve areas which are intimately connected with these structures.

The scant investigations at hand are naturally still open to doubt, and may be modified or rejected after later researches. Whether, in the future, we must be content with an explanation such as has just been given, or whether we shall be able to determine the more minute pathogenetic processes in Addison's disease depends upon the results of continued investigations. In my opinion, judging by what has preceded, these researches must be based less upon physiological experiment than upon increased and minute histologic investigation, especially in the areas of the nervous system that are implicated; we also need research in the field of the chemisiry of the adrenals, of the blood, and of metabolism. By histological study the question of the constancy of a lesion of the nerves in Addison's disease will be solved; by chemical research the probable implication of the adrenals in pigmentation of the skin will have to be decided. For histological investigation, the atypical cases without disease of the adrenals will be of the greatest importance. As such cases are particularly rare, these advances in our knowledge will be very gradual, and it will be all the more necessary, therefore, for us minutely to investigate each available case in the future.

\section{DIAGNOSIS}

A few words regarding the diagnosis of Addison's disease may be added, particularly as the view has lately been expressed that diagnosis is not possible during the life of the patient. Such a view refers of course only to the determination during life of the pathologico-anatomical changes which are expected in the adrenals, or (and this we must emphasize according to our experience) in their surrounding areas, especially in the sympathetic nervous system. The clinical picture of the disease in question, even when it does not present itself in typical form, is usually not difficult to recognize. It has previously been stated that this affection is very similar to many other discolorations of the skin combined with cachexia, for instance, to certain forms of tuberculosis, carcinosis, malaria, pellagra, vagabond's disease, and the like. These affections, under some circumstances, may show similarity to the Addison symptom-complex. But a longer clinical observation of most of them will lead to a correct conclusion, since in these discases (as has been mentioned above) the pigmentation differs by certain irregularities, and by the absence of implication of the mucous membranes, from true bronzing of the skin, and, further, because the symptoms directly referable to the suprarenal region 
are absent. Above all, the correct etiology may usually be recognized in the other cliniical conditions. If, however, these pseudo-Addisonian affections can be excluded, and if the true Addison symptom-complex is present, we must remember the presence of changes which, according to experience, are frequently present; viz., caseation of the adrenals, a presumption which may be confirmed by the determination of tuberculous disease in other organs, particularly a modcrately advanced pulmonary tuberculosis. In a similar manner, in a small number of cases, secondary symptoms which favor carcinosis or kindred affections may, perhaps, be combined with a palpable tumor in the adrenal region, this directing the diagnosis to a malignant growth of the adrenals. Only where both these forms of adrenal disease are unlikely will the assumption of any other and rarer form of adrenal disturbance be justified. In all cases it must be remembered that, besides disease of the adrenals, an affection of the surrounding nerve areas, particularly of the abdominal sympathetic plexus, with a greater or less distribution to the central nervous system, may be present; that moreover under some circumstances, these diseases of the nervous system may take the place of the affection of the adrenals; and that, finally, in rare exceptional cases, both may be absent. That the finding of the latter cases, however, is not opposed to the clinical diagnosis, "Addison's disease," I need not reiterate.

On the other hand, the cases of profound adrenal degeneration without the symptoms of Addison's disease, particularly without bronzing of the skin (which are not very rare), will generally be impossible of diagnosis. That these do not belong to Addison's disease, in the strict sense of the term, I have already stated. Lately it has been proposed to differentiate them by the term "adrenal insufficiency." Only in instances in which the disturbances point definitely to the adrenal region, and particularly where palpation shows a tumor or other abnormal condition in this area, can this type of disease of the adrenals be suspected. Where this is not the case, the picture of a general cachexia is present, a picture which, as a rule, is not characteristic enough to separate it from other constitutional disturbances.

\section{THERAPY}

The doubts which envelop the pathogenesis of Addison's disease will naturally also appear in the views which dominate the treatment of this affection. Accordingly we note from the time at which the clinical picture became known up to within the last few years but few endeavors to adopt a specific or methodical treatment of the affection, and these few have not been further developed. Thus, by some observers, remedies such as faradization, hydrotherapy, amyl nitrite, nitroglycerin (on account of its action in raising blood-pressure) and transfusion have been employed and advised without having obtained general recognition. In some of these instances, and by these methods of treatment, a decided improvement is said to have resulted, and some cases have been reported even as "almost cured," and to have died only during a relapse. There are intercurrent improvements to which I have already referred when discussing the spontaneous tendency of the disease to a fluctuat- 
ing course. In these remissions a cessation of all the clinical symptoms not infrequently precedes the fatal terminal stage, but the improvement cannot be looked upon as the effect of treatment. However, it is quite likely that such spontaneous remissions may be favored and increased by active symptomatic treatment and general hygiene.

That up to the present time no cure of a case of Addison's disease has been authenticated has already been remarked. The few cases which have been assumed to be such by their observers are doubtful as regards the diagnosis. Under these circumstances, the opinion of Potain, who is said to have seen a case cured and who believes that the disease in one-tenth of the cases is curable, is incomprehensible. Similar doubts are expressed in regard to a case recently reported (by Oestreich), which is looked upon as an "operative cure" of Addison's disease, and which I shall briefly mention: In a woman whose pulmonary apex showed the symptoms of an old focus of infiltration, and who suffered from marked cachexia, there was difficulty in digestion, gastric and lumbar pains, and a tumor the size of a small apple was found in the gastric region. This was removed, and after a good recovery from the operation the symptoms of the patient disappeared, strength increased, and in the following nine months she was looked upon as well. The tumor was found to be a tuberculous, degenerative adrenal organ surrounded by a fibrinous covering; the medullary substance of the organ was almost entirely destroyed. That in this case extirpation of the tuberculous adrenal led to recovery cannot be doubted; but unilateral, adrenal tuberculosis with cachexia and gastric disturbance is not Addison's disease; and that the patient without an operation would have succumbed to Addison's disease is open to doubt.

Thus, until recently, the symptomatic treatment of the clinical symptoms has been the only therapeutic indication in Addison's disease, and besides observation of the gastric and intestinal symptoms (in which we were warned quite properly against the use of extremely active or drastic remedies, as by this means uncontrollable diarrhea may be produced), on account of the prominence of the asthenic phenomena, the use of tonics was the main indication. Iron and arsenic here played an important rôle, and I must mention that in the two cases observed by me the longest quiescence of the disease with transitory improvement of all the symptoms, also of the discoloration of the skin, occurred after the continued use of Fowler's solution in large doses.

An advance occurred in the treatment of the affection when the study of the internal secretion of certain organs, above all of the so-called blood-vessel glands, was combined with an attempt to introduce organotherapy. In fact, a malady such as Addison's disease, in which evidently in the majority of cases the main and occasionally the only known pathological basis consists in the destruction and gradual functional deterioration of a blood-vessel gland necessary to life, appeared to be particularly suitable for the modern therapy of compensation. Theoretic and experimental investigations, above all the trials with injections of suprarenal extract in animals, already mentioned and described, show the conspicuous and important actions of this secretion. I shall only add that in these animal researches the value of adrenal therapy was in part directly confirmed: thus in experimental animals, after bilateral 
renal extirpation, life could be prolonged for twenty-four hours by injections of suprarenal extract (Andersson and Hultgren). Moreover, experience proved that in disease of the thyreoid gland, which may be looked upon as an analogous condition, by a simple substitution therapy, the deleterious consequences of the absence of the thyreoid could be combated, and a fatal general affection, such as myxedema, could thus be transformed into a harmless condition. Therefore, it is not surprising that in the last eight or nine years, following the English physicians, various investigators have attempted to employ suprarenal therapy in Addison's disease, in which either the gland in substance or preparations obtained from the glands were introduced into the body. Besides the amelioration or cure of the affection, they hoped in this manner to demonstrate, more conclusively than by the animal experiments, the direct dependence of the disease upon the disappearance of the adrenals, and also to show that the physiology and the pathology of the adrenals were directly analogous to the thyreoid gland and athyreoidism.

Unfortunately the results of suprarenal treatment in Addison's disease have not justified these hopes. No cure of the disease in this way has been recorded up to the present time. Yet a certain number of cases have been reported in which by this treatment transitory improvement followed, and was so conspicuous that it could not be disregarded entirely. Among these cases, perhaps the most convincing are those in which, after cessation of the specific treatment, an aggravation of the general condition occurred, and, eventually, pigmentation of the skin followed; of these, two are particularly worthy of mention:

Thus Schilling (1897) reports the case of a boy, aged fifteen, who was ill for two years and showed quite decided bronzing. For three months raw adrenal substance was administered to him (a fresh gland from a sheep or lamb daily). During this time an almost complete disappearance of the abnormal pigmentation occurred (only a slight discoloration upon the mucous membrane of the mouth remaining) and an increase in strength and of weight (33 pounds) so that he was looked upon as almost well. After a three months' cessation of the treatment, and following sudden symptoms of intoxication, death occurred (perhaps in consequence of a beginning pneumonia). The adrenals were contracted almost to cicatrices, and permeated by caseous foci. More striking, perhaps, is the case observed by Edel (1900): A locksmith, aged thirty-three, in whom the symptoms of rapidly increasing weakness and bronzing, particularly of the face, dorsum of the hands, and penis, had existed for six months without showing any remission. Suprarenal tablets were administered (one gram each, two given twice daily), and after only two to three days his strength began to increase, so that after fourteen days he was able to return to work; moreover, the bronzed areas of the skin began to improve from the third day of treatment, and five weeks later hands, face and penis, with the exception of isolated areas, were again of a normal color. After a month and a half of improvement, tubercular meningitis set in and caused death in seven days. From the beginning of this complication the adrenal tablets had been stopped, the bronzing reappeared, and a short time prior to death had almost completely returned. The autopsy showed, besides 
distributed caseation of the glands, a tuberculous nodule in only the right adrenal, while the solar plexus and the semilunar ganglion were embedded in callous connective tissue.

In the study of such examples the previously mentioned tendency of certain cases of Addison's disease to spontaneous remission with consequent fatal exacerbation is to be remembered. But it must be admitted that here the rapidity and the complete amelioration of the symptoms appears to be out of proportion with what is observed in the spontaneous course of the disease. Particularly, in the second case, the complete recurrence in a few days of the dark bronzing of the skin can only be understood by assuming a preceding artificial modification of the pigment metabolism of the body. It appears that these two cases, and a few similar ones, are sufficient to prove the possibility of a favorable influence upon the principal symptoms of Addison's disease by the artificial introduction of suprarenal substance.

It is true that we have records of quite a number of cases opposed to thesecases in which suprarenal treatment had no influence upon bronzing of the skin, nor upon the general symptoms. And from the reports at hand I believe that the number of these negative cases is at least equal to those in which positive results have been obtained. Sometiries indeed a direct aggravation of the symptoms has been noted from the beginning of this treatment. Nor has it been possible to prove by chemistry that metabolism in the organism is decidedly influenced by the introduction of adrenal substance. For even though one investigator (Pickardt), after a nine days' research in metabolism in a case of Addison's disease, during which time suprarenal tablets were administered, found an increase of albumin decomposition, in an analogous research by another investigator (Senator), nothing abnormal could be determined. In the case of two dogs who were fed with adrenals (with the raw gland as well as with the glandular extract) similar experiments showed no decided change of the nitrogen balance (Blum).

Perhaps the form and amount in which the adrenal substance is administered is important for its supplementary action. Apart from a few unsuccessful or disastrous investigations with the implantation of animal adrenals under the skin, the glands have been administered in a natural condition (as fresh as possible), usually chopped up with necessary additions. They have been occasionally given in a dry condition, as adrenal powder, but in most cases they have been used as extract, which in rare cases has been subcutaneously injected, but has generally been given internally in the form of adrenal tablets (particularly in England, but also in Germany and other countries). The doses administered varied. according to the reports, with the fresh adrenal gland, from one to two glands daily; with the extracts, an amount corresponding to $0.2-4.0$ of the dried glandular substance. It will be noted that the best method of introducing the glandular substance has not been definitely determined. Many further observations will be necessary to decide this. The opinion expressed by some authors (for example, by Lewandowsky) that the administration of the raw glandular substance is more effective than the exhibition of the extract appears to be confirmed by a number of clinical histories. But it must be pointed out that in the second of the cases men- 
tioned above the conspicuous intercurrent improvement occurred after the use of adrenal tablets. Whether an intravenous injection in man of suprarenal extract, which has been advised, will produce a more intense action than other methods, and whether it will not also produce dangerous symptoms, appears to be questionable.

That the results by means of adrenal treatment in Addison's disease are no more definite and gratifying than those mentioned above is not surprising, nor can they be looked upon as final. For, in the first place, the number of investigations is much too small to determine this therapeutic question. I estimate the number of cases that have been accurately reported as much less than 100; and in most of these it must be remembered that there was opportunity for suprarenal treatment only for a short time, or only very late in the course of the disease, so that the condition was an unfavorable one for therapeutic results. Therefore a long continuance of observations will be necessary before a definite opinion can be given regarding the value of organotherapy in Addison's disease, and to decide whether the apparent favorable results in individual cases will be guarantecd by further observation.

But even if organo-therapeutic results should not improve with increasing experience, this circumstance cannot be used to nullify the importance of the adrenal function in the pathogenesis of Addison's disease. The therapeutic introduction of adrenals from animals, or of extracts obtained from these, can only substitute in a very feeble way for the secretion furnished by the living gland in the human organism. Action similar to that which is exerted directly by the adrenals upon the fluids of the body can hardly be expected from the peripheral absorption (through stomach, skin, etc.) of the finished products, especially since the latter, as all physiologico-chemical investigations have shown, are subject to great destruction in the tissues of the body. Comparison with the remarkable action of the extract of thyreoid gland which, even in the first investigations of substitution therapy was shown to cure myxedema, should not lead us to expect similarly easy and surprising results in all analogous investigations (even although they appear to be pathogenetically well founded), since the physiologic and pathologic conditions in many other organs are certainly much more complicated than in the case of the thyreoid gland and its functions.

As regards the influence of organotherapy in Addison's disease, we must content ourselves for the present with stating that the experiences which have been gathered are not sufficient to permit a conclusion. They are also insufficient to determine the likelihood of improvement or cure, or to decide theoretically whether or not the artificial introduction of adrenal secretion is a proof of the adrenal theory in Addison's disease. But neither do the reports at hand require that we should cease to hope that further and more exact therapeutic investigations will furnish better results.

From this, future observers may learn the practical lesson to test the adrenal treatment of Addison's disease upon every opportunity. If we begin the treatment in individual cases as early as possible, and compare the influence of the various modes of administration, we shall gradually be able to determine what to expect from this method of treatment, and to recognize 
whether there is any well founded hope of successful substitution therapy in Addison's disease. As in the pursuit of pathologico-anatomical knowledge of the disease, so here a long time may elapse, as cases of the disease which are especially suitable for favorable treatment are very rare in almost all countries.

Thus we see that in the realm of practical therapy in Addison's disease much is still obscure and much must be left for further investigation.

If we now return to the question at the beginning of this article, whether according to present knowledge we may declare that Addison's disease may be explained by an absence of the adrenal function, we must give the answer already indicated more than once, that for several reasons this is impossible. The numerous experiments which have been carried out in clinical, anatomical, physiological, chemical and therapeutic realms in the last half century to determine the basis of the disease have given us much knowledge regarding the clinical picture as well as in regard to many properties of the adrenals. But, as we have seen, important links are absent for the explanation of Addison's disease as an affection of the adrenals alone. Hence, in disease of the gland in combination with that of other organs, particularly of parts of the nervous system, it now appears impossible to find this explanation without separating the rôles of these parts in the development of the definite clinical symptoms. This conception will not appear strange to those who have endeavored to explain the disease as a physiologico-chemical consequence of adrenal disease; and I believe it to be more satisfactory than the subjugation of the pathologic process to a restricted theory which, at least for the present, leaves several phases unexplained. Whether this view will be accepted in the future, or whether the adrenal theory in a purer form will be found sufficient for the explanation of the disease, will depend upon the results of future experience and investigation. That these researches, together with our therapeutic endeavors, will in my opinion aid in the further explanation of the disease upon a pathologico-anatomical and also upon a chemical basis has already been indicated. Thus continued investigations in the interesting clinical picture of Addison's disease, and the elaboration of the previous observations, are bequeathed to the next decades of the new century as an object worthy of their labors. 


\title{
A C ROM E G A L I A
}

\author{
By C. BENDA, BerLIN
}

THE erroneous idea that the gigantic is to be looked upon as an expression of prehistoric times-primordial-is firmly rooted in the human mind. When we think of the seas and forests primeval as inhabited by monsters like the plesiosaurus and the megatherium we readily conclude that a primordial race of gigantic people must have represented the survivors of the animal kingdom who vanquished these leviathans. We are content with the rôle of dwarfed epigones, and in the specimens of unusual size occasionally seen we acclaim the revival of this primordial power. Science has proven these fantasies to be incorrect. We can prove, or at least infer, that prior to these giant beasts, as well as to-day, there existed prolific races of smaller beings. Prominent scientists assume that the original forms of the vertebrate animals were dwarfed forms. Nothing conflicts with this hypothesis, and much favors the probability that the apelike proanthropos also, and some at least of the earliest races of man, were dwarfs, even though other primordial races may have early attained a larger structural development. Thus our views regarding giants have been fundamentally changed, since it is unlikely that giants are to be considered as atavistic or primordial types. In the year 1872, Carl $\nabla$. Langer first proved by careful studies that besides a normal form of giant skulls a pathologic one may be distinguished. To-day the opinion is expressed that giant growth is commonly the result of a pathologic process.

This change of conception is intimately associated with our increasing knowledge of that process of pathologic growth which is the subject of this article-acromegalia. The investigations of Pierre Marie in the year 1886 are to be regarded as the foundation for this knowledge. Although cases of the disease had been observed prior to this time and to some extent well described, for instance, by the Italian Verga, it was, as Sternberg says, the labors of the French investigator that introduced the disease into the clinic from medical monstrosity and curio cabinets. Marie characterized the affection by means of the peculiar symptoms which had also been noted by other observers, and of which now one, now another, proved most interesting. $\mathrm{He}$ also worked out the principal clues for the differentiation of this process from other affections which had some symptoms in common with it. Marie deserves still further credit for giving to the disease its characteristic, euphonious, and easily remembered name, for we must remember how readily the human mind adheres to a scheme, and by adopting a distinctive name grasps the conception which the name embodies. Nevertheless there is an impor- 
tant point, not brought out in the name, which must be borne in mind if the pathologic process and the autopsy finding are to be accurately remembered: It is not the size of the extremities which represents the most striking symptom of the disease, as might be concluded from the name; it is the enlargement, the abnormal, disproportionate growth of these parts. If Cyrano de Bergerac, besides his celebrated nose, had had large hands and feet, regarding which history probably gives no reliable reports, this would not have been a sufficient reason for calling him acromegalic, provided he had had these deformities from birth, or by heredity. In contradistinction to some descriptions (even very recent ones) of "unquestioned" acromegalic symptoms in the bony skeleton, it must again be pointed out that the characteristic symptom of the disease is not the unusual form of certain parts but their advancing change, and that in any case in which anamnestic and clinical data in regard to this symptom were absent only the existence of all the other factors and the resemblance to the picture of acromegalia can be considered presumptive evidence. This is a concession to clinical medicine which requires a certain amount of self-denial on the part of the pathological anatomist, but the historical honor must in this case be accorded to the clinician who founded the conception of the disease.

By Pierre Marie's pioneer labors, by the long series of his thorough investigations and by those of his pupils, as well as by a great number of clinical and pathological researches in which the most prominent scientists have taken part, such an exhaustive description of the disease has been given that it may almost be regarded as complete. Excellent comprehensive treatises, among which those of M. Sternberg are to placed in the front rank, have disseminated this knowledge throughout the medical profession.

\section{ETIOLOGY}

The tendency to this disease is an unfortunate prerogative of early maturity; one-half of all the known cases develop in the third decade of life. Less frequently cases occur between the thirtieth and fortieth years, and a few begin later; rarely has an onset before the twentieth year been observed. Special stress must be laid upon the point that the disease, although usually beginning after the body has attained its normal growth, nevertheless occurs at a time of life when there is still decided plasticity of the body, and the form thus presented must be separated from that of the true senile period.

Both sexes are affected to the same extent, but in the cases that begin late a decided predominance of women has been noted. The disease is quite rare. I believe that, including the four typical cases upon which I held an autopsy; I have had more cases than any other pathologist. These are included in about 8,000 autopsies which I have seen in a service of eight years in the Urban Berlin Hospital ; curiously, three of these cases occurred in a period of thirteen months from March, 1896, to April, 189\%, and only one occurred before or after this period.

As may be seen from the previously mentioned observations of $v$. Langer, there are obvious relations between acromegalia and giant growth since it 
appears that acromegalia most frequently attacks individuals characterized already by great bodily size. According to Sternberg 20 per cent. of the "giants" are attacked by acromegalia. Among my four cases two were above medium size. But, as is noticeable from the figures, the relation is not a necessary one. At this point I should like to call attention to the fact that in the skeleton of a dwarf which is in the Berlin Pathological Museum, and which I had an opportunilty of-examining through the kindness of Professor Waldeyer, I found suspicious acromegalic stigmata, so that, with all due regard to what has been expressed above, I may presume that even dwarfs are not exempt from the disease.

The cause of the disease is enveloped in obscurity. Of course trauma and psychical emotion, as in all diseases which involve the nervous system, are mentioned as starting points by the patients and their relatives, and are suggested as causes of the affection.

\section{SYMPTOMS}

The onset of the disease is accompanied by very various neuropathic phenomena. There are reports of extreme lassitude, of drawing pains and paresthesia in the head and in the extremities; in short, mild symptoms such as almost every patient, whatever his disease, will admit if they are suggested to him, such also as almost any healthy person will also acknowledge. Of importance in women is the invariable cessation of menstruation, and in some males the appearance of impotence.

The peculiar changes, as a rule, are first noticeable in the face. A distortion of the features occurs which renders the patient unrecognizable to his nearest relatives, and this in a few cases can also be objectively shown by photographs taken prior to the disease if the pictures and the patient are brought together for comparison. I must further take this opportunity to warn physicians against photographs of acromegalic patients. I regret that I must sow the seeds of doubt in what the laity believe to be the absolute art of photography, but I am a photographer myself, and I know how the products of this art are attained. Besides pathologic acromegalia, there is also (as I must reveal) an artificial one, which in portraits by novices in photography is almost epidemic. This always occurs when a picture is taken of the entire sitting figure, full face, with arms and legs extended. This depends upon a simple perspective effect due to the use of photographic objectives of short focus, and to our habit of placing the object at a very short distance from the camera as is necessary to obtain large pictures. The first cause of this faulty perspective is the misplacement of the object, which is seen in almost all pictures of acromegalics that are known to me. In such pictures full face reproduction, similar to that of the statues of Egyptian kings, has almost become typical. How far the other effects of this faulty perspective extend can only be determined by pictures in which the distortion, even of a single extremity, is recognizable at once, as in a picture in my possession, in which the extended great toes are almost as broad as all the rest of the foot. The photographer has unconsciously intensified the impression of the abnormal- 
ity that is to be depicted; if he had used a normal hand as a comparison for the pathologic condition he would have perceived that the former, to make the comparison possible, would have to be brought the same distance from his apparatus as the latter, and not be left perhaps half a yard behind, as was the case in the picture portraying the condition of the feet.

But, to return from this digression, aside from this little photographic trick, a practised eye is not necessary to note in the advanced stages of the disease the very characteristic condition of the face. The prominent superciliary ridge, with the bushy eyebrows which occasionally almost meet in the median line, gives the face a threatening expression; the swollen lips, the very prominent zygomatic arch and the lower jaw give a somewhat bestial expression, and the enormous nose with its grim humor forms the only redeeming feature.

A similar and progressive deformity in the extremities gradually becomes noticeable. The feet become plump, larger, and broader, and the hand which is deformed till it is like a bear's paw appears especially conspicuous. This impression is further increased by the comparative slenderness of the lower arm. The X-ray picture shows that the bones are covered with exostoses; these are particularly noticeable at the end phalanges of the fingers and toes. According to Pierre Marie two forms of alteration of the hand are to be differentiated, one of which consists in a general enlargement, the other in a thickening of the bones of the hand. In the latter the exostoses are more prominent.

Regarding the enlargement of the internal organs, one above all others becomes clinically conspicuous, and must be counted among the most important and constant symptoms of the disease-I mean the tongue, which may assume extraordinary dimensions. In some cases closing of the mouth is no longer possible. Enlargement of the thyreoid gland is often recognizable.

Of great importance, but not always present, are the symptoms which point to a tumor-like enlargement of the hypophysis. These symptoms are principally those of a basal cerebral tumor, but from the description of the autopsy finding we shall readily understand that these symptoms need not occur, even in very large tumors of the hypophysis, so long as the tumor does not invade the cavity of the skull. In many cases the symptoms in question are, however, well developed. They consist, first, in severe headache which occurs in paroxysms, vertigo, vomiting, decrease of intelligence and, in particular, somnolence. The direct pressure of the tumor of the hypophysis acts most constantly upon the optic nerves, or upon the optic chiasm, and shows itself in hemianopsia, amblyopia even to amaurosis, due to atrophy of the stretched and compressed optic neryes. More rarely the muscles of the eye are implicated, especially those supplied by the oculomotor nerve. At this point I must also mention exophthalmos which has been observed in many cases, and which is usually referred partly to the change in the bones of the orbit, and partly to enlargement of the bulbus oculi. I believe, however, that the fact that acromegalic exophthalmos occurs periodically may best be explained by the assumption that it is not due to organic changes in the 
carity of the eye, but to stasis in the sinus cavernosus, as might readily occur from the pressure of a hypophyseal tumor.

How many of the general nervous disturbances are to be referred to the tumor, and how many to an independent disease of the nerves cannot be accurately determined. But among other symptoms I must here refer to the prominence of general nervous phenomena, such as pains, weakness, sensations of heat and the like. An important rôle is played by increased secretion of sweat, polydipsia, and polyuria, which in many cases are probably the sign of a co-existing diabetes. Occasionally, however, they certainly occur without this condition. In the further course of the disease a conspicuous loss of strength is observed, and nephritis and myocarditis often supervene, so that we find the picture of a severe general intoxication. Death results in such cases either from paralysis of the heart or from bronchopneumonia. A remarkable and frequent complication of the disease is diabetes, which, for example, occurred in two of the four cases observed in the Urban Hospital, and in one of the patients was the cause of death by coma. It is questionable whether the diabetes of acromegalics is to be ascribed to the previously mentioned symptoms of intoxication, or whether it is directly due to the tumor of the hypophysis. I incline to the latter view, for in the two cases in which the largest tumors of the hypophysis were found by us it was present, and disease of the pancreas was absent. Not infrequently the tumor of the hypophysis causes death by edema of the brain. Finally, in a disease which runs such a slow course it is not remarkable that intercurrent affections in no way related to the main disease frequently cause death. For example, in one of our cases a perforating peritonitis, complicating cancer of the stomach, led to the fatal issue.

The symptoms most dangerous to life are the immediate effects of pressure from the tumor of the hypophysis, and those which present the general symptoms of intoxication. This is favored, as I may mention here, from a clinical standpoint, by the great importance of disease of the pituitary body, and also by the fact that in all the cases mentioned in literature in which death occurred in a short time without any intercurrent affections, the phenomena of the tumor of the hypophysis were most prominent in the clinical picture, and the autopsy always showed an extremely large tumor. Even though one may not agree with Sternberg's view that these cases are due to a special type of tumor (see below in the account of investigations by Hanau and myself), the claim of Sternberg must be recognized that these cases are to be differentiated as a "malignant" form of acromegalia. My only objection is that Sternberg defines this differentiation by such an arbitrary mark-the duration of the disease for four years. I do not doubt that at least one of our cases, the case of A. Fränkel, who after six years succumbed to the disease without complications, must also be included in this "malignant" group. Another of our cases of the same duration would also be included here if we assume the fatal diabetes which existed in this case to be a symptom of the tumor of the hypophysis, as I unquestionably do. It is somewhat arbitrary, therefore, to divide the other cases, as proposed by Sternberg, into an "ordinary" and a "benign" form, as they may last thirty, or even up to 
fifty, years. It appears to me more rational to regard as malignant or acute all cases which pursue an uncomplicated and progressive course leading to death. In this type of disease the cases on record show that a duration of about six years is the rule. It is best to regard as benign or chronic all cases in which death is probably caused not by the acromegalia but by complications which are not connected, or but slightly connected with the main disease.

\section{PATHOLOGY}

A somewhat wider, but by no means complete knowledge of the nature of this remarkable disease has been obtained by autopsies and microscopic investigations, of which quite a number are already at hand. Beginning with the skeleton, we shall first direct our attention to the bones of the hands and feet, which form the nucleus of the astonishing anomalies of growth that appear during life. It is unquestionable that the bones in many of the cases that have been described showed great enlargement. A case lately reported by 0 . Israel is a prominent example of this kind. I believe, however, that in most cases a sensation of slight disappointment cannot be suppressed when the skeleton of the hand or foot is compared with our recollection, or, better, with a plaster cast of the fleshy member. Above all we are then convinced that the most prominent disturbance of proportions in many cases has almost disappeared, and only minute comparison with normal skeletons will enable us to note the abnormalities. The flattening and irregularities of the end phalanges, which at the onset attract attention, as Sternberg correctly states, are but slightly different from the normal skeleton, but it appears to me that in acromegalia, they are found somewhat more constantly than in normal skeletons. In a foot in our collection the second phalanx of all the toes from the second to the fifth is somewhat elongated. Otherwise, however, only the insertions of the muscles and tendons are roughened, thickened and covered with osseous proliferations. It is these which at first sight give the impression of gross deformity. Upon minute investigation we discover smaller exostoses, irregular porosities of the cortical layer, bony outgrowths upon the ends of the joints and the like, but we must admit that even all of these collectively cannot produce the picture of the "bear's paw," as we see it in life. The same impression is conveyed on examining the long tubular bones of the extremities, as well as those of the trunk. Everywhere the important point is the decided roughness of the insertion of muscles which have proliferated; otherwise the bones show only moderate change in form. As an exception I must mention the clavicle, which in most cases of acromegalia shows a decided increase in bulk. But we must not permit ourselves to be led astray by occasional investigations of individual bones. One femur in our collection shows enormous thickness, but it belongs to Stadelmann's case, and must be correctly looked upon as a combination of acromegalia with gigantism, and may be entirely independent of the former disease.

The vertebral column occasionally shows ankylosis of the vertebral bodies by bony bridges, but the commonest lesions are the hyperostoses and exostoses of the vertebral processes, which perhaps form the main substratum of the 
frequently observed kyphosis of acromegalics, unless, as Arnold supposes, it is habitual and due to the weight of the head.

The lesions in the bony skull are of quite different significance. The roof of the skull shows no gross changes except early obliteration of the sutures and flat exostoses. Upon the base of the skull the sphenoid bone is of chief interest, and always shows more or less deformity due to the tumor of the hypophysis. Its upper surface almost always shows a widening of the fovea hypophyseos, with disappearance of the dorsum sellæ. In other cases the floor is decidedly deepened and eroded, so that the sinus of the sphenoid bone is laid bare above. In several cases reported in literature, and in one of our collection, the usure also involves the partition and the floor of the sphenoid cavities so that a continuous bony defect is seen at the base of the skull. Otherwise the inner surface of the cavity of the skull shows no constant changes. The lower surface of the occipital bone is usually markedly deformed by exostoses at the insertion of the muscles. In the skull in our collection just mentioned, the external occipital protuberance hangs like a bent plug. The frontal bone is greatly thickened, particularly at the squamous base, so that the superciliary ridges stand out prominently. This thickening, however, is not solid. It is combined with a widening of the air spaces which may spread to such an extent that the frontal sinus extends throughout the greater part of the squamous portion of the frontal bone. In the bones of the face it is particularly the increase in size of the zygomatic arch and the lower jaw which, corresponding to the deformities determined during life, appears in the bony skeleton. Besides the gradual thickening and elongation of the lower jaw anteriorly, exostoses at the insertions of the muscles are noted. The upper jaw is much less often involved in this elongation; hence the lower jaw protrudes. These changes which are clearly noted only in the skeleton show that the shape of the living acromegalic's skull must not be called recedent and prognathous, as was common before Sternberg corrected the view. In a skull in my possession the alveolar process of the incisor teeth is implicated so that by an anterior curvature of the lower teeth it is opposed to the projecting lower jaw.

The microscopic examination of the diseased bones shows only that we are not dealing with a specific process, but with a deposit and absorption of osseous substance occurring in the same manner as in normal development. In this connection it may also be pointed out that according to the results of Langer, Klebs, and Sternberg, the mysterious influence of the disease upon certain portions of the osseous system may at least be in part understood when we recognize that some of the changes are secondary to other and more prominent alterations. This may be most clearly recognized upon the head. "The marked development of the apparatus of mastication requires a strong foundation upon the head. This may be furnished through thickening of the solid supports, or by dilatation of the hollow supports, or by an extension of the weight over a larger surface. The first requirements are fulfilled by the hypertrophied zygomatic arches, the second by the widened air spaces, and the third by the general increase in the size of the bones of the skull" (Sternberg). 
This author also attributes the changes in the vertebral column, in the clavicle, in the muscular and ligamentous insertions upon the occiput to one predominating factor, the increase in weight of the head. This view is more reasonable than that which explains the pathologic process as due to a general irritative growth of bony tissue. There is another beautiful proof of Sternberg's theory which, so far as I know, has not been noticed by former investigators. The pneumatic spaces in the bones of the skull are produced by an absorption of the bony substance. The bone is of the same type as that found in the so-called "functional" bony structures which are best recognized in the spongiosa, and which become modified by the slightest pressure or the slightest added weight of the bones so as to produce a structure suited to the mechanical necessities of the situation. Since the functional structures in acromegalia (the frontal sinuses) develop so enormously, we may look upon this as a proof that the new formation of bone in these cases, unlike that in leontiasis ossea, or in general hyperostosis, is not due to a pathologic osteoplastic activity, but is an adjustment to special requirements of the bone.

If, then, we accept Sternberg's view, and suppose that such a secondary bony proliferation accounts for the hypertrophy of the zygomatic arches, at the base of the skull, in the vertebral column, etc., then the hyperplasia of the apparatus of mastication, especially of the lower jaw, would have to be assumed as the primary change. I, however, see no reason to stop here; I think we may assume that the hyperplasia of the lower jaw is itself secondary, since this bone, too, shows throughout a uniform extension, so that its deformities may be explained as chiefly the effect of an increased strain on the insertions of the muscles and ligaments.

In this case the actual primary change would consist in an enlargement of the soft parts, and especially of the tongue, which may be considered responsible for the position of the jaws from the fact that by its increased volume, for which the oral cavity is no longer adequate, it exerts pressure upon the jaws. In fact the macroglossia is a characteristic feature of the clinical picture, and to this I should like to ascribe a dominant rôle in producing the change in the face, including the soft parts, namely, the lips and the nose. The tongue has repeatedly been examined microscopically by others as well as by myself. A prominent part in the hyperplasia is made up by the mucous membrane, which is completely covered with wart-like proliferations of the papillæ which are also distributed in the sub-papillary layers. Within the muscular strata broad, abnormal, connective tissue strands appear. Whether the musculature itself is increased cannot be decided. From the examination of my preparations I do not, however, concur in the view sometimes expressed that degeneration of the muscular fibers is present. I was able, by new staining methods, to show the transverse striation with such clearness that I have utilized the preparations of acromegalic tongues as objects with which to demonstrate transverse striped muscles. I must assume, therefore, that degeneration of the tongue muscles, like that more frequently mentioned in the muscles of the body, must be accounted for as one of the late marantic phenomena of the affection.

With Strümpll I shall mention the changes in the soft parts of the ex- 
tremities, as well as in the face, which have also been noted by previous investigators, and shall regard then as a feature more prominent than disease of the bones. I have already mentioned how slightly in some cases the bones take part in the enlargement. The principal mass of the so-called "bear's paw" is formed by the soft parts, so that here the conviction is almost forced upon us that the enlargement of the bones, the thickening as well as the elongation, arises only secondarily as a support for the increased mass of the soft parts, and that in the exostoses at the insertions of the ligaments and muscles we may also recognize the reaction of the enormous increase in weight which is to move the extremities. The hypertrophy of the soft parts, as we may convince ourselves both macroscopically and microscopically, is due to extremely tense connective tissue strands richly permeated with elastic fibers. These first thicken from the cutis itself downward. Afterward, enlargement of the papillæ and thickening of the epidermis become visible. The connective tissue proliferation is most massive in the subcutis, next in the deep fatty layers of the hands and feet. Each fat globule appears to be surrounded by its own thick connective tissue capsule, and connective tissue forces its way also between the fat-cells in thick layers, although we cannot recognize any decided atrophy of the fat-cells. In one of my cases I found the fat globules permeated in many places by a loose fibrous connective tissue rich in mucin. Nevertheless, by their outlines and by isolated, well preserved fat-cells, the distribution of the lobules is still easily recognizable, also their sharp line of demarcation from the tense connective tissue of the surroundings. This remarkable condition of the fatty tissue accounts for the fact that, in fresh sections, it exudes like a compressed filling, and gives the impression, described by several authors, that the skin is too scant for its contents. The sweat-glands and nerve trunks of the cutis and of the subcutis have their own special thickened layers of connective tissue, and we may note in the cutaneous nerves, what Arnold first described, viz. : that connective tissue forms between the individual fibers. The degeneration particularly described by P. Marie and Marinescu, I could find in only one of the cases investigated by me, but I do not doubt that it is frequently a natural consequence of the previously described alterations. The same may also be said of the muscles, in which also the hypertrophy of connective tissue is probably the primary factor and degeneration the occasional consequence. Besides these diffuse changes, fibromata mollusca, papillomata, and abnormal pigmentation are also observed. The sebaceous glands and the hair remain quite normal, nor was I able to find any decided abnormalities in the ressels.

\section{PATHOLOGICAL ANATOMY}

Of the pathological findings in internal organs I shall first briefly mention a number of the less constant ones. The internal organs may be implicated in the hypertrophy so that splanchnomegalia has been spoken of. We cannot, however, determine in these findings in how far the observers were dealing with simple gigantism, and how far it was a progressive enlargement caused by acromegalia. This doubt applies to the large kidneys and livers 
of many acromegalics. In other organs, as in the heart and the spleen, pathologic enlargements occur, yet they must not be looked upon as consequences of the acromegalic process, but as independent diseases.

Corresponding to the clinical symptoms, the frequently determined hyperplasias or degenerations of the internal female genitalia and of the testicles are perhaps in intimate relation to the pathologic process, while the cutaneous parts of the external genitalia are frequently implicated in the acromegalic enlargements.

In the pancreas, connective tissue hyperplasia has been described several times. On account of the frequent complication of acromegalia with diabetes, we might easily interpret this as a result of the acromegalic process. But in the cases investigated by me, particularly in the one combined with diabetes, the pancreas was perfectly normal, while, in another case in which connective tissue increase was present, diabetes did not exist.

The central nervous system, i. e., the base of the brain, may be involved directly and to a marked extent by the tumor of the hypophysis, particularly the infundibular region, the pons, and the optic chiasm. All other findings, such as column degeneration of the spinal cord, represent only accidental complications. In the cervical sympathetic, Marie and Marinescu found connective tissue increase and ganglion degeneration, and this was several times confirmed by other observers. In my cases I was unable to recognize decided changes in this region.

The chief interest of pathological researches has been concentrated for a long time upon the so-called "blood-vessel glands." Of these the thymus gland first attracted attention for a short time after Klebs had ascribed to it a predominant rôle in the disease. The enlargement of the thymus gland is, in fact, a very frequent finding. The gland may extend into the anterior mediastinum, so as to correspond with that of the new-born, though in comparison it is actually larger. The adrenals are large but normal. The thyreoid gland may develop into a massive goiter, or may be decidedly atrophic. The enlargement may be of any of the types usually found in goiters, without any predominant specific form being recognizable. The degenerative forms evidently have nothing in common with those characteristic of myxedema. In my case the glomus carotideum was examined, and found to be small and normal.

In the pathology of acromegalia our greatest interest is in the hypophysis. Since the first undoubted autopsy findings of Verga in a case of acromegalia, the characteristic findings in the skeleton by $\nabla$. Langer, and the observation of tumors of the mucous gland (pituitary body), the autopsies in acromegalia have been more numerous. In accordance with the size and character of the tumor, the description of the findings varies. There are extant descriptions of the pituitary gland which show that the authors did not consider this to be at all enlarged, and we can only base our assumption of an enlargement upon the fact that masses were mentioned as present. In other cases the enlargements are distinct, but only of moderate degree. Thus, in two cases, I have found tumors about the size of a cherry, one of which eroded the groove of the pituitary body and the other the dorsum sellæ. The enlargements are of every degree up to the size of a goose's egg or even an apple. These either 
develop downward and penetrate the entire sphenoid bone to the pharynx, as in a case of Hansemann and in one of my cases, or develop upward and compress and displace the organs at the base of the brain, as well as the nerves and venous sinuses situated upon both sides of the sella turcica. This has been variously described; in one of my cases the development was upward and also, to a slight degree, downward. The tumor invariably develops from the anterior lobe of the gland, and in a few cases has been described as simple hyperplasia of the gland. In other instances, adenoma or struma hyperplastica of the gland is directly mentioned. Besides these, in still other cases, proliferation, softening, cysts or connective tissue hyperplasia have been found. Finally, in a great number of cases the tumors have been regarded as maljgnant. Among these, isolated cases of gliomata have been mentioned. Claus and van der Stricht found a lymphadenoma. The majority of malignant tumors of the pituitary gland have been found to be sarcomata. Sternberg has laid particular stress upon the fact that in the six cases of malignant acromegalia which were known up to the time of the publication of his monograph, the tumors were all sarcomata. In the meantime Gubler, in a case of malignant acromegalia histologically investigated by Hanau, and I myself in at least two cases, found tumors of the hypophysis which were similar to the malignant variety, and which upon superficial examination might also have been looked upon as sarcomatous. But we recognized the tumor cells as originating from the epithelium of the hypophysis, and designated these tumors as hyperplastic, and eventually as malignant adenomata. Hanau expressed the suspicion that in the cases described as sarcomata the same adenomatous form of tumor was really present. I was able to demonstrate in my four cases that the character of the tumor was in the main the same, and that its origin could be found in the glandular epithelium of the anterior lobe of the pituitary body. I wish to lay special stress upon a fact (also noted by Tamburini) that in at least three of my cases, a large part of the tumor consisted of a very peculiar form of epithelium, the "granular cells," which correspond to the so-called chromophilic cells of Flesch. These are the cells which are looked upon as the essential functionating cells of the normal gland. I presume that in every case of hypophysial tumor the hyperplastic proliferation of the cells is the basis for a neoplasm, and that, in the further course, a malignant degeneration of the tumor starts in these cells; or a malignant proliferation of other tissue elements may replace this primary new formation and so destroy it.

When we consider the supposed importance of the hypophysial tumor in the clinical picture of acromegalia, we must admit that it is by no means free from objections. Several hypotheses in marked contrast to each other may be mentioned. The most extreme (in one direction) assumes that the tumor of the hypophysis is only a symptomatic enlargement of the organ which may be markedly developed, slightly developed, or entirely absent. In opposition to this is the conception that an abnormal function of the hypophysis is the sole cause of the disease. A more conservative view ascribes the disease to a primary anomaly of the "blood-vessel glands," among which the hypophysis is to some extent implicated. 
The question cannot now be determined with absolute certainty because we must admit that it is not yet fully recognized that the hypophysis is diseased in all cases of acromegalia. No less an authority than R. Virchow has maintained from the beginning that the changes in the hypophysis are a secondary finding and were absent in some of his characteristic cases. A case from Virchow's Institute has lately been published by O. Israel, which apparently proves this. These extraordinary differences in opinion can only be reconciled by the fact that the differential diagnosis of the disease is by no means so exact as might appear from the publications of P. Marie and Sternberg. It must be borne in mind that any of the individual symptoms of the disease, the peculiar osseous growth, the changes in the soft parts, the macroglossia, and even the tumor of the hypophysis may occur independently. Hence in many cases a difference of opinion may arise as to what symptomcomplex justifies the diagnosis of acromegalia. Virchow lays great stress upon the changes in the bones of the extremities, and undoubtedly has furnished incontestable proof, which is confirmed by the case of 0 . Israel, that they also occur without a tumor of the hypophysis. This was also recognized by Pierre Marie who diagnosticated a similar disease of the extremities as hypertrophic osteoarthropathy.

I believe, however, that if we adhere to the name acromegalia for the classical, clinical and anatomical symptom-complex of P. Marie alone, we must admit that the changes in the soft parts and in the bones of the face have at least as much importance as those of the bones of the extremities. In the case of Israel clinical observation together with the autopsy findings in the bone reveal, as the celebrated author himself emphasized, that other important symptoms of acromegalia besides the tumor of the hypophysis were absent. It appears to me most important that there was no record of the acromegalic macroglossia and the corresponding deformity of the lower jaw. We may, therefore, safely deny that this case is acromegalia, and must agree with the author that it is inadvisable to deduce from it any far-reaching conclusions as to the nature of acromegalia.

The fact is much more important that in literature quite a number of cases are mentioned in which true adenomata of the hypophysis were found without acromegalia. I still entertain the hope that by careful investigation with the most approved methods we may find these tumors to deviate from the type of those occurring in acromegalia. In a case recently examined by me, the question arises whether the age of the patient, sixty years, might not explain the absence of corresponding general symptoms. Opposed to the view that the tumor of the hypophysis is a symptom and not the cause of acromegalia is the fact that the common type of hypophysial tumors differs from the normal structure of the gland (as was especially apparent in all of my four cases which were minutely investigated) as well as from the other acromegalic hyperplasias, which in the main are composed of connective tissue.

The opinion that disease of the hypophysis produces the symptoms of acromegalia only by co-operation with the other blood-vessel glands is not in accordance with the fact that no other blood-vessel gland is so constantly involved in the disease as the pituitary body. Indeed this is not even approxi- 
mately the case. The symptoms which have been recognized as consequences of diseases of the other blood-vessel glands are generally absent in the clinical picture of acromegalia; or, if they do occur, they represent distinct complications, and the characteristic changes of acromegalia are not produced by disease of any of them. Finally, the especial, peculiar, histological structure of the hypophysial disease favors the opinion that this plays a rôle entirely different from that of the other blood-vessel glands.

In so far as we can arrive at logical conclusions by exclusion and without experiment, we may maintain the view which was first expressed by Pierre Marie that disease of the hypophysis is the etiologic factor in acromegalia.

We will now consider in what manner the hypophysis causes the disease. The original view of Pierre Marie that the changes are due to an absence of function of the degenerated hypophysis-as in myxedema by an absence of function of the thyreoid--is not substantiated by our more exact knowledge of the character of the glandular proliferation. Somewhat more reasonable and better founded are the hypotheses of Hansemann and Uthoff, who hold that the relation between the enlargement of the hypophysis and the affected parts is an altruistic hyperplasia. In whatever way the progressive development of the hypophysis is produced, in that way the progressive development of other parts (extremities, bones of the face and internal organs) is also brought about. According to Hansemann, altruistic hyperplasia may occur with organs that have an embryologic connection, as between the anterior lobe of the hypophysis and the tongue, but we can hardly say that there is any such relation between the gland and the extremities. This view becomes even less tenable when it is clearly demonstrated that neither the changes of the hypophysis nor those of the extremity represent simple progessive hyperplasia, and that in both we are dealing with new tumor-like formations of entirely atypical nature.

In my previously published articles, I have accepted the view of Tamburini, as do also Hanau and Woods Hutchinson, that the pathologic development of the hypophysis is due to a hyperactivity and over-production of the secretion which is to be utilized internally. Tamburini, at any rate, attributes the phenomena of growth to these causes, while the cachexia observed in the later stages of the disease is regarded as a consequence of the adenomatous and cystic degeneration of the gland. For several reasons this theory must now be somewhat modified. First, in its original form, the division of the disease into two phases is impossible. On the contrary, in the purest type of acromegalia, Sternberg's malignant form, the development of the tumor of the hypophysis is unquestionably combined with symptoms of peripheral hyperplasia and the injurious effects of this upon the entire organism. Tamburini's theory is also difficult of acceptance because its adherents, as well as its opponents, have been forced to the conclusion that if his views are correct, the secretion of the hypophysis must also influence the normal growth of the body; Woods Hutchinson has gone so far as to declare that the hypophysis is the center for body growth.

These difficulties lessen when the processes of peripheral growth are recognized as constant but less important consequences of acromegalia (which is 
clear to me), and the true nature of the disease is conceived as a tumor-like formation in the connective tissue which, as Erb and Ponfick have already pointed out, resembles that of elephantiasis and also myxedema. It might be more easy to ascribe the symptoms of the disease to the action of a specific toxic secretion which, under normal circumstances, possesses an unknown function, and which is present in the circulation in such slight amounts that no deleterious effect is produced. I refer to the zymogen-like granules secreted in the chromophilic cells which, in at least three of my cases, I found enormously increased, while in the normal gland their action appeared of less importance. I believe it may be strictly maintained that if there is a lessening of the normal supply of the bodies to the circulation they may affect the activity of digestion as profoundly as the blood plasma as shown by recent investigations. Hence an over-production of them may cause severe damage to the tissues of the body.

\section{TREATMENT}

In conclusion I shall refer briefly to the therapy of the affection. I have only negative points to report, since, up to the present time, it has been absolutely without effect. Yet a knowledge of the pathology of the disease may prevent error in the choice of therapeutic measures. The uncritical way in which organotherapeutic "curative" results have been published led, as a matter of course, to the production of hypophysis tablets and their administration to acromegalics as soon as it became known that in acromegalia the hypophysis appeared to be affected. Fortunately, it has been demonstrated both by the use of these and by animal experiment that the administration of the pituitary gland by mouth is quite harmless; we may assume that its active constituents are rendered inert by the digestive fluids. Otherwise, we would realize the unpleasant truth that this therapy must aggravate the disease by increasing the materia peccans. Until a hypophysis antitoxin is produced, the only rational treatment, based on the pathology of the disease, must be the extirpation of the hypophysial tumor. We cannot understand why the hand of the fearless surgeon who has dared to attack the neighboring Gasserian ganglion should halt at this operation. 


\title{
CHRONIC ARTICULAR RHEUMATISM
}

\author{
By W. HIS, BASEL
}

IN attempting to describe a disease so familiar and commonplace as chronic articular rheumatism (this appears to be the prevalent opinion in regard to it), it is almost necessary to begin with a captatio benevolentiox. A few years ago, when I began to study this disease somewhat in detail, I learned that it had long attracted the attention of many scientists. The last of the greater compilations, that by Pribram, although by no means exhaustive, contains notes of over 500 investigations which refer to chronic articular rheumatism, and among the authors are found the most prominent names in medical science, of whom I shall mention Charcot, Bouchard, Lancereaux, Pierre Marie in France, the Garrods, father and son, in England, Richard Volkmann, Senator, Bäumler, and Albin Hoffmann in Germany.

The disease is by no means a new one; it was familiar to the ancients who confounded it with gout and designated it by the collective term arthritis. It was not until the year 1800 that Landré-Beauvais expressly pointed out that the disease was a distinct entity. Alfred Garrod in his celebrated book on gout proved that the deposit of urate salts which is the materia peccans in the joints affected by gout never occurs in chronic articular rheumatism. Nevertheless, there are still some authors who, even in recent years, have endeavored to merge the two diseases, believing them to be one. This attempt is due to the fact that certain phenomena in the joints, and many of the accompanying and subsequent symptoms in both diseases, may be the same; we shall refer later to this point more in detail.

The manifestations of chronic articular rheumatism are very various, and if the descriptions of different authors are compared with one another, it is often difficult to believe that they are portraying the same disease. What one experienced observer describes as a frequent finding, another, no less experienced, has scarcely ever seen. Similar to this is the variety of opinions in regard to the etiology; greater diversity of views can scarcely be conceived. According to some authors, for instance, M. Schïller and Bannatyne, chronic rheumatism is an infectious disease in which there can be invariably demonstrated certain microörganisms; to others (Bouchard, Lancereaux) the disease is the expression of a constitutional anomaly which is markedly hereditary, running in families, and showing itself by numerous other symptoms. Other writers believe that the affection is in the main a local process confined to the joints and periarticular tissues. Finally, there are some who consider it a disease of the central nervous system. 
Just as diverse is the nomenclature. Almost every author has attempted to arrange the varying pathological pictures in distinct groups (all mentioned by Pribram), and of the names that have been chosen some refer to the clinical course (for instance, Charcot's rheumatisme articulaire chronique progressif). some to the shapes assumed by the joint (arthritis deformans of Virchow and R. Volkmann, rheumatisme noueux of Trousseau), some to the anatomical findings (M. Schüller's polyarthritis villosa) and some to their authors' etiologic views, for example, rheumatisme goutteux, diathésique (Pierre Marie) and infecticux (Teissier and Roque).

This confusion is increased by the fact that the same title is applied to quite opposite pathological conditions. Thus, the rheumatisme deformant of the French, with effusion, proliferation of the capsule, and spindle-shaped swelling of the joint, is identical with what the German surgeons and clinicians (following R. Volkmann) call chronic rheumatism in contrast with arthritis deformans, which is characterized by atrophy of the capsule and proliferation of the bone, in many joints or in one alone (for example, the malum senile coxæ).

This confusion can only be cleared up by describing briefly all the principal types of joint disease.

1. When a young person, after brief prodromal symptoms or a tonsilitis with fever, is attacked with painful swelling of the joints, the inflammation spreading from one joint to another, affecting large and small joints alike, and without predilection for any-when the endocardium, the myocardium, the pericardium, the pleuræ, perhaps even the meninges are involved-we recognize in this picture an infectious disease, acute articular rheumatism, and we know that, as a rule, it runs its course without leaving permanent changes in the joints. Exceptionally, however, swelling of one or more joints and moderate pain and stiffness remain. After weeks or months the joint function is more or less completely restored or the diseased condition becomes permanent. This is chronic arthritis, resulting from an attack of typical acute articular rheumatism.

Now as the primary forms of chronic arthritis may begin with acute febrile attacks, their differentiation is often difficult. Davaine closely studied the disease, and laid especial stress upon possible prodromes, splenic tumor, visceral complications, the ease with which the effusion in the joint may be displaced, the absence of trophic disturbances (atrophy of the muscles), and the absence of family predisposition to acute articular rheumatism. Unquestionably Davaine has arranged this in too schematic a manner, and, therefore, in any individual case we may waver for a time in our decision; the occurrence if secondary chronic rheumatism is, however, recognized by all later authors.

2. The second form is prone to attack persons between thirty and forty years of age. The disease is ushered in with fever and pains in the joints, which are swollen but not to the same extent as in acute rheumatism. They have an elastic, tense feeling, but fluctuation is obtained with difficulty; the skin is slightly reddened, and is edematous above and below the joint so that the affected joint shows a spindle-shaped swclling. Gradually new joints are 
attacked or those affected improve, but a number of those first attacked always remain permanently diseased.

The joints exhibit conspicuous regularity in the order of their involvement. First, the smaller joints of the body are attacked; the phalangeal and interphalangeal joints of the fingers with the exception of the thumb, the corresponding joints of the toes, then those of the hands, of the elbows and knees; the shoulder and hip are almost always exempt. The disease, however, shows a preference for certain joints which are usually spared by acute polyarthritis, such as the jaw, the sternoclavicular joint and the sternocostal joints. ${ }^{1}$ Secondly the affection is conspicuously symmetrical: Almost always both hands, both feet, both knees are attacked; only the joints mentioned above are, as a rule, attacked unilaterally. The further course of the disease varies. Either the joints return more or less to the normal, to be similarly attacked after months or years (often acutely and with fever) or the affection is "chronic from the onset," and in time changes occur in the joints which prefigure the third form now about to be described.

3. The third form generally attacks elderly persons, particularly women during the menopause; the disease begins with indistinct nervous symptoms, drawing or tearing pains, furry sensations or formication, sensations of cold, etc., in the hands and feet. The patients notice that the motility of the fingers gradually decreases. Fine movements such as sewing, knitting, writing, become difficult, particularly in the morning and during cold weather. Careful observers note that the ends of the joints and the basic and interphalangeal joints are slightly thickened; they feel hard and are not very tender on pressure. The fingers gradually deflect toward the ulnar side, and this is first noticeable in the proximal joint with the extended interphalangeal joints ; finally complete subluxation results in the basic joint, though in this, as well as the other changes, the thumb is rarely implicated. The interosseous spaces show deep grooves; the ball of the thumb and little finger are atrophied. The same changes take place in the toes. Muscular contractures appear early and cause abnormal positions of the extremities. Charcot, with his artistic mind, attempted to arrange these positions according to a system: A flexion and an extension type (more correctly a hyper-extension type) each type with several sub-varieties. If, with Vidal, we accept also an extension type (straight line) every imaginable form results, and if we reflect that the fingers of the same hand may present various types side by side, the value of this schematic division will not appear great. Besides the muscular contracture, changes in the joints add to the immobility; the capsule shrinks to fibrinous strands, the surfaces of the joints coalesce by connective tissue ligaments, exostoses and ecchondroses appear: The final result in well marked cases is complete immobility of the joints in abnormal positions; the unfortunates are condemned to permanent invalidism, and fill a large part of our almshouses and homes for incurables.

- This form differs from the two first mentioned by its gradual and afebrile

1 These, however, although rarely, are sometimes attacked in acute polyarthritis; indeed, the jaw may be the only joint attacked (Hamm, Manasse). 
onset, its slow course, the absence of effusion into the joints, cutaneous edema and capsular swelling. It resembles them in a preference for the small joints and in the conspicuous symmetry; moreover, every possible transitional form occurs, so that they can only arbitrarily be differentiated. More correctly, perhaps, we may call them different developments of the same disease. Therefore I have not yet accepted the division which is often made (lately by Curschmann) into chronic articular rheumatism and arthritis deformans. I agree with Charcot, who designates the two as the exudative and the dry form of primary chronic progressive polyarthritis.

4. The fourth form is characterized by appearing in elderly persons and in the aged, by a markedly chronic course, by invariable limitation to one or more large joints, and frequently, although not always, by its connection with trauma. This is the arthritis deformans of $\mathrm{R}$. Volkmann, the rheumatisme chronique partiel of Charcot, the best known type of which is the malum senilc coxæ. But this affection is not infrequently noted in the fourth and fifth decades of life, most often, perhaps, in the shoulder after a fall or contusion.

Effusion is almost always absent. When the patient is at rest the pains are moderate or cease entirely; movement is limited to a great extent, muscle contractions or, at least, constrained positions, generally occur and atrophy is frequent (in the shoulder, mostly of the deltoid and triceps). Upon movement, friction and cracking are heard and felt in the ends of the joints. After the disease has lasted a long time deformities due to exostoses and ecchondroses invariably appear. These will be described below.

5. The fifth form includes the chronic, deforming and ankylosing diseases of the vertebral column (Pribram). Julius Braun, in 1875, was the first to collect a large number of cases. Strümpell described the implication of the hip-joint in arthritis of the vertebral column. Pierre Marie in 1898 worked out the symptomatology of "spondylose rhizomélique" which appears in men immediately after the completion of their growth, and consists in complete adhesion of all vertebræ, scoliosis and kyphosis of the shoulder- and hipjoints, but with intact extremities. Another form was described by Bechterew in 1892: Limited movement in the vertebral column with anterior curvature, particularly of the upper parts; associated with this pareses of the musculature of the neck, trunk and extremities, and atrophy of the muscles of the back and scapula. Common to both of these forms are nervous disturbances -anesthesia and paresthesia, neuralgia, paralyses and muscular atrophies in varying form and extent. The Strümpell-Marie form distributes itself from below upward; Bechterew's variety from the shoulder to the hip.

In the meantime, in a large number of observations, particularly those compiled by W. Anschütz, it was noted that a sharp differentiation of these forms is impracticable, that, on the contrary, numerous transitional stages are observed. Some of these begin at the upper or lower end of the vertebral column, with or without involvement of the joints of the trunk, or, finally, in combination with chronic arthritis of the extremities in one or another order. Joh. Müller described a case with extreme stiffness of the articulations of the ribs, respiration being maintained entirely by the diaphragm and the 
abdominal muscles; Clarke described a disease confined to the costovertebral joints of the twelfth pair of ribs. Anatomically the process produces changes in the ligaments of the intervertebral sheaths and of the vertebral joints in varying combinations (Schlesinger); the nervous disturbances are partly due to compression of the nerve roots, partly to chronic meningitis (Bechterew). Etiologically we must consider primary and secondary chronic arthritis, arthritis deformans, gonorrhea, syphilis and other infections. Whether the affections confined to the ligamentous apparatus and intervertebral sheaths are to be classed as chronic arthritis may be questionable. Bäumler calls attention to the fact that stiffness of the hip-joints and lower vertebral articulations in consequence of the patient's increased weight may lead to disease of the upper vertebræ. R. Bennecke lays stress upon the action of frequent, though insignificant, trauma upon the vertebral sheaths and bony structures. In conclusion, similar clinical pictures are presented by contracture of the lumbar

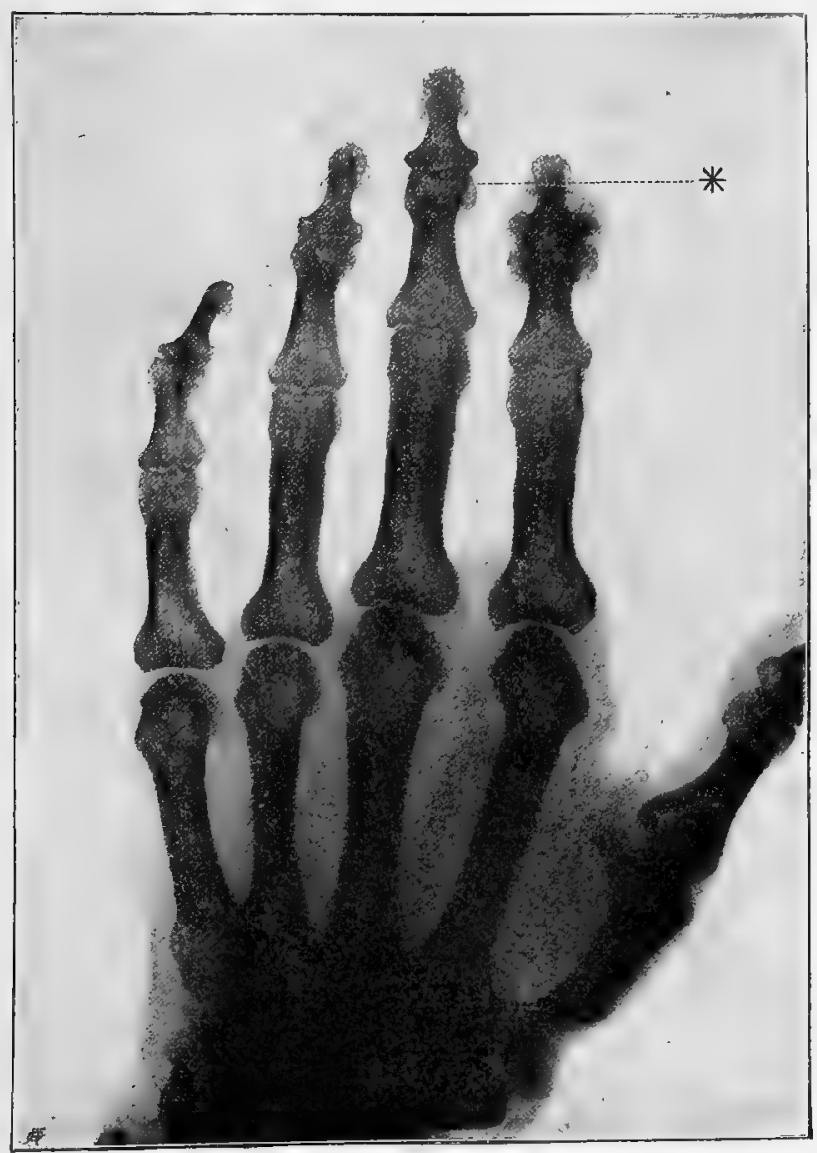

Fig, 4.-Röntgen Picture Showing Heberden's Nodes upon the Index and Little Finger, with Exostoses of the Second and Third Phatanges. 
muscles and those of the back. Beer, Zenner, and lately Cassirer and Senator have described such "myogenous" vertebral stiffness, some of which may be due to hysteria and some to acute fibrous myositis.

These conditions indicate that chronic rigidity of the vertebra may be due to very different causes, but we may be certain that those forms which occur in connection with acute or chronic articular rheumatism or with disease of the joints of the extremities should be classed as chronic arthritis.

6. As the sixth and last type, I must mention those almost painless or only temporarily painful nodes which occur in the terminal joint of the threejointed fingers, appearing upon their posterior surface, giving them an oval shape, hard to the touch, and gradually, from rigidity of the joint, fixing the fingers in a position of flexion or abduction. They are often looked upon as the signs of uric acid gout; $\mathrm{E}$. Pfeiffer believes them to be a certain guide in diagnosis even in the absence of other gouty symptoms.

Heberden, who discovered them, did not attribute them exclusively to gout, and I coincide in the opinion of the majority of later authors who find these Heberden's nodes occasionally in gout, occasionally in chronic arthritis, but most often as the only arthritic symptom in otherwise healthy individuals. The Röntgen picture shows them to be exostoses, and they form a connecting link between chronic rheumatism and arthritis deformans; with the former they have in common symmetry and multiplicity, with the latter, the bony, marginal proliferations, and the usually painful and chronic course. It is well to follow Charcot and to separate them as special forms of disease.

This finishes the description of the most common types; but it must be added that in individual cases transitional stages occur which tend to merge the types into each other. For this reason clinical findings do not permit a fine differentiation, and an attempt has been made to found a classification upon the anatomical changes.

\section{PATHOLOGY}

The anatomical changes extend to all parts of the joint, cartilage, bone and capsule, and frequently also to the adjoining muscles, tendons and tendon sheaths. In the earlier stages these changes are relatively but little known; they are found as accidental lesions, and often run their course during the life of the patient without symptoms. Thus, the patient from whom illustration 5 was taken was a chorus girl and dancer until a few weeks before her death. Moderate changes are noted by surgeons, who look upon chronic rheumatism as a border-land which should be gradually brought under their dominion. The later changes are well known anatomically, but they illuminate the problem of their origin as poorly as ulcerative phthisis explains the onset of pulmonary tuberculosis.

Even to-day it is not quite clear whether the affection begins in the cartilage of the joint or in the synovial membrane. Sometimes the one, sometimes the other, appears to be the case; nevertheless both are early attacked. In the cartilage the basic substance appears completely detached, the cartilage cells proliferated, and sometimes discharged from their capsules into the joint fluid. 
In some forms the amount of synovial fluid is increased, occasionally turbid but-and this is characteristic of chronic arthritis-never purulent. The villi of the synovial membrane are hyperemic (violet red), showing

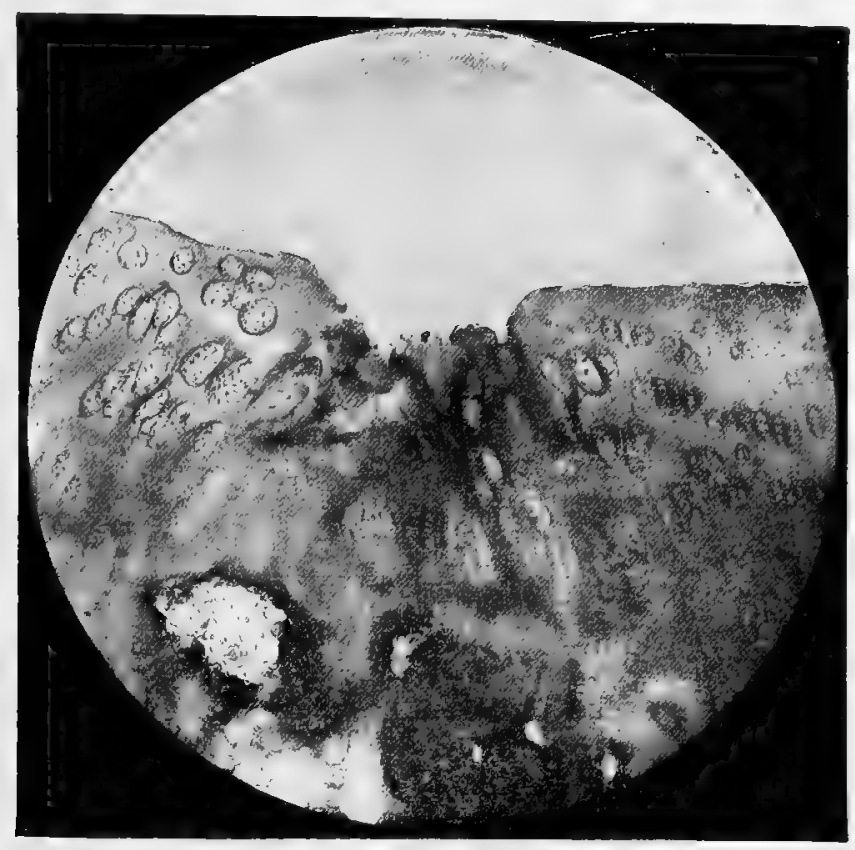

Fig. 5. -Metatarso-Phalangeal Articulation of a Chorus Gird, Aged Forty-Five, with Beginning Chronic Arthrits without Symptoms; Death from Contracted Kidney. Fibrillation of the cartilaginous basic substance, proliferation of the cartilage cells, the capsules of which partially open into the joint cavity. The capsule of the joint is intact.

marked proliferation; occasionally fatty degeneration is noted (lipoma arborescens), and in well developed cases present the appearance of sheep's wool; the capsule and its surroundings show edematous infiltration. The bone appears unchanged in the early stages of the disease.

In later stages the smooth surfaces are denuded of cartilage, the bone is bare, and, if joint movements have still been performed, shows grooves, resembling the crevasses of a glacier. Often the bared surface is dense and ivory-like, or it is covered by connective tissue which extends from the borders of the synovial membrane, from transformed cartilage cells, or even from the surfaces bare of cartilage. This connective tissue covers the joint surface (Kachel), and frequently adheres to the opposing joint ends (fibrous ankylosis). These connective tissue striæ may calcify and finally ossify (bony ankylosis). Upon the border of the cartilage, wherever it is covered by synovial membrane, ecchondroses develop which are transformed into osteoids and finally into bony tissue. By these exostotic marginal proliferations, the joint end attains a mushroom form and resembles the crown of the antler of a deer. 
The bony substance becomes rarefied and fragile, the capsule, which was at first flaccid, proliferated, and infiltrated with serum, is transformed into fibrous tissue which cicatrizes around the ends of the joint and limits motion. In the proliferated villi, islands of cartilage or bony tissiue form, and these may be thrown off as free joint bodies. The final stage is complete osseous or fibrous ankylosis of the joint which is fixed in more or less unnatural position and greatly deformed.

All these processes are common to the various forms of chronic arthritis; at one time one, at another time another, becomes prominent. Thus, in the exudative form of progressive chronic polyarthritis, proliferation and edematous infiltration of the synovial membrane and its surroundings dominate the situation, hence the spindle-shaped form of the joints. The cartilage often appears to be secondarily implicated.

In polyarthritis "sicca" which has a chronic beginning, the proliferation of the joint is not as marked as the fibrous transformation of the cartilage. The outlines of the joint ends as a consequence of this become prominent, and exostoses, if present, may be felt through the tense skin as hard, prominent nodules which may sometimes be seen. This form also shows a tendency to fibrous ankylosis. In senile deforming monoarthritis these osseous border proliferations with disappearance of cartilage play the main rôle in the limitation of movement; the capsule forms no essential part of the clinical picture. Finally, Heberden's nodes are exostoses which at first slightly limit motion.

If the anatomical descriptions of later authors are compared (particularly Schuchardt and Weichselbaum) we are forced to agree with Charcot who declines sharply to separate the forms according to the anatomical findings. $\mathrm{He}$ regards them as in some sense branches of the same trunk.

\section{CLINICAL SYMPTOMS}

The clinical picture would be incomplete if only the joint lesions were considered. Very frequently there is early impairment of the general health. The cases occurring in young persons with fever are particularly apt to show from the onset emaciation and conspicuous cachexia. This can hardly be attributed to the fever. Occasionally the fever lasts for weeks and reaches $102^{\circ} \mathrm{F}$. and higher, but there is no proportion between the height or duration of the fever and the emaciation. Indeed even after the temperature falls the patients remain weak. The inactivity of the patient, the want of fresh air, and the constant pain have been urged in explanation. But if we bear in mind that patients with disease of the spinal cord become abnormally fat despite the fact of constant pain and immobility, we cannot concur in this view, but must look upon these forms of arthritis as conspicuous instances of wasting disease. Amyloid degeneration (Roese) which frequently appears confirms this. It is true other cases of arthritis belong to the fat plethoric type and here also transitional cases are recorded. Frequently, particularly in the young, there is moderate anemia and oligocythemia.

Most of the muscles of the body take part in the emaciation; in some cases the entire musculature suffers but almost invariably the muscles in the neigh- 
borhood of the joints are involved, and among these the extensors are most seriously affected." If rheumatism attacks the hand the interossei atrophy very early, often before the patients notice any hindrance in movement. This occurs in the exudative as well as in the dry form. In the latter we find a very characteristic ulnar abduction of the basic joint of the fingers from the first to the fourth. An attempt has been made to explain this by contractures of the muscles or from the flaccid condition of the capsule; this, however, I believe to be incorrect. For in older persons this is frequently the first symptom noted, even before there is any abnormal distention of the capsule or any muscular contractions. It is more reasonable to assume that the lumbricales atrophy simultaneously with the interossei, which normally have the property of adduction, besides that of extension of the basal phalanx. Later this deviation terminates in well marked subluxation. That the bones take part in the atrophy is well known; in the senile monoarthritic deforming varicty this begins in the end of the joint; but in the juvenile polyarthritic form the Röntgen picture often shows a conspicuous coalescence and disappearance of the spongiosa in the neighborhood of the affected joint. Perhaps this is the effect of immobilization. But since Sudeck has shown that in every form of arthritic inflammation, distortion or trauma may produce within a few weeks decided atrophy of the bones, transient or permanent, similar conditions should also be looked for in chronic arthritis. The skin and its structures take part in the process, and Herz has described a case in which, with every new attack, a glove-like desquamation of the skin of the hand and shedding of the nails occurred; such cases are rare. The changes resembling scleroderma are more frequent; the shining ivory-like skin adheres to the deformed joint, smooth and immovable. In the juvenile cases there is frequently an excessive functional hyperhidrosis. The contractures which occur particularly in the flexor muscles of the fingers and toes are important, but these may also be noted in the extensor groups which are to a high degree responsible for the terrible and incurable deformities in some of which the knees are drawn up to the chin.

Frequently the tendons and tendon sheaths are involved in the process and along these structures tough subcutaneous nodules occasionally appear which may be temporary or permanent; these are also noted in acute rheumatism (see a dissertation by Rabinowitsch). Pribram asserts that he has only observed them in acute rheumatism; Fig. 6, however, is an example of such nodules in a case of undoubted chronic polyarticular arthritis.

In conclusion, I must mention intermuscular or intramuscular, dense, callous infiltrations which are rarely alluded to in literature, but are well known to orthopedists and masseurs, and are skilfully treated by them.

The visceral complications are especially interesting on account of their importance in the conception of the disease. That the endocardium may be attacked has been admitted, and the frequency with which this happens varies in different reports from 4 per cent. to 80 per cent. of the cases. The secondary and the senile deforming varieties do not attack the heart. In the statistics of primary chronic polyarthritis there are differences of opinion as to whether functional heart murmurs or only the genuine valvular affections have been included. Pribram, who was the first to call attention to this variation, 
gives the following statistics of his cases. In the forms beginning acutely, there were 53.4 per cent. of heart murmurs and 20 per cent. of valvular disease; in cases chronic from the outset only 13.5 per cent. of heart murmurs and 4 per cent. of valvular disease.

Chronic nephritis of benign character and showing but slight tendency to uremia is not infrequently observed.

Among other complications those affecting the eye, such as iritis, iridocyclitis and episcleritis, and the skin (various forms of erythema, eczema, urticaria and psoriasis) are to be mentioned. The occurrence of multiple symmetric lipomata has frequently been observed in connection with chronic arthritis, as well as bronchial asthma, dyspepsia, hemorrhoids and numerous nervous disturbances, and this gives rise to some confusion. These affections are spoken of particularly by authors who look upon chronic arthritis as an expression of a general constitutional anomaly.

Lancereaux, in 18\%0, divided rheumatism into two classes, of which one, "qui ne laisse jamais des traces sur son passage" corresponds to acute articular rheumatism; the other he said was: "pas une maladie, mais un syndrôme,

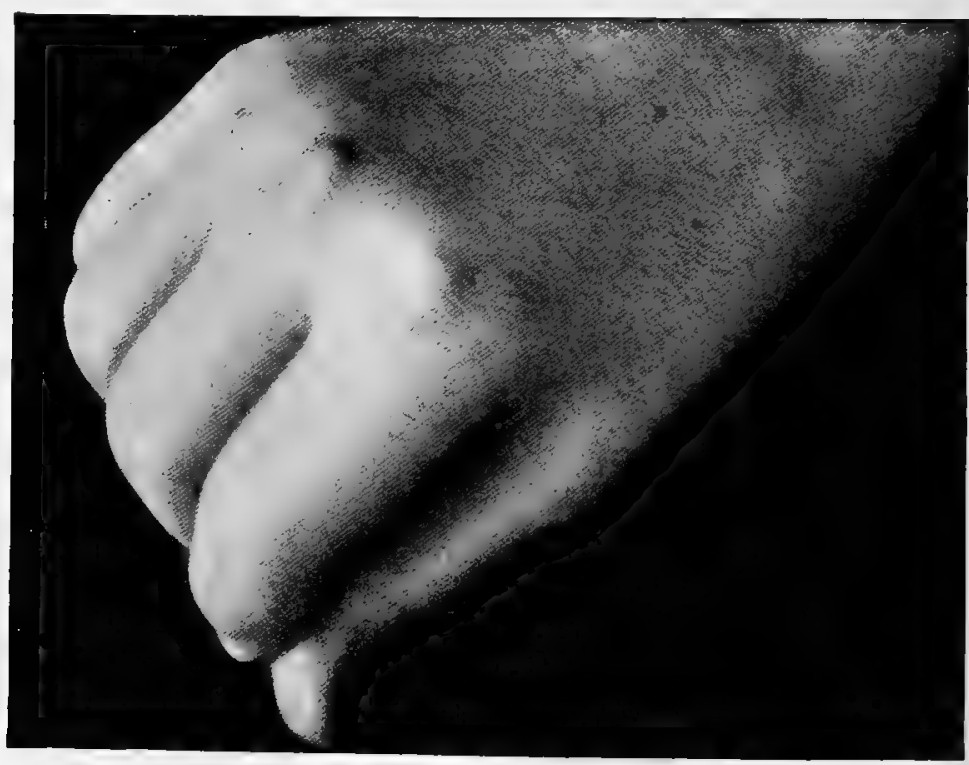

Fig. 6.-Subcutaneous Nodules Situated upon the Dorsal Tendon Sheates in a Case of Chronic Exudative Polyarthritis in a Young GirL.

une manifestation d'un état constitutionel," "le branche d'un grande famille pathologique," "herpétisme," a vasomotor trophic neurosis, expressing itself in dynamic (migraine, epistaxis, neuralgias) and material disturbances in the skin and of the tissues deficient in blood-vessels. In this group is also included the triad of diabetes, obesity, and gout. Bouchard gives a similar definition of "arthritisme"; both authors emphasize its conspicuous family and hered- 
itary character, and even so careful an observer as Potain believes: "Ce n'est pas telle ou telle forme de rheumatisme qui se transmet, mais une prédisposition générale exposant à un groupe commun d'affections dans laquelle il faut même ranger la goutte."

On this point it is difficult to arrive at a definite conclusion. When we read the clinical reports of German, French, English and American authors

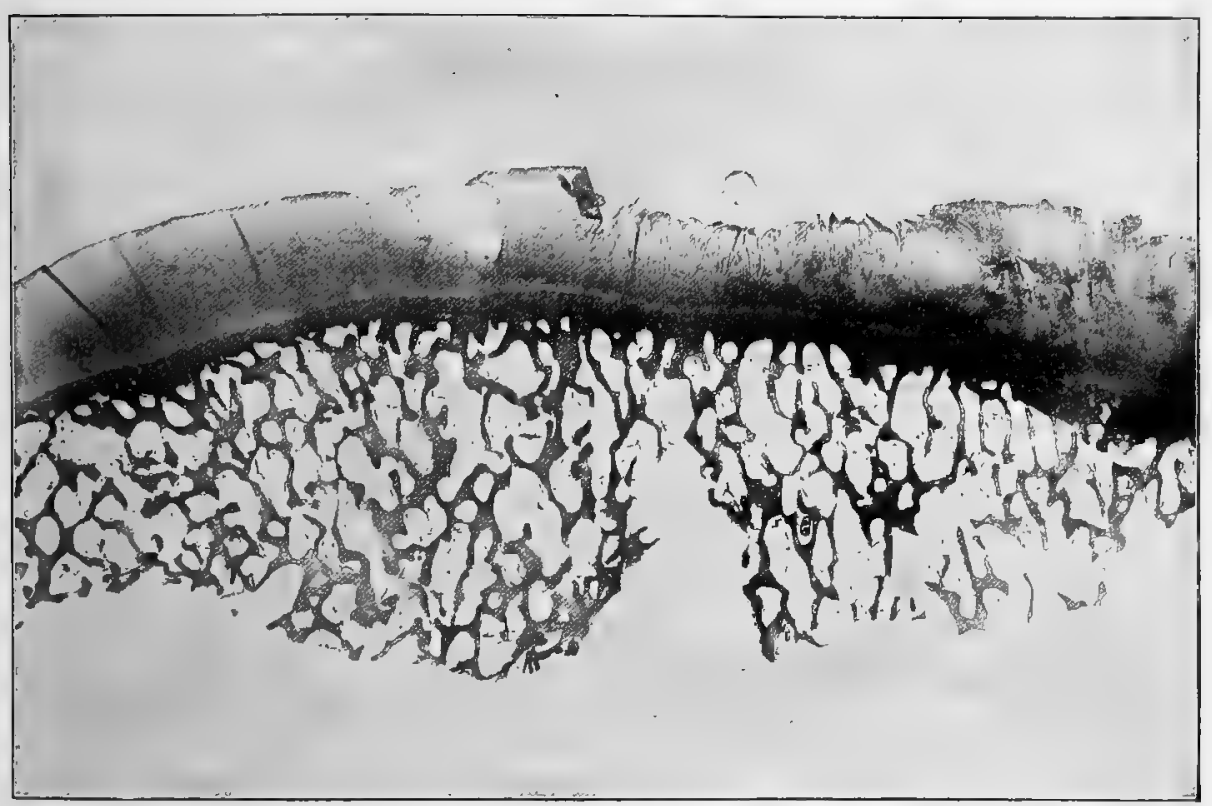

Fig. 7.-Patglifar Cartilage of a Young Man who, after Passing Through an Attack of Gout, Succumbed to Croupous PNeumonia. The cartilage shows a velvety fibrillar tion of the matrix similar to the condition in chronic arthritis. The joints contained no deposits of urate salts.

we receive the impression that certain forms of chronic polyarthritis frequently attack debilitated persons. One author has, however, gathered his clinical material in the hospital, among feeble, anemic, poorly nourished patients, cases of true arthritis pauperum, whereas the cases of others were among those living in affluence, the portion of the population debilitated by luxury and close intermarriage.

Under such conditions we cannot regard the simultaneous appearance of two or more diseases as a proof of their identity, and since decisive statistics are not to be had, the critical skepticism of A. Hoffmann and other German authors is certainly justified.

Yet the relation of arthritis to apparently dissimilar affections-and here I must mention psoriasis-becomes constantly more obvious. Adrian has recently reported 94 cases of this combination, which has been known in France for some time. Gerhardt was the first to observe the condition in Germany, 


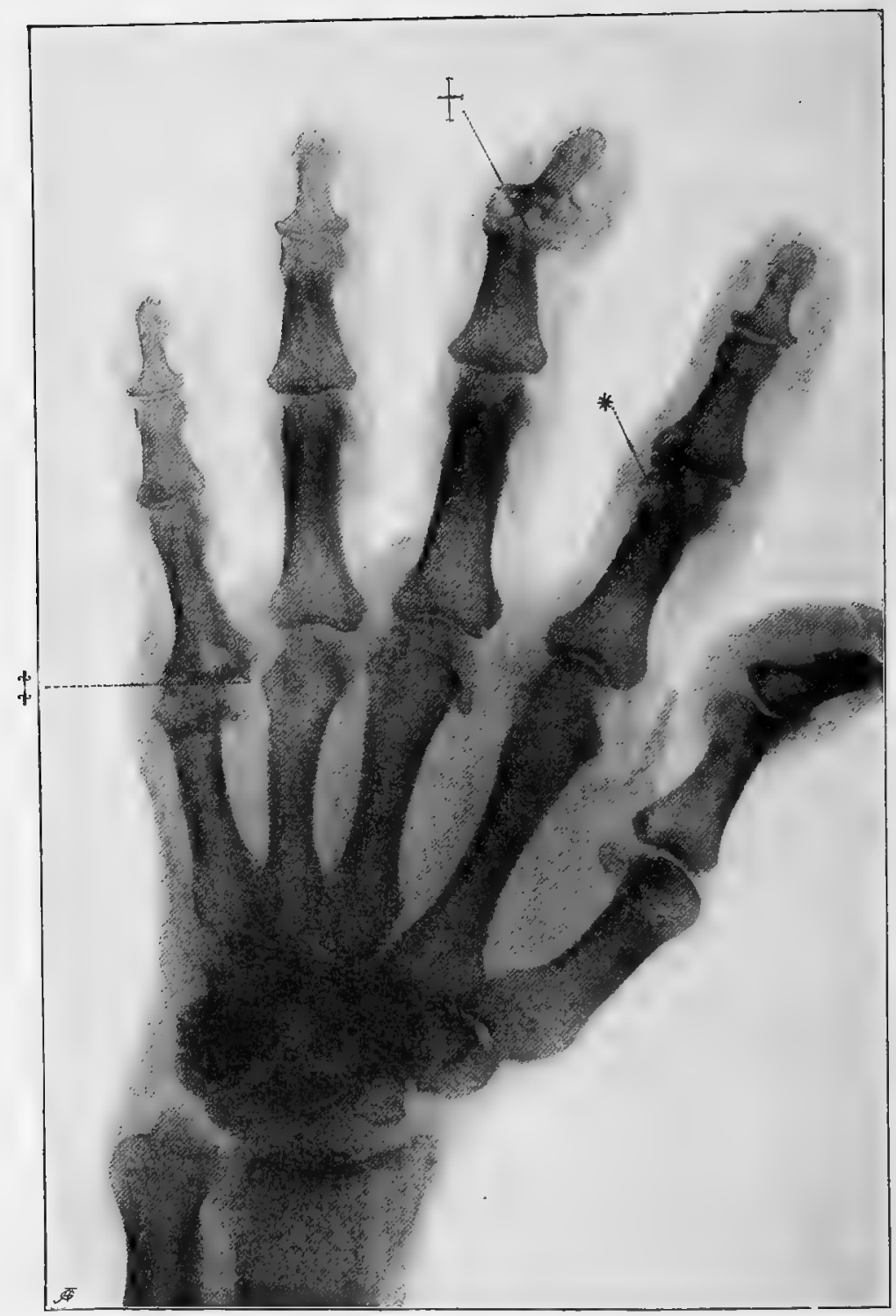

Fig. 8.-Radiograpi of the Hand of a Gouty Patinnt. At the point marked by the asterisk, gouty tophi are seen as translucent foci; at the dagger, exostoses similar to Heberden's nodes; at the double dagger, subluxation in the basal joint of the little finger. 
and after a critical analysis he came to the conclusion that coincidence can be wholly excluded, since in his and other cases the affections show simultaneous exacerbations and improvement.

Here, also, the relation existing between chronic arthritis and gout must be considered. This much is certain: 1 . That after gout has existed for a long time in a joint, proliferation of the cartilage, exostoses and disappearance of the capsules are found; 2 . That atypical gout may give rise to polyarticular swelling, resembling the exudative form of arthritis; 3 . That in gouty families, individuals may present symptoms resembling chronic arthritis. But if we do not wish to lose ourselves in a realm of unfruitful speculation, we must adhere to the belief that gout is an affection characterized by a deposit of uric acid salts in the body and by the presence of uric acid in the blood. The latter point will decide the differential diagnosis of doubtful cases, in which we should place our dependence not upon the uncertain thread test of Garrod, but upon the more difficult chemical analysis.

We may, therefore, look upon gout as one of the causes of chronic arthritic changes, while recognizing that the two diseases are by no means identical.

The constitutional predisposition appears to be of especial importance in one form of chronic arthritis, namely, that marked by Heberden's nodes. They are found isolated, especially in the aged, and then are frequently hereditary; very often they are associated with asthma, migraine, neuralgia, sciatica and muscular rheumatism and particularly with gout. Bouchard was the frst to describe nodosities of the middle joints of the fingers due to swelling of the second phalanx and occurring in cases of gastric dilatation; he gave them the name of "comptodactylie," and showed that this swelling disappeared with improvement in the gastric affection. Pribram also observed one case of this malady.

I do not believe that it will be possible hereafter to deny the existence of a constitutional arthritis; but it is not necessary to adopt the scheme of the French, who look upon every non-infectious arthritis as an expression of an "herpétisme or arthritisme." In the future accurate weighing of all the circumstances will show whether definite anatomical lesions exist or an arthritis runs a particular course in persons with a predisposition of the type described by the French.

The infectious theory of arthritis has many more supporters than has dyscrasia. The febrile form which comes in paroxysms with a relatively frequent endocarditis and severe general disturbances gives strong support to this view, and on several occasions microörganisms have been cultivated (by M. Schüller, Bannatyne, Blaxall and Wohlmann) from the contents of the joint and their pathogenicity has been proven by animal experiment. Unfortunately, the microörganisms described are not all the same, and so careful an investigator as Pribram found it impossible to detect any of them in the cases he examined. The pathogenic agent, therefore, as in the case of articular rheumatism, is not yet determined and the infectious nature of the disease is only a hypothesis, although a very probable one.

As a third possibility, disease of the central nervous system has been considered. This is suggested by the symmetry of the affection, the atrophy and 
contractures of the muscles, the trophic cutaneous disturbances, and the similarity of the chronic rheumatic joint changes to unmistakable nervous arthropathies occurring in tabes, syringomyelia, progressive muscular atrophy, hemiplegia, progressive paralysis and also in peripheral neuritis. In the latter disease rarefaction of the bony substance with swelling of the distal ends of the extremities and the formation of club finger tips is observed (pulmonary osteoarthropathy of P. Marie, see the compilation of W. Berent). In diseases of the central nervous system disfiguring deformities appear with atrophy and proliferation of the bones and cartilage, and these show a great resemblance to senile monoarthritis, differing, however, by their more rapid course, absence of pain, fissures in the capsule, and extra-capsular, osseous and cartilaginous proliferations.

To explain the muscular atrophy the assumption of a primary nervous affection is quite unnecessary, since Charrier has shown that this atrophy accompanies all joint inflammations, especially when they run their course with effusion (Kremer). Hoffa proved that they do not occur when the centrifugal nerves are severed; they are, therefore, trophoneuroses which are produced by reflex action initiated by the diseased joint surfaces.

As anatomical investigation of the spinal cord has given positive results only in rare, exceptional cases (R. Wichmann) the neurotic explanation seems scarcely probable. Under certain circumstances, some forms of arthritis might be looked upon as infectious trophoneuroses, following Teissier and Roque.

It is, however, quite unlikely that the various forms of chronic articular rheumatism are due to any single cause. In the first place, we may exclude monoarthritis deformans, which is so closely allied to other senile changes (Weichselbaum) and which is so frequently produced by trauma. But it is also unlikely that there is any one cause for all cases of chronic polyarthritis, if we bear in mind the forms which are similar in all these symptoms, and yet are produced by various well known and quite distinct infectious diseases. Gerhardt in 1896 originated the term acute rheumatoid or pseudo-articular rheumatism, and defined it as follows: "Pseudo-articular rheumatism is that form of disease in which it may be proven, or where it is very likely, from its external appearance, that it is produced by the special pathogenic organisms of a definite infectious disease; the remaining cases are included under true articular rheumatism." These pseudo-rheumatisms have in common that they occur only in a minority of the individuals who are attacked by the infection in question and that the same organism which produces the infectious disease also produces joint pain, arthritic swelling or suppuration of the joint. In this sense we may speak of chronic rheumatoid ("pseudo-rheumatism" of Pribram) as an arthritis in which the exciting cause is one of the acute exanthemata, influenza, or pneumococcus infection, particularly gonorrhea, syphilis and tuberculosis. Gonorrhea is a clear example of the fact that the same microörganism, according to its virulence and the individual constitution of the affected person, may cause any grade of the disease from a transitory arthritic pain and serous effusion to an incurable, chronic deforming arthritis and spondylitis. I should like to call particular attention to rheumatic tuberculoid or tuberculous rheumatoid, a condition which lately has been 
frequently described (Poncet, Maillard, H. Strauss, Barjon, Roma, Potal). The usual course of such cases is this: the disease begins as a subchronic or chronic articular rheumatism which finally becomes localized to one or more joints, and there develops typical tuberculous changes. This course resembles osteomyelitis, of which we observed a case in the clinic at Basel in an individual aged seventeen, in whom, after a fever lasting several months, accompanied with wandering multiple joint swellings, muscular contractures, and exostoses, the epiphyses became loosened from the neck of the left femur, thus confirming the diagnosis.

True chronic polyarthritis may owe its origin to various infections, or to constitutional causes of the type suggested by Lancereaux and Bouchard. Perhaps in some cases nervous disturbances may play a rôle. These cases cannot be diagnosed by rules, no matter how skilful the reasoning processes, but only by following the advice given by Archibald Garrod, to inquire into the family history of each individual case, its nervous disturbances, etc., and by carefully investigating the bacteria present. Perhaps in this manner a rational differentiation of the varying forms, which to-day is impossible, may in time be attained.

\section{TREATMENT}

As in all chronic diseases for which we have no specific, the number of remedies is legion. But, in giving a synopsis, I shall divide them into groups.

First, internal remedies. I take for granted the knowledge that the antirheumatics, the salicylates, antipyrin and allied remedies often diminish pain, but never have the specific action which is the case in acute articular rheumatism. The salts of iodin are very useful, not only in gonorrheic and syphilitic rheumatoid, but also in the exudative polyarthritic form. Tonics are advised by the best authorities, and since experience has shown that in these affections the local difficulties are closely related to the general health of the patient, cod liver oil, iron, arsenic, quinin and strychnin preparations are of decided benefit in anemic, feeble, emaciated individuals, especially after febrile paroxysms or after active treatment. The salts of lithia, and mineral waters containing lithia, have been advised on account of their action in gout, but there is no rational indication here for their use. In fact, in a disease in which excessively chronic and decided yet spontaneous changes occur, the value of any curative agent is always very uncertain.

Menzer's successful trials of streptococcus serum, which were based upon the theory of an infectious etiology, are interesting but by no means conclusive.

The principal rôle in therapy is played by external physical remedies.

Water must be mentioned first. That energetic applications of cold water are harmful in the first stages of inflammation is a common experience. Only after acute exacerbations have run their course, and after long continued sweating procedures, may cold douches, needle baths, and affusions be made use of by an experienced and careful hand, and then serve a useful purpose as a hardening process. 
Warm water in all forms is frequently employed, from the Priessnitz pack to a hot bath. The latter are used particularly in the form of natural springs, which are of ancient repute. Their effect is to be ascribéd more to the temperature of the water and the duration of the bath than to the chemical constituents of the water (hydrogen sulphid, alkaline sulphates, calcium, gypsum, sodium chlorid, etc.). In rheumatism, the unaccountable experience is, that natural mineral waters may bring about improvement after the effect of hot water baths in the home has been exhausted. This effect is chiefly due to the cutaneous irritation of mineral waters rich in carbonic acid, such as those of Nauheim and Oeynhausen, or of mud baths combined with the thermic effect of the water. To these must be added the effect of applications of peatsoil, fango, natural sulphur, etc., and heat applied in various ways, sweat procedures, hot air and steam douches (the latter often producing a relaxation of muscle contractures and stiffness), sand baths, local and general hot air baths and, finally, local inflammatory applications; painting with tincture of iodin or ichthyol, compresses with iodin-potassium iodid salve $(1: 10: 100)$, vesication, etc.

All these applications have in common a tendency, to produce hyperemia, and August Bier has contributed other original therapeutic methods. The irritating or anodyne effect of derivatives and counterirritants has always been explained on the hypothesis that the blood is drawn from the distant diseased parts to the surface. Bier has shown that this is incorrect; that, on the contrary, the deeper parts partake in the hyperemia, and that this produces the anodyne and absorbent effect. Bier stimulates this action still further by hot air and stasis. The former produces an intense, highly active, i.e., arterial, hyperemia, which is greater the higher the temperature of the agent.

A simple apparatus, a wooden box with two openings to permit the entrance and the exit of the hot air, and bandages on which to rest the affected member are sufficient for the purpose; the hot air is produced by a Quincke sweat tube, or a phénix à air chaud, the tube being introduced into the opening of the box. Similar apparatus has been employed by Tallermann, and Lindemann has used electric heat; the effect of the electric light baths now in vogue, in which the electric bulbs radiate not only light but heat, is the same. For institutions these are very serviceable, as they are not dangerous and are easy to regulate. Bier's apparatus, however, has the enormous advantage that it may be constructed by a carpenter, at very slight cost, in the house of the patient, and be employed at the bedside. The effect of this remedy is excellent, as $I$ am able to testify.

The value of passive hyperemia attracted the attention of Bier on account of the rarity with which tuberculosis of the lungs is found associated with valvular disease of the heart. He attempted artificial blood stasis in tuberculosis of the joints, and was so gratified with the success attained that he employed this method also in other joint diseases. Above the diseased area an elastic (rubber) bandage is applied so tightly that the extremity becomes edematous.

However, the pressure must not be so great as to compress the arteries; the member must be warm, and the stasis must not produce the slightest pain. 
The compression may be continued for a long time. I have carried on this treatment for several months at a time. Bier has lately, however, advised us to use the method for only one or two hours daily.

The effect is at first to decrease the pain to such a degree that, for example, the tearing pains of gonorrheal arthritis soon cease, giving way to a feeling of well-being.

The rule which Bier has expressly emphasized is this, that the stasis itself must never produce pain; as soon as this appears, the bandage must be loosened or removed.

The further effect of stasis is to increase absorption. It is evident at once that joint effusion, edematous infiltrations of the capsule, and proliferation of the joint villi are more amenable to some treatment than are ecchondroses and osteophytes, that, therefore, the dry atrophic form of chronic arthritis is less susceptible to treatment than the hypertrophic proliferating forms with their spindle-shaped joints. Bier's hot air and stasis treatment, judged by the results, is by no means a panacea. The physician treating a case of chronic rheumatism must decide which symptoms most urgently call for relief. Fresh joint and capsule swellings require rest, the alleviation of pain, and the application of remedies which increase absorption, such as the salicylates, iodin, and heat. Older capsular contractures, in which immobility and fibrous ankylosis have occurred, require active and passive movements and the condition is often relieved by stasis. Muscular atrophy is benefited by faradic treatment and, above all, by massage. Contractures require applications of heat, prolonged baths, hot air or steam douches, peat or fango poultices, and protection from cold.

The earlier the treatment is begun, the better the result.

Firm ankyloses and hyperostoses, particularly of the larger joints, require surgical and orthopedic treatment; practice with suitable apparatus may improve the gait, and prevent the bad consequences of too great weight upon the vertebral column. The operative treatment of mono- and polyarthritis, according to the few results reported by W. Müller and his pupil Elter, deserves further trial.

\section{LITERATURE}

A very complete compilation has recently been given by Pribram in Nothnagel's Handbuch, vii, 2.

C. Adrian, "Ueber Arthropathia psoriatica." Grenzgebiete der Medicin, 1903, xi, p. 237.

W. Anschütz, "Ueber die Versteifung der Wirbelgelenke." Grenzgebiete der Medicin, viii, p. 461.

Bannatyne, Wohlmann and Blaxall, Lancet, 25, April, 1896.

Barjon, "Radiographie appliquée à l'étude des arthropathies déformantes," Paris, 1897.

Bechterew, "Steifigkeit der Wirbelsäule." Neurologischés Centralbl., 1893, und Zeitschr. f. Naturheilkunde, 1899, xv.

Beer, "Rigidität der Wirbelsäule." Wiener med. Blätter, 1897, Nr. 8 u. 9. 
W. Berent, "Zur Aetiologie osteoarthropathischer Veränderungen." Berliner klin. Wochenschr., 1903, Nr. 4.

A. Bier, "Hyperämie als Heilmittel," Leipzig, 1903; ferner 19. Congress für innere Medicin in Berlin, 1901; Münchener med. Wochenschr., 1898 und 1901, Nr. 48.

Bouchard, "Leçons sur les autointoxications," 1887.

I. Braun, "Klinische und anatomische Beiträge zur Kenntnis der Spondylitis deformans," Hannover, 1875.

R. Cassirer, "Ueber myogene Wirbelsteifigkeit." Berliner klin. Wochenschr., 1902, Nr. 10 u. 11.

Charrin, "Progrès Méd.," 1894.

I. I. Clarke, "Note of a Painful Condition of the Twelfth Pair of Ribs." Clinical Soc. of London, October 11, 1902.

H. Curschmann, "Berichte der med. Gesellschaft zu Leipzig." Schmidt's Jahrbücher, 1895.

G. Davaine, "Etude comparée du rheum. art. aigu. et des poussées aigues du rheum. chronique." Thèse, Paris, 1897.

J. Elter, "Weitere Beiträge zur chirurgischen Behandlung der Arthritis deformans." Zeitschr. f. Chirurgie, 1903, 66, p. 387.

Sir Alfred Garrod, "Natur und Behandlung der Gicht." German by Eisenmann. Würzburg, 1861.

Archibald Garrod, "Clinical and Pathological Relations of the Chronic Rheumatic Affections." Lancet, March 16, 1901.

C. Gerhardt, "Ueber Rheumatoidkrankheiten." 14. Congress für innere Medicin in Wiesbaden, 1896, p. 169 . "Verhältnis der Schuppenflechte zu Gelenkerkrankungen." Berliner klin. Wochenschr., 1894, Nr. 38.

A. Hoffman, "Lehrbuch der Constitutionskrankheiten," Stuttgart, 1893.

Herz, "Ueber das gleichzeitige Vorkommen von chronischen Haut- und Gelenker" krankungen." Wiener klin. Wochenschr., 1896, Nr. 26.

Hoffa, "Zur Pathogenese der arthritischen Muskelatrophien." Volkmann's Sammlung klin. Vorträge, 1892, N. F., Nr. 50.

M. Kachel, "Untersuchungen über Polyarthritis chronica adhæsiva." Ziegler's Beiträge, 1903, xxxiii, p. 327.

o. Kremer, "Pathogenese der arthritischen Amyotrophien." Inaug.-Diss., Greifswald, 1902.

Lancereaux, "Atlas d'anatomie pathologique," Paris, 1871. "Traité de l'herpétisme," Paris, 1883; "Leçons de Clinique médicale," 1892, I.

Landré-Beauvais, "Doit-on admettre une nouvelle espèce de goutte sous le nom de goutte asthénique primitive?" Thèse, Paris, an viii (1800).

Lindemann, "Locale Behandlung von Gelenkrheumatismus, etc., mit elektrischen Heissluftapparaten." Therapeut. Monatshefte, März, 1900.

L. Maillard, "Rheumatisme tuberculeux." Gazette hebdomad., 1900, No. 88.

K. Manasse, "Zwei Fälle von isolirter rheumat. Erkrankung der Kiefergelenke." Münchener med. Wochenschr., 1902, Nr. 20.

Menzer, Deutsche med. Wochenschr., 1903, Nr. 67 und Zeitschr. f. klin. Medicin, 47, p. 109.

Joh. Müller, "Beobachtung über reine Zwerchfell-Bauchathmung bei ankylosirender Wirbelgelenkentzündung." Verhandlungen der Würaburger phys.-med. Gesellschaft, 1901, p. 41.

W. Müller, "Zur Frage der operativen Behandlung der Arthritis deformans." Archiv f. klin. Chirurgie, 47, 1894. 
Pribram, "Chronischer Gelenkrheumatismus und Osteoarthritis deformans." Nothnagel's Handbuch der spec. Pathologie und Therapie, vii, 2.

E. Pfeiffer, "Ueber Gichtfinger." Berliner klin. Wochenschr., 1891, Nr. 15.

Potain, Semaine méd., 20. Mai, 1891, p. 210.

A. Poncet, "Rheumatisme tuberc. abarticulaire." Lyon méd., 1902, No. 29; Gazette hebdomad., 1902, Nr. 58.

Potal, "Rheum. artic. tub. chronique." Gazette hebdomad., 1902, No. 10.

H. Rabinowitsch, "Beiträge zur Kenntnis des Gelenkrheumatismus mit Knötchenbildung." Inaug.-Diss., Berlin, 1899.

Roma, "Rheumat. tuberculeux." Gazette hebdomad., 1902, No. 93.

Roese, "Ueber amyloidentartung bei chronischer Arthritis." Inaug.-Diss., Leipzig, 1897.

Schuchardt, "Krankheiten der Knochen und Gelenke." Deutsche Chirurgie, 28. Lieferung.

M. Schüller, Berliner klin. Wochenschr., 1893, Nr. 36, 1900, Nr. 5 bis 7; 15. Congress für innere Medicin, Berlin, 1897.

Schlesinger, "Chronische Steifigkeit der Wirbelsäule." Grenzgebiete der. Medicin, 1900.

Senator, "Krankheiten der Bewegungsorgane." Ziemssen's Handbuch der spec. Pathologie, xiii.

Sudeck, "Acute (trophoneurotische) Knochenatrophie nach Entzündungen und Traumen der Extremitäten." Deutsche med. Wochenschr., 1902, Nr. 19.

H. Strauss, "Acute Miliartuberculose unter dem Bild einer Polyarthritis." CharitéAnnalen, xxiv.

Tallermann und Mendelsohn, "Behandlung des chronischen Gelenkrheumatismus." Deutsche med. Wochenschr., 1898, Nr. 11.

Teissier et Roque, "Rheumatisme chronique." Traité de méd. de Brouardel. Gilbert et Girode.

Trousseau, "Clinique médicale."

R. v. Volkmann, "Krankheiten der Gelenke." "Pitha-Billroth'sche Chirurgie."

Vidal, "Considérations sur le rheum. chron. primitiv." Thèse, Paris, 1855.

Weichselbaum, Virchow's Archiv, lv.

Wohlmann, Brit. Med. Jour., November 11, 1900. 


\section{PENTOSURIA}

\section{By F. BLUMENTHAL, BerLin}

PRIOR to the last few years all urine which gave a distinct and unquestionable reduction test was considered to contain sugar, and permitted us to conclude the presence of grape sugar; this view is no longer tenable. We know now that a variety of sugars are found in human urine all of which react to the recognized sugar tests (Trommer's, Nylander's, the phenylhydrazin test). Besides grape sugar we find mill sugar, which appears during the puerperal period in a woman who has an abundant secretion of milk yet does not nurse her child; this substance may persist in the urine for months post partum, in fact as long as the secretion of milk continues. Here the differential diagnosis from grape sugar is accomplished very simply by the fermentation test; milk sugar does not ferment with yeast, while grape sugar does. Robinson and Lépine, and also Rosin and Laband, have lately described cases of levutosuria which is characterized by its independence of the ingestion of carbohydrates, and this condition may be recognized by the decided levorotatory power developed during the fermentation test of the urine. Of less importance is the occurrence of maltose, which occasionally appears in the urine in diseases of the pancreas, and the presence of which can scarcely be detected even by the most delicate chemical methods. Of more importance are the conbined glycuronic acids and the pentoses.

We refer here to the former substances because the combined glycuronic acids may be considered as pentose-carbonic acids, $\mathrm{C}_{5} \mathrm{H}_{10} \mathrm{O}_{5} \mathrm{CO}_{2}$. Combined glycuronic acids are found in the urine after the administration of numerous drugs, especially such as contain the aldehyd and ketone groups (Neubauer), and these may then be excreted in the urine in combination with glycuronic acid. Among the best known of these drugs are: morphin, chloral, turpentine, menthol, antipyrin, etc. Glycuronic acid is also found in the urine combined with indoxyl and with phenol. As some of the combined glycuronic acids respond to the Trommer and Nylander tests, it may be of importance in such cases-particularly with doubtful tests-to search for the cause, and it is then not infrequently found that, at least in human urine, we are dealing with a very decided indicanuria. If the patient who voids this reducing urine has taken any drug, the supposition is natural that it has been excreted as glycuronic acid and has thus caused the reduction.

The urine coming from a case of pentosuria gives a distinct but somewhat delayed reaction with Trommer's test, and usually (as E. Salkowski first 
noted) the reduction with Trommer's test only takes place upon cooling, but then quite suddenly.

The phenylhydrazin test is also positive with pentose; Nylander's test is not quite so distinct, and thus it happens that a person applying for life insurance is sometimes rejected on the ground that he is supposed to be a dia'betic. This happened in the case of a patient, aged thirty, who had been married for but a short time, and who, on account of his rejection for life insurance, was very unhappy. In great excitement he consulted a physician for a probable diabetes. Upon examination of the urine the physician at once became suspicious, for the urine was optically inactive in the polariscope and did not ferment with yeast. When the urine was examined more accurately (Prof. Salkowski) it was found that the sugar it contained was pentose.

Here was a case in which the examination of a urine having reducing properties but optically inactive and non-fermentative led to the discovery of pentose; the question may quite properly be asked, "How can such a diagnosis be confirmed with certainty?"

A urine that contains pentose, and only such a one, gives the ORCIN TEST. This is done in the following manner: 3 c.c. of urine are decomposed with about 6 c.c. of fuming hydrochloric acid; to this is added a few granules of orcin and the mixture is then heated to the boiling point. As soon as the mixture begins to boil, a bluish green color appears which is proof positive of pentose. Urine containing grape sugar or milk sugar does not give this reaction; urine containing glycuronic acid gives the test only upon prolonged boiling, and then the precipitate is never greenish blue but more of a violet color.

Before the orcin test was introduced into the chemistry of urine, the phloroglucin test was used but it was much less positive. According to E. Salkowski, this test may be carried out in the following manner: 3 c.c. of urine are decomposed with 3 c.c. of hydrochloric acid of a specific gravity of 1.019, to which a few granules of phloroglucin are added and the mixture is heated to the boiling point. Even after slight heating a cherry red color develops which gradually becomes more distinct, and finally (a point that is characteristic) turns greenish black. If amyl alcohol is then added, and the mixture shaken, the coloring matter is dissolved, and shows an absorption band between D and E, i. e., between yellow and green. Urines which contain large amounts of glycuronic acid show a brownish black color, but present the same absorption lines; urines which contain only traces of glycuronic acid show no absorption lines nor the characteristic colors indicative of pentose.

I believe that the orcin test is more reliable than the phloroglucin test, as most of the glycuronic acids yield no reaction with the latter; at least, not if the test is done in the manner I have indicated. In using the orcin test, one or two drops of liq. ferri sesquichlor. may be added, according to Bial; this produces a beautiful blue color. Or Bial's reagent may be used (acid. muriat. conc. 250.0 ; orcin 0.5 ; liq. ferri sesquichlor. 10 drops). Confusion with pentose, provided the urine is examined twenty-four hours after it has been voided, is only possible with two of the glycuronic acids at present known, 
namely, with mentholglycuronic acid and turpentinglycuronic acid. Both of these glycuronic acids have the property of decomposing spontaneously, and as free glycuronic acid also reacts to the orcin test, they may be confounded. We are prevented from making this mistake, first, by the history, as mentholglycuronic acid and turpentinglycuronic acid are only excreted after the ingestion of menthol and turpentine; secondly, by the odor of the urine; menthol urine smells of peppermint, turpentine urine of violets. Therefore, in human urine, since the introduction of the orcin test, there can scarcely be any diffculty in the recognition of pentose. The circumstances are different in the urine of the herbivora. They frequently take up with their food a mass of pentosan, that is, the anhydrid of pentose. The pentosans have the same relation to pentose that glycogen has to grape sugar. These pentosans are in part excreted as such and give the orcin test, as does pentose, for by heating with hydrochloric acid pentose is developed from pentosan.

As the urine of the herbivora very frequently has reducing properties and therefore gives a more or less distinct orcin test, confusion with pentose is not impossible. In these cases the phenylhydrazin test must be employed; if this is positive while the fermentation test is negative, pentose is present. If the fermentation test is positive, after fermentation has ceased the phenylhydrazin test must also be positive if pentose is present.

After this slight digression, we return to the chemical analysis of the urine. If we have under consideration a urine that gives a positive reaction with Trommer's test, the phenylhydrazin test, the orcin and phloroglucin tests for pentose, if the urine is optically inactive and does not ferment, then the patient excreting such a urine has pentosuria. The question now arises, What does this condition indicate? As it is certain that he is excreting sugar, i. e., pentose, there is unquestionably a disturbance in sugar metabolism., We are then confronted with another question, whether we are dealing with a variety of diabetes mellitus, a pentose diabetes, or something else. If we are dealing with a variety of diabetes mellitus, the combustion of carbohydrates must be diminished, as in the case of diabetes. Such a connection must be thought of, all the more so as Ruff has produced pentose from derivatives of grape sugar by oxidation with potassium permanganate and hydrogen peroxid, and it is possible that the human organism may also carry on this process of oxidation. Further, as it is known that the pentose which occurs in nature and enters the body with the food, such as the l-arabinose and l-xylose, undergoes only partial combustion even in the healthy, it is not unnatural to explain pentosuria by conceiving that the patient, from the forms of sugar with six carbon atoms, forms pentose. This he incompletely oxidizes, and hence excretes a portion of it. If this view were correct, with the withdrawal of starch from the food pentosuria should disappear or at least lessen, and, on the other hand, with the profuse administration of starch or grape sugar the pentosuria should increase.

But when we withdraw carbohydrates from patients who excrete pentose, it is always observed that the pentosuria continues, and apparently in the same degree as formerly, while the administration of even 100 grams of grape sugar after a strict diet free from sugar does not lead to a decided increase of the 
pentosuria. From these results it is obvious that pentose in the animal organism is not formed by the oxidation of hexose, i. e., the varieties of sugar with six atoms of carbon or their multiples.

This to some extent also answers the question whether pentosuria is a variety of diabetes mellitus. The latter disease is characterized loy the fact that the power to burn starches and hexoses is diminished. That this is not true of pentosuria, at least not of all cases of pentosuria, was shown by Bial and myself, for the administration at once of 100 grams of grape sugar caused no glycosuria. The combustion of d-galactose in our case of pentosuria was also entirely normal. It follows from this that the patient with pentosuria has no greater tendency to alimentary glycosuria than the healthy; hence if we look upon alimentary glycosuria as a proof of the existence of diabetes mellitus, the urine of the pentosuric is in this respect also entirely negative. There is good ground for the supposition that pentosuria is a pentose diabetes, in which metabolism for sugar with six atoms of carbon is normal, while the property of combustion for pentose is diminished or has entirely ceased. With food in the form of grain and fruit, and in beer and tea we ingest a certain amount of pentosans; it is quite possible that pentosans are changed into pentose in the stomach by the action of hydrochloric acid; but the organism is incapable of oxidizing them and they are therefore excreted. This hypothesis is accepted by Naunyn and Lüthje, and by others. Can this view be possible?

The pentose which we ingest with our food is the dextro-rotary l-arabinose, while, as we know from the investigations of Carl Neuberg, inactive arabinose also occurs in the urine. Since inactive arabinose, as the researches of Emil Fischer have shown, can only occur by the combination of $d$-arabinose and l-arabinose, the organism must, in addition to l-arabinose, also furnish d-arabinose; so that i-arabinose may be formed.

The question whether the organism contains pentose groups has been discussed for a long time. As is well known, Hammarsten found in the nucleoproteid of the pancreas a reducing substance which he assumed to be pentose. E. Salkowski has produced phenylosazone, and, upon the basis of an analysis of the same, has determined with certainty that the reducing substance is pentose. On account of the abundant furfurol formation which is said to be characteristic of pentose, and which upon decomposition was shown to be present in the nucleo-proteids from various animal organs, and on account of the production of phenylosazones at the melting point of pentosazone, $157^{\circ}$ to $160^{\circ} \mathrm{C}$., I have maintained that all animal nucleins contain a pentose group, and that the pentose group is characteristic of nucleins just as is the group of xanthin bases, for I found no proteids which contain pentose except nucleins. I have also held that when an albumin body gives the phloroglucin test for pentose we are justified in declaring it to contain nuclein. This view at the time appeared to be decidedly opposed to the prevailing opinion. A. Kossel and Neumann had, two years previously, found no pentose group in nucleinic acid of the thymus gland. The former denied absolutely the presence of a carbohydrate group in the spermatic nucleinic acid. Noll, the pupil of A. Kossel, forbore entering into this discussion. He believes that we must dis- 
criminate sharply two reducing carbohydrates. One is loosely combined with the nucleins, and in the formation of nucleinic acid separates from nuclein; the second, a firmly combined atomic group with a molecule of nucleinic acid, causes the production of levulinic acid and formic acid, the latter of which does not form a reducing carbohydrate. In view of this, Kossel's adherents admitted the presence of a reducing carbohydrate in animal nuclein, but refrained from any expression regarding the nature of the reducing substance. Later, Friedrich Müller questioned the opinion, then prevalent, that pentose was the sugar of the vegetable kingdom and did not occur in the animal kingdom. He believed there was often a confusion of pentose with glycuronic acid, a confusion which was by no means impossible on account of the test then in vogue. This diversity of views led Bergell and myself to make further researches in regard to pentosuria. From a urine supposed to contain pentose, he and I obtained a barium combination of the questionable sugar, the analysis of which showed we were, in fact, dealing with pentose. By this means, the presence of pentose in the urine of animals was further confirmed by Jastrowitz and Salkowski, and the production by Carl Neuberg of pure r-arabinose from so-called pentose urine furnished proof incontestable in every respect. Furthermore, Wohlgemuth, who at my suggestion examined the nucleo-proteid of the liver, produced from this a chemically pure phenylosazone which by analysis proved to be phenylpentosazone. The presence of pentose in animal organs was later confirmed by other competent observers. Thus Neumann, the former co-worker of Kossel, found that, with an improved technic, nucleinic acid obtained from the thymus gland gave a decided pentose reaction. The labors of Bang, Jacob, and Bergell, Friedenthal, Umber, Grund and others, proved the correctness of the theory first suggested by me that not only all vegetables but also all animal nucleo-proteids contained carbohydrates belonging to the pentose group.

The question now arises, How was it possible that these-groups were so long overlooked in the nucleins by prominent investigators, and that my earlier results were so long unconfirmed? This must be ascribed to the fact that in the nucleo-proteid of the thymus, as Umber and I determined in the pancreas proteid, the pentose group is very loosely combined. To obtain the nucleoproteid of the thymus a solution of the same in an alkali and precipitation with acetic acid are sufficient to separate the greater portion of the pentose and to obtain a phosphorus-containing albumin body that no longer shows pentose but still contains some xanthin bases. And for the nucleo-proteid of the pancreas Umber has shown that the pentose group is one of the first products of pepsin digestion which enters solution. This view might very readily coincide with that of Kossel's adherents who believe that the reducing carbohydrate is very loosely attached to the nucleo-proteid, for on the solution of thymusnuclein in alkali a phosphorus-containing albumin body which still contains xanthin bases, i.e., a nuclein, is retained. I believe, therefore, that such a body free from pentose should, at most, be designated an atypical nuclein.

That the pentose group is but loosely combined with the nucleins is certainly not true of the pancreas nuclein, for Bang has obtained pentose from its nucleinic acid. 
According to recent investigations of Neumann, pentose is present in the nucleinic acid of the thymus; hence it follows that the pentose group is an integral constituent of at least some of the nucleinic acids, if not of all.

Of the pentoses which occur in animal nucleins, that of the pancreas has been most minutely investigated. Bang supposed it to be dextro-rotary, and Neuberg has lately arrived at the surprising conclusion that the pancreatic pentose is l-xylose. This latter theory is of great importance as bearing on the origin of pentose in chronic pentosuria. For, if on other grounds we were inclined to believe that the pentose of urine originates from the pancreatic nuclein such an opinion would by this result be proven to be erroneous. It is impossible to understand how xylose could be changed into arabinose. It is true that we have only proven for pancreatic nuclein that its pentose is l-xylose; the other nucleins have not been investigated in this respect.

The researches in metabolism by Bial and myself have also made it appear unlikely that pentoses are formed in the pentosuric patient by an imperfect nuclein decomposition, since the metabolism of the pentosuric shows no such increased destruction of nucleins. Neither the excretion of uric acid nor the excretion of phosphoric acid is increased in pentosuria. It also appears to be impossible that the inactive arabinose in pentosuria originates from other nucleins, and not alone from the pancreas nucleins, and we must search elsewhere for an explanation of the origin of pentose.

Carl Neuberg has given us important and interesting conclusions in this feld. He demonstrated that the l-xylose which is found in pancreatic nuclein originates from grape sugar; now we have assumed for a long time that glucose is partly oxidized from glycuronic acid. If we consider glycuronic acid as pentose carbonic acid, $\mathrm{C}_{5} \mathrm{H}_{10} \mathrm{O}_{5} \mathrm{CO}_{2}$, it need only give off its carlo-xylose group to produce dextro-rotary l-xylose. This the organism requires for the construction of nucleins. On the other hand, in his opinion, the r-arabinose, the urinary sugar of pentosuria, originates in a very different manner.

As is well known, milk sugar is split up in the intestine into dextrose and galactose; a portion of the galactose is certainly utilized for glycogen production. Another portion enters into cerebrin, for Thierfelder was able to demonstrate the presence in cerebrin of galactose. The galactose contained in cerebrin, however, is dextro-rotary, like the galactose contained in food. But it is very easy to change this d-galactose to its inactive form, and then from the inactive galactose inactive arabinose may readily be produced by oxidation. Carl Neuberg is therefore of the opinion that inactive arabinose originates from derivatives of galactose. How far this view is correct is still uncertain, for the behavior of i-galactose must be tested in the organism of a pentosuric patient. The behavior of the ordinary d-galactose has been studied by Bial and myself, but we were unable to determine an increase of pentose excretion.

It is evident from these considerations regarding pentosuria that prior to its discovery by $\mathrm{E}$. Salkowski we had but slight understanding of the sugar metabolism of the human organism, and that in pentosuria we are dealing with an anomaly of sugar metabolism which must be assumed to be an independent one. It has nothing in common with diabetes, and is also not to be regarded 
as a pentose diabetes, for the property of combustion of the pentoses of the food is nowise altered. The same conditions are present here as in levulosuria, which Rosin and Laband have lately determined represents an anomaly in the production of levulose, not, however, dependent upon a disturbance of its combustion. I do not believe it can be doubted that in true diabetes we shall ultimately reach the point of separating a group of cases in which the formation of grape sugar in the organism is disturbed; while there is no anomaly of combustion. I mean the cases with slight excretion of sugar (1 per cent. or less) and this almost entirely independent of the carbohydrate constituents of the food. Perhaps, also, in many severe cases such a disturbance in the synthesis of sugar may be found.

Pentosuria is therefore an independent disturbance of metabolism, which is characterized by the fact that in this condition an inactive sugar has been found in nature for the first time. Thereby the law that the animal organism and plants can produce only active varieties of sugar has been proven erroneous. A second law, which for a long time was considered incontestable, namely, that the pentoses are the sugar of the vegetable kingdom only, and do not occur at all in the animal kingdom, has also been nullified.

We must separate true chronic pentosuria from alimentary pentosuria. A large number of individuals do not possess the property of oxidizing large amounts of pentosan introduced with the food, but excrete a portion of these pentosans as pentose. This is alimentary pentosuria. The amount of pentose excreted is about 0.2 to 0.5 per cent. This pentose always arises from the pentosans which are taken up with the food, for the most part in the dextrorotary l-arabinose contained in fruits. This phenomenon we generally see in the summer when fruit is freely eaten (cherries, strawberries, whortleberries, plums).

This alimentary pentosuria has nothing in common with chronic pentosuria, in which arabinose also occurs; 'here not the dextro-rotary but the inactive appears in the urine. We now find, however, that besides chronic pentosuria the alimentary type may also exist, and I am in possession of records of cases in which both the inactive arabinose and the dextro-rotary appear in the urine. The proof of this has been deduced as follows: first, the urine without fermenting was dextro-rotary; secondly, only pentosazone could be produced from it; and thirdly, according to Neuberg, a solution of the osazone in pyridin alcohol proved dextro-rotary.

True chronic pentosuria, as we have seen, is characterized by the fact that, independently of food, inactive arabinose is excreted continuously. The amount of inactive arabinose varies in this condition between 0.3 and 1 per cent. by Knapp's method of titration.

We find, however, cases also of chronic diabetes that are complicated by pentosuria, i. e., showing a slight excretion of pentose. To this group belong a number of diabetics in whose urine very small amounts of pentose have been demonstrated, as shown by Külz and Vogel. In these cases the pentose has not the slightest clinical importance so far as can be determined at this timè. It occurs in such minute traces that its presence cannot be detected by our 
common tests, and the utilization of several liters of urine is necessary to find the substance at all. We have no knowledge as to which pentose appears in the urine in diabetes (whether pentose of the food, or inactive arabinose, or pancreas pentose, i. e., l-xylose), nor do we know whether it is particularly in the severe cases of diabetes that pentose is excreted.

To quite a different group belong those cases of pentosuria in which, besides pentose, glycose appears transitorily in the urine. In this instance the glycosuria may have an accidental cause (morphin), as in the case of Jastrowitz and Salkowski which led to the discovery of pentosuria. We may, however, encounter the combination of true pentosuria with mild diabetes. I have seen such a case. The quantity of grape sugar amounted to 0.6 to 1 per cent., the amount of pentose was 0.3 to 0.5 per cent. As the urine was sent to me for investigation only a few times, I can say nothing further about the course of the case. Only this much could be determined, that the pentose found in the urine was inactive, therefore probably an inactive arabinose.

The second group of chronic cases of pentosuria includes the pure cases, in which no other sugar than pentose is found. Up to this time the following cases are reported in literature: first, the case of Jastrowitz and E. Salkowski in which the glycosuria disappeared after morphin was stopped, while the pentosuria proved chronic; secondly, the two eases described by Salkowski and myself. The first of these occurred in a merchant, thirty-six years of age, and always healthy, who was married and had four living children. The amount of pentose he excreted varied between 0.7 and 1 per cent., with an average amount of urine of a liter to a liter and a half per day. This case occurred in 1895 in the practice of Dr. L. Feilchenfeld who still has the patient under observation. Up to this time the pentosuria has never been associated with any serious symptoms. The patient repeatedly suffered from hydrocele, and the fluid obtained by puncture was examined by me and found to contain grape sugar but no pentose. The urine has also been utilized by C. Neuberg for the preparation of $\mathrm{r}$-arabinose. The patient was decidedly thin.

The third case occurred in a banker, aged sixty-five. This patient (according to the report of Dr. Blumenthal, who treated him for over twenty years before the pentosuria was discovered) is said to have repeatedly had reducing substances in his urine, but grape sugar was never found until 1895, when the pentosuria was discovered. This case is especially interesting as, in the family of the patient, numerous chronic diseases occur, particularly diabetes and nervous diseases. Up to two years before death (which occurred in 1900) the urine in this case constantly showed about one per cent. of pentose. For some time before death he was treated by another physician; his death was due to arteriosclerosis. The autopsy showed calcification of the coronary arteries; the pathologist told me that nothing of special interest was found in the pancreas, but unfortunately he had not been informed of the existence of pentosuria.

The fourth and fifth cases were published by Dr. Bial. The fourth was that of a merchant from Warsaw, aged thirty-seven, who suffered from mild 
gastric and intestinal symptoms and supposed himself to be a diabetic. Small amounts of sugar had been frequently found in his urine, and a suitable diet had been advised. The report from a Warsaw chemical laboratory shows slight quantities of sugar found by Trommer's test and a positive reaction to phenylhydrazin. This patient was also conspicuously thin. Bial determined that pentose was present in the urine in July, 1898, as well as constantly for twenty days in July, 1899, but no grape sugar could be found.

Case V (Bial). A druggist, twenty-eight years old, perfectly well. The amount of pentose in Case IV amounted to 0.3 per cent., in Case V, 0.35 per cent.

Case VI. Reported by Dr. Fritz Meyer. A merchant, aged thirty-nine, never previously ill, was rejected by a life insurance company six years ago, and before the discovery of pentosuria, on account of diabetes. He was treated in Carlsbad, was on a strict diet, and was declared to be cured as the examination of his urine by the polariscope showed optical inactivity. Five years ago he married, and is now the father of a healthy child. His health, with the exception of a mild attack of perityphlitis, has always been good. In April, 1900 , symptoms appeared which gave rise to the suspicion of a constitutional disease; his weight is said to have decreased decidedly; it amounted to 150 pounds. He complained of headache, lumbar pains, lassitude, vertigo, and severe neuralgia, particularly in the region of the sciatics. The urine amounted to 1,800 c.c.; it was clear, contained no formed elements, and was without albumin. The urine reacted positively to Trommer's and Moore's tests; being optically inactive it was examined for pentose, and pentosuria was determined.

In the seventh case, the patient was an American lady, who had been several times under treatment in Carlsbad on account of supposed diabetes. Her case was always considered to be a severe one, for, in spite of the strictest diet, it was never possible to render the urine aglycosuric. In this case quite a decided amount of pentose was found, over 1 per cent., but not the slightest trace of grape sugar.

The eighth case (Dr. Brat) occurred in a lady, aged sixty-two, who for several years was under professional treatment on account of a presumably mild diabetes. She had a slight degree of fatty heart, otherwise, however, she was quite well. This lady has been under my observation for more than a year, and in this entire time has never excreted grape sugar. In her case, however, the urine was somewhat dextro-rotary, 0.2 per cent., and I believe it therefore not unlikely that, besides inactive pentose, a dextro-rotary pentose was present in slight degree.

Case IX. The brother of the patient just described (Case VIMI), aged about fifty, perfectly well. His urine showed about 75 per cent. of pentose. According to analyses made by several chemists since $1892,0.2$ to 2 per cent. of sugar was determined. One found only a reducing property in the urine but no sugar. There is no question in my mind that the gentleman was not diabetic, for a breakfast containing large amounts of sugar gave no results. The case is otherwise of no clinical interest; the other brothers and sisters present neither diabetes nor pentosuria. 
Additional cases of pentosuria have been described by Colombini in morphin habitués; by Caporelli in xanthoma, others by Reale and Romme, and a case of alimentary pentosuria with glycosuria by Barszewsky.

It is not remarkable that so many cases of pentosuria have been confounded with diabetes, for the qualitative tests are the same. The important question is, and remains: Has pentosuria anything in common with diabetes? Have pentosurics on account of their pentosuria a special liability to become diabetics? On account of what has been above stated these questions must be answered negatively.

Pentosuria is a disturbance of metabolism which is connected perhaps with cerebrin metabolism, perhaps with the formation of galactose. Negativety the principal point is that there is no evidence of insufficiency in the utilization of the carbohydrates consumed with the food.

Another question now arises: What is the prognosis of pentosuria? It is unwise to express a dogmatic opinion on this point since we have known the disease for only a decade. Notwithstanding this, it may be said that the prognosis is probably much more favorable than in mild diabetes, for the pentosuric utilizes fully the starches and other carbohydrates which are administered with the food, and the amount of pentose which he forms and excretes is small, at most from 15 to 20 grams per day. This, therefore, represents no more serious prognosis than the mildest cases of diabetes. Upon the other hand it must not be forgotten that sugar is circulating in the blood. That this is true is obvious from the investigations conducted by Bial and myself, as we have proven the presence of arabinose in the blood. But the presence of a large quantity of sugar in the blood leads to arteriosclerosis, and may give rise to other changes.

Whether the pentosuric is more susceptible to infections than a healthy person, as is the case with diabetics, is very difficult to say, for up to the present time the data are very scanty. Upon the whole, and in the majority of cases, the prognosis of pentosuria can certainly not be termed very serious.

Regarding therapy, such treatment as we employ in diabetes is out of place in pentosuria, as is obvious from all that has been stated. In general only this much is to be said: (1) A pure meat diet, according to the experience of Fritz Meyer and myself, is not well borne by pentosuries, for neuralgic symptoms, if present, are increased. (2) A mill diet is found to be particularly advantageous.

In conclusion, a word regarding the frequency of the disease. Although it may be assumed from the, as yet, scant publications that pentosuria is a rare affection, I cannot admit that it is to be regarded as a curiosity like maltosuria. Such a comparison is incorrect, for pentosuria, in the first place, gives us a very interesting insight into disturbances of metabolism; and secondly, it cannot be considered immaterial that a person, as in almost all of the cases that have been cited here, should be supposed for years to be a diabetic, and subjected to dietetic and mineral spring treatment which is absolutely out of place. In life insurance the decision is of the greatest importance. The pentosurics must at least be admitted to have as favorable a prognosis as the mild cases of diabetes. 
If it be further remembered that nine cases have been detected in Salkowski's laboratory and in the First Medical Clinic of Berlin, the affection cannot be so rare as many believe. Hence a knowledge of this disturbance and of the means to its diagnosis is an absolute necessity for every physician.

Briefly the most important diagnostic factors are as follows: Positive orcin test with negative fermentation test proves pure pentosuria; positive orcin test and positive fermentation test denote pentosuria and glycosuria.

A turning to the right of the polariscope does not prove the non-existence of pentosuria, for dextro-rotary pentose also occurs in the urine. 


\section{DISEASES OF THE BLOOD}




\title{
BLOOD AND BLOOD EXAMINATION
}

\author{
By A. LaZaruS, Charlottenburg (Berlin)
}

Tris examination of the blood, in comparison with other clinical investigations, has very slowly forced its way into practice. While the conscientious physician rarely fails to make an examination of the urine or of the sputum in cases in which it seems necessary or at all useful, examinations of the blood, even the simplest, have up to the present been resorted to by a very small number of practitioners.

The object of this article is to call attention to the necessity of clinical blood investigation, and to describe the simplest methods. It will then become a matter of routine to examine the blood no less frequently than the various other secretions, excretions, or inflammatory products.

Of course, the importance of the results of blood investigation in different diseases of the blood is not always equally great. In the cases in which a disease of the blood or of the blood-producing organs comes into question, an examination of the blood is more important than any other clinical research, and often this alone will guide us to a definite opinion. This is true of the various forms of anemia, of leukocytosis and leukemia, of many diseases of the bone-marrow, and in certain parasitic diseases of the blood. [There can hardly be said to be "many diseases of the bone-marrow" recognized to-day, and the blood has never yet helped much to advance our knowledge in this direction.

Parasitic diseases of the intestine and other internal organs should be mentioned here among those in which blood examination is of great diagnostic value.-ED.]

The number of cases is, however, disproportionately greater in which an examination of the blood, although actively complementing other methods, is alone not decisive. It may be of value in the differential diagnosis between various acute infectious diseases; it may make clear the nature of many cases of poisoning; in the prognosis of many bacterial diseases we may under some circumstances find points of support in the condition of the blood; the prophylaxis and therapy of malaria, according to Robert. Koch's investigations, can only be made certain by regular examinations of the blood, and to these many other examples might be added [e.g., trichiniasis, filariasis, uncinariasis, trypanosomiasis.-ED.].

That the history under some circumstances may become enriched by an examination of the blood is obvious if we remember that after recovery from 
certain infectious discases protective bodies may be demonstrated in the blood serum.

After these brief indications it will be seen that we have quite a large field of activity, even if we limit ourselves to such an investigation of the blood as is of immediate clinical importance. It is impossible, however, to omit a brief explanation of the normal physiology and anatomy of the blood, as this enables us to measure the degree of pathological change liable to occur.

We must first decide upon the best method of obtaining the amount of blood necessary for investigation. Of course for clinical purposes only those methods of examination are valuable which need no more than one or two c.c., or-if there be a necessity for frequent repetition-a few drops of blood. Consequently, many clinical methods, compared with strict physiological processes, suffer more or less in reliability. This fact has lately given rise to an endeavor to modify certain physiological methods which hitherto have necessitated large quantities of blood. The results have, in the main, been rather unsatisfactory. This is true, for example, of the "clinical" estimation of the alkalinity of the blood, and of the iron and phosphorus in the blood. If large quantities of blood cannot be obtained, it is better to refrain entirely from such estimations than, by an appearance of exactness, to produce figures of questionable value.

Fortunately, however, for many important clinical investigations, only a few drops of blood are necessary, and these are best obtained under aseptic precautions by a simple prick of the finger, of the toe, or of the lobe of the ear. [In America the finger is rarely used, as the pain of puncture is much greater than in the ear. An ordinary glover's needle (bayonet-pointed) is convenient, cheap and efficient. A sewing needle cán be used but does not answer nearly as well.-Ev.] By friction or by active muscular movement these portions of the body may previously be made somewhat hyperemic. In general the choice of the place is immaterial; for smear preparations the finger is preferred. In very sensitive patients, provided the investigation is to be repeated frequently, the ear is chosen. The prick is made with half of a steel pen or with a Soennecken's lancet; but we may also use the so-called "pistol knife" in which the needle-shaped knife may be adjusted, and, by pressure of the spring, inserted to an exact depth.

If larger quantities of blood, i. e., a few cubic centimeters or more, are necessary it is advisable, provided there is no contra-indication, to obtain the material by wet cups; in which proceeding it must of course be remembered that blood thus obtained is more or less admixed with lymph. Finally puncture of a dilated vein of the arm by the aid of a Pravaz syringe, which is very readily performed, or an ordinary venesection may be resorted to.

The withdrawal of blood, if performed aseptically, is entirely without danger and, as a rule, does not disturb the patient in the least. We should never forget, before procceding, to assure ourselves that we are not dealing with a hemophilic. In such persons, even with a simple puncture of the finger, there may be great difficulty in stopping the flow of blood. [In such cases a mere touch of the needle point to the skin will give us all the blood needed without producing any troublesome hemorrhage.-ED.] 


\section{HEMOGLOBIN}

The exuding drop of blood shows even to the naked eye a number of properties. The redder it is, the richer it is in oxyhemoglobin; the darker, the greater the amount of reduced hemoglobin. Accordingly, it is at once recognized from these properties whether the blood originates from the arterial and capillary vessels or from the venous system. In carbonic acid poisoning the color of the blood is particularly bright and, as a rule, is designated as cherry-red.

In the red oxygen-containing blood differences in the intensity of the coloring are readily determined, but these are frequently not easy of recognition in very dark venous blood. Such differences become more noticeable if the drop of blood is caught and spread out upon white linen or upon white blotting paper; the greater or lesser staining power of different drops of blood is very well demonstrated in this simple manner, and, with some practice, from the color of the stain a conclusion may be drawn as to the AMOUNT OF HEMOGLOBIN in the blood. Following this principle, Tallqvist has described a "hemoglobin scale" by the aid of which gross differences in the amount of hemoglobin may be quite accurately estimated. [The Tallqvist scale gives us not ideal accuracy but all the accuracy that we can use in diagnosis, prognosis and treatment. Its crrors rarely exceed 10 per cent., and in the hands of the unskilled other and more "accurate" instruments often show more errors than this. The cheapness of the scale and the ease and quickness of using it are also important recommendations.-ED. 7

Accuracy is greater with apparatus especially constructed for the estimation of the coloring power of the blood, the so-called hemoglolinometer. This apparatus is quite properly named; for, in the main, it is the amount of hemoglobin contained which determines the coloring property of the blood; the importance of a few other coloring substances contained in the blood is in comparison quite insignificant.

For clinical purposes a large number of blood colorimeters have been described; on account of their simplicity Gowers's hemoglobinometer and Fleischl's hemometer are most used, and will now be described (Dare and Oliver).

The principle of the Fleischl hemometer, as modified by Miescher, is the following: By the aid of a pipette which is furnished with the apparatus (Fig. 9 $\mathrm{Mel}$.) an exactly determined amount of blood is dissolved in a measured quantity of distilled water; the color of this solution is compared with that of a colored glass wedge which, by its gradually increasing thickness, represents a scale of blood concentrations. The point in the wedge is now searched for which is just as intensely colored as the solution of hlood that is to be cxamined, and the number is read off which is found at this point of the glass wedge. If, for example, the color of the blood solution is equal to that designated as 75 in the glass wedge, it means that the examined blood contains 75 per cent. of the normal amount of hemoglobin. Besides the estimation of the percentage of hemoglobin, this apparatus also makes it possible to 
reckon the absolute amount of hemoglobin in milligrams in 1 c.mm. of blood. [It is essential to remember that percentage readings are very misleading when applied to children, since their normal is about 75 per cent. of the normal of adult men. Women's blood contains 10-15 per cent. less coloring matter than men's. All hemoglobin instruments should be graduated in milligrams per c.mm. of blood instead of in percentages of a supposed "normal."-ED.] The investigation is rather laborious because it can only be carried out with artificial illumination. The apparatus is quite expensive on account of the difficulty in manufacturing the glass wedge. Its use, however, is valuable, for an investigator in constant practice is able to reduce the errors to 5 per cent. and less.

The little apparatus of Gowers works on the following principle: A tube contains a standard color solution which possesses the tint of a diluted watery

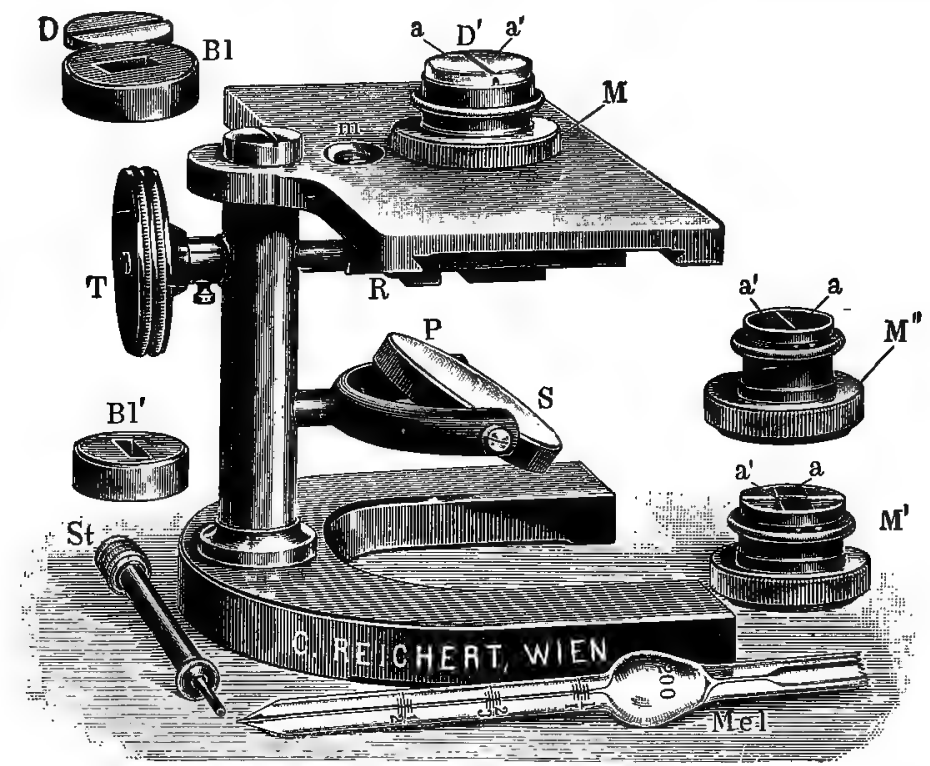

Fig. 9.-Hemometer. (After v. Fleischl-Miescher.)

hemoglobin solution. In a graduated glass tube of the same size a small but accurately determined amount of blood is diluted with water until it is the same color as the color in the test-tube; naturally this occurs the sooner the thinner the blood is at the beginning. If, for example, an equalization of color is reached at 60 , this indicates that the blood only contains 60 per cent. of hemoglobin in comparison to normal blood.

This test is quite simple and it may be completed in a few minutes during the office hour; the apparatus is very cheap. With a well made instrument the errors may be reduced to from 10 to 5 per cent. I must call attention to the fact that the manufacture of this apparatus is carried on by some quite unreliable manufacturers, so that, for example, test solutions are furnished in 
color tints such as thin watery blood solutions never show. Probably because of these poor instruments, there has been a partial opposition to the use of the Gowers apparatus.

A short time ago Sahli made a change in the Gowers hemoglobinometer, which appears to have added greatly to the value of the apparatus. For this

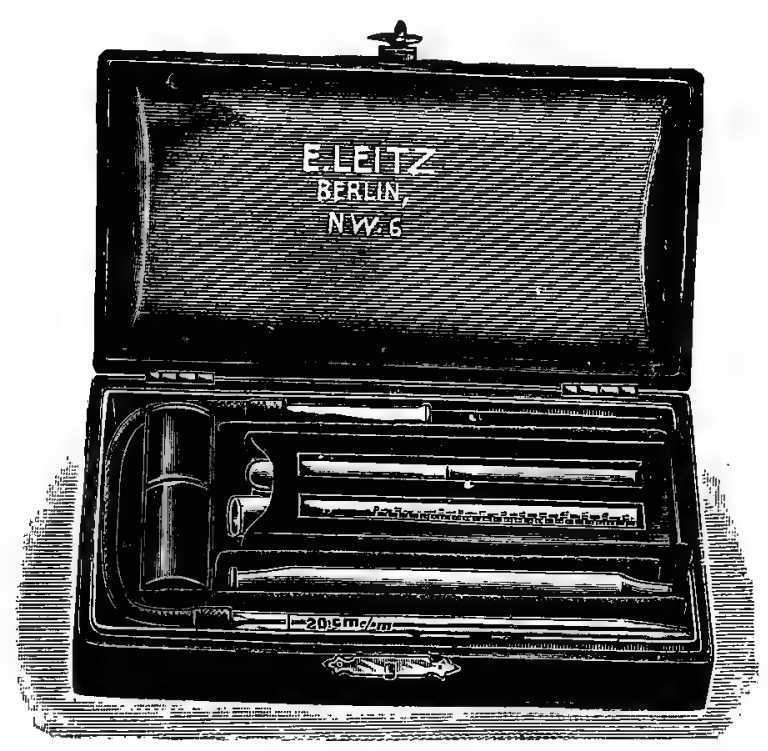
purpose the measured quantity of blood is first placed in a slightly diluted hydrochloric acid solution which produces a dark brown solution of hydrochlorate of

Fig. 10.-Немомeter. (After Sahli.)

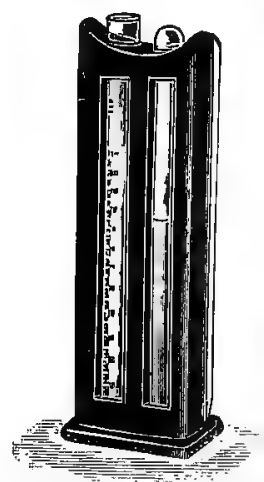

Fig. 11.-Henometer. (After Sahli.)

hematin. This mixture is then diluted with water until it corresponds exactly in color to a test solution which is furnished with the apparatus, which also consists of hydrochlorate of hematin in a definite dilution. From the amount of water necessary to produce this correspondence in color, we may estimate the amount of hemoglobin in the drop of blood that has been tested. In its mechanism the apparatus is much superior to the original, as may be seen in the two illustrations which are placed side by side (Figs. 10 and 11).

Among other instruments (of which there is a great number, each investigator having his favorite one) I should like to mention one of the latest, the practical importance of which upon a large scale must still be proven, and the use of which is somewhat more difficult than of those previously depicted; the principle, however, is interesting, and differs greatly from that of the colorimetric ones that have been described; for this reason it is necessary to compare its results with those obtained by the aid of other instruments. I refer to the hemophotograph of Gaertner (see Figs. 12 and 13). Gaertner started from the observation that the permeability of a diluted watery blood solution for the photographic rays of sunlight was in inverse proportion to its hemoglobin content. For this reason he spreads the blood solution to be examined in a layer of definite and uniform thickness, lays photographic paper under it, and per- 
mits the sunlight to penetrate this for a definite time. The degree of blackening which the paper shows under the blood layer is compared with a standard scale, and then the hemoglobin is read from a table which has been obtained empirically.

In quite a series of investigations I have compared the results obtained with the apparatus of Gowers and Gaertner and have found reasonable uniformity.
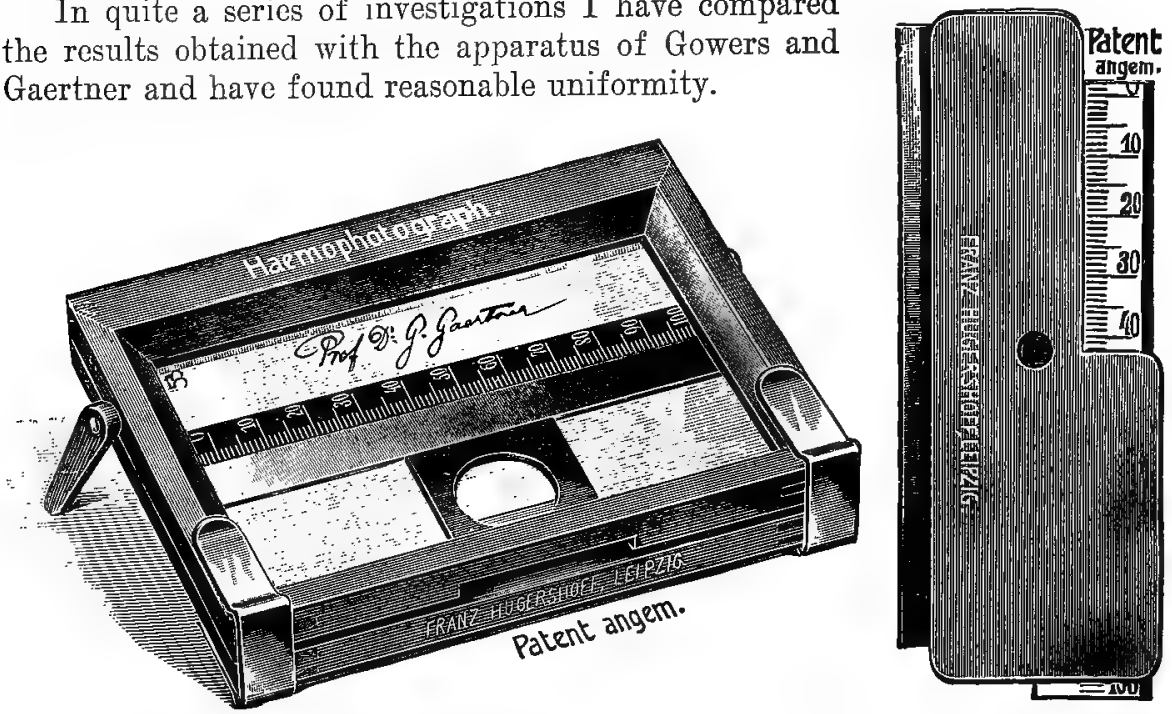

Fig. 12.-HeMophotograph. (After Gaertner.)

Fig. 13.-HEMOPHOTOGRAPH.

(After Gaertner.)

When by the aid of some of these instruments a result has been obtained, it must always be borne in mind that no exact test has been made, that, on the contrary, all sorts of errors may be present. Those which are due to the imperfections of the instrument I have already pointed out; and we must always assume 5 to 10 per cent. of errors in the examination of a definite drop of blood, even although we are quite expert in the examination; with the novice this percentage may be much greater. Unfortunately, it is impossible by the use of more exact so-called physiologic methods to avoid these errors in the estimation of hemoglobin, partly because the methods are complicated, and partly because they require too large amounts of blood, and, therefore, cannot be utilized in practice.

To these errors, which are, however, due to the methods or instruments, still others must be added which are due to a certain changeability in the blood itself. It is well known that the most varied influences may change the caliber of the blood-vessels, by stimulating the vaso-dilators or the vasoconstrictors; for example, light, heat, cold, muscular activity, etc. According to the caliber of the vessels-of course within certain narrow limitsthe number of corpuscles in the capillary blood will vary, and with this the amount of hemoglobin, the specific gravity, the total solids and the albumin contents of the blood. Moreover, if the blood to be examined is not obtained by puncture or incision of the blood-vessel directly, but, as in the great majority of cases, by a prick in the finger or by wet cups, the unavoidable admixture 
of tissue lymph to the blood means another source of error, although in itself not a great one.

In spite of all these objections, which should be constantly borne in mind, the clinical estimation of hemoglobin is of great value; in practice it is unquestionably more important than all other methods of blood examination. The decision of the most important question, whether the patient be anemic or not, can only be made positively by a hemoglobin test. The physician should accustom himself to look upon an examination of a patient as complete only after the blood has been cxamined by the aid of a hemoglobinometer; then a surprisingly large number of persons will be found whose blood will show quite a different condition from what might have been expected before the examination from their general appearance. My estimation is rather too low than too high when I say that fully one-half of those who are pale, who have pale mucous membranes and cool extremities, and for this reason usually designate themselves as "anemic," show a perfectly normal amount of hemoglobin. Such persons do not suffer from an abnormal composition of the blood, but from its abnormal distribution. That the greatest differences in regard to treatment will result from this in individual cases certainly requires no further demonstration. I shall only point to the futility of many a treatment by iron; due to the fact that the treatment was begun without any indication for it, i. e., without proof of a diminution in the amount of hemoglobin. A careful test of the amount of hemoglobin in the blood would in many cases cause us to relinquish the iron treatment and lead to the use of other curative methods.

In conclusion I must add that a diminution in hemoglobin may occasionally be discovered where the external appearances by no means indicate anemia; of course a correct estimate of the degree of anemia in such cases must depend upon a test of the hemoglobin.

The chief value of the clinical investigation of hemoglobin depends upon the fact that it soon informs us whether and to what degree the blood may be looked upon as anemic; from this we can determine whether to investigate the blood for other changes or not.

\section{COUNTING THE BLOOD-CORPUSCLES}

For this purpose we use exclusively the Thoma-Zeiss counting apparatus (Fig. 14). This consists of a pipette $G$ with a mixing chamber $E$, in which the one or ten per cent. blood dilutions are made; and of a counting chamber $D$ and $c$ in which under the microscope 400 divisions may be recognized, each containing $\frac{1}{400}$ c.mm. In counting the red blood-corpuscles the following method is pursued: The blood is sucked up to a definite mark in the capillary tube, and then diluted with a preserving fluid. We use exclusively Hayem's solution, the composition of which is the following:

Mercuric chlorid ..................... 0.5

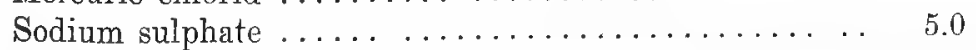

Sodium chlorid ......................... 1.0

Aq. dest. . . . . . . . . . . . . . . . 200.0 
A drop of the diluted solution is allowed to fall upon the counting chamber, the cover-glass is placed over it, and this is permitted to sediment for a short time. [Türck's emendation of this step in technic is of value. Before

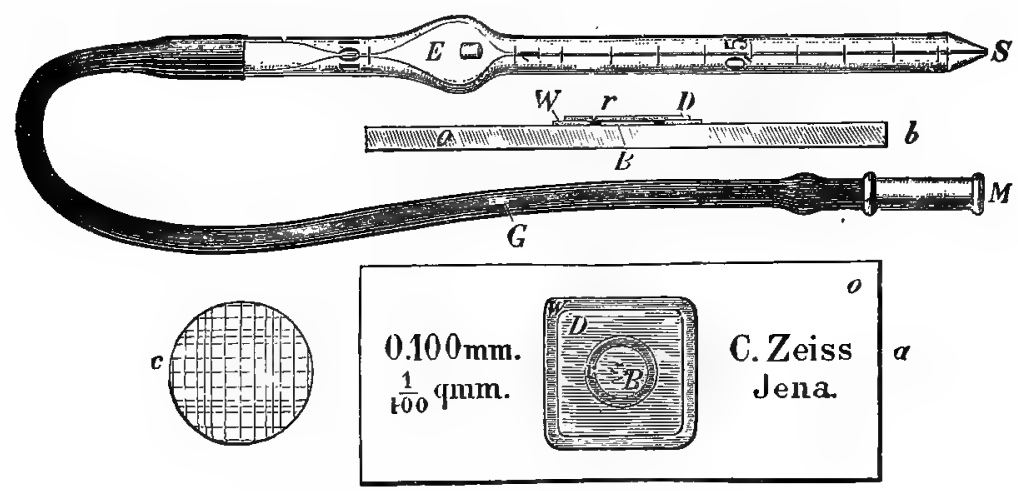

Fig. 14.-Hemocrtometer. (After Thoma.)

blowing out a drop of diluted blood upon the counting-dise, he puts on 2 corners of the shelf ( $W$, Fig. 14), that is, to support the cover-glass, a minute drop of water. The diluted blood drop is then adjusted on the counting disc and as rapidly as possible the cover-glass is let down and pressed firmly into

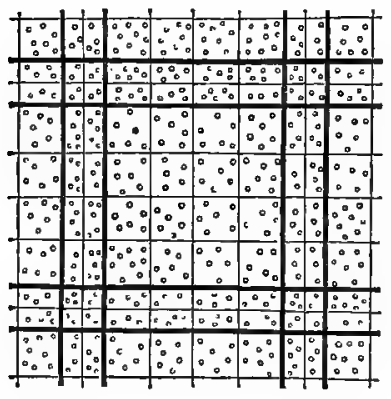

Fig. 15. - The Mrcroscopic Prcture wrth Blood-CorPUscles. (After Laandois.) position by strong pressure with the thumbs. Newton's rings are then visible at once, and the cover-glass is sealed firmly in position.-Ep.] After which (using about 300 diameters magnification) as many as possible of the squares in view should be counted. If, for example, we have diluted the blood $1: 100$ and then count in 32 squares (Fig. 15) 300 red blood-corpuscles, the following calculation is made (we must multiply with 100 to compensate for the blood dilution):

$$
\frac{300 \times 4000 \times 100}{32}=3: 750,000 ;
$$

This is the number of red blood-corpuscles contained in a c.mm.

Any one in constant practice with this instrument possesses an exact measuring apparatus in which the danger of error is very slight; naturally, the aggregate of inaccuracies is less when more squares are counted. [Of course the amount of error depends on the number of squares counted. With men properly trained no constant practice is needed. My own practice is to count 100 squares. The error is then negligible.-ED.]

It must be noted that, particularly in extreme anemia, the number of blood-corpuseles may easily be reckoned as too low, since the microcytes which occur here are apt to be missed if we are using a low power. Obviously the observations we have made in regard to the variation in the composition of 
the blood, in speaking of the hemoglobin test, are also true as regards the blood-count.

In practice the value of the determination of the number of red bloodcorpuscles is not very great, but it is important in any careful study of cases. This is obvious if we realize that the amount of hemoglobin and the number of blood-corpuscles are not always exactly proportional; that, for example, a diminution in the hemoglobin contents to 50 per cent. does not necessarily imply a diminution of erythrocytes from 5,000,000 to 2,500,000; they may be above or below this. We designate as the average "value" (V) of the individual corpuscle $\frac{\mathrm{Hb}}{\mathrm{E}}$. i. e., the division of the percental hemoglobin figure by the percental diminution or increase of the erythrocytes. ["Color-index" is the word generally used in Amcrica instead of "value."-Ev.] If, for example, the amount of hemoglobin has been reduced to 50 per cent., the erythrocytes being $2,500,000$ to the c.mm., the average "value" has remained 1; if, however, with 50 per cent. hemoglobin only 200,000 red blood-corpuscles are present, the $V$ has even risen to $1.25\left(\frac{50}{4} 0\right)$. On the other hand, the "value" is only 0.8 if we have only 40 per cent. hemoglobin and 2,500,000 red blood-corpuscles. This "value-estimation" is of importance for the reason that the various types of anemic conditions are distinguished by the "value" [or color-index] of the blood; for example, in chlorosis or in posthemorrhagic anemia [or, in fact, in any well-marked secondary or symptomatic anemia.-En.], $V$ is often decidedly below 1, while in progressive pernicious anemia it is frequently decidedly above 1.

The number of red blood-corpuscles to the c.mm. upon the average amounts in men to $5,000,000$, in women to $4,500,000$. [Hewes and other Americans have noted that in healthy American adults the number of red cells is usually near 6,000,000, often above that figure.-ED.] In most anemic conditions this is reduced, and values below $1,000,000$, even as low as 300,000 , have been seen without absolutely precluding the recovery of the patient. On the other hand it must be emphasized that a normal number of erythrocytes is not proof against the presence of anemia, unless the amount of hemoglobin is also normal.

Under certain circumstances, some of which have not yet been sufficiently explained, a decided increase of red blood-corpuscles occurs, a hyperglobulia. This is, for example, the case in the various forms of stasis of the circulation, but it is also the expression of an actual increase of blood formation. Under such circumstances, Türk, for instance, found 9,150,000 erythrocytes.

[Cases of this type were first described by Cabot in 1899, later by Osler who noted particularly the enlargement of the spleen associated with the polycythemia.-ED.]

\section{COUNTING THE LEUKOCYTES}

To count the white blood-corpuscles we use a dilution of only 1 to 10 , which may be done in the large-bore mixing pipette in the Thoma apparatus. In order that counting may be facilitated the red blood-corpuscles are 
destroyed, and for this purpose the blood is diluted with 0.5 per cent. acetic acid solution. This process has only one disadvantage, viz., that the nuclei of erythroblasts which may be present cannot be differentiated from white blood-corpuscles. In general, however, this error is quite without importance.

The normal number of white blood-corpuscles in a c.mm. of blood varies betwcen 5,000 and 10,000 .

By an estimation of the absolute number of white and red blood-corpuscles we simultaneously learn their proportion to each other, the importance of which we shall describe somewhat more minutely at another place.

\section{SPECIFIC GRAVITY}

The most exact method, one easily carried out with some practice, is that of Schmaltz. For this purpose we require one or more drops of blood, about 0.1 to 0.2 c.c. This is sucked up into small glass capillaries that have been previously weighed upon an accurate chemical scale, noting how much they weigh when empty, and how much after having been filled with distilled water at a temperature of $15^{\circ} \mathrm{C}$. Then the weight of the tube filled with blood is determined, and the specific gavity of the blood is calculated by dividing its weight by the weight of the same amount of distilled water.

Hammerschlag's method has been the most extensively used from the fact that it does not necessitate an expensive scale, but only a simple aërometer with divisions from 1.010 to 1.070 .

This process is based upon the physical law: "A body floats in a fluid of the same specific gravity." According to Hammerschlag a medium-sized drop of fresh blood is permitted to fall into a benzol chloroform mixture; if the blood after being dropped into the fluid sinks still lower, it is heavier than the mixture, and by the addition of a corresponding amount of chloroform an attempt is made to compensate for this. If the drop of blood remains upon the surface it is lighter than its menstruum, which is now made lighter by the addition of more benzol. If, finally, the blood drop remains at the height at which it is first dropped, it possesses the specific gravity of the benzol chloroform mixture, which may easily be read off by the aid of the aërometer. As the blood drop under the influence of the fluid surrounding it readily changes its weight, splitting into many small particles, this process must be carried on very rapidly or fresh drops must be constantly made use of. The benzol chloroform mixture can always be utilized for new estimations, as it is readily freed from blood by filtration.

In exactness, Schmaltz's pycnometer is decidedly superior to Hammerschlag's method.

In normal males the specific gravity of the total blood is 1.059 , in normal women 1.056 upon the average.

In general, the estimation of the specific gravity only complements or confirms the results obtained by hemoglobin estimation. According to minute exact investigations the amount of hemoglobin and of the specific gravity 
are equal to one another, so that by this somewhat more complicated method of examination we learn no more than may be more simply determined. There are also tables from which we may read off directly the specific gravity of the blood with a definite amount of hemoglobin, and vice versa. This intimate dependence is clear without further explanation: The hemoglobin, as the principal constituent of the red blood-cell, determines the greatest fluctuation in the gravity of the total blood, especially since the fluid of the blood is of an extraordinary constancy in its composition. Only when the specific gravity of the blood serum becomes decidedly lighter than the normal will the parallelism between hemoglobin and specific gravity of the total blood be destroyed.

Closely related to the estimation of the specific gravity are the quantitative estimation of the total solids and the estimation of the amount of albumin or nitrogen of the blood. Neither method has as yet been adopted in practice, partly because they are much too difficult, and partly because they do not enable us to form any opinion which we cannot otherwise arrive at much more readily. It must be added that the result of the estimation of nitrogen in some cases cannot be utilized at all, for, besides the hemoglobin and the white blood-corpuscles, other nitrogen-containing substances, of other origin, may be present in the blood and in an indefinite amount. In revicwing what has been written in special works upon the subject regarding these methods of investigation, we must say that they have added nothing distinctly new to hematology.

The investigations which have been made with the total blood have been extended for scientific purposes to the individual constituent parts, also particularly to the estimation of the amount of albumin and of the specific gravity. The separation of the two integral parts, BLOOD-CORPUSCLES AND SERUM, may be made in a simple manner either by sedimentation or centrifugation. Material for investigation may be procured even from a few drops of blood; it is sucked up into small glass tubes in which the serum, at the latest in twenty-four hours, may be spontaneously expressed.

As the most important result of these researches, it must be emphasized that the serum in some pathologic conditions does not take part in the changes of the total blood but retains its composition. Thus, according to Grawitz, the serum in chlorosis and in progressive pernicious anemia does not ake part, or to a very slight extent, in the hydremia of the total blood, and invariably shows normal quantities of total solids. In chronic nephritis, the hydremia of the total blood is chiefly attributed to the decided increase of water in the serum.

I shall mention still other methods of blood examination, although they have not as yet been considered of importance in practice; because it seems advisable briefly to describe some conditions of which mention is occasionally made. For, since the purpose of this article is the stimulation of more frequent hematologic investigation in practice, we must consider not only those methods which have been found of practical value, but those with which, in spite of great efforts, little or nothing has been demonstrated either theoretically or practically. 


\section{ALKALINITY}

The estimation of the ALKALINITY of the blood must be first mentioned. That fresh blood has an alkaline reaction cannot be directly determined by the use of litmus paper on account of the color of the blood, but a particularly sensitive red litmus paper must be moistened with a dilute sodium chlorid solution, then the blood to be tested must be dropped upon it and the paper be rinsed again with a sodium chlorid solution. It is very difficult to determine the degree of alkalinity with any accuracy. In the newer methods the blood to be used is made of a lacquer color, and this is titrated against a normal tartaric acid solution with lacmoid paper. For this purpose we usually require somewhat larger quantities of blood, 5 to 8 c.c.; C. S. Engel has, however, constructed an "alkalimeter" which makes it possible to carry out the test with $\frac{1}{20}$ c.c. of blood.

How slight is the importance of these investigations, even to-day, is shown by the reports of various authors that even the normal values vary within wide limits; for instance, from 203 (Canard) to 508 (Loewy) mgm. NaOH in 100 c.c. of blood. With such differences in the normal value, it is not surprising that results obtained under pathologic conditions give rise to conclusions still less definite. Thus, one author found increased alkalinity in certain diseases in which another found decided diminution.

In the present status of the question, we must be cautious in introducing such an uncertain method of investigation into practice; for, since the result is finally expressed in figures which have a deceptive appearance of exactness, it is much more misleading than the most subjective method of examination.

We are by no means convinced of the biologic importance of the alkaline reaction of the blood. Many authors are inclined to look upon the bactericidal properties of the blood as bearing a certain parallel relation to the alkaline reaction of the blood, but are unable to prove the correctness of this view. Even with such a proof little appears to be gained. For it is self-evident that a definite degree of alkalinity means a relative optimum for bacteria, since the increase of alkalinity at once produces for them unfavorable conditions.

I must call attention to the fact that Brandenburg may perhaps have opened a way out of these uncertainties by his recent investigations. Brandenburg demonstrated that we must differentiate between the alkali combined with albumin and that combined with carbonic acid. These elements can be separated from one another since the latter is capable of diffusion, while the alkali combined with albumin is not. Thus it was shown that the diffusible part of alkali represents a very constant value corresponding to about $60 \mathrm{mgm}$. $\mathrm{NaOH}$ - while the non-diffusible part is exposed to the greatest variations. It is now conclusively shown that under pathological conditions even when there is great variation of the total alkalinity from the normal, the amount of diffusible alkali-" the alkali tension"-remains almost unchanged. 


\section{VOLUME OF RED CELLS}

Some authors attach a certain value to the determination of the volume of the red blood-cells in a definite amount of blood. For the correct estimation of this various instruments have been invented, and either those in which the blood-corpuscles are separated from the serum by centrifugation ("hematokrit") or those in which this occurs by spontaneous sedimentation, can be employed. Every method should be rejected in which salt or a salt solution is used to prevent the coagulation of the blood. For the volume of red corpuscles remains uninfluenced only when it comes in contact with an isotonic salt solution; as, however, particularly in pathological conditions, a special concentration is isotonic for each kind of blood in each individual case, the estimation of the volume would first depend upon a previous determination of the isotonic salt solution; and such a difficult process can be of no use in practice.

The method which we owe to Köppe is very valuable because coagulation is prevented in the centrifugal tubes of his hematokrit by the absolutely smooth walls of the tubes. As the blood, therefore, remains unmixed we may perhaps assume that, after complete centrifugation, the proper proportion of plasma and cells as it exists in the blood-vessels is shown.

If, however, the pathology of the blood is investigated by estimations of volume, the figures obtained by these instruments show nothing that may be looked upon as enriching our knowledge or promoting our understanding of diseases of the blood; and I only refer to this method for the sake of completeness, with the express statement that at present it cannot be considered a clinical method of investigation.

\section{SPECTROSCOPIC EXAMINATION}

For certain methods of examination of the blood the use of the sPECTrosCOPE must be understood, for in the recognition of various hemoglobin combinations which are characteristic of certain conditions, particularly of poisoning, the spectroscopic investigation of the blood is absolutely necessary and enables us to reach conclusions not to be arrived at by the microscope or any other methods. The principle of this method must be considered as understood; but it may be mentioned that in qualitative investigations, as a rule, a so-called pocket spectroscope is sufficient, and that it is well to place the blood for examination in watery solutions of varying concentrations between two plane parallel glasses.

The determination of $\mathrm{CO}-\mathrm{Hb}$ and of methemoglobin, the former occurring in carbonic acid poisoning, the second in various forms of intoxication, particularly with potassium chlorate, is of the greatest practical importance. The spectroscopic determination of methemoglobin, as may be noted from the adjoining table, is possible by characteristic absorption lines (Fig. 16). (The determination of $\mathrm{CO}-\mathrm{Hb}$ is difficult on account of the similarity of the spectrum with that of $\mathrm{O}-\mathrm{Hb}$. The differentiation is only certain when we observe 
that the line of $\mathrm{CO}-\mathrm{Hb}$ does not disappear upon addition of reduction agents, for example, ammonium sulphate, because $\mathrm{CO}$ is firmly combined with hemoglobin; while the spectrum of $\mathrm{O}-\mathrm{Hb}$ is changed by the same agent to that of reduced hemoglobin.

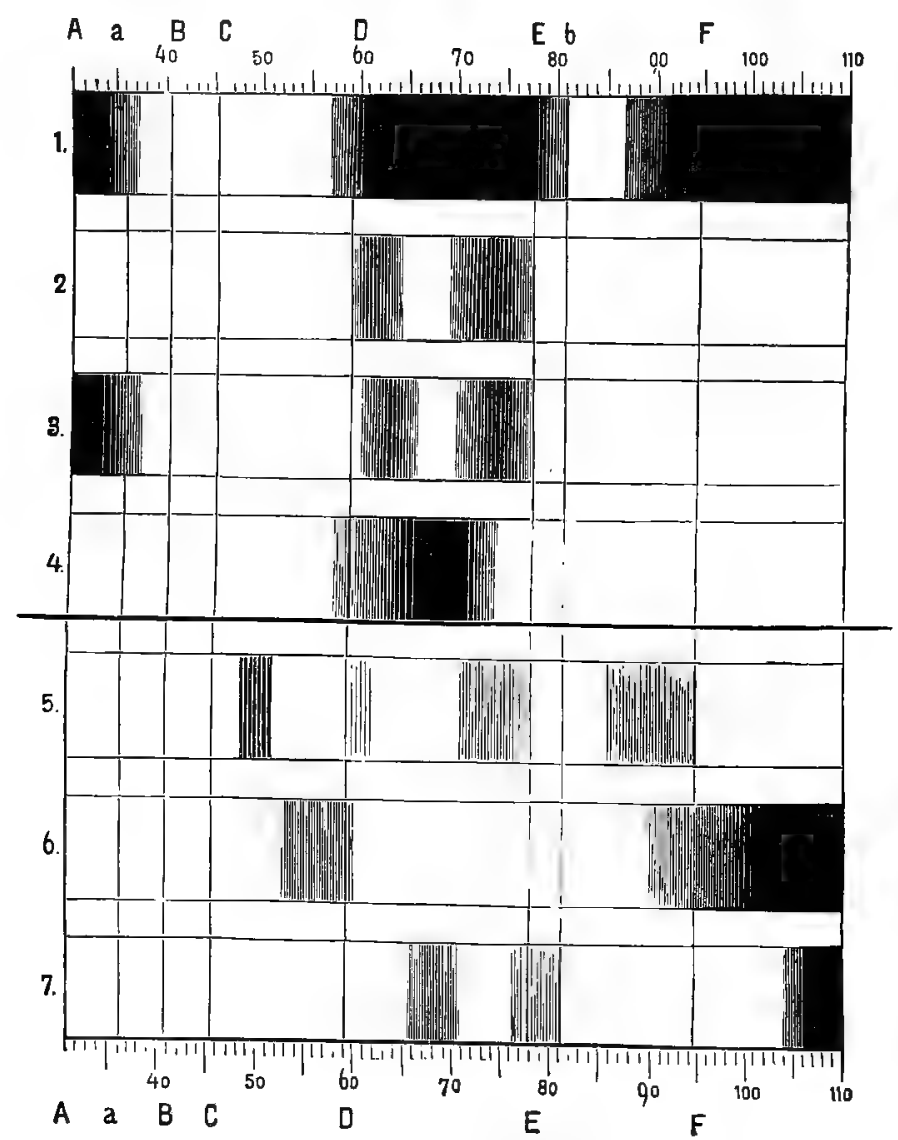

Fig. 16.-The Vartous Absorption Spectra of Hemoglobin. In all of the spectra the various Fraunhofer lines and a scale in millimeters are drawn. (After Landois.)

Finally, the slightest degree of hemoglobinemia may be demonstrated with certainty by the spectroscope in those cases in which ocular examination leaves a doubt as to whether or not the serum contains hemoglobin.

\section{AGGLUTINATIVE REACTIONS}

To the law of immunity which has made us acquainted with the vital importance of functional changes in the blood we owe a valuable diagnostic method. We know that in the natural infections of man, as well as in animal experiment, peculiar changes occur in the blood serum, under the 
influence of specific bacteria or bacterial toxins. Among these we may differentiate two classes: The antitoxic sera and the bactericidal sera; the first variety is represented by the serum containing diphtheria antitoxin, the second by the energetic bacteriolytic action of the blood serum in convalescence from cholera.

As a variety related to the last group the agglutinating serum may be included, which occurs during the course of, and after recovery from, many infections. We are most familiar with this condition in enteric fever, in which it first led to valuable and practical diagnostic results.

The essential part of the phenomenon is that, in a mixture of such serum with typhoid bacilli in a culture medium, they are deprived of their motility and clump, i. e., "agglutinate." To determine this fact a few drops of serum, 1 to 2 c.c., are sufficient, and these may be most easily obtained by centrifugation or sedimentation in thin glass tubes. The phenomenon may be demonstrated macroscopically as well as microscopically (Gruber-Iridal reaction). In the former case, with nutritive bouillon after the addition of the serum, we see how a profuse uniform turbid accumulation of typhoid bacilli clear. completely in from twelve to twenty-four hours, and a delicate floceulent precipitate forms which, even by powerful shaking of the test-tube, can no longer be disseminated.

The microscopic test is carried out in the following manner: A drop of the typhoid culture which is to be utilized for the test is arranged as a hanging drop, so that we may convince ourselves of the active motility of the bacilli. If serum in an exact proportion is then carefully added to the collection of bacilli, and one drop is placed under the microscope in a positive reaction, we notice even after a few minutes, at latest after a quarter of an hour, a complete cessation of movement, a clumping of bacteria in large groups, as well as an indistinctness in their outlines.

[Another method is the following: A 3-inch test-tube of small caliber is carried to the bedside of the patient together with a clean medicine dropper. Ten drops of water are allowed to fall from this dropper into the test-tube. The patient's ear is then punctured in the ordinary way and a full drop of blood is sucked into the dropper and then expelled into the water in the test-tube. The test-tube is then corked and carried to the laboratory where the culture is kept, and one drop of the blood and water mixture is mixed with one drop of the culture and examined between slide and cover. There is no need of separating serum and corpuscles in this test; the whole blood is equally serviceable.

For public health work the blood may be dried on tin foil, folded in, and mailed to the State Laboratory where dilution is made by weight. This method is extensively and most successfully used by the State Board of Health in Michigan.-ED.]

The value of this reaction test can only be properly appreciated if it is carried out with certain precautions. First, we must assure ourselves of the proper composition of the typhoid culture to be used in testing, and must be sure that it is a fresh one, not older than a day. The serum requires, as already stated, a corresponding dilution, because even normal serum if undi- 
luted may cause agglutination; on the other hand, the dilution should not be too weak, as the agglutinating substances in some cases of enteric fever are not present in very large amounts, and if the serum be too greatly diluted they are inactive. It is best to begin with a dilution of one part of serum to thirty parts of bouillon, and, if a positive reaction be obtained, it should then be determined with how weak a dilution we can still secure a reaction. In some cases, a positive reaction has been obtained even after concentrations of 1-5,000. If the test gives a positive result with a high dilution, the diagnosis of enteric fever is thereby assured, with the one limitation that the serum may perhaps have received its agglutinating properties from a typhoid fever some time previously, even several years before.

If, however, the result of the test is negative with a dilution of $1: 30$, it is well to repeat it in the course of the disease, daily if possible. Frequently it is noted that in the early course of the disease the serum, which at first showed no reaction while diluted, gradually in slighter concentration betrays the presence of agglutinating substance. But the diagnosis of enteric fever cannot be excluded with certainty even in case of a continued negative result of the reaction. The appearance of the reaction may be expected at the earliest upon the eighth day of the disease. ${ }^{1}$ [Since it is impossible to fix in any way-except arbitrarily-the first day of the disease, it is hard to make any accurate statement about the earliest appearance of Widal's reaction. At the time when patients first consult a physician, two-thirds of them show a positive agglutination reaction in the blood, but in a few it is delayed as late as the fifth or sixth week, or it may never appear. Over 95 per cent. of cases, however, show a reaction at some time in the course of the disease. Different epidemics vary greatly in these points, some showing a large proportion of early reactions, while in others physicians complain of the high percentage of negative reactions in the earlier weeks of the disease.-Ev.]

In view of the very valuable diagnostic success of serum diagnosis in enteric fever, it may be readily understood that an attempt would be made to reach the same results in other diseases, but, unfortunately, there has been as yet no practical success. Endeavors specially directed to the recognition of tuberculosis by its specific agglutinating reaction may be looked upon as futile, since Robert Koch reached entirely negative results in this direction.

\section{BACTERIOLOGICAL EXAMINATION}

The diagnosis and also the prognosis may, under some circumstances, be helped by the bacteriological investigation of the blood. There are quite a

1 Lately Próscher (Centralbl. f. Bakteriologie, xxxi) has proposed a modification which indicates a decided practical improvement. According to this it is particularly valuable that a typhoid bouillon may be utilized in which the bacilli have been killed by the addition of one part of a forty per cent. formalin to 100 parts of bouillon. This bouillon may be used for weeks without losing its agglutinating property. For the action of the serum upon the bacilli Pröscher allows from one to two hours at blood heat; for viewing the serum bouillon mixture which is kept in a bowl we employ a low power dry lens (about $\frac{1}{50}$ enlargement). 
number of diseases in which, particularly in especially severe cases, the pathogenic agents enter the blood. Formerly we contented ourselves with a microscopic investigation of fresh or stained blood to determine the presence of bacteria. But this process should only be resorted to for the determination of the spirilli of relapsing fever, or, perhaps, of the bacilli of anthrax. In other cases it may lead to errors, first because of the small number of germs that may be present, and, secondly, because confusion with accidental bacterial contaminations may readily occur. It is therefore advisable to puncture a vein of the arm under strict antiseptic precautions, to withdraw 1 to 2 c.c. of blood, and to prepare cultures, best in bouillon, or to determine by animal experiments the probable presence of pathogenic bacteria.

In this manner we are able to determine with certainty in the circulating blood the presence of streptococci, staphylococci, pneumococci, the bacilli of enteric fever, of the plague, of tuberculosis and others. [Typhoid bacilli have repeatedly been cultivated from the blood of doubtful fehrile cases before the Widal reaction had appeared. Hence this procedure may be of great value in obscure cases. The same is true of the cultivation of pneumococci from the blood in obscure pneumococcus infections and "central pneumonias."-ED.]

The determination of the pathogenic agents just mentioned can only be designated as an auxiliary factor in diagnosis, but the recognition of spirilli in the blood is the only means for a positive diagnosis of relapsing fever. As a rule this determination is very easy, for the peculiar curved form of the spirilli (see Fig. 17) precludes confusion with other organisms. Their number is usually very large; our detection of them is greatly facilitated by their motility, and, finally, they stain readily with various anilin colors, for example, intensely with fuchsin, so that they may be easily found in a dry preparation even if present in the blood in very small numbers.

Probably in no other disease is the practical importance of blood examination greater than in

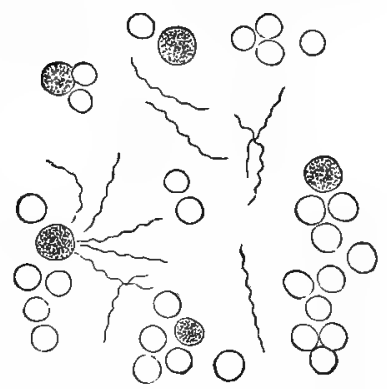

Fig. 17.-RELAPSing FeVer SPIRILLI. (After v. Jaksch.) malaria. This is naturally not the place in which to describe the entire devclopment of the malarial parasite, nor even to mention all of the varieties which occur in the blood, as a special article has been devoted to this important subject. ${ }^{1}$ Here it need only be mentioned that, in many cases, we should look for the plasmodia in the blood, and should familiarize ourselves with the most important points in the technic of examination.

In the investigation of the blood for malarial parasites for diagnostic purposes, the stained dry specimen is unquestionably the most suitable, and we shall explicitly describe its preparation in another place. For when very few parasites are in the blood, this method alone assures their detection, and

1 Compare the article by Loeffer in the volume on "Infectious Diseases." 
with very little practice it protects us more fully from error than an investigation of the fresh blood.

The withdrawal of blood had best be made in the afebrile period; and even here it must be borne in mind that, for as long a time as possible prior to this, no quinin should be administered.

Fixing the preparation is best accomplished by the use of absolute alcohol for five minutes. [It seems likely that all the fixing methods described in this article will soon be replaced by the fixation produced by methylic alcohol which is the menstruum in which the various "Romanowsky" stains are dissolved. Using Leishman's or Wright's modification of the Romanowsky method we fix the specimen in the same fluid which later (when water has been added) stains the specimen. This saves much time, trouble, the apparatus and expense. The technic is given in detail below.-ED.] Staining is best done either by a one per cent. methylene-blue solution, or by the Chenzinsky methylene-blue eosin mixture mentioned in another place, or after the method especially proposed for the study of malaria by Romanowsky-Ziemann.

With an enlargement of about $500^{-}$and the use of an oil immersion, it is not difficult even for the novice to recognize the fully developed plasmodium within the red blood-corpuscles. First, the blue tint which they acquire from the methylene-blue readily distinguishes them from the nucleus of the cell; this is an uncommonly delicate sky-blue in contrast with the darker blue of the nucleus, which more closely resembles the actual color of the material used in staining. [With the Romanowsky stain which is now widely used in this country for all blood-specimens, the nucleus is colored crimson and its contrast both with the blue of the parasite and the yellow of the surrounding corpuscle forms thus one of the most recognizable features of the organism. - ED.] By the amount of pigment the recognition of the plasmodium is also decidedly facilitated.

It is more difficult if only the very young forms of the plasmodia are present, those which are still very small, and have not as yet formed pigment. Occasionally the characteristic seal-ring shape facilitates their recognition; frequently, however, the apparently structureless forms may be confused with the nuclei or the fragments of a nucleus of the erythrocytes, or with the dots of "stippled" erythrocytes. Here the character of the staining in particular gives a point of support for their recognition. Observations made at a more favorable time, and eventually an examination of the fresh blood to determine the motility of these bodies, furnish convincing proof.

How important it is to carry out an examination for plasmodia is shown by the fact that, according to Robert Koch, the prophylaxis as well as the therapy of malaria is intimately connected with the results of the blood findings.

The investigation itself, however, is so simple that it should never be omitted in any case in which long protracted, perplexing fever is present, or in which the history, or the condition of the spleen, or other clinical phenomena point to the possibility of a malarial infection. Especially in regions in which malaria is quite rare valuable time for the patient is frequently lost, because the physician has neglected the examination of the blood, supposing 
a septic disease or a tuberculosis, etc., to be present, and malaria has not been recognized as the true cause of the morbid phenomena. [In this country the opposite mistake is more common. Many cases of tuberculosis are miscalled malaria because they have chills, and no proper examination of the chest or of the blood is made.-ED.]

Among all the methods of blood examination used in the last decade unquestionably the most far-reaching results have been attained by the investigation of the NORMAL AND PATHOLOGICAL HISTOLOGY of the blood; this was particularly fostered by the technic of the dried preparation and the differential stains introduced by Ehrlich.

If a drop of fresh blood is placed under the microscope, enlarged about 500 times and examined with an oil immersion a number of important points may be observed. There will first be noted, provided the layer of blood is thin enough, the shape of the red blood-corpuscles, their depression, their hemoglobin staining; by this method also the rouleaux formation of the blood discs becomes plain. The structure of the white corpuscles may be recognized by this simple process, and various forms may be differentiated from one another.

Thus the lymphocytes are perceived as cells about the size of a normal red blood-corpuscle, though some attain double or even three times this size; their nucleus is circular, takes up the greater part of the cell, and is sharply demarcated from the narrow protoplasmic border surrounding it.

The majority of the leukocytes are conspicuous by their dense, decidedly refractive, fine granulations; one, two, or three faint nuclei deposited in them may be recognized. These cells are twice or three times as large as the erythrocytes. In a few of the leukocytes present (eosinophiles) the granulations are much more decidedly refractive, and the individual granules are much coarser. These two last named forms are alike in size, as well as in the conditions and the number and shape of their nuclei.

The blood-plaques are most easily recognized by their agglutination in clumps and slight refractive power.

Studies of the more minute structure of the leukocytes can only be made with difficulty by this simple method, as the forms not yet described are usually only present in very small numbers. Moreover, in a prolonged examination, the blood soon suffers changes which produce disturbance. In consequence of this the investigation of the fresh blood is of very little service, and we are usually compelled to preserve the blood by a proper method and to stain it. For these purposes there is no better process than the DRIED STAINING PRocrss after Ehrlich [except the methylic alcohol fixation described below.-ED.], and this is as simple in its operation as it is serviceable. It has the practical advantage that it allows the investigator to work quite independently of the presence of the patient, to choose the time and place of examination, and permits at any time a repetition of the investigation and a demonstration of the result obtained.

The specimen is made as follows: Cover-glasses of about $18 \mathrm{~mm}$, having a quadrangular shape and of special thinness $(0.1$ to $0.08 \mathrm{~mm}$.) are used; 
these are carefully cleansed in ether and alcohol so that fat, fiber and all particles of dust are removed. [Water is sufficient for cleansing cover-glasses provided they are properly polished with silk or tissue paper afterwards.-ED.] On a cover-glass of this kind a small drop of blood which has exuded from a prick of the finger is collected; this glass is dropped, blood side downward, upon a second glass, with the result that the blood soon spreads out spontaneously in a thin film between the two very flexible glasses to about threequarters of their extent, as if in a capillary space. After a complete distribution of the drop of blood, the glasses are removed from one another [by sliding off the upper of the two covers without any lifting or tilting, but strictly in the plane of their surfaces.-Ev.], and the blood is seen distributed

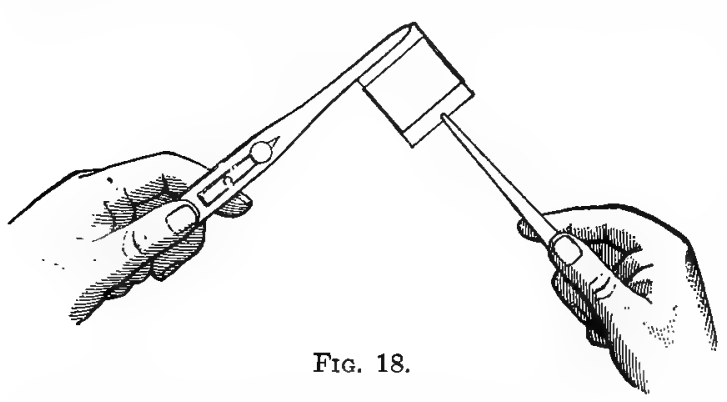
upon each in a uniform layer. If the drop taken was small enough, the blood cells are found in an even layer side by side without overlapping each other. In view of the fact that the red blood-corpuscles are damaged with extraordinary ease, especially by moisture, this maneuver should not be attempted with the unaided hand; for the moisture of the tip of the fingers is capable of changing the outlines of the red blood-corpuscles and of extracting their hemoglobin. For this reason, in the preparation of these specimens two forceps should be used in the manner depicted in Fig. 18. [For any one whose fingers are not habitually and obviously damp the use of forceps is unnecessary. -ED.7

When the blood films have dried, which usually happens within twenty to thirty seconds, it is necessary before subjecting them to the stain to fix the blood in a suitable manner. For practical purposes it is sufficient usually if the specimens are placed for five minutes in a solution of 1.0 formol in 100 parts of absolute alcohol. After this method of fixation almost all the stains which need practically be considered may be employed. If we intend, however, to prepare especially beautiful specimens, or to make investigations for seientifie pur-

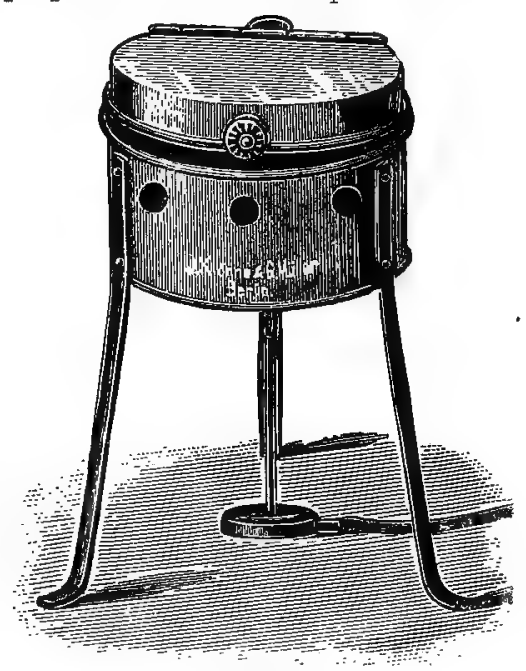

Fig. 19. poses, then Ehrlich's old method-fixation by heat alone-had better be utilized. Most serviceable for this purpose is a small copper kettle (Fig. 19) inside of which a small quantity of toluol is brought to the boiling point. 
The thin copper lid of the kettle will, a few minutes after the toluol has begun to boil, reach the temperature of the boiling point of toluol $\left(111^{\circ} \mathrm{C}\right.$.). Upon the plate heated in this manner the cover-glasses with the dried blood are placed for thirty to sixty seconds, and this fixation is quite sufficient for most of the staining methods.

Among the methods of staining the blood those of Ehrlich have shown themselves to be the most successful. In particular his triacid solution, which in its application is quite simple, brings out a great many points, and furnishes reliable conclusions. The formula for its preparation is the following:

The three stains, orange $G$, acid fuchsin and methylene green, are prepared in saturated aqueous solutions, and are mixed in the following amounts and order (with thorough shaking during the process):

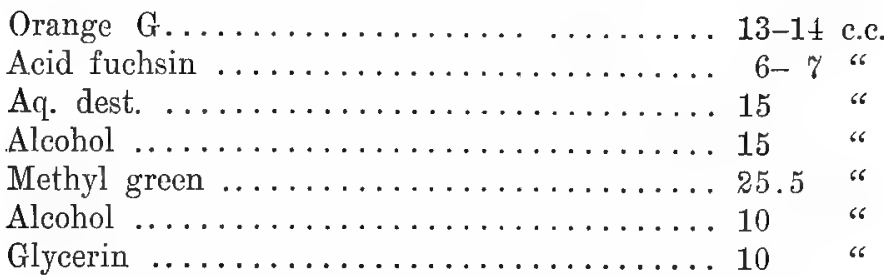

If the fixed blood preparation is allowed to float for about five minutes upon a few drops of this staining solution, the staining is finished. There is no danger of over-staining, even in a decidedly longer duration of staining. The cover-glass is then thoroughly rinsed in water, carefully dried between blotting papers and mounted in Canada balsam.

For certain purposes, as we shall see later on, this triacid solution is not suitable, and we are compelled occasionally to use complementary stainings with methylene-blue, or with methylene-blue eosin mixtures. Formulæ for such stains are the following:

Very instructive pictures are obtained if we stain for half a minute with a solution of 0.5 eosin to 100 c.c. of a 60 per cent. alcohol, rinse in water, and subsequently stain for two minutes with a watery methylene-blue solution of $1-250$.

Specimens of great clearness and elegance are obtained by a careful application of the Chenzinsky's methylene-blue eosin mixture:

Concentrated watery methylene-blue solution....... 40 c.c.

0.5 per cent. eosin solution in 70 per cent. of alcohol... 20 "6

Distilled water......................... 40 "

This solution must be filtered each time prior to use; the staining requires from six to twenty-four hours and, to prevent evaporation and the precipitation of the staining material, must be carried out in air-tight closed cups.

By the aid of these staining methods we are enabled to recognize a number of the peculiarities of the blood cells, which are indistinct or entirely unrecognizable in fresh blood. Some of the newer, particularly the so-called "universal," stains have such glaring faults that,' at least for the present, they 
appear to be unsuitable for practice. [After using and teaching Ehrlich's method as just described for eight years, I have recently discarded it in favor of one of the "universal "stains which our author here condemns. Leishman's "Romanowsky" stain made up by Wright's method (see Journal of Med. Research, 1902, vol. vii) is much quicker and easier to use, needs no fixing fluid or heating apparatus, and gives pictures which in 99 cases out of 100 are superior to those obtained by Ehrlich's method.

The technic is as follows: Adjust the cover-glass film in Cornet's forceps. Flood it with the stain, using a medicine dropper and keeping the bottle tightly corked when not in use. After one minute add water drop by drop until a greenish iridescent scum appears on the surface. About "\% drops are needed with a $\frac{7}{8}$ inch cover-glass. Allow the stain, so diluted, to act two minutes. Wash in water, and allow the preparation to stand one minute more in water. Dry in blotting paper and mount in Canada balsam.

This stain brings out all that Ehrlich's method does and, besides this, stains the blood-plates, the granules of mast-cells, the chromatin of malarial parasites and the basophilic granules in abnormal red cells-all points of value.

The only weak point of the Romanowsky stains is the deceptive resemblance between certain megaloblasts, certain lymphocytes, and certain myelocytes. In perhaps 1 case in 100 this troubles a beginner; in perhaps 1 in 1,000 it troubles an expert; but in no case. does this difficulty affect the essentials - the diagnosis, prognosis, or treatment of the case.-Ev.]

In examining normal blood which has been stained according to these methods we note the following important peculiarities: The red bloodcorpuscles have been wholly stained with orange, that is, with eosin, and this causes the unstained depression which is free from hemoglobin to stand out prominently.

Among the white blood-corpuscles the lymphocytes must be first mentioned (see colored plate, Row VII, $a$ and $b$ ). Their nuclei show the above described configuration, and on staining with a triacid solution they are moderately well stained with methylene green, the protoplasm, however, being of a very pale yellowish color. [The nuclei are often so faintly stained as to be nearly invisible. The protoplasm frequently remains unstained or shows a faint gray color.-ED.] In methylene-blue eosin preparations the nucleus is deeply stained with the blue stain, which is very frequently, however, found to be more marked in the blue color of the rim of the protoplasm. This is an expression of the fact that the protoplasm of the lymphocytes is more basophilic than its nucleus. In the not infrequent cases in which it is difficult to determine the relation of a white blood-corpuscle to a definite cell group, this condition is always a positive sign that the cell must be looked upon as a lymphocyte; however, the absence of this reaction is not absolute proof that the cell is not related to the lymphocytes, for, as may be noted from the colored plate, the protoplasm occasionally shows a weaker stain with the basic staining material than the nucleus.

The cells which, in fresh blood, we have recognized as finely granular and polynuclear, are shown, in staining with the triacid stain $(\operatorname{IX}, b)$ to have pale green colored nuclei and very fine, almost dust-like granulations of a 
reddish violet color, filling the entire protoplasm. The granulation owes this color to the chemical action of a combination of the acid fuchsin and the methylene-green produced by a "neutral" stain; for this reason these granulations are designated as neutrophilic, and this variety of cells as polynuclear neutrophitic leukocytes. As this granulation only stains in "neutral" dyes, it remains unstained in the methylene-blue eosin mixtures that do not contain a "neutral" dye stuff, and we therefore find in the polynuclear leukocytes in this stain an entirely unstained protoplasm, while the nucleus shows an intense blue (IX,a).

A small number of white blood-corpuscles by their staining and morphology show a close relationship to the group described above; they only differ in that the granulation is much more sparse, and that instead of a polymorphic and intensely stained nucleus a larger, less indented, quite faintly stained nucleus is present. These cells we designate as transitional forms (see colored plate VIII, $c$ ). They are the stages immediately prior to the polynuclear neutrophilic leulkocytes which in part are formed from the following groups:

Large mononuclear leukocytes. This cell form is one which is most difficult to differentiate from the lymphocytes; nevertheless this differentiation is absolutely necessary. In the triacid stain they are remarkable for their size, being three times as large as a normal red blood-corpuscle. Their nucleus is large, comprising about one-third of the cell, usually eccentrically placed, round, of a pale green stain; their protoplasm is almost unstained and free of granulations (VIII, $b$ ). The cells stained in methylene-blue (VIII, $a$ ) are often decidedly more characteristic. We have one prominent sign which greatly facilitates the differentiation of this variety of cells from the larger forms of the lymph-cells; namely, that while (as has been mentioned above, and as may be noted from the colored plate) in the lymphocytes the boundary between the nucleus and the protoplasm is sharply marked, in the large mononuclear leukocytes the dividing line is indistinct, so that frequently we cannot determine where the nucleus begins and the protoplasm ends. The protoplasm of this cell variety is apparently never more decidedly basophilic than its nucleus.

The three last named cell forms constitute an intimately connected group, that is, links in a chain of development the beginning series of which are the large mononuclear leukocytes, and from these, by transformation and division of the nucleus as well as by the extension of granulations, the transitional forms and, later, the polynuclear neutrophilic leukocytes, arise.

The cell forms which in fresh blood are conspicuous by their coarse granulations may also be readily differentiated from the others in the stained preparation. Their nuclei stain with nuclear stains with medium intensity; the granulations which almost completely fill the protoplasm. in the triacid solution are orange or copper color $(\mathrm{X}, b)$, and in eosin-containing solutions they are stained a brilliant red $(\mathrm{X}, a)$; unfortunately, in the reproduction they are not very well depicted. On account of this property of acid dyes (especially eosin) to attract these granulations, the entire cell is called oxyphilic, acidophitic or eosinophilic. 
Another variety of the white blood-corpuscles is the mast-cell (XI, $a, b, c)$, the peculiarities of which cannot be recognized in fresh blood, but only upon the use of very definite methods of staining. [Mast-cells are beautifully stained by the Romanowsky method mentioned above.-En.] These cells are characterized by a quite coarse, often irregular, granulation which as a rule only partly fills up the protoplasm. Their distinctive feature, however, is the basophilic reaction of the granulations and their power to modify the color tone of most of the substances that stain them ("metachromasia"), so that, for example, with kresyl-violet $R$ they stain almost pure brown. They also differ from other granulations by their ready solubility in water, so that they can only be stained in alcoholic, not in watery, stain solutions. Therefore, the two staining mixtures mentioned above which are in most common use are not suitable for staining the granulations of the mast-cells, but we can best use for this purpose alcoholic solutions of methylene-blue, dahlia, or kresyl-violet R. [See last note-_En.] In the preparations stained with triacid the mast-cells are recognizable, since the insufficient staining of the granulation causes the cell to appear as if permeated by vacuoles (XI,c). They are frequently so sparse in normal blood that they can only be discovered by examining several preparations.

These various cell forms may be determined in all normal blood by the aid of different stains, and in the adult they are found in about the proportion shown in the last division of TABLE $\mathrm{A}$, where the large mononuclear leukocytes and the transitional forms, on account of their close relationship, are included in one group.

In the arrangement of TABLE $\mathrm{A}$ the fact is disclosed that the lymphocytes occupy a unique position among all the white blood-corpuscles. This is mainly due to their origin: They originate in the lymphatic tissue of the body, the principal mass of which consists of the lymph-glands; lesser amounts of this variety of tissue are, however, also present in the bone-marrow, and it therefore, perhaps, contributes a small portion of the lymphocytes of the blood. The groups from the second to the fifth, however, originate exclusively in the bone-marrow; only under definite pathological conditions does the production of these varieties of cells also occur in other regions. The point of origin of the mast-cells, which is chiefly in the bonc-marrow, must also be the connective tissue where they are formed in large quantities, particularly in certain pathological conditions.

A second comprehensive difference between the lymphocytes and the five other varieties of cells consists in the fact that the latter have the property of active movement which the lymphocytes do not possess; we shall see later that this difference is of great importance for the conception of pathological processes. [It has been conclusively shown, I think, that lymphocytes do possess the power of ameboid movement.-ED.] 
TABLE A

THE WHITE BLOOD-CORPUSCLES OF THE NORMAL BLOOD

I. Lymphocytes (large and small)

Per cent.

II. Large mononuclear leukocytes ?

III. Transitional forms

IV. Polynuclear neutrophilic leukocytes

V. Eosinophilic leukocytes

$22-25$

VI. Mast-cells

The blood-plaques are recognizable in fresh blood by their lesser size $(1-3 \mu)$, their clump formation, and their slight refractive power; they stain a pale blue in methylene-blue solutions, and in the triacid stain from gray to grayish brown. [In most triacid preparations the blood-plates are invisible. With Romanowsky stains they are dark plum color and are easily seen.-ED.]

Before entering upon the physiologic and pathologic importance of individual cell forms we must first describe a few coll anomalies. In different pathological conditions decided changes in the shape and size of the red blood-corpuscles are noted, and these we designate by the term poikilocytosis (Plate, Row III). The average normal size of the red blood-corpuscle is from 8-9 $\mu$; in some conditions of the blood, besides these "normocytes" there are also very small forms which may be only 2 to $3 \mu$ in size ("microcytes"); on the other hand we may observe large forms which are frequently from $14-15 \mu$, in rarer cases may even reach 18,20 and $22 \mu$ (macrocytes, megalocytes, gigantocytes). Besides the changes in size, the manifold changes in the shape of the bloodcorpuscles are important, and may be looked upon as an expression of segmentation and decomposition.

Occasionally combined with these pure morphologic changes, but quite as frequently entirely independent of them, we note in the erythrocytes deviations from normal conditions which are shown by a diminution of their hemoglobin contents. For example, forms of various size are noted in which the central depression is very markedly extended so that only a comparatively small part of the disc, often only a quite narrow rim, appears to contain hemoglobin; i. e., to stain in the corresponding solution (sce colored Plate, Row III).

A diminution in the amount of hemoglobin of the individual blood-corpuscle is also occasionally shown by a generally weaker power of staining.

Besides these slight deviations in tinctorial properties, the red blood discs frequently show marked qualitative anomalies; for instance, we note very frequently blood dises which do not take a pure stain with eosin or orange, but a mixed one, so that in staining with the triacid stain they are more or less grayish brown, in methylene-blue eosin solution from bluish red to lilac, and occasionally they may appear pure blue. This behavior, which now is generally designated as "polychromatophitic legeneration," may be explained by the fact that foreign substances which stain with the nuclear colors have 
been deposited in the plasma of the red blood-corpuscle (see Plate, Row II). [There are many, and on the whole convincing, reasons for regarding these granules as evidence of regeneration or of unripeness, but controversy is still active on the subject, and it seems best for the time to use some neutral term such as "basophilic stippling."-ED.]

Such a deposit of substances which, for example, stain in methylene-blue but not, however, in the triacid solution, is found in a granular or dust-like, form in the protoplasm of the red blood-cells, whether this is normally stained or degenerated in the manner above described (polychromatophilia) (see Plate, Row VI). We should be cautious in assuming the identity of these deposits with the granules of the white blood-corpuscles; therefore we should also avoid the designations granular, granulation, granulated erythrocytes, etc., and choose the non-implicating designation "stippled erythrocytes."

Much less frequent than these staining anomalies of the red blood-corpuscles is an additional one, the appearance in the circulating blood of nucleated. red blood-corpuscles, "erythroblasts." It is well known that normally in the red bone-marrow nucleated red blood-corpuscles appear. These are about the size of the normal non-nucleated blood dises, but contain a nucleus of an extraordinarily intense stain and a protoplasm that usually appears in the color of the stains that color hemoglobin, more rarely in that of polychromatophilic degeneration. They are unquestionably the early stages of the normal erythrocytes, and for this reason are designated as normoblasts (see Plate, Row IV). On the other hand, only under pathological conditions in the adult do we find another form of erythroblasts which are decidedly larger than the normal red blood dises, and have a diameter of 14,16 , even $20 \mu$ and more. Their nucleus, as a rule, is but feebly stained by nuclear dyes, their protoplasm almost invariably shows the condition of polychromatophilic degeneration. We designate this variety of cells as megaloblasts, or gigantoblasts (see Plate $\mathrm{V}, \grave{a}, b$ ), and recognize in them the embryonic stages of megalocytes and gigantocytes.

In the white blood-corpuscles changes in form or in staining property are of slight importance. It should be mentioned that very frequently the protoplasm of the lymphocytes at its border shows proliferation and segmentation, processes which indicate a degenerative condition without our being able to assign to them definite pathologic importance.

Much more interesting is the appearance in the circulating blood of a cell form which, under normal conditions, is met with only in the bone-marrow. This is a mononuclear leukocyte of varying size ( 8 to $20 \mu$ in diameter) (see Plate, Row XII), the protoplasm of which is filled by a dense neutrophilic granulation; Ehrlich has given this corpuscle the name " myelocyte."

The white blood-corpuscles show variations of great importance in their number, both as regards their total number per cubic millimeter and in their proportion to the red blood-corpuscles $\left(\frac{\mathrm{WC}}{\mathrm{RC}}\right)$ and particularly, in the proportion of the individual forms of the white blood-corpuscles to one another. 'The estimation of the proportion of $\frac{\mathrm{WC}}{\mathrm{RC}}$ is possible by the aid of Thoma's apparatus for counting the absolute numbers; this may also be accomplished with 
the stained dry preparation. For this purpose we use the movable quadratic Ehrlich eye-piece (see Fig. 20). The principle of this diaphragm depends upon its making it possible to count the red and white blood-corpuscles in different large divisions of the field of view, so that, for example, the leukncytes are counted in a field six or nine times as large as the field in which the

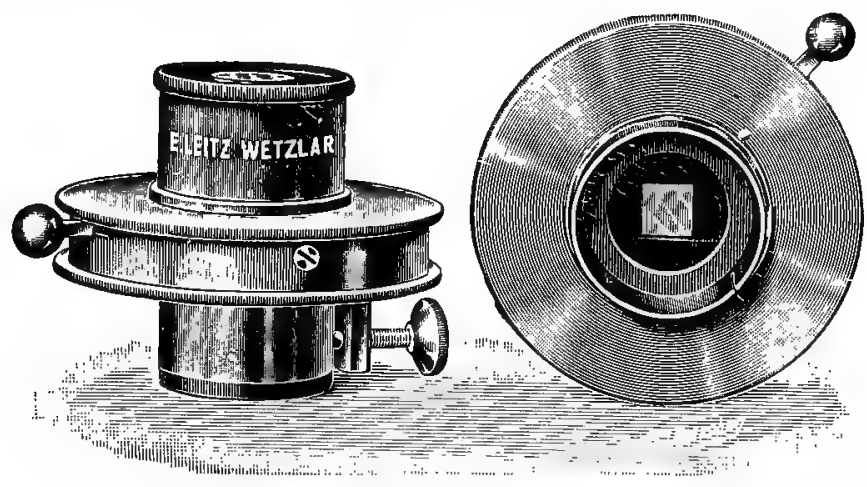

Fig. 20.-Ehrlich's Eye-piece with Iris Diaphragm.

reds are counted. The total number determined after counting a larger series of fields of the red is then directly compared with the sum of the white by multiplying by six or nine. A prerequisite for a correct result by this method of estimation is an absolutely uniform layer of blood upon the cover-glass.

The proportional relation of the individual varieties of blood-corpuscles to one another may be determined by a simple calculation of the cells when examining a dry preparation.

We have described the fundamental characteristics of the blood cells in their normal and pathological condition, omitting everything that depends upon purely theoretic investigation and which has not yet been directly applied to practice, as well as that which is still being debated, and concerning which the views of competent observers are still at variance. How has it been possible to utilize this material in general and special pathology?

In the article upon Anemia (see this volume) due stress has been laid upon the pathological changes which the red blood-corpuscles undergo in the various anemias, and we may now limit ourselves to a description of the changes in the white blood cells in the same diseases.

In the first place, as regards the appearance of pathological cell forms, for the reasons indicated we have included only the myelocytes in our description. But the circumstances under which these are found in the circulating blood agree in so many points with the circumstances under which the number of white blood-corpuscles increases or decreases that they require no special elucidation.

Consequently, the most important chapter in the pathology of the white blood-corpuscles is that which treats of the changes in their total number and the relative proportions of the individual leukocyte forms. Since, in general, we refer to the presence of leukocytes as leukocytosis, an increase of their 
total number we speak of as hyperleukocytosis, and a diminution, as hypoleukocytosis. [I prefer the term "leukocytosis" for an increase, and the term "leukopenia" for a decrease, in the circulating leukocytes.-ED.] Even at the normal, the absolute number of white blood-corpuscles and the proportion of their different varieties show distinct variation; in different pathologic conditions, however, and especially in infectious diseases, the deviation of these conditions from normal becomes more marked. We find the explanation for this in the principle of chemotaxis, according to which bacteria and their products of metabolism, as well as numerous other toxic substances, are capable of attracting by chemical irritation the cells which have accumulated in the blood-forming organs, a condition which we designate as positive chemotaxis; on the other hand under quite similar circumstances the leukocytes are repelled by the previously mentioned poisons, so that the number in the circulating blood is decreased, and this process we call negative chemotaxis. The self-evident presupposition for this attraction and repulsion of the leukocytes (since the condition is a notable example of action at a distance) is the property of the leukocytes to show active movement. For this reason we give to this state the name active leukocytosis.

Active leukocytosis furnishes the most varied pictures, not only quantitatively but qualitatively, because of the fact that the chemotaxic irritability of the different leukocyte varieties shows specific differences. Thus there are substances which only influence one variety of cell in a positive chemotaxic manner, being inert toward all others. This shows itself most frequently in a decided increase of the polynuclear neutrophilic leukocytes alone in numerous infectious conditions, as in septic diseases, diphtheria, and articular rheumatism; other infections have a negative chemotaxic property for this cell form so that, for example, in enteric fever, we often find them decidedly diminished. On the other hand the eosinophilic leukocytes show marked power of attraction for the blood in bronchial asthma, in pemphigus, in trichinosis, and in several varieties of helminthiasis. More rare, but nevertheless certain, is a chemotaxis of the mast-cells exhibited according to this principle.

It is a peculiarity of the chemotaxic reaction that all leukocytes endowed with one type of granulations are attracted simultaneously even if the composition of the nucleus varies; for example, the myelocytes react in the same way as the polynuclcar neutrophilic leukocytes. For this reason, in accordance with the variety of the cell that is influenced, we speak of neutrophilic hyperleukocytosis or hypoleukocytosis of eosinophilic or mast-cell leukocytosis.

Besides these specific leukocytoses, we diagnosticate another form of hyperleukocytosis which we designate as a mixed form, because in this all cells that are capable of chemotaxic reaction are increased in the blood. This is myelogenous leukemia; we therefore arrive at the hypothesis, no matter how obscure to us the etiology and pathogenesis of myelogenous leukemia may still be, that in this disease a toxic substance is active which has the property of influencing all myelogenous cell varieties in a positive, chemotaxic manner.

A form of white blood-corpuscles must still be mentioned which does not possess the active property of locomotion: these are the lymphocytes. [See note at foot of $\mathrm{p}$. 298.] It is therefore evident that this form is not subject to 


\section{EXPLANATION OF THE COLORED PLATE}

\section{cells drawn with Leitz one-twelfth oil immersion.)}

I. Erythrocytes, stained with triacid and with the Chenzinaky stains.

II. Polyehromatophilic "degeneration."

III. Poikilocytosis; pessary forms.

IV. (a) Normoblasts.

(b) Free normoblast nucleus.

V. (a) Megaloblast.

(b) Gigantoblast.

VI. "Stippled" erythrocytes. wentions

VII. Lymphocytes.

VIII. Large mononuclear leukocytes:
(a) with Chenzinsky.
(b) with triacid.
(c) transitional form.

IX. Polynuclear neutrophilic leukocytes.

X. Eosinophilic leukocytes:

(a) with Chenzinsky.

(b) with triacid.

XI. Mast-cells :
(a) with methylene-blue.
(b) with dahlia.
(c) with triacid.

XII. Myelocytes. 


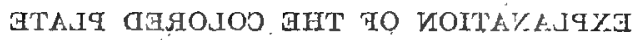

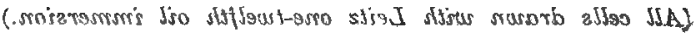

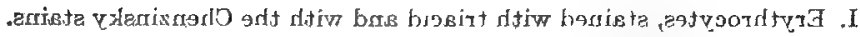

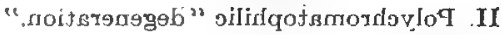

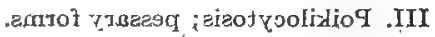
.ajesidomio $V_{\perp}$ (o) VI

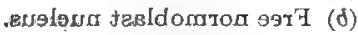
.tessidolsog $(\mathrm{s})$. I itesldotaggin (d) .agtygoreltrya "balqaite". IV

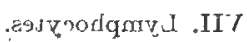




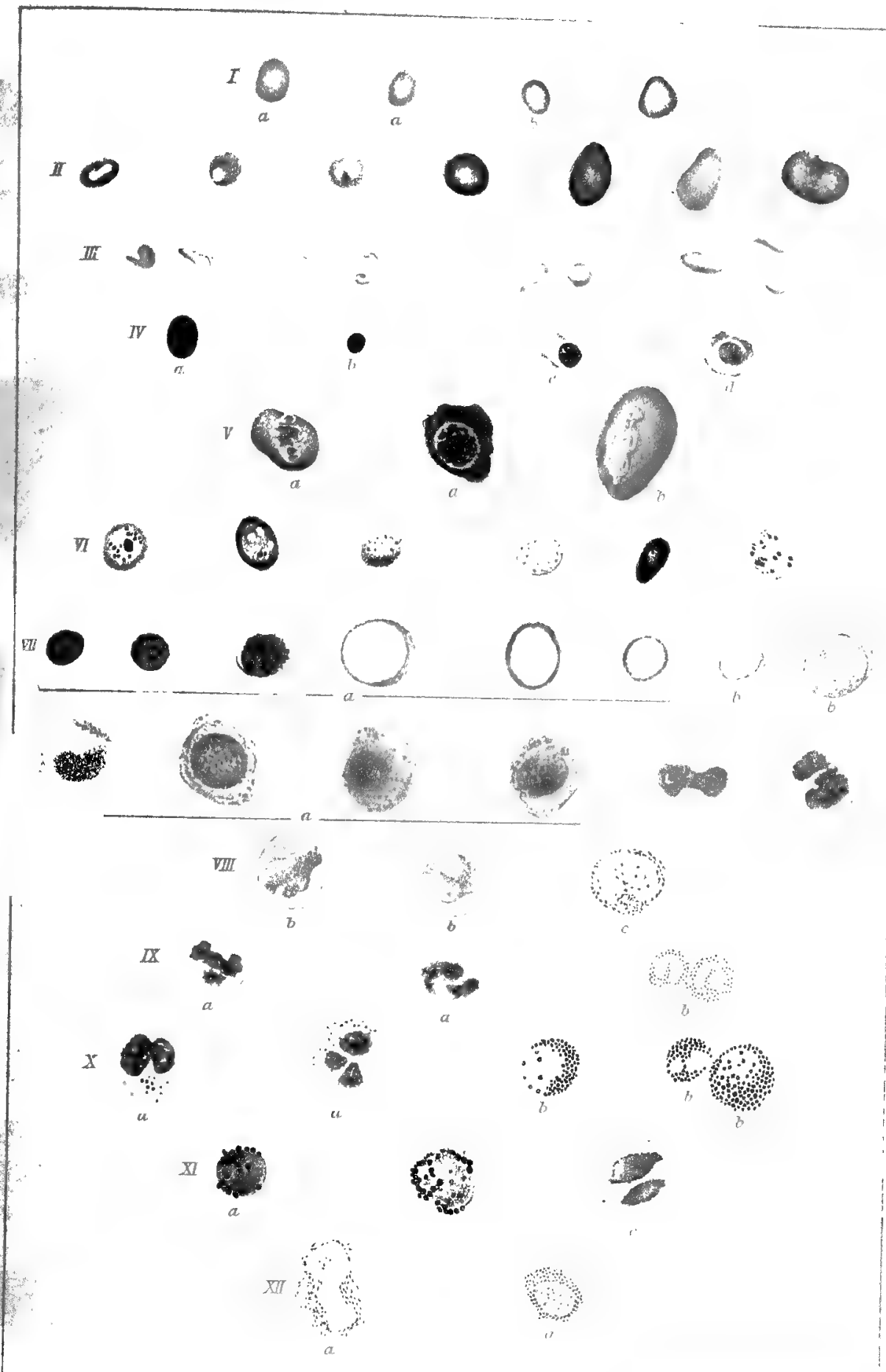


1

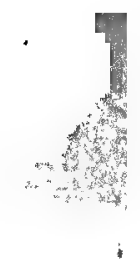

?

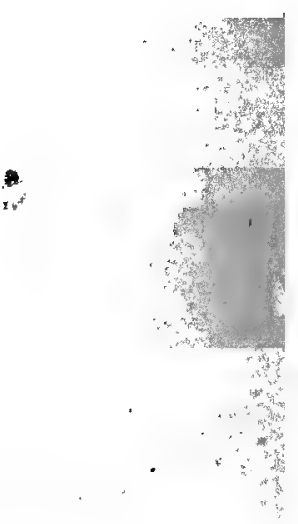

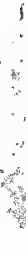

$4^{4}$

耀 
the laws of chemotaxis. Since, in spite of this, there are often transitory or permanent variations in the number of lymphocytes found in the blood, these require another explanation. We find this either $(a)$ in the increased production of lymph-cells, due to hyperplasia or inflammation of the lymphatic tissue, or $(b)$ in an influx of this variety of cells by increased lymph circulation. The lymphocytes themselves are entirely passive in these processes, and for this reason we designate their increase as passive leukocytosis. Extreme degrees of this condition we designate as lymphatic leukemia. (A special description of the various forms of leukemia will be found in this volume, the article being by Professor v. Leube.)

I cannot close this article without showing by a few examples that the researches described here are of importance in the diagnosis of diseases other than the actual blood diseases.

The number of the leukocytes, for instance, is decidedly important in the differential diagnosis of the infectious diseases. If we remember how difficult the differentiation of enteric fever from other affections occasionally is, any sign which facilitates the diagnosis will be a great help. The affections which may be confounded with enteric fever, such as osteomyelitis, general sepsis, pneumonia, etc., are characterized by a decided increase of the neutrophilic leukocytes, while in uncomplicated typhoid fever this variety of cell is decidedly diminished or is at most present in only normal numbers.

In the differentiation of inflammations of the cerebral meninges, an examination of the blood may often be of benefit; and if, in a case of undoubted meningitis, we find a normal relation of the leukocytes, the assumption of the tubercular form instead of a purulent one is justified.

The occasionally extremely difficult differentiation between scarlatina and measles may often be facilitated by a blood examination owing to the fact that a distinct diminution of the polynuclear leukocytes, or an almost normal condition of the same, is quite characteristic of measles, while in scarlatina a decided polynuclear neutrophilic hyperleukocytosis is the rule.

Lately Curschmann and subsequent investigators have reported that in numerous cases of perityphlitis, the diagnosis of an abscess which could not be determined by palpation was made from an increase of leukocytes to 20,000 and more, and this prevented all necessity for exploratory incision. [ "Lately" the Germans have wakened up to these facts which have been known and used in America since described by me in 1894.-ED.] The subsequent surgical operation absolutely confirmed the reliability of this symptom. On the other hand, clinicians have in numerous cases discountenanced a surgical operation on account of an almost normal leukocyte finding. Here, also, the further development of the disease justified the diagnosis. Almost as much importance may be ascribed to the phenomenon of leukocytosis in the diagnosis of abscesses localized elsewhere, which otherwise could not be determined with certainty.

In a case in which the diagnosis was otherwise obscure, Brown concluded from a decidedly marked eosinophilic hyperleukocytosis that the underlying condition was trichinosis; the further course of the disease justified this opinion. 


\section{THE ANEMIAS}

\section{By P. EHRLICH AND A. LAZARUS \\ FrankForT-ON-MaIN \\ Charlottenburg (Berlin)}

Is reviewing the earlier literature of anemia, very exact descriptions of the general clinical symptoms may be found, while the changes in the blood seem to have been somewhat overlooked. The object of this article is to acquaint physicians with the advance in knowledge along these lines; points which have long been established facts will be passed over rapidly, and the special consideration of hematology will be our aim.

Easy as it may be to distinguish the different forms of anemia, mistakes in their recognition have sometimes occurred, and do still occur, chiefly through a disregard of hematology. The fact that there can be no anemia without changes in the blood seems to have been long ignored. Disturbances in the circulation of the blood, especially, lead to incorrect diagnoses, because these disturbances cause important clinical symptoms common also in anomalies in the composition of the blood. Such symptoms are: Intense pallor of the skin and mucous membranes, small pulse and its increased frequency, and among subjective phenomena weakness and vertigo. These acute symptoms appear, for instance, under such psychical influences as fear, terror, etc., or in acute somatic disorders such as affections of the stomach, or from fatigue, or from excesses in "Baccho et Vencre." They may also be present in chronic conditions, especially in neurasthenia, in gastric affections, and in heart disease. Mistakes are therefore prone to occur, because in a few instances the symptoms mentioned are also present in true anemia.

The presence of anemia is, therefore, only positively established after blood-changes have been proven. We never find that the blood deviates from the normal in one respect only; in every case thorough examination shows a group of changes. Still, a determination of the hemoglobin alone is sufficient to demonstrate the existence or non-existence of an anemia. We say a person is anemic if on testing his blood an amount of hemoglobin smaller than normal is found, and we adhere to this designation even if other important properties of the blood and the clinical symptoms appear entirely normal. On the contrary, when a patient has the general clinical symptoms of anemia while his blood shows a normal percentage of hemoglobin, we do not call him anemic. In practice, every case of anemia is identical with oligochromemia.

The reduction of hemoglobin may be due to the fact either that too little is formed or too much consumed. A formation of hemoglobin that does not keep pace with the normal consumption may be either the expression of an 
affection of the hematopoietic organs, or in consequence of the fact that too little material, or an inferior material, is at the disposal of these organs.

An exaggerated consumption of hemoglobin takes place in hemorrhages and, indirectly, also in wasting processes of all kinds. The processes of blond regeneration and blood consumption are so intimately related that a disturbance of one of these processes is likely to bring about a disturbance of the other.

Often the blood regeneration is only relatively insufficient; that is, the blood-forming organs, especially the bone-marrow, furnish less blood than is required for the momentarily and abnormally increased demand. In these cases it often occurs that the hematopoiesis is more considerable and more energetic than in health, entire portions of the bone-marrow, physiologically passive, now becoming active; the consumption of blood may, however, remain so great that even an increased production does not maintain the balance. In these cases, whether the work of the blood-making organs is absolutely increased or diminished, the amount only, not the manner, of blood formation is changed.

On the contrary, we see conditions in which the type of blood-formation in larger or smaller regions of the bone-marrow has changed, and hematopoiesis has assumed a type which is abnormal in the adult organism.

Those anemic conditions in which blood restitution takes place according to a normal type are called SIMPLE ANEMIAS; and those in which the process is partially or wholly different from the normal type which will be considered more in detail later, are called PROGRESSIVE PERNICIOUS ANEMIAS. [The old term, "simple anemia," to which Ehrlich here reverts, does not seem to me likely to come back into use. It seems most reasonable to retain our present current term, "secondary or symptomatic anemia," for trouble of this type, and to use the terms, "pernicious anemia" and "chlorosis," with the consciousness that they, too, are secondary, but secondary to a cause which in most cases is wholly unknown.-ED.]

\section{SIMPLE ANEMIA}

As a result of extensive observations of the blood changes, and of supplementary studies of the changes in the bone-marrow, we know that in by far the larger number of cases of anemia the normal mode of blood regeneration is preserved; hence, these are classed as STMPLE ANEMIAS.

The causes of simple anemia are, first of all, acute, subacute, and chronic posthemorrhagic conditions. The supervention of anemia in these instances from the loss of considerable blood is readily understood.

Many constitutional or organic diseases lead to simple anemia, either by reducing the appetite, or by the loss of blood and tissue-juices, but especially through suppuration and albuminuria. Poisons also play an important rôle. Here the large group of autointoxications play a part and another damage is done by toxic substances developed by bacteria and other parasites. We may explain in two ways the effect which brings about the anemia without being able, in the special instance, to determine with certainty which of the two active causes, or whether, perhaps, both together, have been operative. Some 
of the poisons appear to destroy the blood directly; others to diminish or to change the function of the hematopoietic organs.

Excluding the diseases accompanied by suppuration, bleeding, and gangrene, a few others deserve especial mention as producing simple anemia. Such, for instance, are chronic digestive disorders, malignant tumors, scrofulosis, syphilis, malaria, and the different forms of helminthiasis.

In addition to the symptoms of the diseases which, according to the old terminology, were called "secondary anemia," the symptoms of simple anemia may be found in cases which are apparently "idiopathic." They are the consequence of faulty hygienic conditions, among which unsatisfactory nutrition plays the greatest rôle, and it may here be remarked that food which leads to a reduction in the percentage of hemoglobin need not necessarily be insufficient in quantity but it is lacking in iron and albuminous substances. [That faulty nutrition is a cause of anemia seems, of course, most plausible, but definite evidence for it is so far wanting.-ED.]

But, whatever the cause of the anemia in individual cases may be, no difference is to be noted in its essential results, the changes in the blood, or in their main clinical symptoms.

The most important symptom is the REDUCTION IN HEMOGLOBIN; this not only characterizes the disease as anemia, but also gives us information as to the degree of the morbid changes. Cases vary greatly in this respect, and vary without any apparent relation to the etiology. We meet with cases of "simple anemia" in which the values are but slightly below the normal. To this class belong also cases which show 20,15 and even only 10 per cent. of the normal amount of hemoglobin.

In the mild cases of simple anemia, the red blood-corpuscles differ little from those of normal blood. In cases somewhat more serious, deviations from the normal number and morphological changes are observed. In extreme cases the number of red cells may amount to less than 10 per cent. of the normal; 50 per cent. is by no means rare.

For the numerical relation between the percentage of hemoglobin and the blood-corpuscles in simple anemia, the etiology seems to be, to some extent, a determinative factor. Parallel deviations of both values from the normal are often seen; for instance, a reduction of 30 per cent. in the amount of hemoglobin corresponds to a reduction of 30 per cent. in the number of red corpuscles. After hemorrhages, however, we find that the amount of hemoglobin is considerably less than the corresponding red corpuscle count; and in cases of uncomplicated chlorosis, the parallel is disturbed in the sense that the number of red corpuscles is often nearer the normal than the hemoglobin value.

The morphological changes of the erythrocytes in simple anemia affect their size and their shape. We find that the individual red corpuscles show considerable difference in size; being mostly smaller than normal. Increase in size is rare, and is then but slight. The. shape of the red corpuscles is frequently more or less distorted and deformed. These conditions of deviation in size and shape are designated "poikilocytosis." Milder degrees of simple anemia do not show it, but the degree of poikilocytosis corresponds to the severity of the anemia. 
The reduction in the hemoglobin value of the individual corpuscle is, in some cases of simple anemia, very striking. This can be seen in fresh blood, but better in the dry stained preparation, from the fact that a larger area of the center remains unstained than would correspond to the physiological depression of the corpuscle. In some forms only a ring of stained portion may be seen (pessary forms).

Furthermore, the red corpuscles often manifest under the microscope a change in reaction to the usual stains. This change is known as "anemic or polychromatophilic degeneration." 1 It may be recognized from the fact that the hemoglobin of the corpuscles does not assume the pure color of the stain (eosin, orange), but a mixed color (blue-red in the methylene-blue eosin solutions, grayish-yellow with the triacid solutions). These changes are often very pronounced even in the mildest degrees of simple anemia.

A rare occurrence, which is probably characteristic only in especial forms of simple anemia, is the presence of granular or punctiform deposits in the protoplasm of the erythrocytes ("stippled erylhrocytes"); these are most apparent when stained with methylene-blue. They are found especially in lead anemias, even in the mild grades, and also in malarial ancmias. In simple anemias of other etiology, even in very grave cases, they are commonly absent, so that to a certain extent specific significance may be attached to them. [The universally applicable Romanowsky stains, described and recommended above, bring out the stippling beautifully.-ED.]

of great importance in the diagnosis of the different kinds of anemia is the fact that in the circulating blood nucleated red corpuscles of the same kind as those found in the normal red marrow ("normoblasts") sometimes appear. Such cells are noted in comparatively few cases of simple anemia. We find normoblasts with fair regularity, though usually only transitorily, in post-hemorrhagic conditions, and sometimes, in these cases, in quite large numbers. Their appearance proves an increase in intensity of the activity of the bone-marrow, and in extent of marrow which is active; for the clinical phenomenon corresponds anatomically to a more or less decided transformation of quiescent fat marrow into functioning red marrow, and an especially marked richness of these portions in normoblasts. We must emphasize the fact that the appearance of normoblasts in the blood is in no relation to the severity of the anemia; sometimes they are found in the mildest conditions, and are often looked for in vain in the severest. Except in acute post-hemorrhagic anemia their number is always small-so that only one nucleated corpuscle is found to one thousand and more non-nucleated red blood-corpuscles.

Besides the presence of normoblasts microblasts are sometimes noted.

Other alterations in the properties of the blood are also found in simple anemia, always in the direction of a reduction in the values. This is true of the SPECIFIC GRAVITY OF THE BLOOD, its total solids, and the amount of albumin it contains; the reduction is the more pronounced the greater the deviation of the hemoglobin from the normal.

The WHITE CORPUSCLES in simple anemia present nothing that is charac- 
teristic. In one case we may find a normal number of their different varieties, in another case a marked neutrophilic hyperleukocytosis may be present, in a third a hypoleukocytosis, or an increase in the number of lymph-cells, etc. So many factors influence the condition of the leukocytes, that in the multitudinous cases of simple anemia due to causes or complications differing from one another, no uniform picture can be described.

Summarizing, we may say that the most important and essential symptoms of the blood-changes in simple anemia are a diminution in the hemoglobin percentage and in the number of red blood-corpuscles, poikilocytosis, and the changes in the staining reaction. By attending to these factors in each individual case we can decide whether or not it belongs to the simple anemias, and how grave the prognosis is.

The general clinical symptomatology of anemic conditions exhibits a few traits so characteristic as to make them in most cases readily recognizable even without an examination of the blood. Owing to their wide distribution, they are so well known to physicians that a brief reference to them will suffice.

The pallor of the skin and of the mucous membranes is an obvious consequence of the diminished hemoglobin pereentage of the blood. It is often intensified by a permanent constriction of the peripheral blood-vessels. The same causes lead to a lessened capacity for muscular work, and readily produce sensations of weakness and fatigue. The most vital internal organs must also suffer in consequence of a reduction in the value of the nutrition; and, in severe cases, all physical and psychical functions are below par.

It is surprising that, in anemic people, THE GENERAL METABOLISM, even the interchange of gases, is not, as a rule, reduced; whereby we may conclude that if, in some cases, these functions are abnormal, it is due not to the anemia, but to some special cause or complication.

Even in severe cases, the adipose tissue is well preserved unless a loss of flesh follows in consequence of some special circumstances (as severe nutritive disturbances). This is a striking phenomenon for which no satisfactory explanation has been given; it was formerly attributed to a reduction in the oxidation processes, but this is usually not present at all; and the reduction in muscular activity and other functions is not sufficient cause.

Related to this phenomenon is FATTY DEGENERATION of some of the organs, especially of the muscles and the circulatory system. Fatty degeneration and fatty infiltration are chiefly found in the muscles of the heart, in the muscles of the eyc, and the intima of the blood-vessels.

To the fatty degeneration of the endothelial cells of the blood-vessels may be attributed the fact that in many anemic conditions a general HEMORRHAGIC DIATHESIS develops, which not infrequently combines with the primary disease to form a vicious circle. By their localization in the organs of special sense, hemorrhages of the eye and the ear are especially alarming, and lasting injury with total destruction of sight and hearing has been observed in severe cases of simple anemia. On this occasion we consider it our duty to differ with the widespread opinion that the symptom of retinal hemorrhage in anemic conditions belongs exclusively to progressive pernicious anemia. 
The diagnosis of simple anemia, therefore, is often easily made from its most manifest features. The careful physician will, however, make a blood examination, or at least a test for hemoglobin, even in cases where a glance is sufficient to show that he is dealing with anemia. In this way alone can we determine with certainty what part vasomotor influences play in shaping the disease. This alone will assure us that the supposition of anemia is not unfounded; and if the test shows a reduction we have then positive information regarding the degree of ancmia. Moreover, in cvery case of severe anemia, a microscopic examination of a stained specimen of the blood is absolutely necessary in order to make the important differential diagnosis between simple and progressive pernicious anemia.

The prognosis depends, in the first place, upon the cause of the disease. If this can be removed there is a good prospect of a cure. Under such circumstances, even in the severest cases in which the hemoglobin and the number of red corpuscles amount to only 10 per cent. of the normal, we have known cases of simple anemia to terminate in complete recovery.

The treatment of simple anemia must in the first place be directed to the cause. In numerous cases in which the cause is found and removed, as, for instance, continually recurring hemorrhages in the intestinal tract, worms, improper hygienic conditions, etc., no special treatment of the anemia is necessary after the removal of the cause; for spontaneously the blood returns to its normal state.

When causal treatment is impossible, or when it fails to influence the anemia beneficially, attention must be paid to the symptoms of anemia as such. In the great majority of cases, medication is far superior to any other mode of treatment. Iron and arsenic, separately or combined, lend a powerful stimulus to blood production, and after their rational administration, often even in a few weeks, the morbid changes in the blood and in the general condition disappear, and make way for a restoration to health. The choice of the special preparation is essentially unimportant; the main point is to continue the treatment long enough, and to administer sufficiently large doses. The general rule that 0.1 gram of iron is to be given pro die may serve as a criterion for the administration of special iron preparations, organic or inorganic. The dose of arsenic varies widely, and it must be determined by the individual tolerance. Usually we succeed best if we administer 3 to 5 milligrams of arsenious acid, divided into several doses, properly diluted and given on a full stomach. The drug should be continued for a long while, even after the normal status has been reached.

But there are other anemic conditions in which iron and arsenic give unsatisfactory results, or are of no use whatever. Here dietetic and physical therapy must be resorted to. As the metabolism of anemia does not deviate from the normal, the normal diet of healthy people may be allowed. Theoretically, preference has been given to food rich in iron (meat, yolk of egg, spinach, apples, etc.). Often, however, owing to the special peculiarities of a given case (for instance, in cases complicated with gastro-intestinal affections) special modifications of the diet must be made which cannot be here considered.

Among the general auxiliary measures in the treatment of anemics, rest- 
physical, mental and psychical-must be accorded the first place. In this connection, certainty in diagnosis will aid materially in the choice of treatment; for instance, the pale neurasthenic, not really anemic, needs considerable physical exercise; while the muscular activity of a true anemic must be limited as much as possible.

Modern hydrotherapy has gradually acquired a prominent place in the treatment of anemia; but we must always bear in mind that the withdrawal of too much bodily heat is to be avoided, because all anemics are sensitive to cold. Cold applications, such as cold ablutions with friction, or partial douches of short duration, are best given in the morning immediately on rising-for then the redilatation of the cutaneous blood-ressels takes place pleasantly and actively after the patient returns to his bed. Here, too, we must remember the distinction between the treatment of anemics and of pseudoanemics; the latter have a perfectly normal composition of the blood, and only suffer from an abnormal contraction of the peripheral blood-vessels.

In protracted cases, a change of climate is frequently very beneficial, and, particularly in excessive anemia, a sojourn in the mountains of medium altitude. More robust patients may be sent with adrantage even to high mountainous regions, which often powerfully stimulates the regeneration of the blood.

In most cases of severe anemia, a sojourn at the seashore is not to be advised; in moderately severe cases it is to be recommended. Great caution must be exercised in the use of sea-baths, as they sometimes aggravate anemia.

\section{PROGRESSIVE PERNICIOUS ANEMIA}

Progressive PeRnicious aNejita must be distinguished from other anemic conditions becanse in blood-regeneration it presents a most striking peculiarity. In opposition to the process in simple anemia, in progressive pernicious anemia blood-regeneration in larger or smaller regions of the bone-marrow takes place in a manner different from the physiological. In the blood-forming organs and in the circulating blood cells we note cells which are never found in the healthy adult organism. Since these cells are found physiologically in the embryonal life of man, we speak of a "reversion of bloodformation to the embryonal type."

Before accurate study of the changes in the blood and in the bone-marrow had been made possible, the consideration of this particular form of anemia as a special type was based upon a more negative foundation. If the symptoms of severe anemia were found without a distinctly palpable cause, the disease was considered idiopathic, and was designated progressive pernicious anemia. Almost always in such cases we observed in the blood the changes alluded to, which are to be accurately described later on, and thus these positive signs, particularly as they are regarded as the expression of marked transformation in the body, are almost universally recognized as the important differential diagnostic points.

The theory of the "primary" nature of progressive pernicious anemia was shattered in the course of time by numerous experiences, and this aided us 
in arriving at our present standpoint. To-day we know that progressive pernicious anemia may undoubtedly often follow other diseases, and not as an independent disease, but only as a symptom; as, for instance, in the case of diabetes. We shall now briefly describe what has been demonstrated with certainty.

The best illustration of severe anemia which we possess is the case of individuals who harbor the bothriocephalus latus in their intestines. Notwithstanding all the controversies on this subject, there can be no doubt that this worm, living or dead, may produce the most severe anemic conditions, and that this bothriocephalus anemia is a true progressive pernicious anemia. We understand fully the mode of action of this parasite, and may regard it as proven that the disease is due to toxic substances that have a hemolytic effect, and thus cause anemia. Further it may also be assumed as likely that the parasite changes the activity of the bone-marrow in a peculiar way. The incontestable fact that a very large number of people are afflicted with bothriocephalidæ without becoming anemic may be explained in different ways. In the first place, there may be a difference in the degree of virulence of the toxins; or, again, a difference in the susceptibility of the host. For there is no doubt that individuals of one species may possess a very different susceptibility to the same poison; for example, the susceptibility of rabbits to crotin has lately been proven to show enormous variations. Finally, the explanation of a gradual autoimmunization to the toxin is quite reasonable.

Leaving now the consideration of bothriocephalus anemia, which is at present understood, we may say that all that is known of the presumable causes of progressive pernicious anemia may thus be summarized: "The same deleterious agents which are capable of producing a 'simple' anemia may also develop the progressive pernicious form." Why apparently identical causes may in some instances produce the common simple anemia and in others the comparatively rare progressive pernicious anemia we do not as yet know.

In considering the symptoms of progressive pernicious anemia, CHANGES OF THE BLOOD, especially morphological alterations, claim our closest attention.

In a typical, well-defined case of progressive pernicious anemia, the first glance at a good stained preparation is sufficient to distinguish it from simple anemia. We find that the majority of erythrocytes, or at least a large number of them, have a diameter largely above the normal, 15 to $18 \mu$ (megalocytes) and, by their stain, these cells disclose to us a great richness in hemoglobin.

On repeated examination, we almost always find some megaloblasts, i. e., erythrocytes with nuclei, the predecessors of the megalocytes. Except in the worst stages of the disease, the number of megaloblasts is always very scant. [The number of megaloblasts usually exceeds the number of other erythroblasts, but presents no close parallelism with the severity of the clinical manifestations or with the intensity of the anemia. It is true that in most cases the number of megaloblasts increases as the symptoms are aggravated, and diminishes in the remissions of the disease. But to this rule there are many exceptions.--ED.] Besides megaloblasts, normoblasts and microblasts are also to be found. Of non-nucleated red corpuscles, besides megalocytes, we also see 
cells of normal size or smaller; the smallest forms, the microcytes, are frequently observed.

All other peculiarities of simple anemia are to be found in progressive pernicious anemia: Poizitocytosis, polychromatophilic degeneration, and "stippled" erythrocytes; contrary to the rule in simple anemia stippled erythrocytes are found with great regularity in progressive pernicious anemia.

The red corpuscles are always noticeably reduced, often to the low figure of 20 per cent. to 10 per cent. of the normal.

The leukocytes are often absolutely reduced, but not always; moreover, a neutrophilic hypoleukocytosis is often found. [Especially in the remissions of the disease when the number of red cells is rapidly increasing and the marrow is very active.-ED.]

The other blood values differ little from those of severe cases of simple anemia. The hemoglobin percentage, the specific gravity, the amount of sol$i d s$, and the amount of albumin show a diminution of their values corresponding with the grade of the disease. [Why the authors do not mention at this point the high color-index, i. e., the relatively increased hemoglobin which is so characteristic (though not invariable) in the disease, I do not understand.-ED.]

According to this, of all the changes in the blood, only the changes in the size of the erythrocytes, and the formation of the nuclei of the erythroblasts denote the variety of anemia. The appearance of megalocytes and megaloblasts is characteristic of progressive pernicious anemia, while their permanent absence is indicative of the simple form. For megaloblasts and megalocytes appear in the blood only when some region of the bone-marrow shows a megaloblastic degeneration. The simultaneous occurrence of normoblasts, normocytes, and smaller forms, as well as a temporary predominance of one or the other of these forms, is not at all surprising when we consider that the transformation of the bone-marrow is always only partial, and is often limited to very small areas, while in the other regions of the bone-marrow the blood elements are produced physiologically.

The other properties of the blood can only be looked upon as denoting the DEGREE of the disease, as in simple anemia. We must expressly state that in undoubted cases of progressive pernicious anemia moderate values of the hemoglobin, a moderate number of red blood-corpuscles, a moderate specific gravity, etc., may be found; while, on the other hand, minimal values are met with in positive cases of simple anemia. It follows that the degree of anemia is, to a certain extent, independent of its type.

In the CLINICAL PICTURE of progressive pernicious anemia marked characteristics are a peculiar pallor and a slight puffiness of the skin, which may enable the physician to guess the diagnosis at the first glance. No word will exactly describe the color of the skin; the designation "faded-yellow" perhaps best portrays the real condition, but no description can take the place of personal observation.

Moderate jaundice is frequently present, and slight edema may be almost always noticed in the face, in the abdomen, and in the legs.

The contrast between the severity of the constitutional symptoms and the 
well-nourished appearance of the patient is still more striking here than in chlorosis and other simple anemias. There is hardly a disease in which, with so great a cachexia, the adipose tissue is so well preserved.

Concerning the METABOLISM in progressive pernicious anemia we have little knowledge. The increase of proteid decomposition, which has been observed in some cases, must be due to some inconstant factor, for in other cases there is no increase at all. The same is true of the oxidation processes.

Fever is present in almost all cases, if only transitorily, but it is by no means characteristic, and bears no relation to the intensity of the disease. Its origin is probably a ferment intoxication from the destruction of red bloodcorpuscles.

The CARDIAC symptoms are prominent. For a long time the disease was considered to be merely a symptom of severe cardiac degeneration. The subjective disturbances which are very often troublesome to the patient consist of (a) palpitation, brought about by the Ieast exertion, which often leads to $(b)$ true precordial distress and $(c)$ severe dyspnea. Auscultation of the heart reveals over all the valve-areas a clear, soft, usually systolic, rarely diastolic murmur. Percussion as a rule is normal. The heart symptoms are often so intense that during life it is impossible to distinguish the condition from genuine endocarditis.

Nevertheless, we must state with emphasis that well-defined severe cases of progressive pernicious anemia have been observed in which the heart showed neither subjective nor objective morbid phenomena.

The weakness of the heart action is best indicated by the character of the PULSE, which usually is of low tension, small, and frequent. [Very frequent and very interesting in this disease is the violent pulsation of the elastic peripheral arteries which often equals that seen in aortic regurgitation. Edwards ${ }^{1}$ has recently reported a case of pernicious anemia in which the abdominal aorta. was so expansile and pulsated so forcibly that aneurism seemed the only explanation. The carotids flap loosely.-ED.]

On the part of the DIGESTIVE ORGANS, marked anorexia, persistent nausea, belching and vomiting, and also unconquerable repugnance to certain foods, for instance, meat, are often noticed. Examination of the gastric contents in the living has not infrequently revealed a complete suspension of the gastric secretion-"achylia gastrica." The abdomen, as a rnle, is everywhere soft and not sensitive upon pressure.

The motor capacity of the intestine, the power of absorption, and the secretion of the intestinal glands seem, as a rule, normal.

The SPLEEN is generally normal or only slightly decreased in size, but there are positive cases recorded in which extraordinarily large splenic tumors complicated the clinical picture.

Some disturbances of the CENTRAL NERVous sYSTEM may be noticed, though in comparatively rare instances. The mind may be in a condition of deep depression or marked exaltation, and the one may repeatedly alternate with the other. Toward the end, disturbances of consciousness and deep coma

1 Trans. of the Assn. of American Physicians, 1903. 
appear. In a number of cases, failure of the memory and lasting impairment of intelligence have been noticed.

In the reports of the last decade, numerous disturbances of the SPINAL CORD - in fact, a symptom-complex-have been observed corresponding exactly to the picture of a genuine tabes dorsalis; namely, pupillary rigidity, loss of knee-jerks, ataxia of the limbs, incontinence of the bladder and rectum, lancinating pains, disturbances of sensation, anesthesia, and paresthesia. In some cases one or another classical symptom of tabes is missing and those of the clinical counter-picture, spastic spinal paralysis, are found. [Occasionally intense neuralgic pains in the extremities occur when locomotion is attempted. These cases are doubtless akin to those of intermittent claudication.-ED.]

It has already been remarked that these affections of the nervous system are not regular accompaniments of progressive pernicious anemia; and it must be added that in all probability they are usually independent of the severity of the anemia, but due, as we suppose, to some special etiology. In any case, we are not justified in regarding these symptoms as consequences of the anemia, but they are presumably co-ordinated consequences of the same cause-the effect of toxins. [Three groups of cases should be here distinguished:

(a) Those in which the nerve symptoms appear late in the course of a case of obvious pernicious anemia.

(b) Those in which there are no nerve symptoms (except peripheral paresthesiæ) during life, but obvious sclerotic changes in the cord post mortem.

(c) Those in which the cord symptoms precede and overshadow the manifestations of anemia. This most interesting group of cases has been studied especially by James J. Putnam of Boston.-ED.]

More exact clinical knowledge of progressive pernicious anemia has led us to give special consideration to the changes in the eye, particularly to the RETINAL HEMORRIIAGES and their consequences. It cannot be denied that these changes are usually found in progressive pernicious anemia, but isolated cases have been met with in which the eyes were quite normal; and besides, in undoubted cases of simple anemia as already mentioned, profuse retinal hemorrhages may occur. Consequently this symptom may be considered as a sign of the degree, and not of the variety, of the anemia.

Disorders of the sense of hearing and of smell are of slight importance and exceedingly uncommon; but these may, under some circumstances, proceed to complete suppression of these functions.

The POST MORTEM FINDINGS in progressive pernicious anemia are of especial interest because in some respects they give important information regarding the nature of the disease.

Besides the extreme anemia of all the organs and the hemorrhages that are always present, though these vary in amount and localization, sIDEROSIs is always found, that is, an abnormally increased amount of iron in the internal organs, especially in the liver, in the spleen, in the bone-marrow, and in the lymph-glands. [Neither the hemorrhage nor the siderosis is invariable, though in the majority of cases they are found.-ED.] This increase of iron 
in progressive pernicious anemia may be considered a proof of the invariably concomitant increased destruction of red corpuscles.

FATTY DEGENeration, the clinical consequences of which have already been alluded to, is found quite often in the muscles of the extremities, of the trunk, and conspicuously in the muscles of the eye. Almost always we find the fatty degeneration of the heart muscle which is such a well-known characteristic of the disease. The fatty degeneration of the arterioles and capillaries is interesting because it occasions hemorrhages in the internal organs.

Attention must be devoted to the DIGESTIVE TRACT, because the achylia gastrica sometimes present leads us to expect definite anatomical changes. In fact a more or less markcd atrophy of the gastric and intestinal glands has been found repeatedly, yet the pathologico-anatomical changes at the autopsy by no means always coincide with the symptoms during life; quite marked degenerative changes have been found in cases where the functions of the stomach and intestines had apparently been perfectly normal, while, on the other hand, in cases in which achylia gastrica undoubtedly had been present no anatomical changes were found. The relations of the gastro-intestinal atrophy to progressive pernicious anemia have not as yet been sufficiently demonstrated. It must be remembered that only comparatively few cases of anemia, and those not always the most severe, show these changes; and furthermore, that in a series of other changes nowise related to progressive pernicious anemia, atrophy of the gastro-intestinal glands has been found. The supposition is reasonable that this atrophy and anemia have no causal relation to cach other, but that they represent the effects of a common cause.

A few instances of progressive pernicious anemia have been reported in which carcinoma of the stomach, in extent about the size of a hazel-nut, and without ulceration or metastases, has been found. The small size of these tumors prevents our recognizing in them the cause of the anemia; but if one is not content to call it "coincidence" the supposition that progressive pernicious anemia has prepared the way for the formation of the tumors is not altogether unreasonable. Of course, the cases in which advanced carcinosis is found combined with progressive pernicions anemia must be judged quite differently; here it is probable that the carcinoma is the cause of the anemia.

On account of the symptoms suggesting tabes and other diseases the CENTRAL NERVOUS SYSTEM has been made the subject of especially thorough researches. Besides the hemorrhages which are most apt to occur in the brain and spinal cord, we find focal and also diffuse degenerations of the nerve fibers in the white substance of the spinal cord, with consecutive proliferation of the connective tissue. These changes, which occur particularly in the posterior columns, are without doubt the cause of the severe clinical symptoms.

The changes in the BONE-MARRow, especially those revealed by the microscopical examination of the organ, are the most important of all, because we find in them convineing proof of the necessity of classifying anemic conditions according to the mode of blood-regeneration. If we consider only the macroscopical evidences, the classification of anemia appears impossible; for in almost all cases we find a transformation of the yellow fat marrow into red 
lymphoid marrow, these changes including sometimes smaller, sometimes larger, portions of the bone-marrow. If, in a case of simple anemia, we were to examine such a portion microscopically, an exact picture of the normal lymphoid marrow would be seen, such as is always found in the ribs, the vertebræ, the sternum, and is besides characterized by the presence of white cells (which we do not take into consideration), and by red blood-corpuscles of the same size as the erythrocytes of normal blood, some without, some with, nuclei. But the picture we obtain in cases of progressive pernicious anemia of all, or of many, of these transformed red bone-marrow regions is entirely different. The nucleated, as well as the non-nucleated, red blood-corpuscles are of such a size, and contain such a rich quantity of hemoglobin, as is never observed in the bone-marrow of the normal adult but only in the embryo. These differences seem to be so essential as to necessitate a special classification of the anemias, even if thry did not bear out what we should expect from the clinical symptoms and the condition of the blood.

In progressive pernicious anemia the whole fat marrow very rarely degenerates into lymphoid megaloblastic marrow, and this is a factor which enables us the better to comprehend certain variations in the blood findings, and is of the greatest importance in practical diagnosis. Often only a portion of the lymphoid marrow shows a megaloblastic structure, while the other regions are lympho-normoblastic. Moreover, only a part of the fat marrow is diseased in most cases. Of course, the number of megaloblasts and megalocytes contained in the circulating blood depends upon the size of the megaloblastic portion of the bone-marrow, and other temporary variations in the composition of the blood may be thus explained.

It is our purpose to discuss in this article only points of practical importance. But we must not forget that a series of questions most intimately related to the changes in the bone-marrow are still awaiting solution. For bothriocephalus anemia, the correct explanation has probably been given, and most authors of the present day maintain the view that the toxin of the bothriocephalus causes the specific megaloblastic changes of the bone-marrow. This gives us a hint as to the etiology of other types of progressive pernicious anemia, and inclines us to the general supposition of a specific toxic influence. It is more difficult to answer the question as to what particular circumstances transform a simple anemia into the megaloblastic form, an occurrence which must certainly be considered infrequent considering the prevalence of simple anemia. At present, the toxin hypothesis does not sufficiently explain the extraordinarily rare cases in which progressive pernicious anemia develops as the consequence of a simple posthemorrhagic anemia.

But it cannot be disputed that progressive pernicious anemia may develop from a simple anemia, and this confirms the view that there must be transitional stages in which it is exceedingly difficult, and often even impossible, to determine to which class of anemias a given case belongs. Still, we meet with such transitions and intermediate stages within the whole domain of pathology, in fact in all departments of natural science; they must not, therefore, be an obstacle to the differentiation of the fully developed.forms, nor are they proofs of the non-existence of special types of the disease. 
Therefore, in spite of many uncertainties, we are warranted in stating that the condition which most distinctively stamps progressive pernicious anemia may be described as follows: Progressive pernicious anemia is NOT AN EXTREMELY MARKED DEGREE OF ANEMIA, BUT A DISTINCTIVE TYPE OF THE DISEASE CHARAOTERIZED BY A PECULIAR MODE OF BLOOD-FORMATION.

\section{THE COURSE OF THE DISEASE}

The course of progressive pernicious anemia presents some remarkable and characteristic features, which are more pronounced than in any other disease. Only occasionally does the disease run a continuously progressive and fatal course; usually the morbid condition is relieved once or several times by a state lasting for weeks or months that may simulate almost perfect health. These "remissions" often follow stages of the disease so severe that they seem to be the immediate precursors of death; during extreme prostration, a change suddenly sets in-spontaneously or under the influence of medication-a change that within a few weeks often leads to apparently perfect recovery. Remissions such as these, as many as five or more, have repeatedly been noticed in the same individual.

The duration of the disease varies from a few weeks to five or ten years; but by far the majority of patients succumb in a year or a year and a half after the symptoms have distinctly developed.

The prognosis of the disease is exceedingly grave; the so-called recoveries have probably been only the beginning of the remissions, which delay, but cannot avert, the fatal issue. According to our present knowledge, the disease must be considered incurable and fatal. [In the vast majority of cases, including most of those published as "cures," death follows within three years, but there are a few well-authenticated cases still alive at the end of ten years. Perhaps 1 in 100 may recover.-ED.]

Bothriocephalus anemia is a striking exception to this rule. This form of the affection is often entirely cured by the expulsion of the worm, even though symptoms of progressive pernicious anemia have already developed to the fullest extent, and the anemia has attained a high degree. We recognize from this that the mere presence in the blood of megaloblasts and megalocytes-i. e., the megaloblastic degeneration of the bone-marrow-does not in itself make the prognosis absolutely unfavorable, but that even with this the disease may lose its progressive character if we can succeed in removing its cause from the organism. So long as we are ignorant of the origin of progressive pernicious anemia, so long are the prospects of a cure decidedly unfavorable.

The most important point in the diagnosis is the differentiation between simple and progressive pernicious anemia. In well-marked cases of the latter, the general clinical picture enables us to make the diagnosis. The peculiar pallor, the extreme weakness, the tired expression, the marked development
of heart symptoms, the retinal hemorrhages, the striking contrast between the asthenia and the well-preserved adipose tissue-all these combine to form a picture that clearly stamps the disease after a merely superficial examination. 
If the patient is under observation for a longer time, the appearance of a remission is a remarkable aid to the diagnosis. Even in such characteristic cases, besides the clinical examination, we must not omit an examination of the blood as a control test; if the general clinical picture of the disease is not perfectly developed, blood examination is even more essential.

If we find in the blood undoubted megaloblasts besides genuine megalocytes, the diagnosis of progressive pernicious anemia is established beyond doubt. [This statement is certainly too strong. Undoubted megaloblasts with a majority of normoblasts occur in some perfectly curable post-malarial anemias. It is the predominance, not the presence, of megaloblasts and megalocytes that is most significant.-ED.] If we do not find them it is more difficult to reach a conclusion. Whoever is careful to bear in mind the above-mentioned changes in the bone-marrow will exclude pernicious anemia in doubtful cases only after repeated examinations of the blood have shown it to be entirely free from these characteristic elements.

We must always remember that the megaloblasts usually pass only in small numbers from the bone-marrow into the blood. Hence we must examine thoroughly several technically perfect preparations before we can positively say that this variety of cell is absent. If the blood is of a pronounced megalocytic character, then the diagnosis of progressive pernicious anemia may be made even though megaloblasts are absent, because the appearance in the blood of large numbers of megalocytes cannot occur without a previous megaloblastic transformation of the bone-marrow.

Another diagnostic difficulty is the fact that there are undoubtedly cell forms which morphologically represent a transitional stage between normoblasts and megaloblasts. If these are sparse, they may be left entirely out of consideration; if they appear in great numbers, they are indicative of progressive pernicious anemia.

To determine the cause of progressive pernicious anemia, an examination of the feces for tape-worm ova is necessarily important; the presence of the bothriocephalus ova or links will aid materially in the prognosis and treatment. [Uncinaria eggs should also be looked for, though the type of anemia produced by uncinariasis is usually somewhat different from that described here.-E.D.]

From the standpoint of differential diagnosis we must bear in mind endocarditis, carcinoma of the gastro-intestinal tract, tabes dorsalis, and Addison's disease, and, during the comatose terminal stages of the malady, other affections in the course of which severe disturbances of consciousness occur. In these cases, the verdict depends mainly upon the blood examination.

\section{THERAPY}

Treatment offers hope of success only in the bothriocephalus anemia. After the anthelmintic (extr. filix mas) has produced its effect, if the anemia is not too far advanced a definite cure will shortly follow. These results encourage us to try the filix mas even in cases in which the bothriocephalus has not been found. 
Marked improvement and even apparent cure has been repeatedly noticed after the stomach and intestines had been thoroughly cleansed by laxatives and irrigations. In such cases we are warranted in the interpretation of the progressive pernicious anemia as an autointoxication from the gastro-intestinal tract.

Organotherapy, the treatment of the disease with bone-marrow, has thus far given no results.

On the contrary, some very surprising, though not always accountable, results have been attained by the transfusion of small quantities $(40-100$ c.c.) of defibrinated human blood. In several cases transfusion has been followed immediately by the onset of a complete remission of the discase and life was prolonged for months, though when transfusion was again performed, after a relapse, it was ineffectual. But if the patient's life is endangered, and if coma has already set in, this operation is our last hope and resource, and should at least be attempted.

The most important and successful treatment is by arsenic. To this remedy we are indebted for an extraordinarily large number of apparent cures, and by its aid in some cases we succeed in bringing about remissions and in delaying the imminent outbreak of a relapse.

Nevertheless there can be no lasting cure, even with the aid of arsenic.

This remedy is administered according to the generally observed rules: Small doses in the beginning and gradually increased, long-continued use, and a gradual decrease. The doses should be adapted to the individual susceptibility and reaction; as a rule, even during the acme of the treatment, we find doses smaller than the maximal to be sufficient. During the remission, when no symptoms are present, it is well to give the remedy from time to time for several wecks. The best indication for a renewal of the treatment is afforded by regular examinations of the blood, which may give warning of a new outbreak before the clinical symptoms suggest it.

An unfortunate idiosyncrasy for arsenic not infrequently develops in the course of trcatment, and may prove disastrous for the patient, inasmuch as the proposed substitutes for this remedy have not yet borne the test of experience.

As for general treatment, rest and comfort are of paramount importance. The diet must be adapted to the individual susceptibility of the stomach and intestine, as wcll as to the sometimes insurmountable repugnance of the patient for meat. In view of the debility of the patient, hydrotherapentic treatment is not advisable, but sponge baths may be given without danger. General massage is preferable to bathing. A change of climate may be indicated during the remissions, and a sojourn in a mountainous region of moderate altitude is more likely to prove beneficial than any other climatic change.

\section{IITERATURE}

The earlier literature of this subject, in all its details, is given by Ehrlich-Lazarus, "Anemia," "Nothnagel's Handbuch," viii, Wien, 1900. More comprehensive works which have appeared are: Strauss and Rohnstein, "The Composition of the Blood in Different Anemias," Berlin, 1901; Schaumann, "Pernicious Anemia in the Light of the Modern Toxin Hypothesis," Volkmann's Clinical Lectures, New Series, No. 287. 


\section{CHLOROSIS}

\section{By E. Grawitz, Charlottenburg (Berlin)}

Among the numerous cases of anemia constantly coming under observation in the hospital, one group stands out prominently, which, in regard to the origin as well as the symptomatology, and the entire course of the affection, occupies a distinctive position, and, since the time of Hippocrates, has been considered to indicate an impoverished condition of the blood. [As Stengel has recently remarked, there scem to be fewer cases diagnosticated as chlorosis in the clinics of the larger American cities than was the case some years ago. Whether there are, in fact, fewer cases or whether better technic makes us reject many that were supposedly, but not actually, chlorotic, I do not know.-ED:]

\section{SYMPTOMS}

This group consists almost exclusively of young girls and young women, in whom the anemia betrays itself by a conspicuous pallor of the skin and the mucous membrane. [I have never seen in adolescent boys any cases of anemia at all resembling those which in girls we call chlorosis, and those reported in literature are mostly a good many years back and vaguely reported.-ED.] In many cases there is also a decided yellowish tinge which may sometimes verge upon green, and, as frequently happens in pathology, from this by no means significant nor even very frequent green color, a term has originated which has long encompassed the entire symptom-complex, and has become current in the language of all countries; namely, the designation "chlorosis"

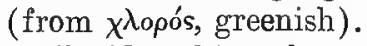

Besides this pale or pale yellow discoloration of the skin, there are in many eases dark gray rings around the eyes; it is especially worthy of note that there is no sign of loss of the subcutaneous adipose tissue; on the contrary, many of these patients have a plentiful layer of fat.

It may be seen, therefore, that this form of anemia is not due to a general cachexia, but occurs in well-nourished persons, a fact which is confirmed by an examination of other organs.

Besides the peculiar color of the skin, the majority of chlorotics show a more or less pronounced edematous infiltration of this tissue, so that, for instance, the lower eyelids often seem swollen, and imprints of the examining fingers on the back and at the ankles of the patient are distinctly persistent.

These signs lend to the patient a peculiar doughy appearance, which, at 320 
the first glance, vividly recalls that of persons affected with acute nephritis, yet this, of course, is not due to the yellowish discoloration.

The circulatory apparatus presents numerous and striking symptoms. In the heart there is almost always an increase of the area of dulness, to the right and to the left. The hand may feel a distinct systolic thrill, especially over the pulmonary artery, and on auscultation systolic murmurs, which commonly have a peculiar blowing character, are very distinctly heard.

These cardiac murmurs have been recognized for a long time, and, in spite of careful study, are still subjects of dispute. The murmurs are usually loudest over the pulmonary artery, and an attempt has been made to explain them by the fact that there is a relaxation of the heart muscle owing to insufficiency of the blood-supply, that the papillary muscles do not contract with sufficient power, that, therefore, during systole there is a relative insufficiency of the auriculoventricular valves which causes a regurgitation of the blood to the auricles during systole in the same manner as in cases of organic mitral insufficiency.

This much is certain; that these systolic murmurs are intimately related with the anemic condition of the blood, that they appear when the disease develops, and disappear when the general clinical picture shows improvement.

Therefore, they have been properly called "anemic murmurs," in contradistinction to heart murmurs produced by valvular lesions which are designated "organic murmurs," and an exact knowledge of these conditions is the more important as it is frequently the duty of the physician to decide whether changes and murmurs in the heart, especially in young persons, are to be attributed to a valvular lesion or whether they are only accompanying symptoms of anemia. [The term, "anemic" or "hemic" murmurs, is not well chosen, for the majority of such murmurs occur not in anemic cases but in fevers, in neurotic or excited conditions, in growing children and after any violent exertion (boat-races, etc.). On the other hand, cases of marked anemia without any murmurs are not very uncommon. I prefer the term "functional murmur."-ED.] As an instance of the importance of this differential-diagnostic distinction, we may mention the requirements for enlistment in military service, in which this question is of momentous importance.

As a criterion in practice the following important points favor anemic murmurs:

1. The existence of distinct anemia.

2. The simultaneous presence of the systolic murmur over all the valves.

3. Especial loudness in the pulmonary area.

4. Absence of that accentuation of the pulmonic second sound which constantly accompanies insufficiency of the mitral valve.

It must, however, be stated with emphasis that simple as this appears in theory it is often difficult in practice to arrive at a decision; for, in the first place, training is necessary to make these examinations correctly, and, further, prolonged observation of the patient is the only decisive criterion, for, as has been stated, anemic murmurs are transitory while organic heart murmurs are permanent.

Neither does the character of the pulse give reliable information in regard 
to this question. The pulse of anemics is for the most part simply accelerated, of varying strength, and often slightly dicrotic; in some cases a crural murmur has been noted. Obviously, then, the pulse of chlorotics presents no characteristic changes.

The acceleration of the heart beat and of the pulse is explained by a diminution of hemoglobin, as the blood, in order to accomplish the required oxidation of the whole organism, must circulate more rapidly than when it contains a normal amount of hemoglobin. As a result of this, acceleration of the heart's action is one of the common symptoms of chlorosis as well as of other forms of anemia.

Subjectively, the patients are often vividly conscious of this acceleration, so that a troublesome sensation of cardiac palpitation develops, a point which has been noted in the earliest literature as a prominent symptom of chlorosis. This sensation becomes aggravated upon excrtion as when they attend to their housework, ascend stairs, etc. As a result they readily become short of breath, and the rapid beating of the heart which appears after these slight exertions often persists afterward as a subjective but very disagreeable symptom.

In these cases it is surprising to note that some chlorotics, who during the day become dyspneic on the slightest exertion, are able in the evening to attend $a$ ball and indulge most immoderately in dancing, and even to continue this exercise for hours without any diffculty in breathing approaching that which occurs during the day, when, for instance, they ascend only one flight of stairs. Of course, upon the following morning, a particularly great prostration is discernible.

Similar to the murmurs in the heart, in such patients "venous murmurs" have for a long time been recognized which are designated "nun's murmurs" or also "bruit de diable." These are best auscultated over the sterno-clavicular articulation; care must, however, be exercised that the neck of the person is not turned too much to one side, for, in this case, the murmur may also be noted in normal subjects. This murmur is due to the circumstance that the jugular vein is held by tense bands situated behind the articulation just mentioned, so that in this region it cannot expand and contract with facility, as it does, for instance, higher up in the neck. Now, since in anemics the lumen of the vein is lessened-being less full-when the blood enters from the narrowed portions into the easily dilatable parts previously mentioned, differences in the blood current arise which give rise to these murmurs. This is the usual explanation of these conspicuous venous murmurs, but it is unsatisfactory, inasmuch as in other anemic conditions in which the blood reveals much greater deterioration than in chlorosis-therefore a more favorable condition for the production of the murmurs-no adventitious bruits are discernible. [It should be mentioned at this point that violent pulsations of the peripheral arteries, especially the carotids, are noticeable and sometimes troublesome in cases of severe anemia of any type. The arteries flap and jump so violently as to remind one of the conditions seen in aortic regurgitation.

Dilatation of the heart is not uncommon and may be demonstrated by radioscopy or by percussion. A beautiful picture of a heart dilated in the course of a case of chlorosis is included in the $\nabla$. Ziemssen-Rieder Atlas.-ED.] 
The respiratory apparatus in chlorotics shows few deviations from the normal. A symptom noticeable in many of the patients is an accelerated respiration rate, which is to be explained, like the acceleration of the heart's action, by a relative deficiency of the blood in oxygen. This, however, is not characteristic of chlorosis, but is also met with in other affections accompanied by deficiency in hemoglobin.

Much more numerous are the symptoms on the part of the digestive apparatus. First of all, as an almost invariable symptom, there is found anorexia and repugnance for certain kinds of food, for instance, meat; and, on the other hand, and this is a characteristic symptom of the disease, a morbid desire for food diffcult to digest or wholly indigestible. Thus, many of these patients have an especial liking for sour foods, vinegar, pickled products, etc., and furthermore, for lime, chalk, slate pencils and the like; longings which remind one forcibly of similar cravings in pregnancy.

Atony of the muscular wall of the stomach is often found, which not infrequently leads to dilatation and displacements of this organ. Still, I cannot coincide in the opinion of Meinert, who considers gastroptosis, that is, the pathological descent of the fundus of the stomach, a constant symptom of chlorosis, and even makes it responsible for the origin of the whole disease.

As a matter of fact, there is often found in young girls a displacement of the stomach from its normal limits, the result of tight lacing and the wearing of corsets; and, as general weakness of the muscles exists on account of the anemia, conditions are very favorable for the development of gastroptosis and dilatation in consequence of the stagnation of food and tight lacing.

In many cases the secretory activity of the stomach is quite normal; in others an excessive production of hydrochloric acid is met with; and in still others acidity is decreased. These, however, are facts that must be ascertained by direct examination of the gastric contents, and should never be guessed at from the subjective complaints, as, for instance, from the inclination to eat chalk.

The activity of the intestines is usually disturbed, and constipation is one of the commonest symptoms; indeed, as will be seen later, theories have been developed which ascribe the origin of the whole discase to this phenomenon.

Concerning the metabolism of chlorotics, exact researches have been made, especially by v. Noorden and his pupils, from which it is ascertained that in these patients no especially increased waste of albumin occurs, that the absorption of oxygen is within normal limits, and that the excretion of urea shows no noteworthy change.

The body temperature is neither increased nor diminished. The appearance of fever is probably to be explained for the most part by the presence of complications. Slight transitory rises of temperature without demonstrable organic changes occur in some cases; but we must consider the close relationship between chlorosis and hysteria- a fact which we shall consider frequently later on-in which fever of like character also is noted, and has thus far not been satisfactorily explained.

In chlorotics the sexual functions are disturbed in various ways; and here 
again the attempt has been made to refer the development of the disease to these disturbances.

Menstruation in chlorotic girls is delayed, or if it has already appeared it becomes scanty or ceases when the chlorosis develops. In contrast to this, other chlorotics suffer from remarkably profuse menstruation, which sometimes may be normal for a few months; at the same time, there is considerable suffering at the onset, such as headache, general nausea, indisposition, pain in the abdomen, etc.

In opposition, however, to the opinion that the menstrual anomalies are the actual cause of the disease, it must be maintained that many chlorotics menstruate in a perfectly normal manner until the onset of the disease, so that, logically, these anomalies which develop during the course of the chlorosis must undoubtedly be considered as secondary conditions. Leukorrhea is also very frequent in chlorotics.

The symptoms referable to the nervous system are numerous, and here we must differentiate two groups, one of which may be easily recognized as solely a consequence of the anemic condition of the blood, while the other has an independent character and is specially significant. To the first group belong vertigo, specks before the eyes, syncope, which can be easily explained as due to a deficient supply of blood to the brain, all the more so as the symptoms ameliorate when the patients are kept in a recumbent posture, so that the access of blood to the brain is facilitated, while they become most prominent as soon as the patients assume the erect position or attempt to walk. These symptoms of anemia of the brain, resulting from unsatisfactory circulation of the blood-at the same time poor in oxygen-become maniisest in a similar manner also in other forms of anemia.

In the second group (nervous symptoms independent of anemia) belong especially psychical anomalies, which manifest themselves by a change in the disposition, and a dislike for mental and physical exertion; on the other hand, when it is specially interesting to the patient, she can endure a considerable amount of exertion, as, for instance, in dancing, as has been previously mentioned. [Insomnia at night and a heavy sleepy condition in the daytime are often seen in chlorosis, but some patients sleep excessively.-ED.]

As regards sensibility, we often find anesthesia of different parts of the skin as well as of the mucous membranes, for example, of the pharynx; in other cases there is hyperesthesia, and especially neuralgic phenomena in the distribution of different nerves. Intercostal neuralgia is quite common, and, if it occur on the left side, is often incorrectly referred to the spleen as the so-called "splenalgia," of which the patients complain.

Motor disturbances, also, are often accompanying symptoms. Paresis of the various muscles of the larynx and also of those of the extremities is often found.

The condition of the reflexes varies; they are frequently normal, sometimes diminished, in other cases exaggerated.

Moreover, disturbances of the sympathetic system 'manifesting themselves by an abnormal innervation of the blood-vessels are very important. Thus we may observe in these patients an abnormal facility for blushing followed 
by a rapidly succeeding pallor. The appearance of erythematous areas upon the trunk may also be grouped with these.

Disturbances of the organs of special sense, particularly often that of vision, manifesting itself by the fact that the patients soon tire in reading, sewing, etc., in most cases are caused by weakness of the eye-muscles and of the mechanism of accommodation.

Besides this, in many instances, serous infiltrations of the retina are found, which then appears strikingly pale, and, especially about the papilla, lacks distinct contour, and looks as if loosened.

Now and then attacks of transitory blindness or partial deficiencies in vision are noticeable, which, although they greatly alarm the patient, offer a good prognosis and disappear completely.

Anomalies of taste have already been mentioned. We also find abnormalities of smell, the same as in hysteria.

\section{THE BLOOD}

The BLOOD of CHLORotics has, for a very long time, been the subject of thorough and extensive researches, and even with the naked eye the watery appearance and the marked pallor of the blood, which flows freely from the slightest puncture, is conspicuous. If blood of this kind is diluted with a definite quantity of water, and this mixture is compared with a similar dilution of a like quantity of normal blood, the difference in color is very decided; this is also shown by all the various instruments which are constructed for the same purpose, i. e., for comparison, by means of transmitted light, of the color of the blood with a standard color, the so-called hemoglobinometer, so as to determine a decided reduction of the coloring matter of the blood.

The exact estimation of the amount of hemoglobin from the amount of iron contained in the blood also shows a considerable reduction of the iron and hemoglobin values, so that there can be no doubt that in chlorosis there is a considerable reduction of the hemoglobin percentage.

In opposition to this, it is of special interest that the count of red blood cells, which in the course of time has been made in many cases and from different standpoints, shows in the majority of cases of uncomplicated chlorosis no considerable reduction in the number of these cells, which are the carriers of the hemoglobin, and it can be stated positively to-day that a reduction in the number of these cells, an "oligocythemia," is not present in chlorosis. The normal number of red corpuscles in a cubic millimeter of woman's blood is about 4,500,000; in chlorotics we find an average of from $3,800,000$ to $4,000,000$, and in some cases even an almost normal number; while a marked reduction in the number of red blood cells is found only in such chlorotics as are debilitated in consequence of a prolonged duration of the disease, insufficient nutrition and complications of some kind.

These two important facts, that, on the one hand, the amount of hemoglobin is always considerably reduced, sometimes to a third of the normal, while the number of red blood cells is changed but very little, allow us to conclude positively, that some individual red blood cells must be poorer in 
hemoglobin. Therefore, we designate this peculiar form of anemia in chlorosis as an "oligochromemia," that is, a deficiency in the. hemoglobin of the blood. This symptom forms one of the most important characteristics of the blood of chlorotics, and is only seldom found so pronounced in the other anemias. [In my experience "chlorotic blood"-excessively low color-index -is not at all uncommon in other forms of anemia, e. g., in "splenic anemia," malarial anemia, cancerous anemia, post-hemorrhagic anemia, etc. Chlorotic blood is in no way peculiar.-ED.]

If attention be directed to the cells of the blood themselves, we find the view corroborated, even on examination of the unstained preparation; each individual red cell has sustained a considerable loss in its hemoglobin. The majority of these cells appear strikingly pale, and absorb stains very imperfectly, a sign that the quantity of coloring hemoglobin in the cells has been considerably reduced.

Morphologically we find striking differences in the size of the red blood cells. Many of them appear remarkably large (macrocytes) and at the same time quite pale, without a distinctly pronounced central umbilication-as though swollen. These large, pale discs have been designated, quite appropriately, "chlorotic" blood-corpuscles. Still other cells, on the contrary, are distinguished by their diminutive size (microcytes). [Finnish observers have proved that the average diameter of the red corpuscles in chlorosis is often diminished. The cells grow smaller as the disease is aggravated, and return gradually to their normal size in convalescence.-ED.]

The stained cells show very distinctly the anomaly designated polychromatophilia; that is, they partially take up the basic stains instead of acid stains; and, therefore, the stained preparation has a peculiar appearance characterized by the fact that many large cells, very faintly stained erythrocytes, alternate with many small intensely stained cells, and with cells showing polychromatophilia.

On the other hand, genuine phenomena of degeneration, such as poitilocytosis and granular "degeneration" (the presence in the red cells of numerous, very small, basophilic granules), do not belong to the blood picture of chlorosis, but occur only in very far advanced cases of this kind when decided disturbances of nutrition are present, or complications which lead to cachexia; as, for instance, habitual constipation with increased intestinal decomposition.

In the same manner, nucleated red blood cells are not, as a rule, present in chlorosis, but occur only in the extreme grades of the disease when intense irritation of the hematopoietic organs, i. e., of the bone-marrow, has taken place.

The condition of the leutrocytes in chlorosis is not uniform. It may be stated positively that these cells do not show any characteristic change in this disease. Very often their number is increased; especially during those stages of the disease in which regenerative processes in blood production are manifested by other signs as well as, for instance, by the appearance of nucleated erythrocytes.

In the experience of the author, no new cell forms appear in this variety of leukocytosis; but we have rather to deal with an increase in number of 
the normal forms of leukocytes, and by preference with an increase in the number of polynuclear neutrophilic forms and of the lymphocytes, while ahnormal forms, as, for instance, the myelocytes (large leukocytes, with one nucleus and with neutrophilic granules) belong to the exceptions.

To sum up, we may say that the leukocytes do not play a rôle of any importance in the blood changes of chlorosis.

The third formed constituents of the blood, the so-called "blood plaques," - attract especial attention in chlorosis because here they appear in remarkably increased numbers, so that large groups of these small colorless structures may often be seen in each field of the fresh preparation.

We are still uncertain as to their significance. Some authors, for instance, the pupils of Arnold, consider them as a segmentation product of the red blood cells, while others believe them to be fragments of disintegrated nuclear substance.

Since these small bodies, as I have repeatedly noted in many examinations, may be partially stained with basic stains, especially well with methyleneblue, and partially with certain nuclear stains, for instance, with Ziemann's [or Leishman's] eosin methylene-blue mixture, I believe that, at least in the majority of cases, they may be considered fragments of nuclear substance, and at best only a small number of them may have originated from red blood cells.

In any case, our knowledge concerning these small structures is so indefinite that from their appearance in increased numbers in the circulating blood we cannot draw any conclusion, either general or special, in regard to chlorosis.

In uncomplicated cases of chlorosis, the blood serum shows, as a rule, no noteworthy reduction in the percentage of albumin. The specific gravity of the serum is on the average 1.028, which corresponds perfectly with the composition of normal serum, and the total solids appear to be normal in amount, averaging about 10 per cent. [The greatest menace to the life of the chlorotic is thrombosis of the cerebral sinuses. Venous thrombosis of the extremities is also much commoner than in any other form of anemia. As a rule, thrombosis occurs in the severest and most chronic cases. It is very possibly connected with the excess of platelets in the blood.-Ev.]

Taking into consideration all these changes, it follows that the blood in chlorosis is of a peculiar composition, and characterized by the fact that the normal number of blood cells is only very slightly reduced while the amount of hemoglobin of the individual cell is considerably diminished, and thus a peculiar relationship between the fuid constituents of the blood and the blood cells develops, which is surprisingly manifest if we puncture a vein in the arm, permit a few cubic centimeters of blood to flow into a tube, and centrifugate it before it coagulates. In doing this we notice (as also when we allow the blood to coagulate spontaneously or to form a sediment) that the mass of the red cells, in toto, is extraordinarily reduced, often to only 95 per cent. of the whole blood mass, compared with 75 per cent. of the blood fluid, while in normal blood both components are present in about equal proportions.

It is obvious from the above mentioned examinations that the blood cells, although present in about normal numbers, are markedly deficient in hemoglobin, while the chief bulk of the blood is formed by plasma, so that this 
condition of the blood has been properly designated "polyplasmia," that is, an increase of the total amount of plasma in contrast with other changes of the blood, such as are found, for instance, in chronic circulatory disturbances in consequence of heart or lung diseases, in which the volume of the blood cells exceeds by far that of the plasma, and in which, therefore, a condition of "oligoplasmia" is present. [The researches of Haldane and Smith regarding the total volume of the blood in chlorosis as measured by capacity to absorb CO gas point to the same polyplasmia referred to by Grawitz.-En.]

All these changes must be carefully considered, as they point the way to a more thorough understanding of the pathologic process in chlorosis, as we shall see later. However, the circumstances in all cases are by no means so simple and so distinctly pronounced that we are able to make the diagnosis of chlorosis from the blood changes alone. We must emphasize explicitly that in general practice the blood examination can only confirm the diagnosis; the disease itself can only be diagnosticated by taking into consideration all the previously described symptoms.

In conclusion let us review the entire symptomatology of chlorosis, and compare it with that of simple anemia, for example-to choose the simplest form-post-hemorrhagic anemia, such as develops after repeated hemorrhages from the stomach or the uterus.

In simple anemia we find general pallor, extension of the cardiac dulness, systolic murmurs and also venous murmurs as in chlorotics; in addition the signs of anemia of the brain are found, specks before the eyes, vertigo and syncope. On the other hand, if there are no complications, the entire group of nervous symptoms are lacking in simple anemia, especially anomalies in the psychical sphere and of the appetite; in spite of the increased frequency of the pulse, as the result of anemia, the troublesome cardiac palpitations are absent, as are also the symptoms referable to the sensory sphere and the vasomotor tissue which give to chlorotics their peculiar puffy appearance.

The changes in the blood, too, are essentially different, for in simple anemia following hemorrhage we always find a considerable reduction in the number of cells, while the amount of hemoglobin in the individual cell is only slightly or not at all decreased. [This is true immediately after the bleeding. Later, the amount of hemoglobin becomes relatively diminished, and the blood may simulate closely the conditions found in chlorosis.-ED.] On the other hand, the serum in these anemias always shows a more or less considerable diminution in the percentage of albumin, so that these two main factors, the blood cells and the blood serum, are entirely different from those of chlorosis. Moreover, in anemias following severe hemorrhage we find nucleated red corpuscles and an increase in the number of leukocytes. [Post-hemorrhagic leukocytosis is a curiously inconstant phenomenon. As a rule it occurs, not immediately after the hemorrhage, but some days later, and its duration is very variable. Unless frequent examinations are made the wave of increase is often missed altogether.

The same is true of the "shower" of normoblasts which usually occurs after hemorrhage, but may be very brief.-ED.] 


\section{PATHOLOGICAL ANATOMY}

The anatomical lesions of this discase have by no means been determined with certainty. The great difficulty in making positive statements regarding organic changes in chlorosis lies in the fact that in uncomplicated chlorosis fatal cases are exceedingly rare, and we are consequently dependent upon the scant observations obtained when chlorotics have perished from an intercurrent disease, and in these cases we are compelled to decide which among the organic changes are to be referred to chlorosis and which are to be attributed to the affection causing the fatal issue.

In these cases, changes in the hematopoietic apparatus of chlorotics, that is, in the marrow of the long, tubular bones, which might be held responsible for the origin of the blood changes, are as yet not understood. The author himself examined thoroughly the tibia of two chlorotic girls, and was unable to find any disease changes, macroscopically or microscopically, in the distribution of the red and fat marrows.

Best known are the changes in the circulatory apparatus, presented by Virchow as the anatomical foundation of chlorosis, and which for a long time were interpreted in this sense by clinicians. According to Virchow, we are dealing in these cases with a defective development of the heart and of the large arterial vessels, with a hypoplasia or dwarfed state in which the changes in the vessels become manifest by abnormal narrowing, thinness, and elasticity of the arteries, and also by irregular ramifications of the vessels.

This hypoplasia of the heart and vascular system, as Virchow teaches, favors the assumption of a predisposition, either congenital or acquired in early youth, which, as a rule, causes actual disturbances having pathological importance only at puberty, and that chlorosis itself, though incurable, may, by proper treatment, particularly by dietetic measures, be kept in a state of latency.

These hypoplasias of the heart and valvular system subsequently became matters of great importance, and the narrow, thin-walled and enormously elastic aorta has briefly been designated "aorta chlorotica," without at all explaining how a diminished amount of hemoglobin in the blood can result from narrowness of the vascular system, secondly, why the chlorotic symptom-complex can in many cases be so readily and permanently overcome, and, finally, the important question why men who, according to Virchow, also frequently suffer from hypoplasia of the heart and of the arteries, are so rarely attacked by chlorosis. In regard to this latter point particularly, i. e., the rare occurrence of chlorosis in men, it is remarkable that Virchow disregarded this in his hypothesis, in spite of frequent anatomically recognizable changes in the vascular system.

As a matter of fact, in many chlorotics, hypoplasia of the heart and of the vessels certainly plays an important rôle, but only in so far as it represents an irreparable and, therefore, very unfavorable complication of chlorasis; for in this condition we are dealing with a faulty structure of the vascular system which is acquired in early youth, which cannot of itself lead to chlorotic 
blood changes, i. e., decrease in the amount of hemoglobin, but which in all likelihood brings with it another pathological condition of the blood which we must designate as oligcenia vera, i. e., a decrease in the total mass of the blood.

Such persons with lessened capacity of the heart and of the ressels and probably of a lessened total quantity of blood, may, provided they do not over-exert themselves, appear to be quite healthy; and, as a matter of fact, we are very frequently surprised to find this state of affairs in a well developed form at the autopsy of persons in whom we could not suspect an anomaly of the vascular system.

In itself it is quite unlikely that hypoplasia of the vascular system is capable of producing chlorosis. It can at most be looked upon as a predisposing factor; aside from this it certainly plays an important rôle in those chlorotics who show an abnormal tendency to relapses, characterized by very slight inclination to improvement, so that such cases have also been designated as "habitual" and "persistent" chlorosis.

At the present time it may be maintained with certainty, that the severe anatomical changes of the vascular system are not characteristic, nor are they even the specific anatomical foundation of chlorosis. In our further discussion, we shall have to consider their effect as factors in prognosis and treatment.

Similar hypoplastic changes have also been found in the genital organs, and these changes have also been considered to bear a causal relation to chlorosis. Yet here we may hold the same view as in the hypoplasia of the vascular system, that these anomalies are to be looked upon only as predisposing factors, and that they cannot be regarded as an explanation of the entire symptom-complex, especially of the blood changes.

All other changes, such as degeneration of the heart muscle, parenchymatous inflammation of the lidneys, and other anatomical alterations, are to be considered as secondary. Hence it must be admitted that at the present time a definite anatomical cause of this disease is absolutely unknown.

\section{FREQUENCY OF CHLOROSIS WITH REGARD TO SEX, AGE AND LOCALITY}

It has been noticed for many years that chlorosis affects preferably the female sex. There are, however, undoubtedly cases of genuine chlorosis in young men, all the symptoms of the disease most distinctly presenting themselves, in the blood as well as in the other organs. As Wunderlich has observed, the symptom-complex is noted usually in young men of poor physique and of sedentary occupation-tailor-apprentices, office clerks, etc. [See Editorial Note II on p. 320.-ED.] We do not as yet know whether or not the above mentioned changes in the heart and vascular system are already existent in these cases and predispose to the outbreak of the disease.

The age at which chlorosis appears in the great majority of cases is that of puberty, from the twelfth or fourteenth year to the twentieth. [From eighteen to twenty-three is, in my experience, the period in which the great 
majority of cases occur. Immediately after the establishment of menstruation chlorosis is rare. An interval of five, or even ten, years often follows.-ED.] The disease does appear, however, in young children, or, on the other hand, and this is not infrequent, may occur in the twenties; after the thirtieth year chlorosis is exceedingly rare, either as a primary or recurrent attack.

Concerning the constitution it may be said that young, delicately built girls, weak in muscle, but comparatively fat, are most likely to be affected with chlorosis; on the other hand, the robust constitution of a girl raised in the country, for instance, a scrvant, offers no protection against the appearance of this disease. It must be confessed, however, that when chlorosis appears in girls of the latter type, as a rule it is milder, and can be remedied sooner and more surcly than in those with a delicate constitution.

Whether chlorosis occurs more often in certain localities than in others is difficult to determine, for it is almost impossible to obtain positive statistics of this disease, and especially because many physicians include under the name of chlorosis all varieties of anemia in young girls. It is certain at any rate that the disease is more frequent in large cities than in the country.

\section{PREDISPOSING INFLUENCES}

Many factors may be looked upon as predisposing causes of chlorosis, and a knowledge of these is of the utmost importance for the thorough comprehension of the entire picture of the disease on the one hand, as well as for the treatment on the other hand.

These deleterious predisposing influences are to be found, first, in the domain of general hygiene, and, secondly, among certain organic diseases.

In the first group, i. e., the hygienic influences, we must include errors in physical and mental training, which, especially among the higher classes, are of great social importance.

Here, to cite a very frequent error, we observe a neglect of the physical development of growing girls as compared with that of boys. Out of regard for their dress, they are not permitted to romp freely, but are taken out to walk by the side of a governess; they are not sufficiently in the fresh air, and have no opportunity of enjoying a rapid, powerful and invigorating exercise of the muscles.

When we consider the view of Virchow, previously referred to, that hypoplasia of the heart and of the vascular system is partially acquired in intrauterine life but is specially developed in extra-uterine existence, the thought involuntarily arises that, in many cases, this lack of development of the heart and vessels may be due to the fact that the normal stimulus for such a development of the vascular system as is unquestionably necessary for free movement of the muscle masses in the limbs and trunk is in these children not sufficiently active, and that this faulty development of the circulatory apparatus may, perhaps, be considered a form of inactivity hypoplasia. [If this be true, the apparent diminution in the number of cases of chlorosis presenting themselves at our clinics might be explained by the more active habits of life which are certainly prevalent in America in the present decade.-ED.] 
As already remarked, although the whole complex of symptoms in chlorosis cannot be explained by these anatomical changes, yet they surely form predisposing factors, and moreover, as we shall observe later, chlorosis is undoubtedly more severe in these abnormally organized girls than in those with a normal circulatory system.

Still more injurious is the pernicious habit of allowing young girls to wear tightly laced corsets which compress the lower parts of the thorax, hinder respiration and the circulation of the blood, and unquestionably, besides producing the well-known changes in the liver, lead to alteration of position, and prevent the motility, of the stomach. [That tight lacing has any definite relation to chlorosis is, I think, quite unproved, though many writers refer to it.-ED.]

Other injurious causes are to be found in the nutrition; these consist of disturbances of digestion with a loss of appetite for wholesome food; instead of nourishing the young girl with milk and other easily digested and nutritious food, she is allowed to feed upon dainties.

Among the lower classes, unhygienic conditions of living are frequently at fault; growing up in damp, badly ventilated rooms into which the rays of the sun rarely penetrate, eating insufficiently and of indigestible food, are conditions potent as predisposing causes of chlorosis. [It has still to be shown, I think, that bad hygiene can or does produce any type of anemia. It is natural to expect that anemia would result from such conditions, but I have never seen any good reason to believe that it does.-ED.]

In all classes, premature indulgence in alcohol must be considered; this is often permitted children through mistaken dietetic priuciples, but conduces to the undermining of the health at an early age.

To these faults in training psychical factors are added, such as the premature awakening of sensuality by exciting literature, exaggerated novels and the like, from which ideas of love develop entirely too early and, in many cases, not only exert an unwholesome influence upon the psychical nature, but upon the whole physical condition.

A peculiar predisposition favoring the development of chlorosis may be observed daily in the life of a large city. This is manifested in young servant girls, who, as a rule to-day, come from the country or from small towns to the large cities in quest of higher wages, and often, after a brief residence, suffer from well-developed chlorosis. This contingent of young girls forms a large proportion of our hospital patients, and it is interesting to inquire into their history. Usually we find that these girls, on the average from eighteen to twenty-two years of age, were while at home always well, strong, and able to work, as may also be inferred from their "service books." After arrival in the large eity, a very brief period sometimes elapses before they become ill and show the fully developed symptom-complex of chlorosis.

The author has taken speeial interest in these cases, and after careful investigation has found that two to four weeles are often sufficient to transform $a$ healthy and robust-looking girl into a patient with pronounced symptoms of chiorosis. It is worthy of note that these patients manifest no anomalies of the stomach or intestines except, perhaps, transitory constipation, nor any 
disturbances of the sexual functions, a point which is important for the comprehension of the entire process.

As factors which favor the outbreak of the affection we may mention the changes in the food and in the mode of life, particularly the late going to bed and the hard work (the climbing of many flights of stairs). Other causes which play an important rôle are the hurry of life, psychical exaltation, especially nostalgia, and finally reduced consumption of fresh air.

Certainly, it is undeniable that the mere removal of a previously perfectly healthy young girl from the country to a large city is sufficient to produce the entire symptom-complex of chlorosis, and that the same girl, if sent back to her former country home, will lose every trace of the disease; for this reason the author suggests the name "city chlorosis" for this variety of the affection.

Besides these factors, organic diseases may lead to the development of chlorosis. As an instance, we may mention "gastroptosis" with the digestive disturbances that accompany it. Meinert, especially, has called the attention of the profession to its frequent presence in chlorosis.

Menstrual abnormalities, too, particularly profuse menstruation, certainly predispose to chlorosis, as also probably does an attack of an acute infectious disease.

Reviewing at a glance all these predisposing factors, beginning with the anatomical changes of the circulatory apparatus, then the unhygienic influences, and, finally, actual organic diseases, it is at once apparent that there exists no specific factor nor one single factor which is found as the cause in all cases, but, on the contrary, taking into consideration the great variety of predisposing causes, we have reason to believe that the points of minor resistance that favor the outbreak of this peculiar disease are manifold. The question arises which, in this large number of diversified and detrimental influences, is the connecting link that may satisfactorily explain the genesis and the true nature of the disease.

\section{THE GENESIS OF CHLOROSIS}

The views that have been expressed with regard to the development and the true nature of this disease vary greatly. First, the theory is to be considered which treats of chlorosis as an essential blood disease, in which all organic changes or symptoms on the part of the organs are of a secondary nature, and the blood itself is the primary seat of the disease. This view is no longer tenable on account of the numerous and exact blood examinations that have been made in the last few decades, the results of which have been mentioned above. According to our present knowledge, a specific disease of the blood must of necessity be combined with changes of the blood cells, and, as we have noted, there is absolutely no support for this view, since the principal and most important morphological changes consist in a decrease of the hemoglobin of the blood-corpuscles, while degenerative changes, as well as characteristic pathological changes of the leukocytes, are entirely absent.

The absence of pathological changes of the bone-marrow, as we have already stated, is against the view of a substantive blood disease, and another 
point must be particularly emphasized to which Becquerel and Rodier, the celebrated investigators in the realm of hematology, called attention about the middle of the last century, namely, that the changes in the blood of chlorotics do not correspond with the severity of the clinical picture; on the contrary, particularly at the onset of the disease, when all of the other symptoms are conspicuously developed, the blood changes are comparatively slight, and only increase upon prolongation of the malady. This late deterioration of the blood, according to our present knowledge, must be referred partly to an insufficient ingestion of food, in some cases also to accompanying symptoms; for example, to profuse menses, sometimes perhaps to autointoxication from the intestines. Therefore, the assumption that in chlorosis the blood itself is the tissue primarily diseased cannot be maintained.

The development of chlorosis has been also attributed to disturbances in the sexual functions; for, as previously remarked, too slight or too profuse menses are observed in most cases of chlorosis, and Trousseau actually refers to menorrhagic chlorosis.

Anatomical anomalies of the sexual organs, which not infrequently appear as hypoplasia of these organs, have, as we found to be the case in the circulatory apparatus, been looked upon as responsible for the development of the disease. Nevertheless, these changes cannot all be looked upon as special causes of chlorosis, but, at most, in many cases as predisposing causes. In the majority of instances, however, they are certainly secondary conditions in the chlorotic pathological picture.

That these anomalies have no general importance in the genesis of chlorosis may be seen clearly from the fact that in numerous chlorotics neither abnormalities nor functional anomalies of the sexual organs can, at any period of the disease, be discovered.

In accordance with modern pathological views, an attempt has lately been made to attribute to the sexual organs the development of chlorosis in another sense. It has been assumed that from these organs (as we know positively in the case of the thyreoid gland) besides their well-known specific function, still another internal secretory activity proceeds, by means of which materials reach the circulation, and that this activity bears a certain relation to the hematopoietic function.

This view is based mainly upon the fact that chlorosis is prone to appear in that period of life in which the sexual organs mature. Yet it is indicative of the uncertainty of these views that, while v. Noorden assumes that there is an absence of the internal secretion in chlorotics, and that as the result of this the material reaching the blood is insufficient to stimulate blood formation, in opposition to this Lloyd Jones holds the opinion that at the time of sexual maturity internal secretions enter the blood in superfluous amounts, and in some complicated manner bring about its chlorotic composition.

These modern views according to which chlorosis is attributed to the hypothetical functional disturbances of the generative organs appear to me as untenable as the earlier ones which were based upon anatomic and well-known functional disturbances. I believe these views of chlorosis to be just as partial as those concerning hysteria, in which the name at once shows that the origin 
of this disease was formerly supposed to be in the uterine sphere, although there can be no doubt that in this morbid state many hysterical women have perfectly normal sexual organs and functions, so that to-day no physician seriously refers the symptom-complex of hysteria to the uterus.

Another opinion which 'was firmly maintained by Clark and Nothnagel is that in chlorotics, who almost invariably suffer from constipation, there is an abnormal process of decomposition in the intestines, and from the resorption of products of this the disease originates.

This theory of autointoxication, however, cannot be considered to explain the entire pathological picture; indeed it may be contidently asserted that many chlorotics never suffer from constipation-neither before nor after the onset of the disease. Nevertheless, I am under the impression that in some cases of chlorosis, autointoxication from the intestines may have an auxitiary deleterious effect, and I believe that various degenerative signs in the erythrocytes which do not conform to the ordinary blood-picture of chlorosis may often be attributed to these auxiliary causes.

Another theory as to the etiology of chlorosis has been proposed by Meinert, who, as has been stated, lays special stress upon displacement and atony of the stomach; this, in his opinion, develops a dragging of the abdominal sympathetic, and is said to cause ancmia, that is, chlorosis.

In reviewing these theories, of which I have indicated only the most important, it may be noted that they are alike unsatisfactory from the circumstance that they do not conform to a uniform principle, active and pathologic in all cases, but, on the contrary, of all of them it may be said that the anatomical or functional changes considered to be causative are in many cases certainly not present.

When we consider the characteristic symptoms which distinguish chlorosis from other simple conditions, and reflect that such symptoms can be produced by the nervous system exclusively, and remember, on the other hand, the peculiar blood finding which shows a conspicuous accumulation of plasma in the blood and a decrease of hemoglobin in the cells without a diminution in the number of the cells, the conviction forces itself upon us that the characteristic changes found in this form of anemia must be referred to the nervous system.

In my opinion chlorosis depends more upon anomalies in the lymphformation than in the blood-formation; abnormal amounts of fluid accumulate in the tissues as well as in the chlorotic blood, as was shown in the description of the symptomatology, and in chlorosis it appears as though the blood-vessels were disturbed in their function, and the systematic exchange of fluid between blood and tissue altered; thus accumulations of fluid arise which, because of disturbed secretory activity, are not excreted in sufficient amount, and in consequence lead to stasis of the lymph.

These disturbances in the circulation of the blood and lymph are, in my opinion, to be referred to anomalies in function of the vasomotor nerves, to which also we may refer the pallor and blushing that in chlorotics appear and disappear so rapidly. Abnormalities in the exchange of fluid between the blood and the tissues of the body are especially dependent upon pathological functioning of the vasomotor nervous system, as my former investigations 
have taught me. I believe, therefore, that in this direction, i. e., in some neurosis of the vasomotors, we must search for the explanation of the peculiar blood findings of chlorosis, for hereby the polyplasmia of the blood and of the tissues may readily be explained, and it is also perfectly comprehensible that from a general accumulation of fluid in the tissues, particularly in the bone-marrow, the supply of hemoglobin to the erythrocytes should be deficient.

Chlorosis, therefore, I consider to be a neurosis, which appears in young adolescents, not exclusively, but usually, in girls and women; its development being favored by various preceding, predisposing causes. The anemic composition of the blood is simply one symptom of this neurosis, even though it is the most invariable and most prominent symptom. The origin of the anemia may be also referred to a faulty function of the nerves, as also are all other nervous symptoms which characterize chlorosis.

The symptomatology, as well as the views regarding the etiologic factors, and also the therapeutic results which are to be later described, in my judgment exclude a specific organic disease of the blood, as well as the idea of an anemia dependent on the formerly mentioned functional and anatomical anomalies; on the contrary, I believe chlorosis to be a link in a chain of neuroses particularly prevalent in females, and to represent a peculiar form of the general hysteric symptom-complex.

\section{DIAGNOSIS}

In regard to the diagnosis of chlorosis, it must first be emphasized that the blood finding alone is not decisive in the recognition of chlorosis; as the blood condition above described occurs not only in chlorosis, but is occasionally found in other anemias, and therefore cannot be looked upon as typical or pathognomonic. In this disease, as well as in other anemias, the blood finding may be utilized to confirm the diagnosis after a previous general examination of the organs, but, on the other hand, the diagnosis cannot be made from the blood condition and the organic changes be, then made to conform to this finding.

The diagnosis in well-developed cases may be readily made when the disease occurs in young, puffy-looking girls, with a yellowish-green color of the skin, who present the manifold symptoms and anomalies on the part of various organs which have been described. There are, however, some difficulties here which not infrequently lead to error, and are therefore of importance in practice.

It must be emphatically pointed out that it is a mistake to make a diagnosis of chlorosis, without further consideration, when young girls showing general pallor come under treatment; on the contrary, this peculiar disease must be distinguished from the usual forms of anemia, and here we may consider particularly the anemia which is the result of internal hemorrhages, for example, from the menses, or hemorrhage due to gastric ulcer which, under some circumstances, may produce even more serious changes in the composition of the blood than chlorosis, but which, for obvious reasons, and chiefly from a therapeutic standpoint, must be differentiated from chlorosis. 
The local examination of the genital organs in the one case, or of the stomach, the vomit, the feces, etc., in the other, will guard us from error. But even before these points are decided, chlorosis may usually be distinguished from simple anemias by the great number of pre-eminently nervous and circulatory disturbances which distinctly separate the one clinical picture from the other.

There are other organic diseases which often occur insidiously in young girls and lead to anemia, so that difficulty in diagnosis may arise, especially in family practice where the aids to diagnosis are not so easily obtained as in the hospital, and where errors may more readily be made.

In the first place I shall name pulmonary tuberculosis, which, at the onset, often causes but slight local disturbances, perhaps only a trifling cough of which but little notice is taken. Nevertheless, the affection in these early stages gives rise to a distinct anemia. [It is important, however, also to recognize that intense pallor without any demonstrable anemia is very prone to occur in tuberculosis.-Ev.] And it becomes an imperative duty most carefully to examine the lungs, especially at the apices, in any doubtful case.

Another organic affection, which in practice frequently resembles chlorosis, is chronic nephritis; this is prone to appear at puberty in those who, as children, have passed through an infectious discase and recovered, especially scarlatina which was later followed by a sequel-renal inflammation-the latter, however, not having been entirely cured but, on the contrary, continuing to affect the patient in later life, although not infrequently the distinct signs, particularly edema, appear first during puberty. It is therefore absolutely necessary in doubtful cases to make a careful examination of the urine, both chemically and microscopically.

\section{COURSE OF THE DISEASE}

The course of the disease in individual cases shows great variation, and from the earliest periods cases of chlorosis have been observed in which there was prompt improvement, whereas others manifested a tendency to relapses, and others again were so stubborn as to appear incurable in spite of any form of therapy.

Therefore, various forms of chlorosis have been differentiated, such as "transitory," "relapsing," and "habitual." These variations in the form of the disease can rarely be recognized at the onset. The course is also dependent on a variety of factors so that these forms can only be differentiated from one another after prolonged observation. The most common form is unquestionably the benign and transitory, in which all of the symptoms and the blood condition are relieved in the course of a few weeks.

The main contingent of this group is of course made up of girls and women of strong constitution, who, before the development of chlorosis, were comparatively well and active. The favorable progress of the disease, as will be more minutely demonstrated in the therapy, is due partly to the fact that the patient is removed from her usual surroundings, and is treated under condi- 
tions which have a beneficial influence upon metabolism in general and upon the nervous system.

The cases which run an unfavorable course, particularly those forms of the malady which are looked upon as habitual, i. e., those not completely disappearing, are unquestionably often complicated by anatomical abnormalities such as have been described; for instance, by an abnormal smallness and narrowness of the heart and vascular system, and, probably, by an unfavorable predisposition of the hematopoietic apparatus, and a diminution in the amount of blood. In these cases we are not dealing with pure chlorosis, but with a complicated anemia due to anatomical and chlorotic changes, and in many of these instances Virchow's opinion must be regarded as correct; namely, that the chlorotic symptoms may be suppressed by suitable therapy, but the disease can never be entirely cured.

It is important to note that these very persons whose vascular system is imperfectly developed offer very slight resistance to other morbific and damaging influences, i. e., that they are usually predisposed to catarrhal affections of the various organs, and that they are more subject to severe acute infectious diseases, as, for example, pneumonia, etc., than persons with a normally developed vascular system. This fact explains the previously mentioned anatomical anomalies found at the autopsy of anemic young girls.

Quite as obstinate as these mixed forms of oligemia and chlorosis due to anatomical changes are the forms of the affection in early youth due to such errors in bringing up as have been described-insufficient nutrition, deficient stimulation of the circulation, and undue irritation of the nervous systemso that these children from their earliest years have never, in fact, had a hale, hearty, rosy-cheeked appearance, and have never shown the normal mental and physical vigor and sprightliness characteristic of a well-developed child that has received proper bringing up under good management.

We meet such girls chiefly in private practice among the better classes; almost from infancy they are markedly languid, at school they are less capable of application, and are easily fatigued; on the other hand, as they grow, they develop a special liking for belles-lettres or for books which excite the imagination, and show but slight inclination to exert their physical powers. It is therefore quite possible that in these girls, on account of insufficient muscular exercise and stimulation, an undeveloped condition of the heart and vascular system is induced, so that in some of them pure chlorotic symptoms no longer appear, but mixed forms of general anemia (oligemia) and chlorosis.

It is interesting to observe these girls when they reach puberty, marry, and attain a riper maturity. We usually find that the chlorotic symptoms, i.e., the puffy appearance and the numerous irregularities on the part of the various organs, especially of the vascular system, disappear. Nevertheless, these women remain pale, are inactive, easily irritated, and form a large proportion of the widely distributed group of hysterics, so that, upon accurate investigation of the history of most hysterics, the report is obtained that in early youth they had been anemic.

This fact bears further testimony, in my opinion, to the close relationship of chlorosis and hysteria. Indeed it may be observed in various realms of 
hematology that diseases of the blood and of the nervous system present many identical etiologic factors, and frequently show a certain parallelism in their course.

Finally, the fact must be borne in mind that in many cases chlorosis appears as a hereditary condition, and we may assume that both the debility of the nervous system, and the anatomical incompleteness in the structure of the vascular system are inherited. But the view may be just as well maintained that errors in bringing up which favor the outbreak of chlorosis may be transmitted from the mother to the daughter in consequence of the faulty manner of life in some families just as a disease may be transmitted.

\section{PROGNOSIS}

The prognosis in general is that of other benign diseases which are readily cured, and consequently it may in general be regarded as favorable from the onset. It is especially so in the case of those girls in whom the symptoms have appeared suddenly, and who previously neither were anemic nor showed other pathological symptoms. Such cases occur in domestics who have grown up in the country or in a small town. More unfavorable are the conditions in girls who from early youth have shown the signs of anemia, who are addicted to all the previously mentioned crrors of education and general hygiene, the importance of which in the development of the disease has been indicated above. Those cases are doubtless particularly unfavorable that are complicated by the existence of anatomical anomalies of the heart and vascular system such as were pointed out. These latter young girls, in consequence of their generally lessened power of resistance, are in greater danger when acute diseases such as pulmonary inflammations, influenza, enteric fever, etc., appear intercurrently.

\section{TREATMENT}

Chlorosis is generally amenable to treatment, but from the onset we must bear in mind the factors which favor the development of the disease, and as every rational treatment must begin by an attempt to remove the cause of the malady, or at least its predisposing elements, it becomes the duty of the physician to try to discover in the individual case what causes have favored the development of chlorosis, a point that is of paramount importance in family practice, where the error must be sought in the general mode of life of the family and particularly in the bringing up of young girls. This is often a difficult and delicate task, and requires great tact and judgment on the part of the physician.

Particularly for this reason, i. e., because the development of the disease is favored by the injudicious manner of life and habits of the family, it is wise in many cases to remove the patient from her usual surroundings and place her in a suitable sanatorium, preferably in a suitable health resort; in manv cases the results will prove that stubborn cases of chlorosis that have resisted all the therapeutic efforts of home practice are speedily cured on removal from their former surroundings. It may be maintained with cer- 
tainty to-day that there is no specific in the treatment of chlorosis, and that even iron, which Felix Niemeyer declared in his time to be a specific, cannot be looked upon as such nor infallible.

On the contrary, experience proves that some chlorotics, who have shown no appreciable improvement under a plentiful iron treatment because their general hygienic surroundings were unchanged, are restored to health in a short time if they-as is frequently the case, for example, with the previously mentioned domestics-are sent from their usual sphere of activity to the country where fresh air and generally favorable hygienic conditions exert a curative influence upon them without a drug therapy of any kind whatsoever.

In keeping with my view that chlorosis is a neurosis in which the blood alteration is not the essential part of the disease, but usually forms the most constant symptom, I have for some time attempted to influence this disease without administering drugs that have a specific action upon the blood composition, and particularly without the employment of iron preparations, but purely by the same dietetic and hydrotherapeutic measures which are employed in other neuroses, especially in hysteria. With this antinervous régime I have completely cured a number of chlorotics in about the same time as with the -ordinary iron therapy, yet it is true that some individual cases were favorably affected after an iron therapy had been instituted.

It is evident, at all events, that under hospital treatment with good nursing and proper nutrition, the use of sweat baths at the beginning, friction with light massage later, and the internal administration of some nervines such as bromide or valerian, but without any iron medication, the same favorable results can be obtained as are observed in girls who regain their health by being sent back to their country homes.

This therapeutic experience strengthens my belief in the view that chlorosis is a peculiar neurosis going hand in hand with a damage in hemoglobin formation, and, to my surprise, I recently found that Sydenham in his excellent "Lectures upon Hysteria," which even to-day are well worth reading, particularly emphasizes this, that chlorosis is curable by the same remedies which are effective in hysteria.

In spite of these experiences, I do not by any means intend to deny the value of iron treatment in chlorosis. This has been demonstrated in practice, time and again, by numerous examples; I generally advise the administration of iron in the treatment of chlorosis. I merely wish to point out that in these cases iron is not to be looked upon as a specific, that we must not commit the error of thinking that enough is done when we prescribe a good iron preparation for a chlorotic girl, but that the main point in treatment undoubtedly consists in good nursing and diet.

The treatment of chlorosis is best begun by ordering complete rest in bed, a laxative to relieve the coprostasis, and something to stimulate the appetite. It is well at the start to ascertain by an examination of the gastric contents the state of the secretory and motor functions of the stomach; for, in some cases, as has been stated, hydrochloric acid is in excess, while in others it is diminished, and in still other cases atony of the gastric walls exists which prevents the timely expulsion of the ingesta. All of these morbid changes 
in the stomach must be relieved by a rational treatment before we proceed with a special therapy; and, as a rule, it is comparatively easy to correct these anomalies of secretion.

The diet in the beginning must consist of easily digested food, of which milk is of the greatest importance, and only in very stout girls is it necessary to limit or entirely withhold this, the best nutritive measure. Easily digested vegetables, especially the green vegetables, such as spinach, which, by the way, contains a small amount of iron, green peas and beans, mashed potatoes, and rice are particularly advisable. Later on, finely scraped raw beef or scraped ham may be allowed, while the use of eggs, at least in large amounts, is not recommended on account of the intestinal decomposition which readily ensues. Very frequently at the onset of treatment it is necessary to remove the edematous swellings which appear in the face, giving the patient a peculiar puffy appearance; these are also seen in the ankles and elsewhere. For this purpose, venesection has lately been employed again, as it was by many physicians at the beginning of the preceding century, though even then, when venesection was in vogue, experienced physicians such as Becquerel and Rodier, on the basis of their exact blood investigations, cautioned the profession against the indiscriminate employment of venesection in chlorosis. To-day, with our knowledge of the more minute constituents of the blood in chlorosis, venesection cannot be looked upon as a justifable therapeutic measure; for the plethora, i. e., the excessive accumulation of fluid in the blood, the removal of which was attempted by the exponents of the venesection therapy, is rather increased by the loss of blood and by the consequent inflow of lymph, and in my opinion the only favorable result of venesection is the reactive outbreak of sweat to which these physicians attached great weight.

This effect of the outbreak of sweat upon the fluid accumulated in the blood and in the tissues can, in my opinion, be brought about more effectively and with less danger by a simple sweat bath. In puffy chlorotics, during the first period of the treatment, I employ hot baths followed by sweating two or three times a week, and I find that the subjective difficulties as well as the objective findings are always favorably influenced thereby.

Massage, too, is effective, particularly as long as the patients remain in bed; it acts very favorably in stimulating the circulation and the entire metabolism.

Iron therapy, as already mentioned, forms an essential adjuvant in the treatment of chlorosis, although to-day we no longer hold the old opinion that iron is directly utilized in the formation of hemoglobin in the body, but it is to be looked upon mainly as a stimulant for the hematopoietic organs. That iron actually reaches the fluids of the body ly absorption, a point that has been questioned even very recently, has lately been positively proven chiefly by the investigations of Quincke.

The number of iron preparations at our disposal for therapeutic purposes is exceedingly large. Besides the inorganic compounds of iron such as ferrum oxydatum, ferrum sulphuricum, ferrum citricum, ferrum carbonicum, ferrum chloratum, and ferrum sesquichloratum, we possess countless newer preparations which contain iron in albumin combinations, and to these lately have 
been added remedies which contain hemoglobin itself in different combinations.

All of the last-named remedies are based upon the hypothesis that iron is absorbed and assimilated more readily when combined with albumin products, especially those prepared from blood itself in accordance with modern organotherapy; these give to the organism as directly as possible a compensation for the loss of hemoglobin in the pathologically altered blood.

Of the organic iron preparations there are to be named ferratin, an artificially produced iron preparation (Schmiedeberg), which is said to be identical with the iron combinations present in the organism and in the food; further, ferrum peptonatum and the various liquores ferri albuminati. Of iron-containing blood preparations new ones are combined and placed upon the market almost daily, so that it is scarcely worth while to mention the names of these, for the most part, ephemeral preparations. Especially fashionable in practice are hematogen, hemaldumin, hemol, sanguinal, etc. It must be remembered in the employment of all these remedies that in all probability the iron, whether given in an organic or inorganic combination, is primarily attacked by the gastric juice and changed into a chlorid combination, and that later it is absorbed in the bowel as an iron chlorid albuminate. It is therefore quite unlikely that the artificial or natural iron albuminates are absorbed in the form in which they are administered, and it may be assumed that, during the time of their absorption into the fluids of the body, great labor is thrown upon the stomach and intestine, much greater than in the administration of inorganic preparations.

Regarding the special activity of these remedies in chlorosis, neither from literature nor from my own experience are many observations of patients of this sort known to me, from which it might be concluded with any certainty that any one of the above-mentioned organic preparations produces a cure more rapidly and with greater certainty than is observed upon the administration of the ordinary iron preparations.

What is particularly lauded by the manufacturers concerning these remedies is the useful action of the albumin contained in them; but it must be mentioned that these amounts of albumin are so small that they play. no rôle in the nutrition of the chlorotic, particularly as in this disease there is no noteworthy deficiency in albumin metabolism.

Unquestionably the majority of these modern remedies act by suggestion, and therefore in practice we can rarely do without them, as the laity nowadays manifest the greatest interest in the products of pharmaceutical industry. In cases, however, in which the therapy remains uninfluenced by these subjective effects upon the patient, as in hospital treatment, there is absolutely no reason for rejecting the old well-tried iron preparations in their various combinations; thus, for example, the excellent Blaud's pills.

According to the suggestion of C. Gerhardt, for some years I employed with success liquor ferri sesquichlorati, three to five drops three times daily, diluted in water.

The numerous iron springs in Germany and neighboring countries contain iron for the most part as a suboxid combined with carbonic acid; and the 
waters are, therefore, suitable for drinking at the spring; not, however, for bottling, as carbonic acid readily passes off and the iron is then precipitated. A few springs contain sulphate of iron, and still fewer, chlorid of iron.

The best-known German iron springs are in Pyrmont in the Duchy of Waldeck, Langenschwalbach at the Taunus, Bocklet (near Kissingen), Charlottenbrunn, Cudowa and Flinsberg in Silesia, Driburg in the Teutoburger Woods, Elster in Saxony, Franzensbad in Bohemia, Kohlgrub in Bavaria, 900 meters above the sea, Muskau in the Lausitz, Liebenstein in Mciningen, Freienwalde on the Oder in Brandenburg, Pulzin in Pomerania, Reinerz, 500 meters high, in the earldom of Glatz, Steben in Upper Franconia.

Of foreign iron springs there are particularly to be named: Farnbühl in Luzerne, St. Moritz in the Upper Engadine, 1,700 meters above the sea, and Val Sinestra in the Lower Engadine; further, Haarlem in Holland, Szliácz in Hungary, $S p a$ in Belgium.

Besides iron, in some cases arsenic is of use as in other anemias; and therefore, besides the preparations of the pharmacopeia, arsenical springwaters must also be considered; the best known are those of Roncegno and Levico in the Southern Tyrol as well as the Guber Spring in Srebrenica in Bosnia.

The beneficial effect of all of these iron and iron-arsenic spring cures certainly does not consist only in their contents of iron and arsenic, but quite as much in the stimulation to metabolism by change of climate and the constant outdoor life, so that, in fact, many extremely chlorotic patients are seen to recover in these regions who show but slight improvement while at home in spite of any iron preparation.

of other drugs quinin is chiefly to be mentioned, while remedies such as phosphorus, manganese, and organopreparations, for example, bone-marrow, thymus, etc., are of no importance.

\section{PROPHYLAXIS}

Although in the direct treatment of the chlorotic symptom-complex a great variety of dietetic, physical and medicinal auxiliary remedies are at our disposal, it must nevertheless be repeated that many cases of chlorosis belong to the diseases which are readily preventable, and particularly where the social position of young girls precludes the necessity of their earning their own livelihood, and the external conditions are favorable for healthy development.

Since, in spite of this, the disease is observed frequently in well-to-do families, it must be attributed wholly to the utter ignorance even to-day in the most highly cultured families regarding the fundamental principles of hygiene, a deplorable condition which must be combated, on the one hand, by a greater dissemination of the laws of hygiene among the people, and, on the other hand, by the activity of each individual family physician.

It is sufficient here, to avoid repetition, simply to refer to the deleterious factors which have been previously mentioned as predisposing, and which may be removed in a large measure by hygienic regulation of the mode of life, by bodily exercise, exercise in the open air, etc. 


\section{LEUKEMIA}

\section{By W. von LEUBE, WürzBURG}

SINCE the discovery of leukemia by Virchow in 1845 , we designate by this term a disease characterized by an increase in the number of white cells in the blood as the result of the morbid activity of the blood-forming organs, and in which the blood alteration forms the essential feature of the progressive and pernicious course of the disease. [The blood changes dominate the clinical picture, and are manifest in many organs post mortem, but it is the belief of most authorities that the heightened activity of glands and marrow to which they are due is itself a result of some toxic (chemotactic) stimulus.-ED.]

This definition is sufficient clearly to differentiate "leukocytosis" from leukemia: In leukocytosis we are, in fact, also dealing with an increase of the leukocytes in the circulating blood; but this is a transitory symptom in the course of manifold diseases. At the same, leukocytosis is not, like leukemia, a morbid condition of a progressive and pernicious nature; on the contrary it is in a measure the expression of the self-protective power of the body against the disease present.

\section{COMPOSITION OF THE BLOOD}

Without a thorough knowledge of the composition of the blood and of the genesis of its different morphologic elements, an insight into the nature of leukemia is impossible. We shall, therefore, describe briefly the normal condition of the blood and of its cellular elements before we discuss leukemia in detail. ${ }^{1}$

The three formed elements of the blood are, as is generally known, the red blood cells, the white blood cells and the blood-plaques. These last have thus far little, or no, importance in pathology.

The red blood cells, "erythrocytes," are flat, circular, biconcave discs, averaging 7.5 micromillimeters in diameter. Normally they do not contain a nucleus, but consist of a protoplasmic stroma the gaps of which are filled with hemoglobin. The formation of the red blood-corpuscles occurs in embryonic as well as in post-fetal life from nucleus-containing, colored blood cells, the

1 This article relating to the diagnosis and pathogenesis of leukemia is based, in the main, upon the complete and recently revised chapter on Leukemia in the sixth edition of my "Special Diagnosis of Internal Diseases" (English edition, Leube-Salinger, D. Appleton \& Co., 1904.) 
hematoblasts. These cells are invariably present in the red bone-marrow of the flat bones, of the sternum and of the ribs, the base of the skull and the vertebræ. The red blood cells which at first contain a nucleus, lose this later by caryolysis, i. e., by dissolution of the nucleus (according to some investigating authors by expulsion) and then enter the circulating blood as nonnucleated red blood-discs. We must at present adhere to the view that in post-fetal life the red (non-nucleated) blood cells are formed exclusively in the red bone-marrow, namely, from the nucleus-containing, red blood cells which are constantly present in these regions. The latter cells, the hematoblasts, appear, at least in embryonic life, to arise from lymphocytes by hemoglobin production, first as megaloblasts, which perhaps are originally the mother cells of the normoblasts. [Several authorities of the first rank take exception to this account of the hematoblasts, and believe that red and white cells arise from different stems.-ED.] However, after the latter have been formed, they increase by mitosis, constantly producing new normoblasts. Besides the bone-marrow, in embryonic life the lymph-glands also probably have an erythropoietic function [probably also the liver, and possibly the spleen.ED.]; but in the adult organism blood-formation occurs only in the bonemarrow, where also the transformation of normoblasts into non-nucleated blood discs occurs (by caryolysis). Under normal conditions only non-nucleated blood cells pass from the bone-marrow into the blood. Whenever an appreciable quantity of nucleated red blood cells is found in the blood, pathological conditions are present: Infections, intoxications, inanition. If large numbers of nucleated blood cells are in circulation, severe anemias, even of the severest character, are present.

When the red blood cells die they are replaced by fresh material coming from the bone-marrow, so that the number of erythrocytes remains at almost a constant figure, namely, about 5,000,000 for men and about 4,500,000 for women to the cubic millimeter, a proportion of about 600 to 1 of the white cells $[5,000,000$ is too low for the average of healthy adult males in America. The correct figure is much nearer 6,000,000.-ED.] The majority of the blood cells, unquestionably, disintegrate in the liver. We are justified in this assumption for reasons which need not be here minutely considered, and of which only one shall be mentioned, viz., that in the liver large amounts of biliary coloring matter are daily produced. But bile, as its chemical composition proves (hematin $\mathrm{C}_{32} \mathrm{H}_{32} \mathrm{~N}_{4} \mathrm{O}_{4} \mathrm{Fe}$ by taking up water and giving off iron$+2 \mathrm{H}_{2} \mathrm{O}-\mathrm{Fe}$-forms bilirubin $\mathrm{C}_{32} \mathrm{H}_{36} \mathrm{~N}_{4} \mathrm{O}_{6}$ ), can only consist of hemoglobin which has been given up from the stroma of the red blood cells. We can in fact artificially increase at will the production of biliary coloring matter by the injection of hemoglobin into the blood.

The white blood cells (leukocytes) represent colorless, membraneless cells with one or more nuclei and a protoplasm variously constituted. Since the introduction of new methods of staining in examining the blood, the first application and completion of which we owe to P. Ehrlich's excellent investigations in the pathology of the blood, the fact has developed that normal blood contains not only two varieties of leukocytes, as was assumed for a long time, but many kinds, differing markedly from each other. It is best to differen- 
tiate two principal varieties, mononuclear and polynuclear (or polymorphonuclear) cells, both of which show various subvarieties:

A. Mononuclear Forms: Cells with one nucleus and varying amounts of basophilic, non-granular protoplasm.

1. Lymphocytes, characterized by a large, round, centrally-placed nucleus and a narrow protoplasmic ring. The latter, as well as the nucleus, reacts basophilically, especially the protoplasm which shows no granulations. [No granulations, that is, with Ehrlich's stain. With the Romanowsky stain a variety of pink, violet, and sometimes a few blue granules are seen in the protoplasm of the lymphocytes. This is true of all sizes of lymphocytes, though commoner in the large forms.-ED.] The lymphocytes have no power of ameboid movement. [I am convinced by the observations of several competent men that lymphocytes (especially, but not only, the larger forms) do have the power of ameboid movement.-ED.] The majority of them scarcely attain the size of the red blood discs; rarely are large lymphocytes found, especially in the blood of children. The number of lymphocytes amounts to about 25 per cent. of the white blood-corpuscles. Some individual examples, especially of the larger forms, show slight segmentation of protoplasm. 2. Large mononuclear leukocytes, twice or three times larger than the lymphocytes, differing markedly from the large lymphocytes, in that the large, usually oval nucleus is generally eccentrically situated and that the non-granulated protoplasm is relatively well developed. This protoplasm and the nucleus have, like the lymphocytes, a basophilic reaction, but, in contrast to the latter, the protoplasm stains less readily than the nucleus. Transitional forms between the first and second variety are not observed, and for this reason, according to Ehrlich, the "large mononuclear" cells are strictly differentiated from the lymphocytes, all the more so as "transitional forms" from the large mononuclear to the polynuclear are observed, i. e., large cells with neutrophilic granulation and with indentations in the nucleus. [All recent and unprejudiced observers seem to me to agree that there are abundant transitional forms between the "large lymphocyte" as here described and the "large mononuclear leukocyte" of the same text. A differentiation of these two varieties is in my opinion impossible.-ED.] The number of the large mononuclears in normal blood is always small (about one per cent.).

B. Polynuclear Forms: Cells with several small nuclei or usually with a polymorphous nucleus, i. e., with marked indentations of the nucleus, so that some of the nuclear segments are connected by thin chromatin threads. The polymorphonuclear ("polynuclear") leukocytes are further characterized by ameboid movement. The protoplasm is granular and shows varying conditions regarding staining which give rise to the differentiation into the three following varieties:

1. Neutrophitic polynuclear leukocytes, usually designated as "polynuclears," characterized by the dense granulation of the protoplasm and the affinity of the same to "neutral" staining material. They form in the normal blood about 70 per cent. of the white blood-corpuscles. [There are no true neutral stains. A special differential acid stain like Ehrlich's "triacid" is what is here referred to. 
The normal percentage of polynuclear leukocytes is in this country more often near 60 per cent. than near 70 per cent.-ED.]

2. Eosinophile cells, characterized by their size and by the coarse granules in their protoplasm which stain intensely by acid stains (eosin). [The size of eosinophiles is not characteristic. They are about the size of a polynuclear cell or often a little smaller, but their irregular shape (as seen in thin smear preparations) renders a definite statement as to their diameter difficult.ED.] They resemble the neutrophilic polynuclear cells and, like these, are markedly contractile; their number amounts to about 3 per cent. of the white blood-corpuscles.

3. Basophilic leuteocytes, "mast-cells" are scanty in normal blood ( 0.5 per cent. of the leukocytes). They are characterized by the intense basophilic reaction of the granules in their protoplasm and by the very slight staining affinities of the nucleus; the granulation does not color with the triacid stain, hence mast-cells appear in triacid preparations as light, non-granular cells.

Opinions are more at variance concerning the origin of the white bloodcorpuscles than of the red, although a number of investigators (Virchow, Kölliker, Max Schultze, Neumann, Heidenhain, Arnold, Mokoff, Rieder, Engel and, above all, P. Ehrlich, and in late years Askanazy, Pappenheim and Rubenstein) have closely studied this subject. At present, it is difficult, almost impossible, to take a positive stand in the mooted question. The following theory is probably more in accordance with present opinions than any other.

After the investigations of the last decade, and especially since the introduction of tinctorial methods of examination by Ehrlich, Virchow's view that the lymphocytes are the young, the leukocytes the old, forms of the cells, the latter arising from the former, can no longer be accepted in its simple, strict conception. On the contrary, it is better to adhere to the view that the lymphocytes, the leukocytes and the hemaglobin-containing cells represent separate stages of development of cells, which probably are only alike in their first elements-namely, cells with non-granular, weakly basophilic protoplasm and one round nucleus. From these the myelocytes develop in the bone-marrow, the protoplasm becoming granular (neutrophilic or eosinophilic), while the nucleus still retains its round form; in the later stages of development the granulation becomes more marked, the nucleus flattened, sinuate and, finally, pigmented (polymorphonuclear leukocytes with neutrophilic or eosinophilic reaction). At this age the cells enter the circulating blood as "polynuclear" leukocytes. A few immature cells, i. e., still basophilic, non-granular and supplied with one nucleus, enter the blood as the so-called "mononuclear" leukocytes, which then mature in the blood, becoming polynuclear leukocytes. The cells which have begun to mature in the bone-marrow remain in the bonemarrow until perfectly mature, becoming polynuclear leukocytes, so that the blood does not normally contain myelocytes. The mature polynuclear cells, however, enter the blood upon chemotactic, physiological irritation in relatively small but usually quite constant numbers, under pathological conditions frequently in very large numbers ("leukocytosis"). The Ioss of the polynuclear cells which pass into the blood is compensated by the formation of new primary stages of the same, the myelocytes, and by the fur- 
ther development of the latter into a correspondingly greater number of polynuclear cells.

In the spieen and lymph-glands leukocytes are not produced, at least not in noteworthy amounts (even in cases where they are much needed, as in leukocytosis). On the other hand, the lymph-glands (the spleen only to a slighter degree) are the points of origin for the formation of lymphocytes, which, as is well known, represent one-fourth of the white blood cells in normal blood. Similarly to the myelocytes they are probably also formed in the bone-marrow from large basophilic, mononuclear cells which are the early stages of the leukocytes and generally do not enter the blood, or at most only as "large lymphocytes" in children, and under pathologic conditions in lymphatic leukemia (where they are met with in the blood in great numbers, particularly in the acute form of the disease). Just as the myelocytes mature ("age") into polymorphonuclear leukocytes, so does the nucleus of the lymphocytes pass through this aging process in that it later undergoes lobulation and fragmentation (Rieder's cells), without the protoplasm losing its basophilic, non-granular character.

The nuclear changes-lobulation and segmentation into several nucleitherefore, indicate the physiological age of the cells; just so may we assume, in general, that a slighter staining quality (amblychromasia), especially of the cell nuclei, characterizes the cells as incomplete, undeveloped, in contrast to the intensely staining ("trachychromatic") mature forms.

As already mentioned, the bone-marrow is almost exclusively the organ of production of the myelocytes and polynuclear cells, although typical myelocytes are found in the spleen and lymph-glands (without having been carried in by the blood stream). On the other hand, the lymph-glands are to be considered the seat of production of the small lymphocytes. Of course, we find lymphocytes in scant numbers in the bone-marrow too; of these it is probable that a fair proportion have been carried in, a part, however, having been formed in the marrow itself.

The origin of the very small number of mast-cells, which, under normal conditions, are found in the blood, has not yet been determined with certainty. Most investigators assume that they originate in the connective tissue; others believe that they develop from lymphocytes.

\section{SYMPTOMS AND BLOOD-CHANGES}

Let us now return to the discussion of leukemia. Marked cases are manifest at the first glance by the extreme pallor of the patient's skin and mucous membranes, and occasionally by the color of the blood as obtained by puncture or incision of the finger tips which may be whitish red. [Cases are pale only if anemia has developed, and this even in well-marked cases is often not notable. In the later stage of the disease, but not until then, anemia becomes severe.-ED.] The microscopic examination of the fresh blood in cases of leukemia of high grade shows even without counting the abnormal increase in the white blood-corpuscles. But a count and a comparison with the number of erythrocytes is absolutely necessary in the less marked cases. Whereas 
normally we find an average of 8,000 leukocytes (in children somewhat more, 9,000 to 10,000 ) in a cubic millimeter of the blood taken from the veins, i. c., one white cell to 600 erythrocytes, the number of leukocytes may so increase in leukemia that the proportion is 1 to 50,1 to 10 , or 1 to 2 . In fact, the red and white blood-corpuscles may even be present in similar amounts in the blood. The count of white blood-corpuscles shows 100,000 and even 500,000 and more, to the cubic millimeter.

In some cases of leukemia, no noteworthy change in the erythrocytes, either in number or appearance, occurs; as a rule, however, they are decreased in numbers to about one-half of the normal or even less, just as in severe anemias. In general the decrease of the erythrocytes is in proportion to the increase in the number of white blood-corpuscles. But the numerical relation of the erythrocytes in leukemia is a secondary consideration, and the diagnosis of the affection depends primarily upon the white blood-corpuscles. There are cases of simple anemia in which the number of red blood-corpuscles is markedly diminished, but the production of white has remained normal; then the proportion of the white to the red cells may become 1 to 25 , etc., without leukemia being present. For example, in pernicious anemia, with a diminution of the erythrocytes to 250,000 to the cubic millimeter, and a normal condition of the white blood cells $(8,000)$, the proportion would be about 1 to 30 and still no leukemia would be present. Leukemia is, however, to be assumed under all circumstances if the proportion falls below 1 to 20, because these figures can only occur when, besides the decrease in the number of the red blood-corpuscles as a result of anemia (even in the most excessive diminution of the same that has been observed up to the present in cases of pernicious anemia), the number of leukocytes is absolutcly increased at the same time.

Besides the diminution in the number of red blood-corpuscles, the blood also contains, as a rule, numerous nucleated red blood-corpuscles in the form of normoblasts, more rarely megaloblasts, or transitional forms between these. The amount of hemoglobin in the blood is diminished in leukemia but the coloring of the individual corpuscles (abnormally decreased in numbers) need not be diminished. An increase in the blood-plaques has also been observed upon several occasions.

The diagnosis of leukemia can be made without further consideration in cases which show an extraordinary increase of the white blood-corpuscles, but in doubtful cases the presence of the disease can only be determined by an exact microscopical examination of the morphology of the loukocytes.

Besides the increase in the white cells, what is particularly conspicuous in the microscopic examination of leukemic blood is that in the majority of cases the polynuclear cells are most numerous; in other cases, however, the lymphocytes form the greater number, and this type of blood alteration persists during the entire course of the illness. [In 41 cases of myelogenous leukemia studied by me the polynuclear cells average $4 \% .5$ per cent. It is the polymorphous condition of the blood, the endless variety among the leukocyte forms, that most impresses one in this disease.-Ev.] We, therefore, quite properly differentiate two different varieties of leukemia. 
1. Lymphocyte leukemia.

2. Leukocyte leukemia.

Since the source of the lymphocytes, as has been mentioned, is the spleen and the lymph-glands, and that of the leukocytes is the bone-marrow, the common designations of lymphatic leukemia (lymphemia) and myelogenous leukemia (myelemia) may be selected for these two basic forms of leukemia. As, however, the bone-marrow also normally produces typical lymphocytes, and as it has been determined in rare cases that an over-flooding of the blood with lymphocytes may occur by proliferative changes in the marrow without enlargement of the spleen and lymph-glands (myelogenous lymphemia), I prefer the less prejudicial division of the leukemic forms into "lymphocyte leukemia" and "leukocyte leukemia."

In the blood at the autopsy, or after prolonged standing of the blood of leukemics, Charcot's crystals are found, usually within the leukocytes, and particularly in the eosinophilic polynuclear cells. Their relation to the lastnamed cells has lately been determined beyond doubt, so that we' may say: Wherever eosinophile cells are present in large numbers, Charcot's crystals are also found. This at once makes it clear that they are absent in lymphocyte leukemia, and, on the other hand, that the crystals are not only found in leukocyte leukemia but in all diseases in which eosinophiles occur in large numbers, nasal polypi, bronchial asthma, etc. The demonstration of Chareot's crystals is, therefore, by no means pathognomonic of leukemia, the less so as they are also found in normal bone-marrow, which is not to be wondered at.

In comparison with the results of the microscopic examination of the blood, all the other morbid phenomena of leukemia are of decidedly subordinate importance, even though in the individual case they greatly aid in the diagnosis. There are cases of leukemia in which, besides the alteration in the blood and the symptoms of anemia in connection therewith, all other objectively demonstrable morbid phenomena are absent, and the diagnosis must be made entirely from the blood findings! These are, however, under all circumstances, as will be shown later, rare and exceptional cases; the rule is that many organs, especially those connected with blood-formation, suffer marked changes which may be demonstrated by physical examination.

In this connection we must mention particularly the enlargement of the spleen which is present in the majority of cases of leukemia. The splenic tumor is usually of considerable size; it extends to the median line or beyond and downward to the hypogastric region. On account of its sharp indented margin, the direction of the growth of the tumor, and especially its evident origin from the left hypochondrium, there is rarely cause to doubt that we are dealing with a tumor of the spleen. The diagnosis may be difficult if, as in one of my cases, the large spleen turns so that the hilus appears at the top. The consistence of the splenic tumor is hard; its circumference, after it has attained a certain size, is usually constant. Rarely transient shrinkages are noted, or, on the other hand, excessive swelling or rupture of the organ. As a rule the swollen spleen is not sensitive to palpation, and the subjective diffculties which the tumor causes are generally slight. At most the patients complain of a feeling of fulness in the abdomen and slight difficulty in respira- 
tion; less frequently pain occurs in the splenic region, or even inflammatory phenomena which manifest themselves in peritoneal friction sounds in this area.

In some of the cases, most frequently in lymphocyte leukemia, lymphgland enlargement occurs. [I have never seen lymph-gland enlargement except in lymphocyte leukemia.-ED.] As a rule, the glands of the neck and axillary region, more rarely those in other areas of the periphery or the internal lymph-glands, are affected. The glandular tumors are moderately hard, the skin covering them is movable and not reddened. If the mesenteric and retroperitoneal glands are hyperplastic, they may be occasionally felt through the abdominal walls. In case the enlargement affects the tracheal and bronchial lymph-glands, at least a probable diagnosis of this result of the leukemia may be made, provided the symptoms of tracheal or bronchial stenosis, of paralysis of the vocal cords, the result of pressure upon the recurrent laryngeal nerve, or difficulty in deglutition, arise. In marked cases of swelling of the tracheo-bronchial lymph-glands or of the persisting thymus gland, the percussion note over the manubrium is dull and the bone is arched outward. In the adenoid tissue of the tonsils and also in the adenoid follicles at the root of the tongue hyperplasia and swelling may likewise be noted.

Besides the spleen, the various lymph-glands, and the other adenoid organs, the bone-marrow is almost always affected by anatomical changes. According to the experiences gathered up to this time, changes in the bonc-marrow are invariably present in leukemia. In lymphocyte leukemia we find, especially in the lymph-glands, in the spleen and eventually in the liver, but invariably also in the bone-marrow, proliferations of the lymphoid tissue, which in these cases may become so plentiful that the production of the polynuclear cells in the bone-marrow and the passage of the same into the blood may be prevented by the overgrowth of the lymphocytes in the marrow. As soon as the lymphocytes begin to enter the blood, floating in from the bonemarrow, hyperplastic lymph-glands and spleen (quite passively as we must assume from Ehrlich's convincing deductions), the picture of lymphocyte leukemia develops.

The implication of the bone-marrow in the leukemic processes can never be determined with certainty intra vitam by the sensitiveness of the bone to pressure, but only when the microscopic examination reveals in the circulating blood mononuclear, granular marrow cells, the origin of which from the bonemarrow is certain, and also large quantities of nucleated red blood cells.

Besides the three above-named organs which are particularly affected in leukemia, other portions of the body, although less frequently, are involved in changes which are in direct relation to leukemia. In the majority of cases the liver, in consequence of leukemic-cell infiltration between the acini, is enlarged. The surface is smooth, its consistence moderately hard. Marked grades of ascites and jaundice are only to be expected if the periportal lymphglands are enlarged and press upon the portal vein and biliary passages. Ascites, that is, the transudation of a large amount of fluid into the peritoneal cavity, may also be caused by a leukemic nodular infiltration of the peritoneal layers and of the omentum. This is, however, not frequent.

Leukemic infiltrations occur also in the stomach and intestine; they arise 
from adenoid tissue areas of the wall (i. e., the solitary follicles). Dyspeptic phenomena and diarrhea point to this complication but do not permit certainty in diagnosis. In some patients a leukemic stomatitis and pharyngitis develop.

It is noteworthy that, in spite of the diminution of the erythrocytes, neither dyspnea nor orthopnea occurs in leukemia, at least not according to my experience (provided there are no complications on the part of the respiratory organs). [I have repeatedly seen dyspnea and even orthopnea in leukemia as soon as anemia became severe or circulatory stasis marked.-ED.] Pettenkofer and Voit first demonstrated in one of their patients, who was suffering from leukemia, the ability of the organism, during rest, regardless of the diminution of the erythrocytes, to assimilate as much oxygen as a healthy person with the same nourishment. Neither do marked disturbances of metabolism occur in the course of leukemia, or, if they are now and then noted, they are at least not dependent upon the leukemic processes as such. The tendency of the leukemic to catarrhal affections of the respiratory passages may favor the production of pulmonary inflammation in some cases; in other cases lymphatic infiltrations form in the lungs. The development of lymphatic nodules has been noted in the epiglottis, in the larynx and in the trachea, also upon the pleura; not rarely do transudates appear in the pleural cavity.

Painful non-ulcerating nodes occasionally develop in the skin, and must be looked upon as leukemic lymphoid formations. [Deafness was produced in one of my cases by nodules in the ear.-ED.] A tendency to profuse sweating is not rare, and adds to the exhaustion of the patients.

The murmurs noted upon auscultation of the heart are of an accidental nature, the result of the marked anemia which occurs in leukemia. [Late in the course of the disease.-ED.] In consequence of poor nutrition and exhaustion of the heart muscle such a dilatation of the heart may occur that the valves though still normal in their length can no longer close. Thus relative mitral and tricuspid insufficiency may develop.

Not only such relative cardiac insufficiencies and accidental murmurs may occur as a consequence of the anemia which develops in leukemia, but also quite a number of other symptoms such as appear more or less regularly in any anemia; e.g., palpitation, weakness and lassitude, vertigo, headache, attacks of syncope, and edema; perhaps also fever which is irregular but may be at times quite high $\left(102^{\circ} \mathrm{F}\right.$. to $104^{\circ} \mathrm{F}$.).

In the course of leukemia, in rare cases, long-continued priapism occurs, due perhaps to a thrombosis in the corpora cavernosa. This symptom has been observed a number of times, and occurs so rarely in other conditions that its presence should be considered suggestive of leukemia.

Of special interest in the diagnosis of leukemia are the changes in the eyeground which occur in the course of the affection. In keeping with the increased number of white corpuscles and the anemia the fundus of the eye has an unusual, pale, orange-yellow appearance. But this discoloration is not conspicuous in all cases; it is always absent if the amount of hemoglobin has not been materially decreased. The so-called retinitis leukemica is character- 
ized by an extremely tortuous condition of the reins, a band-like opacity of the retina, and hemorrhages into the same, as well as by opacity, indistinct contours and bluish-yellow color of the optic nerve papilla. Not rarely the veins are accompanied by white streaks, and white areas intermixed with hemorrhages are visible in the macula; the hemorrhages are circular in shape, with a prominent ycllowish-white center. Occasionally there is a tendency to the development of numerous large hemorrhages which may also occur in the vitreous body, so that the ophthalmoscopic picture may resemble that of thrombosis of the central vein of the retina. Such a thrombosis may occur simultaneously with hemorrhages around the optic nerve and in the latter itself, and may also develop in the orbital portion of the optic nerve. Functional disturbances are present to only a slight extent.

The changes in the urine observed in leukemia are of great importance in diagnosis, as well as in the study of the disease.

Apart from the occasionally observed albuminuria, which is in part the result of the anemia, in part the consequence of lymphomatous infiltration of the renal substance (which is indicated by the presence of casts and of a large number of leukocytes in the sediment of the urine), a change in the excretion of the solid constituents of the urine is more or less frequently found in leukemia. An absolute and relative increase of the excretion of uric acid (up to 8 grams per diem) has been almost invariably observed.

This increase of uric acid in leukemia is certainly not, as was formerly supposed, the result of insufficient oxidation in the organism. This opinion is contradicted not only by the results of the investigations of Pettenkofer and Voit, but also by the fact determined by Stadthagen, that in the body of the patient suffering from leukemia the sodium urate which has been administered per os is capable of further oxidation. Neither can the excessive production of uric acid be considered due to the enlargement of the spleen commonly present in leukemia, as patients with chronic splenic tumor excrete uric acid in normal amounts in contrast to leukemia with enlargement of the spleen, in which the excretion of uric acid is markedly increased. As we know now that uric acid originates from the nuclein which is liberated in the decomposition of the cellular nuclei, especially by oxidation of alloxur bases which are contained in nucleinic acid, the conclusion is obvious that the increase of uric acid excretion in the course of leukemia may be referred to the destruction of a relatively greater number of leukocytes than under normal conditions. However, the increased excretion of uric acid may also be an expression of increased function, that is, of metabolism of the superfluous leukocytes, so that an increase of uric acid excretion in the course of leukemia is by no means a proof of great decomposition of leukocytes and their nuclei.

The excretion of urea usually does not differ from the normal; in two cases of severe leukemic cachexia in my clinic Fleischer and Penzoldt noted an increase of urea excretion, but this was in the late stages of leukemia, when, as in carcinomatous cachexia, a marked decomposition of organic albumin with increase-of the excretion of nitrogen occurs. Hematuria is also observed in the course of leukemia.

This symptom is connected with the general hemorrhagic diathesis of the 
leukemic, one of the most important symptoms of leukemia as regards diagnosis, and especially as regards prognosis. Hemorrhages may occur in the urinary passages and in various parts of the body, i. e., in the respiratory passages, in the digestive tract, in the skin (purpura), in the subcutaneous connective tissue (sometimes in the form of colossal hemorrhagic tumors), in the muscles, in the internal parts of the ear, etc.; hemorrhages are also noted in the central nervous system in the course of leukemia, and may give rise to apoplectic attacks with paralysis, or they may result in sudden death; these intercurrent hemorrhages are usually the cause of the fatal outcome of leukemia, or, at least, they predispose to it. Multiple effusions of blood occasionally occur in the peripheral nervous system, in the sheaths of the nerves or in the nerves themselves, and these result in fatty degeneration of the nerves and the muscles supplied by them. In some cases of leukemia the hemorrhagic diathesis may so dominate the clinical picture that the cases appear to be purpura hemorrhagica until the examination of the blood discloses the error. [This is especially true of the cases of acute lymphatic leukemia.-ED.]

The symptoms of leukemia-the increase in the number of leukocytes in the blood as well as the enlargement of the spleen and lymph-glands-may for a time ameliorate greatly in the course of intercurrent infectious diseases. [Or even without any known cause. Some of these spontaneous remissions happen to coincide with the inception of a special method of treatment and lead to the reports of curative drugs, etc.

Of the remissions under X-ray treatment, mention will be made later. -ED.]

The classification generally in vogue until lately by which individual cases of leukemia were grouped as "myelogenous" or as "lymphatic" leukemias, according as the spleen or the lymph-glands were enlarged, can no longer be maintained from a hematological standpoint, chiefly because of the important researches of Neumann and Ehrlich. For some time we differentiated not only between splenic and lymphatic leukemias, but also between cases of the splenic-medullary variety, or those of the pure medullary form (" myelogenous leukemia"). But these designations for the individual varieties of leukemia, based as they are on the changes prominent in the clinical picture in organs more or less implicated in the formation of the blood, are not justified either from a hematological or from a clinical standpoint. For, in both forms, the lienal and the lymphatic, the bone-marrow is primarily implicated in the changes of the hematopoietic process, and, on the other hand, the characteristic blood picture, which in one case may show a marked preponderance of lymphocytes, in another a great majority of leukocytes and their primary stages, is usually not at all altered, whether the lymph-glands or the spleen are enlarged or not. We therefore recur to my chosen differentiation of only two varieties, according to the point of origin of the cell forms which are markedly increased in the blood-lymphocyte leukemia and leukocyte leukemia. The differentio-diagnostic points of these two varieties of leukemia are the following:

1. Lymphocyte Leukemia. The blood picture is characterized by a conspicuous preponderance of large and small lymphocytes in comparison to the 
leukocytes. Nucleated red blood cells and megaloblasts, although found in the blood in lymphocyte leukemia, yet, compared with their usual abundance in leukocyte leukemia, are by no means prominent.

According to the course of the affection, two varieties must be differentiated, the acute and the chronic.

Acute lymphocyte leukemia ("acule lymphemia"), the recognition of which we owe above all to Ebstein and A. Fraenkel, is characterized by its rapid, often febrile, course, resembling a severe infectious disease. Besides the usually insignificant enlargement of the spleen and lymph-glands, the other clinical phenomena of leukemia are present, stomatitis, retinitis, etc. Most prominent in the clinical picture is the hemorrhagic diathesis, so that the affection has the appearance of purpura hemorrhagica and may be confounded with this, all the more so as the hemorrhages occasionally occur before the characteristic blood picture of acute lymphemia is formed. The blood picture itself is highly characteristic: Preponderance of the lymphocytes, especially of the large ones, very rarely of the smaller, with a vesicle-like, sometimes deeply indented nucleus; erythroblasts are present although few in number; the polynuclear cells are not only relatively but also absolutely decreased. The number of white blood cells compared to the red ones may be extraordinarily great (up to 1 to 1 !). [In the majority of cases, however, it is not excessive-much less than in myelogenous cases.-ED.] The affection may terminate fatally in a few days or a few weeks.

Chronic lymphocyte leukemia is differentiated from the above-described form by its greatly protracted course: Gradual enlargement of the Iymphglands, especially those of the neck, also enlargement of the spleen to a greater or less extent. In rare cases, even in a very chronic course (in one case under my observation the affection lasted at least two years), only insignificant enlargements of the glands appear. The lymphoid hyperplasia of the bonemarrow is present here, as in the acute form. The hemorrhagic diathesis is also quite marked in the chronic form of leukemia, and as consequences of anemia there may be a great variety of symptoms, such as dilatation and insufficiency of the heart, etc., which may threaten life. The blood picture in the main resembles that of the acute form, i. e., mononuclear lymphocytes, but usually the small forms are most prominent, whereas the other colorless blood cells are almost absent. Large lymphocytes are also found in chronic lymphocyte leukemia during the entire course of the affection, and in rare cases, as in the acute form, they may even dominate the blood picture.

2. LEUKOCYTE LEUKEMIA, by far the most frequent form of leukemia, may be easily differentiated from both of the last-mentioned varieties of leukemia by its entirely different blood picture. The increase of the white blood cells is usually very marked; here, however, the polymorphonuclear leukocytes are greatly increased in the microscopic picture: neutrophites, and, above all, eosinophitic polynuclear cells are, as Ehrlich has found, always absolutcly increased. There is invariably an absolute increase of the mast-crlls which occasionally are twice as numerous as the eosinophiles, and their determination is of great importance in a diagnostic respect because a marked increase of the mast-cells is only observed in leukemia. [The absence of mast-cells was espe- 
cially noted in 3 of my 41 cases.-ED.] The phenomenon, however, which especially characterizes leukocyte leukemia, and shows its origin to be the changes in the bone-marrow ("myelogenous leukemia"), is that, besides the polynuclear cells, their ancestors, the mononuclear granular leukocytes, i. e., the neutrophilic and eosinophilic myelocytes, are always found in the blood and occasionally are found in such great numbers (up to 100,000 per c.mm.) as to simulate at first sight the blood picture of acute lymphemia with its large mononuclear cells; some of the myelocytes show, as has already been stated, coarse eosinophilic granules (eosinophilic myelocytes). The cells which have been described, the polynuclear cells as well as the mononuclear, are occasionally large, at other times remarkably small ("dwarf forms"). Besides these immature leukocytes (the myelocytes), which normally are not met with in the circulating blood, other immature forms of erythrocytes also originating in the bone-marrow are found in the circulating blood stream of patients with leukocyte leukemia, such as normoblasts and, more rarely, megaloblasts in varying amounts, at times in great numbers.

The enlargement of the spleen in this variety is especially well marked, the enlargement of the lymph-glands is sometimes quite prominent, at other times less so. [See editorial note on page 351.-ED.] It is remarkable that we rarely have an opportunity to observe the development of the tumors step by step. Usually the physician sees the disease in its full intensity, so that we must assume that leukocyte leukemia rapidly reaches the acme of its development. Besides the splenic tumor and the enlargement of the glands, the other clinical phenomena have already been fully described: The hemorrhagic diathesis, the retinitis, the increase of uric acid in the urine, etc.

From an etiological standpoint there is little of value in the diagnosis of leukemia. In some cases leukemia appears to follow infectious diseases (malaria, diphtheria, the puerperium, influenza, etc.), or occurs in connection with trauma, as concussions (perhaps especially of the bones). These "causes" of leukemia, considered as direct agents producing the disease, are of very questionable nature. This, therefore, justified the sensation which followed the "discovery" of Löwit (who had already distinguished himself by his studies regarding the leukemic process) that the disease was due to the presence of amœbr in the blood and in the organs producing the blood cells. In leukocyte leukemia Löwit found a form of ameba which he believed to increase in the blood by sporulation (hæmamœba leucæmiæ magna); in lymphocyte leukemia another form of parasite differing from the former by its active motility (hæmamœba leuc. parva vivax). Doubtless Löwit's discovery, if confirmed, would be of the greatest value in the explanation of the nature of leukemia; but, unfortunately, we cannot as yet reckon with this factor, as the investigations are by no means concluded and have called forth many contradictory opinions. [There are, I think, no contradictory opinions among those who have studied Löwit's preparations, and repeated his technic. Competent observers are unanimous in believing that Löwit's supposed parasites were, in fact, artefacts. No competent investigator has confirmed Löwit's work.-ED.]

In the author's opinion, leukemia is due to a specific poison, which impairs the process of blood formation, especially that of the white blood cells. In 
his opinion, the important question, in all blood diseases, is this: What kind of poison is it which is affecting the process of blood formation in each individual case? On this depend the changes manifest in the blood.

1. If the damage chiefly affects the red blood cells, simple "anemia" results, in which the erythrocytes deviate more or less from the normal in their number, shape, maturity and hemoglobin percentage, while the white corpuscles are affected only incidentally, and especially as to their number.

2. If the poison affects chiefly the process of gencration of the white corpuscles, so that their number is increased and their morphotic relation is pathologically altered, the blood disease presents itself in the form of a leukemia.

3. If the damage affects all components of the blood equally, blood diseases result that represent a complete upsetting of the process of blood formation with changes in the number and form of both the white and the red blood cells-blood diseases usually described as transitional or mixed forms of pernicious anemia and leukemia. It is difficult to classify satisfactorily the individual cases of this group as anemia or leukemia, if the usual terms, anemia and leukemia, are used as mutually exclusive. I believe, therefore, that it is advisable to give them a special name, for instance, "leukanemia" or something similar. ${ }^{1}$

In this form of blood disease, as already remarked, the production of red and white blood-corpuscles is wholly upset, and accordingly, in the blood picture, decided changes appear in the leukocytes as well as in the erythrocytes, such changes as we are accustomed to note, on the one hand in leukemia, and on the other hand in pernicious anemia. Blood formation may cease entirely, and this deficiency can no longer be compensated in any manner, so that the organism soon succumbs to the pernicious blood disease. Clearly to illustrate the affection which I designate as leukanemia I shall quote the complete history of a case observed in my clinic:

A boy, K. L., aged ten, entered the hospital May 6th, and died May 9, 1900. Patient is said to have been always weak, but never seriously ill, and attended school regularly up to April 22d. Mild symptoms; twice vomiting occurred, so that the patient was compelled to remain at home from April 22d to April 29th. On April 29th he sang as a choir boy in church, and on May 1st again attended school, but on May 5th, on account of his pallor, which the

1 In my opinion, it is well to carry out, in the main, this classification of blood diseases, in spite of the fact that in typical leukemias not only normoblasts are found but also now and then megaloblasts, and although, vice versa, apart from the alterations in the differential count (the predominance of lymphocytes), a few myelocytes are occasionally found in severe anemias. But these findings are of subordinate and secondary importance compared with the primary condition, according to which the disease, in consonance with the fundamental principle of nosology " a potiori fiat denominatio," is to be spoken of in one case as leukemia, in another as anæmia gravis, and by all diagnosticians is thus commonly designated. A disease can only receive the name of "leukanemia" when both leukocytes and erythrocytes are uniformly and decidedly damaged in their development, when the case can neither be put in the category of leukemia nor in that of pernicious anemia, and when the division of blood diseases into two groups is no longer possible. 
teacher noticed, he was sent home. From May 3d to May 6th he still took his food with appetite; then, four days before his death, epistaxis occurred, with yellow discoloration of the skin; pain in the nape of the neck and toothache appeared, as well as pain in the epigastrium. In the course of the day severe apathy became more and more noticeable, increasing to complete loss of consciousness, so that the patient was brought by his relatives to the Julius Hospital.

Status prosens: The appearance of the patient denoted serious illness; skin and mucous membranes deathly pale; eyelids and hands markedly edematous; the skin over the rest of the body slightly bloated, showing yellow discoloration but not jaundice; conjunctiva snow-white. Coma. No opisthotonus, no rigidity of the neck and no spasms. Pupils dilated, reacting normally. Liver and spleen moderately enlarged and hard; sensitive to pressure. The muscles of the calves of the legs not especially sensitive to pressure, but the bones of the thigh as well as the sternum extraordinarily so. Lungs normal. The cardiac dulness extended to the right sternal border; heart sounds clear; gallop rhythm, undulation of the veins of the neck. Urine contained traces of albumin; no albumoses, no casts. The ophthalmoscopic examination showed feeble filling of the vessels, especially of the arteries, which were mere threads_- " marantic thrombosis of the central artery with numerous disseminated hemorrhages." Temperature $102^{\circ} \mathrm{F}$. to $104^{\circ} \mathrm{F}$.

As the symptoms of extraordinary impoverishment of the blood were present, besides a slight enlargement of the liver and spleen, the diagnosis was set down as "severe anemia." On account of the edema of the skin and the presence of albumin in the urine, a nephritis was at first thought to be the cause. This assumption, however, was dropped when it was found that only traces of albumin, but neither blood cells nor epithelium, nor even casts, were present. An acute infection seemed much more likely, and the rapid course of the disease, the severe disturbance of the general condition, the high fever, the enlargement of liver and spleen and the albuminuria were in favor of this. Yet the clinical picture did not correspond to any of the usual infectious diseases. It was obvious that only an exact examination of the blood would clear away these diagnostic difficulties.

The blood count showed an excessive diminution in the number of erythrocytes: 256,000 per c.mm.! The blood-corpuscles varied in size (with some megalocytes), and the triacid stain showed that some red corpuscles contained nuclei : Normoblasts 76, megaloblasts 152, in a c.mm. The amount of hemoglobin of the entire blood was not more than 10 per cent., the hemoglobin contents of the individual blood-corpuscles (color-index) was therefore relatively increased.

The absolute number of white blood cells was scarcely increased, 10,600, though when compared with the total number of the erythrocytes it seemed high, 1:24. The morphology of the leukocytes and the proportion of the individual leukocyte varieties to each other deviated greatly from normal conditions, so that the blood picture resembled that of leukemic blood. Besides the usual neutrophilic, polynuclear leukocytes which were present in diminished amounts $(4,680)$, many myelocytes were seen $(1,380$ neutrophiles and 
76 eosinophiles); mast-cells were absent; however, there were large mononuclear leukocytes (228) and remarkably numerous lymphocytes $(4,260)$, large and small-almost as many as polynuclear cells. Expressed in percentage the blood contained: Lymphocytes 40.2 per cent. (large 35.3 per cent., small 4.9 per cent.); polynuclears (neutrophilic leukocytes) 44.1 per cent.; myelocytes 13.6 per cent. (neutrophiles 13 per cent., eosinophiles 0.6 per cent.) ; large mononuclears 2.1 per cent.; and but very few mast-cells (only one found in all the blood preparations examined).

The course of the affection during the four days in which the patient was in the hospital was extraordinarily rapid. The temperature, which varied between $102.2^{\circ}$ and $104^{\circ} \mathrm{F}$. from the first to the second day, dropped on the third day to $100.4^{\circ} \mathrm{F}$., falling upon the fourth day, when death occurred, to 91.1 $1^{\circ}$ F.; consciousness, which had been lost prior to his admission to the hospital, returned on the second day, and continued until death. The energy of the heart, however, weakened from hour to hour. The cardiac dulness, which was normal at the onset, became more and more extended without the appearance of murmurs. Weakness increased until death occurred on May 9th.

The clinical diagnosis entered at the Pathological Institute was: "Progressive pernicious anemia, enlargement of the liver and spleen, infectious degenerative processes in the bone-marrow with disturbances in the formation of red and white blood-corpuscles; dilatation of the heart, myocarditis (?)."

The main points in the autopsy report (v. Rindfleisch) may be mentioned as follows: Excessive anemia of the entire body. The marrow of the femur in its lower half everywhere red, that of the sternum reddish. Spleen somewhat enlarged, $13.5 \mathrm{~cm}$. in length, $8 \mathrm{~cm}$. in breadth, $3 \frac{1}{4} \mathrm{~cm}$. in thickness; upon section lighter than normal, consistence somewhat soft; Malpighian bodies enlarged, indistinct. Liver of firm consistence, gives a distinct amyloid reaction, which, however, soon disappears when the organ is placed in water. The heart is distinctly dilated, particularly in the right ventricle; the left ventricle somewhat less so. The muscles of the heart are very anemic, parts showing grayish discoloration and punctiform hemorrhages; fatty degeneration of the muscular trabeculæ in the left heart. No enlargement or change in the lymph-glands. Kidneys pale, in part showing a dappled anemia; capsule peels easily; consistence somewhat firmer than normal.

The anatomical diagnosis (v. Rindfleisch) was as follows: Ancemia maxima corporis totius; Hypercemia ossium cum infiltratione cellulari partim leukocytica partim erythrocytica. Lien hyperplasticus ex intumescentia leukocytica corpusculorum Mfalpighii. Hepar modice auctum degeneratio hepatis amyloidea. Degeneratio cordis adiposa prosertim ventriculi sinistri, dilatatio ventriculi sinistri. Myocarditis. Edema pulmonis, atelectasis.

Microscopical examinations (v. Rindfleisch) showed: In the spleen areas of softening, in the internal parts of which were abscess-like accumulations of polynuclear cells but without pyogenic cocci; a softening of all the Malpighian bodies with transformation of their lymphocytes into pus cells. Amyloid degeneration of the liver not affecting the interstitial tissue but merely the liver cells proper, which alone gave the amyloid reaction. The blood- 
vessels as a whole were free from amyloid but contained numerous detached large myelocytes besides red corpuscles. As a curious finding may be mentioned small foci of liquefaction about one-fourth the diameter of an acinus filled exclusively with polynuclear leukocytes and liver cells in the process of gradual dissolution, but containing no cocci. These foci were situated sometimes near the edge of the acinus, sometimes near the center, and were rarely confluent. No siderosis could be determined in several examinations. The heart muscle showed fatty degeneration with fragmentation of the fatty muscle fibers and hemorrhages the size of a millimeter. Kidneys principally anemic. The bone-marrow contained chiefly large myelocytes with large nuclei, slightly or not at all granular. Normoblasts were found mostly around the small hemorrhages; megaloblasts were more scanty.

Epicrisis.-The blood findings, in so far as they relate to the red bloodcorpuscles $(250,000$ erythrocytes, 76 normoblasts, 152 megaloblasts in a c.mm.), would class this affection as pernicious anemia, and at that the usual form-the metaplastic-with transition of the yellow bone-marrow into red, with formation of profuse, nucleated red blood-corpuscles, and especially of megaloblasts in the marrow, and their entrance into the circulating blood. The erythrocytes were relatively rich in hemoglobin, corresponding to the usual finding in pernicious anemia.

The condition of the white corpuscles in the case in question is more important than that of the red. We note, point for point, that the findings correspond to leukemic blood, yet there is no absolute increase worth mentioning in the white blood cells.

The great number of myelocytes in the blood (and also in the bonemarrow) is primarily conspicuous; they form one-seventh of all the leukocytes. According to our present knowledge of the genesis of the leukocytes, myelocytes may be looked upon as the product of blood cell formation in the bone-marrow, and as the prior stage of the polynuclear leukocytes of the blood. It can hardly be disputed that in our case the transportation into the blood of these immature leukocytes (the mononuclear neutrophilic and eosinophilic myelocytes) represents a process in the blood analogous to the emigration of the immature erythrocytes, the normoblasts and the megaloblasts. This analogy is so natural that the question may well arise whether this condition -emigration of immature leukocytes with the simultaneous emigration of the immature erythrocytes from the bone-marrow into the blood-does not occur frequently in pernicious anemia, and even whether it does not invariably occur. To decide this question, only the latest investigations, based upon methods of staining that are in use to-day, should be considered. From researches which are in this respect decisive it is shown that the appearance of myelocytes in the blood of patients suffering from pernicious anemia is not usual; generally none or apparently but few (at the utmost up to 0.5 per cent.) of these immature leukocytes are found. In our case, however, the blood was rich in myelocytes-they represented one-seventh (!) of the white blood cells and the onc-hundred-and-eighty-fourth part of all the blood cells. We must, therefore, assume in this rare case, that we were dealing with an insufficiency in the function of the bone-marrow, affecting simultaneously the 
formation of the red as well as of the white cells, so that the embryonic forms of the red and white cells did not come to maturity. These cells, i. e., of the (nucleated) normoblasts and megaloblasts as well as of the myelocytes, appear to have emigrated into the blood before their transformation into normal, finished blood cells could be completed.

Another striking feature of the case was that (besides the neutrophilic or eosinophilic granular myelocytes) non-granular myelocytes and even, what is more conspicuous, non-granular polynuclear cells could be demonstrated with certainty in the circulating blood—a fact that may perhaps be explained by supposing that in this rapid and abbreviated hematopoietic process not even granulation could take place, much less complete maturity of the leukocyte forms produced in the bone-marrow.

While the individual points in the blood picture of our case which have been described up to this time can be brought into genetic connection only with a severe damage in the function of the bone-marrow, this source of disturbance of the blood formation is questionable because of the remarkable relative increase of the lymphocytes in the blood ( 40 per cent. of lymphocytes compared with 44 per cent. of polynuclears).

We might at first suppose the latter to be only an expression of the markedly diminished production of the polynuclears in the bone-marrow, i. e., a relative increase of the lymphocytes because the formation of polynuclears in the bone-marrow is reduced, while that of the lymphocytes in the spleen and lymph-glands continues unhindered-and this corresponds to the important fact which Strauss determined-the relative increase of the lymphocytes in pernicious anemia. The circumstance that 35 per cent. of large, and only 5 per cent. of small, forms were found in the blood is opposed to this view. Therefore a disturbance in the formation of lymphocytes must in this case be assumed. It has lately been regarded as certain that lymphocytes are normally produced not only in the spleen and in the lymph-glands but also in the bone-marrow; hence it appears rational to hold the damaged function of the bone-marrow responsible also for the disturbance in lymphocyte formation which, in our case, no doubt existed. But, in my opinion, this is impossible, since the lymphocytes in the bone-marrow, examined post mortem, were very few, while, in contrast, the tissue fluid taken from the spleen one hour after death contained profuse amounts of large and small lymph-cells. It appears to me, therefore, that any other view than the following would be forced, namely, that the increase of lymph-cells in the blood was due to a greater formation of these cells in the spleen. This idea is the more plausible since the lymph-glands in our case were everywhere absolutely unchanged.

The results of the analysis in this case may, therefore, be summarized as follows:

A simple pernicious anemia was not present, in spite of the fact that the blood showed the characteristic changes of pernicious anemia in the hemoglobin-containing cells circulating in the blood, the erythrocytes. Against pernicious anemia was the enormous production of myelocytes and their transportation into the blood, the scanty presence of megaloblasts in the bone-marrow, and the absence of siderosis of the liver. But the diagnosis of lenkemia does 
not fit the facts any better; for, in this case, there was no absolute increase of the white blood-corpuscles and no absolute increase of the eosinophile cells; in fact, the number of them in this case was decidedly diminished $(0.6 \mathrm{per}$ cent.); moreover, Charcot's crystals were not present in the bone-marrow. These facts scarcely coincide with the assumption of a leukemia which, in this case, must certainly have originated in the bone-marrow (it must be remembered that the myelocytes alone constitute one-seventh of all the leukocytes).

We may, therefore, say that in this case we were dealing with a severe, perhaps infectious, disturbance of the process of formation of the blood cells in the bone-marrow, and that this affected the red as well as the white bloodcorpuscles, with the result that both remained in an immature condition, the white cells not even being perfectly granular. This reduction of the function of the bone-marrow in a few days led to the complete cessation of blood formation, and this to the destruction of the vitality of the organism. Besides the cessation of the function of the bone-marrow, the function of the spleen was severely damaged as regards the production of lymphocytes, which were produced in a hurry and hence entered the blood chiefly as large lymphocytes.

The essential feature of this case of leukanemia is the fact that the development of both red and white corpuscles was affected. In leukemia, on the other hand, it must be supposed that the specific poison is concentrated essentially upon the white corpuscles, sometimes the lymphocytes, sometimes the leukocytes, according to the nature of the irritant. The process in leukemia is probably as follows:

By the continuous, not transitory, action of the poison, a more rapid production and washing out of white cells is brought about, and during this process, not only mature forms but also immature elements, sometimes more, sometimes less, pass into the blood-in the leukocytic leukemia the myelocytes, in the lymphocytic leukemia large lymphoid cells (which may be regarded at least with probability as immature elements, normally not yet fit to pass into the blood). The emigration of the complete polynuclear cells (and partly perhaps of the myelocytes) may be easily explained as a consequence of their ameboid contractility, whereas the enormous emigration of the lymphocytes which in lymphocyte leukemia are incapable of ameboid movement is apparently difficult to understand. However, I believe there is nothing against the assumption that the lymphocytes, because of their production in excessive numbers, may also enter the circulating blood in large numbers, as they do under normal conditions, i. e., by "passive exudation," owing to an unusually strong lymph circulation, etc., provided the processes which normally cause the entrance of the lymphocytes are more active, which is true to a varying extent in different cases.

This of itself leads us to discuss the question of the part in the leukemic process which is taken by the various organs in which blood cell formation normally takes place. It may to-day be regarded as certain that the bonemarrow is particularly implicated in leukemia, not only in leukocyte leukemia but also in lymphocyte leukemia. 
We may assume that normally the majority of the lymphocytes are produced in the spleen and lymph-glands, and only a few in the bone-marrow. As, however, hyperplasias of the spleen and lymph-glands occur without lymphemia ("pseudo-leukemia"), as well as with it (lymphocyte leukemia), and since, moreover, there are cases of leukemia with lymphadenoid changes of the bone-marrow but without enlargement of the spleen or lymph-glands, we may conclude that the inplication of the bone-marrow, i.e., the supervention of increased marrow activity in simple hyperplasia of the spleen and lymph-glands, represents the most important element in the development of leukemia (Neumann). However, in my opinion this does not explain why an increase of the lymphocytes in the blood may not occur in hyperplasia of the spleen and lymph-glands without an affection of the bone-marrow. As the small lymphocytes which are formed in the lymph-glands under normal conditions permanently enter the circulating blood, it is not apparent why, with an increased production in the lymph-glands and with a marked migration into the blood, they should not appear there in greater numbers. On the other hand why do we always have lymphemia when the bone-marrow is implicated? The explanation that in hyperplasia of the tissue of the spleen and lymph-glands their capsule also dilates, and therefore (in contrast with the conditions in hyperplasia of the bone-marrow), no mechanical migration of the lymph-cells into the blood occurs, is contradicted by the reflection that if this were true every disturbance in the expansile power of the capsule should result in a marked overflow of lymph-cells into the blood. In my opinion, it is better for the present not to consider pseudo-lenkemia at all in connection with leukemia, and also not to look for the nature of leukemia in the increase of the white blood cells alone.

We cannot, I believe, refrain from asking what becomes of the white blood cells which are so enormously increased in the organism in leukemia. That in health the leukocytes are partly excreted and partly destroyed in the internal parts of the body is not to be doubted. If, with the enormous increase of white cells in the blood of leukemics, the consumption does not go hand in hand with the increased production, a surplus of white cells in the blood must result. But there is no cogent proof as yet for the view that there is any such increase in the consumption of leukocytes, which in this instance would have to be enormous in order to keep pace with the increased production.

My conception of the pathogenesis of leukemia is, therefore, the following: The action of a specific agent causes a pathologically great stimulus to the growth of the hematopoictic tissues of the body, which especially affects the production of white blood-corpuscles. As a result of this, a flooding of the blood, at one time with lymphocytes, at another time with leulkocytes, with mature and immature forms of the same, occurs. This change in the blood is continued, partly by the pathologic irritation of the blood-forming organs, partly because the superfuous white blood cells are insufficicntly used up in the economy of the body. 


\section{DIAGNOSIS}

The diagnosis of leukemia is in the majority of cases not difficult; indeed it is very easy in well marked cases of the disease. We are always dealing with leukemia when the proportion of white cells to red is 1 to 15 or less, and the absolute count of leukocytes or lymphocytes (according to the case) is 30 to 50 times greater than normal, and when, besides the relative and absolute increase in the number of white cells, an immaturity of the cells is strikingly manifest. One finds in the blood besides mature leukocytes and lymphocytes many large mononuclear cells and abundant myelocytes, large lymphocytes and also immature forms of erythrocytes, i. e., nucleated red blood-corpuscles in large or small amounts, and in most striking profusion in leukocytic leukemia. In leukocytic leukemia we are struck, moreover, by the absolute increase of eosinophiles and mast-cells.

Having diagnosticated leukemia, we must now determine to which form it belongs, whether to the lymphocytic or leukocytic type, which is not diffcult if we remember the above mentioned points of distinction.

If the immature forms of blood-corpuscles are absent from the circulating blood, and if a "polymorphia" of the white corpuscles is lacking, we are not dealing with a case of leukemia, at least not of leukocytic leukemia, but with a leulkocytosis in which myelocytes are never found (except in the very rare, isolated cases in which there is an excessively hasty production of blood cells). [Myelocytes are not at all infrequently found in leukocytosis, i. e., in peritonitis, in diphtheria, in pneumonia and in malignant diseases.-ED.] As the blood pictures of leukocytosis and leukemia resemble each other in so far as an increase in the number of leukocytes is concerned, we must now discuss more thoroughty the manner in which leukocytosis originates, and the blood picture of leukocytosis.

\section{LEUKOCYTOSIS}

In some physiological processes, viz., during digestion, pregnancy, after bodily exercise, etc., we find, upon examination of the blood, an increase of the white cells. [That there is no leukocytosis of digestion or of pregnancy seems to me demonstrated by the more careful researches of recent years. There is a midday leukocytosis in most persons and this may coincide with the after-dinner period. But after breakfast and after supper there are no changes to speak of.

The supposed leukocytosis of pregnancy is not observable, as a rule, until a few days or hours before labor; sometimes not until labor has begun. After parturition there is usually a well marked leukocytosis lasting several days. -ED.] In this "physiological" leukocytosis the proportion of the various forms of leukocytes to one another does not vary greatly from the normal, whereas it is characteristic of pathological leukocytosis that, according to the nature of the disease in the course of which a leukocytosis appears, marked alterations are noted in the proportionate numbers of the various leukocyte forms. According to whether the lymphocytes or the leukocytes are increased, 
we speak of "lymphocytosis" or "leukocytosis" in a restricted sense, and in this way, according to the prominence of one or the other leukocyte forms in the blood picture, we may differentiate between a polynuclear neutrophitic, and an eosinophilic, leukocytosis.

It has been declared at various times that the lymplocytes have no independent, active motility; if, therefore, in the course of pathological conditions a one-sided (symptomatic) increase of lymphocytes is noted in the blood, we must consider that large numbers of them have reached the circulating blood by reason of an increased cell production in the cytogenic tissue of the lymph-glands (and of the bone-marrow to a much less extent) and by a more active lymph circulation ("passive leukocytosis," Ehrlich). This form of leukocytosis, "lymphocytosis," is quite rare, occurring especially in catarrhal affections of the stomach and intestines of nurslings, in pertussis, in severe rickets, syphilis, etc., after injections of pilocarpin and tuberculin, and after extirpation of the spleen.

More frequent is the second form of leukocytosis, polynuclear leukocytosis. Various irritations (infectious agents, chemical poisons, etc.) cause these leukocytes, which are always present in great amounts in the bone-marrow [as well as in the capillaries of the liver and especially of the lungs.-ED.] and which, on account of their ameboid movement, are always ready to pass into the blood, to emigrate, and to enter the blood by "chemotaxis" ("active" leukocytosis). As a rule, it is the neutrophilic polynuclear leukocytes which circulate in the blood in large numbers in leukocytosis; i. e., almost all intoxications and infections lead to this, the "usual" form of leukocytosis. Such causes are: Poisoning by potassium chlorate, phenacetin, oil of turpentine, albumoses, arseniuretted hydrogen, etc.; also cachectic conditions as a result of malignant tumors, phthisis, severe blood loss; and, above all, the various infectious diseases-pneumonia, sepsis, diphtheria, erysipelas, etc. On the other hand, in certain pathological conditions we note a one-sided increase of the eosinophilic cells (eosinophilic leukocytosis). This is the case in bronchial asthma, in various diseases of the skin, in malignant tumors, and especially also in helminthiasis (due to the presence of tenix, ascarides, ankylostoma, etc., in the intestine. [As well as in echinococcus disease, trichiniasis, Bilharziosis, trypanosomiasis and practically all the diseases due to animal parasites.-ED.]

Leukemia represents a morbus sui generis, the nature of which, as we have seen, depends upon a pathological blood formation, i. e., not only upon an excessive increase of the white blood cells but also upon their qualitative change (the passage of immature forms into the blood). In leukocytosis, on the other hand, the condition is only a functional alteration of the bloodforming organs, and in the majority of cases (leukocytosis in the restricted sense) exclusively of the bone-marrow, which reacts to pathological irritation by the formation and expulsion of large numbers of normal and mature colorless blood-corpuscles. This explanation of leukocytosis, the strict conception of which as an inereased function of the bone-marrow was formulated by Ehrlich, has lately been fully confirmed experimentally by Rubinstein [and still more fully worked out by Longcope in his studies of the marrow 
in typhoid, pneumonia, and peritonitis.-ED.]. After the injection of an agent causing leukocytosis, he followed step by step the changes in the bonemarrow. As soon as the leukocytes appeared in the blood in large numbers, the granular, mature elements in the bone-marrow almost wholly disappeared, and, instead of these, in a few days, the immature forms, especially the myelocytes, increased. The latter, therefore, covered the loss due to the enormous emigration of the polynuclears into the blood as a result of the leukocytosis. The spleen and the lymph-glands, however, in Rubinstein's experiments, took no part in making up the loss of white corpuscles in polynuclear leukocytosis, but, according to our present opinion regarding the separate development of leukocytes and lymphócytes, this is not to be wondered at.

From what has been stated, it is clear that the diagnosis of leukocytosis rests primarily on the discovery of a disease which is recognized as usually giving rise to leukocytosis. The diagnosis becomes more certain if the increase of the white blood cells is a transitory one (i. e., disappearing with the cessation of the underlying affection), and remains from the onset within moderate limits. While in leukemia ratios of 1 white to 10 red corpuscles, and even 1 to 2 , are quite usual, in leukocytosis there is a much slighter relative increase of leukocytes, and only in exceptional cases, as in cachectic conditions when there is a decided decrease of the erythrocytes simultaneously with leukocytosis, does it happen that proportional figures of 1 to 20 are observed for a long time. Under such conditions these cases may simulate leukemia, but here the recognition of morphological alterations in the white. bloodcorpuscles leads to the correct diagnosis, because the excessive appearance of myelocytes, eosinophiles, and mast-cells in the circulating blood (in short, a markedly polymorphous condition of the blood elements which is quite foreign to leukocytosis) directly favors the existence of leukemia. The diagnosis of the latter disease is also aided by the decided enlargement of the spleen and lymph-glands, and the absolute and relative increase of uric acid excretion in the urine, etc.

These last named factors especially must be taken into consideration when we are dealing with a lymphocytosis, because here the most important point of distinction between leukemia and (polynuclear) leukocytosis, the polymorphia of the blood cells, is of no avail. In practice, however, the diagnosis of lymphocytosis is never doubtful, the difficulty in differentiating between a lymphocytosis and a feebly developed lymphocytic leukemia being theoretic rather than actual. [On the contrary, mistakes have actually occurred. See Steven (Lancet, 1902, vol. xx) and Cabot ("Clinical Examination of the Blood," 5th edition, 1904, p. 194).--ED.]. For, in the acute form of lymphocytic leukemia, the large lymphocytes almost always predominate, and this alone is sufficient to prevent confusion. between lymphocytic leukemia and lymphocytosis; even in chronic lymphocytic leukemia the large lymphocytes are not entirely absent in the microscopical preparations, although, in the great majority of cases, only small leukocytes are found.

Above all, the usually enormous increase of lymphocytes in lymphocytic leukemia, the progressive, pernicious nature of the disease compared with the 
transitory, benign character of the increased number of lymphocytes in lymphocytosis, determines the correct diagnosis. On the other hand, the following symptoms point to leukocytosis: The absence of enlargement of the spleen and of the lymph-glands which is almost always present in lymphocytic leukemia; and, secondly, the presence of a primary disease which would lead to leukocytosis (gastro-enteritis infantum, etc.).

\section{PROGNOSIS}

The prognosis of leukemia may be briefly summed up: it is absolutely unfavorable; death is the only outcome of the disease. This is true not only of the acute form of leukemia in which death occurs usually in a few days or weeks, but also of chronic leukemia, of lenkocytic leukemia, as well as of chronic lymphocytic leukemia. However, in both forms of chronic leukemia, especially leukocytic leukemia, remissions may occur with considerable improvement of symptoms, and the disease may sometimes last for from one to four years. But since we have learned positively to recognize leukemia by new methods of blood examination, no actual cure has as yet been observed. From this standpoint we must look with suspicion on "recoveries" reported from time to time, and I do not hesitate to pronounce as doubtful a case of cure which came under my observation twenty-five years ago, since the diagnosis made at that time does not conform to the requirements of to-day.

This gloomy view concerning the prognosis of leukemia is, however, based on the present state of our experience, and it is to be hoped that when we obtain more exact knowledge of the nature of the specific poison causing this disease, our therapy will become more effectual, and the prospects of cure more favorable.

\section{TREATMENT}

At the present time there is nothing that can be regarded as the fulfilment of an indicatio morbi. All that has been thus far attempted in treatment may be regarded merely as efforts to feel our way, and these have so far been abandoned as soon as undertaken. A few drugs only have held their place in the treatment of leukemia, and by most physicians, as well as by myself, are employed faute de mieux, though it is true that the long duration of a few cases treated in this manner, and the transitory but decided remissions in the course of the disease may, at least in part, be referred to the action of the remedy.

Arsenic is the drug which most deserves our confidence. During its administration, we see in some cases an improvement in the condition of the blood (not only an increase in the number of red but also a decrease in the number of the white corpuscles), an arrest of the splenic enlargement, and the disappearance of the hemorrhagic diathesis.

The best way of administering arsenic is in the form of Fowler's solution with two parts of aqua cinnamomi: 
R Solutio kalii arsenicosi............... 10.0

Aq. cinnamomi ................... 20.0

M., D., S.: Gtt. xv-xxx, three times daily.

We begin with $15 \mathrm{gtt}$. t.i.d. (taken after meals), and increase gradually, i. e., after one week $20 \mathrm{gtt}$. t.i.d., after the second week $25 \mathrm{gtt}$. t.i.d., and after the third week $30 \mathrm{gtt}$. t.i.d.; this dose we maintain for some time. I am not enthusiastic regarding the subcutaneous use of arsenic. The injection of arsenic into the circulating blood could only be desirable if the results obtained by this method were incomparably more brilliant than after the administration of arsenic by the mouth, and this is decidedly not the case.

Another remedy producing appárently favorable results is quinin. Its action is asserted to be remarkable, especially if it be combined with arsenic or iron. In this respect, the mineral waters containing arsenic and iron, as Levico and Roncegno water, and the Levico water used in the form of baths, deserve consideration.

In the majority of cases the erythrocytes decrease to one-half their number or less with the increase of white corpuscles (at least in the latter course of both leukocytic and lymphocytic leukemia), and here the administration of arsenic, iron and quinin, whose favorable influence on the red corpuscles cannot be denied, is warranted from a theoretical standpoint, as well as empirically.

Iodin preparations, formerly prescribed to some extent, have recently been less used; no appreciable results of their use have been noticed. The same is true of phosphorus in doses of 0.001 to 0.005 , which has been praised, especially by English physicians.

Two therapeutical measures which were recently hailed with enthusiasm, oxygen inhalations and organotherapy (preparations made from lymph-glands and bone-marrow), have produced no striking results, although amelioration and a slower progress of the disease have been supposed to follow their administration. In general, what has been said of the administration of arsenic, iron, and quinin is true also of this method of treatment. We may use these remedies, indeed, we must use them, but we can only hope for a partial success; the disease up to this time has been invariably fatal, but, as a rule, lasts a year or longer, and calls for a display of humanity on the part of the physician which is not shown by quietly folding his hands and rejecting any method of treatment which offers the patient the slightest hope of recovery or even improvement.

Although I strongly recommend the trial of the above-mentioned therapeutic measures in the treatment of leukemia, just as strongly must I condemn local procedures. Not only does the lack of results militate against their use, but we are thoroughly convinced that the nature of leukemia is not to be found in the affection of the spleen or of any individual lymph-gland, nor in any other organ accessible to local treatment. I believe that the time has come to discontinue all efforts to cure the disease by injections of arsenic, ergotin, etc., into the spleen, by faradization or galvanopuncture of the organ, or even by extirpation of the glands, or by splenectomy which, without an exception, has been rapidly followed by death. 
It is much more sensible to retard the increased albumin waste in the organism by rational diet, or, in its later stages, to compensate it, at least partially, by increased nutrition. A nourishing diet, rich in nitrogen, is therefore to be advised because a well-nourished organism will always offer more resistance to the disease than one debilitated by cachexia. To strengthen the body, besides judicious diet, moderate systematic exercise in the open air and the avoidance of too great physical exertion must be insisted upon.

Of course, the indicatio symptomatica may require different expedients. Marked diarrhea must be combated by tannigen and opium, dyspeptic symptoms by regulation of the diet, lavage, etc., exhausting sweats by camphoric acid and atropin, a marked hemorrhagic diathesis by ergotin or other preparations of ergot in large doses (ergotin 0.2, five times daily, secale 10.0 to 150 , to be used in two days), cardiac asthenia by camphor, etc. It is unnecessary to enumerate these indications in greater detail, for the treatment of individual symptoms and complications is in accordance with the same rules as in other discases. Only I must caution against the too liberal use of drugs in leukemia; we must not forget that we are dealing with the debilitated, the desperately ill, in whom it might be dangerous to act according to the principle "anceps remedium melius quam nullum."

[The treatment of leukemia by X-rays certainly deserves mention. Since Senn's original observation in 1902, 30 or 40 cases have been published in this country in which the disease has been treated by exposure of the splcen or (in lymphatic cases) of the spleen and glands to the Roentgen rays.

Cases are treated daily, biweekly, or weekly according to the condition of the skin and its ability to resist "burns." In a few cases, the rays have been made to fall upon the long bones.

In the majority of these cases improvement, sometimes remarkable and (so far) lasting, has followed the treatment. A few cases have been altogether unaffected, a number have relapsed after temporary improvement, and a small number have becn worse after the treatment. In acute febrile cases there has been no success.

The rationale of the treatment is not at all clear, for the exposures have usually been made over the spleen, and not over the marrow where the disease is most active. We know from the researches of Warthin and others that the X-rays have a specific leukocytolytic effect on the blood forming organs of animals, and if the over-production of leukocytes were occurring in the leukemic spleen instead of in the marrow the effect of the X-ray would be comprehensible. At present it remains a mystery, but it is none the less advisable that all subacute and chronic cases of either variety of leukemia should be given X-ray treatment, which at present offers the best possibility of retarding (or curing?) the disease.-ED.] 


\title{
PSEUDO-LEUKEMIA (HODGKIN'S DISEASE AND BANTI'S DISEASE)
}

\author{
By H. SENATOR, Berlin
}

WE often meet with cases characterized by the following lesions: a more or less well-developed anemia, together with multiple lymph-gland enlargements, a very decided enlargement of the spleen, or both. This glandular and splenic enlargement also occurs in leukemia, which, however, differs from pseudo-leukemia in possessing a characteristic condition of the blood, particularly a great increase of leukocytes, which is not present in pseudo-leukemia.

To this difference from leukemia, which these cases often resemble greatly, Bonfils and Wilks in the year 1856 were the first to call attention, though Virchow had described leukemia eleven years previously, in 1845.

Soon the publication of cases of this kind increased, and new names were repeatedly invented for them. Bonfils designated his cases as "cachexia without leukemia"; Wilks called his "anemia lymphatica," and later "Hodgkin's disease," since Hodgkin had described such cases explicitly, long before the discovery of leukemia, that is, in the year 1832. The counterpart to "anemia lymphatica" (in which enlargement of the lymph-glands is the only lesion or is much more marked than that of the spleen) is the term "anemia splenica," a condition in which the lesions are confined to the spleen. The combination of both conditions has been called "anemia lymphatico-lienalis." Still other names have been proposed which I do not intend to mention, since they are based wholly on the anatomical condition without regard to the clinical phenomena, and are not adapted to all the cases belonging in this category. They have all been superseded by the term introduced by J. Cohnheim in the year 1865, "pseudo-leukemia," which, in spite of all objections, has maintained its place until to-day and will probably maintain its vogue for a long time.

It is true that "pseudo-leukemia" has become a collective name for a number of different affections, but since these affections cannot always be separated from one another, and since this name is short and conveniently expresses what is common to them all (namely, that they resemble leukemia but still are not leukemia, at least clinically), the name pseudo-leukemia will be employed in this article.

The difficulties in the nomenclature of this disease are the same as, for example, in "ileus." Every physician knows that the clinical picture designated by this name is brought about by various anatomical changes of the 
intestine. But as we are not always able to differentiate these changes we cannot discard the name which describes the typical clinical picture.

This, of course, must not prevent us from investigating so far as is possible the anatomical conditions in each individual case of pseudo-leukemia, and finding, if we can, the exact lesion present, particularly as support may be gained in this way for prognosis and therapy. [The stndies of Dorothy Reed, confirmed by those of Longcope and of Simmons, have, I think, established Hodgkin's disease upon a definite histological basis, and differentiated it alike from tuberculosis, from sarcoma, and from leukemic infiltrations. In view of these findings, the diagnosis can and should be made by the excision of one (or more if need be) of the superficial lymph-glands and examining it histologically._Ev.]

The organs which are to be considered in pseudo-leukemia, the lymphglands and the spleen, are almost always simultaneously affected, although not often to the same extent. In pseudo-leukemia we know but little regarding the third organ which is important, or probably important, in blood formation, namely, the bone-marrow; it has sometimes been found hyperemic and of the same consistence as in leukemia, but in regard to this further investigation is necessary.

In the majority of cases the enlargement of the lymph-glands is more conspicuous than that of the spleen. Hence some clinicians recognize as "pscudoleukemia" only those multiple lymph-gland enlargements first described by Hodgkin, and distinguish sharply the cases in which enlargement of the spleen is more prominent, or is present alone. To me it appears that this limitation of the conception "pseudo-leukemia" is unwise, for an enlargement of the lymph-glands alone or of the spleen alone rarely occurs. Even though during life we find many cases with decided enlargement of the glands, and no apparent enlargement of the spleen, still I believe that at the autopsy the spleen is almost invariably found enlarged. On the other hand, in cases with predominant enlargement of the spleen, some enlarged lymph-glands are almost always found at the autopsy, if not the superficial ones then those of the abdomen or of the thoracic cavity. A sharp distinction therefore cannot be drawn here, and there are many cases in which the spleen and lymph-glands are equally-or, rather, proportionately enlarged.

If, however, we wish to lay special stress either upon lymph-gland enlargement or upon the spleen, in the former case we may use the terms "pseudoleukemia lymphatica," or true Hodgkin's disease, in the latter case "pseudoleukemia lienalis" or "splenica"; finally the cases with equal implication of the lymph-glands and of the spleen may be designated as "pseudo-leukemia lymphatico-lienalis" or "spleno-lymphatica."

We shall first describe the more common form, pseudo-leukemia lymphatica or Hodgkin's disease.

\section{HODGKIN'S DISEASE}

The most conspicuous and usually the first symptom is the enlargement of a gland or of a few glands in the neck, oftenest at the angle of the jaw. This enlargement occurs as a rule quite gradually, so that only in the course of 
several weeks or even months does it become noticeable. By this time the individual gland has attained the size of a cherry or a small walnut, or a group of swollen glands has become conspicuous to sight and touch.

The swellings are painless. After a latent period of varying duration they begin to increase following a definite course next to be described. First, more glands are involved, usually those in the neighborhood of the one first affected, i. e., those in the lower part of the neck or in the back of the neck, later those in the axilla, at the eibow, in the inguinal region, occasionally those in the popliteal space. Next comes an increase in the size of the individual glands, which may become as large as a man's fist or even larger. The glands of the neck and axilla, which are those first affected, are always most advanced in growth, while those of the inguinal region and of the thigh are less markedly enlarged.

The enlarged glands can usually be felt and defined with ease; in other cases they are more or less matted together; they are more frequently soft than hard in consistence, and in many cases they may be readily "moved about. As a rule the skin above the glands can easily be raised, but shows no other change. The spleen, as has been said, in this form of pseudo-leukemia, Hodgkin's disease, is almost always implicated, but to a much less degree than the glands, so that its enlargement cannot always be determined with certainty during the life of the patient.

Add to these facts the vista of a gradual increase in the glandular enlargement, while almost exactly in proportion to this the general condition of the patient deteriorates so that he becomes anemic, loses his strength and emaciates, and we are in possession of the most important features of the clinical picture.

But in these outlines the entire symptom-complex is by no means revealed. Indeed it may be very complicated.

The symptoms are in part purely local consequences of the existence of the tumors, and hence depend particularly upon the pressure which these glands exert, or upon the metastases which they produce. But there are other symptoms of a general nature, which result partly from the anemia and cachexia, partly from the actual nature of the disease which is wholly unknown to us.

Before recounting the symptoms I must add that, besides the glandular enlargements which are externally visible and palpable, there is almost invariably an enlargement of the internal lymph-glands, those of the thoracic and abdominal cavities. These internal glands are sometimes large, sometimes small and therefore not always clinically recognizable. Cases do occur in which only the internal lymph-gland's are enlarged, the external ones not at all or to an exceedingly slight degree. I have myself seen a very remarkable case of this type, to which I shall recur later. We must admit that such cases are not likely to be diagnosticated clinically; the pathologist designates them as multiple lymphomata or as lymphomatosis, the same title given by him to the external enlargements which occur in Hodgkin's disease.

It is these internal glandular enlargements which bring about the most severe pressure phenomena, especially in the thorax where there is less room for growth than in the abdominal cavity. Enlarged glands in the anterior 
mediastinal space press particularly upon the innominate vein, and thus produce swelling of the veins of the neck and the wall of the chest with cyanosis and edema of the face, to which may be added dropsical effusions into one or both pleuræ. These phenomena, however, are not so frequent or so marked as with other tumors and tumor-like formations in the thoracic cavity, for the glandular swellings are usually softer and less resistant than other tumors. It is true that harder forms occasionally occur depending upon histological variations the discussion of which will follow later on, and these hard tumors may produce all the phenomena of pressure and stasis.

The development of dropsical effusions may be produced in three ways: (1) by venous stasis; (2) by displacement of the lymphatic trunks, causing a hindrance to the absorption of tissue fluid; and (3) by progressive anemia and $c a-$ chexia.

More common than stasis phenomena are diffculties in respiration. These are caused, (1) by direct pressure which the glandular swellings exert upon the larynx, trachea or bronchi: (2) by pressure upon the vagus, or (very frequently) upon the recurrent laryngeal nerve; (3) by the decrease of erythrocytes which are the necessary oxygen carriers in the function of respiration. Difficulty in deglutition as the result of pressure upon the pharynx or esophagus is rare.

Enlargements of the thoracic lymph-glands also produce both dropsical effusions into the pleura, and true inflammations of the pleura (unilateral or bilateral), which cannot always be differentiated from dropsy, since there are fluids intermediate between dropsical effusions (transudates) and inflammatory effusions (exudates).

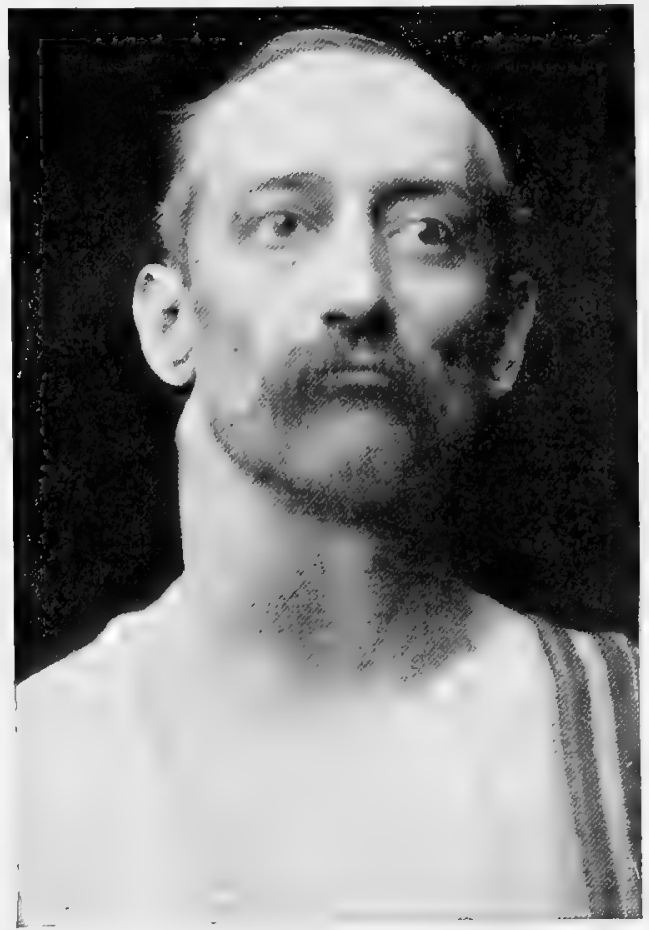

Fig. 21.-Case of Hodgrin's Disease in Dr. SalINGer's Ward IN the Jefreerson Medical College Hospital. Operated upon by Prof. W. W. Keen for the removal of large masses of lymphglands pressing upon the trachea, which produced attacks of suffocation.

Another symptom which is probably to be referred to the local pressure exerted by the glandular tumors is neuralgia. I have scen it most frequently in the lower extremities, especially in the course of the sciatic nerve or the crural nerve. In a case of this kind in which the patient was troubled with painful contractions in the left ilio-psoas muscle, I could feel in the corre- 
sponding abdominal region distinct glandular enlargements which probably exerted pressure upon the muscle and the nerve which supplied it, and thus caused the pains and contractions.

The urine shows nothing special; its composition is that which is usual in anemia provided no complications are present. Occasionally, as in leukemia, it is found conspicuously rich in alloxur bodies, particularly uric acid. These bodies, together with the nucleo-histon which was once found in the urine in pseudo-leukemia by A. Jolles, are explained by an increased destruction of leukocytes. ${ }^{1}$

Finally, as a frequent but not invariable symptom, we must mention hemorrhages from various organs, particularly from the nose and in the skin in the form of petechix of varying size, rarely as larger effusions of blood. It appears to me that the tendency to hemorrhage is particularly great in cases with glandular enlargements in the internal parts of the body, in the thorax, and, above all, in the abdominal cavity. The case previously referred to, in which only these internal glands were enlarged (and very decidedly so), ran its course with the clinical picture of Werlhof's disease, i. e., with hemorrhages from the nose and mouth, hematemesis, and blood in the urine, but without other noteworthy symptoms except slight fever. The autopsy showed hemorrhages in almost every organ, and numerous lymphomata in the thorax and abdominal cavity of which we had no suspicion during the patient's life.

Shall we look upon these glands as the cause of the hemorrhages? This is hardly possible, for they are invariably absent in Werlhof's disease, while, on the other hand, glandular enlargement is often present without any tendency to extensive hemorrhages. We can only say that glandular enlargements sometimes lead to a radical change in the blood in consequence of which hemorrhages occur, and that these changes are particularly noted when the lymph-glands of the internal parts of the body are diseased.

In this case, as I have said, only the internal lymph-glands were enlarged; in other eases the lymph-gland chains situated in the digestive canal, i. e., the tonsils, the glands of the mucous membranes of the mouth and cheek, the solitary and agminate glands of the intestine may be affected, with or without involvement of the external lymph-glands. In consequence of these enlargements all sorts of otherwise inexplicable disturbances occur.

Ferer does not belong to the typical picture of Hodgkin's disease. Now and then slight rises in temperature occur, particularly when fresh glandular enlargements develop rapidly, but fever is not characteristic, and in some cases may be entirely absent until the anemia becomes very grave, or until near the end of life. This last type of fever corresponds to the febrile conditions which are observed in other sevcre anemias. Of course there may be at any period in the disease a pyrexia due to complications.

I am not unmindful of the fact that some isolated cases have been described in which fever was a most prominent symptom, but which, nevertheless, have shown in the main the clinical course of Hodgkin's disease. In these cases

1 From the communication of Jolles it could not be determined whether in his case a lymphatic or a lienal pseudo-leukemia was present. 
the fever is usually remittent or intermittent, with regular or irregular pauses some of which last several days (recurring). Such cases have been described by Pell, Ebstein and others as "chronic relapsing fever" or as "recurring glandular fever." "But in many, perhaps in the majority, of these cases, tubercle bacilli and other changes indicating tuberculosis have been found in the glands, and the question has therefore arisen and been discussed whether these cases should be designated as "pseudo-leukemia."

\section{DIAGNOSIS}

This brings us to the difficult realm of diagnosis, difficult for the reason mentioned at the beginning of the article, viz., because the conception of "pseudo-leukemia" is not a sharply defined one, or, more accurately expressed, because there is no unanimity as to which glandular enlargements are to be considered as belonging to the disease. There is general agreement only on one point, that all cases of secondary or symptomatic adenitis, i. e., all those which appear in the course of other infections and are in causal relation with them, are not to be designated as "pseudo-leukemia," but only those appearing as primary, multiple, glandular enlargements which dominate the clinical picture.

Such lymph-gland enlargements, however, are by no means always of one type; indeed, from a pathological standpoint they vary greatly. I have already mentioned that in the cases described as "chronic relapsing fever," or as "recurring glandular fever," tuberculosis is often found in the enlarged lymph-glands. But it is also true that in a large number of the cases described as "pseudo-leukemia" and showing the typical clinical picture of that disease without fever or with only an occasional rise in temperature, tuberculosis of the glands has been found. In other cases anatomical investigation has revealed sarcomatous changes, and the disease for this reason has been designated lympho-sarcomatosis (Kundrat). Finally, in still other cases the gĩandular swellings resembled leukemic lymphomata.

This latter form of multiple lymph-gland enlargement, and this alone, Ehrlich and Pinkus desire to have designated as "pseudo-leukemia." They define the latter as a disease with lymphadenoid cell-accumulations which in every respect resemble the lymphatic leukemic form, running its course with an increase of the lymphocytes at the expense of the multinuclear leukocytes but without any material increase in the total number of the leukocytes. Their proportion to the erythrocytes is said not to exceed 1 to 200 or 1 to 100 . In such cases the lymphadenoid proliferations occur, not only in the lymph-glands, but in the organs of the chest and abdominal cavity and in the skin, just as in lymphatic leukemia. Whether the bones are affected in pseudo-leukemia as in leukemia is, as I have already remarked, still questionable.

This discovery represents (if true) an essential advance in the conception of pseudo-leukemia, even although for the time it has no great practical importance, particularly for clinical diagnosis. For, in the first place, if the blood condition required by Ehrlich and Pinkus is actually present, the differentiation of pseudo-leukemia from leukemia may be difficult or even impossible. 
The ratio of white cells to red cells which is set up as a limit (namely, 1 to 200 or 1 to 100) allows wide space for variations in individual opinions; indeed, such a ratio is often found in well-recognized cases of leukemia.

Further, it is not easy to determine during life that the glandular enlargement actually depends upon lymphadenoid proliferation. Even if an enlarged gland is extirpated, and these proliferations are found in it, we cannot conclude with certainty from this that in all the other enlarged glands the same changes, and only these, are present, and that the condition is not due in part to other processes, particularly tuberculosis, a combination which has been actually observed (Askanazy, Fischer, Freudweiler and others). Further, the blood conditions designated as characteristic by Ehrlich and Pinkus, an increase of lymphocytes without much if any increase of the leukocytes is not constant, a fact of which I have convinced myself in at least one case after prolonged observation. The proportion of the mononuclear to the polynuclear leukocytes varies, and for a time may be even normal. The increase in lymphocytes is not always present from the onset. Finally, I do not believe that we can exclude the possibility of finding the blood-picture required by Ehrlich and Pinkus, in connection with glandular enlargements which do not show the pure leukemic character, i. e., with tuberculosis or sarcomatosis of the glands, and especially with the complicated or mixed forms previously mentioned. [The Ehrlich-Pinkus formula certainly is not present in every case of pseudo-leukemia—and is not at all uncommon in other diseases-e. g., in debilitated and neurasthenic conditions, in variola, in some syphilitic cases, and in some acute septic enlargements of the lymph-glands.-ED.]

The greatest difficulty is always experienced in the differentiation of tuberculosis of the lymph-glands from true lymphomata, and it is rarely possible (except when a gland has been extirpated and examined) to decide this question with certainty. As points of support for a probable diagnosis the following may serve: Tuberculosis of the glands is rare in those beyond the twenty-fifth year of life. The appearance of glandular enlargements without any other symptoms in later life would therefore be evidence against the tubercular nature of the affection. Pain and tenderness in the enlarged glands is frequently observed in tuberculosis; it is rare or does not occur at all in lymphomata. Tuberculosis of other organs is greatly in favor of the assumption of a glandular tuberculosis, and also fever, which, as I have already said, is certainly absent or appears very rarely in the non-tubercular form. Enlargement of the spleen, in the absence of ferer, is against tuberculosis. Finally, tubercular lymph-gland enlargements adherent to their surroundings usually break down from caseation, and if they are superficially situated rupture externally. In a case of this kind it is easy to make the diagnosis. But caseation and abscess formation do not occur at the onset, but only, if at all, after a lapse of time; hence until this happens this criterion for diagnosis is not present. Apart from this there is a form of glandular tuberculosis which leads to very slight caseation or none at all; I refer to the indurative form. The nature of such cases is obviously difficult to determine. In short, the differentiation of tubercular adenitis from lymphoma, or the so-called lymph adenomata, is very frequently difficult or impossible during life. 
The same is true of the third previously mentioned form of glandular enlargement, lympho-sarcomatosis. According to Virchow, a hard and a soft form of lympho-sarcoma have been differentiated, depending upon whether or not the proliferation of the reticulum of the gland and of the connective tissue predominates over the cell proliferation. The softer forms, however, show gradual transition stages to the pure lymphomata or lymphadenomata, so that even for the pathologist the decision between these varieties of tumors may be very difficult. Hence, the harder composition of the lymphosarcomata, compared to the lymphomata, has very little diagnostic importance, as the difference is not decisive. Somewhat more valuable in diagnosis is the fact that lymphosarcoma has a greater tendency to invade the capsule of the gland and the neighboring tissue, and to rupture through the skin, which never occurs in pure lymphomata, but which does occur, as has just been stated, in tubercular adenitis. The tendency to metastasis, which has been mentioned as characteristic of lymphosarcoma, is of very slight value in diagnosis during life, for three reasons: First, adenitis of other types readily causes metastases in internal organs, either by way of the lymph stream or blood channel; secondly, metastases are frequently not recognizable by symptoms; and thirdly, in the presence of certain symptoms it may be difficult, even impossible, to decide whether these symptoms are due to actual metastatic tumors in an organ or are only caused by the pressure and irritation which glandular tumors in the cavities of the body exert upon a neighboring organ. Thus, for example, symptoms which point to an affection of the respiratory apparatus, dyspnea, cough, etc., may be caused by metastases in the lungs or in the bronchi, as well as by pressure of enlarged mediastinal glands.

To all this we must add that, even in quite unquestionable cases of lymphosarcoma, we find the blood-picture which Ehrlich and Pinkus have designated as characteristic of pseudo-leukemia produced by multiple lymphomata.

It will, therefore, not be looked upon as an exaggeration when I say that only in a small minority of cases is it possible to recognize with certainty the anatomical nature of the glandular enlargements which are the foundation of the clinical picture of pseudo-leukemia. [See note on page 3\%1.-ED.]

\section{ETIOLOGY}

As to etiology very little has been determined with certainty. The affection appears in childhood and in adolescence, somewhat more frequently in the male than in the female sex. Occasionally several cases have been noted in the same family, for instance, among brothers and sisters or in one of the parents and a child, and this suggests a hereditary or family predisposition.

Not infrequently pseudo-leukemia occurs in connection with other diseases as, for example, malaria, and often in my experience, after diseases which are combined with an inflammatory irritation of the nasal cavities and of the tracheo-bronchial mucous membrane, i. e., after inflammations of the throat. measles, whooping-cough, influenza and the like. One might assume that from the areas of mucous membrane just mentioned an irritation is exerted upon the glands which causes enlargement, but then it remains unexplained 
why these inflammatory affections which are so exceedingly common rarely cause much glandular enlargement, and it is also inexplicable how the enlargement of the glands originally irritated can distribute itself to distant parts, and, finally, to all or nearly all the glands of the body. The irritation theory must be supplemented by assuming a special predisposition to disease of the lymph-glands, and this view must serve to elucidate those cases in which no causal factor that could produce irritation has preceded the glandular enlargement. More plausible is the view that a poison is at work in the blood, and that the glandular lesions are only symptomatic. In the cases which appear to derelop spontaneously and without any evidence of such a poison, it is probable that there has been noticed irritation from the tonsils, or from the whole pharyngeal mucous membrane. Many years ago I pointed out the fact. that the so-called "vestibulum pharyngis" was a "vestibulum malorum," an ante-chamber by which innumerable pathogenic agents found their entrance into the body. According to this hypothesis, in Hodgkin's disease a damaging agent finds its way from the pharynx through the lymph-glands into the blood and thus causes enlargement, first in the glands of the neck, and later in the others.

That in the later stages of the disease a deleterious agent must actually be present in the blood, is proven by the almost invariable implication of the spleen, which of late has very aptly been designated as a lymph-gland of the blood.

\section{COURSE, DURATION AND RESULT}

I have very little to add to what has been said regarding the course, duration and outcome of Hodgkin's disease. The disease runs its course with paroxysms of varying duration; as a rule it lasts from one to five years, very rarely longer. Two years is an average duration. The usual outcome of the disease is death, which is the result either of the previously mentioned pressure and stasis, or of an increase in the anemia, producing exhaustion which is not infrequently intensified by copious hemorrhages. A termination in recovery is exceedingly rare. Occasionally, prior to death, a transition into true leukemia has been observed, with the addition, therefore, of characteristic blood-changes to the glandular enlargements.

\section{THERAPY}

Treatment of the fully developed disease is not very effectual, as an actual and permanent recovery can scarcely ever be brought about, but, at the most, only a cessation in the glandular enlargement with more or less prolonged improvement of the general condition. Whether early treatment accomplishes more, i. e., complete recovery, is questionable. Nevertheless, an effort should be made to combat the glandular enlargement as early as possible.

For this purpose various remedies are at our disposal to which a certain beneficial effect cannot be denied; besides extirpation of the gland.

Among the remedial agents, Billroth has advised arsenic which has proved most efficacious. This is given either internally, or, more generally, in injections subcutaneously or in the parenchyma of the gland. For internal use 
we give Fowler's solution, one to five drops once or twice daily, best given diluted with twice or three times this amount of ordinary or aromatic water. I believe arsenious acid to be even more effective, either in the form of the pilulæ asiaticæ ( $\mathrm{B}$ Acid. arsenicos. 0.06, piperis nigri pulv. 1.5, rad. liquir. pulv. 3.0, mucilag. gummi arab. q. s. ad pil. Ix) of which one pill is taken once to three times daily To avoid irritating the stomach, these, like all other arsenic preparations, are not to be given upon an empty stomach, but only while eating or immediately after a meal. A very serviceable arsenic preparation is sodium cacodylate, also known under the name of arsycodile, which contains considerable arsenious acid (nearly two-thirds) and is well borne. The dose for adults is 0.025 to 0.1 , and this is gradually increased in pills (sodium cacodylate 0.25 to 0.1 , sacch. et gummi arab. q. s. ad pil. $x x$, daily one to five pills) or for children in solution (1:15, according to age three to ten drops). After prolonged use of this preparation, in some cases a disagreeable garlicky odor of the breath appears and causes us to desist from its use.

Arsenic acts more readily by subcutaneous or parenchymatous injections (into the glandular substance) than by internal administration. Usually Fowler's solution is employed (beginning with 0.1 and cautiously increasing even up to one gram) or the solution of sodium arsenite advised by $\nabla$. Ziemssen, of which a somewhat larger dose is injected. But even here cacodylic acid is to be preferred on account of its greater solubility and larger contents of arsenic. A 10 per cent. solution of sodium cacodylate in sterilized water is used for about a week (one-half of a Pravaz syringeful being injected) ; it is then stopped for a few days, and then resumed, the same dose being injected, until gradually, with pauses of a few days, the dose is increased to an entire syringeful.

Recently a preparation containing a large quantity of arsenic atoxyl (anilidmetarsenite) has been advised by Walter Schild, and this seems to deserve preference even above the cacodylate. Of a solution of two parts in ten of water, two to ten divisions of the hypodermic syringe are injected and the dose is gradually increased. The injections are almost painless, and are well borne. In the only case of Hodgkin's disease that I treated by this method the result appeared to be satisfactory, but the duration of observation was too brief to permit me to speak of a permanent success.

A good auxiliary remedy in every arsenic treatment is formed by the mineral waters containing arsenic and iron, those of Roncegno, Levico, Srebrenica (Guber spring), and of Cudowa (Eugene spring), which, on account of the arsenic they contain (and this is greatest in the Roncegno water and smallest in the Cudowa water), must be administered with all the caution with which we prescribe other arsenic preparations (therefore not upon an empty stomach), in from one to five tablespoonfuls for adults and as many teaspoonfuls for children; these waters are best administered in milk or Seltzer water.

Besides, preparations of iodin have been administered internally and used as inunctions over the enlarged glands. They have, however, not proven particularly serviceable.

More effective are inunctions with potassium soap which, as is well known, 
is frequently beneficial in scrofulous glandular tumors. Ordinary green soap (sapo kalinus venalis) or the somewhat more agreeable fluid potassium soap (sapo kalinus) may be cmployed; of the former, according to the size and number of the enlarged glands, a piece the size of a cherry to that of a walnut, of the latter a teaspoonful to a tablespoonful and more, may be daily rubbed into the skin with a pellet of cotton until redness and burning are produced, when the rubbing is stopped and is not resumed until these irritative symptoms have disappeared.

Since it is often impossible to determine the nature of the glandular swelling, as I have previously stated, it is well to add to the soap substances which have a more or less specific action in particular diseases, for example, iodoform in certain tuberculous processes (and also in many syphilitic ones). The potassium in the soap loosens the epidermis and the cement edges in the skin, and in this way the direct entrance of iodoform into the diseased tissue is facilitated. An inunction of this kind that I have used in other affections consists of: Iodoform 5.0, sapo kalinus and lanolin or unguentum adipis lanæ or vaselin, $\bar{a} \bar{a}$ 20.0.

Extirpation of the glands, in so far as they are susceptible to operative interference, is unquestionably indicated in those cases in which a single gland or the pressure of several glands in this area causes disturbance or becomes dangerous. Opinions differ as to whether extirpation is advisable at an early period when only one or a few glands are slightly affected, since the results of such early excisions have varied. Some observers believe that extirpation of the glands, performed once or several times, induces a slower course or a long cessation of the disease, while others, on the contrary, have observed a more rapid growth of the glands to follow. The explanation of this variation may be that frequently, besides the external visible glands that may be reached, others lie more deeply hidden, or cannot be reached by the knife of the surgeon, and the operative removal of the former, perhaps also of others which up to that time had not been implicated in the morbid processes, causes irritation.

Early extirpation, therefore, can only be looked upon as an experiment which may meet with success, and this is the more likely the earlier it is attempted.

Besides this treatment which is especially directed to the glandular swelling we must try by every means to reduce the anemia and improve the general condition. Above all, the patients should have the advantage of the most favorable hygienic conditions, nutritious food, fresh air either in the country or other suitable climate, as well as such measures as are possible in the individual circumstances for stimulating metabolism from the skin, such as friction, baths, etc.

All this may be most readily secured in properly chosen sanatoria. The most popular among these, probably on account of their well-known effect upon scrofulous glandular affections, are the sodium chlorid springs (particularly the stronger salt springs), and especially the iodin-and bromin-containing salt springs, such as those at Hall in Upper Austria, Krankenheil, Königsdorff-Jastrzemb, Kreuznach and others. These springs are used for bathing, for poultices, and, with necessary caution, also for drinking. 
The most useful remedial measures for the latter purpose are tonics and roborants, and chiefly the preparations of iron and quinin. Arsenic when properly used has a tonic effect, and so improves the nutrition that if it is administered internally other remedial agents may be dispensed with, or simply combined with arsenic. Lately a combination with ferrum cacodylicum in doses of $0.25-0.3$ gram daily has been advised according to the following prescription: Ferr. cacodyl. 1.0, Aqu. cinnam. 25, of which 20 to 40 drops are taken three times daily.

I need hardly say that in every dietetic and drug treatment attention to the digestive organs is of great importance, and if these show any disturbance of function, amelioration must here be first attempted.

It is also self-evident that febrile conditions or possible complications are to be treated according to their special indications. [The X-ray treatment of Hodgkin's disease seems at this time to promise more than any other. The number of eases is not large, but the results are often favorable. The technic is very simple; the affected glands are exposed to the X-ray for as long a period (every day or every second day) as the skin will stand without sustaining any "blush." Pressure symptoms due to substernal glands have thus been very notably relieved.-ED.]

\section{BANTI'S DISEASE}

We turn now to the description of the second form of pseudo-leukemia, the lienal or splenic variety, in which enlargement of the spleen is predominant or apparently occurs alone, i. e., without glandular enlargement. I say apparently, for, according to my investigations, the lymn̨h-glands are almost always implicated. It is true that sometimes the enlargement affects only the deeper ones, particularly those glands situated in the abdominal cavity, and therefore recognizable only at autopsy. There are numerous cases, as I have mentioned previously, in which the spleen and lymph-glands are attacked simultaneously, and these cases are designated as pseudo-leukcmia lymphaticolienalis, and constitute a transitional stage between the two other forms.

Aside from the glandular swelling and the course of the disease the clinical picture in the lienal form scarcely differs from that of the lymphatic form (Hodgkin's disease), and the description may consequently be brief.

Besides the enlargement of the spleen, or even before this becomes noticeable, the earliest symptom is anemia, and Gretsel (Griesinger) has therefore designated the disease as "splenic anemia." But this name is not distinctive for, as later researches have shown, the blood finding does not, or at least not always, correspond morphologically to that of simple anemia. On the contrary, manifold changes, particularly in regard to the number and proportional relations of the leukocytes, are met with, but never such a decided increase as in leukemia. At one time the blood may, in fact, resemble simple chronic anemia, i. e., the number of erythrocytes may be more or less decidedly decreased, the hemoglobin to the same degree or even more decidedly diminished, and the proportion of leukocytes to erythrocytes, although varying not markedly, exceeds normal limits. [The very low color-index has been especially 
emphasized by Osler.-ED.] At other times, with. the same relation of the erythrocytes and of the hemoglobin, leukopenia is present; that is, there is a conspicuous diminution of the leukocytes below the lowest normal limits. In still other cases there is a relative decrease in the polynuclear neutrophilic leukocytes, which normally make up about three-quarters of the leukocytes. Sometimes the cells designated as lymphocytes are predominant, i. e., a lymphocythemia is present. In conclusion, a blood change is found which in some respects resembles pernicious anemia; namely, nucleated erythrocytes of normal or larger size (normoblasts and megaloblasts) are seen, and with this an increase in the leukocytes, sometimes of the polynuclears, sometimes of the lymphocytes.

This latter blood composition is observed pre-eminently in infancy, and has been described by $\nabla$. Jaksch as anemia infantum pseudoleukemica. This is a pseudo-leukemia which has special characteristics in the infantile organism. Normally the infant's leukocytes are more numerous than those of the adult; and in the child the lymphocytes also are more profuse, and from the hyperemic red marrow of the growing bones nucleated young erythrocytes more readily enter the circulation. These nucleated erythrocytes, according to our present views, are to be regarded as immature corpuscles.

Other blood lesions such as poikilocytosis, increase of the blood plaques, etc., are not characteristic.

We must not expect that the same blood condition will be present in every case and at all times for, in the first place, these changes arise gradually in the blood with an increase of the anemia, and probably also with the growth in the spleen, corresponding to the growth in the glands, as in Hodgkin's disease. Thus, in this malady, as in the other form, periods of transitory improvement in the condition occur and the blood composition improves. Further febrile conditions may arise under the influence of which the number of leukocytes may change; for example, a leukopenia may give place to a leukocytosis; or, again, profuse hemorrhages in the disease may entirely alter the blood picture.

In the splenic form of pseudo-leukemia, certainly in adults, hemorrhages are even more frequent than in the lymphatic form, especially hemorrhages from the nose and from the gastro-intestinal canal ; but there are also hemorrhages from the gums, from the skin, and from the vitreous body of the eye, etc. [Osler has explained the gastric hemorrhages as due to the inability of the stomach veins (anastomosing with those of the spleen through the vasa brevia) to empty themselves on account of the cirrhotic process which forms part of the changes in the spleen.-ED.]

The urine is very similar to that of the lymphatic form.

The implication of the liver in splenic pseudo-leukemia is very interesting. In the lymphatic form enlargement of the liver also occurs, due to the previously mentioned metastatic lymphomata or lymphomatoid formations. But these lymphomata are rarely of decided extent and rarely give rise to marked disturbance. In the splenic form, however, the liver is very frequently and conspicuously involved. Banti deserves credit for having first called attention to the combination of pseudo-leukemic enlargement of the spleen with cirrhosis of the liver, and for having remarked the causal connec- 
tion between them; therefore quite properly this combination is designated as Banti's disease.

The chief symptom by which cirrhosis of the liver may be recognized is ascites. Yet this may occur, as I have scen, in pseudo-leukemia in which the liver is not attacked, or to but an insignificant extent, so that besides cirrhosis of the liver, to which Banti refers the ascites in all cases, other causes may be operative. As such a cause I have mentioned displacement of the lymph channels in the abdominal cavity by enlarged lymph-glands, which, especially if combined with an anemic composition of the blood, and perhaps with stasis due to the marked enlargement of the spleen, may give rise to ascites. In some cases, disease of the portal vein may possibly be the cause. [A calcified thrombus of the portal vein has been found by Warthin in two cases of this disease (splenic anemia).-ED.]

Banti reports that in the disease named after him the intima of the portal vein from the anastomosis of the splenic veins to the liver has been found covered with coarse plaques similar to the sclerotic and atheromatous coats of the aorta. He assumes that toxic substances from the originally diseased spleen have reached the portal vein through the veins of the spleen, and later reached the liver, thus causing the pathological changes. In two cases belonging to this category upon which autopsies were held, and in which neither ascites nor liver cirrhosis was present (which, therefore, did not strictly represent Banti's disease), these changes were not found either in the portal vein or in the splenic vein. They belong perhaps to a more advanced stage of the disease.

But even if ascites is present the clinical picture deviates in many essential points from ordinary hepatic cirrhosis, that form known as "alcoholic liver." The enlargement of the spleen in pseudo-leukemia is much greater than in the latter, and the skin does not show the grayish yellow discoloration which is so usual in cirrhosis of the liver. On the contrary, it is pale, as in anemia, provided unusual circumstances do not bring about a darker pigmentation. For example, Osler has observed in some cases a melanotic discoloration due to an old malaria. Furthermore, the urine is usually of a different composition, without bilirubin and urobilin, provided special complications, such as decided stasis due to ascites and the like, are not present.

We must, however, agree with Banti that in the clinical condition named after him the enlargement of the spleen is the primary affection and not the result of enlargement of the liver. In the other cases of splenic pseudo-leukemia not associated with hepatic cirrhosis, it can no longer be doubted that the enlargement of the spleen is the primary affection to which the other disturbances, particularly the anemia and the abnormal composition of the blood, may be attributed.

The anatomical condition of the splenic tumor is usually reported as dependent upon hyperplasia, but microscopic investigation shows variations which depend upon the extent to which the pulp and the Malpighian bodies as well as the trabeculæ are implicated in the hyperplasia. It appears, or so I conclude from a case observed by me, that in early cases only the tissue of the pulp is hyperplastic owing to a decided increase of the lymph-cells in the 
reticulum. Afterward an enlargement of the Malpighian bodies with an in.crease in their cells is observed, so that they stand out as grayish white nodules of the size of a pea, having the characteristics of lymphomata. Finally, with the lapse of time the connective tissue proliferates, the capsule of the spleen becomes thickened, the spleen itself hardens and is permeated by more or less - tense connective tissue strands by which the pulp is more and more obscured or absorbed. The follicles become thickened and enlarged and gradually lose their reticular structure and cellular stratification, being changed into tough fibro-cellular nodules. At last the normal structure of the spleen disappears to a greater or less degree, and only tough trabecular tissue remains.

In this process, the larger vessels, the arteries, and particularly the veins, are often found thickened, sometimes also covered with chalk plates, such as Banti observed in the trunk of the splenic vein in cases complicated with hepatic cirrhosis.

The cause of the enlargement of the spleen, i. e., the actual cause of the disease, is unknown. In the relation of the spleen to the blood, which, as I have already stated, may be compared to that of the lymph-glands to individual areas of tissue, it may certainly be considered that a deleterious element present in the blood exerts an irritation upon the spleen. What the nature of this irritation is we do not know. Specific bacteria or toxins, which nowadays so readily come into consideration, have not been found. Much favors the view that the damage originates in the gastro-intestinal canal, and thence reaches the blood. The circumstance that digestive disturbances, especially diarrhea, frequently precede enlargement of the spleen, and the fact that swelling of the lymph-glands in the abdominal cavity is rarely absent, favor this hypothesis.

From this point of view the assumption is certainly justified that the disease of the liver is not necessarily caused by enlargement of the spleen, but that it is the independent consequence of the same deleterious process. By this we do not intend to deny that the abnormally constituted blood which circulates from the spleen to the liver may cause pathological effects. It may be in consequence of the simultaneous action of both poisons-that of the gastro-intestinal canal and that of the spleen--that the hepatic cirrhosis sometimes develops very early, at other times hardly at all.

As a contribution to our knowledge of the etiology of pseudo-leukemia, it must be stated that in a fair proportion of cases the malady develops in the course of diseases in which enlargement of the spleen or a chronic splenic tumor already exists, or in the course of which it appears, e. g., in malaria, syphilis, particularly in that form which occurs in children, hereditary syphilis, and in rickets. The characteristic symptoms of the latter diseases gradually retrograde, while the splenic tumor persists or even increases; anemia and cachexia become more prominent, and gradually the boundary line between these maladies and pseudo-leukemia disappears, and it becomes impossible to say where the one begins and the other ceases.

The character of the blood may in such instances decide the diagnosis, particularly if the change described by Ehrlich and Pinkus is present-or, at all events, a relative increase of the lymphocytes with a low count of leuko- 
cytes such as would exclude leukemia. But I have already mentioned that a hematological condition of this kind is not always present, even in cases that are to be strictly considered pseudo-leukemia. In these, another state of the blood combined with a characteristic enlargement of the spleen is of importance in the diagnosis, namely, a simple high-graded anemia, i. e., oligocythemia and oligochromemia and this is especially so if leukopenia be also present.

The recognition of splenic enlargement in cases of this kind can hardly be difficult, for the spleen early attains a size that is scarcely ever seen except in leukemia. Its lower border may reach anteriorly to the true pelvis, to the median line and cven beyond. The surface is smooth, and upon pressure the organ is but slightly or not at all painful.

In the differential diagnosis all other hypertrophies of the spleen must be excluded, and this is usually not difficult, for acute enlargements do not come into consideration. The chronic enlargements, including tuberculosis and amyloid degeneration, which are somewhat rare, may be readily recognized, provided the etiologic factors and the condition of the other organs are considered. ["Idiopathic" splenic enlargement with anemia very slight or absent and no other symptoms at all is at times difficult to separate from some of the cases in this group.-ED.] The diagnosis is made with less ease when ascites is present, not because there is any difficulty in determining the enlargement of the spleen, but because the question then arises whether hepatic cirrhosis is also present, and whether this or the enlarged spleen is the primary affection, i. e., whether Banti's disease has or has not developed. Here again an examination of the blood will aid in the decision provided the above-mentioned changes are present. The enlargement of the spleen in primary hepatic cirrhosis is never so great as in Banti's disease, and, according to my experience, the appearance of the skin and the condition of the urine in the latter affection are not the same as in hepatic cirrhosis.

The course and termination, and therefore the prognosis of splenic psendoleukemia, do not differ greatly in adults from the lymphatic variety. The condition is different in children. Here not infrequently it is possible to bring about a decided improvement in the anemia and in the general condition, and even a diminution in the size of the spleen. I do not know whether or not this retrogression may be complete, but in cases not too far advanced I believe it possible. This difference in the nature of the disease in adults and in children may depend upon the action of the blood-forming organs, particularly the bone-marrow, which in children shows a more vigorous function. It is for this reason that children react more readily to deleterious agencies, and for this reason also an improvement is more easily brought about in children than in adults.

In the treatment of this form of pseudo-leukemia the same factors must guide us as are decisive in the other variety, only that here not the enlargement of the lymph-glands but that of the spleen is to be combated. Unfortunately drugs have even less $p$ ower to influence this process than in the former instance. There are a certain number of so-called "splenic remedies," but they owe their reputation chiefly to their efficacy in malaria and in the enlargement of the spleen dependent upon this disease. Quinin is to be men- 
tioned first among these drugs, then piperin and eucalyptus. But, even in cases in which malaria has preceded, they have no effect upon the remaining splenic tumor; and, besides, these patients have already taken quinin, arsenic or other remedies to a considerable extent. If not, arsenic in one of the forms previously mentioned may be employed, at least internally, on account of its stimulation to metabolism, and perhaps also because of its power in blood production. Subcutaneously atoxyl, which has already been mentioned, is especially worthy of a trial. Parenchymatous injections of arsenic, of carbolic acid, etc., have been resorted to, but as these are not absolutely harmless, and are of questionable value, they are not advisable. Inunctions in the splenic region are useless, nor have I seen any benefit from massage or electricity. Somewhat more effective, it appears to me, is the application of cold to the splenic area, particularly in the form of the cold douche, the jet douche after Fleury, or the fan douche. These are best employed while the patient lies upon the right side of the body in a warm bath, with the left side of the abdomen exposed.

The most certain means of overcoming the splenic tumor and (in so far as this is considered to be actually the first of the main symptoms of the disease) to bring about recovery, is splenectomy. But this is neither so easy nor so harmless as the removal of the enlarged lymph-gland. Adhesions of the tumor to surrounding organs and marked hemorrhage present great difficulties in the operation, and endanger the life of the patient. Nevertheless lately, owing to improvements in technic, extirpation of the spleen has been successfully accomplished in pseudo-leukemia, and in a number of cases has brought about the disappearance of the anemia and of the cachexia; for what length of time is another question. Of course success is to be expected only when the disease has not existed for too long a time, and severe sequelæ, such as cirrhosis of the liver, multiple lymph-gland enlargement, etc., have not appeared.

If ascites is present the attempt may be made to prevent its recurrence, and to bring about a decrease in the size of the spleen, producing a collateral circulation with the venous system of the body, by attaching the omentum and the spleen to the abdominal wall (after Talma or Schiassi).

Extirpation of the spleen in children is less likely to be considered, for in them, as mentioned, the hygienic, dietetic and drug treatments are more effectual. Iodin and iron have proven serviceable, as well as arsenic; baths and a change of climate are to be recommended as in the lymphatic form. The same treatment is of course to be employed in adults.

\section{IITERATURE}

Askanazy, Ziegler's Beitr. zur path. Anatomie, Jena, 1888.

Bonfils, Société méd. d'observation, Paris, 1856.

G. Banti, "Lo Sperimentale," 1894, 1895; Ziegler's Beitr. zur allg. Path., etc., 1898, xxiv.

Clarke, Brit. Med. Journ., 1901, ii, p. 701.

J. Cohnheim, Virchow's Arch., xxiii.

W. Ebstein, Berl. klin. Wochenschrift, 1887. 
P. Ehrlich and F. Pinkus in Nothnagel's Spec. Path., 1901, vii, i.

Fischer, Arch. f. klin. Chir., 1897, lv.

Freudweiler, Deutsches Archiv f. klin. Med., 1897, lxiv.

Gretsel, Berl. klin. Wochenschrift, 1866, Nr. 20.

E. Grawitz, Klin. Pathologie des Blutes, Berlin, 1902, 2 Aufl, p. 351 ff.

R. v. Jaksch, Wiener klin. Wochenschr., 1889, Nr. 22, 23.

A. Jolles, Zeitschr. f. klin. Med., 1898, xxxiv.

Kundrat, Wiener klin. Wochenschr., 1893, Nr. 12, 13.

J. H. Musser, Transactions of the Association of Amer. Physicians, 1901.

Osler, Amer. Journ. of Med. Sc., 1900, January.

Pel, Berliner klin. Wochenschr., 1885, 1 und 1887, 35.

M. Reed, Johns Hopkins Hosp. Reports, x, 1902, Nos. 3-5.

W. Schild, Berliner klin. Wochenschr., 1902, Nr. 13.

H. Senator, Berliner klin. Wochenschr., 1901, Nr. 46.

Sternberg, Centralbl. f. d. Grenzgeb. Med. u. Chir., 1899, ii.

Wilks, Guy's Hosp. Reports, 1856, ii.

Talma, Berliner klin. Wochenschr., 1898, Nr. 38.

B. Schiassi, "Un nuovo trattamento del Morbo del Banti," Bologna, 1902. 


\title{
THE HEMORRHAGIC DIATHESES
}

\author{
By M. LITTEN, Berlin
}

THERE is a group of diseases in which the essential symptom is a tendency to more or less extensive hemorrhage, which distributes itself over various organs and thus becomes dangerous to life.

We designate this tendency to external and internal hemorrhages, which probably depend upon a change in the blood or in the blood-vessels, or in both, as the hemorrhagic diathesis. The diseases which belong to this group, which in some cases resemble one another so closely that it has sometimes been thought possible to combine them as one disease, were formerly designated "scurvy." But to-day there is much diversity of opinion as to how far this division is justified.

In accordance with the present state of our knowledge, the following groups will be considered separately: 1. Scurvy; 2. Hemophilia; 3. Morbus maculosus Werlhofii. But even with these subdivisions it must be emphasized that our knowledge does not often permit a sharp separation; the boundaries are partly artificial, being neither etiologically nor pathologico-anatomically defined with accuracy.

\section{SCURVY}

By scurvy we understand a general disturbance of nutrition which rarely occurs sporadically but usually epidemically, and almost always under the influence of unfavorable, unhygienic circumstances, particularly that of improper food; it is usually of insidious onset and slow course, and may terminate either in complete recovery or in death.

The disease is characterized by a severe general cachexia and by a series of local disturbances chiefly due to a transitory hemorrhagic diathesis; this may present symptoms that completely coincide with those of hemophilia, of purpura hemorrhagica, or purpura rheumatica, but is sharply differentiated from the first by the fact that the changes are hereditary in the former affection and permanently present in the individual, while in the latter and in scurvy we are always dealing with an acquired disease which is generally transitory, and terminates in recovery or death, although frequently many relapses take place.

\section{HISTORY}

The history of scurvy is exceedingly interesting and important, as it demonstrates most forcibly the progress of hygiene and of scientific investigation. 
When, after the discovery of America, shipping acquired new interest, when voyages which formerly were limited to the coasts of countries were extended over the open, wide sea, the brave seafaring men were confronted with new and quite peculiar conditions of life. Cut off for many months from land, exposed to the mercy of the winds and the waves, limited to the narrow confines of their ship, where they huddled together in large numbers, often exposed to great hardship, in the choice of food and drink they were entirely restricted to that brought from their homes, and particularly to such food as could be kept for a long time. Frequently they were compelled to subsist on food more or less tainted. It is obvious that such conditions would inevitably result in disease; and, great and brilliant as are the discoveries of that time, the great and hideous figure of scurvy, the disease which developed from these voyages of discovery, and which caused the failure of many expeditions, cannot be forgotten.

In the year 1498, when Vasco de Gama undertook his celebrated voyage around the Cape of Good Hope, the crew was attacked by scurvy, and of 160 persons he lost in a short time more than one-third. We know well the decimating character of the disease which occurred in the expedition of Cartier in 1535, and in those of v. Monts, Pontgrave and Poutrincourt to Canada toward the end of the sixtcenth century; in the French expedition of Dellon to India; in the journey of the English fleet under Lord Anson around the world (1740-1744), in which voyage the disease recurred repeatedly in various latitudes, and 380 out of 500 men succumbed to the malady; in the North Polar expedition of Ellis (1\%46-1\% $1 \%$ ) in search of the northwest passage to Hudson Bay; in the fleet of the English admiral, Gleary, who in 1780 returned with 2,400 scurvy patients, and in other expeditions.

The reports of these expeditions are so definite that there can be no doubt of the identity of the disease. We have less information regarding the occurrence of scurvy upon land, the first reliable report of which dated about a hundred years later, at which time the name scurvy or "scharbock" for the first time appeared. Our knowledge of the occurrence of the disease in antiquity is very limited, although it may be assumed that the peculiar conditions which favored the appearance of the pest, as later investigation has taught, must also have produced scurvy in earlier epochs.

The best historical accounts of scurvy we owe to August Hirsch, whose description we have mainly followed.

He succeeded, however, in finding in old medical writings only one form of the disease described which so far corresponds to the picture of scurvy that their identity may be assumed; namely, in the Hippocratic collection, an affection described as cilcòs aimarírys. Although the disease des-

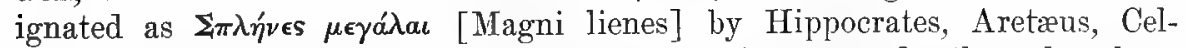
sus, Cælius, Aurelianus, Paulus Aggineta, Avicenna and others has becn by some authorities taken for scurvy, Hirsch demonstrates that this disease was malaria. Pliny mentions two diseases, stomatokaké and skalotyrbé, the first in particular running its course with an affection of the mouth resembling scurvy.

But it is quite as likely that this was "stomatite ulcéreuse," an army dis- 
ease, as the herba Britannica was used for stomatokaké and skalotyrbé. Some have considered oscedo (a disease mentioned by Marcellus) as probably scurvy, although the nature of this affection is otherwise not known, and we do not know what plant was meant by herba Britannica.

The occurrence of scurvy in the Middle Ages is quite certain. Several descriptions exist of decimating diseases which followed the massing of great armies, as during sieges, etc.

Thus, Jacques de Vitry reports the outbreak in 1218 and 1219, in the Army of the Crusaders while besieging Damiette, of a disease in the course of which the gums became gangrenous, the extremities painful, the tibia later turned frightfully black, and the patients were unable to eat; thus the disease ran its course, the patients suffering intense pain from which most of them were only relieved by death. Joinville explicitly describes a similar disease which appeared in the army of Louis IX while besieging Cairo in 1250. If we can conclude from these descriptions that the disease was scurvy, we may also assume that this disease had certainly occurred previously.

Cordus was the first to use the term "scharbock" for this malady. But Hirsch believes it questionable whether he himself ever observed the disease. On the other hand, quite accurate descriptions are given by Olaus, Magnus, Echthius, Ronsseus, Wierus, Dodonæus, and Brucæus. Most of these accounts refer to epidemics that occurred in northern countries bordering on the sea, in North Germany, Scandinavia, and The Netherlands. From these descriptions it also appears that the disease usually occurred under circumstances of great stress, such as famine, war, sieges or other unfavorable social conditions. Descriptions of certain diseases as scurvy, to which their symptoms show only a partial similarity, are unreliable. This is the case with an epidemic which is said to have occurred in the year 1486 in Saxony, Thuringia, and some neighboring countries.

The earliest description of scurvy by Fabricius, rector of the princely school of Meissen, is found at the beginning of the eighteenth century, but Hirsch proves that the condition was ergotism, the nature of which was still unknown to physicians of that time. Medical history of the seventeenth century, and especially that of the eighteenth, inclines us to believe that during this entire period Europe was afflicted with seurvy which was more prevalent than any other disease. The fact that during so long a period of time a single disease should have predominated to such a marked extent is at once suspicious, and close investigation has shown that the exact opposite was really the case; that persons who had rarely seen scurvy declared almost everything to be scurvy, and thus brought about tremendous confusion in medical science. The cause of this must be ascribed to Eugalenus, whose book, "De morbo scorbuto liber," which appeared in the year 1720, Hirsch declared to be patchwork which could not be equalled in medical literature, either in the ignorance of its author, or in the results which it nevertheless achieved, since for more than a century it remained the canon regarding scurvy, and the best physicians of the age were unable to free themselves from its influence. The book made a great sensation, particularly the teaching that all children were born with a predisposition to scurvy (Drawitz), and that the cause and root 
of every disease could be recognized in scurvy (Bontekoe). Not even Boerhaave was able to rise entirely superior to these views, but he was sufficiently critical to state that the disease had, during his time, appeared much more rarely in The Netherlands. Keen and earnest crities were not lacking who not only were opposed to the scurvy delusion, but went to the other extreme, and denied absolutely the existence of scurvy. Sydenham with others protested against this, and asserted the rare occurrence of scurvy, while Kramer furnished an accurate description of cases actually observed by him in which he also declared that the disease was lcast known by those who most frequently described it. In the year 1752 Lind's masterly treatise appeared, which up to the present time remains one of the best descriptions of scurvy; in this he compared the first accounts of the disease (which he characterized as chaos) with the later views maintained regarding the affection, and anew so accurately portrayed the clinical picture that it subsequently admitted of little change.

Notwithstanding Lind's proof that scurvy was not an infrequent disease in the previous century, the fact still remains that it occurred far more frequently in the period in which Lind wrote than in our time. Hirsch has zealously investigated these epidemics, and collected the records of all others occurring on land, from 1556 to $187 \mathrm{r}$, in so far as they have borne the test of scientific criticism.

Although we must admit that this compilation is not a complete history of outbreaks of scurvy, since not every pandemic, and not nearly all of those limited to a small area, or occurring in a single institution, have been described, still it enables us to draw conclusions regarding the frequency of the disease, its geographical distribution, and the probable causes of its development. In all 143 epidemics are mentioned, of which two occurred in the sixteenth, four in the seventeenth, thirty-three in the eighteenth, and one hundred and four in the nineteenth century. These figures make it strikingly apparent that the views of Eugalenus and his adherents were exaggerated.

Of the epidemics in the course of the previous century, that of Paris is to be particularly mentioned; it occurred in $1870-\% 1$ during the siege of the city, and spread owing to the scarcity of food. Among the French troops who, in 18\%1, were held as prisoners of war in Germany, an epidemic oceurred in Ingolstadt which, however, according to Döring, did not attain great dimensions; among 10,000 prisoners only 159 were attacked. In the Milbank Penitentiary in London scurvy prevailed in 1824 and 1840; also in the prison in Prague in 1831,1836 and 1842; in the workhouse of Ludwigsburg in Württemberg in four successive years, from 1850 to 1853, and in the prison of the same city, in 185\%. The last epidemic in Germany worth mentioning occurred in 1875-76 in a penal institution in the city of Moringen; the last in France in the spring of $18 \% 7$ in the prison at Mazas. Epidemics occur more frequently in Russia, even to-day, usually under the influence of extraordinary circumstances, such as crop failure, famine, or other social evil.

The majority of the epidemics in Hirsch's tabulations are found to have occurred in Russia, and this country also furnishes interesting details regarding the geographic distribution of the disease. Russia with 35 epidemics is followed by Germany with 19 epidemics, France with 15, Sweden, Norway 
and Denmark with 14, and England with 11. The other European countries present much smaller figures. Of foreign countries India heads the list with 14 epidemics, then follows North America with 1\%, and Algiers with \%. The disease may even to-day be looked upon as endemic in Russia, and occasionally it shows extraordinarily wide distribution, as is evident from the reports of the Obuchow Hospital in St. Petersburg.

In the great epidemic of 1849 which distributed itself over a wide area of the Russian Empire, Krebel reports that among 260,444 persons 60,958 died. In Asiatic Russia also, especially along the coast of the Arctic Sea, in the Siberian-Chinese boundary land, and upon the peninsula of Kamchatka, scurvy is frequently seen to-day. In northwestern Europe scurvy never played a prominent rôle, and although, for example, a famine with scurvy appeared in Iceland in the years 1836-3\%, the scurvy did not assume an endemic character. The conditions are the same in other European countries; only now and then localized epidemics are reported from prisons. Epidemics have prevailed extensively in Algiers without assuming a distinctly endemic character; scurvy appeared among the French troops in Egypt in 1801; in Abyssinia the disease has been observed almost exclusively in travellers, the natives being spared, although they usually live under decidedly more unfavorable and insanitary conditions than the strangers, and these conditions are powerful factors in the distribution of the disease. On the other hand, in the Eastern Soudan and in the entire rain zone of East Africa the disease is said to occur frequently among natives as well as strangers. In South Africa, on the contrary, the disease is reported to be quite unknown among the negroes.

In Asia, India in particular is subject to the disease; a great number of epidemics there are reported; these occurred chiefly among the poorer population, and were widespread. Upon the coast of Dschemenia (Arabia) the disease is endemic; in 1839 the English troops in Aden were attacked by it; in China, especially in the northern provinces in which the population live in a squalor elsewhere unequalled, epidemics are not rare; also among the poorer population in Japan scurvy is quite frequent.

In Australia, in numerous expeditions to the interior which were undertaken for purposes of discovery, the affection has been very serious, and lately it has appcared endemically among the shepherds upon the wide grazing plains of that country.

In the southern parts of America the disease appears to be unknown, and in the north the natives show no susceptibility to the affection. The epidemics which have been reported occurred among United States troops who were exposed to great privations at outlying stations, in lumber camps in the interior of Canada, but particularly in California during the gold fever, among adventurers who had collected from all parts of the earth and who lived under circumstances of great privation. These reports now have renewed interest because great numbers of people have rushed to the gold fields of Alaska, where, after Greenland, scurvy is more prone to occur than in any country of the Arctic regions, and where all the conditions are favorable for the outbreak of an epidemic.

Among the members of the well-known Nansen expedition, from July, 
1893, to August, 1896, which was one of the most important voyages of scientific investigation of all times, not a single case of this disease occurred. Later, when considering the prophylaxis, we shall refer to the important measures by which the celebrated leader prevented an outbreak of the disease which might have brought the expedition to an end.

An important American investigation of scurvy in children, in the year 1898 (the American Pediatric Society's Collective Investigation of Infantile Scurvy in North America), has given the following results: Of 372 cases, 367 occurred in the white, 4 in the black race, and 1 in the Chinese; 51 per cent. were males, 49 per cent. were females. The greatest number of cases occurred between the seventh and fourteenth months. We shall refer to this again.

\section{ETIOLOGY}

In spite of many endeavors it has been impossible to discover a cause which can be considered at all reliable; all search for the pathogenic agent of the disease, though it is often assumed to be of miasmatic, or even of infectious origin, has been without result.

The question of food remains the alpha and the omega in the etiology of scurvy, as in so far as the food supply is influenced by the conditions of weather and the seasons the latter will always have a more or less decisive influence in the production of the disease.

It is generally assumed that improper nutrition in a certain direction is pre-eminently responsible for the affection, particularly a deficiency of vegetable acid potash in the daily food. It has repeatedly been noted that during long sea voyages in which salted or pickled meat exclusively was consumed scurvy appeared. As the ash of pickled meat contains much less potassium than that of fresh meat, an attempt has been made to ascribe scurvy to the deficient intake of potassium, but perhaps quite improperly so, for in prisons where the diet consists almost exclusively of vegetables rich in potassium, scurvy is not infrequently noted. On the other hand, it has been remarked that many races in the far north are often compelled for months continuously to live on pickled meat or salted fish without being attacked by scurvy.

Later investigations, in which an amelioration of the scorbutic affection is said to have followed the addition of fat to the diet, appear to indicate that scurvy is due rather to a diet limited in variety and deficient in fat than to a lessened amount of potassium salts in the food.

Besides this, a number of oiher causes are considered to be active: General deficiency in food, the close huddling of many persons in cramped, unclean and wet dwellings, excessive corporeal labor and psychical depression, and, above all, the ingestion of tainted food (ptomain poisoning).

In considering more minutely the factors which are mentioned, we must first consider the view that scurvy is a miasmatic or infectious disease.

The first experimental investigations of this subject were made by Murri in Bologna, who injected subcutaneously rabbits with blood taken from scurvy patients. A rise in temperature and small petechiæ developed in the lobes of the ears of these animals. Upon killing the animals hemorrhages were 
also found in the serous membranes and in the internal organs. Murri quite properly avoids drawing conclusions regarding the transmissibility of the disease from these results. Analogous findings were obtained by Contù and Mari in similar experiments. Further investigations resulted in the cultivation of the pathogenic agent of the disease from the blood of scurvy patients. Wieruszskij obtained from scurvy blood in numerous cultures on various media, partly negative results and partly well-known bacteria, so that he concluded that scurvy could not be regarded as an infectious disease due to microorganisms which could be found in the blood. Babes conducted further experiments. He started with the presumption that scurvy is an infectious disease, and that the point of entrance for the pathogenic agent must be sought for in the mucous membrane of the alveolar processes. He therefore extirpated small portions of the gums of scurvy patients, and in the preparations, which had been hardened in alcohol he demonstrated a definite bacillus with which he infected rabbits by intravenous injection. Hemorrhagic foci occurred in various organs in which the bacillus was also found. Babes describes the bacillus as an elongated, bent organism, pointed at the ends, about $0.3 \mu$ wide and of about the same length, often forming waving threads twice as long and of various lengths, somewhat thinner and decidedly longer than the cholera bacillus. The youngest specimens are double structures, and show the tendency to form metachromatic bodies which stain dark violet with methylene-blue, and are thicker than the rods. The rods themselves stain only weakly with rubin, and do not stain according to Gram. The bacilli resemble the bacillus $\epsilon$ described by Miller, and are probably always present in the oral cavity. The investigations of Babes certainly require further confirmation, for they are assuredly open to doubt. He himself admits that he has not found the pathogenic agent of scurvy, but only speaks of a "bacillus that causes gingivitis and hemorrhages in scurvy." The findings of Rosenell and Bornträger are quite unreliable; the latter does not even consider the cocci found by him as the pathogenic agent of scurvy.

Jackson and Harley ${ }^{1}$ regard scurvy as a ptomain poisoning. To prove this view experimentally they have attempted to produce scurvy in monkeys by feeding them with tainted meat. Some of the animals had rice and maize given to them with fresh meat; a second group was fed on the same food except that the meat was tainted by prolonged standing; a third group ate the same food as the second with the addition of fresh fruits. Prolonged observation of the animals gave the following results: The first group of animals showed no characteristic symptoms except slow emaciation and diarrhea which appeared after some time. The monkeys of the second group showed decided diminution in strength, and in the majority of them there was a muco-hemorrhagic diarrhea as wcll as, in some of them, spongy, bleeding, and ulcerative gums. In two of these animals the blood examination revealed a decided decrease of hemoglobin with a slight decrease in the number of erythrocytes and marked leukocytosis; a decrease of specific gravity dependent particularly upon a diminution of proteids; increase of fibrin and of coagulabil-

I"An Experimental Inquiry into Scurvy." Lancet, April 28, 1900. 
ity; therefore, in the main, a picture which resembled the anemia of human scurvy. The authors mentioned, therefore, believed themselves justified in declaring the disease of the monkeys to be scurvy, although the autopsies established no proof. In the third group the addition of fresh fruits but slightly influenced the disease of the animals. These investigations, although requiring further demonstration, may be looked upon as proof that contaminated meat has deleterious effects even in monkeys and as confirmation of the practical experience that only the intake of fresh meat can prevent the outbreak of scurvy (compare prophylaxis).

These investigations in connection with recently expressed views as to the importance of oral affections in the development of pernicious anemia (?) led Home ${ }^{1}$ to assume that an affection of the gums is the origin of scurvy. He believes that microörganisms still unknown (which may develop from tainted food) infect the digestive tract, first producing a disease of the gums, and then an anemia which leads to hemorrhages and ulceration. Outbreaks of scurvy occurring under circumstances which preclude cleanliness of the oral cavity are in conformity with this theory.

Turner, ${ }^{2}$ according to the data gathered by him during the great scurvy epidemic in the eastern provinces of Russia during the year 1899, believes scurvy to be due to a specific pathogenic agent which is still unknown, and to be an infectious disease which is transmissible by contagion. In this he reasons only from a clinical standpoint, whereas bacteriologic investigations in this epidemic have given no positive results. The foundations for his belief that the disease results from infection and not from errors in diet are: 1 . The epidemic character of the disease; 2 . Its appearance in definite localities while neighboring districts or those with which there is but slight communication remain free; 3 . The frequency of the disease among the rich; 4 . The common occurrence of the disease in persons who are in daily contact with the patients. He relates his experience with these conditions in a collection of villages in the Government of Kazan which are populated by various races (Russians, Tartars, Tchuktchis), living isolated from each other. While the Russian villages remained almost free from the disease, the villages in which there was but little communication with other races showed many cases of the affection, particularly among the rich Tartar population. But in this region, a village in which very poor heathen Tchuktchis lived entirely isolated remained uninfected. The fact that the Tartars and their relatives ate salted meat almost exclusively and no vegetables is, in his opinion, not proof to the contrary. He lays the greatest stress upon the cases in which the disease was contracted through nursing; among eight nurses who were active there, four were attacked, and even the author himself had a mild form of the disease.

The previously mentioned collective American Report shows that so-called infantile scurvy must be regarded as the consequence of a chronic intoxication (chronic ptomain poisoning) produced by unsuitable food, for which

1 “"The Etiology of Scurvy." Lancet, August 4, 1890.

${ }_{2}$ "Le Scorbut est-il une maladie infectieuse ou contagieuse?" Arch. génér. de Méd., Aout, 1900 . 
intestinal fermentative processes have prepared the way. In 167 of 372 children in the first year of life, scurvy was preceded by another disease, usually disturbance of the digestive organs. The nutrition prior to the appearance of scurvy had consisted in 10 children of breast milk exclusively, in 4 of unsterilized, in 68 of sterilized, milk, and in the remainder of artificial foods.

We shall now consider what other factors may also be regarded as causes of this disease.

It is certain that age and sex do not play a rôle in scurvy, although it has been erroneously maintained that in single epidemics particularly persons of a definite age, or of one sex, have been attacked (for example, according to Fauvel, in the Salpetrière in Paris in 1847 only old women had the disease). Scurvy occurs more frequently in men than in women; the reason for this is quite obvious, for it is men who are chiefly exposed to the causes of the disease.

The same circumstances make it apparent why the disease usually appears during middle life, but no age can be said to be exempt, as the disease has been noted in the aged, occurring epidemically, and also in foundling asylums which were improperly conducted. The so-called infantile scurvy occurs in nurslings in the first year of life, particularly in the ninth and tenth months; it has so far been impossible to determine that any particular variety of food is the cause; breast-fed children are affected as well as those brought up on sterilized cow's milk or artificial foods. It has lately been maintained of Barlow's disease that milk which has been cooked too long, or that which has been subjected to too high a temperature, may be the cause. Those who adhere to this view may easily assign the same cause for infantile scurvy.

It has been shown in most scurvy epidemics that individual predisposition plays a certain rôle as well as the constitution of the patient. It is unquestionable that persons who have recently recovered from other diseases, or are still suffering from them, are particularly liable to an attack of scurvy. As predisposing diseases we may mention malaria, dysentery, enteric fever, tuberculosis, trauma as well as syphilis, especially after a very active mercurial treatment (Krebel). It is needless to enumerate all of the predisposing diseases, but I should like to point out that I have repeatedly observed isolated cases of severe scurvy in immediate connection with influenza. Quite recently, too, I treated three cases of the severest form of scurvy, two fatal cases in female children of thirteen and fifteen years, in whom the disease was connected with measles and tuberculosis, the third case in a diabetic aged sixtyfour. Moreover, I should like to emphasize that occasionally cases of scurvy with very extensive cutaneous hemorrhages and intense oral implication occur in absolutely healthy and well-nourished individuals, living under the most favorable circumstances, in whom not one of the recognized causes can come into question at all. Such obscure and entirely incomprehensible cases convinec us that up to the present time we have no absolute knowledge regarding the cause of the disease; they make it probable, however, that we should consider the view, sometimes expressed and constantly rejected, that predisposition to scurvy may be hereditary, even though to-day a discussion of this question does not appear to be timely. The facts mentioned have nevertheless been 
proven beyond doubt, and I could cite other typical and convincing cases from my own experience.

That a particular climate cannot be considered etiologically is evident from the geographical distribution of the disease. Great stress has been laid upon the prevalence of damp, cold weather during the time of epidemies, yet it appears from numerous communications that in winter and in summer, with a moist as well as with a dry air, the disease has been widely distributed. The conditions of the soil have little absolute importance.

According to our present knowledge the disease appears to be induced by unhygienic, above all by improper alimentary, conditions. The enumeration of these and the history of the disease show that by far the greatest number of epidemics have occurred upon long sea voyages, in camps, in besieged fortresses, in barracks, in prisons, in almshouses, in foundling asylums, etc. Under such circumstances a great number of injurious factors act conjointly, and it is a question to which of these the greatest importance is to be attached. Besides insufficient clothing, contaminated air as the result of over-crowding of rooms, and other influences that take part in the etiology of the disease, there is great unanimity among observers of all times and countries as to the decided influence of faulty nutrition in the causation of scurvy. However, the views diverge widely as to what errors in diet are most important. Scurvy has seldom occurred after a famine, although very frequently after a failure of crops. On the contrary, it has been attributed to the almost exclusive use of salt meat; this was the prevailing opinion before modern methods of preserving made it possible to supply better food to ships, and at a period when sea voyages were much longer than at present, so that a crew for months at a time subsisted exclusively on meat which had been salted. Many races in the far north, however, year in and year out, live almost wholly upon salt meat and fish, and in spite of this scurvy is almost unknown among them; numerous observers, too, have reported epidemics in which there was absolutely no deficiency of fresh meat. The lack of fresh water has also been mentioned, but only in isolated cases (the report of Beckler of the expedition of Burke to the interior of Australia in 1861).

The number of observations showing the importance of a deficiency of fresh vegetables in the food is almost overwhelming. We regret we cannot enter into the details of these, but refer the reader to Hirsch, who, in a "short" compilation to which he devotes many pages, relates the full particulars.

We shall only briefly state that Bachstrom was the first to point out the influence of a deficiency of fresh vegetables in the food. Lee tells us that the severe epidemic in 1823 in the south of Russia began when great swarms of grasshoppers devastated the fields. Almost all the reports of the epidemic of scurvy during the siege of Paris in 18\%0-71 agree in stating that it developed only when the supply of fresh vegetables, especially of potatoes, was exhausted, in spite of the unhygienic and evil social conditions previously existent.

Delpech cites the case of a wine merchant, aged forty-five, who was attacked by scurvy although living in easy circumstances, in a sanitary, dry, and wellheated house, and partaking plentifully of fresh meat, yet in his dietary no 
fresh vegetables were found. He recovered on eating vegetables and juicy fruits. We can rarely prove the post hoc, propter hoc with such certainty as here, where the eating of fresh vegetables, particularly those belonging to definite categories, is the best preventive, and in an existing scurvy is the best remedy.

The fact that certain vegetables, above all potatoes, have such a positive action caused Garrod to note that potatoes in particular contain a great amount of potassium carbonate. He then examined other foods to ascertain the amount of potassium carbonate (potash) which they contained. According to him they contain in one ounce (= about 30 grams) as follows:

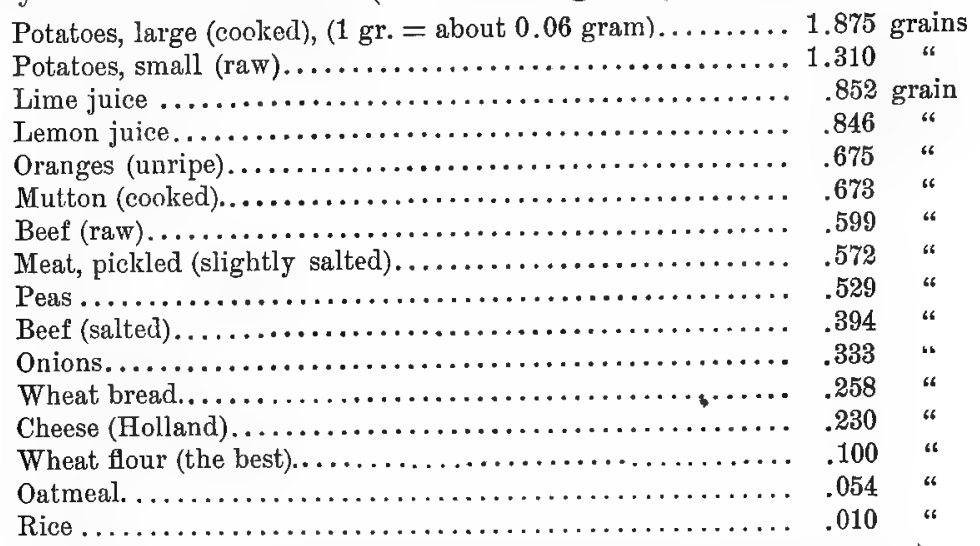

In consequence of these investigations Garrod supposes that a too slight intake of acid vegetable potassium is the primal cause of scurvy, and celebrated authors, above all J. v. Liebig and Hirsch, coincide with him.

The part that the potato plays in regard to scurvy may be recognized from the fact of the decrease of the disease with the increase of potato culture. How much the cultivation of vegetables was neglected in former times is shown by Hirsch's statement that Katharine of Arragon, the wife of Henry VIII, in - order to obtain a salad, was obliged to send her gardener to The Netherlands for the material.

But scurvy has not developed exclusively where there was a deficiency in vegetables, and particularly those rich in potash. Hirsch relates a great number of examples confirmatory of this. Indeed no one will now maintain that other causes are no longer to be considered; on the contrary, the exceptions prove that a great predisposing importance may be attached to them. We may perhaps suppose that in the above-mentioned exceptions sufficient potassium was consumed in the food, but for some reason, perhaps as the result of other deleterious causes, the organism was unable to assimilate it. Bunge believes that a cause may be found in the immoderate and extensive use of salted meat, for the reason that in pickling the meat the salts and also the potassium are extracted from it.

A. E. Wright ${ }^{2}$ had previously expressed the opinion that the disease de-

1 "On the Pathology and Therapeutics of Scurvy." Lancet, August 25, 1900. 
pends upon a kind of acid intoxication, owing to the fact that the diet which experience shows is a cause of scurvy contains a great excess of mineral acids in comparison with the bases. This agrees with the fact that antiscorbutic remedies in general contain a superfluous amount of bases in comparison to mineral acids. In conformity with this view the readily oxidizable salts contained in organic acids would be particularly adapted to the prophylaxis and therapy of the disease. Wright tested this in 7 cases of scurvy (for the most part in soldiers during the siege of Ladysmith). He gave appropriate salts, particularly sodium lactate (also acetates, carbonates and oxalates), and tested simultaneously the alkalinity of the blood by a method which he proposed, now known as the "hemoalkalimetric." In all of these cases the alkalinity of the blood was at the onset very low; and by this treatment an increase almost or quite to the normal occurred remarkably soon in all (with the exception of a fatal case) and at the same time a retardation of the other symptoms of the disease. These results confirm the view that there is an acid intoxication in scurvy.

However fascinating the potassium theory may be, it is by no means absolutely proven, and it does not contradict the view that scurvy may, in spite of this, be an infectious disease. Scurvy may perhaps be assumed to be an infectious disease of a non-contagious nature produced by a microörganism which finds in a body deficient in potassium a favorable culture medium for its development.

\section{PATHOLOGICAL ANATOMY}

The cadavers are characterized usually by only a slight degree of post mortem rigidity, and by the appearance of numerous livid areas. There is also a tendency to rapid cadaverous alterations. The various cutaneous hemorrhages present during life remain distinctly visible after death. The lower extremities are frequently edematous. Upon microscopic investigation of the hemorrhagic areas we note that in the smaller (petechiæ) the point of exit of the blood is usually the capillary network in the area surrounding the hair follicle; the larger hemorrhages (suggillations and ecchymoses) originate quite differently from superficial or deeper layers of the corium according to their seat and their extent. The erythrocytes which have exuded show all stages of decolorization and of decomposition. The surrounding tissue is permeated by hemoglobin of various colors.

As the cause of the hardenings and ecchymoses in the subcutaneous connective tissue and in the muscles, various infiltrations of blood are found, partly diffuse, in part sharply demarcated, and, according to the time of their appearance, of different colors and consistence. In the old foci where the tissues are not yet regenerated, instead of the fibrin coagula we find tough cicatricial masses of connective tissue in the surroundings of which the muscular tissue is partly rigid, partly atrophic, and the tendons either to a great extent adherent to the muscles or so hardened that movement is impossible. In this way ankylosis and deformities occur, such as club-foot, which will be discussed later. In the cavity of the joints a serous or even hemorrhagic effusion is occasionally found. The walls are frequently unchanged. If, how- 
ever, hemorrhages have occurred, more or less extensive ulcerations of the capsule of the joint, hemorrhagic effusions between the bones and the cartilage, and hemorrhagic softening of the epiphyses are noted. Corresponding to the clinical symptoms, we find between the periosteum and bones effusions of blood in different stages of coagulation or decomposition, ulceration of the periosteum, wasting and even suppuration of the cartilage of the joint, and necrotic changes in the bones. Sometimes the insertions of the muscles are loosened by these. In the interior of the bone also, particularly in the. spongiosa, hemorrhagic effusions take place.

In the bone-marrow Iymphoid transformation has not infrequently been observed.

In infantile scurvy an epiphyseal separation is occasionally observed, and in about 45 per cent. of the cases rickets also is present.

Very important changes occur in the digestive tract. The mucous membrane of the mouth, except that of the gums, is but slightly altered; now and then hemorrhagic areas and erosions are noted. The gums are invariably swollen, moderately reddened, and completely permeated by red blood-corpuscles. In older cases there is a coarse thickening as the result of proliferation of the connective tissue. Frequently the surface is ulcerated or necrotic. Babes differentiates microscopically five layers in a sequence from without inward which he describes as follows: 1. The upper layer for the most part is denuded of epithelium, moderately thick, pale, resembling a diphtheritic membrane, and permeated with but few nuclear fragments and by various bacteria, particularly streptococci; 2. A layer about $0.1 \mathrm{~mm}$. in thickness, without structure; upon staining with Löffler blue it is seen as a film of bent, extraordinarily fine bacilli, often showing long wavy forms which may reach the deeper tissues and the superficial layer in the form of sheaves or lines, and in the latter form they permit the recognition of granular decomposition; 3. Mononuclear and polynuclear round cells; 4. Mucous membrane tissue with edematous swelling, and a granular exudate with numerous bacilli of the variety described under 2 . In the walls of the vessels and in their surroundings swollen spindle cells with reticulated protoplasm which may be well stained by methylene-blue; 5. Markedly dilated larger vessels with large spindle cells in their walls.

Upon the gastric and intestinal mucous membranes infiltrations of blood are frequently found; also ulcerative losses of substance, besides necrotic and diphtheritic changes. In those cases which run their course with bloody diarrhea, special pathological processes are found in the colon. The mucous membrane is swollen, friable, and covered with a hemorrhagic mass which may be readily peeled off. The tissue lying beneath is softened or completely decomposed.

Cases are also noted in which the follicles show pathological changes, and in which the alterations have been limited to these. They are frequently ulcerated, surrounded by dense hemorrhagic infiltration.

In the large glandular organs of the abdomen, the liver and the pancreas, no special changes are found except hemorrhages and fatty degeneration. The spleen is usually enlarged, soft, even fluctuating, and occasionally exhibits 
infarcts over which the capsule of the spleen shows the remains of an inflammation that has run its course (circumscribed perisplenitis). The kidneys rarely reveal pathologic changes; only when much albumin has been excreted in the urine a more or less advanced parenchymatous nephritis may be noted, while milder grades of albuminuria leave no trace.

Amyloid degeneration of the kidneys I have never seen in scurvy, and this as well as infarcts appears to occur very rarely. However, hemorrhages under the capsule, and into the mucous membranes of the urinary passages, are not rarely met with.

The pleuræ and the pericardium are very frequently covered with hemorrhages. The pleural cavities and the pericardium occasionally contain very large amounts of a slightly hemorrhagic fluid or even pure blood. Fibrinous deposits are not rare.

The heart miuscle is pale, flaccid, often permeated by hemorrhages, occasionally showing fatty degeneration. The valves are intact, provided an acute endocarditis has not complicated the course of the disease; if so, the signs of an acute endocarditis verrucosa or ulcerosa may be met with.

In the lungs there is usually hemorrhagic edema and hypostatic congestion in the lower lobes posteriorly; occasionally, as severe complications, croupous pneumonia or hemorrhagic infarcts, but rarely gangrene. The mucous membrane of the respiratory passages is often covered with petechiæ and with a hemorrhagic mueus; there is edema of the larynx.

Besides fatty degeneration and occasionally hemorrhagic imbibition of the heart muscle, the same changes are found in the muscles of the body, particularly those of the back, thigh and arms.

\section{SYMPTOMATOLOGY}

The disease is characterized by two main groups of symptoms: 1. By an intense swelling of the gums combined with loosening and hemorrhage, to which are often added ulceration and decomposition with extreme fotor ex ore; 2. By numerous extravasations of blood under the skin, in the mucous membranes, in the coats of the eye, in the muscles, into the cavities of the body, into the joints, under the periosteum, etc.; these give rise to further disturbances which are to be looked upon partly as mechanical, as, for example, the pressure of the intrapericardial effusion of blood upon the heart. In severe cases a general cachexia of the most serious nature is added. This cachexia is usually the first, and for a long time the only, symptom of the disease, and as a rule appears gradually. At first the patients are languid and show a loss of energy, without, at the onset, being unable to follow their usual occupations; the lassitude, however, increases steadily, even slight excrtion becomes difficult, and gives rise to palpitation and dyspnea. To this is added as a very important and almost constant symptom pain in the limbs, and particularly arthritic pains, which are of marked rheumatic character and may occur in all the joints, but which, as a rule, are generally localized to the lower extremities, and are, therefore, not infrequently confounded with Schönlein's peliosis rheumatica. Increasing weakness and sensitiveness upon exertion, and absolute loss of appetite are combined with a growing sensation of chilliness, 
as well as a great tendency to somnolence, the latter symptom, however, beir by no means relieved by frequent slumber.

Corresponding to the advancing symptoms of the disease, the extern: signs of the affection become noticeable. The expression of the face denot suffering, the lines are flaccid, the freshness of the complexion gives way to cyanotic pallor, the visible mucous membranes are of a livid color, the eyt are dull, sunken in the orbits and are surrounded by rings. The skin of th body loses luster and smoothness, it becomes dry and fissured, and occasionall desquamates as in pityriasis of the aged. Upon the skin brownish spots ar observed, similar to the bronze discoloration in Addison's disease. At th onset fever is usually absent. With loss in strength advancing emaciatio goes hand in hand. To the same extent the pulse loses in tension and volum and is slow, but becomes for the time full and frequent whenever the patien makes a comparatively slight bodily exertion. The subjective sensation 0 palpitation of the heart is combined with this, without, however, either i rest or after movement, any conspicuous change being revealed by physice examination of the heart.

The constitutional phenomena just mentioned, to a certain extent prc dromes of the disease, may be entirely absent, and the patient be attacked sud denly-without a prodromal stage-with the characteristic symptoms. Usu ally, however, these symptoms precede by a considerable time, the perio varying between several days, usually from eight to fourteen, and, in excep tional cases, several weeks.

The most conspicuous site for the characteristic symptoms of scurvy is th gums, and we must emphasize that only those areas of the gums are attacker which correspond to points in the jaw in which the teeth are inserted; so that for example, in children and in the aged, in those areas in which no teeth hav appeared, or in those from which they have already fallen out, the gums shor no lesion. Where, however, through carious processes the teeth have partiall: decayed, or where, after disappearance of the crown the alveoli still posses roots, the disease preferably localizes itself. The anterior gums usually, an especially at the onset of the disease, are decidedly implicated, but not the res of the mucous membrane of the mouth. At first the free border of the gums particularly the bit which projects in the intermediate space between two teeth begins to swell, rising above its surroundings, at the same time assuming deep bluish-red color. This color is not only the expression of an edematou hyperemia but usually of a hemorrhagic infiltration as well, whereby it differ distinctly from other forms of stomatitis.

The affection of the gums rapidly advances, the swollen areas are fre quently extremely painful, and a very characteristic symptom appears; mod erate pressure, a mere touch, causes profuse hemorrhage. The longer thi duration of the disease, and the more severe its form, the more extensive th affection of the gums. At the same time, the swelling becomes greater anc greater, so that frequently nothing of the teeth can be seen.

Simultaneously an intensely disagreeable odor of decomposition proceed from the mouth, and in the later course of the disease becomes quite unbear able. The inflammatory swelling increases still further, deposits of a dirty 
grayish white color resembling those of diphtheria form, adhering closely to the mucous membrane, and after their removal the mucous membrane suffers a more or less decided loss in substance, and a bleeding, very painful surface remains. Sometimes the mucous membrane even becomes gangrenous. The surface is coated with a dirty, fetid deposit, the upper layers of which slough off, and are expectorated with the saliva. If this state continue for a long time the gums may become gangrenous and necrose, the alveoli may be exposed to a great extent, and the teeth may become loose and fall out. The unfortunate patient now suffers tortures, for these symptoms increase in severity and usually many others go hand in hand, and particularly the extremely severe cachexia. The latter is probably not a consequence of the various severe local symptoms. On the contrary, it ushers in the affection, forms a principal factor in all the phases of the disease, and is the primal cause of the intensity of the individual symptoms.

In many patients, and according to the severity of the disease of the gums, there is an increase in the secretion of saliva, probably due to reflex causes, so that a hemorrhagic fetid fluid, in which infusoria and low fungi may be recognized, streams almost continuously from the mouth.

If the affection terminate in recovery, complete restitution may occur, and the teeth which were previously loose may again become firmly rooted. More frequently a tough tissue resembling cicatrix forms and remains for life.

Mechanical effects produced by chewing and mechanical irritation have been assumed to be the cause of the almost invariable inflammation of the gums in scurvy; to these may be added the inherent tendency of the disease to inflammation.

In children, besides the usual form of simple stomatitis, another variety designated as aphthous stomatitis, an inflammatory affection of the mucous membrane of the mouth, sometimes occurs, particularly during the period of dentition, i. e., between the ninth month and the middle of the third year. Henoch says regarding this: In some of the cases a disagreeable odor from the mouth is added to the symptoms described, and minute investigation always reveals a hyperemic gum which bleeds readily, which may even show a grayish yellow detritus of friable character that may be readily loosened with a spatula. This form of stomatitis, which is of a decidedly contagious nature, usually runs a favorable course; more rare is another which is designated by the name of ulcerative stomatitis. While the fibrinous plaques which are invariably present in the latter affection are absent in the former, the implication of the gums requires the careful attention of the physician. These are dark red or bluish red and swollen, they bleed readily and decompose from the edge surrounding the tooth, gradually forming a grayish yellow paste; the crown of the tooth is exposed, and finally becomes loosened. Upon pressure a purulent fluid exudes from the space between the loosened gums and the teeth, a fetid odor comes from the mouth, and the surrounding soft parts. The cheeks and the submaxillary connective tissue frequently show edematous swelling. Occasionally the process attacks the periosteum of the jaw, extending down into the alveolar processes, causing the teeth to fall out, and finally gives rise to partial necrosis of the jaw. 
This description, after Henoch, corresponds to analogous processes in scurvy with the exception of the necrosis of the jaw, and this probably occurs very rarely in scurvy. Henoch, one of our greatest pediatric clinicians, in his description of stomatitis fails to mention scurvy, and assigns no place to this disease in his lectures upon diseases of children; I conclude from this fact that he is of the opinion that the stomatitis of infancy has nothing in common with scurvy and, moreover, that he has not observed scurvy in nurslings or in the years of infancy. ${ }^{1}$

That there is, however, such a condition as infantile scurvy may be seen from the American Collective Report, in which it is stated that among 372 cases which occurred during infancy, particularly during the period between the seventh and fourteenth months, in only 16 cases were the gums unaffected; in the remaining 313 cases there is mention of either swelling and loosening of the gums or of ulcerative processes in the gums. Certainly, Henoch's cases were not scurvy, for he reports in them no extravasations of blood into the skin or the mucous membranes; neither can we doubt that cases of ulcerative stomatitis which are not of a scorbutic nature may occur also in adults.

In view of the entirely different etiology of stomatitis and scurvy we must clearly separate the oral affections of the two diseases, and we shall only refer to scurvy if, besides the stomatitis, other symptoms, to be more minutely described later, are also present. Among these are hemorrhages into the skin, into the subcutaneous connective tissue, in the mucous and serous membranes, into the joints, muscles, etc.

The hemorrhages into the skin usually occur in the form of petechiæ which vary greatly in size. They occur early and most profusely upon the lower leg, particularly upon the extensor surfaces; the trunk and the other extremities are often affected later, the face invariably remaining entirely exempt. If they appear in great numbers the impression is given that a paint brush has been dipped in blood and spattered over these areas. Traumatic influences, a blow, pressure, especially such as is caused by the wearing of tight clothing (garters, girdles, belts, etc.), not infrequently produce these hemorrhages and give to them a definite, under some circumstances a characteristic, form and shape; for example, the form of streaks (vibices). Often an extremity is profusely covered with them, and, according to their age, they present a dark red, brownish red, green or yellowish color, corresponding to the well-known changes in color which extravasated blood or hemoglobin presents when undergoing alteration. These hemorrhages have a special predilection for the gluteal region, where they occasionally develop extensively.

The first effusions into the skin frequently form around a hair follicle, so

: In the latest edition of Henoch's book, in the description of Barlow's disease, there is mention of a change in the gums, as follows: "To these symptoms almost invariably is added a spongy swelling of the gums with fetor and tendency to hemorrhages as in the case of scurvy, and this is most marked in the cases where teeth are already present." Then follows another statement of importance: "For the present we may only assume that we are here dealing (in Barlow's disease) with a form of the hemorrhagic diathesis that has much in common with scurvy but does not appear to be identical with it." A special description of scurvy is, however, not found in his latest edition. 
that apparently the capillary vessels of the latter may be looked upon as the starting point of the hemorrhage. The effusions of blood appear as though punctured by the little hairs in this area. The hair then becomes dry, fibrillates, and falls out. The region about the nails is also frequently the seat of hemorrhages; when these suppurate, the process often extends to the bed of the nail so that it leads to paronychia, that is, an onychia scorbutica, as the result of which the nail dies. The ulcers upon other parts, particularly upon the lower extremities and the buttocks, may be of extraordinary size. Some are covered with dark, firm crusts, some have a dirty base which is covered with decomposed purohemorrhagic shreds of tissue, or flat granulations may be seen which bleed at the slightest touch. Usually the ulcers secrete continuously a thin hemorrhagico-purulent, sometimes even ichorous, fluid which has a most offensive odor. Unless cicatrization of the ulcers occurs previous to general improvement (and this is rare) these frequently spread to the surrounding tissues, or even invade them deeply, thus causing the erosion of larger vessels and hemorrhages which may terminate fatally. But even without this serious outcome these cutaneous ulcers are of bad prognosis, for their profuse secretion greatly debilitates the organism.

The hemorrhages into the subcutaneous connective tissue and into the muscles may be widely distributed, and attain the size of a plate or even be larger. Sometimes they develop acutely, sometimes slowly; in the former case they are usually accompanied by pain and an increase in temperature. The skin above them, as a rule, can be but slightly moved or not at all, it has a doughy sensation, is painful upon pressure and feels hot. In the case of these subcutaneous hemorrhages the lower extremities are the chief seat, as they are of petechiæ, particularly the region of the tendo Achillis and the popliteal space. At the onset there is noticed a soft swelling which later becomes harder, and is finally as hard as wood. The margins of the tumor are not always sharply defined. The skin above the swelling is not movable and the swelling is often not sharply marked off from the surrounding tissues. Over the swelling the skin shows decided redness and edema; it is shiny, hot and painful. After a few days these symptoms ameliorate, and the redness turns to a dull brown. Then absorption occurs, the skin desquamates, and always retains a dark pigmentation. But the swelling may soften and eventually rupture, and a quantity of necrosed gangrenous tissue mixed with blood sloughs away. The result is a deep ulcer. The course is, however, not always so acute. The swelling and hardening may occur much more gradually; pain and fever may be absent. Upon the skin there may appear the signs of a more or less well-developed suggillation, according to whether the process runs its course superficially or in the deeper tissue. As a matter of course such foci decidedly limit the function of the muscles, whether they are located in the muscles themselves or in the neighboring connective tissue. When disease of the muscle is combined with disease of the connective tissue, it is often impossible for the physician to make a differential diagnosis. In the muscles quite isolated foci may be found which are characteristic in that the integument over them scarcely shows change. These foci vary greatly in the symptoms which they produce; some are circumscribed, some diffuse, 
some painful, some painless, some acute and accompanied by fever, some gradual in onset and afebrile in course.

Hemorrhages from the mucous membranes are decidedly less frequent, but they may cause death or hasten death by inanition. We must mention hemorrhages from the nose, hematemesis, enterorrhagia, hematuria, metrorrhagia and hemoptysis. For a long time, severe epistaxis has been greatly dreaded; it is, however, not very frequent, but can rarely be stopped without packing, and sometimes causes death. These hemorrhages rarely occur spontaneously, but are usually the result of slight injuries to the nasal mucous membrane; they may follow strenuous blowing of the nose. The fact that an external cause is usually required to produce the hemorrhages which are so characteristic of scurvy, Immermann believes will explain the circumstance that the gums are usually the seat of these symptoms. He is of the opinion that the stomatitis marginalis of scurvy is also secondary, inasmuch as the gums, considering the delicacy of their histologic structure, are so frequently and decidedly exposed to mechanical, chemical and thermic irritations-particularly as the development of the affection of the gums is always apparently associated with the presence of teeth in the jaw, and therefore with the process of chewing. It may also be here emphasized that the gums are not exclusively the seat of the disease, nor are they the first typical points, but severe cases may run their course without an implication of the gums, and in general the sequence of the individual symptoms and the number of the implicated organs may be very variable.

Hemorrhage from the mucous membranes of the stomach and intestines is decidedly more rare than from the mucous membrane of the nose, and is prone to occur when the bowel is stimulated to decided peristalsis; as, for example, after the administration of purgatives. Its development may, therefore, be compared with epistaxis after violent blowing of the nose. In rare cases dysentery is simultaneously present. Hemoptysis occurs only occasionally owing to a coexisting tuberculosis or a fibrinous pneumonia, the latter disease then having its starting point in a hemorrhagic pulmonary infarct.

Besides these hemorrhages which appear upon the surface, or from organs with an external outlet, others may occur in the internal cavities, or there may be bleeding even into the organs themselves. These represent a severe form of the disease; they cause extreme debility or may even be the immediate cause of death. Among these we must mention first the pleural and pericardial hemorrhages some of which may be looked upon as forms of noninflammatory hemothorax which occasionally leads to an inflammation of the serosa, some as inflammatory hemorrhagic effusions which, by compression of the lungs or paralysis of the heart, decidedly increase the danger to life. That these effusions into the serous cavities (which may also occur in the peritoneum) are susceptible of absorption is unquestionable.

Meningeal hemorrhages have also been observed, and may be diagnosticated by pain, paresthesia, spasms, contractures, paralyses, and apoplectiform attacks. In some cases death occurs with the symptoms of apoplexy, but actual cases of true cerebral hemorrhage appear to be extraordinarily rare. More frequent are scorbutic changes in the eye. Hemorrhages and inflammation of the con- 
junctiva, hemorrhages into the anterior chamber of the eye, or choroiditis hemorrhagica have been noted. The subconjunctival hemorrhages may cause loosening of the conjunctiva, and to such an extent that the membrane swells out under the eyelid, frequently covering the eye-ball to a great extent. Occasionally iritis also occurs. All of these symptoms may improve or may lead to corresponding permanent changes. In very severe cases, bilateral panophthalmitis usually occurs and leads to entire loss of sight. These cases invariably terminate fatally. Seggel observed in a mild case hemorrhages into the vascular wall of the retinal vessels, besides frequent repetitions of subconjunctival hemorrhages, and a swelling due to hemorrhage into the pia sheath of the optic nerve with a slight cloudiness of the papilla. Not infrequently in the course of the disease, as prodrome, or as. sequel, hemeralopia or night-blindness is observed-a condition in which the sense of sight is more or less lost during dusk and at night. Nothing is known of the causal relation of the underlying affection to these phenomena.

A particular and important group of symptoms is that affecting the tissues constituting the joints, including the cartilage and the bone. Besides the more or less widely distributed rheumatoid pains in the joints, painful swellings of the latter, due to effusions, are observed. These latter may be purely serous, but more frequently consist of a sanguinolent fluid. Sometimes there is also suppuration of the joint with subsequent erosion and deformity, and finally true ankylosis. Inasmuch, however, as the affection usually terminates in recovery, these effusions are generally absorbed without ankylosis developing. In the bones, particularly after mechanical injuries not necessarily at all severe, hemorrhages occur, or inflammatory periosteal effusions between the periosteum and the bone arise. Indurated and very painful swellings result, which, however, may slowly subside, or may lead to local necrosis and the formation of sequestra. At the epiphyses the coverings of the cartilage become detached. The long tubular bones of the lower extremities are chiefly attacked, next those of the upper extremity, frequently also the ribs, in which case the process leads to detachment from the sternum. The kind of aid given by scurvy to many intercurrent affections may be suspected if we notice how in fresh cicatrices, or in those previously formed, it produces a softening of the callus.

During the progress of the symptoms described the constitutional condition of the patient deteriorates. He becomes cachectic looking, the fat and the musculature gradually disappear. Fever may be absent, in other cases may be slight, without conforming to any definite type. Decided rises in temperature are generally due to complications or to suppuration. Sometimes swelling of the spleen is noted; this, however, can by no means be looked upon as specific. The symptoms on the part of the heart correspond with those of other anemic conditions-systolic murmurs and dilatation. Clinically the most prominent symptoms are cardiac palpitation and dyspnea upon comparatively slight movement and exertion. The small, frequent, and slightly irregular pulse is notable. In the course of the disease endocarditis occasionally occurs, but clinically, in the majority of the cases, can scarcely be recognized, being anatomically characterized by a delicate circle of excrescences upon the free border of the mitral valve or the aortic valve. Under some circumstances 
it runs its course with the symptoms of ulcerative endocarditis. It is to br hoped that we may succeed in discovering in the endocardial deposits the pathogenic agent of the disease, which so far has been unsuccessfully lookec for in the blood. On the part of the digestive tract we have occasional diar. rhea which may be of a dysenteric character. Several times the combinatior of scurvy with true dysentery has been observed. Kidney troubles are com. paratively slight; occasionally transitory albuminuria is met with, without ou being able to conclude from this the presence of genuine nephritis, which is a rare complication. Reports of marked hematuria are rare. The urine is fre. quently of a dark color, which Kretschy ascribes to an increase of the norma] coloring material due to a greater destruction of red blood-corpuscles. In severe cases the quantity of the urine, especially during the progress of the disease is greatly diminished; peptonuria occurs as well as albuminuria. According to $\mathrm{v}$. Jaksch the peptone does not originate in the blood itself, but from the hemorrhages of the skin, of the subcutaneous cellular tissue, etc. To this he ascribes the origin of the profuse urobilin.

Of course, special importance has been attached to the amount of potash in the urine, but the reports regarding the quantity found are very contradictory. Phosphoric acid is said to be increased; unquestionably the amount of uric acid is great, especially at the height of the disease, but it rapidly decreases with beginning convalescence.

In the blood, apart from Klebs' questionable nomads, nothing characteristic has been found. The findings correspond in the main with those of severe anemia. The amount of hemoglobin is decreased, and in severe cases not only the number of erythrocytes but also their hemoglobin contents; poikilocytes and microcytes I myself have frequently found, some with basophilic granulations. Particularly common is the form of achromic cell described by me resembling a pessary. Penzoldt also reports the presence in the blood of granular, and some markedly refractive, bodies which he looks upon as embryonic forms of red corpuscles. Macrocytes also are said to have been found in large numbers. According to the reports of Laboulbène the number of leukocytes is increased; this I am unable to confirm from my own observations. The reports are contradictory regarding the amount of iron in the blood. According to Opitz and Schneider it is somewhat increased; according to Duchek it is nearly normal, and according to Becquerel, Rodier and Chalvet it is decidedly decreased. The latter has also determined a decrease in the amount of potassium.

As a result of our review of the great number of accounts of severe symptoms in past epidemics we must emphasize our impression that, at least in Germany, the severe forms of the disease are now only very rarely observed.

Frequently the symptoms are limited to great lassitude, pain in the joints and limbs, extensive hemorrhages into the skin and elsewhere, and more or less decided hemorrhagic inflammation of the gums. In these cases the entire course is comparatively short.

Scurvy may terminate in death or in complete or partial recovery. Generally recovery is exceedingly slow, and weeks and months pass before the rlifferent processes have run their course. The disease of the gums, if great 
loss of substance has not occurred, steadily improves, the swelling disappears, and restitutio ad integrum takes place. On the other hand, permanent changes in the form of cicatricial retractions or hyperplastic thickening of the tissue may remain, which, however, do not give rise to any difficulties. Of the processes in the skin, the small hemorrhages improve most readily. They go through all the changes that hemoglobin shows, and finally disappear. With an improvement in the general condition, the cutaneous ulcers also pursue a favorable course. Their surfaces become clean, and finally skin is formed in which a dark pigmentation remains for a long time. The swelling in the muscles and connective tissue also gradually recedes, although very slowly, so that a callus is often evident long after complete recovery. Not rarely, too, connective tissue proliferation occurs (as in the gums), the result of which is permanent contraction or fixation of the muscles and joints, with, for example, the formation of club-foot.

\section{DIAGNOSIS}

In view of the marked symptoms, the conspicuous epidemic character of the disease, and the extraordinary circumstances under which it usually arises, the diagnosis of scurvy can scarcely be difficult.

Difficulty arises only when we are dealing with sporadic cases, and even in these a careful history of the previous conditions of the life and residence of the patient will lead to a recognition of the affection. If we remember that the greatest number of epidemics occur upon ships, in fortresses, among isolated bodies of troops, or in penal and eleemosynary institutions, that quite definite etiologic factors such as insanitary conditions of life, and, above all, improper food have preceded the outbreak, no further proof is needed for us at once to recognize the disease. The disease may break out in so many places that it is only very rarely that symptoms are at all scanty in a given case; almost always a number of the usual symptoms appear simultaneously. Serious diagnostic difficulties will, therefore, only occur in isolated cases of unusually mild character. But these are just the ones which to-day present themselves for professional treatment.

First among the external symptoms we have learned to recognize those which almost always precede the local phenomena of the disease and increase with its advance; i. e., the general cachexia. Next come the various effusions of blood into the skin, into the deeply lying connective tissue and the muscles, the rheumatoid arthritic pains occasionally present, and, above all, the affection of the gums. The number of diseases which may be confounded with it is very small. First, those diseases which form a common group with sceurvy (hemophilia and morbus maculosus Werlhofii). Neither is epidemic in any sense; and as both are to be described later they need not now be considered. I will state here, however, that, in spite of the external similarity of many cardinal symptoms, the disease of the gums is the predominant feature in scurvy, and is not present in either of the other diseases mentioned.

Cutaneous hemorrhages, especially petechiæ and small ecchymoses, are met with in a number of cachectic diseases. When associated with rheumatoid or arthritic pains a confusion of scurvy with peliosis rheumatica may occur. If, 
in such cases, the diagnosis is difficult, it must be remembered that scorbutic hemorrhages are generally of an inflammatory character, while in the other diseases this is by no means the case. Finally, it must be borne in mind that in scurvy we have excellent therapeutic measures under the action of which the symptoms usually disappear rapidly, while in the majority of the other diseases therapy is for the most part of no avail.

\section{PROGNOSIS}

While the prognosis during past historic epochs was usually very grave, to-day, fortunately, it is very favorable, especially in cases other than the most severe. Naturally, we must be cautious in prognosis, for even in mild and moderately severe cases intercurrent affections may bring about death. The earlier the therapy is begun the more favorable the ultimate result and the shorter the course, although even under favorable circumstances it always requires considerable time for the patient completely to regain his strength. Complete recovery is, of course, only to be expected in those cases in which the disease has shown itself to be mild; in other cases recovery is usually incomplete. Only in cases in which no loss of substance has occurred can a complete restitutio ad integrum be expected.

In cases which terminate fatally, the patient usually suffers for a long time, and passes through many complications before he finally succumbs to general exhaustion. Yet many circumstances may lead to an early death. Among these by far the commonest are hemorrhages of lethal extent. These may be from the gums, from the nasal mucous membrane, from cutaneous ulcers, eroded arteries, or from the digestive tract.

More frequently death results from complications, particularly dysentery and croupous pneumonia, more rarely malignant endocarditis, the cause of which is to be sought for in the entrance of infectious germs from the ulcers. Quite often excessive effusions into the pleural cavity or into the pericardium threaten life; sometimes extreme cardiac asthenia leads to a fatal result, and if the patient makes an unusual exertion or rises suddenly, he collapses lifeless, as occasionally happens in children who have had an attack of diphtheria.

\section{PROPHYLAXIS AND TREATMENT}

Garrod's teaching that a deficiency in acid vegetable potassium is the chief cause of the disease indicates the direction in which a prophylaxis in scurvy is to be attained. The theory of Garrod, although correct in its fundamental principles, does not appear to be absolutely well-founded throughout, for cases of scurvy develop where there is no deficiency of potassium in the food. In the epidemic in Rastatt (1851-1852), for example, there was no deficiency in fresh vegetables; nor in that of Ingolstadt where, in spite of good food, such as meat and potatoes, an epidemic occurred among the prisoners of war. It has therefore been assumed that it is not the deficient administration of potassium, but the insufficient retention of potassium in the organism, which causes the disease. Unquestionably, among other conditions, the increased consumption of potassium in the body plays an important rôle. If the theory of Bunge 
is correct, the excessive ingestion of table salt produces an increased excretion of potassium. Thus the fact can easily be accounted for that the continued use of salted meat frequently causes scurvy, although this is denied by many.

In consonance with opinions generally held, plentiful amounts of acid vegetable potassium should be administered to the organism in the form of fresh green vegetables such as new potatoes, spoonwort (scurvy grass), cabbage, spinach, water cress, radishes, sorrel, sauerkraut, carrots, turnips, onions, artichokes, asparagus and lettuce, also juicy fruits, oranges, milk, fresh meat, meat extract, meat juice, particularly puro (from the laboratory of Scholl in Munich), meat juice, essence of beef, and good potted meats. In view of the comparatively low price of vegetables, the daily diet may be well supplied with acid vegetable potassium even in the feeding of prisoners for whom but a small allowance is made. Lately the diet in prisons has been decidedly improved by the addition of fish.

It is more difficult to prevent scurvy at sea. In long sea voyages the supply of fresh vegetables is soon exhausted, and a portion is likely to spoil. But canned vegetables may readily take their place without decidedly increasing the cost, owing to the abundance of good material upon the market. Upon long trips a substitute for fresh meat is furnished by meat extract.

On many ships in order to furnish the requirement of acid vegetable potassium, lemon juice is regularly given to the crew. In the English marine it is obligatory that the crew shall receive a corresponding amount of lime juice in a mixture of ten parts of lime juice to one part of spirits. The best method is to carry the fresh fruit upon the trip, and prepare lemonade from it. Artificial lemon juice is sometimes adulterated, and decomposes very readily. Nordenskjöld advises preserved mulberries for expeditions, as these are said to have a particularly favorable action. Neale believes fresh meat (with the blood) to be the most efficient antiscorbutic. When this is not at hand, green or canned vegetables and lemon juice must be used. Potatoes and milk are also advised, as well as cider and Moselle wine.

Great stress is also laid upon drinking-water and, if necessary, this must be obtained from salt water by distillation. Nansen advises that in Polar regions the ice which projects above the surface be melted for drinking, since these parts have been exposed during the summer to the rays of the sun, and have to a great extent lost their salt. He believes it unnecessary to distil this water for drinking in order to escape the danger of scurvy, and considers the belief erroneous that a small amount of salt is harmful. Good beer (pine beer) is useful for the prevention of scurvy, and the present status of brewing makes it possible to take large amounts of inexpensive beer upon long sea voyages.

Pickled meat, as universal experience has shown, has a deleterious effect, and for this reason its use is to be limited as much as possible.

In regard to epidemics at sea, the conditions of seafaring in modern times have changed; steamers have greatly shortened the voyages, and provisions are carried in smaller quantity than formerly. These causes, and also the excellent naval regulations in which England has shown itself superior to all seafaring nations, have made the disease so rare in the English Navy that in 
the years from 1856 to 1861 only 1.05 per thousand of marines were attacke by the disease. In the Austrian Marine the number of affections from $1863 \mathrm{t}$ $1870=1$ per cent., but in $18 \% 1$ and $187 \%$ it declined to 0.34 per cent. I the German Marine during the period from April, 1875, to March, 1880, onl 16 cases of scurvy and 76 cases of scorbutic gum affection occurred, thes groups collectively giving a proportion of 0.475 per cent.

The occurrence of scurvy upon ships depends neither upon sailing in north ern nor in southern latitudes, in this nor in that season, but particularly upor the supply of provisions in proportion to the length of the voyage. Thi readily explains why conditions are more unfavorable upon sailing ships thar upon steamers. In the former only the most careful ship hygiene can preven an outbreak of the disease, and upon such ships general hygienic conditions ar still unsatisfactory.

Besides proper kinds and sufficiency of food, general hygiene is most impor tant in prophylaxis, particularly perfect cleanliness of body as well as of hab. itation, avoidance of excesses, protection against cold, etc. Isolation of the patients when possible, and the greatest attention to cleanliness on contact witl them, form the best means for limiting the spread of the disease, that is, for arresting it. Too much stress cannot be laid upon exercise in the open air, by which the psychical condition of the patients is improved. Where a change of residence is possible we should always take advantage of it.

I should like to mention a few points of importance from the interesting book of Nansen, "Farthest North," which reports one of the greatest sea voyages of all times, in which during three years no case of scurvy occurred. In regard to provisions he holds the view that in prolonged Arctic expeditions meat and fish that have been preserved by salting, smoking or incomplete drying are to be regarded as objectionable, and are to be rejected. The leading thought in provisioning must be to preserve the food either by careful and complete drying, or by sterilization. "What I wished to attain was to have not only a nourishing and wholesome stock of provisions, but to see to it that there was as much variety as possible. We took fish of all sorts in hermetically closed tins, dried fish and preserved fish, potatoes, dried as well as in tins, all sorts of dried and preserved vegetables, cooked and dried fruit, preserves and marmalade in large amounts, sweetened and unsweetened condensed milk, preserved butter, dried soups of various kinds, etc., even bread, dried vegetables, etc., in zinc cases. As drink we used at breakfast and in the evening chocolate, coffee and tea, occasionally milk. For supper at first beer, later lemon juice with sugar or syrup. Aside from beer and some malt extract the expedition had no alcoholic drinks. Occasionally a grog was made from mulberries or other fruit syrup with the addition of some spirits." Meat and fish were carried in a desiccated condition, free from fat, cartilage, etc., and mixed with kidney fat, also Vaage's fish flour; flour (previously stewed), so that it could be eaten without further preparation; dried cooked potatoes, pea soup, chocolate, bread (carefully dried, hard, wheat bread) and aleuronat bread (wheat flour with 30 per cent. of aleuronat) and butter.

It may be interesting to quote the menu of the 25th of December, 1893: 1. Oxtail soup, 2. Fish-pudding, with potatoes and melted butter, 3. Reindeer 
roast with peas, French beans, potatoes and preserved cranberries, 4. Mulberries with cream, 5. Cake with marchpane.

The general treatment of scurvy is entirely analogous with the prophylactic diet. Warm baths often have a favorable action.

Special treatment: In the drug treatment of scurvy, cochleariæ has long been prominent. A formula greatly employed is:

R Herb. cochl. rec. conc................. 50.0

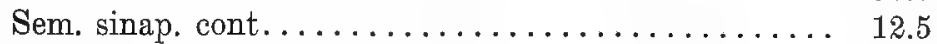

Vin. gall. alb.................... 300.0

Macera per biduum, colat. adde spir. æth. chlor..... 6.0

M., D., S.: Half a wine-glassful three times daily.

There are further employed: Astringents, such as tonica amara, and aromatics, such as tannic acid, quinin, cascarilla, myrrh, ratanhia, calamus, and gentian.

The employment of beer yeast, pure or with the addition of water and sugar, $\bar{a} \bar{a} 200$ to 300 grams, daily, is very popular.

Disease of the gums is best prevented by the early removal of all carious teeth and deposits of tartar; later this is not so feasible, and the affection of the gums must then be treated locally with astringent solutions. Tincture of myrrh, potassium chlorate and potassium permanganate in weakened solution are especially employed.

In scorbutic cutaneous ulcers, poultices containing potassium permanganate in solutions of 1 to 300 and bandages are serviceable.

Hemorrhages are treated by styptics and tampons, as well as by ergot. Surgical interference may be very dangerous; even simple bandages that cause pressure may bring about deep tissue hemorrhages and deep ulcerations.

In case of constipation all purgatives which have a decided action are to be avoided unless absolutely necessary. The bowels must be opened by the cautious administration of enemata, or by the employment of the mildest laxatives, such as tamarinds, for by the too active stimulation of peristalsis fatal intestinal hemorrhages may be produced. In the treatment of such bleeding little or nothing is gained with styptics such as ergot and sesquichlorid of iron; ice externally or internally serves the purpose best.

\section{HEMOPHILIA (BLEEDER'S DISEASE)}

By the term hemophilia we mean a peculiar hereditary anomaly of constitution, characterized on the one hand by traumatic hemorrhages of extraordinary stubbornness, and on the other hand by a conspicuous tendency to spontaneous and repeatedly recurring hemorrhages, for which, up to the present time, no plausible anatomical substratum has been determined. Combined with this is a marked predisposition to "rheumatic" affections which is manifested by painful arthritic swellings. In the recognition of the disease, the congenital and hereditary factors must be considered as well as the habitual tendency to bleeding, and in the present conception of the disease these two peculiarities dominate the clinical picture. They form a basis, too, for the dif- 
ferentiation of hemophilia from related clinical pictures which are included ir the conception of the hemorrhagic diathesis, i. e., from scurvy and morbu: maculosus Werlhofii. The former differs from hemophilia decided]y by thi fact that it is rarely sporadic, but usually endemic or epidemic. Still morr characteristic, however, is its dependence upon external conditions; it is the expression of severe disturbance of nutrition produced by obscure diseases of by long-continued insufficient and improper nourishment. Morbus maculosus Werlhofii differs from hemophilia particularly in that it unquestionably belong: to the acquired diseases, in which the factor of heredity plays no part. This affection is never congenital as is the case with hemophilia, and this differentia] peculiarity also applies to scurvy. Unlike the scurvy patient, the bleeder is frequently well nourished and strong, and except for his tendency to spontaneous hemorrhages he might almost be considered healthy.

In comparing hemophilia with related diseases in the group of the hemorrhagic diatheses, one peculiarity of hemophilia stands out prominently. In contrast with what is found in the diseases previously named, it is not a pathologic process but a permanent condition which manifests itself sometimes after recognizable causes (traumatic hemorrhages), at other times following unknown ones (spontaneous hemorrhages). For this reason hemophilia has been designated as a "vitium primæ formationis."

Hemophilia is probably in all cases a congenital, and also a hereditary condition. The intensely hereditary character of this pathologic condition has been from the earliest times a source of great interest. Grandidier calls it "the most hereditary of all hereditary diseases." Only very exceptionally is a single case of hemophilia observed in a family. The most striking point is the frequency of the cases in one and the same family. From a study of the total number of known hemophilia cases and their distribution in individual families, it may be noted that in every family of bleeders there are at least three bleeders. Sometimes the transmission is immediate from the parents to the child. Much more frequently, however, the transmission of the disease follows a peculiar law of heredity, peculiar because it reveals a remarkable sex difference in the frequency with which hemophilia is acquired and transmitted. Females show a greater tendency to transmit the disease; while, in contrast to this, in the male sex a predisposition to acquire the disease is prominent. The latter predisposition is evident from the fact that the majority of bleeders are of the male sex. The tendency of the female to transmit hemophilia is characterized by another peculiarity. A woman from a bleeder family may transmit hemophilia to her offspring without herself being hemophilic, the disease skipping a generation. This order of transmission is indeed the rule. Formerly, when fewer reports of cases were at hand, it was believed that only males were affected by the disease, females being exempt, and that it was women exclusively who transmitted the disease. On this assumption first the laity and subsequently also scientists designated the women in families of bleeders as "conductors." Close investigation and increasing experience have, however, taught us that females also may be attacked by hemophilia, although far less frequently than males. For every thirteen males there is one female bleeder. The viow that women exclusively transmit the disease 
can no longer be strictly maintained on account of many exceptions to this rule. Grandidier has described the predisposition, transmissibility, and the hereditary sequence in hemophilia in the following paragraphs:

1. Men from families of bleeders who are bleeders themselves, whose wives are not descended from bleeder families, by no means always beget hemophilic children; on the contrary, in these cases the children are frequently healthy with no tendency to the disease. Vice versa, however, it appears that hemophilia quite invariably appears among the children of women who are bleeders.

2. Men descended from bleeder families without being bleeders themselves, and whose wives are from normal families, rarely beget hemophilic children; on the other hand, women belonging to bleeder families who are not themselves bleeders, give birth almost invariably to children who suffer conspicuously from hemophilia.

On account of the gravity of hemophilia, its continuity in families, and the fatal outcome which is usually due to severe hemorrhage, also because of popular interest and the mysterious character of the affection, it is natural that records regarding bleeder families extend far back into the past.

Grandidier, in his well-known monograph, cites 200 bleeder families with 609 male and 48 female bleeders (=13 to 1 ). In the bleeder family described by Stahel there were 24 males, all bleeders, in four generations. Although females are much more rarely attacked by the disease than males, yet transmission most certainly occurs through the female members of the family. In Bollinger's collection of cases it appears to be the rule, as in color-blindness (Daltonism), that the sons of women whose fathers were bleeders are most liable to hemophilia.

Otto and Nasse have published reports regarding the first recorded bleeder families. Among those that have become especially well known are the families at Tenna in Graubündten, the American family, Appleton-Browe, and the Mampel family at Kirchheim near Heidelberg, whose genealogical tree was first described by Chelius in the year 182\%, then by Mutzenbecher in 1841, and lately by Lossen (Fig. 22). This ancestral tree shows that the tendency to bleed is transmitted exclusively through the female members of the family who themselves without exception remained unaffected, a peculiarity which we shall later discuss in another affection, hereditary Daltonism. Vice versa, from marriages between male bleeders and female non-bleeders healthy children are born.

Worthy of note also is the proportion between boys and girls in the various generations: In the first generation from the common ancestors, there are four boys and two girls; three of these boys (or 75 per cent.) are bleeders, but none of the girls. In the second generation there are 14 boys and 9 girls; of the former 13 (or 93 per cent.) are bleeders, of the latter, none. Finally, in the third generation, among at least 50 children, there is only one bleeder, a male who was the offspring of a non-bleeder mother belonging to the same ascending line. There is, therefore, a conspicuous diminution of the disease in the third generation, and this is probably due to intermarriage with healthy families. 
Another tree of a well-marked bleeder family was described by M. Fischer in 1889 in his dissertation on this disease. This family, comprising more than four generations, lived in a village in Württemberg, and though otherwise not of distinction was characterized by a few remarkable facts. Thus the apparent male ancestor who was a bleeder (he married twice, one wife evidently not being hemophilic, there being bleeders among the children of both marriages) himself directly transmitted the predisposition, whereas this is ordinarily the case only with the female members of the family. Furthermore, among the female "conductors," there were two who were themselves bleeders, which is contrary to the rule. On the whole, among 114 members of the family there were 17 bleeders, 13 males and 4 females, the implication of the female sex being excessively high. The length of life of these bleeders was from nine months up to sixty-two years. In most members of the family, especially among the bleeders, there was another pathologic predisposition, namely, to rheumatic affections, headache, congestions, and affections of the teeth.

Only continuous and prolonged study of the genealogical history of a bleeder family will reveal whether the disease is likely to assert itself for a longer time, or whether the tendency is so diminished in the mothers of the third generation that it will no longer betray itself.

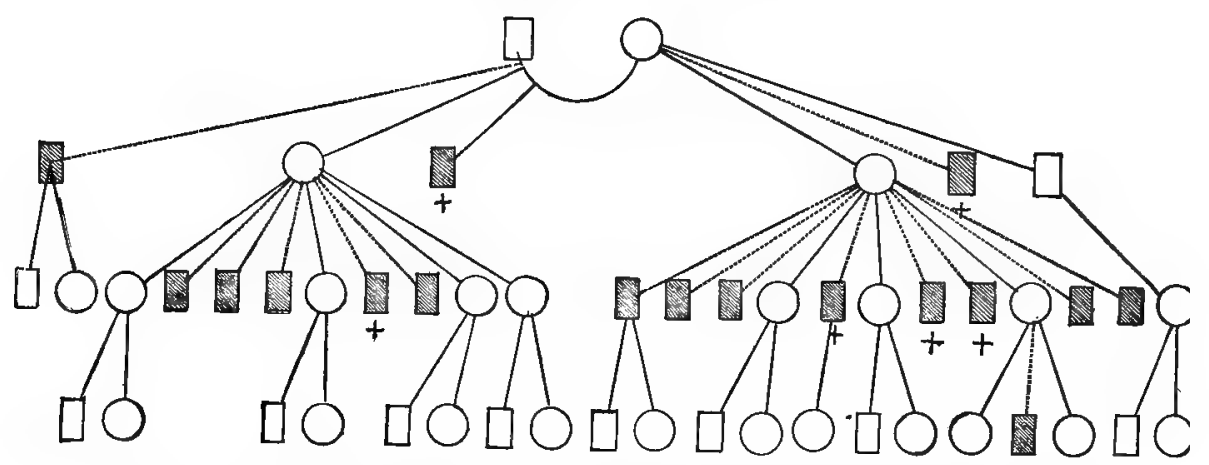

Fig. 22.-Genealogical Tree of the Bleeder Family Mampel. (After Lossen.) Bleeders are shaded.

GUIDE TO FIGURES 22, 23, 24, AND 25.

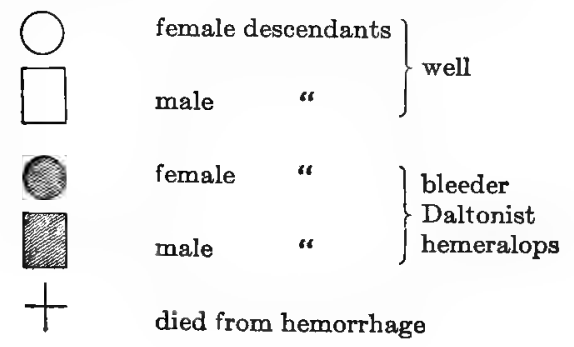


The fact that hemophilia may be directly transmitted also by the male descendants forms an exception to the rule that women, even healthy women, transmit the disease, that is, act as conductors. In a family in Bremen the affection was transmitted by the father to the male members through three generations. Also in the bleeder family from Wald in the Canton Zurich such a tendency is noted, while on the other hand, the transmission from the healthy mother occurs in the sons. In this case, in the first generation that showed bleeders, among 16 persons there were $\%$ bleeders. In the following generation, among 28 members there were 16 bleeders. In the third generation, there was a noteworthy decrease in that this showed only 1 bleeder and

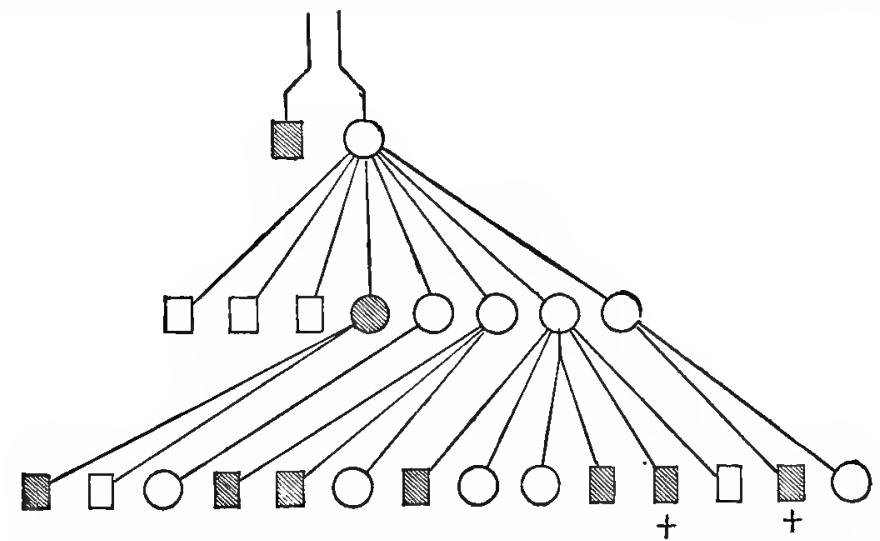

Fig, 23.-Genealogical Tree of a Bleeder Family. (After H. Gocht.) Bleeders are shaded.

12 non-bleeders. That from a hemophilic mother bleeders as well as nonbleeders may be born is demonstrated by the following ancestral tree which is taken from a communication of H. Gocht (Arch. f. klin. Chir., Bd. 59) (Fig. 23).

The disease proved itself most stubborn in a family in the little village of Tenna in Graubündten, consisting of about 170 persons, whose family tree was first described by Grandidier and Vieli, and then carefully revised by Hösli who eliminated many incorrect statements of the first communication. Here the hereditary transmission could be followed through six or seven generations. However, bleeders twice married into the family, which may explain the long persistence of the pathologic predisposition. Several times the disease skipped two generations in this family, then reappeared in the third. In the direct descendancy still longer pauses occurred; but probably only the severest cases have been recorded.

The disturbances to which we may ascribe hemophilia may be designated as "parablastic," if we with His ascribe the development of the connective tissue and of the blood and lymph apparatus to the parablasts in opposition to the archiblasts, this latter designation being reserved for the epithelial tissues that compose the ectoderm and the entoderm.

Although the development of the parablasts may still be questionable, 
and it may not be as yet quite certain, as His assumes, that this is an excl sively maternal formation, still pathology furnishes a number of remarkab facts emphasized by Klebs, which assign to the connective tissue germ ("par: blasts") a definite position in the structure and economy of the body. As 1 the controversy in regard to the origin of the parablasts and their compariso with the archiblastic tissue, no matter how it may be decided, His has prove the entrance of the parablastic endothelia into the cavities of the heart an the vessels (a view which has been given new support by Kölliker and by ( Hertwig) and this indicates a great advance in pathology. If this specii position of the parablasts is recognized, quite new view-points are presente for the production of pathologic predispositions during fetal development, 1 which Klebs particularly refers. For it may be proven that in a varyin development of the connective tissue extraordinarily important congenital an pathological processes are combined, and that these are processes in which tr transmission of both sexes plays throughout a very different rôle, the fema] element representing the carrier of the disturbance, without in all cases pas ticipating in the disturbance itself, while the male element is the passive par We might be inclined from this general fact to ascribe to the parablasts $\varepsilon$ least a predominant feminine nature, as might easily be possible if they actuall arose from leukocytes that had wandered in. As this, however, has not yt been proven we must content ourselves with looking upon the parablast as structure upon which the male pronucleus (sperm cell) in the main has slight influence, much less than the female pronucleus.

We incline to this view for the reason that individual parts, particularly th bony tissue, by reproducing paternal forms from the parablastic tissues, as $\mathrm{i}$ very frequently seen, may be recognized as under the influence of the mal pronucleus. Nevertheless, we may assume that in certain cases this influenc is much less operative in females than in males, and thus the non-appearanc of the disease in the female members of a hereditarily affected family may fin an explanation.

The same peculiarity of the transmission of properties through the mothes who herself may show no traces of them, is observed (as may be mentioned her in passing) also in other disturbances which cannot be traced exclusively $t$ the parablasts, such as hereditary Daltonism. In this, as in bleeder families the law of transmission to the male descendants is seen, as was mentioned b: Ribot and Darwin. Horner reports two very conclusive ancestral trees, th correctness of which is due to his personal knowledge of the members of th family. The ancestral tree is here given for comparison (Fig. 24).

This affection occurs exclusively in the male descendants, and is trans mitted by the female who remains free. The single exception of a transmis sion from father to son, which occurred in the fifth generation, is only al apparent one, for the mother belonged to a collateral line affected with th same defect. It is noteworthy that from this marriage a great number 0 grandchildren who were color-blind were begotten, the mothers of all, corre sponding to the law, remaining unaffected, although in this case the hereditar. transmission appears to have attained an unusual development.

The same law of heredity as in hemophilia and in color-blindness is als 
operative in another remarkable affection-night-blindness (hemeralopia). Although this disease, strictly considered, is not within the realm of our theme, nevertheless it is so interesting that this slight digression is permissible. Our knowledge regarding the etiology of night-blindness, in so far as heredity is concerned, is limited to the following points: First, that hemeralopia, if it is hereditary, attacks more men than women, and that the disease is frequently met with when the parents are blood relatives; further, that the disease has been observed in several children of the same family, and has been followed through several generations, from four to six. In literature we find that where the disease has appeared in successive generations partly sons and partly daughters were attacked (Sédan, Recueil d'ophthalm., 1885: Two fam-

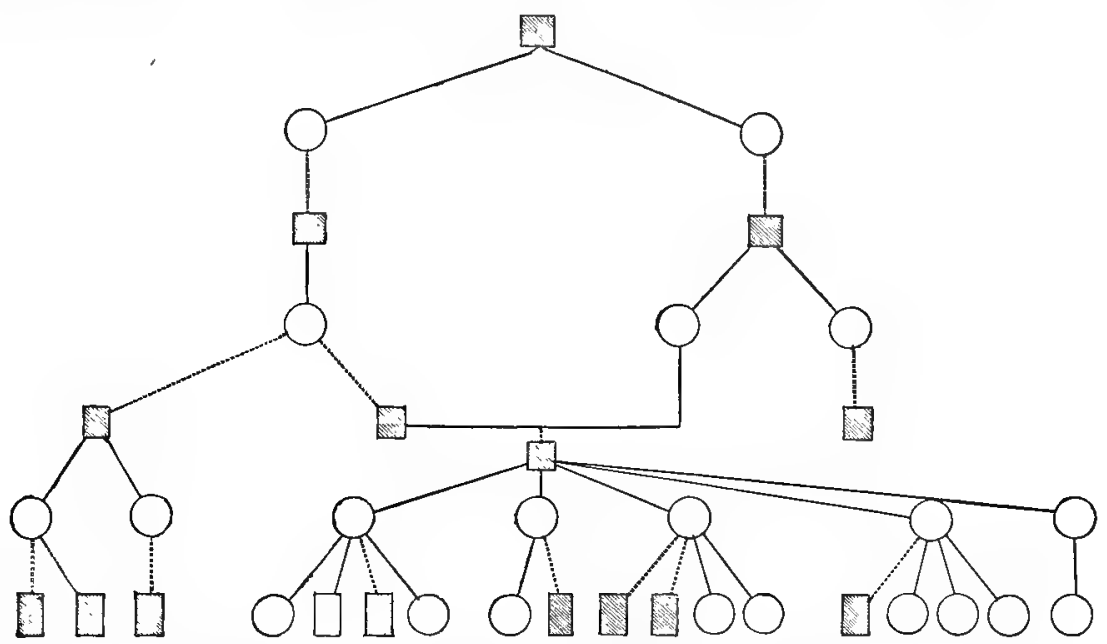

Fig. 24.-Genealogical Tree of a Famity showing Daltonism. (After Horner.) Daltonists are shaded.

ilies: in the first there are only 12 members, 5 females and 7 males, that were affected, and in the second family, of 9 that were affected, 5 females and 4 males. Savenzy (Irish Hospital Gazette, 18\%2) reports a family of 5 brothers and 5 sisters, of whom 2 brothers and 3 sisters had night-blindness); or the disease may attack only the sons in a generation, and in such cases we occasionally find the type of heredity which is characteristic of hemophilia and of Daltonism.

We owe to E. Ammann, eye specialist in Winterthur, the knowledge of the fact that night-blindness is transmitted according to the same laws as hemophilia and Daltonism. Ammann reports the family tree of a hemeralopic family from which the law of heredity may be determined with absolute certainty (Fig. 25). At the same time the observation that night-blindness and myopia are often combined receives an important confirmation from the fact that he determined the constant occurrence of both these affections in this fanily.

It was demonstrated that in the latter family only the hemeralopes were nearsighted (Ammann found nine dioptrics), while the members who were 


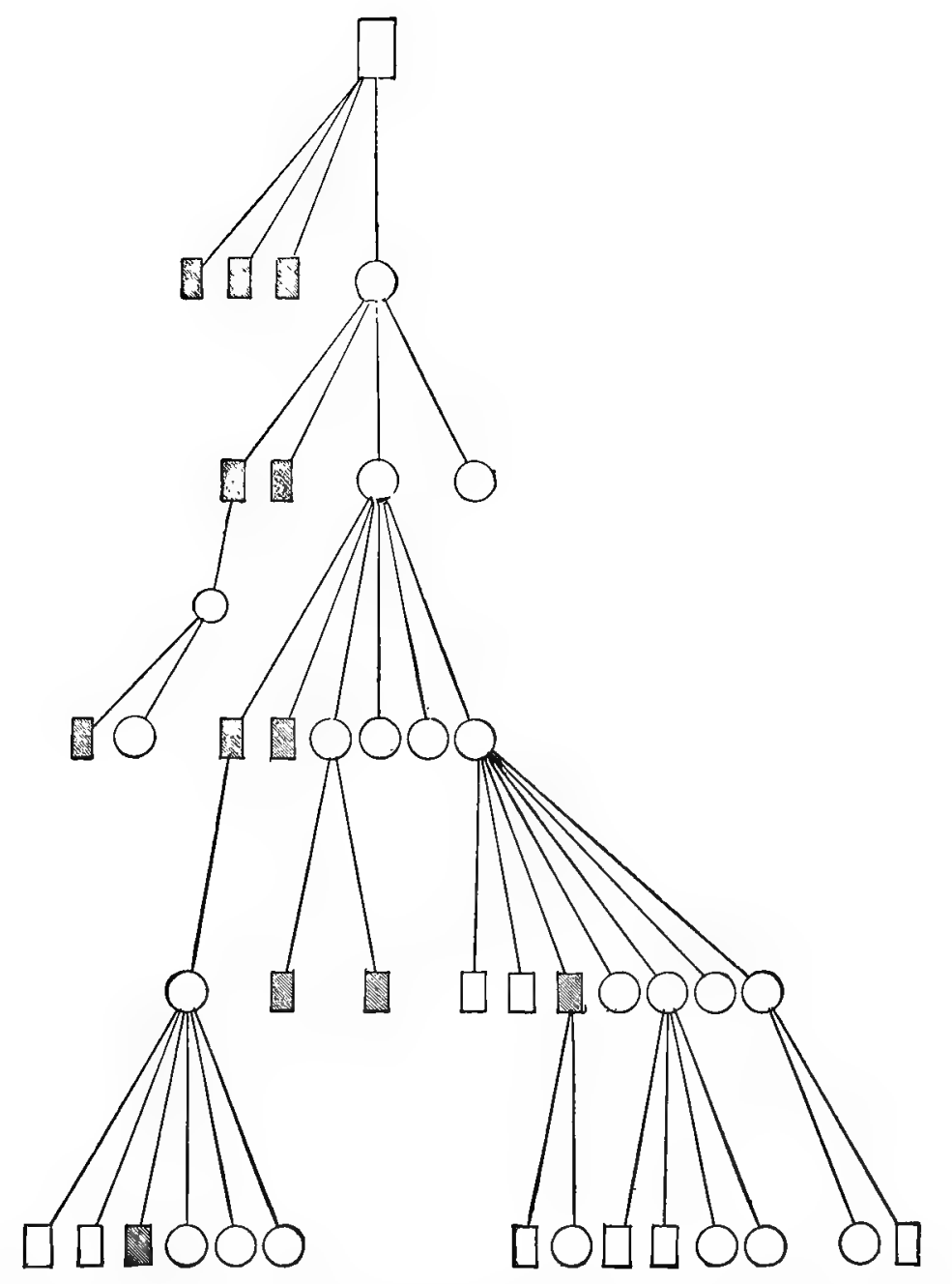

Fig. 25.-Genealogical Tree of a Hemeralopic Family. (After E. Ammann.) Hemeralops are shaded. 
not attacked by night-blindness showed almost normal refraction. If we consider the genealogy of this family, which is shown under Fig. 25, it is noted first that the ancestor of the family, Andreas St., born in 1750, was healthy. Whether he was the son, or his wife the daughter or sister, of a person who had night-blindness, cannot now be ascertained. His three sons were all night-blind, but did not marry. His daughter, Mrs. Lehmann-Steiner, unfortunately became the ancestress for the further transmission of the disease. She transmitted the affection to her two sons; of her two daughters, one (Mrs. Vogel-Lehmann) transmitted the disease to two of her four sons. Therefore, not all of the sons are night-blind, as in the second and third generations, but only one-half of them; of the four married daughters only. two - became conductors for the following generation, and of these two only one transmitted the disease to her two sons, while the other (Mrs. Frey) had three sons of whom one only showed the disease. The four daughters of Mrs. Frey have very large families, but the sixth generation of this line remains completely exempt from the disease.

Therefore a decided diminution of the disease is here apparent, as had been previously observed by Cunier in the year 1838. The condition is different in the families where the disease is transmitted from the male side, i. e., from the affected father through the daughter to the grandchild: Walter Reifer, the grandchild of Adolf Vogel, is a descendant of the sixth member, and inherits the disease to so high a degree that he can scarcely go about alone at night. In the fifth generation also there is a grandchild affected by night-blindness, in whose line the affection perhaps may be propagated; therefore the disease may not rapidly die out in this family, but it is certainly on the decline. It would be interesting to know the further fate of the family; whether a member of the family with night-blindness in a manifest or latent form may, for some unknown reason, become the progenitor of a race that will show a decided tendency, or whether the disease will gradually disappear. The question also interests us whether the affection may arise spontaneously without any hereditary predisposition, and may then be only accidentally transmitted in this or that way, or whether such sporadic cases as are actually observed depend upon a poorly maintained family tradition, or upon insufficient observation of the individual members of the family. To decide this, further accurate professional observations will be necessary.

The hereditary form of hemophilia is unquestionably and by far the most frequent, but a so-called congenital form of the disease also appears to exist. We understand by this that from the marriage of healthy persons originating from healthy families children are born who are bleeders, and from these latter the disease may be further transmitted.

The importance of marriage between blood relations in favoring the development of hemophilia has been emphasized, as well of psychical influences (such as fright, anger) during pregnancy. Up to this time there is no scientifically founded theory in support of these views.

Spontaneously, and without heredity, the disease certainly occurs only in a very small number of cases, and in these the twenty-second year of life is looked upon as the absolute limit of time up to which persons previously 
healthy may develop symptoms of hemophilia. These conditions, which in themselves cannot be accounted for, are increased in importance by the fact that spontaneous cases of " hemophilia " which develop in later life occur only as quite localized hemorrhages limited to an individual organ.

Regarding the geographic distribution of the disease it appears that Germany furnishes the main contingent of cases; yet other countries are not entirely exempt from the disease. The following table, compiled by Grandidier, gives the following figures:

\begin{tabular}{|c|c|c|c|c|}
\hline COUNTRIES. & $\begin{array}{c}\text { Bleeder } \\
\text { Families. }\end{array}$ & $\begin{array}{l}\text { Individual } \\
\text { Bleeders. }\end{array}$ & \multicolumn{2}{|c|}{$\begin{array}{l}\text { Bleeders. } \\
\text { Males. } \quad \text { Females. }\end{array}$} \\
\hline 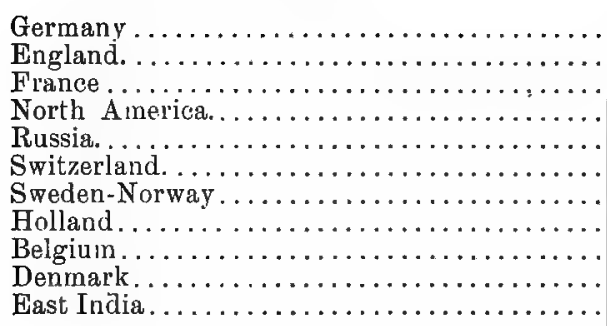 & $\begin{array}{r}93 \\
46 \\
20 \\
15 \\
7 \\
5 \\
3 \\
2 \\
1 \\
1 \\
1\end{array}$ & $\begin{array}{r}258 \\
141 \\
80 \\
61 \\
11 \\
48 \\
9 \\
9 \\
4 \\
3 \\
6\end{array}$ & $\begin{array}{r}236 \\
134 \\
75 \\
60 \\
7 \\
48 \\
6 \\
7 \\
4 \\
2 \\
5\end{array}$ & $\begin{array}{r}22 \\
7 \\
5 \\
1 \\
4 \\
\ddot{3} \\
2 \\
\ddot{1} \\
1\end{array}$ \\
\hline Total. & 194 & 630 & $\begin{aligned} & 584 \\
= & 92.6 \%\end{aligned}$ & $\begin{aligned} & 46 \\
= & 7.4 \%\end{aligned}$ \\
\hline
\end{tabular}

The actual causes of hemophilia are entirely unknown to us; everywhere we meet with hypotheses. If the disease, as is assumed, be a parablastic disturbance this may take place in the course of fetal development in two ways; either in a greater or less development of the connective tissue germ or because individual portions of this structure suffer particularly. Of special importance is the fact that it is the maternal element which transmits the disease without being itself necessarily involved, this property being an inherent function of the maternal body, and much more marked in the feminine descent than in the masculine. This faculty can exist only in the parablastic tissue, and especially in the vascular system which arises from it. Consequently it is very likely that the parablast, of all the tissues of the organism, is most influenced by the mother, being less subject to paternal impressions than any of the other constituents.

There are two factors which stand out prominently in the investigation of the causes of the disease: the structure of the vascular system and the composition of the blood itself. As hemophilia is not to be considered a temporary but a permanent pathologic condition which is congenital and hereditary, we are compelled to assume a disturbance in "the first formation," which affects a portion of the connective tissue germ. Nevertheless, hypotheses of this nature are not at present capable of verification by scientific investigation; therefore, in regard to the actual condition, we have been compelled to rely upon the scant anatomical findings which have now and then been reported, but which, considered collectively, have not solved the puzzling nature of the disease in question. 
The abnormal composition of the vascular system is said to consist in the greater tendency of the vascular walls to rupture, and this has been thought to be due to a peculiar thinness and narrowness of the arteries, as well as to their very superficial situation. Even were this condition more common than is actually the case, these vascular alterations would not sufficiently explain the spontaneous hemorrhages, for we find similar changes in the narrowed aorta of chlorosis but no hemorrhages. In discussing the pathological anatomy we shall revert to this; at present it need only be stated that some investigators such as Hooper, Liston, Fischer and others found the walls of the arteries in hemophilia very thin and showing fatty degeneration. Virchow laid special stress upon the fact that in a bleeder, aged twenty-four, the aorta was not only very thin and elastic but also very narrow, almost of infantile dimensions, while the capillary vessels showed no change. Elastic arteries which are too narrow propel the blood into the capillaries with too great force, and this circumstance favors the hemorrhagic diathesis, or at least the continuance of the bleeding. This narrowing of the arteries cannot be ascribed to any special disease of the walls of the vessel, but to a disturbance of development, analogous to the condition in chlorosis; this is consistent with another special characteristic of hemophilia, namely, the marked hereditary tendency of the affection. Whether the fatty degeneration of the intima, which has been found in some cases but by no means in all, is not rather the result of the posthemorrhagic anemia than the cause of hemophilia is also questionable. At the autopsy of some cases of hemophilia, hypertrophy of the left ventricle with extreme thinness of the right has been found, and this circumstance has been suggested as an explanation of the hemorrhages. If the blood be propelled with great force through the narrow, thin-walled arteries, the increased pressure of the hypertrophied left ventricle may cause a rupture. Apart from the comparative rarity of hypertrophy of the left ventricle, it must be remembered that a great number of the hemorrhages are of a diapedetic nature, and cannot be referred to rupture of the vessels alone; besides, we note the same symptom-complex in congenital narrowing of the aorta without the sequence of extensive hemorrhages such as frequently recur in hemophilia!

The fallacy of these mechanical explanations became more evident as opinions multiplied that an insufficient coagulability of the blood was the cause. The latter conclusion concerning the blood was the result of experience that in hemophilia all hemorrhages, even with the most insignificant beginning, are difficult to control. The blood does coagulate, however, as may be seen from the surface of wounds; spontaneously it forms coagulated masses from beneath which new quantities of blood exude, and these also soon coagulate, and thus enlarge the blood clot. Gradually the oozing of blood ceases, and the hemorrhage is arrested. Sometimes, however, in more severe hemorrhages, there is complete cessation of the production of the fibrin ferment, the blood gradually loses its power of coagulation, the hemorrhages progressively drain the tissues, and death results from excessive loss of blood. Any form of hemorrhage may be fatal, but this is most frequent in epistaxis; next, after extraction of teeth; and, occasionally, in intestinal hemorrhages, etc.

Microscopic and chemical examination of the blood has shown normal 
conditions in by far the great majority of cases, so that the expectation of finding there the actual cause of the disease has not been realized. Neither is it possible to determine any deviation from the normal in the amount of salts in the blood, in the quantity of fibrin producers, in the corpuscular constituents of the same, in the numerical relation of the blood-corpuscles to one another, or in the amount of hemoglobin. The anticipation of finding the amount of fibrin decreased is also unconfirmed; Heyland found 5 to 1,000 , Gavoy-Ritter 2.6 to 1,000 , and Otte 4.3 to 1,000 . In the numerous controversies regarding hemophilia the coagulability of the blood continues to be the main and interesting question, and has played a great rôle. While some authors, such as Grandidier, Lossen and others, found diminished coagulability of the blood, according to other authors this occurs only in the later stages after severe hemorrhages (Hoffmann, "Text-Book of Constitutional Diseases"). Grawitz does not consider these findings contradictory, for, under ordinary circumstances, after prolonged hemorrhage, an increase of coagulability occurs, and the blood which exudes last in hemorrhage frequently coagulates immediately. Therefore, the slowing of coagulation in the later stages proves conclusively the diminution in the power of coagulation.

These theories of the coagulation of the blood have been recently confirmed by Alex. Schmidt, and applied by him to explain the conditions existing in hemophilia. According to his observations, the blood of a patient coagulated four and a half minutes after exuding, and in consideration of the amount of blood previously lost he pronounces the time preceding coagulation as an abnormally long one. In his patient the action of a "zymoplastic substance," produced by him, was first tested for its power in increasing coagulation; it was placed in a test-tube with the blood of a hemophilic, and coagulation occurred after ten seconds, whereas previously the time was four and a half minutes. Locally applied to bleeding gums, as soon as contraction of the vessels and a momentary cessation of bleeding had been brought about by an injection of cocain, the "zymo-plasma" also proved an excellent styptic, and therefore holds out the possibility of a substance which may be used to increase coagulation.

The deterioration of the blood in functionating erythrocytes, as an explanation of the hemorrhages, which Cohnheim assumed, is absolutely unproven; I have repeatedly taken specimens of the blood of hemophilics which had been stained according to various methods, and have shown them to blood experts who could detect nothing abnormal in them. Blood counts have repeatedly shown normal conditions, as well in regard to the erythrocytes as to the leukocytes. The blood-plaques are said to be increased in amount. My own experience of hemophilia, which in all amounts to four cases, has been gained by the same methods of blood investigation which we employ in all other blood diseases. In two cases in which there was no marked anemia, no definite changes were found. In both of the other cases, in which repeated hemorrhages caused severe anemia, the patients being respectively eight and sixteen years of age, I found the same changes as in cases of anemia after acute and profuse losses of blood; namely, conspicuously pale color of the erythrocytes, absence of well-developed rouleaux formation, decrease of hemo- 
globin (30 to 35 per cent., according to Sahli), macrocytes, poikilocytes, microcytes, increase of blood-plaques; no leukocytosis. These conditions improved under suitable treatment (good nutrition, fresh air, processes for hardening the body, avoidance of everything detrimental to health). I should like to mention here that frequent inhalations of oxygen did not show the slightest influence upon the general condition or upon the composition of the blood.

G. Cohen reports a very remarkable blood finding in a bleeder (Zeitschr. f. klin. Med., Festschrift, 1890): The blood was almost colorless, did not coagulate upon beating, and upon standing left only isolated, friable, white coagula of fibrin of the size of a bean; microscopically the well-known polymorphia of the red blood cells was found, such as occurs in anemic conditions, but no rouleaux formation.

Various theories have been proposed in literature as to the origin of hemophilia. Among the best known that of Immermann is the most important and most widely circulated. It depends upon certain views of Virchow to which we have already in part referred, but to which we must now recur. Immermann expounds this theory in the following way:

Hemophilia is a form of the hemorrhagic diathesis which is chiefly characterized by a hereditary predisposition ${ }^{1}$ and a habitual manifestation; the hemorrhages which are readily produced, frequent, severe, stubborn, and dangerous, are chiefly caused by a hereditary and habitual disproportion between the blood volume and the capacity of the vascular apparatus, which results in an unusual increase of secondary pressure in the latter. Functional erethism of the heart and unusual development of its muscles may in some cases of the affection be responsible for the production of hemorrhages, as well as for its abnormal clinical course, and for the tendency to fluxion in the affected individual; possibly other neurotic influences may occasionally arise to increase periodically the continuously fluxionary diathesis.

Oertel expresses himself likewise regarding the nature of hemophilia. $\mathrm{He}$ is of the opinion that hemophilia is to be regarded as a hydremic plethora of high degree. Following the same train of thought as Immermann and Oertel, G. Cohen (Zeitschrift f. klin. Med., Festschrift, 1890) has founded a general treatment of hemophilia, the main object of which is to combat the hydremic plethora by an energetic cataphoresis and diuresis. The decided improvement which Cohen brought about-this refors only to a single case-furnishes some evidence of the correctness of this theory.

Cohen's patient was the ninth of eleven children and was born in 1852; at the time she came under treatment she was thirty-eight years old. Her father suffered repeatedly from severe epistaxis; twice tampons had to be used. A grandchild of her sister suffered from morbus maculosus with effusion of blood into the joints and renal hemorrhages and died of this disease when twenty years of age. There is no other history of hemophilia in the family, except a severe hemorrhage after extraction of a tooth in an older sister of the patient. This patient was a delicate chlorotic girl, with scant menstrua-

: Differently expressed: "The heredity of certain pathological properties." 
tion and without tendency to hemorrhages. A far more decided hereditary taint was noted in the nervous system; the father was a person easily upset by the slightest psychical irritation, e. g., if he were going upon a journey, vomiting and marked agoraphobia would occur. He died of heart disease. One sister of the father suffered from hysterical spasms, and another was very "nervous." Among the children, one daughter cannot walk upon the street without an attendant, and the patient in question suffers from cardiac palpitation and an ill-defined feeling of fear unless accompanied by her brothers and sisters. In this patient's twelfth year the first marked hemorrhage occurred after the extraction of a tooth; a few months later menstruation began, at first quite regular and not especially profuse. A year later, after exertion in walking, upon the fourth day of the period, the first prolonged metrorrhagia occurred, with syncope, palpitation of the heart, nervous attacks of fear and excitement; the menses now became irregular, very profuse and prolonged. During her attendance at school attacks of epistaxis were first noted, and these were repeatedly followed by spasms. In the year 1869 the patient was bedridden almost the entire summer, using iron waters, whey and baths. She was in bed most of the time in 1870 on account of frequent epistaxis and severe uterine hemorrhages; for the first time marked edena of the feet was present. Toward the end of the year epistaxis was again severe. In $18 \% \%$ there were frequent attacks of epistaxis, but injections of ergotin checked the hemorrhages for from six to eight weeks. In the next year, after a trifling wound of the finger, hemorrhages occurred, lasting for several weeks. Uterine bleeding was almost continuous. Ergotin was of no avail. In 1874 hemorrhage occurred after the extraction of a molar. Two years later, following a very severe attack of dysentery with hematemesis and enterorrhagia such a severe epistaxis occurred that it was necessary to keep a tampon continuously in the nose. Later, bleeding from the nose lasted several hours. Galvanization of the sympathetic, combined with ergotin treatment, temporarily improved the general condition. In the year 1881, for the first time, large purpuric spots with decided, painful swelling appeared upon the left upper arm. Menstruation lasted uninterruptedly from November of the same year until February, 1882. Vaginal tampons were continuously necessary. In March, 1882, for the first time, and then very frequently, there was hemorrhage from the uninjured tip of the finger. During January of the next year several intact fingers and the nose bled daily. Subcutaneous hemorrhages followed, the blood in large areas of the right thigh and of the left upper arm finding its way through the skin. In 1884 there was profuse diarrhea, with frequent vomiting accompanied by massive hemorrhages, and prolonged elevation of temperature which lasted for months. The urine became exceedingly scant, one-eighth to a quarter of a liter in twenty-four hours; it was pale, free from albumin, of very low specific gravity (1.002 to 1.005 . The average loss of blood in twenty-four hours amounted to one pound (358 grams). Galvanic baths decreased the number of hemorrhages. Improvement was brought about exclusively by treatment based upon the decreased amount of urine and an energetic diaphoresis was persistently carried out for over three years; injections of pilocarpin which for a time were substituted by packs 
lasting for hours, by effusions of folia jaborandi, flor. tilix, and the administration of large doses of digitalis. The patient was cured at the age of thirty-eight after suffering from the disease for twenty-five years.

In the study of the etiology of hemophilia, those who adhere to the Immermann-Oertel theory doubt much the prominent rôle which the hereditary narrowing of the aortic system is said to play. This hereditary anomaly also produces chlorosis, a disease which, in spite of the similarity of individual anatomical relations, has nothing in common with the manifestations of hemophilia. It may possibly be opposed to this that a second injurious factor, an abnormal composition of the blood, is added to the smallness and narrowness of the vascular system, and from this combination hemophilia arises, or, more correctly, some of the symptoms of the affection. In opposition to this is the fact that the examinations of the blood in hemophilia up to the present time have shown nothing constant nor positive; only in quite isolated instances have microcytosis, macrocytosis, poikilocytosis, and absence of rouleaux formation been observed. Similar deficiency of hemoglobin in the erythrocytes and in the total blood has been repeatedly noted in anemic individuals after successive hemorrhages (especially of a traumatic or neoplastic nature) without the tendency to an acquired hemorrhagic diathesis, so that a characteristic or pathognomonic significance cannot be ascribed to it. All hypotheses which attribute hemophilia to an abnormal composition of the blood are lacking in the proofs upon which a scientific proposition should be based.

That hemophilia is a neuropathic diathesis is v. Recklinghausen's hypothesis (Handbuch der Allg. Pathol. des Kreislaufes und der Ernährung). In the next article, when describing morbus maculosus, we shall refer more minutely to the spontaneous hemorrhages due to the vasomotors, and shall here only mention that this author ascribes to these hemorrhages (in support of his theory) the prominent nervous symptoms which occur in certain cases of hemophilia and occasionally in entire families of bleeders. But the question arises whether in a general disease such as hemophilia the spontaneous and unexpected manifestations which occur may not naturally kecp the patient in a constant state of tension and expectancy, and thus implicate the nervous system, and whether or not the nervous disturbances are of a secondary nature.

Finally, we must mention another view, already referred to in scurvy, that hemophilia is a toxic infectious disease. This is the opinion of W. Koch of Dorpat, but it has received but slight attention in scientific circles. Koch, in his book "Bleeder's Disease and Its Varieties," analyzes the evidence on which is based the conception of hemophilia as an independent affection. $\mathrm{He}$ criticises adversely the reports of the disease which have been obtained up to the present time. He points out how faulty these are, and their many contradictions. The conclusion which he reaches is, approximately, that hemophilia is not an independent disease, but that it is an infectious discase and identical with scurvy. "I believe," says Koch, "that the blood of hemophilics exudes through the walls of the vessels, which give no evidence of anatomical change, because specific toxins are mixed with the blood; I therefore believe hemophilia to be an infectious disease, and, on account of the coincident symp- 
tomatology and pathological anatomy, I believe it to be the same infectiou disease as scurvy. In order to prove its parasitic nature, I must first refe to the supposedly congenital form of hemophilia, to that form in which th symptoms are present from the time of birth, and which can only be ascribe to transmission from hemophilic parents. This hereditary condition can neve be explained by the theory of a pes varus congenitus or a meningocele con genita, i. e., by a vitium primæ formationis in which definite observable ana tomical changes (no matter how complicated in origin these may appear $\mathrm{t}$ the eye of the observer) result in disturbances of function. For nearly century such anatomical conditions have been sought for in hemophilia, anc in spite of improved methods of investigation, only vascular anomalies ca: now be found, a lesion the unimportance of which admits of no dispute, par ticularly as in only a few cases has it been found. In opposition to thi theory, I believe congenital hemophilia to be quite similar to congenital syph ilis, and from the standpoint of infection I note the association of hemophili with wounds and ulcerative processes such as humid, benign, tuberculous an syphilitic skin eruptions, glandular tuberculosis, discharges from the ear, sup puration of the navel, etc.

"Some hemophiliacs have a marked and abnormal desire to eat sand, earth chalk, peat, acid and pungent vegetables. Some have febrile attacks; som perish with surprising rapidity after only slight hemorrhage or other localiza tion; almost all have an enlarged spleen. I lay great stress upon these fact: as well as on one which, so far as I know, has not yet been mentioned, muc] less discussed, namely, the connection between hemophilia and infection. Fur ther we must consider the presumption that, in those hemophilic familie who made no change of residence for generations, particularly if the hemor rhages occurred only in later life, the process may often be referred to influ ences of the ground instead of to heredity."

Koch's theory of hemophilia as a toxic infectious disease, both as a whol and in its premises, is so purely visionary that it scarcely appears worth dis cussion. The association of hemophilia with glandular tuberculosis, tubercula and syphilitic cutaneous ulcers, discharge from the ear, febrile states and th other pathologic conditions which he mentions is so extremely rare as to b. scarcely noted at all by other authors. The same is true of the splenic tumor which Koch says hemophiliacs almost invariably show, and upon which $h$ especially bases his theory of the infectious nature of the disease. Othe symptoms which he emphasizes are not due to hemophilia, but to the anemi: produced by the hemorrhages, as is the case with neuropathic symptoms Above all, his theory of an infectious etiology is untenable inasmuch as th presence of pathogenic agents has never been proven; all investigations as $t$ this point have been negative. Still more unwarranted does it appear to $\mathrm{m}$ to ascribe the pathologic processes of hemophilia to telluric influences instea of to heredity.

Fully as I agrce with Koch that the origin of hemophilia cannot be looker for in purely anatomical causes-that is to say, be dependent upon themcannot concur in his assumption of a toxic infection. Even if this coul explain the nature of the congenital form of hemophilia, it can never explair 
the mystery of the transmission of the disease in a hemophilic family, why the mother herself should remain unaffected while she transmits the disease, why the male members of the family only are attacked, why men from bleeder families do not beget hemophilic children, whether they themselves are bleeders or not, etc. Moreover, congenital hemophilia is much rarer than that form in which children in the second year or adolescents manifest the signs of the disease! Are the pathogenic agents latent in such cases? And what causes them to assert their virulence?

In rare cases mothers with relapsing fever have given birth to children with relapsing fever! This is probably analogous to the "toxic infection" which Koch assumes as the cause of congenital hemophilia.

In the last few years, Kolb, Babes, Gärtner, Tizzoni and Giovannini have demonstrated bacteria in patients suffering from purpura hemorrhagica as well as in hemophilia of the newborn; these bacilli were apparently also pathogenic in animals, and upon inoculation produced a disease characterized by hemorrhages. These maladies are probably analogous to the hemorrhages often seen in other infections, and we may assume these hemorrhages to be due partly to local changes in the vessels caused by collections of bacteria, and partly to the toxic effect of bacterial products. These may, therefore, to some extent be classed with toxic hemorrhages, but have not the slightest element in common with human hemophilia.

That with the hemophilic predisposition severe or even uncontrollable hemorrhages follow trifling occasional causes such as never produce hemorrhage in the healthy is a generally well-known fact, and this has led to a differentiation between spontaneous and traumatic hemorrhage. But obviously in this division the conception of "trauma" must be very elastic. The hemophilic tendency to hemorrhage shows itself during the period of physiologic development, particularly in the two periods of dentition, at puberty, and at the climacterium. Even in tying and severing the umbilical cord we sometimes produce severe, even fatal hemorrhage, and we recognize by this and other signs the hemophilic predisposition of the newborn.

Severe hemorrhages from the gums are prone to occur in the first period of dentition, and we are forced to look upon the cutting of the teeth as trauma. Quite slight corporeal punishment may produce cutaneous hemorrhages and infiltrations of blood out of all proportion to the nature and extent of the injury. Blowing the nose may cause uncontrollable epistaxis; profuse conjunctival hemorrhages may be produced by touching the eye. Purgatives may result in severe intestinal hemorrhages. At the menstrual period and in normal labor extraordinarily severe hemorrhages have been observed.

Especially frequent are effusions of blood into the joints.

From the observations of surgeons it is evident that the first hemorrhages into the joints, as well as also the great majority of the later ones, are caused by external conditions. This explains why children are not attacked by hemorrhages into the joints before they learn to walk; the "trauma" is absent. But when they first begin to walk, when the joints are utilized and the entire muscular system comes into action, trauma after trauma occurs, and arthritic hemorrhages are apt to appear. We quite often observe severe hemorrhages 
into the joints which occur while the patients are in bed, and when we are unable to detect the slightest external cause. In such instances we are almost always dealing with joints which have repeatedly been the seat of effusions of blood.

Most bleeders perish in infancy, a large proportion before the eleventh year. Rarely do they reach old age, seventy and above. The hemophilic predisposition diminishes with advancing years.

If we study the reports up to the present time, we are convinced that the true causes of hemophilia are absolutely unknown. Even of the two etiologic factors most frequently and invariably mentioned-an abnormal composition of the vascular walls (which is said to consist in great fragility) and the insufficient power of coagulability of the blood, to which I shall again referwe do not possess a single positive proof which will bear criticism.

Heredity assumes such importance in the etiology of this disease that it is well to point out that a highly nervous temperament on the part of the ancestors has often been noted. Of course, the reports of the patients and their relatives must be accepted and utilized with extreme caution; yet a number of well-authenticated cases are on record which certainly prove the neuropathic predisposition of the ancestors of bleeders.

\section{PATHOLOGICAL ANATOMY}

The anatomical examinations in fatal cases of hemophilia have, up to the present time, shown nothing that is characteristic or invariable; in particular, very little has been revealed to explain the tendency to spontaneous hemorrhages, except in the case of the joints. Here, by the tireless researches of surgeons, we are permitted a deeper insight into the mechanism of arthritic hemorrhages.

The number of autopsies by experts on the cadavers of bleeders is very small; consequently the number of anatomico-pathological findings is also very scant. In general the following is reported: The cadaver is conspicuously anemic; the skin is waxy pale. In cases in which death has occurred from hemorrhage after trauma, petechiæ, ecchymoses, suffusions of blood and signs of injury are found upon the skin itself with comparative frequency. The internal organs throughout are extremely anemic. The small amount of blood flowing from severed vessels is decidedly watery. Of the abdominal organs, in which there are often signs of preceding hemorrhages, the spleen is particularly interesting; W. Koch maintained-as we have seen-that it is invariably enlarged; it has occasionally been found enlarged, but is usually normal. This enlargement of the spleen in hemophilia has sometimes been deemed especially important, particularly as proving the infectious nature of the disease; but the knowledge that this finding is inconstant has led to the rejection of the theory.

The blood-ressels of the skin which are superficially situated have been often described, the arteries as well as the veins. The muscle of the heart frequently, but by no means invariably, shows fatty degeneration. Its size has in some cases been found normal; in other cases it is hypoplastic, and in still others hypertrophic, especially as regards the left ventricle. In a number 
of instances the large arteries and their first branches show an unusually narrow lumen. In the examination of the structure of the larger and smaller arteries, the extremely thin, sometimes actually transparcnt condition of the intima is often conspicuous. Partial fatty degeneration is not rarely obscrved.

Virchow called attention to the smallness of the heart in hemophilia, to the narrowness and thinness of the walls of the vessels, which is similar to that in chlorosis. On account of the rarity of autopsy reports in this disease, it is highly interesting to refer. to this finding in a case observed by Virchow, upon which he held an autopsy, and which he has detailed (Deutsche Klinik, 1859, and Canstatt's Jahresb., 1859). "The blood, as in other cases of hemophilia, was not deficient in fibrin; it formed a decided buffy coat. The arteries and veins showed no large ruptures-in fact, no conspicuous changes; the hemorrhages, therefore, must have been from the capillaries. The vcins were very wide, the arteries very elastic and narrow. The capillaries and nerves showed no decided alterations. In the central part of the vascular apparatns an abnormality was found which was certainly congenital; the thymus gland was still very large, the heart pale and small, the aorta narrow, its walls thin and very elastic, with wavy rises due to slight sclerosis and fatty degeneration of the intima, particularly in the descending thoracic aorta. This entire finding closely resembled chlorotic conditions to which I (Virchow) have previously called attention. The development of the heart from the aorta is much retarded in chlorotics; enlargement of the heart is late and occurs only occasionally. Hemorrhages are very frequent and severe in chlorotics, and these can probably be traced to the arterial changes which have been observed; at all events the round ulcers of the stomach which occur chiefly in chlorotics certainly bear a relation to individual arteries. There is much in favor of the congenital, or at least very early, development of the predisposition to chlorosis; and this may be positively maintained in the case of hemophilia. The blood collected from this case rapidly decomposed, depositing xanthoglobulin crystals as well as triple phosphates and leucin. This case teaches us that in hemophilia it is not an unusual fluidity, a dissolution of the blood, which produces the tendency to hemorrhage, because blood rich in fibrin, just as in scurvy, may be found in the hemorrhagic diathesis. Nowhere was tendency to rupture of the blood-vessels noted. Therefore the question arises whether, in this case, as in chlorosis, the narrowness and extreme clasticity of the vessels (arteries) so increase the blood-pressure in the capillaries that a predisposition to rupture and consequent hemorrhage is produced. In this case the cause of the homorrhagic diathesis was to be sought in a deficiency of the vascular formation, and it is certainly noteworthy that the thymus gland had persisted for so long a time."

Virchow, therefore, considered retardation of vascular development to be an especial pathologico-anatomical sign in hemophilia. He observes, however, that exactly the same conditions as were noted by him in hemophilia occur also in chlorosis. The question why retardation in vascular development causes two such entirely different diseases as chlorosis and hemophilia has not yet been answered. If we are inclined to be critical, we may assume from this condition of affairs that retardation of vascular formation cannot 
be the determining cause of hemophilia. Nevertheless, the descriptions of Virchow are highly important, as they form the basis of the ImmermannOertel theory of hemophilia.

But the positive findings mentioned above which have been observed in a number of autopsies in cases of hemophilia are opposed-few as they are-by a much greater number in which, after minute investigation, experienced pathologists were unable to determine anything noteworthy.

More widely known are the changes in the bleeder's joint, a knowledge of which we owe to the valuable work of surgeons. In the first stage of the arthritic disease, we find the symptoms of a simple effusion of blood. The joint cavity is filled with fluid blood and dark coagula of fibrin. The latter are partly free, partly adherent to the walls, and by proliferation of the celllayers of the synovial membranes they are made integral parts of the wall of the joint. The capsule is thickened, shows bloody infiltration, and is discolored. Moreover, fibrin deposits are found upon the capsule and upon the surface of the cartilage, causing the formation of brownish pigmented villi and cartilage proliferations. If the effusion of blood is not absorbed the joint swelling remains, and inflammation is produced which pathologically resembles the condition König has designated as hydrops tuberculosus fibrinosus. The contents of the joint are either serous or hemorrhagico-serous, and of a light brownish color. The perisynovial connective tissue is sclerotically thickened. The synovial intima shows swelling and reddish-brown or brownish discoloration, and a great number of synovial villi of a brownish tint. The blood coagulum in some cases attains the thickness of a finger. The cartilage has lost its white color and its luster, and appears of a brownish hue. Coagulated masses of blood are found in layers which show organization and connective tissue change. The cartilage is partly softened by the disappearance of its upper layer. Continuous small and large depressions, sharp-edged and maplike, invade deeply the surface of the bone surrounding the cartilage. The surface of the cartilage is uneven on account of an irregular disappearance of substance. Following this second inflammatory stage, a third occurs in which regenerative changes play the principal rôle. By adhesion of the joint surfaces, and by processes of shrivelling in the soft parts and capsules, stiffness of the joints supervenes. The joint cavity becomes denuded, the joint ends deformed; subluxation takes place; contracture and ankylosis may occur.

Examination with the Röntgen rays has, according to Gocht (Arch.f. kl. Chir., 1899), given constant and important findings: It is at once evident that the lower end of the femur upon the affected side is very much smaller than that of the opposite side. The bones upon the diseased side are atrophic and decidedly too permeable, as may be noted in the lighter color of the Röntgen picture-the epiphysial lines upon the femur and the tibia lack the normal rounded curves, and are irregular, often serrated, with a double contour. While upon the normal side a broad, open space marks the presence of normal cartilage, and the osseous ends of the femur and the tibia present their wellretained smooth surfaces, the conditions upon the diseased side are quite different. A decreased joint space is found, owing to destruction of the cartilage. The ends of the bones appear completely changed. The arthritic 
portion of the femur is serrated and irregular, the cavity normally existing between the two condyles has been obliterated; the same is also true of the intercondylar eminences of the tibia which are irregular and broadened. Still more advanced lesions are seen in older patients; the space in the joint cartilage disappears entirely, the tibia seems dislocated toward the femur outwardly. Occasionally adhesions may be noted between the femur and the tibia. The condition of the capsule cannot be accurately determined on account of the effused blood.

The microscopic investigations of the vascular changes in hemophilia, made by Buhl and Birch-Hirschfeld, deserve attention.

The former found in a preacher, aged seventy-four, suffering from chronic dermatoses and presenting hemophilic symptoms, an immoderate growth of the capillary vessel loops with a great increase and subsequent transformation of their nuclei. Buhl himself does not believe this anomaly to be peculiar to hemophilia. Birch-Hirschfeld examined the heart and the large vessels, a portion of the spleen, the kidney and the skin of a child, aged one year, who had succumbed to congenital hemophilia, and remarks concerning these organs: "In the heart and in the vessels connected with it I discovered no changes. It may be stated that the vessels of the heart, considering the age of the child, were quite of normal size. In the heart muscle there were but slight indications of fatty degeneration. The valves, as well as the intima of the larger vessels, were delicate and normal in structure; the same was also true of the muscularis and media of the arteries. In the kidneys the cortical canals were swollen and there was slight granular cloudiness of the epithelium. There was nothing noteworthy in the spleen except moderate hyperplasia of the stroma of the pulp. But I must mention-with reserve-a finding which I noted in the capillaries and the transitional vessels of other organs, particularly of the liver and kidncys. In some areas the endothelia were obviously enlarged, their nuclei swollen; here and there were granular deposits in the protoplasm. In some rather poor preparations which were stained with silver, the epithelial arrangement appeared to me irregular, disarranged, with occasional wide spaces between the endothelia. I do not lay special stress upon this condition. In delicate changes of this kind it is not always possible to determine how much is artifact, and it must not be forgotten that such changes are, perhaps, not rare in the last stages of other chronic cases." Kidd described in the finer vessels of the subcutaneous connective tissue and of the muscles an increase of the endothelia with dropsical swelling of the muscularis and proliferation of their nuclei-changes which Legg in another case was unable to recognize. I should like to mention the simultaneous occurrence of hemophilia with multiple sarcomata.

Obviously pathological anatomy affords no clue for the comprehension of the clinical picture. The belief that blood formation is periodically increased, which is mentioned by some authors, has but little to support it, and the same is true of the hypothesis that the overfilling of the narrow, perhaps friable, vascular system of hemophiliacs by a hypertrophied heart through which newly formed blood is occasionally forced in large amounts leads to a rupture of the markedly distended capillaries. 


\section{SYMPTOMATOLOGY AND COURSE}

The phenomena of the disease are quite often revealed purely by accident; the patients are attacked either spontaneously, or, in consequence of very slight injuries, by profuse hemorrhages or such as are very difficult to control. Among such cases I wish to mention the hemorrhages which occasionally follow ritual circumcision, and sometimes cause the surgeon great difficulty, as well as those which occur after perforating the lobe of the ear for earrings, and, finally, the muscular hemorrhages which occur in older children of a hemophilic predisposition, when they are chastised by the teacher in school; these, under some circumstances, bring the guiltless teacher into court.

The disease shows various grades of development, and by no means the same intensity of symptoms in all cases. The history of families of bleeders has shown that in individual members all types of the disease, from the mildest rudimentary forms up to the severest ones, may be found. The milder forms are characterized by an evident tendency to hemorrhages, but the bleeding never assumes such proportions as directly to threaten life.

In nearly three-fourths of the cases the first hemorrhage occurs before the end of the second year, and the latest period for its occurrence is the twentysecond year of life; only in quite isolated cases has the first hemorrhage happened at a later age. A great tendency to hemorrhage is noted in bleeders at about the period of physiological development. Most hemophiliacs die in the first year; a large proportion before the end of the tenth year. Only very rarely is old age reached-seventy years and beyond. Experience teaches that with increasing years the hemophilic predisposition declines, until finally it disappears entirely.

As has been stated, the physician or the family often becomes aware of the hemophilic tendency of an individual quite accidentally, by the difficulty of controlling a hemorrhage, which returns again and again. This is especially apt to be the case if the patient is not a member of a bleeder family, and has not older relatives, particularly brothers, in whom severe hemorrhages sometimes occur as the result of quite trivial injuries while at play. In other cases, surgeons while performing operations make the unpleasant discovery that the hemorrhage from an operative wound does not cease, and that they are dealing with a bleeder. The hemorrhagic or hemophilic predisposition of the newborn is earliest recognized if hemorrhages appear after severing the umbilical cord; these occasionally are uncontrollable, and result in the death of the child. However, we can by no means refer all hemorrhages from the cord of the newborn to a hemophilic predisposition of the child. It has been proven that umbilical hemorrhages in the newborn may also be due to the influence of bacterial blood infection. The next event which may lead to the discovery of the disease is ritual circumcision, which, as is well known, is performed upon the eighth day after birth. On the other hand, vaccination, according to usual experience, is relatively harmless. The disease may, however, not show an early spontaneous development but may be latent, and only appear after an exciting cause and upon occasion. The most common time for this is the period of first dentition, and thus we frequently note decided 
signs of the disease in the form of severe hemorrhages from the gums. Sometimes in girls, although quite rarely, there may be no evidence of the disease throughout infancy and childhood, the first symptoms appearing at the time of puberty and recurring at each menstrual period, the menses being unusually profuse and persisting for a long time. Even after an easy and uncomplicated labor occasionally severe, even fatal, hemorrhages occur, so that Kehrer has proposed the interruption of pregnancy in hemophilic women by artificial labor.

The most obvious and important sign in the clinical picture of hemophilia is the appearance of well marked hemorrhages either without an assignable cause or as the result of very slight external injuries. In about 50 per cent. of the cases the mucous membrane of the nose is the seat of the hemorrhages; next follow bleeding from the gums and intestinal hemorrhages (each about 12 per cent.), then pulmonary, gastric and renal hemorrhages (each about 6 per cent.). Most frequent are the hemorrhages from the skin and mucous membrane, next from the joints, to which I shall later refer explicitly, and finally, metrorrhagia. Apart from arthritic hemorrhages, hemorrhages from the serous membranes without assignable cause are quite rare. On the other hand, hemorrhage from the conjunctiva is occasionally noted, apparently occurs without cause, and may sometimes be so severe as to result fatally. This was seen in the case of two brothers, both bleeders, who were quite young. Occasionally the hemorrhages are so profuse that death takes place in a few hours. It is often astonishing to observe what enormous amounts of blood hemophiliacs may lose, and how rapidly they recover from this. In Cohen's case the patient lost a pound of blood ( 358 grams) per hour, and in another case of hemophilia, within eleven days twenty-four pounds of blood were lost. Occasionally syncope, the result of cerebral anemia whereby bloodpressure falls to a minimum, causes a cessation of hemorrhage. With an increase of the blood-pressure, hemorrhage begins anew. The disproportionately rapid regeneration of the blood must be referred to the previously mentioned increase of the hematopoietic function of the bone-marrow in hemophilia (Fischer) which, however, is of a somewhat hypothetical nature.

It is customary in hemophilic bleeding to differentiate between spontaneous and traumatic hemorrhages. All hemorrhages are designated as spontaneous for which no plausible reason can be assigned. But it must also be considered, in the study of the hemorrhagic diathesis, that there are conditions in which the organism of the hemophiliac reacts by hemorrhages to very slight influences-influences of such an insignificant character that the person who suffers a lesion scarcely notes it at all or but very slightly. Without doubt some of the hemorrhages which in this diathesis have been looked upon as spontaneous are in reality traumatic. We differentiate in these hemorrhages (spontaneous as well as traumatic) two forms: The superficial and the interstitial.

Spontaneous hemorrhages are occasionally preceded by prodromes such as cardiac palpitation, congestion of the head, vertigo, tinnitus aurium, anxiety, nausea and even vomiting. Prior to very severe hemorrhages, decided redness of the face is often noted; in the visible arteries rapid and strong pul- 
sations may be seen. The general condition, also, is decidedly influenced; the patient becomes restless; he is filled with fears or there is lassitude. For a long time the fleeting character of these hemorrhages has been noted. After the hemorrhage has ceased, the affected persons usually feel relieved and easy in mind. In other cases these premonitory phenomena are entirely absent. The hemorrhages may come on without any noteworthy prodromes. Superficial spontaneous hemorrhages occur most frequently from the mucous membranes; first from the most exposed, as those of the nose, then the mucous membrane of the mouth. Spontaneous hemorrhages may also occur from the mucous membrane of the urinary organs and from the female.sexual organs, also from the lungs, the stomach and the intestines. The hemorrhages from the skin are most likely to occur in cicatrices, or from cuts or ulcers, and the hemorrhage then takes place during a period in which the rupture of continuity is beginning to heal. Spontaneous mucous membrane hemorrhages are occasionally combined with those of the surface of the skin. In subcutaneous effusions of blood which embrace a wide area occasionally suppuration is noted, leading to gangrene of the skin and external perforation, after which a slough consisting of brownish and gangrenous shreds is discharged. The blood in the subcutaneous hematomata remains fluid for a long time similar to the condition in arthritic hemorrhages, and the careless opening of such foci may lead to uncontrollable and fatal hemorrhages; the latter may even occur when these hematomata rupture spontaneously.

Interstitial spontaneous hemorrhages occur most frequently in the scalp and in the face, next in the scrotum, more rarely in the extremities, and least frequently upon the trunk. Often the tips of the fingers are implicated, and the blood may exude or spurt from them as from a sponge dipped in blood. Although these hemorrhages appear spontaneously, i. e., without visible external cause, it may be regarded as certain that they, as well as the "traumatic" ones now to be described, are due to very slight, scarcely determinable, mechanical insults. Intraparenchymatous hemorrhages of internal organs, perhaps with the exception of the kidneys, very rarely occur in areas which are protected from external influence, a circumstance which, according to Strümpell, forms an essential difference between hemophilia and the acquired hemorrhagic diathesis.

Superficial traumatic hemorrhages may take place in any superficially lying part of the body, in all areas of the skin, and upon the superficially situated mucous membranes, as well as upon those mucous membranes whose secretions are discharged por vias naturales. To these must be added the serous membranes of the thoracic and abdominal cavities. Hemorrhages from the surface and from the mucous membranes which are susceptible to ordinary inspection may be produced by a laceration, a puncture, a bite or other wound, also by a blow, a fall, and by surgical operations. It is to be remarked in this connection that accidental ruptures of continuity, particularly those of an irregular form, cause hemorrhage in hemophiliacs much more readily than those produced by design, as in surgical operations. Cutaneous areas with ulcerating processes and cicatrices are loci minoris resistentice. Of the various regions of the body, the head is the one in which the hemorrhage of hemo- 
philiacs assumes an exceptionally severe character. Probably the most copious hemorrhages occur after the extraction of teeth. In one case reported, the rupture of the hymen was followed by lethal hemorrhage. It is a peculiar fact that small wounds in hemophiliacs bleed in proportion much more profusely than larger wounds. Fordyce controlled a hemorrhage by enlarging the wound with a knife. This method appears to be the best in the case of lacerated wounds which bleed profusely. Traumatic surface hemorrhages usually occur from only one isolated point. The blood exudes from the wound as from a sponge saturated with blood; nowhere can a spurting artery be found.

Interstitial hemorrhages after trauma occur particularly in the skin and the subcutaneous connective tissue. They follow the slightest external causes. A push, a blow in sport, or prolonged pressure as in sitting or lying, may produce interstitial hemorrhages. Blood tumors are formed (hematomata). Those areas on the surface of the body are most liable to interstitial hemorrhages, which, from the habit of life, are most exposed to pressure, the buttocks and the upper posterior parts of the leg, as well as the back.

Diffuse hematomata are found especially in the soft parts of the arms and thighs, as well as occasionally in the sheath of the psoas muscle. They occasionally resemble an abscess, with tense, glistening, hyperemic skin, and are very painful. The subeutaneous effusions of blood may extend over a wide area; upon suppuration, gangrene of the skin and external rupture have been observed as in spontaneous hemorrhages; a chocolate-colored mass intermingled with gangrenous shreds is then discharged.

The other most important symptom of the disease is the great difficulty with which the hemorrhage is controlled. This forms the chief danger of the malady, and is the reason why bleeders very rarely attain old age. The open, hemophilic bleeding reveals perfectly the characteristics of parenchymatous hemorrhage. From the entire surface which has been exposed by the traumatic rupture of continuity, the blood oozes continuously for many hours. Even by the most careful observation it is impossible to find a spurting vessel.

How long the hemorrhage may continue in the individual case cannot be foretold. It is quite uncertain, but the excessive loss of blood in itself acts favorably in controlling the hemorrhage. The bleeding hemophiliac is attacked by syncope; shortly afterward the hemorrhage ceases. Frequently, however, it lasts for so long a time as to bring about a fatal issue. During the hemorrhage, frequently there is at first increased cardiac activity; later, in consequence of increasing anemia the pulse becomes small, sometimes imperceptible, the patient is pale and feeble; in very severe cases there are delusions, convulsions and delirium.

The exuding blood at first appears normal; if, however, the hemorrhage is long continued, it constantly becomes lighter and of a watery, serous character, owing to the increasing anemia. Chemical and microscopical investigations of the blood have not given noteworthy results. There is no evidence to support Immermann's theory that the hemorrhages are due to a plethora.

The interstitial hemorrhages under the surface of the skin form blood- 
tumors (hematomata), which, in accordance with the transformation of the hemoglobin, may show the well known changes of color observed in all deposits of blood. Now and then a hematoma of this kind suppurates and ruptures. Such hemorrhages damage the nutrition and constitution of the patient all the more as they lead to certain complications which will be more minutely described later. Moreover, the knowledge that he is affected with such a disease as hemophilia severely disturbs the psychical condition and the nervous system of the patient.

Among the characteristic complications of hemophilia the tendency to "rheumatic" muscular and arthritic disease occupies the most important place, partly for the reason that we recognize in this a close analogy to the hemorrhagic diathesis in general. The arthropathies which may appear in any of the joints are the most familiar complications of this affection; first, on account of their frequency, and secondly, because their recognition, partly by means of the Röntgen rays, and partly by local surgical treatment which frequently permits direct inspection of the open joints, has led to more accurate knowledge of them. They occur spontaneously as well as in consequence of trauma which may be extremely slight, for they occur even in bedridden patients who can scarcely move. Frequently they are of undoubted "rheumatic" nature, for the tendency of hemophiliacs to be affected by "rheumatic irritation" is very great. The peculiar relation which exists between the hemorrhagic diathesis and diseases of the joints will be more closely considered in the next chapter. In hemophilic arthropathies the knee- and elbowjoints are particularly often implicated. The disease begins with pain and swelling which may lead to stiffness and a position of flexion, as in subacute arthritic inflammation, or with a tumor albus. The diagnosis is not always easy, and sometimes can be made only by noting the other hemophilic phenomena which have preceded the joint disease. The latter occurs most frequently in isolated attacks which may be recognized by the pain and swelling of certain joints as well as by fever, exactly as in the case of polyarthritis rheumatica. The course of the disease is naturally a very chronic one. In the majority of cases it is young males who are affected by hemophilic arthritis.

F: König divides the arthritic affection of bleeders into three stages: First, the stage of initial hemorrhage, the hemarthros of bleeders. With proper care the hemarthros may heal and the disease may terminate in this stage. If this is not the case the blood acts as an irritant, more blood is added, and a peculiar form of inflammation develops (second stage) which shows itself as a panarthritis, and which in the pathology as well as in its clinical symptoms shows great similarity to tuberculosis of the joints. This stage is to be designated as the inflammatory, the hemophilic tumor albus. The third stage is the retrogressive, the stage which leads to permanent deformity of the joints, the contracted bleeder joint with dissolution of the joint cavity, adhesions, and displacements of the arthritic surfaces, and finally ankylosis and deformity of the joint ends.

König's division appears to have found general acceptance among surgeons. The first stage is not to be viewed as though we were dealing with but a single 
hemorrhage. On the contrary, the most characteristic feature of this stage is the fact that one or more effusions of blood are promptly absorbed without any external derangement or even diminution of function remaining which can be recognized by disturbance or change in the joints. Gocht, in Hoffa's Clinic, observed in different hemophilic youths quite a number of more or less severe arthritic hemorrhages, either without a determinable trauma or as its direct consequence. The knee-joint, which he mentions as the one most frequently affected, at times appeared to be enormously thickened by a large effusion which occurred very suddenly, sometimes in from five to ten minutes. The joint was sometimes in slight flexion, at other times also in extension; sometimes there was pain, at other times none. Sometimes the joint was fixed and immovable, again the function was not at all disturbed. A patient in Hoffa's Clinic had more than 45 severe hemorrhages in the right knee-joint without suffering the slightest inconvenience in the use and function of the joint. The left knee-joint which was contracted had sustained about 40 hemorrhages; the first 39 gave rise to no injury; only the last effusion of blood led to permanent contracture. If the effusion is not absorbed, the swelling in the joint remains and is not amenable to treatment, as, for instance, was the case in this last mentioned knee-joint which led to contraction. Pains appear, and the function is much interfered with. According to König we are then dealing with that peculiar form of inflammation (second stage) which, as well in its pathology as in the clinical symptoms, closely resembles tuberculosis of the joint, and which was called by this author hydrops tuberculosus fibrinosus. The picture of the latter disease is simulated even to the most minute detail. In such cases the contents of the joint are hemorrhagicoserous or serous with a brownish color. The synovial intima shows swelling and brownish discoloration, and a large number of fluctuating, brownish, discolored, synovial villi. The coagula of blood in some areas may attain enormous thickness. The cartilage loses its white color and its luster, and shows a brownish discoloration; it is partially softened because its upper layer has been lost. It also shows defects and unevenness in its surface on account of an irregular loss of substance. The investigation of such joints with the Röntgen rays has been described by Gocht as follows: We recognize that the lower end of the femur upon the deformed side, in contrast to the other side, is decidedly smaller. The difference amounts to more than $\mathbf{1} \mathrm{cm}$. The bones upon the diseased side are atrophic and too permeable to the Röntgen rays, as may be recognized from the lighter color in the skiagram. The epiphysial lines upon the femur and tibia do not show the normal rounded edge, but they are irregular, serrated, with double contours. Upon the epiphysis of the femur anomalous indentations are shown. While upon the normal side an open space denotes the presence of normal cartilage, and the osseous ends of the femur and tibia present their normal forms, the conditions upon the diseased side are quite different: Corresponding to the loss of cartilage, a decidedly decreased joint space is found. The ends of the bones appear completely changed; the end of the femur is serrated and irregular; the indentation normally between the condyles is lost, and the intercondylar eminence of the tibia is irregular and extended. The recent losses in structure 
and the recent deposits fit each other. The capsular shadows upon the inner side of the contracted knee-joint are darker than upon the normal side.

In the case of an older boy, in whom remarkable changes had taken place, the cartilage space in the joint had disappeared entirely, the tibia was markedly dislocated outwardly. Between the bones adhesions could be seen. The relations of the capsule could not be judged from the picture, for at the time the plate was made an enormous effusion of blood had occurred in the joint, which was revealed in the picture as an oviform shadow the size of a hand.

In the diagnosis of bleeder affections of the joint, in accordance with König's views, Gocht differentiates three groups as follows:

1. Individuals who are well-known bleeders,

2. Those who have not been known to be bleeders, but who present characteristic symptoms of hemophilia besides the arthritic affection,

3. Those in whom neither the history nor any prominent symptom indicates the general affection.

If there is no history of hemophilia, such as preceding severe hemorrhages, the very rapid development of an effusion into the joints without noteworthy trauma and an accurate examination may lead to the proper diagnosis. The pains are often out of all proportion to the severity of the case, and the function of the implicated joint may be scarcely impaired. This is especially the case if the patient is seen in the first stage of the disease. If, on the other hand, a joint is found in the stage of inflammation, the diagnosis becomes more difficult, as the clinical picture of the bleeder joint is identical with that of hydrops fibrinosus tuberculosus. The difficulty lies in the fact that the investigator rarely considers the possibility that he is dealing with the diseased joint of a bleeder. For, if hemophilia is considered at all, an error in diagnosis is virtually excluded. The following phenomena point to a hemophilic joint: subacute hemorrhages and scleroses about the joints; freshly developed effusions into the joints, or hemorrhages under the skin into the muscles, etc. ; last, and above all, the signs of previous disease in other joints. König also declares that in hemophilia the patients are generally youthful males with a conspicuous pallor of the face. This latter symptom Gocht quite properly denies, for, in the first place, bleeders may show a healthy color of the face, and, on the other hand, patients with tumor albus are for the most part extremely pale, though of course abscess and formation of fistula, which are common with tumor albus, are exceedingly rare in hemophiliacs. In doubtful cases one or more injections of tuberculin may be used for diagnostic purposes.

From what has been said, it is evident that the recognition of a bleeder's joint may sometimes be very easy, at other times difficult but still possible, occasionally impossible.

Gocht agrees with Iinser that those bleeders who do not suffer from disease of the joints are exceptions. Almost invariably the arthritic affection is localized in one knee-joint. This may be due to the fact that the knee-joint is most exposed to injury on account of the great extent of its surface and its exposed position. The first hemorrhages, as well as the great majority of the later ones, are due to external causes. That children are not attacked by 
hemorrhages of the joints prior to learning to walk is explained by the absence of trauma. If, however, during the period in which they learn to walk, the joints are brought into use as well as the muscles, the time for hemorrhage of the joint has come, for the physiologic function of the joint is to be looked upon as a series of traumatic influences! Quite often surgeons note the appearance of arthritic hemorrhages of the most severe type even though the patient remains in bed, and is apparently not exposed to the slightest external injury. In such instances the joints affected are those that have been repeatedly the seat of effusions of blood. Probably because of the hyperplasia of the synovial villi, in consequence of changes in the surface of the cartilage, and on account of the great predisposition to hemorrhages, any slight motion may cause a laceration of such synovial villi, and so give rise to severe hemorrhages.

The question whether the predisposition to arthritic hemorrhages decreases with time must generally be answered in the affirmative. For, as we have already pointed out, according to the general views of hemophilia, the tendency to bleed declines in later life, and disappears entirely in the aged. We must likewise assume that a predisposition to arthritic hemorrhages gradually decreases, especially since the children become less active as they grow up and greater care is taken to prevent injuries. Another point which Gocht also considers in his observations is, whether the joints finally, in consequence of pathologico-anatomical changes, lose their tendency to hemorrhage. If a joint enters the third stage, the retrogressive, if destruction of the joint cavity has developed, if the former hyperplastic villi have contracted, or if adhesions in the connective tissue of a cartilaginous or osseous character have occurred, hemorrhages will be much rarer, in the first place, for anatomical reasons, and, secondly, because irritation due to motion is no longer present.

Still another factor must be considered-the variation in the individual predisposition. If, as we have seen, one hemophilic patient is capable of withstanding more than 45 severe arthritic hemorrhages without apparently suffering in function, while another readily develops a panarthritis, it follows that the power to absorb the effusion of blood in the joints also varies greatly in different patients. The question of the coagulability of the blood in the hemophiliac, which is still open and undecided, need not enter into this discussion.

According to Gayet and Th. Hirsch, the clinical phenomena of the arthritic affections of the hemophiliac resemble especially an acute or subacute arthritis (on account of pain and swelling, fever, stiffness, and the flexed position). On the side of pathogenesis, these authors believe that, besides hemorrhagic processes, we cannot deny a rheumatic influence in the joint affections.

Of other complications, the neuropathic predisposition is to be mentioned, which in some patients, chiefly women, presents itself in manifold ways. Here the hereditary neuropathic predisposition is especially prominent. Neuralgia is a relatively frequent occurrence in bleeders, and occasionally neuritis has also been noticed. In some cases a long-continued fever without local changes has been observed. Finally, I must mention the appearance of circumscribed, hard, painful infiltrations into the skin and the subcutaneous connective tissue, above which the skin remains unchanged in color; later 
these may undergo the characteristic changes which occur in the extravasations, or they may disappear without these changes.

\section{LOCAL HEMORRHAGES UPON A HEMOPHILIC BASIS}

In the last decade a number of cases have been published in which the common characteristic was a more or less profuse hemorrhage from a kidney, the absolute integrity of the organ being determined either by clinical observation or by operation. In these important cases the question arises: What factor is the cause of the hemorrhage? Although Lauenstein published his fundamental case in the year $188 \%$, and a large number of similar observations followed (those of Sabatier, Schede, Anderson and Leguen in the years 1889 and 1890), only Senator's article ("Regarding Renal Hemophilia," 1891) can be accorded scientific, authoritative importance. I shall therefore give a synopsis of the case:

A girl, aged nineteen, in the year $188 \%$, noted blood in the urine immediately after menstruation. The examination of the voided hemorrhagic urine at that time showed a large amount of hemoglobin although no red bloodcorpuscles. After the lapse of two years, during which time decided debility and periodic cough gave rise to the suspicion of tuberculosis, the hemorrhage recurred; this time it was more profuse, and lasted with slight intervals more than six months. The analysis of the urine showed actual hematuria; the blood discharged through the kidneys differed but very little from pure unmixed blood. Toward the end of February, 1890, Senator determined the following: Patient is well formed, very pale, no trace of emaciation. The internal organs present nothing abnormal; lungs and kidneys apparently healthy. Urination painless, perhaps somewhat more frequent than normal, but without tenesmus. The urinary sediment consists exclusively of erythrocytes; crystals, pus and other pathologic constituents are absent. No fever. The examination carried out under an anesthetic shows no changes, neither in the kidney nor in the bladder nor in the sexual organs, but it was demonstrated by means of the cystoscope that the blood flowed from the right ureter. Aftcr the usual causes of hemorrhage, such as stone, tumor and tuberculosis, had been excluded, Senator made a diagnosis of hemophilia which was confirmed by the history. It was ascertained that the patient was a member of a family in which hemorrhages occurred frequently. Four sisters, and a brother aged seventeen, showed great tendency to epistaxis; the father, who at the time was quite healthy, as a child had been frequently attacked by nose bleed and hemoptysis without showing any pulmonary lesions. Eleven brothers and sisters of the father suffer or have suffered from epistaxis; an uncle of the pationt, who for a long time suffered from epistaxis, in his twentieth year had an attack lasting twenty-four hours, after which purpura appeared over the entire body and hematemesis occurred; the disease ended fatally after fourteen days. A second uncle is the father of two children who have inherited epistaxis from him. The mother of the father, up to the time of her death, menstruated profusely. Unquestionably, therefore, the patient was a member of a bleeder family. Although no symptoms of hemo- 
philia had previously appeared, Senator believes, in the absence of other causes, that he may assume a hematuria of hemophilic origin. The continued hemorrhage resulted in a severe anemia which resisted all internal remedies, and for this reason nephrectomy was proposed and performed. The organ was laid bare and removed, although apparently unchanged. The further course of the disease was very favorable. Upon the second day after the operation the blood disappeared from the urine and never recurred. Four weeks later the patient left the hospital entirely well. The examination of the extirpated kidney showed small inflammatory foci and extravasations; otherwise the organ was absolutely normal.

I shall add to this two other analogous cases observed in v. Leyden's clinic and described by G. Klemperer :

1. A man, aged thirty-five, admitted to the clinic on the 15th of April, 1893. The father of the patient died of enteric fever, the mother is living and since earliest childhood has suffered from frequent subcutaneous hemorrhages and marked bleeding even after insignificant injuries. A brother died of hemorrhage during an amputation. Another brother of the patient is also predisposed to hemorrhages. The patient himself, immediately after birth, had a severe hemorrhage from the umbilical cord. In childhood hemorrhages frequently occurred from the nose and other parts of the body after insignificant trauma. From his third year, he suffered from swelling of the joints of the upper and lower extremities. As a rule these swellings occurred suddenly without cause, usually early in the morning; they were extremely painful, but often disappeared on the same evening; complete motility of the limbs, however, did not return until some months after the attack. At fifteen the patient had a fracture of the thigh and severe subcutaneous hemorrhages delayed normal recovery. His first attack of hematuria occurred at sixteen, accompanied by dull pain in the right renal region, which soon became coliclike; this was followed by nausea and vomiting. The urine was of a bloody red or black color. This condition lasted for several months, the hemorrhages recurring at intervals of from six months to two years. One attack of hematuria lasted thirteen weeks. The patient did not seek professional aid, as he believed himself to be a bleeder, and looked upon the hematuria as a symptom of the general affection. During the attacks he remained quiet, and relieved the pain by large doses of morphin which had first been used at the time of the enlargement of the joints. The patient came to the clinic principally for the treatment of his morphinism. He is pale, nervous; the internal organs are normal. Upon the fourth day after admission a renal hemorrhage occurred which lasted for two weeks. The urine contained pure blood. The patient was discharged eured upon the 15th of May, 1893. Toward the end of his stay in the hospital two hemorrhages into the wrist-joints occurred. Subsequently this happened quite frequently. Up to March, 1896, the hematuria had not recurred.

2. The other case was that of an official aged twenty-six years, the history showing but slight hereditary predisposition. The patient himself was a bleeder. From the age of sixteen, almost every year attacks of hematuria occurred, which occasionally were repeated several times in the year, and lasted 
for hours or weeks. With these the patient had insignificant pain in the right renal region. He consulted a physician only when a hemorrhage lasted longer than eight days. Upon the $2 d$ of November, 1895, pain occurred in the right renal region, and upon the 9 th of November he voided bloody urine. Examination showed nothing abnormal; no pathologic constituents except blood were found in the urine. In spite of numerous remedies the hemorrhage continued, and the anemia constantly became more marked. Upon the 28th of December all drugs were stopped, and hydrotherapy was begun. The patient had a bath each day lasting ten minutes, followed by affusions to the renal region. The bath was begun at a temperature of $95^{\circ} \mathrm{F}$., the water being gradually cooled to $75.2^{\circ} \mathrm{F}$. The affusions were at a temperature of $82.4^{\circ} \mathrm{F}$. to $60.8^{\circ} \mathrm{F}$. Gradually the hemorrhages became slighter, and the urine finally cleared. Upon the 15th of January the patient left the clinic cured.

A fourth case is published by S. Grosglik: "Ueber Blutungen aus anatomisch unveränderten Nieren" (Sammlung klin. Vorträge, Nr. 203). An army officer, aged thirty-six, of a bleeder family on both paternal and maternal sides. The patient was of healthy appearance, but suffered frequently from marked epistaxis and hemorrhage from the rectum. In September and December, 1896, he had severe hematuria for which clinically no cause could be assigned. As the patient's history was unknown at that time, the diagnosis wavered between a beginning tumor and tuberculosis of the kidney. After an observation of six months, as no further point of support could be determined for either diagnosis, and after the patient had related his family history; Grosglik made the diagnosis: "Idiopathic renal hematuria upon a hemophilic basis."

In other cases of "néphralgie hématurique" in which the hematuria could not be assigned to a hereditary hemophilic constitution, it has been assumed that the cases were due to vasomotor or traumatic renal hemorrhage (i.e., due to corporeal over-exertion). Accordingly, varieties of hemophitic vasomotor and traumatic renal hemorrhage might be described in which the possibility of other causes for hemorrhages than an anatomical substratum could not be excluded.

In regard to the diagnosis, if there is no enlargement of the kidney, and if the composition of the urine and the accompanying symptoms do not favor an organic affection, the following facts are of importance: The proof of a hereditary hemophilic predisposition, a preceding exertion, or disturbances of the nervous system (hysteria, neurasthenia, etc.) by which the vasomotor center for the kidney might be implicated. If the history reveals anything positive, the diagnosis of essential hematuria becomes likely. It only becomes certain, however, when, after prolonged observation, no distinct signs of anatomical change have appeared.

The treatment of hemophilic renal hemorrhage, according to Grosglik, should be expectant. If the course of the affection is severe internal remedies are powerless, and if the hemorrhage threatens to prove fatal the bleeding kidney must be removed as soon as possible. In so-called vasomotor renal. hemorrhages the diagnosis should be immediately confirmed by surgical interference, which should not only embrace the exposure and palpation of the 
organ but also an incision into the kidney, and a minute investigation of the parenchyma. If the incised kidney is found to be anatomically unchanged, it should be closed by suture and replaced. If this procedure affords no relief, a secondary nephrectomy is to be performed. Primary extirpation is not warranted in these cases.

How little data the exploration of an incised kidney in vivo may furnish, Zondek recently demonstrated in a discussion before the Society of Internal Medicine, when he arrived at the following conclusions:

1. Even in a kidney laid open by operation the presence of a small stone cannot be excluded with certainty.

2. Even when surgical exploration of the organ gives a negative result, inflammatory changes of the renal tissue may be due to a stone in a concealed calyx.

3. Complete proof of the diagnosis of angio-neurotic hemorrhage from the kidney necessitates the most careful investigation of the entire kidney after it has been removed from the body, and also of the ureter down to its point of entrance into the bladder.

Harris (Philadelphia Medical Journal, March 19, 1898) published two cases of "essential" renal hematuria. In one of the patients the kidney was exposed. In both cases the hemorrhage ceased.

Harris has collected 16 similar cases from literature. The majority of the patients were cured by simple nephrotomy. He comes to the following conclusions: 1. Renal hemorrhages occur in which none of the usual causes, acute nephritis, tuberculosis, sepsis, neoplasms, malaria, etc., are present. 2. The pathological changes arising in the kidney are unknown to us. 3. These hematurias are not controlled by ordinary styptics. 4. If tonics, cold baths, etc., are without effect, nephrotomy must be performed. 5. Primary nephrectomy is in no wise indicated.

$J$. Israel writes on the curative influence of renal incision in cases of unilateral colic and unilateral renal hemorrhages in the obscure pathologic condition which up to the present has been called néphralgie, néphralgie hématurique, renal hematuria without known lesions, essential or angio-neurotic renal hemorrhage, all of which, according to his investigations, most frequently depend upon chronic inflammatory changes of the kidney. Up to the year 1898 he had operated upon 14 cases of this kind. Five kidneys were examined microseopically, and four of these showed different forms of nephritis. In eight of the remaining nine patients, microscopic investigation of the kidneys showed organic changes. Of the fourteen cases treated by nephrotomy three died, of whom two had severe bilateral nephritis; six were completely and permanently cured; three after apparent recovery suffered from mild relapses of varying duration; in two patients the operation was designated as useless.

Israel has summed up the important knowledge obtained from these observations in the following: 1. There are cases of unilateral nephritis. 2. There are cases of renal colic due to nephritis which may completely simulate colic from calculi. 3. There are cases of bilateral nephritis which produce only unilateral colic. 4. There are cases of severe nephritis with absence of albumin in the urine and absence of casts. 5. The urine may be free from albumin 
in spite of a profusion of hyaline, granular and epithelial casts. ${ }^{1}$ 6. There are cases of nephritis with paroxysmal, profuse hemorrhages. 7. Nephritic hemorrhages may occur and run their course with or without colic; the hemorrhage is not the cause of the colic. Both phenomena are the results of renal - congestion. 8. A great number of pathologic conditions designated up to now as néphralgie hématurique are to be referred to nephritic processes. 9 . Incision into the kidney in many cases influences the nephritic process and its symptoms favorably. - In a lecture, " Hemorrhage from a Normal or Apparently Normal Kidney," Naunyn in the main agrees with Israel that interstitial nephritis is the most frequent cause of this form of renal hemorrhage.

Pousson in two cases of apparently spontaneous renal hematuria extirpated the affected kidney. The microscopic examination of the congested kidney revealed inflammatory foci in the renal cortex without other abnormalities. Poirier and Picqué advise in such cases only the operation of nephrotomy, as frequently the other kidney also presents signs of chronic nephritis. In contrast to this Regnier believes that in such cases we are invariably dealing with a beginning renal tuberculosis (?); he therefore strongly advises early nephrectomy.

Hofbauer (Mitth. a. d. Grenzgeb. d. Med. u. Chir., 1899, V, 3) describes a case of essential unilateral hematuria in a girl aged thirteen: It was especially difficult to determine in this case the etiology of the hematuria which had persisted for two years. It had appeared without an assignable cause, and, in spite of this great duration, had produced no sequelæ with the exception of anemia and its consequences. The examination of the urine revealed no pathological constituents except an admixture of blood. Upon cystoscopy the urinary bladder was found normal; the blood was seen to exude from the left ureter while from the right ureter clear urine was being excreted. No signs upon palpation. An exact clinical diagnosis was not made. On account of marked loss of blood there was an exploratory incision only; this did not furnish the hoped-for, absolute certainty. On exposure, the kidney was found to be normal in size, and palpation revealed no pathologic changes.

When, to facilitate more exact inspection, the kidney was drawn forward, a laceration of one of the larger vessels made extirpation necessary. The hemorrhage ceased. Recovery followed. Macroscopically, the kidney, the renal pelvis, and the ureter were perfectly normal. Microscopical investigation of the extirpated organ revealed a chronic glomerular nephritis. In this case the diuresis following the nephrectomy was very interesting. The amount of urine a few days after the operation not only equalled the former amount but decidedly exceeded it.

In 1891 Senator, in his case of renal hemophilia (Berliner klin. Wochenschr., 1891, Nr. 1), tested the activity of the remaining left kidney. For this purpose he examined the urine daily for four weeks from the second day after nephrectomy, in regard to amount, specific gravity, and its contents of

1 I should like to remark in this connection that upon the occasion of the Tenth Congress of Internal Medicine in 1891, when I demonstrated the centrifuge in Wiesbaden for the first time, I called attention to the fact that "casts oceur quite frequently in urine free from albumin, and can scarcely be looked upon as pathological." 
nitrogen. The reaction was always acid. With the exception of the first day upon which the urine still contained some blood, and upon the succeeding day when a small amount of albumin was present, abnormal constituents were never noted. The examination for nitrogen was conducted according to the well-known process of $\mathrm{Kjeldahl,} \mathrm{and} \mathrm{several} \mathrm{times} \mathrm{urea} \mathrm{was} \mathrm{estimated} \mathrm{accord-}$ ing to Pflüger's modification of Liebig's method. It was shown from the table, published in Senator's report of his case, that immediately after the operation the remaining left kidney performed all the work of both kidneys, perhaps at first functionating even a little more, for a daily excretion of nitrogen of over 16 grams (such as was seen in this case) is the normal for an adult person with a plentiful supply of food, and for a young girl in such a condition (lessened amount of food after the operation) is a decided and abnormal increase.

Floderus in Lennander's Clinic observed a case of renal hematuria with macroscopically unchanged kidneys. He examined microscopically the kidney extirpated by Lennander, and demonstrated a disseminated sclerotic glomerulitis with advancing sclerosis in the adjacent connective tissue, also fatty degeneration and atrophy of the epithelium. In consequence of this finding Floderus studied minutely the previously published cases of so-called essential renal hemorrhage. He divides these cases into the five following groups:

1. Renal hemorrhage in hemophilia.

2. Renal hemorrhage due to vasomotor disturbance.

3. Hematuria from mechanical causes.

4. Hemorrhage from kidneys which are the seat of nephritic processes.

5. Imperfectly described cases of essential renal hemorrhage.

After these literary deductions Floderus enters upon a discussion of most of the cases published, minute reports of which are entirely lacking. For this reason the last group must include the majority of the cases assigned to the four other groups. His criticism of the cases described in literature permits the assumption that in scarcely a single instance was the bleeding kidney shown to be intact histologically.

In a woman aged forty-five, who for two years and six months suffered from a left-sided renal hematuria, de Keersmaecker performed nephrectomy. The kidney revealed interstitial and parenchymatous inflammatory nephritis (with hemorrhage).

Rovsing has written a long article regarding unilateral hematuria of questionable origin and its treatment by nephrectomy.

Before making the diagnosis, "hemorrhage from normal kidneys" we must determine by all methods at our command: 1 . That the hemorrhage actually originates from the kidney; 2. That all pathologico-anatomical changes which may be assumed to be the cause of renal hemorrhage are absolutely excluded. The cases published may be divided into two groups: 1 . The cases treated exclusively by medication; 2. The cases in which the kidney was directly palpated by operation, was incised, or was removed. Only the last-named cases, of which Rovsing collected reports of 13, are of particular importance. In all of these observations the authors unanimously state that 
they were unable to find any material or assignable cause for the hemorrhage; while, from the circumstance that the hemorrhage ceased after operative interference, whether by a simple exploration, a nephrotomy or a nephrectomy, proof was given that the hemorrhage actually arose from the kidney in question. For the explanation of the hemorrhage, therefore, the assumption of a "local hemophilia" or an "angioneurosis" is in these cases held sufficient.

If, however, the published clinical histories are critically examined, we arrive at the conclusion that, as a matter of fact, in by far the majority of cases, pathological conditions of the kidney or of the pelwis of the kidney might have been determined which would explain the hemorrhage. Finally, in the cases in which the kidney has been found apparently normal, the possibility was not considered that the starting point of the hemorrhage was not in the kidney substance at all, but in the pelvis of the kidney, or in the ureter.

Rovsing communicates four personal observations, in three of which pathological changes were observed, and comes to the following conclusions: 1 . Many of the published cases of hemorrhage of nervous origin do not stand the test of critical analysis, as in some of them pathological conditions were actually present which very readily explain the hemorrhage, while in other cases the investigation was not thorough enough to exclude other affections, not only those of the kidney but also those of the bladder, as cystoscopy was not performed to determine the origin of the hemorrhage from only one ureter. 2. There is no doubt that in a number of cases of hematuria from normal kidneys, dislocation of the kidney with torsion of the pedicle or kinking of the ureter plays an important rôle. 3. The first case mentioned by Rovsing demunstrates the fact, unknown till then, that lacing and the pressure of a corset may directly cause very marked disease of the kidney. 4. After a careful sifting of the cases a few still remain in which decided hemorrhage, which was proved to originate from one ureter, disappeared permanently, although the exploratory incision of the kidney in question showed no demonstrable pathologic changes. 5. Such cases are so rare that they may be considered purely as exceptions, and since serious disease can never be excluded clinically as a cause for obscure cases of hematuria, in all doubtful cases an exploratory lumbar incision should be made, especially since, according to experience, this may have a curative effect upon the hematuria.

In the first session of the Fourth French Congress for Urology (October, 1899) a symposium upon "essential hematuria" took place. Malherbe and Legueu maintained that, besides hemorrhages occurring in tuberculosis, carcinoma, and lithiasis, a so-called essential hematuria had also been described which is apparently independent of disease of the urinary organs. In an accurate analysis of the cases published up to that time, particularly those cases which had been pathologico-anatomically investigated, pathological changes were almost invariably noted even though often slight, for example, calcareous incrustation of a pyramid, or slight tuberculous disease. The most frequent cause of so-called "essential hematuria" is evidently chronic nephritis. The quite insignificant sclerosis of the renal parenchyma, as, for instance, after contusion of the kidney, in floating kidncy, and in pregnancy, may remain unilateral, and not necessarily give rise to further symptoms of Bright's disease. 
The assumption of a "hemophilic" and an "angio-neurotic" hematuria is not justifiable. “On peut dire, qu'il n'y a pas d'hématurie essentielle: toutes les hématuries sont symptomatiques et relèvent d'une cause générale (toxique ou infectieuse) ou d'une affection rénale."

Tédenat also questions the occurrence of true essential hematuria. His own experience relates to four cases of which two were very probably connected with nephrolithiasis; nephrotomy produced a cure. In another case the hemorrhage ceased after the removal of a small hydatid cyst of the kidney. In the fourth case the cause of the hematuria was a carcinoma of the colon compressing the ureter, after the removal of which the hemorrhages permanently ceased.

Pousson also denies the existence of essential hematuria; he calls attention particularly to the hemorrhages which result from the retention of urine, in consequence of floating kidneys, or from other causes. For instance, he observed an apparent renal hematuria in a patient in whom there was at the left renal opening of the ureter a mucous membrane valve which caused, from time to time, a more or less complete retention of urine. After division of the valve and fixation of the kidney the hemorrhages ceased. In another case a stone embedded in the mouth of the ureter produced the hemorrhage.

In this connection Albarran remarks that the cases of so-called essential hematuria may be divided as follows: 1 . Hematuria in floating kidney; 2. In hydronephrosis; 3 . Hematuria in early stages of chronic nephritis. Seventeen cases of this latter form are already mentioned in literature. He adds another case occurring in a patient aged fifty-three. The hemorrhage ceased after nephrotomy. The diagnosis of diffuse parenchymatous and interstitial nephritis was confirmed by the microscopical examination of an excised portion of the kidney. Boursier emphasizes that so-called essential hematuria may for a long time be the only symptom of a nephrolithiasis. In the urate and oxalate diathesis severe hematuria is also observed without any stone being present; it may be that the small crystals produce an irritation of the renal parenchyma and so eventually lead to periodic hematuria.

Hamonic calls attention to hematuria as a premonitory symptom of certain renal affections (parenchymatous nephritis, nephrolithiasis, tuberculosis, carcinoma).

Galland-Gleize also confirms this fact. He communicates reports of four cases in which for a year and a half to seven years hemorrhage had preceded the disease later determined (kidney-bladder stone, tumor of the bladder).

Loumeau agrees with those who do not believe in essential hematuria except when the microscopic investigation demonstrates that the "renal epithelium" is normal. But we should require even more than this; not only the integrity of the epithelium but also that of the entire parenchyma should be determined after a portion of the kidney has been excised and examined. This will be still insufficient for an anatomical diagnosis, as the pathologic process is not always uniformly distributed over the entire organ. But, apart from the actual, diffuse disease of the kidney, this objection is much more valud for those diseases of the renal tissue which arise from a focus, such as a stone, etc. Here the investigation of one, or even of several, portions of the kidney 
might show perfectly normal conditions, while in the immediate vicinity of the excised portion changes might have taken place which gave rise to the hemorrhage!

Pasteau has described a typical case of so-called essential hematuria in floating kidney. Here the hemorrhages were connected with severe attacks of renal colic, and were permanently relieved by nephropexy.

In opposition to many other authors, Castan believes in an actual essential hematuria, as a proof of which he mentions the following typical case: A woman, during the menopause, and for a period of several months, had very profuse hematuria without the slightest symptom of nephritis being evident. Complete and permanent cure was effected by means of baths, massage, etc. In Castan's opinion, a disturbance of menstruation may cause the retention in the body of certain toxins which, without causing demonstrable disease of the renal parenchyma, may give rise to hematuria. To this category the hematuria of the pregnant also belongs: "La femme est en état de toxhémie permanente."

Guyon declares that among the causes of severe threatening renal hemorrhages congestive conditions occupy the front rank. The kidneys, however, macroscopically and microscopically, may present no changes to explain the congestion. He admits, of course, that renal hemorrhages may be due to inflammation, neoplasms, stone, etc. Guyon mentions cases of profuse congestive renal hemorrhages in which neither at the operation nor at the autopsy could pathological changes be detected in the kidney. Especially noteworthy are three observations in which, during pregnancy and lactation, severe congestive conditions led to profuse renal hemorrhages.

Briefly, the cases are as follows:

1. A woman, aged thirty-five, previously healthy, in the eighth month of pregnancy voided for fourteen days urine of a coffee-brown color. In the eighth month of her third pregnancy hematuria recurred and lasted for three weeks. During this pregnancy there were no signs of nephritis. Three months after this last labor, sudden, severe, continuous hematuria appeared, and debilitated the patient greatly. There was a discharge of coagula, with severe pain in the right renal region. Up to this time the patient had been nursing her child. Upon advice she ceased nursing the child, and the hemorrhages immediately stopped. Right kidney somewhat enlarged; irregular shape. Diagnosis: Neoplasm of the right kidney. Upon lumbar incision and section, kidney found to be perfectly normal. Suture; recovery. Hemorrhage has not recurred.

2. Hematuria in the course of the fourth and fifth pregnancies; otherwise perfectly normal conditions.

3. Severe hematuria at the end of pregnancy; sudden stoppage after termination of labor.

The same author mentions two other cases of hematuria during pregnancy. A total of twelve cases of this kind is found in literature. Guyon does not believe that these pregnancy hematurias are to be looked upon as true essential hematurias. Besides mechanical causes, inflammatory changes which lead to congestion evidently play a great rôle. 
Desnos has seen in a large number of gouty patients hematuria which either preceded or followed the attacks of gout. This gouty hematuria was either accompanied by renal colic or ran its course without pain; the condition may be brought about by "congestion," but may find its explanation frequently in the influence of stone.

On account of limitation of space, it is impossible for me to discuss more minutely the literature of the last few years, and I shall refer only to the very comprehensive discussion in the Berlin Society of Internal Medicine in January, 1902, of the excellent article of Professor Senator, "Renal Colic, Renal Hemorrhage and Nephritis," which gives succinctly the views regarding these questions of such prominent authors as Senator, James Israel, G. Klemperer, and L. Casper. Any one desiring to learn the present status of this question will find in this source material upon which to form his opinion, although it is impossible at this time to state a definite conclusion. The subject is still open to discussion, and the combined labors of the surgeon and the internal clinician will be required to clear it from all doubt.

I must emphasize a fact which is frequently observed, that in hemophilia, hemorrhages from other parts of the body cease when hematuria occurs.

Senator was the first to throw light on this dark subject, and it is certainly owing to his labors that some renal hemorrhages have been recognized as of hemophilic origin. Although some have doubted whether, in his case, the condition was actually renal hemophilia or, on the contrary, hemorrhage due to another and, at that time, unrecognizable cause, there is much in favor of his view that cases of hereditary renal hemophilia have lately been observed (Attlee and Guthrie, St. Bartholomew Hospital Journal, December, 1901, and The Lancet, May 3, 1902, xviii ; compare also Senator, "Renal Diseases," $2 \mathrm{~d}$ edition, p. 515, additions). No matter how we view this condition it is greatly to Senator's credit, and will remain so, to have been the first to recognize the just importance of, and to throw the proper scientific light upon, the underlying cause!

Hematuria must not be looked upon as the only local hemorrhage in hemophilia. 'Under similar circumstances pulmonary and gastric hemorrhages are occasionally noted in bleeders, at the age of puberty or even later, who have until then been free from hemorrhage, and for these there is not the slightest anatomical basis. Cases of hematuria have awakened interest especially by their being unilateral, and also because it has been possible by operative procedures to make an exact and certain diagnosis during life, which is impossible in the case of other organs, particularly of the stomach and lungs. In the future the diagnosis of essential hematuria will necessitate much greater exactness.

\section{PROGNOSIS}

The prognosis of hemophilia is unfavorable: 60 per cent. of bleeders succumb before the eighth year of life and only 11 per cent. reach the twentysecond year. After puberty the chances are somewhat better; but, even in later life, a slight injury may terminate fatally. In a family in Finland in five generations from a stock not previously hemophilic fourteen male descend- 
ants succumbed directly to hemorrhage. The first symptoms of the disease were noted after the children had reached the age of six months.

\section{TREATMENT}

General prophylaxis. On account of the hereditary nature of the disease, and in view of the fact that the affection is mainly transmitted by the female, it is obvious that the proper restriction of marriage would lessen hemophilia. From considerations of this kind laws have been framed in accordance with which the question of marriage in bleeder families should be considered. The fundamental rules, based upon Grandidier's rich experiences in this respect, are as follows:

1. All females, members of bleeder families, whether they themselves are bleeders or not, are to be advised not to marry.

2. All male members who themselves are not bleeders may unquestionably be permitted to marry.

A male bleeder is only to be dissuaded from marrying if it can be proven that in his family hemophilic males have reared hemophilic children, although the males in question married healthy women from healthy families.

A factor which is said to favor hemophilia is the intermarriage of relatives. Whether this is justifiable or not is difficult to determine. So far as we have positive knowledge, the conditions are the same as in the intermarriage of relatives generally. There can be no doubt that professional prohibition of marriage has very little effect. The desire of a hemophilic family to see a daughter married may be much. stronger than the fear that from the marriage bleeders may be born.

Of paramount importance in combating hemophilia is individual prophylaxis. This is to begin as soon as the child is born, and during the years of infancy it must be very strict in accordance with the experience that at this age the hemorrhages of hemophiliacs are especially prone to be exceedingly serious. In nurslings in whom there is a suspicion of hemophilia, all attempts to remove slight congenital defects are absolutely prohibited. To this category belong the operations for hare-lip, malformation of the palate, severing the frenum of the tongue, the operation for syndactylism, the removal of nævi, etc. Ritual circumcision of the children of Jewish and Mohammedan families is to be strictly prohibited. In girls the usual piercing of the lobe of the ear for earrings must be omitted. Experience shows that vaccination of hemophiliacs does not give rise to threatening hemorrhage; it may therefore be practised in infants (and also re-vaccination) without danger. Great attention must be paid to the teeth in the children of bleeder families. The periodical observation of the teeth by a thorough and experienced dentist, and the careful treatment of even the slightest affections of the teeth are necessary in order to preserve them. In view of the extreme danger of surgical operations upon the oral cavity, particularly extraction of the teeth, care must be exercised to prevent such operations in so far as possible in children of bleeder families. The application of leeches, blisters, wet-cups, in children as well as in adult bleeders, is to be avoided. When the children begin to go about by them- 
selves, their play with other children is to be watched. The nurse must be instructed to see that the children do not injure themselves; their toys are to be so chosen that injury to the skin may be avoided. Hemophilic children must not be chastised. As soon as the children are intelligent enough, they and their playmates are to be instructed that injuries by falling or blows, a prick with a needle or pen, should be avoided. If the children are sent to school the parents should inform the teacher that the pupil is a member of a bleeder family. It is advisable that the physician instruct the teacher, in so far as may be necessary for the welfare of the child while in school, as to the symptoms of hemophilia. The teacher will then understand how to conduct himself toward a hemophilic pupil, and what instructions he should give the other pupils as to their behavior to the bleeder. Hemophilic children should not take part in gymnastic exercises; they should never be subjected to corporeal punishment.

In the choice of an occupation the bleeders are limited at once by the circumstance that in comparison with others they are weak. They must, therefore, choose occupations in which great bodily strength is unnecessary. Those without means should be advised to become clerks or designers. Among the lighter trades they should not be taught those trades in which slight injuries are apt to occur, such as watch-making, engraving, paper-hanging, and that of goldsmith and barber. Bleeders whose circumstances permit may with advantage pursue the learned occupations. Such students should not take part in games, nor should they be accepted in the army.

General Treatment. Hemophiliacs should be placed on a special diet, and fluids which slightly stimulate the vascular system (alcohol, tea and coffee) are to be avoided. The use of milk, lemonade, orangeade, etc., is to be advised. Solid food is to be of the bland kind; spiced foods should be prohibited. Vegetables (particularly fresh vegetables, fruit, salads) are beneficial. The general nutrition is to be stimulated by baths, cold ablutions, occasionally sea baths, and by residence in the country.

Special Treatment. Attempts have been made to control the disease by the administration of drugs. In a case reported by Wickham Legg, the use of iron chlorid is said to have brought about improvement, but in a case of my own this was ineffectual. The following drugs have also been tested: Mineral acids, sugar, lead acetate, magnesium sulphate and sodium sulphate. of the mineral acids, chicfly upon Werlhof's advice, sulphuric acid in the form of the elixir acidi Halleri has been employed. The freshly expressed juice of lemons has been warmly advised. From this the deeply-rooted, popular belief in the lemon treatment, and, in milder cases, the orange treatment, has developed, in which in a gradually increasing number, from one to ten lemons daily, that is, their juice, are consumed. This "treatment" is continued for from six to eight weeks. But its benefits are as slight as from the long-continued use of Haller's acid.

The salines are perhaps of use in the sense that they relieve the congestive conditions which play a rôle in hemophilia. General strengthening and tonic remedies have frequently been resorted to during the periods free from hemorrhage; and during this time, and also when any signs portend an approach- 
ing hemorrhage, ergot, lead acetate, hydrastis canadensis, as well as opium and silver nitrate, have been employed to combat the hemorrhagic diathesis. Upon the whole little is to be expected from the drug treatment of hemophilia!

Regarding the treatment of special hemorrhages in the hemophiliac, attempts must first be made to control the hemorrhage by mechanical means. The member is to be elevated. Occasionally this alone is sufficient. Secondly, the employment of local styptics comes into question, iron chlorid and gelatin; under some circumstances the cautery may also be employed. Sometimes we succeed with tampons; sometimes by the application of a rubber bandage to the bleeding member and by compression of the nearest large artery. 'Sometimes the tying of a large vessel becomes necessary. Hémard, to control the hemorrhage after extraction of a tooth, tied the common carotid artery.

D. McKenzie (British Medical Journal, April 27, 1901) reports the occurrence in a hemophiliac of an apparently uncontrollable epistaxis which yielded at once to a tampon of cotton soaked in suprarenal extract.

R. Heymann (Münchener med. Wochenschr., 1899) reports the case of a bleeder aged twenty-eight, who, after removal of the tonsil, suffered from severe hemorrhage which could not be controlled. Heymann injected under the skin of the thorax 180 c.c. of a 2.5 per cent. neutralized and sterilized solution of gelatin in a physiologic salt solution warmed to $40^{\circ} \mathrm{C}$. After fifteen minutes the hemorrhage ceased, and did not recur for thirty hours. The injection was twice repeated, and the hemorrhages invariably became slighter. Heymann concludes from this that an increasing coagulability of the blood was produced.

Nichols (Medical News, 1898) reports the history of a hemophiliac, twentyfour years old, who, while intoxicated, fell upon broken glass and received extensive incised wounds. For seven days the hemorrhage could only be partially controlled, and the patient showed symptoms of a dangerous anemia; Nichols then poured the contents of a culture tube filled with 10 per cent. gelatin into the wound. The hemorrhage permanently ceased.

My own numerous experiments with gelatin for the purpose of controlling severe hemorrhages and in the treatment of aneurism lead me to doubt the correctness of these observations, although I do not wish to discountenance the occasional use of gelatin for styptic purposes. But, in doubtful cases, we must not expect great results from its employment.

Adrenalin locally has of late been successfully used in controlling bleeding, and is worthy of trial in hemophilia under similar circumstances.

of internal remedies ergot and its derivatives have been used. Their effect, however, in the fully developed disease is very questionable.

During pregnancy in hemophilic women hemorrhages indicate artificial abortion or early induced labor (Kehrer). Broock (Transactions of the Chirurgical Society of London, 1900, page 103) reports the case of a woman, a member of a bleeder family, who had borne two children with well-marked symptoms of hemophilia, and, as she had suffered greatly from hemorrhage in each labor, he endeavored to prevent it in the third. He instituted a treatment which had for its purpose, on the one hand, a general strengthening of 
the entire system (change of air in combination with the administration of iron, quinin and arsenic) and, on the other hand, the increase of the coagulability of the blood (calcium chlorid). The course of the labor was in exact accordance with his expectations. The loss of blood was very slight, the child showed no signs of hemophilia, and was stronger than the children previously borne.

The treatment of the hemophilic joints is especially important. F. König advises the following method: A fresh hemarthros, above all in a blecder, is to be so treated that the patient cannot use the affected joint; moderate compression decidedly promotes absorption. The question whether any operative treatment of the diseased joint is permissible König answers affirmatively, in so far as puncture of the joint is concerned. König has performed puncture in the knee-joint three times, twice with carbolic acid washing. The operation was always harmless, one patient was much improved, and the other patient was cured; but König advises that this be the limit of operative procedure. Of three patients into whose joints he opened, by mistake, supposing the condition to be tuberculosis, two bled to death, and one after many hemorrhages recovered, but had a stiff joint remaining. According to Guyot, the treatment of the hemophilic arthropathies in the acute stage must be entirely expectant; later, however, puncture of the joint and incision, with removal of coagula, is said to facilitate recovery, after which recurrences of the bleeding are less to be feared.

The latest reports regarding the therapy of blecder's joints are from Hoffa's Clinic, and in the article by Gocht, previously referred to, great importance is attached to the prophylaxis of hemophilia. Improvement of the general condition by good food, residence in pure air, and hardening of the body, as well as the avoidance of all injuries which may give rise to hemorrhages, are the most important measures. Boys must be trained to this from the onset, and as early as possible they must be taught to avoid running, jumping, and other active children's games. If it is known that the child is a bleeder before it learns to walk, the greatest care must be exercised. The joint hemorrhages which follow the most insignificant, scarcely avoidable, injuries are numerous enough, aside from the greater injuries which may bring about hemorrhage, and must be carefully guarded against.

The first lesion to be described in Gocht's article is a fresh effusion of blood into the knee-joint. In marked cases enormous swelling arises. The joint is so tense and so filled with fluid that the resistance of the capsule of the joint checks a further exudation of blood. The symptoms are the same as after severe contusions of the joint, except that in many cases from the onset the function is scarcely disturbed. Sometimes the knee assumes the mediumflexure position, at other times not so, or only for a few hours until the capsule has accommodated itself to the exudation. The treatment varies. Where there is decided pain, absolute rest, moist compresses, ice, or compression with bandages may be beneficial; in other cases the patients are kept perfectly quiet or are permitted to walk about carefully. Gocht determined from his experience in the Clinic that generally a moderate use of the joint in walking has no injurious effect upon the absorption of the effusion in the joint. 
The same period of time, about two to four weeks, is required for the disappearance of the effusion, whether there is absolute rest or use of the joint. A few days after the development of the effusion massage is to be cautiously begun, not upon the joint itself, but in parts centrally situated from the joint. This causes no pain to the patient, and by action upon the peripheral joint, absorption is stimulated. At the same time this treatment prevents the muscular atrophy, particularly of the extensor muscles, which so readily appears in all diseases of the joints.

In the majority of cases these measures will suffice to bring about absorption of the effusion of blood, especially if the hemorrhages have not as yet been frequent. The older the effusions and the more frequently the joint has been attacked, the less rapidly does absorption of the hemorrhage take place. Nevertheless, here also the treatment must be expectant, and usually recovery will ensue.

If a joint has entered into the stage in which it resembles hydrops fibrinosus tuberculosus, where inflammatory phenomena dominate the clinical picture, orthopedic treatment becomes necessary. In opposition to König, Gocht maintains that only when the patient suffers great pain, and provided the physician is absolutely certain in the diagnosis, should puncture be performed. As experience has taught that absorption, even although slowly, nevertheless almost invariably occurs, removal of the blood need not be resorted to except for imperative reasons. Here, on the contrary, orthopedic treatment should be employed. Besides the measures which have already been mentioned, such as a position of rest, compression, and (particularly for the beginning contracture) extension, plaster of Paris is to be employed, and, above all, portable apparatus so as not to keep the patient too long in bed. A brisement forcé, in fact all force employed for extension of a contracture in a bleeder, is entirely contraindicated. Rail-brace apparatus, well manufactured and accurately fitted to the extremity, is of even greater use in the third stage of the disease in which permanent deformity of the joints has sometimes occurred. The many extreme and violent stretchings of the capsule, and the inflammatory processes following these, cause an increasing damage (i. e., flaccidity) of the capsule of the ligamentous apparatus. Just as in tuberculosis, not only flexion but subluxation and abduction of the tibia posteriorly and outwardly gradually occur.

Changes in the ends of the bones are added to those of the capsule of the joint, so that we have a mixed form of destruction and distention-luxation. The processes of contractures which occur in the capsule, ligaments, muscles, fascia, and skin increase the contracture and form a decided hindrance to correction. As to the different methods of extension, surgical monographs must be consulted. Here it will only be stated that the patients in Hoffa's Clinic, during the entire time of extension, are out of bed and move about. When the leg is completely extended, the apparatus is worn, according to the severity of the case, for three months to six months and even longer to prevent relapses.

The employment of the rail-brace apparatus, according to the published reports, has given excellent results, so that this method of treatment can be warmly recommended. 


\title{
MORBUS MACULOSUS WERLHOFII (PURPURA)
}

\author{
(PURPURA SIMPLEX, HEMORRHAGICA, RHEUMATICA SEU PELIOSIS \\ RHEUMATICA SCHOENLEINII)
}

\section{DEFINITION}

Purpura, on account of its obvious manifestations, has been known to physicians for a very long time. Superficial hemorrhages arising from every possible cause have hitherto been grouped together. Hence affections in which, according to the present state of our knowledge, the purpura forms only an auxiliary or symptomatic phenomenon (for example, diseases with jaundice and hemorrhages) have been regarded as independent diseases. From the mass of diseases in which purpura occurs upon the external skin, Werlhof, in the year 1775 , isolated a distinct clinical picture which he designated purpura hemorrhagica, and which was subsequently named after him.

Later, other divisions were made in the group of hemorrhagic diseases. Purpura simplex was isolated as an independent affection, and a variety called purpura urticans was also described.

Finally, upon the authority of Schönlein, the term peliosis rheumatica came into use. - It must be admitted that such a division was justified at that period; it was necessary at a time when the conception of disease entities rested chiefly on a subtle differentiation of clinical symptoms. From this point of view great importance was attached to a single symptom, as is shown by the fact that the term "peliosis rheumatica" rests solely on the observation that, in some cases of purpura, an affection of the joints is occasionally noted. The fact was lost sight of that the latter often occurred in combination with all hemorrhagic diseases, not only in those whose clinical pictures belong strictly to purpura, but also in the course of scurvy and hemophilia. Of course the old divisions of the different forms of purpura are no longer justified. As a matter of fact, in the last twenty-five years a change in the conception of purpura has taken place, and there is now a tendency to regard all purpuric diseases as related, and the individual forms, previously supposed to be independent, are regarded from a common standpoint. Upon the basis of my own experience, which extends over many years and includes exceedingly numerous observations, I believe strictly and absolutely that the individual purpuric diseases are not essentially different, but are due to the same cause, and only vary in degree; that is, the "varieties" depend upon the intensity of the affection.

Occasionally these differences are clinically presented in such a manner that the inexperienced believes he has before him entirely different clinical pictures that have nothing in common with one another. This is a difficult field for polemics, and convincing arguments are useless if another is fully convinced to the contrary. The only method leading to conviction is minutely, carefully, and without prejudice to weigh all the facts bearing upon the question. It will then become manifest that all these variations of the disease form indefinable transitional stages which merge into each other, and only by great circumspection can differentiations be made. The standard of identity 
accepted by me has been exceeded by other authors (e. g., Schwimmer and Scheby-Buch) who are not satisfied with the inclusion of the various forms of purpura, but desire also to include scurvy in this clinical group; other authors even add hemophilia.

Apart from the facts demonstrated by bacteriology, which it is hoped will be more certainly confirmed in the near future, purpura is an affection due to the action of unknown deleterious agents, occurring sporadically and showing a transitory tendency to different varieties of hemorrhage. In contrast to hemophilia there is no congenital or hereditary factor, and, unlike scurvy, there is no tendency to epidemiologic and endemiologic distribution, and the hemorrhages are not associated with severe constitutional manifestations. Scurvy is distinguished by the fact that it not only occurs sporadically but also epidemically or endemically. More characteristic, however, is its dependence upon external conditions; it is almost invariably the expression of severe nutritive disturbance produced by obscure diseases or by the long continuance of insufficient or improper food.

Morbus maculosus Werlhofii differs essentially from hemophilia in the fact that it unquestionably belongs to the acquired diseases, in which the factor of heredity is never operative. It has no congenital characteristics, such as are so peculiar to hemophilia.

This is also a distinguishing factor between scurvy and hemophilia. Unlike the scorbutic patient, the bleeder is frequently well nourished and strong. Except in his tendency to spontaneous hemorrhage he may be looked upon as quite normal. In the comparison of hemophilia with related diseases in the group of the hemorrhagic diatheses, one peculiarity of hemophilia is particularly prominent; it is not a pathologic process, but a permanent condition which manifests itself sometimes in consequence of known, at other times of unknown, causes.

In accordance with these views, we shall consider the purpuric affections together, but we shall not forget the older clinical divisions; we shall bear in mind that between the individual forms of the disease transitional stages occur, and that the barriers between them are artificial.

The conception of purpura as a particular chapter of special pathology presupposes the exclusion of all the affections which are combined with hemorrhages of the skin, which hemorrhages, however, prove nothing essential or definite, but are only a single symptom in the entire picture. Among such affections are: Hemorrhagic variola, typhoid, acute atrophy of the liver, phosphorus poisoning, sepsis, ulcerative endocarditis, pernicious anemia, leukemia, yellow fever, icterus gravis, snake-bite poisoning, etc.

Purpura should be differentiated only when the hemorrhages exist alone or dominate the cntire clinical picture. Of course, in investigating the disease, a sequel, such as the resulting anemia, is of minor importance.

\section{ETIOLOGY}

In most cases it is impossible to determine any immediate cause for the disease; the affection appears in typical cases spontaneously. Cases of this kind have led to the belief that scurvy, which may arise under similar condi- 
tions, is identical with purpura, so that the latter has been designated as the milder and more acute form of scurvy. This view, as we have shown, is incorrect, even though there is occasionally a mild affection of the gums in Werlhof's disease. Often the symptoms of purpura hemorrhagica appear during convalescence from severe infectious diseases, particularly after enteric fever and malaria.

In rachitic children, as well as during pregnancy and in labor, the affection has also been noted. Isolated reports have been given of toxic forms of purpura. The affection has also been observed after the inhalation of poisonous gases.

In the ecchymoses which are distributed so numerously over the entire body it is often possible to demonstrate, in the substance which occludes the capillary vessel, the cause of the entire affection. In the multiple capillary hemorrhages of septic affections, which show to the naked eye white centers in the midst of the effused blood, occlusion of the capillaries and capillary veins with emboli of micrococci may frequently be determined. So in hemorrhagic smallpox bacteria are found in the hemorrhagic and purulent foci of the external skin and in the internal organs, the spleen, kidneys and lungs. In the retinal hemorrhages of metastatic ophthalmia, I was also able to find masses of bacteria in the occluded vessel, centrally or removed from the foci, whereas in other cases which were evidently of septic nature, this proof could not be demonstrated. This attempt to find thrombi in the vessels has failed entirely in many cases of hemorrhagic exanthems. In many other diseases and intoxications the most important clinical symptoms of which consist of hemorrhagic conditions (cholera, plague, yellow fever, anthrax, poisoning from snake-bite, petechial typhus, etc.) occlusion of the capillaries in the surroundings or in the center of the ecchymoses have not been manifest, and we have been forced to the conclusion that zymotic substances, ferment bodies, ptomains or toxins produce the hemorrhagic diathesis.

How this occurs, whether they alter the blood directly and thus cause occlusion of the capillaries, or whether they affect the walls of the vessels in their structure, or in their functions as muscular and nervous apparatus, we do not know.

Whether in morbus maculosus, that is, purpura hemorrhagica, microörganisms or their toxins circulate in the blood, exerting a deleterious effect upon it or upon the membranes of the vessel, and lead to the multiple ecchymoses which are typical of this disease, has not been ascertained with certainty, although such a view is likely. The facts at hand are not generally accepted.

Few authors have found well-characterized microörganisms as the cause of purpura. Ajello, who in a case of purpura demonstrated methemoglobin in the spectrum of the blood, assumes that purpura hemorrhagica may arise by autointoxication from the intestinal tract as the result of absorbed products of proteid decomposition; Schwab also believes an action of toxins to be the cause of this disease. In other forms of purpura which arise in connection with infectious diseases, the blood has been examined for bacteria, only in part with a positive result. Earlier authors, such as Batemann and Grisolle, have looked upon certain forms of purpura as infectious and contagious; and, 
in a number of cases of purpura, as is evident from later investigations, the proof of bacillary disease has been shown. Bacilli were found by Klebs, Ceci, Reher, Demme, Vessalle, Gendre, Ginnard, Simon-Legrain, Jones, Tizzoni, Giovannini, Kolb-Petrone, Babes and Letzerich; Hanot and Luzet, Widal and Thérèse found streptococci; Lebreton and I each found staphylococci in one case; negative findings on the other hand have been mentioned by Marfan, Legendre, Demys and others.

The opinion, especially maintained by Petrone, that purpura hemorrhagica in an infectious disease, Letzerich believes he has proven to be correct by bacteriologic investigation. In the case of a woman, aged twenty-five, who suffered from a prolonged attack of this disease, who was, however, cured, he found, in 1884, small, glistening, round granules in the blood. By culture these proved to be the spores of a bacillus which he described and depicted minutely as bacillus purpuræ. With several generations of this culture a large number of rabbits were infected by injection into the abdominal cavity, and always with a positive result; the animals showed a short time afterward, especially in the ears, circumscribed dilatation of the capillaries with succeeding hemorrhage, injection of the gums, etc.; if the animals were killed, similar hemorrhages and vascular dilatations were found in various portions of the body. The microscopic investigation showed in the blood-vessels in many areas the previously described bacilli, that is, their spores. These were found most constantly, and were most numerous in the enlarged liver.

Letzerich in this connection points to the frequent enlargement of the liver in the human affection. This is not to be construed as a frequent occurrence, however; in the organs of the experimental animals he found very marked accumulations of erythrocytes, and stasis in the capillaries due to this. Further, in the areas of the dichotomous branching of the smallest vessels hyaline plugs developed by the action of the chemical poison produced by the bacteria upon the albumin of the blood. To these vascular plugs he refers on the one hand the hemorrhages into the organs, and on the other hand the decided circulatory disturbance. The bacillus enters the human body, probably through the mucous membrane of the oral and pharyngeal cavities.

Letzerich believes these investigations demonstrate with certainty that purpura hemorrhagica is a chronic infectious disease, and points to its analogy with syphilis and malaria. It is noteworthy that three years after these primary researches, and, as he states, in consequence of them, Letzerich himself was attacked by a very prolonged purpura, running its course with marked enlargement of the liver, and from his blood the previously described bacillus was cultivated.

The bacillus described by Letzerich has some similarity to the bacillus anthracis, particularly in regard to the conditions of its growth. In Koch's stab cultures both formed more or less irregular colonies (flocculi), the middle point consisting of net-like threads which, toward the border, ran off in sheathlike bundles. They differ decidedly, however, by their size and the form of the spores. Although in both bacilli a segmentation of the apparent threads occurs in various lengths, those of purpura are much smaller in their length and breadth. Added to this the spores of Letzerich's bacillus, in comparison 
with the large oval structures of the bacillus anthracis, are very small and perfectly round. The bacillus purpuræ liquefies gelatin with great difficultyscarcely at all -and only at high temperatures $\left(25^{\circ}\right.$ to $30^{\circ} \mathrm{C}$.) in the surrounding of the flocculi (colonies).

In the petechiæ of man and of the experimental animals a sheath-like arrangement of the bacilli, sometimes with segmentation, is noted in foci; these frequently are found equidistant from each other, not lying closely together, and are spores beginning to develop.

The surface of the stab in Koch's nutritive gelatin shows flat crescentic grooves which are only recognizable upon close observation.

The chemical differences must also be mentioned. The bacillus purpuræ does not form so toxic a substance (toxin) as the bacillus anthracis, but only a feebly acting ptomain corresponding with the slight evening rises in temperature that now and then occur. Pure purpura differs in this respect from the acute forms of purpura (petechiæ) usually observed in the course of the infectious diseases, as these owe their origin to other microörganisms.

Even with cultures of the third relapse, Letzerich produced purpura in rabbits. In sections, through small petechiæ in the transverse section of the capillary net, and also where dichotomous branches pass into the lumen of the same, dense proliferations of purpura bacilli were readily recognized by simple methylene-violet staining.

The bacillus purpuræ is not found in all the forms of its development in all of the petechiæ. Toward the end of the disease, or during the relapse, only clumps of free spores and individual bacilli are observed. Examination of numerous petechiæ is then necessary to find the cycle of development of the microörganism as described. In the relapses of the disease, particularly, we frequently note emboli of spores which, upon superficial observation, may be looked upon as micrococei emboli, but, upon closer study, merely from the circumstance that they are embedded in gelatinous plugs, their development from threads and rods can be determined.

Lockwood also believes purpura to be of an infectious nature, and the bacillus described by Letzerich to be the carrier of the infection and pathogenic agent. According to this author, the very acute development and the course, which is similar to that of an infectious disease, favor the view of an infectious, development, and he quoted 17 cases from literature. Of these 13 died in from forty-seven hours to twenty-one days. $\mathrm{He}$ is also inclined to look upon purpura simplex and purpura rheumatica as due to the same infection, but showing a different intensity. Cases of purpura which ran a fatal course in seven hours have never to my knowledge been published by others, and under no circumstances can these be included among the cases of pure purpura; the most severe case of fulminant purpura that I ever saw (to be detailed later) terminated fatally in two days.

H. Neumann cites cases of hemorrhagic diathesis of the newborn. In the first case, besides the staphylococcus pyogenes aureus as in former cases, the bacillus pyocyaneus $\beta$ was found. He does not admit a direct relation of the latter to the hemorrhagic diathesis, but attributed the diathesis to syphilis which was present. 
In another case (with melena) the bacillus lactis aërogenes was found, but to this he ascribes no pathologic importance.

Lebreton describes in a young girl a case of fatal purpura which developed very acutely after great fright. Large confluent ecchymoses appeared, from the blood of which, upon culture, the staphylococcus albus and the staphylococcus aureus were obtained in mixture. The disease was therefore looked upon as infectious purpura.

Wikner also found the staphylococcus pyogenes albus in a case of morbus maculosus Werlhofii.

In a number of cases of purpura I have examined small areas of the skin covered with petechiæ which were excised during life, and I have never been able to determine the presence of microbes.

Later investigations and reports of this malady are not at hand; in the last four volumes of the Virchow-Hirsch Year-Books, I have not observed a single report of purpura in man. In this article publications up to the year 1898 have been referred to.

We now come to another series of reports which had their origin partly in anatomical studies of the vessels in the course of the hemorrhage, and partly in certain experiments.

Silbermann, who conducted one series of experiments, started from the well-known researches of Armin Koèhler, who, in his work at Dorpat upon thrombosis and its relations to the fibrin ferment, mentions a series of experiments in dogs, in which, by infusion of blood very rich in ferment, a disease resembling Henoch's purpura (see below) was produced, inasmuch as, in a short time after the transfusion, multiple capillary ecchymoses were noted in the subcutaneous cellular tissue, and hematemesis, hemorrhagic diarrhea, and intestinal colic occurred. The fulminant, and almost invariably fatal, course of the pathologic process produced by Koehler, on account of the great amount of ferment contained in the injected blood, caused Silbermann to choose a method in which the animals either did not die or did not succumb so rapidly, and in which the purpuric spots appeared more numerously and were distributed over the entire skin. He tried this plan: The dogs, prior to the ferment intoxication, received small doses of pyrogallic acid ( 0.05 per kilogram of weight of the dog), by which only a moderate stasis in the veins and capillaries occurred.

This property of pyrogallic acid in small amounts depends upon a very slight damage to the blood, a slight "shadow formation" and fragmentation of the erythrocytes, by reason of which the circulatory disturbance occurs.

In a number of dogs, after stasis of the veins and capillaries of the entire circulation had been produced by suitable doses of pyrogallic acid, Koehler's experiments were repeated, with the expectation that the cutaneous capillaries, which, like all other capillaries of the body, were under high pressure with decided strain on their walls, would become more permeable by the ferment blood. As a result of these experiments it was shown that in the animals thus prepared-by injections of ferment blood-thromboses and hyaline vascular changes could be invariably produced in the internal organs, less constantly: in the skin. The consequences of these vascular changes were multiple hemorrhages into the internal organs and partially also into the skin. 
Since a rupture of the vessels could not be found microscopically (the proof of this can only be certainly demonstrated with great difficulty) these hemorrhages were assumed by Silbermann to be due to diapedesis, and this to distention of the vessel walls. The vascular changes as well as the extravasations are themselves consequences, not causes, of the circulatory disturbance, for alteration of the vessel wall never occurs alone but is always observed in combination with thrombosis, while the opposite is very often the case. The frequent closure of the capillary area by thrombosis may be explained by the lessened rapidity of circulation thercin, a factor which greatly favors the occurrence of coagulation. The stasis which occurs in mild cases, and is followed by thrombosis in the more severe ones, damages the vascular wall, however, so that it makes it permeable to the passage of blood. Indeed this stasis may give rise to fatty degeneration and even necrosis of the vascular cells.

Since "purpura" with swelling of the joints, hematemesis, hemorrhagic diarrhea and intestinal colic had been experimentally produced in animals by means of a dyscrasic blood the question arose whether human purpura-no matter in what form-might also be explained as a primary disease of the blood. Silbermann believes that this question must be answered in the affirmative, for the reason that, apart from certain important blood findings, the. essential connection of the pathologic phenomena can only be uniformly explained upon the basis of a primary disease of the blood.

The hemorrhages into the skin and into the gastro-intestinal tract, the hemorrhagic vomiting, the colic, and the swelling of the joints, which occur in severe cases of purpura, Silbermann explains as due to a primary blood alteration which leads to a slowing of circulation, stasis and formation of thrombi. Upon the basis of these circulatory disturbances congestive hemorrhages occur, and also hemorrhages which follow as the result of the occlusion of vessels, and give rise to necrosis of the tissues. These stases and thrombi damage not only the gastro-intestinal canal, but also the tissue of the liver, the kidneys and the myocardium, and may cause fatty degeneration of the cells and necrobiosis. All of these processes were observed lately by Tizzoni and Giovannini in the organs of a girl suecumbing to severe purpura. There was occlusion of the vessels which had produced hemorrhage and necrosis of tissue, and also caused the vascular changes previously observed in man and animal attacked by purpura. Silbermann regards it as certain that this degeneration of the walls of the vessels is secondary, because, in his experiments, all stages of the obliteration of vessels have been followed, and the hyaline deposits have been shown to be results of this obliteration.

In literature similar views in regard to the nature of purpura are constantly met with. Thus Green, du Castel, Dusch,' Mackenzie, Krauss and others believe that there are alterations of the blood in certain forms of purpura. Leloir, who has also observed stasis in the vessels, differentiates a purpura "par modification des vaisseaux" and a purpura "par modification du sang." Riehl and $\nabla$. Kogerer, on the other hand, believe the alteration in the vessels to be the primary condition; the change in the blood, i. e., the thromboses, the secondary. The latter says, in his article regarding the development of cutaneous hemorrhages: "On the whole there can be no doubt that the thrombi 
which have been found are the direct cause of the hemorrhage. The thrombi themselves are due to the combined action of local and general causes; hence vascular changes, as is evident from the constancy of the finding, play the main rôle even if they do not form a conditio sine quâ non." The view of $\mathrm{v}$. Kogerer that the thrombi are due to general and local causes is also the opinion of Silbermann who, however, looks upon the alteration of the blood as the general cause, and the pathologic change in the blood current of the capillaries (due to ferment blood) as the local cause. On account of these constant anatomic findings $v$. Kogerer calls purpura a primary disease of the vessels, while Silbermann believes these changes to be secondary. Other observers, for example, Leloir, have not noted the constant occurrence of disease of the vessels. He believes the occlusion of the vessels to be embolic, while v. Kogerer and Silbermann declare this to be thrombotic.

$\nabla$. Kogerer defines the result of his investigations in purpura in the following scheme:

(a) Vascular disease,

(b) Thromboses,

(c) Extravasations of blood,

(d) Pigmentation.

While Silbermann comes to the following conclusions:

(a) Thromboses,

(b) Vascular disease,

(c) Extravasations of blood,

(d) Pigmentation.

As will be noted these authors agree that the disease of the vessels is the underlying cause of the hemorrhages, but Silbermann looks upon stasis and thrombus-formation (in the small veins and capillaries) as the primary condition which leads to disease of the vessels; while v. Kogerer believes thrombusformation to arise as the result of the disease of the vessels. The latter condition in either case causes hemorrhage.

v. Kogerer examined 13 cases which ran their course with cutaneous hemorrhages; in these he excised particles of the skin, and studied microscopically the changes in the different layers of the cutis and subcutaneous cellular tissue. The diseases represented were scurvy, morbus maculosus, tuberculosis pulmonum, carcinoma ventriculi, marasmus senilis, peliosis rheumatica, sepsis, vitium cordis, etc. In all cases in which careful search was made thrombi were found. These were mostly situated in the small venous trunks, but were also found in the small arteries. Besides this many minute arteries and capillaries were filled with fibrin coagula and blood-corpuscles, and it was impossible in some cases to decide whether the condition was one of actual thrombosis or only a vessel surcharged with blood (!). The thromboses were occasionally numerous, at other times more scant, and frequently repeated search was necessary to find them. In some cases the degeneration of the vascular wall was conspicuous.

Riehl, even prior to $\mathrm{v}$. Kogerer, invariably found changes in the blood- 
vessels of the cutis and of the subcutaneous connective tissue in scurvy, morbus maculosus, and purpura rheumatica, as well as in the purpura of cachectic individuals.

In cases of scurvy and morbus maculosus, diffuse endarteritis and thickening of all the coats of the vessels, hyaline degeneration, and partial fatty degeneration of the same, narrowing of the lumen and proliferation of the endothelium, could (he said) be invariably demonstrated. Besides, it was found that in some areas the connective tissue surrounding the ressels and the fatty tissue showed decided round-cell infiltration.

In the hemorrhagic areas fresh and more or less altered extravasations of blood were observed, i. e., coarsely granular or flaky blood pigment, embedded between the bundles of connective tissue and in the cell infiltrations. On the other hand, Riehl could never detect pigmented leukocytes, as in the case of Addison's disease, etc. In both processes the cross-striated muscles which were examined showed fatty degeneration.

In purpura rheumatica and purpura cachecticorum as well as in a case of hemorrhage into the skin occurring after sepsis, similar changes could be determined, but the vascular alterations and infiltrations of the perivascular connective tissue were less marked. In all instances the reticular stratum of the cutis showed the greatest implication of the large arteries; next to these the vessels of the adipose tissue, and least marked the vascular layers of the subpapillary tissues.

In all cases examined by v. Kogerer the vascular changes which have been described could be demonstrated, and he therefore concludes their certain connection with the hemorrhages. He assumes further that the hemorrhage results per rhexin. But in order that the pathologically changed layers of the vessels shall rupture during life, other local conditions, he believes, must be simultaneously active. These, in his opinion, consist in thromboses of the smallest arteries, and he refers to the hemorrhages in the centers of which, in septic endocarditis and similar processes, emboli of micrococci are found (Weigert, Litten and others).

Besides the early investigations of Hayem and Stroganow, which have been referred to, the more recent ones of Leloir and Riehl must be mentioned. They confirm the finding of vascular changes in the reticular stratum of the cutis and in other areas of the body, the changes consisting of thickening, hyaline degeneration, fatty degeneration of the endothelium, and formation of thrombi. v. Recklinghausen also finds these causes of hemorrhage; I shall quote two of his remarks relating to this condition (Handbook of General Pathology of the Circulation and of Nutrition): "As regards many cases of multiple capillary hemorrhage, formerly included in the eategory of the hemorrhagic diathesis or of blood dissolution, etc., we are able to-day to prove that they occur mechanically, by occlusion of the smallest vessels with indifferent material, or with one of specially septic constitution," and further: "Occasionally, as the result of obstruction of a vein, capillary hemorrhages also appear in the area of stasis; this is, however, quite rare, and we may therefore conclude that here complications, for example, senile vascular changes, are operative." 
Despite the report that by anatomical investigations vascular changes have been found in the ecchymoses of neighboring capillaries as well as in the smallest arterial and venous vessels, in my opinion no clinician will admit these to be a constant etiologic factor in purpura. In order to reject absolutely the theory that the described vascular changes are the cause of such processes, one must have witnessed how the normal skin of the entire body may be covered by hemorrhages in the course of a few hours, so that the entire surface appears spotted like the skin of a tiger or a leopard. I refer here to the illustration I gave in Nothnagel's "Special Pathology and Therapy," Part III, vol. viii. Such a possibility must be constantly borne in mind. We shall discard absolutely the assumption of a vascular disease distributed over the entire cutaneous surface, if we consider the appearance and disappearance of the affection in the briefest time, the recurrence and repeated recurrence after varying intervals, and the final complete recovery from the disease. That the vessels in the hyperemic areas present the phenomenon of stasis is self-evident; the exit of blood per diapedesin is itself the final and most extreme phenomenon and expression of each extensive stasis. But a purpura hemorrhagica widely distributed over the entire body, appearing acutely, and sometimes involving the mucous membranes and the serous membranes, can never be due to a hyaline vascular degeneration. How could this arise so suddenly and disappear so rapidly? I admit the vascular degeneration as a cause in certain cases running a severe and fatal course, particularly the forms which run a chronic course, in which the cutaneous hemorrhages represent one of the secondary symptoms of the disease, as, for instance, in senile marasmus, peliosis cachecticorum or in pulmonary tuberculosis. But the mild cases (ordinary purpura) which run a more or less rapid course, repeatedly appearing and disappearing again, certainly do not depend upon stasis and hyaline vascular degeneration but on internal causes entirely unknown to us at the present time.

The many microscopic investigations which I have made in cutaneous hemorrhages in all forms of the hemorrhagic diathesis, and particularly my numerous investigations of the blood-vessels of the eye (the optic nerve, the choroid and the retina) in pernicious anemia, leukemia, scurvy, diabetes and sepsis, I must admit have given results which are by no means satisfactory. In many hemorrhages of the retina in these diseases, in spite of the most minute and careful examination of numerous sections so thin that they permitted the use of an immersion lens, I have not succeeded in detecting vascular changes in the arterioles or capillaries supplying them. The conducting vessel was quite intact, and each blood-corpuscle contained in it, as well as the nuclei of the walls, and the patent lumen, could be distinctly recognized almost up to the bleeding point, where frequently a dichotomous division occurred. The two branches given off could be seen for a short distance and were quite intact until they disappeared in the hemorrhage. The eye of the investigator, no matter how practised, is unable to see in the midst of the hemorrhage which completely covers the arteriole or capillary what has become of the vessel, and especially whether the hemorrhage has occurred per diapedesin or per rhexin. Hence I must absolutely reject this theory, and can only compliment those observers who were able to demonstrate a vascular change 
in "every" case. At this point, I wish to refer to my later investigations of the vascular changes in the retinitis of Bright's disease, which are soon to be published.

The conditions are not very dissimilar in hemorrhage, occurring as the result of general septic disease, although they really do not belong to the same category. There is no doubt that in cases of sepsis and malignant endocarditis, in the midst of cutaneous hemorrhages, occasionally a white center is seen which is analogous to the same processes in the retina. Although I have sometimes succeeded in recognizing these white centers as small infarcted blood-vessels filled with micrococci, this finding is not constant, and cannot be proved to be a process which occurs during life; under no circumstances can conclusions be drawn from this to explain the multiple cutaneous hemorrhages in the various forms of purpura.

How far an infection may be assumed to cause the disease in these latter cases must remain an open question until it is proven whether, and to what extent, the bacillus purpuræ or some other pathogenic microbe is a constant finding in the blood of patients suffering from morbus maculosus. If later investigations should substantiate this, the view would also be permissible that the toxins or ptomains circulating in the blood lead to an extensive stasis formation in the capillaries and small veins, or that they directly alter the blood, thus causing occlusion of the capillaries, or, finally, that they affect the vascular walls in their structure or in the functions of their muscular and nervous apparatus so that, for example, a stasis as the result of paralytic dilatation of the smallest vessels might take place.

That the severest cases of this disease, designated as purpura fulminans, terminating fatally in the briefest time, are of an infectious character, and are probably due to the entrance of pathogenic microörganisms which have a very deleterious effect, I believe to be true beyond doubt, although, unfortunately, this has not as yet been proven.

As a rule the disease is not frequent; females, according to reports, appear to be somewhat more predisposed than males. An age limit does not exist, but at middle life attacks appear to be most numerous. The aged and nurslings are very rarely affected. Nevertheless, the report that prior to the fifth year of life scarcely any cases occur is incorrect. I have certainly seen quite a number of cases which occurred between infancy and the fifth or sixth year. These cases were either pure "purpura simplex" or "purpura hemorrhagica," or such forms as are complicated with disease of the joints (peliosis rheumatica), or, lastly, forms in which not only cutaneous hemorrhage but intestinal symptoms, particularly vomiting, intestinal colic, and watery or hemorrhagic diarrhea, are prominent. In quite a large number of cases which occurred in rachitic children in the second, third and fourth years of life the only symptom was attacks of cutaneous hemorrhage, and after repeated relapses these children made a complete recovery. Here also the female sex was in the majority.

Immediate causes can be determined only in the rarest cases. The disease seems to appear "spontaneously and primarily," and either to exhaust itself 
in one attack or to extend through several relapses. In isolated cases the duration of the attack may be many months or even years.

Chilling or wetting of the body, damp dwellings and insufficient food are cited as causes, but without sufficient reason. Since unfavorable circumstances of life and unhygienic surroundings reduce the bodily resistance and increase the predisposition to all diseases, this is naturally the case also in the homorrhagic diathesis. We see poorly nourished individuals with poor constitutions forming by far the great contingent of sufferers from this disease, but we also find the disease in millionaires who live in Iuxurious palaces, and who can command all the advantages of life and the best of care.

The affection always occurs sporadically and as an isolated disease, never, as in the case of scurvy with which it is often confused, showing an endemic or epidemic distribution.

In isolated cases intoxication with marsh gas has been reported, but probably this is erroneous. On the other hand, it appears to be absolutely proven that in convalescents from enteric fever and malaria, or after these diseases have run a prolonged course, purpura sometimes follows. I have also observed purpura as a sequel of scarlatina and measles, as well as in the late course of diabetes mellitus and in pulmonary phthisis. Bright's disease also belongs to those affections in the course of which cutaneous hemorrhages occasionally occur. Evidently the conditions are here somewhat more complicated. I should like to refer to Mathieu's view that the purpura which is seen quite frequently in Bright's disease is of vital importance, and develops under the same conditions as uremia. We must recognize the absolute untenability of this assumption. Purpura arises in all forms of nephritis, but especially in interstitial nephritis; during the course of the latter it often recurs (a few spots or a great many), and again disappears without being of decisive importance in prognosis.

I shall revert later to the views of Bamberger, a very careful observer, but here I may mention my own opinion based upon numerous observations. Purpura and renal affections appear to me to bear double and varying relationship. The evidence of this is the fact that after long continued, frequently relapsing purpura, we often observe albuminuria (usually not exceeding 0.1 per cent.) with or without a very scant number of casts. After a shorter or longer duration these signs gradually disappear without developing into chronic nephritis. In a second group of cases the relation is unquestionably the opposite, inasmuch as with a contracted kidney, repeated attacks of simple or hemorrhagic purpura occur. But in such cases the prognosis does not assume a more serious character.

Dohrn once observed a child born of a woman suffering from purpura, the child also being attacked by the disease. If this was not really a case of hemorrhagic diathesis as a result of septic infection, the conclusion is justified that the vascular system (blood and vessels) of mother and child suffered from the same deleterious influences.

In conclusion, we must mention that in individual cases as an immediate consequence of severe nervous shock, pain, fright and fear, severe cases of purpura arise. In this connection I shall mention the case of Lebreton, who 
saw purpura in a young girl after great fright, appearing very acutely and rapidly running a fatal course. Upon the skin, large, confluent hemorrhagic spots were found.

Bobrizki saw in a girl aged twelve, as the result of severe nervous shock (an attempted rape), an immediate attack of morbus maculosus. The same observer noted a second case in a boy aged ten, who, in consequence of a fire, was severely frightened and was immediately attacked by the same disease.

Bobrizki is of the opinion that the irritation of the nervous centers produced by fright caused a paralysis of the vasomotor nerves which permitted the extravasation of blood per diapedesin.

\section{GENERAL CLINICAL PICTURE}

By purpura we understand an affection which appears spontaneously, its chief characteristic consisting in transitory hemorrhages of the external skin, the serous and mucous membranes, as well as hemorrhage into the parenchyma of the internal organs.

Under purpura we include only such hemorrhages as denote the character of the disease and stamp it as an independent affection, while the purely symptomatic extravasations of blood which occasionally occur in the course of cachectic or febrile affections (as, for example, in the course of sepsis or acute articular rheumatism) are to be left entirely out of consideration. The first form of the disease, in which bleeding occurs exclusively in the skin, is designated "purpura simplex." When bleeding occurs not only in the skin but in the mucous membranes, the serous membranes, and the internal (parenchymatous) organs it is designated purpura hemorrhagica. Finally, when the hemorrhages run their course with pain and swelling in the joints, the disease is called peliosis or purpura rheumatica. This terminology might be extended much further. According to the etiology or clinical symptoms, we might speak of peliosis or purpura gonorrhoica or of a purpura abdominalis or dyspeptica (see below). But, as has already been stated, all these apparently different forms depend upon a general cause, the so-called hemorrhagic diathesis, which is probably due to a microparasitic pathogenic agent (up to the present time entirely unknown) and which finds expression in forms that are only clinically different and merge into each other in many ways. Altogether they form one and the same essential hemorrhagic disease of varying grade and varying intensity. But it must be always remembered that, besides the hemorrhagic symptoms, no other primary affection is present in the clinical picture. Purpura simplex is to be looked upon as the mildest form of the disease; we can, however, never premise with certainty whether it will remain mild during the entire course of the disease, or will change gradually into severe purpura hemorrhagica, or whether disease of the joints or other complications may eventually appear. For this reason we cannot look upon the various forms of the hemorrhagic diathesis (mentioned by various authors) as distinct. They are all aspects of one disease, produced in each individual case by separate conditions. This disease we call morbus maculosus.

In describing briefly the history and development of this disease, we need only follow a case step by step to demonstrate the difficulties which arise in 
an attempt to classify these hemorrhagic diseases in accordance with the usual reports and designations.

For example, we will take a case of purpura with joint affection and intestinal hemorrhage. Purpura with joint affection, provided it is not also complicated by intestinal hemorrhage, would ordinarily be designated as peliosis rheumatica. As most authors mention neither internal hemorrhage in peliosis rheumatica nor joint affections in purpura hemorrhagica, embarrassment would surely ensue as to the category in which to place the case if the teachings of these authors were followed. But it is absolutely certain that such a case should be included in the great group of the hemorrhagic diatheses.

According to the classic descriptions of the clinicians of the middle of the previous century, it was generally accepted that purpura simplex, purpura hemorrhagica, and peliosis rheumatica were to be viewed as entirely different diseases, that in peliosis rheumatica no internal hemorrhages occurred, and further, that in the different forms of purpura no joint affection was observed.

I need only mention here the revered names of Rayer, Willan, Wunderlich, Schönlein, Hebra and Cannstatt. The last, in complete unanimity with Wunderlich and Hebra, teaches that in purpura hemorrhagica, in contrast to peliosis rheumatica, no joint affection occurs, and, moreover, that the absence of joint affection is an important diagnostic point in the differentiation between purpura and scurvy.

A differential sign between purpura simplex and purpura hemorrhagica, according to the previously named authors, is the presence of large ecchymoses upon the external skin and the occurrence of blecding from the mucous membrane and in the internal organs in the latter affection.

Accurate observation of typical cases among a great number of patients has shown the unreliability of all of these views, and has demonstrated especially that the points in the history of the individual case regarding the form, extent and seat of the hemorrhages, as well as in regard to the implication of the joints, hitherto held to be diagnostic, can no longer be so regarded. As we shall have to consider more minutely these important questions which dominate the situation, I will only, at this point, demonstrate by some typical examples to what confusion it would lead if, upon the basis of such purely external symptoms and signs, the cases of hemorrhagic diathesis were to be classified. I shall now show that other complications may occur in hemorrhagic diseases by which the external picture of the affection may be apparently changed, while its essential nature remains the same.

I shall first mention two cases that came under my own observation: In the first there were recurring paroxysms of petechiæ, large ecchymoses and suggillations (particularly in the extremities) with painful arthritic swellings, which passed rapidly away; besides these, there were severe gastric disturbances with bilious vomiting, attacks of colic and recurrent hemorrhagic diarrhea. The gums were swollen and bled readily, the teeth were loosened.

With what were we dealing? Without practical experience, it would be very difficult to recognize the condition among the earlier classifications, and we should lose our way in the devious paths of complicated and apparently non-related symptom-complexes, Purpura, acute rheumatic fever, scurvy, 
peliosis, dysentery, all these would be considered, and formerly would unquestionably have been diagnosticated.

Yet we know now, from careful investigations, that all of these symptoms, apparently so different, may run side by side within the conception of a severe purpura.

Which qualifying adjective we attach to the purpura in this case- "rheumatica," "intestinalis," "hemorrhagica," or perhaps, on account of the implication of the gums, "scorbutica"-is quite immaterial; now one, now another symptom-group may assume greater prominence among the phenomena. As we shall see, however, all of the symptoms which have been mentioned may, and occasionally do, appear in purpura.

The second case also is a characteristic example of the diagnostic difficulties which may arise under some circumstances. Besides the extensive cutaneous hemorrhages which were of paramount interest, there were repeated multiple and painful joint swellings, appearing and disappearing suddenly, and relapses which almost always occurred if the patient attempted to leave the bed. Symptoms on the part of the digestive apparatus, colic, bilious and hemorrhagic vomiting, and hemorrhagic diarrhea were also present. There can be no doubt that the case was one of so-called purpura rheumatica.

Cases with extensive cutaneous hemorrhages and multiple arthritic affections are often described in literature as "anomalous" articular rheumatism. Occasionally these are combined with inflammation of the serous membranes, particularly with pericardial exudates presumably of a hemorrhagic nature. A conspicuous feature of these cases is that the joint affection runs a more rapid and more favorable course than it ordinarily does in pure acute articular rheumatism. As similar cases originated during the period when the favorable action of salicylic acid and its salts upon diseased joints was unknown, it must be suspected that the authors in question failed to recognize the much more rapid and more favorable course of the arthritic affections in purpura. This should have attracted their attention to the fact that they were not dealing with genuine articular rheumatism "with an atypical course," but with a complication of purpura.

Another complication of purpura which met with tardy recognition exhibits phenomena on the part of the stomach and intestines, such as intense colicky attacks with vomiting and hemorrhagic diarrhea.

The simplest and mildest forms of purpura are those in which isolated hemorrhagic spots frequently appear in the form of minute petechiæ upon the surface of the skin and are the only clinical symptom. These sometimes occur very suddenly without being preceded by prodromes. More often this eruption is accompanied by gastric disturbance, loss of appetite, epigastric pressure, lassitude, vomiting and slight fever. The affection sometimes lasts but a few hours, sometimes for a few days, and, exceptionally, from one to two weeks. The cutaneous hemorrhages usually present themselves in the form of numerous small and large dusky red or bluish red circular spots; they appear particularly upon the lower legs and feet, often also upon the abdomen and the arms, and frequently exhaust themselves in a single crop. In fresh cases, they do not disappear upon pressure with the finger, and they 
differ from flea-bites by the absence of the circumscribed circular areola, which is only seen in acute cases and soon disappears. The face almost always remains entirely exempt. Upon the extremities the extensor areas are invariably more markedly implicated than the flexor areas. In form and size these cutaneous hemorrhages usually resemble petechiæ, i. e., they are of the circumference of the head of a pin. Occasionally isolated, more extensive hemorrhages are observed, which are scarcely larger than a pea. In a short time the hemorrhages change their color, and undergo the well-known alterations in color of extravasated hemoglobin, or may cease quite suddenly, without leaving any pigmentation. After a few days at the longest, during which time relapses frequently occur, they become pale and disappear, and the disease may be looked upon as having run its course. The patients, who are sometimes a little anemic, convalesce and the disease terminates in complete restoration to health; or one or several relapses of the same mild nature may occur. Nevertheless, after the process has run its course upon the skin, it is well to make regular and careful examinations of the urine for albumin.

The disease designated as purpura hemorrhagica represents a severe and tenacious type in which, besides cutaneous hemorrhages, bleeding also occurs into the mucous membranes. This variety may also develop without prodromes, and run an afebrile course. It differs from the previously described form, above all, by the fact that the hemorrhages are more profuse and more extensive, so that the body looks as if it had been sprinkled with blood. Besides punctiform hemorrhages, extensive suggillations occur which sometimes reach as deep as the cutis, forming hard infiltrations. Sometimes large confluent blood spots, sometimes streaks and globular or concentric figures, or confluent masses of irregular arrangement, are noted. Thus large areas, or the greater part of the entire cutaneous surface, may be implicated so that only small patches of skin between the hemorrhagic spots remain entirely free. The deeply situated striæ-like hemorrhages, over which the skin shows all the colors of the rainbow, are very characteristic. These are frequently found in the popliteal space, also upon the upper arm and thighs, occasionally upon the buttocks, and give the impression, by their yellowish green or bluish red color, that they were produced by a blow, a fall, or a contusion.

Similar discolored cutaneous areas are found in scurvy due to deeply-seated extravasations which have undergone the same changes in color that extravasated hemoglobin shows. These extravasations may be deeply situated in the muscles and cause decided hematomata.

Fever may be entirely absent; if evening rises in temperature occur they are usually slight, the temperature rarely exceeding $101.3^{\circ} \mathrm{F}$; more frequently, however, this form runs its course without fever. Occasionally, but by no means always, hemorrhages take place in the mucous membranes. The earliest are in the nasal mucous membrane, and may produce more or less severe epistaxis.

This is followed by hemorrhages of the mucous membranes of the lips, cheeks, palate, and gums, without, however, as in the case of scurvy, giving rise to loosening of the teeth or to ulcerative processes in the gums; yet occasionally there is a loosening or swelling of the gums and also even hemorrhage. 
Severe gastric conditions, or decided pain and swelling of the jounts are more rarely noted, at most in 40 per cent. of the cases. But the process may last a long time (six to fifteen months) owing to repeated relapses, and may cause great debility. Severe grades of anemia combined with palpitation, vertigo and syncope are not infrequently observed in such cases.

The debility is markedly increased when the disease runs its course with fever and albuminuria. It is to be mentioned that in bed-ridden patients relapses take place as soon as the patients attempt to leave the bed; it is at this time also that joint affections are apt to appear or recur. The severe cases of purpura hemorrhagica with gastric symptoms will be considered later on.

Purpura urticans has been described by authors as a special variety in which, besides cutaneous hemorrhage, there is an urticarial eruption of the external skin, the individual wheals of which appear to be hemorrhagic. Occasionally these urticarial eruptions occur simultaneously with petechiæ, in other cases the two conditions alternate.

Strictly we should use the term "purpura urticans" to designate only such cases of urticaria as leave behind them true petechiæ or purpuric areas. I have observed such cases in which each time, after the patient had left his bed, new patches of urticaria alternating with purpura appeared, and in which the urticarial eruption invariably left purpuric areas behind. Such cases last for many weeks, or even for months. Gastric symptoms are often present in purpura urticans, as in true urticaria.

The forms of purpura which I have described thus far (including the milder forms of the so-called erythema nodosum-boil-like, deep infiltrations with hemorrhages) usually appear without conspicuous prodromata, and without other local disturbances. In contrast with these forms of "pure" purpura are others in which, a few days prior to the appearance of the hemorrhagic phenomena, mild prodromes of an indefinite nature, lassitude, headache, anorexia, may be observed. Finally a drawing sensation in the limbs and pain in the joints may usher in the malady; then moderate fever, lasting several days, with pain in the joints, particularly of the lower extremity, appear and last until the outbreak of the true purpuric affection. Thus we reach the form which has been designated peliosis or purpura rheumatica. It is to Schönlein's credit that he first recognized the unquestionable and intimate relation existing between the hemorrhagic diathesis and certain arthritic affections; but he did not discern that this connection is a very general one. On the contrary, he declared it to be only a limited one, and thus he failed to apprehend the true nature of the hemorrhagic diathesis, the proper understanding of which was delayed for a long time.

The designation peliosis ( $\dot{\eta} \pi \epsilon \lambda \dot{i} \omega \sigma \iota s$ ) is first found in the works of Hippocrates and signifies "blood under the skin." As is well known, Schönlein appropriated this term as a designation for the clinical picture, and added to it the epithet "rheumatica."

According to Schönlein's well known lectures, which were published by "some of his pupils," the following are the main symptoms of peliosis rheumatica: "The areas never coalesce. The patients have either formerly suffered from rheumatism, or then exhibit rheumatic phenomena, mild periodic 
pains in the joints (in the ankles, in the knees, or in the joints of the hand and shoulder). These joints are edematous and painful to the touch. The spots characteristic of the disease appear in the majority of cases first upon the extremities, particularly upon the lower, and then only up to the knees. The hemorrhagic areas are of the size of a lentil or a millet-seed, light red, not raised above the skin, and gradually turn a dirty brown and desquamate. (The latter symptom in my experience is most often absent!) The eruption occurs in crops during a period lasting often for weeks. Any change of temperature, no matter how slight, may cause a new eruption. The affection is mostly accompanied by fever, which is of the remittent type.

"The disease has been confounded with morbus maculosus Werlhofii. The absence of the so-called purpuric phenomena in the mouth which shows no changes, the absence of free hemorrhages, the absence of nervous phenomena, the character of the exanthem (never reaching the size typical of Werlhof's disease, never becoming confluent, and being a light red), the affection of the joints which is lacking in maculosus, the great debility and loss of strength, confirm the diagnosis.

"The clinical picture is seen in individuals with a delicate, vulnerable skin, who have either previously suffered from rheumatism or in whom, as the result of chilling, symptoms of peliosis simultaneously with those of rheumatism appear." So much for Schönlein's view.

Notwithstanding the great master's beautiful and exact portrayal of the clinical picture which has been named after him, it is evident that his experience in purpuric diseases was not extensive. We find in this description all the errors which have so long dominated this department of clinical medicine; they. have been propagated in the text-books for three decades, and have retarded the recognition of the true nature of this group of diseases.

Particularly fallacious is the idea that the situation and the size, as well as the configuration of the hemorrhages, are characteristic of this or that form, as well as the isolation or the confluence of the purpuric spots. It is equally unimportant whether the hemorrhages occur only upon the external skin or also upon the mucous membranes. But the erroneous interpretation of the presence or absence of arthritic affections in the hemorrhagic diathesis had more influence than any other factor in preventing a true understanding of the nature of the malady. The recognition of the fact that in every form of the hemorrhagic diathesis the joints may be implicated did not come till much later. The correction of this error may properly be looked upon as the most important advance in the knowledge of the hemorrhagic diseases.

Literature furnishes but a single autopsy report of the disease described under the misleading term peliosis rheumatica. This case is from Traube's Clinic, and is reported by Leuthold. It deserves special consideration, because Traube was for a long time Schönlein's clinical assistant, and knew exactly what Schönlein meant by the designation "peliosis rheumatica." The case is briefly as follows: A carpenter, aged thirty-nine, after prolonged lifting in the Clinic, developed slight pain in the joints. There was edema of the feet, and upon the dorsum of both several small dark red areas about the size of a pin's head appeared; these areas did not rise above the level of the skin nor 
disappear upon pressure with the finger. At the malleoli (which, like all the joints of the feet, were particularly tender to the touch) there were areas of the same kind but of larger circumference. The temperature rose. A few days later there was distinct swelling in the right knee-joint; in the following night there were tearing pains in the joint and a further eruption of petechiæ, which was most profuse upon the forearm and lower extremity, occurred in groups or isolated in parts of the body excepting the thorax and the face. Besides this, marked edema of the hands and erythema of the finger-joints developed; in the same region after the appearance of petechiæ in the following night with swelling of the left knee-joint, large extravasations of blood occurred, raising the skin in the form of tense vesicles.

While some of the petechiæ became pale and disappeared, new crops of hemorrhagic patches appeared simultaneously with an improvement in the arthritic swelling. The fever during this time was partly of the continued and partly of the intermittent type. Albuminuria was present. Among the autopsy findings I shall only mention those of the diseased joints: In the right knee-joint, which was somewhat distended, much colorless, viscid, ropy fluid; the synovial membrane very pale, only in isolated areas in the upper part here and there brownish points. The fatty areas show decided injection. The semilunar cartilage is transparent, somewhat yellowish upon the border. In the left knee-joint also much clear fluid and around the patella fenestration, transudation and vascular injection; under the tendon of the extensor of the knee, brownish extravasations, partly fresh, partly fading.

"The pathologico-anatomical findings in this obscure process which Schönlein has described as peliosis rheumatica, differ in no respect from the changes shown at autopsy in the joints in acute rheumatism." Near this the important note is found: "Not markedly different from the findings in gonorrheal rheumatism."

A patient in the course of gonorrhea was attacked by ileotyphus, which caused death at the beginning of the eighth day. In the autopsy report the following is noted: "The right knee is perhaps somewhat wider than the left externally; otherwise very little that is abnormal. Internally there was a very marked, fine, dark-red injection of the ligamenta cruciata, as well as of the entire capsule of the joint, besides a gelatinous edema of the same, most developed around the patella. The cartilage of the joint and the semilunar cartilage present nothing abnormal. The fluid in the joint is scant, somewhat more viscid and darker than normal. In their sequelæ also, genuine rheumatism and gonorrheal rheumatism appear to have a certain similarity, as may be noted from the observation of an endocarditis, which occurred in connection with the rheumatism of a long-existing gonorrhea, and, therefore, must be looked upon as dependent upon it."

Thus far the facts! Whether or not we recognize the existence of a clinical picture such as Schönlein described and designated as peliosis rheumatica must be left to the individual judgment. Probably all will agree on one point, viz. : that if we adopt the name introduced by Schönlein we must understand by it what he understood. Nevertheless we frequently find the name used without any clinical picture in the remotest degree resembling the condi- 
tion described by Schönlein. I shall mention here only the well-known cases of Bamberger, ${ }^{1}$ the description of which the author introduces with the words: "The so-called peliosis rheumatica in the lower extremities often occurs in Bright's disease. I find five cases of this kind among my observations. Usually the purpuric areas remain unchanged for many months. What connection exists here, if we do not use general expressions, is not at present clear. In two instances an autopsy was held: I shall briefly describe the cases:

"1. A man aged thirty-nine, always well, admitted to the hospital on account of pain and hemorrhagic areas upon the lower extremities. After some days edema; great amount of albumin. Pleurisy. Death. At the autopsy hemorrhages were found in the skin, upon the mucous membrane of the stomach, and in the kidneys.

"2. A woman aged thirty-six. Besides the usual symptoms of Bright's disease, there was stenosis of the mitral valve, also engorgement of the liver. Purpuric areas upon the lower extremity had existed over a year. Death due to apoplexy. Autopsy. Fresh hemorrhagic focus in the brain; stenosis of the mitral valve; wedge-shaped infarct in the spleen; pneumonia and chronic nephritis."

These cases do not in the least resemble the clinical picture of Schönlein; in each case he was dealing with a chronic nephritis with purpura the result of cachexia and the hemorrhagic diathesis due to this (peliosis cachecticorum). Bamberger here uses the designation "peliosis" as synonymous with "purpura," and has no reason to speak of a rheumatic form as he in no way indicates any disease of the joints. If one searches for the etiologic relationship of the symptoms, one must surely find it in the loss of albumin which caused the cachexia, and in the hemorrhagic diathesis dependent upon this.

I have seen great numbers of cases with hemorrhages such as Bamberger described, in chronic nephritis as well as also in old valvular lesions of the heart, and have only here described these in order to show that the clinical picture presented by prominent clinicians a comparatively short time after Schönlein's teaching was so obscured that nothing was left of it but a peculiar type of cutaneous hemorrhage. That this has been generally looked upon as the main symptom of the affection is shown by an examination of the literature. Here we are forcibly impressed with the fact that, step by step, from the great group of purpura varieties which ran their course with arthritic symptoms, one particular form was arbitrarily separated and presented as an independent disease. As the number of cases accumulated it became clearer that arthritic affections may occur, and do occur in every variety of hemorrhagic disease. Soon, without an exception, all those cases were described as peliosis rheumatica in which the two symptoms were found. If the cases happened to differ very widely from Schönlein's description they were explained as a form of peliosis with "an atypical course." Thus it came to pass that neither a definite form of hemorrhage nor any special type of joint affection was considered typical of the clinical picture in question. and on the other hand no importance was attached to etiology. 
In consequence of these slovenly methods of classification cases of ulcerative endocarditis with purulent arthritis and multiple cutaneous and internal hemorrhages finally came to be designated as peliosis rheumatica, and hemorrhage in the latter disease was assumed to be embolic. But if we leave out of consideration altogether the results of these blunders, we find in literature an increasing number of cases described as peliosis rheumatica which have nothing in common with the original Schönlein clinical picture except the combination of hemorrhages and joint affection. Attention to a particular type of hemorrhage soon ceased, and instead of the isolated, non-confluent, lentil-sized petechiæ upon the lower extremities, described by Schönlein, we find writers including in their descriptions every form of cutaneous hemorrhage, of mucous membrane hemorrhage, and of hemorrhage into internal organs. If we attempt to bring order out of the chaos of pathologic entities and clinical pictures described in literature under the name of peliosis rheumatica we find simple purpura, purpura hemorrhagica and purpura urticans, morbus maculosus Werlhofii, scurvy, true peliosis rheumatica and, finally, erythema nodosum.

Occasionally we find each of these maladies, running their course with cutaneous hemorrhage, complicated with joint affections, as will be later explained in detail. Hence the decision as to the nature and character of the disease should not depend upon the seat, nor the size, nor the form and confluence of the hemorrhages, nor upon the implication of the mucous membranes, the serous membranes or the internal organs, nor even upon the sequence of the individual symptoms, but wholly upon the etiology. This, however, in the group of diseases above mentioned, must be looked for in those changes in the blood which we have designated by the term hemorrhagic diathesis. Patients suffering from this disease have a pale, delicate skin which upon the slightest cause shows a tendency to hemorrhage; they also present the well-known symptoms of chlorosis and anemia. It is probably due to this vulnerability of the skin, which is also unusually sensitive to changes in temperature, that such individuals are more readily attacked by rheumatoid affections than others. It seems arbitrary to isolate from this great complex of cases which have a common cause, a single group particularly characterized by the behavior and localization of the petechiæ, and to assign a particular name to it. In any large batch of clinical material many cases will be met with which correspond minutely to the description of Schönlein, with the sole difference that the form of the hemorrhages varies, or that they have their seat in the mucous membranes. How can the latter be reasonably described as peliosis rheumatica, and the former not? I have a large number of clinical histories which I have been for a long time collecting because I was interested in the question of the relation between the hemorrhagic diseases and affection of the joints. I shall refer later to the conclusions based on these, and at this point shall only state that I concur in the opinion at which Scheby-Buch arrived after careful study: "The history of peliosis rheumatica is of itself sufficient to show the untenability of the view that it is an independent disease, even if we did not have other weighty reasons for the same conclusion."

No one would think of designating scurvy combined with arthritic affec- 
tion as peliosis rheumatica, yet carefully studied cases of purpura hemorrhagica with joint affection have been described by some writers as peliosis rheumatica, and by others as acute articular rheumatism with hemorrhages. French and English authors were among the first to describe all such cases of extensive hemorrhagic diathesis with articular affection as "acute articular rheumatism with atypical course," and thus to a certain extent constructed a new clinical picture. This example shows what the results might be if, without the protective guidance of etiology, and solely on the basis of obvious symptoms, one should attempt to classify these cases! With a sharply defined clinical picture such as scurvy usually presents, only an unusually slovenly habit of mind could make it possible to mistake the relation of the symptoms. But the case is quite different when we attempt to deal with the group of cases last indicated. Some authors describe them as purpura or peliosis rheumatica, according to whether the cutaneous phenomena or the joint affections are more prominent; others call them rheumarthritis, without realizing that the clinical picture does not correspond to Schönlein's conception, and that in acute articular rheumatism cutaneous hemorrhages do not occur except in the presence of certain severe complications!

One who has seen much of acute articular rheumatism knows that it has in uncomplicated cases no association with cutaneous hemorrhages, and will not confound this disease with any other. Of great importance is the tendency of rheumatism to produce secondary inflammations of the serous membranes and of the endocardium, which is absent or extremely rare in the pure form of peliosis and in the hemorrhagic diathesis. Further, the absence in all forms of purpura of the very severe and exhausting sweats of acute rheumatism, as well as the absence of severe joint phenomena, is important. Cutaneous or internal hemorrhages (particularly of the retina) occurring in acute rheumatism depend upon a complicating acute endocarditis, as I have explicitly pointed out in my monograph upon septic diseases. ${ }^{1}$ Such cases are also to be clearly differentiated from those in which, with a simultaneous septic or so-called ulcerative endocarditis, purulent joint metastases with numerous petechiæ distributed over the entire skin and retinal hemorrhages appear. The cardinal difference between these groups is that, in the former, acute articular rheumatism introduces the scene and represents the original disease, while in the latter a wound affection (for example, diphtheria of the placental area, etc.) or internal suppuration (such as thrombophlebitis suppurativa of the pelvic veins due to abortion, or of the spermatic veins in the course of gonorrhea) is the cause. In the last-named group of cases the joint affections are invariably secondary. Many examples of both varieties of cases have been given in my article upon septic disease. (See p. 380 et seq., and p. 588.)

Having now proven, as I believe, that there is no justification for Schönlein's clinical picture of peliosis rheumatica, and that the condition was a joint affection combined with a hemorrhagic diathesis, I must mention that there are cases which run a course exactly as described by Schönlein, except that they are due to another cause, namely, gonorrhea. I have used the designa-

1 Zeitschr. f. klin. Med., Bd. ii, 1881. 
tion "peliosis gonorrhoica" in my article upon this subject, ${ }^{1}$ because I wished to point out to the profession the external connection, and also that there might be no misunderstanding of what I mean by the expression, but I shall not consider further the form of joint affection with cutaneous hemorrhage which arises in connection with gonorrhea.

In the main, Schönlein's description is applicable to the milder forms of rheumatic purpura. Some further explanation will be given in the description of the so-called Henoch's purpura. With the appearance of purpura the arthritic pains generally cease. Not infrequently the purpuric spots are associated with urticarial eruptions and edema of the dorsum of the feet and ankles, as well as with erythema multiforme exsudativum. The disease may run its course in a few weeks. Frequently, however, relapses occur with renewed arthritic pain and fresh eruptions of purpura, and the disease sometimes lasts for months, even from eighteen months to two years and longer. Fever may be present or completely absent.

The more intense the affection of the joint the higher the evening rise of temperature. In very severe cases, in which the clinical picture may resemble acute articular rheumatism, we find prolonged febrile rises of a remittent character. The affection differs from true typical articular rheumatism, above all, by the absence of profuse sweats and of a tendency to endocardial complications, to which the hemorrhages occasionally seen in true rheumatism are to be referred. Nevertheless in rare and severe cases of rheumatic purpura, the appearance of the diseased joints may very closely resemble that in true acute articular rheumatism, even to such an extent that a differentiation is impossible. In a case from Traube's Clinic reported by Leuthold, which ran a fatal course, the condition of the joint differed nowise from the changes which are observed at the autopsy in acute rheumatism (see above). However, no report of purpura rheumatica is known to me in which a purulent effusion was met with in the joint cavities. If the disease is very much prolonged, anemic symptoms become noticeable as in acute rheumatism, among others anemic cardiac murmurs. Only rarely is enlargement of the spleen observed.

Hemorrhages into the mucous membranes are not absent; thus Kaposi found in one case hematuria, in another case, which terminated lethally, ecchymosis with subsequent gangrene of the mucous membrane of the palate, while Duhring described a bloody discharge from the genitalia. These cases resembled the form, now to be delineated: Henoch's "purpura abdominalis."

Henoch first noted in the year 1868 as a complication of purpura the fact that the eruption of purpura and the arthritic phenomena may be accompanied by a number of abdominal symptoms: Vomiting, hemorrhagic diarrhea and colic. Henoch represented as characteristic the appearance of these symptoms in paroxysms with an interval usually lasting several weeks, and occasionally even as long as a year. In the earlier literature upon purpura, with the exception of a case by Wittan, there is no definite report of the occurrence of this disease in combination with joint troubles and severe abdominal symptoms. We 
therefore regard this not as a special form of purpura but as a special manifestation of the same transitory hemorrhagic diatheses which in all cases have one and the same pathogenetic cause.

According to v. Dusch and Hoche the affection occurs especially in youth. In earliest childhood, from the first to the third year, and in advanced age, beyond the forty-sixth year of life, no case is known; after the third year the number of cases gradually increases to its maximum between the ninth and twelfth years. The frequency then remains about the same up to the twenty-fourth year. Beyond this age the affection again becomes quite rare.

Males are more frequently attacked than females; of forty patients whose sex is mentioned 33 were males and 7 females. These reports regarding age and sex differ decidedly from those of other authors. Regarding sex, I have noted a decided preponderance of the affection in males.

As predisposing causes, besides recovery from a previous acute rheumatic attack, unfavorable hygienic conditions, damp dwellings and insufficient nutrition are sometimes noted, but among the cases there are also patients who have not been subjected to these influences. It is necessary to emphasize this, as purpura is still occasionally spoken of as a disease of the proletariat, and as the expression of a certain general cachexia.

With more or less disturbance in general health of varying duration, accompanied by headache, lassitude, anorexia, etc., rheumatoid pains appear which are drawing or tearing in character, not strictly localized, but in various parts of the body, having their seat particularly in the lower extremity and in the back. At times there is a transitory edematous swelling in the affected regions.

Some of the patients now take to bed, others continue to follow their ordinary pursuits. Soon, however, severe pains occur in one or more joints, usually without any perceptible external change in the affected area; in other cases one or more joints begin to swell, the skin reddens, becomes hot, and in the surrounding areas edematous infiltrations appear, quite as in acute articular rheumatism. In this stage sometimes mild fever is noted; temperatures reaching $101.3^{\circ} \mathrm{F}$. are rare, as are high temperatures in the later course of the disease.

As a rule, the pains and immobility of the joints cause the patient to send for a physician, and frequently he is the first to note the purpuric areas, which are at the onset isolated, arising without any subjective symptoms. Usually the eruption causes itching only when it begins with urticaria. Generally a period of several days intervenes between the occurrence of the joint pains and the appearance of the first eruption of purpura.

The purpura itself, in the beginning usually bright red and consisting of small, irregular and isolated spots, or groups formed of these, gradually coalesces into larger, irregular areas, which in the course of a few days show a change in color and become bluish or yellow or dark brown.

From the lower leg (where they frequently but by no means always appear first) they distribute themselves gradually by continuity, or in jumps to the upper thigh, buttocks and gluteal regions. New eruptions appear upon both arms, or upon the trunk, so that sometimes the entire body, particularly the region of the larger joints, appears covered with purpuric spots. 
While the pupura itself causes the patient great anxiety but does not actually give rise to suffering, other symptoms soon become prominent and are much more troublesome, e.g., disturbances on the part of the intestinal tract which are characteristic in that they generally resist all treatment. Patients complain of severe colie-like pains in the abdomen, particularly in the umbilical region, which are frequently so severe as to bend the patient's body, and evoke loud cries. The abdomen is also retracted and sensitive to pressure; the bowels at the beginning of the attack are constipated. These difficultics are increased by a stubborn vomiting, which at first contains the food last introduced, then consists of yellowish green bilious masses frequently admixed with blood. The pulse becomes small and rapid; the facial expression shows anxiety; the entire condition is a pitiable one.

The constipation which is present at the onset soon gives way to a more or less profuse discharge of thin yellowish feces often admixed with blood, the occurrence of which sometimes coincides with the cessation of the pain.

The colic and the vomiting occasionally last for days; the patient eats little or nothing. Now and then under the influence of severe retching, epistaxis occurs. Gradually all of the phenomena ameliorate, the vomiting ceasing first, then the abdominal pains, while the thin fecal discharges continue to be voided from time to time until the period of apparent convalescence.

The pains in the joints have in the meantime ceased, and the purpura has faded. In case none of the complications which are to be described have appeared, the patient, aside from a certain exhaustion, is comparatively well and believes that convalescence has begun. Occasionally no other attack occurs, and convalescence proceeds uninterruptedly ; in the majority of cases, however, the symptoms are repeated, wholly or in part, after a period which may vary from one day to several weeks, until finally recovery ensues. In relatively rare cases death takes place.

But a schematic course such as described is not the rule; in children the typical clinical picture is most often seen. The frequent deviations and manifold peculiarities observed in the course of most adult cases can best be explained by a description of the individual symptoms.

These will be minutely discussed under the special symptomatology, so that at this place only a few concluding remarks will be made concerning this form of purpura.

Among the peculiarities, a group of cases must be mentioned (rare and in part insufficiently described) in which the arthritic affection is entirely absent, and, besides the purpura, only marked intestinal disturbances exist. The duration of Henoch's purpura varies within wide limits; numerous cases have been recorded lasting from seven days up to nine months; as an average duration, six to twelve weeks may be mentioned.

The prognosis in chitdren in the main is good-among nineteen cases there was but one fatal case, and this was in consequence of acute nephritis. In adults it is less favorable. In twenty-two cases recorded, five were fatal. A definite opinion cannot be given on account of the paucity of records.

We now reach a special manifestation of the disease, which was also described by Henoch in 1887 under the name of purpura fulminans. I described 32 
an analogous clinical picture nine years previously, in the year $18 \% 8$, and also described it and pictured it accurately in 1881, without, however, giving the condition this very descriptive name. In all of these cases there is an extensive cutaneous hemorrhage which rapidly leads to death, of which Henoch himself has seen three cases, while a fourth was described in 1886 by Charron in Brussels.

All these cases have in common the feature that hemorrhages upon the mucous membranes are wholly absent, but with great rapidity, extensive ecchymoses appear which, in a few hours, change the entire skin of the extremities to blue and dark red, and represent a dense bloody infiltration of the cutis. The formation of hemorrhagico-serous vesicles upon the skin occurred in two cases, but gangrene has never been noted and no fetid odor has been observed. The course is exceedingly rapid; within twenty-four hours after the formation of the first purpura death occurred; the longest duration was four days. No complication was present, and, with the exception of general anemia, the autopsy showed an entirely negative condition; there was no sign whatever of embolic or thrombotic processes. One of Henoch's cases developed two days after the crisis in pneumonia, another one and a half weeks after a very mild scarlatina. In both of the other cases, as well as in mine, there was absolutely no known etiology. Two analogous cases have been since published by Ström and Arctander. The first of these followed scarlatina; there is no autopsy report. According to Hervé three similar cases were previously reported (1888) by Guelliot.

Before describing my own case, I shall briefly mention one of Henoch's: A boy, aged five; crisis in pneumonia upon the 22d of November. Since then complete euphoria. During the night of the 24th, sudden pains in the left leg; toward morning purpuric areas upon the chest and thighs, an hour later upon the arms and lower legs. At eleven o'clock in the morning the entire posterior and lateral area of the left thigh was of a bluish black color; toward evening also the left calf and the right knee. Temperature $101.8^{\circ} \mathrm{F}$. In no organ could anything abnormal be detected. During the night of the 24th the entire right leg with the exception of the foot became bluish black. Great apathy and weakness; urine normal. At two o'clock in the morning death in collapse. Autopsy absolutely negative.

My own case occurred in a man, aged twenty-eight, a tinsmith, who upon the 23d of March, 1878, was admitted to Frerichs' Clinic in a comatose condition, and died upon March 25th. No history could be obtained from the patient nor from the relatives. Only this much was certain, that he was well and able to work two days previously. The disease began upon the morning of the 21st. At that time the patient is said to have had a severe chill which forced him to go to bed. A physician who was called the next morning noted high fever and ordered his removal to the Charité. The diagnosis of typhus fever was made.

March 23d, nine o'clock. Patient, a very muscular man, is comatose, constantly mumbling, beating the air with tremulous hands, and continually throwing himself about in bed. The face appears congested, markedly cyanotic, and icy cold. The middle of the upper lip shows a protrusion resembling a 
proboscis. This prominence is due to a deep hemorrhagic suffusion of the mucous membrane beginning at the border of the lip, almost 2 c.c. in breadth, and reaching posteriorly almost to the gums. The mucous membrane in this area appears eroded and covered with thick crusts, upon the removal of which the base of a superficial ulcer of bad color is seen. The mucous membrane around the border of the ulcer is in the form of rolled-up shreds. The gums are bluish red, swollen, in part hemorrhagically infiltrated. The lips are covered with sordes, and from between them a blood-stained mucus exudes. Under the conjunctivæ of both eyes there are circular flattened hemorrhages. The skin is smooth and dry. The color is chiefly yellowish, but is modified by a great number of pale to dark violet, irregularly shaped hemorrhages, which appear partly as small spots and partly as flat extravasations. In some areas these flakes disappear on pressure; at most points, however, they persist. Aside from these spots the skin, on account of stasis and uneven filling of the vessels, assumes a marbled appearance.

Upon the lower extremities quite large suffusions are found which attain an area of several square inches, and also hemorrhages which almost give the impression of being of traumatic origin. Upon the external surface of the thigh they are quite symmetric; there is also a certain symmetry upon the back. In the joints of the feet, and upon the dorsum of each foot, there are extensive suffusions, reddish blue in color, almost the size of a silver dollar. Upon the right lower leg, and upon the dorsum of the left foot there are also large suffusions besides ulcers covered with crusts. The sensorium shows coma; there is no reaction upon deep pricks with a pin. The apex of the heart cannot be felt; cardiac dulness is not increased; heart sounds clear. Lungs normal. Spleen moderately enlarged, reaching $3 \mathrm{~cm}$. beyond the anterior axillary line. In the eye-ground the arteries and veins are dilated, the papillary limits obliterated. No retinal hemorrhages. Temperature reaches 106. $9^{\circ} \mathrm{F}$.

Evening, eight o'clock. Patient is comatose with closed eyes, moaning, and assumes the dorsal decubitus. Respiration increased (32 per minute) and stertorous. Patient very restless. The muscles markedly contracted. There is anesthesia of both cornea.

Half-past eleven at night. Complete unconsciousness. Marked prostration. The face is covered with sweat and feels cool, whereas elsewhere the skin is dry and burning hot. Temperature $107.6^{\circ} \mathrm{F}$. Upon the left thigh, situated externally, a single bluish red suggillation the size of two hands, which has resulted from the confluence of a number of smaller foci. When I saw the patient three hours earlier, about eight o'clock, the individual points of the eruption were still separated by large intervening areas of healthy skin. Since eight o'clock, several fresh, bright red hemorrhages have appeared, among them one the size of a silver dollar. Respiration greatly increased, loud, snoring in character; the pulse very small and soft, 120 per minute. Paresis upon the right side affecting the face and extremities. Ptosis of the right eyelid. In the eye-ground nothing abnormal. Sedes inscice. Urine acid, contains much albumin and numerous broad casts. 
March 24th, nine o'clock in the morning. But little change in the clinical picture. Complete insensibility. Temperature $106.9^{\circ} \mathrm{F}$. Upon the skin many new hemorrhages have appeared. To retain the picture of the skin, I asked the artist, E. Eyrich, to paint a water-color of the case. He began upon the morning of the 24th at a time in which the hemorrhages were so close together that the individual flakes upon the arms, which he had already indicated in contour, assumed an entirely different size and configuration from those I had previously seen. While he was working on his picture the individual flakes coalesced and enlarged. As this condition appeared extremely strange to Mr. Eyrich, he sent for me in order that I might note the coalescence and enlargement of the individual areas. What, however, lent a peculiar character to these hemorrhages was the circumstance that the larger ones were grouped in concentric circles, and that each of these circles showed an entirely different color tint. This peculiar arrangement was caused by the striking rapidity with which the individual areas developed and enlarged. The older center appeared much darker than the fresher peripheral hemorrhage; I myself observed this change in different areas. Thus, areas developed in concentric arrangement which consisted of three to four rings of different colors. The development of these hemorrhages occurred with such enormous rapidity that the change in color from the brightest red to the darkest brownish red could be noted in the course of a few hours.

Evening, eight o'clock. Patient in collapse. The investigation of the eyegrounds shows in the right a small centrally situated retinal hemorrhage, and in the left two more.

12.30 A.M. Death. The temperature taken post mortem showed a rise to $108.8^{\circ} \mathrm{F}$.

The result of the autopsy was very unsatisfactory. The changes worthy of mention were the following:

Cloudy swelling of the liver, spleen and kidneys. White striæ of the medullary substance of the kidneys. Very slight deposits of the finest ex, crescences upon the free border of the mitral leaflet. Multiple hemorrhages in all serous membranes.

The microscopic investigation of the skin gave an entirely negative result; there were neither vascular emboli nor microörganisms inside the vessels. Enormous masses of streptococci were found in the glomeruli and in the intertubular capillaries of the kidneys as well as in the deposits upon the mitral. valve.

That the diagnosis in such a case is not a very simple one, and that great diagnostic errors are liable to occur, is taught by a case of hemorrhagic variola in which the clinical symptoms closely resembled those of the case just described, and only late in the course of the disease several distinct umbilicated pustules appeared which in a brief time broke down, learing ugly ulcers.

The diagnosis of hemorrhagic smallpox, if the latter runs its course without pustule formation, and appears during a period in which other cases of smallpox have not occurred, may give rise to great difficulties on account of the similarity to the clinical picture of purpura fulminans. In the course of severe 
septic diseases, and in acute leukemia also, extensive cutaneous hemorrhages occur.

To the forms of the hemorrhagic diathesis which have been described we must add still another disease which lately has become quite prominent, and which by some authors is looked upon as infantile scurvy. Yet it differs from the latter affection in many important peculiarities. The disease was described in $185 \%$ by Möller in Königsberg as "acute rachitis" and was particularly studied by Barlow in 1883, since which time it has been called by his name. In the last few years this affection has been repeatedly the subject of close investigation, and particularly on account of the increasing frequency of the disease among the children of families of the better class, has become important. It occurs exclusively in children between the middle of the first year and the third year of life. The onset is often, but not always, acute. After a few days of general ill health, occasionally following an attack of diarrhea, sensitiveness and difficulty of movement in one or both lower extremities appear, with sensitiveness to touch. The children are usually found with their limbs extended or retracted upon the abdomen, immovable in bed. Either active or passive motion is painful. Very soon a spindle-shaped, sensitive, smooth, white tumor of elastic consistency is noted in the course of the diaphysis of one or both thighs, rarely of the lower leg or of the upper extremities. Occasionally crepitation is noted in the epiphysial border, which is due to a loosening of the latter.

This deeply situated subperiosteal or subperichondrial tumor in the tract of the long tubular bones is the pathognomonic, peculiar sign of this disease, compared with which the rachitic or scorbutic symptoms are entirely secondary. Neither clinically, by puncture or incision, nor after death, has pus ever been found under the periosteum of the diseased bones, but invariably pure blood, and there is no case on record in which it has ever been observed that the capsule of the joint, in spite of its close proximity, was implicated in the process.

To these previously mentioned symptoms frequently, but by no means always, the symptoms of the hemorrhagic diathesis, of scurvy and of rickets, are added. To the latter has been attributed the great tendency to sweating, particularly of the occiput, as well as the swelling of the epiphyses. Scurvy is thought to explain the loosening and spongy swelling of the gums with fetor, and the tendency to hemorrhage, especially in the cases where teeth are already present. Fever and gastric symptoms are frequent. Occasionally purpura, hemorrhages into the mucous membranes, and albuminuria occur; Henoch mentions hemorrhages under the periosteum of the frontal bone, into the eyelids, and into the retrobulbar tissue with exophthalmos. The appearance of the children is anemic, but very rarely, either by hemorrhage or other complications, does death occur.

Regarding the etiology of this important and interesting disease, it has been maintained more and more lately that improper nourishment is responsible. While by some authors the too great uniformity of the nutrition has been looked upon as the cause, others emphasize the absence of fresh food. In the exhaustive American collective investigation (Boston Med. and Surg. Journal, 1898), and in a great number of individual observations, it has been 
shown that Pasteurized and sterilized milk if used exclusively may cause Barlow's disease, and that sterilization in the Soxhlet apparatus is sufficient to produce it. Lately it has been assumed that the duration of the heating process of the milk is the most important factor, but Heubner believes that heating from ten to fifteen minutes is not productive of harm.

H. Neumann " states in regard to this matter: "From my own experience of the conditions in Berlin for the last five years, the following may be maintained: In nutrition with artificial milk preparations Barlow's disease occurs very rarely, either from the fact that the prolonged use of these foods has become rarer, or that more care is taken in sterilization. In the year $1899 \mathrm{I}$ saw but one case which was due to Gärtner's 'fat-milk.' Considering the mode of preparation of this food only the sterilization, not the chemical treatment, could play a rôle; in my case the milk was sent for weeks in the summer to the seashore, and for this purpose was previously heated to a very high degree. In another case, in the year 1899, cow's milk prepared in a laboratory was powerfully heated-up to the point of browning. Up to the year 1900 , I had seen four children who were taken ill in spite of the fact that the thoroughly reliable raw milk was in two cases heated only for fifteen minutes, and in two cases for only ten minutes in the Soxhlet sterilizer. In two of these cases infant food was added to the milk, but I do not desire to attach any importance to this. I should like to mention here, however, that in two of these cases intestinal catarrh preceded the disease; in the third case dyspepsia was present which may have hastened the appearance of Barlow's disease. On the other hand a case occurred in the entirely normal child of a colleague, and in this case the preparation of the milk must be held entirely responsible for the disease (fifteen minutes in the Soxhlet apparatus). Since the year 1901 Barlow's disease, at least according to my experience (which is also confirmed by Heubner) has increased in frequency in Berlin, and this makes it possible minutely to investigate a very remarkable fact: For the fourteen children that I had an opportunity of seeing in this time the Soxhlet apparatus was always used, and in seven cases the milk was sterilized for ten minutes, in two cases for fifteen minutes, and in five it was boiled for a longer period than this. In spite of the fact that among our well-to-do classes infant's milk may be obtained from a number of reliable dairies, the milk for the fourteen children treated by me was all obtained from the same dairy, and from a dairy which since that time has employed its entire product in a laboratory for heating, from which no exact records can be obtained. While, according to my experience, the mere boiling of this milk does no harm, the combination of sterilization according to Soxhlet with previous Pasteurization may produce Barlow's disease.

"Besides the cases cited above, I later saw seven others, the infants being from six to ten months of age when they came under treatment. Six of these patients had been fed from birth on B's milk; it was boiled for ten or fifteen minutes in the Soxhlet apparatus, or ten to twenty minutes in Hartmann's milk boiler; in one child only did the disease develop while exclusively nour-

1 "Verh, des Vereines für innere Medicin in Berlin," 1903. 
ished by condensed Swiss milk; but with the use of B's milk it developed to a great extent. Therefore, to summarize, Neumann has observed in the last eighteen months twenty-one cases of Barlow's disease, of which twenty may be referred to the use of the same mill. If these cases were left out of consideration Barlow's disease would be as rare as formerly. Therefore, its increased incidence may be referred to a purely accidental and perfectly recognizable cause."

\section{PATHOLOGICAL ANATOMY}

Cases of simple purpura which terminate fatally are rare. If death occurs, this is either the result of profound anemia after profuse hemorrhage or in consequence of complications or sequels. For this reason autopsy findings are very scanty. The cadavers are extremely pallid, also somewhat bloated, and usually covered with hemorrhagic blotches, which have taken on the livid post mortem discoloration of altered hemoglobin. The muscles and fatty tissue are in most of the cases unchanged; only in very protracted cases is the latter decreased. According to the intensity of the disease we find more or less extensive hemorrhages upon the mucous and serous membranes, and under certain circumstances these cover large areas. Occasionally these hemorrhages are distributed to an astonishing degree upon the mucous membrane of the bronchi, of the digestive canal, in the renal pelvis, ureter, bladder, etc. At times the mucous membrane, if we strip off the inspissated blood crusts, may show superficial erosions. Freshly effused blood is also occasionally found in the mucous membranes of the bronchi with bloody mucus. This is also noted in the renal pelvis and in the intestinal canal. In the cavities of the serous membranes, in the pericardium, in the pleura, and in the peritoneum, as well as in the cavities of the joints, besides small ecchymoses, we occasionally find larger effusions of a purely hemorrhagic character, so that we are dealing with hemopericardium, hemothorax, hemarthrosis, etc. We also find parenchymatous hemorrhages, particularly in the liver and kidneys; in isolated cases the adrenals have been found containing bloody infarcts. In the museum collection at my hospital I have two such hemorrhagic adrenal infarcts, each about the size of a medium-large apple. The spleen in a certain number of cases, but by no means invariably, has been found enlarged; in isolated instances it contains the same wedge-shaped hemorrhagic infarcts. In the protracted cases running a febrile course there is cloudy swelling of the large parenchymatous glands besides swelling of Peyer's patches and of the mescnteric glands. The same is observed in the bone-marrow, upon the endocardium, the vascular intima and the neurilemma. The bone-marrow has been found traversed by profuse and small hemorrhages. Pigment infiltration, due to the decomposition of extravasated blood, has also been observed in different organs, particularly in the lymph-glands. The pigment appears in flaky masses and consists entirely of hydrated oxid of iron.

Nothing is known of other constant changes; minute reports are lacking in regard to the condition of the membranes of the joints and cavities, particularly in those cases in which during life rheumatic pains were present. The knee- and foot-joints are the ones which are chiefly attacked with arthritic 
symptoms in purpura; for the present we must content ourselves with this fact, and await further knowledge and resuits from the autopsy findings of the milder forms of the hemorrhagic diseases. In a case of peliosis rheumatica from Traube's Clinic, upon which an autopsy was held, the findings in the joints did not differ from those of acute articular rheumatism and gonorrheal rheumatism (see above). The autopsy report is as follows: The right knee appears somewhat thicker than the left. In the external parts there is little that is abnormal; but in the interior of the joint is a very marked, fine, dark red injection of the ligamenta cruciata, as well as of the entire capsule of the joint, besides a gelatinous swelling of the same, especially marked about the patella. The cartilage of the joints and the semilunar cartilage show no abnormality. The fluid of the joint is scant, somewhat viscid, and darker than normal.

In the main no constant changes have been found, not even such as might be referred to anemia. The valvular apparatus of the heart is invariably intact; in one case of fulminant purpura described by me there was a slight deposit of fine warty excrescences upon the free border of the mitral leaflet, as occurs so frequently in any disease running an acute course. But even these have in other cases been entirely absent.

I shall revert later to the changes in the blood, that is, in its composition; here I will only briefly mention that some authors have found the coagulability of the blood to be diminished, a finding which still requires confirmation.

The spleen and lymph-glands show no constant alterations, but the former organ has repeatedly been found enlarged, and pappy softening of the pulp has been noted (Billroth). It remains a question whether this is not due to post mortem change.

The kidneys occasionally reveal a condition of hemorrhagic inflammation. In cases of chronic albuminuria I several times observed isolated small-celled foci of infiltration in the cortex. In other cases in which albuminuria had existed for many months these were absent.

Hemorrhages upon the retina, as well as upon the cornea, have been determined during life and confirmed post mortem. Upon microscopic investigation of the eyes in question I found the vessels intact in both membranes of the eye, which is by no means the case in analogous hemorrhages in the eyes of patients with renal disease. More frequently meningeal and cerebral hemorrhages have been found at the autopsy, but these have been looked upon as the cause of the epileptiform attacks and paralyses which had occurred during life.

The changes in the vessels in the course and in the vicinity of ecchymoses have been described explicitly in the etiology, and the investigations of v. Kogerer, Riehl and Leloir have been mentioned. Hayem states that thromboses have formed in the finer arteries by the agglutination of leukocytes. Other authors found amyloid degeneration of the capillaries in the vicinity of the petechiæ (?). Stroganow, whose investigations I have already referred to, discovered in the aorta, in the vena cava, and in the veins of the liver infiltrations of the intima with red blood-corpuscles which from the 
lumen of the vessels appeared to have directly penetrated the intima per diapedesin. But this latter observation explained the nature of the disease and the occurrence of the hemorrhages as little as any of the previously mentioned researches.

Among the complicating processes which occasionally result in death I shall enumerate the following: Large effusions into the pleura and abdomen, pulmonary infarcts, purulent peritonitis, croupous-diphtheritic processes of the small intestine, pneumonia, necrosis of the intestine, perforative peritonitis, gangrene of the large intestine with swelling of the mesenteric glands, ecchymoses and ulcers of the descending colon.

Regarding the bacteriologic findings, besides those described in the etiology, I should like to discuss here some further points. Tizzoni and Giovannini isolated from a case of purpura hemorrhagica in which secondary impetigo contagiosa developed, and upon which autopsy was held, a bacillus, the bacillus hemorrhagicus velenosus. In the pustules of impetigo surrounded by the purpuric areas of the skin this bacillus was found with the staphylococcus pyogenes aureus; it was also found in the liver and in the venous blood, but not in the spleen nor in the kidneys. The staphylococcus was present in the pure hemorrhagic foci of the skin, as well as in the kidneys. The bacillus hemorrhagicus velenosus is immotile, $0.2-0.4 \mu$ broad, $0.7-1.3 \mu$ long; it stains well with anilin colors, but not according to Gram. Spore formation was not observed; but there was a certain resistance to drying. The colonies showed irregular contours which resembled coiled locks of hair, and did not bscome fluid. In a stab culture they showed granular growth, and upon agar a growth similar to that upon gelatin. In older cultures a pungent odor is noticeable. Upon potato only a superficial growth of an indistinct nature with a dark yellowish discoloration at the point of inoculation is observed. Upon culture in bouillon moderate turbidity appears, later becoming mucoid. The bacillus is pathogenic in rabbits, dogs, and guinea-pigs, but not in pigeons and mice. The bacilli increase only locally with formation of edema. They, however, give rise to fever, hemorrhagic nephritis, vomiting, hemorrhagic diarrhea, cutaneous hemorrhages. Upon autopsy of the infected animals there are found incoagulability of the blood, coagulation necrosis of the liver and renal epithelium, with a normal condition of the spleen. Cultures sterilized at $70^{\circ} \mathrm{C}$. produce albuminuria. Repeated injections of such cultures confer immunity to subsequent infection.

Kolb, ${ }^{1}$ of the Imperial Bureau of Health, investigated bacteriologically five cases of true idiopathic purpura: Among these were three cases of purpura fulminans which terminated fatally after a brief course; the other two cases recovered. Microscopic investigation, cultures and inoculation of the blood taken from the living patient into mice, guinea-pigs, rabbits and pigeons, gave no results. Positive results were, however, obtained by the bacteriologic investigation of the cadavers. There were examined: $(a)$ Blood from the heart and from the portal vein; $(b)$ some areas of the skin containing characteristic purpura; $(c)$ particles of hemorrhagic portions from the lung; $(d)$ 
the liver, spleen and kidneys; $(e)$ hemorrhagic portions of the intestine; $(f)$ lymph-glands from the thoracic and abdominal cavities.

In the sections from the large hardened glands of the abdomen, as well as in the hemorrhagic cutaneous areas, there was found, after staining with methylene-blue and after the Gram-Weigert process, a moderately large bacillus averaging from $1-2 \mu$ in length and $0.8 \mu$ in breadth with rounded ends. The bacilli were especially profuse in the spleen, some in the small blood- and lymph-vessels forming larger clumps, some also in the interstitial tissue, though here not so numerous as in the ressels, but found lying separated from one another; now and then longer threads were found due to a juxtaposition of the individual bacilli end to end. For the most part two rods were found placed together lengthwise (diplobacilli). In the kidneys the bacilli were mostly met with in the glomeruli, but were here not so numerous as in the spleen. It was also possible to find the bacillus in sections from the liver, and in sections of cutaneous hemorrhagic areas in which, even in the lowest cellular layer of the corium, scattered bacilli were found. Particularly instructive pictures were furnished by sections of hemorrhagic glands. In fresh sections from organs the same variety of bacteria was demonstrated. The bacillus hemorrhagicus Kolb flourishes upon gelatin. The matured colonies are circular in form, with many constrictions and serrations; in the interior fine furrows are seen, and toward the border a more granular appearance becomes prominent. In stab culture after a few days colonies are found partly isolated, partly coalescent, with superficial flat, hyaline extensions along their serrated borders. Upon inoculation a thin leaf-like distribution of a whitish blue color and of porcelain-like transparency with indentations and serrated borders is observed along the entire course. Kolb's bacillus grows upon agar, as in the gelatin stab culture, and somewhat more slowly upon blood serum. Upon potato a white, moist, glistening streak is noted along the course of inoculation. In bouillon cultures a feebly alkaline medium is the best. Even on the first day the solution shows a general cloudy turbidity. As the growth advances the bacteria sink to the bottom. In pure culture the bacillus appears as a short, oval, somewhat plump rod with rounded ends; usually two are found together. Its length amounts to $0.8-1.5 \mu$. The apparent threads which are frequently observed may attain in pure culture a length of $30 \mu$. The bacillus has no motility, and is a facultative aërobe.

Kolb's bacillus is pathogenic in mice, and rabbits and pigeons are susceptible; on the other hand, guinea-pigs very rarely or never succumb to the infection. In susceptible animals the clinical picture corresponds to human purpura hemorrhagica. In rabbits the characteristic purpuric spots may be produced with cultures free from bacteria and mice may be killed in this way.

\section{SPECIAL SYMPTOMATOLOGY}

The most characteristic and prominent symptom of the disease, forming its distinguishing feature, and attracting most attention, is the purpura which develops upon an entirely normal skin without producing any inflammatory disturbance. As the areas of the skin upon which the hemorrhages appear are up to the moment of their development entirely intact, we may assert with 
great probability that these hemorrhages have their origin chiefly in internal causes, and are the expression of the so-called hemorrhagic diathesis. Although the intensity and extent of the hemorrhagic efflorescence is no exact indication of the severity of the disease, observation nevertheless proves that the small, isolated, and rapidly disappearing petechiæ represent the milder type, while the more distributed and very diffuse suggillations, with a protracted course and a tendency to repeated relapses, indicate a more severe form of the disease. At the same time, all the transitional stages from the mildest to the severest of cutaneous affections may occur, and cause the body to appear as if spattered with a large brush dipped in blood. There may be extravasations of the size of a plate, which occasionally leave but few large areas unaffected, and are of a violet to a dark red color. Deep-seated flat hematomata may also arise and extend into the muscles, and over these the skin presents the well-known changes of extravasated hemoglobin, showing all the colors of the rainbow. Finally, streaks and striæ-like "vibices" appear, particularly in the flexure of the knees, which resemble analogous conditions in scurvy, and which go through all the changes in tint and shade from dark blue to greenish yellow.

We have still to consider the relation of the hemorrhagic efflorescences to the complications, particularly to the arthritic affections and to the gastric phenomena.

The appearance of the purpuric areas in the complicated cases differs in no respect from ordinary purpura. Occasionally the eruption begins with the appearance of typical urticaria with vesicles, which gradually become filled with a hemorrhagic fluid, and soon dry in the way so well known in urticaria; the pustules then collapse and disappear without leaving residua. In other cases the urticarial vesicle leaves behind it a hemorrhagic area. Occasionally in the same individual alternating attacks of urticaria and purpura are noted. It has often been remarked that patients who suffer from conditions of this kind have previously suffered from urticaria. Sometimes the development of petechiæ is preceded by the formation of diffuse erythema, in the course of which occasional miliarial vesicles appear.

The changes which hemoglobin undergoes in the hemorrhagic eruptions of the skin correspond as a rule to the well-known processes. Occasionally, however, we see very extensive suggillations of enormous size and of very different ages; in such cases extraordinarily striking pictures are noted which confuse the inexperienced, particularly so if recent hemorrhages are superadded to old ones, which represent all the color modifications and all the tints of altered blood. After absorption pigment is noted with the naked eye only in those cases in which huge extravasations of blood have occurred. Even this, however, disappears after some time, so that with the lapse of weeks or months nothing remains to indicate the process which has taken place. The conditions are different in the microscopic investigation; the pigment deposited and accumulated in the rete Malpighii remains there for a long time, so that after some months-after the disease has run its course-we are still able to recognize in this area that the skin was once the seat of a decided effusion of blood. 
Besides small hemorrhages, not infrequently other forms of deeply situated hemorrhages take place in the subcutaneous tissues; indurated, reddish or bluish, scarcely movable suggillations and infiltrations appear between the periosteum and the external skin (for example, upon the tibia and the vault of the skull), also in areas in which no bones lie just beneath the so-called. erythema nodosum. These larger and smaller infiltrations, over which the skin shows a bluish discoloration and is but slightly movable, are found particularly in areas upon which external pressure has been exerted for some time. These are found occasionally in the course of purpura with and without arthritic affection, but they do not make the slightest impression upon the course of the disease.

Regarding the seat of the petechiæ, the lower leg is most frequently attacked; next, the abdomen, the back and the upper extremities; most rarely, the face and the mucous membrane of the mouth.

I must not omit to mention that in undoubted simple purpura the gums are sometimes very decidedly affected, while, as is well known, cases of scurvy occur without disease of the gums. But we should judge very superficially if we drew from this the conclusion that the latter is a mild form of scurvy, or the former a severe purpura. In this connection I will refer to the description of scurvy (see page 402), and will here simply state the fact that in the course of purpura disease of the gums occasionally occurs, and may lead to severe changes therein with fetor but without the sponginess and ulceration and destruction of the gums or the loosening of the teeth which is so common in the case of scurvy.

The number of successive hemorrhagic eruptions on the skin varies within wide limits from one to twenty attacks; on an average, four attacks have been mentioned.

The relation between the time of the appearance of cutaneous hemorrhages to the other symptoms is a very changeable one; usually, it is true, the former is the earlier symptom. But in children, according to the descriptions of $\checkmark$. Dusch, this rule does not hold and very frequently attacks of severe intestinal phenomena without the simultaneous appearance of purpura are observed, or vice versa. An inflammatory effusion is present in one or more joints which, on account of its long existence, may remain until several crops of purpura have appeared and disappeared. An effusion of this type in one or another joint is somewhat more rare in children than in adults, in whom it appears in about 50 per cent. of the cases; edema in the surroundings of the affected joint is met with particularly in adults.

The effusion is rarely very marked, never becomes purulent, and leaves no disturbances of motion behind. The visible swellings are preceded in almost all cases by arthritic pains or by sensitiveness upon pressure and movement. The joints of the lower extremities are most often attacked, after this the joints of the hands. The differential diagnosis between purpura rheumatica and purpura gonorrhoica may be very difficult under some circumstances, especially if the patient denies a preceding gonorrhea. The prognosis of the former is usually favorable. Peliosis rheumatica differs from acute articular rheumatism in its entire clinical picture, in the course of its fever, particularly in 
the absence of a tendency to profuse sweats and endocardial or pericardial implication, and, finally, by the fact that very frequently the joint phenomena are quite transitory and appear much milder than in the other affection, although this cannot be designated as a constant, invariable rule.

The relation of the arthritic affection to the hemorrhagic disease appears, apart from the arthritic hemorrhages, to be by no means clear. It becomes somewhat less obscure if we consider that in the hemorrhagic diathesis the serous membranes are especially liable to attack and we know from the analogy of other arthritic affections that the serous membranes bear a certain relation to the joints, so that both are often attacked at once.

It is hard to decide whether the implication of the joints of the knee and of the foot, so frequently observed, can be explained by the suggestion that the general predisposition to purpura would naturally fall heaviest on those joints whose function it is to carry the weight of the body, and which for this reason are particularly exposed. Of course this explanation should be similarly operative in other multiple joint affections.

I suppose that the sum of our positive knowledge is this: There is an anatomic relationship between the joint cavities with the serous membranes; hemorrhages into these occur under the influence of the hemorrhagic diathesis. Yet, as regards the arthritic affections in purpura hemorrhagica and in morbus maculosus Werlhofii, it appears to me by no means so firmly established as in the analogous joint disease in scurvy and hemophilia that these are invariably and absolutely hemorrhagic. The clinical symptoms, results of occasional joint-puncture and the autopsy findings in the Traube-Leuthold case (already mentioned three times) are all opposed to this.

Of the intestinal symptoms in severe and complicated forms of the disease painful colics are the most characteristic. These resemble the form seen in chronic lead-poisoning. The seat of pain is referred to the region of the navel, from which it radiates to various places. Although at the autopsy hemorrhagic infiltrations of the intestine with ulcer formation in various areas have repeatedly been found, v. Dusch advises caution in the interpretation of reports from the patient: "Bloody vomiting occurred," or, "Tarry hemorrhagic discharges occurred." $\mathrm{He}$ is of the opinion that such reports prove nothing, and especially not that the seat of hemorrhage is actually within the intestinal canal. It must be remembered that in small children, in sleep, and in comatose patients who maintain the dorsal decubitus considerable quantities of blood may flow unnoticed from the nose into the stomach and be discharged by rectum. I cannot suppress the opinion that this explanation seems very forced and artificial, and I do not doubt that in the cases of severe colic actual enterorrhagia often occurs, a view which is more firmly founded upon the fact that the colics decrease decidedly in severity after profuse hemorrhage from the intestine.

Albuminuria is by no means a rare complication in the course of the various forms of the hemorrhagic diathesis, for the most part without an inflammatory condition of the kidneys. In some cases I have seen the excretion of albumin in every attack, and occasionally it extends into the intervals. In other cases the excretion of albumin lasts for ten months and longer, but 
finally disappears spontaneously without any medication. In other (but much rarer) cases of morbus maculosus Werlhofii, contracted kidney develops, becomes chronic and gradually leads to death. According to my observations of many cases, even after a simple albuminuria (i. e., without casts and leukocytes) has existed for many months, the hope of recovery must not be given up, for this condition may disappear as suddenly as it arose.

In regard to the changes in the eye, retinal hemorrhages have occasionally been observed; they sometimes show white centers. Hemorrhages have also been noted in the choroid and in the sclera. The flow of tears may also show a reddish discoloration. Nettleship saw bilateral neuritis after purpura.

We have no definite knowledge of the causes of febrile phenomena which either precede the cutaneous hemorrhages or occur in the course of the same, and which also are not rarely absent. A more exact insight into these conditions can only be obtained when the etiology of the disease has been established. One fact which I have demonstrated repeatedly appears worthy of mention: even high temperatures may not be influenced in the slightest degree by extremely profuse hemorrhages from internal organs (kidney, lungs, intestines).

In a case of intermittent fever with temperatures up to $102.2^{\circ} \mathrm{F}$. which Kaltenbach observed for a long time, defervescence occurred by lysis. The case was not a typical one. Sudden appearance of high temperature is always suspicious, and points to the existence of some complication.

Hemorrhages from internal organs are, in the main, rare; apart from the likewise quite rare epistaxis, renal hemorrhages occasionally occur as the expression of a recent hemorrhagic nephritis; very scanty hemorrhagic casts with normal and abnormal red blood-corpuscles in the urine determine the diagnosis. The amount of albumin in such cases is always very great. Besides the hemorrhagic form of nephritis, we may also see during the course of, and in immediate connection with, the disease, a nephritis without hemorrhagic constituents. This form very frequently gets well; in rare cases, however, it may lead to edema and uremia.

Putmonary hemorrhages rarely occur in persons with previously healthy lungs. If it is certain that hemorrhagic sputum is present, the first assumption should be that the blood may come from the bronchi. Among other hemorrhages which are, however, most uncommon, I must mention meningeal and cerebral hemorrhages.

Regarding the condition of the spleen but very little can be said; it is certain that in some cases distinct enlargement has been determined and the organ has been felt below the ribs as a more or less soft tumor. In the great majority of cases, however, this enlargement is absent during the entire course of the disease, even in the severest type, of hemorrhagic purpura running a fulminant course.

The composition of the blood in cases of pure hemorrhagic purpura is mentioned by some authors as having shown a slight decrease of the red and an increase of the white blood-corpuscles.

Ajello found the red decreased to $2,500,000$ to $3,000,000$, while the specific gravity of the blood was 1.043 . The erythrocytes are said to undergo an 
especially rapid regeneration and show no morphologic changes, except that Spietschka found after protracted hemorrhages nucleated red-blood cells with polychromatophilic protoplasm. In two cases of hemorrhagic purpura he made frequent blood-examinations, counting the blood-corpuscles, estimating the hemoglobin, and staining permanent preparations with gentian violet and aurantia. In one case the number of blood-corpuscles in the hemoglobin remained constant; in another only showed transitory variations. In both cases after each severe hemorrhage a conspicuously large number of crythrocytes showed distinct nuclear staining. Spietschka looks upon these nucleated blood-corpuscles as juvenile forms, and regards their appearance as a sign of a markedly increased regeneration of the blood.

In a child which died of anemia following purpura hemorrhagica, Billings found in the blood only 500,000 to 700,000 red, 4,000 white corpuscles, and 17 per cent. of hemoglobin; 75 to 80 per cent. of the leukocytes showed no mononuclear forms; no poikilocytosis, no nucleated reds. The blood findings, in the absence of signs of blood regeneration, resembled those of pernicious anemia.

According to my own numerous investigations, the hemoglobin is frequently greatly decreased; more so than would correspond with the diminution in the absolute number of the red blood-corpuscles.

Silbermann found in a case of Henoch's purpura most of the red bloodcorpuscles normal, a few containing but little hemoglobin, others entirely without color ("shadow-corpuscles"). The leukocytes were numerous and rapidly deliquesced; the blood-plaques were markedly increased. To determine any functional damage in the morphologically intact red blood-corpuscles, their properties were tested according to the method of Maragliano: 1. After enclosure in paraffin; 2. After heating; 3. After compression; 4. After mixture with 0.6 per cent. salt solution. The result of this investigation was the following: In the fresh blood examined under paraffin, he found normal erythrocytes with few exceptions, and very numerous leukocytes. Two hours later, in the same preparation, numerous "shadow-corpuscles" were seen, many feebly stained red blood-discs, and a few microcytes; the majority of the white bloodcorpuscles were destroyed. In their place there was found a granular, grayish white heap. Five hours after the enclosure of the blood in paraffin but few blood-corpuscles were to be seen, the majority of them being completely destroyed. In the fresh, undiluted blood the red dises were destroyed by slight pressure exerted upon the cover-glass with a needle; the same occurred upon heating of the blood at $30^{\circ} \mathrm{C}$. In 0.6 per cent. NaCl solution a great number of the red discs lost color at once; the white blood-corpuscles were very soon destroyed, and formed glassy clumps. In sharp contrast with the results just described are the blood-findings during the days of the disease when the patient was still quite well. In this blood neither shadows nor numerous lenkocytes are to be seen, nor even greatly increased blood-plaques; under the influences previously mentioned the erythrocytes show a normal resistance, i. e., they neither lose color very rapidly, nor are many of them destroyed.

For many years I have examined minutely the blood of patients attacked by various forms of purpura, and have found by no means uniform results. 
In many cases very few deviations from the normal were recognized, in many others the signs of anemia were distinctly observed. Anemia is the sum and substance of the blood changes in this affection, if such changes exist at all. Microcytosis, poikilocytosis, numerous pessary forms, a relative and absolute decrease of hemoglobin, blood-plaques increased, occasionally very much so, and profuse cylinder-formation in the blood, such are the important changes which I have observed. An increase of leukocytes, a slight destruction of the same, and the appearance of isolated, nucleated red blood-corpuscles are occasionally but by no means constantly seen. Moreover, the number of leukocytes is subject to extraordinarily rapid change. The many "pessary forms" of red cells which are quite frequently found, in fresh preparations as well as in smears stained with eosin, are only a sign of the scant hemoglobin contents of the erythrocytes, and it is, therefore, not remarkable that in spite of a normal number of red cells the hemoglobin may be decidedly diminished. In the investigation of the fresh blood of patients who suffered from purpura, upon a warm slide (heated to $30^{\circ} \mathrm{C}$.), no change in the corpuscles, either in the red or in the white, was perceptible; they resembled perfectly the blood-corpuscles of healthy persons or were, for example, like those in malaria patients whose blood I had used for comparison.

\section{TREATMENT}

Prophylaxis.-On account of the spontaneous and unexpected appearance of purpura there can be no question of prophylaxis. The frequency of relapses should be a warning to those who have recovered once or repeatedly from the disease, to protect themselves for a long time against deleterious external influences, particularly chilling the body. Change of climate has proven very beneficial.

General Treatment.-The patient must remain in bed for a long time, even if no fever is present. The more rigidly this rule is adhered to, the more certainly will relapses be prevented. He is to move about just as little as possible. The more experience I have with cases of this kind, the more thoroughly I am convinced of the great value of rest in bed. The absolute necessity of this must be impressed upon the patient, so that, from conviction and not merely to please his physician, he will submit and adhere to this very tiresome régime. Every arrangement must be made for the patient's comfort during this prolonged rest in bed; he should be taken down into the garden, or upon a shady veranda, balcony, or the like. Further, the nurses must see that no part of the patient's body is subjected to external pressure, and that he does not bruise or injure himself. With this end in view his bed must be very carefully prepared. Above all, folds in the bed sheets must be prevented. The covering is to be light, the sick room to be kept cool. Psychical excitement and mental exhaustion are to be strictly avoided under all circumstances.

The nutrition must be bland. All foods are to be given cool. Strong coffee, tea, and alcohol are prohibited; only with symptoms of collapse may the latter be made use of. Of foods, milk is most important, perhaps mixed with somatose. Lemonade or orangeade may be used as a drink. 
It is important that attention be paid to the fecal discharges on account of possible hemorrhages and intestinal parasites (or their ova). In case of constipation no salines should be used, but only mild regetable laxatives, such as castor oil, tamarinds, rhubarb, cascara sagrada and others; enemata and irrigations of water may also be utilized. Laxatives should be changed frequently so that the intestine may not become accustomed to a certain remedy.

Special Treatment.-Upon the authority of Werlhof, sulphuric acid is given, even to-day, in the form of Haller's acid elixir, which Werlhof praised as a specific. He also advised in purpura decoctum cortex chinæ (8-10:200, a tablespoonful every two hours). The administration of ergot, of lead acetate, turpentine, and liquor ferri sesquichlorati has been based upon the pathology of the disease. In very stubborn, frequently relapsing cases, I have seen good results from arsenic in the form of Fowler's solution, especially in combination with carbonated or warm baths rich in salt. I can highly recommend this treatment, particularly in the cases in which all other methods have been without result.

Henoch has used ergot in the following manner:

h Ext. secal. cornut.................. 1.5

Aq. destill....................... 150

M., D., S. : For children a teaspoonful, for adults a tablespoonful, every three hours.

I cannot so strongly recommend this. Liquor ferri sesquichlorati may be given in gruel, from one to five drops three times daily.

The following medication is also advised, although $I$ have never seen any results from it: Twenty to thirty drops of the fluid extract of hydrastis canadensis every two to three hours.

In peliosis rheumatica the employment of sodium salicylates, aspirin and antipyrin is worthy of trial.

In epistaxis, dorsal decubitus with the head low, cold to the nape of the neck, and liquor ferri sesquichlorati are indicated. The insufflation of cold water, to which a few drops of the previously mentioned iron salt are added, will often control the hemorrhage. Tampons of cotton dipped in the iron solution may also be necessary. Gastric and intestinal hemorrhages are to be combated by ice in combination with opium. Therapeutic requirements are, however, rarely necessary, as the condition is infrequent. The same is true of renal hemorrhages.

With symptoms of collapse, stimulants are to be used (wine, coffee with brandy, champagne, camphor, ether). The application of heat may become necessary (hot bottles, hot sand bags). In severe cases hypodermoclysis of physiologic salt solution, to which oxygen has lately been added with success, may be resorted to.

During convalescence the patient must be placed on a nourishing diet. Residence in the country, by the sea, or in the mountains is advisable. Of drugs, iron is the best. The urine is to be frequently examined even after the actual disease has run its course, for sometimes in immediate sequence or a 
long time afterward (weeks and months) albuminuria may appear. Occasionally this disappears with a stimulating diet and warm baths; in other cases it remains and passes into a chronic (interstitial) nephritis. For the treatment of this condition, the article upon renal disease may be consulted; but I may remark here that, even in such cases, I have seen favorable results from cold sea baths, though only in well-nourished and robust individuals. 
THE ANIMAL PARASITES 


$$
\text { , }
$$




\title{
THE ANIMAL PARASITES OF MAN
}

\author{
By E. PEIPER, Greifswald
}

BY " animal parasites of man" we mean the organisms which live temporarily or permanently on or in the body of man and derive their nourishment from it. There is no difference, properly, between animals that live as parasites and those that live free. For, on the one hand, there are parasites-e. g., the ascaris nigrovenosa (this species, indeed, does not live on man) - which produce mature offspring capable of living free, and, on the other hand, animals which usually live free may appear as parasites. Thus, the larvæ of flies, which usually live on decaying organic substances, are found "occasionally" as parasites in man, either in the intestinal canal or in suppurating wounds. These occasional parasites must not be classed with the so-called "pseudoparasites." By the latter we generally mean foreign bodies of various kinds and origin which are manifest per vias naturales.

As stated, we distinguish between a temporary and a permanent parasitism. Fleas and bed-bugs infest man only transiently. The development of these organisms is independent of the human body. Temporary parasites include the ectoparasites (or epizoa) which inhabit the skin, the conjunctival sac, the mouth, the nose and its accessory cavities. It is true, the ectoparasites also include some permanent parasites. Most of the permanent or stationary parasites are found as entoparasites or entozoa in the internal organs: in the intestinal canal and the glands belonging to it, in the lungs, in the heart, in the brain, and in the muscles. But the permanent parasites are not all entoparasites, for many of them live also on the skin. Lice and sarcoptes hominis rarely leave the body of man upon which they develop from the ova and multiply.

Many parasites, such as tenia, ascarides, and ankylostoma inhabit man only when mature; others, such as the echinococcus, only during a certain period of its development. Man, therefore, is either the actual host or only the intermediate host. For many parasites, such as tenia solium and tenia saginata, man has the doubtful honor of being the only host. But, man also becomes the host of parasites which, as a rule, select another host animal. Thus, the cysticercus cellulosæ occur not only in the pig, deer, and cat, but by autoinfection also in man. Balantidium coli and echinorhyncus gigas are specific parasites of the pig, but occasionally they are found in man.

Since remote times physicians and zoologists have attempted to ascertain the origin of the parasites. As a rule, this has been easy with the ectoparasites; but exceedingly difficult with the entoparasites. For not only in the 
intestine, but in the organs of the human body that are beyond the reach of external contact, parasites are found whose origin baffles conception, and most remarkable suppositions and opinions have arisen regarding this origin. The most simple and most natural explanation of this secret of nature was the assumption of a generatio cequivoca. It was believed that spontaneous generation, which was supposed to be common among the lower animals, occurred also among the entoparasites. Endeavors were made to demonstrate that the parasites developed from the intestinal mucus, from inspissated fecal masses, and from decomposed blood. Step by step, the proof was slowly furnished that not only highly organized animals, but also the lower were endowed with sexual powers, and that the parasites developed only from fecundated ova. Investigators have had to contend with one great difficulty, namely, the occurrence of young, asexual forms. But the transition of these into intermediate hosts, their return to the specific host, and their development into mature forms was finally established experimentally.

With certain exceptions, sexual maturity and parasitism coincide for those entoparasites which are under discussion. The ova or embryos of the mature parasites reach the outside world, and here, protected by a covering which is very resistant to external influences, they continue their development, and finally reach an intermediate host. Many exceptions occur. The embryos of the trichina spiralis pass from the intestinal wall of the host immediately into his tissues. Others, slipping from the sac of the ova, live free for some space of time; still others migrate directly into the final host, there to reach their maturity. Asexual forms first pass into an intermediate host, where they are harbored and still further developed, until at last they reach their final host; or, after the lapse of some time, they become subject to retrogressive changes.

Our knowledge of the distribution of the animal parasites in man is very incomplete. It is certain that oxyuris, ascaris and trichocephalus, as well as the ectoparasites, pediculus, cimex and pulex, are found throughout the world, and are probably the most common animal parasites. Others, such as echinococcus, ankylostoma, filaria, are observed only in certain countries, outside of these only sporadically, and then introduced from infected districts. Precise statistical reports regarding the distribution of the parasites, which might assist in the explanation of various questions, are very limited in number.

Aside from the influence of climate and soil, the frequency of the occurrence of parasites in man is due also to the customs and habits of a country, and, no less, to the personal cleanliness of the individual inhabitants. I do not refer merely to ordinary cleanliness of the body, which is of importance in the transmission of the ectoparasite, but particularly to cleanliness in kitchen and household. There can be no doubt that the majority of the parasites are transmitted by food and drink, by ingestion from contaminated vessels, soiled hands; etc. In countries where, as in Abyssinia, beef is eaten raw or at least not sufficiently well cooked, the beef tape-worm is greatly disseminated. The tenia solium is not rarely found in Central Germany, where raw or half-cooked pork is often eaten. For the same reason endemics and epidemics of trichinosis have not yet been completely exterminated in these districts. The intake of the 
flukes need not necessarily be with measly meat; it may be brought about by secondary conditions. It is impossible to enumerate all the accidental ways by which the flukes may be transmitted to food, especially in butcher shops and restaurants in which proper cleanliness is lacking. The transmission of the bothriocephalus latus occurs in a manner similar to that of the cysticerci, the hooklets of which are shown to retain great vitality in smoked or frozen pike.

The rôle assumed by insects, especially the common house-fly, in the dissemination of parasites is very interesting. Grassi has shown that the ova of the tenia solium, oxyuris vermicularis, and trichocephalus dispar, pass through the intestine of the fly without change. But Stiles reports that the ova of ascarides undergo further development in the intestinal canal of the fly. Owing to the very general prevalence of flies and their habit of settling upon various food-stuffs, on which they defecate, there can be no doubt that these insects contribute materially to the distribution of the ova of the helminthes. It is certain that vegetables of various kinds and sources, if not thoroughly washed, or if eaten raw, may become the carriers, the same as meat if it has but come in contact with the ova of helminthes.

We know, from the interesting observations of Lutz, how the ascarides develop. The rural population especially, and, above all, the children by playing upon infected soil, acquire the embryos of the ascaris lumbricoides from the soil, whereas the parasites are far less frequent among city-bred people, even among the children. The affection is often transmitted by food contaminated with infected earth, often by means of the drinking-water into which germs capable of development have lodged.

The rôle of drinking-water in the distribution of the entoparasites is undoubtedly of great importance. I do not refer only to the transmission of occasional parasites, but, above all, to the protozoa, the ova of ascaris and of trichocephalus, especially the immature forms of the trematodes, which reach the human intestinal canal by polluted drinking-water. Water has an influence also in the development and distribution of the ankylostoma duodenale, as we shall see later. Since the investigations of Loos, it can no longer be doubted that the last-named parasite is able to force its way into the human organism through the uninjured skin. A number of authors believe this true also of the filiaria medinensis (guinea worm). So manifold are the ways in which the young forms of the parasites, or the parasites themselves, migrate into man that we cannot enter upon them here.

When we endeavor to ascertain whence the former originate, we note that the majority of parasites are derived from animals, especially from our domestic animals, dogs, cats, pigs, and cattle. I refer only to the group of the cestodes, to the trichina, and to numerous epizoa. The more intimate the relations between animal and man, the greater the danger of transmission. Furthermore, man himself is often not only the carrier, but the disseminator of the parasites. Not only does he infect his surroundings with the young, but occasionally he infects himself, as shall be demonstrated later in the discussion of oxyuris vermicularis.

In regard to the symptomatology, there is perhaps no realm of pathology in which so many contradictory opinions have been expressed as have been 
called forth by the animal parasites, especially by the entozoa. Formerly, when certain morbid phenomena could not be explained, enigmatical symptoms were ascribed to the accidental presence of one or several parasites, thus giving rise to many vague conceptions; later there was an evident inclination to ascribe little, or no, reaction upon the affected organism to animal parasites, especially to the entozoa. Undoubtedly, both tendencies were wrong. The phenomena observed in many persons infected by parasites, especially the nervous manifestations, cephalalgia, pruritus, disturbances of sight and hearing, mydriasis, sensations of general weakness and lassitude, should not always be regarded as the consequence of helminthiasis. The decision regarding the causal connection depends also upon whether the symptoms have occurred only after the appearance of the parasite and have ceased soon after the expulsion of the unwelcome visitor. Thus, Cobbold and Davaine report a number of cases in which various nervous symptoms disappeared after the expulsion of a tape-worm. In a case of epilepsy which had persisted for three years Marx saw a cure take place after the expulsion of tenia solium. In a patient who suffered from tabetic phenomena, and in whom there was marked temporary myosis and loss of the pupillary reaction, Denti noted that all symptoms were cured by the expulsion of a tenia solium. Noticeable, too, are the frequent reports of disturbances of the visual apparatus in helminthiasis. In short, it is certain that considerable disturbance of health, sometimes even serious danger to life, may be caused by the presence of parasites.

It is obvious that delicate, nervous, anemic persons suffer much more from the presence of parasites than robust individuals, whose power of resistance is greater, and that the reaction in the former is much more violent. The withdrawal of food-stuffs which are easily assimilated but which often cause the expulsion of the tape-worm chains, meters in length, or numerous ascarides from the intestinal canal, must inevitably, apart from the local reaction, be followed by injurious sequels, especially in weak persons and children. The presence of hundreds, even thousands, of blood-sucking ankylostomata cannot fail to produce serious disturbances. Parasites which have lodged in vital organs, in the liver, lungs, heart and in the eyes, in the brain and the spinal cord, even if relatively small, will certainly cause serious functional disturbances and disease.

The investigations in the last decade by Reyher, Runeberg, Dehio, Schapiro, Schaumann, and others, have demonstrated that the animal parasites, especially the bothriocephali, produce toxins which, taken into the blood, may lead to the severe manifestations of pernicious anemia. The secretion of toxic substances by other animal parasites, such as echinococci, ascaris lumbricoides, ankylostoma duodenale, etc., is also probable. We do not as yet know the toxin itself, but we recognize its deleterious effects.

The number of parasites occurring in man is very large. Every year furnishes reports of the appearance of animal parasites until then unknown. In the following I shall review the fauna of man. It seems most practicable to discuss them in a systematic order. I begin, therefore, with those organisms which can only with difficulty be separated from the lowest vegetable organisms. 


\section{PROTOZOA}

We designate as protozoa the primordial animals, those microscopically small but living organisms which, as monocellular elements, singly or in colonies, collectively represent the simplest animal type. The substance of the body, sarcode, consists of a contractile, finely granulated protoplasm which is mononuclear or polynuclear. The viscid, hyaline ectosarc is capable of motion by contraction and expansion, or by extension and retraction of pseudopodia, by cilia and flagella. There can be no doubt that it is concerned in the ingestion and excretion of food. Fissures and openings in the ectosare take the place in some protozoa of the functions of mouth and anus. The soft endosare serves for the digestion of the food consumed; the contractile vacuoles, occurring in many protozoa, are intended for the accumulation of fluids to be excreted. Propagation takes place by segmentation, gemmation or sporulation.

We know that all three varieties of the protozoa, the rhizopoda, the sporozoa and the infusoria, occur as parasites in man.

The rhizopoda, " root-footed" organisms, include the amoba coli (Loesch), discovered in 1875, the amoba coli felis of Quincke in 1893, the amœba coli mitis of Quincke, which is minutely described in the interesting treatise of Hoppe-Seyler, "Dysentery and Ameba Enteritis," ${ }^{1}$ and the amoba coli vulgaris of Quincke. The investigations of Quincke and Roos have proven that the latter are not pathogenic either in man or in cats. The severe and wellknown dysenteric symptoms caused by the first-named ameba may be materially complicated by the supervention of liver abscesses. Cases of infection by amœba coli mitis are considerably milder, but of a distinctly chronic course. For details regarding these parasites I refer to the previously mentioned article.

Amebre in man are not confined to the intestines. I have referred to their occurrence in the pus of liver abscesses in dysentery. Jjima demonstrated

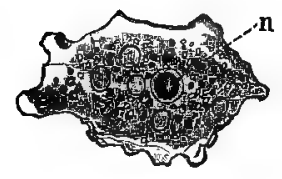

Frg. 26.-AMEBa AT REST.

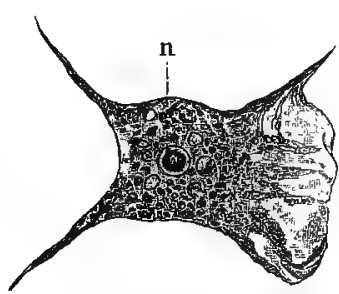

Fig. 27.-Ameba iN Motion.

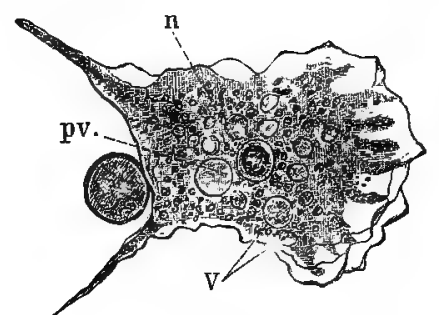

Fig. 28. - Ameiba FlatTENED.

Figs. 26-28.-Leynenia Gemmipara. (After von Leyden-Schaudinn.) $n$, nucleus; $v$, vacuole; $p v$, pulsating vacuole.

amebæ in pleuritic and peritoneal exudates. Gros and Sternberg have reported the presence of amebæ in the tartar of the teeth. Flexner found amebæ in an abscess in the oral cavity, Kartulis in the pus and in an extracted portion of 
the lower jaw. Baelz observed amebæ in the urine, Jürgens in the mucous membrane of the bladder, Kartulis in a tumor of the bladder, Wijnhoff and Zeehuisen in mucohemorrhagic urine, and their causal relation to the morbid phenomena is not yet explained. This detection of amebæ is undoubtedly very interesting, but a definite opinion regarding their significance cannot be arrived at until the culture of amebæ in pure culture has been accomplished. Then we shall be able by experiments to estimate their true significance as generators of disease.

v. Leyden and Schaudinn found in the ascitic fluid of two cases of carcinoma structures of an irregular polygonal or globular form, which, in a contracted condition, measured from 3 to $36 \mu$. Uncontracted, they possess the power of ameboid movement, and send forth processes which are either hyaline or lamellar or granular. The plasma is thickly studded with very refractive granules of a yellowish luster. Several vacuoles for fluid, among them one pulsating, are generally found, and one nucleus. Propagation occurs by gemmation and segmentation. Similar structures were found by Lauenstein and Behla in the ascitic fluid of patients with carcinoma. It is still undecided whether the Leydenia gemmipara Schaudinn, 1896, has any relation to carcinoma.

The second class of the protozoa, the sporozoa, includes parasites which have also been discussed by the above-mentioned authors, such as the coccidium oviforme, Leuckart, 1879, the coccidium perforans, Leuckart, 1879, and the coccidium bigemimum, Stiles, 1891. These parasites are rarely found in man, it is true, but, when present, they cause obstinate intestinal catarrhs which are difficult to cure. Undoubted cases of coccidiosis of the kidneys and of the ureters have also been described by Lindemann, Bland-Sutton, and Targeff. In the case reported by Clarke numerous small, greenish-brown cysts containing the parasites in varying sizes were found in the renal pelvis, in the ureters, and in the bladder.

Miescher's or Rainey's tubes, which became known from the microscopical examination of pork, are of no pathogenic significance and are only rarely found in man. Apart from the doubtful cases of Lindemann and Rosenberg, such structures have been found in man only by Kartulis in the muscles of the abdomen, and by Baraban and Saint-Remy in the muscles of the larynz.

The most important group of the sporozoa are the hemosporidia which include, as is well known, the malarial parasites of man. The highly interesting history of the development of these parasites, and also the pathological condition produced by them, have been exhaustively discussed in the chapter on Malaria (which see in volume "Infectious Diseases").

In concluding this study of the sporozoa, I desire to mention that in the future we may possibly be able to attribute other parasitic affections of man to the parasitic nature of the protozoa. Some well-known authors assume that the pathogenic germs of carcinoma, sarcoma, scarlatina, measles, smallpox, pertussis, etc., are represented by certain protozoa yet to be found or to be investigated. Unfortunately, the researches undertaken up to the present have not led to a positive, indisputable result.

The third class of the protozoa embraces the infusoria, which are structures 
of more or less constant form, and covered with cilia in varying number and arrangement. The infusoria include the flagellates and the ciliated parasites.

Of the flagellates, trichomonas vaginalis, Donné, $183 \%$, is frequently found in yellowish, markedly acid vaginal mucus, whereas the parasite is not present in the normal secretion of the vagina. The parasite, which is elliptical in shape, and 0.01 $\mathrm{mm}$. in length, is elongated into a delicate process at the anterior border of which there are usually four long, slender, whip-like flagella. An undulating membrane, the contracting wave of which is continually directed anteroposteriorly, extends spirally from the point of origin of the flagella. The parasite was found in purulent urine by Marchand, Miura and Dock; in human feces by Janowski and Skaller; in the contents of the stomach in carcinomæ cardiæ by Strube. It also appears that the flagella found by Davaine, Grassi, Eckekrantz, Cunningham, Tham, Marchand, Zunker, Epstein, May, Roos, Schuberg, Schürmayer, and others, which, following Davaine (1854), are designated as trichomonas hominis, are not essentially different from trichomonas vaginalis. Similar structures were also found in the sputum in gangrene of the lungs by Kannenberg, Streng and Schmidt, in serous pleural exudate by Litten, in putrid pleurisy by Roos. It appears that the parasite designated plagiomonas urinaria by Künstler, in 1883, also belongs in this class. The cilia observed by earlier authors were probably only

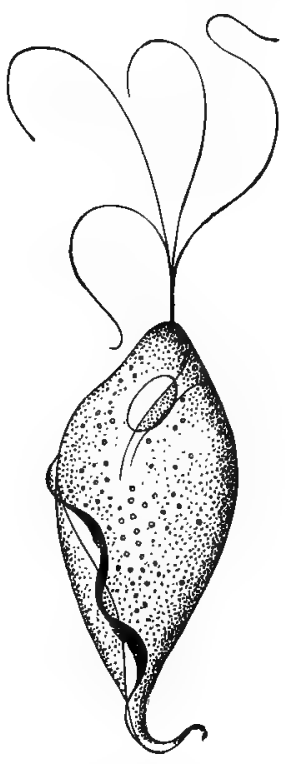

Fig. 29.-TRichomonas VAGINalis, Donné; ConsiderABLY ENLARGED. (After Künstler.) simulated by the continuous motion of the undulating membrane. The number of the flagella is by no means characteristic.

No positive statements can be made regarding the significance of the trichomonades as generators of disease. Yet it is quite conceivable that, if present in enormous quantities, they might lead to long-continued suppuration and catarrh of the intestine. As a rule, they are easily exterminated by repeated doses of $0.1-0.2$ of calomel.

Megastoma entericum Grassi, 1881, and s. Lamblia intestinalis Lambl, 1859 , are of the same significance. The exact description of this parasite will be found in the chapter on Dysentery, ${ }^{1}$ where it is also stated that the parasite, if occurring in large numbers, may cause an intestinal catarrh which is usually cured without difficulty unless complicated by carcinoma or tuberculosis.

Of other flagella, monas globulus, Dujardin; monas lens, Dujardin; monas elongata, Dujardin; bodo intestinalis, Ehrenberg; cercomonas acuminata, Dujardin; cercomonas globulus, Dujardin; cercomonas biflagellata, Stein- 
berg; trichomonas elongata, Steinberg; trichomonas caudata, Steinberg; and trichomonas flagellata, Steinberg, have been probably found as simple commensals.

Of the cilia inhabiting man, cilia infusoria, we are most interested in the balantidium coli, Malmsten, $185 \%$, the pathogenic significance of which as the generator of severe dysenteric intestinal catarrhs is also described in the chapter on Dysentery. Stockvis has also found balantidia in the sputum of a soldier who had returned from the Sunda Islands.

Jacoby and Schaudinn have recently found a new balantidium minutum n. sp. in diarrheic stools, and also the nyctotherus faba n. sp., illustrations of

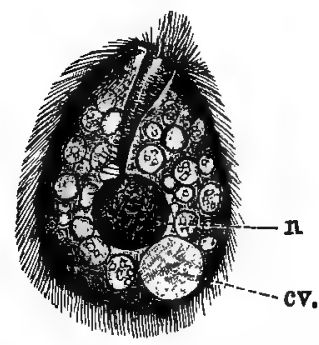

Fig. 30.-Balantidium Minutum.

$n$, nucleus; $c v$, contractile vacuole. (After Schaudinn.)

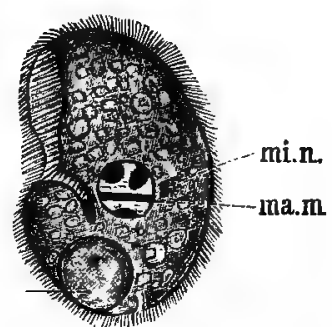

Fig. 31.-Nxctothrerus Faba.

mi.n, micronucleus; ma.m, macronucleus. (After Schaudinn.)

which follow. Vorticelli, which, according to Lindner, are said to infest man, cannot yet be recognized as parasitic structures.

This embraces the principal protozoa which occur in man.

We now come to the more highly-organized class, the plathelminthes or flat-worms. To this group, which embraces the two classes of trematodes, or sucking-worms, and cestodes, or tape-worms, belong the "vermes intestinalis" of the earlier authors or "helminthes" which-sit venia verbo-represent the true intestinal worms.

\section{TREMATODES}

The tongue- or leaf-shaped trematodes include the amphistomum hominis, Lewis and McConnell, 1876.

I wish to include this parasite for the sake of completeness. Observed but a few times in the tropics, it possesses no actual interest for us, especially since its significance as a parasite is by no means accurately determined.

Of greater importance is the distomum hepaticum, Linné, 1758.

The liver-fluke is found in the intestines and in the bile-ducts of a great number of mammals, especially in the sheep. Rot or liver disease in many districts creates great ravages among sheep. The parasite is also often noted to occur sporadically. Its body is oblong, flat, leaf-like, with a proboscidiform projection on the cone-shaped head. One of the two suctorial discs is situated at the point of the cone-like head, and contains the opening of the mouth. The other suctorial disc belongs to the abdominal surface. The oval eggs are 
of a considerable size: Breadth 0.075 to $0.09 \mathrm{~mm}$., length 0.14 to $0.15 \mathrm{~mm}$. The anterior pole is flatter and has a lid.

The development of the liver-fluke, explained by Leuckart and Thomas, is a very complicated one. The ova are discharged with the feces. After a prolonged stay in water, the elongated miracidium develops and after some time breaks the cover of the egg. By means of its ciliary sac it floats about, and finally is taken up by its first intermediary hosts, small water snails, the limnæus minutus and limnæus cahuensis, which are found in large numbers in small pools. In this host the miracidia become transformed into sporocysts, which are generally found in the respiratory cavity of the snail. The sporocysts produce a second generation of germ tubules, redia, from which, finally, the true young of the distoma develop in the form of caudate cercaria. The cercaria then emerge, adhere to grass and water plants and, with these, enter their final host in an

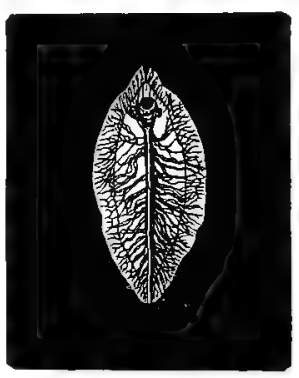

Fig. 32.-Distomum Hepaticum, Linné. encysted form. From the intestine they migrate into the bile-ducts, where they develop into mature animals:

It is obvious, in view of this mode of development, that man is but rarely inhabited by distoma, and even then only by a small number. The entrance of the cercaria into man occurs principally by their ingestion with polluted drinking-water or with raw vegetables, salad, or water-cress, which have grown in inundated places. In many other ways they may enter the body; we need only consider how often children and even grown people put meadow-plants into the mouth.

About 18 cases of disease of the liver in man from distoma have been recorded. As only very few parasites were present, no special symptoms were caused in the majority of these cases. Yet the cases of Bostroem and Biermer, in which the fatal outcome of the disease was due to the parasite, prove that even a single parasite may produce severe phenomena.

It seems that the parasites remain latent for considerable time. The general condition of the patient changes gradually for the worse, he emaciates, obstinate jaundice and uncontrollable, sometimes hemorrhagic, diarrhea develop. Then follow vomiting, violent pains in the region of the liver and general dropsy until, with progressing cachexia, death takes place. The autopsy often shows numerous changes in the liver: Enormous dilatation and occlusion of the bile-ducts with ulceration of the same; excessive increase of the connective tissue in the area surrounding the focus of the parasite; obliteration of the large bile-ducts, occlusion of the hepatic duct, etc. If, therefore, the diagnosis should be difficult, an examination of the stools will enable the physician to recognize the disease. Bostroem and Perroncito succeeded in finding the ova of distomata in the stools.

Owing to the difficulty of the diagnosis, the treatment must always remain symptomatic; but if the diagnosis be correctly made, the expulsion of the liver-fluke may be attempted by the administration of mineral waters or other remedies promoting the secretion of bile. 
Occasionally the liver-fluke may pass from the liver into other organs. Duval found the parasite in the trunk of the portal vein, and Friedberger in the portal vein. It' was found in abscess cavities upon the plantar surface of the foot by Giesker; behind the ear, by Fox; in the right hypochondriac region, by Dionis des Carrières; and it was extracted from the shoulder region by Malherbe. Raillet reports that a French officer in Rio coughed up a Senegal liver-fluke.

The bile-ducts of various mammals contain, together with the distomum hepaticum, the distomum lanceolatum, Rudolphi, 1803, which, like the distomum crassum Busk, 1850 , has been found but rarely in man.

Besides these, I shall also mention the distomum heterophyes, $\nabla$. Siebold, 1852, and the distomum ophthalmobium, Diesing, 1850, as they have been rarely observed in man.

In Formosa, Korea, and, particularly, in Japan, the distomum Westermanni, Kerbert, 1878 , is frequently found, and this is also designated distomum pulmonale, Baelz, 1883, distomum Ringeri, Cobbold, 1880.

This reddish-brown parasite, according to Baelz, attains a length of 8-10 $\mathrm{mm}$., a breadth of $5-6 \mathrm{~mm}$; it is oval, slightly rounded at the ends; a cross section is almost circular; the suctorial discs of the mouth and abdomen are

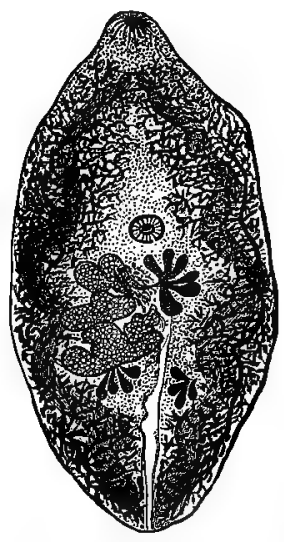

Fig. 33.-Distomum Westermanni ANd Ovum. (After Katsurada.)

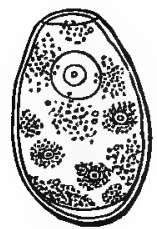

almost of equal size. The ova, thousands of which are often found in bloody sputum, are of a brown color, 0.08 to $0.01 \mathrm{~mm}$. long and $0.05 \mathrm{~mm}$. broad, thin-shelled, and showing a lid at the blunt end. Besides in man, the parasite has been found in the dog and also in animals of the cat family, especially in Japan.

Distomatosis is widely disseminated in Japan, and is the cause of an apparently harmless hemoptysis. The parasites are found in cavities; in one case dissected by Baelz there were twenty specimens. These cavities contain a reddish, pulpy mass consisting of mucus, red blood-corpuscles and numerous distoma ova. The cavities are connected with the bronchi by sieve-like openings through which the ova, sometimes in enormous numbers, reach the spu- 
tum. The patient's general health is not materially impaired; but there is danger of fatal hemorrhages. The diagnosis is determined by the demonstration of the ova. The ova of the distoma may, according to Yamagiva and Otani, be carried to the brain, and there give rise to serious disease.

No special therapy is known as yet. But it seems advisable that persons infected by distoma should leave the regions where these are found in order to avoid further infection.

The distomum spathulatum, Leuckart, 1876, or distomum sinense, Cobbold, 1875, is very common in man, and also in the cat, in China and in some parts of Japan.

This parasite is reddish, almost transparent, flat, $10-18 \mathrm{~mm}$. long, with pointed anterior and posterior ends; the skin is smooth. The suctorial disc of the mouth is larger than that of the abdomen; the latter is situated at the line of the first quarter of the body. The oval eggs, $0.028-0.03 \mathrm{~mm}$. long and $0.016-0.017 \mathrm{~mm}$. broad, are encapsulated, and gradually become black.

The parasites are generally found in large numbers in the bile-ducts, but rarely in the stomach and in the intestines. They produce severe clinical disturbances. According to Baelz, the trouble begins with enlargement of the liver, and a sensation of pressure and weight in its region; this is accompanied by a morbid appetite. After some years the nutrition becomes defective, and uncontrollable diarrheas make their appearance and later become hemorrhagic. Gradually dropsy, ascites, and anasarca develop, and the patients finally succumb with severe cachectic phonomena. The autopsy shows the walls of the gall-bladder and bile-duct studded with cyst-like sinuses the size of a nut, which often contain numerous parasites. The sinuses communicate with the bile-ducts. The liver is enormously enlarged but of normal color. The tissue in the neighborhood of the parasitic foci is atrophied. The ova of the parasites are always found in the feces. We know but little regarding the development of the parasite, only that the ovum while in utero develops a ciliated miracidium. The young forms of the parasite probably live in mollusca. In later stages of development they must reach the intestinal tract of man with raw vegetable, and possibly also with animal, food.

The distomum conjunctum, Cobbold, 1859, has been found, so far, only twice, in individuals in India who had succumbed to severe dysentery.

Distomum felineum, Rivolta, 1885, sive distomum sibiricum, Winogradoff, 1892, deserves special mention.

According to its contractions, its length is $8-18 \mathrm{~mm}$., and its breadth 1.5-2.5 mm. The parasite, according to Braun, in fresh condition, is of a reddish color, almost transparent, flattened, and pointed at the anterior end; the skin is not weaponed. The suctorial discs are of almost equal size. The pharynx is immediately adjacent to the suctorial dise of the mouth; the esophagus is as long as the pharynx. The intestinal shanks, which do not branch out, extend to the posterior end of the body, and are filled with dark brown, granular masses. The eggs are oval, and have a lid which is sharply defined; the length is $0.02-0.03 \mathrm{~mm}$., the breadth $0.011-0.015 \mathrm{~mm}$.

The parasite Winogradoff, which until 1892 was only found in the cat, in that year was noted to be a very frequent parasite of man, being present in 
the liver. In the nine cases observed by Winogradoff, death was not due to the parasites. Yet the changes produced by it are quite considerable, viz., dilatation and inflammation of the bile-ducts, and foci of cirrhosis. In one case there were small pus foci; jaundice and contraction of the liver existed in five cases, ascites in three; the liver was twice found to be enlarged.

Braun's assumption that, owing to the great dissemination of distomum felineum among cats, it must also be present in man, was soon confirmed. Askanazy reported such a case from East Prussia, in 1900. It occurred in a man who had died of colloid carcinoma, which originated in the epithelium of the bile-ducts. The microscopical examination revealed ova and about 100 specimens of distomum felineum in the regions not affected by the tumor. The same author reported a second case, also from East Prussia.

Askanazy supposes the infection in man to be due, as in the cat, to the consumption of raw, or only partially cooked, fish.

Of great interest is another trematode, distomum hæmatobium, Bilharz, 1852 , or the Bilharzia hæmatobia, Bilharz, 1852. The parasites live sexually
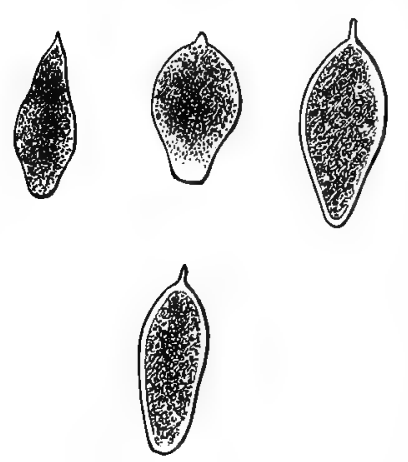

Fig. 35.-Ova of Distomum HAMATOBIUM.

apart. The male is thicker (up to $1 \mathrm{~mm}$.) and shorter (12-14 mm.) than the more slender (up to $0.13 \mathrm{~mm}$.) and longer female $(16-18 \mathrm{~mm}$.). The anterior part of the body which contains the suctorial dises is flattened. The posterior part of the body of the male, by a turning up of the lateral borders, forms a tube (canalis gynæcophorus) which serves for the reception of the female. The surface of the male is covered with small spiny warts, that of the female is smooth. The ova, which are often found in large numbers in the vagina, are slender and quite long, about 0.12 $\mathrm{mm}$. long and $0.04 \mathrm{~mm}$. broad. They are pointed at one end, or, more rarely, supplied with a pointed side tooth. The shell of the ova is thin, and there is no lid. The full grown parasites are found in man in the portal, splenic and renal veins, also in the venous plexus of the urinary bladder, and in the rectum. The ova lie in small, white masses in the various organs, especially in the urinary passages, in the vesicula seminalis, and in the mucosa and submucosa of the large intestine. The primary seat of the ova is probably. the vascular system. The ova, deposited in the veins which are distended to small diverticuli, enter the tissues only after rupture of the vessels.

The history of the development of the parasite which occurs mostly and quite frequently in Africa, and especially in Egypt, is not yet clear. We know only the following: The ova are discharged with the urine. Sometimes empty sacs of ova are found in the urine synchronously with free embryos. Evidently a miracidium, completely ciliated, escapes from the ovum in urine that is greatly diluted with water and floats for some time in the water. It appears likely that there is an intermediate host; but all attempts to infect mollusca, crustacea, and fish have been fruitless. Loos doubts the transmission by an intermediate host. The embryo, in the opinion of this author, enters directly 
into man, and develops into a sporocyst which then infects a patient with its young.

It has been assumed that man becomes infected with the parasite by drinking infected water. Yet the observations and the experimental investigations of Loos make this assumption doubtful. Attempts to infect monkeys by giving them infected water to drink did not prove successful. Brock and Loos assume that the parasites gain access directly through the skin during bathing. Men and boys are affected much more frequently than women and girls who bathe but rarely. Brock found that the newly arrived in the Transvaal who went bathing were soon infected, whereas others who did not bathe remained free from the parasite. Loos rejects, however, the assumption of Harley and Allen that the parasite finds entrance into the body through the urethra. The incubation period, according to Loos, is four weeks; according to Brock, four months.

In addition to the parasites at the points of predilection in the vascular system, autopsies reveal the most marked changes in the bladder and in the ureters. In freshly developed cases there is a marked catarrh of these organs with a viscid, yellowish-red mucus in which, as also in the mucous membrane itself, numbers of distoma eggs are found. In chronic cases the mucous membrane shows the signs of a severe chronic catarrh, and has a sandy appearance. Characteristic excrescences, from the size of a pea to that of a bean, and having the appearance of hemorrhagic ecchymoses, are formed in the swollen submucous tissue. The excrescences sometimes become encrusted, and then represent stony polypi which are broken off and form the nuclei of bladder stones. Pyelitis, nephritis and hydronephrosis follow in severe cases. Kartulis, Albarran-Bernard and Harrison quote cases from their experiences which show in the tissues permeated with distomata the tendency to proliferation, that is, to formation of carcinoma. Not the parasites, but the ova, cause the severe irritation. They are also found in the lungs, in the liver, in the prostate gland and in the mesenteric glands.

Clinically we encounter at first the manifestations of a hematuria associated with burning pain in the urethra. At first intermittent, it gradually becomes permanent. The characteristic ova are found very early in the urine. If the infected individual does not leave the country, the disturbance is aggravated by affections of the renal pelvis and of the kidneys. Stones in the bladder and in the kidneys develop frequently. We have mentioned the tendency to the formation of carcinoma. Cole Madden describes a papilloma of the vagina produced by the ova of distoma. The mucous membrane of the rectum shows similar polypoid excrescences as the bladder. The nutrition becomes defective and, finally, after a course lasting years, death occurs with cachectic manifestations. It seems that severe cases are most frequent in Egypt. In South Africa, after protracted intermittent attacks of hematuria and cystitis, the disease often, at least apparently, ends in recovery. The destruction of the parasite does not in itself lead to recovery. Sonsino estimates the duration of its life at two to three years. If the changes have been very extensive, the morbid symptoms continue to develop after the death of the parasite. 
If the views of Loos are correct, the prophylactic measures are plainly indicated. In the infected countries energetic measures must be taken to prevent the urine of infected individuals from ever reaching the rivers. The embryos will soon perish in closed sewers. The greatest precautions are necessary in regard to bathing, especially in the neighborhood of habitations in which infected persons are living.

A therapy directed to the expulsion of the worms will probably always remain useless. Efforts to expel them by turpentine oil or extractum filicis maris have been, according to the reports of Brock, unsuccessful. We are, therefore, entirely restricted to symptomatic treatment.

I may mention another trematode, the

Monostomum lentis of Nordmann, 1832,

which was once found in an extracted lens; this form is probably identical with the distomum oculi, Ammon, 1833.

Hexathyridium pinguicola, Treutler, 1793, and hexathyridium venarum are also counted among the trematodes.

\section{CESTODES}

Cestodes, tape-worms, have been known from antiquity as parasites of man. Following the definition of Leuckart, we understand by cestodes flatworms without mouth or intestine, which develop by generation and by gemmation upon a scolex, and remain united for some length of time in a ribbonlike colony.

The scolex, known under the name "head of the tape-worm," is supplied with two to four suctorial dises, and generally also with hooks that are bent like a claw.

The head serves as the adhesion apparatus. The flat, two-edged links of the chain (proglottides) grow away from the point of junction. The smallest links contain the ova in which develop the embryos (oncospheres), which in the bothriocephali are ciliated. Only these, reaching the outside world, perforate the shell of the ovum, and swarm in the water until they finally reach an intermediary host. The ova of the other cestodes emerge into the outside world with the feces, or still enclosed in the proglottid, and are at last taken up by a suitable host. The oncospheres, enclosed in small cysts or embedded in closed carities of the body, develop in the host into scolices which, subsequently, are taken up by the actual host and grow into tape-worms.

The best-known of the tape-worms parasitic in man are the tenia solium, Rudolphi, 1810, and the tenia saginata, Goeze, $1^{1782 .}$

I may forego a detailed description of these two parasites, since they are well known. I shall only briefly outline their differentio-diagnostic characteristics.

Tenia solium is distinguished by a head which is supplied with a rostellum, a double row of hooklets and four suctorial discs. The proglottides, numbering 800 to 900 , contain the testicles and the easily recognizable uterus consisting of a medial trunk and of seven to ten lateral branches upon either side. The marginal sexual papillæ alternate quite regularly. The rounded 
ova, the shells of which are radially striated, enclose the oncosphere which is supplied with six hooklets.

Malformations are rarer than in the tenia saginata. I wish to mention only the three-edged or prismatic tenia which is produced, not as was frequently assumed from a twelve-hooked oncosphere, but by the coalescence of two scolices with one-half of their dorsal surfaces, whereas the other two halves remain free; it appears that the dorsal parts of the coalescent halves are at the same time greatly retarded in growth.

The ascxual immature form is the cysticercus cellulosæ, the finn of swine, which is found principally in the pig, but also in other mammals. The mode of infection of the pig and of man is well known.

The cysticercus cellulosæ occasionally also shows malformations. Such are the cysticercus (tenia) acanthotrias Weinland and the cysticercus racemosus which is formed like a bunch of grapes and possesses many branches; the latter is found in the brain and in the heart. This upsets (according to the views of Redon, Blanchard, Raillet and Braun) the hypothesis of a special genus of tenia (cysticercus) acanthotrias.

The second variety, the tenia saginata, is more rounded and larger than the tenia solium, and has the following characteristics: The head is larger, the row of hooklets and the rostellum are absent; the suctorial dises are markedly developed. The proglottides are supplied with irregularly alter-

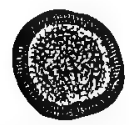

Fig. 36.-Ova of Tenia Solium.

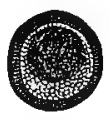

The enlargement is the same in Fig. 36 and Fig. 37.
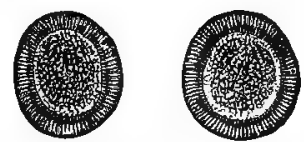

Fig. 37.-Ova of Tenia Saginata.

nating sexual papillæ; they show a uterus with 20 to 30 or even more lateral branches; the sexually mature members which are cast off spontaneously are frequently found without ova, because the latter have been discharged prematurely owing to a lesion of the uterus. The ova are similar to those of the tenia solium (see illustrations). Malformations in the chain of links occur more frequently than in the former variety.

Cysticercus bovis, which is the young form of this parasite, is principally found in cattle. Whereas the cysticercus cellulosæ is sometimes found in the organs of man, particularly in the brain and in the eye, generally through autoinfection, only four cases have been reported in which the cysticercus bovis has been found in man. It is probable, however, that these four cases were not due to the cysticercus bovis at all, but to malformations of the cysticercus cellulosæ.

Man is considered to be the only host of these two forms. Deffke succeeded recently, by feeding the cysticercus cellulosæ to three dogs, in obtaining in two of the dogs small specimens of tenia solium. The delicate specimens gave the impression that they were in the process of expulsion.

Tenia solium has for several decades become steadily rarer. This is evi- 
dently due to meat inspection during which, owing to its size, cysticercus cellulosæ is less often overlooked than cysticercus bovis.

Tenia cucumerina, Bloch, 1786, sive elliptica Batsch, 1782, is also well known.

This tape-worm is frequently found in large numbers in the dog and in the cat. The small rhomboid head shows a club-like rostellum studded with about sixty hooklets arranged in four rows. The cucumber-shaped proglottides are easily detected in the feces of the dog.

The cysticercoid young form inhabits, according to Leuckart and Melnikoff, the abdominal cavity of the dog-louse, of the dog-flea, and, more rarely, of the human flea. The habit which dogs and cats have of swallowing the parasites that infest their hairy skins sufficiently explains the frequency of the infection. This tenia occurs rarely in man, and is only observed in small children. Blanchard reported the infection of an adult who always shared his resting place during the night with his dog.

Quite as rare, at least in Germany, is the following tenia :

Tenia nana, v. Siebold, 1852.

This small tenia, only $2.5 \mathrm{~cm}$. long and $0.5-0.7 \mathrm{~mm}$. broad, is characterized by a globular head supplied with a rostellum and four suctorial discs. The rostellum is covered with a row of $22-27$ hooklets. The proglottides, amounting to about 200, are of an extended oblong form, the lower 20-60 of a yellowish color, well-filled with ova. The sexual papillæ are all on one side. The ova are covered with three structureless, transparent shells which are far apart from each other.

Nothing is as yet known regarding the origin and development of this parasite.

Leuckart supposes the intermediate hosts to be insects. According to Grassi, the parasite develops without an intermediate host, as does the tenia murina (Dujardin), which by many was formerly considered identical with the former. The anatomical differences between these teniæ were recently pointed out by v. Linstow.

The teniæ live in the small intestine in colonies, burrow their way deeply into the mucous membrane of the gut, and, according to Grassi, produce important changes in it.

The parasite has been observed only four times in Germany, and in all four cases Cologne appeared to be the infected locality. It may possibly often be mistaken for the oxyuris vermicularis, owing to their similarity. The parasite has been observed in Egypt, Russia, Servia, Italy, Japan and South. America.

Very few cases of the

\section{Tenia Alavopunctata, Weinland, 1858,}

have been reported. Its identity with tenia diminuta, Rudolphi, and tenia leptocephala Crepl. is established. The very small head is club-shaped, with a rudimentary unprotected rostellum. The proglottides, $2.5-3.5 \mathrm{~mm}$. broad, 0.6-0.7 mm. long, present in their center, posteriorly, a yellow area which 
corresponds to the male sexual organs. The intermediate hosts are, according to Grassi, small insects, such as akis spinoza, anisolabis annulipes, asopia farinalis, scaurus striatus, by the accidental ingestion of which man may also become infected. These observations were confirmed by infection experiments in man and in rats. The parasite has been so far found only seven times in man, and almost exclusively in children.

Quite as rare is the

\section{Tenia madagascariensis, Davaine, 1869.}

This tape-worm is about $25-30 \mathrm{~cm}$. long, with about $660-700$ segments. The head is supplied with four large, circular suctorial dises, and a rostellum with a row of 90 peculiarly formed hooklets. The mature proglottides are $2 \mathrm{~mm}$. long, $1.4 \mathrm{~mm}$. broad. The sexual openings are situated on one side. Each proglottid contains 120-150 small round ova. We are ignorant of the exact development of the parasite and only know that species similar to this tenia dwell in birds. Only five cases of this kind have been observed so far in the tropics, among them one in British Guiana (by Danicls).

It appears to be very doubtful whether the tenia marginata, Balsch, 1786, the tenia serrata, Goeze, 1\%82, and the tenia crassicollis Rudolphi, 1810, or their cysticerces, have been observed in man, but five unknown teniæ occurring in man have been reported, and have been called the

Tenia confusa, Ward, 1896.

Ward, in a preliminary report, has described a tenia found in man. Its length was about five meters. The end proglottides measured $27-35 \mathrm{~mm}$., the breadth $3.5-5 \mathrm{~mm}$. This tenia is considerably more slender than the tenia saginata. The head has four suctorial discs, and a retractile rostellum with six or seven rows of small hooks, those in the front row being the largest.

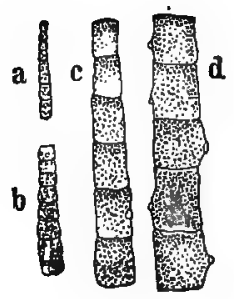

FIG. 38.

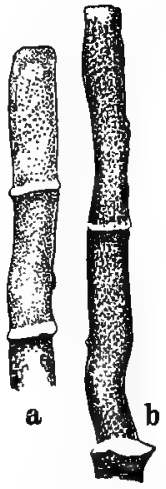

FIG. 39.

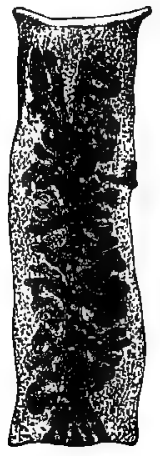

Fig. 40.
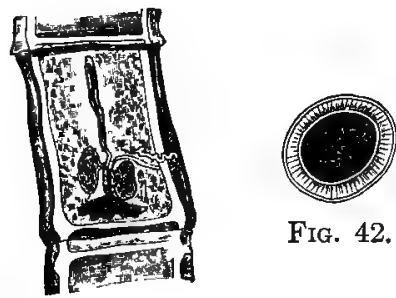

FIG. 42.

FIG. 41.

Figs. 38-42.-Tenta confusa Ward. (After Guyer.)

Fig. 38, $a, b, c, d$, Chain of proglottides. Fig. 39, $a$ and $b$, Mature proglottides. Fig. 40, Female sexual organs. Fig. 41, Male sexual organs. Fig. 42, Ovum of the tenia confusa.

The proglottid-chain is distinguished from tenia solium and tenia saginata by continuous longitudinal musculature, by the presence of a vesicula seminalis 
and a well-developed receptaculum seminis, and of a vaginal sphincter. The ovarian tube opens into the lateral wall of the uterus. Fourteen to eighteen lateral branches issue from each side of the uterus but are not perpendicular to the main trunk. The yellowish ova are of ovoid form.

\section{Tenia africana, v. Linstow, 1900.}

The German colonial possessions have given us the knowledge of a new tenia. v. Linstow described a large tenia of man which Dr. Fülleborn found in a negro soldier at Lake Nyassa. The scolex is unprotected, and is smaller than the following chain of segments. The proglottides, even the terminal ones, which, besides the uterus filled with ova, contain no other sexual organs, are broader than they are long. The uterus consists of a longitudinal trunk extending in an antero-posterior direction with 15-24 transverse branches on either side which radiate toward the center. The interior organization is different from that of tenia saginata in all essential points. The suctorial dises are enclosed by rounded projections from which rays extend to the periphery.

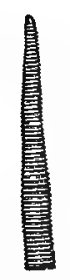

$a$

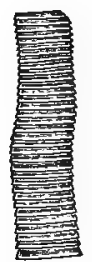

$b$

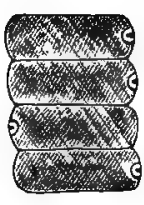

$c$

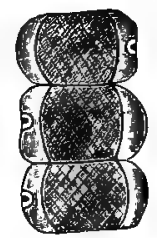

d

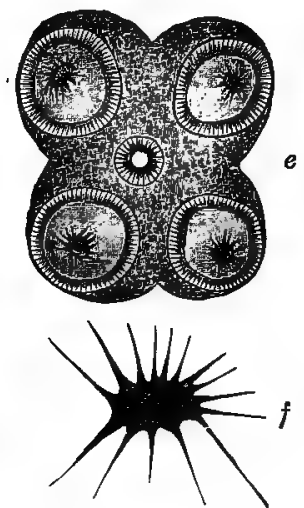

FIG. 43. $-a-d$, Segment chains of the tenia africana; $e$, scolex from the vertical plane; $f$, lumen of a suctorial disc. (After v. Linstow.)

The length of the three fragments of a tenia was $1,375 \mathrm{~mm}$; w whereas the segments of the tenia africana which are two $\mathrm{mm}$. long show full maturity; there is no trace of sexual organs in the segments of the tenia saginata which are $3.16 \mathrm{~mm}$. long and $5.84 \mathrm{~mm}$. broad. The ova are ovoid, $0.042 \mathrm{~mm}$. long and $0.034 \mathrm{~mm}$. broad.

Tenia asiatica, v. Linstow, 1901.

von Linstow recently described a tenia of man which was found by Anger in Aschabad. The specimen is $298 \mathrm{~mm}$. long; the segments are broader than they are long and their chain is narrow. The foremost proglottides have a breadth of only $0.16 \mathrm{~mm}$. with a length that cannot be estimated. Later they increase in breadth from $0.6 \%-1.78$, and in length gradually to $0.99 \mathrm{~mm}$. The posterior border of the segments slightly overlaps the beginning of the following one. Seven hundred and fifty proglottides were present; the scolex was absent. The formation of the uterus, as well as that of the other sexual organs, suggests that this variety belongs to the tenia madagascariensis. 
Recently, in the Centralblatt f. Bact. und Parasit., 1902, Band xxxi, page 770, v. Linstow described a

\section{Tenia hominis n. spr.}

in the collection of helminthes of the Musée zoologique de l'Académie Impérial des sciences de St. Petersbourg. This tenia was found by Anger in a girl in Aschabad.

The scolex was $2.01 \mathrm{~mm}$. long, $1.34 \mathrm{~mm}$. broad. The vertex contained a rudimentary but not prominent rostellum, $0.24 \mathrm{~mm}$. Iong, entering deeply

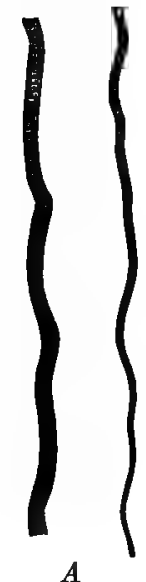
into the scolex, without hooks. The Iumen of the suctorial discs extended antero-posteriorly, so that they formed regular circles upon cross sections. A ring-shaped prominence was noted behind the suctorial discs.

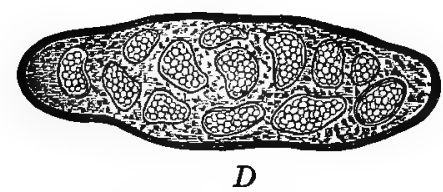

Fig. 44.-Tenia astatica. (After v. Linstow.)

4, Tenia asiatica, natural size; $B$ and $C$, schematic cross sections: $B$, with male, $C$, with female, sexual organs; $g$, vessel; $n$, nerve; $h$, testicles; $v a$, vas deferens; $c$, cirrus bag; $o$, ovary; $d$, yolk sac; $r$, receptaculum seminis; $v$, vagina; $D$, cross section through a segment with masses of ova.

The chain of the proglottides was $70 \mathrm{~mm}$. long, $1.11 \mathrm{~mm}$. broad behind the scolex, $1.97 \mathrm{~mm}$. posteriorly. The sexual organs were not developed.

Hymenolepis (drepanitotonia) lanceolata, Bloch,

a parasite found in many water-fowl, was recently expelled in two specimens by a boy twelve years old. The scolex was not found. The length of the tenia is $85-99 \mathrm{~mm}$., its greatest breadth behind the center of the body length is $8.5-12 \mathrm{~mm}$. The proglottides are all considerably broader than long. The leaf-like thin strobila slightly tapers toward the anterior end; posteriorly it extends in uniform breadth and its termination is rounded. The ova possess the shells which are characteristic of the genus hymenolepis, being far apart from each other.

This parasite uses, as intermediate hosts, fresh water crabs; the cercocysts are found espe-

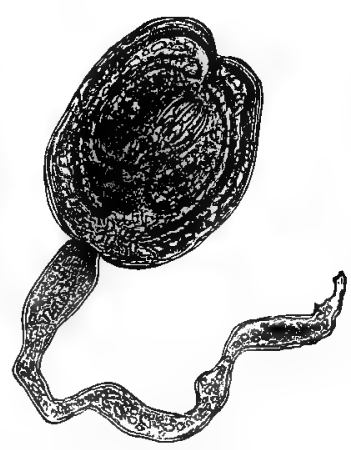

Fig. 45.-Cercocyst From CypRinotus InCONGRUENS, 1900. 
cially in the cyclops serrulatus, Fischer, and diaptomus spinosus, Daday; and since these are frequently found in stagnant waters, the occurrence of the

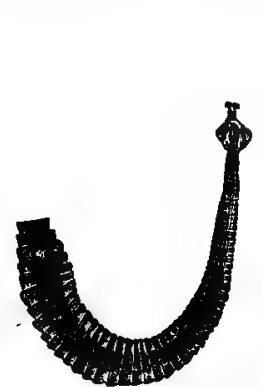

FIG. 46. - HYMENoLEPis La NGEOLATa, Bцосн. Enlarged abouttwentytimes.

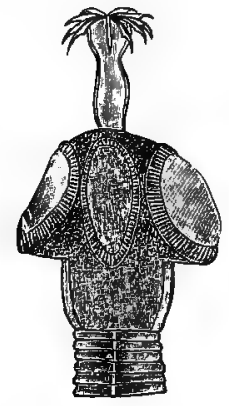

Frg. 47.-Head, Greatit EnLARGED. parasite in geese and ducks is quite natural. The crabs, which are often difficult to recognize, occasionally migrate with the drinking-water into the intestinal canal of man; the cercocyst inhabiting the crabs develops into hymenolepis lanceolata.

The most familiar of the bothriocephali is the

Bothriocephalus latus, Bremser, 1819.

This broad bothriocephalus, called also the pit-head, measures 5-9 meters and is the longest tape-worm of man. The elub-shaped head shows on its lateral border on each side an elongated, slit-like pit. The individual proglottides, 3,000 to 4,200 in number, are remarkably broad in proportion to their length. A grayish-blue centerpiece of rosette-like form bordered by two narrower bands is distinctly visible upon both surfaces. On the ventral surface are the male sexual opening and the mouth of the vagina. The mature ova are ovoid in form and surrounded by a brownish shell with a lid. The ova are rarely found in the last mature segments of the chain. Large numbers of proglottides are often expelled at once. They are voided with the feces, having been previously discharged from the uterus. If the ova reach the water, ciliated embryos develop within them, which slip from the shell of the ova and float for some time in the water. The ciliated covering perhaps seeks the intermediary host, which, as we learn from Braun's brilliant investigations, was found to be the pike (esox lucius), the burbot (lota vulgaris), the perch (perea fluviatilis), the trutta vulgaris and lacustris, the thymallus vulgaris, and the onchorhynchus Perryi. It is still undecided whether this list exhausts the number of intermediate hosts.

The young of the bothriocephalus, plerocercoides, dwell encapsulated in various parts of the intestines and of the musculature, and usually in large numbers.

Bothriocephali have been cultivated by feeding experiments with plerocercoides in the dog, the cat, and also in man.

The transmission of the parasite undoubtedly occurs by means of fish. It takes place frequently in infected districts where fish are eaten raw, half cooked, or after any other mode of preparation which does not effect a destruction of the ova. It is remarkable that living ova have been demonstrated even in fish that were frozen stiff. 
The geographical distribution of the bothriocephalus is a limited one. It has been frequently noted in the Russian Baltic provinces, in the northeastern part of Sweden, in Denmark, East Prussia, Russia and Poland. Its presence has also been determined in the coast districts of Belgium, Holland, northern France, and in Ireland. It is remarkably common in the western parts of Switzerland, on the shores of Lake Geneva and Lake Neufchâtcl, and in those parts of France and Italy bordering upon Switzerland. The parasite is often seen in Japan. It is found sporadically in Germany, in Rhenish Hesse, Berlin, Nuremberg, Munich and Greifswald, and has been found in North America.

Of the other bothriocephali we find in man the

\section{Bothriocephalus cordatus, Leuckart, 1862.}

This variety is characterized by a short, broad, heart-shaped head which is supplied with two suctorial disc-like pits. The parasite which is chiefly found in various mammals in Greenland and in Iceland is occasionally also noted in man.

The tape-worm described as

\section{Bothriocephalus cristatus, Davaine, 1874,}

may possibly represent only another form of the bothriocephalus latus. Its head is characterized by two extremely long, helmet-like points.

Of another variety of bothriocephalus,

$$
\text { Bothriocephalus Mansoni, Cobbold, 1883, }
$$

only plerocercoides have as yet been observed, and these apparently wander in the body of man. They finally reach the skin, and there cause abscess formations, or they enter the urinary passages whence they are discharged with the urine.

\section{Bothriocephalus grandis, Blanchard, 1894.}

Ijima and Kurimoto have observed a bothriocephalus previously unknown, which lacks a head portion. It measures in breadth, anteriorly, $1.5 \mathrm{~mm}$., pos-
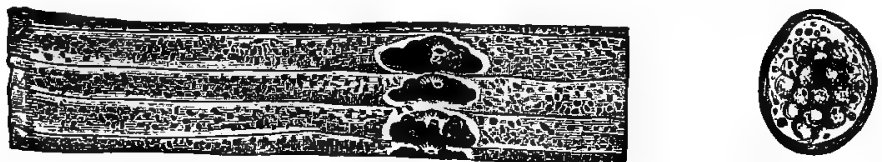

Fig. 49.-Bothriocephalus Grandis. Proglottid and Ovum. (After Ijima and Kurimoto.)

tcriorly, $25 \mathrm{~mm}$. The proglottides are extremely short, and are supplied with a double genital apparatus.

The ventral surface contains the genital pori, anteriorly the mouth of the cirrus and that of the vagina, behind these that of the uterus. The ova possess a lid, are thick-shelled and brown; their length is $0.063 \mathrm{~mm}$., their breadth, $0.048 \mathrm{~mm}$. They contain a mulberry-like mass of fine globules.

There can be no doubt that parasites infect some persons in whom no deleterious influence upon the health is manifest. The patients in such cases 
are often unaware of the presence of their guest until they note the discharge of proglottides. The distressing thought of being the host of such a guest causes the carricrs of the parasite to seek medical aid. Strong, robust persons, owing to their good constitution, are often uninjured by the parasite. 'A weak constitution and sensibility, or other factors which are especially the cause of disintegration of the blood leading to pernicious anemia, will produce symptoms in the affected individuals, sometimes even the most severe morbid phenomena.

There are patients who harbor the parasite for years without presenting the slightest sign of helminthiasis. On the other hand, in sensitive persons the symptoms may be numerous. In all cases the most positive indication is the passage of segments of the worm, which occurs at times spontaneously, at other times with the feces. With tenia saginata the spontaneous discharge of proglottides appears to be more frequent than with tenia solium. In the case of the bothriocephalus segments in chains amounting to several meters in length are passed.

Not infrequently the ingestion of very salty food, of blueberries, strawberries, salad, or of alcoholic drinks, causes the passage of segments. In very rare cases, proglottides are vomited. By reason of abnormal protrusion of the intestines or of the pelvic organs from the abdominal coverings, proglottides occasionally appear externally.

Besides these symptoms the microscopic examination of the feces is of especial importance in the diagnosis. This should never be neglected if the presence of tape-worm is suspected. Very frequently by careful, oft repeated examinations of fecal masses indistinct symptoms on the part of the digestive apparatus, of the nervous system, or of the general nutrition, have been explained, and treatment directed into the proper channels. As the uterus of the tenia has no exit, the eggs can only find egress when the uterus of the mature proglottid is injured. In the case of tenia saginata, discharge of the eggs is almost the rule. The proglottides which are discharged are usually without eggs. The ova of the bothriocephalus are extruded into the feces from the uterus which has an external mouth. These may readily be differentiated from the eggs of teniæ, whereas the eggs of tenia saginata and tenia solium only differ by their variation in size.

Besides the ova, Charcot-Robin's crystals are found in the feces as in the case of other helminthiases.

Whereas the passage of ova and proglottides is the most positive, and occasionally the only, symptom of an existing tape-worm, the other symptoms of tenia permit us to recognize the presence of a tape-worm with but slight probability. In some patients there are disturbances of the digestive apparatus. I shall only mention here pyrosis, bulimia, nausea, a longing for spicy food, etc., symptoms which, as is well known, are grossly exaggerated in the pamphlets of quacks to attract the attention of patients. In others there are colicky pains which are occasionally more severe after taking sour food, and better after fluids. It is evident that the tape-worm acts as a foreign body in the intestinal canal, and may exert a powerful irritation in the region it inhabits. Probably the hooklets which some teniæ possess may share in this 
irritant action. If the tape-worm is only seen after it has been expelled, or after it has died, it is difficult to understand what energy and motility the parasite possesses under normal circumstances in the warm intestine of its host, and how greatly it may irritate the intestinal mucous membrane. This may be noted sometimes as pain, at other times as a digestive disturbance. Intestinal catarrh, constipation, marked irregularity of the bowels are, in fact, very frequent accompaniments of tape-worm disease. We have repeatedly noted the disappearance of all these symptoms after the removal of the parasite.

Besides these local symptoms, in a number of patients suffering from tape-worm there are also general phenomena and so-called reflex symptoms, existing alone or together with the gastric phenomena. It is quite certain that formerly in investigating these symptoms, as the result of incorrect views and on account of erroneous pathological deductions, diagnoses have been made which would not bear strict critical analysis. To deny them utterly would, however, not be correct. There are to be mentioned: Vertigo, and attacks of syncope, headache, stubborn singultus, pupillary differences, rapid change of color in the face, pruritus, spasmodic attacks which occasionally have the characteristics of epilepsy. According to the observations of Grassi, in the case of tenia nana epileptic attacks are especially frequent. In deciding upon the connection of phenomena of this kind, it will aid us in every case to note whether they persist after the disappearance of the worm, and are therefore to be looked upon as a purely reflex condition. If this be the case they are, of course, only an accidental complication.

How far the symptoms on the part of the nervous system are to be regarded as reflex, cannot always be immediately decided. We must first exclude those cases in which cysticercus invasion has to be considered, as in the case of tenia solium. We know positively from autopsies that the brain is the seat by preference of cysticerci. On the contrary, it must be questioned whether the central nervous organs are not under the influence of toxic substances produced by the parasite. It is more than likely that these animal parasites engender toxic products which have a particularly deleterious effect upon the nervous system as well as upon blood formation.

Reyher, Runeberg, Schapiro, Dehio and others have called attention to the fact that the severe anemic conditions occurring in persons affected with the bothriocephalus may assume the character of pernicious anemia and run a course as such. These symptoms may certainly be referred to the presence of the parasite. There is no longer any doubt of the etiologic connection. The circumstance that the majority of infected persons are not anemic suggests the thought that the poison is found only under special circumstances as, for instance, the illness or death of the worm, or the presence of the parasite for a considerable time. Lately Schaumann and Talqvist fed dogs with segments of the bothriocephalus taken from a case of pernicious anemia, and produced undoubted dissolution of blood in the dog. The forms of bothriocephalus which do not produce anemia also contain the hematogenous poison. Occasionally this is present in other teniæ. Eisenlohr soon effected a cure of a case of severe anemia by expulsion of a tenia saginata. 
In conclusion I must mention the fact that persons who have previously been the hosts of parasites, and even persons who have never been affected by tape-worm, but who have read or heard of the symptoms of helminthiasis, occasionally suffer from hypochondria verminosa or teniaphobia. Nervous disturbances which appear incidentally are referred to the presence of a tapeworm. That hysterical or hypochondriacal individuals are particularly predisposed to these conditions is so obvious that it need not be especially dwelt upon.

On account of the great number of local and general symptoms caused by the presence of this parasite, considerable experience must have been acquired to make one suspect the presence of a tape-worm. Certain symptoms which are occasionally present in helminthiasis arouse this suspicion. Among these are the complaints that the symptoms are most distressing when the stomach is empty, and that the disturbances are less on taking sweet food, but are increased by salty or acid food. However, I never begin a tape-worm treatment until after the presence of the parasite has been proven by the passage of proglottides or the eggs in the feces. In examining these the physician must be careful not to confound remnants of undigested food, mucus casts, and shreds of tendons, often mistaken by the laity for proglottides, and thus make an erroneous diagnosis. Dry proglottides after being soaked in water soon assume their characteristic form. As a rule, the microscopic determination of the eggs of the tenia enable us to make the diagnosis earlier and with greater certainty than by the macroscopic proglottides. The encapsulated eggs of the bothriocephalus are readily recognized, but, as has already been mentioned, the differentiation between the eggs of tenia solium and tenia saginata is more difficult.

To recognize from the shape of the proglottid which variety is present it is advisable to fix this between two glass slides. The proglottid of the tenia solium is more delicate and more transparent than the tougher segments of the tenia saginata. In the former the structure of the branching uterus is more plump, the number of lateral twigs only amounts to from 7 to 10 , while the uterus of the tenia saginata shows from 20 to 50 and more lateral twigs. For the recognition of the less frequent parasites special text-books on the subject must be consulted.

\section{PROGNOSIS}

The prognosis is generally favorable. Even in cases of severe bothriocephalus anemia cure has generally taken place after the removal of the parasite. Parasitism with the tenia solium necessitates special care on account of the danger of autoinfection with the hooklets. It is particularly apt to occur from the appearance of ripe proglottides in the stomach as the result of antiperistaltic movements. Prolonged presence of the proglottides in the stomach explains why, in some persons, the cysticercus is found with such extraordinary frequency in the brain and in the musculature. Even in the eye occasionally more than one cysticercus is found. The host of the tenia solium is also a certain menace to those about him. By insufficient cleanliness, hy careless handling of the proglottides which have been passed, and by 
contact with fecal masses, the possessor of a tenia solium may spread the infection.

\section{THERAPY}

Prophylactic measures are based on the history of the development of these parasites. In the first place the spread of cysticercus disease among animals used for food must be prevented, and not only the tape-worms which are voided, but also the feces of the patient with tape-worm disease should be effectually destroyed, best by fire. All who harbor the parasites should be placed under treatment as soon as possible, particularly such persons as come in contact with animals used for food. The patients must be instructed never to defecate in the open air.

The dissemination of tape-worm is actively combated by the erection of abattoirs and the introduction of compulsory meat inspection. It is true this protection is not absolute, for even with experienced meat inspectors some cysticerci which are deeply hidden in the muscles may be overlooked. I refer particularly to the small cysticerci which are few in number, as in the case of the tenia saginata. Cleanliness in the kitchen and in the household, especially in handling raw meat, is absolutely necessary. Vessels in which raw meat is kept should not be used for other food substances. Thorough boiling kills the cysticercus as well as corning, with or without subsequent smoking. The process of smoking, alone, by no means prevents the development of the cysticerci. The heat in the inner parts of the meat to be smoked certainly does not reach $50^{\circ} \mathrm{C}$. and an absolute protection is therefore not obtained.

The removal of the tape-worm in delicate children, in the aged, during the course of acute diseases, particularly severe intestinal diseases, and during menstruation and pregnancy, is usually contraindicated.

I carry out the treatment in the course of a day in which I may personally superintend it. For a long time the actual treatment is preceded by a preparatory one. I have been unable to convince myself of the value of a prolonged preparation. On the day before the treatment is begun, I give milk, and prohibit food cantaining sugar, so that the worm will have no favorable nutritive material. After the midday meal, if at all possible, I give only coffee; in the evening, bouillon, eggs, a little wheat bread, perhaps some red wine diluted with water. At night the patient is given a tablespoonful of castor oil or an enema.

Among numerous vermifuges, which I do not intend to enumerate here, I prefer radix filices or the ethereal extract of male fern, and order extr. fil. mar. æth. 5.0-6.0-7.5-10.0, syr. simpl. 40.0, M.D.S., to be taken within ten minutes. Children receive a correspondingly smaller dose. The tape-worm is to be stupefied by the remedy, and to be subsequently exposed to its action. If nausea or vomiting occur, I give small pellets of ice, some brandy, or a little lemonade. I am also quite fond of employing Helfenberg's Tape-worm. Remedy, which consists in the administration of eight capsules that contain one gram of fil. mar. and 2 grams of castor oil. According to the patient, I give 6 or 8 capsules at once, and, after an hour, 7 capsules which contain the castor oil. I will briefly describe my method of treatment. 
The patient is required to go to bed. A bed-pan, irrigator, lukewarm water and a black pus basin are to be ready at hand. The remedy must be given in the morning before food is taken. To prevent nausea and vomiting, small pellets of ice, brandy, or lemonade are taken. After an hour, one or two tablespoonfuls of castor oil are administered. If, after one or two hours; the worm has not been passed, an attempt should be made by the injection of a large amount of lukewarm water into the rectum, to expel the parasite, which, very frequently, has only been stunned. These injections are absolutely necessary if large portions of the parasite are passed, as they readily break upon manual extraction. If the head of the worm remain in the intestine, an attempt must be made to expel this by the injection of a larger amount of water.

This treatment may be regarded as successful when the head, or, more correctly, the heads, have been found. The search for the head, which is often detached, is facilitated by placing the worm which has been passed in the flat black basin. In spite of the greatest vigilance, it is occasionally impossible to find the head. The result of the treatment is then questionable. It should, however, only be repeated if, after eight to ten weeks, new proglottides are passed, showing that the previous treatment has been unsuccessful.

\section{ECHINOCOCCUS DISEASE}

Echinococcus disease in the human subject is of increasing importance, many cases having come under observation. I shall here consider the subject as briefly as is consistent with accuracy.

The echinococcus polymorphus is the encysted cysticercus of the tenia echinococcus discovered by $\nabla$. Siebold in 1853 , and is found chiefly in the small intestine of the dog, the jackal and the wolf. The assumption that the fox also may be a carrier and distributer of the tenia echinococcus has not yet been proven.

The tape-worm has a length of from 4 to $5 \mathrm{~mm}$. The head is armed with a rostellum and a double row of hooklets. The number of joints is only three; immediately after the last joint is desquamated a new portion forms. The last joint, which contains the mature eggs, comprises almost two-thirds of the entire length of the worm; it is of extremely delicate texture, and is easily lacerated.

If parts of the infective proglottides reach the stomach of man and of certain animals, the tissue of these undergoes digestion by the gastric juice, but the eggs which have been set free are not digested, nor is the embryo. This has six hooklets which, emerging from the egg, penetrate the intestinal wall, and thence in a more or less direct way reach the point where they later develop. It is quite probable that many of the eggs and embryos perish on account of the digestive power of the gastric juice, and are eliminated with the fecal masses, for, otherwise, multiple echinococci would not be so exceedingly rare -much rarer than the solitary form. According to van Beneden and Leuckart, the embryos forcibly penetrate the walls of the intestine, and finally reach the blood and lymph-vessels, in which they are carried onward. The fact that 
they breed in the capillaries of the portal circulation explains the frequent implication of the liver. Often the embryos lodge permanently in the mesenteric glands. Others, by means of the lymph-vessels, or by various avenues of communication, invade the peritoneal and pleural cavities; others enter the thoracic duct, thence find their way into the jugular vein and to the right heart, and are retained in the lung or are distributed to near or even distant organs.

After the echinococcus embryo has reached its destination it develops very slowly; we would digress too far if we described the individual phases of its development.

After the lapse of weeks or months, we again find the echinococcus in a round white cyst which gradually increases in size. Its walls are composed of a characteristically layered cuticula and a parenchymatous layer. After a certain time the latter shows upon its inner surface the so-called breeding capsules, which hang upon a short pedicle from the parenchymatous layer, and are surrounded by a transparent fluid not containing albumin. Some of the

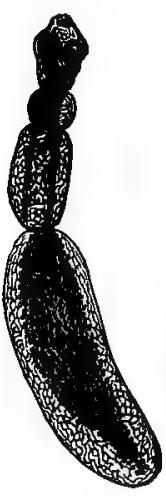

Fia. 50.-Tenta Echinococcus. Greatly enlarged. breeding capsules develop internally, some externally, producing echinococcus heads, the so-callcd scolices.

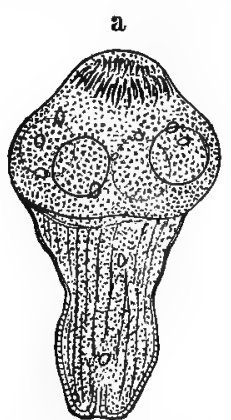

Fig. 51.-Head of the Echinococcus: $a$, with projected, and $b$, with retracted, head.

In many cases daughter-cysts are formed. These originate from embryos which probably represent original parts of the parenchymatous layer, but have remained intralamellar. They lead to cyst formation surrounded by its own cuticula. Gradually bulging inward, they finally reach the cyst cavity. We then speak of echinococcus hydatidosus or endogenus. This endogenous daughter-cyst development is opposed by the exogenous, which occurs mostly among our animals used for food, but may even occur in man. The daughtercysts may later form other cysts. The contents of the cyst are colorless and clear, or slightly opalescent. The fluid contains albumins which are not precipitated by boiling, also common salt, succinic acid and sugar; occasionally inosite, leucin and tyrosin.

The echinococcus cyst at any stage of its development may become sterile; i. e., the formation of scolices (acephalocysts) ceases. Not rarely the echinococcus worm becomes diseased and dies. The fluid becomes turbid, the walls of the cyst collapse, the parenchymatous layer softens and fatty degeneration 
takes place. The head falls off, is partly destroyed or undergoes more or less change, and swims about in the fluid. The contents thicken. A considerable deposit of calcium salts occurs and the shrunken sac may finally be represented by a single calcified nodule.

The echinococcus multilocularis, which in form so strongly resembles an alveolar colloid of the liver that hitherto its original nature has often been entirely misunderstood, must next be mentioned. It is found chiefly in the liver, but also occasionally in the brain, the heart, the diaphragm, and the pleural cavity; in the psoas muscle, the lung, the gall-bladder, the kidney, and the adrenals; in the uterus and anterior wall of the chest; in the duodenum; in the portal and peribronchial lymph-glands and in the bones. The multilocular or alveolar cchinococcus is characterized by small irregularly formed cysts which are separated from one another by masses of connective tissue enclosing a gelatinous plug. In section we recognize a honeycomb-like structure. All the cysts possess the properties of echinococcus cysts except for slight deviations. Upon the inner surface there are frequently layers of granular fatty material or pigment, besides balls with radiating stripes which consist of an organic base impregnated with calcium salts. Not rarely needle-shaped or sheaf-like crystals and hematoidin crystals are also noted. Scolices are often absent.

The limits of the tumor are quite sharply circumscribed. Now and then smaller isolated tumors are found adjacent. Virchow saw strands resembling a pearl necklace, the parts of which projected like processes. The capsule is invariably of a cartilaginous hardness, and adherent to the surrounding tissues.

This echinococcus was for a long time held to be only a variation in the form of the cystoid echinococcus, due to its growth in the blood-vessels, lymphressels, and biliary channels.

Its characteristic alveolar structure, as well as the conspicuous variation in its geographic distribution, has raised doubts as to the identity of the multilocular with the hydatid forms. To decide this question, experiments in feeding dogs with the echinococcus multilocularis were undertaken. Klemm, Vogler, Mangold, and Müller raised teniæ which in the form of their hooklets, as well as in the position of the eggs in the terminal section, differed from the tenia of the cystoid form. Mangold also succeeded, by feeding with a tenia which he had raised, in developing two tumor foci in the liver of a pig; these were recognized as echinococcus multilocularis. The occurrence of multilocular echinococcus in certain geographical regions is well known. According to Vierordt, it is found frequently in Württemberg, Switzerland, Bavaria, also in Austria and Russia, occasionally in Prussia, Baden and North America. Recently Posselt has called attention to a previously unknown region of distribution in the Tyrol; Pichler described a case of echinococcus multilocularis in Kärnten. It is especially interesting to note that in the "classical countries" of the hydatid echinococcus, Iceland, Australia, Mecklenburg and New Pomerania, Dalmatia and Argentina, the echinococcus multilocularis does not occur at all or has been very rarely observed. On the other hand, in the specific regions of distribution of the multilocular echinococcus, in Bavaria, Württemberg, and Northern Switzerland, the hydatid form is quite rare. In conse- 
quence of Posselt's very interesting investigations, I incline more and more to the view that there are two forms of tenia echinococcus.

I should like here to refer to the fact that bone echinococci, which in their distribution show great similarity to the multilocular echinococcus, are frequently identified with these. In bone echinococcus we are dcaling with the exogenous proliferation of small and very small cysts deposited close beside each other; but, besides these, there are also larger cysts with encapsulated daughter-cysts in the surrounding soft parts. Now such larger cysts with daughter-cyst formation are a characteristic of the hydatid form in which it differs markedly from the multilocular form. For this reason it cannot be assumed that every osseous echinococcus belongs to the multilocular form.

Besides being found in man, the echinococcus has been noted particularly in the lung and the liver of sheep, in cattle, and in pigs; more rarely in the dog, the cat, the mule, the horse, the zebra, the dromedary, the camel, the giraffe, etc.

Since it has been demonstrated with certainty that the echinococcus cysticercus occurring in man and his domestic animals represents one and the same tape-worm, there can no longer be any question as to the propagation of echinococcus disease in animal and man-that the dog takes up the grown cysticerci which develop in the domestic animals. The possible modes of dissemination of echinococcus disease in any country will therefore depend upon the number of its domestic animals, and the distribution of the echinococcus pest among them. As a matter of fact, those countries, such as Iceland, Victoria, Mecklenburg and New Pomerania, in which the echinococeus occurs most frequently, are noted for an excessively large number of animals, particularly cattle. A number of years ago I endeavored to investigate the distribution of this parasitic disease, and from the reports of 52 slaughter-houses I decided that the echinococcus occurs, upon the average, in 10.79 per cent. of cattle, in 9.83 per cent. of sheep, and in 6.47 per cent. of pigs. Pomerania and Mecklenburg in Germany are first in the frequency of the echinococcus disease. In Greifswald the dissemination of the pest is enormous. In one period of six months 68.58 per cent. of cattle, 51.02 per cent. of sheep, and 4.93 per cent. of pigs were found diseased. The conditions are similar in Mecklenburg. From my previous investigations I have concluded that the prevalence of the echinococcus infection in the inhabitants of a country is in proportion to the distribution of the echinococcus pest among its domestic animals.

In man, as well as in domestic animals, the dog is the usual, if not the only, transmitter of the infectious germ. The greater the number of dogs in any infected country, the greater the danger of contagion. In my own vicinity, the number of dogs, particularly in the rural districts, is very great. Apart from hunting dogs and shepherd dogs, almost every family possesses a house dog. He accompanies his master as he works about the farm, especially when milking cows, which in the summer occurs twice during the day, hence he has repeated opportunities to visit the grazing cattle, and to deposit his feces upon the feld. Here, on the small grazing places, the focus of infection must be sought for the grazing cattle, which remain here for months, by day and by night. 
But how does the dog become infected? Every one who has made observations in the rural districts knows perfectly well. The butchers as well as the shepherds-the latter, as a rule, performing the duty of slaughter in the country-are familiar with the danger of the echinococcus. Any one who has been in a farming country at slaughter time knows that the parts of the meat which are unsuitable for human food, among these frequently the liver and the lungs which are permeated with echinococci, are used as food for the dogs. Where there is careful management, organs of this kind are buried, but rarely so deep that the dog who sniffs about cannot finally reach them. A vicious circle is formed: the infected cattle, sheep and swine furnish the scolices to the dog; the dog furnishes the ova of the tenia.

If we observe the frequently intimate relation of children as well as adults with dogs, and how the dog will lick the face and the hands, we can scarcely doubt that in this way, as well as by kissing, infection can be conveyed. Dogs of every species sniff at their own ani as well as at those of other dogs; often they carry around upon their noses collections of mature proglottides without these being noticed on account of their diminutive size. Toys which small children often lick, pieces of bread scattered about in different corners of the nursery, may readily become contaminated by the mature eggs of the tenia. The dogs probably discharge the mature proglottides with the feces. In the feces of other dogs they are fond of sniffing around. Thus the dog's nose becomes contaminated, and the transmission of the ova of the tenia may occur. Frequently the dog's feces are discharged in the vicinity of pumps and wells. The proglottides and their ova continue to live for a long time on account of the moisture here constantly present, so that they reach the human stomach with the drinking-water as a still germinating brood. The teniæ may also be readily distributed by means of vegetables that are eaten raw, such as lettuce, cabbage, berries and fruits which grow upon the earth. The number of ways in which an infected material, or the eggs of the tenia, finally reach the intestinal tract of man is infinitely great, and it is unnecessary to presuppose an intimate association of the infected individual with dogs.

The number of children infected appears very small when we consider the fact that at this period of life, in consequence of close association with dogs, they are particularly endangered. This observation is confirmed by all statistics. In the Pomeranian investigation, among 139 patients only 13 were under fifteen years of age. The majority of infections occur between the twentieth and fortieth years of life. Probably in consequence of the slow development of the echinococcus in the human organism, the germs acquired in youth, as a rule, produce definite symptoms only in later years. That the course may be chronic is shown by my collection of cases. Among others, a midwife, aged sixty, from her twenty-fifth year (therefore for thirty-five years) had periodically coughed up echinococci. A farmer, aged forty, mentioned as the probable period of invasion the time from his ninth to his twelfth year, since during this period he always slept in a bed with several dogs. He had suffered since his thirty-fifth year with frequent and repeated attacks of irritative cough with expectoration that contained echinococcus cysts.

It is difficult to find proof from the statistics of Mecklenburg and Pome- 
rania that certain customs and habits of the people, their sex, social condition and occupation, their residence in the city or in the country, create a special predisposition to acquire the parasites. Echinococcus disease represents a slow, often insidious process, running its course for several decades, and the occasional cause, the place and time of infection, cannot be determined.

In the majority of cases only one organ is attacked. Unilocular echinococcus disease most frequently has its seat in the liver. If several organs are attacked-echinococcus multiplex-the liver is found to be one of the organs affected.

Several series of statistics have been compiled with regard to the frequency of " dog tape-worm." These indicate clearly that the liver is the organ most frequently affected; in the Mecklenburg investigation there were 69 per cent. of such cases, in that of Pomerania, 67.33 per cent. As the next organ to be implicated, the lung may be mentioned, showing 11.9 per cent. and 10.6 per cent., following this the kidncys with 3.5 per cent. and 4.6 per cent.

The abdominal cavity, skin, and musculature are also quite frequently attacked, that is, in about 6 per cent. of cases; but, in fact, any organ may be the seat of an echinococcus cyst. The view that traumatism favors the accumulation of the infectious agents may probably be due to the fact that after injuries the echinococci, which until then have been latent, manifest clinical symptoms. The conditions are certainly different with the appearance of multiple cchinococci, especially in the abdominal cavity. The opinion that the simultaneous invasion of many organisms may here be the cause will often prove correct. But another mode of development may also be observed; namely, autosemination after puncture or rupture of an echinococcus sac. Since $\nabla$. Volkmann first called attention to this danger, I have observed it clinically in many cases. The colonizing and propagation of cysts in the abdominal cavity has been absolutely proven by the experiments of Riemann under the direction of Garré. Similar experiments undertaken by other investigators and by myself failed of success, for some unknown reason. Either on account of the danger of disseminating the echinococcus parasite, or because of the toxicity of the echinococcus fluid, exploratory puncture has fallen into disuse.

The danger that the subsequent oozing of the echinococcus fluid from the point of puncture may bring about serious accidents and even death has been demonstrated by numerous observations. The appearance of urticaria after aspiration is a not infrequent occurrence. In a patient in Mosler's clinic, a few hours after the aspiration of an echinococcus of the liver, an eruption of urticaria appeared, soon followed by cyanosis and dyspnea. Only after the lapse of several hours did the threatening symptoms disappear. Other authors report the appearance of fever, singultus, nausea, arthritic pains, etc. A patient of Jenkins's died with these symptoms; Martineau and Bryant report similar cases. Experimental investigations also confirm the view that the echinococcic fluid may have a toxic action. Brieger, for instance, isolated among other substances one which he obtained in the form of salt of platinum, and, after separating it from the metal, he injected it in solution into mice with rapidly fatal result. Aspiration is not advisable, unless the radical oper- 
ation be performed immediately after the exploratory puncture; indeed, it is a question whether aspiration should be performed at all.

Echinococcus disease is not always easy to diagnosticate. Frequently at the autopsy, in the liver or other organs, cysts the size of a child's head and even larger are found which had not produced the slightest symptoms during the life of the patient. The gravity of the disease depends upon the location of the cyst, the size, the effect upon neighboring organs, and, possibly, the purulent character of the contents of the cyst. Should it suppurate, small echinococcus cysts in the brain and spinal cord may cause severe symptoms; on the contrary, occasionally, large sacs which have no deleterious effect upon the surrounding area exist for a long time without giving rise to serious symptoms. In general, the symptomatology may be described as follows:

\section{SYMPTOMS}

The embryo which has entered the body causes a reaction of the tissue which leads to the formation of the wall of a cyst. With continued growth the effect of pressure upon the neighboring tissue becomes apparent, and decided disturbances in circulation, secretion and excretion are manifest. Particularly dangerous is the development of the echinococcus in cavities where the growing tumor cannot fail to cause serious disturbance, as for instance in the pelvis, by compression of the organs situated in this region. When the development of the echinococci occurs in the respiratory tract, the symptoms there are also severe. The symptoms, however, become especially threatening if suppuration supervene, or the contents of the cyst should perforate its walls. If the contents of the cyst be emptied into the abdominal or thoracic cavities, fatal disease usually follows. When there is adhesion among neighboring organs, after penetrating the wall of the cyst the parasite enters these, and permeates them in the same way as the organs primarily affected. Thus the parasite traverses remarkably great distances. The course is most favorable when perforation occurs through the skin. Discharge into the stomach and intestine and into the pelvis of the kidney, into the bladder or into the vagina is relatively favorable. A cyst of this kind may very readily become the starting point of a fatal pyemia. Perforation into the trachea, into the bronchi, and particularly into the circulatory system is always most serious. Echinococcus of the superficial organs runs a less dangerous course.

The general condition of the patient is usually most affected when the echinococcus exerts an influence upon the neighboring organs by its size, or when suppuration occurs. Only after attention has been thus called to the affection does the patient seek professional aid. A number of conspicuous phenomena, such as the slow, steady, painless growth of the tumor, the absence of cachexia, the uniform roundness of the tumor, the smooth surface, the absence of fever, the elasticity, the presence of fluctuation, and the possible demonstration of a hydatid thrill, in general favor the existence of an echinococcus sac. By a hydatid thrill we mean that sensation which the tumor conveys to the percussing finger or to the palpating hand, a sensation similar to that obtained by pressing upon a cushion with springs. The cause of the hyda- 
tid thrill appears to be the waves of the fluid contents of the cyst; it is, therefore, to a certain extent only a distinct fluctuation. This condition also occurs in cysts of the ovaries, in ascites, etc. Santini and Rovighi have observed that the echinococcus cysts, besides the hydatid thrill, convey also, upon simultaneous percussion and auscultation, a characteristic, deep, sonorous tone, the so-called hydatid resonance. This phenomenon is said not to be present in deep seated cysts with thickening and degenerating walls. The size and extent of the cyst, as was demonstrated by Manasse in a case of renal echinococcus, may be determined by the Röntgen rays. Confusion of the echinococcus cyst with other tumors or abscesses, such as cystic degeneration of the kidneys, ovarian cysts, tumors of the uterus, tumors of the omentum, of the pancreas, subphrenic abscesses, etc., can, of course, never be entirely avoided. In spite of the most minute examination of the history, of the results of inspection, of palpation, and of percussion, and of the relation of the tumor to neighboring organs, the diagnosis in some cases cannot be made with absolute certainty unless some definite points of support are obtained by rupture of the cyst and an admixture of its contents with the sputum, with the vomitus, with the contents of the intestines, and with the urine. In cases which offer great diagnostic difficulty, an exploratory puncture may at once clear the entire clinical picture by the recognition of a fluid which is non-albuminous, and which contains scolices or hooklets. But the danger of this process will certainly always limit its use in diagnosis.

The prognosis depends upon the location, the size, and the relation of the echinococcus cyst to its surroundings. Although a benign tumor in itself, it may, particularly by suppuration, lead to phenomena which endanger life. In multiple echinococei the prognosis is correspondingly more unfavorable.

\section{TREATMENT}

Prophylactic measures must be premised with a caution, which cannot be too emphatically expressed, against intimate association with dogs. In infected regions their number should be limited as much as possible. Whether methodic tape-worm cures, on account of the perhaps brief parasitism of the tenia in the dog, are of any use, is doubtful. On the contrary, the important point in prophylaxis, it appears to me, is to protect the dogs from acquiring the tenia echinococcus. This may be best attained by the introduction of the compulsory inspection of meat. All organs infected with echinococci must be destroyed by cremation. Meat inspection in the country districts among cattle raisers is not likely to find favor when they learn that annually in Prussia about 880 cattle directly succumb to the echinococcus pest. The indirect harm arising from this is that animals infested with echinococcus suffer in their nutrition, and are prematurely slaughtered, and this may be an important point in agriculture.

Internal treatment is ineffectual, and successive punctures with aspiration of the contents as well as injections of medicated fluids should be rejected for the above-mentioned reasons.

On principle I am opposed to the method introduced by Baccelli of injec- 
tions of corrosive sublimate, although a number of cases have been reported with favorable results. The process consists in aspiration of the cyst contents with a succeeding injection of a 1-1,000 corrosive sublimate solution. I have seen recovery occur after puncture, even without injection of drugs, but much more frequently suppuration followed and finally made laparotomy necessary. Recovery through surgical means can only take place by the removal of the echinococcus sac.

The seat of the diseases is usually the liver. For this reason I should like briefly to describe the treatment of echinococcus of the liver. Madelung defines extirpation of the cyst including the capsule as the unquestioned "ideal" method of treatment. If of moderate size, with a superficial seat, and there is no suppuration, this operation will bring about the best results. In the majority of cases, the choice between a radical operation or one performed at two different times comes into question. If danger is present, the single or less radical operation is to be preferred on account of its greater rapidity. This is also recommended if the cyst is insufficiently exposed in the field of operation, or if, with multiple cysts, after the removal of one cyst the others are more easily reached. The method also by which an incision is made has the advantage of being less dangerous. As a rule, this method is resorted to if there are no special indications for more rapid interference.

Multilocular echinococcus has in only a few cases been treated by operation; namely, by Brunner, Terillon and Bruns: The latter advises cuneiform excision. Only the smaller multilocular echinococci can be successfully removed by operation.

\section{ECHINOCOCCI OF VARIOUS ORGANS}

I shall briefly review the echinococci found in different organs:

Echinococci of the brain are rare. Davaine mentions 32, Neisser 68 cases. Upon the whole about 90 cases are known, and among these is one case of multilocular echinococcus. Usually the parasite is solitary. It is generally of moderate size. The cysts are situated in the white as well as in the gray substance, and are surrounded by connective tissue capsules; the neighboring cerebral substance is anemic, partly atrophic. After a fairly prolonged time of growth a hemisphere may resemble a sac with thick walls. Several times after penetrating the skull, growth and discharge externally through the nose, ear, etc., have taken place. Westphal observed a case in which about 90 cysts were discharged. The seat and the size of the tumor determine the symptoms. General cerebral and focal phenomena appear until we have the symptoms of cerebral tumor. The diagnosis of the nature of the tumor will be possible only when echinococcus disease of another organ is present, or if, in the course of the disease, external perforation occurs. If the nature of the tumor and the localization of its seat are determined, surgical treatment may be successful. In the main the prognosis is unfavorable.

Echinococcus of the spinal cord is even rarer than that of the brain. About 22 cases in all are known. Usually the parasite is found between the dura and the vertebra, more rarely in the dural sac; or it may develop in the verte- 
bra itself, penetrating externally or internally. In some cases the parasite grows outwardly into the vertebral canal. Maguire mentions two cases in which the parasite developed in the spinal cord itself.

The symptoms are those of compression. The first phenomena frequently arise from disease of the vertebra, and from compression of the nerve trunks. The symptoms develop gradually, and resemble those of a compression myelitis. The entire absence of pain and the free movability of the vertebral column are conspicuous; this, however, may also be the case in tumors. Diagnosis becomes possible when the parasite appears. The prognosis has usually been unfavorable. In the case reported by Szkekeres, operation was attempted after a piece of bone one-half a centimeter in length had been exfoliated.

Echinococcus of the orbit. Echinococcus of the eye-ball has as yet not been determined with certainty, and echinococcus of the orbit is also rare. Krämer has collected 68 and Golowin 93 observations. To these may be added the cases of Ziegler, Wagenmann and Blaschek. It is noteworthy that, according to Krämer, the disease occurs three times as frequently in men as in women. In two-thirds of the cases the patients were between the eleventh and twentyfirst years of life. As a rule, the echinococcus develops primarily in the orbit, in rarer cases it finds it way into neighboring organs after destruction of the wall of the orbit. The parasite is found in both orbits with about the same frequency, and almost always in the base; rarely in the anterior lateral parts. The development is usually insidious, seldom fulminant. The first symptoms are commonly pain and the development of an exophthalmos. The conjunctiva and lids become inflamed. Decrease in motion of the bulbus oculi, disturbances of vision, and loss of sight in varying grades appear gradually. Suppuration is not rare. In cases in which no operation is performed, early atrophy of the optic nerve, necrosis of the cornea, and panophthalmia occur. The diagnosis is often very difficult. Confusion with malignant neoplasms has several times led to enucleation. Krämer cautions us against exploratory puncture on account of the danger of confounding the condition with that produced by an encephalocele. The slow development of a tumor in the orbit without fever, severe pain deep in the orbital cavity, a tumor with distinct or indistinct fluctuation, and the early appearance of disturbances of sight are all strongly indicative of echinococcus. The treatment is surgical.

The echinococcus has been twice observed in the frontal sinus, which had ruptured into the orbit.

In the nose, in the oral cavity, in the tongue, in the gums, in the pharynx and in the parotid the echinococcus has also occasionally been observed.

Echinococcus in the neck is a rare occurrence. Guiterbock has collected 26 cases of this kind. Besides these I found cases recently published by Reich, Jürgens, Thévenot and Steinbrück. The parasite generally chooses as its seat the region of the external border of the sternocleidomastoid; this without doubt accounts for its originating in the sheaths of the large vessels of the neck. The echinococcus sac slowly raises the sternocleidomastoid and appears at the internal border of the muscle as a small tumor, while the larger portion of the sac is situated upon the external border. The connection of both tumors is proven by the continuance of the fluctuation. Later, with increased growth, 
the characteristic lobulated tumor disappears. Decided displacements of the organs of the neck may develop in such cases. Confusions may arise from echinococci which are situated in the muscle itself or that have developed in the thyreoid gland. In three cases after successful extirpation death resulted from secondary hemorrhage.

Echinococcus of the thyreoid gland is rare. Henle reports 18 cases; in three other cases the diagnosis is questionable. Vitrac has collected 21 reports of echinococcus of the thyreoid. Another case is reported by Posadac. The echinococcus is usually unilocular and may attain the size of a fist. Increasing growth causes atrophic conditions of the glandular tissue. The consequences of displacement, that is, compression, of the neighboring organs, in which particularly the trachea, the esophagus and the recurrent laryngeal nerve are to be considered, may be serious. Suppuration of the cyst is frequent. The sac surrounding the echinococcus becomes adherent by inflammation to the adjacent areas, and these are to a certain extent involved in the echinococcus sac, so that this represents only a part of the echinococcus. This enlargement results in increased erosion of the surrounding areas in which the trachea particularly may be implicated. Adhesions to the muscles and the skin are less frequent. The echinococcus grows very slowly. Its form is usually globular. The difficulty in respiration which occurs, to which difficulty in deglutition and paralysis of the recurrent laryngeal nerve may be added, causes the patient to consult a.physician. The diagnosis can rarely be made with certainty. Early operation improves the prognosis decidedly. According to Henle wide opening of the sac is most frequently advisable, as enucleation is often very difficult. If this operation be attempted, it is not always possible to spare the recurrent laryngeal nerve which may be situated in the wall of the sac.

A case of echinococcus of the larynx has been described by Schüssler.

Primary echinococcus of the pleura is rare. Maydl and. Winzerling have collected 30 cases from literature. In these the echinococcus was situated either between the pleura pulmonalis and the costal pleura, in the pleural cavity, or external to the pleura costalis. Much more frequent are secondary pleural echinococci which have ruptured into the pleural cavity from neighboring organs.

The echinococcus is usually found upon the right side, surrounded by a thin wall of connective tissue. It generally attains great dimensions. In the main the symptoms differ but little from those of a pleurisy with effusion. The patients complain of stitch in the side and an irritative cough, accompanied by scant mucus expectoration. Fever is absent. If the echinococcus is large it leads to dilatation of the affected side of the thorax, frequently giving a flaring shape to the lower part of the thorax, with retardation or immobility in respiration. Percussion reveals a more or less extensive area of dulness, frequently bow-shaped. Absence or diminution of the respiratory murmur and of fremitus is noted; occasionally bronchial respiration is present. In small cysts egophony and bronchophony are observed. Neisser quite properly emphasizes as characteristic the conspicuous proximity of normal and abnormal auscultatory phenomena. In right-sided disease, the liver is dis- 
placed downward and the heart to the left. Left-sided echinococci lead to dislocation of the spleen and kidney. In bilateral echinococcus the heart is forced into the mediastinal space. If the echinococcus has a tendency to grow outwardly it forces its way through the intercostal spaces, leads to erosion of the ribs, and appears as a semiglobular tumor beneath the skin. The skin is usually movable over the tumor, but occasionally adheres to it, and shows decided inflammation. Secondary pleurisy is said to be invariably absent. In the main, the echinococcus shows but slight tendency to perforation. If rupture occurs, it usually takes place through the bronchi.

The diagnosis is mostly difficult. Differentiation from solid tumor is, however, generally possible. If a collection of fluid can be detected, the question arises whether this is situated above or below the diaphragm. In favor of a subdiaphragmatic seat is the bell-shaped prominence of the lower parts of the thorax, and the absence of the respiratory displacement of the liver downward. On account of atrophic paralysis of the diaphragm the upper bow-shaped line of dulness prevents the recognition of respiratory displacement. Besides, the history usually points to a disease of the liver. Yet in many cases a positive diagnosis is almost impossible. To distinguish echinococcus from pleurisy with effusion is important. The latter is accompanied by more marked constitutional and local phenomena, chills, fever, cough, stitch in the side and dyspnea. Later the dyspnea is less marked, while in the case of echinococcus it is one of the most prominent and troublesome symptoms. Pleural exudates lead to a uniform bulging, pleural echinococcus to a protuberance, of the lower aperture of the thorax. In pleurisy the line of dulness follows the well-known characteristics, while in echinococcus the line of dulness shows irregularities. The demonstration of another tumor in the liver is not without importance. The course of pleurisy is much more rapid than that of the slowly growing echinococcus. Exploratory puncture is not advisable on account of the danger associated with it, unless an immediate operation is to follow.

Echinococcus of the lung is next in frequency to that of the liver. According to Madelung 11.9 per cent. of all cases occurred in the lungs, and according to the Pomeranian statistics, 10.6 per cent. The parasite may develop in any part of the lung; most frequently, however, it is met with in the right lower lobe. The latter circumstance is explained by the frequent simultaneous affection of the liver. The thinness of the walls of its capsule, as elsewhere, and the yielding of the pulmonary tissue make the decided growth of the echinococcus possible, and it not rarely fills the pleural cavity, leading to displacement of the heart, of the diaphragm, the liver and the spleen. Symptoms of chronic inflammation, of hepatization, of atrophy, or of gangrene, may develop and complicate the pathology. Erosion of the bronchi and of the pulmonary vessels, and rupture into the pleural cavity, are not infrequent. As the result of destruction of larger blood-vessels, severe, often fatal, attacks of hemoptysis occur.

The passage of daughter-cysts into the blood-vessels with the formation of emboli has been observed. Upon opening into the bronchi, gangrenous sputum appears in which daughter-cysts, scolices or shreds of membrane are found. 
In other cases the symptoms of pyopneumothorax develop. Not rarely rupture into the diaphragm occurs, and thus the echinococcus finds entrance into the parenchyma of the liver. Rupture into the intestine and into the walls of the abdomen etc., has also been observed. Secondary pulmonary echinococci are rarer than primary ones. Usually originating in the liver, they penetrate the diaphragm and the pleural cavity and enter the lung. Rupture into the bronchi or into the pleural cavity produces severe symptoms. The simultaneous perforation of the biliary passages and the intestine has been repeatedly observed.

The symptoms for a long time may be indefinite and point only to an affection of the respiratory organs. With increasing growth a very trouble-

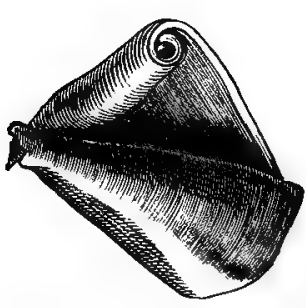

Fig. 52.-EXPECTORATED ECHINOCOCCUS MEMBRANE. some cough and attacks of dyspnea usually occur. As the first symptom I have repeatedly observed hemoptysis with cough, so that the clinical picture resembled phthisis pulmonum. Severe paroxysms of cough may cause perforation by the echinococcus, mostly unilocular, into the bronchi, and the discharge of cysts, large, rolled-up masses of membrane or shreds of membrane and scolices. The seat of the disease, and sometimes even the side which is affected by the disease, may be difficult to recognize, if the cyst is centrally situated or is surrounded by a thick layer of pulmonary tissue containing air. Not rarely, prior to rupture, suppuration or putrefaction of the contents of the cyst occurs, and this always takes place after rupture of the cyst.

If the seat of the echinococcus is at the periphery of the lung, especially if it has attacked an upper lobe, the symptoms resemble chronic pulmonary tuberculosis; but the fair condition of nutrition, in spite of repeated hemoptysis, and the absence of the characteristic expectoration are opposed to this view. Occasionally a complication with tuberculosis is present. Even when the disease attacks a lower lobe, no signs are usually manifest other than those of a pleural effusion. The nature of the affection is frequently determined only by exploratory puncture, which is performed with the expectation of finding a pleural exudate or an empyema.

The prognosis of pulmonary echinococcus is serious, but by no means absolutely unfarorable. In the Pomeranian statistics, among 16 cases spontaneous discharge of the cyst was followed by recovery in eight, and two were cured by operative treatment. Death resulted from exhaustion in two cases. One that was operated upon died. In another, pulmonary echinococcus was only an accidental autopsy finding. Surgical interference appears to be advisable in all cases in which physical examination reveals suppuration and putrefaction in the cyst.

Echinococcus of the mediastinum is uncommon. About six cases only are on record. On account of the proximity to the heart and lungs, the echinococcus here causes grave danger.

Echinococcus disease of the circulatory apparatus is rare. According to Huber about 40 cases have been observed. To these may be added the cases 
of Demantke, Lehne and Klehmet. The echinococcus is usually unilocular. The right heart is more frequently implicated than the left. The seat of the parasite is the heart muscle, and here it attains its growth. For the most part its course is marked by no symptoms, at least of parasitism. The conditions are different, however, if the distended cyst grows into the cardiac cavities and bursts its sac. The symptoms of cesto-embolism in this case do not differ decidedly from emboli as the result of thrombi. According to the size and extent of the embolus, according to its benign or malignant (putrid) composition, and according as the smaller or larger vessels are obstructed, the disturbances due to emboli of cestodens show varying characteristics. We should digress too far if we further discussed these extremely complicated conditions. Death often results suddenly without preceding symptoms. In some cases there are antecedent signs of disease of the heart and of the lungs; in other cases death takes place after a long illness with complications such as perforation and embolism of daughter-cysts.

In emboli into the greater circulation the perforating cyst is usually situated in the left heart, or the cysts develop from a pulmonary echinococcus which has burrowed into the pulmonary vein. Death, as a rule, occurs suddenly. It may be readily understood that such cases as these are rarcly under clinical observation for a long time; the disease is found at the autopsy in cases where death has occurred suddenly.

Echinococcus of the blood-vessels and lymph-vessels as well as of the pericardium is extremely rare.

Echinococcus of the liver is the form most frequently met with. In the Mecklenburg statistics they amounted to 69 per cent. of all observed cases, in the Pomeranian statistics to $6 \% .33$ per cent. They are for the most part unilocular, rarely multiple. They are found in all parts of the liver, but most frequently in the right lobe. When centrally situated the cyst grows but slowly, while echinococci peripherally situated give rise to great enlargement of the organ, even to six or seven times its normal size. The liver substance is ruptured, and the cyst appears with single and multiple semiglobular elevations upon the surface. If the echinococcus develops upon the convex surface it forces the diaphragm far up into the pleural cavity, and causes compression of the lung and displacement of the heart.

Occasionally the echinococcus penetrates the diaphragm and extends into, or perforates into, the pleural cavity or the bronchi. When the cyst spreads into the abdominal cavity, it causes displacement of the stomach and intestines. It occasionally adheres to these organs, and, finally, ruptures into them; or, in the absence of adhesions, it may perforate the abdominal cavity. This is, however, rare. In such cases fatal peritonitis is the immediate consequence, while with rupture into the stomach, intestines, etc., ultimate recovery is possible. The paths which the echinococci may make for themselves are numerous.

Echinococci of smaller size often produce no symptoms, or, at least, not for a long time. Usually only with increasing growth does the parasite manifest its presence. Symptoms are at first local, such as a sense of pressure, of heaviness, or occasionally pain. Frequently echinococci of considerable size 
are seen which have grown almost without symptoms. They finally, however, lead to displacement of adjacent organs, particularly of the lung and the heart, and a prominence appears in the hepatic region and in the lower parts of the thorax. Protuberances from the size of an apple to that of a child's head are not rare, and they may be recognized by inspection and palpation. Palpation is almost always painless. Hydatid thrill, a symptom which has been mentioned so often, is occasionally present. I have only rarely been able to elicit it. Jaundice is by no means frequent. As the size of the tumor increases it gives rise particularly to gastric difficulty: disturbance of appetite, nausea, a tendency to vomit, constipation. Dyspnea, cough, attacks of fear, cardiac palpitation, etc., also occur.

While the course has previously been afebrile, when suppuration of the cyst occurs high fever, chills, and great pain appear, and, if suppuration be long-continued, pyemic phenomena in the lungs, spleen and kidneys may bring a threatening change. Rupture into the pleural cavity, into the lungs, and into the abdominal cavity may also give rise to a condition of collapse, pyopneumothorax, peritonitis, etc., and may seriously endanger the life of the patient. Or perforation into the stomach or intestine may lead to vomiting or discharge of the characteristic contents of the cyst. Rupture into the biliary passages we have several times known to cause the discharge of bilestained cysts or shreds of membrane with the symptoms of a colitis hepatica.

In rare cases the echinococcus is felt beneath the abdominal walls far removed from its original seat, and here finally leads to rupture.

The course described is not invariable in echinococcus disease. Fortunately, the echinococcus not seldom develops to only moderate size. It dies, the sac contracts, and the contents are absorbed. Calcareous nodes and broken cheesy masses in which portions of the echinococcus may be recognized are occasional autopsy findings. In the Pomeranian report, compiled by Wiedemann at my instigation, and including more than 153 cases, echinococcus of the liver occurred 34 times $=33.01$ per cent. as an accidental autopsy finding.

The same compilation gives data concerning the prognosis of echinococcus cases. In 28 instances there was no operative treatment. Twelve patients died of chronic invalidism. Spontaneous recovery by perforation into the lung and intestine occurred in 8 patients. In 8 other cases the further course was unknown. In 41 operative cases recovery followed in $34=82.92$ per cent. Improvement occurred in $4=9.75$ per cent.; 3 died $=7.31$ per cent.

In the differential diagnosis, besides amyloid fatty liver and hypertrophic cirrhosis-diseases which are sufficiently characterized by the accompanying symptoms-we must consider: Hepatic abscess, cancer of the liver, syphilis of the liver, dropsy of the gall-bladder, hydronephrosis, etc.

Lennhof calls attention to a symptom he observed in cysts of the lower part of the liver-an "inspiratory furrow." Upon deep inspiration, according to his observation, the prominence of the abdomen due to the tumor moves downward while the skin retracts above this, and between the arch of the ribs and the prominence a flat furrow is formed.

Abscess of the liver usually develops acutely, and is accompanied by a chill, 
by decided pain and rapid loss of strength. Differentiation between hepatic abscess and a suppurating echinococeus cyst is frequently very difficult. Neither is differentiation between a cyst and a soft cancer nodule easy, for such nodules may frequently produce the signs of fluctuation. Confusion with dropsy of the gall-bladder may readily be avoided, as its position remote from the liver, the form of the tumor, and the anemia will point out the correct diagnosis.

The oval form of hydronephrosis, the immobility in respiration, its position in relation to the colon, palpation in the knee-elbow position, and an examination of the urine will prevent us from confounding echinococcus with hydronephrosis.

Multilocular echinococcus also frequently exists for a long time without symptoms, and then the sensation of fulness and heaviness in the epigastrium and stubborn jaundice appear. Bulging in the hepatic region constantly increases as the result of the decided enlargement of the organ. Palpation reveals a tumor of hard consistence like cartilage; the surface is smooth and nodulated. Fluctuation at first is limited to individual parts. The splecn is usually enlarged. Nutrition gradually suffers; ascites, anasarca and attacks of fever occur. Death results after one or several years, accompanied by the symptoms of marasmus.

In form and size, and in the hardness of the liver, multilocular echinococcus resembles hepatic cancer. But the course of the disease is slower, and nutritive disturbances develop only in the advanced stages. Syphilis of the liver presents other forms and degrees of enlargement. The chills and marked jaundice which usually occur in abscess of the liver will differentiate this affection from multilocular echinococcus. Hepatic cirrhosis and amyloid liver will, as a rule, be easily differentiated from the hydatid. The prognosis has usually been considered absolutely unfavorable. We have already called attention to the results recently obtained by operation. Salis.

Echinococcus of the pancreas has been described by Subboitic, Pericic and

Echinococcus of the spleen is rare; according to the Pomeranian statistics it occurred in 4 per cent. of the cases, in the Mecklenburg statistics in only 1.96 per cent. The parasite finds a favorable medium for its growth in the soft splenic tissue. In the main, the symptoms of splenic echinococcus resemble those of hepatic echinococcus, but with the difference that the formation of tumor is only upon the left side. Splenic echinococcus is not rarely complicated by echinococcus of other organs.

Echinococcus of the kidney, which in the Mecklenburg statistics constitutes 3.5 per cent. of the cases, and in the Pomeranian 4.58 per cent., usually develops in the cortical substance. Renal echinococci for the most part are unilateral. As it grows all parts of the kidney may be implicated in the disease, and the kidney be transformed into a large echinococcus cyst. This cyst extends into the abdominal cavity, and leads to dislocations and to adhesions to neighboring organs. Diseases of the most varied kind may thus be simulated. Small tumors produce no symptoms; larger ones cause pressure phenomena, difficulty in respiration, etc. After years the symptoms of maras- 
mus develop, provided rupture into the pelvis of the kidney and discharge of the cysts have not occurred. The urine which may be voided prior to, with, or after, the discharge of the cyst is turbid, occasionally of a soapy-alkaline, milky, or turbid appearance, and in the sediment the characteristic contents of the cyst are found. After discharge the palpable tumor disappears, to recur after some time. The affection may last for many years. Occasionally the echinococcus takes other roads; perforation into the pleural cavity and abdominal cavity has repeatedly been observed.

Renal echinococcus differs from cysts of the liver and of the spleen by the immobility at its base, by its outlines which may be determined by percussion from the borders of these organs, and by dislocation of the colon. The differential diagnosis between renal echinococcus and hydronephrosis is very diffcult. The history of the development of the tumor, the proof of a hindrance to the discharge of urine, the previous presence of renal calculi or of a stasis pyelitis, point to the presence of hydronephrosis.

The prognosis of renal echinococci is considered by most authors to be very grave. Fortunately, with the advance of modern surgery, operative treatment gives better results than formerly.

In the adrenals only one case each of the cystic and of the multilocular form has up to the present been found.

Echinococcus of the urinary bladder has been reported by Hinsworth and Schönfeld.

Winterberg has described a case of echinococcus of the prostate gland.

Echinococci in the abdominal cavity. Primary cysts of the peritoneum appear to be rare. They are always multiple. Their origin may be referred to a profound general infection or to an autoinfection. The parasite is usually found simultaneously in other organs. Numerous adhesions may frequently preclude the determination of the point of origin. The symptoms which result are due to the pressure exerted upon the abdominal organs. Perforation is rare.

Primary retroperitoneal echinococci are not often found except in the connective tissue of the pelvis and in the female genitalia, where the cysts are situated subserously. In the Pomeranian statistics we find only one case of this kind described by Bitter. Karewski recently published two cases. The symptomatology is usually obscure, and when suppuration takes place it points to a perinephritic abscess. On account of the pressure of the thick layers of muscles and of the bones of the trunk, held by tense fascia, the echinococcus finds but little room for growth, and is forced to develop from the surface by daughter- and granddaughter-cysts of exogenous growth. Conspicuous symptoms appear only when suppuration occurs. To facilitate the diagnosis, Karewski advises exploratory puncture, all danger being obviated by the extraperitoneal position.

Echinococci of the mesentery are somewhat infrequent, and their diagnosis is not easy. In regard to their differentiation from cysts of the omentum, Hahn states that the latter can come into question only when fixed loops of intestine can be determined between the tumor and the abdominal wall, or in the surroundings of the tumor, or in the limits which may be defined by 
palpation or by adhesions. If the intestine is in front the tympanitic note of the latter is the most important diagnostic symptom.

Echinococcus of the omentum is rarely unilocular. Cases of this kind have been published by Grünig and Lüitkemüller.

Echinococcus of the true pelvis and of the genitalia. In the male, echinococci in the true pelvis develop in the space between the rectum and the bladder. Their growth leads to disturbances of function of the rectum and bladder. Now and then perforation into the rectum occurs.

In the female we find echinococcus in the uterus, in the ovaries, in the ligamenta lata, in the anterior and posterior halves of the petvis.

In the uterus the echinococcus usually develops in the submucous tissue. Scanzer's view that the embryo in its wanderings reaches the abdominal opening of the Fallopian tube grasped by the fimbrix, enters the cavity of the uterus, and develops fully there, is quite properly rejected by Schatz for the majority of cases. The submucous seat is explained by Schatz from the circumstance that the embryo, owing to greater strength of the blood current in that direction, particularly at the time of menstruation, is forced against the interior muscular layer near the mucosa. It evidently develops in the muscles, and finally, when labor pains occur, enters the cavity of the uterus through the muscles which have gradually become atrophic. The hydatid has also been found in a subserous position.

The echinococcus lodged in the wall of the uterus is commonly mistaken for a myoma, if echinococci have not appeared in other organs. The circumstance that the wall of the uterus, which in the case of myoma is usually decidedly hypertrophied, is not found so in the case of echinococcus (Schatz) may be utilized in the diagnosis. Subserous myomata develop only a moderate muscular hypertrophy; their consistence is not characteristic. Thirteen cases are mentioned in literature in which the echinococcus sac was an impediment at birth (Schmidt, "Veber Ech. in weibl. Becken," Diss. 1893).

Echinococci of the ovaries and ligamenta lata are rare. Schultze could collect from literature only eleven cases of echinococcus of the ovary, to which he added two cases that came under his own observation. Schatz remarks concerning the diagnosis that a tumor of about the size of an orange, very deeply seated near the uterus, should cause us to suspect echinococcus. Later a group of such tumors, of equal size and closely situated, forms an important diagnostic symptom. Extrauterine pregnancy on account of its rapid progress, and disease of the tubes on account of the characteristic form of the enlargement, can scarcely be confounded with echinococcus. Beowit recently saw both tubes transformed into echinococcus sacs.

Echinococcus of the cellular tissue of the petris is in the majority of cases found upon the inner surface of the posterior wall, less frequently upon the anterior. The diagnosis is greatly facilitated by the ease with which the diseased area may be reached. Echinococcus of the pelvis may be suspected if one or several smooth, tense, elastic but slightly movable tumors, not painful upon pressure, are found, and the ovaries may be distinctly determined. In many cases the cyst is situated between the uterus and the rectum. Fever 
and cachexia are absent. The simultaneous presence of the parasite in other organs facilitates the diagnosis.

Echinococci of the scrotum, the epididymis and the tunica vaginalis have been observed in a few cases.

Echinococcus of the bones is rare. In the Pomeranian statistics not a single case is mentioned.

The parasite is found more frequently in the long bones than in the short bones. In the cases collected by Poppe, the frequency with which different bones were affected was as follows: Humerus 23 per cent., pelvis and tibia 18 per cent., vertebral column and femur 13 per cent., frontal bone 5 per cent., sphenoidal bone, scapula, index finger, sternum, and ribs each 1 case.

The parasite develops in the medullary cavity, i. e., in the spongy substance, and is at first entirely latent. Only with increasing growth do characteristic changes occur. In the long tubular bones the medullary cavity dilates, the wall of the bones becomes eroded, the external wall thin. Often there is severe pain. Slight trauma leads to spontaneous fracture without any previous symptom of disease of the bone. The fracture does not heal, a fact which usually enables us to recognize the nature of the affection. Schnitzler's case proves that occasionally, even in echinococcus of the long tubular bones, decided changes in form and circumference of the bones may take place.

\section{NEMATODA, THREAD-WORMS}

The nematoda are slender, cylindrical, curved, thread-like or tubular worms. Their surface is smooth and curled, occasionally supplied with hooklets, hairs, or bristlets. The anterior end of the body in which the mouth cavity is situated is somewhat slender, the posterior end is pointed or rounded. The anus is usually found upon the ventral surface. The nematoda, at least those which are parasitic in man, are of different sexes. The males, as a rule, are shorter and more slender than the females. In the males the posterior end of the trunk is usually curved, while in the female it shows a straight elongation. In the male the sexual opening and the anus are one, while in the female the former is usually found at about the center of the body. Of the nematoda which are parasitic in man I shall briefly mention:

Rhabditis terricolæ, Dujardin, 1845 , which was once found in the cadaver.

Rhabditis pellio, Schneider, 1866, was found in acid urine containing albumin, blood and pus. I believe that I once saw this parasite with Westphal.

Rhabditis Niellyi, Blanchard, 1885, in a cabin boy produced an itching skin eruption.

Anguillula putrefaciens, Kühn, 1879, was once observed in vomitus. The parasite was evidently taken into the stomach of the affected individual with onions, and then had produced vomiting.

of decidedly greater interest is:

Anguillula intestinalis et stercoralis, Bavay, $187 \%$.

In 1876 Normand, a French marine surgeon, found, in the feces of a soldier who suffered from the so-called Cochin-China diarrhea, anguillula which 
were designated by Bavay as anguillula stercoralis. Another parasite soon afterward found by Normand in patients of this kind was described by Bavay as anguillula intestinalis. By the investigations of Leuckart, Grassi, and Leichtenstern, the relations of these parasites have been determined with certainty. For purposes of study, I may refer to the investigations of the previously mentioned authors as well as to those of Askanazy and Zinn.

According to these investigations the development occurs in the following manner: the hermaphroditic mother animal of the anguillula intestinalis, according to Askanazy, lodges in the intestinal wall, chiefly in the mucous membrane, and frequently bores its way into the epithelium of the gland, there to take up nutritive products. The females at the same time deposit their eggs here. The ova are transformed into embryos which then enter the intestinal cavity. These are met with in fresh feces, often in great numbers, and, according to Zinn, in about twelve hours are transformed into filarialike larvæ. These again enter the intestinal canal of man, and are here formed into parasitic anguillula. This process is called direct metamorphosis.

Other embryos of anguillula intestinalis develop outside the body in about two to three days into sexually ripe male and female animals (rhabditis stercoralis). The direct descendants of these are the embryos of the rhabditis stercoralis, from which the filaria-like larvæ are again formed. The latter wander into the intestinal canal of man, and grow into mother animals of the parasitic anguillula intestinalis. This mode of development is designated by Leuckart as heterogeny. This heterogeny, as was first emphasized by Leichtenstern, does not occur in the majority of cases, particularly in those of European origin.

Anguilluliasis occurs not only in southeastern Asia, Martinique and Brazil, but was met with in 1878 and 1879 by Grassi and Parona in Italy, simultaneously with anchylostomiasis. Lately Askanazy found them in a gamekeeper in East Prussia who had never left his home, and who never came in contact with Rhenish brickmakers.

The parasites live in the juices of the small intestines. Askanazy also found them in the tissues of the mucosa, sometimes stretched out or rolled together. They distribute themselves in the muscularis mucosa, and are met with in great numbers in the region of Lieberkühn's glands. The parasite enters the walls of the intestines to partake of the chyle. Here, according to Askanazy, the eggs are deposited, and the parasite goes through its stages of development. Teissier has observed that the embryos occasionally enter the circulation. Askanazy seems to favor the view that the parasites are to be regarded as no more than simple commensals.

Of special interest is the:

\section{Filaria medinensis Velsch, $16 \% 4$.}

The females attain a length of from 60 to $80 \mathrm{~cm}$. and in form and appearance resemble a catgut string. The male, according to Charles, has also recently been found. It is only $4 \mathrm{~cm}$. in length. Dracontiasis, as the pathologic phenomena produced by the parasite were called by Galen, is due to the fact that the worm penetrates the human organism, and may remain for 
a long time under the skin of the connective tissue entirely latent. Only after completing its full growth-the period of incubation appears to be from eight to ten months-does it leave the papillary bodies of the corium by the formation of an abscess.

The medina worm is most frequently found in tropical countries, particularly in the Old World: in Arabia, around the Persian Gulf, upon the Ganges, the Caspian Sea, in Upper Egypt, Abyssinia and Guinea. It has also been carried to South America.

The filaria embryos enclosed in the uterus can only secure their freedom by rupture of the mother animal. How their further dissemination in the human organism takes place has not yet been definitely determined. It has been thought that the embryo finds ingress through the skin. Some authors assume that infection is conveyed by drinking-water. Probably the worm finds a hold around the lower extremities, especially about the malleoli. It is much more rarely found upon the upper parts of the body.

The symptoms resemble the formation of a furuncle, at the base of which the parasite is seen. Upon extraction of the worm, rupture of the parasite should be avoided, and care should be taken to leave no fragments behind, for, otherwise, decided inflammation soon appears.

A more delicate and smaller parasite is:

\section{Filaria loa Guyot, 1778,}

which lodges between the conjunctiva and bulbus oculi, and gives rise to abscess formation in this region. The parasize is found chiefly in negroes upon the west coast of Africa, also in South America and in the Antilles.

The other filaria are not of special interest. Among these I may mention the filaria lentis, Diesing, 1851, the filaria hominis bronchialis, Rudolphi, 1819, the filaria labialis, Pane, 1864, the filaria inermis, Grassi, 188\%, the filaria immitis, Leidy, 1856, the filaria rectiformis, Leidy, 1880, the filaria hominis oris, Leidy, 1850, the strongylus subtilis, Loos, 1895, and the filaria volvulus, Leuckart, 1893.

\section{Filaria Bancrofti, Cobbold, $188 \%$.}

The embryos of this parasite were found in the fluid of a hydrocele by Demarquay in 1863 in a Havanese. They were found later in the blood, the urine, and in the chyle in so-called lymph-scrotum and in elephantiasis. Bancroft and Lewis found the mature sexual forms in the lymph-vessels. The females bear living young, which enter the blood with the lymph-stream. According to Manson they enter the blood only after sunset; after midnight their number again decreases. If the patient sleep during the day, the embryos appear in the blood during the daytime.

This periodic appearance is explained by $v$. Linstow by the circumstance that the peripheral cutaneous vessels dilate during sleep, and thereby permit the entrance of the embryos, which otherwise would only be present in the larger vessels. Manson states that the appearance of the embryos is simultaneous with the swarming of mosquitoes, which suck the blood of the affected person, and are then filled with embryos. A part of the young brood develop 
with the mosquitoes, and a part are destroyed. The female mosquitoes die after depositing their eggs in water. But the mature filaria, meanwhile, have acquired the power of living for a time in water. With the water they enter the human host and there, chiefly in the lymph-vessel system, they become sexually mature animals.

The male parasite is about $83 \mathrm{~mm}$. in length, and has the thickness of a hair of the head, while the female is $155 \mathrm{~mm}$. in length and $0.7 \mathrm{~mm}$. in

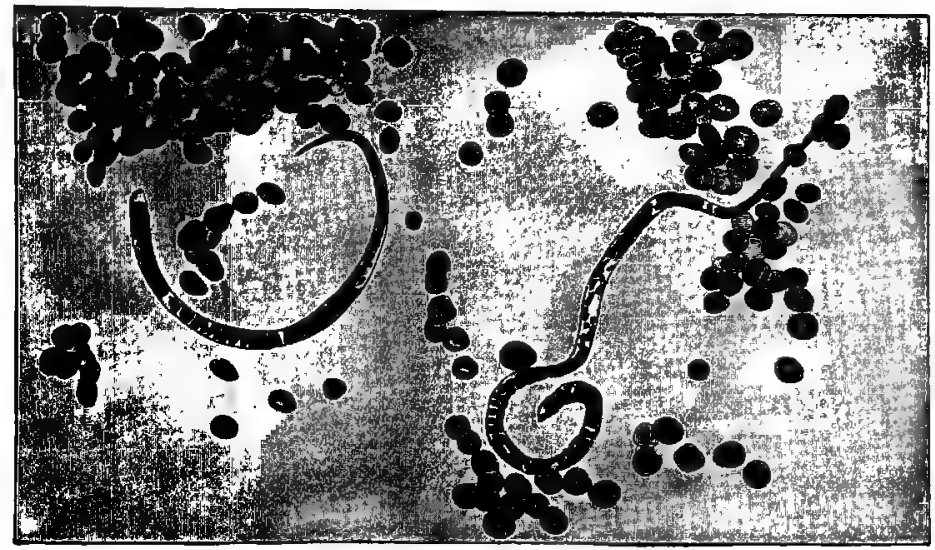

Fig. 53.-Filaria Embryos.

This preparation I owe to the kindness of Professor Magathães of Rio de Janeiro.

breadth. The posterior end of the male is pointed, and rolled in a spiral. The sexual opening of the female is close to the head. In fresh blood the embryo shows active movements of both ends; it is perfectly transparent, and only in the middle of a parasite may a few granule clumps be recognized. Scheube found them upon the average $0.216 \mathrm{~mm}$. long and $0.004 \mathrm{~mm}$. broad.

The home of the filaria is tropical Asia, Africa, America, and Australia.

Besides the filariæ of Bancroft and Cobbold, other blood filariæ are also found. The filaria Magathãesi must first be mentioned. It is decidedly larger than those previously named, and in Brazil lives in the heart of man. Furthermore, Manson has described the filaria sanguinis hominis major-the filaria diurna, Manson; the filaria sanguinis minor-the filiaria perstans, Manson; the filaria, Demarquayi; the filaria, Ozzardi. The sexually ripe form has not yet been found, and v. Linstow believes these filaria to be developmental phases of one and the same embryonic larval form.

The filaria produce marked symptoms in the lymphatic system, the walls of which show inflammatory changes. Thrombosis and cicatricial closure of the lymphatics cause varicose dilatation of the peripheral vessels and the formation of lymph-cysts. These rupture and their contents are set free in the urinary bladder, under the cutis, or in other tissues. The perilymphatic connective tissue is also inflamed. Finally symptoms are produced which resemble elephantiasis. 
The filaria embryos appear to circulate for a long time in the blood before they produce symptoms. Gradually general malaise develops, also pain in the small of the back and renal region, and in the scrotum, followed by an intermittent hematochyluria. Fever and enlargement of the spleen often accompany the symptoms. After continuing for days and weeks these phenomena cease, to reappear at the end of a certain period. During the attack the urine is of a peach-red color. Blood and blood coaguli are found in the sediment, while the urine above this is turbid, whitish, and has a tint of yellow not unlike diluted cream. Upon the surface a cream-like fatty layer may frequently be noticed. Sugar and peptone are absent; cholesterin, lecithin, neutral fats, and fatty acids are present. Besides the embryos, red and white corpuscles and large and small fat globules are found. Gradually the lymphglands enlarge, in the males the scrotum, in the female the labia. The symptoms disappear, to return in a short time in an exaggerated form. Elephantiasis-like swellings form upon the lower, and later upon the upper, extremities. The disease finally causes death, after running a course for years with the symptoms of marasmus.

Whether recovery, which occasionally is spontaneous, is due to remedies or not is very questionable. Drugs appear to have no influence upon the parasite. Surgical interference has been frequently attempted, but, according to Scheube, does not prevent relapses. The utmost stress is to be laid upon prophylaxis, and in this the question of drinking-water is of special importance. The habit of drinking unfiltered water from ditches and cisterns in the tropics is undoubtedly a cause of the distribution of the parasite. Physicians living in these regions strenuously advise that only filtered water, or drinking-water from closed conduits, be used.

It is evident that our knowledge of filariasis is far from complete, and it is hoped that future investigations may shed light upon some of its dark problems. For example, John O'Neill observed a filaria which produced pruriginous vesicles and nodules upon the fingers and elbows with severe itching, and of this parasite nothing further has been heard.

Among the most frequent human parasites are the ascaris lumbricoides, Linné, 1758, trichocephalus dispar, Rudolphi, 1801, and the oxyuris vermicularis, Linné, $1 \% 6 \%$. known.

A zoologic description of these parasites is unnecessary as they are well

The ascaris lumbricoides resembles the rain-worm, and is unquestionably the most common parasite of man. Its embryology was first clearly described by Grassi in the year 188\%, and he proved that it does not require an intermediary host. The fact of direct infection with embryo-containing ova has been confirmed by the further investigations of Lutz and Epstein.

Besides the ascaris lumbricoides, the ascaris mystax, Zeder (the nematode of the cat or $\operatorname{dog}$ ), has also been occasionally found in man. This parasite is much smaller than that of man; and upon the end containing the head two wing-like processes are situated which at once distinguish this worm from those mentioned above. 
Ascaris maritima, Leuckart, 1876 , has up to this time been found only once-in Greenland.

Wherever ascarides hosts, including swine and cattle, distribute the ova in the vicinity of human habitations, man, whose habits and customs bring him into close contact with the foci of infection, acquires the ova of the nematode. The agricultural population, in particular, by their occupation, and, above all, the children, by playing in the infected earth take up the cmbryo. Children in cities are much more rarely contaminated. By the slaughter of pigs and cattle at home or in the immediate vicinity, the infectious germs are widely distributed about the house and in the garden, which is the ordinary playground of the children. In the first and second years children are much more rarely attacked than in the third year and upward. In later life ascarides are more rarely met with but no age is exempt. Often infection occurs through drinking-water or food, such as fruit, vegetables, and the like.

The usual seat of the parasite is the small intestine. A single parasite is rarely found; usually they collect in numbers.

The parasites frequently produce no symptoms, and their accidental discharge betrays their presence. When the parasites are numerous, they produce more or less decided disturbance. In the milder cases only gastric symptoms appear: Anorexia, nausea, salivation, irregular bowel action. In children there is frequently a slightly swollen appearance, deeply sunken cyes, itching of the nose, nocturnal grinding of the teeth. Various nervous symptoms, such as convulsions, epileptiform attacks, headache, pain in the back of the neck, etc., may occur, although these are less frequent. Demme saw cases of severe anemia due to the presence of numerous nematodes; Baelz also believes that ascaris may occasionally produce severe ancmia. Leichtenstern saw severe anemia, and in another case larval malaria with enlargement of the spleen, improve after expulsion of the ascarides. These, as well as many other observations, do not permit us to regard this nematode as the harmless guest which it is usually assumed to be. The severe nervous symptoms have, for the most part, been looked upon as reflex. The observations of Huber, v. Linstow and others have shown that this nematode contains a poison which may give rise to urticaria and conjunctivitis. It has a sharp odor, manifest even on handling of the parasite. As I have explained elsewhere it is quite possible to look upon the nervous symptoms which accompany infection by nematodes not as reflex but as toxic.

Decided local disturbances may also appear when numerous ascarides are present. Sometimes, rarely it is true, the worms may form a ball so that the symptoms of intestinal occlusion may arise. Leichtenstern questions this, but from the observations of Mosler, Pelczynski, Stepp, Sperling and others, it may be considered as proven. The view that nematodes may perforate the intact mucous membrane has not yet been positively demonstrated by investigation, but if ulcer formation is present it is quite possible that the parasites may perforate the ulcerated surface.

It is a remarkable fact that at certain times, especially in serious febrile diseases, ascarides leave their usual seat, the small intestine, and begin to wander. Either they enter the large intestine, are voided from this independently, 
and are later found rolled up in the bed, or they are voided with the fecal masses. In other cases they enter the stomach, thence the esophagus, and find their exit by means of the mouth and nose. If they effect an entrance into the larynx, severe suffocative phenomena are produced which may cause death. Not rarely they find ingress to the excretory ducts of the large abdominal glands, particularly the common gall duct, and thence reach the gall-bladder or the liver and here produce serious lesions. More rarely they invade the pancreatic duct or the vermiform appendix. The tendency of ascarides to lodge in preformed foramina explains their occurrence in the abdominal cavity after perforation of the intestine. If nematodes are capable of thus penetrating into intra- or extra-peritoneal abscesses, in their further course they may be found in very remote situations. Thus, nematodes have been discharged from the bladder, from the uterus, and from abscesses of the abdominal cavity.

If there is a suspicion of helminthiasis, the microscopical investigation of the feces and the finding of the characteristic ova will, as a rule, enable us to make a positive diagnosis.

The prognosis is generally favorable; only exceptionally, by the wandering of the parasites, are threatening symptoms produced.

The administration of santonin will generally lead to the expulsion of the parasite. This drug should never be given upon an empty stomach, however, as rapid absorption permits its toxic properties to appear before its anthelmintic. As is well known, the symptoms of santonin poisoning consist of yellow sight, yellow discoloration of the urine, general malaise, vomiting and spasms, which may readily become dangerous in delicate children. Laxatives soon cause the discharge of the poison.

Santonin is given to children in doses of $0.025-0.05$, to adults in doses of 0.1. Lozenges which usually contain $0.025-0.05$ of santonin may be obtained in pharmacies, and one of these should be taken two or three times daily. Upon the third day a laxative is administered, and frequently the worms, which have been only stupefied, are discharged.

The oxyuris vermicularis, Linné, $176 \%$, is also a parasite that is well known. The direct development of the oxyuris without intermediary host, which was indicated by the investigations of Leuckart, is generally accepted. By the swallowing of oxyuric ova, Leuckart, Grassi and others have directly infected themselves.

It does not appear to me to be sufficiently recognized that the ova are not deposited in the intestine of man, but that the mature females leave the intestine independently or with the feces. We can never conclude from the absence of oxyurisova in the feces that this parasite is not present in the intestine. Only outside of the intestinal canal does the female discharge its ova in the fecal masses. Frequently the surroundings of the anus are thickly covered with oxyuris ova which have been deposited there by female parasites which have been independently discharged. In the ova an embryo soon develops which, however, does not extrude from its sac until after retransportation to the human intestinal tract. Unpared fruit, fresh regetables, berries and other fruits which have come into the slightest contact with infected feces often bring about this infection. In other cases transmission is brought about by clothing which is 
used in common, or by various utensils which may be contaminated with ova. Ova have been repeatedly found in the dirt of the finger nails of oxyuris hosts. This observation sufficiently explains why oxyuris hosts are rarely found isolated in families, pensions, ete., and the same finding also explains the wellknown tenacity of the affection, which has been known to physicians for a long time. The oxyuris host evidently infects himself anew almost daily, or even every night.

The embryos which have been set free in the stomach develop to scxual maturity in the small intestine. The impregnated females enter the cecum, and there await the time for depositing their ova. When this period has arrived, they wander toward the rectum, or beyond this, and are discharged.

No age is exempt from the parasite, but it is particularly frequent in children. Great uncleanliness, huddling together, and sleeping with infected persons favor contagion. The expulsion of parasites is so constant because of their rapid increase that opportunity for the entrance of new ova may be long delayed, and it is, therefore, not rare for persons to harbor a parasite for a great many years.

If the number of parasites is small, they betray their presence only now and then by itching and burning in the rectum. In sensitive individuals, after passing through the anus, a few parasites only may cause a number of symptoms. These disturbances are decidedly increased by the presence of considerable numbers of the oxyuris. By their serpentine movements in the lower portion of the intestine they produce intense itching in the anus. This is particularly marked as soon as the patients go to bed. Sleep is prevented or is interrupted. The periodicity of these symptoms is conspicuous, and as yet no sufficient cause for this has been assigned. Large numbers of oxyuris cause marked catarrhal irritation of the intestinal mucous membrane, perhaps also erosions, which are brought about by the active boring movements of the head extremity of the animals. The venous vessels dilate as in the case of hemorrhoids. Frequently oxyuriasis is complicated with hemorrhoidal conditions and chronic catarrh of the rectum. In consequence of this the bowels not rarely become irregular and diarrheic, and the symptoms of tenesmus appear. A number of consensual symptoms may also develop, which in children readily lead to onanism. Oxyuris may cause pruritus; in the vagina in girls a leukorrhea, in the prepuce in boys a balanitis. Marro found in a pus cyst well-preserved ova of oxyuris vermicularis.

Reflex symptoms of the most varied kinds, due to oxyuris, are said to occur with particular frequency in neuropathically predisposed individuals; I have gathered a number of observations of this kind. At all events, the presence of numerous parasites is not without an influence on the affected organism. In feeble persons, particularly in children, we note that the nutrition suffers, the patient becomes weak and anemic, and there is loss of weight. After expulsion of the parasite, however, the entire condition of the carrier often improves suddenly. Occasionally the oxyuris wanders into the stomach, from here to the esophagus, to the mouth or to the nose. Hartmann saw the frequent expulsion of oxyuris from the nose. It was accompanied by the most severe irritative symptoms, epileptiform attacks, and psychical disturbances. 
These were actually produced by the parasite, for after its destruction by injections into the nose of corrosive sublimate solution and the internal administration of antipyrin, these nervous symptoms at once disappeared.

The diagnosis where oxyuriasis is suspected depends upon investigation of the feces, or examination of the anus and its surroundings. If the finding is negative, the patient is told to use a small enema of cold water immediately upon the appearance of the symptoms. In the return flow of the water, the parasites, if present, will be readily recognized. The microscopic examination of the soiled parts around the anus readily reveals eggs if present. Ova are found in the feces only if simultaneously also the macroscopically recognizable female oxyuris is present.

The removal of oxyuriasis often requires great patience. In the first place it is necessary to prevent the importation of ova by all tendencies and habits which favor this. The sleeping together of well and sick children, the common use of clothing, sponges, towels is to be prohibited. Frequent change of bedding and cleanliness of the finger nails, etc., are to be advised. Moreover, the parasites which are present in the small intestine, and particularly in the cecum, must be expelled by purgatives, such as castor oil, rhubarb, compound infusion of senna, etc. At the same time I employ santonin. But the simultaneous employment of intestinal infusions is absolutely necessary in order to expel the oxyuris which have entered the large intestine. In severe cases I add to the water one to two teaspoonfuls of chlorin water, vinegar, 0.2 to 1 per cent. of medicated soap. As a common household remedy garlic is also employed.

Among the most frequent and also, as a rule, most harmless intestinal parasites is the

\section{Trichocephalus dispar, Rudolphi, 1801.}

This organism is well known, and therefore a zoologic description is unnecessary.

The parasite does not require an intermediary host. We have little knowledge of the method by which it enters the human intestinal tract. Its long period of development and the great resistance of the ova to external influences evidently favor the invasion. Dried and pulverized to dust, they are readily consumed with raw fruit, vegetables or drinking-water, and thus enter the human organism. After the young embryos have left the membrane of the ovum, they attain their development in the small intestine, and finally lodge in the cecum. The parasite is usually found in but few specimens. If found numerously the signs of chronic catarrh may develop in that part of the intestinal tract which they inhabit. The parasite adheres to the mucous membrane in such a way that the anterior end of the head, which is curled in several folds, surrounds the individual parts of the mucous membrane. In other cases it has been proven that the entire anterior thin portion of the body-more than two-thirds of the worm-penetrates the surface of the intestinal mucous membrane. Accordingly it is often very difficult to loosen the parasite from its hold in the mucous membrane.

In regard to the symptoms, in general little is known. In many cases, 
perhaps in the majority, there are apparently no symptoms. Only when a massive collection becomes lodged in the cecum or in the colon, local difficulty may result from the irritation which the worms produce by boring their way deep into the mucous membrane. Moosbrugger, Burchard, Federolf have seen severe symptoms due to trichocephales: Extreme anemia, enteritis with profuse hemorrhagic stools, emaciation and loss of strength. Since Askanazy has shown that the trichocephalus invariably has an iron-containing pigment in its intestinal epithelium, the origin of which is the hemoglobin of the blood, this form of helminthiasis will evoke much greater interest.

The diagnosis may readily be made by the microscopic demonstration of the characteristic ova, thick-shelled brown eggs, at both poles of which there is a knob-like elevation.

The trichocephales cannot readily be expelled, but the combined administration of anthelmintics and purgatives will give the best results. Besides santonin, we have employed successfully the ethereal extract of male fern. From the basis of his experience, Lutz advises thymol.

Among the nematodes which are of great pathogenic importance belong the trichina spiralis, Owen, 1835, and the anchylostoma duodenale, Dubini. 1843. I shall first describe

\section{Trichina spiralis,}

the appearance of which in the musculature of man was observed by Peacock in 1828 , and by J. Hilton in 1833 . These and other findings, which were looked upon only as interesting secondary findings at autopsies, attained their true importance in 1860 through $\nabla$. Zenker. In a case observed by this author, of a girl who was dying from symptoms resembling enteric fever, numerous recently entered trichinæ were found in the muscles, as well as many in the intestine, while no other possible cause for her disease was evident. The same author ascertained that in the district in which the patient had lived a number of milder affections had occurred. Sausages and hams which were still there permitted the detection of massive encapsulated trichina. From this time, the attention of physicians was directed to trichiniasis. It was soon possible, even during the life of the patient, to demonstrate the cause of the disease in the musculature. Abundant material was furnished by the "trichina epidemics" which appeared, particularly in Middle Germany; and the study of these rapidly added to our knowledge of the previously absolutely unknown disease, both clinically and pathologically, and enabled us to reach definite conclusions.

But not only in Germany was trichinosis observed; its existence was soon demonstrated in Switzerland, England, Denmark, Sweden, Russia, France, Italy, India, Syria, America and Australia. Wherever the cause could be ascertained, a connection with the ingestion of pork containing trichina was proven. With the determination of this fact the question arose as to the source of trichina for the swine. The theory that pigs were infected by the ingestion of rain-worms, frogs and moles containing trichinæ was soon shown to be erroneous. Leuckart's view, promulgated in 1862, that rats were the actual source of infection for the pig was of far-reaching importance. We are also 
indebted to $\nabla$. Zenker whose labors have proven that rats become infected by the ingestion of other animals containing trichina. Contagion occurs in pigs, as a rule, by "eating trichina-containing meat of other pigs." As is well known, some parts of the meat are often utilized for feeding swine, particularly during slaughter time in the country, and when animals are skinned offal containing trichinæ is often given to pigs. In such localities it is obvious that sufficient waste material remains to infect at the same time the entire colony of rats that are present. The latter continue to be for years a further source of infection for the pigs, who are usually expert rat catchers, and who often eat the enemy which they have killed. Wherever pigs infected with trichina are slaughtered without the complete destruction of their cadavers, there is danger of distribution of trichina. Mature trichinæ are found in the small intestine of the rat, the wild boar, the fox, the polecat, the marten, the raccoon and the cat, as well as in man and in swine. Man infects himself almost exclusively by the ingestion of raw or insufficiently cooked pork, which contains the so-called muscle trichina in a condition capable of development. Thorough boiling or frying will kill the trichina, provided the temperature in the inner portions of the meat is $50^{\circ}$ to $55^{\circ} \mathrm{R}$. Neither decomposition nor cold diminishes the property of life in the trichina. Muscle trichinæ retain their power of development for many years (up to 31). Thorough salting destroys the life of the trichina, but it is certainly not destroyed by cold smoking nor in the so-called rapid smoking process.

The encapsulated trichinæ found in the musculature appear as small gray nodules, rarely over $1 \mathrm{~mm}$. in length, and consist of a capsule which, as in the case of man, after the lapse of years becomes calcified. The capsule encloses the trichina. This is a spiral worm of 0.6-1 mm. in length and 0.01-0.03 mm. in breadth; the head is pointed, the tail is rounded; upon the head is the mouth opening, upon the tail the anus. If parts of muscles permeated with living trichinæ reach the stomach of man, of the pig or of any other suitable animal, the gastric juice dissolves the capsule; in from two to three days the parasites which have entered the intestine attain their sexual maturity. Soon after copulation the males die, while the females, according to the observations of Askanazy, actively bore into the intestinal mucous membrane, and seven to nine days after infection has taken place produce living young. Thiș act of bringing forth takes place usually in the tissue of the intestinal mucous membrane, that is, close to the central chyle vessels of the villi. Probably the majority of the embryos enter the circulation directly through the chyle and lymph-vessels; carried by the blood-stream they wander into the transverse striped muscles. Up to this time the usual mode of distribution has been assumed to be migration, i. e., by boring through the intestinal walls, the parasites enter the peritoneal cavity, and thence the connective tissue parts of the musculature; or the path has been traced through the submucosa, through the mesentery, and the retroperitoneal connective tissue. According to Askanazy, distribution of the parasites by active wandering is only secondary. About the ninth or the tenth day after infection, the first influx of parasites reaches the musculature. The female is so immensely reproductive that, for about seven weeks, new groups of parasites constantly find their way there. 
The young embryos have a length of $0.1 \mathrm{~mm}$., a breadth of $0.006 \mathrm{~mm}$. The anterior end of the body is thicker than the posterior. After entering the muscle, they penetrate still more deeply into its interstitial tissue, in a longitudinal direction, toward the insertions of the tendons. Their path through the fibers causes a number of changes in them. The trichina has a destructive action upon the finer constituents of the fibers. These lose their transverse striæ and are changed into a fine granular mass. Without doubt the embryo is nourished by the decomposed muscular substance. I do not wish to be wearisome with the finer histologic details, and shall emphasize only the following points in the development of the muscle trichina: The development occurs in about fourteen days. The muscle trichina gradually grows to a length of $0.12-0.16 \mathrm{~mm}$., simultaneously rolling itself into a spiral. The sarcolemma distributes itself about the embryo, and thickens. In the interstitial tissue, as well as in the muscular substance, such an increase of the muscle nuclei occurs that the trichina appears completely embedded in them, and surrounding it is an areola of granular material which stains more intensely than the remaining contents of the nodule. After about four weeks the contents of the nodule permeated by nuclei enter upon a process of reconstruction, which, beginning at both ends, rapidly reduces these ends to thin threads. Around the spiral swelling, as well as around the threads, is a gelatinous sheath which Leuckart recognized as thickened sarcolemma. Outside of this is a zone of inflammatory connective tissue richly supplied with connective tissue corpuscles and leukocytes. Later the disorganized muscular mass in the region of the thread-like processes disappears. The connection with the material surrounding the trichina is interrupted. The formation of a clearly defined trichina capsule now begins; starting from the surrounding connective tissue cells, probably in the course of the old gelatinous sheath, the new and firmer cyst is separated from the encroaching connective tissue cells. Fat cells develop at the poles of the capsules. At about the sixth month the capsule begins to calcify owing to a deposit of calcium carbonate. This calcification may also affect the enclosed trichina. According to Langerhans, the trichinæ as well as their capsules are capable of retrogression and may finally, although only after many years, be completely eliminated from the body.

In man, the source of infection is almost exclusively the pig, very rarely the wild boar and the bear. The danger of transmission of trichinæ capable of development from the ingestion of infected pork depends upon the preparation of the meat. People who partake of infected pork raw or insufficiently cooked show the most severe symptoms. Raw meat in the form of so-called hash, boiled fresh pork from a newly killed pig, roast sausage or German sausage (Knackwurst) in the preparation of which the heating is insufficient to insure the destruction of the trichina appear to be especially dangerous. The ingestion of meat of this kind, which is particularly common in Middle and North Germany, causes the frequent appearance of the disease in endemics and epidemics. In the sixth decade of the last century severe epidemics of this kind were observed in Plauen, Hettstädt, Hedersleben. In South Germany, and in other countries, France, England, Belgium, America, etc., this custom 
does not prevail; pork is either well cooked or boiled or smoked, and trichina epidemics are rare. The question is much discussed, whether infection may be brought about by the importation into Germany of American ham and bacon. According to Billings trichinosis is met with in American pork in 4-5.7 per cent., but the above question. has been answered in the negative by a majority of authors. Some observers, however, hold a directly contrary opinion. Caution is therefore enjoined. Nevertheless, sporadic cases may and will occur everywhere in the future.

No age, no sex is exempt; all who partake of infected meat, either raw or in a half-cooked state, are attacked. The severity of the disease depend: upon the amount of meat and the number of trichinæ consumed. Man appears capable of harboring a certain number of muscle trichinæ without symptoms. This is, at least, indicated by the accidental finding of muscle trichinæ at the autopsy of individuals who during life had shown no symptoms.

The syмттомs produced by trichinosis correspond to the phases of development of the trichinæ. Frequently, a few hours after the ingestion of meat containing trichinæ, severe gastric and intestinal symptoms appear: Nausea, vomiting, cardialgia, vertigo, headache, heaviness in the limbs, constipation or diarrhea. If the infection be severe the symptoms often increase and simulate a severe intestinal catarrh, which in isolated cases may run its course with choleraic symptoms. With increasing lassitude a more or less decided fever develops. The patients become bedridden. v. Linstow quite properly regards the severe initial intestinal symptoms as toxic, due to the toxic products which have been set free by the dissolution of the capsules. The presence of intestinal trichinæ in the dejecta has up to the present been rarely. demonstrated.

In many cases, initial intestinal symptoms are wholly absent, and it is therefore impossible to determine the period at which infection occurred. There are indistinct symptoms; rheumatic pains in the limbs and fever; individual muscles begin to swell and become edematous. Briefly, the symptoms are those of myositis. Simon, Kratz and Rupprecht mention as characteristic, even from the first days of .the disease, the so-called " sympathetic muscle lameness." This consists in a drawing sensation in the limbs, tension and tenderness in the muscles, such as is observed otherwise only after unusual muscular exertion. The flexors, the muscles of the nape of the neck, and those of the lumbar region are particularly involved. Sometimes in the first, more frequently in the second, week, Kratz observed neuralgic pains in the abdomen which, recurring at irregular periods, sometimes as often as six times in the twenty-four hours, resembled a neuralgia cœliaca.

The gastric and intestinal disturbances are frequently accompanied byfever. Only in mild cases is this absent during the second half of the second week, and it is decidedly increased when the muscular symptoms become manifest. Chilliness frequently accompanies the fever, though marked chills are rare. The maximum rise of temperature $\left(104^{\circ}\right.$ to $105.8^{\circ} \mathrm{F}$.) is usually observed from the ninth to the eleventh day after the beginning of the malady. At first continuous, the temperature finally shows a remittent type. The return to nor- 
mal is extraordinarily slow. This is markedly different from the temperature course of enteric fever. In mild cases, even during the third week, the temperature falls to normal, while severe cases continue to show a febrile range from four to seven weeks. The pulse frequency corresponds to the height of the fever.

In the course of the second week, edema of the face, particularly of the eyelids, is characteristic. After several days this may disappear, but it often returns in the later stages. The affection of the muscles is accompanied by marked edema; the genital region often remains free.

Usually from the ninth or tenth day-sometimes earlier or later-with the entrance of the embryo into the muscles, symptoms occur there. The affected muscles are tensely swollen, as hard as wood, and very sensitive to touch or pressure. In mild cases only the sensation of painful tension is felt, and the patients are still able to walk about. In severe cases the slightest movement is accompanied by the most intense pain; locomotion and standing are impossible. As a rule, the muscles of the extremities and particularly the flexors are the most painful. In arder to relax their muscles as much as possible the severe cases assume a characteristic position: Permanent dorsal decubitus with an acute angle contracture in the shoulder, elbow and wrist joint; stiffness and muscular rigidity are observed in the masseters, in the muscles of the neck and of the upper extremities. Implication of the muscles of deglutition and of the tongue often causes great difficulty in swallowing. Hoarseness and aphonia result from the involvement of the muscles of the larynx; and in severe cases pain in the muscles of the eye is rarely absent. The mobility of the eye-balls is affected, and this gives to the face a conspicuously rigid appearance. In severe cases, the masses of trichinæ cause difficulty in respiration; extreme dyspneic conditions may develop with very severe asthmatic attacks, and may be a direct cause of death.

Even at the beginning of the second week Kratz noted inactivity of the diaphragm upon inspiration. The condition noted by Askanazy is very interesting. This author found under the pleura pulmonalis and in the lung sereral disseminated red flakes or points which closely resembled the punctiform ecchymoses upon the human pleura. These were demonstrated to be young trichinæ which had formed emboli.

Changes in the skin are not rare. Besides edema, pruritus, measle-like exanthems, and in the later stages acne, furunculosis, herpes, etc., appear. The sweats which occur in the mild as well as the severe cases are particularly annoying. Desquamation of the skin takes place in convalescence. Formication and cutaneous anesthesia have been observed in isolated cases.

Stubborn bronchial catarrh develops early. In the later stages hypostatic and catarrhal pulmonary inflammations occur. In debilitated patients these not infrequently cause death.

In general there are no characteristic changes in the cardiac activity. In severe cases and in the later course of the affection thromboses develop, particularly in the lower extremities. The appearance of decided leukocytosis with a conspicuous increase of the eosinophiles, which show an increase of from 30 per cent. to 60 per cent., is interesting. This demonstration has repeatedly 
aroused a suspicion of trichinosis which has been later confirmed. Perhaps the eosinophilic cells originate from the polymorphonuclear neutrophilic cells of the diseased musculature.

Lately, in isolated cases, the temporary absence of the patella tendon reflex as well as anomalies of the electric contractility of the muscles has been determined.

The urine shows the usual characteristics of fever urine. Nonne and Höpfner have recently called attention to the not infrequent occurrence of parenchymatous nephritis. Menstrual irregularities are common in some epidemics. In pregnant women abortion is not rare.

Insomnia is one of the chief complaints of the patient. The mind, as a rule, is perfectly clear; only in severe cases associated with high fever does delirium occur.

It is obvious that the nutrition must suffer, particularly in grave cases.

In mild cases the course of the disease is from one to two weeks; in all other cases one to two months intervene before recovery takes place. Death is not rare. The mortality varies in different epidemics. In Hedersleben the mortality amounted to 29.8 per cent., in Calbe 21 per cent., in Burg 20 per cent., in Klein-Quenstedt 14 per cent. In children a favorable course of the affection is frequent; recovery often takes place at the end of the third week. Complications are rare. The slight predisposition of children to trichinosis may be accounted for, first, by the lesser activity of their gastric juice, and secondly, by their more frequent fecal movements in consequence of which large portions of meat containing trichinæ may be discharged.

At the AUTOPSY the intestines reveal but slight changes, and these consist principally in a catarrhal condition of the small intestine. Swelling of the solitary glands, of Peyer's patches, and of the mesenteric glands is observed. In the intestinal mucus, up to the seventh week, intestinal trichinæ are found. Spleen is normal or enlarged. Fatty liver is frequent. Heart muscles and kidneys often show granular opacity. Fatty-liver and nephritis, according to v. Linstow and Askanazy, are due to the toxic products of metabolism of the trichinæ. The lungs usually reveal the signs of a marked bronchitis. The bronchial mucous membrane is intensely reddened, and has a tenacious mucus coating. In the lower posterior portions lobular hepatization is revealed; rarely hemorrhagic infarcts or metastatic abscesses. The color of the muscles often resembles "smoked goose breast"; the smaller muscles are much paler and "wax-colored." From the end of the fifth week macroscopic changes in the form of pale gray striæ of from $\frac{1}{2}$ to $1 \mathrm{~cm}$. in length may be recognized. Upon microscopic examination these are seen to be dark, granular, detrituslike masses. Granular and fatty degeneration, sometimes also waxy degeneration, are found in the surrounding muscle fibers. Not rarely giant celllike structures are noted. Nonne and Höpfner describe the appearance of vacuoles in the muscle fibers. In the later stages this musculature disappears to a great extent and is pale. The number of muscle trichinæ may be enormous. The most intense permeation, according to Cohnheim, is found in the diaphragm, in the intercostals, in the muscles of the neck, of the larynx and of the eyes, of the biceps and triceps. In milder cases 
the presence of a single trichina may be demonstrated only after a search of hours.

The Prognosis cannot be determined in the early stages of the disease. Severe, fulminant phenomena, especially on the part of the digestive apparatus, are not necessarily followed by a severe course, as the diarrheal discharges remove a portion of the infectious material. Cases which appear mild at the onset may subsequently become very severe. After a course of three to four weeks, those cases in which the patient retains a good appetite and the ability to sleep, as well as the integrity of the respiratory organs and of the heart, permit the hope of a favorable outcome. In children the prognosis is more favorable than in adults from the onset.

During the prevalence of epidemics, the DIAGNosis is more readily made than in sporadic cases.

Mild cases may simulate gastric catarrh and muscular rheumatism; severe cases resemble acute gastro-intestinal catarrh, and in their further course may be looked upon as acute articular rheumatism. The edema, the painfulness of the affected muscle groups, the impairment of respiration, are important in diagnosis. The differentiation from acute progressive polymyositis is not always easy. In doubtful cases it is well to search the dejecta, particularly the intestinal mucus, for intestinal trichinx. In severer infections the trichinæ may certainly be determined by the excision of small portions of muscle, which are best taken from the lower end of the biceps. Direct excision is decidedly preferable to the formerly practised harpooning.

The aim of the PROPHYLAXIS should be the prevention of trichinosis in the pig. Stall feeding of swine gives in this respect a certain protection, especially if care is taken never to feed them with uncooked offal. In regions where trichinosis frequently occurs a thorough inspection and radical destruction of meat containing trichinæ should be obligatory. The keeping and fattening of swine in slaughter-houses should be prohibited.

Man is best protected from the disease by eating only thoroughly boiled or well-fried pork. The most intense smoking or pickling will kill the parasite, but these precautionary measures are not everywhere taken. There may .be exposure to infection, for example, upon a journey. The most important preventive measure, therefore, is the erection of slaughter-houses and the introduction of compulsory meat inspection. The microscopic demonstration of muscle trichinæ is not difficult, and this may be carried out by thoroughly competent meat inspectors. Absolute protection, naturally, cannot be conferred by meat inspection if only a few muscle trichinæ are present. It is well, therefore, to carefully abstain from partaking of any but thoroughly cooked or highly pickled or smoked pork.

We are very rarely in a position to begin treatment soon after infection has taken place, but, when this is possible, emetics and purgatives are advisable. Theoretically, an attempt should be made to expel the infectious masses by the administration of castor oil, calomel, etc. Experimentally, it is true, no remarkable results have followed this or any other method of treatment. Neither potassium, nor sodium picronitrate, nor oil of turpentine, camala, extract of male fern, glycerin, etc., has prevented infection. 
Mosler, upon the basis of experimental investigation, has advised benzine:

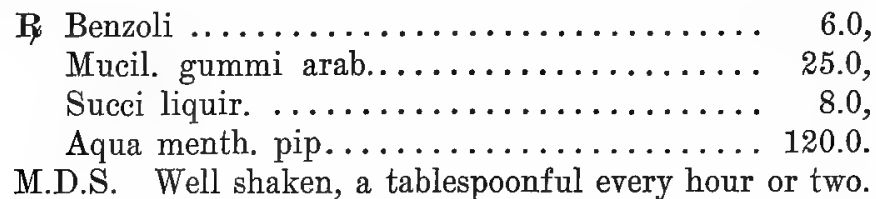

Besides this Mosler advises daily for several days an enema to which is added three to eight grams of benzine. In the main the treatment is symptomatic.

For the sake of completeness I shall mention among other nematodes the eustrongylus gigas, Rudolphi, 1802, a parasite which is found in the renal pelvis of the dog, the wolf, the fox, the horse, the marten, the otter, etc., and has been observed in man in about nine different cases. The parasite has frequently been confounded with other nematodes, with coagula of blood, or with mucus. According to experiments conducted in the dog, the eustrongylus causes severe difficulty in urination. The ova of the parasite may be recognized in the urine. The strongylus paradoxus, Mehlis, 1831, has been even less frequently observed in man.

Gnathostoma siamense, Levinsen, 1889, has up to the present been seen by Levinsen only as one female specimen in a Siamese and in two other persons.

\section{ANKYLOSTOMIASIS-UNCINARIASIS}

One of the most important entozoa of man is the

$$
\text { Ankylostoma duodenale, Dubini, } 1843 .
$$

The parasite was first ciscovered by Dubini in 1838 in Milan in a human cadaver. Soon its occurrence was also noted by Pruner, Bilharz and Griesinger in Egypt. The parasite was recognized by Bilharz and Griesinger on account of its hematophagous properties as the actual cause of "Egyptian chlorosis," which is so extraordinarily prevalent in Egypt.

Wucherer, in 1866, recognized the parasite as the cause of "tropical chlorosis." Its presence was soon afterward determined in the anemia of brickmakers, which had been known for a long time in Italy. Parona, Perroncito, Bozzolo found the parasite associated with the anemia which attacked the workmen engaged in constructing the Saint Gothard tunnel, and looked upon it as the causal factor. Soon the proof of its connection with anemia montana which occurred in the Belgian, Sardinian and French mines in 1882 was established. The occurrence of the parasite was recognized by Mencke in the brickmakers in the neighborhood of Bonn, and a few weeks later by Leichtenstern among the workmen in the brick fields at Cologne.

Since that time its occurrence has also been proven in the miners of the Rhine provinces, Westphalia and Silesia, sometimes in wide distribution.

The male parasite is usually of a white, the females of a whitish yellow, brownish-red, or reddish color. The length of the male averages from $7-11.2$ $\mathrm{mm}$., the thickness $0.46 \mathrm{~mm}$; the females are $16.5 \mathrm{~mm}$. long and $0.63 \mathrm{~mm}$. in 
thickness. The parasite tapers toward the anterior extremity. The intestinal tract begins with a wide slanting mouth, turned toward the back and having six papillæ; it has a bell-like mouth capsule, upon the border of which are six tooth-like chitin ridges. The posterior end of the body of the female terminates in a short ten-pin-like point; that of the male is somewhat bent, terminating in a broad umbrella-like ribbed bursa copulatrix, upon which frequently two long spicula of about $2 \mathrm{~mm}$. in length protrude. The bursa copulatrix serves, on account of its contractile parenchyma ribs, as an apparatus, by means of which the males attach themselves to the body of the female during copulation. The female sexual opening is close behind the middle of the body, and is continued as a short vagina. The parts next to the vagina contain the impregnated ova. These have an oval shape and a smooth surface. Their length is 0.063 and their thickness from $0.036-0.04 \mathrm{~mm}$. They are present in enormous numbers in the human dejecta and, according to the time in

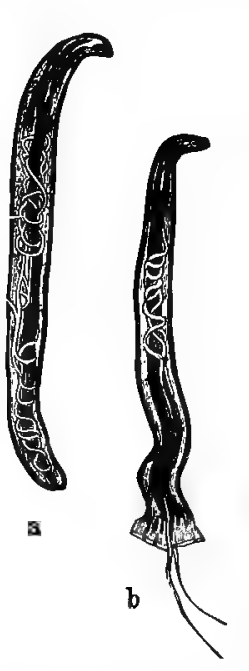

Fig. 54. - Ankylostoma Duodenale: $a$, male; $b$, female. which they have remained in the intestinal canal, 2 or 4-8 segmentation globules may be recognized. Further development occurs only outside of the human organism. For this development the ova require a temperature of from $25^{\circ}$ to $30^{\circ}$ C., moderate moisture, and a surface distribution. Under these circumstances, after three to four days at the latest the embryo may be recognized. After twenty-four to fortyeight hours more, it finds its way through the pole of the ova, and shows active movements.
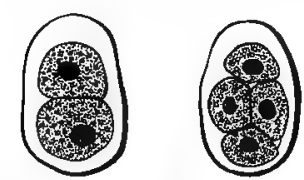

Fig. 55.-OVA OF THE ANKTLOSTOMA Duodenale in Various Stages of Development.

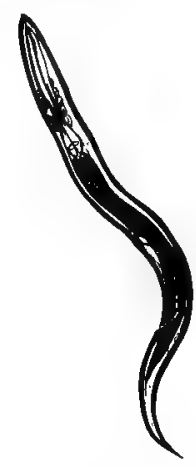

Fig. 56. - Larva of Ankylostoma DuoDENALE.

The young larva is $0.2-0.25 \mathrm{~mm}$. in length and $0.015-0.017 \mathrm{~mm}$. in thickness. The posterior end of the body gradually tapers anteriorly to a pouchlike point. The short and narrow opening of the mouth, the spindle-shaped pharynx, and the globular stomach may be distinctly recognized; in the latter a Y-shaped figure, composed of the three pharyngeal teeth, is found in active movement. The intestine terminates in a fine tube, the anus. The embryo soon begins to grow, and after having attained a length of $0.7-0.8 \mathrm{~mm}$. it encapsulates itself. The larva finally lies in a transparent sheath which uniformly surrounds it. The sheath protects the embryo from external damage, so that for months in this encysted state it may retain its power of further development. Introduced into the human intestine, after dissolution of its chitin capsule in the small intestine, the parasite develops to maturity. Five 
to six weeks after infection has taken place the first ova appear in the human dejecta.

The parasites, as well as the symptoms which are included under the name ankylostomiasis, have been found exclusively in those persons who labor in any way in the earth; miners, tunnel workmen, brickmakers, laborers upon fortifications, etc. The dejecta voided by an ankylostoma host are exceedingly rich in ova, as shown by the interesting observations of Leichtenstern in the brick fields of Cologne, and they are the source of infection for other workmen. The fecal masses deposited in the outskirts of the brick fields, with the gradual advance of the work contaminate the layers of clay and become mixed with the water. Thus there is abundant opportunity for the importation of encysted larvæ. The workmen eat their meals with dirty clay-covered hands, and in their various manipulations of the clay and earthworks, in cleaning their tools, in the use of drinking-water and water for other purposes, there are numerous opportunities for the encysted ankylostoma to find their way into the human organism. Miners and tunnel laborers, and especially the country population in Egypt, Brazil and other countries, infect themselves in a similar manner. In the country, particularly, with its limitations and the absence of systematic hygiene, there are numerous favorable conditions for the propagation of the parasites. In a damp, flat country where the earth is only slightly permeable and there is an absence of suitable latrines, discharge of the dejecta filled with ova in the immediate vicinity of laborers and living places must, under any circumstances, convey the germs to the inhabitants and laborers. Loos recently called attention to the fact that the young embryo may enter the human organism by means of the skin.

The number of parasites is frequently very great: One hundred, 500, 1,000 and more have been found in the small intestine. They are blood-suckers, $\kappa a \tau^{\prime}{ }^{\prime} \xi o x \eta^{\prime} \nu$; and when we consider that their duration of life is. relatively long--Leichtenstern estimates the maximum at five years-and that repeated infection in one and the same individual in infected regions is probably the rule, we can clearly understand the effects of this parasite, which is a bloodsucker.

Three to four weeks after infection the first symptoms appear. Besides dyspeptic difficulties, these consist especially of colic, diarrhea, increasing lassitude, disinclination to work, pallor of the face. Tinnitus aurium, vertigo and cardiac palpitation are added. The continuous withdrawal of blood in the course of weeks or months leads gradually to the most extreme symptoms of anemia. Edema, dyspnea, and attacks of syncope develop upon the slightest bodily exertion. The dejecta, often even five to six weeks after infection, have a hemorrhagic appearance; they are grayish red, brownish red or black. Usually the first hemorrhagic diarrheic discharges occur from five to six weeks after invasion of the larvæ; during a time, therefore, in which the parasites frequently change their situation for the purpose of copulation. In the later course of the affection symptoms of extreme anemia develop in the patients who have become bedridden.

The pulse is rapid and thready. The carotids show active pulsation. In the heart, which is frequently enlarged to percussion, there are systolic mur- 
murs; over the jugular vein distinct nun's murmurs may be recognized. Occasionally an organic cardiac affection develops. As a rule, there is a diminution in the number of red blood-corpuscles, the amount of hemoglobin is decidedly. reduced; sometimes there is leukocytosis. The lungs are usually normal, provided accidental complications are not present. The microscopic examination of the feces shows, besides more or less altered blood cells, the presence of countless ova which, as a rule, are uniformly distributed through the entire fecal mass. Almost invariably Charcot-Leyden crystals are found. The parasite itself is generally only detected in the feces after the administration of anthelmintics.

With increasing severity of the disease the patients show the distinct symptoms of progressive anemia. Edema develops in the extremities and in the face, and to this are added frequent attacks of fever. Changes in the eyeground, edema of the papilla, and effusions of blood into the retina have frequently been observed. In some cases bone pains are noted; Leichtenstern in such a case observed simultaneously a decided enlargement of the spleen.

The majority of the severe anemic phenomena may probably be explained by the blood-absorbing properties of the parasite. The ankylostoma are, as it were, luxurious blood-absorbers, since they nourish themselves only from the blood-plasma, while the red blood-corpuscles leave the intestinal canal of the parasite in an unchanged condition. The loss of blood by secondary hemorrhage from the wounds, particularly during the time in which the parasites frequently change their position for the purpose of copulation, is perhaps even greater than the amount of blood absorbed. Lussano, by very interesting investigations, believes he has proven that the ankylostoma give off like other entozoa toxic products of metabolism which have a deleterious effect upon the blood. Ervant, Arstan, Verdun, Zinn and Jacoby and others have expressed the opinion that, besides a blood-sucking activity, there is probably a toxic effect upon the blood which is an important factor in the origin of anemia. Bohland has determined an abnormally great decomposition of albumin in ankylostoma patients.

If the disease is not recognized, if there is no relief, the debilitated patient suffers for weeks and months, and finally succumbs with the symptoms of general marasmus; or intercurrent affections terminate life. Only rarely does a change of climate and of the mode of life bring about a spontaneous recovery.

The cadavers of patients who die of ankylostomiasis are usually but little emaciated, provided the cases have been uncomplicated, but they are invariably pale and waxy-yellow. The panniculus adiposus, the fat in the mesentery, in the heart, and in the mediastinum, is often decidedly increased. The muscles are pale; general dropsical phenomena, including hydrothorax and hydropericardium, are common. There is marked anemia of all organs. The heart muscle is often dilated, hypertrophied, pale or grayish-brown. The liver and spleen are more or less atrophic, and occasionally show amyloid degeneration. Similar changes are often noted in the kidneys. The small intestine is in some cases decidedly narrowed, the colon sometimes shows signs of 
chronic catarrh. In the duodenum, but especially in the jejunum and the upper portion of the ileum, there are numerous small punctiform ecchymoses. If the necropsy is carefully conducted, an extraordinary number of red-stained ankylostoma are occasionally found. These ankylostoma have gained entrance into the mucosa, and cannot be removed without a certain amount of force. At the point of adherence the mucosa reveals ecchymosed red plaques of a punctated, dappled appearance which vary in size from a five-cent piece to a half-dollar. It is more difficult, as a rule, to find the males than the females, because the former have a more delicate structure, and are therefore less noticeable in the mucus. The mesenteric glands are sometimes enlarged. Masius and Françotte found that the bone-marrow of the long tubular bones was grayish red; occasionally it is rich in fat and is, therefore, more yellow. Numerous medullary cells and nucleated red corpuscles are found upon microscopic examination.

Secondarily other entozoa have been found: Among them anguillula intestinalis and stercoralis ascaris lumbricoides, oxyuris vermicularis and tenia saginata.

The aim of prophylaxis is, first, to prevent the entrance of the parasite into regions not yet affected, and, secondly, to limit its spread in the infected areas. To attain the first object, in the future no workmen should be employed in brickyards, in mines, in tunnels, and other earthworks until their state of health has been carefully investigated, and their feces microscopically examined for the presence of ankylostoma ova. Furthermore, the workmen should be taught that if necessary care is exercised in constructing privies the contamination of working places with infected dejecta from an accumulation of feces can be entirely prevented. Those who know the habits of laborers and their carelessness as to where they discharge their feces, will understand that even most stringent hygienic rules will be imperfectly carried out. The best prophylaxis, therefore, consists in employing only healthy workmen, i. e., those not infected with ankylostomiasis.

If ankylostomiasis has been diagnosticated by examination of the feces, the patient must enter the hospital, and undergo treatment for the expulsion of the parasite. Leichtenstern, who has probably had the largest experience in Germany in the treatment of ankylostomiasis, advises the administration, without preliminary treatment, of the ethereal extract of felix mas which he gives in a dose of ten grams. The drug is given after breakfast. From one to two hours later a suitable dose of castor oil is administered to the patient. The entozoa voided with the dejecta will have been killed. If, in the course of a few days, ankylostoma ova are still found in the feces, the same treatment must be repeated, a second or even a third time, until the ova are no longer detected in the feces. Bozzola advises thymol ( 2 to 10 grams per day); other authors also look upon this drug as a useful anthelmintic. It is evident that, on acccount of the extreme anemia, a symptomatic treatment for this condition must in many cases be employed during convalescence.

$\nabla$. Linstow recently described a representative of the genus physaloptera, physaloptera caucasica n. spr., as a parasite of man. This was found by v. Linstow in the previously mentioned helminthic collection. 
Blanchard recently called the attention of physicians to the occurrence of some varieties of gordius-small, elongated, thin worms having the shape of filarix-as occasional parasites of man. He spoke particularly of the gordius aquaticus, gordius argillaceus and gordius medinensis, which live in fresh water, notably in the springs of mountainous regions. They are introduced into the human intestinal tract by drinking-water, as well as by the ingestion of fruit, and are finally voided. In all about seven transmissions have been observed.

Almost as rare in man is the occurrence of the acanthocephala, a nematodelike parasite without intestines whose anterior end is armed with a proboscis. The echinorhynchus gigas, Goeze, 1782, is found in great numbers in the small intestine of swine. According to Leuckart its presence in man has been proven in a few cases. Lambl once observed the echinorhyncus hominis. It has been shown by artificial infection experiments that echinorhyncus moniliformis, Bremser, 1819, may occur in man.

Hirudines have been observed as occasional parasites of man, and may be transmitted to man. Even now they are frequently used for medicinal purposes. Among them have been noticed: Hirudo medicinalis, Linné, 1758, Hirudo troctina, Johnston, 1816, Hirudo ceylonica Bl., Limnatis nilotica, Savigny, 1820. These are found upon the skin or in the oral cavity of man, and soon begin their blood-sucking activity.

\section{ARTHROPODA}

The arthropoda, particularly the arachnoids, the spiders and the insects, are found in great numbers as parasites of man. Owing to their manner of life they appear chiefly as ectoparasites. To these belong the

\section{Leptus autumnalis, Shaw, 1790.}

The so-called gooseberry or harvest acarus, which is $0.3-0.5 \mathrm{~mm}$. long, and of a reddish color, lives in great numbers through the summer in immature forms on grass and shrubs, and occasionally as parasites upon man and other mammals. The ova of the trombidium holosericum, which are conspicuous in many gardens by their red color, are deposited in June or July. From these a larva develops which we recognize as the leptus autumnalis. This larva bores into the skin with its head and produces a severe cutaneous inflammation with itching which may be relieved by washing with soap, by inunctions with balsam of Peru, or 5 per cent. of carbolic solution or benzine.

Similar varieties of acarus such as tetranychus molestissimus, Weyenbergh, 1886, pediculoides ventricosus, Newport, 1850, tarsonemus intectus, Karpelles, 1885, pygmephorus uncinatus, Flemming, 1884, have been found in workmen who had handled Indian, Bulgarian or Russian grain, and suffered from an itching skin eruption. Other varieties of acarus cheyletus eruditus (Schrank, 1781), and tydeus molestus (Monig) which are found in rags, stables, and tobacco stores, also attack man and give rise to distressing itching.

The dermanyssus avium (Dugès, 1834), the bird acarus, is better known. It occurs in chickens, pigeons and in our song birds when kept in cages; it 
occasionally becomes parasitic in man, and produces very intense itching, erythema and eczema. The acarus which is found in swallows, dermanyssus hirudinis, Hermann, 1804, also occasionally becomes parasitic in man.

Of the ticks the ixodes ricinus Linné, 1758, is parasitic in man. The parasite lives upon low bushes and generally attacks sheep, cattle, dogs, cats, and, not rarely, also man. The female bores with its proboscis deeply into the skin to suck blood. It is necessary to exercise caution in removing the insect, as the proboscis tears readily, and if left in the wound causes inflammation. The application of benzine, petroleum, or oil of turpentine readily removes the parasite.

Not quite so harmless is the bite of the argas reflexus, Fabricius, 1794. It lives in woodwork or masonry, and in some regions attacks pigeons. It occasionally becomes parasitic in man. The parasite produces local inflammation and also certain general symptoms, among which are nausea, pyrosis, diarrhea, irregular heart action and dyspnea. The poison is said to be contained in the powerfully developed salivary glands. The bite of the argas persicus, Fischer de Waldheim, 1824, and of the argas tholozani, Laboulbène et Mégnin, 1882, which live in Persia, is said to be dangerous. In the tropics still other members of the family of argas are known (such as the argas turicata Dugès, argas chincha, Gervais, etc.), which occasionally attack man and produce disagreeable symptoms. Of the family of the tyroglyphides only the tyroglyphus farinæ, de Geer, and the tyroglyphus siro, Linné, the tyroglyphus longior, Gervais, are to be mentioned as occasionally parasitic in man. More important, on account of the extent of its distribution, is the family of sarcoptes:

\section{Sarcoptes scabiei, Linné, 1748.}

The knowledge of scabies dates back to antiquity. Aristotle was apparently acquainted with the organism. But although the insect was familiarly known for centuries, its natural history having been correctly described and depicted, its parasitic character was not recognized, and the itch was looked upon as a disease produced by an acrimonia sanguinis. This opinion became the prevalent one. It was not until 1834, when the completely forgotten art of catching the acarus was revived, that the pathology of the itch was reconstructed, and the acarus generally recognized as the cause of the affection. It is hardly necessary to give here a description of the acarus. A microscopic preparation of the acarus may readily be obtained; in a patient with the itch we look for a large pit which may frequently be seen with the naked eye. This pit is seen upon the hands as a blackish line, and upon the trunk as a whitish punctated line. At the end of this groove, where a whitish discolored point may. be recognized, the epidermis should be carefully removed with a cataract needle, and the insect, which has been laid bare, can be extracted and put upon a slide.

We can easily recognize upon the head the scissors-like jaw feelers with which the impregnated female cuts or bores its way into the epidermis. Gradually it penetrates the deeper layers of the rete Malpighii. The bite is followed by the deposit of an exudate between the cutis and the newest epidermis 
layer from which the acarus is extracted; decided itching immediately arises, perhaps in consequence of the discharge of an irritating fluid. Boring its way transversely from without inward to deposit its eggs, the acarus makes a passage parallel with the first to the surface of the skin. From the eggs, about 50 in number, deposited in this passage an embryo develops, which after from four to seven days makes its own opening to the external skin. After a short time it again bores its way into the skin to pass through a desquamation process, which is repeated several times, and about fourteen days later it appears as a mature insect upon the skin. The male does not bore its own opening, but only hollows out a funnel-like groove in which it lives.

The transmission of the acarus from man to man occurs occasionally by means of clothing, but, as a rule, by intimate contact with the diseased skin, and in almost every case by sleeping with a scabies patient. Among the poorer classes, therefore, a scabies patient is likely soon to infect the entire family. The disease is often spread by prostitutes.

The acarus is apt to attack the flexor surfaces of the joints of the hand and fingers, the interdigital folds, the lateral surfaces of the fingers; in children with a delicate skin the palm of the hand, the surroundings of the elbowjoint, the anterior axillary fold; and in women the nipple. The navel, the skin of the penis and scrotum, the trochanter region, the flexor of the knee and the internal border of the foot are attacked. The acarus also prefers all those portions of the body upon which the underclothing is tight. The face and hairy scalp are rarely attacked. Sämisch has described a case in which scabies gave rise to severe inflammation of the eyes ("keratitis," "Büschelform ").

The irritation from the itching which the parasite produces by its burrowing in the skin is very acute, especially at night, for at this time, stimulated by the warmth of the bed, the mite nourishes itself. The affected individual attempts to relieve the irritation by vigorous scratching. Not only are the small nodular efflorescences produced by the parasites irritated by this scratching, but, in addition, new papular elevations are formed at the exit of the hair follicles, and small vesicles like millet seeds with purulent contents. Continued scratching causes new eruptions, while the older ones are torn away, and become covered with striæ-like blood crusts, from which finally ulcers covered with eschars develop. Marked pustular eruptions often follow even in young people and eczema develops in all those who suffer for a long time from the disease. The diagnosis of scabies may readily be made from the localization of the excoriations, as eczema upon the nipple, upon the fold of the axilla, upon the penis, etc.

A peculiar picture resembling the mange of animals is produced by scabies norvegica s. crustosa. This shows a thick, dirty-gray induration composed of deposits of epidermis upon the palm of the hand, on the sole of the foot, on the elbows, on the face and the hairy scalp. These indurations contain the parasites and their ova often in enormous numbers. According to Fürstenberg this form of scabies is due to the smaller sarcoptes scabiei crustosæ, Fürstenberg.

The disease usually develops typically in one and a half to three months 
after infection. The eczema due to scratching has by this time spread over the entire body; the longer the disease lasts the more marked it becomes. Aside from the irritation and the loss of sleep due to this, no further symptoms arise.

Spontaneous recovery does not result, but recovery from scabies in the course of concurrent febrile diseases is noted as the consequence of the death of the parasite. The ova, however, do not always die. Therefore, after recovery from the disease, the itch frequently reappears.

The DIAGNosis is easy. The location of the efflorescences is particularly characteristic. We can hardly err in making a diagnosis of the itch when a markedly itching cutaneous eruption is characterized by the presence of isolated nodules, particularly upon the anterior surface of the trunk and extremities, of small vesicles upon the fingers and interdigital folds, in the palm of the hand and on the sole of the foot, with pustules upon the fingers, especially in young people, in whom eczema shows itself chiefly in those areas between the nipple and the knees for which the parasite shows a preference. The diagnosis becomes certain by the discovery of the acarus grooves, or of the parasite itself. In all doubtful cases microscopic investigation is necessary.

Prurigo and scabies are often confounded. Leaving out of consideration the time and place of development of the first prurigo efflorescences, prurigo differs essentially by the localization of the eruption. This is found chiefly upon the lower extremities, especially upon the extensor surfaces of the trunk, but never upon the flexor surfaces of the joints.

After scabies has been recognized in an individual, it is wise to examine carefully all other members of the family, and to treat simultaneously all who are affected. 'The aim is to "kill the parasite and heal the eczema. In former times, when the parasitic nature of the itch was not yet understood, the disease was looked upon as almost incurable; we can to-day rapidly cure the affection by a number of remedies such as sulphur, tar, naphthol, balsam of Peru, styrax, petroleum, etc.

It is sufficient to mention a few among the great number of prescriptions:

Re Florum sulphuris,

Olei fagi,

$\bar{a} \bar{a} \ldots \ldots \ldots \ldots \ldots \ldots+20.0$

$\left.\begin{array}{l}\text { Saponis viridis, } \\ \text { Axungiæ porcinæ, }\end{array}\right\} \bar{a} a \bar{a} \ldots \ldots \ldots \ldots \ldots . \quad 40.0$

Cretæalbæ ................. 15.0

or

$\left.\begin{array}{l}\text { B. Styracis liquidi, } \\ \text { Florum sulphuris, }\end{array}\right\} \bar{a} \bar{a} \ldots \ldots \ldots \ldots \ldots \ldots 20.0$

Saponis viridis............... 40.0

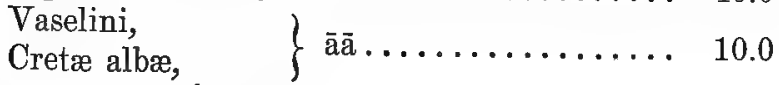

or $\quad \mathbf{R} \beta$-Naphtholi,

Cretæ albæ, $\} \bar{a} a . \ldots \ldots \ldots \ldots \ldots \ldots \ldots . \ldots \ldots$

Saponis viridis................ 50.0

Axungiæ porcinæ................ 100.0 
Spiritus vini..................... 10.0

Olei olivarum.................. 65.0

When there is no eczema the best results will be obtained with inunctions of balsam of Peru. The patient takes a bath in which he rubs himself briskly with green soap. After an hour, when the skin has become entirely dry, balsam of Peru is rubbed into the entire surface of the body. About ten grams are sufficient for this purpose. The next day the same process is repeated. After forty-eight hours the patient takes another bath, which completes the process.

In the majority of cases it is well to give a bath to soften the epidermis before beginning the actual treatment. In private practice $I$ prefer, as a remedy for scabies, the above-mentioned naphthol salve, which on the first day is twice rubbed into the body and completely allays the itching during the succeeding night. If the itching should return, a third inunction is given upon the second day. The patient is able to follow his ordinary occupation. It is best for him to wear woolen underwear as the salve will less readily adhere to this. Upon the sixth day a bath completes the antiscabies treatment. If there is eczema this necessitates further therapeutic measures upon which I cannot here enter.

The "rapid cures" were formerly much in vogue. In this treatment the patient is put in a warm bath and is rubbed with black soap. The bath should last an hour and a half and be followed by an inunction of

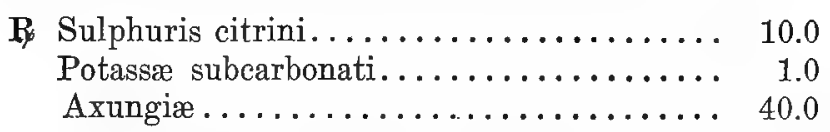

This finishes the treatment. Sensitive persons readily react to this, as well as to other rapid cures, by an eruption of eczema.

Certain forms of scabies occur in various domestic and wild animals, in the horse, the sheep, the goat, the camel, the llama, the pig, the dog, the fox and the lion. These parasites occasionally attack man.

According to Carron du Villards, there are two varieties of acarus in the Antilles which may infect the eye. Fischer saw a decided inflammation of the eye produced by a chicken louse (lipeurus variabilis, Nitzsch) which had found its way into the conjunctival sac. According to Mégnin, another acarus, the holothyrus coccinella, Gervais, produces in birds a dangerous disease, which, when transmitted to man, causes itching of the skin and inflammation. Another acarus, called by the inhabitants of the Antilles the arador, burrows into the skin of the eyelids, forming brown, tortuous furrows which may be recognized with the naked eye. Its presence produces severe itching.

An acarus nephrophages sanguinarius has been found by Miyake and Scriba in the hemorrhagic urine of a Japanese. How important this acarus, which is said most closely to resemble the dermatocopter communis, may be, cannot at present be determined. The significance of the

Demodex folliculorum, Simon, 1842, 
is still debated. This acarus lives in the hair follicles in comedones and in the glands of Meibomius in the skin of the face of man. In the dog, the pig, the cat, and in cattle, hair follicle acari have been observed and are said to be varieties of the parasite of man. The views of authors are divided as to the importance of this parasite as a causative agent of disease. We incline more and more to the opinion that it is one of the harmless parasites, and that its occurrence in acne pustules is of no pathogenic significance. Nevertheless, it is found with extraordinary frequency-according to Hunsche even in 92.5 per cent.-in the sebaceous glands of perfectly healthy individuals. The clinical conception formulated by Raelmann of blepharitis acarica can no longer be maintajned. The acarus is found with great regularity in the ciliary follicles of normal, as well as of abnormal, eyelids.

Of the linguatula, or tongue worms, which are elongated, vermiform, flattened or cylindrically curled worms whose head is armed with an elliptical chitin mouth and two retractile claws, one is parasitic in man. This is the

\section{Pentastoma tanioides, Rudolphi, 1810.}

The sexually mature parasite lives in the nasal cavity, the forehead bones and jaw of the dog, the wolf, the horse, the mule, the goat, and, occasionally, also in man. The eggs discharged with the nasal mucus contain embryos which reach the open air where they are taken up by such animals as rabbits, hares, cattle, horses, etc., and also sometimes by man. They penetrate the intestinal wall, and reach the liver where they become encysted. For a long time the larva pentastoma denticulatum, Rudolphi, was taken for an independent parasite. After a certain time the larvæ may wander further, and in this way reach the respiratory tract, especially the nose. In other cases infected organs are eaten by carnivora. The larvæ which reach the intestinal tract finally find their way to their preferred points of lodgment. Man unquestionably infects himself in a similar manner. The pentastoma either accidentally reaches the nose or is introduced into the intestinal canal by contaminated food.

The sexually mature form is rarely met with in man. Laudon found the parasite in a locksmith, who suffered for years from jaundice and gastric disturbance, and later from epistaxis. Usually the larva (the pentastoma denticulatum), when observed in man, is found particularly in the liver, the lung, the spleen and small intestine, the peritoneum, the heart muscle and the kidneys. Special symptoms are not present in these cases. The pentastoma can be diagnosticated only when they give rise to irritative symptoms in the nose or its auxiliary cavities or when their ova are found in the nasal secretion.

Pruner was the first to find in the liver of two negroes the

$$
\text { Pentastoma constrictum, v. Siebold, } 1852 .
$$

Observations regarding the occurrence of this parasite are very scanty. It appears that when present in great numbers it may be the cause of fatal
peritonitis. 
In conclusion I must mention the

\section{INSECTS}

which are parasitic in man, and I should first like to consider the rôle which they play in the transmission of infectious diseases. The reports concerning this are scanty and widely scattered.

From the interesting investigations of Nutall it appears that it is only rarely that anthrax, chicken cholera and mouse septicemia are transmitted by bed-bugs and fleas, probably because the insects which absorb the microörganisms with their sting, soon rid themselves of them. It cannot, however, be denied that by crushing the sucking animals which contain these microörganisms in their blood, and by scratching at the point of the bite, these germs may be conveyed to man. But it must be remembered that the infectious germs which are present in the dejecta of bed-bugs and fleas lose their toxic properties very rapidly. This is especially noticeable in fleas.

Plague bacilli, transmitted to bed-bugs and fleas, appear to die in the intestinal canal of these insects. The danger lies in the fact that if any one crushes an insect thus infected, and the bite is then rubbed or scratched, the bacilli may be transmitted. It is certain from the experiments or Simmond that flies which have fed upon the dejecta or the bodies of cholera patients take up cholera germs, and transmit them to food. Relapsing fever spirilli taken into the intestine of bed-bugs appear to die with relative rapidity. The immediate inoculation of apes with bed-bugs that have sucked themselves full and have been crushed, has been followed by positive results. Typhoid bacilli may apparently be carried by flies. It has been further proven that flies may take tubercle bacilli into the intestinal tract. The bacilli have been found in the dejecta (fly specks) upon walls, in closets, and on doors. It is not unlikely that in regions where leprosy exists it is transmitted by means of sarcoptes. The bubo disease, Egyptian ophthalmia, and conjunctivitis blennorrhoica may be transmitted by flies. Favus and impetigo are said to be transmitted by lice. That infectious diseases are transmitted to man by means of the ixodes is more than doubtful. The rôle played by the ixodes bovis, Riley, 1869, or the boöphilus bovis, Curtis, 1890, and by the tsetse fly in Texas fever, the tsetse fly disease, has not come within the range of our observation.

Of the parasitic insects I shall only refer briefly to the head louse (pediculus capitis, de Geer). This parasite is distributed over the entire world. It generally lives upon the hairy scalp, but in cases of great uncleanliness also infects other parts of the body. The ova of the head louse are rarely found in the cilia. From the excrement of the lice which is conveyed to the conjunctival sac by the hands, conjunctivitis and blepharitis may apparently be produced. In cases of stubborn head eczema, especially in children, the presence of lice should invariably be looked for. It is known that plica polonica usually develops from a preceding pediculosis capitis with accompanying eczema. The plica polonica (Polish plait) must be removed with the scissors. To exterminate lice, washings with vinegar, petroleum, or corrosive sublimate $(1-1,000)$ and combing the hair by means of a fine comb are advisable. The scalp should then be painted twice daily with Ziett-Lassar zinnober salve: 
B Hydrargyri sulfurati rubri............ 1.0

Sulfuris sublimati .................... 24.0

Olei bergamottæ ..................... gtt. $\mathbf{x x \nabla}$

Vaselini flavi, ad................... 100

In children, Joseph advises:

R Acidi salicylici ...................... 1.0

Tincturæ benzoes................... 2.0

Vaselini flavi, ad.................. 50.0

to be rubbed into the scalp twice daily.

The clothes louse, pediculus vestimenti, Burmeister, lives upon the throat, neck, and trunk of man. The eggs are deposited in the lanugo hair of the skin, as well as in the underclothing. The bite of the louse causes severe itching, and by scratching striæ-like wounds are produced. If the condition exists for a long time, open wounds and ulcers of various kinds appear, and finally a permanent pigmentation of the skin which is particularly noticeable in those areas in which the clothes are worn close to the body. So-called lousiness is produced by the abundance of the clothes louse. Cleansing the clothes and disinfecting the same, and washing the body with vinegar or petroleum will rapidly remove the disease.

The body louse (phthirius inguinalis, Redi, 1668) is most frequently found in the hair surrounding the pubes. Thence it is distributed over the trunk to the axillary cavity, into the beard, into the cilia and into the eye-brows, but is very rarely found upon the hairy scalp. The exanthem produced by scratching is usually found in corresponding places. The maculæ cæruleæ, composed of reddish brown flakes, which are found upon the anterior lateral aspect of the thorax, on the upper thighs and buttocks, are of a peculiar character, and are probably produced by a poison contained in the salivary gland. The parasite may be removed by inunctions of gray salve followed by a bath. Inunctions with balsam of Peru or white precipitate salve will also rapidly remove the parasites. The phenomena produced by bed-bugs (cimex lectularius, Merrett, 1667, cimex ciliatus, Eversmann, 1841, cimex rotundatus, Signoret, 1851) and those produced by the flea, pulex irritans, Linné, 1758, are sufficiently well known.

The sand-flea (sarcopsylla penetrans, Linné, 1758) has lately become of more interest to Germany on account of its appearance in the colonies. Carried from its home in South America to West Africa, the parasite has gradually spread itself from west to east, until it reached German East Africa. By 1897 it became a land plague in Uhehe.

After the female (the male lives independently) has bored its way under the toe-nail, it appears on the first day as a small harmless point, and may easily be removed without pain. Early removal is the only protection from serious consequences; it is therefore advisable to inspect the feet daily, and this is the more necessary in infected regions, as the sand-flea at first causes no symptoms. 
In eight to fourteen days the posterior portion of the abdomen of the parasite swells to the size of a pea. It contains from 60 to 100 eggs. After depositing the eggs the parasite dies. By incautious manipulation in its removal, or by other trauma which affect the toes and cause the abdomen of the para-

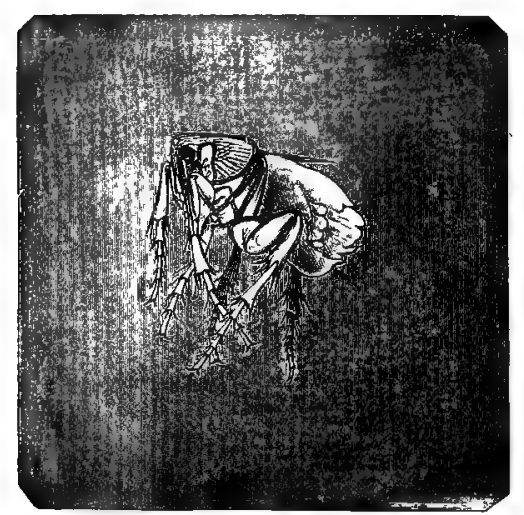

Fig. 57.-Sarcopstlla Penetrans, SAndflea, Prior to its Entrance Under the Nail of the Toe.

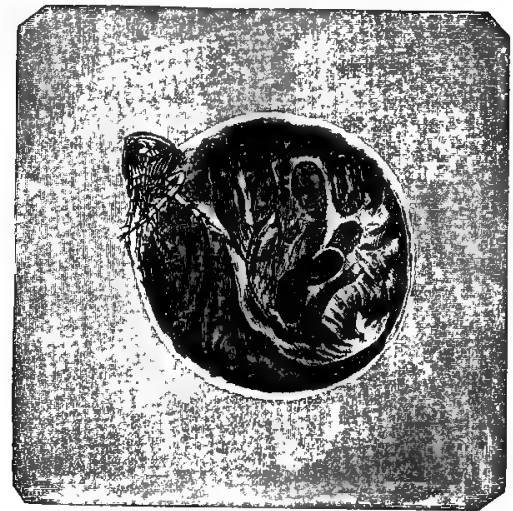

Fjg. 58.-Sarcopsylua Penetrans, Removed FROM A WOUND OF THY NAIL.

site to rupture, the ova are liberated, and stubborn ulcers develop which, if neglected, cause the loss of the toes. Wound infection not rarely complicates the further course. The parasite should be removed by carefully lifting it out with a dull forceps.

\section{FLY LARVA (Maggots)}

Fly larvæ are occasionally observed as parasites in man. The mother animals belong to the family of cestrides or of the muscides, the actual flies. The clinical picture produced by larvæ is known as myiosis externa or interna s. intestinalis.

In Europe myiosis externa is mostly produced by the larva of the muscides, more rarely by the larva of cestrides. For the latter the larva of the hypoderma bovis, de Geer, or hypoderma diana, Brauer, are responsible. In the tropics the gadfly boil of man is due to the dermatobia noxialis, Brauer, or the ochromyia anthropophaga. As soon as the eggs of these flies have been deposited upon the skin, the larvæ pour forth, bore into the skin, and in the course of a few months cause the affection which is known as the "gadfly boil" of cattle, of the stag and of the roe. In cattle larvæ are taken up by the mouth and finally find their way under the skin; whether the same course is followed in man has not yet been proven. Recovery occurs spontaneously, by the egress of the parasites, or by surgical opening and extraction of the larvæ from the carbuncle-like cutaneous swelling. Krautner observed a dipteren larva in an isolated location. He extracted a larva of the hypoderma bovis from the anterior chamber of the eye.

The occurrence of cestrus larvæ in the digestive apparatus of man has not 
yet been determined with certainty. Some authors, however have thought that they produce another disease, the "creeping disease."

In the hot summer months it is noticed-the number of cases observed is still limited-that upon the uncovered portions of the body irregular, serpentine, linear elevations appear, which never branch, and these give rise to itching and burning, and cause a red elevation, slightly raised above the skin. The line is elongated, and tapers rapidly to a point $(1-15 \mathrm{~cm}$. in twenty-four hours). Occasionally at intervals of from $1-2 \mathrm{~cm}$. small nodules are noticeable, which have been declared to be the fecal mass discharged by this insect. That the disease is caused by an animal parasite is certain. The parasite itself, which has been thought to be a gastrophilus larva, has not yet been zoologically and definitely determined.

The majority of affections of myiosis externa are caused by muscides larvæ. In Europe it is particularly the sarcophila Wohlfart, Portschinsky, 1875 , in America the lucilia macellaria, Fabricius, 1794. Those persons are most endangered who sleep in the open air and who suffer from any purulent process: Pus from the ear, nose, or similar affections. The fly is otherwise extremely timid and never enters living rooms, but is attracted by pus and deposits its eggs. The larvæ, which soon come forth, cause remarkable destruction by their enormous appetite. Entering the auditory passages, they eat the external covering, penetrate the tympanic cavity, and pass through the Eustachian tube into the pharynx. Great destruction is wrought in the nose and its auxiliary cavities, where ulcers and purulent wounds are produced. Autopsy findings confirm the extraordinary virulence of these parasites.

In the conjunctiva, in the oral cavity, even in the urethra and the bladder, maggots have occasionally been observed. Formerly, before the introduction of antiseptic surgery, maggots in wounds were not very uncommon; to-day they appear only in such as are grossly neglected. Besides the parasites mentioned above, in man larvæ of calliphora vomitoria Robineau-Desvoidy, cimex limensis, sarcophaga carnaria, Meigen, musca cadaverina, musca domestica, musca stabulans, and others have been observed.

I should like to say here that numerous occasional parasites, such as galleria mellonella, dermestes lardarius, geophilus sodalis, geophilus hortensis, thousand-leggers, have been removed by physicians from the nose. Laboulbène mentions a case in which the gammarus pulex was vomited. This by no means exhausts the number of the occasional parasites.

For their removal, corrosive sublimate injections and chloroform or turpentine mixtures are advised.

The myiosis intestinalis is a much-debated parasite. Although numerous well-observed cases of this kind are on record, the presence of the larvæ of flies in the digestive apparatus is still doubted. We shall touch lightly on these doubts which are nowise justified. Those that have been observed up to now are the larva of musca vomitoria, musca domestica, musca nigra, musca corvina, musca pendula, musca meteorica, calobata cibaria, hydrothorea meteorica, sarcophaga affinis, sarcophaga carnaria, sarcophaga hæmorrhoidalis, sarcophaga hæmatodes, anthomyia canicularis, anthomyia scalaris, etc. 
On account of the myriads of flies about our houses and their habit of resting preferably upon food where they deposit their eggs and on account of the great production of fly larvæ in water, it is quite remarkable that larvæ of flies do not more frequently reach the gastro-intestinal canal of man. There are many avenues by which the larvæ of the anthomyia varieties which deposit their ova in salad, radishes, berries, etc., may reach the intestinal canal. Many of the larvæ are destroyed in the digestive tract, or do not find the conditions suitable for their existence, but they generally possess such extraordinary resistance as to be able to withstand the digestive process.

There is no specific symptomatology. In the history of these patients complaints of nausea, tendency to vomit, vomiting, sour eructations, more or less severe pain in the epigastrium, and attacks of colic may appear. At one time there is constipation, at another time diarrhea, with or without blood. Severe spasmodic attacks, cramps, sensations of fear are observed, which cease at once when the larvæ are passed. There are no sequels, however, as the existence of the parasite is so brief. The larvæ are voided per anum with the stools or by the mouth in vomitus; sometimes more than one hundred larvæ are discharged.

As soon as the discharge of larvæ has been recognized, an attempt should be made by therapeutic measures to effect the speedy removal of the parasite. If the larvæ are still present chiefly in the stomach, they may be removed by lavage. In naphtholin (0.5-1.0 in single doses, up to 5.0 as a daily dose for adults) we no doubt possess a remedy which causes the death of the Iarvæ, that is, brings about their rapid removal.

Besides those mentioned, there are a number of other insects which are " occasional" parasites of man. I need mention here only the different varieties of flies, mosquitoes, wasps, bees, hornets, etc., which occasionally attack man, and by their sting cause marked pain. G. Braun has lately reported the bite of poisonous spiders (Latodectus lagubris) which may bring about serious symptoms. The bite of the tarantula is harmless, but that of scorpions causes severe inflammatory cutaneous symptoms. In conclusion, it must be mentioned that various caterpillars supplied with hairs containing retroserrates and spinules may cause severe irritative phenomena upon the skin, the connective tissue, the corium and iris. Gray or grayish yellow nodules the size of a lentil, being round or oval, form in the iris and subconjunctival cellular tissue. Laudon mentions that in girls inflammation of the sexual apparatus, swelling of the labia and leukorrhea may be caused by the hairs of caterpillars, and also that the toxic substances contained in the cavities of the hairs of caterpillars may, by penetrating or tearing the human skin, produce severe irritative phenomena.

For the relief of the bites or stings of insects, painting with sal ammoniac, ichthyol, or ichthyol salve or the application of ichthyol plaster, is advised.

The severe affections of the eye produced by the hairs of caterpillars require the extraction of the hairs which have entered; in iritis, atropin and warm compresses. Unfortunately, the prognosis is not favorable. The cutaneous irritation may be lessened by vaselin or inunctions with lanolin. 


\section{LITERATURE}

R. Leuckart: "Die Parasiten des Menschen," II. Aufl., Leipzig, 1886-91. Braun: "Die thierischen Parasiten des Menschen," II. Aufl., Würzburg, 1895. Huber: "Bibliographie der klinischen Helminthologie," Jena.

Mosler und Peiper: "Thierische Parasiten." "Nothnagel's specielle Path. u. Ther.," VI. Bd., Wien, 1894.

E. Peiper: Ergebnisse der allgemeinen Pathologie und pathologischen Anatomie, III. Jahrg. "Thierische Parasiten des Menschen."

E. Peiper: "Die Verbreitung der Echinokokkenkrankheit in Vorpommern," Stuttgart, 1894.

E. Peiper: "Fliegenlarven als gelegentliche Parasiten des Menschen," Berlin, 1900. 


\section{TOXICOLOGY}





\title{
IMPORTANT POISONS AND THEIR TREATMENT
}

\author{
By R. v. JAKSCH, Prague
}

Ir is a difficult undertaking to confine within the limits of an article all the data of importance in the study of the poisons with which the practising physician is likely to come into contact.

In modern clinical medicine, the conception of poisoning must be very widely extended. The amount of material to be considered is enormous, because every pathogenic agent which finds entrance into the human organism, including also every contagium vivum, may produce toxic action. This great group of poisons, the endogenous toxicoses, we shall not here consider, but shall content ourselves with the discussion of the exogenous toxicoses which are most important to the physician.

In the definition of the word poison, we are at once confronted with difficulties which need not be further dwelt upon. Our object is to consider a number of symptoms; and the treatment of a series of diseases all of which are due to the introduction within the body of certain well-known chemical agents from without. These agents include the acids, the alkalies, the metalloids, the metallic salts, gases, bodies that belong to organic chemistry, such as the fat derivatives, bodies of the aromatic group, the camphors and balsams, the alkaloids, the glucosides, bitter and indifferent bodies, the toxalbumins and, finally, vegetable and animal poisons, the composition of which is only partially known to us.

From this almost endless series it is apparent that we cannot describe all of the pathologic pictures produced by these poisons, but it will be our object to take, as an example, an important representative of each of the previously mentioned groups, and describe its effects.

We will first direct our attention to the acids. The pathologic processes produced by these poisons may very properly be designated as acidosis. The differences between these acids and in their composition, if we include the organic and inorganic varieties, are parallel with the difference in the clinical picture which develops after their introduction into the human organism. As the type of acidosis, I shall consider inorganic acid, sulphuric acid, and shall discuss the important symptoms of this poison as acidosis. I may remark that I choose this particular acid for the reason that this acid toxicosis is observed with relative frequency by the physician.

There can be no doubt that by the introduction of large amounts of acids, nervous symptoms may be produced; but symptoms of this kind are rarely observed at the bedside, as the local symptoms produced by the acid toxicosis 
completely dominate the scene. Those acids whose corrosive effects are less prominent, as, for example, hydrochloric acid, may show nervous symptoms which can be referred to the acid, but even this is relatively rare. If an individual takes large quantities of free acid, e. g., sulphuric acid, what symptoms are produced? The deeply invading eschars which cover all parts of the mucous membrane are characteristic, particularly in those areas which are brought in contact with the acid in deglutition. Severe retching and the vomiting of brown pulpy masses, excessive pain in the gastric region, intense burning in the esophagus, and marked salivation, are the immediate consequences of such a toxicosis. The vomited material has an acid reaction; within a few hours the symptoms of marked renal irritation develop.

If the amount of acid which has reached the stomach is relatively large, and the acid is concentrated with the continuance of vomiting, symptoms of perforative peritonitis soon appear, due to the erosion of the walls of the stomach by the acid, and the patient succumbs in a short time, often within a few hours. But even if the amount of acid swallowed was not large, days, even weeks, must elapse before the eschars heal. Dysphagia, salivation, pain on deglutition continue, and often fever follows, the wounds which have been produced by the acid becoming the point of entrance by which pyogenic organisms find their way into the body. An important fact is that all poisonings with corrosive acids, even in cases which apparently run a mild course, may lead to stricture of the esophagus; indeed this is an almost invariable result. Of course the frequency of stricture depends on the variety of the acid. Poisoning by sulphuric acid almost always produces this symptom, nitric acid less frequently, hydrochloric acid relatively seldom, but there can be no doubt that strictures also occur from hydrochloric acid toxicosis. They are less often seen after poisoning with organic acids, such as acetic acid, etc., perhaps because poisoning with concentrated solution of these acids is relatively rare.

An important symptom which must be discussed is renal irritation caused by acid toxicosis, which, in my experience, often leads to the typical signs of acute toxic nephritis. Acid nephritis may occur in all acid toxicoses; most frequently, however, we meet with it after poisoning with sulphuric acid. The symptoms are exactly the same as in acute nephritis due to other causes, but I must emphasize the fact that edema does not accompany this form of nephritis, and, furthermore, that dissolved hemoglobin frequently appears in the urine.

The prognosis in poisoning with acids should always be considered grave, as, even in the mildest cases, it can never be asserted at the onset that stricture of the esophagus, which is invariably so dangerous, may not develop. Even with judicious treatment, stricture may result in under-nutrition, or in actual starvation with all its consequences.

In regard to the diagnosis, the fact that an acid toxicosis is present may be readily determined from the markedly acid composition of the vomited material, and from the previously described eschars. We must determine with which acid we are dealing by a testing for the presence of that acid to which the pathologic picture points. If, for example, the eschars are yellow or yellowish, it may be presumed that nitric acid was the toxic agent. If the mucous 
membrane looks as though it had been scalded, hydrochloric acid should be thought of; deeply invading, leather-like eschars are peculiar to sulphuric acid toxicosis, white eschars are peculiar to tartaric acid, etc. The presence of the special acids must, however, be proven by chemical analysis, the consideration of which is not permissible within the scope of this article. Examinations of this kind should, if possible, be made by each physician for himself. But we cannot demand that the practising physician make these tests for himself because complicated chemical apparatus is necessary, and therefore I limit mysclf in this article to a few general indications. Certain inorganic acids, such as sulphuric acid and nitric acid, may be very readily determined; the proof of the presence of hydrochloric acid is more difficult, since it is normally present in all stomach contents. But in most cases it is sufficient to demonstrate by titration with an alkali a large amount of free acid in the vomited material.

Regarding the therapy, our sovereign remedy is milk, which possesses in a high degree the power of acid neutralization; if this is not immediately at hand (for rapidity of action is the most important and first requisite in the treatment of acid toxicosis) then soap suds is to be administered; solutions of albumin are also advisable; of drugs as antidotes, magnesia usta in water is advisable, also lime water. The severe pain which these poisonings cause justifies the use of opiates, morphin and cocain. Unfortunately, painting the mucous membranes with the last-named remedies has repeatedly proved disappointing to me.

Lavage of the stomach in such cases necessitates great care; lavage of the stomach in poisoning from sulphuric acid, particularly, involves certain danger to the patient, for it cannot be denied that by such a process a gastric perforation may be produced. It must also be remembered that even the act of washing out the stomach in such patients produces severe pain, and other forms of distress.

I must briefly describe those acid intoxications which, on account of their not infrequent occurrence and their clinical picture, occupy a special position, and, therefore, have certain interest for the physician. These are poisoning by oxalic and prussic acids.

We shall first describe oxALIC ACID TOXICOSIS. Oxalic acid itself, above all its salts, which are used for polishing metal ware, and to remove ink spots from the skin, is in common use, and is a frequent cause of this toxicosis.

Oxalic acid and its salts produce like.mineral acids a corrosive effect upon the mucous membranes which is shortly followed by a considerable reaction and subsequent inflammation in the surrounding area; there results a picture which more closely resembles poison by alkalies than by acids (see below).

The nervous symptoms which invariably appear in the course of poisoning from this acid, such as convulsions, trismus, and tetanic attacks, are quite noteworthy. In other cases, however, very threatening symptoms, oliguria and anuria, become prominent as signs of severe acute toxic renal insufficiency due to infarction of the kidney with calcium oxalate.

The prognosis in this form of poisoning is always serious; and in all cases a threatening clinical picture results. The diagnosis may be made from the symptoms sketched above, and is usually easy. It becomes certain, however, 
only when it is possible to demonstrate oxalic acid or its salts in the excretions, above all, in the vomited material. In cases of poisoning, in which we have the relatively slight corrosive effects which oxalic acid produces, energetic gastric lavage, best of all with lime water, which changes oxalic acid and its soluble salts into insoluble calcium oxalate, may be resorted to; lime water may also be given internally. The use of sucrate of lime has been especially praised.

As with oxalic acid toxicosis, a special position has been assigned to poi- soning with PRUSSIC ACID. In concentrated solution it is one of the most violent poisons known, and in the course of a few minutes causes death. With a loud cry, the person poisoned collapses, the respiratory frequency rises enormously, convulsions occur, and in a few minutes death results; so rapid is the course that the physician rarely has an opportunity of attempting relief. If there is time, immediate washing out of the stomach, with the addition of hydrogen peroxid, is, at all events, in place; subcutaneous injections of atropin, etc., have been advised, but, in the main, we are compelled to say that in severe cases of poisoning therapy is ineffectual, and only in the mild cases may we count upon success.

We now turn to the second group of poisons, that of the alkalies, salts of alkaline metals, and metalloids. We shall describe only three forms of poisoning, namely: 1, Lye toxicosis ; 2, poisoning with potassium chlorate; 3, poisoning with salts of barium.

Next to poisoning with sulphuric acid, unquestionably poisoning with LYE is undoubtedly most often observed by the physician. We never get poisoning by pure caustic soda, or pure caustic potash, but with mixtures which contain, besides these bodies, potassium carbonate and other salts, i. e., with fluids which are called in commerce soda solution and caustic soda.

What symptoms follow the introduction of these substances by the mouth?

In a short time vomiting of glassy, smeary, soap-like masses occurs and may continue for hours, or even days; the vomited material has an intensely alkaline reaction, and later often assumes a brown to brownish-red color due to decomposed hemoglobin. The patient is tortured by great pain, particularly upon deglutition, the areas of the mucous membrane which have come in contact with the caustic substance appear softened, oozing, swollen, and covered with pseudo-membranes which desquamate in a few days, and then cause the dreaded formation of strictures, particularly in the esophagus. Peritonitis, due to perforation of the stomach by the corrosive action of the alkali upon its walls, may also appear. The result of poisoning of this kind is always serious, and in apparently mild cases the possible sequel of stricture of the esophagus renders the prognosis unfavorable. I may add that alkalies, ceteris paribus, give rise to stricture in decidedly slighter concentration than acids.

The treatment consists in the immediate neutralization of the alkali, which is best accomplished by a lemonade containing tartaric acid. Prompt gastric lavage is contraindicated on account of the danger of perforation of the stomach; on the other hand, on account of the severe pain, the use of morphin and other opiates will be necessary. Cocain has here been as ineffective as in the treatment of acid toxicosis. 
Of special importance in this group of poisons is POTASSIUM CHLORATE; hence we shall briefly consider it. The drug is of special significance for the physician, as poisoning by its medicinal use has been frequently observed in the last few years. The quantity of the salt necessary to bring about this condition varies very widely in different individuals. The lethal dose in children is from 5 to 6 grams and in adults 15 grams or more.

This toxicosis differs particularly from poisoning by alkalies in that there are no local corrosive effects.

Such patients, however, show extreme cyanosis, they complain of a sense of burning and oppression in the chest, the skin assumes a yellowish hue, hemoglobin (hematin) appears in the urine without any other sign of nephritis. In the further course of the affection there are severe nervous symptoms, such as clonic and tonic spasms followed by delirium and then by deep coma; in this condition the patient succumbs. Moreover, there are undoubted cases of poisoning in which the typical symptoms of acute nephritis appear, and the patient dies of uremia.

The prognosis is always dubious; but recovery may occur even in the severest cases.

The diagnosis is made more certain by the demonstration of methemoglobin in the blood, and proof positive is the presence of the salt in the fluid obtained by gastric lavage. If the amounts introduced were very large, the characteristic vapors of chlorin gas are at once given off upon the addition of hydrochloric acid. The treatment requires immediate and thorough washing of the stomach, and the avoidance of the introduction of acids, for the latter generate in the stomach a very dangerous chlorin gas. The use of enemata is also indicated to eliminate the poison which perhaps may already be present in the lower portions of the intestine.

A few words in regard to the COMbinations OF BaRIUM.

All of the soluble salts of barium are very poisonous! Even the insoluble barium carbonate may be changed in the stomach into the soluble barium chlorid, and then have a toxic effect.

The principal symptoms are: Severe pain in the abdomen, profuse diarrhea, signs of acute nephritis and an entire host of nervous phenomena.

The therapy consists in immediate gastric lavage and in the administration of magnesium sulphate and sodium sulphate.

The prognosis is very grave!

I have included this form of poisoning because the barium salts are remedies which enormously increase blood pressure, and therefore barium chlorid has been employed in place of calomel in disturbances of cardiac compensation.

The severe symptoms of this poisoning, I might say the absolute impossibility of relying upon the action of these salts, forces me to advise against their use, even should these salts, as heart tonics, find eulogists in the future.

We now proceed to another group of poisonous agents, the metalloids. The first to be considered are chlorin, bromin and iodin, with their combinations. Only two of the CHLORIN COMBINATIONS are of practical importance, namely, chloral hydrate and chloroform. The first is used as a hypnotic; but it is not a harmless remedy, since upon long-continued use it may give rise 
to psychical depression, and according to all appearances, to progressive paralysis; and, moreover, in persons affected with disease of the heart, even in relatively small doses (2 to 3 grams) it may cause death. We should, therefore, be most cautious in the use of this remedy, sometimes so necessary, for example, in the treatment of certain forms of delirium tremens! In cardiac diseases, particularly, the use of chloral hydrate is contraindicated!

The second remedy to be discussed is chloroform. Every one knows the blessing of its effect, and it is to be hoped that the physician may be spared any exhibition of its deleterious action during his professional career! Chloroform is a potent cardiac poison, and the sudden deaths in narcosis which occur from time to time may be referred to this circumstance. There can be no doubt that chloroform narcosis may produce permanent damage to the organism, such as fatty degeneration of the heart and of the liver, and for this reason our motto must be: Be cautious in the use of chloroform, whether employed as an anesthetic or internally! I shall not detail the symptoms. These are generally known. Fatalities can never be entirely prevented in chloroform anesthesia, but careful, minute investigation of the heart of the patient who is to be anesthetized with chloroform will certainly limit them to a great extent. We must hope that the time is not far distant when less dangerous anesthetics will be substituted for chloroform narcosis. A beginning has already been made in this direction by Schleich's infiltration anesthesia.

In the second halogen, the BROMIN SALTS shall be briefly mentioned; these are extensively used in medicine, and thus cause the development of that pathologic picture which we appropriately designate as bromism. Although we cannot avoid using the salts of bromin in the treatment of epilepsy, and although recent observations have shown that the administration of the salts of bromin in food free from chlorin or, at least, almost free from it, is relatively well borne, on the other hand we cannot deny that, particularly in predisposed individuals, even the therapeutic use of bromin may cause serious symptoms of bromism. It is possible that the administration of bromin in sesame oil may mitigate these symptoms, but it cannot be expected to prevent their appearance entirely. The symptoms are anorexia, metallic taste, dyspepsia, decided emaciation, dermatoses of many varieties; also a series of psychical and nervous disturbances, particularly loss of memory, and these may lead to the development of the severest psychical symptoms. The saLTS OF rODIN act in a similar manner, except that here, as a rule, emaciation, I might say all the somatic symptoms, take precedence of the psychical element.

We now turn to another of this group of poisons, to PHOSPHOROs, which, at least in Austria, assumes uncommonly great importance, for, in certain cities-especially in Prague, next in Vienna, less frequently in the countrythe number of poisonings by this agent, which, in the course of the year, come under the observation of the physician, is an unusually large one. Almost exclusively the cases are suicidal, although the poison is often taken to bring about abortion. But the clinical picture of poisoning by phosphorus has additional interest from the fact that a number of other toxicoses, even a number of diseases for the most part infectious, present or may present a picture 
resembling poisoning from phosphorus. Mushroom poisoning (which see) is an example of such toxicosis, and, of other diseases, acute yellow atrophy of the liver.

What symptoms are produced by poisoning with sticks of phosphorus? A few hours after the ingestion of the poison, nausea and a tendency to vomit, more rarely actual vomiting, appears; the patient is uncommonly restless; jaundice, which may occur upon the first or the second day after the poisoning, indicates the severity of the intoxication. Usually we see decided enlargement of the liver, which may even appear a few hours after the poisoning. The evidences of the hemorrhagic diathesis and fever are added to this, and in a few days the patient succumbs from his suffering. In the majority of cases of poisoning with phosphorus it is fatty degeneration of the heart which causes death. The later jaundice appears, the more favorable the prognosis; a good appetite, or even a sensation of hunger, is a favorable sign in the course of phosphorus toxicosis. In the severe cases the urine contains: Biliary coloring matter and albumin; not rarely, if large amounts of grape sugar be administered to the patient, sugar is also present; that is, an alimentary glycosuria arises, a symptom which, according to my experience, is intimately connected with the changes produced in the liver by this toxicosis.

What is the best treatment for phosphorus poisoning? I know of but one reliable remedy: Thorough gastric lavage performed at once. The earlier and the more actively this is carried out the more certain the result; nevertheless, even cases in which enormous amounts, up to 0.7 gram, of phosphorus have been taken may terminate in recovery, as is shown by a case of my own. I also use sulphate of copper, solutions of old oil of turpentine, hydrogen peroxid, and potassium permanganate, and I must admit that these agents produce certain favorable effects, but, as a sovereign remedy in the treatment of poisoning from phosphorus, they do not equal gastric lavage, in which 30 to 40 liters of water may be used, and this should be continued until the fluid no longer has the faintest smell of phosphorus. The chemical proof of phosphorus in the vomited material or in the feces is very easy: Two pieces of filter paper are moistened, one with a solution of nitrate of silver, the other with a solution of lead acetate; both are then dipped in the material to be tested. If the silver nitrate portion, owing to the reduction of salt of silver, shows a black discoloration, this proves that phosphorus is present. When the lead portion becomes black the result is uncertain, for then the reaction may have been brought about by hydrogen sulphid, and this is particularly prone to occur if the feces are examined. If the test is positive, i. e., if the lead portion is not blackened, certain proof is furnished that poisoning by phosphorus is present. I must remark here that a positive result in the test of the feces always renders the prognosis unfavorable, for this reaction shows that the phosphorus has passed through the entire intestine, and so has in all probability been taken up into the organism. In such cases we must endeavor to eliminate the phosphorus from the intestine as rapidly as possible, the best means being by the administration of an infusion of senna. Much that is interesting regarding phosphorus poisoning might still be mentioned, such as the relations between poisoning by phosphorus and acute yellow atrophy of 
the liver and certain forms of mushroom poisoning, but, unfortunately, these are beyond the limits of this article.

Some reference must be made to chronic phosphorus poisoning which is the terror of the workman in the manufacture of phosphorus matches; and presents itself in the form of a phosphorus necrosis of the lower jaw. In Austria, on account of the excellent hygienic measures enforced by the supervision of the Government, this form of the disease has become a rarity.

We now turn to another agent, sULPHUR and its combinations, of which poisoning by sulphureted hydrogen and carbon bisulphid are the most important.

Poisoning with sulphureted hydrogen is rare, but occurs most frequently in chemical laboratories and among workmen in latrines. If the gas is present in great amounts, and the air is, therefore, relatively deficient in oxygen, the affected individual soon shows the severest symptoms, and rapidly perishes. In less severe cases there is debility, weakness and vomiting occur, followed by fibrillary muscular contractions, etc. Generally this form of poisoning is of slight importance. On the other hand, poisoning by carbon bisulphid occurs more frequently, and leads to very interesting changes in the retina which until recently have been but little studied.

We now turn to a very important toxicosis, that of ARSENIC and its combinations.

All soluble combinations of arsenic are poisonous! The symptoms vary according to the amount of the poison ingested, the ready solubility or insolubility of the substance, and the state of the stomach; if taken on a full stomach the phenomena appear less rapidly than on an empty stomach.

In the severest form, asphyxia arsenicalis, symptoms arise which very closely resemble the clinical picture of cholera Asiatica and in a few hours may cause death. I refer to such symptoms as vomiting, diarrhea, rice-water-like stools, and cramps in the calves of the legs; in less severe cases the same symptom-complex is produced-although less rapidly-and the symptoms of toxic enteritis (purulent stools, tenesmus, etc.) are more prominent.

A positive diagnosis can only be made when arsenic is found in the romited material and in the excretions, for which purpose it is necessary to determine the presence of arsenic by positive methods, and to isolate it: If arsenious acid has been employed, an accurate microscopic investigation will occasionally demonstrate that this substance is present: The white particles are extracted with a forceps from the romited material, rinsed with water, and dissolved in hot water; upon cooling, the characteristic small octahedra of arsenious acid are found as crystals.

What must be done in the treatment of this condition? The answer is this: Immediate and thorough lavage of the stomach, administration of calcined magnesium in water, also washing out the stomach with this preparation and the administration of Bunsen's remedy, namely, hydrated oxid of iron; if at hand, lime water may be employed; by frequent and copious enemata, the intestine must be emptied, and the poison thus expelled from the bowel.

Chronic arsenic poisoning is very interesting on account of the extreme cachexia, the severe nervous disturbances such as polyneuritis, etc., and the 
very peculiar cutaneous melanoses to which these conditions lead and which have lately been much studied. The therapy must be strictly adapted to each individual case. It is impossible to enter into its details.

Poisoning by arseniureted hydrogen must be briefly referred to. This is, of late, not rare in chemical laboratories and in chemical factories. The cases run a severe course, but frequently terminate favorably. Jaundice, hematuria, various spasms form the most important symptoms, i. e., the course is entirely different from that of poisoning with the salts of arsenic! The therapy consists only in strict prophylaxis: Avoidance of all chemical processes in open spaces in which arseniureted hydrogen can possibly develop.

We now turn to another form of toxicoses: Poisoning with metallic salts, metallism. From the enormous number of metallic salts which could be mentioned here, I shall only emphasize poisoning with lead and mercury as of practical importance.

Acute poisoning with salts of lead is, upon the whole, of comparatively slight interest, but much more important are the chronic LEAD TOXICOSES.

Regarding the former, a metallic taste, a sensation of dryness and burning in the mouth and throat, and severe colic-like pains are the most important symptoms; later obstinate vomiting occurs, and the vomited material often shows a hemorrhagic discoloration. A diagnosis of acute poisoning with the salt of lead cannot be certainly made from these symptoms, but the presence of lead salts in the vomited material, the history, the corpus delicti, i. e., the investigation of the salt which may be at hand, the fluid, etc., render the diagnosis of acute lead poisoning possible. The immediate administration of milk, of solutions of albumin, of sodium sulphate and magnesium sulphate which reduce lead combinations to an insoluble lead sulphate, is recommended.

It is most important for the physician to recognize chronic lead poisoning, partly because of the frequency of its occurrence, and partly because it manifests itself in such various ways.

Lead poisoning from occupation, as in type-setters, painters, gas-fitters, etc., is usually preceded by symptoms of lead colic: The patients are seized with paroxysms of severe cutting pains in the abdomen, an attack in its greatest intensity rarely lasting longer than a quarter of an hour, and after a shorter or longer interval the severe paroxysms of pain set in anew. The abdomen is retracted, there is constipation, the pulse is slow. The physician must act at once. A lukewarm bath will often relieve the paroxysms of pain in the briefest time; but the patient should not be allowed to suffer, and if this is not effective, morphin subcutaneously should be employed; 0.005 gram, certainly 0.01 gram, hypodermically employed, will in a short time relieve the attack. But the serious symptoms of chronic lead toxicosis can only be averted if the patient, in the future, carefully avoids contact with combinations of lead or working with salts of lead. If he fail to do this, the first attack is succeeded by a second, a third, etc., and the serious tragedy of chronic lead poisoning begins.

Sometimes, besides the pale appearance, the excruciating pains in the joints (arthralgia), paresthesia, various disturbances of sensation torment the patient; in many cases he is spared these symptoms, but weakness in the 
hands occurs, the hands are moved with difficulty, or, finally, can no longer be extended: The unfortunate condition of bilateral radial paralysis develops (wrist-drop). Sometimes the havoc ceases there; no other symptoms appear; but radial paralysis renders the patient incapable of working for a long time, and frequently for life.

The condition becomes more serious if there is evidence of grave cerebral disease; symptoms of encephalitis, epilepsy, mania, briefly, mental disturbances of all kinds, indicate how seriously this poison influences the brain. Because of these disturbances the prognosis is very serious; and unless disease of another organ, consequent upon this toxicosis, particularly the lead kidney, terminates the life of the unfortunate patient, the insane asylum is likely to be his final resort.

The terrible nature of lead poison is thus apparent, and yet I have mentioned but a few signs of this toxicosis. It must be added that metabolism is most unfavorably influenced by lead, in that it leads to an increased formation and excretion of uric acid, and thus produces symptoms which are also observed in arthritis urica (gout).

What remedial agents does therapy furnish with which to combat these conditions, and what treatment must be adopted if the phenomena described have already appeared? Regarding the first point, only a careful prophylaxis is in order. Workmen in lead who have once recovered from lead colic must always change their occupation; the dangers to which they are subjected must be fully explained to them, and they should be induced to seek other means of support which do not bring them into contact with combinations of lead. If, however, the unfortunate symptoms are present, i.e., radial paralysis, we may attempt to improve the condition by the employment of electricity, massage, particularly vibratory massage, also by the administration of preparations of iodin, such as sodium iodid, iodipin (10 per cent. solution), and furthermore, by the use of baths. In the main we must admit that in radial paralysis, encephalitis, epilepsy, etc., due to lead toxicosis, we are almost powerless.

A word regarding the diagnosis. In the characteristic "lead line," the peculiar bluish discloration of the mucous membrane of the mouth in those areas in which the teeth are implanted in the jaws, we have a cardinal symptom of lead toxicosis. It is true this symptom may be explained in many ways, since various factors, for example, tooth powder which contains wood charcoal, chronic mercury poisoning in workmen in iron and iron salts, may produce a symptom which sometimes very closely resembles this. Hence a diagnosis should never be made from the lead line alone. If, however, other symptoms, particularly colic, favor the view that we are dealing with lead toxicosis, the presence of the lead line may be considered to confirm this diagnosis, the more so as the chemical proof of lead in the secretions, for example, in the urine, is frequently negative, particularly in the late stages of the
disease.

In practical importance mercurial poisoning may be placed side by side with saturnine poisoning. Since the soluble combinations of mercury have been utilized as antiseptics in therapy, the number of cases of acute as well as 
chronic poisoning by mercury has been enormously increased, although recently, since the recognition of the danger in this antisepsis, the number has been lessened by a more judicious use of mercury.

All salts of MERCURY are poisonous! It is true the number of poisonings which have been observed, for example, with mercuric sulphate, with mercuric iodid, etc., are exceedingly small in comparison with the number of poisonings with corrosive sublimate, the hydrargyrum bichloratum corrosivum (mercuric chlorid). These should not be confounded with mercurous chlorid, calomel, which, in much smaller doses, also has a toxic effect. I saw a case in which six grams had been taken with suicidal intent, yet it was followed by recovery in a few days. The lethal dose of chlorid of mercury in our population is about 0.2 gram; I say in our population, because the opium smokers in China can bear enormous doses of corrosive sublimate.

In acute toxicosis with mercury, therefore, we shall discuss only the clinical symptoms produced by corrosive sublimate. In the chronic form we shall concern ourselves more or less with all of the salts, and metallic mercury, even the fumes of mercury, will be taken into consideration.

What are the symptoms of poisoning by corrosive sublimate?

If the poison-as in the majority of cases-is introduced through the mouth, widely distributed eschars at once appear upon those areas of the mucous membrane which have come in contact with the poison. Violent vomiting appears; the vomitus is admixed with blood, and later with shreds of mucous membrane. Colicky pains and profuse diarrhea follow, there is suppression of urine or else but a small amount of hemorrhagic urine is voided. Even at this stage a fatal termination may ensue; the subnormal temperature and the small pulse with low tension indicate the approaching end. Usually, however, the sufferings of the patient are decidedly prolonged. The deeply penetrating eschars cause agonizing pain. Ulcerative stomatitis, parotitis, annoying salivation increase the distress. Gastritis and ulcerative enteritis with all of their consequences supervene. The symptoms on the part of the kidney become more intense. The scanty urine shows the characteristics of acute nephritis. After days of misery the patient succumbs to uremic toxicosis.

In all cases of poisoning with corrosive sublimate the prognosis is serious. Even apparently mild cases may develop acute nephritis and terminate fatally.

For certainty in diagnosis the presence of mercury must be proven in the vomited material, in the urine, and perhaps in the feces. In a number of cases the history and the typical symptoms will at once indicate the correct diagnosis.

The therapy consists in immediate and thorough lavage of the stomach with milk and solutions of albumin. Table salt increases the solubility of corrosive sublimate and therefore cannot be employed. Stomatitis is to be treated with solutions of potassium chlorate, the anuria with lukewarm baths and by venesection. The severe pain necessitates the use of opiates.

We now turn to chronic mercurial poisoning. This occurs most commonly among workers in certain industries.

I should like here to emphasize the fact that mercurial treatment care- 
fully conducted and based upon a correct diagnosis has never produced in any patient the symptoms of chronic mercury toxicosis!

The symptoms in the beginning resemble those of lead poisoning: pallor, dyspepsia, the appearance of a black line upon the gums (see p. 588), but the typical colic of lead poisoning is absent. The patient emaciates. Diarrhea, stomatitis and salivation, and various dermatoses, such as erythema, eczema, furunculosis, and ulcers of the skin, appear. Nervous symptoms, such as tremor and polyneuritis, follow. These are the signs of increased psychical irritability (erethismus mercurialis), and finally, severe psychical depression (coma). All this makes the life of the patient miserable, and may shorten it. But a greater danger consists in the fact that the kidney may be attacked, and after years of invalidism the patients often succumb to the symptoms of contracted kidney or of chronic nephritis, and even without nephritic symptoms some patients may succumb to marasmus præcox relatively early in life.

The prognosis in the first stages, in case no nephritic symptoms are present, is not unfavorable; in the later stages, particularly if psychical disturbances have appeared, it is always serious. In treatment, preparations of iodin and bromin (sodium iodid, iodipin, etc.) are most efficacious. Sulphur baths are also used. With intact kidneys, pilocarpin, in the form of subcutaneous injections, may render good service.

We now turn to another chapter: Poisoning with gases.

For the physician only CARBONIC OxID poisoning has acquired great importance. This toxicosis offers a fruitful field, and it is necessary for him to be acquainted with its pathology and therapy.

Illuminating gas, which contains from 7 per cent. to 10 per cent. of carbonic oxid gas, and the fumes of charcoal, which contain about 0.2 per cent. to 0.4 per cent., are the sources of this poisoning, the former by the presence of the gas in rooms where it is in use, the latter by the escape of charcoal fumes. Danger may therefore arise from defective or misused gas fixtures and from badly constructed stoves, and in case of fire.

Of the initial symptoms of the poisoning little is known. Vomiting occurs frequently in this stage, and often, if the patient be unconscious, the entrance of food or vomitus into the trachea may cause death by suffocation. In the stage at which the physician usually arrives, the patient is unconscious, profoundly asphyxiated. The temperature is low, cardiac action weak and irregular, the pulse quickening, and in a few hours death occurs. Elevations of temperature up to $102.2^{\circ} \mathrm{F}$., and nervous irritative symptoms, such as trismus, tonic and clonic spasms, are not rare. Because of the anesthesia coincident with coma, patients may be severely burned during treatment. The sequels which may follow this toxicosis are particularly serious. These are chorea, poliomyelitis, trophic disturbances of all kinds, gangrene, etc. Metabolism is also seriously deranged; alimentary glycosuria is an invariable symptom and spontaneous glycosuria frequently occurs.

The diagnosis is easy on account of the characteristic symptoms. For the detection of carbonic oxid in the blood I advise the examination of blood obtained by venesection. A portion should be diluted with about 20 times as much water, and then boiled with caustic potash. If carbonic oxid be pres- 
ent the solution becomes first turbid, then of a light red color. Normal blood under these circumstances always assumes a dirty brownish hue. The presence of carbonic oxid hemoglobin in the blood, determined by the spectroscope, may also facilitate the diagnosis.

The treatment of this condition may be considered as fairly established. The sovereign remedy is profuse venesection with a subsequent normal saline infusion. This is to be followed by a lukewarm bath with cold affusions. I have rarely found it necessary to proceed to the latter measures, for since I have treated every case of carbonic oxid toxicosis by venesection and normal salt infusion-whether by accident or as an actual result of therapy -in my hospital service I have lost no case of carbonic oxid toxicosis. In private practice, 'however, I had two cases of carbonic oxid poison, illuminating gas, which ran their course with very peculiar nervous symptoms and after a duration of several days terminated fatally. Chronic carbonic oxid poisoning is a result of inhaling for a long time an atmosphere which contains slight amounts of carbonic oxid. Dyspepsia, anemia, jaundice, splenic tumor, and various symptoms of a nervous nature form the interesting pathology of this toxicosis.

We now proceed to the discussion of poisoning by those substances which belong to the fat bodies-the methane derivatives.

The number of these derivatives is legion. Those interesting to the physician are few: namely, poisoning with ethyl alcohol, with sulphonal, trional and tetronal.

ErHYL ALCOHOL is one of the most violent poisons. We may say that there is no organ, no cell of the human organism, which is not subject to deleterious influence from alcohol.

Even a single acute intoxication with alcohol, a debauch, may cause incalculable harm and lead to severe damage of the organism. It may give rise to a long-continued gastric catarrh, to symptoms of polyneuritis and of renal irritation-although thousands and thousands of cases of acute alcohol intoxication do not produce such serious consequences. The symptoms of alcohol intoxication are: At first a stage of exaltation, with a series of nervous symptoms, among which spasms, which are observed particularly in infancy, must be emphasized. This is followed by deep coma, in which the patient succumbs to the toxicosis. The greatest enemy of such patients is cold, and a low external temperature not infrequently causes death.

The treatment of acute alcohol intoxication consists in the immediate washing out of the stomach and the employment of lukewarm baths with cold affusions. Cutaneous irritants, such as sinapisms, and even the subcutaneous use of atropin, may be indicated.

A much more serious affection, and one peculiar to alcoholics, is delirium tremens. This is an acute psychosis which in persons addicted to alcohol frequently appears as an ominous and dangerous complication of acute diseases.

In the treatment of delirium trements chloral hydrate and opium in the form of enemata are beneficial.

The symptoms of chronic alcoholism are of grave import. Primarily, it is the kidney which is attacked, and with the result particularly of contracted 
kidney. The abuse of alcohol at a relatively early age may produce arteriosclerosis also. Cutaneous changes of all kinds-acne, dilatation of the veinsmay be due to chronic alcoholism. The influence upon the liver is particularly prominent and typical; alcoholic cirrhosis with ascites, caput Medusæ, the small, tough degenerated liver, form a clinical picture peculiar to this disease. An entire array of nervous diseases as well, such as polyneuritis, etc., and apoplexy due to arteriosclerosis, threaten the alcoholic, who frequently indeed betrays himself as such by the tremor of the hands.

The proper therapy of chronic alcoholism requires primarily the avoidance of alcohol in any form. In such cases, however, this abstinence usually comes too late, for alcohol has already produced irreparable organic changes. For this reason all our efforts must be directed toward lessening the abuse of alcohol.

The modern movement against the use of alcohol is calculated to produce a change in this direction. It will be long in taking deep root, but its principle is correct. Only by an example of total abstinence may we hope to bring about results. In writings and popular lectures, etc., this standpoint should be defended, and finally it will enable us to combat the dire consequences of chronic alcoholism.

A word regarding SULPHONAL, TRIONAL and TETRONAL. All of these remedies are useful hypnotics, but they are also poisons, and the prolonged, continued use of them will lead to hematoporphyrinuria, a very serious disturbance of metabolism. For this reason the greatest care is necessary in their use, and the continued employment of them should be prohibited.

We shall now devote our attention to cases of poisoning produced by agents of the aromatic group.

The fact that our modern antipyretics, also our modern antineuralgics, belong to this category gives them special importance.

In this brief article, I can emphasize few symptoms, but must confine myself to the discussion of some general phenomena of this poisoning.

Above all, I must reiterate the fact that all of these remedies are bloodpoisons; all have the property of forming methemoglobin in the blood, to which we may probably attribute the cyanosis which intoxication with these remedies produces. Furthermore, the majority of these agents are combined with sulphuric acid or glycuronic acid in the organism, and are excreted from the organism as combinations.

The symptoms of poisoning by NITRO-BENzOL (mirbane oil, artificial bitter oil of almonds) and ANILIN are of unusual interest. There is extreme cyanosis. Loss of consciousness recurs repeatedly in paroxysms-a symptom due to the changes in the blood produced by nitro-benzol toxicosis and peculiar to such poisoning. Although these toxicoses show exceedingly threatening symptoms-for example, pulmonary edema-they may, nevertheless, run a favorable course. Nitro-benzol poisoning and amido-benzol poisoning have lately been observed frequently. They are incident to certain occupations, and it is not too much to expect that since the danger which these industries entail has been recognized proper prophylaxis will in future make such cases of poisoning much more rare. 
Among the bodies of this group greatest interest is attached to PHENOL (hydroxybenzol, carbolic acid), first, because, on account of the extensive medicinal use of the drug, these cases of poisoning have become uncommonly frequent, and secondly, because the course of this poisoning is similar in type to that of poisoning by the hydroxylens of benzol.

Phenol in concentrated solution causes the formation of eschars upon the skin and mucous membrane; even local necroses may be produced or stubborn acute eczemas which may be distributed over the entire body. In spite of this, however, the dangers would be slight if these were the only effects of the poison. But, introduced into the organism in somewhat larger quantities, it gives rise to headache, vertigo, spasms, mydriasis, tinnitus aurium, severe cyanosis and cardiac collapse, to which the patient may succumb in a short time. Even a 2 to 3 per cent. solution injected in large quantity into the pleural cavity, into the rectum, etc., may produce this effect.

The diagnosis of pheno-toxicosis is easy. The characteristic odor of the breath, the very dark, discolored urine, deficient in sulpho-sulphuric acid, will at once identify the case.

In regard to treatment, if the poison has been taken through the mouth, the stomach should be washed at once. This is best done with water and wine vinegar in equal amounts. The comatose stage should be treated with lukewarm baths and cold affusions. Sugar of lime and sodium sulphite should be employed as antidotes.

The best practice is to be cautious in the use of carbolic acid and its preparations for medical purposes, and the sale of unauthorized preparations should be forbidden. Even dilute solutions should never be used for washing out the cavities of the body.

Chronic carbolic toxicosis is an evil to which many physicians were particularly exposed at the time when operations were carried on under the carbolic spray, a method which has now been abandoned. Dermatoses of all varieties, loss of memory, renal affections-briefly, a marasmus præcox-are the distressing symptoms of this toxicosis, which has now almost disappeared. The prognosis is always serious. A number of physicians have succumbed to this poisoning, but it is now rare.

Analogous affections are produced by other derivatives of the phenol group, particularly by the dioxybenzoles. Picric acid (trinitrophenol) causes, as an important symptom, a yellow discoloration of the skin which is not due to biliary coloring matter.

Particular stress must be laid upon poisoning with FREE SALICYLIC ACID, as this has a highly corrosive effect upon the mucous membranes, and thus gives rise to serious gastroenteritis. I emphasize this because, apparently through ignorance of these facts, free salicylic acid is repeatedly advised medicinally, and I desire to caution physicians against its use! The salicylates are also poisons, although local irritative effects are not peculiar to them; taken in very large doses, or by individuals who are very susceptible to the action of salicylic acid, they produce toxic phenomena consisting of transitory amaurosis, difficulty in hearing, hematuria and hemoglobinuria. In contrast with free salicylic acid, however, their effect is relatively slight: 
Before leaving this group of poisons, I must refer to another remedy which, on account of its extensive use in therapy, often gives rise to toxicosis. This drug is ANTIPYRIN. Antipyrin, at one time the favorite and most extensively employed antipyretic and antineuralgic, is also a poison which may cause severe symptoms of intoxication; in particular, dermatoses of all kinds, the hemorrhagic diathesis, and many cases of so-called hemorrhagic scarlatina which are not due to the intensity of the disease, but to the intensity of the therapy employed. There can be no doubt that antipyrin, besides producing exanthematous eruptions, also produces enanthemas of the most varied kinds. In persons who show an idiosyncrasy to antipyrin-and such individuals are not rare-even small doses may produce a variety of eruptions. It is true that no serious phenomena have as yet been noted among the symptoms of antipyrin toxicosis; nevertheless, the therapeutic employment of this remedy necessitates care, all the more so as its continued use may give rise to the symptoms of chronic antipyrin toxicosis. This is as yet but little known, and has been but little studied, the condition running its course with dyspepsia and gastric and intestinal catarrh.

The group of camphors and terpins, ethereal oils, balsams and resins, we shall mention but briefly. In the main but slight importance is to be attached to them. Only Japanese camphor, commonly called simply CAMPHOR, and employed internally as the oil of camphor, is of importance to the physician. There is no doubt that the introduction of large quantities of camphor into the organism causes extreme cerebral irritation, delirium, spasms and convulsions, even albuminuria and severe renal irritation, and for this reason it is not to be indiscriminately used. But also by the subcutaneous employment of the oil of camphor-at least in certain affections, such as enteric feverlocal abscesses may be produced, even with the most cautious asepsis. I do not deny the favorable effect of camphorated oil in cardiac collapse, but the experience of recent years shows that particular care is necessary in the employment of even this oil!

Some remedies which are in common use, such as BALSAM OF COPAIBA and GAMBOGE, belong to this group, and if indiscreetly employed may give rise to toxic symptoms. Toxic nephritis and toxic enteritis may be produced by these agents, and for this reason they are by no means to be looked upon as indifferent substances. They are enumerated here only that I may recommend care in their medicinal employment.

The next group of agents with which we shall concern ourselves is that of the alkaloids. After prussic acid, this group contains the most violent poisons for the human organism, poisons which, even in minimal doses, may. occasion the severest symptoms. Chemically, the alkaloids are basic bodies which contain a pyridin nucleus, and combine with acids. Their action is independent of the nature of the acid, but depends upon the nature of the free base, and, therefore, upon the nature of the alkaloid. All these agents are characterized by the fact that they have only slight local action; they therefore rarely give rise to gastritis and enteritis, but they act intensely, even in small doses, upon the heart and the brain. paralyzing the respiratory center, and in this way rapidly cause death. 
A number of these alkaloids, such as caffein, thein, and nicotin, are contained in substances which are in general use, such as tea leaves after steeping, coffee grounds, and tobacco; therefore, chronic poisoning by these alkaloids is commonly observed. The scope of this article does not permit me to enumerate all of the alkaloids familiar to us, to which additions are made from year to year by the discovery of new alkaloids in the vegetable kingdom. I shall cite the effects of only those alkaloids which are of medical importance. such as nicotin, morphin, atropin, and cocain. The symptoms of all poisonings due to alkaloids is quite similar, and the therapy in the majority of cases is in the main analogous.

NICOTIN is the active alkaloid of tobacco leaves (nicotiana tabacum) and the different products manufactured from them. Poisoning with this exceedingly virulent free base rarely occurs, and, therefore, is of no practical importance. Most significant are those forms of poisoning by tobacco in which, besides nicotin, other deleterious agents, such as the pyridin bases, carbonic oxid and prussic acid, etc., collectively give rise to the conditions known as acute and chronic nicotinism.

We shall first describe acute nicotinism.

I may be brief, for every one who has smoked knows the disagreeable consequences of the first attempt, and has retained the effects in memory. Headache, nausea, vomiting, diarrhea, great debility and weakness are the symptoms with which the unaccustomed organism responds to the poisonous properties of tobacco. Baths with cold affusions and the administration of alcohol will rapidly relieve the symptoms. Tannin and dilute lugol solution may also be employed internally in severe cases. Usually, however, such poisoning runs a favorable course in a few hours, and only a headache, which sometimes continues for days, may remain.

More important than acute nicotin poisoning due to the use of tobacco is chronic nicotin poisoning which presents itself to the physician in a different aspect. Tachycardia, arrhythmia, and cardiac weakness are the principal and common symptoms in this toxicosis, though these symptoms are susceptible of different explanations. How far severe anatomical changes of the heart are related to chronic tobacco toxicosis I shall not attempt to decide; the possibility of such an occurrence cannot be disputed. Other and very important symptoms are disturbances on the part of the eyes; amblyopia, amaurosis, miosis and spasm of accommodation. Weakness of memory and melancholic conditions are also among the signs of chronic nicotinism.

The treatment consists only of prophylaxis, i. e., the moderate use of nicotin. Disturbances of sight are to be relieved by keeping the patient in a darkened room, and by injections of pilocarpin and strychnin.

The next alkaloid to be considered is morphin. OPIDM, the dried juice of the papaver somniferum, contains a number of alkaloids, but the symptoms of poisoning which it produces are all closely akin to those due to morphin, so that we may consider collectively the symptoms which these agents produce.

Morphin and opium are two of the most valuable treasures of our materia medica. By their judicious use, the physician has brought amelioration to countless numbers of sufferers, but no less has their improper use caused in- 
calculable damage. Opium is employed extensively in the Orient and slightly in our country as an intoxicant in the form of smoking. Thus used it causes severe chronic poisoning to which I shall later refer.

We shall first consider the consequences of the introduction into the body of large quantities of opium or morphin. What constitutes a large quantity is always relative: in a child a milligram of morphin may give rise to the severest symptoms, while the morphinomaniac addicted to morphin may take 0.5 gram and more. At first sopor appears. This passes into deep sleep. The temperature of the body falls, the respirations and the pulse become slow and irregular, the pupils are narrowed, the scleræ injected, and in a few hours the adult patient succumbs in deepest coma. With children, as shown by the observations of Eschle and myself, spasms are prominent, and the child succumbs to paralysis of the respiratory center.

If the individual withstands the shock of the first few hours, or if, by proper treatment, the dangerous stage has been fortunately passed after days of coma, consciousness may return. The patient is, however, greatly debilitated; headache, vomiting, stypsis and dyspepsia continue, and frequently in the period of convalescence death occurs suddenly from cardiac paralysis.

The urine frequently shows glycosuria, alimentary as well as spontaneous.

How is acute morphinism, or opium poisoning, to be treated?

First, in all cases, the stomach is to be washed out, even though morphin has been injected subcutaneously, for it is excreted into the stomach.

The sopor must be combated; a warm bath with cold affusions gives the most certain results. If this procedure has no effect, renesection and the inhalation of oxygen should be resorted to. In desperate cases tracheotomy is also to be performed, and oxygen may be introduced through a tracheal cannula. If, as in the case of a child, respiratory disturbances are most prominent, artificial respiration and even phrenic faradization is to be employed. Threatening cardiac collapse is to be met with injections of camphorated oil as well as hypodermics of strychnin.

So much, briefly, in regard to acute morphin poisoning.

A very important rôle is played by CHRONIC MORPHIN TOXICOsIs, which is widely prevalent.

The diagnosis is not always easy, for often the patient makes every effort to mislead the physician. Numerous deeply situated scars on the skin from abscesses of the subcutaneous cellular tissue are suspicious. Dyspepsia, stypsis, also the symptoms of marasmus præcox, increase the likelihood of the diagnosis, but it becomes absolutely certain only when the patient confesses that he uses morphin, or when solutions of morphin are found in his possession.

The treatment of chronic morphinism can be successfully carried out only in a hospital or in sanatoria adapted to this purpose. A withdrawal treatment must be more or less slow, according to the somatic condition of the individual. Hitzig's gastric lavage with Carlsbad water, and the simultaneous subcutaneous injection daily of decreasing quantities of morphin, are advisable. The employment of so-called substitution methods, i. e., the substitution of alcohol, cocain, etc., for morphin, has produced no good results.

If, under this withdrawal treatment, signs of collapse appear, the treatment 
is to be discontinued. Larger doses of morphin must be administered, or, if cardiac weakness intervene, digitalis or subcutancous injections of camphorated oil, etc., are to be employed.

We now turn to ATROPIN POISONING.

The cases of poisoning where atropin has been administered as medicine are very rare: Poisoning by belladonna (atropa belladonna) is more frequent. These cases of poisoning occur usually in children. The characteristic symptoms are dryness of the mouth, burning of the throat, and difficulty of deglutition. There is also extreme psychical alteration. The cardinal symptom, however, is maximal dilatation and absence of reaction of the pupils, accompanied by marked acceleration of the pulse. In cases terminating fatally, deep coma supervenes, then paralyses of all kinds, and the patients succumb in a few hours.

In all such forms of poisoning immediate gastric lavage is indicated, as well as the employment of emetics, and subcutaneous injections of apomorphin.

The best antidote is morphin subcutaneously injected in doses of $0.02-0.03$ gram.

Among the poisons of special interest to the physician is that of COCAIN, an alkaloid produced only within the last two decades of the last century, but which has found employment in medicine to a great extent as a local anesthetic. Intradural injections of cocain have lately been advised, and, unless the greatest care is exercised, these will soon increase the numbers of poisonings from this remedy.

The first symptom of cocain poisoning is local anesthesia of the areas of the body coming in contact with the cocain, a symptom which the physician is frequently desirous of producing. Unfortunately this symptom does not appear alone, but the respiratory and pulse frequency are increased, arrhythmia of cardiac action occurs, the pupils are dilated, there is cyanosis combined with pallor, and the patient succumbs to cardiac collapse. Occasionally such cases are characterized by the appearance of severe mental disturbance prior to the final collapse; not rarely, however, even after these very severe symptoms, final recovery takes place. Treatment: Gastric lavage if there is severe collapse; warm baths with cold affusions.

A disease which has only lately originated from the abuse of cocain is cocainism, which, in its disastrous consequences, is scarcely less important than chronic morphinism; the symptoms are generally referable to the nervous system, and their number is legion; rapidly increasing marasmus causes the early death of the patient.

In the group of the glucocides only one toxicosis plays a leading rôle, and this is poisoning from the leaves of DIGITALIs PURPUREA, which, as is well known, contains two glucocides (digitalin, digitalein), besides digitonin and digitoxin, a soap-like substance.

The curative effects of infusion of digitalis and tincture of digitalis, etc., are known to all physicians--the increased diuresis, the stronger cardiac contraction, the slowing of the pulse. It is perhaps not so well known that infusion of digitalis may become decomposed by the action of mold fungi and bacteria and toxic substances appear in consequence. 
In summer we should make it a rule to ascertain that an infusion of digitalis has not decomposed; but, besides this, digitalis in too large a dose, or in too large a dose in a special case, may cause symptoms undesirable to both the patient and the physician. Among these we may mention vomiting, gastric oppression, marked diminution of the pulse frequency, slight muscular trembling, symptoms of depression, irregular pulse, cold sweats, severe urticarialike exanthems. In the severest cases death occurs in collapse.

Mild cases run a favorable course in a few days without treatment, or by simply stopping the administration of digitalis. The severe and most extreme cases require active treatment. The stomach and intestines must be emptied, caffein and theobromin given, and serpentaria virginica may be employed as an antidote. If the signs of cardiac debility are threatening, strychnin and atropin are to be subcutaneously injected.

I may remark that severe forms of digitalis poisoning are rarely observed by the physician.

Of poisonings with bitters and indifferent agents, only ALOIN toxicosis need be mentioned.

Employed in small doses (0.2-1.0 gram) aloes cause copious diarrheic discharges. Very large doses give rise to gastritis, enteritis, and nephritis. Treatment consists in the administration of opiates and mucilaginous drinks. The best way to prevent poisoning of this kind is for the physician to refrain from prescribing aloin in too large doses.

A word in regard to ARNICAN, the active constituent of the tincture of arnica, from arnica montana. It was formerly medicinally employed, but is no longer used. Its property of markedly irritating the skin, and, upon internal use, of producing stomatitis, gastritis and enteritis, has robbed it of its usefulness, and I only mention it at this place to warn the physician against its employment.

We must now refer to a long series of vegetable and animal poisons.

These are related partially to poisonous albumin bodies, such as toxalbumins, phytalbumoses, etc., as, for example, in some poisonous toad-stools, in snake poison, in spider poison. They are partly related also to bodies previously unknown which, sooner or later, will be assigned to one of the previously described groups.

From the great number of poisonings by these agents I shall briefly mention a few which are of special interest to the physician.

First we shall consider poisoning from ERGOT (secale cornutum), the permanent mycelium of claviceps purpurea. The views of physicians are not yet in accord as to the poisonous principle of this drug. According to Kobert, sphacelinic acid, cornutin, according to Jacobi, sphacelotoxin is the toxic principle. Poisoning, as a rule, simultaneously affects groups of persons who have eaten food prepared with flour contaminated with ergot. The toxicosis may run an acute or a chronic course.

Acute ergot poisoning runs its course with vomiting and abdominal pain, pain in the epigastrium, the chest and extremities. There are various nervous symptoms: above all, paresthesia and tonic contractures of the extremities, as well as ataxia, epileptiform attacks, and severe psychical disturbances. 
Cyanosis, diarrhea and fever may occur. In cases running an unfavorable course, the temperature falls in the later stages, and severe syncope appears. The patient succumbs in a few days, sometimes even after a few hours. The prognosis of ergotin poisoning is always grave, as the sequelæ of such poisoning, even in cases running a favorable course, may be noticeable for years afterward.

For a positive diagnosis of the affection, ergot must be found in the vomited material, in the remains of the flour which has been ingested. For this purpose we use the coloring matter contained in ergot-erythrosclerotin.

Treatment.-As a primary measure the proper instruction of the public should be enjoined. The treatment itself consists in washing out the stomach, emptying the intestine by the employment of enemata or of purgatives such as castor oil and calomel; as antidotes, tannin and chlorin water; for the collapse, warm baths with cold affusions; in milder cases stimulants, such as coffee, tea, alcohol. In severe cardiac collapse injections of camphorated oil are recommended.

The phenomena of chronic ergotism relate particularly to the nervous system. The most marked symptoms are the paresthesias, in consequence of which the disease is still popularly called itching disease (Kribbelkrankheit). First tonic, then clonic, spasms occur (convulsive ergotism); the patient emaciates to an extreme; trophic disturbances of all kinds, such as furunculosis, loss of hair, loss of the nails, etc., follow. The most dangerous form is that known to the physician as gangrenous ergotism. Gangrene of an extremity in a short time leads to septic infection to which the patient succumbs.

The treatment of the intestinal symptoms is the same as in acute ergotism. The severe pains are relieved by sodium salicylate, bromids, and opiates, and protracted lukewarm baths are also beneficial; gangrene necessitates surgical measures, although they are usually of no avail.

Following ergotism, we must consider peltagra (mal de sol) due to the ingestion of spoiled maize. Besides a number of dyspeptic difficulties, an erythema presents itself particularly upon the exposed portions of the body and recurs year after year in summer. The patient emaciates, nutrition suffers greatly, and he becomes anemic. Nervous symptoms follow; pareses, paralyses, atrophies of the musculature, ptosis, amaurosis, etc. The patient improves in winter; but anemia, dropsy, and finally fever and delirium often set in, and after suffering for years he succumbs, provided an intercurrent affection such as tuberculosis or sepsis does not cause death before this.

The prognosis is exceedingly unfavorable, the treatment unfortunately ineffectual, and only by a proper prophylaxis and the avoidance of spoiled maize as food can we prevent the appearance of this dreaded plague. It is endemic in Friaul and Upper Italy as well as in Bukowina.

Poisoning by TOAD-STOOLS is of practical interest. We will consider only the amanita phalloides and the agaricus phalloides. These are often confounded with the field champignons which resemble them. The plum agaricus is the most frequent cause of this form of poisoning.

The symptoms are profuse diarrhea, vomiting, anuria, spasms and collapse, to which the patient may succumb even in the first stage. In the fur- 
ther course of the affection, jaundice and nephritis occur. The disease is very serious. Seventy-five per cent. of those attacked die. Treatment: Gastric lavage, venesection with subsequent salt infusion, and subcutaneous injection of strychnin.

Poisoning with AGARICUS MUSCARIUS must also be considered. The symptoms are vomiting, hemorrhagic diarrhea, a condition resembling alcohol intoxication, followed by tonic and clonic spasms to which the patient may succumb in a few hours or a few days. The prognosis is decidedly more favorable than in poisoning with agaricus phalloides. Treatment: Gastric lavage, emetics, apomorphin, then tannin; perhaps also venesection and normal salt infusion.

In poisoning with HEBELOMA, on account of the muscarin-like effect, injections of atropin are in place.

We must still refer to poisoning with mushrooms. The edible mushroom is non-toxic. All poisonings of this kind are due to the toxic mushroom, which greatly resembles it.

The symptoms are similar to those produced by amanita phalloides. But the course is generally more favorable and briefer. The treatment is the same as in poisoning with amanita phalloides.

It must be borne in mind that non-toxic mushrooms may also give rise to poisoning, if they have decomposed. The best prophylaxis against mushroom poisoning is proper instruction at school in the recognition of the poisonous mushrooms; the inspectors in markets should by strict supervision prevent the sale of the toad-stool which so closely resembles the non-toxic mushroom.

In discussing FisH PoISONING we may be brief. It is of but little consequence. If the fish itself is non-poisonous, poisoning is only brought about by the fact that it was already decomposed when eaten. Such poisoning will be included under meat poisoning.

Fish, however, may be poisonous. For example, eel blood is poisonous, the roe of the barbel and of the pike (esox lucius) contains toxic substances, and this is also said to be true of the head of the sheat fish (silurus glanis). The tetrodon varieties indigenous to Japan contain a poison resembling curare. The ingestion of fish may also have a deleterious effect if the fish itself has been diseased (bacillary infection, plasmodia, invasion of entozoa). The history will for the most part point to the diagnosis. The symptoms are manifold; usually the signs of gastritis toxica dominate the situation, but nervous phenomena such as pupillary symptoms, spasms and unconsciousness also appear. The course of the disease is generally severe, and frequently the termination is fatal.

The treatment consists in gastric lavage, the earlier performed the better; but this should also be employed in the later stages if vomiting is present. Toxic gastroenteritis is to be relieved by opiates and mucilaginous drinks.

OYsTERs and MUSSELS may produce similar effects, if the bivalves were themselves diseased, or had fed on poisonous substances.

In conclusion, we proceed to the discussion of MEAT POISONING.

Although the various forms of meat poisoning differ in their etiology, there is but little difference in their pathology; they may be due to eating 
poisonous animal food or the meat of diseased animals. We shall investigate particularly that form produced by the ingestion of decomposed meat of any kind. This we usually designate as alantiasis or botulism. This form is the most important, as it is the one most frequently observed by the physician.

We must distinguish three quite different clinical forms: (a) That form which presents predominant paralytic symptoms; $(b)$ that form running its course with symptoms of atomatropin poisoning; $(c)$ that form running its course with symptoms of gastroenteritis. The ingestion of decomposed sausage, in particular, causes the second form, while the ingestion of liver and kidney causes the gastroenteritic symptoms.

The affection is always to be regarded as serious; the mortality is very high.

The treatment must be in accordance with the form of the intoxication. In any case, the immediate washing out of the stomach is always in place. The treatment of the second form is the same as that of fish poisoning, while in the third form the treatment of gastroenteritis, repeatedly mentioned, is here of the utmost importance. In conclusion it must be borne in mind that other foods also, such as milk and cheese, may be the cause of similar poisoning, and poisoning after the ingestion of foods containing vanillin may be included in this category. 



\section{DIET TABLE ${ }^{1}$}

Diet List, Showing the Nutritive Value per Ounce of Various Foodstuffs Expressed in Calories, the Weight Required for a "Portion" of 100 Calories Each, and the Number of Calories of Each Food Principle in an Ordinary Serving of Food.

\begin{tabular}{|c|c|c|c|c|c|c|c|c|c|c|}
\hline \multirow{2}{*}{ 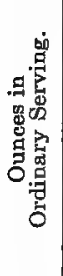 } & \multirow[b]{2}{*}{ Foon. } & \multicolumn{3}{|c|}{ Calories Per Ounce. } & \multirow[b]{2}{*}{ 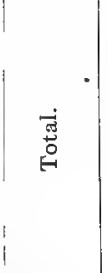 } & \multirow[b]{2}{*}{ 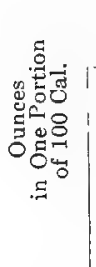 } & \multicolumn{3}{|c|}{ Calortis Strryet. } & \multirow[b]{2}{*}{ 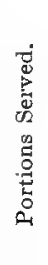 } \\
\hline & & $\begin{array}{l}\text { 离 } \\
\text { 莡 } \\
\text { w }\end{array}$ & 悹 & 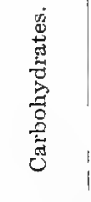 & & & 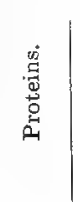 & 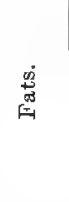 & 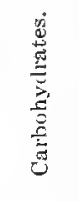 & \\
\hline & Almonds. & 24.5 & 146.4 & 20.2 & 191.1 & .52 & 7 & 38 & 5 & \\
\hline $3 \frac{2}{x}$ & Apples, Bake & 6 & 1.3 & 28.4 & 30.3 & 3.3 & 2 & 4 & 94 & 1 \\
\hline $5 \frac{1}{2}$ & Apples, Fresh & 2.75 & 7.15 & 91.3 & 101.1 & .98 & 2 & $\frac{4}{7}$ & 91 & 1 \\
\hline 6 & Apple Juice. . & & 0 & 17 & 17 & 5.8 & 0 & 0 & 100 & 1 \\
\hline $3 \frac{1}{2}$ & Apple Sauce & .3 & .8 & 20.65 & 21.78 & 4.6 & 1 & 3 & 71 & ${ }_{3}^{3}$ \\
\hline 3 & Apricots. . & 1.3 & 0 & 15.6 & 16.9 & 6 & 4 & 0 & 46 & \\
\hline $1 \frac{3}{4}$ & Asparagus (cooked & 2.5 & 8.8 & 2.6 & $13.9^{\circ}$ & 7.1 & 5 & 15 & 5 & \\
\hline $3 \hat{2}$ & Bananas.... & 1.5 & 1.6 & 25.7 & 28.8 & 3.47 & 5 & 6 & 89 & $1^{4}$ \\
\hline $3 \frac{1}{2}$ & Bananas, Bake & 3.2 & 4.73 & 32.37 & 40.3 & 2.5 & 8 & 11 & 81 & 1 \\
\hline 6 & Barley Gruel. & 1.5 & .7 & 10.2 & 12.4 & 8.1 & 9 & 4 & 62 & 1 \\
\hline $3 \frac{1}{2}$ & Barley, Pearl. & 2.97 & .87 & 27.24 & 31.08 & 3.2 & 9 & 3 & 88 & $1^{4}$ \\
\hline $4 \frac{3}{4}$ & Bean Broth. & 4.3 & 3.2 & 11.6 & 19.1 & 5.2 & 22 & 16 & 62 & 1 \\
\hline $3 \frac{1}{4}$ & Beans, Bakec & 10 & 12.5 & 33.6 & 56 & 1.7 & 32 & 38 & 105 & $1_{4}^{3}$ \\
\hline $3 \frac{1}{2}$ & Beans, Baked (car & 8 & 6.6 & 22.9 & 37.5 & 2.6 & 27 & 22 & 76 & $1 \frac{4}{4}$ \\
\hline 3 & Beet Greens (cooked). & 2.6 & 9.1 & 3.7 & 15.4 & 6.5 & 8 & 30 & 12 & 4 \\
\hline $2 \frac{1}{\ddagger}$ & Beets (cooked). & 2.7 & .3 & 8.6 & 11.6 & 8.6 & 5 & 1 & 19 & \\
\hline $1 \frac{1}{7}$ & Biscuit, Beaten. & 8.4 & 47.3 & 79.2 & 134.9 & 75 & 10 & 61 & 104 & $1 \frac{4}{4}$ \\
\hline $2 \frac{1}{3}$ & Biscuit, Cream & 10.3 & 27.5 & 49.6 & 87.5 & 1.1 & 24 & 64 & 112 & 2 \\
\hline 1 & Biscui & 48.5 & 2.8 & 56.1 & 107.4 & .93 & 45 & 3 & 52 & 1 \\
\hline 1 & Biscuit, Rice. & 8.5 & .9 & 96.6 & 106 & .94 & 7 & 1 & 02 & 1 \\
\hline 3 & Black & 1.5 & 2.6 & 12.7 & 16.8 & 6 & 4 & 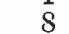 & 38 & \\
\hline $3 \frac{1}{2}$ & Blanc Mange (Chocolate) & 3.4 & 22.86 & 16.25 & 42.52 & 2.35 & 12 & 81 & 57 & $1 \frac{2}{2}$ \\
\hline $5^{2}$ & Blood Oran & 9 & 5 & 13.5 & 14.9 & 6.7 & 4 & . & 69 & 2 \\
\hline $4 \frac{3}{4}$ & Bouillon, Ts & 3.62 & 7.98 & 4.77 & 16.37 & 6.1 & 17 & 36 & 22 & \\
\hline $4_{4}^{\frac{3}{4}}$ & Bouillon, Veget & 7 & 4.3 & 7 & 18.3 & 5.5 & 29 & 18 & 28 & $\frac{3}{4}$ \\
\hline$\frac{1}{2}$ & Brazil Nuts. & 19.8 & 178.1 & 8.2 & 206.1 & .48 & 9 & 87 & 4 & $1^{4}$ \\
\hline 3 & Bread Custard Pudding. & 8.75 & 46.08 & 67.24 & $=122.07$ & .81 & 23 & 133 & 194 & $3 \frac{1}{3}$ \\
\hline 2 & Bread, Corn. & 8.5 & 12.3 & 52 & 72.8 & 1.3 & 17 & 25 & 108 & $1 \frac{1}{2}$ \\
\hline$\overline{2}$ & Bread, Gluter & 10.8 & 3.7 & 58.1 & 72.6 & 1.4 & 18 & 70 & 112 & 2 \\
\hline 2 & Bread, Graham & 10.4 & 4.8 & 60.8 & 76 & 1.3 & 20 & 9 & 121 & $1 \frac{1}{2}$ \\
\hline 2 & Bread, Rye & 10.5 & 1.6 & 62.1 & 74.2 & 1.3 & 21 & 3 & 126 & $1 \frac{1}{2}$ \\
\hline 2 & Bread, White.. & 9.3 & 3.7 & 63.4 & 76.4 & 1.3 & 18 & 7 & 125 & $1 \frac{1}{2}$ \\
\hline 2 & Bread, Whole Wheat. & 11.3 & 2.4 & 58 & 71.7 & 1.4 & 24 & 5 & 121 & $1 \frac{1}{2}$ \\
\hline $4 \frac{3}{4}$ & Broth, Bear & 4.39 & 3.16 & 11.58 & 19.13 & 5.2 & 22 & 16 & 62 & 1 \\
\hline $4 \frac{3}{4}$ & Broth (Mock Chicken). & 3.7 & 3.3 & 4.7 & 11.7 & 16 & 14 & 16 & 20 & 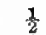 \\
\hline $4 \frac{3}{4}$ & Broth (Vegetable)... & 2.8 & 0 & .5 & 3.3 & 30.3 & 13.3 & 0 & 2.4 & $\frac{6}{6}$ \\
\hline$\frac{1}{2}$ & Butter (Dairy). & 1.2 & 226.6 & 0 & 227.8 & 4 & 1 & 99 & 0 & 1 \\
\hline
\end{tabular}

1 From the diet list of the Battle Creek Sanitarium, Battle Creek, Michigan, J. H. Kellogg. Medical Superintendent. 


\begin{tabular}{|c|c|c|c|c|c|c|c|c|c|c|}
\hline \multirow[b]{2}{*}{ 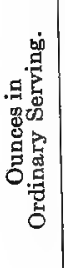 } & \multirow[b]{2}{*}{ FooD. } & \multicolumn{3}{|c|}{ Calories Per Ounce. } & \multirow[b]{2}{*}{ 焉 } & \multirow{2}{*}{ 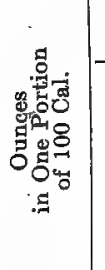 } & \multicolumn{3}{|c|}{ Calortes Sarved. } & \multirow[b]{2}{*}{ 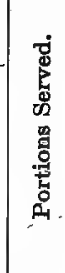 } \\
\hline & & 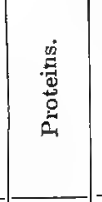 & $\overbrace{=1}^{\infty}$ & 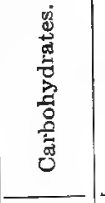 & & & $\begin{array}{l}\text { 总 } \\
\text { 总 } \\
\text { 出 }\end{array}$ & 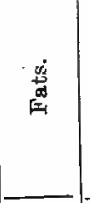 & 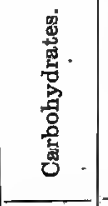 & \\
\hline 6 & Buttermilk & 3.5 & 1.3 & 5.6 & 10.4 & 9.6 & 25 & 10 & 40 & \\
\hline 4 & & .8 & 6.1 & 1.9 & 8.8 & 11.3 & 3 & 19 & 5 & \\
\hline 2 & Cake, Coffe & 7.6 & 40.9 & 66.8 & 114.6 & 87 & 15 & 80 & 130 & 0 \\
\hline $1 \frac{1}{2}$ & Cake, Spon & 12.4 & 14.2 & 94.2 & 120.8 & .8 & 20 & 23 & 157 & \\
\hline $6 \frac{3}{8}$ & Can & $\begin{array}{l}.7 \\
.12\end{array}$ & & 9 & 11.6 & 8.6 & 5 & & 70 & \\
\hline $3 \frac{3}{3}$ & & 2.01 & 7.5 & 10.3 & 19 & 5.05 & 7 & 29 & 39 & \\
\hline 3 & Cauliflower, S & & 6.2 & .3 & 10.2 & 9.8 & 3 & 15 & 7 & \\
\hline 1 & & 1.3 & .3 & 3.9 & 5 & 18.1 & 1 & & 5 & \\
\hline 2 & $\mathrm{Co}$ & 19.9 & 12.4 & 5.1 & 37. & 2.6 & 40 & 25 & 10 & \\
\hline 21 & $\mathrm{Ch}$ & 1.2 & 2.2 & 19.5 & 22 & 4.4 & 3 & 5 & 42 & \\
\hline $2^{2}$ & $\mathrm{Coc}$ & 6.6 & 134.9 & 32.5 & 174 & .6 & 132 & $269.8 \mid$ & 65 & 3 \\
\hline 3 & Cor & 13.9 & 28.8 & 52.8 & 95. & 1 & 42 & 90 & 168 & \\
\hline$\frac{3}{4}$ & Corn Flakes. & 10.8 & 1.4 & 91.3 & 103.5 & .97 & 7 & 1 & 67 & \\
\hline $2 \frac{3}{4}$ & $\begin{array}{l}\text { Corn, Green, Sweet } \\
\text { (cooked)............... }\end{array}$ & 3.6 & 2.9 & 22 & 28.5 & 3.5 & 10 & 7 & 58 & \\
\hline 1 & Crackers, Graham. & 11.7 & 25.1 & 86.1 & 122.9 & .8 & 12 & 25 & 88 & 1 \\
\hline 3 & rries (cooked). & .2 & .7 & 47.8 & 48.7 & 2.1 & 1 & 2 & 147 & 1 \\
\hline 21 & Cre & 2.9 & 49.3 & 5.3 & 57.5 & 1.7 & 6 & 107 & 12 & \\
\hline 2 & $\mathrm{Cu}$ & .9 & .5 & 3.6 & 5 & 20 & 2 & 1 & 7 & \\
\hline 3 & $\mathrm{Cu}$ & 1.8 & & 14.9 & 16.7 & 6 & 5 & 0 & 45 & \\
\hline 31 & Custard, Plain.. & 5.7 & 13 & 12.5 & 31.2 & 3 & 18 & 42 & 40 & 1 \\
\hline 3 & $\begin{array}{r}\text { Dandelion } \\
\text { (cooked)............. }\end{array}$ & 2,8 & 2.7 & 12.5 & 18 & 5.4 & 8 & 8 & 34 & \\
\hline $1 \frac{3}{4}$ & Date & 2.5 & 7.5 & 91.5 & 101.5 & 1 & 3 & 12 & $\begin{array}{r}04 \\
160\end{array}$ & 1 \\
\hline $1 \frac{1}{2}$ & $\mathrm{Eg}$ & 6.63 & 26.43 & 37.9 & 71 & 1.4 & 8 & 38 & 54 & 1 \\
\hline $1 \frac{1}{4}$ & $\mathrm{Eg}$ & 16.3 & 32 & & 48.3 & 2.1 & 26 & 42 & & \\
\hline 8 & $\mathrm{Eg}$ & 5.9 & 23 & 9 & 38 & 2.6 & 46 & 183 & 71 & \\
\hline 3 & & 3.3 & 1 & 4.93 & 9.3 & 10.7 & 9 & 3 & 13 & \\
\hline$\frac{1}{2}$ & Waln & 19.4 & 169.2 & 18.2 & 206.8 & .48 & 9 & 82 & 9 & \\
\hline $3 \frac{1}{2}$ & Farina..... & 2.6 & .8 & 18 & 21.4 & 4.7 & 9.1 & 2.8 & 63 & \\
\hline 2 & & .5 & 8 & 86.6 & 92.4 & 1.1 & 1 & 16 & $\mid 173.2$ & $\begin{array}{l}2 \\
1\end{array}$ \\
\hline$\frac{1}{2}$ & Fi & 18.2 & 174.1 & 15.2 & 207.5 & .48 & $\begin{array}{l}9 \\
0\end{array}$ & 84 & 7 & \\
\hline $1^{\frac{2}{3}}$ & Fr & $\begin{array}{c}0 \\
48.5\end{array}$ & $\begin{array}{r}198 \\
2.8\end{array}$ & $\begin{array}{r}2.85 \\
56.1\end{array}$ & $\begin{array}{l}200.9 \\
107.4\end{array}$ & $\begin{array}{l}.5 \\
.9\end{array}$ & $\begin{array}{r}0 \\
45\end{array}$ & $\begin{array}{r}99 \\
3\end{array}$ & $\begin{array}{r}1 \\
52\end{array}$ & \\
\hline 2. & Go & & & 18.9 & 19 & 5.15 & 1 & & 49 & \\
\hline $3 \overline{3}$ & & .9 & 5 & 11 & 13 & 7.6 & 3 & 2 & 45 & \\
\hline 6 & & 0 & 0 & 23 & & 4.2 & 0 & 0 & 150 & \\
\hline 5 & & 1.16 & 3.2 & 16. & 20 . & 4.7 & 5 & 16 & 79 & \\
\hline 6 & $\mathrm{Gr}$ & 1.48 & .7 & 10 & 12.39 & 8.07 & 9 & 4 & 6 & \\
\hline 61 & $\mathrm{Gr}$ & .8 & 4 & 6. & 8 & 12.5 & 5 & 3 & 42 & \\
\hline 6 & Gruel, Oatn & 1.3 & 1.4 & 5.8 & 8.5 & 11.7 & 7 & 8 & 35 & \\
\hline $3 !$ & $\begin{array}{l}\text { Hash, Potatoes, and } \\
\text { Onions... }\end{array}$ & 2.35 & 10.79 & 18 & 30 . & & 7 & 36 & & \\
\hline $4 !$ & $\mathrm{Ho}$ & 2.6 & .5 & 20.7 & 23 & 4. & 11 & $\begin{array}{r}36 \\
2\end{array}$ & 87 & \\
\hline $1 \frac{5}{3}$ & & & 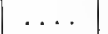 & 94.7 & 95.2 & 1.05 & 1 & & 149 & $1 \frac{1}{2}$ \\
\hline 1 & Jel & 1.2 & & 90.1 & 91.3 & 1.09 & 1 & & 99 & \\
\hline 5 & & 3.3 & 5.6 & 6.3 & 15.2 & 6.6 & 16.5 & 28 & 31.5 & \\
\hline 5 & & & & 14.2 & 14.2 & 7 & & & 75 & \\
\hline 3 & Le & 8.5 & .0 & & 31.7 & 3. & 25.5 & & 68.7 & \\
\hline 3 & Maca & 5 & 3.86 & 17.16 & 26 & 3. & 15 & 11 & 49 & \\
\hline 23 & & 10.8 & 15.88 & 17.85 & 44. & 2. & 30 & 45 & 50 & \\
\hline 1 & $\mathrm{Ma}$ & 0 & 0 & 96.6 & 96. & 1.1 & 0 & 0 & 100 & $l$ \\
\hline $2 \frac{1}{2}$ & & 2.8 & & 48. & 51 & 1. & 6 & 1 . & 118 & \\
\hline $1 \frac{1}{4}$ & Mayonnaise, Cooked.. & 6.87 & 67.1 & 2.85 & 76.8 & 1.3 & 9 & 88 & 3 & \\
\hline
\end{tabular}


DIET TABLE-Continued

\begin{tabular}{|c|c|c|c|c|c|c|c|c|c|c|}
\hline \multirow{2}{*}{ 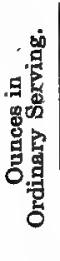 } & \multirow[b]{2}{*}{ FOOD. } & \multicolumn{3}{|c|}{ Carortes Per Ounce. } & \multirow[b]{2}{*}{ 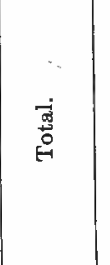 } & \multirow[b]{2}{*}{ 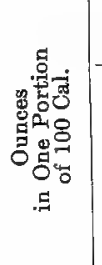 } & \multicolumn{3}{|c|}{ Calories Senved. } & \\
\hline & & 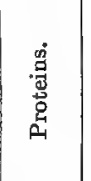 & 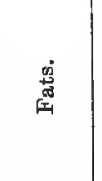 & 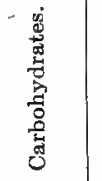 & & & 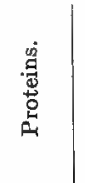 & 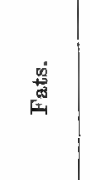 & 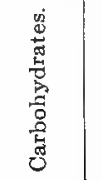 & \\
\hline $6 \frac{1}{2}$ & Milk, Skimmed & 4 & .8 & 6 & 10.8 & 9.3 & 26 & 5.2 & 39 & \\
\hline 6 & Milk, & 3.8 & 11 & 5.8 & 20.6 & 4.9 & 22.8 & & 34.8 & \\
\hline$\frac{1}{4}$ & Nuts, Almo & 24.5 & 146.4 & 20.21 & 191.1 & .52 & 7 & 38 & 5 & \\
\hline & Nuts, Hicko & 18 & 179.7 & 13.3 & 211 & & & 89.8 & 6.6 & \\
\hline 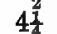 & Oatmeal (Cooked). & 3.3 & 1.3 & 13.4 & 18 & 5.6 & 14 & 5 & 56 & \\
\hline & Olive Oil.. & 0 & 264.1 & 0 & 264.1 & .4 & 0 & 100 & 0 & \\
\hline $1 \frac{3}{3}$ & Olives, Ripe & 2 & 69.1 & 5 & 76.1 & 1.3 & 2 & 91 & 7 & \\
\hline 4 & Ome & 14 & 59 & 1 & 74 & 1.4 & 56 & 236 & & \\
\hline $2 \frac{1}{2}$ & Boile & 1.13 & 4.29 & 5.1 & 10.52 & 9.5 & 3 & 10 & 12 & \\
\hline 5 & Oran & & 0 & 15.1 & 15.1 & 6.6 & 0 & 0 & 75 & \\
\hline 5 & Oras & .9 & .5 & 13.5 & 14. & 6.7 & 4 & 2 & 69 & \\
\hline 3 & Parsi & 1.7 & 5.9 & 9.5 & 17. & 5.8 & 5 & 17 & & \\
\hline $2 \frac{1}{2}$ & Patti & 14.9 & 39.04 & 77.24 & 131.2 & .76 & 37 & 97 & 191 & 3 \\
\hline 4 & Peaches, Fre & .9 & .3 & 11.6 & 12.8 & 7.8 & 3 & 1 & 46 & \\
\hline$\frac{3}{4}$ & Pear & 30.1 & 102.9 & 8.5 & 161.5 & .6 & 22.5 & 77.1 & 6.3 & \\
\hline $4^{4}$ & $\mathrm{Pe}$ & .7 & 1.3 & 16.5 & 18.5 & 5.4 & 3 & 5 & 67 & \\
\hline 3 & Peas & 7.8 & 9.1 & 17.5 & 34. & 3 & 23 & 26 & 51 & \\
\hline $4 \frac{1}{2}$ & Pie, Apple & 7.5 & 18 & 37.2 & 62 . & 1.59 & 33 & 80 & 162 & \\
\hline $5^{2}$ & Pie, & 15.09 & 22.2 & 35.1 & 72.39 & 1.3 & 73 & 108 & 169 & \\
\hline 5 & Pie, Pumpki & 3.25 & 7.26 & 24.88 & 35. & 2.8 & 36 & 71 & 16 & \\
\hline 4 & Pineapple, Fresh. & .5 & .8 & 11.3 & 12.6 & 8 & 2 & 3 & 45 & \\
\hline 4 & Plur & 1.2 & & 23.5 & 24.7 & 4 & 5 & & 95 & \\
\hline$\frac{1}{2}$ & Pop & 12.5 & 13.3 & 91.8 & 117.6 & .85 & 5 & 5 & 40 & \\
\hline 3 & Pot: & 3.4 & 4 & 28.9 & 32. & 3 & 11 & 1 & 88 & \\
\hline $3 \frac{3}{4}$ & Pru & & & 26. & 27 & 3.65 & 3 & 1 & 96 & \\
\hline $2 \frac{1}{2}$ & Puc & 4.8 & 21.8 & 20 & 46 & 2.1 & 13 & 58 & 5 & \\
\hline $2 \frac{1}{2}$ & Pudding, Apple Tapioca & 4. & 17.58 & 26.44 & 48 . & 2.07 & 12 & 45 & 68 & \\
\hline 1 & Radishes. . & 1. & .3 & 6.7 & 8. & 11.7 & 1 & & 5 & \\
\hline 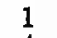 & Raisi & 3 & 8.8 & 88.8 & 100.6 & 1 & 3 & 9 & 88 & \\
\hline 4 & $\begin{array}{r}\text { Raspberries, } \quad \text { Fres h } \\
\text { Black................ }\end{array}$ & 2 & 2.6 & 14 & 19 & .2 & 8 & 10 & 57 & \\
\hline $3 \frac{1}{6}$ & Raspberries, Fresh Red. & 1.2 & -10 & 14.7 & 15 & 6.3 & 4 & & 46 & \\
\hline 4 & Rice, Boile & 3.3 & .3 & 28.5 & 32 & 3. & 13 & 1 & 111 & \\
\hline $1 \frac{3}{4}$ & $\mathrm{Sa}$ & .7 & 51.7 & 2.3 & 51. & 1. & 2 & 93 & 5 & \\
\hline $2 \frac{1}{2}$ & Sala & 4. & 4.8 & 9.2 & 18 & 5. & 6 & 6 & 13 & \\
\hline $4 \sqrt{3}$ & Sou & 9. & 4 & 24.7 & 38.1 & 2 . & 25 & 15 & 65 & \\
\hline & Com & 3. & 7 & 8.9 & 19 & 5. & 17 & 36 & 47 & \\
\hline$A^{\frac{2}{3}}$ & So & 7.1 & 1.85 & 18.07 & 27.1 & 3. & 34 & 8 & 83 & \\
\hline & Sp & 3 . & 1 & 4.93 & 9.3 & 10.7 & 9 & 3 & 1 & \\
\hline $2 \frac{1}{2}$ & $\mathbf{S e}_{\mathrm{c}}$ & 1 . & 2.2 & 16 & 19.8 & 5 & 4 & 6 & 40 & \\
\hline & & 1. & 1.6 & 8. & 11.4 & 8.5 & 5 & 7 & 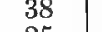 & \\
\hline & $\mathrm{Su}$ & 0 & 0 & 116.6 & 116.6 & .9 & 0 & 0 & 25 & \\
\hline $3 \frac{1}{2}$ & Sweet Pots & 3. & 5.6 & 49 & 58.2 & 1.7 & 12 & 19 & 169 & \\
\hline$\frac{2}{2}$ & Toast, Bre & 11.4 & 27.5 & 86.2 & 125.1 & 8 & 4 & 12 & 4 & 21 \\
\hline $5^{2}$ & Toa & 4.15 & 29.9 & 13.6 & 47.65 & 2.1 & 18 & 143 & 64 & \\
\hline 4 & Tor & 1 & 1.1 & 4. & 6. & 16 & 4 & 4 & 17 & \\
\hline 4 & Tur & .0 & 2.8 & 2 . & 6. & 16.4 & 2 & 12 & 11 & \\
\hline $1+$ & $\overrightarrow{W a}$ & 1.4 & .8 & 3.4 & 5 . & 17. & 2 & 1 & 7 & \\
\hline 8 & Wa & & .5 & 7.8 & 8. & 11.4 & 4 & 4 & & \\
\hline $5 \frac{3}{4}$ & Wheat, & 3.3 & 1 & 22 & 26 . & 3.8 & 18 & 5 & & \\
\hline & Whey & 1.2 & .8 & 5.8 & 7.8 & 12.8 & 0. & & & \\
\hline & Whole-wheat Wafers or & 11.4 & 26 & 84.5 & 122.7 & 81 & 12 & 27 & 86 & \\
\hline 1 & Zwieback & 11.4 & 26.4 & 85.8 & 123.6 & 8 & 11.4 & 26.4 & 85.8 & \\
\hline
\end{tabular}


FLESH FOODS.

\begin{tabular}{|c|c|c|c|c|c|c|c|c|c|c|}
\hline \multirow{2}{*}{ 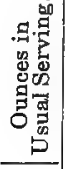 } & \multirow[b]{2}{*}{ Foon. } & \multicolumn{4}{|c|}{ CALORIES PER OUNCE. } & \multirow{2}{*}{ 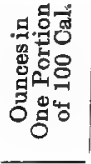 } & \multicolumn{4}{|c|}{ CALORIES SERVED. } \\
\hline & & 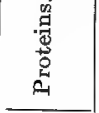 & 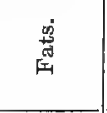 & 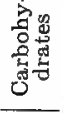 & 㺃 & & 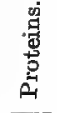 & 离 & 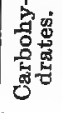 & 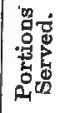 \\
\hline $3 \frac{1}{2}$ & Beef Juice & 5.42 & 1.71 & 0 & 7.13 & 14 & 19 & 6 & 0 & \\
\hline $2 \frac{1}{2}$ & Beef, Roasted (Fat) & 18.14 & 136.85 & 0 & 155.26 & 65 & 48 & 352 & 0 & 4 \\
\hline $2 \frac{1}{4}$ & Beef, Round (Boiled, Lean). & 40.9 & 4.54 & 0 & 45.6 & 2.2 & 90 & 10 & 0 & 1 \\
\hline 6 & Bouillon....... & 2.3 & .3 & 3 & & 33 & 23 & 1 & 1 & $\frac{1}{4}$ \\
\hline 31 & Chicken (Broilers & 24.6 & 6.56 & 0 & 31,16 & 3.2 & 79 & 21 & 0 & 1 \\
\hline $3 \frac{3}{4}$ & Clams..... & 7.5 & 1.08 & 0 & 8.58 & 11.6 & 28 & 4 & 18 & $\frac{1}{2}$ \\
\hline 5 & Cod Fish & 19.3 & 1.02 & 0 & 20.32 & 4.9 & 95 & 5 & 0 & 1 \\
\hline $2 \frac{3}{4}$ & Goos & 18.1 & 95.4 & 0 & 113.5 & .88 & 48 & 252 & 0 & 3 \\
\hline 3 & Halibut & 21.78 & 13.9 & 0 & 35.68 & 2.8 & 61 & 39 & 0 & 1 ' \\
\hline 2 & Lamb Chops (Boilec & 25.3 & 79.7 & 0 & 105 & .96 & 48 & 152 & 0 & 2 \\
\hline $3 \frac{1}{2}$ & Lamb (Leg, Roast) & 22.2 & 33.3 & 0 & 55.5 & 1.8 & 80 & 120 & 0 & 2 \\
\hline 3 & Liver (Veal). & 21.78 & 13.9 & 0 & 35.68 & 2.8 & 61 & 39 & 0 & 1 \\
\hline 2 & Lobst & 19 & 4.8 & 0 & 23.82 & 4.1 & 39 & 10 & 1 & $\frac{1}{2}$ \\
\hline $2 \frac{1}{2}$ & Mutton (Leg, Boiled). & 29.1 & 54.1 & 0 & 83.2 & 1.2 & 70 & 30 & 0 & 1 \\
\hline 3 & Oysters & 7.2 & 3.23 & 0 & 10.43 & 6.8 & 24 & 12 & 14 & $\frac{1}{2}$ \\
\hline 1 & Pork (Bacon, Medium Fat) & 11.3 & 177.3 & 0 & 188.6 & .53 & 12 & 188 & 0 & 2 \\
\hline $2 \frac{1}{4}$ & Pork (Ham, Boiled). & 25.4 & 65.4 & 0 & 90.3 & 1.1 & 56 & 144 & 0 & 2 \\
\hline 3 & Pork (Loin, Chops) & 18.5 & 84.5 & 0 & 103 & .97 & 54 & 246 & 0 & 3 \\
\hline $2 \frac{1}{1}$ & Salmon (California) & 20.4 & 46.6 & 0 & 66.6 & 1.5 & 45 & 105 & 0 & 13 \\
\hline 2 & Shad...... & 21.9 & 25.71 & 0 & 47.61 & 2.1 & 46 & 54 & 0 & 1 \\
\hline & Trout (Broo & 22.2 & 55.5 & 0 & 77.7 & 3.6 & 40 & 10 & 0 & $\frac{1}{2}$ \\
\hline $1 \frac{1}{4}$ & Turkey.. & 24.1 & 59.1 & 0 & 83.2 & 1.2 & 29 & 71 & 0 & 1 \\
\hline $2 \frac{1}{2}$ & Veal (Leg, Boil & 30.4 & 11.2 & 0 & 41.6 & 2.4 & 73 & 27 & 0 & 7 \\
\hline
\end{tabular}

FOOD UNITS OR CALORIES PER OUNCE OF UNCOOKED FOODSTUFFS.

None served in the uncooked state. Value given only for the purpose of comparison.

\begin{tabular}{|c|c|c|c|c|c|c|c|c|c|}
\hline FOOD & $\begin{array}{l}\text { Pro- } \\
\text { teins. }\end{array}$ & Fats. & $\begin{array}{c}\text { Car- } \\
\text { bohy- } \\
\text { drates. }\end{array}$ & Total. & Focd. & $\begin{array}{l}\text { Pro- } \\
\text { teins. }\end{array}$ & Fats. & $\begin{array}{c}\text { Car- } \\
\text { bohy- } \\
\text { drates. }\end{array}$ & Total. \\
\hline Asparagus. & 2.1 & .5 & 3.9 & 6.5 & Gluten Meal (20 & & & & \\
\hline Barley, Pearled & 9.9 & 2.9 & 90.8 & 103.6 & per cent.)..... & 18.4 & 1.7 & 83.6 & 103.7 \\
\hline $\begin{array}{l}\text { Beans (dried)..... } \\
\text { Beans, Butter }\end{array}$ & 26.3 & 4.8 & 69.5 & 100.6 & Hominy... & 9.7 & 1.6 & 92.2 & 103.5 \\
\hline (green)...... & 11 & 1.6 & 34 & 46.6 & Macaroni. & $\begin{array}{r}30 \\
3\end{array}$ & $\begin{array}{l}2 \\
4\end{array}$ & $\begin{array}{ll}69.1 \\
18.4\end{array}$ & 101.8 \\
\hline Beans, Lima (dried) & 21.1 & 4 & 76.9 & 102 & Mushrooms. & 4.1 & $\begin{array}{l}4 \\
1.1\end{array}$ & $\begin{array}{r}18.4 \\
7.9\end{array}$ & $\begin{array}{l}2 b .9 \\
13.1\end{array}$ \\
\hline Beets. . . . . . . . . & 1.9 & .3 & 11.3 & 13.5 & Oatmeal. & 18.8 & 19.2 & 78.8 & 116.8 \\
\hline & 2.7 & .8 & 8.6 & 12.1 & Onions. & 1.9 & .8 & 11.6 & 14.3 \\
\hline $\begin{array}{l}\text { Cabbage......... } \\
\text { Carrots... }\end{array}$ & 1.9 & 8 & 6.5 & 9.2 & Parsnips & 1.9 & 1.3 & 15.8 & 19 \\
\hline $\begin{array}{l}\text { Carrots........... } \\
\text { Cauliflower........ }\end{array}$ & $\begin{array}{l}1.3 \\
2.1\end{array}$ & 1.1 & 10.9 & 13.3 & Peanuts & 22.8 & 77.6 & 21.6 & 122 \\
\hline $\begin{array}{l}\text { Caulhtower........ } \\
\text { Cocoa........... }\end{array}$ & 25.2 & & 5.5 & 8.9 & ied). & 28.7 & 2.7 & 72.3 & 100.7 \\
\hline Corn, Green. . & 3.6 & $\begin{array}{r}7.1 \\
2.9\end{array}$ & $\begin{array}{l}44 \\
23\end{array}$ & 146.3 & Peas, Green. & 8.2 & 1.3 & 19 & 29.2 \\
\hline Cornmeal. & 10.7 & 5.1 & $\begin{array}{l}23 \\
87.9\end{array}$ & $\begin{array}{r}29.5 \\
1037\end{array}$ & Potatoes.. & 2.6 & & 21.5 & 24. \\
\hline Cor & & & $105^{\circ}$ & $\begin{array}{l}103.7 \\
105\end{array}$ & Prunes. & 2.5 & $\mathbf{0}$ & 85.8 & 88 \\
\hline Cranberries. & .6 & 1.6 & 11.5 & $\begin{array}{r}100.7 \\
13.7\end{array}$ & Rumpk & 1.2 & .3 & 6.1 & 7.6 \\
\hline Egg Plant. & 1.4 & .8 & 6 & 8.2 & $\mathrm{R}$ & 9 & .3 & $\begin{array}{l}0.8 \\
92\end{array}$ & $\begin{array}{r}8.0 \\
1018\end{array}$ \\
\hline Flour, Corn. . & 8.3 & 3.5 & 91.5 & 103.3 & Spinach. & 2.5 & .8 & 3.7 & $\frac{10}{7} \cdot 0$ \\
\hline Flour, Graham. . & 15.5 & 5.9 & 83.3 & 104.7 & Sque & 1.6 & 1.3 & 10.5 & 13.4 \\
\hline $\begin{array}{l}\text { Flour, Rye........ } \\
\text { Flour, Whegt }\left(\text { Sn }^{2}\right.\end{array}$ & 7.9 & 24 & 91.8 & 102.1 & Sweet Potatoes. & 2.1 & 1.9 & 32 & 36 \\
\hline $\begin{array}{l}\text { Four, wheat (En- } \\
\text { tire Wheat).... }\end{array}$ & 16.1 & 5.1 & 83.8 & 105 & $\begin{array}{l}\text { Turnips ...... } \\
\text { Vegetable Oys- }\end{array}$ & 1.5 & & 9.5 & \\
\hline Flour, Wheat & & & & & ters or Salsify. & 1 & 5.3 & 4.1 & 10.4 \\
\hline Flour, Wheat (Pat.) & $\begin{array}{r}9.2 \\
12.6\end{array}$ & $\begin{array}{l}3.7 \\
2.9\end{array}$ & $\begin{array}{l}89.1 \\
87.7\end{array}$ & $\begin{array}{l}102 \\
103.2\end{array}$ & Wheat, Cracked. & 13 & 4.5 & 88.1 & 105.6 \\
\hline
\end{tabular}




\section{INDEX OF AUTHORS}

A

A Bet, 210.

Abelous, 205, 206.

Adpison, 199, 200, 202, 205, 214.

Adrian, C., 253, 259.

AJELLO, 459, 494.

Albarran, 449, 513.

ALBU, 14, 40.

ALexander, 209.

ALLEN, 513.

Ammann, E., 419.

AMmoN, 514.

ANDERson, 442.

ANdersson, 225.

ANGER, 518, 519.

ANschütz, W., 246, 259.

Arctiander, 482.

ARnold, 235, 237, 327, 347.

Arstan, 563.

Askanazy, 347, 376, 386, 512, 545, 553, 554, $557,558$.

Atrlee, 451.

Atwater, 20, 24.

AULd, 204.

AVERBECK, 214.

\section{B}

BABCOCK, 195.

BABES, 216, 394, 400, 429, 460.

BACCELi, 533.

BaChSTROM, 397.

BAELZ, 506, 510, 511, 549.

BaLSCH, 517.

BAMBERGER, 468, 476.

BANCROFT, 546, 547.

BANG, 266, 267.

BanNatyNe, 243, 255, 259.
BANTI, 382, 383, 384, 386.

BANTING, 70, 72, 73, 74, 163, 164, 165, 166, $173,175$.

Baraban, 506.

BARJON, 257, 259.

BARLOW, 485.

BARSZEWSKY, 271.

Batemann, 459.

BATSCH, 516.

BaUter, 39.

Baumann, 2, 189, 190, 191.

BÄUMLER, 243, 247.

BavaY, 544, 545.

BAZIN, 143.

Bechterew, 246, 247, 259.

BECKL.er, 397.

BeCQUerel, 334, 341, 408.

BeER, 248, 259.

BEHLA, 506.

Benda, C., 229.

van Beneden, 526.

Benedict, 20, 24.

BenNecke, R., 247.

BEOWIT, 543.

Berent, W, 256, 260.

Bergell, 18, 266.

Bernard, Claude, 111, 114, 115, 116.

Bettencourt, 189.

Bial, 263, 267, 269, 270, 271.

BidDer, 4, 10.

BIEDERT, 47.

BIEDL, 208.

Bier, August, 258, 259, 260.

Biermer, 509.

BilHARz, 512, 560.

Bilimngs, 495, 556.

BiLlRoth, 378, 488.

BiNeT, 30.

Birch-Hirsch FELd, 433. 
Bircher, 188.

Bischoff, E., 4, 56.

BitTer, 542.

BjierRe, 89.

BLANChARD, 515, 516, 521, 544, 564.

Bland-SutTon, 506.

Blascher, 535.

Blaxall, 255, 259.

Bleibtret, 56.

Bloch, 516, 519.

BLUM, 189, 209, 226.

Blumenthal, F., 262, 269.

BoBrizKI, 469.

Boer, 219.

BoerhaAve, 162.

BoHLANd, 563.

Bollinger, 415.

BonfiLs, 370, 386.

BoNTEKoe, 391.

Bornstein, 19, 20, 56, 57.

BORNTRÄGER, 394.

Bostroem, 509.

Bouchard, Сh., 22, 26, 33, 143, 243, 252, $255,257,260$.

Boursier, 449.

Bozzolo, 560, 564.

Bramwell, Byrom, 180, 185, 195.

Brandenburg, 286

Brat, 270.

BraUer, 573.

Braun, G., 511, 512, 515, 520, 575, 576.

Braun, Julius, 246, 260.

Brehmer, 63.

Breisacher, 14.

Breithaupt, 14.

Bremser, 520, 565 .

Brieger, 531.

Brillat-Savarin, 170.

Brock, 513, 514.

Broden, 25.

BROOCK, 454.

Brown, 303.

Brown-SÉquard, 205, 206.

BRUCE, 195.

BRUNNER, 534.

v. Bruns, 192, 193, 534.

BRYANT, 531.

BUHL, 433.

Bunge, 153, 154, 155, 398, 410.

BUNSEN, 586.

BURChaRd, 553.

BURMeister, 572.
BURRESI, 216.

Busk, 510.

\section{C}

САвот, 194, 195, 283, 366.

CAMerer, 36, 37.

Canard, 286.

Cannstatt, 470.

Cantani, 98, 164, 165.

Caporelli, 271.

CASParI, $18,20,38,51$.

CASPER, L., 451.

Cassirer, R., 248, 260.

Castan, 450.

du CAstel, 463.

CECI, 460.

Celusus, 175, 389.

Chalvet, 408.

Chambers, Thomas K., 163, 165.

Charcot, $140,179,243,244,245,246,248$, $250,522$.

Charles, 545.

Charrier, 256.

Charrin, 260.

Charron, 482.

Chelius, 415.

Chenzinsky, 295.

Chossat, 4.

Clark, 335, 386.

Clarke, I. I., 247, 260, 506.

Соввоld, 504, 510, 511, 521, 546, 547.

Cohen, G., 425, 435.

Connheim, J., 370, 386, 424, 558.

Colombini, 271.

Contù, 394.

Cordus, 390.

Cornet, 296.

Cremer, 18.

Cunier, 421.

Cunningham, 190, 507.

Curschmann, H., 246, 260, 303.

Curtis, 571.

CyBulski, 205, 208.

v. CYon, 208.

\section{D}

DADAY, 520.

DANIELS, 517.

DAPPER, 56, 73.

DARE, A., 277. 
DARIER, 208.

DARWIN, 418.

Davaine, G., 244, 260, 504, 507, 517, 521, 534.

Davies, Y., 194.

Debove, 161.

DefFKe, 515.

DenIo, 504, 523.

Delpech, A., 126, 397.

DemantKe, 539.

Demarquay, 546, 547.

Demme, 460, 549.

Demys, 460.

Dentr, 504.

Desnos, 451.

DetTweiler, 63.

Deucher, 46, 47, 48.

Dimsing, $510,546$.

Dock, 507.

DoHRN, 468.

DE Domenicis, 205.

Donders, 166.

DonDONAUUS, 390.

DoNné, 507.

DöRING, 391.

Drawitz, 390.

Drechsex, 189.

DRYer, 208.

Dubini, 553, 560.

DUCHEK, 408.

Duckworth, Sir Dyce, 128.

Dugìs, 565, 566.

DuHRING, 479.

Dujardin, 507, 516, 544.

DünschmanN, 40.

v. Dusch, $463,480,492,493$.

Duval, 510.

\section{$\mathrm{E}$}

EBSTEIN, W., 56, 70, 72, 73, 74, 125, 151, $152,167,211,355,375,386$.

EснтнIUS, 390.

Eckekrantz, 507.

EckHard, 114, 115.

EDEL, 225.

EDWARDS, 313.

EHRENBERG, 507.

EhrhardT, 113.

EHRLICH, P., 293, 294, 295, 300, 304, 305, $319,345,346,347,351,354,355,365$, $375,376,377,384,387$.
EICHHORST, 39.

EISENLOHR, 523.

Elter, J., 259, 260.

Engel, C. S., 286, 347.

EPSTEIN, 507, 548.

ERB, 242.

ERBEN, 47.

ERVant, 563.

Eschle, 596.

Eugalends, 390, 391.

EversmanN, 572.

Ewald, C. A., 179, 180, 190, 193, 194, 212.

F

FabriciUs, 566, 574.

Fauvel, 396.

FEderolf, 553.

FeILChENFELd, L., 269.

Ferria, 195.

Fischer, EMrL, 265.

Fischer, M., 416, 423, 435, 520, 569.

FLEINER, 216, 220.

FleisCher, 42, 353.

FLEISCHL, 277.

Flemming, 565.

FLesch, 239.

FLEURY, 386.

FleXNer, 505.

Floderus, 447.

Foג, 209, 219.

FORDYCE, 437.

Forster, 8.

Fox, 510.

Francotte, 564.

Frank, Johann Peter, 144, 160, 162.

FräNKEL, A., 130, 210, 233, 355.

Fraenkel, S., 189.

FreRICHS, 10.

FREUDWEILER, 376, 387.

FRIEDBERGER, 510.

Friedenthal, 266.

Füldeborn, 518.

FÜRGTENBERG, 567.

v. FürTH, 210.

\section{G}

Gaertner, 279.

GaLeN, 545.

Galland-Gleize, 449.

GarRé, 531. 
GarRod, 128, 132, 136, 146.

Garrod, Alfred, 243, 255, 260, 398, 410. Garrod, Archibald, 243, 257, 260.

GÄRTIG, H., 39.

GärtNeR, 429, 486.

GAUThIER, 195.

Gavoy-RitreR, 424.

GaYer, 441.

DE GEER, 566, 571, 573 .

Geiget, 124.

GeNDRE, 460.

GePPERT, 8, 27, 34.

GerhardT, 38.

GerhaRdt, C., 253, 256, 260, 342 .

Gerhardt, D., 110.

Gervais, 566, 569.

Giesker, 510.

Gilbert, A., 144.

GinNaRd, 460.

Giovannini, 429, 460, 463, 489.

GLEY, 187.

Gоснт, 417, 432, 439, 440, 441, 455, 456.

GoEze, 514, 517, 565.

Goldmann, 190.

Golowin, 535.

GotTlieb, 190, 208.

Gottstein, 195.

GraNdidier, 414, 415, 417, 422, 424, 452.

GrAssI, 503, 507, 516, 517, 523, 545, 546, 548,550 .

Grassmann, 48.

Gratiolet, 205.

Grawitz, E., 29, 320, 328, 387, 424.

Green, 463.

GreENFELD, 180.

Greenhow, 214.

Gretsel, 381, 387.

GRIESINGER, 381, 560.

Griffouillères, 119.

Grisolle, 459.

Gros, 505.

Grosglik, S., 444.

GrUBER, 13.

GRUNd, 266.

GRÜNIG, 543.

Gubler, 239.

Guelliot, 482.

GULL, SIR WM., 179.

GüTeRbock, 535.

Guthrie, 451.

GuYon, 450.

GUYOT, 455, 546.

\section{$\mathrm{H}$}

Hagler, 157, 158.

HAHN, 542.

HaLdANe, 328.

HaMM, 245.

Hammarsten, 265.

HAMMERSCHLAG, 284.

HAMONIC, 449.

HanAU, 233, 239, 241.

HANOT, 460.

Hansemann, 239, 241.

Harley, 205, 394, 513.

HARRIS, 445.

Harrison, 513.

HartmanN, 551.

HARVEY, 70, 72, 163, 165.

HAYEM, 465, 488.

Heberden, 248.

HEBRA, 470.

HEIDENHAIN, 347.

HEINSHETMER, 180.

Helmholtz, 2.

HÉmARD, 454.

HeNle, 536.

HENNEBerg, 19.

HenNig, 190.

HENOCH, 403, 404, 479, 481, 482, 485, 497.

HERMANN, 566.

Hertoghe, 192, 194.

Hertwig, O., 418.

Hervé, 482.

Herz, 251, 260.

Heubner, 36, 47, 486.

Hewes, 283.

HeYland, 424.

Heymann, R., 454.

Hilton, J., 553.

HinsworTH, 542.

Hippock.tTes, 147, 166, 389, 473.

Hirsch, AUgust, 389, 390, 391, 397, 398.

Hirsch, Th., 441.

HirschFeld, F., 14, 40, 56, 70, 72, 73, 74, 174.

HIs, JR., 130.

His, W., 243, 417, 418.

Hitzig, 596.

Hochs, 480.

Hodgkin, 370.

Hofbauer, 446. 
HoFfa, 256, 260, 439, 455, 456.

Hoffmann, Albin, 88, 243, 253, 260, 424.

Hofmeister, 3 190, 196.

Home, 395.

HOOPER, 423.

HÖPFNER, 558.

HoPPe-SEYLER, 1, 8, 36, 49, 505.

HoRsLey, 189.

HösLI, 417.

v. HössLin, 23, 48, 175, 176.

Huber, 538, 549, 576.

Hultaren, 225.

HuN, 185.

Hundhausen, Johannes, 167.

HuNSCHE, 570.

Hutchinson, Woods, 241.

HyRTL, 152.

\section{I}

IJIMA，505, 521.

IMMERMANN, 164, 406, 425, 427, 432 .

ISRAEL, J., 445, 446, 451.

ISRAEL, O., 234, 240.

\section{J}

JACKSON, 394.

$\mathrm{J}_{\mathrm{ACOB},}, 18,266$.

JACOBI, 207, 598.

$\mathrm{J}_{\mathrm{ACOBY}, 508,563 .}$

v. JAKSCH, R., 382, 387, 408, 579.

JANOWSKI, 507.

JANZEN, 120.

JAQUET, 34, 35, 190.

JASTROWITZ, 266, 269.

JENKINS, 531.

JOHNSTON, 565.

JoINVILle, 390.

JoLLES, A., 374, 387.

JONES, LlOYd, 334, 460.

JoSEPH, 572.

JÜRGENS, 216, 506, 535.

\section{K}

KACHEL, M., 249, 260.

V. KAHLDEN, $213,216$.

KAHLER, 115.
KALINDERO, 216.

Kaltenbach, 494.

KANNENBERG, 507.

KA POSI, 479.

KaREWSKI, 542.

KARPELLES, 565.

KARTULIS, 505, 506, 513.

KAST, 186.

KatZenstein, 24, 27.

KaufmanN, 18.

KAYSER, 18, 21.

de Keersmaecker, 447.

KeHRER, 435, 454.

KERBERT, 510.

KIDD, 433.

KISCH, 70, 72, 73, 74, 177.

KJELDAHL, 447.

KLeBS, 235, 238, 408, 418, 460.

KLehmet, 539.

KLEMM, 528.

Klempereir, G., 14, 31, 39, 40, 443, 451.

KNAPP, 268.

Kobert, 598.

Косн, RoвeRt, 275, 290, 292, 460, 461.

Косн, W., 427, 428, 429, 430.

KOCHER, 187.

KOCHER, JR., 189.

Koehler, Armin, 462.

v. Kogerer, 463, 464, 465, 488.

KoLB, 489.

KöLLIKER, 347, 418.

KöNIG, F., 432, 438, 439, 440, 455, 456.

KöPPE, 287.

KoRKUNOFF, 10.

Kossel, A., 265, 266.

Kramer, 391.

KRÄMER, 535.

$\mathrm{K}_{\mathrm{RATZ}}, 556,557$.

KraUS, Fr., 27, 28, 30, 33.

Krauss, 463.

Krautner, 573.

KrEBeL, 392, 396.

Kremer, O., 256, 260.

Kretschy, 408.

KrUG, B., 15, 16, 17, 19, 56, 57, 61.

KrukenberG, 209.

KüHN, 544.

Kù̀z, 112, 268.

Kumagawa, 14.

KUNDRAT, 375, 387.

KÜNSTLE, 39, 507.

Kurimoto, 521. 


\section{L}

LABAND, 262, 268.

LABOULBÈn: $408,574,566$.

LAMBL, 507, 565 .

LaNCEREAUX, 243, 252, 257, 260.

LANDAU, 197.

LANDRÉ-BeatUAis, 243, 260.

v. LANGER, 229, 230, 235, 238.

LANGERHANS, 555 .

LANGLOIS, 205, 206.

LARREY, 151.

LaUdon, $570,575$.

LAUENSTEIN, 442, 506.

LAVES, 28, 33.

LAVOISIER, 2.

LazARUS, A., 275, 304, 319.

LEBRETON, 460, 462, 468.

LeE, 397.

Le Gendre, 128, 460.

LegG, Wickham, 433, 453.

LEGUEN, 442, 448.

LehmanN, C. G, 10, 32.

LehNe, 539.

Leichtenstern, 29, 194，545，549，560, $562,563,564$.

LEIDY, 546.

Leishman, 292, 296.

LELOIR, 463, 464, 465, 488.

LENNANDER, 447.

LenNhoF, 540.

LEO, 28, 33.

LÉon, 163.

Lépine, 262.

LetZerich, 460, 461.

VON LEUBE, 303, 344.

Leuckart, R., 506, 509, 510, 514, 516. $521,526,545,549,550,553,555,565$, 576.

LEUTHOLd, 474, 479, 493.

LEVINSEN, 560 .

LEVy-DoRN, 195.

LEWANDOWSRI, 208, 226.

LEWIN, G., 214, 219.

LEwIS, 508, 546.

v. LEYden, 39, 40, 443, 506.

LICHTENFELDT, 20.

LIEBERMEISTER, 25.

von Liebig, Justus, 2, 3, 4, 50, 51, 398, 447.

LiND, 391.

LinDEMANN, 258, 260, 506.

LINDNER, 508.
LINNÉ, 508, 548, 550, 565, 566, 572.

LINSER, 440.

v. Linstow, 516, 518, 519, 546, 547, 549, $556,558,564$.

LIPMANN-W ULFF, 39.

Liston, 423.

LitTen, M., 388, 465, 507.

LOCKWOOD, 461.

LOEFFLER, 291.

LoEsch, 505.

LoEwT, 51.

Lowwy, A., 25, 196, 286.

LOHSE, 25.

LONGCOPE, 365, 371.

Loos, 503, 512, 513, 514, 546, 562.

Lossen, 415, 424.

LOUMEAU, 449.

LöwIT, 356.

LÜCKE, 172.

LuDwig, 1.

LUFF, 128.

Lussano, 563.

Lüтнје, $16,17,18,19,21,40,41,56,57$, 265.

LÜTKEMÜLLER, 543.

LUTZ, 503, 548, 553.

LUZET, 460.

\section{M}

Mabilite, 192.

MaBON, 195.

Mackenzie, 189.

Madden, Cole, 513.

Madelung, 534, 537.

Magnus, 390.

Magnus-Levr, A., 23, 27, 28, 29, 30, 33, $34,35,64,130,185,192$.

MAGUIRE, 535.

Maillard, L., 257, 260.

MalHerbe, 448, 510

Malmsten, 508.

Manasse, K., 209, 245, 261, 533.

MaNGOLd, 528.

ManN, 51.

Manson, 546, 547.

Maragliano, 495.

Marchand, 206, 507.

Marfan, 460.

MARI, 394. 
Marte, Pierre, 229, 230, 232, 237, 238, $240,241,243,244,246,256$.

MARINESCU, 237, 238.

MARISCHLER, 43.

Marquardsen, E., 167.

Marro, 551.

Martineau, 531.

MARX, 504.

MasiUS, 564.

Matthes, M., 167.

Mathied, 117, 468.

MAY, 30, 40, 507.

MAYDL, 536.

MoConnetu, 508.

McKenzie, D., 454, 463.

McLennan, 190.

McMuns, 209, 212.

Mégnin, 566, 569.

Mehlis, 560.

Meigen, 574.

Meinert, 323, 335.

MeIscher, 277.

MelNikoff, 516 .

Mencke, 560.

Mendelsohn, 261.

Menzer, 257, 260.

v. Mering, 24, 25, 48.

MerRetT, 572.

Meyer, Fritz, 270, 271.

Meyer, R., 2, 33.

Miescher, 506.

Mikulicz, 193.

MiLleR, 394.

MjtCheLL, S. WeIR, 58, 65, 66.

MrURA, 507.

MiyaKe, 569.

MöвiUs, 64.

Mокотғ, 347.

MöLleR, 485.

Monig, 565 .

Moore, 208.

Moosbrugger, 553.

MorazeWSKi, 47.

MosLeR, 531, 549, 560, 576.

Muhlmann, 209.

MüLleR, Fr., 29, 31, 39, 45, 46, 47, 61, $64,266,528$.

MÜLleR, Joh., 246, 260.

MÜlleR, W., 259, 260.

MurRaY, 189:

MuRRI, 393, 394.

MUTZENBECHER, 415.
$\mathrm{N}$

NASsE, 415.

NAUNYN, B., 31, 39, 77, 265, 446.

NeAle, 411.

Nebelthau, 31.

NEHRING, 27, 28, 33.

NeIsser, 536.

NETTLESHIP, 494.

Neuberg, Carl, 265, 266, 267, 268, 269.

NeumanN, H., 265, 266, 267, 347, 354, 363, $461,486,487$.

Neusser, 203, 220.

NEWPORT, 565.

NichOLS, 454.

Nicolaier, A., 148.

Niemeyer, Felix, 340.

Nitzsch, 569.

NOLL, 265.

NONNE, 558.

v. Noorden, C., 15, 16, 28, 34, 35, 37, 38, $39,40,41,42,47,54,56,68,72,73$, $74,194,323,334$.

NORDENSKJOLD, 411.

NORDMANN, 514.

NoRmand, 544, 545.

NothNaGel, 205, 213, 335.

Nutall, 571.

0

OnRtel, $56,70,72,73,74,75,174,175$, $425,427,432$.

Oestreich, 224.

OlaUS, 390.

Oliver, Thomas, 131, 207, 277.

O'NeILL, JoHN, 548.

OPITZ, 408.

ORd, William, 179, 184, 185.

Osler, William, 151, 283, 382, 383, 387.

OSWALD, 189, 190.

OTANI, 511.

OTTE, 424.

OTTO, 415.

OWEN, 553.

OzarKieWICZ, 43.

Ozzard, 547.

\section{$\mathbf{P}$}

PANe, 546.

Pappenheim, 347. 
PARK, 196.

Parona, 545, 560.

Pasteat, 450.

Peiper, E., 501, 576.

PeL, 180, 375, 387.

Pelczynskr, 549.

Penzoldi, 353, 408.

PEPPER, 221.

Penicic, 541.

Perroncito, 509, 560.

Peschel, 14.

Petrone, 460.

v. Pettentzofer, 4, 7, 33, 36, 154, 352, 353.

PFEIFTER, E., 19, 248, 261.

Pflüger, E., 1, 15, 16, 18, 19, 20, 22, 38, $41,50,57,447$.

Philippeaux, 205.

Pichler, 528.

Pickardt, 226.

PicQué, 446.

Pinkus, 375, 376. 377, 384, 387.

Playfair, 58, 65, 66.

Pliny, 163, 389.

Polrier, 446.

Poncet, A., 257, 261.

Ponfick, 186, 196, 242.

POPPE, 544.

Portschinsky, 574.

Posadac, 536.

Posselt, 528, 529.

Potain, 224, 253, 261 .

Potal, 257, 261.

Pousson, 446, 449.

Pribram, 243, 244, 246, 251, 255, 256, 259, 261.

Pröscher, 290.

Prout, 96.

PRUdDen, 185.

Pruner, 560, 570.

Purinton, 208.

Putnam, James J., 314.

QUENO, 195.

Q

QUINCKE, 112, 341, 505.

$\mathrm{R}$

Rabinowitsch, H., 251, 261.

Ramlmann, 570.
RaIllet, 510, 515.

RAINeY, 506.

RAYER, 470.

RAYMOND, 214.

REALE, 271.

v. Reckitinghadsen, 427,465 .

Reclus, 195.

REDI, 572.

REDON, 515.

ReED, Dorothy, 371.

Reed, M., 387.

ReGNIER, 446.

REHER, 460.

ReICH, 535.

ReINBaCh, 193.

REINHOLD, 195.

REISS, L., 199.

Renaut, 213.

REVERDIN, 187.

REYHER, 504, 523.

Rівот, 418.

RichTER, 196.

Rieder, 322, 347.

RIEHL, 213, 463, 464, 465, 488.

Riemann, 531.

Riethus, 27, 30.

Ralex, 571.

v. RINDFLEISCH, 359.

RItTER, 42.

Rivolta, 511.

RoBERTS, 127, 137.

Robin, A., 30, 157, 164, 522.

Robinson, 262.

RodIER, 334, 341, 408.

Roese, 250, 261.

RÖHMANN, 18.

Rohnstein, 319.

Roluso, 87.

Roma, 257, 261.

RoMaNoWsKx, 292.

Romme, 271.

Ronsseus, 390.

Roos, 189, 505, 507.

ROQUE, 244, 256, 261.

Rosemann, R., 42, 50.

Rosenberg, 506.

Roseneld, 394.

ROSENQUUST, 39.

Rosin, 262, 268.

RosITzKr, 191.

Rost, 18.

Rovighi, 533. 
Rovsing, 447, 448.

Rubenstein, 347, 365, 366.

RUBNER, 2, 3, 4, 5, 6, 9, 12, 21, 23, 34, 36, $37,44,47,98$.

RUDOLPH, 510, 514, 516, 517, 546, 548, $552,560,570$.

RUFF, 264.

Runeberg, 504, 523.

RUPPRECHT, 556.

\section{S}

SABATIER, 442.

SAHLI, 279, 425.

SAINT-REMY, 506.

SalIs, 541.

SALKowskI, E., 262, 263, 265, 266, 267, $269,272$.

SALOMON, 25.

SÄMISCH, 567.

SANTINI, 533.

SAVAGE, 180.

SAVENZY, 419, 565.

Scanzer, 543.

SCHÄFER, 207.

Schapiro, 111, 504, 523.

SchattenfRoH, 25.

Schatz, 543.

SchaudinN, 506, 508.

SchaumanN, 319, 504, 523.

Scheby-Buch, 458, 477.

Schede, 442.

Scheude, 547, 548.

Schiassi, B., 386, 387.

SchIFF, M., 187, 188, 205.

SChILd, WALTER, 379, 387.

SCHILling, 225.

SCHLEICH, 584.

Schlesinger, 247, 261.

Schmaltz, 284.

SchmidT, Ad., 4, 10, 45, 46, 47, 48, 507, 543.

Schmidt, Alex., 424.

SCHMIEDEBERG, 342.

Schmitz, 80, 81.

Schmoll, 42.

Schnemoer, 408, 544.

Schnitzler, 544.

SCHOLZ, 29.

SCHÖNFELD, 542.

SchöNLern, 154, 457, 470,473, 474, 475, $476,477,478,479$.
SCHRANK, 565.

SCHREIBER, E., 169.

SchröDT, 194.

Schuberg, 507.

Schubert, 43.

Schuchardt, 250, 261.

SCHÜLLER, M., 243, 244, 255, 261.

Schultze, Max, 347, 543.

SCHÜRMAYER, 507.

SCHÜSSLER, 536.

SCHWAB, 459.

Schwalbe, 167.

SCHWENINGER, 70, 75.

Schwimmer, 458.

Scriba, 569.

SÉDAN, 419.

SEGGEL, 407.

SENATOR, H., 115, 226, 243, 248, 261, 370, $387,442,443,446,447,451$.

SEnN, 369.

Serrano, 189.

SHAW, 179, 565.

v. Siebold, $510,516,526,570$.

SigNORET, 572.

SilbermanN, 462, 463, 464, 495.

Simmons, 371 .

SIMON, 556, 569.

Simon-Legrain, 460.

SIVEN, 14, 15.

Straller, 507.

Sмiтh, 328.

SomNneCKen, 276.

SONDEN, 36, 37.

Sonsino, 513.

SPECK, 25.

SPERling, 549.

SPIETSChKa, 495.

StABel, 193.

StadelmanN, 34, 234.

STADTHAGEN, 353.

STAHEL, 415.

Steinbacher, J., 174.

STEINBerg, 508.

STEINBRÜCK, 535 .

STENGEL, 320 .

STEPP, 549.

STERNBERG, 229, 230, 231, 233, 234, 235, $236,239,240,241,387,505$.

STEVEN, 366 .

STEYSKal, 47 :

STILES, 503, 506.

STOCKVIS, 508. 
Stoermer, 113.

Strassburger, J., 46, 48.

Strauss, H., 47, 112, 257, 261, 319, 361.

STRENG, 507.

van der Stricht, Claus, 239.

Stroganow, 465, 488.

STRÖM, 482.

STRUBe, 507.

STRUBell, 113, 120.

STRÜMPELL, 39, 236, 246, 436.

Stüve, 27, 28, 33.

Subboitic, 541 .

Sudeck, 251, 261.

Svenson, 27, 32, 34.

SydenhaM, Thomas, 126, 147, 340, 391.

SzKekeres, 535.

Szymonowicz, 205, 208.

\section{$\mathbf{T}$}

Tallermann, 258, 261.

TallQuist, 21, 277, 523.

TALMA, 386, 387.

TAMBACH, 189.

Tamburini, 239, 241.

TARGEFF, 506.

TÉDENAT, 449.

Terssier, 244, 256, 261, 545.

Terillon, 534.

Terrier, 118.

Tham, 507.

ThérÈse, 460.

Thévenot, 535.

ThIELe, 27, 28, 33.

THIERFELder, 267.

Thomas, 509.

Thompson, 218.

Tigerstedt, 8, 36, 37.

Tizzoni, 205, 206, 429, 460, 463, 489.

Tollens, 127, 137, 142.

Traube, L., 31, 39, 152, 474, 488, 493.

Treutler, 514.

Trousseau, 112, 126, 244, 261, 334.

Tschinkoff, 212.

TürCK, 282, 283.

TURner, 395.

\section{$\mathrm{U}$}

UMBER, 266.

UTHOFF, 241.
VELICH, 208.

VELSCH, 545.

VERDUN, 563.

VERGA, 229, 238.

VERWORN, 52.

VESSALLE, 460 .

VIDAL, 245, 261.

VIELI, 417.

VIERORDT, 528.

Virchow, 204, 209, 240, 244, 329, 331, 338, $344,347,370,377,423,425,431,432$, 528.

Vitrac, 536.

VOGEL, 42, 268.

VOGLER, 528.

v. VoIт, $1,4,6,7,9,10,11,13,14,15,16$, $18,19,20,21,22,23,33,41,44,45$, $50,51,56,57,352,353$.

VOLHARD, 46.

v. Volkmann, R., 243, 244, 246, 261, 319, 531.

VulPIAN, 209.

W

WADD, 163.

WAGENMANN, 535.

WALDEYER, 231.

De Waldheim, Fischer, 566.

WALLACH, 80.

WARD, 517.

WARTHIN, $369,383$.

WEICHSELBAUM, 250, 256, 261.

WEIGERT, 465.

WEIL, EMIL, 119, 144.

WEINLAND, 515, 516.

WeINTRadd, 1, 28, 33, 47.

Wells, Gideon, 190, 193, 195.

Wendelstadt, 192.

WerLHOF, 453, 457.

WESTPHAL, 124, 534, 544.

Wichmann, R., 256.

Widal, 460.

WiedemanN, 540.

Wierus, 390.

WiERUSzskiJ, 394.

WisnhofF, 506.

WIKNER, 462.

WILKs, 370, 387.

WILLAN, 470.

WinogradoFF, 511, 512.

Winterberg, 542. 
Winternitz, H., 25.

WINZERLING, 536.

WITTAN, 479.

WoHLGeMUTh, 266.

WohlmanN, 255, 259, 261.

WOLPERT, 25.

Wright, A. E., 292, 296, 398, 399.

WUCHERER, 560.

WUNDERLICH, 330, 470.

$\mathrm{Y}$

Yamagiva, 511.
Z

ZEDER, 548.

ZEEHUISEN, 506.

v. ZENKeR, $553,554$.

ZENNER, 248.

ZIEGLER, 535.

ZiemanN, 292.

v. Ziemssen, $322,379$.

ZINN, 80, 81, 563, 545.

ZONDEK, 445.

ZUNKER, 507.

ZuNTZ, 8, 18, 22, 23, 24, 30, 32, 34, 35. 
เ

$+$ 


\section{N D E X}

\section{A}

Abdominal cavity, echinococcus in, 531. lymphadenoids in, 375 .

Abdominal lymph-channels, displaced, 383 .

Abdominal sympathetic, disease of, 111.

Abortion causing thrombophlebitis, 478 .

- Abscess of liver in dysentery, 505.

Absorption, increase of, by stasis, 259.

of food, disturbances of, 43 .

of food, insufficient, 6 .

Acanthocephala, 565.

Acarus, harvest, 565.

of birds, 565 .

transmitted by prostitutes, 567 .

Acetone reaction, 102.

Acetonuria, 91.

Achylia, 47.

gastrica, 313.

Acid, glycuronic, 262, 267.

Acid poisoning, diagnosis and prognosis of, 580.

Acidosis, 87, 90, 95, 97, 102, 579.

Acids, use of, in obesity, 178.

Acquired diseases, 414.

Acromegalia, 195, 229.

autopsy reports of, 230 .

benign, 233.

bony enlargement in, 234 .

combination of, with diabetes, 233 .

development of jaw in, 235.

disproportion of bodily parts in, 230 .

duration of, 234.

enlargement of soft parts in, 237.

etiology of, 230.

eye changes in, 232 .

functional structures in, 236.

malignant, 233, 239, 241.

nervous phenomena caused by, 233 .

pathological anatomy of, 237.

pathology of, 234.

photographs of cases of, 231.
Acromegalia, symptoms of, 231.

therapy of, 242 .

Addison's disease, 199.

aggravation and improvement of, 225 .

atypical cases of, 218 .

autopsy reports of, 214.

cardiac weakness in, 211.

cases of remission in, 225.

changes of the nervous system in, 215, 216.

clinical picture of, $200,210,215,222$.

course and duration of, 210.

cutaneous complications of, 202.

dependence of, upon tuberculosis, 221 .

deviations from type of, 214 .

diagnosis of, 222.

experimental investigation of, 206 .

gastric and intestinal symptoms in, 211.

general cachexia, a cause of, 221.

general conditions in, $203,221$.

histology of, 222.

intestinal symptoms in, 218.

muscular weakness in, 200.

nature of, 210.

nervous and toxic theories of, 221.

nervous symptoms in, 201, 206.

operative cure of, 224.

pathologico-anatomical lesions of, 202.

pigmentation of skin in, 218.

recognition of, 222.

spontaneous course of, 226.

statistics of, 214.

treatment of, 223, 224, 228, 229.

urine in, 212.

Addison's disease with fluctuating course, 223.

Addison's disease without adrenal affection, 214.

without bronzing, 214.

Adenomata, malignant, 239.

Adipose tissue, development of, 54 .

Adipose tissue, in anemia, 308. 
Adipositas, 151.

Adolescents, chlorosis in, 320, 336.

obesity of, 68,154 .

Adrenal glands, tumors of, 219.

Adrenal insufficiency, 223.

Adrenal parenchyma, chemical investigations of, 209.

Adrenal tablets, 226.

Adrenal therapy, results of, 227.

Adrenals, absence of function of, 228. accessory, 206, 220.

anatomy and physiology of, 204.

animal, implantation of, 226.

ascending disease of, 220 .

atrophy of, 202.

cessation of function of, 199, 222.

changes of, in Addison's disease, 203.

chemistry of, 222.

degeneration of, $214,223$.

disease of, 199, 202.

differing from typical Addison's, 215, 220.

extirpation of, 205, 206, 219 .

function of, 203, 207.

functional disturbance of, 218. organic extract of, 197, 207, 226 (see also Suprarenal).

inflammation surrounding, 203.

internal secretion of, 209.

malignant tumors of, 220 .

nature of, 203.

necessity of, to life, 205.

neoplasms of, 202.

parenchyma of, 221.

physiologic function of, 205.

pigment in, 209.

protective functions of, 207.

supplementary, 206.

unchanged condition of, in Addison's disease, 219.

use of, in natural condition, 226.

Adrenalin to arrest bleeding, 454 .

Adynamia, 200.

Affections of bones and joints in the obese, 69.

Agaricus poisoning, 599 .

Aged, metabolism of the, 36 .

Aglycosuria, 93, 95.

dietetic, 87.

Air, fresh, reduced consumption of, 333 .

Albumin, absorption of, in the sick, 47. accumulation of, 41 .
Albumin, circulating, 11, 57 .

dead and living, 3.

decomposition of, 7, 11, 39 .

in ankylostomiasis, 563.

deficiency and superfluity of, 11 .

introduction of, 11.

nuclear, 52.

organic, 16,43 .

reserve, 16, 41, 57.

splitting of, 13.

superfluous amounts of, 12.

toxogenous decomposition of, 39, 40 .

Albumin and iron preparations, 341.

Albumin as a "working mass," 16.

as basis of metabolic products, 2 .

Albumin bodies, 10, 70 .

Albumin deposition, 15.

Albumin in food, indispensability of, 10 .

minimal amount of, 74 .

Albumin in urine of diabetics, 121.

Albumin metabolism, 35.

Albumin minimum, 10, 13.

hygienic and physiologic, 11.

Albumin of body, 43, 55 .

Albumin of the food, 43, 73 .

action of, in nutrition, 71 .

Albumin savers, $15,40$.

Albuminuria, 86.

in diabetes, 86 .

in leukemia, 353.

in morbus maculosus, $475,498$.

in purpura, 493.

Alcohol, energy carried by, 50 .

excess in, a cause of diabetes, 119 .

gout caused by, 127, 130.

in beer, 106.

premature indulgence in, 332 .

use of, in the cure of obesity, 74 .

in diabetes, 101.

Alcoholism, chronic, 591.

Aleuronat, 70, 167.

Alkali tension, 286.

Alkalies, salts of, poisoning by, 582 .

Alkalimeter, 286.

Alkaloids, poisoning from, 594 .

Alloxur bodies, 374 .

Aloin poisoning, 598 .

Alopecia in gout, 143.

Amanita phalloides, 600 .

Amebr, 505.

occurrence of, in various parts of body, $505,506$. 
Ameboid movement, 346.

Amenorrhea in myxedema, 185.

American Pediatric Society's investigation of infantile scurvy, 393, 404, 485.

American remedy for gout, 135.

Amido-benzol poisoning, 592 .

Amphistomum hominis, 508.

Amyloid degeneration, 250.

Anacidity of stomach, 47.

Anatomical changes in chronic articular rheumatism, 248.

Anematosis, 221.

Anemia, 304.

absence of nervous symptoms in, 328 .

ascarides, a cause of, 549 .

blood changes in, 301, 306, 328 .

blood examination in, 307, 309.

bothriocephalus, 311,317 .

caused by uncinaria, 318 .

change of climate in, 310,319 .

clinical symptomatology of, 308.

cold applications in, 310 .

combination of, with obesity, 156 .

decreased metabolism in, 33 .

destruction of tissue in, 39 .

differentiation of simple from pernicious, 317.

excessive, 359 .

fatty degeneration of, 308 .

faulty nutrition a cause of, 306 .

general metabolism of, 308 .

general treatment of, 319 .

hemorrhagic diathesis in, 308 .

idiopathic, 306.

infantum pseudoleukemica, 382 .

jaundice in, 312 .

leukemia, caused by, $352,357,367$.

lymphatica, 370 .

lymphaticolienalis, 370 .

montana, 560 .

of brickmakers, 560 .

pernicious, $47,305,314,382$.

post-malarial, 318 .

profound, 203.

progressive pernicious, $305,310,563$.

cardiac symptoms in, 313 .

clinical picture of, 312 .

course of, 317 .

forms of, 314 .

metabolism in, 313 .

post mortem findings in, 314 .

primary, 310 .
Anemia, progressive pernicious, remission in, 317.

symptoms of, 311 .

tumor formation in, 315.

secondary or symptomatic, 305 .

severe, $357,358$.

simple, 305 .

cause of, 305 .

diagnosis of, 309 .

prognosis of, 309 .

treatment of, 309.

chronic, 381 .

splenic, 326,381 .

therapy of, 318 .

variety of, indicated by blood changes, 312.

well-nourished appearance in, 313.

Anemia in Hodgkin's disease, 373.

Anemia from tape-worm, 523.

Anemics with normal hemoglobin, 281.

Angina pectoris, 141.

Angina pectoris in diabetes, 85 .

Angioneurosis, 448.

Anguillula, development of, 545 .

intestinalis et stercoralis, Bavay, 544, 545 .

putrefaciens, 544.

Anilin poisoning, 592 .

- Animal economy, 4.

Animal poisons, 598.

Animal parasites of man, 501 .

Animal protoplasm, decomposition of, 52 .

Animals, echinococcus disease among, 529.

fattening of, 153 .

parasites derived from, 503.

Ankle, sprain of, 146.

Ankylosis, 259.

fibrous, 250.

of bleeders, 438 .

Ankylostoma, 501.

disturbances caused by, 504 .

duodenale, 561 .

Ankylostomiasis, 560 .

autopsy findings of, 563 .

prophylaxis and treatment of, 564 .

symptoms of, 562 .

Anorexia, 323.

in gout, 140.

Anthrax, 571.

Antifat cures, 72, 160, 170.

indication for, 69 .

irrational, 55 .

metabolism in, 56 . 
Antipyrin poisoning, 594.

Antirheumatics, in gout, 149.

Antitoxic action in adrenals, 221.

Anus, itching of, 551.

Aorta, aneurism of, 216.

chlorotica, 329.

of hemophiliacs, 431 .

Apepsia. See Achylia. gastrica, 47.

Apparatus, use of, in rheumatism, 259.

Appetite, 29.

caprices of, $60,323,428$.

diminished by decreased fluid intake, 75 . enormous, in diabetes, 113.

Appetite in the healthy and the sick, 22.

Appetite of the obese, 157.

Arabinose, 267.

Argas reflexus, Fabricius, 566.

Argas, varieties of, 566 .

Arnican poisoning, 598 .

Arnica, tincture of, 598.

Aromatics, poisoning by, 592 .

Arsenic, idiosyncrasy for, 319 .

in treatment of anemia, 309 .

chlorosis, 343 .

gout, 149 .

Hodgkin's disease, 378.

myxedema, 192.

pseudo-leukemia, 386.

poisoning by, 586 .

Arsenic atoxyl, 379 .

Arsenious acid in Hodgkin's disease, 379.

Artefacts, 356.

Arteries, atheromatous degeneration of, 157 . central, marantic thrombosis of, 358 . compression of, for hemorrhage, 454 . condition of, as indication of age, 81 . peripheral, pulsation of, 322 .

pulsation of, in anemia, 313 .

Arteriosclerosis, differentiation of, from diabetes, 121 .

in diabetes, 85.

Arthritic gout, primary, 131.

Arthritis, 125, 493.

chronic, Heberden's nodes in, 248.

deformans, 244, 246.

gouty, 125.

hereditary, 253.

in hemorrhagic diseases, 476.

pauperum, 253.

urica, 125.

Arthritisme, 252, 255.
Arthropathies, hemophilic, 455.

Arthropoda, 565.

Articulation, sterno-clavicular, 322.

Ascarides, causing suffocation, 550.

expulsion of, by santonin, 550 .

occurrence of, in various parts of body, 549,550 .

Ascaris, infection of children by, 549 .

lumbricoides, Linné, 548.

maritima, Leuckart, 549.

mystax, Zeder, 548.

Ascites, nitrogen balance after, 43. occurrence of, in pseudo-leukemia, 386 .

Asphyxia arsenicalis, 586.

Assafetida, in diabetes, 122.

Assimilation, insufficient, 44.

Asthenia, in Addison's disease, 210.

Asthma, 174.

Athyreoidism, 225.

Athyreosis chronica, 188.

Atomatropin, 601.

Atoxyl, 386.

Atrophy in a nursling, 47 .

Atrophy of ganglion cells, 217.

Atropin, in diabetes, 123.

Atropin poisoning, 597.

Auscultatory phenomena, 536.

Austrian marine, scurvy in, 412.

Autointoxication, 199.

Autopsy findings in Addison's disease, 203.

Azoturia, 22.

\section{B}

Babes, bacillus found by, 394 .

Bachstrom's theory of scurvy, 397.

Bacillus anthracis, 461.

hemorrhagicus Kolb, 490.

hemorrhagicus velenosus, 489 .

of plague, 571.

of typhoid, 571 .

producing scurvy, 399.

purpuræ, 460, 461, 467.

pyocyaneus $\boldsymbol{\beta}, 461$.

Balanitis of prepuce, 551.

Balantidium coli, 501. minutum, 508.

Balsam of Peru, for scabies, 569.

Banting, cure for corpulence, 70, 72, 73, 74, $163,164,165,166,173,175$.

Banti's disease, $370,381$. clinical picture of, 381 . 
Banti's disease, lienal and splenic, 381. urine in, 382.

Bandage, rubber, in rheumatism, 258.

Barium, poisoning by combinations of, 583 .

Barlow's disease, 396, 485, 486, 487. increase of, in Berlin, 486.

Basedow's disease, 64, 65, 193. See also Graves' disease.

Bath cure, 101, 148.

for gout, 135 .

in diabetes, 101.

Baths, hot, for chloroties, 341.

mud, 148.

peat, 148.

sulphur mud, 148.

sun, of Celsus, 175.

thermal, 148.

value of, in diabetes, 122 .

with electric light, 258.

Bear's paw of acromegalia, 234.

Bed-bugs, transmission of parasites by, 571 . varieties of, 572 .

Beer, as preventive of scurvy, 411 . consumption of, in Munich, 130.

effects of, in diabetes, 100 .

use of, in obesity, 170.

Beer glycosuria, 79 .

Belles-lettres, influence of, on girls, 338 .

Benzine, in trichinosis, 560 .

Bernard, Claude, experiments of, 115, 116.

Bial's reagent, 263. tests for pentose, 267 .

Bicycling, 67, 72. in obesity, 176.

Bidder and Schmidt's investigations in metabolism, 4.

Bier's hot air treatment, 259.

Bile, chemical composition of, 345 . stasis of, 46.

Bile-ducts, dilatation and inflammation of, 512.

Bilharzia hæmatobia, 512.

prevalence of, in Africa, 512.

Biliary passages, inflammation of, 140 .

Biogen molecule, 52.

Bird acarus, 565.

Bites of insects, treatment of, $\mathbf{5 7 5}$.

Bitters, poisoning by, 598 .

sugar contained in, 101.

Biuret reaction, 51.

Bladder, distoma infection in, 513 .

echinococci of, 542 .
Bleeder families, 415, 416, 417.

Bleeder's disease, 413 . See also Hemophilia. joint, 432, 439, 440.

Bleeders, marriage of, 452 .

Blindness, color, 418.

night, 419.

transitory, 325 .

Blood, abnormal composition of, 427 .

abnormal distribution of, '281.

agglutination of, 290 .

albumin in, 307, 312.

alkalinity of, 276,286 .

anemic composition of, 324, 336 .

bacteriology of, 290 .

changes of, in progressive pernicious anemia, 311.

chlorotic, 326.

circulation of, 304 .

coagulability of, 454 .

coloring matter of, 325 .

composition of, favoring hemophilia, 422 .

in purpura, 494.

condition of, in hemophilia, 424.

in purpuric child, 495 .

in scurvy, 408.

dilution of, 282.

diseases of, 273.

fixing methods of examination of, 292.

flooding of, with sugar, 79.

fluid constituents of, 327 .

impoverished, 33 .

injection of, 206.

inspissation of, 124.

insufficient coagulability of, 423 .

intensity of color of, 277.

internal secretions in, 334.

leukemic, composition of, 344 .

lymph-cell formation in, 361.

methemaglobin in, in poisoning, 583.

morphological alterations of, 311, 333 .

pallor of, in chlorosis, 325 .

pathology of, 345 .

pigment originating in, 213.

processes for staining, 293, 294.

protective bodies of, 276 .

regeneration of, 305.

shadow-corpuscles in, 495.

shadow formation in, 462 .

solids of, 307,312 .

specific gravity of, $284,307,312$.

spectroscopic examination of, 287.

the seat of chlorotic disease in, 333 . 
Blood, total solids in, 285.

transfusion of, 319 .

uric acid in, 255.

Blood cell formation, infectious disturbance of, 362 .

Blood cells, 326 .

acidophilic, 297.

anomalies of, 299.

circulating, 310 .

eosinophilic, 297.

mononuclear, 346 .

non-nucleated, 345.

nucleated, 326.

oxyphilic, 297.

red, counting of, 325 .

reduction of, 327 .

varying size of, 326 .

Blood changes in Addison's disease, 212.

Blood-corpuscles, 281.

condition of, in chlorosis, 325 .

counting of, 281,325 .

normal numbers of, 283.

nucleated, 349.

percentage of, in anemia, 306 .

pessary forms of, 496 .

red, counting of, 325 .

condition of, in anemia, 306.

formation of, 344 .

reduction of, 312 .

volume of, 287.

white, $283,307$.

condition of, in leukemia, 360 .

increase of, 344, 355 .

number of, 300 .

polymorphia of, 364 .

Blood count in Addison's disease, 204.

Blood-counting, apparatus for, 281.

Blood examination, 275.

in diseases of bone-marrow, 275 .

in malaria, 291.

in parasitic diseases, 275 .

method of, 276.

Blood-formation, anomaly of, 335 .

embryonal type of, 310 .

impairment of, 356 .

morbidly active, 344 .

pathological, 365 .

peculiarity of, in anemia, 317 .

Blood-kindred, intermarriage between, 421.

Blood plaques, 327.

recognition of, 293, 299.

Blood-poisons, 592.
Blood preparations containing iron, 342.

Blood-pressure raised by adrenal therapy, 207, 209.

Blood serum, agglutination of, 289.

dilution of, 289.

investigation of, 285.

methods for testing, 289.

reduction of, 327.

total solids in, 327 .

Blood-staining, by Chenzinsky's method, 295.

by Ehrlich's method, 293, 296.

universal method of, 296.

Blood tumors, 437.

Blood-vessel glands, 238.

Blood-vessels, echinococcus in, 537, 539.

rupture of, 431.

Blood volume, in hemophiliacs, 425.

Blushing of chlorotics, 325, 335 .

Body, activity of, 5 .

composition of, 11.

Body-albumin, 7.

Body landmarks, displacement of, in obesity, $159,160$.

Body nitrogen, increase of, 57.

Body-weight as a control in experiments, 7 . increase of, 59.

Boerhaave's cases of obesity, 160, 162.

Bone-marrow, cell development in, 347, 348. changes in, 351.

in anemia, 315 .

condition of, in pseudo-leukemia, 371 .

functional alteration in, 365 .

insufficient function of, 360 .

lymphoid hyperplasia of, 355 .

pathological changes of, 333 .

red, 345 .

reduced function of, 362 .

Bones, affection of, by gout, 139. atrophy of, 251.

changes in, of hemophiliacs, 456.

condition of, in scurvy, 407, 408.

deformities of, in acromegalia, 232.

echinococcus of, 544 .

enlargement of, in acromegalia, 234.

long, tumor in tract of, 485.

sensitiveness of, to pressure, 358 .

$\mathrm{X}$-ray examination of, 432 .

Bothriocephalus anemia, prognosis of, 524 . treatment of, 318.

Bothriocephalus cordatus, Leuckart, 521. cristatus, Davaine, 521. 
Bothriocephalus grandis, Blanchard, 521.

latus, Bremser, 520 . distribution of, 521.

Mansoni, Cobbold, 521. passage of segments of, 522 .

Botulism, 601.

Boys, chlorosis of, 320,330 . gout in, 129.

Brain, distoma in, 511. echinococei of, 534. effusions of blood into, 158 . injury to, as cause of diabetes, 115.

Bread, casein, 170. containing aleuronat or ergon, 168. diabetic, 99 .

rye, in obesity, 173.

wheat, for the obese, 168, 169.

Breithaupt and Cetti, the fasters, 14.

Brickmakers, disease of, 560 .

Bright's disease, 467, 468.

Brisement forcé, 456.

British Medical Association, Congress of 179.

Bromin salts, poisoning by, 584 .

Bromism, 584.

Bronchial catarrh in trichinosis, 557.

Bronzing in Addison's disease, 201.

Bruit de diable, 322.

Bubo disease, 571.

Buffy coat, 431.

Bulimia in Addison's disease, 200.

Bunge's theory of seurvy, 410.

Burns under X-ray treatment, 369, 381.

Büschel-form of keratitis, 567 .

\section{C}

Cabot's, researches in blood examination, 283.

Cachexia associated with pigmentation of skin, 203.

from parasites, 511 .

in Hodgkin's disease, 373.

myxedematous, 191.

strumipriva, 187, 188.

thyreopriva, 187, 188.

tubercular, 221.

Cachexie pachydermique, 179.

Calculi, uratic, 157.

Calorimetry, direct, 9.

in $\operatorname{man}, 6$.
Calory estimations, of Rubner, 44.

Calory requirement of body, 6,7 .

Calory supply, diminution of, 53.

Calves, cramps in, 128, 136.

Camphor, in diabetes, 122.

Camphor poisoning, 594.

Cancer, hepatic, 541.

Cane-sugar, 78.

Cantani's obesity cure, 165 .

Capillaries, rupture of, 433 .

Carbohydrates, absorption of, 48 .

allowance of, in diabetes, 98 .

in the diet, 74.

in feces, estimation of, amount of, 45 .

percentage of, in beer, 106 .

in food, tables of, 104.

in wines and spirits, 106.

tolerance of, 88 .

value of, as albumin savers, 40 .

Carbolic acid poisoning, 593.

Carbon balance, 7 .

Carbon bisulphid, 586.

Carbonic acid, excretion of, 8 .

Carbonic acid poisoning, 590.

chronic, 591.

Carcinoma, colloid, 512.

from parasitic infection, 513 .

Carlsbad, treatment at, in diabetes, 101.

Casein, 70, 169.

Castor oil, in trichinosis, 559.

Castration, as a cause of obesity, 154 .

Cataphoresis, 425.

Catarrh, intestinal, due to coccidia, 506.

tendency to, in obesity, 157.

Caterpillars, toxic effect of, 575 .

Cattle, mortality among, from echinococcus, 533.

Cautery, to control hemorrhage, 454.

Cell accumulations, lymphadenoid, 375 .

Cell, protoplasm of, 3 .

destruction of, 3 .

Cells, chemical activity of, 3 .

decomposition in, 3.

dwarf, 356 .

growth energy of, 57.

katabolic activity of, 61 .

nuclei of, disease of, 131.

parenchyma, 204.

regenerative energy of, 57.

Cerebral affections, due to gout, 142.

Cerebral diseases, polyuria in, 115 .

Cerebral disturbances in myxedema, 184. 
Cervical ganglia, neuritic disease in, 216. Cestodes, 514.

Cesto-embolism, 539.

Cetti and Breithaupt, the fasters, 14, 32 .

Chambers's cure of corpulence, 163.

Champagne as cause of gouty attack, 135. Charcoal in calorimetric investigations, 6 .

Charcot on rheumatism, 244.

Charcot-Leyden crystals, 350, 563 .

Cheese, in diet of diabetes, 100.

Chemical analysis, in case of poisoning, 581.

Chemical curative agents, 197.

Chemical processes as source of heat, 2 .

Chemotactic stimulus, 344 .

Chemotaxis, 365 .

negative and positive, 302 .

Cherry cure, 147.

Chicken cholera, 571 .

Children, metabolism of, 36 .

Chin, double, 159.

China, organotherapy in, 196.

Chiragra, 125.

Chlorin combinations, poisoning by, 583 .

Chloroform poisoning, 584 .

Chlorosis, 305, 320.

anatomical foundation of, 330 .

appetite in, caprices of, 323 .

blood changes in, $325,327,334$.

blood examination in, $325,328,336$.

body temperature in, 323 .

cardiac murmurs in, 321.

cardiac palpitation in, 322 .

causes of, 333 .

changes of skin in, 320 .

combination of, with obesity, 156 .

complications of, 329 .

course of, 337.

derangement of nervous system a cause of, 335,336 .

development of, 339 .

diagnosis of, 328,336 .

diet in, 341 .

digestive symptoms in, 323 .

diseased blood as a cause of, 334 .

Egyptian, 560.

extreme pallor in, 336 .

forms of, 337 .

frequency of, in cities, $331,333$.

genesis of, 333 .

gigantea, 154 .

habitual, 330 .

hereditary, 339.
Chlorosis, independent symptoms of, 324 . latent, 329.

metabolism in, 33 .

mode of life favoring, 339 .

murmurs in, 321.

nervous disturbances of, 324,325 .

no specific treatment for, 340 .

occurrence of, among servant girls, 332 .

in boys, 320,330 .

in fat people, 320 .

in men, 329 .

in time of Hippocrates, 320 .

pathological anatomy of, 329 .

persistent, 330 .

predisposing causes of, 331 .

prognosis of, 329 .

prophylaxis of, 343 .

rapidity of pulse in, 322 .

relapses of, 330 .

relation of, to sex, 330 .

respiratory apparatus in, 323 .

sexual functions in, 323 .

symptomatology of, 328 .

tight lacing a cause of, 332 :

treatment of, 339 .

by suggestion, 342 .

tropical, 560.

vagaries of appetite in, 323.

Cholelithiasis, 140.

Cholera Asiatica, simulated by arsenical poisoning, 586.

Choleraic symptoms, in trichinosis, 556.

Chorea, thyreoid therapy in, 195.

Chronic articular rheumatism, 243.

Chyle tracts, 48 .

Cimex, varieties of, 572 .

Circulatory apparatus, changes in, 329 .

condition of, in chlorosis, 321.

echinococcus in, 538 .

Circulatory disturbances as result of obesity, 69.

Circumcision, of hemophiliacs, 434, 452.

Cirrhosis, hepatic, 43, 382 .

hypertrophic hepatic, 141.

of liver from parasitic changes, 512 .

Claudicatio intermittens, 85 .

Climacterium, use of oöphorin in, 197.

Climate, influence of, in producing scurvy, 397.

Clothing, use of, in common, 550.

Club finger tips, 256.

Club-foot, as sequence of scurvy, 409. 
Coal gas poisoning, 79 .

Cocain poisoning, 597.

Cocainism, 597.

Coccidium, bigemimum, 506.

oviforme, 506.

perforans, 506.

Colchicum in gout, 149.

Cold and heat, effect of, in metabolism, 25.

Cold, extreme, a cause of diabetes insipidus, 119.

use of, in diabetes, 122.

Colic, lead, 587. renal, 451 .

Collapse, cardiac, 596. treatment of, 497 .

Colloid masses, excretion of, 204.

Color-blindness, 418.

Color-index, 283.

Coma, acid, 92.

diabetic, 80, 91, 102.

fatal, in anemia, 319 .

in purpura, 483 .

opium, 596.

Combustion, a source of energy and heat, 2 . increase and decrease of, 26.

Compensation, process of, 88.

Comptodactylie, 255.

Congress, British Medical Association, 179. Forth French, for Urology, 1889, 448.

International Medical, at Paris, 126, 127.

Congress of Internal Medicine, 1885, 56.

Connective tissue, 348 . changes of, in acromegalia, 237.

Connective tissue hyperplasia, 237, 238.

Constipation in chlorosis, 323.

in gout, 140, 148.

in scurvy, 413 .

obstinate, in the obese; 157.

stubborn, 172.

Consumption, occurrence of, in small eaters, 63.

Contractures of fingers and toes, 251.

Convalescence, abundance of food in, 40 .

in morbus maculosus, 497.

Copaiba, balsam of, poisoning by, 594 .

Coprostasis in chlorosis, 340 .

Corium, pigmentation in deep layers of, 213.

Cornutin poisoning, 598 .

Coronary artery, calcification of, 269.

Corpulence, 151. See also Obesity.

Wadd's views on, 163.

Corrosive sublimate, tolerance of, 589 .
Corrosive sublimate poisoning, symptoms of, 589.

Corsets, injurious effects of, 332 .

Country life for chlorotics, 340 .

Cramps in legs, 139.

Cream, in diet of diabetics, 100 .

Creeping disease, 574.

Cremation of infected meat, 533.

Cretinism, 30, 187.

endemic, 188, 189.

sporadic, 187, 188.

Cretinoid state in women, 180.

Crick in the back, 139.

Crusades, scurvy during, 390.

Crystallose, 109.

Cure, Nature-healing, 60 .

Oertel, 174.

of obesity, 177.

Cures, dietetic, 54 .

mineral water, in diabetes, $92,101$.

weakening, 67 .

Cyanosis in blood poison, 592.

Cyclops serrulatus, Fischer, 520 .

Cyprinotus incongruens, 519 .

Cystericus bovis, 115 .

cellulosæ, 501, 515 .

Cystoid echinococcus, 528.

D

Daltonism, hereditary, $415,418$.

Deafness from lymphatic nodules, 352 .

Debove's treatment of obesity $y_{5} 161$.

Decomposition, alterations of, 4.

nuclein, 52 .

of food, 6 .

Deformity, in chronic articular rheumatism, 246.

of bones in arthritis, 256.

Degeneration, amyloid, 47.

granular, 326.

hyaline, vascular, 466 .

phenomena of, 326.

Deglutition, difficult, 373.

Delirium tremens, 591.

Demodex folliculorum, Simon, 569.

Dentition, a critical period in hemophiliacs, 434.

Depression, hypochondriac, 142.

paroxysms of, 143.

Dextro-rotary pentose, 267. 


\section{Dextrose, 77.}

Dextrosuria, 77.

Diabète azoturique, 113.

Diabetes, actual cure of, 82 .

arteriosclerotic, 84.

arthriticus, 144 .

cerebral causes of, 122.

chronic, 81 .

coexistent with acromegalia, 233.

combination of, with pentosuria, 268 .

decipiens, 144.

diet in, 33.

disease of the brain a cause of, 114 .

drug treatment of, 122.

gastric disturbances in, 102.

hysterical, 117.

increased appetite in, 113.

insipidus, 110.

clinical picture of, 110 .

constitutional condition in, 120.

diagnosis of, 121.

etiologic therapy of, 122 .

etiology of, 112.

hereditary form of, 119.

idiopathic form of, 118.

infectious diseases, followed by, 119 .

low temperature in, 114.

normal, 117.

organic disease of brain, a cause of, 111.

over-nutrition in, 64 .

prognosis of, 120.

psychical treatment of, 122 .

special varieties of, 115 .

symptoms and course of, 112.

typical cases of, 120 .

mellitus, 77, 83, 167.

after scarlatina, 80 .

combination of, with gout, 130 .

hereditary, 82.

metabolism in, 32 .

mild and severe forms of, 83.

occurrence of, in the young, 81 .

over-nutrition in, 64 .

practical therapy of, 92 .

regulated diet for, 96 .

theoretic treatment of, 87.

valuable foods in, 89 .

various forms of, 83 .

mild and severe, 83 .

nutrition in, 113.

occurrence of, in a chemist, 80 .
Diabetes, occurrence of, in the aged and in the young, 80,81 .

in the obese, 83.

organic, 81 .

pancreatic, 81 .

pure, 84.

quantitative diet in, 98.

relations of, to gout, 144 .

saccharin, 115.

sugar excretion in, 52.

tables of foods in, 103 .

thirst in, 113.

treatment of, 87, 101.

typical cases of, 94 .

Diaceturia, 91.

Diaphragm, inactivity of, 557 .

rupture into, 538.

Diaptomus spinosus, Daday, 520.

Diarrhea, fatty, 47.

in leukemia, 369.

in scurvy, 408.

Diatheses, hemorrhagic, 388.

Diathesis, gouty, 130.

hemorrhagic, in anemia, 308.

oxalate, 449.

uric acid, 126.

Didymin, 197.

Diet, amylaceous, 80.

antifat, grades of, 72 .

calculation of nutrition and calory value of, 107.

effect of, in metabolism, 44 .

employment of, in chlorotics, 340,341 .

for the obese, 167.

in diabetes, $87,96,122$.

in leukemia, 369.

maintenance, of the sick, 37 .

mixed, 80.

nutrition and calory value of, 107 .

preventive of scurvy, $411,412$.

quantitative regulation of, 96 .

researches in, 48.

schemes, adherence to, 76 .

tests in, 48.

vegetable, 61, 147.

Diet-tables of Chambers, 163.

Dietary for diabetics, 108.

of Banting, 72, 74 .

of Chambers, 163.

of Dapper, 73.

of Ebstein, 72, 74.

of Harvey, 72. 
Dietary of Hirschfeld, 74 .

of Kisch, 74.

of Oertel, 72,74

of v. Noorden, 74 .

Dietetic cures, 54, 69.

Dietotherapy, 2, 39 .

dangers of, 102.

Digestion, condition of, in Hodgkin's disease, 381 .

impaired by tape-worm, 522 .

physiology of, 1.

vicarious intestinal, 47.

Digestive apparatus, disorders of, in diabetes, 114.

Digestive canal, catarrh of, 140 .

disturbances of, in Addison's disease, 200.

Digestive organs in anemia, 313.

Digestive tract, anemic changes in, 315 .

Digitalis and ite glucocides, poisoning by, 597.

Digitalis infusion, decomposition of, 597 .

Dioptrics, 419.

Dioxybenzole poisoning, 593.

Diplobacilli, 490.

Distoma, 509, 510.

fatal disease caused by, 512 .

occurrence of, in China, India, and Japan, 511.

Distomatosis, 510.

Distomum felineum, Rivolta, 511.

hæmatobium, Bilharz, 512.

hepaticum, Linné, 509.

pulmonale, Baelz, 1883, 510.

sibiricum, Winogradoff, 511.

spathulatum, Leuckart, 511.

Westermanni, 510.

Dog, as transmitter of echinococci, 529 .

Dog tape-worm, 531.

Drinking-water as disseminator of parasites, 503 .

Dropsy, oily, 151.

Drugs, production of hysterical polyuria by, 118.

Dupuytren's contracture, 145.

Dwarf, acromegalic skeleton of, 231.

Dwarfs, prehistoric, 229.

Dysentery, distoma found in cases of, 511 . intercurrent, with scurvy, 408.

Dyspepsia, intestinal, 140.

nervous, 60 .

Dyspnea, 352.

\section{E}

Ear, gouty processes in, 143.

gouty tophi in, 135.

Earth, diseases caused by working in, 562 .

Ecchondroses of rheumatism, 246, 248.

Ecchymoses of purpura, 459, 470, 484, 488.

Echinococei, autosemination of, 531 .

of various organs, 534 .

Echinococcus, 501.

areas infected by, 529 .

cysticercus, 529 .

cysts of, 527,532 .

expectoration of membrane of, 538 .

multilocular, 534.

multilocularis, 528 .

multiplex, 531 .

occurrence of, in bones, 529 .

in female genital organs, 543.

in oral cavity, 535 .

prevalence of, among animals, 526 .

prevalence of infection of, 529 .

propagation of, 527 .

pulmonary, 538 .

renal, 541.

toxicity of fluid of, 531 .

transmission of, by dog, 529 .

tumors of, 528 .

Echinococcus disease, 526.

course and temperature in, 540 .

prognosis of, 533, 540 .

prophylaxis of, 533 .

symptoms of, 532 .

treatment of, 533 .

unilocular, 531, 539.

with duration of thirty-five years, 530 .

Echinococcus sac, an impediment at birth, 543.

Echinorhyncus gigas, Goeze, 501, 565.

Ectoparasites, 501, 565.

Edema in trichinosis, 557.

Edema of face, 341.

Effusion, hemorrhagic, 492.

in scurvy, 400.

into knee-joint, 455.

Effusions in gout, 134 .

Eggs, in diet of diabetics, 100, 108.

Ehrlich eye-piece, 301.

Ehrlich-Pinkus formula, 376.

Ehrlich's method of staining, 293, 294, 296. 
Ehrlich's investigations of the blood, 345, 351.

Electricity to spleen, 386.

Electricity, use of, in diabetes, 123.

Elephantiasis, swellings resembling, 548.

Emaciation, cure of, 161 .

in diabetes insipidus, 120.

in rheumatism, 250.

significance of, 38,39 .

Emboli, from echinococcus cysts, 537. in trichinosis, 557.

Embonpoint, 153, 155.

Emphysema, pulmonary, 142.

Endocardium involved in rheumatism, 251.

Enemata of benzine, 560 .

Energy, law of conservation of, 2. loss of, 43,44 .

transferrence of, 3,11 . metabolism of, 5 .

Energy requirement of body, 11. of the healthy, 9 .

English navy, absence of scurvy in, 411.

Enlargement, in acromegalia, 230. glandular and multiple, 375 .

Enteric fever in chlorotics, 339.

Entoparasites, 501.

Entozoa, 501.

Enuresis nocturna, 119.

Enzymes, action of, 13.

Eosinophiles, 347, 355. in trichinosis, 557.

Eosinopbilie leukocytosis, 365.

Epidemies of trichinosis, 553. of scurvy, 390, 391, 392.

Epididymis, echinococci of, 544 .

Epilepsy, 116. from tenia solium, 504 .

Epinephrin, 210.

Epinephrectomy in animals, 206.

Episcleritis, 252.

Epistaxis, occurrence of, in hemophiliacs, 454.

treatment of, 497.

Epizoa, 501.

Equilibrium, nitrogenous, 10.

Ergon in bread for the obese, 167.

Ergot, use of, in hemorrhages, 454 .

Ergot poisoning, 598.

chronic, 599.

Ergotin, in diabetes, 123.

Ergotism, convulsive, 599. gangrenous, 599.
Eruption, itching, 544.

Erythema, nodosum, 473, 477.

Erythroblasts, 300 .

in anemia, 311.

Erythrocytes, 307, 344.

decrease of, 349 .

increased formation of, 283.

morphological changes in, 306.

normal proportions of, 345 .

recurrence of, in chlorotic blood, 326 .

stippling of, 292, 300, 312 .

Erythrosclerotin, 599.

Eschars, from acid poisons, 580.

Esophagus, ascarides in, 550.

Ethyl alcohol, poisoning by, 591 .

Eucalyptus, in pseudo-leukemia, 386.

Eucasin, 48.

Eustrongylus gigas, Rudolphi, 560 .

Excretion by skin, stimulation of, 123.

Excretions, estimation of, 6.

Exercise, bodily, during antifat cures, 56 . muscular, in diabetes, 92. in obesity, 175, 176.

in production of flesh, 58 . passive, 177.

Exercises, free, of German turners, 176.

Exophthalmic goiter, 64, 66, 193.

Exophthalmos in acromegalia, 233.

Expired air, estimation of, 6 .

Extremities, neuralgia of, 314 .

size of, in acromegalia, 230.

Eye, affections of, in rheumatism, 252. changes of, in acromegalia, 232. cysticercus in, 524 .

disease of, from acarus, 569.

from caterpillars, 575 .

specks before, 324 .

Eye-ball, echinococcus of, 535 .

Eye-ground in leukemia, 352.

Eyrich's picture of purpura, 484.

\section{F}

Face, cyanosis of, 373 .

edema of, 373.

Faradization, 66 .

Fasters, professional, 14.

Fasting values, 9 .

Fat, combustion of, 75 .

danger of, in various diseases, 55 .

development of, in prime of life, 55 . 
Fat, divisibility of, 12.

estimation of, in feces, 45.

formation of, 12, 18, 21, 34 .

excessive, 151, 159.

increase of, 55.

losses and accumulations of, 58 .

mal-assimilation of, 46 .

nausea caused by, 167 .

necessity of, as a food, 166.

repugnance to, 40 .

satiety caused by, 167.

significance of, 54 .

splitting of, 46 .

sugar formation from, 52 .

tables of, in various foods, 103.

place of, in diet, 21,74 .

of the diabetic, 89 .

value of, in diabetes, 100 .

in various diseases, 55 .

Fat of normal children, 153.

Fat absorption, decrease of, 46 .

Fat-albumin diet, 175.

Fat bodies, poisoning by, 591 .

Fat marrow changes in pernicious anemia, 316.

Fat metabolism, 75 .

Fat-milk, Gärtner's, 486.

Fats, neutral, 46.

Fatigue, sensation of, in anemia, 308 .

Fattening of animals, 153.

Fatty degeneration in progressive pernicious anemia, 315.

Features, change of, in myxedema, 184.

Feces, albumin losses in, 47.

analysis of, 45 .

calory value of, 44 .

elementary analysis of, 4 .

estimation of, 6 .

fatty acids in, 46 .

mieroscopic examination of, 522

parasites in, 507.

Feeding forced, 16, 19, 40, 56.

Female breast in obesity, 151.

Females, hemophilia occurring in, 414.

Fermentation test, 262, 264.

Ferratin, 342.

Ferrum carbonicum, 341.

Ferrum chloratum, 341 .

Ferrum citricum, 341.

Ferrum oxydatum, 341.

Ferrum sesquichloratum, 341 .

Ferrum sulphuricum, 341.
Fever, chronic relapsing, 375 .

destruction of tissue in, 39 .

gastric, 80.

intermittent, 494.

recurring, glandular, 375 .

relapsing, pre-natal, transmission of, 429 .

with glandular enlargement, 376 .

Filaria Bancrofti, Cobbold, 546.

embryos, 547.

loa Guyot, 546 .

Magathæsi, 547.

medinensis Velsch, 545.

varieties of, 546 .

Fingers, gout in, 138.

nodosities of, 255 .

Finland, hemophilia in, 451.

Fischer's test, 77.

Fish in diabetes, 108.

Fish, raw, parasitic infection from, 512.

Fish poisoning, 600.

Fixation, methylic alcohol, 293.

Flagellates, 507.

Flat-foot occurring in the gouty, 144, 158 .

Flat-worms, 508.

Fleischl's hemometer, 277, 278.

Flesh, increase of, 55.

Flesh production by stock raisers, 19 .

Fluid in blood, increase of, 341 .

Fluids, digestive, in metabolism, 4.

intake of, limited in diabetes, 124.

in obesity, 174.

simultaneous intake of, with solid food, 75.

Fly, as carrier of infection, 571.

Fly larvæ, 501, 573.

in remote parts of body, 574 .

Follicles, destruction of, 186 .

Food, albuminous, reduction of, 97 . assimilation of, 45 .

changes in, ill effects of, 333 .

consumption of, in the healthy, 4 .

in tubercular disease, 38 .

contamination of, by flies, 575 .

decomposition of, 3,49 .

diminished consumption of, 32 .

energy value of, 37 .

faulty absorption of, 44 .

fresh, as preventive of scurvy, 485 .

indigestible, craving for, 323.

intake of, influence of, 23.

limitation of, in obesity, 154 .

nature and quantity of, 21. 
Food, necessity of, for maintenance, 59 . oxidation of, 3. parasites disseminated by, 511 . passage of tape-worm caused by, 522 . repugnance for, 323 .

source of energy in, 2. sudden withdrawal of, 31 . unclean, as disseminator of parasites, 503. uniform limitation of, 174.

Food molecule, as containing nitrogen, 51.

Food products, disintegration of, 2 . divisibility of, 12 .

plastic, 2. respiratory, 2.

Food requirement of the sick, 21.

Food surplus, 61.

Food values, compilative table of, 14 . standard, 8.

Foods, kinds of, for the obese, 167. percentage of fat in, 103. sugar-producing, 89.

Foot, gout in, 125.

Force, in living organism, 2.

Force, interchange of, $22,37$. permutation of, 2.

Forced feeding, a cause of cardiac weakness, 65 .

in hysteria and neurasthenia, 65, 66 .

in production of flesh, 58 . indications for, 62. technic of, 70.

Fowler's solution in Addison's disease, 224. in morbus maculosus, 497.

Fractures, treatment of, by thyreoid gland, 195.

Freiburg, prevalence of goiter in, 190.

Friction as source of heat, 2. in chlorosis, 340.

Frontal sinus, echinococcus in, 535 .

Fruits, 108.

carbohydrate contents of, 105.

dried, in diabetes, 99.

in diabetes, 99.

pentosan in, 268.

\section{G}

Gadfly boil, 573.

Gaertner's hemophotograph, 279.

Galactose, 267.

Ganglia, semilunar, inflammation and degeneration of, 216.
Gangrene, of extremity, from èrgotism, 599.

Garrod's views of scurvy, 398, 410.

Gases, interchange of, 30, 36, 308 .

interchange of, in obesity, 154.

respiratory, 8.

poisoning by, 590 .

Gastric chemism, disturbance of, 47 .

Gastric contents, examization of, 323, 340 .

Gastric dyspepsia in gout, 140.

Gastric hunger, 60.

Gastric juice, action of, on echinococci, 526.

Gastrophilus larva, 574.

Gastroptosis, 323, 333.

Gelatin to control hemorrhage, 454.

Genital organs, echinococcus in, 543.

examination of, 337 .

hypoplastic changes in, 330 .

implication of, in acromegalia, 238.

German marine, scurvy in, 412.

Germany, parasitic disease in, 529 .

Ghida, 125.

Giant growth, 195, 230.

Giant skull, pathologic, 229.

Giants, prehistoric, 229.

Ciicht. See Gout.

Gigantism, 234, 237.

Gigantoblasts, 300 .

Gigantocytes, 299, 300.

Gingivitis, bacillus causing, 394 .

Girls, neuroses appearing in, 336.

Glands, enlargement of, 371.

extirpation of, 380 .

intestinal atrophy of, 47 .

pain in, 376.

tuberculosis of, 375,376 .

Glandular disease caused by poison in blood, 378.

predisposition to, 378 .

Glandular organs, in scurvy, 400.

Glaucoma in gout, 143.

Gliomata, 239.

Glucose, 77.

Glycogen deposit, 12, 16.

Glycogenic area of brain, 114.

Glycogenic center, 111.

Glycosuria, 77.

alimentary, $77,78,79$.

in gout, 144 .

combination of, with pentosuria, 269.

diabetic, 87.

e saccharo, 79.

experimental, 79 . 
Glycosuria, nature of processes in, 78 . physiologic, 77.

spontar.eous, 79,81 .

transitory, 81.

Glycosuria in morphin poisoning, 596 .

Gnathostoma siamense, Levinsen, 560.

Goiter, occurrence of, in infants, 190. massive, 238.

parenchymatous, 192.

Gonagra, 125.

Gonorrhea, 256, 478. gouty, 142.

Gordius, varieties of, 565 .

Gout, 125.

annual attack of, 139 .

cachectic stage of, 149 .

cancer, caused by, 143 .

causes of, 126, 127.

cerebral affections in, 142.

clinical cases of, 128, 134 .

clinical history of, 126.

combined with hematuria, 451 .

condition of heart in, 141.

confounding of, with rheumatism, 243.

course of, 145.

curative resorts for, 148 .

decreased metabolism in, 33 .

diagnosis of, 146, 150 .

diet in, 147.

edema in, 134.

exemption of women from, 147.

faulty action of kidney a cause of, 128 .

fever in, 134.

foci of, 133.

Garrod's views of, 136, 243.

Heberden's nodes in, 248.

hereditary, 128, 144.

intermediate symptoms of, 133, 139.

"internal," 145.

larval, 132, 149.

latent, 132.

location of, in extremities, 131, 145. in right toe, 128.

lymphangitis in, 143.

materia peccaus in, 132 .

metabolism in, 42.

occurrence of, in adolescents, 129 .

in ancient times, 126.

in England, 130, 131.

in Munich, 130.

in upper and lower classes, 130.

pain in, 134 .
Gout, paralysis in, 143.

paroxysms of, 136.

pathogenesis of, 126.

predisposition to, 139 .

premonitions of, 133,139 .

primary arthritic, 127, 138, 140.

treatment of, 146.

prognosis of, 145,150 .

prophylaxis of, 147.

relation of, to chronic arthritis, 255 .

respiratory conditions in, 142 .

retrocedent, 132.

skin affections in, 143.

specific treatment of, 148.

Sydenham's description of, 126.

symptomatology of, 132, 133, 139 .

treatment of, 146, 148, 150.

by "patience and cotton," 149.

typical attack of, 131, 133.

visceral, 140.

Gowers's hemoglobinometer, 277, 279.

Gram-Weigert process, 490.

Grandidier's views of hemophilia, 415, 452.

Granular cells of epithelium, 239.

Grapes in diabetes, 99.

Grape cure, 148.

Grape-sugar, 12, 264, 270.

in the urine, $77,78,262$.

Grasshopper plague in Russia, 397.

Graves' disease, 28, 29, 38, 70, 193.

forced feeding in, 65 .

Growth, excessive, in acromegalia, 230.

Gruber-Widal reaction, 289.

Gull, Sir Wm., on myxedema, 179.

Gummata of skin in diabetes, 121.

Gums, disease of, 413 .

fetor of, 492.

gangrene of, 403 .

in purpura, 459,470 .

lead line of, 588,590 .

scorbutic, 485 .

spongy, 394 .

the seat of scurvy, 402 .

Gymnastics, 67, 176.

in obesity, 72 .

\section{$\mathrm{H}$}

Hair in acromegalia, 237.

in scurvy, 405.

Haller's acid, 497.

in hemophilia, 453 . 
Hammerschlag's method for testing specific gravity of the blood, 284.

Hardening processes, 257.

Harvey's cure of obesity, 70, 72, 163, 165.

Hayem's solution, 281.

Head, weight of, in acromegalia, 235.

Health, calory requirement in, 35. consumption of food in, 4 . energy-value of food in, 45.

Health resorts, advantages of, 339 . of Europe, 343.

Heart and vascular system in gout, 141 .

Heart, defective development of, 329,331 . dilatation of, 158 . in chlorosis, 322 .

failure of, 75 .

fatty, 152, 174 .

fatty degeneration of, from phosphorus poisoning, 585.

hypertrophy of, 121.

hypoplasia of, 329.

weakness of, in obesity, 176.

Heart muscle, degeneration of, 330 . disease of, 75 .

Heart, hypoplasia of, 329 .

Heat, independent sources of, 2. loss of, in metabolism, 25. production of, in metabolism, 36 .

Heat and cold in metabolism of the sick, 25.

Heat equivalents, 44.

Hebeloma poisoning, 600 .

Heberden's nodes, 247, 248.

Helminthiasis, 504, 522. distressing symptoms of, 524 . prognosis of, 550 .

Hematoblasts, 345 .

Hematogen, 342.

Hematokrit, 287.

Hematology, 304, 354.

Hematomata, 491. diffuse, 437 .

Hematopoietic apparatus of chlorotics, 329 .

Hematopoietic function, 435.

Hematopoietic organs, 341 . affection of, 305.

Hematopoietic tissues, 363 .

Hematuria, angioneurotic, 449. caused by parasitic disease, 513 . essential, 448, 449.

gouty, 451.

hemophilic, 449.

idiopathic renal, 444 .
Hematuria, in leukemia, 353.

in pregnancy, 450 .

unilateral, 446, 447.

Hemeralopia, 419.

transmission of, 419.

Hemoalkalimetric test of alkalinity of the blood, 399.

Hemocytometer, 282.

Hemoglobin, 277.

absorption spectra of, 288 .

consumption of, 305.

formation of, 305 .

percentage of, in anemia, 306, 312 .

in purpuric blood, 495.

reduction of, $288,305,306,326,327,349$.

in chlorosis, 325,333 .

remedies containing, 342 .

Hemoglobin scale, 277.

Hemoglobinemia, 288.

Hemoglobinometer, 277, 325.

Hemometer, 277, 279.

Hemophilia, 413, 445, 446.

amount of blood loss in, 426 .

autopsy findings in, 430, 433 .

appearance of, after trauma, 429 .

blood examination in, 423, 424.

blood-vessels unruptured in, 431 .

causes of, unknown. 430 .

congenital, 421, 428, 429.

course of, 434 .

development of, by intermarriage, 421 .

diagnosis of, 440 .

discovery of, at operations, 434 .

geographical distribution of, 422 .

hereditary, 414, 415, 416, 417.

human, 429.

identity of, with scurvy, 427 .

joints in, 432, 439, 455 .

local, 448.

origin of, 425 .

prognosis of, 451.

prophylaxis of, 452,455 .

renal, 442.

revealed by accident, 434 .

seat of hemorrhage in, 435 .

stages of, 438 .

statistics of, 415 .

symptomatology of, 434 .

three forms of, 440 .

transmission of, in families by females, 414-417.

treatment of, 452,453 . 
Hemophilia as independent disease, 427. as toxic infectious disease, 427,428 .

Hemophiliacs, capricious appetite of, 428 . care of, in childhood, 453.

choice of occupation for, 453 .

diet of, 453.

early death of, 430,434 .

longevity of, 416 .

remarkable blood finding in, 425 .

Hemophotograph, 279, 280.

Hemoptysis in echinococeus disease, 537.

Hemorrhage, arrest of, 454.

arthritic, 435, 441.

conjunctival, 429 .

control of, by zymo-plasma, 424 .

cutaneous, 409, 466, 471, 476.

difficult control of, 437 .

embolic, 477.

essential renal, 445, 447.

gastric, 451.

internal, 336, 494.

interstitial, 435, 436, 437.

leopard appearance of skin caused by, 466.

multiple capillary, 465.

occurrence of, after extraction of teeth, $452,454$.

in hemophilia, 423.

in progressive, pernicious anemia, 314 .

in scurvy, 399, 401, 404, 409.

without external cause, 436.

prodromes of, 435 .

pulmonary, $451,494$.

renal, $444,447,450,451$.

retinal, 308, 314, 478, 488, 494.

spontaneous, 414, 429, 430, 436 .

superficial, $435,436$.

toxic, 429 .

traumatic, 414, 429, 436, 437.

treatment of, 497 .

Hemorrhages, local, upon a hemophilic basis, 442 .

striæ-like, 472 .

Hemorrhagic diathesis, 388, 477.

of leukemics, 353, 355 .

of the newborn, 461.

Hemosodium-urate, 127.

Hemosporidia, 506.

Henoch's purpura, 462, 479, 481, 482, 495.

Hereditary transmission of disease, 415, 416, 417.

Herpétisme, 252, 255.
Heterogeny, 545.

Hexathyridium, 514.

"Hexenschuss," 139.

Hexose, oxidation of, 265.

Hippocrates, deseription of peliosis by, 473 . treatment of obesity by, 152.

Hirschfeld on antifat cures, 56 .

Hirschfeld's diet rules, 174.

Hirudines, 565.

History of gout, 126.

of seurvy, 388.

Hodgkin's disease, 370.

clinical picture of, 372 .

course, duration, and outcome of, 378 .

diagnosis of, 375 .

hereditary predisposition to, 377 .

local symptoms of, 372 .

therapy of, 378 .

Hoppe-Seyler-Tigerstedt apparatus, 8 .

Host, parasitic, autoinfection of, 551 .

Hot-air bath, influence in metabolism, 25.

House-fly, as disseminator of parasites, 503 .

Hunger cure, 161, 175.

Hurry of life, a cause of chlorosis, 333 .

Hydatid echinococcus, 528.

resonance, 533 .

thrill, 532,540 .

Hydrochloric acid, action on pentose, 265.

Hydrogen, arseniureted, poisoning by, 587 . sulphureted, poisoning by, 586 .

Hydronephrosis, 541.

Hydrops tuberculosis fibrinosus, 439.

Hydrotherapy in diabetes, 122.

in obesity, 175, 176.

modern, 310.

Hygiene, errors in, 306.

general, 331.

ignorance of principles of, 343

lack of, among earth workers, 562 .

as cause of chlorosis, 333 .

observance of, in Hodgkin's disease, 380 .

Hymenolepis (drepanitotænia) lanceolata, Bloch, 519.

Hyperacidity of stomach, 47.

Hypercompensation, 88.

Hyperemia, 258.

Hyperglobulia, 283.

Hyperglycemia, diabetic, 78, 86.

Hyperglycosuria, 78.

Hyperhidrosis, 251.

Hyperleukocytosis, 302, 308.

Hypernutrition. See Over-nutrition. 
Hyperplasia, acromegalic, 240.

altruistic, 241.

Hypertrophy of soft parts in acromegalia, 237.

Hypnosis, as cause of hysterical polyuria, 118.

Hypnotism, questionable use of, 122.

Hypochondria verminosa, 524.

Hypoderma bovis, 573 .

Hypodermoclysis, saline, 497.

Hypoleukocytosis, 302 .

Hyponutrition. See Under-nutrition.

Hypophysis, adenomata of, 240.

disease of, 195.

hypertrophy of, 195.

tumor of, 232, 233, 235.

Hypoplasia, inactivity, 331.

Hypoplasia of heart and vascular system, 329.

Hysteria, association of, with diabetes, 117. combination of, with chlorosis, 338 .

origin of, 335 .

polyuria in, 116, 118.

stigmata of, 117, 119.

Sydenham's views of, 340 .

symptom-complex of, 336 .

\section{I}

Ileus, causes of, 370 .

Immermann-Oertel theory of hemophilia, $427,437$.

Inactivity of intestines, intervals of, 9.

Inanition, 148. producing fever, 30 .

Infarcts, hemorrhagic, 401.

Infection, conveyance of, by food, 509 .

by mice, 571 .

by water-cress, 509 .

through wounds, 478.

leukemia caused by, 356 .

morbus maculosus caused by, 459,460 , 461.

Inflammation, pulmonary, 339.

Influenza, a cause of gout, 127.

combined with scurvy, 396.

occurrence of, in chlorotics, 339.

Infusoria, 506.

Ingesta and excreta compared, 6 .

Innervation apparatus, 205.

Inosite, 112.
Insane, muscular action in the, 44.

Insects, parasitic, 571

parasites propagated by, 503 .

sting of, 575 .

Insomnia, 324.

in trichinosis, 558.

Inspiratory furrow in liver, 540 .

Intermarriage, debilitation by, 253. influence of, among hemophiliacs, 415 , 421.

of relatives favoring disease, 452 .

Internal secretion, absence of, 199.

Intervertebral ganglia, disease of, 216.

Intestines, abnormal decomposition in, 335.

activity of, 323 .

autointoxication from, 335 .

paresis of, 219.

leukemic infiltration of, 351.

Intestinal canal, assimilation of food in, 45.

Intima, fatty degeneration of, 431 .

Intoxication, acid, 399, 581.

Inunctions, splenic, 386.

Iodin, ingestion of, 42 . poisoning by, 584 .

use of, in therapy of Hodgkin's disease, 379.

in therapy of leukemia, 368 .

Iodothyrin, 189.

Iridocyclitis, 252.

Iritis, 252.

Iron, food rich in, 309.

inorganic, 341.

use of, in anemia, 309.

in gout, 149 .

in morbus maculosus, 497 .

Iron chlorid, action of, on adrenals, 209.

in hemophilia, 453.

Iron springs of Europe, 343.

of Germany, 342, 343.

Iron therapy in chlorosis, 340,341 .

Ischiagra, 125.

Isodynamic action of carbohydrates and fats, 51 .

Isodynamics, law of, 5 .

Isolation in treatment of diabetes, 124.

Israel's case of acromegalia, 240.

Itch, eruption with, 565 .

Itching disease, 599.

Itching, in scabies, 567.

Ixodes ricinus Linne, 566. 


\section{$\mathrm{J}$}

Jaundice in phosphorus poisoning, 585.

Jaw, hyperplasia of, in acromegalia, 235, 236.

periosteum of, in scurvy, 403.

Jecorin, 209.

Joint, hemophilic, 432, 439, 455 .

Joint, of knee, puncture of, 455 .

Joints, affection of, secondary, 478.

disease of, types of, 244.

- effusions of, in scurvy, 399.

hemorrhages into, 429 .

in rheumatism, 244.

inflammation of; 125.

puncture of, 456 .

stiffness of, 247 .

swellings of, in purpura, 471.

symptoms of, in gout, 132, 136 .

tuberculosis of, 258.

\section{K}

Katherine of Arragon's salad, 398.

Keratitis from scabies, 567 .

Kidney, amyloid, simulating diabetes, 121. circulation of, 123.

coccidiosis of, 506 .

contracted, altered function of kidney in, 112.

differentiated from diabetes, 121 .

echinococcus of, 541 .

floating, 450 .

functional disturbance of, 128.

genuine contracted, 142.

increased excretion of water by, 111 .

insufficiency of, 132.

necrosis of, in gout, 142.

neoplasm of, 450.

normal, hemorrhage from, $446,447$.

parenchymatous inflammation of, 330 .

Kisch's cure for obesity, 177.

Knee, gouty attack in, 135.

stiff, from gout, 128.

Koch's theory of hemophilia, 428.

Kolb's views of purpura, 489.

König's views of hemophilia, 438 .

Koppe's method of blood examination, 287.

Kreatinin 112.

Kribbelkrankheit, 599.

Krug's experiments in feeding, 15, 17.
Kyphosis of acromegalia, 235. of shoulder and hip, 246.

L

L-arabinose, 264.

L-xylose, 264.

Lactose, 78.

Lactosuria, 77.

Lancereaux's views of rheumatism, 252.

Larrey, observations of, on obesity, 151.

Larynx, echinococcus of, 536 . gout in, 142.

Lavoisier's study of chemical processes, 2.

Law of the conservation of energy, 2, 53 . of the development of the surface, 5 . of the permutation of forces, 2 .

Lead acetate, in diabetes, 123.

Lead colic, 587.

Lead line, $588,590$.

Lead poisoning, 131, 587 .

Lecithin in adrenals, 209.

Lemon cure, of gout, 148.

of hemophilia, 453.

Leontiasis ossea, 236.

Leprosy, 571.

Leptus autumnalis, Shaw, 565.

Leucin, 209.

Leukanemia, 357, 362.

Leukemia, 345 .

acute, cutaneous hemorrhages in, 485 .

autopsy reports of, 359 .

blood-changes in, 348 .

blood examinations in, 358,360 .

causes of, 357 .

diagnosis of, $349,364,366$.

fatal course of, 367 .

fever in, 352 .

leukocyte, $350,354,355$.

local treatment of, 368 .

lymphatic, 303, 350, 351, 354, 362 .

myelogenous, 302, 350, 354, 356.

parasites found in, 356 .

pathogenesis of, 363 .

prognosis of, 367 .

proportions of blood-corpuscles in, 366 .

specific poison as cause of, 356 .

splenic-medullary, 354 .

symptoms of, 348 .

true, 378.

treatment of, 367.

varieties of, 349 . 
Leukocytes, basophilic, 347. condition of, in chlorotic blood, 326. large mononuclear, 297. mononuclear, 346, 356. morphology of, 349 . neutrophilic polynuclear, 346 . normal proportion of, 349 . polynuclear neutrophilic, 297. reduction of, 312 .

Leukocytosis, 301, 302, 303, 344, 347. Addison's disease accompanied by, 204. phenomenon of, 303. post hemorrhagic, 328 . trichinosis accompanied by, 557 . varieties of, 364 .

Leukopenia, 302, 385.

Leukorrhea from parasites, 551.

Levulose, 109.

Levulosuria, 77, 262.

Letzerich's views of purpura, 460 .

Leydenia gemmipara Schaudinn, 506.

Lice, of body, clothes, and head, 501, 571, 572.

Life insurance and obesity, 157, 160.

Life insurance and pentosuria, 271.

Life, mode of, as cause of disease, 333.

favoring chlorosis, 339 .

unhygienic, 332 .

process of, a mystery, 49 .

Ligamenta lata, echinococcus in, 543.

Lind's description of scurvy, 391.

Linguatula, 570.

Lip, hemorrhagic prominence of, 483.

Lipoma arborescens, 249.

Lipomatosis cordis, 152. universalis, 152, 154.

Liver, abscess of, 540 . alcoholic, 383.

cirrhosis of, 43,382 .

coagulation necrosis of, 489 .

condition of, in splenic pseudo-leukemia, 382.

echinococcus of, $530,531,534$.

enlargement of, 351 .

functional disturbance of, 140 .

Liver, as food, forbidden in diabetes, 99.

Liver-fluke, 508.

in various parts of body, 510 .

Living cell substance, 41.

Lithia, salts of, 257.

Lithium in gout, 148.

Löwit's researches in leukemia, 356 .
Longevity of the obese, 157.

Lumbago in gout, 146.

Luxury consumption, 10, 53.

Lungs, echinococcus of, 537. hemorrhagic edema in, 401. hypostatic congestion of, 401.

Lüthje's experiments, 16.

Lymph-formation, anomalies of, 335 .

Lymph=glands, adhesion of, 376. enlargement of, 351,370 . internal, enlargement of, 372 . lymphocyte production in. 348 . rupture of, 376 .

Lymph-vessels, echinococcus of. 539.

Lymphadenoid proliferations, 375 .

Lymphadenoma, 339.

Lymphangitis, 143.

Lymphatic trunks, displaced, 373.

Lymphemia, acute, 355 .

Lymphocytes, 293, 296, 302, 346, 382. increase of, 361.

Lymphocythemia, 382 .

Lymphocytosis, 365, 366.

Lymphomata, 384.

leukemic, 375 .

Lymphomatosis, 372.

Lympho-sarcomatosis, $375,377$.

Lye, poisoning by, 582 .

\section{M}

Macrocytes, 299.

Macroglossia in acromegalia, 236.

Maggots. See Fly-larvæ.

Magnus-Levy, experiments of, 23. researches of, in metabolism, 34 .

Maintenance of life, law of, $\mathbf{5}$. on low nutrition, 53.

Mal de sol, 599.

Malaria, blood examination in, 291. enlarged spleen in, 384.

melanotic discoloration from, 383.

Males, hemophilia in, 415 .

Malnutrition, 161.

Malperforant, 85.

Maltose, 262.

Maltosuria, 271.

Mange of animals, 567.

Marasmus in cocainism, 597.

Marriage, of chlorotic girls, 338. of hemophiliacs, 452. 
Massage, 66.

in chlorosis, $340,341$.

of spleen, 386.

Mast-cells, 298, 347, 355.

Meals, daily number of, 171.

Meat, compulsory inspection of, 533.

decomposed, poisoning from, 601 .

fresh, a preventive of scurvy, 395.

kind and amount of, in diet of diabetics, $99,108$.

of poisonous animals, 601 .

prevention of infection by, 525, 554 .

salt, as cause of scurvy, 397, 398, 411.

value of, in diet, 74 .

Mediastinum, echinococcus of, 538.

Medical Congress, Paris, 1900, 126, 127.

Medication in anemia, 309.

Medicine, Internal, Congress of, 56.

Medullin, 197.

Megaloblasts, 300, 311.

in anemia, 311.

Megalocytes, 299, 300.

Melanotic discoloration, 383.

Memory, failure of, in anemia, 314.

Men, chlorosis in, 329, 330. development of fat in, 159.

Meningitis, tubercular, in Addison's disease, 225.

Meningocele congenita, 428 .

Menopause, hematuria accompanying, 450. obesity, as caused by, 155 .

occurrence of rheumatism during, 245 .

use of oöphorin in, 155, 196, 197.

Menstruation, abnormalities of, 333 .

condition of, in chlorosis, 324 .

in hemophiliacs, 435.

disorders of, use of oöphorin in, 197.

Mental changes, in myxedema, 185.

Mentholglycuronic acid, 264.

Mercury, poisoning by, 587,589 .

Mesentery, echinococci of, 542 .

Metabolism, Caspari's investigations in, 51.

chemical processes of, 4 .

condition of, in children, 36 .

in chlorosis, 323 .

in gout, 42 .

in progressive pernicious anemia, 313 .

diagram of, 35 .

disturbance of, in myxedema, 185.

experiments in, of Kayser, 21.

from dynamic point of view, 49 .
Metabolism, hereditary weakness of, 82

increased, 192.

intermediary, 53.

Krug's experiments in, 61.

limited labor of, in diabetes, 96 .

modern laws of, 4 .

perversion of, 32 .

proportion of, to body surface, 5 .

quantitative analysis of disturbances of, $1,4,37$.

quantitative estimation of, 8 .

ratio of, in rest, 5 .

researches in, of Bischoff, 56.

of Jaquet, 35.

of Lüthje, 40, 41, 56 .

of Rubner, 36.

of Sonden, 36, 37 .

of Tigerstedt, 36,37 .

respiratory, $4,25$.

slowing of, 26,31 .

stimulation of, 343 .

sugar, 267.

total energy of, 22.

toxic products of, 207.

Metachromasia, 298.

Metallic salts, poisoning by, 587 .

Metalloids, poisoning by, 582, 583 .

Metals, alkaline, poisoning by, 582 .

Metamorphosis, direct, 545 .

Methemoglobin, 287.

Microblasts, 307.

Microcytes, 299, 326.

Miescher's tubes, 506 .

Migraine in gout, 143 .

Milk, condensed Swiss, for infants, 487.

Pasteurization of, 486 .

value of, for chlorotics, 341 .

in diet of diabetics, 100, 108.

in acid toxicosis, 581.

Milk-sugar, 78, 262.

Mineral spring cures, 148.

for Hodgkin's disease, 379 .

in rheumatism, 258.

Minute-kilo-values in metabolism, 34.

Mitchell, S. Weir, on treatment of neurasthenia and hysteria, 65.

Mode of life as cause of gout, 128.

Monkeys, experimental production of scurvy in, 394.

Monoarthritis, senile, 256.

Monosodium urate, 127.

Monostomum lentis, Nordmann, 514. 
Morbus maculosus Werlhofii, 414, 425, 457. autointoxication a cause of, 459 . autopsy report of, 484 . bacteriology of, 489,490 . composition of blood in, 494. confounding of, with typhus, 482. cutaneous hemorrhages accompanying, 464.

diagnosis of, 484 .

distinction of, from scurvy, 458.

etiology of, 459,477 .

febrile phenomena in, 494.

frequency of, in youth, 480 .

infectious, $459,460,461$.

intestinal symptoms in, 492, 493.

nervous shock a cause of, 468 .

occurrence of, after fright, 462.

pathological anatomy of, 487 .

predisposing cause of, 480 .

prognosis of, in children, 481.

prophylaxis of, 496 .

relapses of, 461.

special symptomatology of, 481, 490 .

stages of, 480.

temperature in, 472 .

treatment of, 496.

Morphin habitués, 271.

Morphin, poisoning, acute and chronic, 595 .

Morphinism, 271, 443.

Motor disturbances in chlorosis, 324.

Mountain climbing, 67, 72.

Mouse septicemia, 571 .

Mucous gland, tumor of, 238.

Mucous membrane, catarrh of, 140 . condition of, in scurvy, 400.

Mucous membrane of mouth, in Addison's disease, 201.

Mucous membrane of respiratory organs, 142.

Murmurs, anemic, 321.

functional, 321 .

hemic, 321 .

nun's, 322 .

organic, 321 .

venous, 322.

Muscides larvæ, 574.

Muscle, accumulation of, 55 . increase of, 57.

Muscle lameness, sympathetic, 556 .

Museles, activity of, 53 .

condition of; 54 .

electric contractility of, 558 .
Muscles, excision of, in trichinosis, 559.

free movement of, 331.

loosening of, by scurvy, 400 .

resemblance of, to smoked goose breast, 558.

swelling of, in trichinosis. 557.

work hypertrophy of, 57 .

Muscular labor, forced, 52.

Muscular power, source of, 15, 51 .

Muscular tension, effect of, 44.

Muscular tonus, 30.

Musculature, increase of, in acromegalia, 236.

trichinæ in, 554

Mushroom poisoning, 585, 600 .

Mussels, poisoning by, 600 .

Myelin, 209.

Myelocytes, 300, 347.

production of, 360 .

Myiosis, 573.

intestinalis, 574 .

Myopathy, progressive, 195.

Myositis, acute fibrous, 248.

Myxedema, 30, 179, 188.

abortive, 192.

autopsy reports of, 186 .

etiology of, 180 .

infantile, 187.

pathology of, 186.

relapses of, 193 .

sensations of cold in, 185.

substitution therapy in, 225.

symptoms of, 180 .

temperature in, 185.

therapy of, 188.

thyreoiodin in, 189, 193, 195.

“Myxodéme fruste," 191, 192.

\section{$\mathrm{N}$}

Nansen Expedition, absence of scurvy in, $393,411,412$.

Nasal cavity, inflammation of, 377.

Natural-Healer, 60.

Nature-cure institutions, 60.

Nausea caused by fat, 167 .

Neck, echinococcus in, 535 .

enlarged glands of, 371 .

Necrosis of jaw in scurvy, 403.

Needle baths, 257.

Nematoda, 544. 
Nernatodes, perforation of ulcers by, 549 .

Nephrectomy, 447. for renal hemophilia, 443 .

Nephritis, 142, 451.

chronic, 337.

differentiated from diabetes, 121.

in rheumatism, 252.

interstitial, 498.

metabolism in, 41, 42.

parenchymatous, 449.

in trichinosis, 558 .

unilateral, 445.

Nephrolithiasis, 449.

Nerve areas connected with adrenals, 222.

Nerve elements, 204.

Nervines in diabetes, 93.

Nervous symptoms in Addison's disease, 200, 211, 218.

in chlorosis, 324 .

Nervous system, central, 255. disturbances of, 313.

in anemia, 315 .

changes in, in Addison's disease, 215.

condition of, in acromegalia, 238 .

in gout, 142.

disease of, a cause of Addison's disease, 213.

simulating adrenal disease, 219 .

hereditary affection of, 426 .

Neuenahr, treatment at, 101.

Neuralgia, gouty, 143.

intercostal, 324 .

local pressure as cause of, 373 .

occurrence of, in chlorosis, 324 .

Neurasthenia, under-nutrition resulting in, 60.

value of fat in, 55 .

Neurasthenic conditions, 142.

Neuritis, in gout, 143.

Neuropathic constitution, 115.

Neuropathic predisposition, 441.

Neuroses, combination of, with corpulence, 69.

functional, 116.

general, 142.

Neurosis of vasomotors, 336 .

Neutral dyes, staining in, 297.

Neutrophiles, 355.

Neutrophilic leukocytosis, 365 .

Neutrose, 48.

Newcastle-on-Tyne, lead poisoning at, 131.

Nicotin poisoning, acute and chronic, 595.
Night-blindness, etiology of, 419 .

Nitro-benzol, poisoning by, 592 .

Nitrogen, elimination of, $12,42,43$.

estimations of, in feces, 45.

food substances free from, 71 .

retention of, 17 .

Nitrogen accumulation, 19.

Nitrogen-balance, 37 .

Nitrogen equilibrium, 7, 20, 29.

Nitrogen excretion in the urine, 11.

Nodules, caused by filariæ, 548 .

Nodules, gouty, 137, 138.

lymphatic, 352.

No-fat cure, 163, 165.

Noorden-Dapper on antifat cures, 56 .

Normal proportions of blood-corpuscles, 283, 284.

Normoblasts, 300, 307, 356 .

shower of, in blood after hemorrhage, 328.

Normocytes, 299.

Nose, copper, in gout, 143.

echinococcus in, 535 .

expulsion of oxyuris from, 551 .

parasitic disease of, 574 .

Nose bleed, 426 .

Nostalgia as cause of chlorosis, 333.

Nourishment during antifat cures, 56 .

Nuclein, atypical, 266.

food rich in, 137.

pancreatic, 267.

Nucleinic acid, 265, 267.

Nucleins, animal, 265.

Nucleo-proteids, 266.

Nursing, in morbus maculosus, 496.

of chlorotics, 340 .

Nutrition, condition of, 54 .

conducing to chlorosis, 332 .

general disturbance of, 206.

individual standard of, 55 .

laws of, 4 .

in carnivora, 4 .

medium state of, 55,59 .

optimum of, 66.

processes of, 50 .

rational therapy of, 1 .

Nutrition, in morbus maculosus, 496.

of convalescents, 31 .

of fever patients, 31 .

of the sick, 35.

Nutritive preparations, 70 .

Nutrose, 70. 
Nyctotherus faba, 508.

Nylander's test, 262.

Obesity, 151.

after menopause, 155 .

after pregnancy, 155.

alcohol, a cause of, 155.

association of, with gout and diabetes, 154.

Banting cure of, $70,72,73,74,163,164$, $165,166,173,175$.

calory requirement in, 35 .

clinical cases of, 171.

combination of, with gout, 171 .

complications of, 69, 156.

composition of urine in, 157.

constitutional, 35 .

cure of, by thyreoiodin, 194 .

dangers of rapid reduction of, 68 .

Debove's treatment of, 161.

degrees of, 68.

diagnosis of, 159.

diet in, 161.

economy of food material in, 34 .

effect of, on life insurance, 158.

effect of perspiration in, 26 .

etiology of, 62, 153.

example of, 151.

fluids unlimited in, 75 .

functional, 156.

gout, as caused by, 130 .

Harvey's cure of, 70, 72, 163, 165.

in ancient times, 151, 152.

influence of, upon feminine fertility, 152.

on mental powers, 159 .

metabolism in, 34 .

muscular exercise in, 173.

occurrence of, in convalescence, 155.

of adolescents, 154.

of eunuchs, 154.

of people of Munich, 155.

of the healthy, 68 .

over-nutrition a cause of, 57 .

plethoric, 62, 177.

predisposition to, 153, 154.

prognosis in, 157.

relation of, to gout, 144.

slow reduction of, 170 .

stages of, 155 .

thirst in, 170.
Obesity, treatment of, 160, 175, 176.

by depletion, 162 .

under-nutrition in cure of, 59.

value of exercise in, 173.

various methods of cure of, 70, 163 .

views of Ebstein and Oertel on reduction of, 56 .

Odor of scorbutic fluid and tissue, 403, 405.

Oertel obesity cure, 174, 175.

Oertel's views of hemophilia, 425 .

Oligæmia vera, 330.

Oligemia combined with chlorosis, 338 .

Oligochromemia, 304, 326, 385.

Oligocythemia, 185, 250, 325, 385.

Oligoplasmia, 328.

Omagra, 125.

Omentum, echinococcus of, 543 .

Onychia scorbutica, 405.

Oöphorin, use of, during the menopause, 155 , 196, 197.

for menstrual difficulties, 197.

Open air exercise, 67.

Ophthalmia, metastatic, 459.

Opium poisoning, acute and chronic, 595, 596.

Opium smokers of China, 589.

Opotherapy, 196.

Oral cavity, amebæ in, 505.

bacilli in, 378, 394, 395.

echinococcus in, 535 .

foul odor from, 403.

Orbit, echinococcus of, 535 .

Orcin test, 263, 272.

Ord's cases of myxedema, 179.

Organic diseases, 331 .

Organism, process of life in, a mystery, 49. waste in, 12.

Organotherapy, 189, 196, 224.

employment of, since antiquity, 196.

in acromegalia, 242.

in anemia, 319.

in leukemia, 368.

practice of, in China, 196.

review of, 197, 198.

Organs, blood-forming, 310.

Orthopedic treatment, 259.

Orthopnea, 352.

Osteoarthropathy, hypertrophic, 240.

Osteomyelitis, 257.

Ovarian extract, 155, 196, 197.

Ovarian therapy, 155, 196, 197.

Ovaries, echinococcus of, 543. 
Over-nutrition, 54

accumulation of fat in, 59 .

amount of food necessary for, 59 .

best method of, 70 .

combination of, with hard manual labor,

62.

with muscular exercise, 58.

consequences of, 61 .

in Basedow's disease, 65.

in chronic wasting diseases, 63 .

in diabetes, 64 .

in exophthalmic goiter, 64 .

in functional nervous diseases, 65 .

in pulmonary tuberculosis, 63 .

in syphilis, 63 .

indications for, 62,66 .

occurrence of, 61 .

technic of, 69 .

Oxalic acid toxicosis, 581 .

Oxidation, changes in, in disease, 43 .

deficient, in myxedema, 185.

degree of, 22 .

after muscular labor, 24 .

in fever, 30 .

diminished, 34 .

increase of, by hot water bath, 25 .

secondary, 91.

Oxybutyric acid in urine, 91.

Oxygen, consumption of, 8.

inhalations of, in leukemia, 368 .

intake of, 8.

treatment by, 497.

Oxyuriasis, diagnosis of, 552 .

treatment of, 552 .

Oxyuris host, 551.

vermicularis, Linné, 548, 550.

Oysters, poisoning by, 600 .

\section{$P$}

Pain in Addison's disease, 218.

in gout, 126.

Palate, gangrene of, 479 .

Pallor, general, in chlorosis, 336 .

Palpitation, cardiac, 322.

Pancreas, carcinoma of, 46.

condition of, in acromegalia, 238.

diseases of, as cause of diabetes, 82,84 .

echinococcus of, 541 .

extirpation of, producing diabetes, 81 .

relation of, to glycosuria, 196.
Pancreas proteid, 266.

Pancreatic juice, action of, 46 . occlusion of, 46,47 .

Panophthalmia, 535.

Parablasts, 417, 418.

Paracentesis, 43.

Paralyses in the obese, 69 .

Paralysis, gouty, 143.

Parasites, anemia caused by, 309 .

animal, distribution of, 502 .

sexual maturity of, 502 .

varieties of, 501.

asexual forms of, 502 .

blood-sucking, 562 .

development of, 502 .

intestinal, 39.

health not injured by, 521 .

occasional, 501, 575.

permanent, 501 .

tenacity of life of, 520 .

virulent form of, 574 .

Parasites of man, 499.

amebr, 505.

balantidia, 508 .

cilia, 508.

coccidia, 506.

distoma, 509.

flagella, 507.

helminthes, 508 .

infusoria, 507.

megastoma entericum, 507.

plathelminthes, 508.

protozoa, 505.

rhizopoda, 505.

trichomonades, 507.

vorticelli, 508 .

Parasitic disease, blood examination in, 275.

prevention of, 525 .

symptomatology of, 503, 509.

Parenchymatous injections, 386.

Parotid, echinococcus in, 535.

Passive exudation, 363.

Pediculus capitis, de Geer, 571.

Peliosis cachecticorum, 476.

gonorrhoica, 479 .

rheumatica, 409, 469, 473, 474, 492 .

autopsy findings of, 488 .

Bamberger's cases of, 476 .

with a typical course, 476.

Schoenleinii, 457.

Pellagra, 599. 
Pelvis, true, echinococcus of, 543.

Pentastoma constrictum, v. Siebold, 570. tænioides, Rudolphi, 570.

Pentose, 77, 262.

combustion of, 265.

dextro-rotary, 272.

in animals, 266.

Pentosuria, 77, 262.

alimentary and chronic, 268.

caused by cherries, plums, beer, 77 .

in morphin habitués, 271.

increase of, by starches, 264 .

prognosis of, 271.

relation of, to diabetes, 271 .

therapy of, 271.

typical cases of, 269 .

Pentosuria and life insurance, 272.

Peptones, calories in, 13.

Pericardium, echinococcus of, 539 . .

Peripheral joints, gout in, 136.

Peripheral nerves, degeneration of, 216.

Peristalsis, intestinal, 207.

Peritonitis, tubercular, 47.

Perityphlitis, examination of blood in, 303 .

Pernicious anemia, 39, 47, 305, 314, 382.

metabolism in, 33 .

parasites as cause of, 504 .

presence of tape-worms a cause of, 523 . toxic influence inducing, 316.

Perspiration, insensible, 26, 113, 184.

Perspiration, sensible, 26.

Pes varus congenitus, 428.

Petechiæe in scurvy, 404.

Pettenkofer respiratory apparatus, 154.

Peyer's patches, in purpura, 487.

Pharmacodynamics, 197.

Phenol poisoning, 593.

Phenylhydrazin test, 262, 263, 264.

Phenylosazone, 265.

Phlegmons of gout, 134.

Phloridzin poisoning, 83.

Phloroglucin test, 263, 265.

Phosphorus in dejecta, chemical proof of, 585.

Phosphorus, retention of, 18 .

Phosphorus poisoning, 584 .

chronic, 586.

Photography in case of acromegalics, 231.

Phthirius inguinalis, Redi, 572.

Physaloptera caucasica n. spr., 564.

Physical exertion, influence of, on metabolism, 24.
Physical remedies, 257.

Picric acid poisoning, 593.

Pierre Marie's study of acromegalia, 229.

Pigment in blood, 292.

Pigment, pathologic deposition of, 213.

Pigmentation of skin, 200.

Pilocarpin, in diabetes, 123.

Pimelosis, 152.

Piperazin in gout, 148.

Piperin in pseudo-leukemia, 386.

Pituitary body, 195.

in acromegalia, 238.

Placental area, diphtheria of, 478 .

Plagiomonas urinaria, 507.

Plasma, accumulation of, in blood, 335.

Plasmon, 48, 70.

Plaster of Paris, for hemophilic joints, 456.

Playfair-Mitchell treatment of neurasthenia, 58,65 .

Plerocercoides, 520.

Plethora, 341.

hemorrhage as caused by, 437 .

hydremic, 425 .

serosa, 121.

vera seu sanguinea, 156.

Pleura, ecchymoses upon, 557. echinococcus of, 536 .

inflammation of, 373 .

Pleural effusions, 373.

Plica polonica, 571.

Pliny on corpulence, 163.

Pliny's rules for obesity, 152.

Pneumonia, central, 291.

followed by purpura, 482 .

Poisoning, acid, 579, 581.

agaricus muscarius, 600 .

allialoid, 594.

anilin, 592 .

animal, 598.

antipyrin, 594 .

arnican, 598.

arsenical, 586.

atropin, 597.

balsam of copaiba, 594 .

barium, 583 .

bromin, 584

camphor, 594 .

carbolic acid, 593.

carbonic oxid, 590.

chloral hydrate, 584 .

chloroform, 584 . 
Poisoning, cocain, 597.

cornutin, 598.

digitalis, 597.

endogenous, 579.

ergot, 598.

ethyl alcohol, 591 .

exogenous, 579.

fish, 600 .

gas, 590 .

iodin, 584 .

lead, 587.

meat, 600 .

mercurial, 587, 589.

morphin, 595, 596.

mushroom, 585, 600 .

mussel, 600 .

nicotin, 595 .

nitro-benzol, 592.

opium, 595, 596.

oxalic acid, 581.

oyster, 600 .

phenol, 593.

phloridzin, 79 .

phosphorus, 584.

picric acid, 593.

prussic acid, 582.

salicylic acid, 593.

sulphonal, 591, 592.

sulphur, 586.

tetronal, 591, 592.

toad-stool, 599 .

trional, 591, 592.

vegetable, 598.

Poisoning caused by alkalies, 582 .

by antineuralgies and antipyretics, 592 .

by arseniureted hydrogen, 587 .

by caffein and thein, 595 .

by chlorin combinations, 583 .

by lye, 582 .

by metallic salts, 587 .

by metalloids, 582,583 .

by potassium chlorate, 583 .

Pocket spectroscope, 287.

Podagra, 125, 140.

Poilkilocytosis, 299, 306, 312, 326.

Polyuria, 111.

after trauma, 116.

hysterical, 116, 118 .

permanent, 115, 119.

pure primary, 117, 118.

transitory, 115.

underlying cause of, 122.
Polyarthritis, chronic, 253.

primary chronic progressive, 246.

sicca, 250.

villosa, 244.

Polychromatophilia, 326.

Polychromatophilic degeneration, 299, 307, 312.

Polycythemia, 185.

Polydipsia, 111.

Polynuclear leukocytosis, 365.

Polypionia infantum, 154.

Polyplasmia, 328.

Polysarcia, 155. adiposa, 152.

Pork, containing trichina, 553.

Portal vein, disease of, 383 .

Potassium, deficiency of, causing scurvy, 399.

acid vegetable, as preventive of scurvy, 410 .

Potassium bromid, in diabetes, 122.

Potassium carbonate, in potatoes, 398.

Potassium chlorate, 583.

Potassium soap, 379,380 .

Potatoes, as preventive of scurvy, 397, 398.

Predisposition to diabetes, 80, 94.

to gout, 126, 139.

to hemophilia, 414 .

to scurvy, $390,396$.

Pregnancy, diabetes insipidus occurring in, 120.

hematuria in, 450 .

hemorrhages of, 454 .

lactosuria in, 79.

psychical influences during, 421.

Priapism in leukemia, 352.

Pribram's statistics of rheumatism, 243, 244.

Priessnitz pack, 258.

Progressive pernicious anemia, 305, 310, $359,563$.

cardiac symptoms of, 313 .

course of, 317 .

distinction of, from various diseases, 318 .

fatality of, 317 .

prognosis of, 317 .

symptoms of, $311,313$.

Prophylaxis in diabetes, 93.

in hemophilia, 452.

Prostate gland, echinococci of, 542 .

Proteid metabolism, 11, 39.

Proteid requirement for the sick, 37. 
Proteids, identity of, 2.

Proteids in metabolism, 36.

Protein substances, 3.

Protoplasm, faulty composition of, 131 . general diseases of, 139, 154, 157. granular, 346.

production of, 40 .

Protozoa, 505.

Prurigo, 568.

Pruritis from oxyuris, 551.

Prussia, cattle disease in, 533.

Prussic acid, 582.

Pseudo-leukemia, 363, 370. clinical picture of, 377 .

lesions of, 371.

occurrence of, in children, 385 .

treatment of, 385.

Pseudo-leukemia lienalis, 371.

lymphatica, 371 .

lymphatico-lienalis, 371 .

Pseudoparasites, 501.

Psoriasis, 143, 253.

linguæ, 140.

Psychic functions in diabetes insipidus, 114.

Psychical anomalies in chlorosis, 324 . in diabetes insipidus, 117.

Psychical causes of chlorosis, 332 .

Psychical influences, symptoms of, 304.

Psychosis, acute, 591.

Ptomain poisoning, a cause of scurvy, 393, 394.

Puberty, chlorosis appearing at, 330 .

Pulmonary artery, murmur over, in chlorosis, 321.

Pulse, in anemia, 321.

Pulse, tension changes in, 121.

Purpura, changes in the blood causing, 463.

changes in vessels causing, 463 .

chilling of body a cause of, 468 .

clinical picture of, 469 .

distinction of, from various diseases, 458 .

experimental production of, 461, 462 .

Henoch's, 462, 479, 481, 482.

idiopathic, 489 .

mildest forms of, 471 .

relapsing, 468, 471, 492 .

remarkable case of, 483,484 .

spontaneous and primary, 467.

successive attacks of, 492 .

toxic forms of, 459.

varieties of, 457,469 .
Purpura, varieties of, abdominalis, 469, 479.

cachecticorum, 465.

dyspeptica, 469 .

fulminans, 467,481 .

gonorrhoica, 469, 492 .

hemorrhagica, 457, 469, 471, 472.

intestinalis, 471, 492 .

rheumatica, $457,465,469,471,473,492$.

scorbutica, 471.

simplex, 457, 467, 469 .

urticans, 457,473 .

Pyelitis, simulating diabetes, 121.

Pyemia from echinococcus, 532.

Pyrocatechin, 209.

\section{Q}

Quinin in leukemia, 368.

in pseudo-leukemia, 385 .

\section{$\mathrm{R}$}

Rachisagra, 125.

Rachitis, 459, 467, 485.

treatment of, by thyreoid gland, 195 .

Radioscopy, 191.

Rags, parasites in, 565.

Rail-brace apparatus, 456.

Rainey's tubes, 506.

Rape, attempted, causing morbus maculosus, 469.

Rats as source of trichina infection, 553.

Rectum, itching of, 551.

polypus of, 513 .

Refrigeration as cause of purpura, 468 .

Régime, antinervous, 340.

Relapsing fever, spirilli of, 291.

Renal diseases, associated with obesity, 69 .

Renal echinococcus, 533.

Renal epithelium, 449.

Renal gout, primary, 132.

Renal hematuria, essential, 445.

Renal hemophilia, 442.

cases of, 443 .

hereditary, 451.

Renal hemorrhage, hemophilic, treatment of, 444.

congestive, 450 .

forms of, 447 .

Renal inflammation combined with gout, 137. 
Renal irritation from acid toxicosis, 580.

Resorts for treatment of diabetes, 101. of gout, 148.

Respiration, difficult, in Hodgkin's disease, 373.

Respiration rate, acceleration of, 323 .

Respiratory apparatus, 7 . of Hoppe-Seyler-Tigerstedt, 8. of Zuntz and Geppert, 8.

Respiratory diseases in the obese, 69 .

Respiratory metabolism, 28, 34.

Respiratory organs, inflammation of, in gout, 142.

Respiratory passages, catarrh of, 352 .

Respiratory quotient, 8 .

Rest energy, 5.

Rest in bed for neurasthenia, 65 .

Rest in treatment of chlorosis, 340 .

Rest metabolism, 29.

Rest values in metabolism, 24, 27, 32 .

tables for, 27.

Restlessness, influence of, in metabolism, 44.

Rete Malpighii, pigmentation in, 213.

Retina, serous infiltrations of, 325 .

Retinal hemorrhages, 308, 314, 478, 488, 494.

Retinitis leukemica, 352.

Retinitis of Bright's disease, 467.

Retroperitoneal echinococci, 542.

Rhabditis, varieties of, 544 .

Rheumatism, 144.

acute, sweats of, 478 .

acute articular, 244, 252.

with atypical course, 478 .

articular, in a chorus girl, 248.

chronic, 244.

Rheumatism, chronic articular, 243, 246.

etiology of, 243.

joint implication in, 244.

pathology of, 248.

treatment of, 257.

combination of, with gout, 148.

gonorrheal, 475 .

internal treatment of, 257.

occurrence of, in hemophiliacs, $413,416$.

pseudo-articular, 256.

secondary chronic, 244.

simulation of, by trichinosis, 559 .

Rheumatisme articulaire chronique progressif, 244.

déformant, 244.

goutteux, diathésique, 244.
Rheurnatisme infectieux, 244.

noueux, 244.

Rheumatoid, acute, 256.

tuberculous, 256.

Rhinagra, 143.

Rickets and enlarged spleen, 384.

Robin's obesity cure, 164 .

Roborants in Hodgkin's disease, 381.

Roborat, 48, 70, 169.

Romanowsliy stains, 292, 296, 299, 307, 346.

Röntgen rays. See X-rays.

Rovsing, views of, on unilateral hematuria, 447.

Rowing, 72 .

Rubner's calculations in metabolism, 9. calorimetric investigations, 5 .

law of the conservation of energy, 2 .

law of the development of the surface, 5

\section{$\mathrm{S}$}

Salicylic acid poisoning, 593.

Salines in hemophilia, 453.

Saliva, increased in scurvy, 403.

Salkowski's case of pentosuria, 263.

discovery of pentosuria, 267.

Sanatoria, dietetics in, 71 .

influence of life in, 67 .

treatment of chlorosis in, 339 .

Sand-flea, disease caused by, 572 .

Santonin for expulsion of parasites, 550 .

for oxyuriasis, 552

Sarcomata, 239.

multiple, 433.

Sarcopsylla penetrans, 572 .

Sarcoptes hominis, 501. scabiei, Linné, 566.

Saturnine poisoning. See Lead.

Sausage poisoning, 601 .

Scabies, diagnosis and treatment of, 568 .

Scarlatina followed by purpura, 482 .

Scharbock, 389.

Sciatica in diabetes insipidus, 114.

Schmaltz's pycnometer, 284.

Schmaltz's test for specific gravity of the blood, 284.

Schmidt's fermentation test, 45.

Schmidt's test diet, 48.

Schönlein's disease, 473.

Scleroderma, 213.

Scrofula, 63. 
Scrotum, echinococci of, 544. excessive development of, 151.

Scurvy, 388, 485.

absence of, in Nansen Expedition, 393, 412.

American Collective Report on, 393, 404, 485.

as an infectious disease, 399 .

bacillus found in, 394 .

constitutional phenomena of, 402.

contraction of, by nurses, 395 .

diagnosis of, 409 .

etiology of, 393 .

experimental study of, 393 .

external signs of, 402, 409.

fatal outcome of, 410 .

faulty nutrition, a cause of, 397 .

frequency of, in Alaska, 392.

geographic distribution of, 391 .

general and special treatment of, 413 .

gums the chief seat of, 402 .

hereditary predisposition to, 396 .

Hirsch's tabulations of, 391 .

history of, 388 .

infantile, 393, 395, 400, 404.

no known pathogenic agent of, 393 .

occurrence of, among seamen, 411, 412.

among Tartars, 395.

among the wealthy, 397.

among United States troops, 392.

during siege of Ladysmith, 399.

during siege of Paris, 397.

in antiquity, 389.

in Russia, 395.

in the tropics, 392 .

pathological anatomy of, 399 .

predisposition to, 396 .

prevention of, in English marine, 411.

production of, in monkeys, 394.

prognosis of, 410 .

prophylaxis of, 395,410 .

rarity of severe forms of, 408 .

recovery from, 408.

symptomatology of, 401 .

treatment of, 410 .

Sea-baths, in anemia, 310.

Seamen, scurvy among, 411.

Sebaceous glands, parasites in, 570 .

Semilunar ganglia, cauterization of, 219. extirpation of, 219.

Seneca's views of gout, 147.

Sensuality awakened in the young, 332 .
Sepsis, myxedema followed by, 186. severe, hemorrhages in, 485.

Septicemia, conveyed by mice, 571 .

Serum of the blood, 285.

Serum, specific gravity of, 327 .

Servant girls, chlorosis among, 332 .

Sexual disturbances, 334 .

Sexual functions in chlorosis, 323.

Sexual organs, anomalies of, 334 .

Siderosis, 314.

Silbermann's views of purpura, 464 .

Silenus as type of obesity, 152, 156.

Siven, experiments of, 15.

Skalotyrbé, 389, 390.

Skin, affections of, in gout, 143.

in rheumatism, 252.

bleaching of, in Addison's disease, 201.

bronzing of, $212,220,226$.

changes of, in trichinosis, 557.

condition of, in gouty attack, 134.

in myxedema, 179, 184.

in rheumatism, 251 .

discoloration of, 201.

doughy appearance of, 320 .

edematous infiltration of, 320 .

faded-yellow, in anemia, 312 .

greenish, 320, 336.

hemorrhages of, 464 .

itching of, in diabetes, 85 .

lymphadenoids in, 375 .

marbling of, 483 .

metabolism stimulated from, 380 .

non-ulcerating nodes in, 352 .

pallid, in anemia, 320 .

pigmentation of, 218.

trophic processes of, 194.

uninjured, hemorrhage from, 426 .

Skull, changes of, in acromegalia, 235 .

Sleepiness in chlorosis, 324 .

Smallpox, hemorrhagic, 484.

Sodium-biurate, 127.

Sodium chlorid in diabetic urine, 112.

Sodium-quadriurate, 127.

Sodium urate, acid, 127.

Solar plexus, changes of, in Addison's disease, 216.

Soxhlet sterilizer, 486 .

Special sense, organs of, 325 .

Spectroscope, use of, 287.

Speech in myxedema, 185.

Spermin, 197.

Sphacelinic acid, poisoning by, 598 . 
Sphacelotoxin, 598.

Sphygmogenin, 210.

Spices, use of, in diabetes, 109.

Spinal cord, changes of, in Addison's disease, 216.

echinococcus of, 534 .

Spinal roots, neurotic processes in, 217.

Spirilli, relapsing fever, 571 .

Splanchnic nerve, degeneration of, 216.

Splanchnomegalia, 237.

Spleen, cold applications to, 386 .

echinococcus of, 541 .

enlargement of, $350,356,370,376,383$, 384.

idiopathic, 385 .

in anemia, 313.

in Hodgkin's disease, 372.

in pseudo-leukemia, 382 .

hyperplasia of, 363,383 .

lymph-cell formation in, 361 .

pappy softening of, 488 .

Splenalgia, 324.

Splenectomy, 386. in children, 386 .

Splenica, 371.

Spleno-lymphatica, 371 .

Spondylose rhizomélique, 246.

Spongiosa, 236.

Sporozoa, 506.

Sport, value of, in obesity, 176 .

Springs, bromin-containing, 380.

iodin, 380 .

sodium chlorid, 380 .

Sputum, gangrenous, 537.

Staining process, of Ehrlich, 293.

Stains, neutral, 346 .

triacid, 346.

universal, 295.

Starvation, 21.

cure of, 161, 162.

losses in, 10, 11.

observations in, 14.

Stasis, circulatory, 352 .

in disease of the heart, 48 .

uric acid, 132.

Statistics of gout, 130 .

Steinbacher's cure of obesity, 174 .

Stiffness of joints in rheumatism, 247.

Stippling, basophilic, 300.

Stomach, affection of, from gout, 140 .

atony and displacement of, 335 .

carcinoma of, 315 .
Stomach, hyperacidity of, 47.

lavage of, $581,597$.

in opium poisoning, 596.

leukemic infiltrations in, 351.

perforation of, 581 .

secretory activity of, 323 .

Stomatite ulcéreuse, 389 .

Stomatitis, aphthous and ulcerative, 403.

from arnica, 598 .

in children, $403,404$.

Stomatokaké, $389,390$.

Stools, fatty, 46.

rice-water, 586.

Strawberry cure, 147.

Styptics, 454.

Substitutiontherapy, 188, 193.

Suffocation, from glandular swelling, 373 .

Sugar, ingestion of, 78 .

forbidden in diabetes, 109 .

presence of, in urine, 44.

producers of, 78 .

Suggestion, as cause of polyuria, 118. effect of, 75 .

Suggillations, enormous, in purpura, 483, 491.

scorbutic, 405.

Suicide by phosphorus poisoning, 584 .

Sulphonal poisoning, 591, 592.

Sulphur, poisoning, 586 .

Sulphuric acid, in morbus maculosus, 497.

Sun baths of Celsus, 175.

Sunstroke, a cause of diabetes insipidus, 119.

Suprarenals. See also Adrenals.

Suprarenal extract, action of, 208.

on central nervous system, 208.

in animals, 224 .

vaso-constricting action of, 208.

Suprarenin, 210.

Surface, law of development of, 5 .

Surgery, danger of, in bleeders, 452 .

Sweat, exhaustive, 478.

reactive outbreak of, 341 .

Sweat apparatus, 258.

Sweat baths, 341 .

in diabetes, 123 .

Sweat formation, 113.

Sweating, 29.

profuse, 44.

in leukemia, 352 .

Sweetbreads, forbidden in diabetes, 99 .

Switzerland, prevalẹnce of goiter in, 190. 
Sydenham's views of gout, 147.

Sympathetic system, diseases of, 216, 218. disorders of, 324 .

neuritic disease of, 216 .

Symptom-complex of gout, 132 .

Syncope, in chlorosis, 324 .

Synovial fluid, non-purulent in rheumatism, 249.

Syphilis, association of, with gout, 145, 148. cerebral, 122.

diabetes as caused by, 120 .

enlarged spleen in, 384 .

hereditary, 384.

joint implication in, 145 .

of brain, 115.

over-nutrition in, 63 .

tertiary, 63.

Systolic murmurs, 321 .

Systolic thrill, 321.

\section{$\mathrm{T}$}

Tabes mesenterica, 47.

Table, comparative, of various diet schemes, 72.

of alcohol and carbohydrates in beer, 106.

of carbohydrates in fruits, 105.

in wines and spirits; 106.

of Dapper's diet scheme, 73 .

of energy metabolism per square meter of body surface, 5 .

of fatty foods and varieties of milk, 104.

of food values necessary for maintenance of $\mathrm{N}$-equilibrium, 14.

of percentage of carbohydrates in bread, flour, vegetables, 104

of fat in cheese, 104.

in eggs, 104.

in meat, 103.

in sausages and preserved meats, 104.

of rest values in different diseases, 27.

Tallqvist scale, 277.

Tamburini's views of acromegalia, 241 .

Tannin, in diabetes, 123.

Tape-worm, 514.

anemia as caused by, 318 .

food causing passage of, 523 .

presence of, without symptoms, 522 .

reflex symptoms caused by, 523 .

removal of, 525, 526 .

Tape-worm disease, prognosis of, 524 .

therapy of, 525 .
Tarantula, bite of, 575 .

Taste, anomalies of, 325 .

Technic of over-nutrition and under-nutrition, 69.

Teeth, extraction of, in bleeders, 452, 454 .

loosening of, 140, 485 .

in scurvy, 403.

tartar of, containing amebæ, 505.

Temperament, phlegmatic in the obese, 159.

Temperature in diabetes insipidus, 114.

in myxedema, 185.

in scurvy, 407.

in trichinosis, 556 .

Tendon sheaths, nodules of, 251.

Tendons, stretching of, 146.

Tenia africana, v. Linstow, 518. asiatica, v. Linstow, 518 .

confusa, Ward, 517.

crassicollis, 517.

cucumerina, Bloch, 516.

echinococcus, 526 .

transmitted by dog, 529, 530 .

flavopunctata, Weinland, 516.

hominis n. spr., 519.

madagascariensis, Davaine, 517.

marginata, 517.

nana, v. Siebold, 516.

saginata, 501, 514, 515 .

serrata, 517.

solium, 514 .

characteristics of, 514 .

prevalence of, in Germany, 502.

Teniaphobia, 524.

Tension, specific energy of, 5 .

Tension energy, consumption of, 53.

Tension power, calculation of, 37 .

Tetanus, thyreoid therapy in, 195.

Tetronal poisoning, 591.

Therapy, dietetic, in anemia, 309.

of diabetes, 92 .

physical, in anemia, 309.

suprarenal, $225,226$.

Thermal baths, 148 .

Thirst, as symptom of diabetes, 113.

endurance of, in obesity, 170.

methods to relieve, 118 .

Thirst cure, 174.

Thoma-Zeiss apparatus, 281, 283.

Thoracic organs, lymphadenoids in, 375.

Thread-worms, 544 .

Thrombophlebitis suppurativa of pelvic veins, 478. 
Thrombosis, as cause of purpura, 462,463 , $464,465$.

Thumb, exemption of, in rheumatism, 245 .

Thymus gland in acromegalia, 238.

of hemophiliacs, 431.

Thymus preparations, 193.

Thyraden, 190.

Thyreo-antitoxin, 189.

Thyreoglobulin, 189.

Thyreoid cure of mental diseases, 195.

Thyreoid extract in myxedema, 180.

Thyreoid gland, absence of, 184.

administration of, 29.

atrophy of, 188.

degeneration of, 180.

echinococcus of, 536 .

enlargement of, in acromegalia, 232, 238.

grafting of, 187.

internal secretory activity of, 334 .

iodin contents of, 190.

strumous degeneration of, 186.

Thyreoid therapy, 188, 191.

toxic action of, 191.

Thyreoidectomy, 187.

in animals, 196.

Thyreoidin in obesity, 175, 176.

Thyreoiodin, 189, 193.

as cure for obesity, 194.

for skin affections, 194 .

Thyreoidism, 189, 192.

Ticks, 566.

Tissue, consumption of, in diabetes, 90 . intoxication necrosis of, 40.

Tissue-building substance, unknown, 19.

Tissue changes in myxedema, 185.

Tissue decomposition in diabetes, 91 .

Tissue destruction, 39 .

Tissue hunger, 60 .

Tissue irritation in gout, 143.

Tissue necrosis in gout, 133.

in scurvy, 400.

Tissue regeneration, 50.

Tissues, parablastic, 418.

Titration method of Liebig, 4.

Toad-stool poisoning, 599 .

Tobacco poisoning, 595 .

Toe, gout in, 136 .

Tongue, condition of, in Addison's disease, 201.

enlargement of, in acromegalia, 236 .

hyperplasia of, 236 .

inky stain of, 201.
Tongue, muscular degeneration of, 236.

Tonies in rheumatism, 257.

Tophi, gouty, 135, 137, 138, 146.

in bursæ mucosæ, 137.

Toxemia from presence of parasites, 523 .

Tracheo-bronchial lymph-glands, swelling of, 351 .

Tracheo-bronchial mucous membrane, inflammation of, 377.

Tracheotomy in opium poisoning, 596.

Training, athletic, 161. physical and mental, 331.

Transmission, pre-natal, of relapsing fever, 429.

Transactions XIIIth International Medical Congress, 126, 127.

Transudates, effect of, in metabolism, 44.

Transvaal, distoma infection in, 513 .

Trauma, as cause of diabetes, 115,119 .

Triacid solution for blood staining, 295 .

Trichina spiralis, 553.

Trichinæ, development of, 554 . in pork, 553 .

Trichinosis, autopsy reports of, 558 . distribution of, 553 .

epidemics of, 555 .

fever in, 556 .

mortality of, 558 .

muscle lameness in, 556 .

origin of, 553,554 .

prognosis of, 559 .

prophylaxis of, 559 .

symptoms of, 556 .

treatment of, 559.

Trichocephalus dispar, Rudolphi, 548, 552. diagnosis of, 553 .

Trichomonas, hominis, 507. vaginalis, Donné, 507.

Trional poisoning, 591, 592.

Trombidium holosericum, 565 .

Trommer's test, 77, 78, 262.

Trophic tracts in Addison's disease, 213.

Trophoneurosis, 142. general, 221.

Trophoneuroses, infectious, 256.

Tropon, 48, 70.

Tsetse fly disease, 571 .

Tubercular adenitis, 376 .

Tuberculoid, rheumatic, 256.

Tuberculosis, adrenal, 219.

chronic, 202.

destruction of adrenals in, 220 . 
Tuberculosis, intestinal, 47. mistaken for malaria, 293.

of glands, 375 .

pulmonary, $31,63,337$.

value of increased nutrition in, 60,63 .

Tumor, hypophysial, 239.

solid, 537.

splenic, direction of growth of, 350 .

Tumors in Hodgkin's disease, 372 .

Tunica vaginalis, echinococcus of, 544 .

Türck's method of blood counting, 282.

Turpentinglycuronic acid, 264.

Typhoid bacilli, 291.

Tyroglyphides, varieties of, 566 .

\section{U}

Uleers, gouty, 137.

of scurvy, 405.

Uncinariasis, 560 .

Uंnder-nutrition, 54, 67.

consequences of, 59 .

employment of, for the obese, 67 .

in diabetes, 91 .

indications for, 62 .

occurrence of, 59.

prolonged, 38.

systematic, 59.

technic of, 69.

when obesity is complicated by other diseases, 69 .

Urates, 137.

monosodium, 137.

Uratica, 125.

Urea as measure of metabolism, 4 .

excretion of, 42.

in diabetic urine, 112.

in leukemia, 353.

Urethra, distoma infection through, 513.

Uric acid as cause of gout, 126 .

Uric acid, deposit of, 255.

condition of, in diabetic urine, 112.

in Hodgkin's disease, 375 .

in leukemia, 127.

excretion of, 131, 136.

in tissues, 127.

percentage of, in gout, 137.

Uric acid engorgement, 149.

Uricemia, 128.

Urina spastica, 117, 119.

Urinary organs, condition of, in gout, 141, 142.
Urinary products, 44.

Urination, painful, from parasitic cause, 560 .

Urine, changes in, in obesity, 157.

condition of, in diabetes, 84 .

in diabetes insipidus, 64 .

in hemophilia, 426.

in leukemia, 353.

in myxedema, 185.

in nephritis, 41.

decrease of, in fevers, 112.

dextro-rotary, 268.

elementary analysis of, 4 .

estimation of excretion of, 6 .

glycose in, 269.

increased excretion of, 110 .

morning flood of, 112 .

of herbivora, 264.

optically inactive, 270.

parasites in, 507.

pentose in, 262.

reducing substances in, 269,270 .

sugar in, in diabetes, 84 .

Urohematoporphyrin, 212.

Urology, Fourth French Congress of, 448.

Urotropin in gout, 148.

Urticaria, 42, 143.

following echinococcus of liver, 531 .

Uterus, echinococcus of, 543 .

\section{V}

Vaccination of hemophiliacs, 434, 452.

Vagina, papilloma of, 513 .

Vagrant's disease, 203.

Valerian, in diabetes, 122, 123.

"Value" in blood counting, 283.

Value-estimation of blood, 283.

Valve, auriculoventricular, insufficiency of, 321.

Valvular disease, uncompensated, 48.

Vanillin poisoning, 601 .

Varices in the obese, 69.

Vascular apparatus, capacity of, in hemophilia, 425.

Vascular disturbances of the obese, 158.

Vascular formation, deficiency of, 431. retardation of, 431.

Vascular system, hypoplasia of, 330 . imperfect development of, 338. structure of, $422,423$.

Vasomotor nerves, anomalous function of, 335. 
Vasomotor tracts in Addison's disease, 213. Vasomotors, neurosis of, 336.

Vegetable acid potash, deficiency of, 393.

Vegetable-albumin preparations, 169.

Vegetable poisons, 598 .

Vegetable potassium, in various foods, 411.

Vegetables, fresh, as preventive of scurvy, 397.

in diet of diabetics, 99, 108, 122, 123.

of chlorotics, 341 .

leguminous, for the obese, 170 .

Veins, of neck, swelling of, 373.

pelvic, 478 .

spermatic, 478 .

Venesection, 341.

Venous thrombosis, 327.

Vermifuges, 525 .

Vertebra, chronic rigidity of, 248.

Vertebral column, ankylosing disease of, 246.

Vertigo in chlorosis, 324 .

in gout, 143.

Vesicles from filaria, 548 . miliarial, 491.

Vessels, dwarfed state of, 329 .

Vestibulum malorum, 378. pharyngis, 378.

Vibices, in scurvy, 404. of purpura, 491 .

Visceral complications in rheumatism, 251.

Vitality, increase of, 20.

Voit's calorimetric method, 6,7 .

experiments, 4.

law of metabolism and nutrition, 11, 50 .

Volkmann's arthritis deformans, 246.

Vomiting of proglottides, 522 .

v. Hoesslin on obesity, 175.

v. Kogerer's views of purpura, 464 .

\section{W}

Wadd's views on corpulence, 163.

Wasting diseases, 63, 250.

Water, allowance of, in diabetes, 98 .

cold, in treatment of rheumatism, 257.

demand of tissues for, 38.

excretion of, 17.
Water, loss of, in blood and tissues, 75.

precautions in use of, 548 .

use of, in the cure of obesity, 74 .

value of, in nutrition, 71 .

Water-cress, infection conveyed by, 509 .

Weakness, sensation of, in anemia, 308 .

Weight, experimental demonstrations of, 4 . in regard to obesity, 158.

loss of, 34 .

in diabetes, 84 .

normally heavy, 158 .

Weight of body, maintenance of, 37 . significance of, 38 .

Werlhof's disease, 457 .

White blood corpuscles, table of, 299 .

Widal's reaction, 290, 291.

Wine in dietary of diabetics, 100 .

Women, as conductors of hemophilia, 414 . diseases of, 155, 196, 197.

neuroses appearing in, 336 .

Working cell substance, 16 .

Worm, medina, 546 .

Wounds, fly larvæ in, 574 .

infection of, 478.

\section{$\mathrm{X}$}

$\mathrm{X}$-rays, burns from treatment by, 369, 381. examination by, of bleeder's joint, 432 . treatment of leukemia by, 354, 369 .

Xylose, 267.

\section{Y}

Yeast fermentation, 262.

Youth, chlorosis a disease of, 320,330 . gout occurring in, 128.

hemophilia appearing in, 415.

life energy in, 36.

\section{Z}

Ziemann's stain, 327.

Zipperlein. See Gout.

Zuntz's researches in metabolism, 24.

Zymoplastic substance, 424. 



\title{
PRACTICAL DIETETICS.
}

WITH SPECIAL REFERENCE TO DIET IN DISEASE.

\author{
By W. GILMAN THOMPSON, M.D.,
}

Professor of Medicine in the Cornell Medical College of the City of New York; Visiting

Physician to the Presbyterian and Bellevue Hospitals, New York.

\section{LARGE OCTAVO, EIGHT HUNDRED AND FOURTEEN PAGES, ILLUSTRATED.}

\author{
Cloth, $\$ 5.00$.
}

\section{Third Revised Edition. Sold only by Subscription.}

"This is at once the best and most exhaustive book upon this subject with which we are familiar. The best because, in the first place, it is written by a teacher of therapeutics who knows the needs of the practicing physician, and yet who has taught in previous years as a professor of physiology all that one needs to know in regard to the principles of digestion and assimilation. For this reason the author is unusually well qualified to prepare a useful manual, but it is not until one has perused the volume that he thoroughly grasps the scope and depth of the manner in which Dr. Thompson has treated his subject."

- Therapeutic Gazette.

"The subject of the dietetic treatment of disease is not an attractive one. This fact explains, no doubt, the comparatively little attention given it in college curriculum and daily study. When one proceeds to examine the valuable contributions to this subject, the scientific investigations which have been made by the United States Department of Agriculture at various experiment stations in all parts of the country, he is convinced that it is high time to look into the matter pretty thoroughly. This book is of value for its summary of the latter and its application of the knowledge to the treatment of the disease."

-Brooklyn Medical Fournal.

"The portion of the work on diet in the various diseases must prove of much value to the practitioner who has sought in vain in so-called 'practices of medicine' for exhaustive discussion of diets for special pathological conditions. In giving applicable diets few diseases are omitted, and the author not only gives his own views, but quotes those of others, especially with regard to such conditions as obesity, rheumatism, gout, and diabetes, in which proper feeding is of such paramount importance. The volume contains nine full-page plates."

-Medical Review of Reviews.

"In the seven years which have elapsed since the first appearance of this standard text-book there have not been the many and important changes in dietetics that other departments of our therapeutics have undergone. Nevertheless, Dr. Thompson has revised much that was published in the first edition, and has introduced into this one the results of more recent studies in the economic values of various dietaries. The general arrangement of the work is unchanged."-New York Medical Fournal.

D. APPLETON AND COMPANY, NEW YORK. 


\section{APPLETONS}

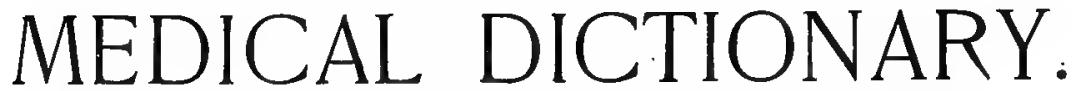

\section{A NEW ILLUSTRATED MEDICAL DICTIONARY.}

An Illustrated Dictionary of Medicine and Allied Subjects in which are given the derivation, accentuation, and definition of terms used throughout the entire field of medical science.

\section{Edited by Frank P. Foster, M.D.}

\section{Numerous Illustrations. One volume, half leather, $\$ 10.00$; thumb indexed, \$1 I.oo.}

"This volume is large, very large, nearly two thousand pages, but it is a single volume, and so its size can be forgiven. As a dictionary the work has many excellencies. The pronunciation is, as a rule, indicated only by accenting the titie words, the derivations are given briefly, and the French and German equivalents, and often the Greek, are inserted when they differ materially from the English. In regard to orthography, we are glad to see that Dr. Foster does not favor what he ralls the fad of substituting the termination 'ic' for 'ical,' dispensing with the final ' $\mathrm{e}$ ' in such terminations as 'ine' and 'ide,' and suppressing diphthonf $s$. The definitions are clear and concise and, so far as we have been able to discover, accurate. In fine, the only serious criticism of the work is its size, which might have been reduced by a careful pruning and rejection of obsolete words; but this counts for little in view of the convenience of arrangement, the accuracy of definition, the completeness, and the beauty of typography and binding."-New York Medical Record, September 24, I904.

"We see few dictionaries without errors or omissions ; in this book we have found none." -The Medical World, Philadelphia, Pa., November, Igo4.

"Probably the most complete and most exhaustive medical dictionary in existence. We lhave tried in vain to think of a word which might be absent in this ponderous volume of 2,000 pages. Everything relating to medicine, pharmacy, chemistry, botany, etc., is there and is explained clearly, concisely, and reliably."

-New York Critic and Guide, October, Iço4.

"It is accurate and up to date in most respects. . . . The work shows the result of careful revision, as the most recent words are to be found in it."

-The Fournal of the American Medical Association, Chicago, Ill., December 17, Igo4.

" This volume before us, of nearly 2,000 pages, seems more of a dictionary and less of an illustrated cyclopedia than almost any other upon the market. It represents conservatism in lexicography, and is prepared on strict lexicological lines. . . The definitions are excellent and can be heartily praised."

-Fohns Hopkins Hospital Bulletin, Baltimore, Md., January, Igo5.

"We have looked over the volume carefully, and have sought for definitions of newer subjects with interest, and in each instance we have not been disappointed. A number of colored plates, as well as ordinary black-and-white illustrations, are employed to illustrate the text. The plate upon malarial fever is an excellent one, and is far better executed than most plates of this character; indeed, it is the best that we have seen since the publication of Thayer and Hewetson's plates some years ago.

"Although the number of medical dictionaries on the market to-day is very great, there is always room for an A No. I book, and those that are already in existence will have to. look to their laurels if they do not wish to be pushed aside by this very notable addition to medical lexicography."-The Therapeutic Gazette.

"I find it a handy, comprehensive, and scholarly work. I have tested it by looking for useful terms recently introduced and thus far have not looked in vain, finding in each instance a succinct, clear, and accurate definition."-Solomon Solis-Cohen, M.D.

MAIL ORDERS PROMP LY ATTENDED TO.

D. A P Plet O N A N D COMPA N Y, Publishers,
436 Fifth Avenue, New York.




\title{
DISEASES OF THE HEART AND ARTERIAL SYSTEM
}

\author{
By ROBERT H. BABCOCK, A.M., M.D.
}

Professor of Clinical Medicine and Diseases of the Chest, College of Physicians and Surgeons (Medical Department of the Illinois State University),

Chicago; Attending Physician to Cook County Hospital for

Consumptives; Fellow and former President of the

American Climatological Association, etc.

Three Colored Plates and One Hundred and Thirty-nine Illustrations in the Text. '8vo. Cloth, $\$ 6.00$

\section{SECOND EDITION \\ SOLD ONLY BY SUBSCRIPTION}

"This treatise is evidently the result, not only of large clinical experience, but of wide reading and careful reflection. The author disclaims any pretense of originality, but it is open to question whether the conclusions and results of a ripe judgment, such as are presented in this volume, are not worth quite as much as some academic socalled original work. Certainly, a large amount of material, both clinical and literary, has been worked out and presented in a most clear, succinct, and practical manner. The author's style is pleasing and without ambiguity, nor is the text over-loaded with unnecessary technical terms. After a careful perusal of Dr. Babcock's book there are two features which strike the reader as characteristic and valuable. These are, on the one hand, the case histories, and, on the other, the unusual rumber and value of the pages devoted to the therapeutics of the subject. The narration of cases, although at times in much detail, does not cause the usual weariness of flesh in the reading, mainly because of the interesting manner in which they are written. They are particularly well chosen to illustrate the manifold varieties of disease and the practical wisdom required in the management of actual cases. Regarding the therapeutic side of the work none but words of praise are required. So far as the reviewer's reading goes there is no more complete and reliable exposition of the treatment of circulatory disease than that found in this volume. That this high commendation is deserved will readily be admitted after reading chapters 16,17 , and 18 upon the treatment of valvular heart disease. These chapters are distinguished by a fulness of detail and a variety of therapeutic resource which cannot but prove of great value, not only to the young practitioner, but, as well, to the clinician of years.

"The book can be unhesitatingly recommended as a distinct acquisition to one's working library."-Brooklyn Medical Journal.

"We commend to our readers this work of Babcock's as a very desirable work for both the specialist and the practitioner, and wish the author a most hearty welcome for his admirable literary endeavor."-Medical News.

D. APPLETON AND COMPANY, NEW YORK. 


\title{
OBSTETRICS.
}

\section{A TEXT-BOOK FOR THE USE OF STUDENTS AND PRACTITIONERS.}

\author{
BY J. WHITRIDGE WILLIAMS,
}

Professor of Obstetrics, Jolins Hopkins University; Obstetrician-in-Chief to the Johns Hopkins Hospital; Gynecologist to the Union Protestant Infirmary, Baltimore, Md.

\section{SIX HUNDRED AND THIRTY ILLUSTRATIONS IN THE TEXT AND EIGHT COLORED PLATES.}

\author{
Cloth, $\$ 6.00 ;$ Half Leather, $\$ 6.50$.
}

\section{SOLD ONLY BY SUBSCRIPTION.}

"The studies of the anatomy of the uterus and its adnexa are unique, and afford the student opportunity for attractive occupation in the acquirement of completer knowledge. The development of the ovum and placenta has never been set forth as well in a work on obstetrics, according to our view, as by Williams. The illustrations showing the structure of the placenta are admirable.

"In the section on obstetric surgery, beginning with induction of abortion and accouchement forcé, including forceps and version, Cesarean section and symphysiotomy, destructive operations, and ending with operative procedures which do not aim at a delivery, is found quite the most ample handling of operative obstetrics that has yet been published in such a treatise. Here, again, illustrations supplement the text in an instructive fashion.

"Williams's dealing with contracted and otherwise deformed pelves, and the management of labor in such conditions, is most satısfactory. Injuries of the birth canal, infection, hemorrhage, and the puerperium are all prepared by one who understands the greatest need of the student, and he tells him in the fewest and plainest words possible what he must know to obtain success in the practice of obstetrics. It is a book made by a clinician, which gives the most advanced exposition of the art and is a distinct addition to obstetric literature.

"Much original work has been done in the way of illustration, as well as in the preparation of the material of many chapters, and the whole subject-matter is presented in an original manner. The book is a credit to both author and publisher."-Buffalo Medical Journal.

"At a certain examination the question was asked: 'Describe the management of a face presentation in the M. D. P. position.' The examined men were like 'sheep before the shearers' $-i . e$., dumb. An investigation showed that the popular text-book made scant reference to the so-called 'undeliverable' position.

"Perhaps a knowledge of this fact led the reviewer of Dr. Whitridge Williams's work to consider, first, the description of the presentation in general, and, second, those of the face in particular. The admirable methods employed in explanation and the ample demonstrating drawings illustrating these fundamentals of obstetric knowledge show that the author is that rare combination, a teacher and a student of students and their requirements.

"Everything is detailed to the required minuteness, and beyond that nothing. The cuts and engravings are many, novel and good. They fill a definite purpose, and that purpose is not 'padding.' As one reads page after page the old-fashioned words, "Pleasure and profit," recur to the mind.

"It has all the earmarks of success; it is crowded with hints of practical value, and it shows what the trained hand and brain adopt as the best methods in overcoming the obstacles of parturition. Surely, if conscientious work and real merit count, we may expect to find this volume upon the lists of the colleges and treasured in private libraries."-New York State Journal of Medicine.

D. APPLETON AND COMPANY, NEW YORK.




\title{
CHEMICAL AND MICROSCOPICAL DIAGNOSIS
}

\author{
By FRANCIS CARTER WOOD, M.D.
}

Adjunct Professor of Clinical Pathology, College of Physicians and Surgeons, Columbia University, New York; Pathologist to St. Luke's Hospital, New York

With One Hundred and Eighty-eight Illustrations in the Text and Nine Colored Plates

8vo. Cloth, $\$ 5.00$ net

"No practitioner of medicine, whatever may be his special work, can afford to be without some book of this sort, and the present volume may frankly be recommended as satisfactory."

-New York Medical Journal.

"We regard this as one of the most important medical books that have appeared of late, and venture the opinion that it will not be long before it will be found in the hands of every laboratory worker, whether teacher or pupil."--Buffalo Medical Journal.

"The work at present before us may be unhesitatingly pronounced one of the best."-Medical Review of Reviews.

"It is unnecessary to emphasize, in the present state of medical knowledge, the importance of microscopical and chemical examinations of the blood and the secretions and excretions of the body in the diagnosis of disease. Indeed, this has been so universally appreciated within recent years that a distinct and increasing demand has been created for works of reference on these and allied subjects. Several such books already occupy honored positions in this literature, and Dr. Wood's book, the subject of this review, is the latest addition'to the library of clinical pathology. Dr. Wood's book is undoubtedly the most complete book of its kind that has 'appeared in the English language."

-California State Journal of Medicine.

D. APPLETON AND COMPANY, NEW YORK 


\title{
THE DIAGNOSTICS OF
} INTERNAL MEDICINE

\author{
A CLINICAL TREATISE UPON THE RECOGNIZED PRINCIPLES OF \\ MEDICAL DIAGNOSIS, PREPARED FOR THE USE OF \\ STUDENTS AND PRACTITIONERS OF MEDICINE
}

\section{By GLENTWORTH REEVE BUTLER, A. M., M. D. \\ Chief of the Second Medical Division, Methodist Episcopal Hospital ; Attending Physician to Departments of Diseases of the Chest and Diseases of Children St. Mary's Hospital, Brooklyn, N. Y. F Fellow of the New York Academy of Medicine; Member of the Medical Society of the County of Kings, etc.}

\section{New Revised Edition, Rewritten and Enlarged}

224 Illustrations, Five Plates, Twenty-two Charts. 8vo, 106 I pages Cloth, $\$ 6.00$

"This is a valuable book upon an important subject. The general plan of the work, the arrangement of subjects, the colored plates, the illustratiuns and diagrams, are alike excellent. The book as a whole is, consequently, a reliable guide for students and practitioners in this very important field of medical practice."- Gournal of Medicine and Science.

"Works on diagnosis are comparatively numerous at the present time, but each new one has its spec al features of interest and value. The present volume covers the greater portion of the field of internal medicine in a very satisfactory way, and undoubtedly will be found by many more acceptable than other works. It is particularly strong in its illustrations; these are generally very well selected to illustrate the text, which itself is clear and readable. It is well worth a place in the physician's library "-Fournal of the American Medical Association, Chicago.

"This volume presents some unusual features, and will find many readers who will appreciate the method by which the subject is treated. The great bulk of the work deals with the evidences of disease, and does so in a most satisfactory manner. The student is taught how to proceed in the examination of his patient, what routine questions are to be asked such as would lead to a preliminary opinion which in turn would suggest certain examinations in detail. The author teaches not only what to do, but how to do it, and his advice and method of diagnosis are to be highly recommended. The smaller details, which only acute observers notice, are pointed out and their significance is weighed. It will be of service as a reference book in suggesting explanations in cases where but one or few symptoms, not pathognomonic, are presented for diagnosis - for each symptom is explained and the ordinary diseases in which it occurs are mentioned, while in the latter part of the book the diseases are treated separately, so that direct suggestions can be immediately considered without conșulting another volume. All the modern laboratory methods are well described, the chapters on the blood and stomach contents being especially good, though brief. The thorough modernness of the instruction is well instanced by drawings of the culex and anopheles genera of mosquitoes. The author is evidently fully conversant with the clinical evidences of disease, for his treatment of the chapters on the signs and symptoms which present themselves in the patient is unusually well balanced with reference to their importance. The section on pain is very thorough; the illustrations are profuse and particularly instructive. "-Medical Record.

D. APPLETON AND COMPANY, NEW YORK 



